Supporting Information for "A model for calculating the density of aqueous electrolyte solutions", by Marc Laliberté and W. Edward Cooper

Part 1: Solutions of one electrolyte in Water

326 pages including this one 
Density of aqueous solutions of $\mathrm{AlCl} 3$

References (23) Dolian, F. E. The Viscosities of Solutions of Chlorides in Certain Solvents. J. Phys. Chem. 1937, 41, 1129-1138

(70) Mason, C. M., The activity and osmotic coefficient of trivalent metal chlorides in aqueous solution from vapor pressure measurements at $25^{\circ} \mathrm{C}, \mathrm{J} \mathrm{Am}$ Chem Soc, 1938, 60, 1638-1647

molality



0.05

0.1
0.2
0.3
0.4
0.5
0.6
0.7
0.8
0.9
1
1.1
1.2
1.3
1.4
1.5
1.6
1.8

$\begin{array}{rrrr}\mathbf{t} /{ }^{\circ} \mathrm{C} \text { mass frac } & \begin{array}{r}\text { Density } \\ \text { exp } /\end{array} & \text { Ref } \\ \mathrm{kg} / \mathrm{m}^{3} & \\ & & 999.5 & (23) \\ 25 & 0.0023 & 991.6 & (23) \\ 25 & 0.0047 & 1001.0 & (70) \\ 25 & 0.00662 & 1003.0 & \\ 25 & 0.0093 & 1005.9 & (23) \\ 25 & 0.01316 & 1008.8 & (70) \\ 25 & 0.02598 & 1020.0 & (70) \\ 25 & 0.03846 & 1031.0 & (70) \\ 25 & 0.05064 & 1041.6 & (70) \\ 25 & 0.06250 & 1052.3 & (70) \\ 25 & 0.07408 & 1063.0 & (70) \\ 25 & 0.08537 & 1073.9 & (70) \\ 25 & 0.09639 & 1084.5 & (70) \\ 25 & 0.10715 & 1094.3 & (70) \\ 25 & 0.11765 & 1104.8 & (70) \\ 25 & 0.12791 & 1114.7 & (70) \\ 25 & 0.13794 & 1124.5 & (70) \\ 25 & 0.14773 & 1134.1 & (70) \\ 25 & 0.15731 & 1143.7 & (70) \\ 25 & 0.16667 & 1153.2 & (70) \\ 25 & 0.17583 & 1162.5 & (70) \\ 25 & 0.19356 & 1181.2 & (70)\end{array}$

\section{App vol App vol} $\exp / \quad$ Calc
Density calc / App Vol Square of Inconsistent Inconsistent $\mathrm{kg} / \mathrm{m}^{3}$ Residual / Residual / Density based on based on $\mathrm{kg} / \mathrm{m}^{3} \quad \mathrm{~kg} / \mathrm{m}^{3} \quad \mathrm{~m}^{3} / \mathrm{kg}$ Residual density $\begin{gathered}\text { apparent } \\ \text { volume }\end{gathered}$

$\begin{array}{llllll}-0.000064 & 0.000131 & 999.04 & 0.4470 & -0.000195 & 0.200\end{array}$

$\begin{array}{llllll}0.000039 & 0.000132 & 1001.13 & 0.4382 & -0.000093 & 0.192\end{array}$

$\begin{array}{lllllll}0.000104 & 0.000133 & 1002.81 & 0.1920 & -0.000029 & 0.037\end{array}$

$\begin{array}{llllll}0.000059 & 0.000134 & 1005.15 & 0.7039 & -0.000075 & 0.496\end{array}$

$\begin{array}{llllll}0.000115 & 0.000135 & 1008.52 & 0.2755 & -0.000021 & 0.076\end{array}$

$\begin{array}{llllll}0.000134 & 0.000141 & 1019.82 & 0.1777 & -0.000007 & 0.032\end{array}$

$\begin{array}{llllll}0.000144 & 0.000145 & 1030.95 & 0.0531 & -0.000001 & 0.003\end{array}$

$\begin{array}{llllll}0.000156 & 0.000150 & 1041.91 & -0.3062 & 0.000006 & 0.094\end{array}$

$\begin{array}{llllll}0.000160 & 0.000154 & 1052.71 & -0.4075 & 0.000006 & 0.166\end{array}$

$\begin{array}{llllll}0.000163 & 0.000159 & 1063.36 & -0.3574 & 0.000004 & 0.128\end{array}$

$\begin{array}{llllll}0.000162 & 0.000163 & 1073.86 & 0.0382 & 0.000000 & 0.001\end{array}$

$\begin{array}{lllllll}0.000164 & 0.000166 & 1084.23 & 0.2736 & -0.000002 & 0.075\end{array}$

$\begin{array}{llllll}0.000171 & 0.000170 & 1094.46 & -0.1562 & 0.000001 & 0.024\end{array}$

$\begin{array}{lllllll}0.000172 & 0.000173 & 1104.56 & 0.2440 & -0.000002 & 0.060\end{array}$

$\begin{array}{lllllll}0.000175 & 0.000176 & 1114.53 & 0.1698 & -0.000001 & 0.029\end{array}$

$\begin{array}{lll}0.000179 & 0.000179 & 1124.38\end{array}$

$\begin{array}{lll}0.000183 & 0.000182 & 1134.12\end{array}$

$\begin{array}{lll}0.000185 & 0.000185 & 1143.74\end{array}$

$0.000188 \quad 0.000188 \quad 1153.25$

$\begin{array}{lll}0.000191 & 0.000190 & 1162.65\end{array}$

$0.000195 \quad 0.000195 \quad 1181.15$

Average Res

Std dev Res

Avg - 4std

Avg +4 st

$0.1170-0.000001 \quad 0.014$

$\begin{array}{lll}-0.0180 & 0.000000 & 0.000\end{array}$

$\begin{array}{lll}-0.0389 & 0.000000 & 0.002\end{array}$

$\begin{array}{lll}-0.0491 & 0.000000 & 0.002\end{array}$

$\begin{array}{lll}-0.1515 & 0.000001 & 0.023\end{array}$

$\begin{array}{lll}0.0548 & 0.000000 & 0.003\end{array}$

$0.0810-0.000019 \quad 1.6551$ Sum Chi sqr

$0.2755 \quad 0.000048$

$-1.0209-0.000210$

$1.1828 \quad 0.000172$

Density of

\section{Curve fit for the model App vol $=\left(w+c 2+c 3^{*} t{ }^{\circ} \mathrm{C}\right) /\left(c 0^{*} w+c 1\right) / e^{\wedge}\left(0.000001^{*}\left(t^{\circ} \mathrm{C}+c 4\right)^{\wedge} 2\right)$}

$\begin{array}{lr}\text { c0 } & 221.6811 \\ \text { c1 } & 160.896 \\ \text { c2 } & 0.151254 \\ \text { c3 } & 0.0025 \\ \text { c4 } & 1500 \\ \text { Min T } & 25 \\ \text { Max T } & 25 \\ \text { Min w } & 0.0023 \\ \text { Max w } & 0.193556 \\ \text { Avg dens res } & 0.080954\end{array}$


$\mathrm{t} /{ }^{\circ} \mathrm{C}$ mass frac

Density

$$
\exp /
$$

$\mathrm{kg} / \mathrm{m}^{3}$

Ref
App vol
$\exp /$
$\exp /$
$\mathrm{m}^{3} / \mathrm{kg}$
App vo
Calc /

$\mathrm{m}^{3} / \mathrm{kg}$

Dens

calc $/$
$\mathrm{kg} / \mathrm{m}^{3}$

Rensidu

$\mathrm{kg} / \mathrm{m}^{3}$

App

$\mathrm{m}^{3} / \mathrm{kg}$ Residual

\section{molality molarity \\ Std dens res \\ 0.275452 \\ Avg app vol res *10^3 -0.019434 \\ Std app vol res \\ 0.047748 \\ No of points in corr \\ 21
2}

Inconsistent

Inconsistent data not used (Residual greater than average +/- 4 standard deviations)

$$
\begin{array}{llllll}
25 & 0.0184 & 1015.4 & \text { (23) } & 0.000020 & 0.000138
\end{array}
$$

1015.4

(23)

$0.000037 \quad 0.000145$

1013.13

1028.83 
Density of aqueous solutions of Al2(SO4)3

References (18) Cupples, H. L. Surface Tension of Aluminium Sulfate Solutions. J. Phys. Chem. 1946, 50, $256-260$.

(90) Perry, R. H.; Green, D. W. Perry's Chemical Engineers' Handbook; McGraw Hill: New York, 7th edition, 1997.

(121) Silva, J. W.; Chenevey, J. E. Specific Gravity of Aluminum Sulfate Solutions. Ind. Eng. Chem. 1945, 37, 1016-1018.

\begin{tabular}{|c|c|c|c|c|c|c|c|c|c|c|c|c|c|c|}
\hline molality & molarity & \multicolumn{2}{|c|}{$\mathrm{t} /{ }^{\circ} \mathrm{C}$ mass frac } & $\begin{array}{r}\text { Density } \\
\exp / \\
\mathrm{kg} / \mathrm{m}^{3}\end{array}$ & Ref & $\begin{array}{r}\text { App vol } \\
\exp / \\
\mathrm{m}^{3} / \mathrm{kg}\end{array}$ & $\begin{array}{r}\text { App vol } \\
\text { Calc / } \\
\mathrm{m}^{3} / \mathrm{kg}\end{array}$ & $\begin{array}{r}\text { Density } \\
\text { calc / } \\
\mathrm{kg} / \mathrm{m}^{3}\end{array}$ & $\begin{array}{r}\text { Density } \\
\text { Residual / } \\
\mathrm{kg} / \mathrm{m}^{3}\end{array}$ & $\begin{array}{r}\text { App Vol } \\
\text { Residual / } \\
\mathrm{m}^{3} / \mathrm{kg}\end{array}$ & $\begin{array}{l}\text { Square of } \\
\text { Density } \\
\text { Residual }\end{array}$ & $\begin{array}{c}\text { Inconsistent } \\
\text { based on } \\
\text { density }\end{array}$ & $\begin{array}{c}\text { Inconsistent } \\
\text { based on } \\
\text { apparent } \\
\text { volume }\end{array}$ & $\begin{array}{r}\text { Density of } \\
\text { water } / \mathrm{kg} / \mathrm{m}^{3}\end{array}$ \\
\hline \multirow[t]{2}{*}{0.0287} & & 15 & 0.0097 & 1009.0 & (18) & -0.000009 & 0.000010 & 1008.81 & 0.1876 & -0.000019 & 0.035 & & & 999.0996 \\
\hline & & 15 & 0.0100 & 1009.3 & (90) & -0.000011 & 0.000010 & 1009.09 & 0.2098 & -0.000021 & 0.044 & & & 999.0996 \\
\hline \multirow[t]{3}{*}{0.0585} & & 15 & 0.0196 & 1019.1 & (18) & 0.000000 & 0.000012 & 1018.84 & 0.2564 & -0.000013 & 0.066 & & & 999.0996 \\
\hline & & 15 & 0.0200 & 1019.5 & (90) & -0.000001 & 0.000013 & 1019.23 & 0.2709 & -0.000013 & 0.073 & & & 999.0996 \\
\hline & & 15 & 0.0400 & 1040.4 & (90) & 0.000008 & 0.000018 & 1039.96 & 0.4429 & -0.000010 & 0.196 & & & 999.0996 \\
\hline 0.1218 & & 15 & 0.0400 & 1040.4 & (18) & 0.000008 & 0.000018 & 1039.96 & 0.4371 & -0.000010 & 0.191 & & & 999.0996 \\
\hline 0.1863 & & 15 & 0.0599 & 1061.7 & (18) & 0.000016 & 0.000023 & 1061.21 & 0.4920 & -0.000007 & 0.242 & & & 999.0996 \\
\hline \multirow[t]{2}{*}{0.2517} & & 15 & 0.0793 & 1082.9 & (18) & 0.000024 & 0.000029 & 1082.45 & 0.4469 & -0.000005 & 0.200 & & & 999.0996 \\
\hline & & 15 & 0.0800 & 1083.7 & (90) & 0.000024 & 0.000029 & 1083.24 & 0.4555 & -0.000005 & 0.207 & & & 999.0996 \\
\hline \multirow[t]{2}{*}{0.3242} & & 15 & 0.0998 & 1106.0 & (18) & 0.000032 & 0.000035 & 1105.64 & 0.3600 & -0.000003 & 0.130 & & & 999.0996 \\
\hline & & 15 & 0.1200 & 1129.3 & (90) & 0.000039 & 0.000041 & 1129.01 & 0.2934 & -0.000002 & 0.086 & & & 999.0996 \\
\hline \multirow[t]{2}{*}{0.4761} & & 15 & 0.1401 & 1153.0 & (18) & 0.000047 & 0.000048 & 1152.91 & 0.0944 & -0.000001 & 0.009 & & & 999.0996 \\
\hline & & 15 & 0.1600 & 1177.0 & (90) & 0.000055 & 0.000054 & 1177.23 & -0.2301 & 0.000001 & 0.053 & & & 999.0996 \\
\hline \multirow[t]{2}{*}{0.636} & & 15 & 0.1787 & 1200.0 & (18) & 0.000063 & 0.000061 & 1200.61 & -0.6110 & 0.000002 & 0.373 & & & 999.0996 \\
\hline & & 15 & 0.2000 & 1227.2 & (90) & 0.000071 & 0.000069 & 1227.81 & -0.6144 & 0.000002 & 0.378 & & & 999.0996 \\
\hline \multirow[t]{3}{*}{0.829} & & 15 & 0.2210 & 1254.7 & (18) & 0.000078 & 0.000077 & 1255.20 & -0.4961 & 0.000001 & 0.246 & & & 999.0996 \\
\hline & & 15 & 0.2400 & 1280.3 & (90) & 0.000085 & 0.000084 & 1280.53 & -0.2337 & 0.000001 & 0.055 & & & 999.0996 \\
\hline & & 15 & 0.2600 & 1307.9 & (90) & 0.000092 & 0.000093 & 1307.58 & 0.3236 & -0.000001 & 0.105 & & & 999.0996 \\
\hline 1.063 & & 15 & 0.2667 & 1317.2 & (18) & 0.000095 & 0.000096 & 1316.72 & 0.4813 & -0.000001 & 0.232 & & & 999.0996 \\
\hline 0.0287 & & 25 & 0.0097 & 1006.8 & (18) & 0.000004 & 0.000018 & 1006.65 & 0.1467 & -0.000015 & 0.022 & & & 997.0449 \\
\hline 0.0585 & & 25 & 0.0196 & 1016.8 & (18) & 0.000010 & 0.000021 & 1016.58 & 0.2234 & -0.000011 & 0.050 & & & 997.0449 \\
\hline 0.1218 & & 25 & 0.0400 & 1037.8 & (18) & 0.000018 & 0.000026 & 1037.47 & 0.3306 & -0.000008 & 0.109 & & & 997.0449 \\
\hline 0.1863 & & 25 & 0.0599 & 1058.8 & (18) & 0.000027 & 0.000031 & 1058.49 & 0.3121 & -0.000005 & 0.097 & & & 997.0449 \\
\hline 0.2517 & & 25 & 0.0793 & 1079.8 & (18) & 0.000034 & 0.000037 & 1079.51 & 0.2915 & -0.000003 & 0.085 & & & 997.0449 \\
\hline 0.3242 & & 25 & 0.0998 & 1102.5 & (18) & 0.000042 & 0.000043 & 1102.45 & 0.0463 & 0.000000 & 0.002 & & & 997.0449 \\
\hline 0.4761 & & 25 & 0.1401 & 1149.0 & (18) & 0.000056 & 0.000055 & 1149.24 & -0.2427 & 0.000001 & 0.059 & & & 997.0449 \\
\hline 0.636 & & 25 & 0.1787 & 1195.8 & (18) & 0.000070 & 0.000067 & 1196.50 & -0.6983 & 0.000003 & 0.488 & & & 997.0449 \\
\hline 0.829 & & 25 & 0.2210 & 1250.3 & (18) & 0.000084 & 0.000083 & 1250.63 & -0.3255 & 0.000001 & 0.106 & & & 997.0449 \\
\hline \multirow{14}{*}{\multicolumn{2}{|c|}{1.063}} & 25 & 0.2667 & 1312.3 & (18) & 0.000100 & 0.000101 & 1311.74 & 0.5634 & -0.000001 & 0.317 & & & 997.0449 \\
\hline & & 34.9 & 0.0493 & 1044.0 & (121) & 0.000030 & 0.000035 & 1043.71 & 0.2850 & -0.000005 & 0.081 & & & 994.0662 \\
\hline & & 34.9 & 0.0985 & 1098.0 & (121) & 0.000039 & 0.000048 & 1096.92 & 1.0819 & -0.000009 & 1.171 & & & 994.0662 \\
\hline & & 34.9 & 0.1479 & 1153.9 & (121) & 0.000064 & 0.000063 & 1154.10 & -0.1983 & 0.000001 & 0.039 & & & 994.0662 \\
\hline & & 34.9 & 0.1977 & 1214.7 & (121) & 0.000082 & 0.000079 & 1215.50 & -0.8022 & 0.000003 & 0.644 & & & 994.0662 \\
\hline & & 34.9 & 0.2460 & 1278.5 & (121) & 0.000096 & 0.000096 & 1278.39 & 0.1066 & 0.000000 & 0.011 & & & 994.0662 \\
\hline & & 45 & 0.0493 & 1039.9 & (121) & 0.000031 & 0.000041 & 1039.37 & 0.5288 & -0.000010 & 0.280 & & & 990.2132 \\
\hline & & 45 & 0.0985 & 1093.0 & (121) & 0.000046 & 0.000054 & 1092.08 & 0.9195 & -0.000008 & 0.845 & & & 990.2132 \\
\hline & & 45 & 0.1479 & 1148.4 & (121) & 0.000069 & 0.000067 & 1148.78 & -0.3845 & 0.000002 & 0.148 & & & 990.2132 \\
\hline & & 45 & 0.1977 & 1209.2 & (121) & 0.000085 & 0.000083 & 1209.77 & -0.5656 & 0.000002 & 0.320 & & & 990.2132 \\
\hline & & 45 & 0.2460 & 1272.6 & (121) & 0.000099 & 0.000100 & 1272.36 & 0.2445 & -0.000001 & 0.060 & & & 990.2132 \\
\hline & & 55 & 0.0493 & 1034.7 & (121) & 0.000040 & 0.000046 & 1034.39 & 0.3055 & -0.000006 & 0.093 & & & 985.6952 \\
\hline & & 55 & 0.0985 & 1087.9 & (121) & 0.000047 & 0.000058 & 1086.65 & 1.2487 & -0.000011 & 1.559 & & & 985.6952 \\
\hline & & 55 & 0.1479 & 1142.6 & (121) & 0.000073 & 0.000071 & 1142.93 & -0.3313 & 0.000002 & 0.110 & & & 985.6952 \\
\hline
\end{tabular}




$$
\begin{array}{rr}
\mathrm{m}^{3} / \mathrm{kg} & \mathrm{m}^{3} / \mathrm{kg} \\
0.000087 & 0.000086
\end{array}
$$

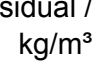

Density
Residual

Inconsistent

Inconsistent Density of
water $/ \mathrm{kg} / \mathrm{m}^{3}$

$\begin{array}{llll}55 & 0.1977 & 1203.3 & (121)\end{array}$

$\begin{array}{rrrr}55 & 0.2460 & 1266.6 & (121) \\ 65.2 & 0.0493 & 1028.8 & (121)\end{array}$

$\begin{array}{llll}65.2 & 0.0493 & 1028.8 \quad(121)\end{array}$

$\begin{array}{ll}0.000087 & 0.000086 \\ 0.000100 & 0.000102\end{array}$

1203.55

$0.000048 \quad 0.000049$

1265.92

1028.70

$0.000061 \quad 0.000061$

1080.53

1136.42

1196.73

$0.000089 \quad 0.000088$

1258.92

$0.000099 \quad 0.000103$

$0.000139 \quad 0.000140$

1395.84

1022.57

0.000060

1074.00

$0.000083 \quad 0.000075$

$0.000094 \quad 0.000089$

$0.000104 \quad 0.000103$

$\begin{array}{ll}0.000141 & 0.000139\end{array}$

$0.000068 \quad 0.000065$

$0.000080 \quad 0.000076$

0.0000920 .000089

$\begin{array}{ll}0.000101 & 0.000103\end{array}$

$\begin{array}{ll}0.000138 & 0.000137\end{array}$

$\begin{array}{ll}0.000086 & 0.000089\end{array}$

$0.000097 \quad 0.000102$

$\begin{array}{lll}0.000155 & 0.000156 \quad 1454.02\end{array}$

Avera

Std dev Res

Avg - 4std

Avg + 4std

$\begin{array}{lll}0.2547 & 0.000001 & 0.065\end{array}$

$0.6803-0.000002 \quad 0.463$

$0.0982-0.000002 \quad 0.010$

$\begin{array}{lll}-0.0290 & 0.000000 & 0.001\end{array}$

$\begin{array}{lll}-0.3175 & 0.000002 & 0.101\end{array}$

$\begin{array}{lll}-0.3275 & 0.000001 & 0.107\end{array}$

$1.3765-0.000004 \quad 1.895$

$\begin{array}{lll}0.9576 & -0.000001 & 0.917\end{array}$

$\begin{array}{lll}-0.3702 & 0.000007 & 0.137\end{array}$

$\begin{array}{lll}0.1979 & -0.000002 & 0.039\end{array}$

$\begin{array}{lll}-1.4390 & 0.000008 & 2.071\end{array}$

$\begin{array}{lll}-1.4814 & 0.000005 & 2.195\end{array}$

$\begin{array}{lll}-0.3579 & 0.000001 & 0.128\end{array}$

$\begin{array}{lll}-1.1277 & 0.000002 & 1.272\end{array}$

$\begin{array}{lll}-0.3434 & 0.000003 & 0.118\end{array}$

$\begin{array}{lll}-0.7469 & 0.000004 & 0.558\end{array}$

$\begin{array}{lll}-0.7467 & 0.000003 & 0.558\end{array}$

$\begin{array}{lll}0.7648 & -0.000002 & 0.585\end{array}$

$\begin{array}{lll}-1.0506 & 0.000002 & 1.104\end{array}$

$0.9393-0.000003 \quad 0.882$

$2.0128-0.000005 \quad 4.051$

$\begin{array}{lll}0.1798 & 0.000000 & 0.032\end{array}$

$0.0667-0.000003 \quad 26.9037$ Sum Chi sqr

$0.6500 \quad 0.000006$

$-2.5334-0.000026$

$2.6668 \quad 0.000021$

985.6952

985.6952

980.4461

980.4461

980.4461

980.4461

980.4461

980.4461

974.7294

974.7294

974.7294

974.7294

974.7294

974.7294

968.3608

968.3608

968.3608

968.3608

968.3608

961.9004

961.9004

961.9004

Curve fit for the model App vol $=\left(w+c 2+c 3^{*} t{ }^{\circ} \mathrm{C}\right) /\left(c 0^{*} w+c 1\right) / e^{\wedge}\left(0.000001^{*}\left(t{ }^{\circ} \mathrm{C}+c 4\right)^{\wedge} 2\right)$

$\begin{array}{lr}\text { c0 } & -0.00172 \\ \text { c1 } & 0.001897 \\ \text { c2 } & -0.030904 \\ \text { c3 } & 0.004087 \\ \text { c4 } & 3804.163 \\ \text { Min T } & 15 \\ \text { Max T } & 95 \\ \text { Min w } & 0.009724 \\ \text { Max w } & 0.398 \\ \text { Avg dens res } & 0.066671 \\ \text { Std dens res } & 0.650021 \\ \text { Avg app vol res *10^3 } & -0.002555 \\ \text { Std app vol res } & 0.005859 \\ \text { No of points in corr } & 64 \\ \text { No of inconsistent poir } & 4\end{array}$

Inconsistent data not used (Residual greater than average $+/-4$ standard deviations)

$\begin{array}{lllllllllllllll}85.4 & 0.0493 & 1029.6 & (121) & -0.000213 & 0.000055 & 1015.79 & 13.8054 & -0.000268 & 190.589 & ! & 968.3608\end{array}$ 
Density Density App Vol Square of Inconsistent $\begin{array}{rrrr}\text { calc / } & \text { Residual / Residual / } & \text { Density based on } \\ \mathrm{kg} / \mathrm{m}^{3} & \mathrm{~kg} / \mathrm{m}^{3} & \mathrm{~m}^{3} / \mathrm{kg} & \text { Residual density }\end{array}$ Inconsistent Density of based on water $/ \mathrm{kg} / \mathrm{m}^{3}$ $\begin{array}{llllll}0.000044 & 0.000077 & 1114.57 & 6.0298 & -0.000033 & 36.359\end{array}$ $\begin{array}{llll}106.2 & 0.3980 & 1433.2 & (121)\end{array}$ 
Density of aqueous solutions of $\mathrm{BaCl} 2$

References (45) Isono, T. Densities, Viscosities, and Electrolytic Conductivity of Concentrated Aqueous Electrolyte Solutions at Several Temperatures.

Alkaline-Earth Chlorides, $\mathrm{LaCl}_{3}, \mathrm{Na}_{2} \mathrm{SO}_{4}, \mathrm{NaNO}_{3}, \mathrm{NaBr}, \mathrm{KNO}_{3}, \mathrm{KBr}$, and $\mathrm{Cd}\left(\mathrm{NO}_{3}\right)_{2}$. J. Chem. Eng. Data 1984, 29, 45-52.

(67) Manohar, S.; Puchalska, D.; Atkinson, G. Pressure-Volume-Temperature Properties of Aqueous Mized Electrolyte Solutions: $\mathrm{NaCl}+\mathrm{BaCl} 2$ from $25 \mathrm{C}$ to $140^{\circ} \mathrm{C}$ J. Chem. Eng. Data 1994, 39, 150-154

(87) Perron, G.; Desnoyers, J. E. Apparent Molal Volumes and Heat Capacities of Alkaline Earth Chlorides in Water at $25^{\circ} \mathrm{C}$. Can. J. Chem. 1974, 52, 3738-3741.

(90) Perry, R. H.; Green, D. W. Perry's Chemical Engineers' Handbook; McGraw Hill: New York, 7th edition, 1997.

(129) Vasilev, Y. A.; Fedyainov, N. V.; Kurenkov, V. V. Specific Heats and Specific Volumes of Isopiestic Aqueous Solutions of BerylliumSubgroup Metal Chlorides. Russ. J. Phys. Chem. 1973, 47, 2799-2803.

\begin{tabular}{|c|c|c|c|c|c|c|c|c|c|c|c|c|c|c|c|}
\hline \multirow[t]{7}{*}{ molality } & \multirow[t]{7}{*}{ molarity } & \multicolumn{2}{|c|}{$\mathbf{t} /{ }^{\circ} \mathrm{C}$ mass frac } & \multicolumn{2}{|l|}{$\begin{array}{r}\text { Density } \\
\exp / \\
\mathrm{kg} / \mathrm{m}^{3}\end{array}$} & \multirow{2}{*}{$\begin{array}{r}\text { App vol } \\
\exp / \\
\mathrm{m}^{3} / \mathrm{kg} \\
0.000103\end{array}$} & \multirow{2}{*}{$\begin{array}{r}\text { App vol } \\
\text { Calc / } \\
\mathrm{m}^{3} / \mathrm{kg} \\
0.000114\end{array}$} & \multirow{2}{*}{$\begin{array}{r}\text { Density } \\
\text { calc / } \\
\mathrm{kg} / \mathrm{m}^{3} \\
1017.89\end{array}$} & $\begin{array}{r}\text { Density } \\
\text { Residual / } \\
\mathrm{kg} / \mathrm{m}^{3}\end{array}$ & \multirow{2}{*}{$\begin{array}{r}\text { App Vol } \\
\text { Residual / } \\
\mathrm{m}^{3} / \mathrm{kg} \\
-0.000010\end{array}$} & \multirow{2}{*}{$\begin{array}{r}\text { Square of } \\
\text { Density } \\
\text { Residual } \\
0.046\end{array}$} & \multirow{2}{*}{$\begin{array}{c}\text { Inconsistent } \\
\text { based on } \\
\text { density }\end{array}$} & $\begin{array}{c}\text { Inconsistent } \\
\text { based on } \\
\text { apparent } \\
\text { volume }\end{array}$ & $\begin{array}{r}\text { Delta density } \\
\qquad / \mathrm{kg} / \mathrm{m}^{3}\end{array}$ & $\begin{array}{r}\text { Density of } \\
\text { water } / \mathrm{kg} / \mathrm{m}^{3}\end{array}$ \\
\hline & & 0 & 0.0200 & 1018.10 & (90) & & & & 0.2148 & & & & & & 999.8395 \\
\hline & & 0 & 0.0400 & 1036.80 & (90) & 0.000109 & 0.000115 & 1036.51 & 0.2871 & -0.000007 & 0.082 & & & & 999.8395 \\
\hline & & 0 & 0.0800 & 1076.00 & (90) & 0.000115 & 0.000119 & 1075.62 & 0.3847 & -0.000004 & 0.148 & & & & 999.8395 \\
\hline & & 0 & 0.1200 & 1117.80 & (90) & 0.000121 & 0.000124 & 1117.36 & 0.4382 & -0.000003 & 0.192 & & & & 999.8395 \\
\hline & & 0 & 0.1600 & 1162.70 & (90) & 0.000125 & 0.000128 & 1161.99 & 0.7115 & -0.000003 & 0.506 & & & & 999.8395 \\
\hline & & 0 & 0.2000 & 1210.50 & (90) & 0.000130 & 0.000132 & 1209.75 & 0.7461 & -0.000003 & 0.557 & & & & 999.8395 \\
\hline 0.05 & & 15 & 0.0103 & 1008.33 & (45) & 0.000112 & 0.000124 & 1008.20 & 0.1330 & -0.000013 & 0.018 & & & & 999.0996 \\
\hline 0.1 & & 15 & 0.0204 & 1017.34 & (45) & 0.000121 & 0.000125 & 1017.25 & 0.0898 & -0.000004 & 0.008 & & & & 999.0996 \\
\hline 0.5 & & 15 & 0.0943 & 1088.46 & (45) & 0.000130 & 0.000133 & 1088.08 & 0.3846 & -0.000003 & 0.148 & & & & 999.0996 \\
\hline 0.7 & & 15 & 0.1272 & 1122.09 & (45) & 0.000139 & 0.000137 & 1122.42 & -0.3253 & 0.000002 & 0.106 & & & & 999.0996 \\
\hline 1 & & 15 & 0.1723 & 1172.52 & (45) & 0.000142 & 0.000142 & 1172.58 & -0.0617 & 0.000000 & 0.004 & & & & 999.0996 \\
\hline 1.2 & & 15 & 0.1999 & 1205.13 & (45) & 0.000145 & 0.000145 & 1205.13 & -0.0031 & 0.000000 & 0.000 & & & & 999.0996 \\
\hline 1.5 & & 15 & 0.2380 & 1251.60 & (45) & 0.000153 & 0.000150 & 1252.63 & -1.0311 & 0.000003 & 1.063 & & & & 999.0996 \\
\hline \multirow{2}{*}{\multicolumn{2}{|c|}{0.05}} & 20 & 0.0103 & 1007.37 & (45) & 0.000117 & 0.000127 & 1007.26 & 0.1065 & -0.000010 & 0.011 & & & & 998.2041 \\
\hline & & 20 & 0.0200 & 1015.90 & (90) & 0.000129 & 0.000128 & 1015.92 & -0.0191 & 0.000001 & 0.000 & & & & 998.2041 \\
\hline \multirow{3}{*}{\multicolumn{2}{|c|}{0.1}} & 20 & 0.0204 & 1016.31 & (45) & 0.000127 & 0.000128 & 1016.28 & 0.0318 & -0.000002 & 0.001 & & & & 998.2041 \\
\hline & & 20 & 0.0400 & 1034.10 & (90) & 0.000132 & 0.000130 & 1034.19 & -0.0890 & 0.000002 & 0.008 & & & & 998.2041 \\
\hline & & 20 & 0.0800 & 1072.10 & (90) & 0.000139 & 0.000134 & 1072.49 & -0.3856 & 0.000004 & 0.149 & & & & 998.2041 \\
\hline \multirow{2}{*}{\multicolumn{2}{|c|}{0.5}} & 20 & 0.0943 & 1087.09 & (45) & 0.000133 & 0.000136 & 1086.78 & 0.3145 & -0.000003 & 0.099 & & & & 998.2041 \\
\hline & & 20 & 0.1200 & 1112.80 & (90) & 0.000142 & 0.000139 & 1113.29 & -0.4880 & 0.000003 & 0.238 & & & & 998.2041 \\
\hline \multirow{2}{*}{\multicolumn{2}{|c|}{0.7}} & 20 & 0.1272 & 1120.55 & (45) & 0.000142 & 0.000140 & 1120.94 & -0.3889 & 0.000002 & 0.151 & & & & 998.2041 \\
\hline & & 20 & 0.1600 & 1156.40 & (90) & 0.000145 & 0.000143 & 1156.81 & -0.4066 & 0.000002 & 0.165 & & & & 998.2041 \\
\hline 1 & & 20 & 0.1723 & 1170.82 & (45) & 0.000145 & 0.000145 & 1170.83 & -0.0065 & 0.000000 & 0.000 & & & & 998.2041 \\
\hline \multirow{2}{*}{\multicolumn{2}{|c|}{1.2}} & 20 & 0.1999 & 1203.20 & (45) & 0.000148 & 0.000148 & 1203.18 & 0.0168 & 0.000000 & 0.000 & & & & 998.2041 \\
\hline & & 20 & 0.2000 & 1203.10 & (90) & 0.000149 & 0.000148 & 1203.27 & -0.1693 & 0.000001 & 0.029 & & & & 998.2041 \\
\hline \multirow{3}{*}{\multicolumn{2}{|c|}{1.5}} & 20 & 0.2380 & 1249.35 & (45) & 0.000156 & 0.000153 & 1250.38 & -1.0269 & 0.000003 & 1.054 & & & & 998.2041 \\
\hline & & 20 & 0.2400 & 1253.10 & (90) & 0.000153 & 0.000153 & 1252.92 & 0.1787 & 0.000000 & 0.032 & & & & 998.2041 \\
\hline & & 20 & 0.2600 & 1279.30 & (90) & 0.000155 & 0.000156 & 1279.02 & 0.2754 & -0.000001 & 0.076 & & & & 998.2041 \\
\hline 0.00388 & & 25 & 0.0008 & 997.76 & (87) & 0.000113 & 0.000129 & 997.75 & 0.0131 & -0.000016 & 0.000 & & & 0.715 & 997.0449 \\
\hline 0.01562 & & 25 & 0.0032 & 999.92 & (87) & 0.000115 & 0.000129 & 999.87 & 0.0471 & -0.000015 & 0.002 & & & 2.871 & 997.0449 \\
\hline 0.02736 & & 25 & 0.0057 & 1002.05 & (87) & 0.000118 & 0.000129 & 1001.99 & 0.0655 & -0.000012 & 0.004 & & & 5.009 & 997.0449 \\
\hline 0.03879 & & 25 & 0.0080 & 1004.13 & (87) & 0.000119 & 0.000130 & 1004.05 & 0.0833 & -0.000010 & 0.007 & & & 7.088 & 997.0449 \\
\hline
\end{tabular}


molality molarity

\section{$t /{ }^{\circ} \mathrm{C}$ mass frac Density}

$$
\exp /
$$

$\exp /$
$\mathrm{kg} / \mathrm{m}^{3}$

0.05
0.07881

0.1

0.1569

0.166667

0.23647

0.31614

0.333333

0.38688

0.5
0.504

0.7

0.8317

0.833333

1
1.2
1.2538
1.5
1.5
1.503
1.6226
0.05
0.1
0.5
0.7
1
1.2
1.5
0.05
0.1
0.166667
0.333333
0.5
0.7
0.833333
1
1.2
1.5

$\begin{array}{lll}25 & 0.0103 & 1006.16 \\ 25 & 0.0161 & 1011.39 \\ 25 & 0.0204 & 1015.07 \\ 25 & 0.0316 & 1025.43 \\ 25 & 0.0335 & 1027.18 \\ 25 & 0.0469 & 1039.55 \\ 25 & 0.0618 & 1053.60 \\ 25 & 0.0649 & 1056.65 \\ 25 & 0.0746 & 1065.96 \\ 25 & 0.0943 & 1085.49 \\ 25 & 0.0950 & 1085.51 \\ 25 & 0.1272 & 1118.85 \\ 25 & 0.1476 & 1140.92 \\ 25 & 0.1479 & 1142.19 \\ 25 & 0.1723 & 1168.80 \\ 25 & 0.1999 & 1201.02 \\ 25 & 0.2070 & 1209.63 \\ 25 & 0.2380 & 1247.01 \\ 25 & 0.2380 & 1249.45 \\ 25 & 0.2384 & 1248.76 \\ 25 & 0.2526 & 1267.20 \\ 30 & 0.0103 & 1004.73 \\ 30 & 0.0204 & 1013.59 \\ 30 & 0.0943 & 1083.73 \\ 30 & 0.1272 & 1116.95 \\ 30 & 0.1723 & 1166.67 \\ 30 & 0.1999 & 1198.76 \\ 30 & 0.2380 & 1244.60 \\ 35 & 0.0103 & 1003.08 \\ 35 & 0.0204 & 1011.91 \\ 35 & 0.0335 & 1023.95 \\ 35 & 0.0649 & 1053.18 \\ 35 & 0.0943 & 1081.83 \\ 35 & 0.1272 & 1114.92 \\ 35 & 0.1479 & 1138.03 \\ 35 & 0.1723 & 1164.46 \\ 35 & 0.1999 & 1196.34 \\ 35 & 0.2380 & 1242.04 \\ 40 & 0.0200 & 1009.60 \\ 40 & 0.0400 & 1027.50 \\ 40 & 0.0800 & 1064.80 \\ 40 & 0.1200 & 1104.70 \\ 40 & 0.1600 & 1147.80\end{array}$

$\begin{array}{lll}40 & 0.1600 & 1147.80\end{array}$
Ref

$\begin{array}{rr}\text { App vol } & \text { App vol } \\ \exp / & \text { Calc / }\end{array}$

ensity Density App Vol calc / Residual / Residual / $\mathrm{m}^{3} / \mathrm{k}$ $\mathrm{m}^{3} / \mathrm{kg}$ $\mathrm{kg} / \mathrm{m}^{3}$

$\begin{array}{llll}(45) & 0.000121 & 0.000130 & 1006.07\end{array}$ (87)

$(67)$
$(87)$ (87) (45) $(129)$
$(45)$

$0.0223-0.000001$

$0.2233-0.000007$

$0.2328-0.000007$

$0.2229-0.000004$

$0.2590-0.000004$

$0.3055-0.000004$

$0.2771-0.000003$

$0.2464-0.000002$

$-0.42090 .000004$

$\begin{array}{ll}-0.3973 & 0.000002\end{array}$

$-0.3200 \quad 0.000002$

$0.6811-0.000004$

$\begin{array}{ll}-0.0847 & 0.000000\end{array}$

$\begin{array}{ll}-0.0484 & 0.000000\end{array}$

$0.0275 \quad 0.000000$

$-0.9838 \quad 0.000003$

$0.000158 \quad 0.000156$

$0.000152 \quad 0.000156$

$0.000155 \quad 0.000156$

1248.45

$0.3051-0.000001$

$0.4913-0.000001$

$0.0917-0.000009$

$\begin{array}{lll}0.0061 & 0.000000\end{array}$

$0.2262-0.000002$

$-0.4133 \quad 0.000003$

$\begin{array}{lll}0.000141 & 1083\end{array}$

0.000150 0.000144

$\begin{array}{llll}0.000150 & 0.000150 & 1166.78\end{array}$

$\begin{array}{lll}0.000160 & 0.000158 & 1245.50\end{array}$

$\begin{array}{ll}-0.1078 & 0.000000\end{array}$

$\begin{array}{lll}-0.0486 & 0.000000\end{array}$

$\begin{array}{ll}-0.8995 & 0.000002\end{array}$

0.000134

1002.99

0.000135

1011.91

$\begin{array}{lll}0.000130 & 0.000136 & 1023.72\end{array}$

$\begin{array}{lll}0.000136 & 0.000139 & 1052.90\end{array}$

$0.0877-0.000008$

0.00290 .000000

$0.2235-0.000006$

$0.2788-0.000004$

$0.2551-0.000002$

$\begin{array}{ll}-0.3850 & 0.000002\end{array}$

$\begin{array}{lll}0.000140 & 0.000143 & 1081.57 \\ 0.000149 & 0.000146 & 1115.30\end{array}$

$\begin{array}{lll}0.000145 & 0.000149 & 1137.38\end{array}$

$0.6469-0.000003$

$\begin{array}{ll}-0.0617 & 0.000000\end{array}$

1164.52

$\begin{array}{ll}-0.0788 & 0.000000\end{array}$

$0.000162 \quad 0.000160$

1242.91

$-0.8665 \quad 0.000002$

$0.000140 \quad 0.000136$

1009.68

$\begin{array}{lll}0.000143 & 0.000138 & 1027.69\end{array}$

$\begin{array}{lll}0.000149 & 0.000142 & 1065.40\end{array}$

$-0.0800 \quad 0.000004$

$\begin{array}{ll}-0.1850 & 0.000004\end{array}$

$\begin{array}{ll}-0.6050 & 0.000007\end{array}$

$\begin{array}{llllll}0.0 .000006 & \end{array}$

$\begin{array}{ll}-0.5516 & 0.000003\end{array}$
Square of Inconsistent Inconsistent Delta density Density of Density based on based on $/ \mathrm{kg} / \mathrm{m}^{3}$ water $/ \mathrm{kg} / \mathrm{m}^{3}$ Residual density apparent

volume

0.019

0.000

0.050

0.054

0.050

0.067

0.093

0.077

0.061

0.177

0.158

0.102

0.464

0.007

0.002

0.968

2.120

0.093

0.24

0.008

0.000

0.051

0.171

0.012

0.002

0.809

0.008

0.000

0.050

0.078

0.065

0.148

0.419

0.004

0.006

0.751

0.006

0.034

0.366

0.740

0.304
997.0449

14.341997 .0449 997.0449

$28.389 \quad 997.0449$

$30.137 \quad 997.0449$

$42.502 \quad 997.0449$

56.554997 .0449

59.61997 .0449

$68.92 \quad 997.0449$

997.0449

997.0449

997.0449

997.0449

$145.147 \quad 997.0449$

997.0449

997.0449

997.0449

997.0449

$252.405 \quad 997.0449$ 997.0449 997.0449

995.6473

995.6473

995.6473

995.6473

995.6473

995.6473

995.6473

994.0319

994.0319

$29.914 \quad 994.0319$

59.151994 .0319 994.0319
994.0319

$143.998 \quad 994.0319$

994.0319

994.0319

994.0319

992.2158

992.2158

992.2158

992.2158

992.2158 
molality molarity

$$
\exp /
$$

$\mathrm{kg} / \mathrm{m}^{3}$

$\begin{array}{lll}40 & 0.2000 & 1193.80\end{array}$

$\begin{array}{lll}40 & 0.2400 & 1243.00\end{array}$

$0.2600 \quad 1268.80$

$0.0103 \quad 999.18$

$0.0204 \quad 1007.97$

$0.0335 \quad 1019.86$

$\begin{array}{ll}0.0649 & 1048.75 \\ 0.0943 & 1077.47\end{array}$

$\begin{array}{lll}0.0943 & 1077.47\end{array}$

$0.1272 \quad 1110.36$

$0.1479 \quad 1132.91$

$0.1723 \quad 1159.47$

$0.1999 \quad 1191.21$

$0.2380 \quad 1236.67$

$0.2380 \quad 1238.11$

$0.0103 \quad 994.62$

$0.0204 \quad 1003.35$

$0.0335 \quad 1015.29$

$0.0649 \quad 1044.14$

$0.0943 \quad 1072.51$

$0.1272 \quad 1105.18$

$0.1479 \quad 1127.90$

$0.1723 \quad 1154.11$

$0.1999 \quad 1185.67$

$0.2380 \quad 1230.94$

$0.2380 \quad 1232.88$

$0.0200 \quad 1000.40$

$0.0400 \quad 1018.10$

$0.0800 \quad 1055.10$

$0.1200 \quad 1094.80$

$0.1600 \quad 1137.30$

$0.2000 \quad 1182.80$

$0.2400 \quad 1231.60$

$0.2600 \quad 1257.10$

$0.0200 \quad 989.00$

$0.0335 \quad 1001.13$

$0.0400 \quad 1006.60$

$0.0649 \quad 1029.71$

$0.0800 \quad 1043.40$

$0.1200 \quad 1082.70$

$0.1479 \quad 1112.51$

$0.1600 \quad 1124.90$

$0.2000 \quad 1170.20$

$0.2380 \quad 1216.39$
Ref

$\begin{array}{cc}\text { App vol } & \text { App vol } \\ \exp / & \text { Calc / }\end{array}$

Density App Vo calc / Residual / Residual / $\mathrm{m}^{3} / \mathrm{kg} \quad \mathrm{m}^{3} / \mathrm{kg}$ $\mathrm{kg} / \mathrm{m}^{3}$ $\mathrm{kg} / \mathrm{m}^{3}$

$\mathrm{m}^{3} / \mathrm{kg}$

$\begin{array}{llllll}\text { (90) } & 0.000157 & 0.000156 & 1193.99 & -0.1941 & 0.000001\end{array}$ $\begin{array}{llllll}(90) & 0.000161 & 0.000161 & 1242.72 & 0.2811 & -0.000001\end{array}$

$\begin{array}{llllll}\text { (90) } & 0.000163 & 0.000164 & 1268.31 & 0.4867 & -0.000001\end{array}$

$\begin{array}{lllll}0.000130 & 0.000136 & 999.12 & 0.0612 & -0.000006\end{array}$

$\begin{array}{lllll}0.000138 & 0.000137 & 1007.98 & -0.0088 & 0.000000\end{array}$

$\begin{array}{lllll}0.000135 & 0.000139 & 1019.72 & 0.1353 & -0.000004\end{array}$

$\begin{array}{lllll}0.000142 & 0.000142 & 1048.72 & 0.0257 & 0.000000\end{array}$

$\begin{array}{llllll}0.000143 & 0.000145 & 1077.21 & 0.2624 & -0.000002\end{array}$

$\begin{array}{lllll}0.000151 & 0.000149 & 1110.72 & -0.3586 & 0.000002\end{array}$

$\begin{array}{lllll}0.000150 & 0.000151 & 1132.65 & 0.2572 & -0.000001\end{array}$

$\begin{array}{llllll}0.000155 & 0.000154 & 1159.61 & -0.1385 & 0.000001\end{array}$

$\begin{array}{lll}0.000158 & 0.000157 & 1191.29\end{array}$

$\begin{array}{lll}0.000164 & 0.000162 & 1237.46\end{array}$

0.000160

$0.000131 \quad 0.000138$

1237.46

$\begin{array}{lll}0.000133 & 0.000140 & 1015.05\end{array}$

$0.000140 \quad 0.000$

$0.000144 \quad 0.000146$

$0.000152 \quad 0.000150$

1043.89

$0.000149 \quad 0.000152$

$0.000156 \quad 0.000155$

$0.000159 \quad 0.000158$

$0.000165 \quad 0.000163$

$0.000160 \quad 0.000163$

$0.000143 \quad 0.000139$

$0.000145 \quad 0.000141$

$0.000151 \quad 0.000145$

$0.000153 \quad 0.000149$

$0.000156 \quad 0.000154$

$0.000159 \quad 0.000159$

$0.000162 \quad 0.000164$

$0.000165 \quad 0.000166$

$0.000134 \quad 0.000138$

$0.000130 \quad 0.000139$

$0.000140 \quad 0.000139$

$\begin{array}{ll}0.000137 & 0.000142\end{array}$

$0.000146 \quad 0.000143$

$0.000151 \quad 0.000148$

$\begin{array}{lll}0.000149 & 0.000151\end{array}$

$0.000154 \quad 0.000152$

$0.000157 \quad 0.000157$

$0.000160 \quad 0.000161$

0.07920 .000000

$\begin{array}{ll}-0.7853 & 0.000002\end{array}$

$0.6519-0.000002$

$0.0666-0.000007$

$\begin{array}{ll}-0.0163 & 0.000001\end{array}$

$0.2411-0.000007$

$0.2502-0.000004$

$0.2786-0.000003$

$\begin{array}{lll}-0.3896 & 0.000003\end{array}$

$0.5141-0.000003$

$-0.1018 \quad 0.000000$

$\begin{array}{lll}-0.0652 & 0.000000\end{array}$

$-0.73750 .000002$

$1.2057-0.000003$

$-0.0772 \quad 0.000004$

$-0.1913 \quad 0.000005$

$\begin{array}{ll}-0.5143 & 0.000006\end{array}$

$\begin{array}{ll}-0.5527 & 0.000004\end{array}$

$-0.4065 \quad 0.000002$

$-0.09210 .000000$

$0.4583-0.000001$

$0.6077-0.000001$

$0.0673-0.000003$

$0.2889-0.000009$

$\begin{array}{lll}-0.0048 & 0.000000\end{array}$

$0.3142-0.000005$

$-0.2510 \quad 0.000003$

$-0.4266 \quad 0.000003$

$0.3303-0.000002$

$\begin{array}{ll}-0.3391 & 0.000002\end{array}$

$-0.0145 \quad 0.000000$

$0.5534-0.000002$
Square of Inconsistent Inconsistent Delta density Density of Density based on based on $\quad / \mathrm{kg} / \mathrm{m}^{3}$ water $/ \mathrm{kg} / \mathrm{m}^{3}$ Residual density apparent

volume

0.038

0.079

0.237

0.000

0.018

0.001

0.069

0.129

0.066

0.019

0.006

0.617

0.425

0.004

0.000

0.058

0.063

0.078

0.152

0.264

0.010

0.004

0.544

1.454

0.006

0.037

0.265

0.305

0.165

0.008

0.210

0.369

0.005

0.083

0.000

0.099

0.063

0.182

0.109

0.115

0.000

0.306
992.2158

992.2158

992.2158

990.2132

990.2132

$29.643 \quad 990.2132$

58.532990 .2132

990.2132

990.2132

$142.695 \quad 990.2132$ 990.2132 990.2132

990.2132

$247.894 \quad 990.2132$ 985.6952 985.6952

$29.592 \quad 985.6952$

$58.447 \quad 985.6952$ 985.6952 985.6952

$142.209 \quad 985.6952$ 985.6952 985.6952 985.6952

247.188985 .6952 983.1989 983.1989 983.1989 983.1989 983.1989 983.1989 983.1989 983.1989 971.7978

$29.33 \quad 971.7978$ 971.7978

$57.915 \quad 971.7978$ 971.7978 971.7978

$140.712 \quad 971.7978$ 971.7978 971.7978

244.588971 .7978 
molality molarity

$\mathrm{t} /{ }^{\circ} \mathrm{C}$ mass frac Density

$\exp /$
$\mathrm{kg} / \mathrm{m}^{3}$

$\begin{array}{rrr}80 & 0.2400 & 1218.60 \\ 80 & 0.2600 & 1244.00 \\ 100 & 0.0200 & 975.50\end{array}$

0.166667

0.333333

0.833333

1.5

0.166667
0.333333

0.333333

0.833333

1.5
.166667

0.333333

0.833333

0.0200
0.0335 975.50

$\begin{array}{ll}0.0335 & 987.52 \\ 0.0400 & 993.10\end{array}$

$0.0649 \quad 1015.89$

$0.0800 \quad 1029.90$

$0.1200 \quad 1069.20$

$0.1479 \quad 1098.48$

$0.1600 \quad 1111.30$

$0.2000 \quad 1156.30$

$0.2380 \quad 1201.41$

$\begin{array}{ll}0.2600 \quad 1229.80 \\ 0.0335 & 972.08\end{array}$

$\begin{array}{rr}0.2600 & 1229.80 \\ 0.0335 & 972.08\end{array}$

$0.0649 \quad 1000.28$

$0.1479 \quad 1082.36$

$0.2380 \quad 1182.83$

$0.0335 \quad 955.18$

$0.0649 \quad 983.40$

$0.1479 \quad 1065.68$

140

$0.2380 \quad 1167.67$

Ref

$\begin{array}{rr}\text { App vol } & \text { App vol } \\ \exp / & \text { Calc / }\end{array}$

$\begin{array}{cc}\exp / & \text { Calc } / \\ \mathrm{m}^{3} / \mathrm{kg} & \mathrm{m}^{3} / \mathrm{kg}\end{array}$

$0.000161 \quad 0.000162$

$\begin{array}{lll}(90) & 0.000161 & 0.000162 \\ (90) & 0.000163 & 0.000164 \\ (90) & 0.000127 & 0.000133\end{array}$

(90)

(67)

$(90)$
$(67)$

$(67)$
$(90)$

(90)

$(67)$
$(90)$

$(90)$
$(90)$
$(67)$

(67)

(90)

$(90)$
$(67)$

$(67)$
$(67)$

$(67)$
$(67)$

$(67)$
$(67)$

$(67)$
$(67)$

$\begin{array}{ll}0.000127 & 0.000133 \\ 0.000125 & 0.000135\end{array}$

$0.000131 \quad 0.000135$

$0.000133 \quad 0.000138$

$\begin{array}{lll}0.000137 & 0.000139\end{array}$

$0.000143 \quad 0.000146$

$\begin{array}{ll}0.000146 & 0.000147\end{array}$

$0.000150 \quad 0.000152$

$0.000157 \quad 0.000156$

$0.000155 \quad 0.000156$

$0.000158 \quad 0.000159$

$\begin{array}{ll}0.000117 & 0.000129\end{array}$

$0.000126 \quad 0.000131$

$0.000138 \quad 0.000139$

$\begin{array}{lll}0.000157 & 0.000149\end{array}$

$0.000097 \quad 0.000121$

$0.000109 \quad 0.000124$

$0.000123 \quad 0.000131$

$\begin{array}{lll}0.000141 & 0.000140\end{array}$
$0.000142 \quad 0.000143$

40

Density Density App Vol

calc / Residual / Residual /

$\mathrm{kg} / \mathrm{m}^{3} \quad \mathrm{~kg} / \mathrm{m}^{3} \quad \mathrm{~m}^{3} / \mathrm{kg}$

$1218.30 \quad 0.3030-0.000001$

$1243.58 \quad 0.4151-0.000001$

$975.38 \quad 0.1236-0.000006$

$987.20 \quad 0.3207-0.000010$

$992.93 \quad 0.1681-0.000004$

$1015.59 \quad 0.2979-0.000004$

$1029.77 \quad 0.1343-0.000002$

$1069.06 \quad 0.1350-0.000001$

$0.4578-0.000003$

$0.2503-0.000001$

$0.3390-0.000001$

$-0.1875 \quad 0.000001$

$0.4370-0.000001$

$0.4005-0.000001$

$0.3511-0.000011$

$999.95 \quad 0.3302-0.000005$

$0.2565-0.000001$

1185.75

954.45
982.49

$-2.9248 \quad 0.000009$

$0.7244-0.000024$

$0.9023-0.000014$

$1064.33 \quad 1.3553-0.000008$

$\begin{array}{lll}1168.05 & -0.3823 & 0.000001\end{array}$

$\begin{array}{lll}\text { Average } & 0.0380 & -0.000002\end{array}$

$\begin{array}{lll}\text { Std dev } & 0.492388 & 0.000005\end{array}$

Avg - 4std $\quad-1.9315-0.000021$

Avg + 4std $\quad 2.0076 \quad 0.000017$
Square of Inconsistent Inconsistent Delta density Density of

Density based on based on $/ \mathrm{kg} / \mathrm{m}^{3}$ water $/ \mathrm{kg} / \mathrm{m}^{3}$

Residual density apparent

$\begin{array}{ll}0.092 & 971.7978 \\ 0.172 & 971.7978 \\ 0.015 & 958.3637\end{array}$

$0.028 \quad 958.3637$

$\begin{array}{lll}0.089 & 57.524 & 958.3637\end{array}$

0.018

0.018

0.210

0.063

0.115

0.035

0.191

0.160

0.123

0.109

0.066

8.554

0.525

0.814

1.837

0.146

33.9022 Sum Chi sqr
958.3637

$140.117 \quad 958.3637$

958.3637

958.3637

243.044958 .3637

958.3637

958.3637

$28.994 \quad 943.0825$

$57.198 \quad 943.0825$

$139.275 \quad 943.0825$

$239.745 \quad 943.0825$

$29.149 \quad 926.026$

57.371926 .026

$139.655 \quad 926.026$

$241.643 \quad 926.026$

\section{Curve fit for the model App vol $=\left(w+c 2+c 3^{*} t{ }^{\circ} \mathrm{C}\right) /\left(c 0^{*} w+c 1\right) / e^{\wedge}\left(0.000001^{*}\left(t^{\circ} \mathrm{C}+c 4\right)^{\wedge} 2\right)$}

c0

$\mathrm{c1}$

c2 4.178544

c3 $\quad 0.068274$

c4 $\quad 3971.864$

Min

$\operatorname{Min} T$

Min $w$

140

Max w

0.000807

Avg dens res $\quad 0.038011$

Std dens res $\quad 0.492388$

Avg app vol res *10^3 $\quad-0.00186$

Std app vol res *10^3 0.004818

No of points in corr

No of inconsistent poir

140
1 
molality molarity

\author{
$t /{ }^{\circ} \mathrm{C}$ mass frac Density Ref$$
\begin{array}{r}
\exp / \\
\mathrm{kg} / \mathrm{m}^{3}
\end{array}
$$$$
\begin{array}{r}
\exp / \\
\mathrm{kg} / \mathrm{m}^{3}
\end{array}
$$
App vol App vol

exp / Calc /
$\mathrm{m}^{3} / \mathrm{kg}$

Density Density App Vol

calc / Residual / Residual/

$\mathrm{kg} / \mathrm{m}^{3}$ $\mathrm{kg} / \mathrm{m}^{3}$

Square of Inconsistent Inconsistent Delta density Density of

Density based on based on Residual density apparent

Inconsistent data not used (Residual greater than average $+/-4$ standard deviations)

$$
1.5
$$

1244.6
$0.000155 \quad 0.000160$

1242.91
$1.6963-0.000005$ 
Density of aqueous solutions of $\mathbf{C a C l} 2$

References (28) Gates, J. A.; Wood, R. H. Densities of Aqueous Solutions of $\mathrm{NaCl}_{1} \mathrm{MgCl}_{2}, \mathrm{KCl}, \mathrm{NaBr}, \mathrm{LiCl}, \mathrm{and} \mathrm{CaCl}_{2}$ from 0.05 to $5.0 \mathrm{~mol} \mathrm{~kg}{ }^{-1}$ and 0.1013 to $40 \mathrm{MPa}$ at $298.15 \mathrm{~K}$. J. Chem. Eng. Data 1985, 30,44-49.

(45) Isono, T. Densities, Viscosities, and Electrolytic Conductivity of Concentrated Aqueous Electrolyte Solutions at Several Temperatures. Alkaline-Earth Chlorides, $\mathrm{LaCl}_{3}, \mathrm{Na}_{2} \mathrm{SO}_{4}, \mathrm{NaNO}_{3}, \mathrm{NaBr}, \mathrm{KNO}_{3}, \mathrm{KBr}$, and $\mathrm{Cd}\left(\mathrm{NO}_{3}\right)_{2}$. J. Chem. Eng. Data 1984, $29,45-52$.

(57) Kumar, A.; Atkinson, G.; Howell, R. D. Thermodynamics of Concentrated Electrolyte Mixtures. II. Densities and Compressibilities of Aqueous $\mathrm{NaCl}-\mathrm{CaCl}_{2}$ at $25^{\circ} \mathrm{C}$. J. Sol. Chem. 1982, 11, 857-870.

(87) Perron, G.; Desnoyers, J. E. Apparent Molal Volumes and Heat Capacities of Alkaline Earth Chlorides in Water at $25^{\circ} \mathrm{C}$. Can. J. Chem 1974, 52, 3738-3741.

(90) Perry, R. H.; Green, D. W. Perry's Chemical Engineers' Handbook; McGraw Hill: New York, 7th edition, 1997.

(113) Saluja, P. P. S.; LeBlanc, J. C. Apparent Molar Heat Capacities and Volumes of Aqueous Solutions of $\mathrm{MgCl}_{2}, \mathrm{CaCl}_{2}$, and $\mathrm{SrCl}_{2}$ at Elevated Temperatures. J. Chem. Eng. Data 1987, 32, 72-76.

(123) Spitzer, J. J.; Singh, P. P.; McCurdy, K. G.; Hepler, L. G. Apparent Molar Heat Capacities and Volumes of $\mathrm{Aqueous}^{\mathrm{E}} \mathrm{Electrolytes:} \mathrm{CaCl}_{2}$, $\mathrm{Cd}\left(\mathrm{NO}_{3}\right)_{2}, \mathrm{CoCl}_{2}, \mathrm{Cu}\left(\mathrm{ClO}_{4}\right)_{2}, \mathrm{Mg}\left(\mathrm{ClO}_{4}\right)_{2}$, and $\mathrm{NiCl}_{2}$. J. Sol. Chem. 1978, 7, 81-86.

(133) Wimby, J. M.; Berntsson, T. S. Viscosity and Density of Aqueous Solutions of $\mathrm{LiBr}, \mathrm{LiCl}, \mathrm{ZnBr}_{2}, \mathrm{CaCl}_{2}$, and $\mathrm{LiNO}_{3}$. 1. Single Salt Solutions. J. Chem. Eng. Data 1994, 39, 68-72.

(136) Zhang, H.-L.; Chen, G.-H.; Han, S.-J. Viscosity and Density of $\mathrm{H}_{2} \mathrm{O}+\mathrm{NaCl}+\mathrm{CaCl}_{2}$ and $\mathrm{H}_{2} \mathrm{O}+\mathrm{KCl}+\mathrm{CaCl}_{2}$ at 298.15K. J. Chem. Eng. Data $1997,42,526-530$.

\begin{tabular}{|c|c|c|c|c|c|c|c|c|c|c|c|c|c|c|c|c|}
\hline \multirow[t]{9}{*}{ molality } & \multirow[t]{9}{*}{ molarity } & \multicolumn{2}{|c|}{$\mathrm{t} /{ }^{\circ} \mathrm{C}$ mass frac } & \multicolumn{2}{|l|}{$\begin{array}{r}\text { Density } \\
\exp / \\
\mathrm{kg} / \mathrm{m}^{3}\end{array}$} & \multirow[t]{2}{*}{$\begin{array}{r}\text { App vol } \\
\exp / \\
\mathrm{m}^{3} / \mathrm{kg}\end{array}$} & $\begin{array}{r}\text { App vol } \\
\text { Calc / } \\
\mathrm{m}^{3} / \mathrm{kg}\end{array}$ & $\begin{array}{r}\text { Density } \\
\text { calc / } \\
\mathrm{kg} / \mathrm{m}^{3}\end{array}$ & $\begin{array}{r}\text { Density } \\
\text { Residual / } \\
\mathrm{kg} / \mathrm{m}^{3}\end{array}$ & $\begin{array}{r}\text { App Vol } \\
\text { Residual / } \\
\mathrm{m}^{3} / \mathrm{kg}\end{array}$ & $\begin{array}{l}\text { Square of } \\
\text { Density } \\
\text { Residual }\end{array}$ & $\begin{array}{c}\text { Inconsistent } \\
\text { based on } \\
\text { density }\end{array}$ & $\begin{array}{c}\text { Inconsistent } \\
\text { based on } \\
\text { apparent } \\
\text { volume }\end{array}$ & $\begin{array}{r}\text { App molal } \\
\text { volume } / \mathrm{cm}^{3} \\
/ \mathrm{mole}^{2}\end{array}$ & $\begin{array}{l}\text { Delta density } \\
\qquad / \mathrm{kg} / \mathrm{m}^{3}\end{array}$ & $\begin{array}{l}\text { Density o } \\
\text { water } \\
\mathrm{kg} / \mathrm{m}\end{array}$ \\
\hline & & 0 & 0.0200 & 1017.10 & (90) & & 0.000187 & 1016.37 & 0.7337 & -0.000035 & 0.538 & & & & & 999.8395 \\
\hline & & 0 & 0.0400 & 1034.60 & (90) & 0.000160 & 0.000190 & 1033.30 & 1.2995 & -0.000030 & 1.689 & & & & & 999.8395 \\
\hline & & 0 & 0.0800 & 1070.30 & (90) & 0.000177 & 0.000198 & 1068.42 & 1.8822 & -0.000021 & 3.543 & & & & & 999.8395 \\
\hline & & 0 & 0.1200 & 1107.20 & (90) & 0.000192 & 0.000205 & 1105.24 & 1.9634 & -0.000013 & 3.855 & & & & & 999.8395 \\
\hline & & 0 & 0.1600 & 1145.40 & (90) & 0.000206 & 0.000213 & 1143.78 & 1.6152 & -0.000008 & 2.609 & & & & & 999.8395 \\
\hline & & 0 & 0.2000 & 1185.30 & (90) & 0.000218 & 0.000222 & 1184.07 & 1.2340 & -0.000004 & 1.523 & & & & & 999.8395 \\
\hline & & 0 & 0.2500 & 1237.60 & (90) & 0.000232 & 0.000234 & 1236.80 & 0.7957 & -0.000002 & 0.633 & & & & & 999.8395 \\
\hline & & 0 & 0.3000 & 1292.20 & (90) & 0.000246 & 0.000246 & 1292.04 & 0.1639 & 0.000000 & 0.027 & & & & & 999.8395 \\
\hline 0.05 & & 15 & 0.0055 & 1003.70 & (45) & 0.000170 & 0.000196 & 1003.55 & 0.1469 & -0.000026 & 0.022 & & & & & 999.0996 \\
\hline 0.1 & & 15 & 0.0110 & 1008.28 & (45) & 0.000171 & 0.000197 & 1007.99 & 0.2936 & -0.000026 & 0.086 & & & & & 999.0996 \\
\hline 0.5 & & 15 & 0.0526 & 1043.62 & (45) & 0.000189 & 0.000204 & 1042.73 & 0.8938 & -0.000016 & 0.799 & & & & & 999.0996 \\
\hline 1 & & 15 & 0.0999 & 1085.34 & (45) & 0.000205 & 0.000213 & 1084.33 & 1.0076 & -0.000009 & 1.015 & & & & & 999.099 \\
\hline 2 & & 15 & 0.1817 & 1161.89 & (45) & 0.000229 & 0.000230 & 1161.53 & 0.3591 & -0.000001 & 0.129 & & & & & 999.0996 \\
\hline 3 & & 15 & 0.2498 & 1229.87 & (45) & 0.000249 & 0.000246 & 1230.92 & -1.0501 & 0.000003 & 1.103 & & & & & 999.0996 \\
\hline 4 & & 15 & 0.3075 & 1290.63 & (45) & 0.000266 & 0.000261 & 1292.89 & -2.2575 & 0.000004 & 5.096 & & & & & 999.0996 \\
\hline 6 & & 15 & 0.3997 & 1391.93 & (45) & 0.000294 & 0.000288 & 1396.57 & -4.6441 & 0.000006 & 21.568 & $!$ & & & & 999.099 \\
\hline & & 18.4 & 0.3471 & 1333.25 & (133) & 0.000277 & 0.000275 & 1334.79 & -1.5422 & 0.000002 & 2.378 & & & & & 998.5208 \\
\hline & & 18.73 & 0.1945 & 1172.50 & (133) & 0.000237 & 0.000236 & 1172.91 & -0.4120 & 0.000002 & 0.170 & & & & & 998.4577 \\
\hline & & 19.26 & 0.4651 & 1466.46 & (133) & 0.000314 & 0.000313 & 1467.19 & -0.7253 & 0.000001 & 0.526 & & & & & 998.354 \\
\hline & & 19.67 & 0.2461 & 1224.17 & (133) & 0.000251 & 0.000248 & 1224.99 & -0.8187 & 0.000002 & 0.670 & & & & & 998.2717 \\
\hline & & 19.74 & 0.5132 & 1516.43 & (133) & 0.000335 & 0.000332 & 1519.59 & -3.1557 & 0.000003 & 9.958 & & & & & 998.2574 \\
\hline & & 19.76 & 0.3958 & 1388.21 & (133) & 0.000291 & 0.000290 & 1388.60 & -0.3905 & 0.000001 & 0.153 & & & & & 998.2534 \\
\hline & & 19.76 & 0.4954 & 1500.08 & (133) & 0.000325 & 0.000325 & 1500.27 & -0.1913 & 0.000000 & 0.037 & & & & & 998.2534 \\
\hline & & 19.78 & 0.2978 & 1278.92 & (133) & 0.000264 & 0.000262 & 1279.80 & -0.8842 & 0.000002 & 0.782 & & & & & 998.2493 \\
\hline & & 19.82 & 0.427 & 1423.84 & (133) & 0.000301 & 0.000301 & 1423.82 & 0.0162 & 0.000000 & 0.000 & & & & & 998.2411 \\
\hline 0.05 & & 20 & 0.0055 & 1002.77 & (45) & 0.000175 & 0.000199 & 1002.64 & 0.1320 & -0.000024 & 0.017 & & & & & 998.2041 \\
\hline
\end{tabular}


molality molarity $\mathrm{t} /{ }^{\circ} \mathrm{C}$ mass frac Density

0.1

0.02976

0.0994

0.10213

0.19769

0.21251

0.42541

0.66613

0.88035

0.01256

0.0203

$$
0.022
$$

0.02235

0.0239

0.03287

0.0334

0.037

0.045

0.0469

0.05

0.0505

0.059

0.0594

0.06635

0.0667

0.0685

0.0724

0.0816

0.0832 $\mathrm{kg} / \mathrm{m}^{3}$

\section{$\exp$ \\ $\mathrm{kg} / \mathrm{m}^{3}$}

$\begin{array}{lll}20 & 0.0110 & 1007.29 \\ 20 & 0.0200 & 1014.80 \\ 20 & 0.0400 & 1031.60 \\ 20 & 0.0526 & 1042.40 \\ 20 & 0.0800 & 1065.90 \\ 20 & 0.0999 & 1083.87 \\ 20 & 0.1200 & 1101.50 \\ 20 & 0.1600 & 1138.60 \\ 20 & 0.1817 & 1159.97 \\ 20 & 0.2000 & 1177.50 \\ 20 & 0.2498 & 1227.54 \\ 20 & 0.2500 & 1228.40\end{array}$

$0.2500 \quad 1228.40$

$0.3000 \quad 1281.60$

$0.3075 \quad 1287.93$

$\begin{array}{ll}0.3500 & 1337.30\end{array}$

$0.3997 \quad 1388.68$

$0.4000 \quad 1395.70$

$0.0987 \quad 1082.68$

$0.0033 \quad 999.97$

$0.0109 \quad 1006.19$

$0.0112 \quad 1006.37$

$0.0215 \quad 1014.75$

$0.0230 \quad 1016.03$

$0.0451 \quad 1034.39$

$0.0688 \quad 1054.40$

$0.0890 \quad 1071.80$

$0.0014 \quad 998.20$

$0.0022 \quad 998.94$

$0.0024 \quad 999.10$

$0.0025 \quad 999.10$

$0.0026 \quad 999.30$

$0.0036 \quad 1000.05$

$0.0037 \quad 1000.08$

$\begin{array}{ll}0.0041 & 1000.47\end{array}$

$0.0050 \quad 1001.20$

0.00521001 .40

$0.0055 \quad 1001.59$

$0.0056 \quad 1001.65$

$0.0065 \quad 1002.50$

$0.0065 \quad 1002.50$

$0.0073 \quad 1003.09$

$\begin{array}{ll}0.0073 & 1003.18\end{array}$

$0.0075 \quad 1003.30$

$0.0080 \quad 1003.70$

$0.0090 \quad 1004.50$

$0.0091 \quad 1004.70$
Ref

App vol
exp / Calc /

$\begin{array}{cc}\exp / & \text { Calc/ } \\ \mathrm{m}^{3} / \mathrm{kg} & \mathrm{m}^{3} / \mathrm{kg}\end{array}$

200

Density Density App Vol Square of
Inconsistent
calc / Residual / $\mathrm{kg} / \mathrm{m}^{3} \quad \mathrm{~kg} / \mathrm{m}^{3} \quad \mathrm{~m}^{3} / \mathrm{kg}$ Residual

nconsistent

App molal Delta density

Density of

$\begin{array}{llllll}0.000179 & 0.000200 & 1007.05 & 0.2384 & -0.000021 & 0.057\end{array}$

$\begin{array}{llllll}0.000183 & 0.000202 & 1014.41 & 0.3902 & -0.000019 & 0.152\end{array}$

$\begin{array}{lllllll}0.000191 & 0.000205 & 1031.00 & 0.6013 & -0.000014 & 0.362\end{array}$

$\begin{array}{llllll}0.000194 & 0.000207 & 1041.63 & 0.7706 & -0.000013 & 0.594\end{array}$

$\begin{array}{llllll}0.000206 & 0.000213 & 1065.35 & 0.5541 & -0.000006 & 0.307\end{array}$

$\begin{array}{lllllll}0.000209 & 0.000216 & 1083.02 & 0.8465 & -0.000007 & 0.717\end{array}$

$\begin{array}{llllll}0.000219 & 0.000220 & 1101.28 & 0.2247 & -0.000002 & 0.050\end{array}$

$\begin{array}{lllllll}0.000230 & 0.000229 & 1138.80 & -0.1972 & 0.000001 & 0.039\end{array}$

$\begin{array}{lllllll}0.000233 & 0.000234 & 1159.77 & 0.1985 & -0.000001 & 0.039\end{array}$

$\begin{array}{llllll}0.000239 & 0.000238 & 1177.89 & -0.3950 & 0.000001 & 0.156\end{array}$

$\begin{array}{llllll}0.000253 & 0.000249 & 1228.69 & -1.1457 & 0.000003 & 1.313\end{array}$

$\begin{array}{lllllll}0.000251 & 0.000250 & 1228.90 & -0.4988 & 0.000001 & 0.249\end{array}$

$\begin{array}{lllllll}0.000263 & 0.000262 & 1282.07 & -0.4672 & 0.000001 & 0.218\end{array}$

$\begin{array}{llllll}0.000269 & 0.000264 & 1290.17 & -2.2351 & 0.000004 & 4.996\end{array}$

$\begin{array}{llllll}0.000276 & 0.000276 & 1337.02 & 0.2759 & 0.000000 & 0.076\end{array}$

$\begin{array}{lllllll}0.000297 & 0.000292 & 1392.87 & -4.1940 & 0.000005 & 17.590\end{array}$

$\begin{array}{llllll}0.000289 & 0.000292 & 1393.17 & 2.5304 & -0.000003 & 6.403\end{array}$

$\begin{array}{llllll}0.000210 & 0.000216 & 1081.91 & 0.7666 & -0.000007 & 0.588\end{array}$

$\begin{array}{llllll}0.000184 & 0.000201 & 999.92 & 0.0539 & -0.000016 & 0.003\end{array}$

$\begin{array}{llllll}0.000190 & 0.000202 & 1006.05 & 0.1348 & -0.000012 & 0.018\end{array}$

$\begin{array}{llllll}0.000195 & 0.000202 & 1006.29 & 0.0788 & -0.000007 & 0.006\end{array}$

$\begin{array}{llllll}0.000199 & 0.000204 & 1014.64 & 0.1136 & -0.000005 & 0.013\end{array}$

$\begin{array}{llllll}0.000200 & 0.000204 & 1015.93 & 0.1031 & -0.000004 & 0.011\end{array}$

$\begin{array}{llllll}0.000205 & 0.000208 & 1034.23 & 0.1568 & -0.000003 & 0.025\end{array}$

$\begin{array}{llllll}0.000214 & 0.000213 & 1054.49 & -0.0828 & 0.000001 & 0.007\end{array}$

$\begin{array}{llllll}0.000220 & 0.000216 & 1072.11 & -0.3149 & 0.000003 & 0.099\end{array}$

$\begin{array}{lllllll}0.000169 & 0.000201 & 998.16 & 0.0449 & -0.000032 & 0.002\end{array}$

$\begin{array}{lllllll}0.000154 & 0.000201 & 998.84 & 0.1049 & -0.000047 & 0.011\end{array}$

$\begin{array}{llllll}0.000156 & 0.000201 & 998.99 & 0.1097 & -0.000045 & 0.012\end{array}$

$\begin{array}{llllll}0.000171 & 0.000201 & 999.02 & 0.0747 & -0.000030 & 0.006\end{array}$

$\begin{array}{llllll}0.000147 & 0.000201 & 999.16 & 0.1419 & -0.000054 & 0.020\end{array}$

$\begin{array}{llllll}0.000174 & 0.000201 & 999.95 & 0.0979 & -0.000027 & 0.010\end{array}$

$\begin{array}{llllll}0.000179 & 0.000201 & 1000.00 & 0.0832 & -0.000023 & 0.007\end{array}$

$\begin{array}{llllll}0.000163 & 0.000201 & 1000.31 & 0.1555 & -0.000038 & 0.024\end{array}$

$\begin{array}{llllll}0.000165 & 0.000202 & 1001.02 & 0.1801 & -0.000036 & 0.032\end{array}$

$\begin{array}{llllll}0.000161 & 0.000202 & 1001.19 & 0.2126 & -0.000041 & 0.045\end{array}$

$\begin{array}{llllll}0.000178 & 0.000202 & 1001.46 & 0.1295 & -0.000023 & 0.017\end{array}$

$\begin{array}{llllll}0.000175 & 0.000202 & 1001.50 & 0.1473 & -0.000026 & 0.022\end{array}$

$\begin{array}{llllll}0.000164 & 0.000202 & 1002.25 & 0.2468 & -0.000038 & 0.061\end{array}$

$\begin{array}{llllll}0.000170 & 0.000202 & 1002.29 & 0.2116 & -0.000032 & 0.045\end{array}$

$\begin{array}{llllll}0.000176 & 0.000202 & 1002.90 & 0.1919 & -0.000026 & 0.037\end{array}$

$\begin{array}{llllll}0.000168 & 0.000202 & 1002.93 & 0.2517 & -0.000034 & 0.063\end{array}$

$\begin{array}{llllll}0.000174 & 0.000202 & 1003.09 & 0.2108 & -0.000028 & 0.044\end{array}$

$\begin{array}{llllll}0.000169 & 0.000202 & 1003.43 & 0.2679 & -0.000033 & 0.072\end{array}$

$\begin{array}{llllll}0.000174 & 0.000202 & 1004.24 & 0.2593 & -0.000029 & 0.067\end{array}$

$\begin{array}{lllllll}0.000168 & 0.000202 & 1004.38 & 0.3163 & -0.000034 & 0.100\end{array}$

!

apparent

998.2041

998.2041

998.2041

998.2041

998.2041

998.2041

998.2041

998.2041

998.2041

998.2041

998.2041

998.2041

998.2041

998.2041

998.2041

998.2041

998.2041

998.1814

20.47

997.2867

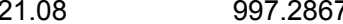

$21.67 \quad 997.2867$

$22.07 \quad 997.2867$

$22.19 \quad 997.2867$

997.2867

$23.72 \quad 997.2867$

997.2867

$1.156 \quad 997.0449$

997.0449

997.0449

$2.051 \quad 997.0449$

997.0449

$3.003 \quad 997.0449$

997.0449

997.0449

997.0449

997.0449

997.0449

$4.607 \quad 997.0449$

997.0449

997.0449

18.64

997.0449

997.0449

997.0449

18.65

997.0449 
molality molarity

$\mathrm{t} /{ }^{\circ} \mathrm{C}$ mass frac

Density

$\exp /$

0.0837

0.0975

0.0999

0.1

0.101

0.1208

0.1301

0.13065

0.1317

0.1406

0.1439

0.1666

0.1854

0.1891

0.1948

0.19623

0.2023

0.2046

0.2515

0.2535

0.2629

0.26325

0.2633

0.2926

0.328

0.3334

0.3372

0.3488

0.3936

0.4007

0.406

0.4164

0.4869

0.4993

0.5

0.5026

0.5295

0.5542

0.5734

0.5843

0.5942

0.6043

0.6139

0.6665

0.6696

0.7154

$\begin{array}{cccc} & & \mathrm{kg} / \mathrm{m}^{3} & \\ 25 & 0.0092 & 1004.60 & (136) \\ 25 & 0.0107 & 1005.90 & (136) \\ 25 & 0.0110 & 1006.13 & (57) \\ 25 & 0.0110 & 1006.10 & (45) \\ 25 & 0.0111 & 1006.21 & (28) \\ 25 & 0.0132 & 1008.13 & (123) \\ 25 & 0.0142 & 1009.00 & (123) \\ 25 & 0.0143 & 1008.85 & (87) \\ 25 & 0.0144 & 1009.00 & (136) \\ 25 & 0.0154 & 1009.70 & (136) \\ 25 & 0.0157 & 1010.10 & (136) \\ 25 & 0.0182 & 1012.04 & (57) \\ 25 & 0.0202 & 1013.80 & (136) \\ 25 & 0.0206 & 1014.10 & (136) \\ 25 & 0.0212 & 1014.60 & (136) \\ 25 & 0.0213 & 1014.67 & (87) \\ 25 & 0.0220 & 1015.45 & (123) \\ 25 & 0.0222 & 1015.50 & (136) \\ 25 & 0.0272 & 1019.58 & (28) \\ 25 & 0.0274 & 1019.70 & (136) \\ 25 & 0.0284 & 1020.83 & (123) \\ 25 & 0.0284 & 1020.55 & (87) \\ 25 & 0.0284 & 1020.60 & (136) \\ 25 & 0.0315 & 1023.20 & (136) \\ 25 & 0.0351 & 1026.18 & (87) \\ 25 & 0.0357 & 1026.59 & (57) \\ 25 & 0.0361 & 1027.37 & (123) \\ 25 & 0.0373 & 1028.00 & (136) \\ 25 & 0.0419 & 1032.00 & (136) \\ 25 & 0.0426 & 1032.50 & (136) \\ 25 & 0.0431 & 1033.37 & (123) \\ 25 & 0.0442 & 1033.90 & (136) \\ 25 & 0.0513 & 1039.80 & (136) \\ 25 & 0.0525 & 1040.68 & (57) \\ 25 & 0.0526 & 1041.00 & (45) \\ 25 & 0.0528 & 1041.26 & (28) \\ 25 & 0.0555 & 1043.50 & (136) \\ 25 & 0.0579 & 1045.50 & (136) \\ 25 & 0.0598 & 1047.20 & (136) \\ 25 & 0.0609 & 1048.10 & (136) \\ 25 & 0.0619 & 1049.00 & (136) \\ 25 & 0.0629 & 1049.70 & (136) \\ 25 & 0.0638 & 1050.60 & (136) \\ 25 & 0.0689 & 1054.96 & (57) \\ 25 & 0.0692 & 1055.20 & (136) \\ 25 & 0.0736 & 1059.00 & (136)\end{array}$

$\begin{array}{lll}0.0736 & 1059.00 \quad(136)\end{array}$
App vol App vol D

$\begin{array}{cc}\exp / & \text { Calc / } \\ \mathrm{m}^{3} / \mathrm{kg} & \mathrm{m}^{3} / \mathrm{kg}\end{array}$

Density App Vol Square of
Inconsistent
calc / Residual / Residual / Density on

Inconsistent App molal Delta density Density of

$\mathrm{m}^{3} / \mathrm{kg} \quad \mathrm{m}^{3} / \mathrm{kg}$

$\mathrm{kg} / \mathrm{m}^{3}$

$\mathrm{kg} / \mathrm{m}^{3}$

$\mathrm{m}^{3} / \mathrm{kg}$ Residual density

$\begin{array}{llllll}0.000183 & 0.000202 & 1004.43 & 0.1748 & -0.000019 & 0.031\end{array}$

$\begin{array}{llllll}0.000178 & 0.000203 & 1005.64 & 0.2635 & -0.000024 & 0.069\end{array}$

$\begin{array}{llllll}0.000177 & 0.000203 & 1005.85 & 0.2830 & -0.000025 & 0.080\end{array}$

$\begin{array}{llllll}0.000181 & 0.000203 & 1005.86 & 0.2442 & -0.000022 & 0.060\end{array}$

$\begin{array}{lllllll}0.000179 & 0.000203 & 1005.94 & 0.2654 & -0.000024 & 0.070\end{array}$

$\begin{array}{lllllll}0.000169 & 0.000203 & 1007.68 & 0.4507 & -0.000034 & 0.203\end{array}$

$\begin{array}{llllll}0.000168 & 0.000203 & 1008.49 & 0.5078 & -0.000035 & 0.258\end{array}$

$\begin{array}{llllll}0.000182 & 0.000203 & 1008.54 & 0.3128 & -0.000022 & 0.098\end{array}$

$\begin{array}{llllll}0.000178 & 0.000203 & 1008.63 & 0.3681 & -0.000025 & 0.136\end{array}$

$\begin{array}{llllll}0.000185 & 0.000203 & 1009.41 & 0.2902 & -0.000019 & 0.084\end{array}$

$\begin{array}{llllll}0.000178 & 0.000203 & 1009.70 & 0.4019 & -0.000025 & 0.162\end{array}$

$\begin{array}{llllll}0.000184 & 0.000204 & 1011.68 & 0.3613 & -0.000019 & 0.131\end{array}$

$\begin{array}{llllll}0.000181 & 0.000204 & 1013.32 & 0.4842 & -0.000023 & 0.234\end{array}$

$\begin{array}{llllll}0.000182 & 0.000204 & 1013.64 & 0.4623 & -0.000022 & 0.214\end{array}$

$\begin{array}{lllllll}0.000183 & 0.000204 & 1014.13 & 0.4667 & -0.000021 & 0.218\end{array}$

$\begin{array}{lllllll}0.000186 & 0.000204 & 1014.26 & 0.4083 & -0.000019 & 0.167\end{array}$

$\begin{array}{lllllll}0.000175 & 0.000205 & 1014.79 & 0.6610 & -0.000029 & 0.437\end{array}$

$\begin{array}{llllll}0.000182 & 0.000205 & 1014.98 & 0.5151 & -0.000023 & 0.265\end{array}$

$\begin{array}{lllllll}0.000187 & 0.000205 & 1019.05 & 0.5308 & -0.000019 & 0.282\end{array}$

$\begin{array}{lllllll}0.000189 & 0.000206 & 1019.22 & 0.4780 & -0.000017 & 0.228\end{array}$

$\begin{array}{lllllll}0.000179 & 0.000206 & 1020.03 & 0.7921 & -0.000027 & 0.627\end{array}$

$\begin{array}{lllllll}0.000189 & 0.000206 & 1020.06 & 0.4843 & -0.000016 & 0.235\end{array}$

$\begin{array}{llllll}0.000188 & 0.000206 & 1020.07 & 0.5311 & -0.000018 & 0.282\end{array}$

$\begin{array}{llllll}0.000188 & 0.000206 & 1022.60 & 0.6040 & -0.000018 & 0.365\end{array}$

$\begin{array}{llllll}0.000192 & 0.000207 & 1025.64 & 0.5399 & -0.000015 & 0.292\end{array}$

$\begin{array}{lllllll}0.000194 & 0.000207 & 1026.10 & 0.4866 & -0.000013 & 0.237\end{array}$

$\begin{array}{lllllll}0.000182 & 0.000207 & 1026.43 & 0.9397 & -0.000025 & 0.883\end{array}$

$\begin{array}{lllllll}0.000193 & 0.000207 & 1027.42 & 0.5763 & -0.000015 & 0.332\end{array}$

$\begin{array}{llllll}0.000191 & 0.000208 & 1031.25 & 0.7463 & -0.000017 & 0.557\end{array}$

$\begin{array}{lllllll}0.000194 & 0.000208 & 1031.86 & 0.6408 & -0.000014 & 0.411\end{array}$

$\begin{array}{llllll}0.000185 & 0.000208 & 1032.31 & 1.0623 & -0.000023 & 1.129\end{array}$

$\begin{array}{llllll}0.000194 & 0.000209 & 1033.20 & 0.7034 & -0.000015 & 0.495\end{array}$

$\begin{array}{lllllll}0.000199 & 0.000210 & 1039.18 & 0.6226 & -0.000011 & 0.388\end{array}$

$\begin{array}{llllll}0.000202 & 0.000210 & 1040.23 & 0.4549 & -0.000008 & 0.207\end{array}$

$\begin{array}{lllllll}0.000197 & 0.000210 & 1040.28 & 0.7158 & -0.000013 & 0.512\end{array}$

$\begin{array}{llllll}0.000197 & 0.000210 & 1040.50 & 0.7581 & -0.000013 & 0.575\end{array}$

$\begin{array}{lll}0.000199 & 0.000211 & 1042.77\end{array}$

$\begin{array}{lll}0.000201 & 0.000211 & 1044.85\end{array}$

$\begin{array}{lll}0.000200 & 0.000211 & 1046.46\end{array}$

$\begin{array}{lll}0.000201 & 0.000212 & 1047.37\end{array}$

$\begin{array}{lll}0.000200 & 0.000212 & 1048.20\end{array}$

$\begin{array}{lll}0.000203 & 0.000212 & 1049.05\end{array}$

$\begin{array}{lll}0.000201 & 0.000212 & 1049.85\end{array}$

$\begin{array}{lll}0.000204 & 0.000213 & 1054.23\end{array}$

$\begin{array}{lll}0.000204 & 0.000213 & 1054.49\end{array}$

$\begin{array}{lll}0.000205 & 0.000214 & 1058.28\end{array}$ $\begin{array}{lll}0.7284 & -0.000012 & 0.530\end{array}$

$\begin{array}{lll}0.6511 & -0.000010 & 0.424\end{array}$

$\begin{array}{lll}0.7399 & -0.000011 & 0.547\end{array}$

$\begin{array}{lll}0.7265 & -0.000011 & 0.528\end{array}$

$\begin{array}{lll}0.7978 & -0.000012 & 0.636\end{array}$

$\begin{array}{lll}0.6531 & -0.000009 & 0.427\end{array}$

$\begin{array}{lll}0.7510 & -0.000011 & 0.564\end{array}$

$\begin{array}{lll}0.7297 & -0.000010 & 0.532\end{array}$

$\begin{array}{lll}0.7122 & -0.000009 & 0.507\end{array}$

$\begin{array}{lll}0.7167 & -0.000009 & 0.514\end{array}$ $\mathrm{kg} / \mathrm{m}^{3}$

997.0449

997.0449

997.0449

997.0449

9.164997 .0449

997.0449

997.0449

$11.808 \quad 997.0449$

997.0449

997.0449

997.0449

997.0449

997.0449

997.0449

997.0449

19.46

997.0449

997.0449

$22.535 \quad 997.0449$

997.0449

19.85

997.0449

$23.504 \quad 997.0449$

997.0449

997.0449

$29.135 \quad 997.0449$

997.0449

997.0449

997.0449

997.0449

997.0449

20.56

997.0449

997.0449

997.0449

997.0449

997.0449

$44.217 \quad 997.0449$

997.0449

997.0449

997.0449

997.0449

997.0449

997.0449

997.0449

997.0449

997.0449

997.0449 
molality molarity

$1{ }^{\circ} \mathrm{C} \mathrm{mas}$

0.775

0.789

0.8528

0.8673

0.9929

1.005

1.0913

1.1578

1.1607

1.1891

1.3226

1.44
1.4754

1.5001

1.5227

1.5406

1.6663

1.6825

1.71

1.7557

1.7575

1.7818

1.8788

1.9034

1.9407

1.979

1.9995

2.006

2.0929

2.1063

2.1167

2.2115

2.226

2.4106

2.5

2.507

2.5885
2.638

2.787

2.8391

2.9091

2.9583

3.017

$\begin{array}{lrrr} & & \mathrm{kg} / \mathrm{m}^{3} & \\ & & \\ 25 & 0.0792 & 1063.90 & (136) \\ 25 & 0.0805 & 1065.10 & (136) \\ 25 & 0.0865 & 1070.30 & (136) \\ 25 & 0.0878 & 1071.40 & (136) \\ 25 & 0.0993 & 1081.11 & (57) \\ 25 & 0.0999 & 1082.22 & (45) \\ 25 & 0.1004 & 1082.47 & (28) \\ 25 & 0.1080 & 1089.40 & (136) \\ 25 & 0.1139 & 1094.70 & (136) \\ 25 & 0.1141 & 1094.90 & (136) \\ 25 & 0.1166 & 1097.10 & (136) \\ 25 & 0.1280 & 1107.50 & (136) \\ 25 & 0.1378 & 1116.50 & (136) \\ 25 & 0.1407 & 1119.20 & (136) \\ 25 & 0.1427 & 1121.11 & (57) \\ 25 & 0.1446 & 1122.70 & (136) \\ 25 & 0.1460 & 1124.10 & (136) \\ 25 & 0.1561 & 1133.60 & (57) \\ 25 & 0.1574 & 1134.70 & (136) \\ 25 & 0.1595 & 1136.80 & (136) \\ 25 & 0.1631 & 1140.00 & (136) \\ 25 & 0.1632 & 1140.20 & (136) \\ 25 & 0.1651 & 1142.10 & (136) \\ 25 & 0.1725 & 1149.10 & (136) \\ 25 & 0.1744 & 1150.90 & (136) \\ 25 & 0.1772 & 1153.60 & (136) \\ 25 & 0.1801 & 1156.30 & (136) \\ 25 & 0.1816 & 1157.96 & (57) \\ 25 & 0.1817 & 1157.99 & (45) \\ 25 & 0.1821 & 1158.21 & (28) \\ 25 & 0.1885 & 1164.50 & (136) \\ 25 & 0.1895 & 1165.40 & (136) \\ 25 & 0.1902 & 1166.20 & (136) \\ 25 & 0.1971 & 1172.80 & (136) \\ 25 & 0.1981 & 1173.90 & (136) \\ 25 & 0.2111 & 1186.60 & (136) \\ 25 & 0.2172 & 1192.27 & (57) \\ 25 & 0.2177 & 1193.20 & (136) \\ 25 & 0.2232 & 1198.70 & (136) \\ 25 & 0.2265 & 1202.10 & (136) \\ 25 & 0.2363 & 1212.00 & (136) \\ 25 & 0.2396 & 1215.40 & (136) \\ 25 & 0.2441 & 1220.10 & (136) \\ 25 & 0.2472 & 1223.20 & (136) \\ 25 & 0.2498 & 1225.24 & (45) \\ 25 & 0.2509 & 1227.01 & (28)\end{array}$

(28)
App vol App vol De

exp / Calc /

ensity

Density

pp Vol Square of calc / Residual / Residual / Density based on

nconsistent App molal Delta density Density o

$\mathrm{m}^{3} / \mathrm{kg} \quad \mathrm{m}^{3} / \mathrm{kg}$

$$
\mathrm{kg} / \mathrm{m}^{3}
$$$$
\mathrm{kg} / \mathrm{m}^{3}
$$

$\mathrm{m}^{3} / \mathrm{kg}$ Residual

based on
density

based on

$/ \mathrm{kg} / \mathrm{m}^{3} \quad$ water

$\begin{array}{llllll}0.000208 & 0.000215 & 1063.23 & 0.6704 & -0.000007 & 0.449\end{array}$

$\begin{array}{llllll}0.000207 & 0.000215 & 1064.35 & 0.7533 & -0.000008 & 0.568\end{array}$

$\begin{array}{lllllll}0.000209 & 0.000216 & 1069.57 & 0.7330 & -0.000007 & 0.537\end{array}$

$\begin{array}{llllll}0.000210 & 0.000217 & 1070.75 & 0.6511 & -0.000006 & 0.424\end{array}$

$\begin{array}{lllllll}0.000217 & 0.000219 & 1080.91 & 0.1955 & -0.000002 & 0.038\end{array}$

$\begin{array}{llllll}0.000213 & 0.000219 & 1081.49 & 0.7347 & -0.000006 & 0.540\end{array}$

$\begin{array}{llllll}0.000214 & 0.000219 & 1081.89 & 0.5799 & -0.000005 & 0.336\end{array}$

$\begin{array}{llllll}0.000216 & 0.000221 & 1088.79 & 0.6109 & -0.000005 & 0.373\end{array}$

$\begin{array}{llllll}0.000217 & 0.000222 & 1094.07 & 0.6335 & -0.000005 & 0.401\end{array}$

$\begin{array}{llllll}0.000218 & 0.000222 & 1094.30 & 0.6041 & -0.000004 & 0.365\end{array}$

$\begin{array}{llllll}0.000218 & 0.000222 & 1096.54 & 0.5619 & -0.000004 & 0.316\end{array}$

$\begin{array}{llllll}0.000222 & 0.000225 & 1106.99 & 0.5091 & -0.000003 & 0.259\end{array}$

$\begin{array}{lllllll}0.000224 & 0.000227 & 1116.06 & 0.4355 & -0.000003 & 0.190\end{array}$

$\begin{array}{lllllll}0.000225 & 0.000227 & 1118.78 & 0.4212 & -0.000002 & 0.177\end{array}$

$\begin{array}{llllll}0.000225 & 0.000228 & 1120.67 & 0.4433 & -0.000002 & 0.196\end{array}$

$\begin{array}{llllll}0.000227 & 0.000228 & 1122.39 & 0.3102 & -0.000002 & 0.096\end{array}$

$\begin{array}{llllll}0.000227 & 0.000229 & 1123.75 & 0.3483 & -0.000002 & 0.121\end{array}$

$\begin{array}{llllll}0.000229 & 0.000231 & 1133.24 & 0.3569 & -0.000002 & 0.127\end{array}$

$\begin{array}{llllll}0.000230 & 0.000231 & 1134.46 & 0.2429 & -0.000001 & 0.059\end{array}$

$\begin{array}{lllllll}0.000230 & 0.000231 & 1136.51 & 0.2867 & -0.000001 & 0.082\end{array}$

$\begin{array}{llllll}0.000232 & 0.000232 & 1139.92 & 0.0831 & 0.000000 & 0.007\end{array}$

$\begin{array}{llllll}0.000231 & 0.000232 & 1140.05 & 0.1494 & -0.000001 & 0.022\end{array}$

$\begin{array}{llllll}0.000231 & 0.000233 & 1141.85 & 0.2467 & -0.000001 & 0.061\end{array}$

$\begin{array}{llllll}0.000234 & 0.000234 & 1149.00 & 0.0973 & 0.000000 & 0.009\end{array}$

$\begin{array}{llllll}0.000234 & 0.000235 & 1150.80 & 0.0960 & 0.000000 & 0.009\end{array}$

$\begin{array}{llllll}0.000235 & 0.000235 & 1153.53 & 0.0739 & 0.000000 & 0.005\end{array}$

$\begin{array}{llllll}0.000236 & 0.000236 & 1156.31 & -0.0098 & 0.000000 & 0.000\end{array}$

$\begin{array}{llllll}0.000236 & 0.000236 & 1157.80 & 0.1650 & -0.000001 & 0.027\end{array}$

$\begin{array}{llllll}0.000236 & 0.000236 & 1157.83 & 0.1588 & -0.000001 & 0.025\end{array}$

$\begin{array}{llllll}0.000237 & 0.000236 & 1158.27 & -0.0524 & 0.000000 & 0.003\end{array}$

$\begin{array}{llllll}0.000238 & 0.000238 & 1164.52 & -0.0201 & 0.000000 & 0.000\end{array}$

$\begin{array}{llllll}0.000238 & 0.000238 & 1165.48 & -0.0794 & 0.000000 & 0.006\end{array}$

$\begin{array}{llllll}0.000238 & 0.000238 & 1166.22 & -0.0229 & 0.000000 & 0.001\end{array}$

$\begin{array}{llllll}0.000240 & 0.000240 & 1172.96 & -0.1614 & 0.000001 & 0.026\end{array}$

$\begin{array}{lllllll}0.000240 & 0.000240 & 1173.99 & -0.0929 & 0.000000 & 0.009\end{array}$

$\begin{array}{llllll}0.000244 & 0.000243 & 1186.89 & -0.2863 & 0.000001 & 0.082\end{array}$

$\begin{array}{llllll}0.000247 & 0.000244 & 1193.04 & -0.7693 & 0.000002 & 0.592\end{array}$

$\begin{array}{llllll}0.000246 & 0.000244 & 1193.54 & -0.3390 & 0.000001 & 0.115\end{array}$

$\begin{array}{lllllll}0.000247 & 0.000246 & 1199.07 & -0.3700 & 0.000001 & 0.137\end{array}$

$\begin{array}{lllllll}0.000248 & 0.000247 & 1202.42 & -0.3170 & 0.000001 & 0.100\end{array}$

$\begin{array}{llllll}0.000250 & 0.000249 & 1212.38 & -0.3795 & 0.000001 & 0.144\end{array}$

$\begin{array}{llllll}0.000251 & 0.000250 & 1215.82 & -0.4235 & 0.000001 & 0.179\end{array}$

$\begin{array}{lllllll}0.000252 & 0.000251 & 1220.42 & -0.3185 & 0.000001 & 0.101\end{array}$

$\begin{array}{llllll}0.000253 & 0.000252 & 1223.63 & -0.4262 & 0.000001 & 0.182\end{array}$

$\begin{array}{llllll}0.000255 & 0.000252 & 1226.33 & -1.0908 & 0.000003 & 1.190\end{array}$

$\begin{array}{llllll}0.000254 & 0.000253 & 1227.43 & -0.4227 & 0.000001 & 0.179\end{array}$ $\mathrm{kg} / \mathrm{m}^{3}$

997.0449

997.0449

997.0449

997.0449

997.0449

997.0449

$85.422 \quad 997.0449$

997.0449

997.0449

997.0449

997.0449

997.0449

997.0449

997.0449

997.0449

997.0449

997.0449

997.0449

997.0449

997.0449

997.0449

997.0449

997.0449

97.0449

997.0449

997.0449

997.0449

997.0449

997.0449

$161.168 \quad 997.0449$

997.0449

997.0449

997.0449

997.0449

997.0449

997.0449

997.0449

997.0449

997.0449

997.0449

997.0449

997.0449

997.0449

997.0449

997.0449

$229.962 \quad 997.0449$ 
molality molarity

$\mathrm{t} /{ }^{\circ} \mathrm{C}$ mass frac Density Ref

$\exp /$
$\mathrm{kg} / \mathrm{m}^{3}$

3.0368

3.1438

3.2268

3.3332

3.4443

3.5941

3.6237

3.8589

3.9093

3.9571

3.9661

4.2137

4.6976

4.7625

4.8394

4.8583

4.98

5.0004

5.3599
5.3683

5.6189

5.7097

5.8689

6
1506

6.2514

6.2858

6.6584

6.8525

7.0766

7.333

7.3414

7.4589

7.8783

$\begin{array}{rrrr} & & \mathrm{kg} / \mathrm{m}^{3} & \\ 25 & 0.2521 & 1227.49 & (57) \\ 25 & 0.2587 & 1235.10 & (136) \\ 25 & 0.2637 & 1240.30 & (136) \\ 25 & 0.2700 & 1247.32 & (57) \\ 25 & 0.2766 & 1254.00 & (136) \\ 25 & 0.2852 & 1263.20 & (136) \\ 25 & 0.2868 & 1265.00 & (136) \\ 25 & 0.2999 & 1279.10 & (136) \\ 25 & 0.3026 & 1282.00 & (136) \\ 25 & 0.3052 & 1284.80 & (136) \\ 25 & 0.3057 & 1285.30 & (136) \\ 25 & 0.3075 & 1285.25 & (45) \\ 25 & 0.3075 & 1288.69 & (57) \\ 25 & 0.3187 & 1299.60 & (136) \\ 25 & 0.3427 & 1326.89 & (57) \\ 25 & 0.3458 & 1329.70 & (136) \\ 25 & 0.3494 & 1333.90 & (136) \\ 25 & 0.3503 & 1334.90 & (136) \\ 25 & 0.3560 & 1341.48 & (28) \\ 25 & 0.3569 & 1343.08 & (57) \\ 25 & 0.3730 & 1360.40 & (136) \\ 25 & 0.3734 & 1360.90 & (136) \\ 25 & 0.3841 & 1372.90 & (136) \\ 25 & 0.3879 & 1377.20 & (136) \\ 25 & 0.3944 & 1384.60 & (136) \\ 25 & 0.3997 & 1385.50 & (45) \\ 25 & 0.4057 & 1397.30 & (136) \\ 25 & 0.4096 & 1401.80 & (136) \\ 25 & 0.4110 & 1403.04 & (57) \\ 25 & 0.4250 & 1419.00 & (136) \\ 25 & 0.4320 & 1426.80 & (136) \\ 25 & 0.4399 & 1435.70 & (136) \\ 25 & 0.4487 & 1445.40 & (136) \\ 25 & 0.4490 & 1445.80 & (136) \\ 25 & 0.4529 & 1450.20 & (136) \\ 25 & 0.4665 & 1465.00 & (136) \\ 28.47 & 0.3471 & 1327.35 & (133) \\ 28.92 & 0.1945 & 1168.13 & (133) \\ 29.31 & 0.4651 & 1459.53 & (133) \\ 29.65 & 0.2461 & 1219.33 & (133) \\ 29.68 & 0.5132 & 1509.46 & (133) \\ 29.75 & 0.3958 & 1381.79 & (133) \\ 29.78 & 0.2978 & 1273.59 & (133) \\ 29.78 & 0.4954 & 1493.08 & (133) \\ 29.87 & 0.427 & 1417.08 & (133) \\ 30 & 0.0055 & 1000.17 & (45)\end{array}$

$\begin{array}{lll}30 & 0.0055 & 1000.17\end{array}$
App vol App vol Den

$\begin{array}{cc}\exp / & \text { Calc / } \\ \mathrm{m}^{3} / \mathrm{kg} & \mathrm{m}^{3} / \mathrm{kg}\end{array}$

Density

Density

calc / Residual / Residual / Density based on

Inconsistent App molal Delta density Density o $\mathrm{kg} / \mathrm{m}^{3}$ $\mathrm{kg} / \mathrm{m}^{3}$ $\mathrm{m}^{3} / \mathrm{kg}$ Residy density

$\begin{array}{llllll}0.000256 & 0.000253 & 1228.71 & -1.2168 & 0.000003 & 1.48\end{array}$

$\begin{array}{llllll}0.000256 & 0.000254 & 1235.56 & -0.4582 & 0.000001 & 0.210\end{array}$

$\begin{array}{lllllll}0.000257 & 0.000256 & 1240.81 & -0.5146 & 0.000001 & 0.265\end{array}$

$\begin{array}{llllll}0.000258 & 0.000257 & 1247.48 & -0.1591 & 0.000000 & 0.025\end{array}$

$\begin{array}{lllllll}0.000260 & 0.000259 & 1254.35 & -0.3500 & 0.000001 & 0.123\end{array}$

$\begin{array}{llllll}0.000262 & 0.000261 & 1263.47 & -0.2732 & 0.000001 & 0.075\end{array}$

$\begin{array}{llllll}0.000262 & 0.000262 & 1265.26 & -0.2568 & 0.000001 & 0.066\end{array}$

$\begin{array}{llllll}0.000265 & 0.000265 & 1279.21 & -0.1086 & 0.000000 & 0.012\end{array}$

$\begin{array}{lllllll}0.000266 & 0.000266 & 1282.15 & -0.1475 & 0.000000 & 0.022\end{array}$

$\begin{array}{lllllll}0.000267 & 0.000267 & 1284.92 & -0.1185 & 0.000000 & 0.014\end{array}$

$\begin{array}{llllll}0.000267 & 0.000267 & 1285.44 & -0.1384 & 0.000000 & 0.019\end{array}$

$\begin{array}{lllllll}0.000271 & 0.000267 & 1287.39 & -2.1419 & 0.000004 & 4.588\end{array}$

$\begin{array}{llllll}0.000265 & 0.000267 & 1287.39 & 1.2981 & -0.000003 & 1.685\end{array}$

$\begin{array}{llllll}0.000270 & 0.000270 & 1299.52 & 0.0767 & 0.000000 & 0.006\end{array}$

$\begin{array}{llllll}0.000275 & 0.000277 & 1325.85 & 1.0386 & -0.000002 & 1.079\end{array}$

$\begin{array}{llllll}0.000277 & 0.000278 & 1329.26 & 0.4355 & -0.000001 & 0.190\end{array}$

$\begin{array}{llllll}0.000278 & 0.000279 & 1333.27 & 0.6268 & -0.000001 & 0.393\end{array}$

$\begin{array}{llllll}0.000278 & 0.000279 & 1334.25 & 0.6474 & -0.000001 & 0.419\end{array}$

$\begin{array}{llllll}0.000280 & 0.000281 & 1340.50 & 0.9720 & -0.000002 & 0.945\end{array}$

$\begin{array}{lllllll}0.000279 & 0.000281 & 1341.54 & 1.5375 & -0.000002 & 2.364\end{array}$

$\begin{array}{llllll}0.000285 & 0.000286 & 1359.41 & 0.9854 & -0.000001 & 0.971\end{array}$

$\begin{array}{llllll}0.000285 & 0.000286 & 1359.82 & 1.0774 & -0.000002 & 1.161\end{array}$

$\begin{array}{llllll}0.000288 & 0.000290 & 1371.80 & 1.1048 & -0.000002 & 1.221\end{array}$

$\begin{array}{llllll}0.000289 & 0.000291 & 1376.04 & 1.1605 & -0.000002 & 1.347\end{array}$

$\begin{array}{llllll}0.000291 & 0.000293 & 1383.36 & 1.2372 & -0.000002 & 1.531\end{array}$

$\begin{array}{llllll}0.000299 & 0.000295 & 1389.28 & -3.7817 & 0.000005 & 14.301\end{array}$

$\begin{array}{lllllll}0.000295 & 0.000297 & 1395.96 & 1.3418 & -0.000002 & 1.801\end{array}$

$\begin{array}{llllll}0.000296 & 0.000298 & 1400.35 & 1.4455 & -0.000002 & 2.090\end{array}$

$\begin{array}{llllll}0.000297 & 0.000298 & 1401.84 & 1.1984 & -0.000001 & 1.436\end{array}$

$\begin{array}{llllll}0.000301 & 0.000303 & 1417.53 & 1.4722 & -0.000002 & 2.167\end{array}$

$\begin{array}{llllll}0.000304 & 0.000305 & 1425.40 & 1.4005 & -0.000002 & 1.961\end{array}$

$\begin{array}{llllll}0.000306 & 0.000308 & 1434.24 & 1.4604 & -0.000002 & 2.133\end{array}$

$\begin{array}{llllll}0.000310 & 0.000311 & 1444.04 & 1.3649 & -0.000001 & 1.863\end{array}$

$\begin{array}{llllll}0.000310 & 0.000311 & 1444.35 & 1.4496 & -0.000002 & 2.101\end{array}$

$\begin{array}{llllll}0.000311 & 0.000313 & 1448.72 & 1.4768 & -0.000002 & 2.181\end{array}$

$\begin{array}{llllll}0.000316 & 0.000317 & 1463.78 & 1.2219 & -0.000001 & 1.493\end{array}$

$\begin{array}{llllll}0.000282 & 0.000280 & 1328.56 & -1.2052 & 0.000002 & 1.453\end{array}$

$\begin{array}{llllll}0.000243 & 0.000241 & 1168.73 & -0.6016 & 0.000002 & 0.362\end{array}$

$\begin{array}{lllllll}0.000318 & 0.000319 & 1458.74 & 0.7932 & -0.000001 & 0.629\end{array}$

$\begin{array}{llllll}0.000256 & 0.000254 & 1220.25 & -0.9168 & 0.000003 & 0.841\end{array}$

$\begin{array}{llllll}0.000338 & 0.000338 & 1510.23 & -0.7712 & 0.000001 & 0.595\end{array}$

$\begin{array}{llllll}0.000295 & 0.000296 & 1381.60 & 0.1946 & 0.000000 & 0.038\end{array}$

$\begin{array}{llllll}0.000268 & 0.000267 & 1274.36 & -0.7734 & 0.000002 & 0.598\end{array}$

$\begin{array}{rrrrrr}0.000329 & 0.000331 & 1491.25 & 1.8315 & -0.000002 & 3.354\end{array}$

$\begin{array}{llllll}0.000305 & 0.000306 & 1416.20 & 0.8818 & -0.000001 & 0.778\end{array}$

$\begin{array}{llllll}0.000181 & 0.000204 & 1000.05 & 0.1240 & -0.000022 & 0.015\end{array}$ $\mathrm{kg} / \mathrm{m}^{3}$

997.0449

997.0449

997.0449

997.0449

997.0449

997.0449

997.0449

997.0449

997.0449

997.0449

997.0449

997.0449

997.0449

997.0449

997.0449

997.0449

997.0449

997.0449

344.431997 .0449

997.0449

997.0449

997.0449

997.0449

997.0449

997.0449

997.0449

997.0449

997.0449

997.0449

997.0449

997.0449

997.0449

997.0449

997.0449

997.0449

997.0449

96.0989

995.9682

995.8535

995.7524

995.7434

995.7225

995.7135

995.7135

995.6864

995.6473 
molality molarity

$\mathrm{t} /{ }^{\circ} \mathrm{C}$ mass frac Density Ref

$\exp /$
$\mathrm{kg} / \mathrm{m}^{3}$

0.1

0.5

2

4

6

0.05

$\begin{array}{lll}0.0400 & 1028.60 & (90)\end{array}$

$\begin{array}{lll}0.0526 & 1039.37 & (45) \\ 0.0800 & 1062.60 & (90)\end{array}$

$0.0999 \quad 1080.40 \quad(45)$

$\begin{array}{lll}0.1200 & 1097.80 \quad(90)\end{array}$

$\begin{array}{lll}0.1600 & 1134.50 \quad(90)\end{array}$

$0.1817 \quad 1155.85$

$0.2000 \quad 1173.00$

$0.2498 \quad 1222.81$

$0.2500 \quad 1223.60$

$0.3000 \quad 1276.40$

$0.3075 \quad 1282.51$

$0.3500 \quad 1331.60$

$0.3997 \quad 1382.30$

$0.4000 \quad 1389.50$

$0.0987 \quad 1079.10$

$0.0055 \quad 998.55$

$0.0110 \quad 1003.02$

$0.0526 \quad 1037.60$

$0.0999 \quad 1078.50$

$0.1817 \quad 1153.68$

$0.2498 \quad 1220.37$

$0.3075 \quad 1279.80$

$\begin{array}{ll}0.3997 & 1379.11\end{array}$

\section{App vol App vol}

$\begin{array}{cc}\exp / & \text { Calc / } \\ \mathrm{m}^{3} / \mathrm{kg} & \mathrm{m}^{3} / \mathrm{kg}\end{array}$

$$
\mathrm{m}^{3} / \mathrm{kg}
$$

$\mathrm{m}^{3} / \mathrm{kg}$

0.0001840 .000205

$0.000193 \quad 0.000206$

$0.000200 \quad 0.000210$

$0.0200 \quad 1012.00 \quad(90)$

$\begin{array}{ll}0.3471 & 1321.38 \\ 0.1945 & 1163.44\end{array}$

$0.4651 \quad 1452.66$

$\begin{array}{ll}0.4651 & 1452.66 \\ 0.5132 & 1502.58\end{array}$

$0.2461 \quad 1214.26$

$0.3958 \quad 1375.32$

$0.4954 \quad 1486.16$

$0.2978 \quad 1268.11$

$0.427 \quad 1410.39$

$0.0200 \quad 1008.40$

$0.0400 \quad 1024.90$

$0.0800 \quad 1058.60$

$0.1200 \quad 1093.70$

$0.1600 \quad 1130.10$

$0.2000 \quad 1168.40$

$0.2500 \quad 1218.60$

$0.3000 \quad 1270.90$

$0.3500 \quad 1325.50$

$0.4000 \quad 1382.60$

$0.0987 \quad 1074.90$
$0.000201 \quad 0.000212$

$0.000213 \quad 0.000217$

$0.000216 \quad 0.000221$

$0.000226 \quad 0.000225$

$\begin{array}{ll}0.000236 & 0.000234\end{array}$

$0.000238 \quad 0.000239$

$0.000245 \quad 0.000243$

$0.000257 \quad 0.000255$

$0.000256 \quad 0.000255$

$0.000268 \quad 0.000268$

$0.000274 \quad 0.000270$

$\begin{array}{ll}0.000280 & 0.000282\end{array}$

$\begin{array}{ll}0.000302 & 0.000297\end{array}$

$0.000293 \quad 0.000297$

$0.000217 \quad 0.000221$

0.0001810 .000206

0.0001850 .000207

$0.000203 \quad 0.000214$

$0.000217 \quad 0.000223$

$0.000240 \quad 0.000240$

0.0002590 .000256

$0.000275 \quad 0.000272$

$0.000303 \quad 0.000299$

$0.000286 \quad 0.000284$

$0.000247 \quad 0.000245$

$\begin{array}{ll}0.000321 & 0.000323\end{array}$

0.0002590 .000257

$0.000299 \quad 0.000299$

$0.000332 \quad 0.000334$

$\begin{array}{lll}0.000272 & 0.000270\end{array}$

$0.000308 \quad 0.000309$

$0.000199 \quad 0.000210$

$0.000204 \quad 0.000213$

$0.000218 \quad 0.000221$

0.0002290 .000229

$0.000239 \quad 0.000237$

$0.000248 \quad 0.000246$

$0.000259 \quad 0.000258$

$\begin{array}{ll}0.000271 & 0.000271\end{array}$

0.0002840 .000285

$0.000296 \quad 0.000301$

0.0002210 .000225
0.0003410 .000341
Density Density App Vol Square of Inconsistent Incor

/ Residual / Residual / Density based on based on volume $/ \mathrm{cm}^{3}$ Delta densit

$\begin{array}{rrrrrr}\text { calc / } & \text { Residual / } & \text { Residual / Density based on } & \text { based on } & \text { volume } / \mathrm{cm}^{3} \\ \mathrm{~kg} / \mathrm{m}^{3} & \mathrm{~kg} / \mathrm{m}^{3} & \mathrm{~m}^{3} / \mathrm{kg} & \text { Residual density apparent } & / \mathrm{mole}\end{array}$ volume

$\begin{array}{llll}1004.42 & 0.2257 & -0.000020 & 0.051\end{array}$

$\begin{array}{llll}1011.72 & 0.2770 & -0.000014 & 0.077\end{array}$

$\begin{array}{llll}1028.17 & 0.4251 & -0.000010 & 0.181\end{array}$

$\begin{array}{llll}1038.72 & 0.6544 & -0.000012 & 0.428\end{array}$

$\begin{array}{llll}1062.22 & 0.3752 & -0.000004 & 0.141\end{array}$

$\begin{array}{llll}1079.74 & 0.6580 & -0.000006 & 0.433\end{array}$

$\begin{array}{llll}1097.82 & -0.0228 & 0.000000 & 0.001\end{array}$

$\begin{array}{llll}1134.98 & -0.4753 & 0.000002 & 0.226\end{array}$

$\begin{array}{llll}1155.73 & 0.1177 & 0.000000 & 0.014\end{array}$

$\begin{array}{llll}1173.66 & -0.6615 & 0.000002 & 0.438\end{array}$

$\begin{array}{llll}1223.87 & -1.0643 & 0.000003 & 1.133\end{array}$

$\begin{array}{llll}1224.08 & -0.4849 & 0.000001 & 0.235\end{array}$

$\begin{array}{llll}1276.59 & -0.1912 & 0.000000 & 0.037\end{array}$

$\begin{array}{llll}1284.58 & -2.0727 & 0.000004 & 4.296\end{array}$

$\begin{array}{llll}1330.80 & 0.8041 & -0.000001 & 0.647\end{array}$

$\begin{array}{llll}1385.80 & -3.5016 & 0.000005 & 12.262\end{array}$

$\begin{array}{llll}1386.09 & 3.4075 & -0.000004 & 11.611\end{array}$

$\begin{array}{lllll}1078.59 & 0.5077 & -0.000004 & 0.258\end{array}$

$\begin{array}{llll}998.41 & 0.1350 & -0.000025 & 0.018\end{array}$

$\begin{array}{llll}002.78 & 0.2423 & -0.000022 & 0.059\end{array}$

$\begin{array}{llll}1036.94 & 0.6564 & -0.000012 & 0.431\end{array}$

$\begin{array}{llll}1077.81 & 0.6870 & -0.000006 & 0.472\end{array}$

$\begin{array}{lllll}1153.49 & 0.1884 & -0.000001 & 0.036\end{array}$

$\begin{array}{llll}1153.49 & 0.1884 & -0.000001 & 0.036 \\ 1281.75 & -1.9598 & 0.000003 & 0.921\end{array}$

$\begin{array}{llll}1281.75 & -1.9470 & 0.000004 & 3.791\end{array}$

$\begin{array}{llll}1382.43 & -3.3236 & 0.000004 & 11.046\end{array}$

$\begin{array}{llll}1322.40 & -1.0233 & 0.000002 & 1.047\end{array}$

$\begin{array}{llll}1164.01 & -0.5655 & 0.000002 & 0.320\end{array}$

$\begin{array}{llll}1451.28 & 1.3754 & -0.000001 & 1.892\end{array}$

$\begin{array}{llll}1502.32 & 0.2644 & 0.000000 & 0.070\end{array}$

$\begin{array}{llll}1215.11 & -0.8523 & 0.000002 & 0.726\end{array}$

$\begin{array}{llll}1374.99 & 0.3332 & 0.000000 & 0.111\end{array}$

$\begin{array}{llll}1483.53 & 2.6311 & -0.000002 & 6.923\end{array}$

$\begin{array}{llll}1268.75 & -0.6385 & 0.000001 & 0.408\end{array}$

$\begin{array}{llll}1409.25 & 1.1362 & -0.000001 & 1.291\end{array}$

$\begin{array}{llll}1008.18 & 0.2187 & -0.000011 & 0.048\end{array}$

$\begin{array}{llll}1024.52 & 0.3816 & -0.000009 & 0.146\end{array}$

$\begin{array}{llll}1058.32 & 0.2751 & -0.000003 & 0.076\end{array}$

$\begin{array}{llll}1093.66 & 0.0403 & 0.000000 & 0.002\end{array}$

$\begin{array}{llll}1130.53 & -0.4280 & 0.000002 & 0.183\end{array}$

$\begin{array}{llll}1168.91 & -0.5079 & 0.000002 & 0.258\end{array}$

$\begin{array}{llll}1218.92 & -0.3157 & 0.000001 & 0.100\end{array}$

$\begin{array}{lllll}1270.97 & -0.0698 & 0.000000 & 0.005\end{array}$

$\begin{array}{llll}1324.69 & 0.8137 & -0.000001 & 0.662\end{array}$

$\begin{array}{llll}1379.46 & 3.1379 & -0.000004 & 9.847\end{array}$

$\begin{array}{lllll}1074.48 & 0.4174 & -0.000004 & 0.174\end{array}$
995.6473

995.6473

995.6473

995.6473

995.6473

995.6473

995.6473

995.6473

995.6473

995.6473

995.6473

995.6473

995.6473

995.6473

995.6473

995.6473

995.6473

995.5806

994.0319

994.0319

994.0319

994.0319

994.0319

994.0319

994.0319

994.0319

992.7585

992.5718

992.4551

992.3453

992.3415

992.3035

992.2959

992.2883

992.2578

992.2158

92.2158

992.2158

992.2158

992.2158

992.2158

992.2158

992.2158

992.2158

992.2158

992.0661 
molality molarity

$t /{ }^{\circ} \mathrm{C}$ mass frac

Density

$\exp /$

0.05
0.1
0.5
1
2
3
4
6

0.02976
0.0994
0.10213
0.19769
0.21251
0.42541
0.66613
0.88035

0.05
0.1
0.5
1
2
3
4
6

$\begin{array}{rrrr}45 & 0.0055 & 994.73 & (45) \\ 45 & 0.0110 & 999.16 & (45) \\ 45 & 0.0526 & 1033.57 & (45) \\ 45 & 0.0999 & 1074.27 & (45) \\ 45 & 0.1817 & 1149.04 & (45) \\ 45 & 0.2498 & 1215.31 & (45) \\ 45 & 0.3075 & 1274.28 & (45) \\ 45 & 0.3997 & 1372.74 & (45) \\ 48.65 & 0.3471 & 1315.31 & (133) \\ 48.75 & 0.0033 & 991.23 & (113) \\ 48.75 & 0.0109 & 997.34 & (113) \\ 48.75 & 0.0112 & 997.53 & (113) \\ 48.75 & 0.0215 & 1005.76 & (113) \\ 48.75 & 0.0230 & 1007.03 & (113) \\ 48.75 & 0.0451 & 1025.07 & (113) \\ 48.75 & 0.0688 & 1044.75 & (113) \\ 48.75 & 0.0890 & 1061.85 & (113) \\ 49.1 & 0.1945 & 1158.43 & (133) \\ 49.51 & 0.4651 & 1445.81 & (133) \\ 49.62 & 0.5132 & 1495.78 & (133) \\ 49.77 & 0.2978 & 1262.48 & (133) \\ 49.81 & 0.3958 & 1368.79 & (133) \\ 49.82 & 0.4954 & 1479.29 & (133) \\ 49.85 & 0.427 & 1403.69 & (133) \\ 49.88 & 0.2461 & 1208.97 & (133) \\ 50.51 & 0.0987 & 1070.17 & (133) \\ 55 & 0.0055 & 990.19 & (45) \\ 55 & 0.0110 & 994.64 & (45) \\ 55 & 0.0526 & 1028.97 & (45) \\ 55 & 0.0999 & 1069.58 & (45) \\ 55 & 0.1817 & 1144.13 & (45) \\ 55 & 0.2498 & 1210.19 & (45) \\ 55 & 0.3075 & 1268.72 & (45) \\ 55 & 0.3997 & 1366.38 & (45) \\ 58.48 & 0.0987 & 1066.13 & (133) \\ 58.63 & 0.3471 & 1309.24 & (133) \\ 58.79 & 0.0987 & 1065.99 & (133) \\ 59.12 & 0.1945 & 1153.15 & (133) \\ 59.51 & 0.4651 & 1439.05 & (133) \\ 59.59 & 0.5132 & 1489.06 & (133) \\ 59.67 & 0.2461 & 1203.50 & (133) \\ 59.8 & 0.3958 & 1362.26 & (133) \\ 59.84 & 0.2978 & 1256.66 & (133) \\ 59.86 & 0.4954 & 1472.51 & (133) \\ 59.94 & 0.427 & 1396.95 & (133) \\ 60 & 0.0200 & 999.40 & (90) \\ & & & \end{array}$

$60 \quad 0.0200 \quad 999.40$
Ref

$\begin{array}{rr}\exp / & \text { Calc / } \\ \mathrm{m}^{3} / \mathrm{kg} & \mathrm{m}^{3} / \mathrm{kg}\end{array}$

Density

Density App Vol Square of Inconsistent In
calc / Residual / Residual / Density on

Inconsistent App molal Delta density Density of

$\begin{array}{rrrrrrr}\mathrm{calc} / & \text { Residual / } & \text { Residual / Density based on } & \text { based on } & \text { volume } / \mathrm{cm}^{3} & / \mathrm{kg} / \mathrm{m}^{3} & \text { water / } \\ \mathrm{kg} / \mathrm{m}^{3} & \mathrm{~kg} / \mathrm{m}^{3} & \mathrm{~m}^{3} / \mathrm{kg} & \text { Residual density } & \text { apparent } & / \mathrm{mole} & \mathrm{kg} / \mathrm{m}^{3}\end{array}$ $\begin{array}{ll}0.000179 & 0.000209 \\ 0.000186 & 0.000209\end{array}$ 994.57 $\begin{array}{llll}90 & 0.2561 & -0.000023 & 0.066\end{array}$ $\begin{array}{llllll}0.000204 & 0.000217 & 1032.85 & 0.7178 & -0.000013 & 0.515\end{array}$ $\begin{array}{llllll}0.000219 & 0.000226 & 1073.46 & 0.8133 & -0.000007 & 0.662\end{array}$ $\begin{array}{llllll}0.000241 & 0.000243 & 1148.63 & 0.4066 & -0.000002 & 0.165\end{array}$ $\begin{array}{llllll}0.000261 & 0.000259 & 1216.01 & -0.7024 & 0.000002 & 0.493\end{array}$ $\begin{array}{llllll}0.000278 & 0.000274 & 1276.02 & -1.7365 & 0.000003 & 3.015\end{array}$ $\begin{array}{llllll}0.000306 & 0.000302 & 1376.02 & -3.2768 & 0.000004 & 10.737\end{array}$ $\begin{array}{llllll}0.000288 & 0.000286 & 1316.36 & -1.0550 & 0.000002 & 1.113\end{array}$ $\begin{array}{llllll}0.000195 & 0.000209 & 991.19 & 0.0461 & -0.000014 & 0.002\end{array}$ $\begin{array}{lllllll}0.000199 & 0.000210 & 997.22 & 0.1260 & -0.000012 & 0.016\end{array}$ $\begin{array}{llllll}0.000203 & 0.000210 & 997.45 & 0.0778 & -0.000007 & 0.006\end{array}$ $\begin{array}{llllll}0.000207 & 0.000212 & 1005.66 & 0.1005 & -0.000005 & 0.010\end{array}$ $\begin{array}{lllllll}0.000208 & 0.000212 & 1006.93 & 0.0999 & -0.000004 & 0.010\end{array}$ $\begin{array}{lllllll}0.000213 & 0.000216 & 1024.93 & 0.1385 & -0.000003 & 0.019\end{array}$ $\begin{array}{llllll}0.000222 & 0.000221 & 1044.83 & -0.0856 & 0.000001 & 0.007\end{array}$ $\begin{array}{lllllll}0.000228 & 0.000225 & 1062.15 & -0.3057 & 0.000003 & 0.093\end{array}$ $\begin{array}{llllll}0.000248 & 0.000247 & 1158.87 & -0.4364 & 0.000002 & 0.190\end{array}$ $\begin{array}{llllll}0.000323 & 0.000325 & 1444.68 & 1.1312 & -0.000001 & 1.280\end{array}$ $\begin{array}{llllll}0.000343 & 0.000343 & 1495.71 & 0.0652 & 0.000000 & 0.004\end{array}$ $\begin{array}{llllll}0.000274 & 0.000272 & 1263.06 & -0.5791 & 0.000001 & 0.335\end{array}$ $\begin{array}{llllll}0.000301 & 0.000301 & 1368.75 & 0.0376 & 0.000000 & 0.001\end{array}$ $\begin{array}{lllllll}0.000334 & 0.000336 & 1476.94 & 2.3510 & -0.000002 & 5.527\end{array}$ $\begin{array}{lllllll}0.000310 & 0.000311 & 1402.94 & 0.7517 & -0.000001 & 0.565\end{array}$ $\begin{array}{llllll}0.000261 & 0.000259 & 1209.57 & -0.5984 & 0.000002 & 0.358\end{array}$ $\begin{array}{llllll}0.000223 & 0.000227 & 1069.74 & 0.4257 & -0.000004 & 0.181\end{array}$ $\begin{array}{llllll}0.000180 & 0.000210 & 990.03 & 0.1625 & -0.000030 & 0.026\end{array}$ $\begin{array}{llllll}0.000183 & 0.000211 & 994.34 & 0.3003 & -0.000028 & 0.090\end{array}$ $\begin{array}{lllllll}0.000203 & 0.000218 & 1028.11 & 0.8601 & -0.000015 & 0.740\end{array}$ $\begin{array}{llllll}0.000218 & 0.000227 & 1068.51 & 1.0718 & -0.000009 & 1.149\end{array}$ $\begin{array}{llllll}0.000241 & 0.000245 & 1143.33 & 0.8017 & -0.000003 & 0.643\end{array}$ $\begin{array}{lllllll}0.000261 & 0.000260 & 1210.42 & -0.2343 & 0.000001 & 0.055\end{array}$ $\begin{array}{llllll}0.000278 & 0.000275 & 1270.22 & -1.4957 & 0.000003 & 2.237\end{array}$ $\begin{array}{llllll}0.000307 & 0.000303 & 1369.98 & -3.5980 & 0.000005 & 12.946\end{array}$ $\begin{array}{llllll}0.000223 & 0.000227 & 1065.61 & 0.5216 & -0.000005 & 0.272\end{array}$ $\begin{array}{llllll}0.000289 & 0.000287 & 1310.48 & -1.2430 & 0.000002 & 1.545\end{array}$ $\begin{array}{llllll}0.000223 & 0.000227 & 1065.44 & 0.5493 & -0.000005 & 0.302\end{array}$ $\begin{array}{llllll}0.000248 & 0.000248 & 1153.34 & -0.1855 & 0.000001 & 0.034\end{array}$ $\begin{array}{llllll}0.000325 & 0.000325 & 1438.92 & 0.1250 & 0.000000 & 0.016\end{array}$ $\begin{array}{llllll}0.000344 & 0.000343 & 1490.22 & -1.1630 & 0.000001 & 1.353\end{array}$ $\begin{array}{llllll}0.000261 & 0.000260 & 1203.99 & -0.4878 & 0.000001 & 0.238\end{array}$ $\begin{array}{llllll}0.000302 & 0.000301 & 1362.87 & -0.6124 & 0.000001 & 0.375\end{array}$ $\begin{array}{llllll}0.000274 & 0.000273 & 1257.20 & -0.5381 & 0.000001 & 0.290\end{array}$ $\begin{array}{llllll}0.000335 & 0.000336 & 1471.33 & 1.1846 & -0.000001 & 1.403\end{array}$ $\begin{array}{llllll}0.000312 & 0.000312 & 1397.05 & -0.0969 & 0.000000 & 0.009\end{array}$ $\begin{array}{llllll}0.000193 & 0.000213 & 998.99 & 0.4053 & -0.000020 & 0.164\end{array}$
990.2132

990.2132

990.2132

990.2132

990.2132

990.2132

990.2132

990.2132

988.6406

988.5963

988.5963

988.5963

988.5963

988.5963

988.5963

988.5963

988.5963

988.4405

988.257

988.2076

988.1401

988.1221

988.1176

988.104

988.0905

987.8049

985.6952

985.6952

985.6952

985.6952

985.6952

985.6952

985.6952

985.6952

983.9737

983.8979

83.8168

983.6492

983.4502

983.4092

983.3683

983.3016

983.2811

983.2708

983.2297

983.1989 
molality molarity

$\mathrm{t} /{ }^{\circ} \mathrm{C}$ mass frac

$\exp$

$\mathrm{kg} / \mathrm{m}^{3}$

$\begin{array}{lll}60 & 0.0400 & 1015.80 \\ 60 & 0.0800 & 1049.20 \\ 60 & 0.1200 & 1084.00\end{array}$

$\begin{array}{ll}0.1600 & 1120.20 \\ 0.2000 & 1158.10\end{array}$

$0.2500 \quad 1207.90$

$0.3000 \quad 1259.70$

$0.3500 \quad 1313.70$

$0.4000 \quad 1370.00$

$\begin{array}{ll}0.3471 & 1303.03 \\ 0.1945 & 1147.62\end{array}$

$\begin{array}{lll}69.21 & 0.1945 & 1147.62\end{array}$

$\begin{array}{llll}69.59 & 0.5132 & 1482.46 & (133) \\ 69.69 & 0.4651 & 1432.32 & (133)\end{array}$

$\begin{array}{llll}69.73 & 0.2461 & 1197.84 & (133)\end{array}$

$\begin{array}{llll}69.91 & 0.3958 & 1355.75 & (133)\end{array}$

$\begin{array}{llll}69.92 & 0.2978 & 1250.77 & (133)\end{array}$

$\begin{array}{llll}69.92 & 0.4954 & 1465.84 & (133)\end{array}$

$\begin{array}{llll}69.99 & 0.427 & 1390.31 & (133)\end{array}$

0.04975

0.05108

0.10015

0.20271

0.49449

0.98452

0.02976

0.0994

0.10213

0.19769

0.21251

0.42541

0.66613

0.88035

0.0752

0.2106

0.4699

\section{App vol App vol}

exp / Calc /

$\mathrm{m}^{3} / \mathrm{kg} \quad \mathrm{m}^{3} / \mathrm{kg}$

Density

Density

App Vol Square of Inconsistent Inconsistent App molal Delta density $\begin{array}{rrrrrr}\mathrm{calc} / & \text { Residual / } & \text { Residual / Density based on based on } & \text { volume } / \mathrm{cm}^{3} \\ \mathrm{~kg} / \mathrm{m}^{3} & \mathrm{~kg} / \mathrm{m}^{3} & \mathrm{~m}^{3} / \mathrm{kg} & \text { Residual density apparent } & / \mathrm{mole}^{2}\end{array}$ volume

Density of

$\begin{array}{llllll}0.000201 & 0.000217 & 1015.16 & 0.6399 & -0.000016 & 0.409\end{array}$

$\begin{array}{llllll}0.000217 & 0.000224 & 1048.62 & 0.5818 & -0.000007 & 0.338\end{array}$

$\begin{array}{llllll}0.000229 & 0.000232 & 1083.60 & 0.3992 & -0.000003 & 0.159\end{array}$

$\begin{array}{llllll}0.000240 & 0.000240 & 1120.12 & 0.0832 & 0.000000 & 0.007\end{array}$

$\begin{array}{llllll}0.000249 & 0.000249 & 1158.15 & -0.0497 & 0.000000 & 0.002\end{array}$

$\begin{array}{llllll}0.000260 & 0.000261 & 1207.74 & 0.1581 & 0.000000 & 0.025\end{array}$

$\begin{array}{llllll}0.000273 & 0.000274 & 1259.42 & 0.2827 & -0.000001 & 0.080\end{array}$

$\begin{array}{llllll}0.000286 & 0.000287 & 1312.82 & 0.8843 & -0.000001 & 0.782\end{array}$

$\begin{array}{llllll}0.000299 & 0.000303 & 1367.37 & 2.6349 & -0.000004 & 6.943\end{array}$

$\begin{array}{llllll}0.000289 & 0.000286 & 1304.52 & -1.4919 & 0.000003 & 2.226\end{array}$

$\begin{array}{llllll}0.000246 & 0.000247 & 1147.39 & 0.2308 & -0.000001 & 0.053\end{array}$

$\begin{array}{llllll}0.000345 & 0.000342 & 1485.65 & -3.1930 & 0.000003 & 10.195\end{array}$

$\begin{array}{llllll}0.000325 & 0.000324 & 1433.70 & -1.3841 & 0.000001 & 1.916\end{array}$

$\begin{array}{llllll}0.000260 & 0.000259 & 1197.99 & -0.1520 & 0.000000 & 0.023\end{array}$

$\begin{array}{llllll}0.000302 & 0.000300 & 1357.17 & -1.4228 & 0.000002 & 2.024\end{array}$

$\begin{array}{llllll}0.000273 & 0.000272 & 1251.21 & -0.4364 & 0.000001 & 0.190\end{array}$

$\begin{array}{lllllll}0.000335 & 0.000335 & 1466.52 & -0.6799 & 0.000001 & 0.462\end{array}$

$\begin{array}{llllll}0.000312 & 0.000310 & 1391.58 & -1.2736 & 0.000002 & 1.622\end{array}$

$\begin{array}{llllll}0.000189 & 0.000210 & 979.12 & 0.1091 & -0.000021 & 0.012\end{array}$

$\begin{array}{llllll}0.000191 & 0.000210 & 979.24 & 0.1063 & -0.000020 & 0.011\end{array}$

$\begin{array}{llllll}0.000200 & 0.000211 & 983.44 & 0.1163 & -0.000011 & 0.014\end{array}$

$\begin{array}{llllll}0.000207 & 0.000213 & 992.15 & 0.1294 & -0.000006 & 0.017\end{array}$

$\begin{array}{llllll}0.000215 & 0.000218 & 1016.48 & 0.2031 & -0.000004 & 0.041\end{array}$

$\begin{array}{llllll}0.000235 & 0.000227 & 1055.85 & -0.8331 & 0.000008 & 0.694\end{array}$

$\begin{array}{llllll}0.000180 & 0.000212 & 987.48 & 0.6241 & -0.000032 & 0.389\end{array}$

$\begin{array}{llllll}0.000189 & 0.000216 & 1003.53 & 1.0712 & -0.000027 & 1.147\end{array}$

$\begin{array}{lllllll}0.000206 & 0.000223 & 1036.78 & 1.4190 & -0.000016 & 2.014\end{array}$

$\begin{array}{llllll}0.000220 & 0.000230 & 1071.59 & 1.4094 & -0.000010 & 1.986\end{array}$

$\begin{array}{llllll}0.000232 & 0.000239 & 1107.98 & 1.2227 & -0.000006 & 1.495\end{array}$

$\begin{array}{llllll}0.000243 & 0.000247 & 1145.94 & 1.1622 & -0.000004 & 1.351\end{array}$

$\begin{array}{llllll}0.000256 & 0.000259 & 1195.54 & 0.9559 & -0.000003 & 0.914\end{array}$

$\begin{array}{llllll}0.000270 & 0.000271 & 1247.39 & 0.4142 & -0.000001 & 0.172\end{array}$

$\begin{array}{llllll}0.000285 & 0.000285 & 1301.15 & 0.1496 & 0.000000 & 0.022\end{array}$

$\begin{array}{llllll}0.000299 & 0.000300 & 1356.32 & 0.7770 & -0.000001 & 0.604\end{array}$

$\begin{array}{llllll}0.000144 & 0.000206 & 961.86 & 0.1869 & -0.000061 & 0.035\end{array}$

$\begin{array}{llllll}0.000161 & 0.000207 & 967.78 & 0.4731 & -0.000046 & 0.224\end{array}$

$\begin{array}{llllll}0.000164 & 0.000207 & 968.01 & 0.4535 & -0.000043 & 0.206\end{array}$

$\begin{array}{llllll}0.000174 & 0.000209 & 976.08 & 0.7096 & -0.000035 & 0.504\end{array}$

$\begin{array}{lllllll}0.000176 & 0.000209 & 977.32 & 0.7376 & -0.000033 & 0.544\end{array}$

$\begin{array}{llllll}0.000184 & 0.000213 & 995.02 & 1.2882 & -0.000029 & 1.659\end{array}$

$\begin{array}{llllll}0.000195 & 0.000217 & 1014.64 & 1.5203 & -0.000021 & 2.311\end{array}$

$\begin{array}{llllll}0.000203 & 0.000220 & 1031.73 & 1.6834 & -0.000018 & 2.834\end{array}$

$\begin{array}{llllll}0.000168 & 0.000207 & 965.63 & 0.3007 & -0.000039 & 0.090\end{array}$

$\begin{array}{llllll}0.000179 & 0.000209 & 977.06 & 0.6564 & -0.000030 & 0.431\end{array}$

$\begin{array}{llllll}0.000180 & 0.000213 & 998.58 & 1.6371 & -0.000033 & 2.680\end{array}$

$\begin{array}{llll}973.96 & 0.8429 & -0.000044 & 0.711\end{array}$ $\mathrm{kg} / \mathrm{m}^{3}$

983.1989

983.1989

983.1989

983.1989

983.1989

983.1989

983.1989

983.1989

983.1989

978.4337

978.2188

978.0032

977.9463

977.9235

977.821

977.8153

977.8153

977.7753

21.03

974.849

974.849

974.849

974.849

974.849

971.7978

971.7978

971.7978

971.7978

971.7978

971.7978

971.7978

971.7978

971.7978

971.7978

959.3157

959.3157

59.3157

959.3157

959.3157

959.3157

959.3157

959.3157

959.2159

959.2159

959.2159

958.3637 
molality molarity

$\begin{array}{ccrc}\mathbf{t} /{ }^{\circ} \mathbf{C} \text { mass frac } & \begin{array}{r}\text { Density } \\ \text { exp } / \\ \mathrm{kg} / \mathrm{m}^{3}\end{array} & \text { Ref } \\ & & \\ 100 & 0.0400 & 991.50 & (90) \\ 100 & 0.0800 & 1025.70 & (90) \\ 100 & 0.1200 & 1061.00 & (90) \\ 100 & 0.1600 & 1097.30 & (90) \\ 100 & 0.2000 & 1135.20 & (90) \\ 100 & 0.2500 & 1184.60 & (90) \\ 100 & 0.3000 & 1235.90 & (90) \\ 100 & 0.3500 & 1289.30 & (90) \\ 100 & 0.4000 & 1345.00 & (90)\end{array}$

\section{App vol App vol}

$\begin{array}{cc}\exp / & \text { Calc / } \\ \mathrm{m}^{3} / \mathrm{kg} & \mathrm{m}^{3} / \mathrm{kg}\end{array}$

Density

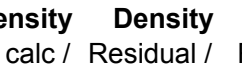
$\mathrm{kg} / \mathrm{m}^{3}$

$\begin{array}{ll}0.000172 & 0.000211\end{array}$

$0.000187 \quad 0.000218$

989.94
1023.07

1023.07

0.0002020 .000226

$0.000218 \quad 0.000234$

0.0002310 .000242

1094.24

1132.31

1182.23

$0.000262 \quad 0.000265$

$0.000278 \quad 0.000278$

$0.000294 \quad 0.000293$

1234.62

1289.24
1345.65

Average Res

Std dev Res

Avg - 4std

Avg + 4std
Density

$\mathrm{kg} / \mathrm{m}^{3}$

App$$
1.5646-0.000040
$$

$1.5646-0.000040$

$2.6257-0.000031$

$3.1702-0.000024$

3.0625

$\begin{array}{lll}2.8870 & -0.000011 & 8.335\end{array}$

$\begin{array}{lll}2.3695 & -0.000007 & 5.615\end{array}$

$\begin{array}{lll}1.2791 & -0.000003 & 1.636\end{array}$

$\begin{array}{lll}0.0617 & 0.000000 & 0.004\end{array}$

$\begin{array}{rrr}-0.6474 & 0.000001 & 0.419\end{array}$

$0.2071-0.000008 \quad 399.298$ Sum Chi sqr

$1.0386 \quad 0.000012$

$\begin{array}{ll}-3.9471 & -0.000058\end{array}$

$4.3614 \quad 0.000042$

Inconsistent

Curve fit for the model App vol= $\left(w+c 2+c 3^{*} t{ }^{\circ} \mathrm{C}\right) /\left(c 0^{*} w+c 1\right) / e^{\wedge}\left(0.000001^{*}\left(t^{\circ} \mathrm{C}+c 4\right)^{\wedge} 2\right)$

$\begin{array}{lr}\text { c0 } & -0.63254 \\ \text { c1 } & 0.939949 \\ \text { c2 } & 4.278471 \\ \text { c3 } & 0.048319 \\ \text { c4 } & 3180.906 \\ \text { Min T } & 0 \\ \text { Max T } & 100 \\ \text { Min w } & 0.001392 \\ \text { Max w } & 0.5132 \\ \text { Avg dens res } & 0.207136 \\ \text { Std dens res } & 1.038555 \\ \text { Avg app vol res *10^3 } & -0.00841 \\ \text { Std app vol res *10^3 } & 0.012498 \\ \text { No of points in corr } & 357 \\ \text { No of inconsistent poir } & 2\end{array}$

Inconsistent data not used (Residual greater than average $+/-4$ standard deviations)
6.6668
$\begin{array}{lll}25 & 0.4253 & 1423.15\end{array}$

$\begin{array}{lll}\text { (57) } \quad 0.000297 & 0.000303\end{array}$

1417.87

$\begin{array}{lll}5.2774 & -0.000006 & 27.851\end{array}$

Inconsistent

App molal Delta density

Density of water /
$\mathrm{kg} / \mathrm{m}^{3}$

$\mathrm{kg} / \mathrm{m}^{3}$

958.3637

958.3637

958.3637

958.3637
958.3637

958.3637

958.3637

958.3637

958.3637

$$
7.4
$$


Density of aqueous solutions of $\mathbf{C d C l 2}$

References (9) Call, T. G.; Ballerat-Busserolles, K; Origlia, M. L; Ford, T. D. Woolley, E. M. Apparent Molar Volumes and Heat Capacities of Aqueous

Magnesium Chloride and Cadmium Chloride at Temperatures from $278.15 \mathrm{~K}$ to $393.15 \mathrm{~K}$ at the Pressure $0.35 \mathrm{MPa}$ : a Comparison of lon-

Ion Interactions. J. Chem. Thermodynamics 2000, 32, 1525-1538.

(23) Dolian, F. E. The Viscosities of Solutions of Chlorides in Certain Solvents. J. Phys. Chem. 1937, 41, 1129-1138.

(37) Herrington, Thelma M.; Roffey, Michael G.; Smith, David P.; Densities of Aqueous Electrolytes $\mathrm{MnCl}_{2}, \mathrm{CoCl}_{2}, \mathrm{NiCl}_{2}, \mathrm{ZnCl}_{2}$ and CdCl${ }_{2}$ from 25 to $72^{\circ} \mathrm{C}$ at $1 \mathrm{~atm}, \mathrm{~J}$. Chem. Eng. Data, 1986, 31, 221-225

(104) Rard, J. A.; Miller, D. G. Densities and Apparent Molal Volumes of Aqueous Manganese, Cadmium, and Zinc Chlorides at $25^{\circ} \mathrm{C}$. J. Chem. Eng. Data 1984, 29, 151-156.

(107) Reilly, P. J.; Stokes, R. H. The Diffusion Coefficients of Cadmium Chloride and Cadmium Perchlorate in Water at $25^{\circ} \mathrm{C}$. Aust. J. Chem. 1971, 24, 1361-1367.

\begin{tabular}{|c|c|c|c|c|c|c|c|c|c|c|c|c|c|c|c|c|}
\hline \multirow[t]{9}{*}{ molality } & \multirow[t]{4}{*}{ molarity } & \multicolumn{2}{|c|}{$\mathrm{t} /{ }^{\circ} \mathrm{C}$ mass frac } & $\begin{array}{r}\text { Density } \\
\exp / \\
\mathrm{kg} / \mathrm{m}^{3}\end{array}$ & Ref & $\begin{array}{r}\text { App vol } \\
\exp / \\
\mathrm{m}^{3} / \mathrm{kg}\end{array}$ & $\begin{array}{c}\text { App vol } \\
\text { Calc / } \\
\mathrm{m}^{3} / \mathrm{kg}\end{array}$ & $\begin{array}{r}\text { Density } \\
\text { calc / } \\
\mathrm{kg} / \mathrm{m}^{3}\end{array}$ & $\begin{array}{r}\text { Density } \\
\text { Residual / } \\
\mathrm{kg} / \mathrm{m}^{3}\end{array}$ & $\begin{array}{r}\text { App Vol } \\
\text { Residual / } \\
\mathrm{m}^{3} / \mathrm{kg}\end{array}$ & $\begin{array}{r}\text { Square of } \\
\text { Density } \\
\text { Residual }\end{array}$ & $\begin{array}{l}\text { Inconsistent } \\
\text { based on } \\
\text { density }\end{array}$ & $\begin{array}{c}\text { Inconsistent } \\
\text { based on } \\
\text { apparent } \\
\text { volume }\end{array}$ & $\begin{array}{r}\text { Apparent } \\
\text { molar volume } \\
/ \mathrm{cm}^{3} / \mathrm{mol}\end{array}$ & $\begin{array}{l}\text { Delta density } \\
\qquad / \mathrm{kg} / \mathrm{m}^{3}\end{array}$ & $\begin{array}{r}\text { Density of } \\
\text { water } / \mathrm{kg} / \mathrm{m}^{3}\end{array}$ \\
\hline & & 25 & 0.0019 & 998.79 & (23) & 0.000081 & 0.000151 & 998.66 & 0.1328 & -0.000070 & 0.018 & & $!$ & & & 997.0449 \\
\hline & & 25 & 0.0038 & 1000.47 & (23) & 0.000095 & 0.000151 & 1000.26 & 0.2128 & -0.000056 & 0.045 & & & & & 997.0449 \\
\hline & & 25 & 0.0076 & 1002.84 & (23) & 0.000240 & 0.000151 & 1003.52 & -0.6825 & 0.000089 & 0.466 & & $!$ & & & 997.0449 \\
\hline & 0.05 & 25 & 0.0091 & 1004.89 & (107) & 0.000144 & 0.000151 & 1004.83 & 0.0665 & -0.000007 & 0.004 & & & & 7.85 & 997.0449 \\
\hline & & 25 & 0.0150 & 1010.55 & (23) & 0.000109 & 0.000152 & 1009.90 & 0.6469 & -0.000042 & 0.418 & & & & & 997.0449 \\
\hline & 0.1 & 25 & 0.0181 & 1012.74 & (107) & 0.000144 & 0.000152 & 1012.60 & 0.1458 & -0.000008 & 0.021 & & & & 15.70 & 997.0449 \\
\hline & & 25 & 0.0296 & 1023.06 & $(23)^{\prime}$ & 0.000141 & 0.000153 & 1022.71 & 0.3510 & -0.000011 & 0.123 & & & & & 997.0449 \\
\hline & 0.2 & 25 & 0.0357 & 1028.40 & (107) & 0.000145 & 0.000153 & 1028.10 & 0.3019 & -0.000008 & 0.091 & & & & 31.36 & 997.0449 \\
\hline 0.2288 & & 25 & 0.0403 & 1032.66 & (37) & 0.000144 & 0.000153 & 1032.24 & 0.4188 & -0.000010 & 0.175 & & & & & 997.0449 \\
\hline 0.24987 & & 25 & 0.0438 & 1035.96 & (104) & 0.000143 & 0.000154 & 1035.45 & 0.5121 & -0.000011 & 0.262 & & & & & 997.0449 \\
\hline & 0.3 & 25 & 0.0527 & 1044.02 & (107) & 0.000146 & 0.000154 & 1043.56 & 0.4671 & -0.000008 & 0.218 & & & & 46.98 & 997.0449 \\
\hline & 0.4 & 25 & 0.0692 & 1059.57 & (107) & 0.000148 & 0.000156 & 1058.97 & 0.6083 & -0.000008 & 0.370 & & & & 62.53 & 997.0449 \\
\hline 0.4453 & & 25 & 0.0755 & 1065.55 & $(37)^{\prime}$ & 0.000149 & 0.000156 & 1064.92 & 0.6339 & -0.000007 & 0.402 & & & & & 997.0449 \\
\hline 0.49994 & & 25 & 0.0840 & 1073.76 & (104) & 0.000149 & 0.000157 & 1073.07 & 0.6931 & -0.000007 & 0.480 & & & & & 997.0449 \\
\hline & 0.5 & 25 & 0.0853 & 1075.05 & (107) & 0.000149 & 0.000157 & 1074.33 & 0.7231 & -0.000007 & 0.523 & & & & 78.01 & 997.0449 \\
\hline & 0.6 & 25 & 0.1009 & 1090.48 & (107) & 0.000151 & 0.000158 & 1089.65 & 0.8307 & -0.000007 & 0.690 & & & & 93.44 & 997.0449 \\
\hline & 0.7 & 25 & 0.1161 & 1105.75 & (107) & 0.000153 & 0.000159 & 1104.95 & 0.8077 & -0.000006 & 0.652 & & & & 108.71 & 997.0449 \\
\hline & 0.8 & 25 & 0.1308 & 1120.95 & (107) & 0.000156 & 0.000160 & 1120.21 & 0.7486 & -0.000005 & 0.560 & & & & 123.91 & 997.0449 \\
\hline 0.9005 & & 25 & 0.1417 & 1132.33 & $(37)^{\prime}$ & 0.000157 & 0.000161 & 1131.66 & 0.6706 & -0.000004 & 0.450 & & & & & 997.0449 \\
\hline & 0.9 & 25 & 0.1452 & 1136.05 & (107) & 0.000158 & 0.000161 & 1135.44 & 0.6167 & -0.000003 & 0.380 & & & & 139.01 & 997.0449 \\
\hline 0.99934 & & 25 & 0.1548 & 1146.47 & (104) & 0.000159 & 0.000162 & 1145.81 & 0.6623 & -0.000003 & 0.439 & & & & & 997.0449 \\
\hline & 1 & 25 & 0.1593 & 1151.14 & (107) & 0.000160 & 0.000162 & 1150.63 & 0.5119 & -0.000002 & 0.262 & & & & 154.10 & 997.0449 \\
\hline 1.3618 & & 25 & 0.1998 & 1197.00 & (37) & 0.000164 & 0.000165 & 1196.67 & 0.3343 & -0.000001 & 0.112 & & & & & 997.0449 \\
\hline 1.5093 & & 25 & 0.2167 & 1217.39 & (104) & 0.000165 & 0.000167 & 1216.91 & 0.4816 & -0.000002 & 0.232 & & & & & 997.0449 \\
\hline 1.627 & & 25 & 0.2297 & 1232.95 & $(37)^{\prime}$ & 0.000168 & 0.000168 & 1232.88 & 0.0734 & 0.000000 & 0.005 & & & & & 997.0449 \\
\hline 1.8475 & & 25 & 0.2530 & 1262.22 & $(37)$ & 0.000170 & 0.000170 & 1262.36 & -0.1364 & 0.000000 & 0.019 & & & & & 997.0449 \\
\hline 2.0004 & & 25 & 0.2683 & 1282.74 & (104) & 0.000170 & 0.000171 & 1282.47 & 0.2696 & -0.000001 & 0.073 & & & & & 997.0449 \\
\hline 2.1395 & & 25 & 0.2817 & 1300.19 & $(37)$ & 0.000173 & 0.000172 & 1300.54 & -0.3494 & 0.000001 & 0.122 & & & & & 997.0449 \\
\hline 2.3412 & & 25 & 0.3003 & 1325.93 & (37) & 0.000175 & 0.000174 & 1326.36 & -0.4276 & 0.000001 & 0.183 & & & & & 997.0449 \\
\hline 2.4884 & & 25 & 0.3133 & 1345.01 & (104) & 0.000175 & 0.000175 & 1344.92 & 0.0917 & 0.000000 & 0.008 & & & & & 997.0449 \\
\hline 2.6401 & & 25 & 0.3261 & 1363.40 & $(37)$ & 0.000177 & 0.000176 & 1363.80 & -0.4023 & 0.000001 & 0.162 & & & & & 997.0449 \\
\hline 2.8474 & & 25 & 0.3430 & 1388.97 & (37) & 0.000178 & 0.000177 & 1389.21 & -0.2433 & 0.000000 & 0.059 & & & & & 997.0449 \\
\hline
\end{tabular}




\begin{tabular}{|c|c|c|c|c|}
\hline molality & molarity & $\mathrm{t} /{ }^{\circ} \mathrm{C}$ & ass frac & $\begin{array}{r}\text { Density } \\
\exp / \\
\mathrm{kg} / \mathrm{m}^{3}\end{array}$ \\
\hline 3.0042 & & 25 & 0.3551 & 1408.05 \\
\hline 3.5665 & & 25 & 0.3953 & 1473.77 \\
\hline 4.1037 & & 25 & 0.4293 & 1533.81 \\
\hline 4.498 & & 25 & 0.4519 & 1576.12 \\
\hline 5.8582 & & 25 & 0.5178 & 1712.59 \\
\hline 6.3608 & & 25 & 0.5383 & 1759.52 \\
\hline 0.2288 & & 35 & 0.0403 & 1029.36 \\
\hline 0.4453 & & 35 & 0.0755 & 1061.99 \\
\hline 0.9005 & & 35 & 0.1417 & 1128.22 \\
\hline 1.3618 & & 35 & 0.1998 & 1192.35 \\
\hline 1.627 & & 35 & 0.2297 & 1228.01 \\
\hline 1.8475 & & 35 & 0.2530 & 1257.04 \\
\hline 2.1395 & & 35 & 0.2817 & 1294.69 \\
\hline 2.3412 & & 35 & 0.3003 & 1320.22 \\
\hline 2.6401 & & 35 & 0.3261 & 1357.40 \\
\hline 2.8474 & & 35 & 0.3430 & 1382.78 \\
\hline 0.2288 & & 45 & 0.0403 & 1025.25 \\
\hline 0.4453 & & 45 & 0.0755 & 1057.60 \\
\hline 0.9005 & & 45 & 0.1417 & 1123.26 \\
\hline 1.3618 & & 45 & 0.1998 & 1186.87 \\
\hline 1.627 & & 45 & 0.2297 & 1222.24 \\
\hline 1.8475 & & 45 & 0.2530 & 1251.05 \\
\hline 2.1395 & & 45 & 0.2817 & 1288.44 \\
\hline 2.3412 & & 45 & 0.3003 & 1313.81 \\
\hline 2.6401 & & 45 & 0.3261 & 1350.78 \\
\hline 2.8474 & & 45 & 0.3430 & 1376.03 \\
\hline 0.2288 & & 55 & 0.0403 & 1020.49 \\
\hline 0.4453 & & 55 & 0.0755 & 1052.59 \\
\hline 0.9005 & & 55 & 0.1417 & 1117.74 \\
\hline 1.3618 & & 55 & 0.1998 & 1180.82 \\
\hline 1.627 & & 55 & 0.2297 & 1215.90 \\
\hline 1.8475 & & 55 & 0.2530 & 1244.48 \\
\hline 2.1395 & & 55 & 0.2817 & 1281.58 \\
\hline 2.3412 & & 55 & 0.3003 & 1306.75 \\
\hline 2.6401 & & 55 & 0.3261 & 1343.46 \\
\hline 2.8474 & & 55 & 0.3430 & 1368.55 \\
\hline 0.2288 & & 65 & 0.0403 & 1015.12 \\
\hline 0.4453 & & 65 & 0.0755 & 1047.01 \\
\hline 0.9005 & & 65 & 0.1417 & 1111.68 \\
\hline 1.3618 & & 65 & 0.1998 & 1174.29 \\
\hline 1.627 & & 65 & 0.2297 & 1209.11 \\
\hline 1.8475 & & 65 & 0.2530 & 1237.49 \\
\hline 2.1395 & & 65 & 0.2817 & 1274.34 \\
\hline 2.3412 & & 65 & 0.3003 & 1299.37 \\
\hline
\end{tabular}

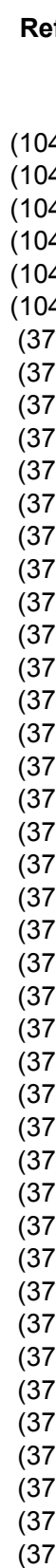

exp / App vol De $\mathrm{m}^{3} / \mathrm{kg}$

Calc/ $\mathrm{m}^{3} / \mathrm{kg}$

$0.000179 \quad 0.000178$

0.000182

0.000185

0.000188

0.000182

0.000185

0.000187

$0.000196 \quad 0.000196$

0.000196

0.000156

0.000162

0.000164

0.000168

0.000169

0.000172

0.000174

0.00017

0.000173

$\begin{array}{ll}0.000177 & 0.000176 \\ 0.000178 & 0.000178\end{array}$

$\begin{array}{ll}0.000178 & 0.000178 \\ 0.000180 & 0.000180\end{array}$

0.000181

0.000180

0.00018

$0.000157 \quad 0.000162$

0.000166

0.000167

0.000172

$0.000178 \quad 0.000177$

$\begin{array}{lll}0.000182 & 0.000181 & 1314.15\end{array}$

$\begin{array}{lll}0.000183 & 0.000183 & 1350.79\end{array}$

$\begin{array}{lll}0.000184 & 0.000185 & 1375.63\end{array}$

$\begin{array}{lll}0.000155 & 0.000161 & 1020.23\end{array}$

$\begin{array}{lll}0.000160 & 0.000164 & 1052.26\end{array}$

$\begin{array}{lll}0.000169 & 0.000170 & 1117.55\end{array}$

$\begin{array}{lll}0.000175 & 0.000175 & 1180.99\end{array}$

$\begin{array}{lll}0.000178 & 0.000177 & 1216.26\end{array}$

$\begin{array}{lll}0.000181 & 0.000180 & 1244.93\end{array}$

$\begin{array}{lll}0.000183 & 0.000182 & 1282.02\end{array}$

$\begin{array}{lll}0.000184 & 0.000184 & 1307.06\end{array}$

$\begin{array}{lll}0.000186 & 0.000186 & 1343.33\end{array}$

$\begin{array}{lll}0.000187 & 0.000188 & 1367.91\end{array}$

$\begin{array}{lll}0.000157 & 0.000163 & 1014.88\end{array}$

$\begin{array}{lll}0.000162 & 0.000166 & 1046.69\end{array}$

$\begin{array}{lll}0.000171 & 0.000172 & 1111.53\end{array}$

$\begin{array}{lll}0.000178 & 0.000177 & 1174.49\end{array}$

$\begin{array}{lll}0.000181 & 0.000180 & 1209.48\end{array}$

$\begin{array}{lll}0.000183 & 0.000182 & 1237.91\end{array}$

$\begin{array}{lll}0.000185 & 0.000185 & 1274.68\end{array}$

$\begin{array}{lll}0.000187 & 0.000186 & 1299.50\end{array}$
997.0449 997.0449 997.0449 997.0449 997.0449 997.0449 994.0319 994.0319 994.0319 994.0319 994.0319 994.0319 994.0319 994.0319 994.0319 994.0319 990.2132 990.2132 990.2132 990.2132 990.2132 990.2132 990.2132 990.2132 990.2132 990.2132 985.6952 985.6952 985.6952 985.6952 985.6952 985.6952 985.6952 985.6952 985.6952 985.6952 980.5548 980.5548 980.5548 980.5548 980.5548 980.5548 980.5548 980.5548 


$\begin{array}{rrrr}\text { molality molarity } & \mathbf{t} /{ }^{\circ} \mathbf{C} \text { mass frac } & \begin{array}{r}\text { Density } \\ \exp / \\ \mathrm{kg} / \mathrm{m}^{3}\end{array} \\ 2.6401 & 65 & 0.3261 & 1335.89 \\ 2.8474 & 65 & 0.3430 & 1360.88 \\ 0.2288 & 75 & 0.0403 & 1009.13 \\ 0.4453 & 75 & 0.0755 & 1040.77 \\ 0.9005 & 75 & 0.1417 & 1104.98 \\ 1.3618 & 75 & 0.1998 & 1167.16 \\ 1.627 & 75 & 0.2297 & 1201.73 \\ 1.8475 & 75 & 0.2530 & 1229.90 \\ 2.1395 & 75 & 0.2817 & 1266.46 \\ 2.3412 & 75 & 0.3003 & 1291.28 \\ 2.6401 & 75 & 0.3261 & 1327.47 \\ 2.8474 & 75 & 0.3430 & 1352.20\end{array}$

Ref
$(37)$
$(37)$
$(37)$
$(37)$
$(37)$
$(37)$
$(37)$
$(37)$
$(37)$
$(37)$
$(37)$
$(37)$

\section{App vol

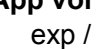

$\exp /$
$\mathrm{m}^{3} / \mathrm{kg}$

0.000188

0.000189

0.000160

0.000165

0.000180

0.000183

0.000185

0.000187

0.000189

0.000190

0.000191
App vol

Calc /

$\mathrm{m}^{3} / \mathrm{kg}$

.000189

0.000191
0.000164

0.000167

$0.000173 \quad 1105.00$

$0.000178 \quad 1167.52$

$0.000181 \quad 1202.25$

$0.000183 \quad 1230.47$

$0.000186 \quad 1266.95$

$0.000188 \quad 1291.57$

$0.000191 \quad 1327.20$

0.000192

\section{Average Res \\ Std dev Res}

Avg - 4std

Avg + 4std
Density App Vol Square of Inconsistent In
calc / Residual / Residual / Density based on

/ Residual /

density

nconsistent

based on apparent

\section{$0.4613-0.000001$}

$\begin{array}{ll}0.4613 & -0.000001 \\ 0.1077 & -0.000002\end{array}$

0.213

$0.1885-0.000002$

0.036

0.000000

0.000

$\begin{array}{ll}0.3649 & 0.000001\end{array}$

$-0.5237 \quad 0.000002$

$-0.57340 .000001$

$\begin{array}{ll}-0.4912 & 0.000001\end{array}$

$\begin{array}{ll}-0.2888 & 0.000001\end{array}$

$0.2670 \quad 0.000000$

$0.8619-0.000001$

$0.105-0.000003$

$0.412 \quad 0.000015$

$-1.5410-0.000061$

$\begin{array}{ll}1.7513 & 0.000055\end{array}$

0.743

Curve fit for the model App vol $=\left(w+c 2+c 3^{*} t{ }^{\circ} \mathrm{C}\right) /\left(c 0^{*} w+c 1\right) / e^{\wedge}\left(0.000001^{*}\left(t^{\circ} \mathrm{C}+c 4\right)^{\wedge} 2\right)$

$\begin{array}{lr}\text { c0 } & -0.09088 \\ \text { c1 } & 0.291159 \\ \text { c2 } & 7.382645 \\ \text { c3 } & -0.03186 \\ \text { c4 } & -3477.52 \\ \text { Min T } & 25 \\ \text { Max T } & 75 \\ \text { Min w } & 0.0019 \\ \text { Max w } & 0.538333 \\ \text { Avg dens res } & 0.105116 \\ \text { Std dens res } & 0.411537 \\ \text { Avg app vol res *10^3 } & -0.0028 \\ \text { Std app vol res *10^3 } & 0.014553 \\ \text { No of points in corr } & 88 \\ \text { No of inconsistent poir } & 90\end{array}$

Inconsistent data not used (Residual greater than average $+/-4$ standard deviations)

$\begin{array}{rrrrrrrrrr}0.01013 & 5 & 0.0019 & 1001.64 & (9) & 0.000097 & 0.000144 & 1001.55 & 0.0865 & -0.000047 \\ 0.02015 & 5 & 0.0037 & 1003.31 & (9) & 0.000093 & 0.000144 & 1003.12 & 0.1882 & -0.000051 \\ 0.04991 & 5 & 0.0091 & 1008.67 & (9) & 0.000048 & 0.000144 & 1007.78 & 0.8897 & -0.000097 \\ 0.1 & 5 & 0.0180 & 1017.53 & (9) & 0.000041 & 0.000145 & 1015.60 & 1.9306 & -0.000104 \\ 0.16987 & 5 & 0.0302 & 1029.95 & (9) & 0.000036 & 0.000146 & 1026.44 & 3.5072 & -0.000110 \\ 0.2483 & 5 & 0.0435 & 1043.51 & (9) & 0.000041 & 0.000147 & 1038.55 & 4.9653 & -0.000105 \\ 0.49935 & 5 & 0.0839 & 1086.61 & (9) & 0.000049 & 0.000149 & 1076.78 & 9.8265 & -0.000100 \\ 0.74558 & 5 & 0.1202 & 1128.94 & (9) & 0.000050 & 0.000152 & 1113.52 & 15.4207 & -0.000102 \\ 0.9986 & 5 & 0.1547 & 1171.94 & (9) & 0.000052 & 0.000154 & 1150.52 & 21.4246 & -0.000103 \\ 0.01013 & 15 & 0.0019 & 1000.88 & (9) & 0.000038 & 0.000147 & 1000.68 & 0.2034 & -0.000110\end{array}$

1
7
0
5
0
2
3

Apparent Delta density Density of $/ \mathrm{cm}^{3} / \mathrm{mol}$

$/ \mathrm{kg} / \mathrm{m}^{3}$ water $/ \mathrm{kg} / \mathrm{m}^{3}$ 980.5548 974.849 974.849

974.849

974.849

974.849

974.849

974.849

974.849

974.849

974.849

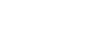

999.9638 999.9638 999.9638 999.9638 999.9638 999.9638 999.9638 999.9638 999.9638 999.0996 


\begin{tabular}{|c|c|c|c|c|}
\hline molality & molarity & \multicolumn{2}{|c|}{$\mathrm{t} /{ }^{\circ} \mathrm{C}$ mass frac } & $\begin{array}{r}\text { Density } \\
\exp / \\
\mathrm{kg} / \mathrm{m}^{3}\end{array}$ \\
\hline 0.02015 & & 15 & 0.0037 & 1002.58 \\
\hline 0.04991 & & 15 & 0.0091 & 1007.85 \\
\hline 0.1 & & 15 & 0.0180 & 1016.62 \\
\hline 0.16987 & & 15 & 0.0302 & 1028.82 \\
\hline 0.2483 & & 15 & 0.0435 & 1042.38 \\
\hline 0.49935 & & 15 & 0.0839 & 1084.86 \\
\hline 0.74558 & & 15 & 0.1202 & 1126.49 \\
\hline 0.9986 & & 15 & 0.1547 & 1169.09 \\
\hline 0.01013 & & 25 & 0.0019 & 998.83 \\
\hline 0.02015 & & 25 & 0.0037 & 1000.61 \\
\hline 0.04991 & & 25 & 0.0091 & 1005.76 \\
\hline 0.1 & & 25 & 0.0180 & 1014.44 \\
\hline 0.16987 & & 25 & 0.0302 & 1026.48 \\
\hline 0.2483 & & 25 & 0.0435 & 1040.00 \\
\hline 0.49935 & & 25 & 0.0839 & 1082.18 \\
\hline 0.74558 & & 25 & 0.1202 & 1123.11 \\
\hline 0.9986 & & 25 & 0.1547 & 1165.34 \\
\hline 0.01013 & & 35 & 0.0019 & 995.81 \\
\hline 0.02015 & & 35 & 0.0037 & 997.52 \\
\hline 0.04991 & & 35 & 0.0091 & 1002.68 \\
\hline 0.1 & & 35 & 0.0180 & 1011.30 \\
\hline 0.16987 & & 35 & 0.0302 & 1023.26 \\
\hline 0.2483 & & 35 & 0.0435 & 1036.58 \\
\hline 0.49935 & & 35 & 0.0839 & 1078.53 \\
\hline 0.74558 & & 35 & 0.1202 & 1118.89 \\
\hline 0.9986 & & 35 & 0.1547 & 1160.75 \\
\hline 0.01013 & & 45 & 0.0019 & 991.99 \\
\hline 0.02015 & & 45 & 0.0037 & 993.69 \\
\hline 0.04991 & & 45 & 0.0091 & 998.81 \\
\hline 0.1 & & 45 & 0.0180 & 1007.37 \\
\hline 0.16987 & & 45 & 0.0302 & 1019.24 \\
\hline 0.2483 & & 45 & 0.0435 & 1032.47 \\
\hline 0.49935 & & 45 & 0.0839 & 1074.17 \\
\hline 0.74558 & & 45 & 0.1202 & 1114.01 \\
\hline 0.9986 & & 45 & 0.1547 & 1155.58 \\
\hline 0.01013 & & 55 & 0.0019 & 987.44 \\
\hline 0.02015 & & 55 & 0.0037 & 989.18 \\
\hline 0.04991 & & 55 & 0.0091 & 994.22 \\
\hline 0.1 & & 55 & 0.0180 & 1002.75 \\
\hline 0.16987 & & 55 & 0.0302 & 1014.58 \\
\hline 0.2483 & & 55 & 0.0435 & 1027.69 \\
\hline 0.49935 & & 55 & 0.0839 & 1069.10 \\
\hline 0.74558 & & 55 & 0.1202 & 1108.57 \\
\hline 0.9986 & & 55 & 0.1547 & 1149.80 \\
\hline
\end{tabular}

\section{Ref}

App vol App

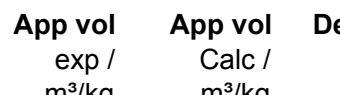

Apparent Delta density Density of $\mathrm{m}^{3} / \mathrm{kg}$ $\mathrm{m}^{3} / \mathrm{kg}$

calc / Residual / Residual /

Density based on

Inconsistent

molar volume

$/ \mathrm{kg} / \mathrm{m}^{3}$ water $/ \mathrm{kg} / \mathrm{m}^{3}$

$\begin{array}{llllll}0.000056 & 0.000148 & 1002.24 & 0.3396 & -0.000092\end{array}$

$\begin{array}{llllll}0.000043 & 0.000148 & 1006.88 & 0.9671 & -0.000105\end{array}$

$\begin{array}{llllll}0.000043 & 0.000149 & 1014.65 & 1.9661 & -0.000106\end{array}$

$\begin{array}{lllll}0.000044 & 0.000149 & 1025.45 & 3.3705 & -0.000106\end{array}$

$\begin{array}{llllll}0.000057 & 0.000153 & 1075.50 & 9.3673 & -0.000096\end{array}$

$\begin{array}{lllll}0.000060 & 0.000156 & 1112.01 & 14.4813 & -0.000096\end{array}$

$\begin{array}{llllll}0.000060 & 0.000158 & 1148.75 & 20.3413 & -0.000098\end{array}$

$\begin{array}{llllll}0.000034 & 0.000151 & 998.62 & 0.2160 & -0.000117\end{array}$

$\begin{array}{lllll}0.000031 & 0.000151 & 1000.17 & 0.4405 & -0.000120\end{array}$

$\begin{array}{llllll}0.000045 & 0.000151 & 1004.78 & 0.9747 & -0.000106\end{array}$

$\begin{array}{lllll}0.000048 & 0.000152 & 1012.51 & 1.9252 & -0.000104\end{array}$

$\begin{array}{lllll}0.000051 & 0.000153 & 1023.24 & 3.2375 & -0.000102\end{array}$

$\begin{array}{lllll}0.000052 & 0.000154 & 1035.21 & 4.7865 & -0.000102\end{array}$

$\begin{array}{lllrl}0.000062 & 0.000157 & 1072.98 & 9.2020 & -0.000094\end{array}$

$\begin{array}{lllrl}0.000067 & 0.000159 & 1109.24 & 13.8681 & -0.000093\end{array}$

$\begin{array}{lllll}0.000067 & 0.000162 & 1145.70 & 19.6424 & -0.000095\end{array}$

$\begin{array}{lllll}0.000034 & 0.000154 & 995.60 & 0.2190 & -0.000119\end{array}$

$\begin{array}{lllll}0.000049 & 0.000154 & 997.14 & 0.3841 & -0.000105\end{array}$

$\begin{array}{lllll}0.000049 & 0.000154 & 1001.72 & 0.9541 & -0.000105\end{array}$

$\begin{array}{lllll}0.000052 & 0.000155 & 1009.41 & 1.8901 & -0.000103\end{array}$

$\begin{array}{lllll}0.000055 & 0.000156 & 1020.07 & 3.1880 & -0.000101\end{array}$

$\begin{array}{lllll}0.000058 & 0.000157 & 1031.96 & 4.6147 & -0.000099\end{array}$

$\begin{array}{llllll}0.000066 & 0.000160 & 1069.48 & 9.0563 & -0.000094\end{array}$

$\begin{array}{lllll}0.000072 & 0.000163 & 1105.48 & 13.4134 & -0.000090\end{array}$

$\begin{array}{llllll}0.000072 & 0.000165 & 1141.66 & 19.0924 & -0.000093\end{array}$

$\begin{array}{lllll}0.000035 & 0.000156 & 991.77 & 0.2207 & -0.000121\end{array}$

$\begin{array}{lllll}0.000051 & 0.000156 & 993.30 & 0.3842 & -0.000106\end{array}$

$\begin{array}{lllll}0.000051 & 0.000157 & 997.86 & 0.9544 & -0.000106\end{array}$

$\begin{array}{lllll}0.000055 & 0.000157 & 1005.49 & 1.8754 & -0.000103\end{array}$

$\begin{array}{lllll}0.000058 & 0.000158 & 1016.09 & 3.1537 & -0.000101\end{array}$

$\begin{array}{lllll}0.000061 & 0.000159 & 1027.90 & 4.5681 & -0.000099\end{array}$

$\begin{array}{lllll}0.000069 & 0.000163 & 1065.16 & 9.0057 & -0.000094\end{array}$

$\begin{array}{llllll}0.000077 & 0.000165 & 1100.90 & 13.1082 & -0.000089\end{array}$

$\begin{array}{llllll}0.000076 & 0.000168 & 1136.81 & 18.7697 & -0.000092\end{array}$

$\begin{array}{lllll}0.000048 & 0.000158 & 987.24 & 0.1990 & -0.000110\end{array}$

$\begin{array}{llllll}0.000043 & 0.000159 & 988.77 & 0.4174 & -0.000116\end{array}$

$\begin{array}{llllll}0.000055 & 0.000159 & 993.29 & 0.9336 & -0.000104\end{array}$

$\begin{array}{llllll}0.000056 & 0.000160 & 1000.88 & 1.8689 & -0.000103\end{array}$

$\begin{array}{lllll}0.000058 & 0.000161 & 1011.40 & 3.1762 & -0.000102\end{array}$

$\begin{array}{lllll}0.000062 & 0.000162 & 1023.14 & 4.5502 & -0.000099\end{array}$

$\begin{array}{llllll}0.000071 & 0.000165 & 1060.15 & 8.9514 & -0.000094\end{array}$

$\begin{array}{llllll}0.000079 & 0.000168 & 1095.64 & 12.9310 & -0.000089\end{array}$

$0.000079 \quad 0.000171$

Residual
0.115
0.935
3.866
11.360
23.942
87.746
209.708
413.767
0.047
0.194
0.950
3.706
10.482
22.911
84.677
192.323
385.825
0.048
0.148
0.910
3.573
10.163
21.296
82.017
179.920
364.519
0.049
0.148
0.911
3.517
9.946
20.868
81.104
171.826
352.300
0.040
0.174
0.872
3.493
10.088
20.704
80.127
167.211
343.473

apparent
volume

$\begin{array}{rr}10.22 & 999.0996 \\ 7.85 & 999.0996 \\ 7.82 & 999.0996 \\ 7.99 & 999.0996 \\ 8.5 & 999.0996 \\ 10.52 & 999.0996 \\ 10.924 & 999.0996 \\ 11.069 & 999.0996 \\ 6.21 & 997.0449 \\ 5.73 & 997.0449 \\ 8.22 & 997.0449 \\ 8.75 & 997.0449 \\ 9.285 & 997.0449 \\ 9.45 & 997.0449 \\ 11.385 & 997.0449 \\ 12.234 & 997.0449 \\ 12.258 & 997.0449 \\ 6.32 & 994.0319 \\ 8.96 & 994.0319 \\ 9.06 & 994.0319 \\ 9.53 & 994.0319 \\ 10.01 & 994.0319 \\ 10.566 & 994.0319 \\ 12.126 & 994.0319 \\ 13.271 & 994.0319 \\ 13.235 & 994.0319 \\ 6.46 & 990.2132 \\ 9.28 & 990.2132 \\ 9.38 & 990.2132 \\ 10.01 & 990.2132 \\ 10.55 & 990.2132 \\ 11.098 & 990.2132 \\ 12.594 & 990.2132 \\ 14.038 & 990.2132 \\ 13.923 & 990.2132 \\ 8.84 & 985.6952 \\ 7.8 & 985.6952 \\ 10.02 & 985.6952 \\ 10.3 & 985.6952 \\ 10.65 & 985.6952 \\ 11.411 & 985.6952 \\ 12.965 & 985.6952 \\ 14.549 & 985.6952 \\ 14.435 & 985.6952\end{array}$




\begin{tabular}{|c|c|c|c|c|c|c|c|c|c|c|c|c|c|c|c|c|}
\hline molality & molarity & \multicolumn{2}{|c|}{$\mathrm{t} /{ }^{\circ} \mathrm{C}$ mass frac } & $\begin{array}{r}\text { Density } \\
\exp / \\
\mathrm{kg} / \mathrm{m}^{3}\end{array}$ & Ref & $\begin{array}{r}\text { App vol } \\
\text { exp / } \\
\mathrm{m}^{3} / \mathrm{kg}\end{array}$ & $\begin{array}{r}\text { App vol } \\
\text { Calc / } \\
\mathrm{m}^{3} / \mathrm{kg}\end{array}$ & $\begin{array}{r}\text { Density } \\
\mathrm{calc} / \\
\mathrm{kg} / \mathrm{m}^{3}\end{array}$ & $\begin{array}{r}\text { Density } \\
\text { Residual / } \\
\mathrm{kg} / \mathrm{m}^{3}\end{array}$ & $\begin{array}{r}\text { App Vol } \\
\text { Residual / } \\
\mathrm{m}^{3} / \mathrm{kg}\end{array}$ & $\begin{array}{r}\text { Square of } \\
\text { Density } \\
\text { Residual }\end{array}$ & $\begin{array}{l}\text { Inconsistent } \\
\text { based on } \\
\text { density }\end{array}$ & $\begin{array}{c}\text { Inconsistent } \\
\text { based on } \\
\text { apparent } \\
\text { volume }\end{array}$ & $\begin{array}{r}\text { Apparent } \\
\text { molar volume } \\
/ \mathrm{cm}^{3} / \mathrm{mol}\end{array}$ & $\begin{array}{l}\text { Delta density } \\
\qquad / \mathrm{kg} / \mathrm{m}^{3}\end{array}$ & $\begin{array}{r}\text { Density of } \\
\text { water } / \mathrm{kg} / \mathrm{m}^{3}\end{array}$ \\
\hline 0.01013 & & 65 & 0.0019 & 982.30 & (9) & 0.000040 & 0.000160 & 982.09 & 0.2146 & -0.000120 & 0.046 & & $!$ & 7.33 & & 980.5548 \\
\hline 0.02015 & & 65 & 0.0037 & 983.93 & (9) & 0.000069 & 0.000160 & 983.61 & 0.3253 & -0.000091 & 0.106 & & $!$ & 12.61 & & 980.5548 \\
\hline 0.04991 & & 65 & 0.0091 & 989.02 & (9) & 0.000057 & 0.000161 & 988.10 & 0.9141 & -0.000103 & 0.836 & & $!$ & 10.52 & & 980.5548 \\
\hline 0.1 & & 65 & 0.0180 & 997.49 & (9) & 0.000058 & 0.000161 & 995.64 & 1.8458 & -0.000103 & 3.407 & $!$ & $!$ & 10.64 & & 980.5548 \\
\hline 0.16987 & & 65 & 0.0302 & 1009.31 & (9) & 0.000058 & 0.000162 & 1006.10 & 3.2048 & -0.000105 & 10.271 & $!$ & $!$ & 10.59 & & 980.5548 \\
\hline 0.2483 & & 65 & 0.0435 & 1022.25 & (9) & 0.000064 & 0.000163 & 1017.77 & 4.4813 & -0.000099 & 20.082 & $!$ & $!$ & 11.811 & & 980.5548 \\
\hline 0.49935 & & 65 & 0.0839 & 1063.37 & (9) & 0.000073 & 0.000167 & 1054.53 & 8.8362 & -0.000094 & 78.079 & $!$ & $!$ & 13.338 & & 980.5548 \\
\hline 0.74558 & & 65 & 0.1202 & 1102.47 & (9) & 0.000082 & 0.000170 & 1089.77 & 12.7004 & -0.000088 & 161.300 & $!$ & $!$ & 15.016 & & 980.5548 \\
\hline 0.9986 & & 65 & 0.1547 & 1143.37 & (9) & 0.000081 & 0.000173 & 1125.15 & 18.2167 & -0.000092 & 331.848 & $!$ & $!$ & 14.908 & & 980.5548 \\
\hline 0.01013 & & 75 & 0.0019 & 976.58 & (9) & 0.000046 & 0.000161 & 976.37 & 0.2036 & -0.000115 & 0.041 & & $!$ & 8.4 & & 974.849 \\
\hline 0.02015 & & 75 & 0.0037 & 978.21 & (9) & 0.000067 & 0.000161 & 977.88 & 0.3321 & -0.000094 & 0.110 & & $!$ & 12.25 & & 974.849 \\
\hline 0.04991 & & 75 & 0.0091 & 983.30 & (9) & 0.000053 & 0.000162 & 982.35 & 0.9489 & -0.000108 & 0.900 & & $!$ & 9.76 & & 974.849 \\
\hline 0.1 & & 75 & 0.0180 & 991.74 & (9) & 0.000055 & 0.000162 & 989.85 & 1.8952 & -0.000107 & 3.592 & $!$ & $!$ & 10.1 & & 974.849 \\
\hline 0.16987 & & 75 & 0.0302 & 1003.45 & (9) & 0.000058 & 0.000163 & 1000.25 & 3.2072 & -0.000106 & 10.286 & $!$ & $!$ & 10.55 & & 974.849 \\
\hline 0.2483 & & 75 & 0.0435 & 1016.32 & (9) & 0.000064 & 0.000164 & 1011.84 & 4.4848 & -0.000100 & 20.113 & $!$ & $!$ & 11.789 & & 974.849 \\
\hline 0.49935 & & 75 & 0.0839 & 1057.16 & (9) & 0.000073 & 0.000168 & 1048.38 & 8.7886 & -0.000095 & 77.239 & $!$ & $!$ & 13.453 & & 974.849 \\
\hline 0.74558 & & 75 & 0.1202 & 1095.97 & (9) & 0.000083 & 0.000171 & 1083.39 & 12.5789 & -0.000088 & 158.228 & $!$ & $!$ & 15.222 & & 974.849 \\
\hline 0.9986 & & 75 & 0.1547 & 1136.60 & (9) & 0.000082 & 0.000174 & 1118.53 & 18.0743 & -0.000092 & 326.680 & $!$ & $!$ & 15.099 & & 974.849 \\
\hline 0.01013 & & 85 & 0.0019 & 970.36 & (9) & 0.000032 & 0.000161 & 970.14 & 0.2253 & -0.000129 & 0.051 & & $!$ & 5.9 & & 968.6203 \\
\hline 0.02015 & & 85 & 0.0037 & 971.93 & (9) & 0.000077 & 0.000161 & 971.64 & 0.2943 & -0.000085 & 0.087 & & $!$ & 14.08 & & 968.6203 \\
\hline 0.04991 & & 85 & 0.0091 & 977.03 & (9) & 0.000052 & 0.000162 & 976.08 & 0.9498 & -0.000110 & 0.902 & & $!$ & 9.55 & & 968.6203 \\
\hline 0.1 & & 85 & 0.0180 & 985.44 & (9) & 0.000054 & 0.000163 & 983.54 & 1.9023 & -0.000109 & 3.619 & $!$ & $!$ & 9.84 & & 968.6203 \\
\hline 0.16987 & & 85 & 0.0302 & 997.09 & (9) & 0.000056 & 0.000164 & 993.87 & 3.2164 & -0.000107 & 10.345 & $!$ & $!$ & 10.318 & & 968.6203 \\
\hline 0.2483 & & 85 & 0.0435 & 1009.90 & (9) & 0.000063 & 0.000165 & 1005.40 & 4.4973 & -0.000102 & 20.226 & $!$ & $!$ & 11.581 & & 968.6203 \\
\hline 0.49935 & & 85 & 0.0839 & 1050.42 & (9) & 0.000074 & 0.000168 & 1041.73 & 8.6898 & -0.000095 & 75.512 & $!$ & $!$ & 13.525 & & 968.6203 \\
\hline 0.74558 & & 85 & 0.1202 & 1088.90 & (9) & 0.000084 & 0.000172 & 1076.53 & 12.3696 & -0.000088 & 153.008 & $!$ & $!$ & 15.4 & & 968.6203 \\
\hline 0.9986 & & 85 & 0.1547 & 1129.29 & (9) & 0.000083 & 0.000175 & 1111.46 & 17.8281 & -0.000092 & 317.841 & $!$ & $!$ & 15.245 & & 968.6203 \\
\hline 0.01013 & & 95 & 0.0019 & 963.62 & (9) & 0.000037 & 0.000161 & 963.41 & 0.2131 & -0.000124 & 0.045 & & $!$ & 6.79 & & 961.9004 \\
\hline 0.02015 & & 95 & 0.0037 & 965.19 & (9) & 0.000076 & 0.000161 & 964.90 & 0.2909 & -0.000085 & 0.085 & & $!$ & 13.96 & & 961.9004 \\
\hline 0.04991 & & 95 & 0.0091 & 970.28 & (9) & 0.000049 & 0.000162 & 969.32 & 0.9572 & -0.000112 & 0.916 & & $!$ & 9.03 & & 961.9004 \\
\hline 0.1 & & 95 & 0.0180 & 978.66 & (9) & 0.000051 & 0.000162 & 976.74 & 1.9188 & -0.000112 & 3.682 & $!$ & $!$ & 9.31 & & 961.9004 \\
\hline 0.16987 & & 95 & 0.0302 & 990.22 & (9) & 0.000055 & 0.000163 & 987.02 & 3.1924 & -0.000108 & 10.191 & $!$ & $!$ & 10.122 & & 961.9004 \\
\hline 0.2483 & & 95 & 0.0435 & 1002.93 & (9) & 0.000063 & 0.000165 & 998.49 & 4.4347 & -0.000102 & 19.666 & $!$ & $!$ & 11.52 & & 961.9004 \\
\hline 0.49935 & & 95 & 0.0839 & 1043.15 & (9) & 0.000074 & 0.000168 & 1034.63 & 8.5218 & -0.000094 & 72.621 & $!$ & $!$ & 13.577 & & 961.9004 \\
\hline 0.74558 & & 95 & 0.1202 & 1081.29 & (9) & 0.000085 & 0.000172 & 1069.25 & 12.0408 & -0.000087 & 144.980 & $!$ & $!$ & 15.579 & & 961.9004 \\
\hline 0.9986 & & 95 & 0.1547 & 1121.40 & (9) & 0.000084 & 0.000175 & 1103.99 & 17.4044 & -0.000091 & 302.915 & $!$ & $!$ & 15.402 & & 961.9004 \\
\hline
\end{tabular}


Density of aqueous solutions of CdSO4

References (6) Asmus, A., "The viscosities of aqueous solutions of strong electrolytes of high valence type", Ann. der Physik, ser 5, 1939, 35, 1-22

(7) Bakeev, M. I.; Zharmenov, A. A.; Baikenova, N. A.; Abdygalimova, S. Sh.; Electrical conductivity and viscosity of the binary systems

MeSO4-H2O (Me=Mn2+, Fe2+, Co2+, Zn2+, Cd2+) at 25-75 degree and the structure of electrolyte solutions, Izv. Nats. Akad. Nauk Resp.

Kaz., Ser. Khim., 1994, 6, 25-30

(117) Schmelzer, N, Einfeldt, J., "Dichtemessungen an einigen...", Wiss. Z. Uni. Rostock, 1989, 38, 81-82

\begin{tabular}{|c|c|c|c|c|c|c|c|c|c|c|c|c|c|c|c|}
\hline molality & molarity & $\mathrm{t} /{ }^{\circ} \mathrm{C}$ & mass frac & $\begin{array}{r}\text { Density } \\
\exp / \\
\mathrm{kg} / \mathrm{m}^{3}\end{array}$ & Ref & $\begin{array}{r}\text { App vol } \\
\text { exp / } \\
\mathrm{m}^{3} / \mathrm{kg}\end{array}$ & $\begin{array}{r}\text { App vol } \\
\text { Calc / } \\
\mathrm{m}^{3} / \mathrm{kg}\end{array}$ & $\begin{array}{r}\text { Density } \\
\text { calc / } \\
\mathrm{kg} / \mathrm{m}^{3}\end{array}$ & $\begin{array}{r}\text { Density } \\
\text { Residual / } \\
\mathrm{kg} / \mathrm{m}^{3}\end{array}$ & $\begin{array}{r}\text { App Vol } \\
\text { Residual / } \\
\mathrm{m}^{3} / \mathrm{kg}\end{array}$ & $\begin{array}{l}\text { Square of } \\
\text { Density } \\
\text { Residual }\end{array}$ & $\begin{array}{l}\text { Inconsistent } \\
\text { based on } \\
\text { density }\end{array}$ & $\begin{array}{l}\text { Inconsistent } \\
\text { based on } \\
\text { apparent } \\
\text { volume }\end{array}$ & $\begin{array}{r}\text { Relative } \\
\text { density }\end{array}$ & $\begin{array}{r}\text { Density of } \\
\text { water } / \mathrm{kg} / \mathrm{m}^{3}\end{array}$ \\
\hline & 0.000069 & 25 & 0.0000 & 997.06 & (6) & -0.000040 & 0.000016 & 997.06 & 0.0008 & -0.000056 & 0.000 & & & 1.000015 & 997.0449 \\
\hline & 0.000118 & 25 & 0.0000 & 997.07 & (6) & -0.000054 & 0.000016 & 997.07 & 0.0017 & -0.000070 & 0.000 & & & 1.000026 & 997.0449 \\
\hline & 0.000193 & 25 & 0.0000 & 997.09 & (6) & -0.000041 & 0.000016 & 997.08 & 0.0023 & -0.000057 & 0.000 & & & 1.000042 & 997.0449 \\
\hline & 0.000268 & 25 & 0.0001 & 997.10 & (6) & -0.000017 & 0.000016 & 997.10 & 0.0019 & -0.000033 & 0.000 & & & 1.000057 & 997.0449 \\
\hline & 0.000343 & 25 & 0.0001 & 997.12 & (6) & -0.000004 & 0.000016 & 997.12 & 0.0014 & -0.000020 & 0.000 & & & 1.000072 & 997.0449 \\
\hline & 0.000395 & 25 & 0.0001 & 997.13 & (6) & -0.000017 & 0.000016 & 997.13 & 0.0027 & -0.000033 & 0.000 & & & 1.000084 & 997.0449 \\
\hline & 0.000483 & 25 & 0.0001 & 997.15 & (6) & -0.000050 & 0.000016 & 997.14 & 0.0066 & -0.000066 & 0.000 & & & 1.000106 & 997.0449 \\
\hline & 0.000577 & 25 & 0.0001 & 997.16 & (6) & 0.000005 & 0.000016 & 997.16 & 0.0013 & -0.000011 & 0.000 & & & 1.00012 & 997.0449 \\
\hline & 0.000677 & 25 & 0.0001 & 997.19 & (6) & -0.000031 & 0.000016 & 997.18 & 0.0067 & -0.000048 & 0.000 & & & 1.000146 & 997.0449 \\
\hline & 0.000804 & 25 & 0.0002 & 997.21 & (6) & 0.000013 & 0.000016 & 997.21 & 0.0006 & -0.000004 & 0.000 & & & 1.000166 & 997.0449 \\
\hline & 0.000943 & 25 & 0.0002 & 997.24 & (6) & 0.000021 & 0.000016 & 997.24 & -0.0010 & 0.000005 & 0.000 & & & 1.000193 & 997.0449 \\
\hline .000968 & & 25 & 0.0002 & 997.24 & (117) & 0.000030 & 0.000016 & 997.24 & -0.0028 & 0.000014 & 0.000 & & & & 997.0449 \\
\hline & 0.001108 & 25 & 0.0002 & 997.27 & (6) & 0.000025 & 0.000016 & 997.27 & -0.0019 & 0.000008 & 0.000 & & & 1.000226 & 997.0449 \\
\hline & 0.001362 & 25 & 0.0003 & 997.32 & (6) & 0.000017 & 0.000016 & 997.32 & -0.0002 & 0.000001 & 0.000 & & & 1.00028 & 997.0449 \\
\hline & 0.001622 & 25 & 0.0003 & 997.38 & (6) & 0.000021 & 0.000016 & 997.38 & -0.0016 & 0.000005 & 0.000 & & & 1.000332 & 997.0449 \\
\hline .001631 & & 25 & 0.0003 & 997.38 & (117) & 0.000011 & 0.000016 & 997.38 & 0.0016 & -0.000005 & 0.000 & & & & 997.0449 \\
\hline & 0.001997 & 25 & 0.0004 & 997.45 & $(6)$ & 0.000016 & 0.000016 & 997.45 & 0.0002 & -0.000001 & 0.000 & & & 1.000411 & 997.0449 \\
\hline & 0.002357 & 25 & 0.0005 & 997.52 & (6) & 0.000024 & 0.000016 & 997.53 & -0.0038 & 0.000008 & 0.000 & & & 1.000481 & 997.0449 \\
\hline .002721 & & 25 & 0.0006 & 997.61 & (117) & 0.000001 & 0.000016 & 997.60 & 0.0087 & -0.000015 & 0.000 & & & & 997.0449 \\
\hline & 0.002787 & 25 & 0.0006 & 997.61 & (6) & 0.000025 & 0.000016 & 997.62 & -0.0052 & 0.000009 & 0.000 & & & 1.000568 & 997.0449 \\
\hline & 0.003264 & 25 & 0.0007 & 997.71 & (6) & 0.000026 & 0.000016 & 997.71 & -0.0063 & 0.000009 & 0.000 & & & 1.000665 & 997.0449 \\
\hline & 0.003787 & 25 & 0.0008 & 997.81 & (6) & 0.000030 & 0.000016 & 997.82 & -0.0109 & 0.000014 & 0.000 & & & 1.000768 & 997.0449 \\
\hline .004254 & & 25 & 0.0009 & 997.95 & (117) & -0.000024 & 0.000016 & 997.91 & 0.0353 & -0.000040 & 0.001 & & & & 997.0449 \\
\hline .004324 & & 25 & 0.0009 & 997.96 & (117) & -0.000018 & 0.000016 & 997.93 & 0.0311 & -0.000035 & 0.001 & & & & 997.0449 \\
\hline & 0.004410 & 25 & 0.0009 & 997.94 & (6) & 0.000028 & 0.000016 & 997.95 & -0.0110 & 0.000012 & 0.000 & & & 1.000896 & 997.0449 \\
\hline & 0.005315 & 25 & 0.0011 & 998.12 & (6) & 0.000028 & 0.000016 & 998.13 & -0.0131 & 0.000012 & 0.000 & & & 1.00108 & 997.0449 \\
\hline & 0.006294 & 25 & 0.0013 & 998.32 & (6) & 0.000027 & 0.000016 & 998.34 & -0.0144 & 0.000011 & 0.000 & & & 1.00128 & 997.0449 \\
\hline & 0.007247 & 25 & 0.0015 & 998.51 & (6) & 0.000031 & 0.000016 & 998.53 & -0.0213 & 0.000014 & 0.000 & & & 1.001469 & 997.0449 \\
\hline & 0.008176 & 25 & 0.0017 & 998.69 & $(6)$ & 0.000033 & 0.000017 & 998.72 & -0.0283 & 0.000017 & 0.001 & & & 1.001653 & 997.0449 \\
\hline .018249 & & 25 & 0.0038 & 1000.76 & (117) & 0.000021 & 0.000017 & 1000.77 & -0.0138 & 0.000004 & 0.000 & & & & 997.0449 \\
\hline .019681 & & 25 & 0.0041 & 1001.10 & (117) & 0.000009 & 0.000017 & 1001.07 & 0.0338 & -0.000008 & 0.001 & & & & 997.0449 \\
\hline 0.16032 & & 25 & 0.0323 & 1029.99 & (117) & 0.000011 & 0.000023 & 1029.59 & 0.4023 & -0.000012 & 0.162 & & & & 997.0449 \\
\hline 0.20177 & & 25 & 0.0404 & 1038.73 & (117) & 0.000006 & 0.000025 & 1037.92 & 0.8125 & -0.000019 & 0.660 & & & & 997.0449 \\
\hline & & 25 & 0.1699 & 1184.90 & $(7)$ & 0.000067 & 0.000062 & 1186.08 & -1.1792 & 0.000005 & 1.391 & & & & 997.0449 \\
\hline & & 25 & 0.2100 & 1237.60 & (7) & 0.000075 & 0.000079 & 1236.21 & 1.3855 & -0.000004 & 1.920 & & & & 997.0449 \\
\hline & & 25 & 0.2967 & 1345.20 & (7) & 0.000128 & 0.000132 & 1343.37 & 1.8341 & -0.000003 & 3.364 & & & & 997.0449 \\
\hline & & 50 & 0.0050 & 993.20 & (7) & -0.000040 & 0.000027 & 992.87 & 0.3295 & -0.000067 & 0.109 & & & & 988.0363 \\
\hline
\end{tabular}


molality molarity

$\begin{array}{crrr}\mathbf{t} /{ }^{\circ} \mathbf{C} & \text { mass frac } & \begin{array}{r}\text { Density } \\ \text { exp } / \\ \mathrm{kg} / \mathrm{m}^{3}\end{array} & \text { Ref } \\ & & 1174.00 \\ 50 & 0.1699 & 17) \\ 50 & 0.2100 & 1225.70 \\ 50 & 0.2967 & 1332.10 \\ 75 & 0.0050 & 979.80 \\ 75 & 0.1699 & 1158.00 \\ 75 & 0.2100 & 1210.20 \\ 75 & 0.2242 & 1227.30 \\ 75 & 0.2967 & 1321.00\end{array}$

Ref

App vol App vol
$\exp / \quad$ Calc

$\begin{array}{cc}\exp / & \text { Calc } \\ \mathrm{m}^{3} / \mathrm{kg} & \mathrm{m}^{3} / \mathrm{kg}\end{array}$

$\begin{array}{ll}0.000068 & 0.000067\end{array}$

$\begin{array}{ll}0.000078 & 0.000082\end{array}$

$0.000131 \quad 0.000129$

$\begin{array}{ll}-0.000011 & 0.000031\end{array}$

$\begin{array}{ll}0.000071 & 0.000066\end{array}$

$0.000076 \quad 0.000079$

$0.000084 \quad 0.000085$

$0.000120 \quad 0.000120$

Average Res

Std dev Res

Avg -4 std
Avg +4 std
Density Density App Vol Square of Inconsistent Inconsisten calc / Residual / Residual / Density based on based on $\mathrm{kg} / \mathrm{m}^{3} \quad \mathrm{~kg} / \mathrm{m}^{3} \quad \mathrm{~m}^{3} / \mathrm{kg}$ Residual density apparent

$\begin{array}{llll}1174.42 & -0.4202 & 0.000002 & 0.177\end{array}$

$\begin{array}{llll}1224.39 & 1.3070 & -0.000004 & 1.708\end{array}$

$\begin{array}{llll}1333.33 & -1.2335 & 0.000002 & 1.521\end{array}$

$\begin{array}{llll}9.60 & 0.2002 & -0.000042 & 0.040\end{array}$

$\begin{array}{llll}159.09 & -1.0941 & 0.000005 & 1.197\end{array}$

$1.0496-0.000003 \quad 1.102$

$0.0624 \quad 0.000000 \quad 0.004$

$0.3175-0.000001$

$0.0839-0.000011$

$0.5465 \quad 0.000025$

$-2.1023-0.000110$

$2.2701 \quad 0.000087$
Relative Density of density water $/ \mathrm{kg} / \mathrm{m}^{3}$

988.0363 988.0363 988.0363

974.849

974.849

974.849

974.849

13.460 Sum Chi sqr

\section{Curve fit for the model App vol $=\left(w+c 2+c 3^{*} t{ }^{\circ} \mathrm{C}\right) /\left(c 0^{*} w+c 1\right) / e^{\wedge}\left(0.000001^{*}\left(t{ }^{\circ} \mathrm{C}+c 4\right)^{\wedge} 2\right)$}

$\begin{array}{lr}\text { c0 } 1 & -1 \mathrm{E}-07 \\ \text { c1 } & 6.11 \mathrm{E}-08 \\ \text { c3 } & -0.00376 \\ \text { c4 } & 0.004108 \\ \text { Min T } & 5007.691 \\ \text { Max T } & 25 \\ \text { Min w } & 75 \\ \text { Max w } & 1.44 \mathrm{E}-05 \\ \text { Avg dens res } & 0.29671 \\ \text { Std dens res } & 0.0839 \\ \text { Avg app vol res *10^3 } & 0.5465 \\ \text { Std app vol res *10^3 } & 0.024114 \\ \text { No of points in corr } & 45 \\ \text { No of inconsistent poir } & 24\end{array}$

Inconsistent data not used (Residual greater than average $+/-4$ standard deviations)

\begin{tabular}{|c|c|c|c|c|c|c|c|c|c|c|c|c|}
\hline 25 & 0.0005 & 998.20 & (7) & -0.001318 & 0.000016 & 997.54 & 0.6644 & -0.001335 & 0.441 & & $!$ & 997.0449 \\
\hline 25 & 0.0050 & 999.30 & (7) & 0.000550 & 0.000017 & 1001.97 & -2.6690 & 0.000533 & 7.123 & ! & $!$ & 997.0449 \\
\hline 25 & 0.0150 & 1006.70 & (7) & 0.000360 & 0.000019 & 1011.89 & -5.1938 & 0.000341 & 26.976 & ! & $!$ & 997.0449 \\
\hline 25 & 0.0299 & 1013.60 & (7) & 0.000456 & 0.000022 & 1027.11 & -13.5145 & 0.000434 & 182.642 & $!$ & $!$ & 997.0449 \\
\hline 25 & 0.0699 & 1081.80 & (7) & -0.000121 & 0.000031 & 1069.50 & 12.2974 & -0.000152 & 151.227 & ! & $!$ & 997.0449 \\
\hline 25 & 0.0990 & 1141.00 & (7) & -0.000275 & 0.000039 & 1101.89 & 39.1134 & -0.000314 & 1529.857 & $!$ & $!$ & 997.0449 \\
\hline 25 & 0.1300 & 1148.40 & (7) & -0.000014 & 0.000048 & 1137.84 & 10.5573 & -0.000062 & 111.457 & ! & & 997.0449 \\
\hline 25 & 0.2242 & 1249.20 & (7) & 0.000100 & 0.000086 & 1254.10 & -4.9030 & 0.000014 & 24.040 & ! & & 997.0449 \\
\hline 25 & 0.2699 & 1309.10 & (7) & 0.000117 & 0.000112 & 1311.44 & -2.3420 & 0.000005 & 5.485 & ! & & 997.0449 \\
\hline 50 & 0.0005 & 990.40 & (7) & -0.003819 & 0.000026 & 988.52 & 1.8820 & -0.003845 & 3.542 & & ! & 988.0363 \\
\hline 50 & 0.0150 & 996.20 & (7) & 0.000458 & 0.000028 & 1002.62 & -6.4161 & 0.000429 & 41.167 & ! & ! & 988.0363 \\
\hline 50 & 0.0299 & 1009.50 & (7) & 0.000293 & 0.000031 & 1017.57 & -8.0671 & 0.000262 & 65.078 & ! & ! & 988.0363 \\
\hline
\end{tabular}


molality molarity
Ref

\section{ensity}

$\exp /$

$\mathrm{kg} / \mathrm{m}^{3}$

$0.0699 \quad 1069.60$

$0.0990 \quad 1321.00$

$0.1300 \quad 1137.70$

$0.2242 \quad 1238.00$

$0.2699 \quad 1288.60$

$0.0005 \quad 976.50$

$0.0150 \quad 983.30$

$0.0299 \quad 993.70$

$0.0699 \quad 1055.70$

$0.0990 \quad 1119.00$

$0.1300 \quad 1122.90$

$0.2699 \quad 1272.10$
App vol App vol Density Density App Vol Square of Inconsistent Inconsistent

$\exp / \mathrm{Calc} /$

$\mathrm{m}^{3} / \mathrm{kg}$

Calc /
$\mathrm{m}^{3} / \mathrm{kg}$

$\begin{array}{ll}-0.000091 & 0.000039\end{array}$

$\begin{array}{ll}-0.001565 & 0.000046\end{array}$

$-0.0000120 .000054$

$0.000100 \quad 0.000088$

$0.000138 \quad 0.000111$

$\begin{array}{ll}-0.002443 & 0.000030\end{array}$

$\begin{array}{ll}0.000436 & 0.000032\end{array}$

$0.000376 \quad 0.000035$

$-0.000097 \quad 0.000042$

$-0.000309 \quad 0.000048$

$-0.0000150 .000055$

$0.000138 \quad 0.000105$ calc / Residual / Residual / Density based on based on $\mathrm{kg} / \mathrm{m}^{3} \quad \mathrm{~kg} / \mathrm{m}^{3} \quad \mathrm{~m}^{3} / \mathrm{kg}$ Residual density $\begin{gathered}\text { apparent } \\ \text { volume }\end{gathered}$

$\begin{array}{llll}1059.25 & 10.3539 & -0.000131 & 107.203\end{array}$ $\begin{array}{lrrr}1091.14 & 229.8568 & -0.001611 & 52834.15\end{array}$ $\begin{array}{llll}1126.63 & 11.0656 & -0.000066 & 122.447\end{array}$ $1242.33-4.3293 \quad 0.000013-18.743$ $\begin{array}{llll}1300.40 & -11.7988 & 0.000026 & 139.212\end{array}$ $\begin{array}{llll}975.32 & 1.1777 & -0.002473 & 1.387\end{array}$ $\begin{array}{llll}989.18 & -5.8802 & 0.000404 & 34.576\end{array}$ $\begin{array}{llll}1003.89 & -10.1856 & 0.000341 & 103.747\end{array}$ $\begin{array}{llll}1044.94 & 10.7596 & -0.000139 & 115.770\end{array}$ $\begin{array}{lllll}1076.43 & 42.5664 & -0.000357 & 1811.898\end{array}$ $\begin{array}{llll}1111.57 & 11.3278 & -0.000070 & 128.318\end{array}$ $\begin{array}{llll}1286.44 & -14.3415 & 0.000032 & 205.677\end{array}$
Relative Density of density water $/ \mathrm{kg} / \mathrm{m}^{3}$

988.0363 988.0363 988.0363

988.0363

988.0363

974.849

974.849

974.849

974.849

974.849

974.849

974.849 
Density of aqueous solutions of $\mathbf{C o C l 2}$

References (37) Herrington, Thelma M.; Roffey, Michael G.; Smith, David P.; Densities of Aqueous Electrolytes $\mathrm{MnCl}_{2}, \mathrm{CoCl}_{2}, \mathrm{NiCl}_{2}, \mathrm{ZnCl}_{2}$ and $\mathrm{CdCl}_{2}$ from 25 to $72^{\circ} \mathrm{C}$ at $1 \mathrm{~atm}$, J. Chem. Eng. Data, 1986, 31, 221-225

(93) Phang, S. The density, Viscosity and Transference Number of Aqueous Cobalt Chloride at 298.15 K. Aust. J. Chem. 1980, 33, 641-645.

(99) Pogue, R. F.; Atkinson, G. Solution Thermodynamics of First-Row Transition Elements. 2. Apparent Molal Volumes of Aqueous MnCl2, $\mathrm{Mn}\left(\mathrm{ClO}_{4}\right)_{2}, \mathrm{CoCl}_{2}, \mathrm{Co}\left(\mathrm{ClO}_{4}\right)_{2}, \mathrm{FeCl}_{2}$, and $\mathrm{Fe}\left(\mathrm{ClO}_{4}\right)_{2}$, from 15 to $55^{\circ} \mathrm{C}$. J. Chem. Eng. Data 1989, 34, 227-232.

(123) Spitzer, J. J.; Singh, P. P.; McCurdy, K. G.; Hepler, L. G. Apparent Molar Heat Capacities and Volumes of Aqueous Electrolytes: CaCl 2 , $\mathrm{Cd}\left(\mathrm{NO}_{3}\right)_{2}, \mathrm{CoCl}_{2}, \mathrm{Cu}\left(\mathrm{ClO}_{4}\right)_{2}, \mathrm{Mg}\left(\mathrm{ClO}_{4}\right)_{2}$, and $\mathrm{NiCl}_{2}$. J. Sol. Chem. 1978, 7, 81-86.

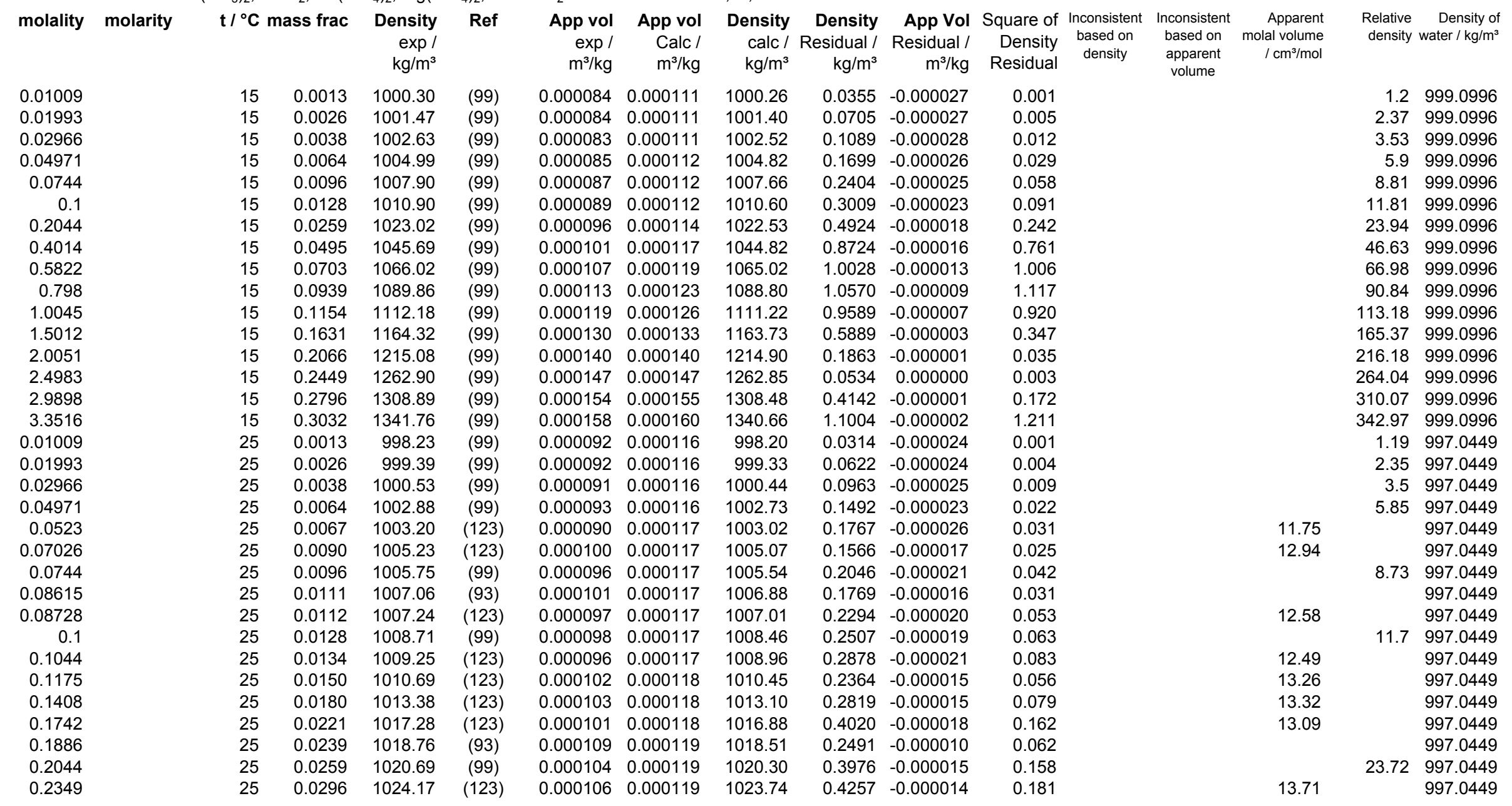


$\mathrm{t} /{ }^{\circ} \mathrm{C}$ mass frac Density

$$
\exp /
$$

$\exp /$
$\mathrm{kg} / \mathrm{m}^{3}$

0.2419

0.4014

0.4483

0.464

0.5822

0.6948

0.798
0.8694

0.8694

1.0045
1.1498

1.351

1.3886

1.5012

1.6373

1.661

2.0051

2.132
2.162

2.314

2.3727

2.4983

2.6227
2.824

2.8825

2.9898

3.29

3.3516

3.68

4.048
0.01009

0.01993

0.02966

0.04971

0.0744

0.1
2044

0.2419

0.4014

0.464

0.5822

0.6948

$\begin{array}{rrr}25 & 0.0305 & 1024.88 \\ 25 & 0.0495 & 1043.11 \\ 25 & 0.0550 & 1047.86 \\ 25 & 0.0568 & 1049.86 \\ 25 & 0.0703 & 1063.23 \\ 25 & 0.0827 & 1075.27 \\ 25 & 0.0939 & 1086.83 \\ 25 & 0.1014 & 1093.50 \\ 25 & 0.1069 & 1099.71 \\ 25 & 0.1154 & 1108.93 \\ 25 & 0.1299 & 1123.82 \\ 25 & 0.1492 & 1143.65 \\ 25 & 0.1528 & 1148.53 \\ 25 & 0.1631 & 1160.64 \\ 25 & 0.1753 & 1173.74 \\ 25 & 0.1774 & 1174.76 \\ 25 & 0.1969 & 1198.62 \\ 25 & 0.2066 & 1211.01 \\ 25 & 0.2168 & 1222.41 \\ 25 & 0.2192 & 1223.61 \\ 25 & 0.2310 & 1237.96 \\ 25 & 0.2355 & 1245.44 \\ 25 & 0.2449 & 1258.47 \\ 25 & 0.2540 & 1268.95 \\ 25 & 0.2683 & 1285.27 \\ 25 & 0.2723 & 1292.97 \\ 25 & 0.2796 & 1304.16 \\ 25 & 0.2993 & 1326.97 \\ 25 & 0.3032 & 1336.74 \\ 25 & 0.3233 & 1360.70 \\ 25 & 0.3445 & 1391.69 \\ 35 & 0.0013 & 995.20 \\ 35 & 0.0026 & 996.36 \\ 35 & 0.0038 & 997.49 \\ 35 & 0.0064 & 999.81 \\ 35 & 0.0096 & 1002.65 \\ 35 & 0.0128 & 1005.60 \\ 35 & 0.0259 & 1017.46 \\ 35 & 0.0305 & 1021.70 \\ 35 & 0.0495 & 1039.66 \\ 35 & 0.0568 & 1046.53 \\ 35 & 0.0703 & 1059.59 \\ 35 & 0.0827 & 1071.79\end{array}$

Ref

\begin{tabular}{|c|c|}
\hline $\begin{array}{l}\text { App vol } \\
\text { exp }\end{array}$ & App vo \\
\hline
\end{tabular}

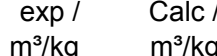

$\mathrm{m}^{3} / \mathrm{kg}$

(37)

$(99)$
$(93)$

(37)

(99)

$(37)$
$(99)$

$(99)$
$(93)$

(37)

$(37)$
$(99)$

(37)

(93)

$(37)$
$(99)$

$(99)$
$(37)$

(93)

(37)

$(99)$
$(37)$

$(37)$
(93)

$(93)$
$(37)$

(99)

(37)

(37)

$(37)$
$(99)$

$(99)$
$(93)$

(99)

(93)

$(93)$
$(99)$
$(99)$
$(99)$

$(99)$
$(99)$
$(99)$

(99)

$(99)$
$(99)$

$(37)$
$(99)$
$(37)$

$(99)$
$(37)$
$(99)$

(99)

(37) $\begin{array}{llllll}0.000108 & 0.000119 & 1024.53 & 0.3509 & -0.000011 & 0.123\end{array}$

$\begin{array}{llllll}0.000109 & 0.000122 & 1042.41 & 0.6994 & -0.000013 & 0.489\end{array}$

$\begin{array}{llllll}0.000119 & 0.000123 & 1047.63 & 0.2308 & -0.000004 & 0.053\end{array}$

$\begin{array}{llllll}0.000115 & 0.000123 & 1049.37 & 0.4872 & -0.000008 & 0.237\end{array}$

$\begin{array}{llllll}0.000115 & 0.000125 & 1062.44 & 0.7905 & -0.000010 & 0.625\end{array}$

$\begin{array}{llllll}0.000121 & 0.000126 & 1074.78 & 0.4882 & -0.000005 & 0.238\end{array}$

$\begin{array}{llllll}0.000120 & 0.000128 & 1086.01 & 0.8230 & -0.000007 & 0.677\end{array}$

$\begin{array}{llllll}0.000131 & 0.000129 & 1093.72 & -0.2208 & 0.000002 & 0.049\end{array}$

$\begin{array}{llllll}0.000127 & 0.000130 & 1099.32 & 0.3858 & -0.000003 & 0.149\end{array}$

$\begin{array}{llllll}0.000126 & 0.000131 & 1108.20 & 0.7285 & -0.000005 & 0.531\end{array}$

$\begin{array}{llllll}0.000132 & 0.000133 & 1123.61 & 0.2062 & -0.000001 & 0.042\end{array}$

$\begin{array}{llllll}0.000141 & 0.000136 & 1144.66 & -1.0085 & 0.000005 & 1.017\end{array}$

$\begin{array}{llllll}0.000137 & 0.000137 & 1148.55 & -0.0232 & 0.000000 & 0.001\end{array}$

$\begin{array}{llllll}0.000136 & 0.000139 & 1160.14 & 0.4959 & -0.000002 & 0.246\end{array}$

$\begin{array}{llllll}0.000142 & 0.000141 & 1174.01 & -0.2680 & 0.000001 & 0.072\end{array}$

$\begin{array}{llllll}0.000148 & 0.000141 & 1176.41 & -1.6457 & 0.000007 & 2.708\end{array}$

$\begin{array}{llllll}0.000146 & 0.000144 & 1199.10 & -0.4819 & 0.000002 & 0.232\end{array}$

$\begin{array}{llllll}0.000145 & 0.000146 & 1210.66 & 0.3490 & -0.000001 & 0.122\end{array}$

$\begin{array}{llllll}0.000150 & 0.000148 & 1223.03 & -0.6191 & 0.000002 & 0.383\end{array}$

$\begin{array}{llllll}0.000156 & 0.000149 & 1225.93 & -2.3217 & 0.000007 & 5.390\end{array}$

$\begin{array}{llllll}0.000158 & 0.000151 & 1240.51 & -2.5530 & 0.000007 & 6.518\end{array}$

$\begin{array}{llllll}0.000154 & 0.000152 & 1246.09 & -0.6480 & 0.000002 & 0.420\end{array}$

$\begin{array}{lllllll}0.000152 & 0.000154 & 1257.91 & 0.5590 & -0.000001 & 0.312\end{array}$

$\begin{array}{llllll}0.000157 & 0.000156 & 1269.48 & -0.5286 & 0.000001 & 0.279\end{array}$

$\begin{array}{llllll}0.000165 & 0.000159 & 1287.89 & -2.6239 & 0.000006 & 6.885\end{array}$

$\begin{array}{llllll}0.000160 & 0.000160 & 1293.18 & -0.2051 & 0.000000 & 0.042\end{array}$

$\begin{array}{llllll}0.000158 & 0.000161 & 1302.78 & 1.3858 & -0.000003 & 1.920\end{array}$

$\begin{array}{lllllll}0.000170 & 0.000166 & 1329.07 & -2.1046 & 0.000004 & 4.429\end{array}$

$\begin{array}{llllll}0.000162 & 0.000167 & 1334.37 & 2.3726 & -0.000004 & 5.629\end{array}$

$\begin{array}{llllll}0.000174 & 0.000172 & 1361.97 & -1.2659 & 0.000002 & 1.603\end{array}$

$\begin{array}{llllll}0.000177 & 0.000177 & 1391.68 & 0.0138 & 0.000000 & 0.000\end{array}$

$\begin{array}{llllll}0.000100 & 0.000118 & 995.18 & 0.0241 & -0.000019 & 0.001\end{array}$

$\begin{array}{llllll}0.000096 & 0.000119 & 996.30 & 0.0575 & -0.000022 & 0.003\end{array}$

$\begin{array}{lllll}0.000097 & 0.000119 & 997.41 & 0.0843 & -0.000022\end{array}$

$\begin{array}{llllll}0.000100 & 0.000119 & 999.68 & 0.1226 & -0.000019 & 0.015\end{array}$

$\begin{array}{llllll}0.000102 & 0.000119 & 1002.49 & 0.1649 & -0.000017 & 0.027\end{array}$

$\begin{array}{lllllll}0.000103 & 0.000120 & 1005.38 & 0.2181 & -0.000017 & 0.048\end{array}$

$\begin{array}{llllll}0.000110 & 0.000121 & 1017.16 & 0.3055 & -0.000011 & 0.093\end{array}$

$\begin{array}{llllll}0.000111 & 0.000122 & 1021.36 & 0.3364 & -0.000011 & 0.113\end{array}$

$\begin{array}{llllll}0.000115 & 0.000124 & 1039.14 & 0.5182 & -0.000010 & 0.269\end{array}$

$\begin{array}{lllllll}0.000118 & 0.000125 & 1046.06 & 0.4679 & -0.000008 & 0.219\end{array}$

$\begin{array}{llllll}0.000120 & 0.000127 & 1059.05 & 0.5403 & -0.000007 & 0.292\end{array}$

$\begin{array}{llllll}0.000124 & 0.000129 & 1071.31 & 0.4754 & -0.000005 & 0.226\end{array}$
Relative Density of

density water $/ \mathrm{kg} / \mathrm{m}^{3}$

997.0449

46.2997 .0449

997.0449

997.0449

$66.38 \quad 997.0449$

997.0449

$90.05 \quad 997.0449$

997.0449

997.0449

112.22997 .0449

997.0449

997.0449

997.0449

$164.08 \quad 997.0449$

997.0449

997.0449

997.0449

$214.6 \quad 997.0449$

997.0449

997.0449

997.0449

997.0449

262.2997 .0449

997.0449

997.0449

997.0449

$308.03 \quad 997.0449$

997.0449

$340.7 \quad 997.0449$

997.0449

997.0449

$1.18 \quad 994.0319$

$2.34 \quad 994.0319$

$3.48 \quad 994.0319$

$\begin{array}{ll}5.81 & 994.0319\end{array}$

$8.67 \quad 994.0319$

$11.64 \quad 994.0319$

$23.57 \quad 994.0319$

994.0319

$45.9 \quad 994.0319$

994.0319

$65.95 \quad 994.0319$

994.0319 
molality molarity

$\mathrm{t} /{ }^{\circ} \mathrm{C}$ mass frac Density

$$
\exp /
$$

$\exp /$
$\mathrm{kg} / \mathrm{m}^{3}$

0.798

0.9215

1.0045
1.1498

1.1498

1.3886
1.5012

1.6373

1.8878

2.0051

2.132

2.3727

2.4983

2.6227

2.8825

2.9898

3.3516

0.01009

0.01993

0.02966

0.04971

0.0744

0.1
0.2419

0.4014

0.464

0.5822

0.6948

0.798

0.9215

1.0045

1.1498

1.3886

1.5012

1.6373

1.8878

2.0051

2.132

2.3727

2.4983

2.6227

2.8825

2.9898

3.3516

$\begin{array}{rrr}35 & 0.0939 & 1082.98 \\ 35 & 0.1069 & 1096.10 \\ 35 & 0.1154 & 1104.91 \\ 35 & 0.1299 & 1120.10 \\ 35 & 0.1528 & 1144.70 \\ 35 & 0.1631 & 1156.16 \\ 35 & 0.1753 & 1169.81 \\ 35 & 0.1969 & 1194.60 \\ 35 & 0.2066 & 1206.17 \\ 35 & 0.2168 & 1218.31 \\ 35 & 0.2355 & 1241.26 \\ 35 & 0.2449 & 1253.27 \\ 35 & 0.2540 & 1264.69 \\ 35 & 0.2723 & 1288.64 \\ 35 & 0.2796 & 1298.51 \\ 35 & 0.3032 & 1330.83 \\ 45 & 0.0013 & 991.39 \\ 45 & 0.0026 & 992.52 \\ 45 & 0.0038 & 993.64 \\ 45 & 0.0064 & 995.94 \\ 45 & 0.0096 & 998.75 \\ 45 & 0.0128 & 1001.66 \\ 45 & 0.0305 & 1017.76 \\ 45 & 0.0495 & 1035.40 \\ 45 & 0.0568 & 1042.50 \\ 45 & 0.0703 & 1055.10 \\ 45 & 0.0827 & 1067.69 \\ 45 & 0.0939 & 1078.25 \\ 45 & 0.1069 & 1091.94 \\ 45 & 0.1154 & 1099.98 \\ 45 & 0.1299 & 1115.88 \\ 45 & 0.1528 & 1140.42 \\ 45 & 0.1631 & 1150.72 \\ 45 & 0.1753 & 1165.46 \\ 45 & 0.1969 & 1190.18 \\ 45 & 0.2066 & 1200.34 \\ 45 & 0.2168 & 1213.80 \\ 45 & 0.2355 & 1236.66 \\ 45 & 0.2449 & 1246.93 \\ 45 & 0.2540 & 1259.98 \\ 45 & 0.2723 & 1283.78 \\ 45 & 0.2796 & 1291.80 \\ 45 & 0.3032 & 1323.47\end{array}$

$\begin{array}{lll}45 & 0.3032 & 1323.47\end{array}$
Ref

$\begin{array}{rr}\text { App vol } & \text { App vol } \\ \exp / & \text { Calc / }\end{array}$

Density

sidual

App Vol

Square of Inconsistent In
Density based on b
density

nconsistent

Apparent al volume $\mathrm{m}^{3} / \mathrm{kg} \quad \mathrm{m}^{3} / \mathrm{k}$ $\mathrm{kg} / \mathrm{m}^{3}$

$(99)$
$(37)$

$0.000126 \quad 0.000131$

1082.47

0.4

$\begin{array}{ll}0.5110 & -0.000005\end{array}$

$\begin{array}{ll}0.4022 & -0.000003\end{array}$

$0.3874-0.000003$

0.261

$\begin{array}{lll}0.000131 & 0.000134 & 1104.52\end{array}$

$0.2782-0.000002$

0.162

0.150

$\begin{array}{lll}0.000134 & 0.000136 & 1119.82\end{array}$

$0.1181-0.000001$

0.077

0.014

$\begin{array}{lll}0.000141 & 0.000142 & 1156.09\end{array}$

$\begin{array}{ll}0.0722 & 0.000000\end{array}$

0.005

0.001

$\begin{array}{llllll}0.000148 & 0.000148 & 1194.74 & -0.1368 & 0.000000 & 0.019 \\ 0.000149 & 0.000149 & 1206.20 & -0.0321 & 0.000000 & 0.001\end{array}$

$\begin{array}{llllll}0.000149 & 0.000149 & 1206.20 & -0.0321 & 0.000000 & 0.001 \\ 0.000152 & 0.000151 & 1218.46 & -0.1520 & 0.000000 & 0.023\end{array}$

$\begin{array}{lll}0.000155 & 0.000155 & 1241.32\end{array}$

0.023

0.055

$\begin{array}{llllll}0.000158 & 0.000159 & 1264.49 & 0.2355 & -0.000001 & 0.055\end{array}$

0.475

1.119

\begin{tabular}{rrrrrr}
0.000166 & 0.000170 & 1328.70 & 2.1267 & -0.000004 & 4.523 \\
\hline
\end{tabular}

$\begin{array}{lll}0.000092 & 0.000119 & 991.36\end{array}$

$0.000100 \quad 0.000119$

992.47

$0.0341-0.00002$

0.002

0.004

0.009

$0.000105 \quad 0.000120$

993.57
995.84

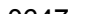

0.0933

$\begin{array}{lll}0.1161 & -0.000012 & 0.013\end{array}$

$\begin{array}{lll}0.000108 & 0.000120 & 998.63\end{array}$

$0.000109 \quad 0.000120$

1001.52

$0.000112 \quad 0.000123$

$\begin{array}{ll}0.000120 & 0.000125 \\ 0.000118 & 0.000126\end{array}$

1017.44

0.020

0.105

0.066

0.217

$0.000126 \quad 0.000128$

1042.03

$.2566-0.000005$

0.018

0.255

$\begin{array}{lll}0.000124 & 0.000130 & 1067.18\end{array}$

$0.000132 \quad 0.000131$

1067.18
1078.29

$0.1340-0.00$

$0.5052-0.000005$

$-0.0387 \quad 0.000000$

0.001

0.222

$\begin{array}{llllll}0.000136 & 0.000134 & 1100.25 & -0.2756 & 0.000002 & 0.076\end{array}$

0.149

0.071

0.797

0.022

0.006

1.390

0.006

0.031

1.492

0.179

0.741

0.338

0.001
Relative Density of

$89.48 \quad 994.0319$ 994.0319

$111.54 \quad 994.0319$ 994.0319

994.0319

163.1994 .0319

994.0319

994.0319

$213.41 \quad 994.0319$

994.0319

994.0319

$260.79 \quad 994.0319$

994.0319

994.0319

306.31994 .0319

338.82994 .0319

1.19990 .2132

$2.33 \quad 990.2132$

$3.46 \quad 990.2132$

$5.78 \quad 990.2132$

8.62990 .2132

$11.56 \quad 990.2132$ 990.2132

$45.63 \quad 990.2132$ 990.2132

$65.53 \quad 990.2132$ 990.2132

88.91990 .2132 990.2132

$110.85 \quad 990.2132$ 990.2132 990.2132

$162.09 \quad 990.2132$ 990.2132 990.2132

212.2990 .2132 990.2132 990.2132

$259.25 \quad 990.2132$ 990.2132 990.2132

$304.57 \quad 990.2132$

$336.55 \quad 990.2132$ 


\begin{tabular}{|c|c|c|c|c|}
\hline molality & molarity & \multicolumn{2}{|c|}{$\mathrm{t} /{ }^{\circ} \mathrm{C}$ mass frac } & $\begin{array}{r}\text { Density } \\
\exp / \\
\mathrm{kg} / \mathrm{m}^{3}\end{array}$ \\
\hline 0.01009 & & 55 & 0.0013 & 986.85 \\
\hline 0.01993 & & 55 & 0.0026 & 987.98 \\
\hline 0.02966 & & 55 & 0.0038 & 989.10 \\
\hline 0.04971 & & 55 & 0.0064 & 991.37 \\
\hline 0.0744 & & 55 & 0.0096 & 994.19 \\
\hline 0.1 & & 55 & 0.0128 & 997.06 \\
\hline 0.2044 & & 55 & 0.0259 & 1008.73 \\
\hline 0.2419 & & 55 & 0.0305 & 1013.15 \\
\hline 0.4014 & & 55 & 0.0495 & 1030.53 \\
\hline 0.464 & & 55 & 0.0568 & 1037.85 \\
\hline 0.5822 & & 55 & 0.0703 & 1050.12 \\
\hline 0.6948 & & 55 & 0.0827 & 1063.01 \\
\hline 0.798 & & 55 & 0.0939 & 1073.11 \\
\hline 0.9215 & & 55 & 0.1069 & 1087.22 \\
\hline 1.0045 & & 55 & 0.1154 & 1094.72 \\
\hline 1.1498 & & 55 & 0.1299 & 1111.13 \\
\hline 1.3886 & & 55 & 0.1528 & 1135.65 \\
\hline 1.5012 & & 55 & 0.1631 & 1145.03 \\
\hline 1.6373 & & 55 & 0.1753 & 1160.65 \\
\hline 1.8878 & & 55 & 0.1969 & 1185.32 \\
\hline 2.0051 & & 55 & 0.2066 & 1194.30 \\
\hline 2.132 & & 55 & 0.2168 & 1208.88 \\
\hline 2.3727 & & 55 & 0.2355 & 1231.65 \\
\hline 2.4983 & & 55 & 0.2449 & 1241.35 \\
\hline 2.6227 & & 55 & 0.2540 & 1254.85 \\
\hline 2.8825 & & 55 & 0.2723 & 1278.48 \\
\hline 2.9898 & & 55 & 0.2796 & 1287.74 \\
\hline 3.3516 & & 55 & 0.3032 & 1319.61 \\
\hline 0.2419 & & 65 & 0.0305 & 1007.96 \\
\hline 0.464 & & 65 & 0.0568 & 1032.65 \\
\hline 0.6948 & & 65 & 0.0827 & 1057.81 \\
\hline 0.9215 & & 65 & 0.1069 & 1082.03 \\
\hline 1.1498 & & 65 & 0.1299 & 1105.96 \\
\hline 1.3886 & & 65 & 0.1528 & 1130.49 \\
\hline 1.6373 & & 65 & 0.1753 & 1155.50 \\
\hline 1.8878 & & 65 & 0.1969 & 1180.15 \\
\hline 2.132 & & 65 & 0.2168 & 1203.68 \\
\hline 2.3727 & & 65 & 0.2355 & 1226.39 \\
\hline 2.6227 & & 65 & 0.2540 & 1249.49 \\
\hline 2.8825 & & 65 & 0.2723 & 1272.97 \\
\hline 0.2419 & & 75 & 0.0305 & 1002.16 \\
\hline 0.464 & & 75 & 0.0568 & 1026.82 \\
\hline 0.6948 & & 75 & 0.0827 & 1052.00 \\
\hline
\end{tabular}

Ref

$\begin{array}{cc}\text { App vol } & \text { App vol } \\ \exp / & \text { Calc / }\end{array}$

$\begin{array}{cc}\exp / & \text { Calc/ } \\ \mathrm{m}^{3} / \mathrm{kg} & \mathrm{m}^{3} / \mathrm{kg}\end{array}$

Density
calc

Density

App Vol sidual

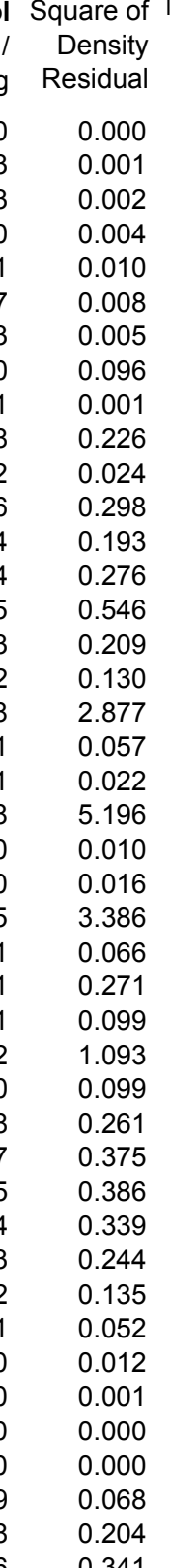

Relative Density of

$1.17 \quad 985.6952$ 2.32985 .6952 $3.45 \quad 985.6952$ $5.76 \quad 985.6952$ $8.62 \quad 985.6952$ $11.53 \quad 985.6952$ 23.37985 .6952 985.6952

$45.49 \quad 985.6952$ 985.6952

$65.36 \quad 985.6952$ 985.6952

$88.68 \quad 985.6952$ 985.6952

110.61985 .6952 985.6952 985.6952

161.65985 .6952 985.6952 985.6952

$211.63 \quad 985.6952$ 985.6952 985.6952

$259.36 \quad 985.6952$ 985.6952 985.6952

$306.43 \quad 985.6952$

$338.76 \quad 985.6952$ 980.5548 980.5548 980.5548 980.5548 980.5548 980.5548 980.5548 980.5548 980.5548 980.5548 980.5548 980.5548 974.849 974.849 974.849 


$\begin{array}{rrrr}\text { molality molarity } & \mathbf{t} /{ }^{\circ} \mathbf{C} \text { mass frac } & \begin{array}{r}\text { Density } \\ \text { exp } / \\ \mathrm{kg} / \mathrm{m}^{3}\end{array} \\ & & & \begin{array}{r}1076.27 \\ 0.9215\end{array} \\ 1.1498 & 75 & 0.1069 & 1076 \\ 1.3886 & 75 & 0.1299 & 1100.24 \\ 1.6373 & 75 & 0.1528 & 1124.83 \\ 1.8878 & 75 & 0.1753 & 1149.88 \\ 2.132 & 75 & 0.1969 & 1174.55 \\ 2.3727 & 75 & 0.2168 & 1198.05 \\ 2.6227 & 75 & 0.2355 & 1220.68 \\ 2.8825 & 75 & 0.2540 & 1243.61 \\ & 75 & 0.2723 & 1266.83\end{array}$

Ref
$(37)$
$(37)$
$(37)$
$(37)$
$(37)$
$(37)$
$(37)$
$(37)$
$(37)$

$\begin{array}{rr}\begin{array}{r}\text { App vol } \\ \text { exp / }\end{array} & \begin{array}{r}\text { App vol } \\ \text { Calc / } \\ \mathrm{m}^{3} / \mathrm{kg}\end{array} \\ \mathrm{m}^{3} / \mathrm{kg}\end{array}$

Density App Vol Square of Inconsistent Inconsistent

calc/

$\mathrm{kg} / \mathrm{m}^{3}$

$\mathrm{m}^{3} / \mathrm{kg}$

Density

density

based on

apparent

Apparent

$\begin{array}{llll}1075.63 & 0.6398 & -0.000005\end{array}$

$1099.62 \quad 0.6236 \quad-0.000004$

0.409

$\begin{array}{llll}1124.27 & 0.5623 & -0.000003 & 0.316\end{array}$

$1174.31-0.2375-0.000001$

$\begin{array}{llll}1198.04 & 0.0081 & 0.000000 & 0.000\end{array}$

$\begin{array}{llll}1220.94 & -0.2573 & 0.000001 & 0.066\end{array}$

$\begin{array}{llll}1244.19 & -0.5808 & 0.000001 & 0.337\end{array}$

$\begin{array}{llll}1267.78 & -0.9503 & 0.000002 & 0.903\end{array}$

$\begin{array}{lll}\text { Average } & 0.1156 & -0.000006\end{array}$

$\begin{array}{lll}\text { Std dev } & 0.6902 & 0.000009\end{array}$

$\begin{array}{lll}\text { Avg - 4std } \quad-2.6452 & -0.000041\end{array}$

Avg + 4std $\quad 2.8764 \quad 0.000028$
Relative Density of density water $/ \mathrm{kg} / \mathrm{m}^{3}$

974.849

974.849

974.849

974.849

974.849

974.849

974.849

974.849

974.849

\section{Curve fit for the model App vol $=\left(w+c 2+c 3^{*} t{ }^{\circ} \mathrm{C}\right) /\left(\mathrm{c} 0^{*} w+c 1\right) / \mathrm{e}^{\wedge}\left(0.000001^{*}\left(\mathrm{t}^{\circ} \mathrm{C}+\mathrm{c} 4\right)^{\wedge} 2\right)$}

$\begin{array}{lr}\text { c0 } 1 & -8.1 \mathrm{E}-08 \\ \text { c1 } & 8.03 \mathrm{E}-08 \\ \text { c3 } & 410.2392 \\ \text { c4 } & 9.180778 \\ \text { Min T } & 5619.75 \\ \text { Max T } & 15 \\ \text { Min w } & 75 \\ \text { Max w } & 0.0013 \\ \text { Avg dens res } & 0.3445 \\ \text { Std dens res } & 0.115629 \\ \text { Avg app vol res *10^3 } & 0.690195 \\ \text { Std app vol res *10^3 } & -0.0061 \\ \text { No of points in corr } & 0.008605 \\ \text { No of inconsistent poir } & 171 \\ & 1\end{array}$

No of inconsistent poir

Inconsistent data not used (Residual greater than average + /- 4 standard deviations) 
molality molarity

Density

$\exp /$

$\mathrm{kg} / \mathrm{m}^{3}$
Ref

$\begin{array}{cc}\text { App vol App vol } \\ \exp / & \text { Calc / }\end{array}$

$\begin{array}{cc}\exp / & \text { Calc/ } \\ \mathrm{m}^{3} / \mathrm{kg} & \mathrm{m}^{3} / \mathrm{kg}\end{array}$
Density Density App Vol Square of Inconsistent Inconsistent calc/ Residual / Residual / Density based on $\mathrm{kg} / \mathrm{m}^{3}$

Phang seems high, Pogue seems low, and Herrington seems average between the two

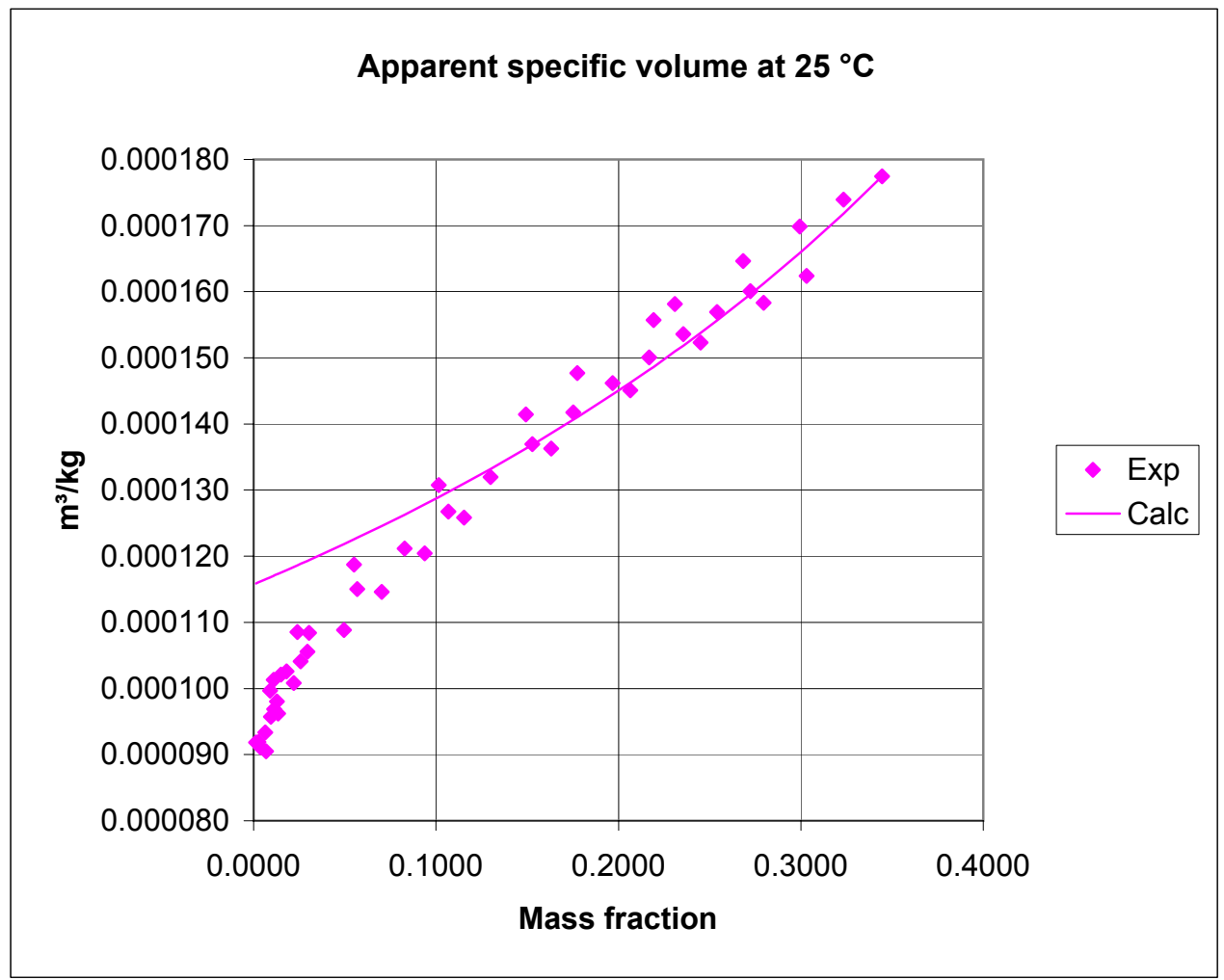


Density of aqueous solutions of CoSO4

References (7) Bakeev, M. I.; Zharmenov, A. A.; Baikenova, N. A.; Abdygalimova, S. Sh.; Electrical conductivity and viscosity of the binary systems MeSO4-H2O (Me=Mn2+, Fe2+, Co2+, Zn2+, Cd2+) at 25-75 degree and the structure of electrolyte solutions, Izv. Nats. Akad. Nauk Resp. Kaz., Ser. Khim., 1994, 6, 25-30

(117) Schmelzer, N, Einfeldt, J., "Dichtemessungen an einigen...", Wiss. Z. Uni. Rostock, 1989, 38, 81-82

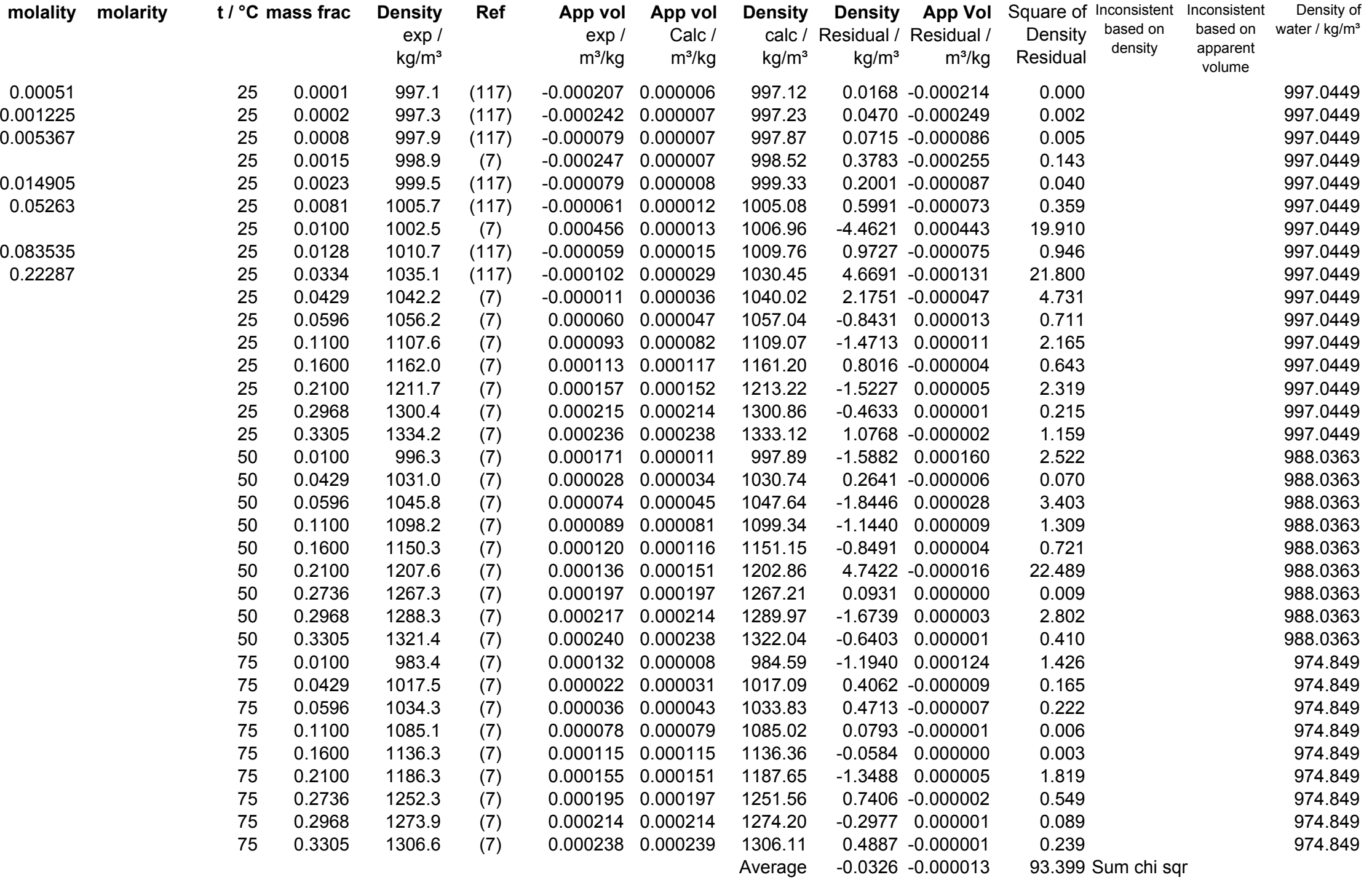


molality molarity

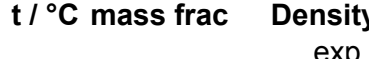

$\exp /$
$\mathrm{kg} / \mathrm{m}^{3}$
Ref

App vol
$\exp /$
$\mathrm{m}^{3} / \mathrm{kg}$

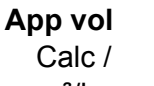

Calc /
$\mathrm{m}^{3} / \mathrm{kg}$

\section{Den}

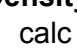

$\mathrm{kg} / \mathrm{m}^{3}$

Resic

Resid

Density

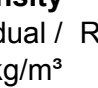

App Vol

Std dev
Avg $-4 s t d$
Avg $+4 s t d$

$\mathrm{m}^{3} / \mathrm{kg}$

$\begin{array}{ll}1.6820 & 0.000117\end{array}$

$-6.7607-0.000481$

$6.6955 \quad 0.000454$

Square of Inconsistent Inconsistent

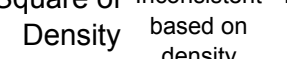

based on

apparent

Density of

$\begin{array}{lr}\text { Curve fit for the model App vol= } \\ \text { c0 } & -118.3645 \\ \text { c1 } & 1368.065 \\ \text { c2 } & 0.013043 \\ \text { c3 } & -0.000145 \\ \text { c4 } & -294.0194 \\ \text { Min T } & 25 \\ \text { Max T } & 75 \\ \text { Min w } & 7.9 E-05 \\ \text { Max w } & 0.3305 \\ \text { Avg dens res } & -0.03259 \\ \text { Std dens res } & 1.682019 \\ \text { Avg app vol res *10^3 } & -0.013421 \\ \text { Std app vol res *10^3 } & 0.116822 \\ \text { No of points in corr } & 34 \\ \text { No of inconsistent poir } & 7\end{array}$

Inconsistent data not used (Residual greater than average $+/-4$ standard deviations)

$\begin{array}{lllllll}0.000158 & 25 & 0.0000 & 997.1 & (117) & -0.000851 & 0.000006\end{array}$

0.37879

0.54945

0.85903

$\begin{array}{rrrrrr}25 & 0.0555 & 1060.2 & (117) & -0.000074 & 0.000044 \\ 25 & 0.0785 & 1094.3 & (117) & -0.000133 & 0.000060 \\ 25 & 0.1175 & 1147.9 & (117) & -0.000119 & 0.000087 \\ 25 & 0.2736 & 1230.1 & (7) & 0.000308 & 0.000197 \\ 50 & 0.0015 & 992.0 & (7) & -0.001702 & 0.000005 \\ 75 & 0.0015 & 979.8 & (7) & -0.002453 & 0.000003\end{array}$

$2)$

$997.07 \quad 0.0209-0.000858$ $105285 \quad 7.3090-0.000118$ $1076.46 \quad 17.8837-0.000193$ $1116.87 \quad 31.0581-0.000206$ $\begin{array}{llll}1277.96 & -47.8595 & 0.000111\end{array}$ $989.50 \quad 2.4967-0.001707$ $976.30 \quad 3.5000-0.002456$

0.000
53.421
319.828
964.608
2290.532
6.233
12.250

997.0449

997.0449

997.0449

997.0449

997.0449

988.0363 
Density of aqueous solutions of $\mathrm{CrCl} 3$

References (90) Perry, R. H.; Green, D. W. Perry's Chemical Engineers' Handbook; McGraw Hill: New York, 7th edition, 1997.

\begin{tabular}{|c|c|c|c|c|c|c|c|c|c|c|c|c|}
\hline${ }^{\circ} \mathrm{C}$ & ass frac & $\begin{array}{r}\text { Density } \\
\exp / \\
\mathrm{kg} / \mathrm{m}^{3}\end{array}$ & Ref & $\begin{array}{r}\text { App vol } \\
\text { exp / } \\
\mathrm{m}^{3} / \mathrm{kg}\end{array}$ & $\begin{array}{r}\text { App vol } \\
\text { Calc / } \\
\mathrm{m}^{3} / \mathrm{kg}\end{array}$ & $\begin{array}{r}\text { Density } \\
\text { calc / } \\
\mathrm{kg} / \mathrm{m}^{3}\end{array}$ & $\begin{array}{r}\text { Density } \\
\text { Residual / } \\
\mathrm{kg} / \mathrm{m}^{3}\end{array}$ & $\begin{array}{r}\text { App Vol } \\
\text { Residual / } \\
\mathrm{m}^{3} / \mathrm{kg}\end{array}$ & $\begin{array}{l}\text { Square of } \\
\text { Density } \\
\text { Residual }\end{array}$ & $\begin{array}{c}\text { Inconsistent } \\
\text { based on } \\
\text { density }\end{array}$ & $\begin{array}{l}\text { Inconsistent } \\
\text { based on } \\
\text { apparent } \\
\text { volume }\end{array}$ & $\begin{array}{r}\text { Density of } \\
\text { water } / \mathrm{kg} / \mathrm{m}^{3}\end{array}$ \\
\hline 18 & 0.0100 & 1007.5 & (90) & 0.000116 & 0.000110 & 1007.56 & -0.0598 & 0.000006 & 0.004 & & & 998.5956 \\
\hline 18 & 0.0400 & 1034.7 & (90) & 0.000128 & 0.000123 & 1034.89 & -0.1943 & 0.000005 & 0.038 & & & 998.5956 \\
\hline 18 & 0.0800 & 1072.2 & $(90)$ & 0.000142 & 0.000140 & 1072.35 & -0.1547 & 0.000002 & 0.024 & & & 998.5956 \\
\hline 18 & 0.1200 & 1111.1 & (90) & 0.000156 & 0.000158 & 1110.94 & 0.1608 & -0.000001 & 0.026 & & & 998.5956 \\
\hline & & & & & \multicolumn{2}{|c|}{ Avg - 4std } & -0.6233 & -0.000008 & & & & \\
\hline & & & & & & $v g+4 s t d$ & 0.4849 & 0.000014 & & & & \\
\hline
\end{tabular}

\section{Curve fit for the model App vol $=\left(w+c 2+c 3^{*} t{ }^{\circ} \mathrm{C}\right) /\left(\mathrm{c} 0^{*} \mathrm{w}+\mathrm{c} 1\right) / \mathrm{e}^{\wedge}\left(0.000001^{*}\left(\mathrm{t}{ }^{\circ} \mathrm{C}+\mathrm{c} 4\right)^{\wedge} 2\right)$}

$\begin{array}{lr}\text { c0 } 1 & 3.146896 \\ \text { c2 } & 232.1545 \\ \text { c3 } & 0.201907 \\ \text { c4 } & 0.0025 \\ \text { Min T } & 1500 \\ \text { Max T } & 18 \\ \text { Min w } & 18 \\ \text { Max w } & 0.01 \\ \text { Avg dens res } & 0.12 \\ \text { Std dens res } & -0.0692 \\ \text { Avg app vol res *10^3 } & 0.003153 \\ \text { Std app vol res *10^3 } & 0.002832 \\ \text { No of points in corr } & 5 \\ \text { No of inconsistent poir } & 0\end{array}$

Inconsistent data not used (Residual greater than average $+/-4$ standard deviations) 
Density of aqueous solutions of $\mathrm{Cr} 2(\mathrm{SO} 4) 3$

References (6) Asmus, A., "The viscosities of aqueous solutions of strong electrolytes of high valence type", Ann. der Physik, ser 5, 1939, 35, 1-22

\begin{tabular}{|c|c|c|c|c|c|c|c|c|c|c|c|c|c|c|c|}
\hline \multicolumn{2}{|l|}{ molality } & \multicolumn{2}{|c|}{$\mathrm{t} /{ }^{\circ} \mathrm{C}$ mass frac } & $\begin{array}{r}\text { Density } \\
\exp / \\
\mathrm{kg} / \mathrm{m}^{3}\end{array}$ & Ref & $\begin{array}{r}\text { App vol } \\
\exp / \\
\mathrm{m}^{3} / \mathrm{kg}\end{array}$ & $\begin{array}{r}\text { App vol } \\
\text { Calc / } \\
\mathrm{m}^{3} / \mathrm{kg}\end{array}$ & $\begin{array}{r}\text { Density } \\
\text { calc / } \\
\mathrm{kg} / \mathrm{m}^{3}\end{array}$ & $\begin{array}{r}\text { Density } \\
\text { Residual / } \\
\mathrm{kg} / \mathrm{m}^{3}\end{array}$ & $\begin{array}{r}\text { App Vol } \\
\text { Residual / } \\
\mathrm{m}^{3} / \mathrm{kg}\end{array}$ & $\begin{array}{c}\text { Square of } \\
\text { Density } \\
\text { Residual }\end{array}$ & $\begin{array}{l}\text { Inconsistent } \\
\text { based on } \\
\text { density }\end{array}$ & $\begin{array}{l}\text { Inconsistent } \\
\text { based on } \\
\text { apparent } \\
\text { volume }\end{array}$ & $\begin{array}{r}\text { Relative } \\
\text { density }\end{array}$ & $\begin{array}{r}\text { Density of } \\
\text { water } / \mathrm{kg} / \mathrm{m}^{3}\end{array}$ \\
\hline & 0.0000673 & 25 & 0.0000 & 997.1 & (6) & -0.002007 & -0.001243 & 997.07 & 0.0076 & -0.000764 & 0.000 & & & 1.00003 & 997.0449 \\
\hline & 0.000345 & 25 & 0.0001 & 997.2 & (6) & -0.001483 & -0.001241 & 997.16 & 0.0123 & -0.000242 & 0.000 & & & 1.000127 & 997.0449 \\
\hline & 0.000497 & 25 & 0.0001 & 997.2 & (6) & -0.001429 & -0.001239 & 997.21 & 0.0139 & -0.000190 & 0.000 & & & 1.000179 & 997.0449 \\
\hline & 0.000593 & 25 & 0.0001 & 997.3 & (6) & -0.001411 & -0.001238 & 997.24 & 0.0151 & -0.000173 & 0.000 & & & 1.000212 & 997.0449 \\
\hline & 0.000763 & 25 & 0.0001 & 997.3 & (6) & -0.001342 & -0.001237 & 997.30 & 0.0119 & -0.000105 & 0.000 & & & 1.000265 & 997.0449 \\
\hline & 0.000954 & 25 & 0.0001 & 997.4 & (6) & -0.001340 & -0.001235 & 997.36 & 0.0147 & -0.000104 & 0.000 & & & 1.000331 & 997.0449 \\
\hline & 0.001084 & 25 & 0.0002 & 997.4 & (6) & -0.001308 & -0.001234 & 997.40 & 0.0118 & -0.000074 & 0.000 & & & 1.000371 & 997.0449 \\
\hline & 0.00131 & 25 & 0.0002 & 997.5 & (6) & -0.001291 & -0.001233 & 997.48 & 0.0113 & -0.000058 & 0.000 & & & 1.000445 & 997.0449 \\
\hline & 0.001727 & 25 & 0.0003 & 997.6 & (6) & -0.001277 & -0.001229 & 997.61 & 0.0121 & -0.000047 & 0.000 & & & 1.000583 & 997.0449 \\
\hline & 0.002181 & 25 & 0.0003 & 997.8 & (6) & -0.001257 & -0.001225 & 997.76 & 0.0103 & -0.000032 & 0.000 & & & 1.00073 & 997.0449 \\
\hline & 0.002852 & 25 & 0.0004 & 998.0 & (6) & -0.001154 & -0.001220 & 997.98 & -0.0277 & 0.000066 & 0.001 & & & 1.000911 & 997.0449 \\
\hline & 0.003348 & 25 & 0.0005 & 998.1 & (6) & -0.001216 & -0.001216 & 998.14 & 0.0000 & 0.000000 & 0.000 & & & 1.0011 & 997.0449 \\
\hline & 0.004599 & 25 & 0.0007 & 998.5 & (6) & -0.001198 & -0.001205 & 998.54 & -0.0050 & 0.000007 & 0.000 & & & 1.001499 & 997.0449 \\
\hline & 0.005956 & 25 & 0.0009 & 999.0 & (6) & -0.001185 & -0.001194 & 998.98 & -0.0078 & 0.000009 & 0.000 & & & 1.00193 & 997.0449 \\
\hline & 0.007628 & 25 & 0.0011 & 999.5 & (6) & -0.001175 & -0.001180 & 999.50 & -0.0062 & 0.000006 & 0.000 & & & 1.00246 & 997.0449 \\
\hline & 0.01009 & 25 & 0.0015 & 1000.3 & (6) & -0.001165 & -0.001160 & 1000.27 & 0.0081 & -0.000005 & 0.000 & & & 1.00324 & 997.0449 \\
\hline & & & & & & & \multicolumn{2}{|c|}{ Average Res } & 0.0051 & -0.000107 & 0.002 & Sum chi sqr & & & \\
\hline & & & & & & & \multicolumn{2}{|c|}{ Std dev Res } & 0.0117 & 0.000194 & & & & & \\
\hline & & & & & & & \multicolumn{2}{|c|}{ Avg - 4std } & -0.0415 & -0.000883 & & & & & \\
\hline & & & & & & & \multicolumn{2}{|c|}{ Avg + 4std } & 0.0518 & 0.000669 & & & & & \\
\hline
\end{tabular}

Curve fit for the model App vol $=\left(w+c 2+c 3^{*} t{ }^{\circ} \mathrm{C}\right) /\left(c 0^{*} w+c 1\right) / e^{\wedge}\left(0.000001^{*}\left(t^{\circ} \mathrm{C}+c 4\right)^{\wedge} 2\right)$

\begin{tabular}{lr} 
c0 1 & 1.00452 \\
c2 & 1.769719 \\
c3 & -0.08502 \\
c4 & 0.0025 \\
Min T & 1500 \\
Max T & 25 \\
Min w & 25 \\
Max w & $1 \mathrm{E}-05$ \\
Avg dens res & 0.001494 \\
Std dens res & 0.005145 \\
Avg app vol res *10^3 & 0.011674 \\
Std app vol res *10^3 & -0.10673 \\
No of points in corr & 0.194055 \\
No of inconsistent point & 16 \\
\hline
\end{tabular}

Inconsistent data not used (Residual greater than average $+/-4$ standard deviations) 
Density of aqeous solutions of $\mathbf{C u C l} 2$

References (23) Dolian, F. E. The Viscosities of Solutions of Chlorides in Certain Solvents. J. Phys. Chem. 1937, 41, 1129-1138.

(26) Ellis, R. N.; Stokes, R. H.; Wright, A. C.; Spiro, M. Transference Numbers and Conductance in Concentrated Copper (II) Chloride Solutions at $25^{\circ} \mathrm{C}$. Aust. J. Chem. 1983, 36, 1913-1921.

(74) Motin, M. A., Temperature and Concentration Dependence of Apparent Molar Volumes and Viscosities of $\mathrm{NaCl}, \mathrm{NH} 4 \mathrm{Cl}, \mathrm{CuCl}$, and MgSO4 in Pure Water and Water + Urea Mixtures, J. Chem. Eng. Data, 2004, 49, 94-98

(90) Perry, R. H.; Green, D. W. Perry's Chemical Engineers' Handbook; McGraw Hill: New York, 7th edition, 1997

(97) Pogue, R. F.; Atkinson, G. Solution Thermodynamics of First-Row Transition Elements. 1. Apparent Molal Volumes of Aqueous NiCl2, $\mathrm{Ni}(\mathrm{ClO} 4) 2, \mathrm{CuCl} 2$, and $\mathrm{Cu}(\mathrm{ClO} 4) 2$, from 15 to $55^{\circ} \mathrm{C}$. J. Chem. Eng. Data 1988, 33, 370-376.

\begin{tabular}{|c|c|c|c|c|c|c|c|c|c|c|c|c|c|}
\hline \multirow[t]{7}{*}{ molality } & \multirow[t]{7}{*}{ molarity } & \multicolumn{2}{|c|}{$\mathrm{t} /{ }^{\circ} \mathrm{C}$ mass frac } & \multicolumn{2}{|l|}{$\begin{array}{r}\text { Density } \\
\exp / \\
\mathrm{kg} / \mathrm{m}^{3}\end{array}$} & \multirow{2}{*}{$\begin{array}{r}\text { App vol } \\
\text { exp } / \\
\mathrm{m}^{3} / \mathrm{kg} \\
0.000043\end{array}$} & \multirow{2}{*}{$\begin{array}{r}\text { App vol } \\
\text { Calc / } \\
\mathrm{m}^{3} / \mathrm{kg} \\
0.000051\end{array}$} & \multirow{2}{*}{$\begin{array}{r}\text { Density } \\
\text { calc / } \\
\mathrm{kg} / \mathrm{m}^{3} \\
1009.42\end{array}$} & \multirow{2}{*}{$\begin{array}{r}\text { Density } \\
\text { Residual / } \\
\mathrm{kg} / \mathrm{m}^{3} \\
0.0793\end{array}$} & \multirow{2}{*}{$\begin{array}{r}\text { App Vol } \\
\text { Residual / } \\
\mathrm{m}^{3} / \mathrm{kg} \\
-0.000008\end{array}$} & \multirow{2}{*}{$\begin{array}{r}\begin{array}{r}\text { Square of } \\
\text { Density } \\
\text { Residual }\end{array} \\
0.006\end{array}$} & \multirow{2}{*}{$\begin{array}{l}\text { Inconsistent } \\
\text { based on } \\
\text { density }\end{array}$} & $\begin{array}{l}\text { Inconsistent } \\
\text { based on } \\
\text { apparent } \\
\text { volume }\end{array}$ \\
\hline & & 0 & 0.0100 & 1009.5 & (90) & & & & & & & & \\
\hline & & 0 & 0.0400 & 1038.7 & (90) & 0.000065 & 0.000066 & 1038.63 & 0.0673 & -0.000002 & 0.005 & & \\
\hline & & 0 & 0.0800 & 1078.8 & (90) & 0.000085 & 0.000085 & 1078.84 & -0.0353 & 0.000000 & 0.001 & & \\
\hline & & 0 & 0.1200 & 1120.8 & (90) & 0.000101 & 0.000101 & 1120.72 & 0.0769 & -0.000001 & 0.006 & & \\
\hline & & 0 & 0.1600 & 1165.3 & (90) & 0.000113 & 0.000116 & 1164.58 & 0.7191 & -0.000003 & 0.517 & & \\
\hline & & 0 & 0.2000 & 1212.1 & (90) & 0.000124 & 0.000129 & 1210.71 & 1.3904 & -0.000005 & 1.933 & & \\
\hline 0.01027 & & 15 & 0.0014 & 1000.4 & (97) & 0.000080 & 0.000069 & 1000.38 & -0.0153 & 0.000011 & 0.000 & & \\
\hline 0.02113 & & 15 & 0.0028 & 1001.7 & (97) & 0.000078 & 0.000070 & 1001.74 & -0.0221 & 0.000008 & 0.000 & & \\
\hline 0.0304 & & 15 & 0.0041 & 1002.9 & (97) & 0.000080 & 0.000070 & 1002.89 & -0.0383 & 0.000009 & 0.001 & & \\
\hline 0.04879 & & 15 & 0.0065 & 1005.1 & (97) & 0.000082 & 0.000072 & 1005.18 & -0.0669 & 0.000010 & 0.004 & & \\
\hline 0.07517 & & 15 & 0.0100 & 1008.3 & (97) & 0.000084 & 0.000073 & 1008.45 & -0.1081 & 0.000011 & 0.012 & & \\
\hline 0.09715 & & 15 & 0.0129 & 1011.0 & (97) & 0.000086 & 0.000075 & 1011.16 & -0.1531 & 0.000012 & 0.023 & & \\
\hline 0.2006 & & 15 & 0.0263 & 1023.5 & (97) & 0.000094 & 0.000081 & 1023.81 & -0.3476 & 0.000013 & 0.121 & & \\
\hline 0.3888 & & 15 & 0.0497 & 1045.7 & (97) & 0.000103 & 0.000092 & 1046.31 & -0.6247 & 0.000011 & 0.390 & & \\
\hline 0.6119 & & 15 & 0.0760 & 1071.4 & (97) & 0.000112 & 0.000103 & 1072.24 & -0.8248 & 0.000009 & 0.680 & & \\
\hline 0.8038 & & 15 & 0.0975 & 1093.0 & (97) & 0.000119 & 0.000111 & 1093.95 & -0.9321 & 0.000008 & 0.869 & & \\
\hline 1.0333 & & 15 & 0.1220 & 1118.3 & (97) & 0.000126 & 0.000120 & 1119.25 & -0.9502 & 0.000006 & 0.903 & & \\
\hline 1.5005 & & 15 & 0.1679 & 1167.9 & (97) & 0.000139 & 0.000135 & 1168.79 & -0.9270 & 0.000004 & 0.859 & & \\
\hline 1.9955 & & 15 & 0.2115 & 1218.2 & (97) & 0.000150 & 0.000148 & 1218.75 & -0.5877 & 0.000002 & 0.345 & & \\
\hline 2.4934 & & 15 & 0.2511 & 1265.9 & (97) & 0.000161 & 0.000159 & 1266.70 & -0.8016 & 0.000002 & 0.642 & & \\
\hline 2.9953 & & 15 & 0.2871 & 1312.0 & (97) & 0.000169 & 0.000168 & 1312.96 & -0.9347 & 0.000002 & 0.874 & & \\
\hline \multirow{7}{*}{\multicolumn{2}{|c|}{3.5741}} & 15 & 0.3246 & 1362.8 & (97) & 0.000178 & 0.000176 & 1363.99 & -1.1937 & 0.000002 & 1.425 & & \\
\hline & & 20 & 0.0100 & 1007.2 & (90) & 0.000107 & 0.000081 & 1007.47 & -0.2665 & 0.000026 & 0.071 & & \\
\hline & & 20 & 0.0400 & 1036.0 & (90) & 0.000088 & 0.000094 & 1035.73 & 0.2706 & -0.000006 & 0.073 & & \\
\hline & & 20 & 0.0800 & 1075.4 & (90) & 0.000103 & 0.000111 & 1074.68 & 0.7235 & -0.000008 & 0.523 & & \\
\hline & & 20 & 0.1200 & 1116.5 & (90) & 0.000117 & 0.000125 & 1115.31 & 1.1908 & -0.000008 & 1.418 & & \\
\hline & & 20 & 0.1600 & 1159.5 & (90) & 0.000131 & 0.000138 & 1157.90 & 1.5999 & -0.000007 & 2.560 & & \\
\hline & & 20 & 0.2000 & 1205.2 & (90) & 0.000141 & 0.000150 & 1202.74 & 2.4615 & -0.000008 & 6.059 & & \\
\hline 0.01004 & & 25 & 0.0013 & 998.3 & (97) & 0.000089 & 0.000084 & 998.28 & -0.0067 & 0.000005 & 0.000 & & \\
\hline 0.02007 & & 25 & 0.0027 & 999.5 & (97) & 0.000088 & 0.000085 & 999.51 & -0.0102 & 0.000004 & 0.000 & & \\
\hline \multirow{2}{*}{\multicolumn{2}{|c|}{0.0301}} & 25 & 0.0040 & 1000.7 & (97) & 0.000091 & 0.000085 & 1000.74 & -0.0216 & 0.000005 & 0.000 & & \\
\hline & & 25 & 0.0042 & 1001.0 & (23) & 0.000067 & 0.000085 & 1000.89 & 0.0790 & -0.000019 & 0.006 & & \\
\hline 0.04899 & & 25 & 0.0065 & 1003.0 & (26) & 0.000090 & 0.000086 & 1003.04 & -0.0228 & 0.000003 & 0.001 & & \\
\hline \multirow{2}{*}{\multicolumn{2}{|c|}{0.05018}} & 25 & 0.0067 & 1003.1 & (97) & 0.000093 & 0.000087 & 1003.19 & -0.0411 & 0.000006 & 0.002 & & \\
\hline & & 25 & 0.0083 & 1004.8 & (23) & 0.000072 & 0.000087 & 1004.66 & 0.1318 & -0.000016 & 0.017 & & \\
\hline
\end{tabular}

Relative Apparent Density of density molar volume water $/ \mathrm{kg} / \mathrm{m}^{3}$ $/ \mathrm{cm}^{3} / \mathrm{mol}$

999.8395

999.8395

999.8395

999.8395

999.8395

999.8395

999.0996

$2.62 \quad 999.0996$

$3.76 \quad 999.0996$

$6.02 \quad 999.0996$

$9.25 \quad 999.0996$

$11.92 \quad 999.0996$

$24.38 \quad 999.0996$

$46.63 \quad 999.0996$

$72.38 \quad 999.0996$

999.0996

$168.92 \quad 999.0996$

$219.26 \quad 999.0996$

$267.04 \quad 999.0996$

$313.21 \quad 999.0996$

$364.02 \quad 999.0996$

998.2041

998.2041

998.2041

998.2041

998.2041

998.2041

$1.23 \quad 997.0449$

$2.46 \quad 997.0449$

$\begin{array}{ll}3.68 & 997.0449\end{array}$

997.0449

997.0449

$6.12 \quad 997.0449$

997.0449 


\begin{tabular}{|c|c|c|c|c|c|}
\hline molality & molarity & $\mathrm{t} /{ }^{\circ} \mathrm{C}$ & ass frac & $\begin{array}{r}\text { Density } \\
\exp / \\
\mathrm{kg} / \mathrm{m}^{3}\end{array}$ & $\mathbf{R e}$ \\
\hline 0.07493 & & 25 & 0.0100 & 1006.1 & (97 \\
\hline 0.08601 & & 25 & 0.0114 & 1007.5 & (26) \\
\hline 0.09909 & & 25 & 0.0131 & 1009.1 & 26 \\
\hline 0.1003 & & 25 & 0.0133 & 1009.2 & 97 \\
\hline & & 25 & 0.0165 & 1011.6 & 23 \\
\hline 0.14524 & & 25 & 0.0192 & 1014.6 & 26 \\
\hline 0.2006 & & 25 & 0.0263 & 1021.1 & 97 \\
\hline 0.24295 & & 25 & 0.0316 & 1026.2 & 26 \\
\hline & & 25 & 0.0325 & 1027.3 & 23 \\
\hline 0.413 & & 25 & 0.0526 & 1045.7 & 97 \\
\hline & & 25 & 0.0635 & 1056.5 & 23 \\
\hline 0.5081 & & 25 & 0.0639 & 1056.9 & 26 \\
\hline 0.5093 & & 25 & 0.0641 & 1057.0 & 26 \\
\hline 0.6219 & & 25 & 0.0772 & 1069.4 & 97 \\
\hline 0.7908 & & 25 & 0.0961 & 1088.6 & 26 \\
\hline 0.816 & & 25 & 0.0989 & 1090.9 & 97 \\
\hline 1.0223 & & 25 & 0.1208 & 1113.3 & 97 \\
\hline 1.195 & & 25 & 0.1384 & 1132.3 & $(26$ \\
\hline 1.5704 & & 25 & 0.1743 & 1170.8 & 97 \\
\hline 2.0791 & & 25 & 0.2185 & 1220.7 & 97 \\
\hline 2.183 & & 25 & 0.2269 & 1231.8 & 26 \\
\hline 2.6006 & & 25 & 0.2591 & 1269.5 & 97 \\
\hline 2.767 & & 25 & 0.2711 & 1286.5 & 26 \\
\hline 3.1291 & & 25 & 0.2961 & 1317.4 & 97 \\
\hline 3.351 & & 25 & 0.3106 & 1338.3 & 26 \\
\hline 3.5741 & & 25 & 0.3246 & 1356.2 & 97 \\
\hline 3.923 & & 25 & 0.3453 & 1386.7 & 26 \\
\hline 4.487 & & 25 & 0.3763 & 1432.4 & 26 \\
\hline 5.394 & & 25 & 0.4204 & 1501.2 & 26 \\
\hline 0.01027 & & 35 & 0.0014 & 995.3 & (97 \\
\hline 0.02113 & & 35 & 0.0028 & 996.6 & (97 \\
\hline 0.0304 & & 35 & 0.0041 & 997.7 & 97 \\
\hline 0.04879 & & 35 & 0.0065 & 999.9 & 97 \\
\hline 0.07517 & & 35 & 0.0100 & 1003.0 & 97 \\
\hline 0.09715 & & 35 & 0.0129 & 1005.7 & (97 \\
\hline 0.2006 & & 35 & 0.0263 & 1017.8 & (97 \\
\hline 0.3888 & & 35 & 0.0497 & 1039.4 & (97 \\
\hline 0.6119 & & 35 & 0.0760 & 1064.3 & (97 \\
\hline 0.8038 & & 35 & 0.0975 & 1085.3 & (97 \\
\hline 1.0333 & & 35 & 0.1220 & 1109.9 & (97 \\
\hline 1.5005 & & 35 & 0.1679 & 1158.0 & (97 \\
\hline 1.9955 & & 35 & 0.2115 & 1206.9 & \\
\hline 2.4934 & & 35 & 0.2511 & 1253.3 & \\
\hline 2.9953 & & 35 & 0.2871 & 1298.3 & \\
\hline 3.5741 & & 35 & 0.3246 & 1347.8 & \\
\hline
\end{tabular}

App vol App vol Density Density App Vol exp / Calc/ calc/ Residual / Residual / $\mathrm{m}^{3} / \mathrm{kg} \quad \mathrm{m}^{3} / \mathrm{kg} \quad \mathrm{kg} / \mathrm{m}^{3} \quad \mathrm{~kg} / \mathrm{m}^{3} \quad \mathrm{~m}^{3} / \mathrm{kg}$

$\begin{array}{lllll}0.000096 & 0.000088 & 1006.20 & -0.0819 & 0.000008\end{array}$ $\begin{array}{llllll}0.000093 & 0.000089 & 1007.54 & -0.0445 & 0.000004\end{array}$ $\begin{array}{lllll}0.000095 & 0.000089 & 1009.13 & -0.0789 & 0.000006\end{array}$ $\begin{array}{lllll}0.000097 & 0.000090 & 1009.28 & -0.1063 & 0.000008\end{array}$ $\begin{array}{lll}0.000128 & 0.000091\end{array}$ 0.000096 $0.000104 \quad 0.000095$ $0.000102 \quad 0.000098$ $0.000095 \quad 0.000098$ $0.000116 \quad 0.000106$ $0.000115 \quad 0.000111$ $0.000115 \quad 0.000111$ $0.000115 \quad 0.000111$ $0.000124 \quad 0.000116$ $0.000125 \quad 0.000123$ $0.000130 \quad 0.000124$

$0.000136 \quad 0.000131$ $0.000137 \quad 0.000137$ $0.000149 \quad 0.000148$ $0.000162 \quad 0.000160$ $0.000160 \quad 0.000162$ $\begin{array}{ll}0.000172 & 0.000170\end{array}$ $\begin{array}{ll}0.000171 & 0.000173\end{array}$ $0.000179 \quad 0.000178$ $0.000180 \quad 0.000181$ $0.000185 \quad 0.000184$ $\begin{array}{ll}0.000187 & 0.000188\end{array}$ $0.000193 \quad 0.000194$ $0.000202 \quad 0.000202$ $0.000095 \quad 0.000098$ $0.000096 \quad 0.000099$ $0.000100 \quad 0.000100$ $0.000099 \quad 0.000101$ $0.000102 \quad 0.000102$ $0.000104 \quad 0.000103$ $\begin{array}{lll}0.000113 & 0.000109\end{array}$ $0.000123 \quad 0.000118$ $0.000132 \quad 0.000128$ $0.000138 \quad 0.000135$ $\begin{array}{lll}0.000145 & 0.000143\end{array}$ $0.000158 \quad 0.000156$ $0.000167 \quad 0.000168$

$\begin{array}{lll}0.000177 & 0.000177\end{array}$

$\begin{array}{lll}0.000185 & 0.000185\end{array}$ $\begin{array}{lll}0.000192 & 0.000192 & 1348.05\end{array}$
Square of Inconsistent Inconsistent Density based on based on Residual density apparent volume

0.007

0.002

0.006

0.011

0.386

0.007

0.060

0.022

0.009

0.276

0.084

0.085

0.078

0.447

0.080

0.545

0.540

0.006

0.090

0.350

0.292

0.958

0.688

0.371

0.881

0.117

0.952

0.773

0.000

0.000

0.000

0.000

0.000

0.000

0.000

0.011

0.055

0.117

0.136

0.115

0.076

0.010

0.002

0.007

0.050
Relative Apparent Density of density molar volume water $/ \mathrm{kg} / \mathrm{m}^{3}$ $1 \mathrm{~cm}^{3} / \mathrm{mol}$

$\begin{array}{rr}9.1 & 997.0449 \\ & 997.0449 \\ 12.16 & 997.0449 \\ & 997.0449 \\ & 997.0449 \\ 24.1 & 997.0449 \\ & 997.0449 \\ 48.82 & 997.0449 \\ & 997.0449 \\ & 997.0449 \\ & 997.0449 \\ 72.57 & 997.0449 \\ & 997.0449 \\ 94.14 & 997.0449 \\ 116.6 & 997.0449 \\ & 997.0449 \\ 174.28 & 997.0449 \\ 224.36 & 997.0449 \\ & 997.0449 \\ 273.22 & 997.0449 \\ 321.3 & 997.0449 \\ & 997.0449 \\ 360.18 & 997.0449 \\ & 997.0449 \\ & 997.0449 \\ 164.92 & 997.0449 \\ 214.1 & 997.0449 \\ 260.85 & 997.0449 \\ 306.09 & 997.0449 \\ 355.92 & 994.0319 \\ 2.57 & 994.0319 \\ 3.68 & 994.0319 \\ 5.91 & 994.0319 \\ 9.07 & 994.0319 \\ 11.69 & 994.0319 \\ 23.87 & 994.0319 \\ 45.61 & 994.0319 \\ 70.72 & 994.0319 \\ 91.83 & 994.0319 \\ 116.52 & 994.0319 \\ & 994.0319 \\ & 994.0319 \\ & 994.0319 \\ & 994.0319 \\ & 994.0319\end{array}$




$\begin{array}{lrrr}\mathbf{t} /{ }^{\circ} \mathrm{C} \text { mass frac } & \begin{array}{r}\text { Density } \\ \text { exp } /\end{array} & \text { R } \\ & & \mathrm{kg} / \mathrm{m}^{3} & \\ & & 1002.0 & (90) \\ 40 & 0.0100 & 1030.5 & (90) \\ 40 & 0.0400 & 103.2 & (90) \\ 40 & 0.0800 & 1068.2 & \\ 40 & 0.1200 & 1107.0 & (90) \\ 45 & 0.0014 & 991.4 & (97) \\ 45 & 0.0028 & 992.7 & (97) \\ 45 & 0.0041 & 993.8 & (97) \\ 45 & 0.0065 & 996.0 & (97) \\ 45 & 0.0100 & 999.1 & (97) \\ 45 & 0.0129 & 1001.7 & (97) \\ 45 & 0.0263 & 1013.5 & (97) \\ 45 & 0.0497 & 1034.9 & (97) \\ 45 & 0.0760 & 1059.4 & (97) \\ 45 & 0.0975 & 1079.8 & (97) \\ 45 & 0.1220 & 1103.7 & (97) \\ 45 & 0.1679 & 1151.0 & (97) \\ 45 & 0.2115 & 1198.9 & (97) \\ 45 & 0.2511 & 1245.2 & (97) \\ 45 & 0.2871 & 1289.4 & (97) \\ 45 & 0.3246 & 1338.4 & (97) \\ 55 & 0.0014 & 986.9 & (97) \\ 55 & 0.0028 & 988.2 & (97) \\ 55 & 0.0041 & 989.3 & (97) \\ 55 & 0.0065 & 991.4 & (97) \\ 55 & 0.0100 & 994.5 & (97) \\ 55 & 0.0129 & 997.1 & (97) \\ 55 & 0.0263 & 1008.9 & (97) \\ 55 & 0.0497 & 1029.9 & (97) \\ 55 & 0.0760 & 1054.2 & (97) \\ 55 & 0.0975 & 1074.6 & (97) \\ 55 & 0.1220 & 1098.2 & (97) \\ 55 & 0.1679 & 1144.7 & (97) \\ 55 & 0.2115 & 1192.0 & (97) \\ 55 & 0.2511 & 1237.1 & (97) \\ 55 & 0.2871 & 1280.8 & (97) \\ 55 & 0.3246 & 1328.9 & (97)\end{array}$

Ref

$\begin{array}{cc}\text { App vol App vol } \\ \exp / & \text { Calc / }\end{array}$

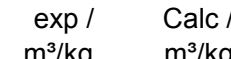

$$
\mathrm{m}^{3} / \mathrm{k}
$$

$\mathrm{m}^{3} / \mathrm{kg}$

Density Density App Vol calc / Residual / Residua / $\begin{array}{rr}\mathrm{kg} / \mathrm{m}^{3} & \mathrm{~kg} / \mathrm{m}^{3} \\ \mathrm{~m}^{3} / \mathrm{kg}\end{array}$

$\begin{array}{lllll}0.000024 & 0.000109 & 1001.14 & 0.8577 & -0.000086\end{array}$ $\begin{array}{lll}0.000072 & 0.000121 & 1028.41\end{array}$ $\begin{array}{lll}0.000112 & 0.000135 & 1066.05\end{array}$ $\begin{array}{lll}0.000137 & 0.000148 & 1105.38\end{array}$ $\begin{array}{lll}0.000103 & 0.000113 & 991.43\end{array}$ $\begin{array}{lll}0.000103 & 0.000113 & 992.71\end{array}$ $\begin{array}{lll}0.000108 & 0.000114 & 993.80\end{array}$ $\begin{array}{lll}0.000109 & 0.000115 & 995.97\end{array}$ $\begin{array}{lll}0.000112 & 0.000116 & 999.06\end{array}$ $\begin{array}{lll}0.000115 & 0.000117 & 1001.63\end{array}$ $\begin{array}{lll}0.000126 & 0.000122 & 1013.61\end{array}$ $\begin{array}{lll}0.000132 & 0.000131 & 1034.96\end{array}$ $\begin{array}{lll}0.000143 & 0.000140 & 1059.59\end{array}$ $\begin{array}{lll}0.000151 & 0.000147 & 1080.25\end{array}$ $\begin{array}{lll}0.000159 & 0.000154 & 1104.37\end{array}$ $\begin{array}{lll}0.000169 & 0.000167 & 1151.67\end{array}$ $\begin{array}{lll}0.000179 & 0.000177 & 1199.47\end{array}$ $\begin{array}{lll}0.000186 & 0.000186 & 1245.43\end{array}$ $\begin{array}{lll}0.000194 & 0.000193 & 1289.82\end{array}$ $\begin{array}{lll}0.000200 & 0.000200 & 1338.84\end{array}$ $\begin{array}{lll}0.000103 & 0.000126 & 986.89\end{array}$ $\begin{array}{lll}0.000107 & 0.000127 & 988.14\end{array}$ $\begin{array}{lll}0.000108 & 0.000127 & 989.22\end{array}$ $\begin{array}{lll}0.000112 & 0.000128 & 991.34\end{array}$ $\begin{array}{lll}0.000116 & 0.000130 & 994.37\end{array}$ $\begin{array}{lll}0.000118 & 0.000131 & 996.89\end{array}$ $\begin{array}{lll}0.000127 & 0.000135 & 1008.65\end{array}$ $\begin{array}{lll}0.000137 & 0.000143 & 1029.61\end{array}$ $\begin{array}{lll}0.000147 & 0.000152 & 1053.82\end{array}$ $\begin{array}{lll}0.000154 & 0.000158 & 1074.13\end{array}$ $\begin{array}{lll}0.000163 & 0.000165 & 1097.86\end{array}$ $\begin{array}{lll}0.000175 & 0.000176 & 1144.43\end{array}$ $\begin{array}{lll}0.000184 & 0.000186 & 1191.52\end{array}$ $\begin{array}{lll}0.000193 & 0.000194 & 1236.83\end{array}$ $\begin{array}{lll}0.000200 & 0.000201 & 1280.62\end{array}$ $\begin{array}{lll}0.000207 & 0.000207 & 1329.00\end{array}$ Average Res Std dev Res Avg - 4std Avg + 4std
Square of Inconsistent Inconsistent Density based on based on Residual density apparen apparen
volume

0.736 $2.0911-0.000049$ $2.1541-0.000024$ $1.6219-0.000011$ $0.0132-0.000010$ $0.0280-0.000010$ $0.0243-0.000006$ $0.0391-0.000006$ $0.0451-0.000005$ $0.0318-0.000002$ $\begin{array}{ll}-0.0925 & 0.000003\end{array}$ $\begin{array}{ll}-0.0741 & 0.000001\end{array}$ $-0.2135 \quad 0.000003$ $-0.4748 \quad 0.000004$ $\begin{array}{ll}-0.6875 & 0.000005\end{array}$ $-0.6588 \quad 0.000003$ $\begin{array}{ll}-0.5500 & 0.000002\end{array}$ $\begin{array}{ll}-0.2232 & 0.000001\end{array}$ $\begin{array}{ll}-0.4538 & 0.000001\end{array}$ $\begin{array}{lll}-0.4649 & 0.000001\end{array}$ $0.0309-0.000023$ $0.0544-0.000020$ $0.0764-0.000019$ $0.1027-0.000016$ $0.1334-0.000013$ $0.1666-0.000013$ $0.2207-0.000008$ $0.3299-0.000006$ $0.3890-0.000005$ $0.4605-0.000004$ $0.3243-0.000002$ $0.2808-0.000001$ $0.5198-0.000002$ $0.2266-0.000001$ $\begin{array}{ll}0.1284 & 0.000000\end{array}$ $\begin{array}{ll}-0.0776 & 0.000000\end{array}$ $0.0042-0.000001$ $0.6222 \quad 0.000012$ $-2.4845-0.000051$ 2.49290 .000049
4.373

4.640

2.631

0.000

0.001

0.001

0.002

0.002

0.001
0.009

0.005

0.046

0.225

0.473

0.434

0.303

0.050

0.206

0.216

0.003

0.006

0.011
0.018

0.028

0.049

0.109

0.151

0.212

0.105

0.079

0.270

0.051

0.016

44.5182 Sum chi sqr
Relative Apparent Density of density molar volume water $/ \mathrm{kg} / \mathrm{m}^{3}$ $/ \mathrm{cm}^{3} / \mathrm{mol}$

992.2158 992.2158 992.2158 992.2158 990.2132 990.2132 990.2132 990.2132 990.2132 990.2132 990.2132 990.2132 990.2132 990.2132 990.2132 990.2132 990.2132 990.2132 990.2132 990.2132 985.6952 985.6952 985.6952 985.6952 985.6952 985.6952 985.6952 985.6952 985.6952 985.6952 985.6952 985.6952 985.6952 985.6952 985.6952 985.6952

$\begin{array}{lr}\text { Curve fit for the model App vol= } \\ \text { c0 } \\ \text { c1 } \\ \text { c2 } & 1868.499 \\ & 0.07137 .233 \\ & \end{array}$


molality molarity

\section{$\mathbf{t} /{ }^{\circ} \mathrm{C}$ mass frac Density}

\section{$\exp /$}

$\mathrm{kg} / \mathrm{m}^{3}$
Ref

$\begin{array}{rr}\text { App vol } & \text { App vol } \\ \exp / & \text { Calc / } \\ \mathrm{m}^{3} / \mathrm{kg} & \mathrm{m}^{3} / \mathrm{kg}\end{array}$

Density App Vol

calc / Residual / Residual /

$\mathrm{kg} / \mathrm{m}^{3}$
Square of Inconsistent Inconsistent Density based on based on Residual density apparent
Relative Apparent Density of density molar volume water $/ \mathrm{kg} / \mathrm{m}^{3}$

$\begin{array}{lr}\text { c3 } & 0.002565 \\ \text { c4 } & 575.6913 \\ \text { Min T } & 0 \\ \text { Max T } & 55 \\ \text { Min w } & 0.0013 \\ \text { Max w } & 0.4204 \\ \text { Avg dens res } & 0.0042 \\ \text { Std dens res } & 0.622171 \\ \text { Avg app vol res *10^3 } & -0.00092 \\ \text { Std app vol res *10^3 } & 0.012407 \\ \text { No of points in corr } & 116 \\ \text { No of inconsistent poir } & 3\end{array}$

Inconsistent data not used (Residual greater than average $+/-4$ standard deviations)

\begin{tabular}{|c|c|c|c|c|c|c|c|c|c|c|c|c|}
\hline 1.7904 & 25 & 0.1940 & 1163.1 & (26) & 0.000265 & 0.000153 & 1193.13 & -30.0810 & 0.000112 & 904.868 & ! & $!$ \\
\hline 0.0625 & 35 & 0.0083 & 999.05 & (74) & 0.000400 & 0.000102 & 1001.54 & -2.4899 & 0.000299 & 6.199 & ! & $!$ \\
\hline 0.125 & 35 & 0.0165 & 1003.95 & (74) & 0.000405 & 0.000105 & 1008.97 & -5.0158 & 0.000300 & 25.158 & $!$ & $!$ \\
\hline 0.25 & 35 & 0.0325 & 1013.61 & (74) & 0.000408 & 0.000112 & 1023.63 & -10.0119 & 0.000297 & 100.237 & $!$ & $!$ \\
\hline 0.5 & 35 & 0.0630 & 1032.08 & (74) & 0.000417 & 0.000123 & 1052.19 & -20.1103 & 0.000294 & 404.424 & $!$ & $!$ \\
\hline 1 & 35 & 0.1185 & 1066.61 & (74) & 0.000428 & 0.000142 & 1106.68 & -40.0682 & 0.000286 & 1605.464 & $!$ & $!$ \\
\hline 0.0625 & 40 & 0.0083 & 997.20 & (74) & 0.000404 & 0.000109 & 999.65 & -2.4534 & 0.000295 & 6.019 & & $!$ \\
\hline 0.125 & 40 & 0.0165 & 1002.07 & (74) & 0.000408 & 0.000112 & 1007.01 & -4.9441 & 0.000296 & 24.444 & ! & ! \\
\hline 0.25 & 40 & 0.0325 & 1011.66 & (74) & 0.000412 & 0.000118 & 1021.54 & -9.8739 & 0.000294 & 97.493 & ! & ! \\
\hline 0.5 & 40 & 0.0630 & 1030.00 & (74) & 0.000421 & 0.000130 & 1049.85 & -19.8533 & 0.000291 & 394.153 & $!$ & $!$ \\
\hline \multirow[t]{3}{*}{1} & 40 & 0.1185 & 1064.17 & (74) & 0.000433 & 0.000148 & 1103.89 & -39.7184 & 0.000285 & 1577.548 & $!$ & $!$ \\
\hline & 40 & 0.1600 & 1151.0 & (90) & 0.000139 & 0.000159 & 1146.67 & 4.3333 & -0.000021 & 18.778 & ! & \\
\hline & 40 & 0.2000 & 1195.3 & (90) & 0.000152 & 0.000170 & 1190.19 & 5.1104 & -0.000018 & 26.116 & ! & \\
\hline 0.0625 & 50 & 0.0083 & 992.97 & (74) & 0.000408 & 0.000122 & 995.33 & -2.3563 & 0.000286 & 5.552 & & $!$ \\
\hline 0.125 & 50 & 0.0165 & 997.80 & (74) & 0.000413 & 0.000125 & 1002.55 & -4.7524 & 0.000287 & 22.586 & ! & ! \\
\hline 0.25 & 50 & 0.0325 & 1007.34 & (74) & 0.000416 & 0.000131 & 1016.81 & -9.4766 & 0.000285 & 89.806 & ! & ! \\
\hline 0.5 & 50 & 0.0630 & 1025.53 & (74) & 0.000425 & 0.000142 & 1044.62 & -19.0953 & 0.000283 & 364.629 & ! & ! \\
\hline 1 & 50 & 0.1185 & 1059.53 & (74) & 0.000436 & 0.000159 & 1097.76 & -38.2280 & 0.000277 & 1461.378 & $!$ & ! \\
\hline
\end{tabular}

997.0449 53.8994 .0319 $54.4 \quad 994.0319$ $54.9 \quad 994.0319$ 56.1994 .0319 $57.6 \quad 994.0319$ $54.3 \quad 992.2158$ $54.9 \quad 992.2158$ $55.4 \quad 992.2158$ $56.6 \quad 992.2158$ 58.2992 .2158 992.2158 992.2158 $54.9 \quad 988.0363$ $55.5 \quad 988.0363$ $55.9 \quad 988.0363$ 57.1988 .0363 $58.6 \quad 988.0363$ 
Density of aqueous solutions of CuSO4

References (84) Pearce, J. N.; Pumplin, G. G. The Apparent and Partial Molal Volume of Ammonium Chloride and of Cupric Sulfate in Aqueous Solutions at $25^{\circ} \mathrm{C}$. J. Am. Chem. Soc. $1937,59,1221-1222$.

(90) Perry, R. H.; Green, D. W. Perry's Chemical Engineers' Handbook; McGraw Hill: New York, 7th edition, 1997.

(95) Demichowicz-Pigoniowa, J. Dependence of Density, Viscosity and Electric Conductivity of Aqueous CuSO ${ }_{4}$ Solutions on Temperature and Concentration. Ann. Soc. Chim. Polonorum 1973, 47, 2183-2190.

(101) Puchalska, D.; Atkinson, G.; Routh, S. Solution Thermodynamics of First-Row Transition Elements. 4. Apparent Molal Volumes of Aqueous $\mathrm{ZnSO}_{4}$ and $\mathrm{CuSO}_{4}$ Solutions from 15 to $55^{\circ} \mathrm{C}$. J. Sol. Chem. 1993, 22, 625-639.

(125) Suryanarayana, C. V.; Alamelu, S. Electrical Conductance of Concentrated Aqueous Solutions of Copper Sulfate. Bull. Chem. Soc. Japan 1959, 32, 333-339.

\begin{tabular}{|c|c|c|c|c|c|c|c|c|c|c|c|c|c|c|c|}
\hline \multirow[t]{5}{*}{ molality } & \multirow[t]{5}{*}{ molarity } & \multicolumn{2}{|c|}{$\mathrm{t} /{ }^{\circ} \mathrm{C}$ mass frac } & $\begin{array}{r}\text { Density } \\
\exp / \\
k g / m^{3}\end{array}$ & Ref & \multirow[t]{2}{*}{$\begin{array}{r}\text { App vol } \\
\exp / \\
\mathrm{m}^{3} / \mathrm{kg}\end{array}$} & $\begin{array}{r}\text { App vol } \\
\text { Calc / } \\
\mathrm{m}^{3} / \mathrm{kg}\end{array}$ & $\begin{array}{r}\text { Density } \\
\text { calc / } \\
\mathrm{kg} / \mathrm{m}^{3}\end{array}$ & $\begin{array}{r}\text { Density } \\
\text { Residual / } \\
\mathrm{kg} / \mathrm{m}^{3}\end{array}$ & \multirow{2}{*}{$\begin{array}{r}\text { App Vol } \\
\text { Residual / } \\
\mathrm{m}^{3} / \mathrm{kg}\end{array}$} & \multirow{2}{*}{$\begin{array}{r}\text { Square of } \\
\text { Density } \\
\text { Residual } \\
0.031\end{array}$} & $\begin{array}{l}\text { Inconsistent } \\
\text { based on } \\
\text { density }\end{array}$ & $\begin{array}{l}\text { Inconsistent } \\
\text { based on } \\
\text { apparent } \\
\text { volume }\end{array}$ & $\begin{array}{l}\text { Delta density } \begin{array}{r}\text { Apparent } \\
/ \mathrm{kg} / \mathrm{m}^{3} \mathrm{molal} \text { volume } \\
1 \mathrm{~cm}^{3} / \mathrm{mol}\end{array}\end{array}$ & $\begin{array}{r}\text { Density of } \\
\text { water } / \mathrm{kg} / \mathrm{m}^{3}\end{array}$ \\
\hline & & 0 & 0.0100 & 1010.40 & (90) & & -0.000028 & 1010.22 & 0.1750 & & & & & & 999.8395 \\
\hline & & 0 & 0.0400 & 1042.90 & (90) & -0.000032 & -0.000022 & 1042.45 & 0.4472 & -0.000010 & 0.200 & & & & 999.8395 \\
\hline & & 0 & 0.0800 & 1088.70 & (90) & -0.000020 & -0.000013 & 1087.98 & 0.7242 & -0.000008 & 0.525 & & & & 999.8395 \\
\hline & & 0 & 0.1200 & 1137.90 & (90) & -0.000011 & -0.000002 & 1136.42 & 1.4788 & -0.000010 & 2.187 & & & & 999.8395 \\
\hline 0.01009 & & 15 & 0.0016 & 1000.80 & (101) & -0.000057 & -0.000014 & 1000.73 & 0.0694 & -0.000043 & 0.005 & & & & 999.0996 \\
\hline 0.05 & & 15 & 0.0079 & 1007.60 & (95) & -0.000066 & -0.000012 & 1007.17 & 0.4268 & -0.000053 & 0.182 & & & & 999.0996 \\
\hline 0.05005 & & 15 & 0.0079 & 1007.39 & (101) & -0.000038 & -0.000012 & 1007.18 & 0.2087 & -0.000026 & 0.044 & & & & 999.0996 \\
\hline 0.07024 & & 15 & 0.0111 & 1010.66 & (101) & -0.000032 & -0.000012 & 1010.44 & 0.2248 & -0.000020 & 0.051 & & & & 999.0996 \\
\hline 0.09946 & & 15 & 0.0156 & 1015.49 & (101) & -0.000033 & -0.000011 & 1015.14 & 0.3522 & -0.000022 & 0.124 & & & & 999.0996 \\
\hline 0.15013 & & 15 & 0.0234 & 1023.53 & (101) & -0.000020 & -0.000010 & 1023.27 & 0.2565 & -0.000010 & 0.066 & & & & 999.0996 \\
\hline 0.20012 & & 15 & 0.0310 & 1031.53 & (101) & -0.000016 & -0.000008 & 1031.28 & 0.2544 & -0.000008 & 0.065 & & & & 999.0996 \\
\hline 0.25 & & 15 & 0.0384 & 1042.00 & (95) & -0.000073 & -0.000006 & 1039.23 & 2.7653 & -0.000067 & 7.647 & & & & 999.0996 \\
\hline 0.30011 & & 15 & 0.0457 & 1047.32 & (101) & -0.000007 & -0.000005 & 1047.20 & 0.1162 & -0.000002 & 0.014 & & & & 999.0996 \\
\hline 0.49998 & & 15 & 0.0739 & 1078.36 & (101) & 0.000005 & 0.000002 & 1078.70 & -0.3402 & 0.000004 & 0.116 & & & & 999.0996 \\
\hline 0.5 & & 15 & 0.0739 & 1080.40 & (95) & -0.000018 & 0.000002 & 1078.70 & 1.6967 & -0.000020 & 2.879 & & & & 999.0996 \\
\hline 0.7002 & & 15 & 0.1005 & 1108.96 & (101) & 0.000015 & 0.000008 & 1109.73 & -0.7675 & 0.000006 & 0.589 & & & & 999.0996 \\
\hline 0.752 & & 15 & 0.1072 & 1118.60 & (95) & 0.000003 & 0.000010 & 1117.66 & 0.9412 & -0.000007 & 0.886 & & & & 999.0996 \\
\hline 1 & & 15 & 0.1376 & 1153.84 & (101) & 0.000026 & 0.000019 & 1155.01 & -1.1738 & 0.000006 & 1.378 & & & & 999.0996 \\
\hline 1.005 & & 15 & 0.1382 & 1157.30 & (95) & 0.000011 & 0.000019 & 1155.76 & 1.5444 & -0.000008 & 2.385 & & & & 999.0996 \\
\hline 1.265 & & 15 & 0.1680 & 1196.50 & (95) & 0.000018 & 0.000030 & 1193.64 & 2.8614 & -0.000012 & 8.187 & & & & 999.0996 \\
\hline 1.33069 & & 15 & 0.1752 & 1202.12 & (101) & 0.000036 & 0.000033 & 1202.98 & -0.8585 & 0.000003 & 0.737 & & & & 999.0996 \\
\hline 0.05 & & 18 & 0.0079 & 1007.00 & (95) & -0.000054 & -0.000010 & 1006.64 & 0.3566 & -0.000044 & 0.127 & & & & 998.5956 \\
\hline 0.25 & & 18 & 0.0384 & 1040.20 & (95) & -0.000042 & -0.000004 & 1038.60 & 1.6000 & -0.000039 & 2.560 & & & & 998.5956 \\
\hline 0.5 & & 18 & 0.0739 & 1080.60 & (95) & -0.000027 & 0.000004 & 1077.93 & 2.6655 & -0.000031 & 7.105 & & & & 998.5956 \\
\hline 0.752 & & 18 & 0.1072 & 1118.30 & (95) & 0.000001 & 0.000013 & 1116.75 & 1.5469 & -0.000012 & 2.393 & & & & 998.5956 \\
\hline 1.005 & & 18 & 0.1382 & 1156.50 & (95) & 0.000012 & 0.000022 & 1154.71 & 1.7876 & -0.000010 & 3.196 & & & & 998.5956 \\
\hline 1.265 & & 18 & 0.1680 & 1194.70 & (95) & 0.000023 & 0.000032 & 1192.46 & 2.2436 & -0.000009 & 5.034 & & & & 998.5956 \\
\hline & & 20 & 0.0100 & 1008.60 & (90) & -0.000031 & -0.000008 & 1008.37 & 0.2349 & -0.000023 & 0.055 & & & & 998.2041 \\
\hline & & 20 & 0.0400 & 1040.10 & (90) & -0.000007 & -0.000002 & 1039.87 & 0.2268 & -0.000005 & 0.051 & & & & 998.2041 \\
\hline & & 20 & 0.0800 & 1084.00 & (90) & 0.000011 & 0.000007 & 1084.32 & -0.3219 & 0.000003 & 0.104 & & & & 998.2041 \\
\hline & & 20 & 0.1200 & 1130.80 & (90) & 0.000023 & 0.000018 & 1131.55 & -0.7537 & 0.000005 & 0.568 & & & & 998.2041 \\
\hline & & 20 & 0.1600 & 1180.00 & (90) & 0.000037 & 0.000031 & 1181.39 & -1.3949 & 0.000006 & 1.946 & & & & 998.2041 \\
\hline
\end{tabular}


molality molarity

$\mathrm{t} /{ }^{\circ} \mathrm{C}$ mass frac Density

$\exp /$

$\mathrm{kg} / \mathrm{m}^{3}$

$\begin{array}{r}0.02993 \\ 0.05 \\ 0.05005 \\ 0.07024 \\ 0.09913 \\ 0.1 \\ 0.15013 \\ 0.2 \\ 0.20012 \\ 0.25 \\ 0.30011 \\ 0.4 \\ 0.49998 \\ 0.5 \\ 0.6 \\ 0.7002 \\ 0.752 \\ 0.8 \\ 1 \\ 1 \\ 1.005 \\ 1.1964 \\ 1.265 \\ 1.33069 \\ 1.4182 \\ 0.0992 \\ 0.1965 \\ 0.2921 \\ 0.3862 \\ 0.4785 \\ 0.5693 \\ 0.6586 \\ 0.7463 \\ 0.8326 \\ 0.9175 \\ 1.001 \\ 1.083 \\ 1.164 \\ 1.244 \\ 1.321 \\ 1.398 \\ 1.51 \\ \hline\end{array}$

$\begin{array}{lll}20 & 0.1800 & 1206.00\end{array}$

$\begin{array}{lll}25 & 0.0048 & 1001.93\end{array}$

$0.0079 \quad 1005.60$

$0.0079 \quad 1005.18$

$\begin{array}{ll}0.0156 & 1013.02\end{array}$

$0.0157 \quad 1013.21$

$0.0234 \quad 1020.94$

$0.0309 \quad 1029.11$

$0.0310 \quad 1028.78$

$0.0384 \quad 1039.40$

$0.0457 \quad 1044.14$

$0.0600 \quad 1060.36$

$0.0739 \quad 1074.50$

$0.0739 \quad 1077.40$

$0.0874 \quad 1090.96$

$0.1005 \quad 1104.32$

$0.1132 \quad 1120.99$

$0.1376 \quad 1150.56$

$0.1376 \quad 1149.08$

$0.1382 \quad 1152.20$

$0.1603 \quad 1179.05$

$0.1680 \quad 1191.70$

$0.1752 \quad 1196.81$

$0.1846 \quad 1210.68$

$0.0156 \quad 1011.00$

$0.0304 \quad 1027.00$

$0.0445 \quad 1042.00$

$0.0581 \quad 1057.00$

$0.0710 \quad 1071.00$

$0.0833 \quad 1085.00$

$0.0951 \quad 1098.00$

$0.1064 \quad 1110.00$

$0.1173 \quad 1124.00$

$\begin{array}{ll}0.1277 & 1136.00\end{array}$

$0.1378 \quad 1149.00$

$0.1474 \quad 1161.00$

$0.1567 \quad 1172.00$

$0.1657 \quad 1184.00$

$0.1741 \quad 1195.00$

$0.1824 \quad 1205.00$

$0.1942 \quad 1223.00$
$0.0111 \quad 1008.42$

$0.1072 \quad 1115.40$
Ref

App vol App vol

$\mathrm{m}^{3} / \mathrm{kg} \quad \mathrm{m}^{3} / \mathrm{kg}$

(90) $\quad 0.000043 \quad 0.000038$

$\begin{array}{lll}\text { (101) } & -0.000026 & -0.000005\end{array}$

$-0.000075-0.000004$

$-0.000021-0.000004$

$\begin{array}{lll}\text { (101) } \quad-0.000017 & -0.000003\end{array}$

(101) $\quad-0.000012-0.000003$

(84) $\quad-0.000015-0.000003$

(101) $\quad 0.000000-0.000001$

(84) $\quad-0.000007 \quad 0.000000$

(101) $0.000003 \quad 0.000000$

(95) $\quad-0.000062 \quad 0.000002$

$\begin{array}{lll}(101) & 0.000013 & 0.000003\end{array}$

(84) $0.000005 \quad 0.000006$

(101) $\quad 0.000025 \quad 0.000010$

(95) $\quad-0.000009 \quad 0.000010$

(84) $\quad 0.000015 \quad 0.000013$

$\begin{array}{lll}(101) & 0.000034 & 0.000016\end{array}$

$\begin{array}{lll}\text { (95) } \quad 0.000010 & 0.000018\end{array}$

(84) $0.000024 \quad 0.000020$

$\begin{array}{lll}(84) & 0.000031 & 0.000027\end{array}$

$0.000039 \quad 0.000027$

$\begin{array}{lll}\text { (95) } & 0.000026 & 0.000027\end{array}$

$\begin{array}{lll}\text { (84) } & 0.000037 & 0.000035\end{array}$

$\begin{array}{lll}\text { (95) } & 0.000028 & 0.000037\end{array}$

$\begin{array}{lll}(101) & 0.000047 & 0.000040\end{array}$

(84) $0.000044 \quad 0.000044$

$\begin{array}{lll}(125) & 0.000026 & 0.000001\end{array}$

(125)

(125)

(125)

(125)

(125)

(125)

(125)

$(125)$
$(125)$

(125)

(125)

$(125)$
$(125)$

(125)

(125) $\begin{array}{rr}0.000026 & 0.000001 \\ -0.000004 & 0.000004 \\ 0.000001 & 0.000007\end{array}$

$\begin{array}{ll}0.000001 & 0.000007\end{array}$

$0.000008 \quad 0.000012$

$0.000011 \quad 0.000015$

$0.000020 \quad 0.000018$

$0.000032 \quad 0.000021$

$\begin{array}{ll}0.000027 & 0.000024\end{array}$

$\begin{array}{ll}0.000033 & 0.000027\end{array}$

0.0000310 .000030

$0.000034 \quad 0.000033$

$0.000040 \quad 0.000036$

$0.000040 \quad 0.000040$

$0.000042 \quad 0.000043$

$0.000048 \quad 0.000046$

$0.000043 \quad 0.000051$
$0.000000 \quad 0.000010$
Density Density App Vol Square of Inconsistent Inconsistent Delta density Apparent Density of calc / Residual / Residual / Density based on based on $/ \mathrm{kg} / \mathrm{m}^{3}$ molal volume water $/ \mathrm{kg} / \mathrm{m}^{3}$ $\mathrm{kg} / \mathrm{m}^{3} \quad \mathrm{~kg} / \mathrm{m}^{3} \quad \mathrm{~m}^{3} / \mathrm{kg}$ Residual density apparent

$\begin{array}{llll}1207.16 & -1.1573 & 0.000004 & 1.339\end{array}$

$\begin{array}{llll}1001.83 & 0.1000 & -0.000021 & 0.010\end{array}$

$\begin{array}{llll}1005.03 & 0.5658 & -0.000071 & 0.320\end{array}$

$\begin{array}{llll}005.04 & 0.1378 & -0.000017 & 0.019\end{array}$

$1008.26 \quad 0.1581-0.000014 \quad 0.025$

$1012.86 \quad 0.1576-0.000010 \quad 0.025$

$1013.00 \quad 0.2051-0.000013 \quad 0.042$

$\begin{array}{llll}1020.96 & -0.0243 & 0.000001 & 0.001\end{array}$

$\begin{array}{llll}1028.86 & 0.2450 & -0.000007 & 0.060\end{array}$

$\begin{array}{lll}-0.1008 & 0.000003 & 0.010\end{array}$

$\begin{array}{lll}2.6458 & -0.000064 & 7.000\end{array}$

0.247

0.009

$0.0957-0.000$

1.655

2.594

0.049

4.600

1.197

0.292

0.454

4.638

0.054

0.380

5.224

3.375

0.013

0.157

0.062

0.065

0.357

0.104

0.150

0.043

2.091

0.126

0.875

0.034

0.008

0.513

0.005

0.019

0.275

4.950
998.2041

997.0449

997.0449

997.0449

997.0449

997.0449

997.0449

997.0449

997.0449

997.0449

997.0449

997.0449

997.0449

997.0449

997.0449

997.0449

997.0449

997.0449

997.0449

997.0449

997.0449

997.0449

997.0449

997.0449

997.0449

995.6473

995.6473

995.6473

995.6473

995.6473

995.6473

995.6473

995.6473

995.6473

995.6473

995.6473

995.6473

995.6473

995.6473

995.6473

995.6473

995.6473 


\begin{tabular}{|c|c|c|c|c|c|c|c|c|c|c|c|c|c|c|c|}
\hline molality & molarity & $\mathrm{t} /{ }^{\circ} \mathrm{C}$ & lass frac & $\begin{array}{r}\text { Density } \\
\exp / \\
\mathrm{kg} / \mathrm{m}^{3}\end{array}$ & Ref & $\begin{array}{r}\text { App vol } \\
\exp / \\
\mathrm{m}^{3} / \mathrm{kg}\end{array}$ & $\begin{array}{r}\text { App vol } \\
\text { Calc / } \\
\mathrm{m}^{3} / \mathrm{kg}\end{array}$ & $\begin{array}{r}\text { Density } \\
\text { calc / } \\
\mathrm{kg} / \mathrm{m}^{3}\end{array}$ & $\begin{array}{r}\text { Density } \\
\text { Residual / } \\
\mathrm{kg} / \mathrm{m}^{3}\end{array}$ & $\begin{array}{r}\text { App Vol } \\
\text { Residual / } \\
\mathrm{m}^{3} / \mathrm{kg}\end{array}$ & $\begin{array}{r}\text { Square of } \\
\text { Density } \\
\text { Residual }\end{array}$ & $\begin{array}{l}\text { Inconsistent } \\
\text { based on } \\
\text { density }\end{array}$ & $\begin{array}{c}\text { Inconsistent } \\
\text { based on } \\
\text { apparent } \\
\text { volume }\end{array}$ & $\begin{array}{r}\text { Delta density } \begin{array}{r}\text { Apparent } \\
/ \mathrm{kg} / \mathrm{m}^{3} \mathrm{molal} \text { volume } \\
\\
1 \mathrm{~cm} / \mathrm{mol}\end{array}\end{array}$ & $\begin{array}{r}\text { Density of } \\
\text { water } / \mathrm{kg} / \mathrm{m}^{3}\end{array}$ \\
\hline 0.01009 & & 35 & 0.0016 & 995.69 & (101) & -0.000036 & 0.000002 & 995.63 & 0.0602 & -0.000038 & 0.004 & & & & 994.0319 \\
\hline 0.02993 & & 35 & 0.0048 & 998.89 & (101) & -0.000023 & 0.000002 & 998.77 & 0.1207 & -0.000025 & 0.015 & & & & 994.0319 \\
\hline 0.05 & & 35 & 0.0079 & 1002.40 & (95) & -0.000055 & 0.000003 & 1001.94 & 0.4584 & -0.000058 & 0.210 & & & & 994.0319 \\
\hline 0.05005 & & 35 & 0.0079 & 1002.11 & (101) & -0.000017 & 0.000003 & 1001.95 & 0.1605 & -0.000020 & 0.026 & & & & 994.0319 \\
\hline 0.07024 & & 35 & 0.0111 & 1005.31 & (101) & -0.000012 & 0.000003 & 1005.14 & 0.1729 & -0.000015 & 0.030 & & & & 994.0319 \\
\hline 0.0992 & & 35 & 0.0156 & 1009.00 & (125) & 0.000049 & 0.000004 & 1009.70 & -0.7026 & 0.000044 & 0.494 & & & & 994.0319 \\
\hline 0.09946 & & 35 & 0.0156 & 1009.99 & (101) & -0.000011 & 0.000004 & 1009.74 & 0.2465 & -0.000015 & 0.061 & & & & 994.0319 \\
\hline 0.15013 & & 35 & 0.0234 & 1017.89 & (101) & -0.000002 & 0.000006 & 1017.71 & 0.1778 & -0.000007 & 0.032 & & & & 994.0319 \\
\hline 0.1965 & & 35 & 0.0304 & 1025.00 & (125) & 0.000007 & 0.000007 & 1024.98 & 0.0176 & -0.000001 & 0.000 & & & & 994.0319 \\
\hline 0.20012 & & 35 & 0.0310 & 1025.67 & (101) & 0.000003 & 0.000007 & 1025.55 & 0.1209 & -0.000004 & 0.015 & & & & 994.0319 \\
\hline 0.25 & & 35 & 0.0384 & 1036.00 & (95) & -0.000056 & 0.000009 & 1033.34 & 2.6571 & -0.000065 & 7.060 & & & & 994.0319 \\
\hline 0.2921 & & 35 & 0.0445 & 1040.00 & (125) & 0.000008 & 0.000010 & 1039.90 & 0.0996 & -0.000002 & 0.010 & & & & 994.0319 \\
\hline 0.30011 & & 35 & 0.0457 & 1040.89 & (101) & 0.000015 & 0.000010 & 1041.15 & -0.2559 & 0.000005 & 0.065 & & & & 994.0319 \\
\hline 0.3862 & & 35 & 0.0581 & 1054.00 & (125) & 0.000020 & 0.000013 & 1054.49 & -0.4855 & 0.000008 & 0.236 & & & & 994.0319 \\
\hline 0.4785 & & 35 & 0.0710 & 1068.00 & (125) & 0.000024 & 0.000016 & 1068.69 & -0.6889 & 0.000009 & 0.475 & & & & 994.0319 \\
\hline 0.49998 & & 35 & 0.0739 & 1071.29 & (101) & 0.000024 & 0.000016 & 1071.98 & -0.6890 & 0.000008 & 0.475 & & & & 994.0319 \\
\hline 0.5 & & 35 & 0.0739 & 1074.10 & (95) & -0.000009 & 0.000016 & 1071.98 & 2.1179 & -0.000025 & 4.486 & & & & 994.0319 \\
\hline 0.5693 & & 35 & 0.0833 & 1082.00 & (125) & 0.000024 & 0.000018 & 1082.56 & -0.5554 & 0.000006 & 0.309 & & & & 994.0319 \\
\hline 0.6586 & & 35 & 0.0951 & 1095.00 & (125) & 0.000031 & 0.000021 & 1096.08 & -1.0832 & 0.000009 & 1.173 & & & & 994.0319 \\
\hline 0.7002 & & 35 & 0.1005 & 1101.39 & (101) & 0.000031 & 0.000023 & 1102.35 & -0.9559 & 0.000008 & 0.914 & & & & 994.0319 \\
\hline 0.7463 & & 35 & 0.1064 & 1109.00 & (125) & 0.000026 & 0.000024 & 1109.26 & -0.2557 & 0.000002 & 0.065 & & & & 994.0319 \\
\hline 0.752 & & 35 & 0.1072 & 1111.40 & (95) & 0.000015 & 0.000024 & 1110.11 & 1.2922 & -0.000010 & 1.670 & & & & 994.0319 \\
\hline 0.8326 & & 35 & 0.1173 & 1121.00 & (125) & 0.000035 & 0.000027 & 1122.10 & -1.1019 & 0.000007 & 1.214 & & & & 994.0319 \\
\hline 0.9175 & & 35 & 0.1277 & 1133.00 & (125) & 0.000040 & 0.000030 & 1134.62 & -1.6206 & 0.000010 & 2.626 & & & & 994.0319 \\
\hline 1 & & 35 & 0.1376 & 1145.30 & (101) & 0.000041 & 0.000033 & 1146.67 & -1.3655 & 0.000008 & 1.865 & & & & 994.0319 \\
\hline 1.001 & & 35 & 0.1378 & 1146.00 & (125) & 0.000038 & 0.000033 & 1146.81 & -0.8108 & 0.000004 & 0.657 & & & & 994.0319 \\
\hline 1.005 & & 35 & 0.1382 & 1147.40 & (95) & 0.000033 & 0.000033 & 1147.39 & 0.0084 & 0.000000 & 0.000 & & & & 994.0319 \\
\hline 1.083 & & 35 & 0.1474 & 1158.00 & (125) & 0.000039 & 0.000036 & 1158.66 & -0.6576 & 0.000003 & 0.432 & & & & 994.0319 \\
\hline 1.164 & & 35 & 0.1567 & 1170.00 & (125) & 0.000040 & 0.000039 & 1170.23 & -0.2322 & 0.000001 & 0.054 & & & & 994.0319 \\
\hline 1.244 & & 35 & 0.1657 & 1181.00 & (125) & 0.000045 & 0.000042 & 1181.53 & -0.5326 & 0.000002 & 0.284 & & & & 994.0319 \\
\hline 1.265 & & 35 & 0.1680 & 1185.50 & (95) & 0.000039 & 0.000043 & 1184.48 & 1.0234 & -0.000004 & 1.047 & & & & 994.0319 \\
\hline 1.321 & & 35 & 0.1741 & 1192.00 & (125) & 0.000047 & 0.000045 & 1192.28 & -0.2799 & 0.000001 & 0.078 & & & & 994.0319 \\
\hline 1.33069 & & 35 & 0.1752 & 1192.94 & (101) & 0.000049 & 0.000046 & 1193.62 & -0.6830 & 0.000003 & 0.466 & & & & 994.0319 \\
\hline \multirow[t]{2}{*}{1.398} & & 35 & 0.1824 & 1203.00 & (125) & 0.000048 & 0.000049 & 1202.89 & 0.1063 & 0.000000 & 0.011 & & & & 994.0319 \\
\hline & & 40 & 0.0100 & 1002.40 & (90) & -0.000016 & 0.000006 & 1002.18 & 0.2247 & -0.000022 & 0.050 & & & & 992.2158 \\
\hline 0.0992 & & 40 & 0.0156 & 1007.00 & (125) & 0.000059 & 0.000007 & 1007.81 & -0.8111 & 0.000051 & 0.658 & & & & 992.2158 \\
\hline \multirow[t]{2}{*}{0.1965} & & 40 & 0.0304 & 1023.00 & (125) & 0.000011 & 0.000010 & 1023.02 & -0.0172 & 0.000001 & 0.000 & & & & 992.2158 \\
\hline & & 40 & 0.0400 & 1033.20 & (90) & 0.000008 & 0.000012 & 1033.05 & 0.1474 & -0.000003 & 0.022 & & & & 992.2158 \\
\hline 0.2921 & & 40 & 0.0445 & 1038.00 & (125) & 0.000010 & 0.000013 & 1037.86 & 0.1366 & -0.000003 & 0.019 & & & & 992.2158 \\
\hline 0.3862 & & 40 & 0.0581 & 1052.00 & (125) & 0.000021 & 0.000016 & 1052.38 & -0.3783 & 0.000006 & 0.143 & & & & 992.2158 \\
\hline \multirow{2}{*}{\multicolumn{2}{|c|}{0.4785}} & 40 & 0.0710 & 1066.00 & (125) & 0.000025 & 0.000018 & 1066.51 & -0.5138 & 0.000006 & 0.264 & & & & 992.2158 \\
\hline & & 40 & 0.0800 & 1076.40 & (90) & 0.000023 & 0.000020 & 1076.60 & -0.2023 & 0.000002 & 0.041 & & & & 992.2158 \\
\hline 5693 & & 40 & 0.0833 & 1080.00 & (125) & 0.000024 & 0.000021 & 1080.31 & -0.3144 & 0.000003 & 0.099 & & & & 992.2158 \\
\hline
\end{tabular}


$\mathrm{t} /{ }^{\circ} \mathrm{C}$ mass frac Density

\section{exp /}

$\mathrm{kg} / \mathrm{m}^{3}$

0.6586

0.7463

0.8326

0.9175

1.001

1.083
1.164

1.244

1.321

1.398
1.8

0.01009

0.02993

0.05005

0.07024

0.0992

0.15013

0.1965

0.20012

0.25

0.2921

0.30011

0.3862

0.4785

0.49998

0.5
0.5693

0.6586

0.7002

0.7463

0.752

0.8326

0.9175

1.001

1.005

1.083

1.164

1.244

1.265

1.321

1.33069

$\begin{array}{cccc}40 & 0.0951 & 1093.00 & (125) \\ 40 & 0.1064 & 1106.00 & (125) \\ 40 & 0.1173 & 1119.00 & (125) \\ 40 & 0.1200 & 1122.20 & (90) \\ 40 & 0.1277 & 1131.00 & (125) \\ 40 & 0.1378 & 1143.00 & (125) \\ 40 & 0.1474 & 1155.00 & (125) \\ 40 & 0.1567 & 1167.00 & (125) \\ 40 & 0.1657 & 1179.00 & (125) \\ 40 & 0.1741 & 1190.00 & (125) \\ 40 & 0.1824 & 1200.00 & (125) \\ 40 & 0.2232 & 1251.00 & (125) \\ 45 & 0.0016 & 991.87 & (101) \\ 45 & 0.0048 & 995.03 & (101) \\ 45 & 0.0079 & 998.21 & (101) \\ 45 & 0.0111 & 1001.35 & (101) \\ 45 & 0.0156 & 1005.00 & (125) \\ 45 & 0.0234 & 1013.79 & (101) \\ 45 & 0.0304 & 1021.00 & (125) \\ 45 & 0.0310 & 1021.47 & (101) \\ 45 & 0.0384 & 1031.50 & (95) \\ 45 & 0.0445 & 1035.00 & (125) \\ 45 & 0.0457 & 1036.71 & (101) \\ 45 & 0.0581 & 1050.00 & (125) \\ 45 & 0.0710 & 1064.00 & (125) \\ 45 & 0.0739 & 1066.54 & (101) \\ 45 & 0.0739 & 1070.00 & (95) \\ 45 & 0.0833 & 1077.00 & (125) \\ 45 & 0.0951 & 1091.00 & (125) \\ 45 & 0.1005 & 1095.03 & (101) \\ 45 & 0.1064 & 1104.00 & (125) \\ 45 & 0.1072 & 1106.70 & (95) \\ 45 & 0.1173 & 1116.00 & (125) \\ 45 & 0.1277 & 1129.00 & (125) \\ 45 & 0.1376 & 1139.54 & (101) \\ 45 & 0.1378 & 1141.00 & (125) \\ 45 & 0.1382 & 1143.00 & (95) \\ 45 & 0.1474 & 1153.00 & (125) \\ 45 & 0.1567 & 1165.00 & (125) \\ 45 & 0.1657 & 1176.00 & (125) \\ 45 & 0.1680 & 1181.20 & (95) \\ 45 & 0.1741 & 1188.00 & (125) \\ 45 & 0.1752 & 1186.77 & (101) \\ & & & \end{array}$

App vol App vol Density Density App Vol Square of Inconsistent Inconsistent Delta density Apparent Density of exp / Calc/ calc/ Residual/ Residual / Density based on based on $\mathrm{m}^{3} / \mathrm{kg} \quad \mathrm{m}^{3} / \mathrm{kg} \quad \mathrm{kg} / \mathrm{m}^{3} \quad \mathrm{~kg} / \mathrm{m}^{3} \quad \mathrm{~m}^{3} / \mathrm{kg}$ Residual density apparent

$\begin{array}{llllll}0.000031 & 0.000024 & 1093.78 & -0.7783 & 0.000007 & 0.606\end{array}$

$\begin{array}{llllll}0.000034 & 0.000027 & 1106.89 & -0.8894 & 0.000007 & 0.791\end{array}$

$\begin{array}{llllll}0.000034 & 0.000030 & 1119.68 & -0.6764 & 0.000005 & 0.458\end{array}$

$\begin{array}{llllll}0.000035 & 0.000031 & 1122.88 & -0.6811 & 0.000005 & 0.464\end{array}$

$\begin{array}{llllll}0.000040 & 0.000033 & 1132.14 & -1.1382 & 0.000007 & 1.296\end{array}$

$\begin{array}{llllll}0.000043 & 0.000036 & 1144.27 & -1.2741 & 0.000007 & 1.623\end{array}$

$\begin{array}{llllll}0.000044 & 0.000039 & 1156.07 & -1.0691 & 0.000005 & 1.143\end{array}$

$\begin{array}{llllll}0.000044 & 0.000042 & 1167.59 & -0.5944 & 0.000003 & 0.353\end{array}$

$\begin{array}{llllll}0.000044 & 0.000045 & 1178.85 & 0.1521 & -0.000001 & 0.023\end{array}$

$\begin{array}{llllll}0.000046 & 0.000048 & 1189.55 & 0.4482 & -0.000002 & 0.201\end{array}$

$\begin{array}{llllll}0.000051 & 0.000051 & 1200.12 & -0.1243 & 0.000000 & 0.015\end{array}$

$\begin{array}{llllll}0.000074 & 0.000068 & 1252.93 & -1.9289 & 0.000006 & 3.721\end{array}$

$\begin{array}{llllll}-0.000039 & 0.000008 & 991.80 & 0.0740 & -0.000047 & 0.005 \\ -0.000018 & 0.000008 & 994.91 & 0.1243 & -0.000026 & 0.015\end{array}$

$\begin{array}{llllll}-0.000018 & 0.000008 & 994.91 & 0.1243 & -0.000026 & 0.015 \\ -0.000011 & 0.000009 & 998.06 & 0.1543 & -0.000020 & 0.024\end{array}$

$\begin{array}{llllll}-0.000003 & 0.000009 & 1001.21 & 0.1370 & -0.000012 & 0.019\end{array}$

$\begin{array}{llllll}0.000057 & 0.000010 & 1005.74 & -0.7352 & 0.000047 & 0.541\end{array}$

$\begin{array}{llllll}0.000006 & 0.000011 & 1013.67 & 0.1211 & -0.000005 & 0.015\end{array}$

$\begin{array}{llllll}0.000009 & 0.000013 & 1020.87 & 0.1299 & -0.000004 & 0.017\end{array}$

$\begin{array}{lllllll}0.000012 & 0.000013 & 1021.43 & 0.0386 & -0.000001 & 0.001\end{array}$

$\begin{array}{llllll}-0.000044 & 0.000014 & 1029.15 & 2.3485 & -0.000058 & 5.516\end{array}$

$\begin{array}{llllll}0.000029 & 0.000015 & 1035.65 & -0.6470 & 0.000014 & 0.419\end{array}$

$\begin{array}{lllllll}0.000019 & 0.000016 & 1036.88 & -0.1707 & 0.000003 & 0.029\end{array}$

$\begin{array}{llllll}0.000020 & 0.000018 & 1050.09 & -0.0946 & 0.000001 & 0.009\end{array}$

$\begin{array}{llllll}0.000023 & 0.000021 & 1064.17 & -0.1651 & 0.000002 & 0.027\end{array}$

$\begin{array}{llllll}0.000032 & 0.000021 & 1067.42 & -0.8846 & 0.000011 & 0.782\end{array}$

$\begin{array}{llllll}-0.000009 & 0.000021 & 1067.43 & 2.5724 & -0.000030 & 6.617\end{array}$

$\begin{array}{llllll}0.000033 & 0.000024 & 1077.90 & -0.9029 & 0.000009 & 0.815\end{array}$

$\begin{array}{llllll}0.000029 & 0.000026 & 1091.31 & -0.3064 & 0.000003 & 0.094\end{array}$

$\begin{array}{llllll}0.000048 & 0.000028 & 1097.51 & -2.4821 & 0.000021 & 6.161\end{array}$

$\begin{array}{llllll}0.000032 & 0.000029 & 1104.36 & -0.3595 & 0.000003 & 0.129\end{array}$

$\begin{array}{llllll}0.000018 & 0.000029 & 1105.20 & 1.4961 & -0.000011 & 2.238\end{array}$

$\begin{array}{llllll}0.000040 & 0.000032 & 1117.09 & -1.0910 & 0.000007 & 1.190\end{array}$

$\begin{array}{llllll}0.000038 & 0.000035 & 1129.50 & -0.4999 & 0.000003 & 0.250\end{array}$

$\begin{array}{llllll}0.000048 & 0.000038 & 1141.44 & -1.9014 & 0.000011 & 3.615\end{array}$

$\begin{array}{llllll}0.000041 & 0.000038 & 1141.59 & -0.5854 & 0.000003 & 0.343\end{array}$

$\begin{array}{lllllll}0.000033 & 0.000038 & 1142.16 & 0.8387 & -0.000005 & 0.703\end{array}$

$\begin{array}{llllll}0.000042 & 0.000041 & 1153.33 & -0.3328 & 0.000002 & 0.111\end{array}$

$\begin{array}{llllll}0.000043 & 0.000044 & 1164.81 & 0.1870 & -0.000001 & 0.035\end{array}$

$\begin{array}{llllll}0.000047 & 0.000047 & 1176.02 & -0.0240 & 0.000000 & 0.001\end{array}$

$\begin{array}{llllll}0.000038 & 0.000048 & 1178.95 & 2.2549 & -0.000010 & 5.084\end{array}$

$\begin{array}{llllll}0.000044 & 0.000050 & 1186.69 & 1.3109 & -0.000005 & 1.719\end{array}$

$\begin{array}{llllll}0.000055 & 0.000050 & 1188.02 & -1.2522 & 0.000005 & 1.568\end{array}$
992.2158

992.2158

992.2158

992.2158

992.2158

992.2158

992.2158

992.2158

992.2158

992.2158

992.2158

992.2158

990.2132

990.2132

990.2132

990.2132

990.2132

990.2132

990.2132

990.2132

990.2132

990.2132

990.2132

990.2132

990.2132

990.2132

990.2132

990.2132

990.2132

990.2132

990.2132

990.2132

990.2132

990.2132

990.2132

990.2132

990.2132

990.2132

990.2132

990.2132

990.2132

990.2132

990.2132 
molality molarity

\section{$\mathrm{t} /{ }^{\circ} \mathrm{C}$ mass frac Density}

1.39

1.95
0.0992

0.1965

0.2921

0.3862

0.4785

0.5693

0.6586

0.7463

0.8326

0.9175

1.001

1.083

1.164

1.244

1.321

1.398

2.12

0.01009

0.02993

0.05

0.05005

0.07024
0.0992

0.09946

0.15013

0.1965

0.20012

0.2921

0.30011
0.3862

0.4785

0.49998

-5
0.5

0.5693

0.6586

0.7002

0.7463

0.752

0.8326

0.9175

$0.0304 \quad 1016.00$ exp

$\mathrm{kg} / \mathrm{m}^{3}$

$\exp /$
$\mathrm{kg} / \mathrm{m}^{3}$

Ref

(125)

$\begin{array}{lll}0.2374 & 1268.00 & (125)\end{array}$

$0.0156 \quad 1003.00 \quad(125)$

$0.0304 \quad 1019.00 \quad(125)$

$0.0445 \quad 1033.00 \quad(125)$

$0.0581 \quad 1047.00 \quad(125)$

$0.0710 \quad 1061.00 \quad$ (125)

$0.0833 \quad 1075.00 \quad(125)$

$\begin{array}{lll}0.0951 \quad 1088.00 \quad(125) \\ 0.1064 & 1101.00 & (125)\end{array}$

$\begin{array}{lll}0.1064 & 1101.00 \quad(125)\end{array}$

$\begin{array}{lll}0.1173 & 1114.00 & (125) \\ 0.1277 & 1127.00 & (125)\end{array}$

$0.1378 \quad 1139.00 \quad(125)$

$\begin{array}{lll}0.1474 & 1150.00 \quad(125)\end{array}$

$\begin{array}{lll}0.1567 & 1162.00 & (125)\end{array}$

$0.1657 \quad 1173.00 \quad(125)$

$\begin{array}{lll}0.1741 & 1185.00 \quad(125)\end{array}$

$0.1824 \quad 1195.00 \quad$ (125)

$0.2528 \quad 1285.00 \quad(125)$

$0.0016 \quad 987.34$

$0.0048 \quad 990.48$

$0.0079 \quad 993.20$

$0.0079 \quad 993.64$

$0.0111 \quad 996.77$

$0.0156 \quad 1001.00$

$0.0156 \quad 1001.39$

$0.0234 \quad 1009.11$

$0.0310 \quad 1016.62$

$0.0384 \quad 1025.50$

$0.0445 \quad 1030.00$

$0.0457 \quad 1031.60$

$0.0581 \quad 1045.00$

$0.0710 \quad 1059.00$

$0.0739 \quad 1060.36$

$\begin{array}{ll}0.0739 & 1063.30\end{array}$

$0.0833 \quad 1073.00$

$0.0951 \quad 1086.00$

$0.1005 \quad 1089.79$

$0.1064 \quad 1098.00$

$0.1072 \quad 1098.90$

$0.1173 \quad 1111.00$

$0.1277 \quad 1124.00$ $\begin{array}{lll}(101) & -0.000037 & 0.000012 \\ \text { (101) } & -0.000016 & 0.000013\end{array}$

(95) $\quad 0.000046 \quad 0.000013$

$\begin{array}{lll}\text { (101) } & -0.000009 & 0.000013\end{array}$

$\begin{array}{lll}(101) & -0.000002 & 0.000014\end{array}$

$\begin{array}{lll}(125) & 0.000019 & 0.000014\end{array}$

$\begin{array}{lll}\text { (101) } & -0.000003 & 0.000014\end{array}$

(101)

(125)

(101)

(125)

(101)

(125)

$(125)$

(95)

(125)

(101)

(125)

$(95)$
$(125)$

(125)

$\begin{array}{ll}0.000009 & 0.000016 \\ 0.000019 & 0.000017\end{array}$

$0.000017 \quad 0.000017$

$\begin{array}{ll}-0.000012 & 0.000018\end{array}$

$0.000035 \quad 0.000020$

$0.000027 \quad 0.000020$

$0.000023 \quad 0.000022$

$0.000025 \quad 0.000025$

$0.000048 \quad 0.000026$

$0.000013 \quad 0.000026$

$0.000024 \quad 0.000028$

$0.000029 \quad 0.000030$

$0.000051 \quad 0.000032$

$0.000040 \quad 0.000033$

$\begin{array}{ll}0.000039 & 0.000033 \\ 0.000039 & 0.000036\end{array}$

$\begin{array}{ll}0.000037 & 0.000039\end{array}$
Den

Density App Vol Square of Inconsistent Inconsistent Delta density Apparent Density of calc / Residual / Residual / Density based on based on $/ \mathrm{kg} / \mathrm{m}^{3}$ molal volume water $/ \mathrm{kg} / \mathrm{m}^{3}$

0.601

0.146

0.237

0.200

0.069

0.417

0.428

0.110

0.459

0.457

0.126

0.081

0.061

0.209

0.011

0.005

1.693

0.637

0.092

0.006

0.018

0.067

0.030

0.031

0.006

0.075

0.029

0.006

0.000

1.482

0.519

0.118

0.002

0.000

3.462

1.159

0.152

0.010

5.134

0.718

0.617

0.228

0.044
990.2132

990.2132

988.0363

988.0363

988.0363

988.0363

988.0363

988.0363

988.0363

988.0363

988.0363

988.0363

988.0363

988.0363

988.0363

988.0363

988.0363

988.0363

988.0363

985.6952

985.6952

985.6952

985.6952

985.6952

985.6952

985.6952

985.6952

985.6952

985.6952

985.6952

985.6952

985.6952

985.6952

985.6952

985.6952

985.6952

985.6952

985.6952

985.6952

985.6952

985.6952

985.6952

985.6952 
$\mathrm{t} /{ }^{\circ} \mathrm{C}$ mass frac Density

$$
\exp /
$$

$\mathrm{kg} / \mathrm{m}^{3}$

1

1.001
1.005

1.083

1.164

1.244

1.265
1.321

33069

1.398
1.3069

2.3

0.0992

0.1965

0.2921

0.3862

0.4785

0.5693

0.6586

0.7463

0.8326

0.9175

1.001

1.083
1.164

1.244

1.321

1.398

2.49

$\begin{array}{lrrc}55 & 0.1376 & 1133.19 & (101) \\ 55 & 0.1378 & 1136.00 & (125) \\ 55 & 0.1382 & 1137.00 & (95) \\ 55 & 0.1474 & 1148.00 & (125) \\ 55 & 0.1567 & 1160.00 & (125) \\ 55 & 0.1657 & 1170.00 & (125) \\ 55 & 0.1680 & 1174.40 & (95) \\ 55 & 0.1741 & 1182.00 & (125) \\ 55 & 0.1752 & 1181.00 & (101) \\ 55 & 0.1824 & 1192.00 & (125) \\ 55 & 0.2685 & 1302.00 & (125) \\ 60 & 0.0156 & 998.80 & (125) \\ 60 & 0.0304 & 1014.00 & (125) \\ 60 & 0.0445 & 1028.00 & (125) \\ 60 & 0.0581 & 1042.00 & (125) \\ 60 & 0.0710 & 1056.00 & (125) \\ 60 & 0.0833 & 1070.00 & (125) \\ 60 & 0.0951 & 1083.00 & (125) \\ 60 & 0.1064 & 1095.00 & (125) \\ 60 & 0.1173 & 1109.00 & (125) \\ 60 & 0.1277 & 1121.00 & (125) \\ 60 & 0.1378 & 1133.00 & (125) \\ 60 & 0.1474 & 1144.00 & (125) \\ 60 & 0.1567 & 1157.00 & (125) \\ 60 & 0.1657 & 1168.00 & (125) \\ 60 & 0.1741 & 1180.00 & (125) \\ 60 & 0.1824 & 1190.00 & (125) \\ 60 & 0.2844 & 1319.00 & (125)\end{array}$

Ref

$\begin{array}{cc}\text { App vol App vol } \\ \exp / & \text { Calc }\end{array}$

$\begin{array}{cc}\exp / & \text { Calc } / \\ \mathrm{m}^{3} / \mathrm{kg} & \mathrm{m}^{3} / \mathrm{kg}\end{array}$

$0.000055 \quad 0.000041$

$\begin{array}{ll}0.000040 & 0.000041\end{array}$

$0.000038 \quad 0.000041$

$0.000041 \quad 0.000044$

$\begin{array}{ll}0.000042 & 0.000047\end{array}$

$0.000050 \quad 0.000050$

$0.000044 \quad 0.000051$

$0.000047 \quad 0.000053$

$0.000057 \quad 0.000053$

$0.000052 \quad 0.000056$

$0.000097 \quad 0.000096$

$\begin{array}{ll}0.000002 & 0.000016\end{array}$

$\begin{array}{ll}0.000001 & 0.000019\end{array}$

$0.000022 \quad 0.000021$

$\begin{array}{ll}0.000029 & 0.000024\end{array}$

$0.000029 \quad 0.000027$

$0.000027 \quad 0.000029$

$0.000032 \quad 0.000032$

$\begin{array}{ll}0.000041 & 0.000035\end{array}$

$0.000034 \quad 0.000037$

$0.000038 \quad 0.000040$

$0.000041 \quad 0.000043$

$0.000047 \quad 0.000045$

$0.000042 \quad 0.000048$

$0.000046 \quad 0.000051$

$0.000043 \quad 0.000054$

$0.000048 \quad 0.000057$

$0.000107 \quad 0.000106$

$06 \quad 1319.07$

Average Res

Std dev Res

Avg - 4std

Avg +4 std

\section{Density}

calc/ $\mathrm{kg} / \mathrm{m}^{3}$

$\mathrm{m}^{3} / \mathrm{kg}$

kg Resi

quare of Inconsistent In

consistent Delta density Apparent Density of 1135.79

1136.36

$\begin{array}{ll}-2.4530 & 0.000014\end{array}$

$0.2140-0.000001$

$0.6424-0.000004$

$0.5511-0.000003$

$1.1500-0.000005$

0.01250 .000000

$1.5098-0.000007$

$1.4135-0.000006$

$\begin{array}{lll}-0.9116 & 0.000004\end{array}$

$0.9390-0.000004$

$\begin{array}{ll}-0.2646 & 0.000001\end{array}$

$0.2877-0.000019$

$0.5544-0.000018$

$\begin{array}{lll}-0.0279 & 0.000001\end{array}$

$\begin{array}{ll}-0.2879 & 0.000005\end{array}$

$\begin{array}{lll}-0.1787 & 0.000002\end{array}$

$0.2556-0.000003$

$0.0165 \quad 0.000000$

$\begin{array}{ll}-0.8806 & 0.000007\end{array}$

$0.5358-0.000004$

$0.2665-0.000002$

$0.3120-0.000002$

$\begin{array}{ll}-0.3133 & 0.000002\end{array}$

$1.3204-0.000006$

$1.2147-0.000005$

$2.6439-0.000011$

$2.1949-0.000009$

$\begin{array}{ll}-0.0661 & 0.000000\end{array}$

$\begin{array}{ll}0.0324 & -0.000004\end{array}$

\begin{tabular}{ll}
0.000017 \\
\hline
\end{tabular}

$-3.8919-0.000072$

$3.9567 \quad 0.000064$
985.6952

985.6952

985.6952

85.6952

985.6952

985.6952

985.6952

85.6952

985.6952

985.6952

983.1989

983.1989

983.1989

983.1989

983.1989

983.1989

983.1989

983.1989

983.1989

983.1989

983.1989

983.1989

983.1989

983.1989

0.004

983.1989

Curve fit for the model App vol $=\left(w+c 2+c 3^{*} t{ }^{\circ} \mathrm{C}\right) /\left(c 0^{*} w+c 1\right) / e^{\wedge}\left(0.000001^{*}\left(t^{\circ} \mathrm{C}+c 4\right)^{\wedge} 2\right)$

$\begin{array}{lr}\text { c0 } & -2 \mathrm{E}-07 \\ \text { c1 } & 1.09 \mathrm{E}-07 \\ \text { c2 } & -0.12506 \\ \text { c3 } & 0.003831 \\ \text { c4 } & 4936.746 \\ \text { Min T } & 0 \\ \text { Max T } & 60 \\ \text { Min w } & 0.001608 \\ \text { Max w } & 0.2844 \\ \text { Avg dens res } & 0.032402\end{array}$

$$
-2 E-07
$$

-07

003831

001608

Avg dens res

0.032402 
molality molarity

$$
\exp /
$$

$\exp /$
$\mathrm{kg} / \mathrm{m}^{3}$

0.981072

Std dens res

$\begin{array}{ll}\text { Avg app vol res *10^3 } & -0.00382 \\ \text { Std app vol res *10^3 } & 0.016932\end{array}$

$\begin{array}{lr}\text { Std app vol res *10^3 } & 0.016932 \\ \text { No of points in corr } & 232\end{array}$

3

No of inconsistent poir

Inconsistent data not used (Residual greater than average $+/$ - 4 standard deviations)

0.01009

$$
1.65
$$

1.65
0.05

$\begin{array}{lll}25 & 0.0016 \quad 997.20\end{array}$

$\begin{array}{lll}35 & 0.2085 & 1249.00\end{array}$

$0.0079 \quad 998.70$

101) $0.000906-0.000005$

$\begin{array}{lll}(125) & 0.000021 & 0.000059\end{array}$

$\begin{array}{lll}\text { (95) } & -0.000074 & 0.000009\end{array}$
998.66

1236.62

998.05 $\begin{array}{ll}-1.4589 & 0.000911 \\ 12.3843 & -0.000038\end{array}$

$0.6521-0.000083$
2.128

153.370

0.425
997.0449

994.0319 990.2132 
Density of aqueous solutions of $\mathrm{FeCl} 2$

References (48) Kaminsky, M. Concentration and Temperature Dependence of the Viscosity of Aqueous Solutions of Strong Electrolytes. II. NaCl, $\mathrm{Li}_{2} \mathrm{SO}_{4}, \mathrm{FeCl}_{2}$, and $\mathrm{CeCl}_{3}$ Solutions. Z. Physik. Chem. N. F., 1956, 8, 173-191

(99) Pogue, R. F.; Atkinson, G. Solution Thermodynamics of First-Row Transition Elements. 2. Apparent Molal Volumes of Aqueous MnCl2, $\mathrm{Mn}\left(\mathrm{ClO}_{4}\right)_{2}, \mathrm{CoCl}_{2}, \mathrm{Co}\left(\mathrm{ClO}_{4}\right)_{2}, \mathrm{FeCl}_{2}$, and $\mathrm{Fe}\left(\mathrm{ClO}_{4}\right)_{2}$, from 15 to $55^{\circ} \mathrm{C}$. J. Chem. Eng. Data 1989, 34, $227-232$

\begin{tabular}{|c|c|c|c|c|c|c|c|c|c|c|c|c|c|c|c|}
\hline molality & molarity & \multicolumn{2}{|c|}{$\mathrm{t} /{ }^{\circ} \mathrm{C}$ mass frac } & $\begin{array}{r}\text { Density } \\
\exp / \\
\mathrm{kg} / \mathrm{m}^{3}\end{array}$ & Ref & $\begin{array}{r}\text { App vol } \\
\exp / \\
\mathrm{m}^{3} / \mathrm{kg}\end{array}$ & $\begin{array}{r}\text { App vol } \\
\text { Calc / } \\
\mathrm{m}^{3} / \mathrm{kg}\end{array}$ & $\begin{array}{r}\text { Density } \\
\text { calc / } \\
\mathrm{kg} / \mathrm{m}^{3}\end{array}$ & $\begin{array}{r}\text { Density } \\
\text { Residual / } \\
\mathrm{kg} / \mathrm{m}^{3}\end{array}$ & $\begin{array}{r}\text { App Vol } \\
\text { Residual / } \\
\mathrm{m}^{3} / \mathrm{kg}\end{array}$ & $\begin{array}{r}\text { Square of } \\
\text { Density } \\
\text { Residual }\end{array}$ & $\begin{array}{l}\text { Inconsistent } \\
\text { based on } \\
\text { density }\end{array}$ & $\begin{array}{c}\text { Inconsistent } \\
\text { based on } \\
\text { apparent } \\
\text { volume }\end{array}$ & $\begin{array}{r}\text { Relative } \\
\text { density / } \\
\text { 1000(rhor-1) }\end{array}$ & $\begin{array}{r}\text { Density of } \\
\text { water } / \mathrm{kg} / \mathrm{m}^{3}\end{array}$ \\
\hline 0.0251 & & 15 & 0.0032 & 1001.93 & (99) & 0.000110 & 0.000124 & 1001.88 & 0.0445 & -0.000014 & 0.002 & & & 2.83 & 999.0996 \\
\hline 0.05136 & & 15 & 0.0065 & 1004.86 & (99) & 0.000113 & 0.000125 & 1004.79 & 0.0781 & -0.000012 & 0.006 & & & 5.77 & 999.0996 \\
\hline 0.07416 & & 15 & 0.0093 & 1007.42 & (99) & 0.000113 & 0.000126 & 1007.30 & 0.1210 & -0.000013 & 0.015 & & & 8.33 & 999.0996 \\
\hline 0.1009 & & 15 & 0.0126 & 1010.36 & (99) & 0.000118 & 0.000127 & 1010.24 & 0.1166 & -0.000009 & 0.014 & & & 11.27 & 999.0996 \\
\hline 0.2501 & & 15 & 0.0307 & 1026.67 & (99) & 0.000126 & 0.000131 & 1026.51 & 0.1649 & -0.000005 & 0.027 & & & 27.6 & 999.0996 \\
\hline 0.5322 & & 15 & 0.0632 & 1056.77 & (99) & 0.000137 & 0.000139 & 1056.60 & 0.1632 & -0.000002 & 0.027 & & & 57.72 & 999.0996 \\
\hline 0.8246 & & 15 & 0.0946 & 1087.02 & (99) & 0.000145 & 0.000146 & 1086.93 & 0.0951 & -0.000001 & 0.009 & & & 88 & 999.0996 \\
\hline 1.045 & & 15 & 0.1170 & 1109.10 & (99) & 0.000152 & 0.000151 & 1109.22 & -0.1207 & 0.000001 & 0.015 & & & 110.1 & 999.0996 \\
\hline 1.5322 & & 15 & 0.1626 & 1156.73 & (99) & 0.000162 & 0.000161 & 1156.90 & -0.1726 & 0.000001 & 0.030 & & & 157.77 & 999.0996 \\
\hline 2.0932 & & 15 & 0.2097 & 1209.26 & (99) & 0.000171 & 0.000171 & 1209.25 & 0.0130 & 0.000000 & 0.000 & & & 210.35 & 999.0996 \\
\hline & 0.00099 & 15.5 & 0.0001 & 999.14 & (48) & 0.000045 & 0.000124 & 999.13 & 0.0099 & -0.000079 & 0.000 & & $!$ & 0.12 & 999.0228 \\
\hline & 0.00198 & 15.5 & 0.0003 & 999.25 & (48) & 0.000085 & 0.000124 & 999.24 & 0.0099 & -0.000039 & 0.000 & & & 0.23 & 999.0228 \\
\hline & 0.00496 & 15.5 & 0.0006 & 999.59 & (48) & 0.000094 & 0.000124 & 999.57 & 0.0186 & -0.000030 & 0.000 & & & 0.57 & 999.0228 \\
\hline & 0.00991 & 15.5 & 0.0013 & 1000.15 & (48) & 0.000101 & 0.000124 & 1000.12 & 0.0286 & -0.000023 & 0.001 & & & 1.13 & 999.0228 \\
\hline & 0.01984 & 15.5 & 0.0025 & 1001.28 & (48) & 0.000102 & 0.000124 & 1001.22 & 0.0559 & -0.000022 & 0.003 & & & 2.26 & 999.0228 \\
\hline & 0.04959 & 15.5 & 0.0063 & 1004.73 & (48) & 0.000093 & 0.000125 & 1004.52 & 0.2075 & -0.000033 & 0.043 & & & 5.71 & 999.0228 \\
\hline & 0.09919 & 15.5 & 0.0124 & 1010.15 & (48) & 0.000115 & 0.000127 & 1010.00 & 0.1529 & -0.000012 & 0.023 & & & 11.14 & 999.0228 \\
\hline & 0.19838 & 15.5 & 0.0246 & 1021.06 & (48) & 0.000124 & 0.000130 & 1020.90 & 0.1617 & -0.000006 & 0.026 & & & 22.06 & 999.0228 \\
\hline & 0.2975 & 15.5 & 0.0365 & 1031.81 & (48) & 0.000131 & 0.000133 & 1031.72 & 0.0877 & -0.000002 & 0.008 & & & 32.82 & 999.0228 \\
\hline & 0.00099 & 18 & 0.0001 & 998.72 & (48) & 0.000045 & 0.000126 & 998.71 & 0.0101 & -0.000081 & 0.000 & & $!$ & 0.12 & 998.5956 \\
\hline & 0.00198 & 18 & 0.0003 & 998.83 & (48) & 0.000085 & 0.000126 & 998.82 & 0.0103 & -0.000041 & 0.000 & & & 0.23 & 998.5956 \\
\hline & 0.00496 & 18 & 0.0006 & 999.16 & (48) & 0.000095 & 0.000126 & 999.15 & 0.0196 & -0.000031 & 0.000 & & & 0.57 & 998.5956 \\
\hline & 0.00991 & 18 & 0.0013 & 999.71 & (48) & 0.000110 & 0.000126 & 999.69 & 0.0205 & -0.000016 & 0.000 & & & 1.12 & 998.5956 \\
\hline & 0.01984 & 18 & 0.0025 & 1000.83 & (48) & 0.000111 & 0.000126 & 1000.79 & 0.0397 & -0.000016 & 0.002 & & & 2.24 & 998.5956 \\
\hline & 0.04959 & 18 & 0.0063 & 1004.15 & (48) & 0.000117 & 0.000127 & 1004.08 & 0.0663 & -0.000011 & 0.004 & & & 5.56 & 998.5956 \\
\hline & 0.09919 & 18 & 0.0125 & 1009.63 & (48) & 0.000122 & 0.000129 & 1009.55 & 0.0808 & -0.000006 & 0.007 & & & 11.05 & 998.5956 \\
\hline & 0.19838 & 18 & 0.0246 & 1020.41 & (48) & 0.000132 & 0.000132 & 1020.43 & -0.0157 & 0.000001 & 0.000 & & & 21.85 & 998.5956 \\
\hline & 0.2975 & 18 & 0.0366 & 1031.07 & (48) & 0.000139 & 0.000135 & 1031.24 & -0.1671 & 0.000004 & 0.028 & & & 32.52 & 998.5956 \\
\hline & 0.00099 & 25 & 0.0001 & 997.15 & (48) & 0.000126 & 0.000131 & 997.15 & 0.0006 & -0.000005 & 0.000 & & & 0.11 & 997.0449 \\
\hline & 0.00198 & 25 & 0.0003 & 997.27 & (48) & 0.000087 & 0.000131 & 997.26 & 0.0112 & -0.000045 & 0.000 & & & 0.23 & 997.0449 \\
\hline & 0.00495 & 25 & 0.0006 & 997.60 & (48) & 0.000110 & 0.000131 & 997.59 & 0.0130 & -0.000021 & 0.000 & & & 0.56 & 997.0449 \\
\hline & 0.0099 & 25 & 0.0013 & 998.15 & (48) & 0.000118 & 0.000131 & 998.14 & 0.0162 & -0.000013 & 0.000 & & & 1.11 & 997.0449 \\
\hline & 0.0198 & 25 & 0.0025 & 999.26 & (48) & 0.000118 & 0.000132 & 999.23 & 0.0332 & -0.000013 & 0.001 & & & 2.22 & 997.0449 \\
\hline 0.0261 & & 25 & 0.0033 & 999.96 & (99) & 0.000117 & 0.000132 & 999.91 & 0.0477 & -0.000014 & 0.002 & & & 2.92 & 997.0449 \\
\hline & 0.04951 & 25 & 0.0063 & 1002.55 & (48) & 0.000123 & 0.000133 & 1002.49 & 0.0578 & -0.000009 & 0.003 & & & 5.52 & 997.0449 \\
\hline
\end{tabular}


$\mathrm{t} /{ }^{\circ} \mathrm{C}$ mass frac Density

$\exp /$
$\mathrm{kg} / \mathrm{m}^{3}$

0.0519

0.07846

0.09903

0.2501

0.5435

0.8401

1.0861

1.4891

2.0932

0.00099

0.00198

0.00494

0.00987

0.01974

0.04944

0.09888

0.19774

0.29649

0.00098

0.00197

0.00494

0.00987

0.01975

0.02526

0.05153

0.04936

0.07635

0.1015

0.09871

0.19739

0.2501

0.29596

0.5291

1.073

1.6069

2.0932

$0.0003 \quad 994.24$

$0.0006 \quad 994.58$

$0.0025 \quad 996.21$

$0.0032 \quad 996.83$

$0.0063 \quad 999.44$

$0.0065 \quad 999.71$

$0.0096 \quad 1002.41$

$0.0125 \quad 1004.77$

$0.0127 \quad 1005.15$

$0.0246 \quad 1015.26$

$0.0307 \quad 1021.05$

$0.0366 \quad 1025.59$

$0.0628 \quad 1050.22$

$0.0907 \quad 1076.53$

$0.1197 \quad 1104.90$

$0.1692 \quad 1156.09$

$0.2097 \quad 1200.73$
Ref

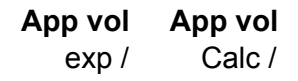

$\mathrm{m}^{3} / \mathrm{kg} \quad \mathrm{m}^{3} / \mathrm{kg}$

$0.000121 \quad 0.000133$

$0.000122 \quad 0.000133$

$0.000124 \quad 0.000134$

$0.000130 \quad 0.000134$

0.0001390 .000137

$0.000134 \quad 0.000138$

$0.000146 \quad 0.000140$

$0.000145 \quad 0.000146$

0.000158

$0.000166 \quad 0.000166$

$0.000176 \quad 0.000176$

$0.000128 \quad 0.000135$

$0.000128 \quad 0.000135$

$0.000110 \quad 0.000135$

$0.000125 \quad 0.000135$

$0.000121 \quad 0.000135$

$0.000127 \quad 0.000136$

$\begin{array}{lll}0.000135 & 0.000137\end{array}$

$0.000147 \quad 0.000140$

$0.000154 \quad 0.000143$

$0.000120 \quad 0.000138$

$0.000165 \quad 0.000138$

$0.000128 \quad 0.000138$

$0.0013 \quad 995.13$

$0.0001 \quad 992.32$
$0.000127 \quad 0.000138$

$0.000123 \quad 0.000139$

$\begin{array}{ll}0.000136 & 0.000139\end{array}$

$0.000126 \quad 0.000139$

$0.000129 \quad 0.000140$

$0.000143 \quad 0.000141$

$0.000130 \quad 0.000141$

$0.000152 \quad 0.000143$

$0.000140 \quad 0.000145$

$0.000160 \quad 0.000146$

$\begin{array}{ll}0.000156 & 0.000157\end{array}$

$0.000163 \quad 0.000163$

$0.000173 \quad 0.000173$

$0.000180 \quad 0.000181$

$0.000122 \quad 0.000141$
$0.000131 \quad 0.000139$

$0.000150 \quad 0.000152$
Density Density App Vol Square of Inconsistent Inconsistent

calc / Residual / Residual / Density based on based on

$\mathrm{kg} / \mathrm{m}^{3} \quad \mathrm{~kg} / \mathrm{m}^{3} \quad \mathrm{~m}^{3} / \mathrm{kg}$ Residual density apparent

Relative Density of

$\mathrm{kg} / \mathrm{m}^{3} \quad \mathrm{~m}^{3} / \mathrm{kg}$ Residual volume

$1002.73 \quad 0.0760-0.000012 \quad 0.006$

$\begin{array}{llll}1005.63 & 0.1086 & -0.000011 & 0.012\end{array}$

$\begin{array}{lll}0.1224 & -0.000010 & 0.015\end{array}$

$1007.92 \quad 0.0529-0.000004 \quad 0.003$

$\begin{array}{llll}8.72 & -0.0625 & 0.000002 & 0.004\end{array}$

$\begin{array}{llll}1024.18 & 0.1276 & -0.000004 & 0.016\end{array}$

$\begin{array}{llll}029.45 & -0.2648 & 0.000007 & 0.070\end{array}$

$\begin{array}{llll}0.0470 & -0.000001 & 0.002\end{array}$

$\begin{array}{llll}0.039 & 0.0332 & 0.000000 & 0.001\end{array}$

$\begin{array}{llll}110.19 & 0.0385 & 0.000000 & 0.001\end{array}$

$\begin{array}{llll}0.31 & -0.0335 & 0.000000 & 0.001\end{array}$

$\begin{array}{llll}996.73 & 0.0122 & -0.000010 & 0.000\end{array}$

$\begin{array}{llll}997.81 & 0.0351 & -0.000014 & 0.001\end{array}$

$0.0585-0.000009$

$006.46 \quad 0.0249-0.000002 \quad 0.001$

$\begin{array}{llll}1017.22 & -0.1718 & 0.000007 & 0.030\end{array}$

$\begin{array}{llll}027.89 & -0.4261 & 0.000011 & 0.182\end{array}$

$\begin{array}{llll}994.14 & 0.0022 & -0.000018 & 0.000\end{array}$

$\begin{array}{llll}994.25 & -0.0067 & 0.000027 & 0.000\end{array}$

$994.57-0.0065-0.000010$

$995.11 \quad 0.0143-0.000012 \quad 0.000$

$\begin{array}{lllll}996.19 & 0.0184 & -0.000007 & 0.000\end{array}$

$996.77 \quad 0.0506-0.000016 \quad 0.003$

$999.42-0.0180-0.000003-0.000$

$\begin{array}{llll}999.62 & 0.0883 & -0.000014 & 0.008\end{array}$

$\begin{array}{llll}1002.30 & 0.1110 & -0.000012 & 0.012\end{array}$

$\begin{array}{llll}1004.79 & -0.0258 & 0.000002 & 0.001\end{array}$

$\begin{array}{llll}005.01 & 0.1346 & -0.000010 & 0.018\end{array}$

$\begin{array}{llll}015.49 & -0.2242 & 0.000009 & 0.050\end{array}$

$\begin{array}{llll}1020.89 & 0.1638 & -0.000005 & 0.027\end{array}$

$\begin{array}{llll}026.12 & -0.5232 & 0.000014 & 0.274\end{array}$

$\begin{array}{llll}0.1460 & -0.000002 & 0.021\end{array}$

$0.1349-0.000001-0.018$

$\begin{array}{lllll}1104.82 & 0.0739 & -0.000001 & 0.005\end{array}$

$\begin{array}{lll}0.0998 & 0.000000 & 0.010\end{array}$

$\begin{array}{llll}992.32 & 0.0023 & -0.000019 & 0.000\end{array}$ $\begin{array}{ll}5.78 & 997.0449\end{array}$

8.72997 .0449

$10.98 \quad 997.0449$

10.96997 .0449

$21.68 \quad 997.0449$

$27.34 \quad 997.0449$

$32.24 \quad 997.0449$

58.33997 .0449

88.84997 .0449

113.52997 .0449

$152.68 \quad 997.0449$

$208.93 \quad 997.0449$

0.11995 .6473

0.22995 .6473

$0.56 \quad 995.6473$

1.1995 .6473

2.21995 .6473

$5.5 \quad 995.6473$

$10.89 \quad 995.6473$

$21.49 \quad 995.6473$

31.96995 .6473

0.11994 .0319

0.21994 .0319

$0.55 \quad 994.0319$

$\begin{array}{ll}1.1 & 994.0319\end{array}$

2.19994 .0319

2.81994 .0319

$5.44 \quad 994.0319$

$\begin{array}{ll}5.71 & 994.0319\end{array}$

$8.43 \quad 994.0319$

$10.8 \quad 994.0319$

11.18994 .0319

$21.36 \quad 994.0319$

$27.18 \quad 994.0319$

$31.75 \quad 994.0319$

$56.53 \quad 994.0319$

$82.99 \quad 994.0319$

111.53994 .0319

$163.03 \quad 994.0319$

$207.94 \quad 994.0319$

0.11992 .2158 
molality

$\begin{array}{rrrrrr} & & & & \mathrm{kg} / \mathrm{m}^{3} & \\ & 0.00197 & 40 & 0.0003 & 992.42 & (48) \\ & 0.00492 & 40 & 0.0006 & 992.76 & (48) \\ & 0.00985 & 40 & 0.0013 & 993.29 & (48) \\ & 0.0197 & 40 & 0.0025 & 994.37 & (48) \\ & 0.04926 & 40 & 0.0063 & 997.57 & (48) \\ & 0.09853 & 40 & 0.0125 & 1002.85 & (48) \\ & 0.19701 & 40 & 0.0246 & 1013.24 & (48) \\ & 0.29539 & 40 & 0.0366 & 1023.47 & (48) \\ 0.02555 & & 45 & 0.0032 & 993.02 & (99) \\ 0.05239 & & 45 & 0.0066 & 995.93 & (99) \\ 0.07629 & & 45 & 0.0096 & 998.51 & (99) \\ 0.09856 & & 45 & 0.0123 & 1000.91 & (99) \\ 0.2501 & & 45 & 0.0307 & 1016.97 & (99) \\ 0.4931 & & 45 & 0.0588 & 1042.13 & (99) \\ 0.8438 & & 45 & 0.0966 & 1077.48 & (99) \\ 1.056 & & 45 & 0.1180 & 1098.39 & (99) \\ 2.0932 & & 45 & 0.2097 & 1194.81 & (99)\end{array}$

\section{App vol App vol}

$\begin{array}{cc}\exp / & \text { Calc } / \\ \mathrm{m}^{3} / \mathrm{kg} & \mathrm{m}^{3} / \mathrm{kg}\end{array}$

$0.000167 \quad 0.000141$

$0.000126 \quad 0.000141$

$0.000143 \quad 0.000141$

$\begin{array}{ll}0.000139 & 0.000142\end{array}$

$0.000143 \quad 0.000143$

$0.000149 \quad 0.000144$

$\begin{array}{ll}0.000159 & 0.000147\end{array}$

$0.000167 \quad 0.000149$

$0.000127 \quad 0.000145$

$0.000132 \quad 0.000146$

$0.000134 \quad 0.000146$

$0.000135 \quad 0.000147$

$0.000145 \quad 0.000151$

$0.000155 \quad 0.000156$

$0.000163 \quad 0.000164$

$0.000167 \quad 0.000168$

$0.000185 \quad 0.000185$
Density Density App Vol Square of Inconsistent Inconsistent calc / Residual / Residual / Density based on based on $\mathrm{kg} / \mathrm{m}^{3} \quad \mathrm{~kg} / \mathrm{m}^{3} \quad \mathrm{~m}^{3} / \mathrm{kg}$ Residual density apparent

$\begin{array}{llll}992.43 & -0.0063 & 0.000026 & 0.000\end{array}$

$\begin{array}{llll}992.75 & 0.0096 & -0.000015 & 0.000\end{array}$

$\begin{array}{llll}993.29 & -0.0017 & 0.000001 & 0.000\end{array}$

$\begin{array}{llll}994.36 & 0.0073 & -0.000003 & 0.000\end{array}$

$\begin{array}{llll}997.58 & -0.0026 & 0.000000 & 0.000\end{array}$

$\begin{array}{llll}1002.92 & -0.0698 & 0.000006 & 0.005\end{array}$

$\begin{array}{llll}1013.56 & -0.3228 & 0.000013 & 0.104\end{array}$

$\begin{array}{lll}1024.14 & -0.6703 & 0.000017\end{array}$

$992.96 \quad 0.0571-0.000018$

$995.83 \quad 0.0919-0.000014$

$998.39 \quad 0.1213-0.000013$

000.77

1016.79

1042.02

1077.43

1098.31

Average Res

Std dev Res

Avg - 4std

Avg + 4std
$0.1422-0.000012$

$0.1749-0.000006$

$0.1128-0.000002$

$0.0534 \quad 0.000000$

$0.0834-0.000001$

$-0.1895 \quad 0.000001$

$0.0141-0.000009$

$0.1398 \quad 0.000016$

$-0.5452-0.000074$

$0.5734 \quad 0.000056$
Relative Density of density / water $/ \mathrm{kg} / \mathrm{m}^{3}$ 1000(rhor-1)

$0.21 \quad 992.2158$

$0.55 \quad 992.2158$

$1.08 \quad 992.2158$

$2.17 \quad 992.2158$

$\begin{array}{ll}5.4 & 992.2158\end{array}$

$10.72 \quad 992.2158$

$21.19 \quad 992.2158$

$31.5 \quad 992.2158$

2.83990 .2132

$5.77 \quad 990.2132$

$8.38 \quad 990.2132$

$10.8 \quad 990.2132$

$27.02 \quad 990.2132$

$52.43 \quad 990.2132$

$88.13 \quad 990.2132$

$109.25 \quad 990.2132$

206.62990 .2132

Curve fit for the model App vol= $\left(w+c 2+c 3^{\star} t{ }^{\circ} \mathrm{C}\right) /\left(\mathrm{c} 0^{*} \mathrm{w}+\mathrm{c} 1\right) / \mathrm{e}^{\wedge}\left(0.000001^{*}\left(\mathrm{t}^{\circ} \mathrm{C}+\mathrm{c} 4\right)^{\wedge} 2\right)$

\begin{tabular}{lr} 
c0 & 98.65403 \\
c1 2 & 199.5046 \\
c3 & 0.336389 \\
c4 & 0.003844 \\
Min T & 1650.129 \\
Max T & 15 \\
Min w & 45 \\
Max w & 0.000125 \\
Avg dens res & 0.209682 \\
Std dens res & 0.0141 \\
Avg app vol res *10^3 & 0.139823 \\
Std app vol res *10^3 & 0.008644 \\
No of points in corr & 0.01622 \\
No of inconsistent poir & 93 \\
\hline
\end{tabular}

Inconsistent data not used (Residual greater than average $+/-4$ standard deviations) 
Density of aqueous solutions of FeSO4

References (7) Bakeev, M. I.; Zharmenov, A. A.; Andamasov, R. S.; Baikenova, N. A.; Abdygalimova, S. Sh. Electrical Conductivity and Viscosity of the Binary Systems MeSO4-H2O (Me=Mn2+, Fe2+, Co2+, Zn2+, Cd2+) at 25-75 Degree and the Structure of Electrolyte Solutions. Izv.

Nats. Akad. Nauk Resp. Kaz., Ser. Khim. 1994, 6, 25-30.

(21) Degremont, "Memento Technique de l'eau.", Paris, 1989

(90) Perry, R. H.; Green, D. W. Perry's Chemical Engineers' Handbook; McGraw Hill: New York, 7th edition, 1997.

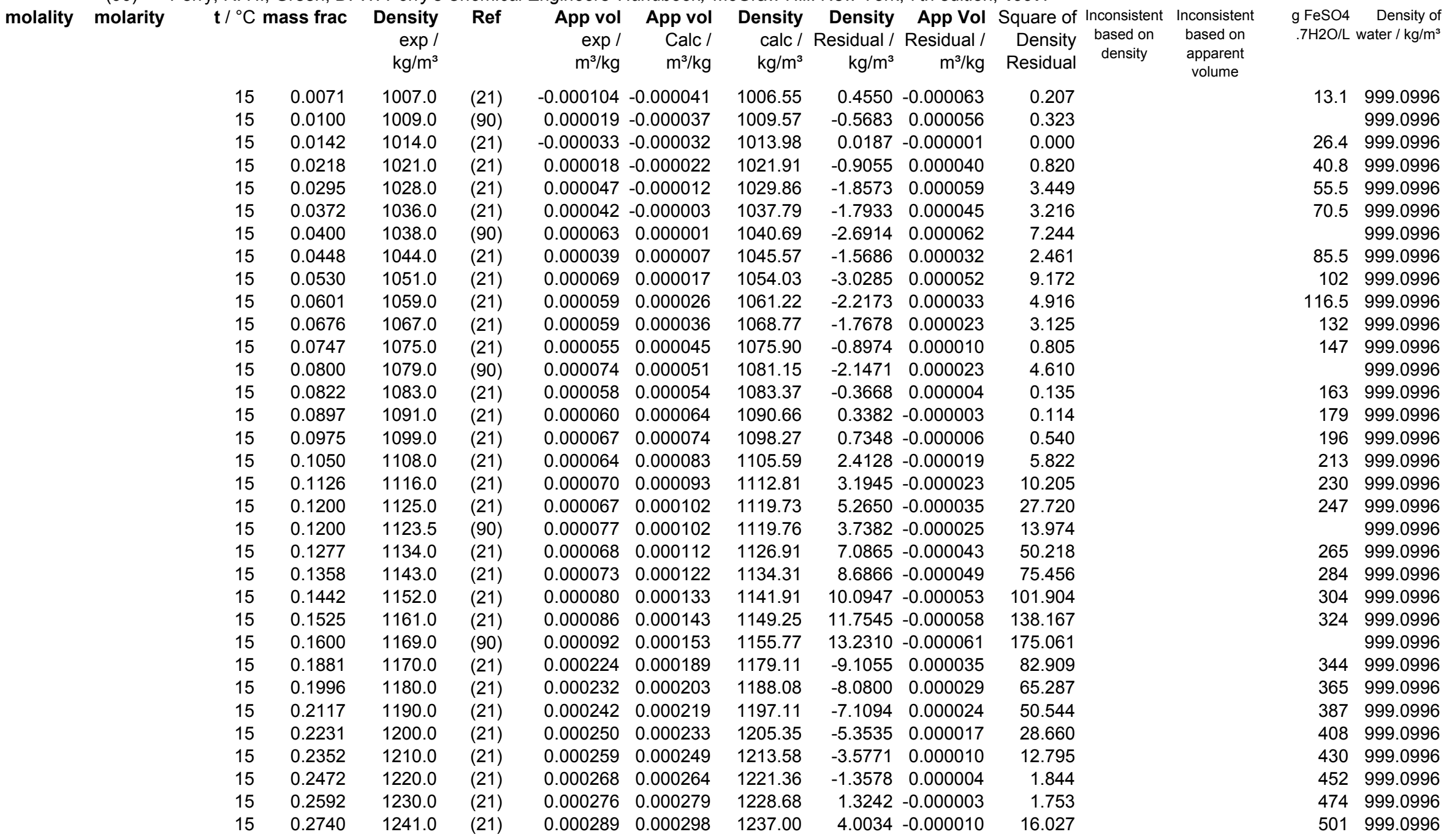


molality molarity

$\begin{array}{crrr}\text { t }{ }^{\circ} \mathrm{C} \text { mass frac } & \begin{array}{r}\text { Density } \\ \text { exp } /\end{array} & \text { Ref } \\ & & \mathrm{kg} / \mathrm{m}^{3} & \\ & & \\ 18 & 0.0020 & 1000.7 & (90) \\ 18 & 0.0040 & 1002.8 & (90) \\ 18 & 0.0080 & 1006.5 & (90) \\ 18 & 0.0100 & 1008.5 & (90) \\ 18 & 0.0400 & 1037.5 & (90) \\ 18 & 0.0800 & 1078.5 & (90) \\ 18 & 0.1200 & 1122.0 & (90) \\ 18 & 0.1600 & 1167.5 & (90) \\ 20 & 0.0020 & 1000.2 & (90) \\ 20 & 0.0040 & 1002.2 & (90) \\ 20 & 0.0080 & 1006.2 & (90) \\ 20 & 0.0100 & 1008.2 & (90) \\ 25 & 0.0054 & 1002.0 & (7) \\ 25 & 0.0100 & 1006.1 & (7) \\ 25 & 0.0247 & 1020.2 & (7) \\ 25 & 0.0458 & 1038.4 & (7) \\ 25 & 0.0935 & 1080.2 & (7) \\ 25 & 0.1178 & 1106.9 & (7) \\ 25 & 0.1400 & 1123.5 & (7) \\ 25 & 0.1695 & 1153.1 & (7) \\ 25 & 0.1895 & 1172.7 & (7) \\ 25 & 0.2109 & 1196.1 & (7) \\ 50 & 0.0054 & 993.4 & (7) \\ 50 & 0.0247 & 1012.1 & (7) \\ 50 & 0.0458 & 1030.8 & (7) \\ 50 & 0.0935 & 1073.5 & (7) \\ 50 & 0.1178 & 1098.9 & (7) \\ 50 & 0.1400 & 1115.2 & (7) \\ 50 & 0.1695 & 1145.7 & (7) \\ 50 & 0.1895 & 1161.7 & (7) \\ 50 & 0.2109 & 1191.2 & (7) \\ 75 & 0.0247 & 997.7 & (7) \\ 75 & 0.0458 & 1017.6 & (7) \\ 75 & 0.0935 & 1060.6 & (7) \\ 75 & 0.1178 & 1090.8 & (7) \\ 75 & 0.1400 & 1106.0 & (7) \\ 75 & 0.1695 & 1133.6 & (7) \\ 75 & 0.1895 & 1150.8 & (7) \\ 75 & 0.2109 & 1181.4 & (7)\end{array}$

\section{App vol App vol D}

$\begin{array}{cc}\exp / & \text { Calc / } \\ \mathrm{m}^{3} / \mathrm{kg} & \mathrm{m}^{3} / \mathrm{kg}\end{array}$

$-0.000042-0.000040$

$-0.000036-0.000038$

$0.000025-0.000033$

$0.000018-0.000030$

$\begin{array}{ll}0.000063 & 0.000007\end{array}$

$0.000074 \quad 0.000056$

0.0000840 .000106

$0.000096 \quad 0.000155$

$0.000002-0.000036$

$0.000003-0.000033$

$0.000007-0.000028$

$0.000009-0.000026$

$0.000078-0.000021$

$0.000100-0.000016$

$\begin{array}{lll}0.000080 & 0.000001\end{array}$

0.0001310 .000026

$0.000177 \quad 0.000082$

$0.000158 \quad 0.000110$

$\begin{array}{lll}0.000197 & 0.000136\end{array}$

$0.000202 \quad 0.000171$

$0.000210 \quad 0.000194$

0.0002120 .000220

$\begin{array}{ll}-0.000007 & 0.000020\end{array}$

$0.000037 \quad 0.000038$

$0.000096 \quad 0.000059$

$0.000150 \quad 0.000104$

$0.000146 \quad 0.000128$

$\begin{array}{ll}0.000188 & 0.000149\end{array}$

$0.000190 \quad 0.000178$

$0.000214 \quad 0.000197$

$0.000194 \quad 0.000218$

$0.000073 \quad 0.000062$

$0.000085 \quad 0.000079$

$0.000138 \quad 0.000116$

$0.000100 \quad 0.000135$

$0.000157 \quad 0.000153$

$0.000178 \quad 0.000176$

$0.000198 \quad 0.000192$

$0.000175 \quad 0.000209$
Density Density App Vol Square of Inconsistent Inconsistent calc / Residual / Residual / Density based on based on $\mathrm{kg} / \mathrm{m}^{3} \quad \mathrm{~kg} / \mathrm{m}^{3} \quad \mathrm{~m}^{3} / \mathrm{kg}$ Residual density apparent

$\begin{array}{llll}1000.68 & 0.0028 & -0.000001 & 0.000\end{array}$

$\begin{array}{llll}1002.76 & -0.0076 & 0.000002 & 0.000\end{array}$

$\begin{array}{llll}1006.91 & -0.4646 & 0.000057 & 0.216\end{array}$

$\begin{array}{llll}1008.99 & -0.4909 & 0.000048 & 0.241\end{array}$

$\begin{array}{llll}1039.92 & -2.4161 & 0.000056 & 5.837\end{array}$

$\begin{array}{llll}1080.17 & -1.6741 & 0.000018 & 2.803\end{array}$

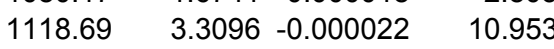

$\begin{array}{llll}1154.73 & 12.7750 & -0.000059 & 163.200\end{array}$

$\begin{array}{llll}1000.28 & -0.0760 & 0.000038 & 0.006\end{array}$

$\begin{array}{llll}1002.35 & -0.1468 & 0.000037 & 0.022\end{array}$

$\begin{array}{llll}1006.48 & -0.2847 & 0.000035 & 0.081\end{array}$

$\begin{array}{llll}1008.55 & -0.3517 & 0.000035 & 0.124\end{array}$

$\begin{array}{llll}1002.53 & -0.5325 & 0.000099 & 0.284\end{array}$

$\begin{array}{llll}1007.28 & -1.1771 & 0.000116 & 1.386\end{array}$

$\begin{array}{llll}1022.23 & -2.0326 & 0.000079 & 4.132\end{array}$

$\begin{array}{llll}1043.62 & -5.2191 & 0.000105 & 27.239\end{array}$

$\begin{array}{llll}1090.69 & -10.4880 & 0.000095 & 109.997\end{array}$

$\begin{array}{llll}1113.89 & -6.9893 & 0.000048 & 48.850\end{array}$

$\begin{array}{llll}1134.29 & -10.7881 & 0.000060 & 116.383\end{array}$

$\begin{array}{llll}1160.18 & -7.0825 & 0.000031 & 50.162\end{array}$

$\begin{array}{llll}1176.82 & -4.1187 & 0.000016 & 16.964\end{array}$

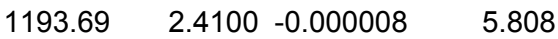

$\begin{array}{llll}993.25 & 0.1452 & -0.000027 & 0.021\end{array}$

$\begin{array}{llll}1012.05 & 0.0460 & -0.000002 & 0.002\end{array}$

$\begin{array}{llll}1032.59 & -1.7944 & 0.000037 & 3.220\end{array}$

$\begin{array}{llll}1078.43 & -4.9256 & 0.000046 & 24.261\end{array}$

$\begin{array}{llll}1101.43 & -2.5304 & 0.000018 & 6.403\end{array}$

$\begin{array}{llll}1121.95 & -6.7544 & 0.000039 & 45.622\end{array}$

$\begin{array}{llll}1148.52 & -2.8189 & 0.000013 & 7.946\end{array}$

$\begin{array}{llll}1165.97 & -4.2698 & 0.000017 & 18.231\end{array}$

$\begin{array}{llll}1184.06 & 7.1354 & -0.000024 & 50.915\end{array}$

$\begin{array}{llll}997.98 & -0.2762 & 0.000011 & 0.076\end{array}$

$\begin{array}{llll}1017.90 & -0.2977 & 0.000006 & 0.089\end{array}$

$\begin{array}{llll}1062.93 & -2.3342 & 0.000022 & 5.449\end{array}$

$\begin{array}{llll}1085.92 & 4.8819 & -0.000035 & 23.833\end{array}$

$\begin{array}{llll}1106.69 & -0.6887 & 0.000004 & 0.474\end{array}$

$\begin{array}{llll}1134.02 & -0.4209 & 0.000002 & 0.177\end{array}$

$\begin{array}{llll}1152.31 & -1.5076 & 0.000006 & 2.273\end{array}$

Average
$9.7943-0.000034 \quad 95.927$

$\begin{array}{lll}-0.3405 & 0.000015 & 1749.09 \text { Sum chi sqr }\end{array}$ $\mathrm{g} \mathrm{FeSO} 4$ Density of

$.7 \mathrm{H} 2 \mathrm{O} / \mathrm{L}$ water $/ \mathrm{kg} / \mathrm{m}^{3}$

998.5956

998.5956

998.5956

998.5956

998.5956

998.5956

998.5956

998.5956

998.2041

998.2041

998.2041

998.2041

997.0449

997.0449

997.0449

997.0449

997.0449

997.0449

997.0449

997.0449

997.0449

997.0449

988.0363

988.0363

988.0363

988.0363

988.0363

988.0363

988.0363

988.0363

988.0363

974.849

974.849

974.849

974.849

974.849

974.849

974.849

974.849 
molality molarity

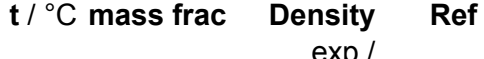

$\exp /$
Ref

App vol
$\exp /$
$\mathrm{m}^{3} / \mathrm{kg}$

\section{App vol \\ Calc /}

$\mathrm{m}^{3} / \mathrm{kg}$

$\begin{array}{rrr}\text { Std dev Res } & 4.9515 & 0.000040 \\ \text { Avg - 4std } & -20.1465 & -0.000144 \\ \text { Avg + 4std } & 19.4656 & 0.000175\end{array}$

Curve fit for the model App vol= $\left(w+c 2+c 3^{\star} t{ }^{\circ} \mathrm{C}\right) /\left(c 0^{*} w+c 1\right) / e^{\wedge}\left(0.000001^{*}\left(t^{\circ} \mathrm{C}+\mathrm{c} 4\right)^{\wedge} 2\right)$

$\begin{array}{lr}\text { c0 } & -3.67 \mathrm{E}-06 \\ \text { c1 } & 0.000125 \\ \text { c2 } & -0.062861 \\ \text { c3 } & 0.00157 \\ \text { c4 } & 3942.992 \\ \text { Min T } & 15 \\ \text { Max T } & 75 \\ \text { Min w } & 0.002 \\ \text { Max w } & 0.274007 \\ \text { Avg dens res } & -0.340468 \\ \text { Std dens res } & 4.951515 \\ \text { Avg app vol res *10^3 } & 0.015094 \\ \text { Std app vol res *10^3 } & 0.039891 \\ \text { No of points in corr } & 72 \\ \text { No of inconsistent poir } & 5\end{array}$

Inconsistent data not used (Residual greater than average + / $\mathbf{4}$ standard deviations)

$\begin{array}{llllll}15 & 0.2000 & 1215.0 & (90) & 0.000112 & 0.000204\end{array}$

$18 \quad 0.2000$

$\begin{array}{llllll}50 & 0.0100 & 1001.8 & (7) & -0.000378 & 0.000024\end{array}$

$\begin{array}{llllll}75 & 0.0054 & 982.2 & (7) & -0.000407 & 0.000047\end{array}$

$\begin{array}{llllll}75 & 0.0100 & 988.5 & (7) & -0.000391 & 0.000051\end{array}$
$1188.37 \quad 26.6335-0.000092$

$1187.50 \quad 25.9996-0.000090$

$997.77 \quad 4.0272-0.000403$

$\begin{array}{lll}979.86 & 2.3400 & -0.000454\end{array}$

$984.20 \quad 4.2955-0.000442$

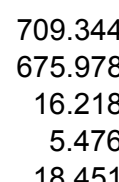

999.0996

998.5956

988.0363

974.849

974.849 
Density of aqueous solutions of $\mathrm{FeCl} 3$

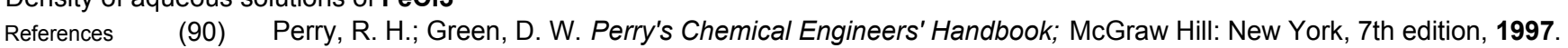

\begin{tabular}{|c|c|c|c|c|c|c|c|c|c|c|c|c|c|}
\hline \multirow[t]{36}{*}{ molality } & \multicolumn{2}{|c|}{$\mathrm{t} /{ }^{\circ} \mathrm{C}$ mass frac } & $\begin{array}{r}\text { Density } \\
\exp / \\
\mathrm{kg} / \mathrm{m}^{3}\end{array}$ & Ref & $\begin{array}{r}\text { App vol } \\
\exp / \\
\mathrm{m}^{3} / \mathrm{kg}\end{array}$ & $\begin{array}{r}\text { App vol } \\
\text { Calc / } \\
\mathrm{m}^{3} / \mathrm{kg}\end{array}$ & $\begin{array}{r}\text { Density } \\
\text { calc / } \\
\mathrm{kg} / \mathrm{m}^{3}\end{array}$ & $\begin{array}{r}\text { Density } \\
\text { Residual / } \\
\mathrm{kg} / \mathrm{m}^{3}\end{array}$ & $\begin{array}{r}\text { App Vol } \\
\text { Residual / } \\
\mathrm{m}^{3} / \mathrm{kg}\end{array}$ & $\begin{array}{r}\text { Square of } \\
\text { Density } \\
\text { Residual }\end{array}$ & $\begin{array}{l}\text { Inconsistent } \\
\text { based on } \\
\text { density }\end{array}$ & $\begin{array}{l}\text { Inconsistent } \\
\text { based on } \\
\text { apparent } \\
\text { volume }\end{array}$ & $\begin{array}{r}\text { Density of } \\
\text { water } / \mathrm{kg} / \mathrm{m}^{3}\end{array}$ \\
\hline & 0 & 0.0100 & 1008.6 & (90) & 0.000131 & 0.000178 & 1008.13 & 0.4707 & -0.000046 & 0.222 & & & 999.8395 \\
\hline & 0 & 0.0200 & 1017.4 & (90) & 0.000137 & 0.000179 & 1016.52 & 0.8775 & -0.000042 & 0.770 & & & 999.8395 \\
\hline & 0 & 0.0400 & 1034.7 & (90) & 0.000158 & 0.000183 & 1033.62 & 1.0752 & -0.000025 & 1.156 & & & 999.8395 \\
\hline & 0 & 0.0800 & 1070.3 & (90) & 0.000177 & 0.000190 & 1069.13 & 1.1680 & -0.000013 & 1.364 & & & 999.8395 \\
\hline & 0 & 0.1200 & 1108.8 & (90) & 0.000181 & 0.000197 & 1106.45 & 2.3474 & -0.000016 & 5.510 & & & 999.8395 \\
\hline & 0 & 0.1600 & 1147.5 & (90) & 0.000196 & 0.000204 & 1145.68 & 1.8207 & -0.000009 & 3.315 & & & 999.8395 \\
\hline & 0 & 0.2000 & 1187.0 & (90) & 0.000212 & 0.000212 & 1186.90 & 0.0956 & 0.000000 & 0.009 & & & 999.8395 \\
\hline & 0 & 0.2500 & 1240.0 & (90) & 0.000225 & 0.000222 & 1241.38 & -1.3837 & 0.000004 & 1.915 & & & 999.8395 \\
\hline & 0 & 0.3000 & 1297.0 & (90) & 0.000236 & 0.000232 & 1299.29 & -2.2937 & 0.000005 & 5.261 & & & 999.8395 \\
\hline & 0 & 0.3500 & 1360.5 & (90) & 0.000243 & 0.000242 & 1360.78 & -0.2822 & 0.000000 & 0.080 & & & 999.8395 \\
\hline & 10 & 0.0100 & 1008.4 & (90) & 0.000137 & 0.000183 & 1007.94 & 0.4635 & -0.000046 & 0.215 & & & 999.6996 \\
\hline & 10 & 0.0200 & 1016.8 & (90) & 0.000159 & 0.000185 & 1016.28 & 0.5248 & -0.000025 & 0.275 & & & 999.6996 \\
\hline & 10 & 0.0400 & 1034.1 & (90) & 0.000168 & 0.000188 & 1033.26 & 0.8369 & -0.000020 & 0.700 & & & 999.6996 \\
\hline & 10 & 0.0800 & 1069.2 & (90) & 0.000188 & 0.000195 & 1068.52 & 0.6806 & -0.000007 & 0.463 & & & 999.6996 \\
\hline & 10 & 0.1200 & 1107.1 & (90) & 0.000192 & 0.000202 & 1105.56 & 1.5435 & -0.000011 & 2.382 & & & 999.6996 \\
\hline & 10 & 0.1600 & 1144.9 & (90) & 0.000207 & 0.000209 & 1144.46 & 0.4371 & -0.000002 & 0.191 & & & 999.6996 \\
\hline & 10 & 0.2000 & 1184.7 & (90) & 0.000219 & 0.000217 & 1185.33 & -0.6265 & 0.000002 & 0.392 & & & 999.6996 \\
\hline & 10 & 0.2500 & 1238.0 & (90) & 0.000230 & 0.000227 & 1239.29 & -1.2882 & 0.000003 & 1.659 & & & 999.6996 \\
\hline & 10 & 0.3000 & 1295.0 & (90) & 0.000240 & 0.000237 & 1296.60 & -1.5975 & 0.000003 & 2.552 & & & 999.6996 \\
\hline & 10 & 0.3500 & 1358.0 & (90) & 0.000246 & 0.000247 & 1357.39 & 0.6100 & -0.000001 & 0.372 & & & 999.6996 \\
\hline & 10 & 0.4000 & 1423.5 & (90) & 0.000256 & 0.000258 & 1421.77 & 1.7310 & -0.000002 & 2.996 & & & 999.6996 \\
\hline & 10 & 0.4500 & 1492.0 & (90) & 0.000267 & 0.000269 & 1489.79 & 2.2103 & -0.000002 & 4.886 & & & 999.6996 \\
\hline & 10 & 0.5000 & 1561.0 & (90) & 0.000281 & 0.000281 & 1561.44 & -0.4388 & 0.000000 & 0.193 & & & 999.6996 \\
\hline & 20 & 0.0100 & 1006.8 & (90) & 0.000146 & 0.000188 & 1006.38 & 0.4177 & -0.000041 & 0.175 & & & 998.2041 \\
\hline & 20 & 0.0200 & 1015.2 & (90) & 0.000163 & 0.000189 & 1014.66 & 0.5394 & -0.000026 & 0.291 & & & 998.2041 \\
\hline & 20 & 0.0400 & 1032.4 & (90) & 0.000172 & 0.000193 & 1031.52 & 0.8771 & -0.000021 & 0.769 & & & 998.2041 \\
\hline & 20 & 0.0800 & 1066.9 & (90) & 0.000195 & 0.000200 & 1066.51 & 0.3923 & -0.000004 & 0.154 & & & 998.2041 \\
\hline & 20 & 0.1200 & 1104.0 & (90) & 0.000202 & 0.000207 & 1103.24 & 0.7568 & -0.000005 & 0.573 & & & 998.2041 \\
\hline & 20 & 0.1600 & 1141.8 & (90) & 0.000214 & 0.000214 & 1141.81 & -0.0149 & 0.000000 & 0.000 & & & 998.2041 \\
\hline & 20 & 0.2000 & 1182.0 & (90) & 0.000223 & 0.000222 & 1182.31 & -0.3069 & 0.000001 & 0.094 & & & 998.2041 \\
\hline & 20 & 0.2500 & 1234.0 & (90) & 0.000236 & 0.000232 & 1235.75 & -1.7463 & 0.000005 & 3.050 & & & 998.2041 \\
\hline & 20 & 0.3000 & 1291.0 & (90) & 0.000244 & 0.000242 & 1292.46 & -1.4611 & 0.000003 & 2.135 & & & 998.2041 \\
\hline & 20 & 0.3500 & 1353.0 & (90) & 0.000251 & 0.000252 & 1352.58 & 0.4222 & -0.000001 & 0.178 & & & 998.2041 \\
\hline & 20 & 0.4000 & 1417.5 & (90) & 0.000261 & 0.000263 & 1416.19 & 1.3103 & -0.000002 & 1.717 & & & 998.2041 \\
\hline & 20 & 0.4500 & 1485.0 & (90) & 0.000272 & 0.000274 & 1483.34 & 1.6584 & -0.000002 & 2.750 & & & 998.2041 \\
\hline
\end{tabular}


molality molarity

$\begin{array}{cccc}\mathbf{t} /{ }^{\circ} \mathrm{C} \text { mass frac } & \begin{array}{r}\text { Density } \\ \exp / \\ \mathrm{kg} / \mathrm{m}^{3}\end{array} & \text { Ref } \\ & & & \\ 20 & 0.5000 & 1551.0 & (90) \\ 30 & 0.0100 & 1004.0 & (90) \\ 30 & 0.0200 & 1012.2 & (90) \\ 30 & 0.0400 & 1029.2 & (90) \\ 30 & 0.0800 & 1063.6 & (90) \\ 30 & 0.1200 & 1100.6 & (90) \\ 30 & 0.1600 & 1138.6 & (90) \\ 30 & 0.2000 & 1178.6 & (90) \\ 30 & 0.2500 & 1229.0 & (90) \\ 30 & 0.3000 & 1285.0 & (90) \\ 30 & 0.3500 & 1347.5 & (90) \\ 30 & 0.4000 & 1411.5 & (90)\end{array}$

\section{App vol App vol D

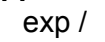

Calc /
$\mathrm{m}^{3} / \mathrm{kg}$$$
\mathrm{m}^{3} / \mathrm{kg}
$$

$0.000288 \quad 0.000285$

$\begin{array}{lll}0.000169 & 0.000192\end{array}$

$0.000183 \quad 0.000194$

$\begin{array}{ll}0.000183 & 0.000194 \\ 0.000186 & 0.000197\end{array}$

$0.000202 \quad 0.000204$

$0.000206 \quad 0.000212$

$\begin{array}{ll}0.000206 & 0.000212 \\ 0.000216 & 0.000219\end{array}$

$0.000225 \quad 0.000226$

0.0002420 .000236

$0.000250 \quad 0.000246$

$0.000255 \quad 0.000256$

$\begin{array}{lll}0.000265 & 0.000267\end{array}$

\subsection{0}

Average Res

Std dev Res

Avg - 4std

Avg + 4std calc / Resi
$\mathrm{kg} / \mathrm{m}^{3}$

Residual

$\mathrm{kg} / \mathrm{m}^{3}$

$-3.014$

0.

$0.2372-0.000024$

$-0.000024$

$0.4928-0.000012$

$0.1918-0.000002$

$0.7670-0.000005$

$0.5360-0.000003$

$0.4172-0.000002$

$\begin{array}{ll}-2.1050 & 0.000006\end{array}$

$\begin{array}{ll}-2.2406 & 0.000005\end{array}$

$0.7909-0.000001$

$1.9028-0.000002$

$0.2134-0.000008$

$1.2408 \quad 0.000014$

$-4.7497-0.000064$

$\begin{array}{ll}5.1765 & 0.000048\end{array}$

Curve fit for the model App vol $=\left(w+c 2+c 3^{*} t{ }^{\circ} \mathrm{C}\right) /\left(\mathrm{c} 0^{*} w+c 1\right) / \mathrm{e}^{\wedge}\left(0.000001^{*}\left(t{ }^{\circ} \mathrm{C}+\mathrm{c} 4\right)^{\wedge} 2\right)$

$\begin{array}{lr}\text { c0 } & -1333.774 \\ \text { c1 } & 4369.203 \\ \text { c2 } & 1.529782 \\ \text { c3 } & 0.007099 \\ \text { c4 } & 829.2131 \\ \text { Min T } & 0\end{array}$

Min $T$

Max T 30

Min w $\quad 0.0100$

Max w 0.5000

Avg dens res $\quad 0.2134$

Std dens res $\quad 1.2408$

Avg app vol res *10^3 -0.0083

Std app vol res *10^3 $\quad 0.0140$

No of points in corr

No of inconsistent poir

47
0

Inconsistent data not used (Residual greater than average +/- 4 standard deviations) 
Density of aqueous solutions of $\mathrm{Fe} 2(\mathrm{SO} 4) 3$

References (14) Chenlo, F. et al., "Viscosidad de disoluciones acusas de Fe2(SO4)3, Fe2(SO4)3-Na2SO4 y Fe2(SO4)3-NaCl a diferentes concentrationes y temperaturas", Afinidad, 1997, 54, 126-128

(75) National Research Council USA, "International Critical Tables of Physical and Numerical Data, Physics, Chemistry, and Technology.", McGraw-Hill, New York, 1928

(90) Perry, R. H.; Green, D. W. Perry's Chemical Engineers' Handbook; McGraw Hill: New York, 7th edition, 1997.

\begin{tabular}{|c|c|c|c|c|c|c|c|c|c|c|c|c|c|c|}
\hline \multirow[t]{34}{*}{ molality } & \multicolumn{3}{|l|}{ molarity } & \multicolumn{2}{|l|}{$\begin{array}{r}\text { Density } \\
\exp / \\
\mathrm{kg} / \mathrm{m}^{3}\end{array}$} & \multirow{2}{*}{$\begin{array}{r}\text { App vol } \\
\text { exp / } \\
\mathrm{m}^{3} / \mathrm{kg} \\
0.000117\end{array}$} & $\begin{array}{r}\text { App vol } \\
\text { Calc / } \\
\mathrm{m}^{3} / \mathrm{kg}\end{array}$ & $\begin{array}{r}\text { Density } \\
\text { calc / } \\
\mathrm{kg} / \mathrm{m}^{3}\end{array}$ & $\begin{array}{r}\text { Density } \\
\text { Residual / } \\
\mathrm{kg} / \mathrm{m}^{3}\end{array}$ & $\begin{array}{r}\text { App Vol } \\
\text { Residual / } \\
\mathrm{m}^{3} / \mathrm{kg}\end{array}$ & \multirow{2}{*}{$\begin{array}{r}\text { Square of } \\
\text { Density } \\
\text { Residual } \\
0.061\end{array}$} & $\begin{array}{c}\text { Inconsistent } \\
\text { based on } \\
\text { density }\end{array}$ & $\begin{array}{c}\text { Inconsistent } \\
\text { based on } \\
\text { apparent } \\
\text { volume }\end{array}$ & $\begin{array}{r}\text { Density of } \\
\text { water } / \mathrm{kg} / \mathrm{m}^{3}\end{array}$ \\
\hline & & 15 & 0.0100 & 1008.0 & (75) & & 0.000142 & 1007.75 & 0.2479 & -0.000024 & & & & 999.0996 \\
\hline & & 15 & 0.0200 & 1018.0 & (75) & 0.000072 & 0.000143 & 1016.53 & 1.4659 & -0.000071 & 2.149 & & & 999.0996 \\
\hline & & 15 & 0.0400 & 1037.0 & (75) & 0.000086 & 0.000145 & 1034.50 & 2.5045 & -0.000058 & 6.272 & & & 999.0996 \\
\hline & & 15 & 0.0600 & 1056.0 & (75) & 0.000102 & 0.000147 & 1053.00 & 2.9968 & -0.000045 & 8.981 & & & 999.0996 \\
\hline & & 15 & 0.0800 & 1076.0 & (75) & 0.000107 & 0.000149 & 1072.08 & 3.9233 & -0.000043 & 15.392 & & & 999.0996 \\
\hline & & 15 & 0.1000 & 1095.0 & (75) & 0.000124 & 0.000152 & 1091.74 & 3.2639 & -0.000027 & 10.653 & & & 999.0996 \\
\hline & & 15 & 0.1200 & 1116.0 & (75) & 0.000127 & 0.000154 & 1112.00 & 3.9981 & -0.000027 & 15.985 & & & 999.0996 \\
\hline & & 15 & 0.1400 & 1137.0 & (75) & 0.000134 & 0.000157 & 1132.89 & 4.1053 & -0.000023 & 16.854 & & & 999.0996 \\
\hline & & 15 & 0.1600 & 1158.0 & (75) & 0.000143 & 0.000159 & 1154.44 & 3.5645 & -0.000017 & 12.706 & & & 999.0996 \\
\hline & & 15 & 0.1800 & 1181.0 & (75) & 0.000144 & 0.000162 & 1176.65 & 4.3548 & -0.000017 & 18.964 & & & 999.0996 \\
\hline & & 15 & 0.2000 & 1204.0 & (75) & 0.000149 & 0.000165 & 1199.54 & 4.4552 & -0.000015 & 19.848 & & & 999.0996 \\
\hline & & 15 & 0.2500 & 1264.0 & (75) & 0.000162 & 0.000172 & 1259.95 & 4.0547 & -0.000010 & 16.440 & & & 999.0996 \\
\hline & & 15 & 0.3000 & 1330.0 & (75) & 0.000171 & 0.000180 & 1325.08 & 4.9161 & -0.000009 & 24.168 & & & 999.0996 \\
\hline & & 15 & 0.3500 & 1400.0 & (75) & 0.000182 & 0.000189 & 1395.20 & 4.7979 & -0.000007 & 23.020 & & & 999.0996 \\
\hline & & 15 & 0.4000 & 1476.0 & (75) & 0.000192 & 0.000199 & 1470.43 & 5.5707 & -0.000006 & 31.033 & & & 999.0996 \\
\hline & & 15 & 0.4500 & 1560.0 & (75) & 0.000201 & 0.000210 & 1550.70 & 9.3011 & -0.000009 & 86.511 & & & 999.0996 \\
\hline & & 15 & 0.5000 & 1647.0 & (75) & 0.000213 & 0.000222 & 1635.62 & 11.3778 & -0.000008 & 129.455 & & & 999.0996 \\
\hline & & 17.5 & 0.0100 & 1007.2 & (75) & 0.000155 & 0.000147 & 1007.28 & -0.0829 & 0.000008 & 0.007 & & & 998.6866 \\
\hline & & 17.5 & 0.0200 & 1015.7 & (75) & 0.000163 & 0.000148 & 1016.01 & -0.3058 & 0.000015 & 0.094 & & & 998.6866 \\
\hline & & 17.5 & 0.0400 & 1032.7 & (75) & 0.000177 & 0.000150 & 1033.84 & -1.1407 & 0.000027 & 1.301 & & & 998.6866 \\
\hline & & 17.5 & 0.0600 & 1049.8 & (75) & 0.000189 & 0.000152 & 1052.21 & -2.4094 & 0.000036 & 5.805 & & & 998.6866 \\
\hline & & 17.5 & 0.0800 & 1067.0 & (75) & 0.000200 & 0.000155 & 1071.13 & -4.1304 & 0.000045 & 17.060 & & & 998.6866 \\
\hline & & 17.5 & 0.1000 & 1084.0 & (75) & 0.000213 & 0.000157 & 1090.62 & -6.6223 & 0.000056 & 43.855 & & & 998.6866 \\
\hline & & 17.5 & 0.1200 & 1102.8 & (75) & 0.000214 & 0.000160 & 1110.70 & -7.9042 & 0.000054 & 62.476 & & & 998.6866 \\
\hline & & 17.5 & 0.1400 & 1121.5 & (75) & 0.000218 & 0.000162 & 1131.40 & -9.8951 & 0.000056 & 97.913 & & & 998.6866 \\
\hline & & 17.5 & 0.1600 & 1140.9 & (75) & 0.000221 & 0.000165 & 1152.71 & -11.8143 & 0.000056 & 139.577 & & & 998.6866 \\
\hline & & 17.5 & 0.1800 & 1160.9 & (75) & 0.000224 & 0.000168 & 1174.68 & -13.7806 & 0.000056 & 189.905 & & & 998.6866 \\
\hline & & 17.5 & 0.2000 & 1181.1 & (75) & 0.000228 & 0.000171 & 1197.31 & -16.2129 & 0.000057 & 262.857 & & & 998.6866 \\
\hline & & 17.5 & 0.2500 & 1241.0 & (75) & 0.000219 & 0.000178 & 1256.92 & -15.9240 & 0.000041 & 253.572 & & & 998.6866 \\
\hline & & 17.5 & 0.3000 & 1307.3 & (75) & 0.000213 & 0.000187 & 1321.06 & -13.7630 & 0.000027 & 189.421 & & & 998.6866 \\
\hline & & 17.5 & 0.3500 & 1376.4 & (75) & 0.000216 & 0.000196 & 1389.91 & -13.5147 & 0.000020 & 182.648 & & & 998.6866 \\
\hline & & 17.5 & 0.4000 & 1448.7 & (75) & 0.000224 & 0.000206 & 1463.54 & -14.8365 & 0.000017 & 220.121 & & & 998.6866 \\
\hline & & 17.5 & 0.4500 & 1527.8 & (75) & 0.000231 & 0.000218 & 1541.77 & -13.9718 & 0.000013 & 195.212 & & & 998.6866 \\
\hline
\end{tabular}


molality

\begin{tabular}{|c|c|c|c|c|}
\hline \multirow[t]{30}{*}{ molarity } & \multicolumn{2}{|c|}{$\mathrm{t} /{ }^{\circ} \mathrm{C}$ mass frac } & $\begin{array}{r}\text { Density } \\
\exp / \\
\mathrm{kg} / \mathrm{m}^{3}\end{array}$ & Ref \\
\hline & 17.5 & 0.5000 & 1612.7 & (75) \\
\hline & 17.5 & 0.5500 & 1702.8 & (75) \\
\hline & 17.5 & 0.6000 & 1798.3 & (75) \\
\hline & 18 & 0.0100 & 1006.6 & (75) \\
\hline & 18 & 0.0100 & 1007.2 & (90) \\
\hline & 18 & 0.0200 & 1015.6 & (75) \\
\hline & 18 & 0.0200 & 1015.7 & (90) \\
\hline & 18 & 0.0400 & 1034.6 & (75) \\
\hline & 18 & 0.0400 & 1032.7 & (90) \\
\hline & 18 & 0.0600 & 1055.4 & (75) \\
\hline & 18 & 0.0800 & 1075.4 & (75) \\
\hline & 18 & 0.0800 & 1067.0 & (90) \\
\hline & 18 & 0.1000 & 1095.4 & (75) \\
\hline & 18 & 0.1200 & 1116.4 & (75) \\
\hline & 18 & 0.1200 & 1102.8 & (90) \\
\hline & 18 & 0.1400 & 1138.2 & (75) \\
\hline & 18 & 0.1600 & 1160.2 & (75) \\
\hline & 18 & 0.1600 & 1140.9 & (90) \\
\hline & 18 & 0.1800 & 1182.2 & (75) \\
\hline & 18 & 0.2000 & 1206.1 & (75) \\
\hline & 18 & 0.2000 & 1181.1 & (90) \\
\hline & 18 & 0.2500 & 1268.9 & (75) \\
\hline & 18 & 0.3000 & 1334.7 & (75) \\
\hline & 18 & 0.3000 & 1307.3 & (90) \\
\hline & 18 & 0.3500 & 1408.5 & (75) \\
\hline & 18 & 0.4000 & 1487.3 & (75) \\
\hline & 18 & 0.4000 & 1448.7 & (90) \\
\hline & 18 & 0.5000 & 1612.7 & (90) \\
\hline & 18 & 0.6000 & 1798.3 & (90) \\
\hline 0.125 & 25 & 0.0480 & 1041.3 & (14) \\
\hline 0.25 & 25 & 0.0925 & 1080.5 & (14) \\
\hline 0.5 & 25 & 0.1712 & 1167.7 & (14) \\
\hline 0.75 & 25 & 0.2404 & 1247.5 & (14) \\
\hline 1 & 25 & 0.3008 & 1329.3 & (14) \\
\hline
\end{tabular}

\section{App vol App vol}

$\begin{array}{cc}\exp / & \text { Calc / } \\ \mathrm{m}^{3} / \mathrm{kg} & \mathrm{m}^{3} / \mathrm{kg}\end{array}$

0.0002390 .000230

$0.000249 \quad 0.000244$

0.0002590 .000260

$0.000205 \quad 0.000148$

$0.000146 \quad 0.000148$

$0.000163 \quad 0.000149$

$0.000158 \quad 0.000149$

$0.000130 \quad 0.000151$

$0.000175 \quad 0.000151$

$0.000103 \quad 0.000153$

$0.000107 \quad 0.000156$

$0.000199 \quad 0.000156$

$0.000116 \quad 0.000158$

$0.000121 \quad 0.000161$

$0.000213 \quad 0.000161$

$0.000124 \quad 0.000164$

$0.000130 \quad 0.000166$

$0.000221 \quad 0.000166$

$\begin{array}{ll}0.000137 & 0.000169\end{array}$

$0.000140 \quad 0.000172$

$0.000228 \quad 0.000172$

$\begin{array}{ll}0.000148 & 0.000180\end{array}$

$0.000161 \quad 0.000188$

$0.000213 \quad 0.000188$

$0.000169 \quad 0.000197$

$0.000179 \quad 0.000208$

$0.000224 \quad 0.000208$

$0.000239 \quad 0.000232$

0.0002590 .000262

$0.000115 \quad 0.000166$

$0.000166 \quad 0.000171$

$\begin{array}{lll}0.000147 & 0.000183\end{array}$

$0.000165 \quad 0.000194$

$\begin{array}{lll}0.000170 & 0.000205\end{array}$

\section{Average Res}

Std dev Res

Avg - 4std

Avg + 4std
App Vol Square of Inconsistent Inconsistent Density of calc / Residual / Residual / Density based on based on water $/ \mathrm{kg} / \mathrm{m}^{3}$ $\mathrm{kg} / \mathrm{m}^{3} \quad \mathrm{~kg} / \mathrm{m}^{3} \quad \mathrm{~m}^{3} / \mathrm{kg}$ Residual density apparent

$\begin{array}{llll}1624.12 & -11.4229 & 0.000009 & 130.482\end{array}$

$1709.57 \quad-6.7662 \quad 0.000004 \quad 45.781$

$\begin{array}{llll}1796.30 & 2.0031 & -0.000001 & 4.012\end{array}$

$0.0192-0.000002 \quad 0.000$

$\begin{array}{lll}-0.2922 & 0.000014 & 0.085\end{array}$

$\begin{array}{lll}-0.1922 & 0.000009 & 0.037\end{array}$

$0.8975-0.000021 \quad 0.806$

$\begin{array}{lll}-1.0025 & 0.000023 & 1.005\end{array}$

$3.3559-0.000050 \quad 11.262$

$4.4646-0.000048 \quad 19.932$

$\begin{array}{lll}-3.9354 & 0.000043 \quad 15.488\end{array}$

$5.0052-0.000042 \quad 25.052$

$5.9590-0.000040 \quad 35.510$

$\begin{array}{lll}-7.6410 & 0.000052 & 58.384\end{array}$

$1131.09 \quad 7.1073-0.000039 \quad 50.513$

$\begin{array}{lll}-11.4689 & 0.000055 & 131.535\end{array}$

$1174.29 \quad 7.9119-0.000032 \quad 62.599$

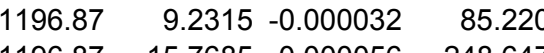

$\begin{array}{llll}196.87 & -15.7685 & 0.000056 & 248.647\end{array}$

$\begin{array}{llll}14.4270 & -0.000027 & 208.138\end{array}$

$\begin{array}{llll}1462.19 & 25.1062 & -0.000029 & 630.322\end{array}$

$\begin{array}{llll}1462.19 & -13.4938 & 0.000016 & 182.082\end{array}$

$\begin{array}{llll}1621.90 & -9.1982 & 0.000007 & 84.608\end{array}$

$\begin{array}{lllll}1792.69 & 5.6130 & -0.000003 & 31.506\end{array}$

$1038.67 \quad 2.6297-0.000051 \quad 6.915$

$\begin{array}{lll}0.6194 & -0.000006 & 0.384\end{array}$

$8.3084-0.000036 \quad 69.030$

$10.6158-0.000029 \quad 112.696$

$\begin{array}{lll}18.5677 & -0.000035 & 344.761\end{array}$

$0.0854-0.0000015670 .073$ Sum chi sqr

$9.2684 \quad 0.000035$

$-36.9881-0.000141$

37.15890 .000139
998.6866

998.6866

998.6866

998.5956

998.5956

998.5956

998.5956

998.5956

998.5956

998.5956

998.5956

998.5956

998.5956

998.5956

998.5956

998.5956

998.5956

998.5956

998.5956

998.5956

998.5956

998.5956

998.5956

998.5956

998.5956

998.5956

998.5956

998.5956

998.5956

997.0449

997.0449

997.0449

997.0449

997.0449

\section{Curve fit for the model App vol $=\left(w+c 2+c 3^{*} t{ }^{\circ} \mathrm{C}\right) /\left(\mathrm{c} 0^{*} w+c 1\right) / e^{\wedge}\left(0.000001^{*}\left(t^{\circ} \mathrm{C}+\mathrm{c} 4\right)^{\wedge} 2\right)$}




\begin{tabular}{|c|c|c|c|c|c|c|c|c|c|c|c|c|}
\hline molality & molarity & $\mathrm{t} /{ }^{\circ} \mathrm{C}$ mass frac & $\begin{array}{r}\text { Density } \\
\exp / \\
\mathrm{kg} / \mathrm{m}^{3}\end{array}$ & Ref & $\begin{array}{r}\text { App vol } \\
\exp / \\
\mathrm{m}^{3} / \mathrm{kg}\end{array}$ & $\begin{array}{r}\text { App vol } \\
\text { Calc / } \\
\mathrm{m}^{3} / \mathrm{kg}\end{array}$ & $\begin{array}{r}\text { Density } \\
\mathrm{calc} / \\
\mathrm{kg} / \mathrm{m}^{3}\end{array}$ & $\begin{array}{r}\text { Density } \\
\text { Residual / } \\
\mathrm{kg} / \mathrm{m}^{3}\end{array}$ & $\begin{array}{r}\text { App Vol } \\
\text { Residual / } \\
\mathrm{m}^{3} / \mathrm{kg}\end{array}$ & $\begin{array}{r}\text { Square of } \\
\text { Density } \\
\text { Residual }\end{array}$ & $\begin{array}{c}\text { Inconsistent } \\
\text { based on } \\
\text { density }\end{array}$ & $\begin{array}{c}\text { Inconsistent } \\
\text { based on } \\
\text { apparent } \\
\text { volumer } / \mathrm{kg} / \mathrm{m}^{3} \\
\text { volume }\end{array}$ \\
\hline
\end{tabular}

$\begin{array}{lr}\text { c0 } & 0.474439 \\ \text { c1 } & -0.646238 \\ \text { c2 } & -713.1004 \\ \text { c3 } & -25.56846 \\ \text { c4 } & 4023.184 \\ \text { Min T } & 15 \\ \text { Max T } & 25 \\ \text { Min w } & 0.0100 \\ \text { Max w } & 0.6000 \\ \text { Avg dens res } & 0.085379 \\ \text { Std dens res } & 9.268376 \\ \text { Avg app vol res *10^3 } & -0.001226 \\ \text { Std app vol res *10^3 } & 0.035048 \\ \text { No of points in corr } & 67 \\ \text { No of inconsistent points } & 0\end{array}$

Inconsistent data not used (Residual greater than average +/- 4 standard deviations) 
Density of aqueous solutions of $\mathbf{H C I}$

References (3) Akerlof, G; Teare, J. A. Note on the Density of Aqueous Solutions of Hydrochloric Acid. J. Am. Chem. Soc. 1938, 60, 1226-1228.

(30) Goldsack, D. E.; Franchetto, A. A. The Viscosity of Concentrated Electrolyte Solutions. III. A Mixture Law. Electrochimica Acta 1977, $22,1287-1294$.

(39) Hershey, J. P.; Sotolongo, S.; Millero, F. J. Densities and Compressiblities of Aqueous $\mathrm{HCl}$ and $\mathrm{NaOH}$ from 0 to $45^{\circ} \mathrm{C}$. The Effect of Pressure on the lonization of Water. J. Sol. Chem. 1984, 13, 825-848.

(90) Perry, R. H.; Green, D. W. Perry's Chemical Engineers' Handbook; McGraw Hill: New York, 7th edition, 1997.

(98) Pogue, Randall; Atkinson, Gordon, Apparent Molal Volumes and Heat Capacities of Aqueous $\mathrm{HCl}$ and $\mathrm{HClO}_{4}$ at $15-55{ }^{\circ} \mathrm{C}, \mathrm{J} \mathrm{Chem} \mathrm{Eng}$ Data, 1988, 33, 495-499

(128) Torók, T. I.; Berecz, E. Volumetric Properties and Electrolytic Conductances of Aqueous Ternary Mixtures of Hydrogen Chloride and some Transition Metal Chlorides at $25^{\circ} \mathrm{C}$. J. Sol. Chem. 1989, 18, 1117-1131.

\begin{tabular}{|c|c|c|c|c|c|c|c|c|c|c|c|c|c|c|c|}
\hline \multirow[t]{35}{*}{ molality molarity } & \multicolumn{2}{|c|}{$\mathrm{t} /{ }^{\circ} \mathrm{C}$ mass frac } & $\begin{array}{r}\text { Density } \\
\exp / \\
\mathrm{kg} / \mathrm{m}^{3}\end{array}$ & Ref & $\begin{array}{r}\text { App vol } \\
\exp / \\
\mathrm{m}^{3} / \mathrm{kg}\end{array}$ & $\begin{array}{c}\text { App vol } \\
\text { Calc / } \\
\mathrm{m}^{3} / \mathrm{kg}\end{array}$ & $\begin{array}{r}\text { Density } \\
\text { calc/ } \\
\mathrm{kg} / \mathrm{m}^{3}\end{array}$ & $\begin{array}{r}\text { Density } \\
\text { Residual / } \\
\mathrm{kg} / \mathrm{m}^{3}\end{array}$ & $\begin{array}{r}\text { App Vol } \\
\text { Residual / } \\
\mathrm{m}^{3} / \mathrm{kg}\end{array}$ & $\begin{array}{r}\text { Square of } \\
\text { Density } \\
\text { Residual }\end{array}$ & $\begin{array}{l}\text { Inconsistent } \\
\text { based on } \\
\text { density }\end{array}$ & $\begin{array}{c}\text { Inconsistent } \\
\text { based on } \\
\text { apparent } \\
\text { volume }\end{array}$ & $\begin{array}{l}\text { Delta density } \\
\qquad / \mathrm{kg} / \mathrm{m}^{3}\end{array}$ & $1000^{*}(\mathrm{RD}-1)$ & $\begin{array}{r}\text { Density of } \\
\text { water } / \mathrm{kg} / \mathrm{m}^{3}\end{array}$ \\
\hline & -5 & 0.0100 & 1004.80 & (90) & 0.000449 & 0.000479 & 1004.50 & 0.3022 & -0.000030 & 0.091 & & & & & 999.2563 \\
\hline & -5 & 0.0200 & 1010.40 & (90) & 0.000449 & 0.000480 & 1009.76 & 0.6370 & -0.000031 & 0.406 & & & & & 999.2563 \\
\hline & -5 & 0.0400 & 1021.30 & (90) & 0.000461 & 0.000483 & 1020.36 & 0.9374 & -0.000022 & 0.879 & & & & & 999.2563 \\
\hline & -5 & 0.0600 & 1032.10 & (90) & 0.000470 & 0.000486 & 1031.05 & 1.0488 & -0.000016 & 1.100 & & & & & 999.2563 \\
\hline & -5 & 0.0800 & 1042.80 & (90) & 0.000478 & 0.000490 & 1041.82 & 0.9752 & -0.000011 & 0.951 & & & & & 999.2563 \\
\hline & -5 & 0.1000 & 1053.60 & (90) & 0.000485 & 0.000493 & 1052.68 & 0.9211 & -0.000008 & 0.848 & & & & & 999.2563 \\
\hline & -5 & 0.1200 & 1064.50 & (90) & 0.000490 & 0.000496 & 1063.61 & 0.8916 & -0.000007 & 0.795 & & & & & 999.2563 \\
\hline & -5 & 0.1400 & 1075.40 & (90) & 0.000495 & 0.000500 & 1074.61 & 0.7921 & -0.000005 & 0.627 & & & & & 999.2563 \\
\hline & -5 & 0.1600 & 1086.40 & (90) & 0.000499 & 0.000503 & 1085.67 & 0.7287 & -0.000004 & 0.531 & & & & & 999.2563 \\
\hline & -5 & 0.1800 & 1097.50 & (90) & 0.000503 & 0.000506 & 1096.79 & 0.7077 & -0.000003 & 0.501 & & & & & 999.2563 \\
\hline & -5 & 0.2000 & 1108.70 & (90) & 0.000507 & 0.000510 & 1107.96 & 0.7362 & -0.000003 & 0.542 & & & & & 999.2563 \\
\hline & -5 & 0.2200 & 1120.00 & (90) & 0.000510 & 0.000513 & 1119.18 & 0.8220 & -0.000003 & 0.676 & & & & & 999.2563 \\
\hline & -5 & 0.2400 & 1131.40 & (90) & 0.000514 & 0.000517 & 1130.43 & 0.9731 & -0.000003 & 0.947 & & & & & 999.2563 \\
\hline & -5 & 0.2600 & 1142.60 & (90) & 0.000518 & 0.000521 & 1141.70 & 0.8986 & -0.000003 & 0.807 & & & & & 999.2563 \\
\hline & -5 & 0.2800 & 1153.70 & (90) & 0.000522 & 0.000524 & 1152.99 & 0.7079 & -0.000002 & 0.501 & & & & & 999.2563 \\
\hline & -5 & 0.3000 & 1164.80 & (90) & 0.000527 & 0.000528 & 1164.29 & 0.5114 & -0.000001 & 0.262 & & & & & 999.2563 \\
\hline & 0 & 0.0200 & 1010.57 & (3) & 0.000469 & 0.000489 & 1010.17 & 0.3977 & -0.000019 & 0.158 & & & 10.73 & & 999.8395 \\
\hline & 0 & 0.0400 & 1021.18 & (3) & 0.000478 & 0.000492 & 1020.59 & 0.5924 & -0.000014 & 0.351 & & & 21.34 & & 999.8395 \\
\hline & 0 & 0.0600 & 1031.78 & (3) & 0.000484 & 0.000495 & 1031.08 & 0.6981 & -0.000011 & 0.487 & & & 31.94 & & 999.8395 \\
\hline & 0 & 0.0800 & 1042.39 & (3) & 0.000490 & 0.000498 & 1041.65 & 0.7394 & -0.000009 & 0.547 & & & 42.55 & & 999.8395 \\
\hline & 0 & 0.1000 & 1053.01 & (3) & 0.000495 & 0.000502 & 1052.29 & 0.7212 & -0.000007 & 0.520 & & & 53.17 & & 999.8395 \\
\hline & 0 & 0.1200 & 1063.67 & (3) & 0.000500 & 0.000505 & 1062.99 & 0.6789 & -0.000005 & 0.461 & & & 63.83 & & 999.8395 \\
\hline & 0 & 0.1400 & 1074.36 & (3) & 0.000505 & 0.000508 & 1073.75 & 0.6085 & -0.000004 & 0.370 & & & 74.52 & & 999.8395 \\
\hline & 0 & 0.1600 & 1085.09 & (3) & 0.000509 & 0.000512 & 1084.56 & 0.5263 & -0.000003 & 0.277 & & & 85.25 & & 999.8395 \\
\hline & 0 & 0.1800 & 1095.83 & (3) & 0.000513 & 0.000515 & 1095.42 & 0.4092 & -0.000002 & 0.167 & & & 95.99 & & 999.8395 \\
\hline & 0 & 0.2000 & 1106.60 & (3) & 0.000518 & 0.000519 & 1106.31 & 0.2848 & -0.000001 & 0.081 & & & 106.76 & & 999.8395 \\
\hline & 0 & 0.2200 & 1117.38 & (3) & 0.000522 & 0.000522 & 1117.24 & 0.1410 & -0.000001 & 0.020 & & & 117.54 & & 999.8395 \\
\hline & 0 & 0.2400 & 1128.17 & (3) & 0.000526 & 0.000526 & 1128.18 & -0.0134 & 0.000000 & 0.000 & & & 128.33 & & 999.8395 \\
\hline & 0 & 0.2600 & 1138.95 & (3) & 0.000530 & 0.000530 & 1139.14 & -0.1892 & 0.000001 & 0.036 & & & 139.11 & & 999.8395 \\
\hline & 0 & 0.2800 & 1149.72 & (3) & 0.000535 & 0.000533 & 1150.10 & -0.3764 & 0.000001 & 0.142 & & & 149.88 & & 999.8395 \\
\hline & 0 & 0.3000 & 1160.46 & (3) & 0.000539 & 0.000537 & 1161.04 & -0.5844 & 0.000001 & 0.341 & & & 160.62 & & 999.8395 \\
\hline & 0 & 0.3200 & 1171.15 & (3) & 0.000543 & 0.000541 & 1171.97 & -0.8218 & 0.000002 & 0.675 & & & 171.31 & & 999.8395 \\
\hline & 0 & 0.3400 & 1181.77 & (3) & 0.000547 & 0.000545 & 1182.87 & -1.0965 & 0.000002 & 1.202 & & & 181.93 & & 999.8395 \\
\hline & 0 & 0.3600 & 1192.82 & (3) & 0.000551 & 0.000549 & 1193.72 & -0.8959 & 0.000002 & 0.803 & & & 192.98 & & 999.8395 \\
\hline
\end{tabular}


molality molarity

$\mathbf{t} /{ }^{\circ} \mathrm{C}$ mass frac $\begin{gathered}\text { Density } \\ \exp /\end{gathered} \quad$ Ref $\exp /$
$\mathrm{kg} / \mathrm{m}^{3}$

0.09273

0.19421

0.29113

0.3884

0.42968

0.51396

0.5994

0.68327

0.76939

0.85291

0.01005

0.02032

0.03001

0.0505

0.07539

0.09273

0.1001

0.124

0.1488

0.19421

0.2303

0.29113

0.38838

0.42968

0.51396

0.59944

0.68327

\begin{tabular}{rr}
0.3800 & 1202.74 \\
0.0034 & 1001.75 \\
0.0070 & 1003.68 \\
0.0105 & 1005.50 \\
0.0140 & 1007.31 \\
0.0154 & 1008.07 \\
0.0184 & 1009.62 \\
0.0214 & 1011.19 \\
0.0243 & 1012.71 \\
0.0273 & 1014.27 \\
0.0302 & 1015.77 \\
0.0200 & 1009.90 \\
0.0400 & 1020.00 \\
0.0600 & 1030.09 \\
0.0800 & 1040.18 \\
0.1000 & 1050.30 \\
0.1200 & 1060.45 \\
0.1400 & 1070.62 \\
0.1600 & 1080.83 \\
0.1800 & 1091.06 \\
0.2000 & 1101.31 \\
0.2200 & 1111.58 \\
0.2400 & 1121.86 \\
0.2600 & 1132.14 \\
0.2800 & 1142.41 \\
0.3000 & 1152.65 \\
0.3200 & 1162.86 \\
0.3400 & 1173.02 \\
0.3600 & 1183.62 \\
0.3800 & 1193.10 \\
0.0004 & 999.29 \\
0.0007 & 999.49 \\
0.0011 & 999.67 \\
0.0018 & 1000.05 \\
0.0027 & 1000.51 \\
0.0034 & 1000.82 \\
0.0036 & 1000.96 \\
0.0045 & 1001.39 \\
0.0054 & 1001.85 \\
0.0070 & 1002.66 \\
0.0083 & 1003.32 \\
0.0105 & 1004.40 \\
0.0140 & 1006.13 \\
0.0154 & 1006.87 \\
0.0184 & 1008.35 \\
0.0214 & 1009.85 \\
0.0243 & 1011.30 \\
\hline & \\
\hline
\end{tabular}

App vol App vol exp / Calc / $\mathrm{m}^{3} / \mathrm{k}$ $\mathrm{m}^{3} / \mathrm{kg}$

$\begin{array}{ll}0.000556 & 0.000553\end{array}$

$\begin{array}{lll}0.000469 & 0.000494\end{array}$

$0.000473 \quad 0.000494$

$\begin{array}{lll}0.000476 & 0.000495\end{array}$

$\begin{array}{lll}0.000478 & 0.000495\end{array}$

$0.000479 \quad 0.000496$

$\begin{array}{ll}0.000480 & 0.000496\end{array}$

$\begin{array}{lll}0.000481 & 0.000497\end{array}$

$\begin{array}{ll}0.000482 & 0.000497\end{array}$

$\begin{array}{ll}0.000483 & 0.000498\end{array}$

$\begin{array}{ll}0.000484 & 0.000498\end{array}$

$0.000495 \quad 0.000503$

$\begin{array}{lll}0.000503 & 0.000507\end{array}$

$0.000508 \quad 0.000510$

$\begin{array}{ll}0.000514 & 0.000513\end{array}$

$0.000518 \quad 0.000517$

$0.000523 \quad 0.000520$

$\begin{array}{lll}0.000527 & 0.000524\end{array}$

$0.000531 \quad 0.000527$

$0.000535 \quad 0.000531$

$0.000539 \quad 0.000535$

$0.000543 \quad 0.000538$

$0.000546 \quad 0.000542$

$0.000550 \quad 0.000546$

$0.000554 \quad 0.000550$

$0.000558 \quad 0.000553$

$\begin{array}{lll}0.000562 & 0.000557\end{array}$

$0.000566 \quad 0.000561$

$0.000569 \quad 0.000565$

$0.000574 \quad 0.000570$

$\begin{array}{lll}0.000476 & 0.000507\end{array}$

$\begin{array}{lll}0.000477 & 0.000507\end{array}$

$\begin{array}{lll}0.000479 & 0.000507\end{array}$

$\begin{array}{lll}0.000486 & 0.000507\end{array}$

$0.000488 \quad 0.000507$

$0.000492 \quad 0.000507$

$\begin{array}{ll}0.000489 & 0.000507\end{array}$

$0.000491 \quad 0.000507$

$0.000492 \quad 0.000507$

$0.000495 \quad 0.000508$

$0.000495 \quad 0.000508$

$\begin{array}{ll}0.000498 & 0.000508\end{array}$

$0.000500 \quad 0.000509$

$0.000500 \quad 0.000509$

$0.000502 \quad 0.000510$

$0.000503 \quad 0.000510$

$0.000504 \quad 0.000510$

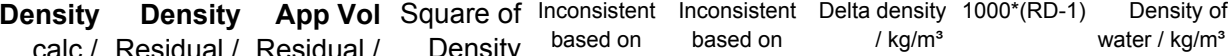
calc / Residual / Residual / Density based on based on $\mathrm{kg} / \mathrm{m}^{3} \quad \mathrm{~kg} / \mathrm{m}^{3} \quad \mathrm{~m}^{3} / \mathrm{kg}$ Residual density apparent

$204.51 \quad-1.7662 \quad 0.000003 \quad 3.120$

$\begin{array}{llll}1001.67 & 0.0823 & -0.000024 & 0.007\end{array}$

$1003.53 \quad 0.1501-0.000021 \quad 0.023$

$\begin{array}{llll}0.205 .30 & 0.27 & -0.000019 & 0.041\end{array}$

$0.2500-0.000018 \quad 0.063$

$\begin{array}{lll}0.2694 & -0.000017 & 0.073\end{array}$

$\begin{array}{lll}0.3058 & -0.000016 & 0.093\end{array}$

$0.3399-0.000016 \quad 0.116$

$0.3736-0.000015 \quad 0.140$

$0.4058-0.000014 \quad 0.165$

$\begin{array}{lll}0.4384 & -0.000014 & 0.192\end{array}$

$0.1697-0.000008 \quad 0.029$

$0.1723-0.000004 \quad 0.030$

$0.1027-0.000002$

$\begin{array}{ll}-0.0242 & 0.000000\end{array}$

0.011

0.001

0.030

0.113

$\begin{array}{lll}-0.3367 & 0.000002\end{array}$

0.270

0.483

0.765

1.103

1.474

1.866

2.269

2.673

3.098

3.892

2.448

4.804

0.000

0.000

0.001

0.002

0.003

0.003

0.004

0.005

0.007

0.008

0.012

0.012

0.016

0.018

0.021

0.024

0.026

202.90

1.791

3.718

5.536

7.345

8.109

9.66

11.222

12.748

14.305

15.809

10.20

20.30

30.39

40.48

50.60

60.75

70.92

81.13

91.36

101.61

111.88

122.16

132.44

142.71

152.95

163.16

173.32

183.92

193.40

$0.1345-0.000009$

$0.1550-0.000007$

$0.1623-0.000007$

1.716









5.561
7.035
7.766
9.25
10.747
12.205

999.8395

999.9638

999.9638

999.9638

999.9638

999.9638

999.9638

999.9638

999.9638

999.9638

999.9638

999.6996

999.6996

999.6996

999.6996

999.6996

999.6996

999.6996

999.6996

999.6996

999.6996

999.6996

999.6996

999.6996

999.6996

999.6996

999.6996

999.6996

999.6996

999.6996

0.192999 .0996

0.388999 .0996

0.57999 .0996

0.947999 .0996

$1.407 \quad 999.0996$

999.0996

1.864999 .0996

2.297999 .0996

$2.753 \quad 999.0996$

999.0996

4.228999 .0996

999.0996

999.0996

999.0996

999.0996

999.0996

999.0996 
molality molarity

$\mathbf{t} /{ }^{\circ} \mathrm{C}$ mass frac $\begin{gathered}\text { Density } \\ \exp /\end{gathered}$ $\exp /$
$\mathrm{kg} / \mathrm{m}^{3}$

0.76938

0.8529

0.01005

0.02032

0.03001

0.0505

0.07539

$\begin{array}{lll}15 & 0.0273 & 1012.80 \\ 15 & 0.0302 & 1014.23 \\ 20 & 0.0100 & 1003.20 \\ 20 & 0.0200 & 1008.00 \\ 20 & 0.0200 & 1008.20 \\ 20 & 0.0400 & 1017.91 \\ 20 & 0.0400 & 1018.10 \\ 20 & 0.0600 & 1027.71 \\ 20 & 0.0600 & 1027.90 \\ 20 & 0.0800 & 1037.51 \\ 20 & 0.0800 & 1037.60 \\ 20 & 0.1000 & 1047.34 \\ 20 & 0.1000 & 1047.40 \\ 20 & 0.1200 & 1057.21 \\ 20 & 0.1200 & 1057.40 \\ 20 & 0.1400 & 1067.10 \\ 20 & 0.1400 & 1067.50 \\ 20 & 0.1600 & 1077.03 \\ 20 & 0.1600 & 1077.60 \\ 20 & 0.1800 & 1086.98 \\ 20 & 0.1800 & 1087.80 \\ 20 & 0.2000 & 1096.95 \\ 20 & 0.2000 & 1098.00 \\ 20 & 0.2200 & 1106.95 \\ 20 & 0.2200 & 1108.30 \\ 20 & 0.2400 & 1116.97 \\ 20 & 0.2400 & 1118.70 \\ 20 & 0.2600 & 1126.98 \\ 20 & 0.2600 & 1129.00 \\ 20 & 0.2800 & 1137.00 \\ 20 & 0.2800 & 1139.20 \\ 20 & 0.3000 & 1146.99 \\ 20 & 0.3000 & 1149.30 \\ 20 & 0.3200 & 1156.97 \\ 20 & 0.3200 & 1159.30 \\ 20 & 0.3400 & 1166.89 \\ 20 & 0.3400 & 1169.10 \\ 20 & 0.3600 & 1177.26 \\ 20 & 0.3600 & 1178.90 \\ 20 & 0.3800 & 1186.54 \\ 20 & 0.3800 & 1188.50 \\ 20 & 0.4000 & 1198.00 \\ 25 & 0.0004 & 997.23 \\ 25 & 0.0007 & 997.42 \\ 25 & 0.0011 & 997.59 \\ 25 & 0.0018 & 997.96 \\ 25 & 0.0027 & 998.41\end{array}$

App vol App vol exp / Calc / $\mathrm{m}^{3} / \mathrm{k}$ $\mathrm{m}^{3} / \mathrm{kg}$

(39) $(39)$
$(90)$

$\begin{array}{ll}0.000527 & 0.000526\end{array}$ $\begin{array}{lll}0.000505 & 0.000511 & 1012.62\end{array}$

0.000514

$0.000515 \quad 0.000515$

$0.000505 \quad 0.000515$

$\begin{array}{ll}0.000512 & 0.000519\end{array}$

$0.000522 \quad 0.000522$

$0.000519 \quad 0.000522$

$0.000526 \quad 0.000526$

$\begin{array}{ll}0.000532 & 0.000529 \\ 0.000531 & 0.000529\end{array}$

$0.000536 \quad 0.000533$

$0.000534 \quad 0.000533$

$0.000540 \quad 0.000536$

$0.000537 \quad 0.000536$

$0.000544 \quad 0.000540$

$0.000540 \quad 0.000540$

$0.000547 \quad 0.000543$

$0.000543 \quad 0.000543$

$\begin{array}{ll}0.000551 & 0.000547\end{array}$

$\begin{array}{ll}0.000547 & 0.000547\end{array}$

$0.000554 \quad 0.00055$

0.0005490 .000551

$0.000558 \quad 0.000555$

$0.000552 \quad 0.000555$

0.0005620 .000559

$0.000555 \quad 0.000559$

$0.000565 \quad 0.000562$

$0.000559 \quad 0.000562$

0.0005690 .000566

$0.000563 \quad 0.000566$

$0.000572 \quad 0.000570$

$0.000567 \quad 0.000570$

$0.000576 \quad 0.000575$

$0.000571 \quad 0.000575$

$0.000579 \quad 0.000579$

$0.000575 \quad 0.000579$

$0.000583 \quad 0.000583$

$0.000580 \quad 0.000583$

$0.000584 \quad 0.000587$

$0.000494 \quad 0.000517$

$\begin{array}{ll}0.000498 & 0.000517\end{array}$

$0.000498 \quad 0.000517$

$\begin{array}{ll}0.000501 & 0.000517\end{array}$

$0.000502 \quad 0.000517$

998.37
Density Density App Vol Square of Inconsistent Inconsistent Delta density $1000^{*}(\mathrm{RD}-1) \begin{array}{r}\text { Density of } \\ \text { based on }\end{array}$ water $/ \mathrm{kg} / \mathrm{m}^{3}$ calc / Residual / Residual / Density based on based on $\mathrm{kg} / \mathrm{m}^{3} \quad \mathrm{~kg} / \mathrm{m}^{3} \quad \mathrm{~m}^{3} / \mathrm{kg}$ Residual density $\begin{gathered}\text { apparent } \\ \text { volume }\end{gathered}$

$0.1707-0.000006$

0.029

$\begin{array}{lll}0.1782 & -0.000006 & 0.032\end{array}$

$\begin{array}{lll}0.1088 & -0.000011 & 0.012\end{array}$

$0.0114-0.000001 \quad 0.000$

$0.2073-0.000010 \quad 0.043$

$\begin{array}{lll}0.0776 & -0.000002 & 0.006\end{array}$

$\begin{array}{lll}0.2635 & -0.000006 & 0.069\end{array}$

$\begin{array}{lll}-0.0162 & 0.000000 & 0.000\end{array}$

$\begin{array}{lll}0.1697 & -0.000003 & 0.029\end{array}$

$\begin{array}{lll}-0.1544 & 0.000002 & 0.024\end{array}$

$\begin{array}{lll}-0.0686 & 0.000001 & 0.005\end{array}$

0.091

$\begin{array}{lll}-0.2453 & 0.000002 & 0.060 \\ -0.4399 & 0.000003 & 0.194\end{array}$

$\begin{array}{lll}-0.4399 & 0.000003 & 0.194 \\ -0.2541 & 0.000002 & 0.065\end{array}$

$\begin{array}{lll}-0.5837 & 0.000004 & 0.341\end{array}$

$\begin{array}{lll}-0.1879 & 0.000001 & 0.035\end{array}$

$\begin{array}{lll}-0.7051 & 0.000004 & 0.497\end{array}$

$\begin{array}{lll}-0.1393 & 0.000001 & 0.019\end{array}$

$\begin{array}{lll}-0.8162 & 0.000004 & 0.666\end{array}$

$\begin{array}{lll}-0.0003 & 0.000000 & 0.000\end{array}$

$\begin{array}{lll}-0.9083 & 0.000004 & 0.825\end{array}$

$\begin{array}{lll}0.1375 & -0.000001 & 0.019\end{array}$

$\begin{array}{lll}-0.9625 & 0.000004 & 0.926\end{array}$

$\begin{array}{lll}0.3834 & -0.000001 & 0.147\end{array}$

0.959

$\begin{array}{rrr}-0.9791 & 0.000003 & 0.959 \\ 0.7468 & -0.000002 & 0.558\end{array}$

$\begin{array}{rrr}0.7468 & -0.000002 & 0.558 \\ -0.9778 & 0.000003 & 0.956\end{array}$

$\begin{array}{lll}1.0381 & -0.000003 & 1.078\end{array}$

0.861

$\begin{array}{rrr}-0.9278 & 0.000003 & 0.861 \\ 1.2681 & -0.000003 & 1.608\end{array}$

$\begin{array}{lll}-0.8576 & 0.000002 & 0.735\end{array}$

$\begin{array}{lll}1.4483 & -0.000004 & 2.098\end{array}$

$\begin{array}{lll}-0.7350 & 0.000002 & 0.540\end{array}$

$\begin{array}{lll}1.5909 & -0.000004 & 2.531\end{array}$

$\begin{array}{lll}-0.5972 & 0.000001 & 0.357\end{array}$

$\begin{array}{lll}1.6086 & -0.000003 & 2.588\end{array}$

$\begin{array}{lll}0.0791 & 0.000000 & 0.006\end{array}$

$\begin{array}{lll}1.7150 & -0.000003 & 2.941\end{array}$

$\begin{array}{lll}-0.2318 & 0.000000 & 0.054\end{array}$

$\begin{array}{lll}1.7240 & -0.000003 \quad 2.972\end{array}$

$\begin{array}{lll}1.7506 & -0.000003 \quad 3.065\end{array}$

$0.0085-0.000023-0.000$

$\begin{array}{lll}0.0142 & -0.000019 & 0.000\end{array}$

$0.0213-0.000020 \quad 0.000$

$\begin{array}{lll}0.0301 & -0.000016 & 0.001 \\ 0.0431 & -0.000016 & 0.002\end{array}$

0.002

$13.696 \quad 999.0996$

15.134

9.80

19.71

29.51

39.31

49.14

59.01

68.90

78.83

88.78

98.75

108.75

118.77

128.78

138.80

148.79

158.77

168.69

179.06

188.34
999.0996

998.2041

998.2041

998.2041

998.2041

998.2041

998.2041

998.2041

998.2041

998.2041

998.2041

998.2041

998.2041

998.2041

998.2041

998.2041

98.2041

998.2041

998.2041

998.2041

998.2041

998.2041

998.2041

998.2041

998.2041

998.2041

998.2041

998.2041
998.2041

998.2041

998.2041

998.2041

998.2041

998.2041

998.2041

998.2041

$0.186 \quad 997.0449$

$0.373 \quad 997.0449$

0.551997 .0449

0.921997 .0449

1.372997 .0449 
molality molarity

$\mathbf{t} /{ }^{\circ} \mathrm{C}$ mass frac $\begin{gathered}\text { Density } \\ \exp /\end{gathered} \quad$ Ref $\exp /$
$\mathrm{kg} / \mathrm{m}^{3}$

0.1001

0.124
0.1488

0.2303

0.582

0.608

0.61316

0.69727

0.79917

0.90026

0.96879

1.229

1.867

1.867
1.96232

2.52
3.0147

3.0147

3.191

3.8527

3.878
4.6314

4.9124

5.316

6.0064

6.6253

6.836

7.616

8.447
9.2949

0.2353

11.2696

12.163

13.177
13.857

14.336

14.856

15.288

15.38

16.37

$\begin{array}{lrrr}25 & 0.0036 & 998.85 & (98) \\ 25 & 0.0045 & 999.28 & (98) \\ 25 & 0.0054 & 999.72 & (98) \\ 25 & 0.0083 & 1001.16 & (98) \\ 25 & 0.0208 & 1007.23 & (128) \\ 25 & 0.0217 & 1007.70 & (30) \\ 25 & 0.0219 & 1007.75 & (39) \\ 25 & 0.0248 & 1009.17 & (39) \\ 25 & 0.0283 & 1010.87 & (39) \\ 25 & 0.0318 & 1012.53 & (39) \\ 25 & 0.0341 & 1013.71 & (128) \\ 25 & 0.0429 & 1018.30 & (30) \\ 25 & 0.0445 & 1018.65 & (39) \\ 25 & 0.0637 & 1028.30 & (30) \\ 25 & 0.0668 & 1029.55 & (128) \\ 25 & 0.0841 & 1038.10 & (30) \\ 25 & 0.0990 & 1045.23 & (128) \\ 25 & 0.1042 & 1047.70 & (30) \\ 25 & 0.1232 & 1057.02 & (128) \\ 25 & 0.1239 & 1057.80 & (30) \\ 25 & 0.1445 & 1067.47 & (128) \\ 25 & 0.1519 & 1071.16 & (128) \\ 25 & 0.1624 & 1076.10 & (30) \\ 25 & 0.1797 & 1084.93 & (128) \\ 25 & 0.1946 & 1092.40 & (128) \\ 25 & 0.1995 & 1094.60 & (30) \\ 25 & 0.2173 & 1103.88 & (128) \\ 25 & 0.2355 & 1113.10 & (30) \\ 25 & 0.2531 & 1122.13 & (128) \\ 25 & 0.2718 & 1131.65 & (128) \\ 25 & 0.2912 & 1141.59 & (128) \\ 25 & 0.3072 & 1149.76 & (128) \\ 25 & 0.3245 & 1158.39 & (128) \\ 25 & 0.3357 & 1163.86 & (128) \\ 25 & 0.3433 & 1167.51 & (128) \\ 25 & 0.3514 & 1171.37 & (128) \\ 25 & 0.3579 & 1174.37 & (128) \\ 25 & 0.3593 & 1175.15 & (128) \\ 25 & 0.3738 & 1181.99 & (128) \\ 30 & 0.0200 & 1005.34 & (3) \\ 30 & 0.0400 & 1014.93 & (3) \\ 30 & 0.0600 & 1024.52 & (3) \\ 30 & 0.0800 & 1034.11 & (3) \\ 30 & 0.1000 & 1043.72 & (3) \\ 30 & 0.1200 & 1053.37 & (3) \\ 30 & 0.1400 & 1063.04 & (3) \\ 30 & 0.1600 & 1072.75 & (3) \\ & & & \end{array}$

$0.1600 \quad 1072.75$

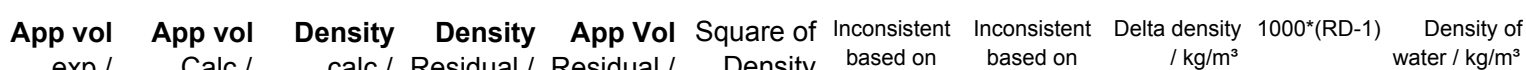
$\exp /$ Calc $/$ $\mathrm{m}^{3} / \mathrm{kg}$ calc/ Residual / Resic ased on $\mathrm{m}^{3} / \mathrm{kg} \quad \mathrm{m}^{3} / \mathrm{kg}$ $\mathrm{kg} / \mathrm{m}^{3}$ $\mathrm{kg} / \mathrm{m}^{3}$ $\mathrm{m}^{3} / \mathrm{kg}$ Residual apparen volume

$0.000504 \quad 0.000518$ $0.000505 \quad 0.000518$

998.80

$0.0509-0.000014$

0.003

$0.000505 \quad 0.000518$

999.22

$0.0586-0.000013$

0.003

$\begin{array}{lll}0.000508 & 0.000518 & 1001.07\end{array}$

$0.0681-0.000013$

0.005

$\begin{array}{lll}0.000515 & 0.000520 & 1007.11\end{array}$

$0.000514 \quad 0.000521$

1007.55

$\begin{array}{ll}0.000516 & 0.000521\end{array}$

$0.0852-0.000010$

0.013

$0.1461-0.000007$

$0.1135-0.000005$

0.021
0.013

0.011

$\begin{array}{lll}0.000517 & 0.000521 & 1009.06\end{array}$

$0.1052-0.000004$

$0.0911-0.000003$

$0.0694-0.000002$

$0.000520-0.0$

$0.000520 \quad 0.000523$

1012.47

$\begin{array}{lll}0.000525 & 0.000524 & 1017.88\end{array}$

$0.000525 \quad 0.000528$

1018.65

$\begin{array}{lll}0.000529 & 0.000528 & 1029.58\end{array}$

0.008

0.005

0.011

0.173

$-0.0062 \quad 0.000000$

$0.2097-0.000003$

$\begin{array}{ll}-0.0348 & 0.000000\end{array}$

0.000

0.044

0.001

0.001

$\begin{array}{lllll}0.000536 & 0.000534 & 1045.46 & -0.2385 & 0.000002\end{array}$

$\begin{array}{ll}-0.3260 & 0.000003\end{array}$

$0.000538 \quad 0.000535$

1045.46

$\begin{array}{lll}0.000541 & 0.000538\end{array}$

$0.000538 \quad 0.000538$

1057.40

$\begin{array}{ll}-0.3260 & 0.000003 \\ -0.3729 & 0.000003\end{array}$

0.057

0.106

0.139

0.003

$\begin{array}{llllll}0.000545 & 0.000542 & 1067.95 & -0.4727 & 0.000003 & 0.223 \\ 0.000546 & 0.000543 & 1071.63 & -0.4756 & 0.000003 & 0.226\end{array}$

0.000549

$\begin{array}{lllll}0.000551 & 0.000549 & 1085.40 & -0.4729 & 0.000002\end{array}$

0.515

0.224

0.154

$\begin{array}{lllll}0.000553 & 0.000551 & 1092.79 & -0.3925 & 0.000002 \\ 0.000555 & 0.000552 & 1095.25 & -0.6464 & 0.000003\end{array}$

$\begin{array}{ll}-0.1937 & 0.000001\end{array}$

$0.0705 \quad 0.000000$

0.418

0.038

0.005

$\begin{array}{lll}0.000559 & 0.000559 & 1113.03\end{array}$

$\begin{array}{lll}0.000561 & 0.000563 & 1121.73\end{array}$

$\begin{array}{lll}0.000564 & 0.000566 & 1130.88\end{array}$

$\begin{array}{lll}0.000567 & 0.000570 & 1140.39\end{array}$

$\begin{array}{lll}0.000569 & 0.000573 & 1148.16\end{array}$

$0.4044-0.000001$

0.164

0.586

$1.2019-0.000003 \quad 1.445$

$1.5980-0.000004 \quad 2.554$

$1.8786-0.000004 \quad 3.529$

$2.0111-0.000004 \quad 4.045$

$2.0297-0.000004 \quad 4.120$

$2.0435-0.000004 \quad 4.176$

$1.9419-0.000004 \quad 3.771$

$2.0696-0.000004 \quad 4.283$

$2.0923-0.000004 \quad 4.378$

$\begin{array}{lll}0.0868 & -0.000004 & 0.008\end{array}$

$\begin{array}{lll}0.0267 & -0.000001 & 0.001\end{array}$

$\begin{array}{lll}-0.0748 & 0.000001 & 0.006\end{array}$

$\begin{array}{lll}-0.2120 & 0.000002 & 0.045\end{array}$

0.129

$\begin{array}{llllll}0.000537 & 0.000535 & 1034.32 & -0.2120 & 0.000002 \\ 0.000542 & 0.000539 & 1044.08 & -0.3586 & 0.000003\end{array}$

$\begin{array}{lll}0.000542 & 0.000539 & 1044.08\end{array}$

$\begin{array}{lll}0.000546 & 0.000542 & 1053.86 \\ 0.000550 & 0.000546 & 1063.65\end{array}$

$\begin{array}{ll}-0.4878 & 0.000004\end{array}$

$\begin{array}{ll}-0.6125 & 0.000004\end{array}$

0.238

0.375

0.497
$1.814 \quad 997.0449$

2.685997 .0449

$4.125 \quad 997.0449$

997.0449

997.0449

997.0449

997.0449

997.0449

997.0449

997.0449

997.0449

997.0449

997.0449

997.0449

997.0449

997.0449

997.0449

997.0449

997.0449

997.0449

997.0449

997.0449

997.0449

997.0449

997.0449

997.0449

997.0449

997.0449

997.0449

997.0449

997.0449

997.0449

997.0449

997.0449

997.0449

997.0449

997.0449

997.0449

995.6473

995.6473

995.6473

995.6473

995.6473

995.6473

995.6473

995.6473
2.241997 .0449 
molality molarity

$\mathrm{t} /{ }^{\circ} \mathrm{C}$ mass frac Density $\exp /$ $\mathrm{kg} / \mathrm{m}^{3}$

$\begin{array}{lll}30 & 0.1800 & 1082.47\end{array}$ $0.2000 \quad 1092.22$ $0.2200 \quad 1102.00$

$0.2400 \quad 1111.79$

$\begin{array}{lll}0.2600 & 1121.57\end{array}$

$0.3000 \quad 1141.12$

$\begin{array}{ll}0.3200 & 1150.87\end{array}$

$0.3400 \quad 1160.56$

$\begin{array}{lll}0.3600 & 1170.73\end{array}$

$0.3800 \quad 1179.77$

$0.0004 \quad 994.21$

$0.0007 \quad 994.40$

$0.0011 \quad 994.57$

$0.0018 \quad 994.94$

$0.0027 \quad 995.36$

$0.0036 \quad 995.82$

$0.0045 \quad 996.24$

$0.0054 \quad 996.68$

$0.0185 \quad 1002.99$

$0.0219 \quad 1004.60$

$0.0255 \quad 1006.32$

$\begin{array}{ll}0.0293 & 1008.12\end{array}$

$0.0363 \quad 1011.46$

$0.0200 \quad 1001.78$

$0.0400 \quad 1011.25$

$0.0600 \quad 1020.69$

$0.0800 \quad 1030.14$

$0.1000 \quad 1039.61$

$0.1200 \quad 1049.10$

$0.1400 \quad 1058.61$

$0.1600 \quad 1068.15$

$\begin{array}{lll}0.1800 & 1077.71\end{array}$

$0.2000 \quad 1087.28$

$0.2200 \quad 1098.67$

$0.2400 \quad 1106.46$

$0.2600 \quad 1116.05$

$0.2800 \quad 1125.63$

$0.3000 \quad 1135.19$

$0.3200 \quad 1144.72$

$0.3400 \quad 1154.19$

$0.3600 \quad 1164.12$

$0.0004 \quad 990.40$

$0.0007 \quad 990.58$

$0.0011 \quad 990.75$
$0.2800 \quad 1131.35$

$\begin{array}{rr}0.0083 & 998.10 \\ 0.0185 & 1002.99\end{array}$

$0.3800 \quad 1172.93$
Ref

App vol App vol

$\mathrm{m}^{3} / \mathrm{kg} \quad \mathrm{m}^{3} / \mathrm{kg}$

$0.000557 \quad 0.000553$

$0.000560 \quad 0.000557$

$0.000564 \quad 0.000561$

$0.000567 \quad 0.000565$

$\begin{array}{lll}0.000571 & 0.000569\end{array}$

$\begin{array}{lll}0.000574 & 0.000572\end{array}$

$\begin{array}{ll}0.000578 & 0.000577\end{array}$

0.0005810 .000581

$0.000585 \quad 0.000585$

$0.000592 \quad 0.000593$

$0.000504 \quad 0.000525$

$0.000501 \quad 0.000525$

$\begin{array}{lll}0.000509 & 0.000525\end{array}$

$0.000508 \quad 0.000525$

$0.000515 \quad 0.000525$

$0.000510 \quad 0.000526$

$0.000510 \quad 0.000526$

$\begin{array}{lll}0.000511 & 0.000526\end{array}$

$0.000514 \quad 0.000526$

$0.000522 \quad 0.000528$

$\begin{array}{lll}0.000523 & 0.000529\end{array}$

$0.000524 \quad 0.000529$

$0.000526 \quad 0.000530$

$\begin{array}{lll}0.000528 & 0.000531\end{array}$

$\begin{array}{lll}0.000527 & 0.000531\end{array}$

$0.000534 \quad 0.000535$

$0.000539 \quad 0.000538$

$0.000544 \quad 0.000542$

$0.000548 \quad 0.000545$

$\begin{array}{lll}0.000552 & 0.000549\end{array}$

$0.000556 \quad 0.000553$

$\begin{array}{lll}0.000560 & 0.000557\end{array}$

$0.000564 \quad 0.000560$

$0.000567 \quad 0.000564$

$0.000564 \quad 0.000568$

$0.000574 \quad 0.000572$

$0.000578 \quad 0.000576$

$0.000581 \quad 0.000580$

$\begin{array}{lll}0.000585 & 0.000584\end{array}$

$0.000588 \quad 0.000588$

$0.000592 \quad 0.000592$

$0.000594 \quad 0.000597$

$0.000599 \quad 0.000601$

$0.000497 \quad 0.000531$

$0.000507 \quad 0.000531$

$0.000508 \quad 0.000531$ exp / Calc /

$\begin{array}{lll}0.000587 & 0.000589\end{array}$
Density Density App Vol Square of Inconsistent
calc / Residual / Residual / Density based on

$\mathrm{kg} / \mathrm{m}^{3} \quad \mathrm{~kg} / \mathrm{m}^{3}$

$\mathrm{m}^{3} / \mathrm{kg}$ Residual

0.619

0.688

0.678

0.607

0.498
0.339

0.166

0.032

0.006

0.833

0.537

0.000

994.38

994.55

995.34

995.76

996.17

996.60

997.99
1002.87

1004.88

1006.19

1008.00

1011.35

1001.69

1011.20

1020.74
1030.32

1039.92

1049.54

1059.17

1068.80

1078.42

1097.61

1107.16

1116.66

1135.48

1144.78

1153.98

1163.08

1172.05

990.56

990.73

$0.1024-0.000012 \quad 0.010$
0.000

0.000

$0.0078-0.000$

$0.0178-0.000024$

$0.0172-0.000016$

$0.0318-0.000017$

0.001

0.001

$0.0550-0.000015-0.003$

$0.0701-0.000016 \quad 0.005$

$\begin{array}{lll}0.0784 & -0.000015 & 0.006\end{array}$

$\begin{array}{lll}0.1196 & -0.000006 & 0.014\end{array}$

$\begin{array}{lll}0.1231-0.000006 & 0.015\end{array}$

$0.1247-0.000005$

$0.1235-0.000004$

$0.1163-0.000003$

$0.0905-0.000005$

$0.0493-0.000001$

$\begin{array}{ll}-0.0581 & 0.000001\end{array}$

$\begin{array}{ll}-0.1857 & 0.000002\end{array}$

$\begin{array}{ll}-0.3170 & 0.000003\end{array}$

$\begin{array}{ll}-0.4452 & 0.000003\end{array}$

$\begin{array}{ll}-0.5630 & 0.000004\end{array}$

$\begin{array}{lll}-0.6525 & 0.000004\end{array}$

$-0.7154 \quad 0.000003$

$-0.7527 \quad 0.000003$

$1.0549-0.000004$

$\begin{array}{ll}-0.7025 & 0.000002\end{array}$

$\begin{array}{lll}-0.6146 & 0.000002\end{array}$

$\begin{array}{ll}-0.4800 & 0.000001\end{array}$

$\begin{array}{ll}-0.2971 & 0.000001\end{array}$

$\begin{array}{ll}-0.0636 & 0.000000\end{array}$

$0.2034 \quad 0.000000$

$8731-0.000002$

$0.0120-0.000034$

$0.0174-0.000024$

$0.0240-0.000022$
0.015
0.016

0.015

0.014

0.008

0.002

0.003

0.034

0.100

0.198

0.317

0.426

0.512

0.567

1.113

0.494

0.378

0.230

0.088

0.004

0.041

1.077

0.762

0.000
0.000

0.001 $\begin{array}{lrr}\text { nconsistent } & \text { Delta density } & 1000^{*}(\mathrm{RD}-1) \\ \text { based on } & / \mathrm{kg} / \mathrm{m}^{3} & \begin{array}{r}\text { Density of } \\ \text { water } / \mathrm{kg} / \mathrm{m}^{3}\end{array}\end{array}$ volume

86.82

96.57

106.35

116.14

125.92

135.70

145.47

155.22

164.91

175.08

184.12

573

12.284

14.093

17.432

9.56

19.03

28.47

37.92

47.39

56.88

66.39

75.93

85.49

106.45

114.24

123.83

133.41

142.97

152.50

161.97

171.90

180.71

$3 / 28 / 2004$

995.6473

995.6473

995.6473

995.6473

995.6473

995.6473

995.6473

995.6473

995.6473

995.6473

995.6473

0.183994 .0319

$0.372 \quad 994.0319$

$0.54 \quad 994.0319$

0.911994 .0319

$1.34 \quad 994.0319$

$1.795 \quad 994.0319$

$2.224 \quad 994.0319$

$2.661 \quad 994.0319$

4.089994 .0319

994.0319

994.0319

994.0319

994.0319

994.0319

992.2158

992.2158

992.2158

992.2158

992.2158

992.2158

992.2158 


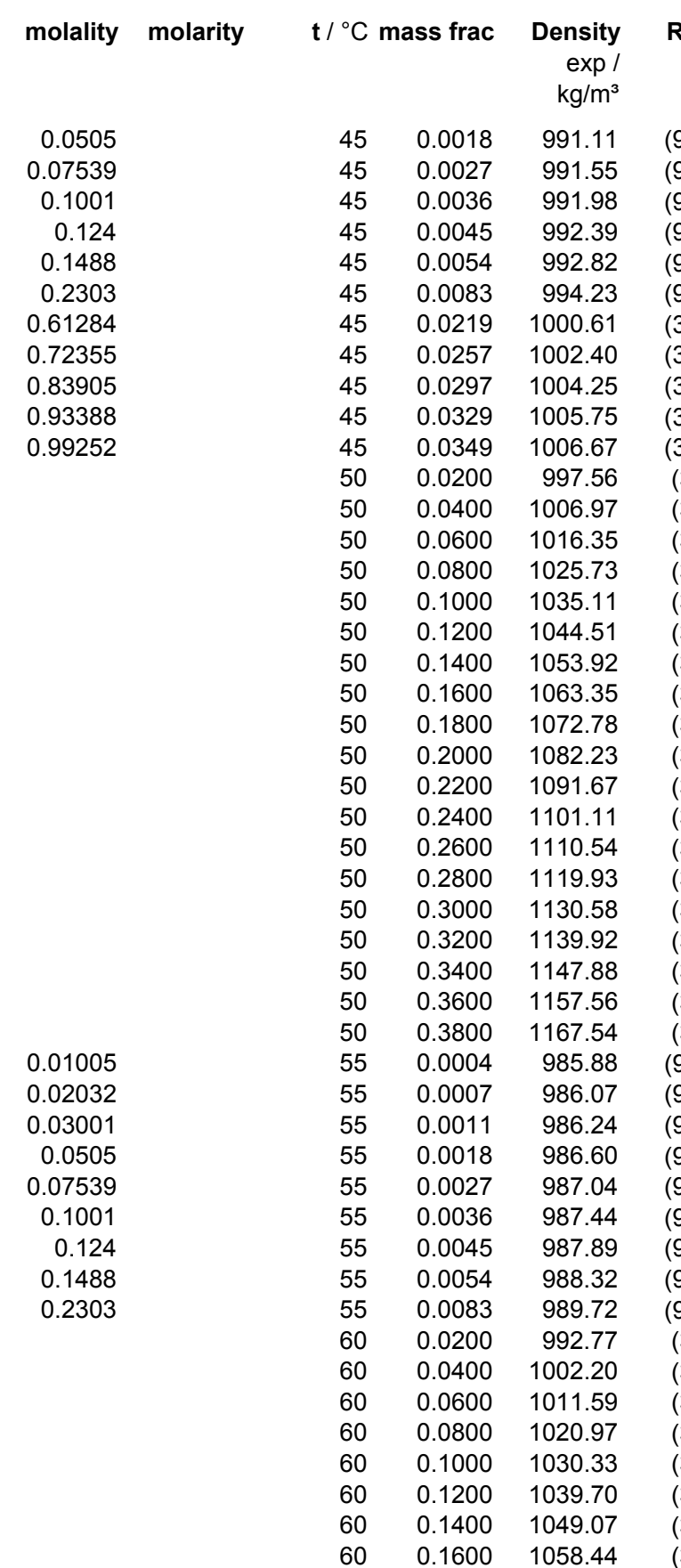

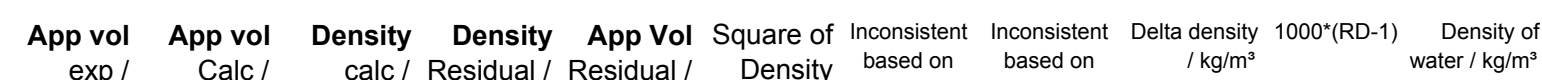
exp/ Calc/ calc/ Residual/ Residual / Density based on based on$$
\mathrm{m}^{3} / \mathrm{kg} \quad \mathrm{m}^{3} / \mathrm{kg}
$$
$\mathrm{kg} / \mathrm{m}^{3}$

$$
\mathrm{kg} / \mathrm{m}^{3}
$$

$\mathrm{m}^{3} / \mathrm{kg}$

991.08

$0.000513 \quad 0.000531$

$0.000515 \quad 0.000531$

991.50
991.92

$0.0352-0.000020$

$\begin{array}{lll}0.0491 & -0.000018 \\ 0.0599 & -0.000017\end{array}$

0.001

$0.000518 \quad 0.000531$

992.33

$0.0613-0.000014$

0.002
0.004

$0.000518 \quad 0.000532$

$0.000520 \quad 0.000532$

$0.000530 \quad 0.000534$

$0.000532 \quad 0.000535$

992.75

994.13

1000.51
1002.33

$0.000534 \quad 0.000536$

$0.000536 \quad 0.000536$

$0.000537 \quad 0.000537$

$0.000529 \quad 0.000536$

1004.21

1005.75

$0.000536 \quad 0.000540$

$\begin{array}{ll}0.000542 & 0.000543\end{array}$

$\begin{array}{ll}0.000547 & 0.000547\end{array}$

$0.000552 \quad 0.000550$

$0.000556 \quad 0.000554$

$0.000560 \quad 0.000558$

$0.000564 \quad 0.000561$

$0.000568 \quad 0.000565$

$0.000572 \quad 0.000569$

$\begin{array}{lll}0.000575 & 0.000573\end{array}$

$\begin{array}{lll}0.000579 & 0.000577\end{array}$

$0.000583 \quad 0.000581$

$0.000586 \quad 0.000585$

$\begin{array}{lll}0.000587 & 0.000589\end{array}$

$\begin{array}{lll}0.000591 & 0.000593\end{array}$

$\begin{array}{lll}0.000598 & 0.000597\end{array}$

$0.000600 \quad 0.000602$

$0.000603 \quad 0.000606$

$0.000494 \quad 0.000534$

$0.000497 \quad 0.000534$

$0.000499 \quad 0.000534$

$0.000507 \quad 0.000534$

$0.000510 \quad 0.000535$

$0.000522 \quad 0.000535$

$0.000514 \quad 0.000535$

$0.000515 \quad 0.000535$

$0.000519 \quad 0.000536$

$\begin{array}{lll}0.000527 & 0.000539\end{array}$

$0.000535 \quad 0.000542$

$0.000541 \quad 0.000546$

$0.000547 \quad 0.000549$

$\begin{array}{lll}0.000552 & 0.000553\end{array}$

$\begin{array}{lll}0.000556 & 0.000557\end{array}$

$\begin{array}{ll}0.000561 & 0.000560\end{array}$

$0.000565 \quad 0.000564$
$0.0737-0.000014-0.005$

$\begin{array}{ll}0.0953-0.000012 & 0.009\end{array}$

$0.1020-0.000005 \quad 0.010$

$0.0735-0.000003 \quad 0.005$

$\begin{array}{lll}0.0381 & -0.000001 & 0.001\end{array}$

$\begin{array}{lll}0.0039 & 0.000000 & 0.000\end{array}$

$\begin{array}{lll}-0.0198 & 0.000001 & 0.000\end{array}$

$\begin{array}{lll}0.1374 & -0.000007 & 0.019\end{array}$

$0.1255-0.000003-0.016$

$\begin{array}{lll}0.0500 & -0.000001 & 0.002\end{array}$

$\begin{array}{lll}-0.0533 & 0.000001 & 0.003\end{array}$

$\begin{array}{lll}-0.1778 & 0.000002 & 0.032\end{array}$

$\begin{array}{lll}-0.2965 & 0.000002 & 0.088\end{array}$

$\begin{array}{lll}-0.4122 & 0.000003 & 0.170\end{array}$

$\begin{array}{lll}-0.5070 & 0.000003 & 0.257\end{array}$

$\begin{array}{lll}-0.6396 & 0.000003 & 0.409\end{array}$

$\begin{array}{lll}-0.6691 & 0.000003 & 0.448\end{array}$

$\begin{array}{lll}-0.6609 & 0.000002 & 0.437\end{array}$

$\begin{array}{lll}-0.6146 & 0.000002 & 0.378\end{array}$

$\begin{array}{lll}-0.5490 & 0.000002 & 0.301\end{array}$

$\begin{array}{lll}0.8477 & -0.000002 & 0.719\end{array}$

$\begin{array}{lll}1.0177-0.000002 & 1.036\end{array}$

$\begin{array}{lll}-0.0961 & 0.000000 & 0.009\end{array}$

$0.6199-0.000001 \quad 0.384$

$1.7597-0.000003 \quad 3.097$

$0.0144-0.000040 \quad 0.000$

$\begin{array}{lll}0.0271 & -0.000038 & 0.001\end{array}$

$\begin{array}{lll}0.0379 & -0.000036 & 0.001\end{array}$

$0.0499-0.000028 \quad 0.002$

$\begin{array}{lll}0.0657 & -0.000025 & 0.004\end{array}$

$\begin{array}{lll}0.0459 & -0.000013 & 0.002\end{array}$

$\begin{array}{lll}0.0935 & -0.000021 & 0.009\end{array}$

$\begin{array}{lll}0.1050 & -0.000020 & 0.011\end{array}$

$\begin{array}{lll}0.1317 & -0.000016 & 0.017\end{array}$

$\begin{array}{lll}0.2325 & -0.000012 & 0.054\end{array}$

$\begin{array}{lll}0.2857 & -0.000007 & 0.082\end{array}$

$0.2652-0.000004-0.070$

$\begin{array}{lll}0.2069 & -0.000002 & 0.043\end{array}$

$\begin{array}{lll}0.1072 & -0.000001 & 0.011\end{array}$

$\begin{array}{lll}0.0031 & 0.000000 & 0.000\end{array}$

$\begin{array}{lll}-0.1081 & 0.000001 & 0.012\end{array}$

$\begin{array}{ll}-0.2187 & 0.000001\end{array}$

0.048 $\begin{array}{lll}-0.5924 & 0.000003 & 0.351\end{array}$ volume
$0.908 \quad 990.2132$

$\begin{array}{ll}1.351 \quad 990.2132 \\ 1.787 & 990.2132\end{array}$

$1.787 \quad 990.2132$

2.199990 .2132

$2.637 \quad 990.2132$

$4.052 \quad 990.2132$

990.2132

990.2132

990.2132

990.2132

990.2132

988.0363

988.0363

988.0363

988.0363

988.0363

988.0363

988.0363

988.0363

988.0363

988.0363

988.0363

988.0363

988.0363

988.0363

988.0363

988.0363

988.0363

988.0363

988.0363

169.52

179.50

$0.188 \quad 985.6952$

$0.378 \quad 985.6952$

0.556985 .6952

0.921985 .6952

1.365985 .6952

1.769985 .6952

$2.227 \quad 985.6952$

2.663985 .6952

$4.08 \quad 985.6952$

983.1989

983.1989

983.1989

983.1989

983.1989

983.1989

983.1989

983.1989 
molality molarity t $/{ }^{\circ} \mathrm{C}$ mass frac $\begin{array}{r}\text { Density } \\ \exp / \\ \mathrm{kg} / \mathrm{m}^{3}\end{array}$

$\begin{array}{lll}60 & 0.1800 & 1067.80\end{array}$ $\begin{array}{lll}60 & 0.2000 & 1077.16\end{array}$

$\begin{array}{lll}60 & 0.2200 & 1086.50\end{array}$

$\begin{array}{lll}60 & 0.2400 & 1095.82\end{array}$

$\begin{array}{lll}60 & 0.2600 & 1105.10\end{array}$

$\begin{array}{lll}60 & 0.2800 & 1114.35\end{array}$

$\begin{array}{lll}60 & 0.3000 & 1124.80\end{array}$

$\begin{array}{lll}60 & 0.3200 & 1132.66\end{array}$

$\begin{array}{lll}60 & 0.3400 & 1141.62\end{array}$

$\begin{array}{lll}60 & 0.3600 & 1151.12\end{array}$

$\begin{array}{llr}70 & 0.0200 & 987.46\end{array}$

$\begin{array}{rrr}70 & 0.0400 & 997.00\end{array}$

$0.0600 \quad 1006.47$

$0.0800 \quad 1015.91$

$0.1000 \quad 1025.32$

$0.1200 \quad 1034.72$

$0.1400 \quad 1044.10$

$0.1600 \quad 1053.46$

$0.1800 \quad 1062.80$

$0.2000 \quad 1072.11$

$0.2200 \quad 1081.39$

$0.2400 \quad 1090.62$

$0.2600 \quad 1099.80$

$0.2800 \quad 1108.90$

$0.3000 \quad 1117.93$

$0.3200 \quad 1126.86$

$0.3400 \quad 1135.66$

$0.3600 \quad 1144.83$

$\begin{array}{ll}0.0200 & 981.71\end{array}$

$0.0400 \quad 991.41$

$0.0600 \quad 1001.03$

$0.0800 \quad 1010.59$

$0.1000 \quad 1019.83$

$0.1200 \quad 1029.59$

$0.1400 \quad 1039.04$

$0.1600 \quad 1048.45$

$0.1800 \quad 1057.81$

$0.2000 \quad 1067.12$

$0.2200 \quad 1076.37$

$0.2400 \quad 1085.54$

$0.2600 \quad 1094.63$

$0.2800 \quad 1103.63$

$0.3000 \quad 1112.50$

$0.3200 \quad 1121.24$

$0.3400 \quad 1129.82$

$0.3600 \quad 1138.74$

$0.0100 \quad 963.60$
App vol App vol

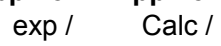

$\mathrm{m}^{3} / \mathrm{kg} \quad \mathrm{m}^{3} / \mathrm{kg}$

$0.000569 \quad 0.000568$

$\begin{array}{ll}0.000573 & 0.000572\end{array}$

$0.000578 \quad 0.000576$

$0.000582 \quad 0.000580$

$0.000586 \quad 0.000584$

$0.000590 \quad 0.000588$

$\begin{array}{ll}0.000590 & 0.000592\end{array}$

$0.000598 \quad 0.000596$

$0.000602 \quad 0.000600$

$0.000605 \quad 0.000605$

$\begin{array}{ll}0.000521 & 0.000539\end{array}$

$0.000530 \quad 0.000543$

$0.000537 \quad 0.000546$

$0.000543 \quad 0.000550$

$\begin{array}{ll}0.000548 & 0.000554\end{array}$

$0.000554 \quad 0.000557$

$0.000559 \quad 0.000561$

$0.000563 \quad 0.000565$

$\begin{array}{lll}0.000568 & 0.000569\end{array}$

$0.000573 \quad 0.000572$

$0.000577 \quad 0.000576$

$\begin{array}{lll}0.000582 & 0.000580\end{array}$

$0.000586 \quad 0.000584$

$0.000591 \quad 0.000588$

$0.000595 \quad 0.000593$

$0.000600 \quad 0.000597$

$0.000605 \quad 0.000601$

$0.000608 \quad 0.000605$

$0.000510 \quad 0.000539$

$0.000520 \quad 0.000542$

$0.000528 \quad 0.000546$

$0.000535 \quad 0.000549$

$0.000544 \quad 0.000553$

$0.000548 \quad 0.000556$

$0.000553 \quad 0.000560$

$\begin{array}{lll}0.000559 & 0.000564\end{array}$

$0.000564 \quad 0.000568$

$0.000569 \quad 0.000571$

$0.000575 \quad 0.000575$

$0.000580 \quad 0.000579$

$0.000585 \quad 0.000583$

$0.000590 \quad 0.000587$

$0.000595 \quad 0.000592$

$0.000600 \quad 0.000596$

$0.000606 \quad 0.000600$

$0.000610 \quad 0.000604$

$0.000476 \quad 0.000531$
Density Density App Vol Square of Inconsistent $\begin{gathered}\text { Inconsistent } \\ \text { based on }\end{gathered}$ Delta density $1000^{*}(\mathrm{RD}-1) \begin{array}{r}\text { Density of } \\ \text { calc / Residual / Residual / }\end{array}$ Density based on $/ \mathrm{kg} / \mathrm{m}^{3}$ calc / Residual / Residual / Density based on based on $\mathrm{kg} / \mathrm{m}^{3}$

$\mathrm{m}^{3} / \mathrm{kg}$ Residual

volume

84.60

93.96

103.30

112.62

121.90

131.15

141.60

149.46

158.42

167.92

9.69

19.23

28.70

38.14

47.55

56.95

66.33

75.69

85.03

94.34

103.62

112.85

122.03

131.13

140.16

149.09

157.89

167.06

9.91

19.61

29.23

38.79

48.03

57.79

67.24

76.65

86.01

95.32

104.57

113.74

122.83

131.83

140.70

149.44

158.02

166.94

$\begin{array}{ll}-2.5113 & 0.000006 \\ -2.6819 & 0.000006\end{array}$

$\begin{array}{lll}0.5036 & -0.000054\end{array}$
3.622

6.307

0.254
983.1989

983.1989

983.1989

983.1989

983.1989

983.1989

983.1989

983.1989

983.1989

983.1989

977.7696

977.7696

977.7696

977.7696

977.7696

977.7696

977.7696

977.7696

977.7696

977.7696

977.7696

977.7696

977.7696

977.7696

977.7696

977.7696

977.7696

977.7696

971.7978

971.7978

971.7978

971.7978

971.7978

971.7978

971.7978

971.7978

971.7978

971.7978

971.7978

971.7978

971.7978

971.7978

971.7978

971.7978

971.7978

971.7978

958.3637 


\section{molality molarity}

$\mathrm{t} /{ }^{\circ} \mathrm{C}$ mass frac Density $\exp /$ $\mathrm{kg} / \mathrm{m}^{3}$

$\begin{array}{lll}100 & 0.0200 & 968.80\end{array}$

$\begin{array}{lll}100 & 0.0400 & 979.10 \\ 100 & 0.0600 & 989.20\end{array}$

$\begin{array}{lll}100 & 0.0800 \quad 999.20\end{array}$

$\begin{array}{lll}100 & 0.1000 & 1009.00\end{array}$

$\begin{array}{lll}100 & 0.1200 & 1018.80\end{array}$

$\begin{array}{lll}100 & 0.1400 & 1028.60 \\ 100 & 0.1600 & 1038.30\end{array}$

$\begin{array}{lll}100 & 0.1600 & 1038.30\end{array}$

$\begin{array}{lll}100 & 0.1800 & 1047.90\end{array}$

$\begin{array}{lll}100 & 0.2000 & 1057.40\end{array}$

$\begin{array}{lll}100 & 0.2200 & 1066.80\end{array}$

$\begin{array}{lll}100 & 0.2400 & 1076.10\end{array}$

$0.2600-1085.30$

$\begin{array}{lll}100 & 0.2800 & 1094.20\end{array}$

$\begin{array}{lll}100 & 0.3000 & 1103.00\end{array}$
Ref

(90)

$(90)$
$(90)$

$(90)$
$(90)$

$(90)$
$(90)$

$(90)$
$(90)$

(90)

$(90)$
$(90)$

$(90)$
$(90)$

$(90)$
$(90)$

$(90)$
$(90)$

$(90)$
$(90)$
App vol App vol
exp / Calc /

$\begin{array}{cc}\exp / & \text { Calc } / \\ \mathrm{m}^{3} / \mathrm{kg} & \mathrm{m}^{3} / \mathrm{kg}\end{array}$

$\begin{array}{ll}0.000481 & 0.000532\end{array}$

$0.000491 \quad 0.000536$

$0.000501 \quad 0.000539$

$0.000510 \quad 0.000543$

$0.000520 \quad 0.000546$

$0.000528 \quad 0.000550$

$0.000535 \quad 0.000554$

$0.000541 \quad 0.000557$

$0.000548 \quad 0.000561$

$0.000555 \quad 0.000565$

$\begin{array}{lll}0.000561 & 0.000569\end{array}$

$\begin{array}{lll}0.000568 & 0.000573\end{array}$

$0.000574 \quad 0.000577$

$0.000581 \quad 0.000581$

$0.000587 \quad 0.000585$

Average Res

Std dev Res

Avg - 4std

Avg + 4std

967.84

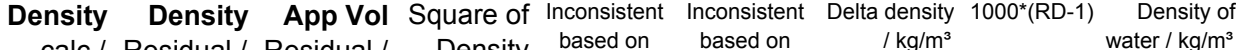
calc / Residual / Residual / Density based on based on based on
apparent

$/ \mathrm{kg} / \mathrm{m}^{3}$ $\mathrm{kg} / \mathrm{m}^{3}$

$\mathrm{m}^{3} / \mathrm{kg}$ Residual density volume

958.3637

958.3637

958.3637

958.3637

958.3637

958.3637

958.3637

958.3637

958.3637

958.3637

958.3637

958.3637

958.3637

958.3637

958.3637

Curve fit for the model App vol $=\left(w+c 2+c 3^{*} t{ }^{\circ} \mathrm{C}\right) /\left(c 0^{*} w+c 1\right) / e^{\wedge}\left(0.000001^{*}\left(t{ }^{\circ} \mathrm{C}+c 4\right)^{\wedge} 2\right)$

c0 -80.0605

c1

255.4204

118.4171

c3 $\quad 1.01639$

c4 2619.464

Min

$\operatorname{Max} T$

Min $w$

-5
100

Max w

100

0.4

Avg dens res $\quad 0.0889$

Std dens res $\quad 0.849655$

Avg app vol res *10^3 $\quad-0.00566$

Std app vol res *10^3 0.010245

No of points in corr

331

No of inconsistent poir

Inconsistent data not used (Residual greater than average $+/-4$ standard deviations) 
Density of an aqueous solution of $\mathrm{HCN}$

References (53) Kortüm, G., Reber, H., Density, viscosity, and dielectric constant of pure hydrocyanic acid and of hydrocyanic acid - water mixtures at $0^{\circ}$, Z. Elektrochem. , 1961, 65, 809

(90) Perry, R. H.; Green, D. W. Perry's Chemical Engineers' Handbook; McGraw Hill: New York, 7th edition, 1997.

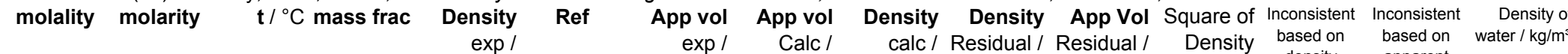

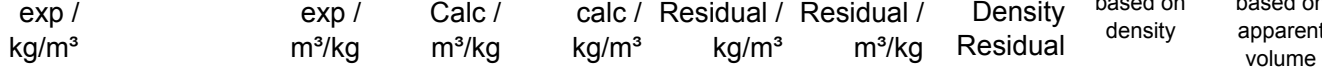

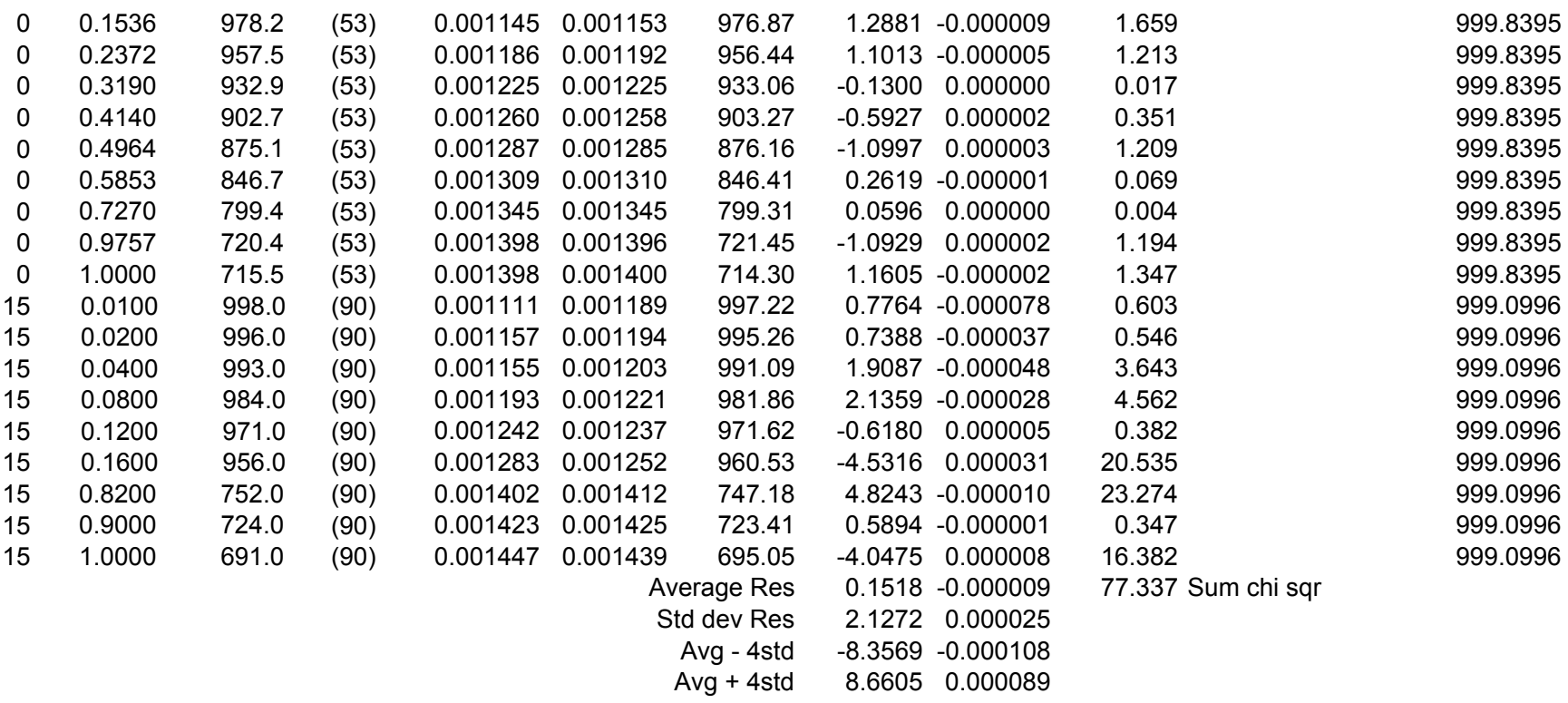

Curve fit for the model App vol= $\left(w+c 2+c 3^{*} t{ }^{\circ} \mathrm{C}\right) /\left(c 0^{*} w+c 1\right) / e^{\wedge}\left(0.000001^{*}\left(t^{\circ} \mathrm{C}+c 4\right)^{\wedge} 2\right)$

$\begin{array}{lr}\text { c0 } & 255.8148 \\ \text { c1 } & 283.1092 \\ \text { c2 } & 0.668883 \\ \text { c4 } & 0.006206 \\ \text { Min T } & 891.0008 \\ \text { Max T } & 0 \\ \text { Min w } & 15 \\ \text { Max w } & 0.0100 \\ \text { Avg dens res } & 1 \\ \text { Std dens res } & 0.151819 \\ \text { Avg app vol res *10^3 } & 2.12717 \\ \text { Std app vol res *10^3 } & 0.00933 \\ \text { No of points in corr } & 18 \\ \text { No of inconsistent poir } & 0\end{array}$

Inconsistent data not used (Residual greater than average $+/-4$ standard deviations) 
Density of aqueous solutions of HNO3

References (35) Haase, R.; Saurmann, P.-F.; Dücker, K.-H. Conductivities of Concentrated Electrolyte Solutions. II. Nitric Acid. Z. Physik. Chem. N. F. $1965,46,129-139$.

(82) Patterson, B. A.; Woolley, E. M.; Thermodynamics for lonization of Water at Temperatures from $278.15 \mathrm{~K}$ to $393.15 \mathrm{~K}$ and at the Pressure 0.35 MPa: Apparent Molar Volumes and Apparent Molar Heat Capacities of Aqueous Solutions of Potassium and Sodium Nitrates and Nitric Acid, J. Chem. Thermodynamics, 2002, 34, 535 - 556

(90) Perry, R. H.; Green, D. W. Perry's Chemical Engineers' Handbook; McGraw Hill: New York, 7th edition, 1997.

\begin{tabular}{|c|c|c|c|c|c|c|c|c|c|c|c|c|}
\hline \multirow[t]{37}{*}{ molality } & \multicolumn{2}{|c|}{$\mathrm{t} /{ }^{\circ} \mathrm{C}$ mass frac } & $\begin{array}{r}\text { Density } \\
\exp / \\
\mathrm{kg} / \mathrm{m}^{3}\end{array}$ & Ref & $\begin{array}{r}\text { App vol } \\
\exp / \\
\mathrm{m}^{3} / \mathrm{kg}\end{array}$ & $\begin{array}{r}\text { App vol } \\
\text { Calc / } \\
\mathrm{m}^{3} / \mathrm{kg}\end{array}$ & $\begin{array}{r}\text { Density } \\
\text { calc / } \\
\mathrm{kg} / \mathrm{m}^{3}\end{array}$ & $\begin{array}{r}\text { Density } \\
\text { Residual / } \\
\mathrm{kg} / \mathrm{m}^{3}\end{array}$ & $\begin{array}{r}\text { App Vol } \\
\text { Residual / } \\
\mathrm{m}^{3} / \mathrm{kg}\end{array}$ & $\begin{array}{r}\text { Square of } \\
\text { Density } \\
\text { Residual }\end{array}$ & $\begin{array}{l}\text { Inconsistent } \\
\text { based on } \\
\text { density }\end{array}$ & $\begin{array}{c}\text { Inconsistent } \\
\text { based on } \\
\text { apparent } \\
\text { volume }\end{array}$ \\
\hline & -10 & 0.1810 & 1116.9 & (35) & 0.000413 & 0.000435 & 1112.0 & 4.8715 & -0.000022 & 23.732 & & \\
\hline & -10 & 0.2212 & 1143.7 & (35) & 0.000425 & 0.000441 & 1139.3 & 4.4355 & -0.000015 & 19.674 & & \\
\hline & -10 & 0.2588 & 1170.8 & (35) & 0.000431 & 0.000446 & 1165.3 & 5.5007 & -0.000016 & 30.258 & & \\
\hline & -10 & 0.2957 & 1195.4 & (35) & 0.000443 & 0.000452 & 1191.3 & 4.0806 & -0.000010 & 16.652 & & \\
\hline & -10 & 0.3308 & 1222.6 & (35) & 0.000446 & 0.000458 & 1216.4 & 6.1625 & -0.000013 & 37.976 & & \\
\hline & -10 & 0.3625 & 1246.4 & (35) & 0.000451 & 0.000464 & 1239.4 & 7.0338 & -0.000013 & 49.475 & & \\
\hline & -10 & 0.4000 & 1271.9 & (35) & 0.000463 & 0.000471 & 1266.7 & 5.2093 & -0.000008 & 27.137 & & \\
\hline & -10 & 0.4267 & 1292.2 & (35) & 0.000468 & 0.000476 & 1286.2 & 5.9931 & -0.000008 & 35.917 & & \\
\hline & -10 & 0.4508 & 1308.6 & (35) & 0.000475 & 0.000481 & 1303.8 & 4.7888 & -0.000006 & 22.932 & & \\
\hline & -10 & 0.4842 & 1332.4 & (35) & 0.000483 & 0.000488 & 1328.1 & 4.3053 & -0.000005 & 18.535 & & \\
\hline & -10 & 0.4985 & 1341.8 & (35) & 0.000487 & 0.000491 & 1338.4 & 3.3819 & -0.000004 & 11.437 & & \\
\hline & -10 & 0.5497 & 1374.8 & (35) & 0.000503 & 0.000503 & 1374.8 & 0.0384 & 0.000000 & 0.001 & & \\
\hline & -10 & 0.6003 & 1403.2 & (35) & 0.000520 & 0.000515 & 1409.2 & -5.9812 & 0.000005 & 35.775 & & \\
\hline & -10 & 0.7011 & 1459.1 & (35) & 0.000550 & 0.000543 & 1469.8 & -10.6697 & 0.000007 & 113.842 & $!$ & \\
\hline & -10 & 0.7533 & 1500.4 & (35) & 0.000557 & 0.000560 & 1494.6 & 5.7644 & -0.000003 & 33.228 & & \\
\hline & -10 & 0.8011 & 1508.8 & (35) & 0.000579 & 0.000577 & 1511.9 & -3.0523 & 0.000002 & 9.316 & & \\
\hline & 0 & 0.0100 & 1005.8 & (90) & 0.000407 & 0.000424 & 1005.6 & 0.1683 & -0.000017 & 0.028 & & \\
\hline & 0 & 0.0200 & 1011.7 & (90) & 0.000414 & 0.000425 & 1011.5 & 0.2327 & -0.000011 & 0.054 & & \\
\hline & 0 & 0.0300 & 1017.6 & (90) & 0.000418 & 0.000426 & 1017.3 & 0.2537 & -0.000008 & 0.064 & & \\
\hline & 0 & 0.0306 & 1018.3 & (35) & 0.000408 & 0.000427 & 1017.7 & 0.5878 & -0.000019 & 0.346 & & \\
\hline & 0 & 0.0391 & 1023.2 & (35) & 0.000417 & 0.000428 & 1022.8 & 0.4425 & -0.000011 & 0.196 & & \\
\hline & 0 & 0.0400 & 1023.6 & (90) & 0.000420 & 0.000428 & 1023.3 & 0.3315 & -0.000008 & 0.110 & & \\
\hline & 0 & 0.0500 & 1029.6 & (90) & 0.000422 & 0.000429 & 1029.2 & 0.3662 & -0.000007 & 0.134 & & \\
\hline & 0 & 0.0700 & 1041.8 & (35) & 0.000425 & 0.000431 & 1041.3 & 0.5129 & -0.000007 & 0.263 & & \\
\hline & 0 & 0.0900 & 1054.3 & (35) & 0.000426 & 0.000434 & 1053.5 & 0.7834 & -0.000008 & 0.614 & & \\
\hline & 0 & 0.1000 & 1060.6 & (90) & 0.000427 & 0.000435 & 1059.7 & 0.8993 & -0.000008 & 0.809 & & \\
\hline & 0 & 0.1100 & 1066.9 & (35) & 0.000429 & 0.000436 & 1065.9 & 0.9796 & -0.000008 & 0.960 & & \\
\hline & 0 & 0.1397 & 1086.2 & (35) & 0.000431 & 0.000440 & 1084.6 & 1.5652 & -0.000010 & 2.450 & & \\
\hline & 0 & 0.1500 & 1092.7 & (90) & 0.000434 & 0.000442 & 1091.2 & 1.4921 & -0.000008 & 2.226 & & \\
\hline & 0 & 0.1600 & 1099.2 & (35) & 0.000435 & 0.000443 & 1097.6 & 1.5704 & -0.000008 & 2.466 & & \\
\hline & 0 & 0.1810 & 1112.9 & (35) & 0.000439 & 0.000446 & 1111.2 & 1.6594 & -0.000007 & 2.753 & & \\
\hline & 0 & 0.2000 & 1125.5 & (35) & 0.000442 & 0.000449 & 1123.7 & 1.8026 & -0.000007 & 3.249 & & \\
\hline & 0 & 0.2000 & 1125.5 & (90) & 0.000442 & 0.000449 & 1123.7 & 1.8026 & -0.000007 & 3.249 & & \\
\hline & 0 & 0.2212 & 1139.7 & (35) & 0.000445 & 0.000452 & 1137.7 & 1.9513 & -0.000007 & 3.807 & & \\
\hline & 0 & 0.2400 & 1152.5 & (35) & 0.000448 & 0.000455 & 1150.3 & 2.1634 & -0.000007 & 4.680 & & \\
\hline & 0 & 0.2500 & 1159.4 & (90) & 0.000450 & 0.000457 & 1157.1 & 2.3215 & -0.000007 & 5.389 & & \\
\hline
\end{tabular}

Apparent Density of molal water / $\mathrm{cm} / \mathrm{mol} \quad \mathrm{kg} / \mathrm{m}^{3}$ 998.117 998.117 998.117 998.117 998.117 998.117 998.117 998.117 998.117 998.117 998.117 998.117 998.117 998.117 998.117 998.117 999.8395 999.8395 999.8395 999.8395 999.8395 999.8395 999.8395 999.8395 999.8395 999.8395 999.8395 999.8395 999.8395 999.8395 999.8395 999.8395 999.8395 999.8395 999.8395 999.8395 
$t /{ }^{\circ} \mathrm{C}$ mass

0.01657

0.03313

0.06637

0.13233

0.27635

0.5498

\section{Density}

exp

$\mathrm{kg} / \mathrm{m}^{3}$

0

$0.2800 \quad 1180.3$

$0.3000 \quad 1194.5$

$0.3308 \quad 1216.6$

$0.3500 \quad 1230.6$

$0.3625 \quad 1239.4$

$0.4000 \quad 1264.9$

$0.4000 \quad 1264.9$

$0.4267 \quad 1283.2$

$0.4500 \quad 1299.0$

$0.4508 \quad 1299.6$

$0.4842 \quad 1322.4$

$0.4985 \quad 1331.8$

$0.5000 \quad 1332.7$

$0.5200 \quad 1345.4$

$0.5497 \quad 1363.8$

$0.5500 \quad 1364.0$

$0.5700 \quad 1375.9$

$0.6000 \quad 1393.1$

$0.6003 \quad 1393.2$

$0.7011 \quad 1444.0$

$0.8011 \quad 1484.8$

$0.0010 \quad 1000.55$

$0.0021 \quad 1001.14$

$0.0042 \quad 1002.38$

$0.0083 \quad 1004.66$

$0.0100 \quad 1005.7$

$0.0171 \quad 1009.81$

$0.0200 \quad 1011.5$

$0.0300 \quad 1017.3$

$0.0335 \quad 1019.28$

$0.0400 \quad 1023.2$

$0.0500 \quad 1029.0$

$0.1000 \quad 1059.4$

$0.1500 \quad 1090.9$

$0.2000 \quad 1123.4$

$0.2500 \quad 1156.9$

$0.3000 \quad 1191.7$

$0.3500 \quad 1227.5$

$0.4000 \quad 1261.3$

$0.4500 \quad 1294.7$

$0.5000 \quad 1327.7$

$0.5500 \quad 1358.3$
Ref

App vol App vol

$$
\mathrm{m}^{3} / \mathrm{kg} \quad \mathrm{m}^{3} / \mathrm{kg}
$$

$0.000454 \quad 0.000461$

$0.000456 \quad 0.000464$

$0.000457 \quad 0.000465$

$0.000458 \quad 0.000466$

$0.000461 \quad 0.000470$

$0.000464 \quad 0.000473$

$\begin{array}{lll}0.000467 & 0.000475\end{array}$

$0.000476 \quad 0.000482$

$\begin{array}{lll}0.000476 & 0.000482\end{array}$

$\begin{array}{lll}0.000483 & 0.000488\end{array}$

$0.000488 \quad 0.000492$

$\begin{array}{ll}0.000488 & 0.000492\end{array}$

$0.000496 \quad 0.000499$

$0.000500 \quad 0.000503$

$\begin{array}{ll}0.000501 & 0.000503\end{array}$

$0.000506 \quad 0.000507$

$0.000515 \quad 0.000514$

$0.000515 \quad 0.000514$

$\begin{array}{ll}0.000521 & 0.000519\end{array}$

$0.000530 \quad 0.000527$

$\begin{array}{ll}0.000530 & 0.000527\end{array}$

$\begin{array}{lll}0.000561 & 0.000555\end{array}$

$0.000592 \quad 0.000589$

0.0004390 .000429

$0.000436 \quad 0.000429$

$0.000422 \quad 0.000429$

$0.000434 \quad 0.000430$

$0.000428 \quad 0.000430$

$0.000430 \quad 0.000431$

$0.000430 \quad 0.000431$

$0.000432 \quad 0.000432$

$0.000434 \quad 0.000432$

0.0004330 .000433

$0.000435 \quad 0.000435$

$0.000439 \quad 0.000441$

$0.000444 \quad 0.000448$

$\begin{array}{lll}0.000451 & 0.000455\end{array}$

$\begin{array}{ll}0.000457 & 0.000462\end{array}$

$0.000464 \quad 0.000470$

$\begin{array}{ll}0.000470 & 0.000479\end{array}$

$\begin{array}{lll}0.000482 & 0.000488\end{array}$

$0.000494 \quad 0.000498$

$0.000506 \quad 0.000509$

$0.000520 \quad 0.000520$
Density Density App Vol Square of Inconsistent Inconsistent calc / Residual / Residual / Density based on based on density apparen

$\begin{array}{llll}1177.5 & 2.8177 & -0.000007 & 7.939\end{array}$

$1188.3 \quad 3.1424-0.000008 \quad 9.875$

$1191.2 \quad 3.2806-0.000008-10.762$

$1198.1 \quad 3.4764-0.000008 \quad 12.085$

$1212.6 \quad 4.0489-0.000008 \quad 16.393$

$1225.9 \quad 4.6635-0.000009 \quad 21.749$

$1234.7 \quad 4.7216-0.000009 \quad 22.294$

$1261.0 \quad 3.9174-0.000006 \quad 15.346$

$1261.0 \quad 3.9174-0.000006 \quad 15.346$

$1279.7 \quad 3.4767-0.000005 \quad 12.087$

$1296.0 \quad 2.9668-0.000004 \quad 8.802$

$1296.6 \quad 3.0081-0.000004 \quad 9.049$

$1319.8 \quad 2.6009-0.000003 \quad 6.765$

$1329.6 \quad 2.1583-0.000002 \quad 4.658$

$\begin{array}{llll}1330.7 & 2.0300 & -0.000002 & 4.121\end{array}$

$1344.3 \quad 1.1054-0.000001 \quad 1.222$

$\begin{array}{llll}1364.2 & -0.3667 & 0.000000 & 0.134\end{array}$

$\begin{array}{llll}1364.4 & -0.3648 & 0.000000 & 0.133\end{array}$

$\begin{array}{llll}1377.4 & -1.5367 & 0.000001 & 2.362\end{array}$

$\begin{array}{llll}1396.5 & -3.3618 & 0.000003 & 11.302\end{array}$

$\begin{array}{llll}1396.6 & -3.4480 & 0.000003 & 11.889\end{array}$

$1453.0-9.0429 \quad 0.000006$

$\begin{array}{llll}1490.8 & -6.0052 & 0.000003 & 36.062\end{array}$

$\begin{array}{llll}1000.6 & -0.0106 & 0.000010 & 0.000\end{array}$

$\begin{array}{llll}1001.2 & -0.0153 & 0.000007 & 0.000\end{array}$

$\begin{array}{llll}1002.3 & 0.0301 & -0.000007 & 0.001\end{array}$

$\begin{array}{llll}1004.7 & -0.0405 & 0.000005 & 0.002\end{array}$

$\begin{array}{llll}1005.7 & 0.0206 & -0.000002 & 0.000\end{array}$

$\begin{array}{llll}1009.8 & 0.0035 & 0.000000 & 0.000\end{array}$

$\begin{array}{llll}1011.5 & 0.0129 & -0.000001 & 0.000\end{array}$

$\begin{array}{llll}1017.3 & 0.0033 & 0.000000 & 0.000\end{array}$

$\begin{array}{llll}1019.3 & -0.0498 & 0.000001 & 0.002\end{array}$

$\begin{array}{llll}1023.2 & -0.0082 & 0.000000 & 0.000\end{array}$

$\begin{array}{llll}1029.1 & -0.0213 & 0.000000 & 0.000\end{array}$

$\begin{array}{llll}1059.2 & 0.2058 & -0.000002 & 0.042\end{array}$

$1090.3 \quad 0.5737-0.000003 \quad 0.329$

$\begin{array}{llll}1122.4 & 1.0042 & -0.000004 & 1.008\end{array}$

$\begin{array}{llll}1155.3 & 1.5922 & -0.000005 & 2.535\end{array}$

$\begin{array}{llll}1188.9 & 2.7733 & -0.000007 & 7.691\end{array}$

$\begin{array}{llll}1223.1 & 4.4347 & -0.000008 & 19.667\end{array}$

$1257.5 \quad 3.8264-0.000006 \quad 14.641$

$1291.8 \quad 2.8748-0.000004 \quad 8.264$

$\begin{array}{llll}1325.7 & 1.9980 & -0.000002 & 3.992\end{array}$

$\begin{array}{llll}1358.6 & -0.2781 & 0.000000 & 0.077\end{array}$
Apparent Density of molal water / volume / $\quad \mathrm{kg} / \mathrm{m}^{3}$

999.8395 999.8395 999.8395 999.8395 999.8395 999.8395 999.8395 999.8395 999.8395 999.8395 999.8395 999.8395 999.8395 999.8395 999.8395 999.8395 999.8395 999.8395 999.8395 999.8395 999.8395 999.8395 999.8395 999.9638 999.9638

$26.58 \quad 999.9638$

$27.37 \quad 999.9638$ 999.9638

$27.117 \quad 999.9638$ 999.9638 999.9638

$27.343 \quad 999.9638$ 999.9638 999.9638 999.9638 999.9638 999.9638 999.9638 999.9638 999.9638 999.9638 999.9638 999.9638 999.9638 


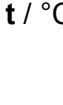

5

$\begin{array}{ll}0.6500 & 1412.8 \\ 0.0100 & 1005.3\end{array}$

$0.0200-1011.0$

$0.0300 \quad 1016.7$

$0.0306 \quad 1017.0$

$0.0391 \quad 1022.0$

$0.0400 \quad 1022.4$

$0.0489 \quad 1027.5$

$0.0500 \quad 1028.2$

$0.0700 \quad 1039.9$

$0.0900 \quad 1051.8$

$0.1000 \quad 1057.8$

$0.1100 \quad 1063.8$

$0.1397 \quad 1082.4$

$0.1500 \quad 1088.7$

$0.1600 \quad 1095.0$

$0.1810 \quad 1108.4$

$0.2000 \quad 1120.6$

$0.2000 \quad 1120.6$

$0.2212 \quad 1134.4$

$0.2400 \quad 1146.9$

$0.2500 \quad 1153.6$

$0.2800 \quad 1173.8$

$0.2957 \quad 1184.7$

$0.3000 \quad 1187.6$

$0.3100 \quad 1194.5$

$0.3308 \quad 1209.0$

$0.3500 \quad 1222.7$

$0.3625 \quad 1231.0$

$0.4000 \quad 1256.0$

$0.4000 \quad 1256.0$

$0.4267 \quad 1273.6$

$0.4500 \quad 1289.0$

$0.4508 \quad 1289.6$

$0.4842 \quad 1311.6$

$0.4985 \quad 1320.6$

$0.5000 \quad 1321.5$

$0.5200 \quad 1333.8$

$0.5497 \quad 1351.6$

$0.5500 \quad 1351.8$

$0.5700 \quad 1363.4$

$0.6000 \quad 1380.1$

$0.6003 \quad 1380.2$
Ref

App vol App vol

$$
\mathrm{m}^{3} / \mathrm{kg} \quad \mathrm{m}^{3} / \mathrm{kg}
$$

0.0005350 .000533

$0.000550 \quad 0.000546$

$0.000439 \quad 0.000435$

$0.000442 \quad 0.000436$

0.0004430 .000438

$0.000445 \quad 0.000438$

$\begin{array}{lll}0.000443 & 0.000439\end{array}$

0.0004450 .000439

$0.000447 \quad 0.000440$

$\begin{array}{lll}0.000447 & 0.000440\end{array}$

$0.000448 \quad 0.000443$

$0.000450 \quad 0.000445$

$\begin{array}{lll}0.000451 & 0.000446\end{array}$

0.0004520 .000448

$0.000453 \quad 0.000452$

$0.000455 \quad 0.000453$

$0.000456 \quad 0.000455$

$\begin{array}{ll}0.000458 & 0.000457\end{array}$

$\begin{array}{ll}0.000461 & 0.000460\end{array}$

0.0004610 .000460

$\begin{array}{ll}0.000463 & 0.000463\end{array}$

$0.000465 \quad 0.000466$

$0.000467 \quad 0.000468$

$0.000470 \quad 0.000473$

$0.000472 \quad 0.000475$

$0.000473 \quad 0.000476$

$0.000474 \quad 0.000478$

$0.000477 \quad 0.000481$

$\begin{array}{lll}0.000479 & 0.000485\end{array}$

$0.000482 \quad 0.000487$

$0.000490 \quad 0.000494$

$\begin{array}{lll}0.000490 & 0.000494\end{array}$

$0.000496 \quad 0.000499$

$0.000501 \quad 0.000504$

$\begin{array}{lll}0.000501 & 0.000504\end{array}$

$0.000509 \quad 0.000511$

$0.000513 \quad 0.000514$

$\begin{array}{ll}0.000513 & 0.000514\end{array}$

$0.000518 \quad 0.000519$

$0.000527 \quad 0.000526$

$0.000527 \quad 0.000526$

$0.000532 \quad 0.000531$

$\begin{array}{ll}0.000541 & 0.000538\end{array}$

$\begin{array}{ll}0.000541 & 0.000538\end{array}$
Density Density App Vol Square of Inconsistent Inconsistent calc / Residual / Residual / Density based on based on $\mathrm{kg} / \mathrm{m}^{3} \quad \mathrm{~kg} / \mathrm{m}^{3} \quad \mathrm{~m}^{3} / \mathrm{kg}$ Residual density apparent

$\begin{array}{llll}1389.8 & -3.0035 & 0.000003 & 9.021\end{array}$

$\begin{array}{llll}1418.6 & -5.7889 & 0.000004 & 33.511\end{array}$

$\begin{array}{llll}1005.4 & -0.0382 & 0.000004 & 0.001\end{array}$

$\begin{array}{llll}1011.1 & -0.1075 & 0.000005 & 0.012\end{array}$

$\begin{array}{llll}1016.9 & -0.1774 & 0.000006 & 0.031\end{array}$

$\begin{array}{llll}1017.2 & -0.2158 & 0.000007 & 0.047\end{array}$

$\begin{array}{llll}1022.2 & -0.1573 & 0.000004 & 0.025\end{array}$

$\begin{array}{llll}1022.7 & -0.2577 & 0.000006 & 0.066\end{array}$

$\begin{array}{llll}1027.9 & -0.3539 & 0.000007 & 0.125\end{array}$

$\begin{array}{llll}1028.5 & -0.3383 & 0.000006 & 0.114\end{array}$

$\begin{array}{llll}1040.3 & -0.3936 & 0.000005 & 0.155\end{array}$

$\begin{array}{llll}1052.3 & -0.4529 & 0.000005 & 0.205\end{array}$

$\begin{array}{llll}1058.3 & -0.4971 & 0.000004 & 0.247\end{array}$

$\begin{array}{llll}1064.4 & -0.5738 & 0.000005 & 0.329\end{array}$

$\begin{array}{llll}1082.6 & -0.2441 & 0.000001 & 0.060\end{array}$

$\begin{array}{llll}1089.1 & -0.3563 & 0.000002 & 0.127\end{array}$

$\begin{array}{llll}1095.3 & -0.3182 & 0.000002 & 0.101\end{array}$

$\begin{array}{llll}1108.6 & -0.1819 & 0.000001 & 0.033\end{array}$

$\begin{array}{llll}1120.7 & -0.1105 & 0.000000 & 0.012\end{array}$

$\begin{array}{llll}1120.7 & -0.1105 & 0.000000 & 0.012\end{array}$

$\begin{array}{llll}1134.4 & 0.0205 & 0.000000 & 0.000\end{array}$

$\begin{array}{llll}1146.6 & 0.2864 & -0.000001 & 0.082\end{array}$

$\begin{array}{llll}1153.2 & 0.4385 & -0.000001 & 0.192\end{array}$

$\begin{array}{llll}1173.0 & 0.8413 & -0.000002 & 0.708\end{array}$

$1183.4 \quad 1.2986-0.000003 \quad 1.686$

$1186.3 \quad 1.3298-0.000003 \quad 1.768$

$\begin{array}{llll}1193.0 & 1.5448 & -0.000003 & 2.386\end{array}$

$\begin{array}{llll}1206.9 & 2.0874-0.000004 & 4.357\end{array}$

$\begin{array}{llll}1219.8 & 2.8532 & -0.000005 & 8.141\end{array}$

$\begin{array}{llll}1228.3 & 2.7139 & -0.000005 & 7.365\end{array}$

$1253.6 \quad 2.3608-0.000004 \quad 5.573$

$1253.6 \quad 2.3608-0.000004 \quad 5.573$

$\begin{array}{llll}1271.7 & 1.9371 & -0.000003 & 3.752\end{array}$

$1287.3 \quad 1.6799-0.000002 \quad 2.822$

$1287.9 \quad 1.7441-0.000002 \quad 3.042$

$\begin{array}{llll}1310.1 & 1.5173 & -0.000002 & 2.302\end{array}$

$1319.5 \quad 1.1099-0.000001 \quad 1.232$

$\begin{array}{llll}1320.5 & 1.0278 & -0.000001 & 1.056\end{array}$

$\begin{array}{llll}1333.5 & 0.3281 & 0.000000 & 0.108\end{array}$

$\begin{array}{llll}1352.4 & -0.7848 & 0.000001 & 0.616\end{array}$

$\begin{array}{llll}1352.6 & -0.7731 & 0.000001 & 0.598\end{array}$

$\begin{array}{llll}1365.0 & -1.5794 & 0.000001 & 2.494\end{array}$

$\begin{array}{llll}1383.0 & -2.8792 & 0.000003 & 8.290\end{array}$

$\begin{array}{llll}1383.2 & -2.9550 & 0.000003 & 8.732\end{array}$
Apparent Density of molal water / $\mathrm{kg} / \mathrm{m}^{3}$

999.9638 999.9638 999.6996 999.6996 999.6996 999.6996 999.6996 999.6996 999.6996 999.6996 999.6996 999.6996 999.6996 999.6996 999.6996 999.6996 999.6996 999.6996 999.6996 999.6996 999.6996 999.6996 999.6996 999.6996 999.6996 999.6996 999.6996 999.6996 999.6996 999.6996 999.6996 999.6996 999.6996 999.6996 999.6996 999.6996 999.6996 999.6996 999.6996 999.6996 999.6996 999.6996 999.6996 999.6996 
$\mathrm{t} /{ }^{\circ} \mathrm{C}$

$\begin{array}{rrr}10 & 0.6500 & 1405.5 \\ 10 & 0.7011 & 1428.9 \\ 10 & 0.7533 & 1456.7 \\ 10 & 0.8011 & 1468.6 \\ 15 & 0.0010 & 999.65 \\ 15 & 0.0021 & 1000.23 \\ 15 & 0.0042 & 1001.42 \\ 15 & 0.0083 & 1003.62 \\ 15 & 0.0100 & 1004.6 \\ 15 & 0.0171 & 1008.50 \\ 15 & 0.0200 & 1010.2 \\ 15 & 0.0300 & 1015.8 \\ 15 & 0.0335 & 1017.60 \\ 15 & 0.0400 & 1021.4 \\ 15 & 0.0500 & 1027.0 \\ 15 & 0.1000 & 1056.1 \\ 15 & 0.1500 & 1086.5 \\ 15 & 0.2000 & 1117.8 \\ 15 & 0.2500 & 1150.3 \\ 15 & 0.3000 & 1183.8 \\ 15 & 0.3500 & 1218.3 \\ 15 & 0.4000 & 1251.1 \\ 15 & 0.4500 & 1283.6 \\ 15 & 0.5000 & 1315.7 \\ 15 & 0.5500 & 1345.5 \\ 15 & 0.6000 & 1373.4 \\ 15 & 0.6500 & 1398.4 \\ 20 & 0.0100 & 1003.6 \\ 20 & 0.0200 & 1009.1 \\ 20 & 0.0300 & 1014.6 \\ 20 & 0.0306 & 1014.9 \\ 20 & 0.0391 & 1019.5 \\ 20 & 0.0400 & 1020.1 \\ 20 & 0.0489 & 1025.0 \\ 20 & 0.0500 & 1025.6 \\ 20 & 0.0600 & 1031.2 \\ 20 & 0.0700 & 1036.9 \\ 20 & 0.0700 & 1036.9 \\ 20 & 0.0800 & 1042.7 \\ 20 & 0.0900 & 1048.5 \\ 20 & 0.0900 & 1048.5 \\ 20 & 0.1000 & 1054.3 \\ 20 & 0.1100 & 1060.2 \\ 20 & 0.1100 & 1060.2 \\ & & \end{array}$

molality

molarity

t $/$

Ref

nsity

(90)

(35)

(35)

(35)

0.01657

0.03313

0.06637

0.13233

0.27635

0.5498 $\begin{array}{rr}\text { App vol } & \text { App vol } \\ \exp / & \text { Calc / }\end{array}$

$$
\mathrm{m}^{3} / \mathrm{kg} \quad \mathrm{m}^{3} / \mathrm{kg}
$$

$0.000556 \quad 0.000552$ $0.000572 \quad 0.000567$ $0.000584 \quad 0.000584$ $0.000602 \quad 0.000601$ $0.000468 \quad 0.000440$ $0.000458 \quad 0.000440$ $0.000445 \quad 0.000440$ $0.000456 \quad 0.000441$ $\begin{array}{ll}0.000449 & 0.000441\end{array}$ $\begin{array}{lll}0.000456 & 0.000442\end{array}$ $0.000452 \quad 0.000442$ $0.000454 \quad 0.000443$ $0.000457 \quad 0.000444$ 0.0004550 .000445 $0.000457 \quad 0.000446$ $\begin{array}{ll}0.000461 & 0.000452\end{array}$ $0.000464 \quad 0.000459$ 0.0004690 .000466 $0.000475 \quad 0.000473$ $0.000480 \quad 0.000482$ $0.000486 \quad 0.000490$ $\begin{array}{ll}0.000497 & 0.000499\end{array}$ $0.000508 \quad 0.000509$ $\begin{array}{lll}0.000519 & 0.000520\end{array}$ $0.000532 \quad 0.000531$ $\begin{array}{ll}0.000546 & 0.000544\end{array}$ $\begin{array}{ll}0.000561 & 0.000557\end{array}$ $0.000459 \quad 0.000447$ $\begin{array}{lll}0.000461 & 0.000448\end{array}$ 0.0004630 .000449 $0.000464 \quad 0.000449$ $\begin{array}{lll}0.000467 & 0.000450\end{array}$ $0.000465 \quad 0.000450$ $0.000466 \quad 0.000451$ $\begin{array}{lll}0.000466 & 0.000451\end{array}$ $0.000467 \quad 0.000453$ $0.000468 \quad 0.000454$ $0.000468 \quad 0.000454$ $0.000467 \quad 0.000455$ $\begin{array}{lll}0.000468 & 0.000456\end{array}$ $0.000468 \quad 0.000456$ 0.0004690 .000458 0.0004690 .000459 0.0004690 .000459
Density Density App Vol Square of Inconsistent Inconsistent calc / Residual / Residual / Density based on based on $\mathrm{kg} / \mathrm{m}^{3} \quad \mathrm{~kg} / \mathrm{m}^{3} \quad \mathrm{~m}^{3} / \mathrm{kg}$ Residual density apparent

$\begin{array}{llll}1410.9 & -5.4120 & 0.000004 & 29.290\end{array}$

$\begin{array}{llll}1435.9 & -7.0413 & 0.000005 & 49.580\end{array}$

$\begin{array}{llll}1456.7 & 0.0483 & 0.000000 & 0.002\end{array}$

$\begin{array}{llll}1470.1 & -1.5377 & 0.000001 & 2.364\end{array}$

$\begin{array}{llll}999.7 & -0.0293 & 0.000028 & 0.001\end{array}$

$\begin{array}{llll}1000.3 & -0.0379 & 0.000018 & 0.001\end{array}$

$\begin{array}{llll}1001.4 & -0.0192 & 0.000005 & 0.000\end{array}$

$\begin{array}{llll}1003.7 & -0.1242 & 0.000015 & 0.015\end{array}$

$\begin{array}{llll}1004.7 & -0.0808 & 0.000008 & 0.007\end{array}$

$\begin{array}{llll}1008.7 & -0.2414 & 0.000014 & 0.058\end{array}$

$\begin{array}{llll}1010.4 & -0.2015 & 0.000010 & 0.041\end{array}$

$\begin{array}{llll}1016.1 & -0.3215 & 0.000010 & 0.103\end{array}$

$\begin{array}{llll}1018.1 & -0.4763 & 0.000014 & 0.227\end{array}$

$\begin{array}{llll}1021.8 & -0.4507 & 0.000011 & 0.203\end{array}$

$\begin{array}{llll}1027.6 & -0.5788 & 0.000011 & 0.335\end{array}$

$\begin{array}{llll}1057.1 & -0.9644 & 0.000009 & 0.930\end{array}$

$\begin{array}{llll}1087.5 & -0.9537 & 0.000005 & 0.910\end{array}$

$\begin{array}{llll}1118.7 & -0.8982 & 0.000004 & 0.807\end{array}$

$\begin{array}{llll}1150.7 & -0.3969 & 0.000001 & 0.158\end{array}$

$\begin{array}{llll}1183.3 & 0.4922 & -0.000001 & 0.242\end{array}$

$\begin{array}{llll}1216.3 & 1.9609 & -0.000004 & 3.845\end{array}$

$1249.5-15626-0.000002$

$\begin{array}{llll}1282.6 & 1.0248 & -0.000001 & 1.050\end{array}$

$\begin{array}{llll}1315.0 & 0.6630 & -0.000001 & 0.440\end{array}$

$\begin{array}{llll}1346.4 & -0.9044 & 0.000001 & 0.818\end{array}$

$\begin{array}{llll}1376.0 & -2.6410 & 0.000002 & 6.975\end{array}$

$\begin{array}{llll}1403.2 & -4.7797 & 0.000004 & 22.846\end{array}$

$\begin{array}{lll}1003.8 & -0.1279 & 0.000013\end{array}$

$\begin{array}{llll}1009.4 & -0.2799 & 0.000014 & 0.078\end{array}$

$\begin{array}{llll}1015.0 & -0.4401 & 0.000014 & 0.194\end{array}$

$\begin{array}{llll}1015.4 & -0.4610 & 0.000015 & 0.213\end{array}$

$\begin{array}{llll}1020.2 & -0.6984 & 0.000017 & 0.488\end{array}$

$\begin{array}{llll}1020.7 & -0.6082 & 0.000015 & 0.370\end{array}$

$\begin{array}{llll}1025.8 & -0.7734 & 0.000015 & 0.598\end{array}$

$\begin{array}{llll}1026.4 & -0.7740 & 0.000015 & 0.599\end{array}$

$\begin{array}{llll}1032.2 & -0.9372 & 0.000015 & 0.878\end{array}$

$\begin{array}{llll}1037.9 & -1.0417 & 0.000014 & 1.085\end{array}$

$\begin{array}{llll}1037.9 & -1.0476 & 0.000014 & 1.097\end{array}$

$\begin{array}{llll}1043.8 & -1.0748 & 0.000012 & 1.155\end{array}$

$\begin{array}{llll}1049.6 & -1.1326 & 0.000011 & 1.283\end{array}$

$\begin{array}{llll}1049.6 & -1.1384 & 0.000011 & 1.296\end{array}$

$\begin{array}{llll}1055.5 & -1.2382 & 0.000011 & 1.533\end{array}$

$\begin{array}{llll}1061.5 & -1.2736 & 0.000010 & 1.622\end{array}$

$\begin{array}{llll}1061.5 & -1.2736 & 0.000010 & 1.622\end{array}$
Apparent Density of molal water / volume / $\mathrm{kg} / \mathrm{m}^{3}$

999.6996 999.6996 999.6996 999.6996

$29.49 \quad 999.0996$

$28.87 \quad 999.0996$

$28.03 \quad 999.0996$

$28.71 \quad 999.0996$ 999.0996

$28.71 \quad 999.0996$ 999.0996 999.0996

$28.825 \quad 999.0996$ 999.0996 999.0996 999.0996 999.0996 999.0996 999.0996 999.0996 999.0996 999.0996 999.0996 999.0996 999.0996 999.0996 999.0996 998.2041 998.2041 998.2041 998.2041 998.2041 998.2041 998.2041 998.2041 998.2041 998.2041 998.2041 998.2041 998.2041 998.2041 998.2041 998.2041 998.2041 


\section{$\mathrm{C}$}

20

20

20

20

20

20

20

20

20

20
20

20

$\begin{array}{ll}0.5497 & 1339.1 \\ 0.5500 & 1339.3\end{array}$

$0.5700 \quad 1350.5$

$0.5700 \quad 1350.5$ $\exp /$

$\mathrm{kg} / \mathrm{m}^{3}$

$\begin{array}{lll}0.1300 & 1066.1 \quad(90)\end{array}$

1072.1

1078.1

$\begin{array}{ll}1084.2 \\ 1600 & 1090.3\end{array}$

1090.3

$1800 \quad 1102.6$

$0.1810 \quad 1103.2$

$2000 \quad 1115.0$

$0.2000 \quad 1115.0$

$2212 \quad 1128.3$

$.2400 \quad 1140.4$

$0.2588 \quad 1152.6$

$.2700 \quad 1160.0$

1166.6
$0.2957 \quad 1177.2$

$3000 \quad 1180.0$

$\begin{array}{ll}0.3200 & 1186.4\end{array}$

$\begin{array}{ll}0.3308 & 1200.7\end{array}$

$\begin{array}{ll}1222.1 \\ 0.3625 & 122.0\end{array}$

$3700 \quad 1227.0$

$.4000 \quad 1246.3$

1259.1

$0.4500 \quad 1278.3$

1278.8

$0.4842 \quad 1300.3$

1309.1

$0.5000 \quad 1310.0$

1321.9
1350.5
App vol App vol

$\mathrm{m}^{3} / \mathrm{kg} \quad \mathrm{m}^{3} / \mathrm{kg}$

$0.000470 \quad 0.000460$

0.0004710 .000462

$0.000470 \quad 0.000463$

$0.000472 \quad 0.000463$

$0.000472 \quad 0.000464$

$0.000473 \quad 0.000466$

$0.000473 \quad 0.000466$

$\begin{array}{ll}0.000474 & 0.000467\end{array}$

$\begin{array}{lll}0.000475 & 0.000469\end{array}$

$0.000475 \quad 0.000469$

$0.000476 \quad 0.000470$

$\begin{array}{ll}0.000477 & 0.000471\end{array}$

$\begin{array}{lll}0.000477 & 0.000471\end{array}$

0.0004790 .000474

$0.000480 \quad 0.000475$

$\begin{array}{lll}0.000481 & 0.000477\end{array}$

$\begin{array}{ll}0.000482 & 0.000479\end{array}$

$0.000483 \quad 0.000480$

$0.000484 \quad 0.000482$

$0.000485 \quad 0.000484$

$0.000487 \quad 0.000486$

$0.000487 \quad 0.000487$

$\begin{array}{ll}0.000488 & 0.000489\end{array}$

$0.000490 \quad 0.000491$

$\begin{array}{ll}0.000491 & 0.000492\end{array}$

$0.000493 \quad 0.000496$

$0.000495 \quad 0.000498$

$0.000497 \quad 0.000499$

$0.000503 \quad 0.000505$

$0.000503 \quad 0.000505$

$0.000508 \quad 0.000509$

$0.000509 \quad 0.000510$

$0.000514 \quad 0.000515$

$0.000514 \quad 0.000515$

$0.000518 \quad 0.000519$

$0.000521 \quad 0.000522$

$0.000525 \quad 0.000525$

$0.000525 \quad 0.000526$

$0.000530 \quad 0.000530$

$0.000530 \quad 0.000530$

$\begin{array}{ll}0.000538 & 0.000537\end{array}$

$0.000538 \quad 0.000537$

$0.000543 \quad 0.000542$

$0.000543 \quad 0.000542$
Density Density App Vol Square of Inconsistent Inconsistent calc / Residual / Residual / Density based on based on $\mathrm{kg} / \mathrm{m}^{3} \quad \mathrm{~kg} / \mathrm{m}^{3} \quad \mathrm{~m}^{3} / \mathrm{kg}$ Residual density apparent

$\begin{array}{llll}1067.4 & -1.3442 & 0.000010 & 1.807\end{array}$

$\begin{array}{llll}1073.4 & -1.3494 & 0.000009 & 1.821\end{array}$

$\begin{array}{llll}1079.3 & -1.2070 & 0.000007 & 1.457\end{array}$

$\begin{array}{llll}1079.5 & -1.3887 & 0.000009 & 1.929\end{array}$

$\begin{array}{llll}1085.6 & -1.3615 & 0.000008 & 1.854\end{array}$

$\begin{array}{llll}1091.7 & -1.3671 & 0.000007 & 1.869\end{array}$

$\begin{array}{llll}1091.7 & -1.3671 & 0.000007 & 1.869\end{array}$

$\begin{array}{llll}1097.8 & -1.4048 & 0.000007 & 1.974\end{array}$

$\begin{array}{llll}1104.0 & -1.3739 & 0.000006 & 1.888\end{array}$

$\begin{array}{llll}1104.6 & -1.3925 & 0.000006 & 1.939\end{array}$

$\begin{array}{llll}1110.2 & -1.3735 & 0.000006 & 1.887\end{array}$

$\begin{array}{llll}1116.4 & -1.4027 & 0.000006 & 1.968\end{array}$

$\begin{array}{llll}1116.4 & -1.4027 & 0.000006 & 1.968\end{array}$

$\begin{array}{llll}1128.9 & -1.3463 & 0.000005 & 1.813\end{array}$

$\begin{array}{llll}1129.7 & -1.4024 & 0.000005 & 1.967\end{array}$

$\begin{array}{llll}1141.6 & -1.1963 & 0.000004 & 1.431\end{array}$

$\begin{array}{llll}1148.0 & -1.0583 & 0.000003 & 1.120\end{array}$

$\begin{array}{llll}1153.6 & -0.9759 & 0.000003 & 0.952\end{array}$

$\begin{array}{llll}1160.7 & -0.7499 & 0.000002 & 0.562\end{array}$

$\begin{array}{llll}1167.2 & -0.5766 & 0.000002 & 0.333\end{array}$

$\begin{array}{llll}1177.3 & -0.1036 & 0.000000 & 0.011\end{array}$

$\begin{array}{llll}1180.1 & -0.0844 & 0.000000 & 0.007\end{array}$

$\begin{array}{llll}1186.6 & 0.1380 & 0.000000 & 0.019\end{array}$

$\begin{array}{llll}1193.1 & 0.3469 & -0.000001 & 0.120\end{array}$

$1200.1 \quad 0.6237-0.000001-0.389$

$\begin{array}{llll}1212.6 & 1.4127 & -0.000003 & 1.996\end{array}$

$\begin{array}{llll}1220.7 & 1.3563 & -0.000003 & 1.840\end{array}$

$1225.6 \quad 1.3605-0.000002-1.851$

$12452 \quad 1.0868-0.000002 \quad 1.181$

$\begin{array}{llll}1245.2 & 1.0868 & -0.000002 & 1.181\end{array}$

$\begin{array}{llll}1258.2 & 0.8725 & -0.000001 & 0.761\end{array}$

$\begin{array}{llll}1262.6 & 0.7240 & -0.000001 & 0.524\end{array}$

$1277.6 \quad 0.6648-0.000001 \quad 0.442$

$\begin{array}{llll}1278.2 & 0.6498 & -0.000001 & 0.422\end{array}$

$1290.5 \quad 0.6424-0.000001 \quad 0.413$

$1299.5 \quad 0.8145-0.000001 \quad 0.663$

$\begin{array}{llll}1308.5 & 0.6003 & -0.000001 & 0.360\end{array}$

$1309.4 \quad 0.5598-0.000001 \quad 0.313$

$\begin{array}{llll}1321.9 & 0.0219 & 0.000000 & 0.000\end{array}$

$\begin{array}{llll}1321.9 & 0.0219 & 0.000000 & 0.000\end{array}$

$\begin{array}{llll}1339.9 & -0.8351 & 0.000001 & 0.697\end{array}$

$\begin{array}{llll}1340.1 & -0.8146 & 0.000001 & 0.664\end{array}$

$\begin{array}{llll}1351.9 & -1.4311 & 0.000001 & 2.048\end{array}$

$\begin{array}{llll}1351.9 & -1.4311 & 0.000001 & 2.048\end{array}$
Apparent Density of molal water / volume / $\quad \mathrm{kg} / \mathrm{m}^{3}$

998.2041 998.2041 998.2041

998.2041 998.2041 998.2041 998.2041 998.2041 998.2041 998.2041 998.2041 998.2041 998.2041 998.2041 998.2041 998.2041 998.2041 998.2041 998.2041 998.2041 998.2041 998.2041 998.2041 998.2041 998.2041 998.2041 998.2041 998.2041 998.2041 998.2041 998.2041 998.2041 998.2041 998.2041 998.2041 998.2041 998.2041 998.2041 998.2041 998.2041 998.2041 998.2041 998.2041 998.2041 
molality molarity

$t /{ }^{\circ} \mathrm{C}$ mass frac

0.01657

0.06637

0.13233

0.27635

0.5498

$\begin{array}{crrr}\mathbf{t} /{ }^{\circ} \mathrm{C} \text { mass frac } & \text { Density } & \text { Ref } \\ & & \mathrm{exp} / & \\ & & \mathrm{kg} / \mathrm{m}^{3} & \\ 20 & 0.6000 & 1366.7 & (90) \\ 20 & 0.6003 & 1366.8 & (35) \\ 20 & 0.6200 & 1376.9 & (90) \\ 20 & 0.6500 & 1391.3 & (90) \\ 20 & 0.7011 & 1413.8 & (35) \\ 20 & 0.7533 & 1434.9 & (35) \\ 20 & 0.8011 & 1452.4 & (35) \\ 25 & 0.0010 & 997.59 & (82) \\ 25 & 0.0042 & 999.24 & (82) \\ 25 & 0.0083 & 1001.42 & (82) \\ 25 & 0.0171 & 1006.12 & (82) \\ 25 & 0.0306 & 1013.4 & (35) \\ 25 & 0.0335 & 1014.92 & (82) \\ 25 & 0.0391 & 1018.1 & (35) \\ 25 & 0.0489 & 1023.6 & (35) \\ 25 & 0.0700 & 1035.3 & (35) \\ 25 & 0.0900 & 1046.6 & (35) \\ 25 & 0.1100 & 1058.0 & (35) \\ 25 & 0.1397 & 1075.8 & (35) \\ 25 & 0.1600 & 1087.9 & (35) \\ 25 & 0.1810 & 1100.7 & (35) \\ 25 & 0.2212 & 1125.4 & (35) \\ 25 & 0.2400 & 1137.4 & (35) \\ 25 & 0.2588 & 1149.5 & (35) \\ 25 & 0.2800 & 1163.1 & (35) \\ 25 & 0.2957 & 1173.5 & (35) \\ 25 & 0.3100 & 1182.9 & (35) \\ 25 & 0.3308 & 1196.8 & (35) \\ 25 & 0.3625 & 1217.9 & (35) \\ 25 & 0.4000 & 1241.7 & (35) \\ 25 & 0.4267 & 1258.5 & (35) \\ 25 & 0.4508 & 1273.7 & (35) \\ 25 & 0.4842 & 1294.9 & (35) \\ 25 & 0.4985 & 1303.4 & (35) \\ 25 & 0.5200 & 1316.0 & (35) \\ 25 & 0.5497 & 1332.9 & (35) \\ 25 & 0.5700 & 1344.1 & (35) \\ 25 & 0.6003 & 1360.1 & (35) \\ 25 & 0.7011 & 1406.5 & (35) \\ 25 & 0.7533 & 1427.0 & (35) \\ 25 & 0.8011 & 1444.2 & (35) \\ 30 & 0.0100 & 1000.9 & (90) \\ 30 & 0.0200 & 1006.1 & (90) \\ & 0.0300 & 1011.4 & (90) \\ & & & \\ & & \\ 25 & \end{array}$

App vol App vol

exp / Calc/

$\mathrm{m}^{3} / \mathrm{kg} \quad \mathrm{m}^{3} / \mathrm{kg}$

$0.000552 \quad 0.000550$

$0.000552 \quad 0.000550$

$0.000557 \quad 0.000555$

$0.000566 \quad 0.000563$

$\begin{array}{ll}0.000582 & 0.000578\end{array}$

$0.000597 \quad 0.000595$

$\begin{array}{lll}0.000611 & 0.000612\end{array}$

$\begin{array}{ll}0.000476 & 0.000451\end{array}$

$0.000474 \quad 0.000451$

$0.000473 \quad 0.000452$

$0.000474 \quad 0.000453$

$0.000474 \quad 0.000455$

$0.000475 \quad 0.000455$

$0.000473 \quad 0.000456$

$0.000471 \quad 0.000457$

$0.000473 \quad 0.000459$

$0.000475 \quad 0.000462$

$0.000478 \quad 0.000465$

$0.000477 \quad 0.000469$

$0.000479 \quad 0.000471$

$0.000481 \quad 0.000474$

$0.000486 \quad 0.000480$

$0.000487 \quad 0.000483$

0.0004890 .000486

$0.000492 \quad 0.000489$

$0.000493 \quad 0.000492$

$\begin{array}{ll}0.000495 & 0.000494\end{array}$

$0.000497 \quad 0.000498$

$\begin{array}{lll}0.000501 & 0.000504\end{array}$

$0.000509 \quad 0.000511$

$0.000515 \quad 0.000516$

$0.000520 \quad 0.000521$

$0.000527 \quad 0.000528$

$0.000530 \quad 0.000531$

$0.000535 \quad 0.000536$

$0.000543 \quad 0.000543$

$0.000549 \quad 0.000547$

$\begin{array}{lll}0.000557 & 0.000555\end{array}$

$0.000587 \quad 0.000584$

$0.000602 \quad 0.000601$

$0.000615 \quad 0.000618$

$\begin{array}{ll}0.000477 & 0.000458\end{array}$

$0.000483 \quad 0.000459$

$0.000483 \quad 0.000460$
Density Density App Vol Square of Inconsistent Inconsistent calc / Residual / Residual / Density based on based on

volume

$\begin{array}{llll}1369.0 & -2.3298 & 0.000002 & 5.428\end{array}$

$\begin{array}{llll}1369.2 & -2.3966 & 0.000002 & 5.743\end{array}$

$\begin{array}{llll}1379.9 & -3.0386 & 0.000003 & 9.233\end{array}$

$\begin{array}{llll}1395.4 & -4.1303 & 0.000003 & 17.059\end{array}$

$\begin{array}{llll}1418.9 & -5.0842 & 0.000004 & 25.849\end{array}$

$\begin{array}{llll}1438.0 & -3.1228 & 0.000002 & 9.752\end{array}$

$1450.2 \quad 2.2449-0.000001 \quad 5.040$

$\begin{array}{llll}997.6 & -0.0257 & 0.000025 & 0.001\end{array}$

$\begin{array}{llll}999.3 & -0.0927 & 0.000022 & 0.009\end{array}$

$\begin{array}{llll}1001.6 & -0.1783 & 0.000021 & 0.032\end{array}$

$\begin{array}{llll}1006.5 & -0.3700 & 0.000021 & 0.137\end{array}$

$\begin{array}{llll}1014.0 & -0.6225 & 0.000020 & 0.388\end{array}$

$\begin{array}{llll}1015.6 & -0.7054 & 0.000020 & 0.498\end{array}$

$\begin{array}{llll}1018.8 & -0.7080 & 0.000017 & 0.501\end{array}$

$\begin{array}{llll}1024.3 & -0.7226 & 0.000014 & 0.522\end{array}$

$\begin{array}{llll}1036.4 & -1.0564 & 0.000014 & 1.116\end{array}$

$\begin{array}{llll}1047.9 & -1.3145 & 0.000013 & 1.728\end{array}$

$\begin{array}{llll}1059.6 & -1.6176 & 0.000013 & 2.616\end{array}$

$1077.2 \quad-1.4364 \quad 0.000009 \quad 2.063$

$\begin{array}{llll}1089.4 & -1.5429 & 0.000008 & 2.380\end{array}$

$\begin{array}{llll}1102.2 & -1.5032 & 0.000007 & 2.260\end{array}$

$\begin{array}{llll}1127.0 & -1.5792 & 0.000006 & 2.494\end{array}$

$\begin{array}{llll}1138.7 & -1.3087 & 0.000004 & 1.713\end{array}$

$\begin{array}{llll}1150.5 & -1.0185 & 0.000003 & 1.037\end{array}$

$\begin{array}{llll}1163.9 & -0.8211 & 0.000002 & 0.674\end{array}$

$\begin{array}{llll}1173.9 & -0.3968 & 0.000001 & 0.157\end{array}$

$\begin{array}{llll}1183.0 & -0.1141 & 0.000000 & 0.013\end{array}$

$\begin{array}{llll}1196.3 & 0.4827 & -0.000001 & 0.233\end{array}$

$\begin{array}{llll}1216.6 & 1.2502 & -0.000002 & 1.563\end{array}$

$1240.7 \quad 0.9972-0.000002 \quad 0.994$

$\begin{array}{llll}1257.8 & 0.7441 & -0.000001 & 0.554\end{array}$

$1273.0 \quad 0.6587-0.000001 \quad 0.434$

$1294.0 \quad 0.9368-0.000001 \quad 0.878$

$1302.8 \quad 0.6041-0.000001 \quad 0.365$

$\begin{array}{llll}1315.9 & 0.1031 & 0.000000 & 0.011\end{array}$

$\begin{array}{llll}1333.6 & -0.6626 & 0.000001 & 0.439\end{array}$

$\begin{array}{llll}1345.3 & -1.1866 & 0.000001 & 1.408\end{array}$

$\begin{array}{llll}1362.1 & -2.0410 & 0.000002 & 4.166\end{array}$

$\begin{array}{llll}1410.5 & -3.9560 & 0.000003 & 15.650\end{array}$

$\begin{array}{llll}1428.9 & -1.9178 & 0.000001 & 3.678\end{array}$

$\begin{array}{llll}1440.5 & 3.7179 & -0.000002 & 13.823\end{array}$

$\begin{array}{llll}1001.1 & -0.1965 & 0.000020 & 0.039\end{array}$

$\begin{array}{llll}1006.6 & -0.4817 & 0.000024 & 0.232\end{array}$

$\begin{array}{llll}1012.1 & -0.7029 & 0.000023 & 0.494\end{array}$
Apparent Density of molal water / volume / $\mathrm{kg} / \mathrm{m}^{3}$

998.2041 998.2041 998.2041

998.2041 998.2041 998.2041

998.2041

$29.98 \quad 997.0449$

$29.85 \quad 997.0449$

$29.83 \quad 997.0449$

$29.886 \quad 997.0449$ 997.0449

$29.952 \quad 997.0449$ 997.0449 997.0449 997.0449 997.0449 997.0449 997.0449 997.0449 997.0449 997.0449 997.0449 997.0449 997.0449

997.0449

997.0449

997.0449

997.0449

997.0449 997.0449

997.0449

997.0449 997.0449

997.0449

997.0449 997.0449

997.0449

997.0449 997.0449 997.0449 995.6473 995.6473 995.6473 
molality molarity

$t /{ }^{\circ} \mathrm{C}$ mass frac

\begin{tabular}{|c|c|c|c|}
\hline & 30 & 0.0306 & 1011.7 \\
\hline & 30 & 0.0391 & 1016.3 \\
\hline & 30 & 0.0400 & 1016.8 \\
\hline & 30 & 0.0489 & 1021.7 \\
\hline & 30 & 0.0500 & 1022.2 \\
\hline & 30 & 0.0700 & 1033.3 \\
\hline & 30 & 0.0900 & 1044.6 \\
\hline & 30 & 0.1000 & 1050.3 \\
\hline & 30 & 0.1100 & 1055.9 \\
\hline & 30 & 0.1397 & 1072.3 \\
\hline & 30 & 0.1500 & 1079.4 \\
\hline & 30 & 0.1600 & 1085.4 \\
\hline & 30 & 0.1810 & 1098.0 \\
\hline & 30 & 0.2000 & 1109.4 \\
\hline & 30 & 0.2000 & 1109.4 \\
\hline & 30 & 0.2212 & 1122.5 \\
\hline & 30 & 0.2400 & 1134.3 \\
\hline & 30 & 0.2500 & 1140.6 \\
\hline & 30 & 0.2588 & 1146.2 \\
\hline & 30 & 0.2800 & 1159.7 \\
\hline & 30 & 0.3000 & 1172.7 \\
\hline & 30 & 0.3100 & 1179.2 \\
\hline & 30 & 0.3308 & 1192.7 \\
\hline & 30 & 0.3500 & 1205.5 \\
\hline & 30 & 0.3625 & 1213.4 \\
\hline & 30 & 0.4000 & 1237.0 \\
\hline & 30 & 0.4000 & 1237.0 \\
\hline & 30 & 0.4267 & 1253.6 \\
\hline & 30 & 0.4500 & 1268.0 \\
\hline & 30 & 0.4508 & 1268.5 \\
\hline & 30 & 0.4842 & 1288.4 \\
\hline & 30 & 0.4985 & 1297.8 \\
\hline & 30 & 0.5000 & 1298.7 \\
\hline & 30 & 0.5200 & 1310.2 \\
\hline & 30 & 0.5497 & 1326.8 \\
\hline & 30 & 0.5500 & 1327.0 \\
\hline & 30 & 0.5700 & 1337.7 \\
\hline & 30 & 0.6000 & 1353.3 \\
\hline & 30 & 0.6003 & 1353.4 \\
\hline & 30 & 0.6500 & 1377.0 \\
\hline & 30 & 0.7533 & 1419.1 \\
\hline & 30 & 0.8011 & 1449.8 \\
\hline 0.01657 & 35 & 0.0010 & 994.54 \\
\hline 0.03313 & 35 & 0.0021 & 995.07 \\
\hline
\end{tabular}

Ref

App vol App vol

$\mathrm{m}^{3} / \mathrm{kg} \quad \mathrm{m}^{3} / \mathrm{kg}$

$\begin{array}{ll}0.000484 & 0.000460\end{array}$

0.0004830 .000461

$0.000482 \quad 0.000461$

$\begin{array}{lll}0.000481 & 0.000462\end{array}$

0.0004830 .000462

$\begin{array}{ll}0.000481 & 0.000465\end{array}$

$\begin{array}{lll}0.000481 & 0.000467\end{array}$

0.0004820 .000469

$\begin{array}{lll}0.000483 & 0.000470\end{array}$

$0.000490 \quad 0.000474$

$0.000485 \quad 0.000475$

$\begin{array}{lll}0.000485 & 0.000477\end{array}$

$\begin{array}{ll}0.000487 & 0.000480\end{array}$

0.0004890 .000483

$0.000489 \quad 0.000483$

$\begin{array}{lll}0.000491 & 0.000486\end{array}$

$0.000493 \quad 0.000489$

$0.000494 \quad 0.000490$

$0.000495 \quad 0.000492$

$0.000497 \quad 0.000495$

$0.000499 \quad 0.000498$

$\begin{array}{ll}0.000500 & 0.000500\end{array}$

$0.000503 \quad 0.000503$

$0.000505 \quad 0.000507$

$0.000507 \quad 0.000509$

$0.000514 \quad 0.000516$

$0.000514 \quad 0.000516$

$\begin{array}{ll}0.000520 & 0.000521\end{array}$

$0.000525 \quad 0.000526$

0.0005250 .000526

$0.000533 \quad 0.000533$

$0.000535 \quad 0.000536$

$0.000536 \quad 0.000537$

$\begin{array}{lll}0.000541 & 0.000541\end{array}$

$\begin{array}{ll}0.000548 & 0.000548\end{array}$

$0.000548 \quad 0.000548$

$0.000554 \quad 0.000553$

$0.000562 \quad 0.000561$

$0.000562 \quad 0.000561$

$\begin{array}{lll}0.000576 & 0.000574\end{array}$

$0.000607 \quad 0.000606$

0.0006120 .000623

$\begin{array}{lll}0.000511 & 0.000462\end{array}$

$0.000504 \quad 0.000462$
Density Density App Vol Square of Inconsistent Inconsistent calc / Residual / Residual / Density based on based on $\mathrm{kg} / \mathrm{m}^{3} \quad \mathrm{~kg} / \mathrm{m}^{3} \quad \mathrm{~m}^{3} / \mathrm{kg}$ Residual density $\begin{gathered}\text { apparent } \\ \text { volume }\end{gathered}$

$\begin{array}{llll}1012.4 & -0.7464 & 0.000024 & 0.557\end{array}$

$\begin{array}{llll}1017.2 & -0.8804 & 0.000022 & 0.775\end{array}$

$\begin{array}{llll}1017.7 & -0.8596 & 0.000021 & 0.739\end{array}$

$\begin{array}{llll}1022.6 & -0.9349 & 0.000018 & 0.874\end{array}$

$\begin{array}{llll}1023.3 & -1.0518 & 0.000020 & 1.106\end{array}$

$\begin{array}{llll}1034.5 & -1.2353 & 0.000017 & 1.526\end{array}$

$\begin{array}{llll}1046.0 & -1.3620 & 0.000014 & 1.855\end{array}$

$\begin{array}{llll}1051.7 & -1.4318 & 0.000013 & 2.050\end{array}$

$\begin{array}{llll}1057.5 & -1.6289 & 0.000013 & 2.653\end{array}$

$\begin{array}{llll}1074.9 & -2.6363 & 0.000016 & 6.950\end{array}$

$\begin{array}{llll}1081.0 & -1.6375 & 0.000009 & 2.681\end{array}$

$\begin{array}{llll}1087.0 & -1.5917 & 0.000008 & 2.533\end{array}$

$\begin{array}{llll}1099.6 & -1.5901 & 0.000007 & 2.528\end{array}$

$\begin{array}{llll}1111.1 & -1.6938 & 0.000007 & 2.869\end{array}$

$\begin{array}{llll}1111.1 & -1.6938 & 0.000007 & 2.869\end{array}$

$\begin{array}{llll}1124.0 & -1.5395 & 0.000006 & 2.370\end{array}$

$\begin{array}{llll}1135.6 & -1.3085 & 0.000004 & 1.712\end{array}$

$\begin{array}{llll}1141.8 & -1.1936 & 0.000004 & 1.425\end{array}$

$\begin{array}{llll}1147.3 & -1.0530 & 0.000003 & 1.109\end{array}$

$\begin{array}{llll}1160.5 & -0.7632 & 0.000002 & 0.582\end{array}$

$\begin{array}{llll}1173.0 & -0.2901 & 0.000001 & 0.084\end{array}$

$\begin{array}{llll}1179.3 & -0.0729 & 0.000000 & 0.005\end{array}$

$\begin{array}{llll}1192.4 & 0.3278 & -0.000001 & 0.107\end{array}$

$\begin{array}{llll}1204.5 & 1.0114 & -0.000002 & 1.023\end{array}$

$1212.4 \quad 1.0179-0.000002 \quad 1.036$

$\begin{array}{llll}1236.0 & 0.9646 & -0.000002 & 0.930\end{array}$

$\begin{array}{llll}1236.0 & 0.9646 & -0.000002 & 0.930\end{array}$

$\begin{array}{llll}1252.8 & 0.8075 & -0.000001 & 0.652\end{array}$

$\begin{array}{llll}1267.3 & 0.6930 & -0.000001 & 0.480\end{array}$

$\begin{array}{lllll}1267.8 & 0.6970 & -0.000001 & 0.486\end{array}$

$\begin{array}{llll}1288.3 & 0.0673 & 0.000000 & 0.005\end{array}$

$\begin{array}{llll}1297.0 & 0.8062 & -0.000001 & 0.650\end{array}$

$\begin{array}{lllll}1297.9 & 0.8030 & -0.000001 & 0.645\end{array}$

$\begin{array}{llll}1309.8 & 0.3668 & 0.000000 & 0.135\end{array}$

$\begin{array}{llll}1327.1 & -0.3313 & 0.000000 & 0.110\end{array}$

$\begin{array}{llll}1327.3 & -0.3030 & 0.000000 & 0.092\end{array}$

$\begin{array}{llll}1338.6 & -0.9009 & 0.000001 & 0.812\end{array}$

$\begin{array}{llll}1354.9 & -1.6138 & 0.000001 & 2.604\end{array}$

$\begin{array}{llll}1355.1 & -1.6726 & 0.000002 & 2.798\end{array}$

$\begin{array}{llll}1380.0 & -2.9978 & 0.000002 & 8.987\end{array}$

$\begin{array}{llll}1420.0 & -0.8812 & 0.000001 & 0.776\end{array}$

$\begin{array}{llll}1431.0 & 18.7543 & -0.000011 & 351.726\end{array}$

$\begin{array}{llll}994.6 & -0.0507 & 0.000049 & 0.003\end{array}$

$\begin{array}{llll}995.2 & -0.0867 & 0.000042 & 0.008\end{array}$
Apparent Density of molal water / volume / $\quad \mathrm{kg} / \mathrm{m}^{3}$

995.6473 995.6473 995.6473 995.6473 995.6473 995.6473 995.6473 995.6473 995.6473 995.6473 995.6473 995.6473 995.6473 995.6473 995.6473 995.6473 995.6473 995.6473 995.6473 995.6473 995.6473 995.6473 995.6473 995.6473 995.6473 995.6473 995.6473 995.6473 995.6473 995.6473 995.6473 995.6473 995.6473 995.6473 995.6473 995.6473 995.6473 995.6473 995.6473 995.6473 995.6473 995.6473 994.0319 994.0319 
molality

0.06637

0.13233

0.27635

0.5498

$\begin{array}{cccc}\mathbf{t} /{ }^{\circ} \mathrm{C} \text { mass frac } & \begin{array}{r}\text { Density } \\ \exp / \\ \mathrm{kg} / \mathrm{m}^{3}\end{array} & \text { Ref } \\ & & & \\ 35 & 0.0042 & 996.21 & (82)\end{array}$

$\begin{array}{rrrrrr}35 & 0.0042 & 996.21 & (82) & 0.000477 & 0.000463 \\ 35 & 0.0083 & 998.25 & (82) & 0.000492 & 0.000463 \\ 35 & 0.0171 & 1002.81 & (82) & 0.000491 & 0.000464\end{array}$

$\begin{array}{lll}35 & 0.0335 & 1011.36\end{array}$

$\begin{array}{rrr}40 & 0.0100 & 997.3 \\ 40 & 0.0200 & 1002.5\end{array}$

$\begin{array}{lll}40 & 0.0200 & 1002.5 \\ 40 & 0.0300 & 1007.7\end{array}$

$\begin{array}{llll}40 & 0.0300 & 1007.7 & (90) \\ 40 & 0.0306 & 1007.9\end{array}$

$\begin{array}{lll}40 & 0.0391 & 1012.4 \\ 40 & 0.0400 & 1012.9\end{array}$

$\begin{array}{llll}40 & 0.0400 & 1012.9 & (90) \\ 40 & 0.0489 & 1017.7 & (35)\end{array}$

$\begin{array}{llll}40 & 0.0500 & 1018.2 \quad(90)\end{array}$

$\begin{array}{llll}40 & 0.0700 & 1029.0 & (35) \\ 40 & 0.0900 & 1039.9 & (35)\end{array}$

$\begin{array}{llll}40 & 0.1000 & 1045.5 \quad(90)\end{array}$

$\begin{array}{llll}40 & 0.1100 & 1051.0 & \text { (35) }\end{array}$

$40 \quad 0.1397 \quad 1068.1 \quad$ (35)

$\begin{array}{llll}40 & 0.1500 & 1073.9 \quad(90)\end{array}$

$\begin{array}{lll}0.1600 & 1079.7 \quad(35)\end{array}$

$0.1810 \quad 1091.9 \quad$ (35)

$0.2000 \quad 1103.1$

$0.2000 \quad 1103.1 \quad(90)$

$0.2212 \quad 1115.7$

$0.2400 \quad 1127.1$

$0.2500 \quad 1133.2 \quad(90)$

$0.2588 \quad 1138.7 \quad(35)$

$0.2800 \quad 1151.9$

$\begin{array}{lll}0.3000 & 1164.5 \quad(90)\end{array}$

$0.3100 \quad 1170.8$

$0.3308 \quad 1184.1 \quad$ (35)

$0.3500 \quad 1196.6 \quad(90)$

$0.3625 \quad 1204.3 \quad$ (35)

$0.4000 \quad 1227.0 \quad$ (35)

$0.4000 \quad 1227.0 \quad(90)$

$0.4267 \quad 1243.0 \quad$ (35)

$0.4500 \quad 1257.0 \quad(90)$

$0.4508 \quad 1257.5 \quad$ (35)

$0.4842 \quad 1277.7 \quad(35)$

$0.4985 \quad 1285.9 \quad(35)$

$0.5000 \quad 1286.7 \quad(90)$

$0.5200 \quad 1297.8$

$0.5497 \quad 1313.9 \quad(35)$

$0.5500 \quad 1314.1 \quad(90)$

$0.5700 \quad 1324.6 \quad$ (35)
App vol App vol $\exp / \quad$ Calc /

$$
\mathrm{m}^{3} / \mathrm{kg} \quad \mathrm{m}^{3} / \mathrm{kg}
$$

0.000491

$0.000494 \quad 0.000469$

$\begin{array}{ll}0.000491 & 0.000470\end{array}$

$\begin{array}{ll}0.000492 & 0.000471\end{array}$

$\begin{array}{ll}0.000496 & 0.000471\end{array}$

$0.000494 \quad 0.000472$

$\begin{array}{ll}0.000493 & 0.000472\end{array}$

$\begin{array}{lll}0.000492 & 0.000473\end{array}$

$0.000493 \quad 0.000474$

$0.000493 \quad 0.000476$

$0.000494 \quad 0.000479$

$0.000494 \quad 0.000480$

$0.000495 \quad 0.000481$

$0.000495 \quad 0.000485$

$0.000497 \quad 0.000486$

$\begin{array}{lll}0.000497 & 0.000488\end{array}$

$0.000499 \quad 0.000491$

$\begin{array}{ll}0.000501 & 0.000494\end{array}$

$\begin{array}{ll}0.000501 & 0.000494\end{array}$

$0.000504 \quad 0.000497$

$0.000505 \quad 0.000500$

$0.000506 \quad 0.000501$

$0.000507 \quad 0.000502$

$0.000509 \quad 0.000506$

$0.000511 \quad 0.000509$

$0.000512 \quad 0.000511$

$0.000514 \quad 0.000514$

$0.000516 \quad 0.000518$

$0.000518 \quad 0.000520$

$\begin{array}{ll}0.000526 & 0.000527\end{array}$

$0.000526 \quad 0.000527$

$0.000536 \quad 0.000537$

$0.000536 \quad 0.000537$

$0.000543 \quad 0.000544$

$\begin{array}{ll}0.000546 & 0.000547\end{array}$

$0.000547 \quad 0.000547$

$0.000551 \quad 0.000552$

$0.000559 \quad 0.000559$

$0.000559 \quad 0.000559$

$0.000564 \quad 0.000564$
$0.000531 \quad 0.000532$
Density Density App Vol Square of Inconsistent Inconsistent calc / Residual / Residual / Density based on based on $\mathrm{kg} / \mathrm{m}^{3} \quad \mathrm{~kg} / \mathrm{m}^{3} \quad \mathrm{~m}^{3} / \mathrm{kg}$ Residual density apparent

$\begin{array}{llll}996.3 & -0.0600 & 0.000015 & 0.004\end{array}$

$\begin{array}{llll}998.5 & -0.2360 & 0.000029 & 0.056\end{array}$

$\begin{array}{llll}1003.3 & -0.4724 & 0.000027 & 0.223\end{array}$

$\begin{array}{llll}1012.2 & -0.8653 & 0.000025 & 0.749\end{array}$

$\begin{array}{llll}997.6 & -0.2517 & 0.000025 & 0.063\end{array}$

$\begin{array}{llll}1002.9 & -0.4217 & 0.000021 & 0.178\end{array}$

$\begin{array}{llll}1008.3 & -0.6256 & 0.000021 & 0.391\end{array}$

$\begin{array}{llll}1008.7 & -0.7618 & 0.000024 & 0.580\end{array}$

$\begin{array}{llll}1013.3 & -0.8941 & 0.000022 & 0.799\end{array}$

$\begin{array}{llll}1013.8 & -0.8631 & 0.000021 & 0.745\end{array}$

$\begin{array}{llll}1018.6 & -0.9304 & 0.000018 & 0.866\end{array}$

$\begin{array}{llll}1019.2 & -1.0338 & 0.000020 & 1.069\end{array}$

$\begin{array}{llll}1030.3 & -1.2684 & 0.000017 & 1.609\end{array}$

$\begin{array}{llll}1041.4 & -1.5375 & 0.000016 & 2.364\end{array}$

$\begin{array}{llll}1047.1 & -1.5750 & 0.000014 & 2.481\end{array}$

$\begin{array}{llll}1052.7 & -1.7377 & 0.000014 & 3.020\end{array}$

$\begin{array}{llll}1069.7 & -1.6329 & 0.000010 & 2.666\end{array}$

$\begin{array}{llll}1075.7 & -1.7865 & 0.000010 & 3.191\end{array}$

$\begin{array}{llll}1081.5 & -1.7949 & 0.000010 & 3.222\end{array}$

$\begin{array}{llll}1093.8 & -1.8796 & 0.000009 & 3.533\end{array}$

$\begin{array}{llll}1105.0 & -1.8905 & 0.000008 & 3.574\end{array}$

$\begin{array}{llll}1105.0 & -1.8905 & 0.000008 & 3.574\end{array}$

$\begin{array}{llll}1117.6 & -1.8992 & 0.000007 & 3.607\end{array}$

$\begin{array}{llll}1128.9 & -1.7604 & 0.000006 & 3.099\end{array}$

$\begin{array}{llll}1134.9 & -1.6781 & 0.000005 & 2.816\end{array}$

$\begin{array}{llll}1140.2 & -1.4883 & 0.000004 & 2.215\end{array}$

$\begin{array}{llll}1153.0 & -1.1313 & 0.000003 & 1.280\end{array}$

$\begin{array}{llll}1165.2 & -0.7018 & 0.000002 & 0.493\end{array}$

$\begin{array}{llll}1171.3 & -0.5027 & 0.000001 & 0.253\end{array}$

$\begin{array}{llll}1184.0 & 0.0839 & 0.000000 & 0.007\end{array}$

$\begin{array}{llll}1195.8 & 0.8328 & -0.000002 & 0.693\end{array}$

$1203.4 \quad 0.8816-0.000002 \quad 0.777$

$1226.3 \quad 0.6763-0.000001 \quad 0.457$

$1226.3 \quad 0.6763-0.000001 \quad 0.457$

$\begin{array}{llll}1242.5 & 0.4699 & -0.000001 & 0.221\end{array}$

$\begin{array}{llll}1256.6 & 0.4472 & -0.000001 & 0.200\end{array}$

$\begin{array}{llll}1257.0 & 0.4682 & -0.000001 & 0.219\end{array}$

$\begin{array}{llll}1276.8 & 0.8601 & -0.000001 & 0.740\end{array}$

$\begin{array}{llll}1285.2 & 0.7129 & -0.000001 & 0.508\end{array}$

$\begin{array}{llll}1286.1 & 0.6427 & -0.000001 & 0.413\end{array}$

$\begin{array}{llll}1297.5 & 0.2500 & 0.000000 & 0.063\end{array}$

$\begin{array}{llll}1314.2 & -0.2830 & 0.000000 & 0.080\end{array}$

$\begin{array}{llll}1314.3 & -0.2480 & 0.000000 & 0.062\end{array}$

$\begin{array}{llll}1325.2 & -0.5955 & 0.000001 & 0.355\end{array}$
Apparent Density of molal water / volume / $\mathrm{kg} / \mathrm{m}^{3}$

$30.06 \quad 994.0319$

$30.98 \quad 994.0319$

$30.97 \quad 994.0319$

$30.955 \quad 994.0319$

992.2158

992.2158

992.2158

992.2158

992.2158

992.2158

992.2158

992.2158

992.2158

992.2158

992.2158

992.2158

992.2158

992.2158

992.2158

992.2158

992.2158

992.2158

992.2158

992.2158

992.2158

992.2158

992.2158

992.2158

992.2158

992.2158

992.2158

992.2158

992.2158

992.2158

992.2158

992.2158

992.2158

992.2158

992.2158

992.2158

992.2158

992.2158

992.2158

992.2158 
$t /{ }^{\circ} \mathrm{C}$ mass frac

0.01657

0.03313

0.06637

0.13233

0.27635

0.5498

$\begin{array}{crrr}\text { t }{ }^{\circ} \mathrm{C} \text { mass frac } & \text { Density } & \text { Ref } \\ & & \mathrm{exp} / & \\ & & \mathrm{kg} / \mathrm{m}^{3} & \\ 40 & 0.6000 & 1339.8 & (90) \\ 40 & 0.6003 & 1339.9 & (35) \\ 40 & 0.7011 & 1383.5 & (35) \\ 40 & 0.7533 & 1403.4 & (35) \\ 40 & 0.8011 & 1425.8 & (35) \\ 45 & 0.0010 & 990.73 & (82) \\ 45 & 0.0021 & 991.26 & (82) \\ 45 & 0.0042 & 992.36 & (82) \\ 45 & 0.0083 & 994.39 & (82) \\ 45 & 0.0171 & 998.88 & (82) \\ 45 & 0.0335 & 1007.26 & (82) \\ 50 & 0.0100 & 993.1 & (90) \\ 50 & 0.0200 & 998.2 & (90) \\ 50 & 0.0300 & 1003.3 & (90) \\ 50 & 0.0306 & 1003.6 & (35) \\ 50 & 0.0391 & 1007.9 & (35) \\ 50 & 0.0400 & 1008.4 & (90) \\ 50 & 0.0489 & 1013.0 & (35) \\ 50 & 0.0500 & 1013.6 & (90) \\ 50 & 0.0699 & 1024.1 & (35) \\ 50 & 0.0899 & 1034.9 & (35) \\ 50 & 0.1000 & 1040.3 & (90) \\ 50 & 0.1100 & 1045.7 & (35) \\ 50 & 0.1397 & 1062.4 & (35) \\ 50 & 0.1500 & 1068.0 & (90) \\ 50 & 0.1600 & 1073.7 & (35) \\ 50 & 0.1810 & 1085.7 & (35) \\ 50 & 0.2000 & 1096.6 & (35) \\ 50 & 0.2000 & 1096.6 & (90) \\ 50 & 0.2212 & 1109.0 & (35) \\ 50 & 0.2400 & 1120.1 & (35) \\ 50 & 0.2500 & 1126.0 & (90) \\ 50 & 0.2800 & 1144.2 & (35) \\ 50 & 0.3000 & 1156.4 & (90) \\ 50 & 0.3100 & 1162.5 & (35) \\ 50 & 0.3308 & 1175.4 & (35) \\ 50 & 0.3500 & 1187.6 & (90) \\ 50 & 0.3625 & 1195.0 & (35) \\ 50 & 0.4000 & 1217.0 & (35) \\ 50 & 0.4000 & 1217.0 & (90) \\ 50 & 0.4267 & 1232.6 & (35) \\ 50 & 0.4500 & 1246.1 & (90) \\ 50 & 0.4508 & 1246.6 & (35) \\ 50 & 0.4842 & 1266.1 & (35) \\ & & & \end{array}$

App vol App vol

$\exp /$ Calc/

$\mathrm{m}^{3} / \mathrm{kg} \quad \mathrm{m}^{3} / \mathrm{kg}$

$\begin{array}{ll}0.000572 & 0.000571\end{array}$

$0.000572 \quad 0.000571$

$0.000601 \quad 0.000600$

$0.000616 \quad 0.000616$

$0.000625 \quad 0.000633$

$0.000503 \quad 0.000473$

$\begin{array}{ll}0.000497 & 0.000473\end{array}$

$0.000485 \quad 0.000474$

$0.000497 \quad 0.000474$

$0.000498 \quad 0.000475$

$\begin{array}{ll}0.000499 & 0.000477\end{array}$

$0.000496 \quad 0.000480$

$0.000497 \quad 0.000481$

$0.000499 \quad 0.000482$

$0.000500 \quad 0.000482$

$0.000502 \quad 0.000483$

$\begin{array}{ll}0.000501 & 0.000483\end{array}$

$0.000502 \quad 0.000484$

$\begin{array}{lll}0.000502 & 0.000484\end{array}$

$0.000502 \quad 0.000487$

$0.000502 \quad 0.000489$

$0.000504 \quad 0.000491$

$0.000505 \quad 0.000492$

$0.000505 \quad 0.000496$

$\begin{array}{ll}0.000507 & 0.000497\end{array}$

$0.000507 \quad 0.000499$

$0.000509 \quad 0.000502$

$0.000511 \quad 0.000504$

$\begin{array}{lll}0.000511 & 0.000504\end{array}$

$\begin{array}{ll}0.000513 & 0.000507\end{array}$

$0.000515 \quad 0.000510$

$\begin{array}{ll}0.000516 & 0.000512\end{array}$

$\begin{array}{ll}0.000519 & 0.000517\end{array}$

$0.000521 \quad 0.000520$

$0.000522 \quad 0.000521$

$0.000524 \quad 0.000525$

$0.000526 \quad 0.000528$

$0.000529 \quad 0.000530$

$\begin{array}{ll}0.000536 & 0.000537\end{array}$

$\begin{array}{ll}0.000536 & 0.000537\end{array}$

$\begin{array}{ll}0.000541 & 0.000542\end{array}$

$\begin{array}{ll}0.000546 & 0.000547\end{array}$

$\begin{array}{ll}0.000546 & 0.000547\end{array}$

$0.000553 \quad 0.000554$
Density Density App Vol Square of Inconsistent Inconsistent calc / Residual / Residual / Density based on based on $\mathrm{kg} / \mathrm{m}^{3} \quad \mathrm{~kg} / \mathrm{m}^{3} \quad \mathrm{~m}^{3} / \mathrm{kg}$ Residual density apparent

$\begin{array}{llll}1340.8 & -1.0325 & 0.000001 & 1.066\end{array}$

$\begin{array}{llll}1341.0 & -1.0846 & 0.000001 & 1.176\end{array}$

$\begin{array}{llll}1385.8 & -2.3449 & 0.000002 & 5.499\end{array}$

$\begin{array}{llll}14027 & 0.7200 & 0.000000 & 0.518\end{array}$

$\begin{array}{llll}1412.9 & 12.8600 & -0.000008 & 165.379\end{array}$

$\begin{array}{llll}990.8 & -0.0306 & 0.000030 & 0.001\end{array}$

$\begin{array}{llll}991.3 & -0.0485 & 0.000024 & 0.002\end{array}$

$\begin{array}{llll}992.4 & -0.0455 & 0.000011 & 0.002\end{array}$

$\begin{array}{llll}994.6 & -0.1842 & 0.000023 & 0.034\end{array}$

$\begin{array}{llll}999.3 & -0.3888 & 0.000023 & 0.151\end{array}$

$\begin{array}{cccc}1008.0 & -0.7624 & 0.000022 & 0.581\end{array}$

$\begin{array}{llll}993.3 & -0.1611 & 0.000016 & 0.026\end{array}$

$\begin{array}{llll}998.5 & -0.3183 & 0.000016 & 0.101\end{array}$

$\begin{array}{llll}1003.8 & -0.5075 & 0.000017 & 0.258\end{array}$

$\begin{array}{llll}1004.1 & -0.5365 & 0.000017 & 0.288\end{array}$

$\begin{array}{llll}1008.7 & -0.7696 & 0.000019 & 0.592\end{array}$

$\begin{array}{llll}1009.1 & -0.7285 & 0.000018 & 0.531\end{array}$

$\begin{array}{llll}1013.9 & -0.8907 & 0.000018 & 0.793\end{array}$

$\begin{array}{llll}1014.5 & -0.8810 & 0.000017 & 0.776\end{array}$

$\begin{array}{llll}1025.2 & -1.1247 & 0.000015 & 1.265\end{array}$

$\begin{array}{llll}1036.1 & -1.2438 & 0.000013 & 1.547\end{array}$

$\begin{array}{lll}10417 & -1.4029 & 0.000013\end{array}$

$\begin{array}{llll}1047.2 & -1.5362 & 0.000013 & 2.360\end{array}$

$\begin{array}{llll}1063.8 & -1.4354 & 0.000009 & 2.060\end{array}$

$\begin{array}{lll}1069.6 & -1.6477 & 0.000010\end{array}$

$\begin{array}{llll}1075.3 & -1.6169 & 0.000009 & 2.614\end{array}$

$\begin{array}{llll}1087.3 & -1.6029 & 0.000008 & 2.569\end{array}$

$\begin{array}{llll}1098.2 & -1.6362 & 0.000007 & 2.677\end{array}$

$\begin{array}{llll}1098.2 & -1.6362 & 0.000007 & 2.677\end{array}$

$\begin{array}{llll}1110.5 & -1.5266 & 0.000006 & 2.330\end{array}$

$\begin{array}{llll}1121.5 & -1.3981 & 0.000005 & 1.955\end{array}$

$\begin{array}{llll}1127.4 & -1.3590 & 0.000004 & 1.847\end{array}$

$\begin{array}{llll}1145.0 & -0.8300 & 0.000002 & 0.689\end{array}$

$\begin{array}{llll}1156.9 & -0.4696 & 0.000001 & 0.220\end{array}$

$\begin{array}{llll}1162.8 & -0.3022 & 0.000001 & 0.091\end{array}$

$\begin{array}{lllll}1175.2 & 0.2400 & -0.000001 & 0.058\end{array}$

$\begin{array}{llll}1186.6 & 1.0239 & -0.000002 & 1.048\end{array}$

$1194.0 \quad 0.9942-0.000002 \quad 0.988$

$\begin{array}{lllll}1216.2 & 0.7677 & -0.000001 & 0.589\end{array}$

$\begin{array}{llll}1216.2 & 0.7677 & -0.000001 & 0.589\end{array}$

$1231.9 \quad 0.6565-0.000001 \quad 0.431$

$1245.5 \quad 0.5726-0.000001 \quad 0.328$

$\begin{array}{lllll}1246.0 & 0.6088 & -0.000001 & 0.371\end{array}$

$\begin{array}{llll}1265.2 & 0.9385 & -0.000001 & 0.881\end{array}$
Apparent Density of molal water / volume / $\quad \mathrm{kg} / \mathrm{m}^{3}$

992.2158 992.2158 992.2158 992.2158 992.2158

$31.7 \quad 990.2132$

$31.32 \quad 990.2132$

$30.54 \quad 990.2132$

$31.29 \quad 990.2132$

$31.37 \quad 990.2132$

$31.471 \quad 990.2132$ 988.0363 988.0363 988.0363 988.0363 988.0363 988.0363 988.0363 988.0363 988.0363 988.0363 988.0363 988.0363 988.0363 988.0363 988.0363 988.0363 988.0363 988.0363 988.0363 988.0363 988.0363 988.0363 988.0363 988.0363 988.0363 988.0363 988.0363 988.0363 988.0363 988.0363 988.0363 988.0363 988.0363 
molality molarity

$\mathrm{t} /{ }^{\circ} \mathrm{C}$ mass frac

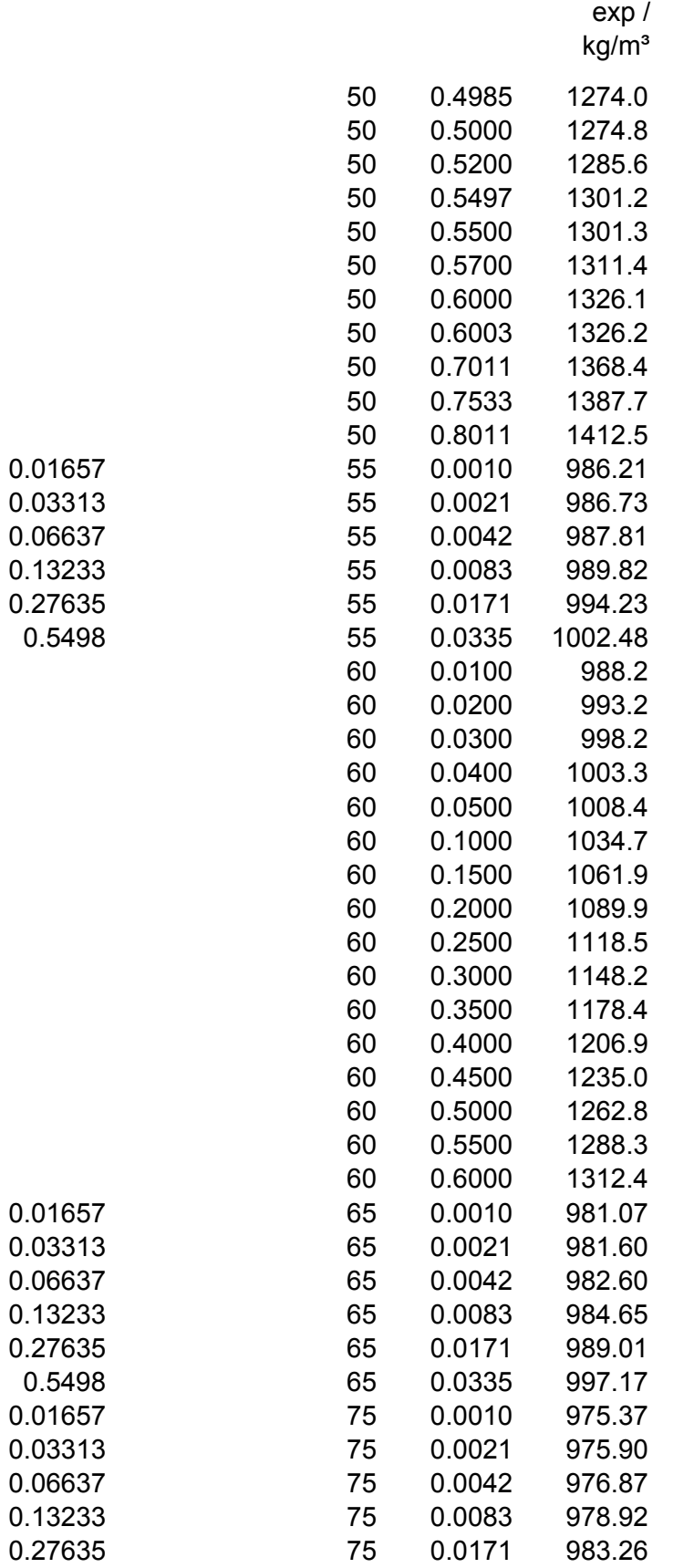

Ref

App vol App vol

$$
\mathrm{m}^{3} / \mathrm{kg} \quad \mathrm{m}^{3} / \mathrm{kg}
$$

$\begin{array}{ll}0.000556 & 0.000557\end{array}$ $0.000557 \quad 0.000558$ $0.000562 \quad 0.000562$ 0.0005690 .000569 0.0005690 .000569 $0.000574 \quad 0.000574$ 0.0005820 .000581 $0.000582 \quad 0.000581$ $\begin{array}{ll}0.000611 & 0.000609\end{array}$ $0.000625 \quad 0.000626$ $0.000632 \quad 0.000643$ $\begin{array}{lll}0.000506 & 0.000484\end{array}$ $0.000505 \quad 0.000484$ $0.000492 \quad 0.000484$ $0.000503 \quad 0.000485$ $0.000506 \quad 0.000486$ $0.000507 \quad 0.000488$ $\begin{array}{lll}0.000502 & 0.000491\end{array}$ $\begin{array}{ll}0.000505 & 0.000492\end{array}$ $0.000508 \quad 0.000493$ $0.000508 \quad 0.000494$ $0.000509 \quad 0.000495$ $\begin{array}{lll}0.000511 & 0.000501\end{array}$ 0.0005150 .000508 $0.000519 \quad 0.000515$ $0.000525 \quad 0.000522$ $0.000530 \quad 0.000530$ $\begin{array}{lll}0.000536 & 0.000539\end{array}$ $\begin{array}{ll}0.000546 & 0.000548\end{array}$ $\begin{array}{ll}0.000556 & 0.000557\end{array}$ $0.000567 \quad 0.000568$ $\begin{array}{lll}0.000579 & 0.000579\end{array}$ $0.000592 \quad 0.000591$ $0.000505 \quad 0.000495$ $0.000499 \quad 0.000495$ $0.000510 \quad 0.000495$ $\begin{array}{lll}0.000507 & 0.000496\end{array}$ $\begin{array}{lll}0.000510 & 0.000497\end{array}$ $0.000512 \quad 0.000499$ $0.000500 \quad 0.000505$ $\begin{array}{ll}0.000496 & 0.000506\end{array}$ $0.000517 \quad 0.000506$ $\begin{array}{ll}0.000510 & 0.000506\end{array}$ $\begin{array}{lll}0.000513 & 0.000507\end{array}$
Density Density App Vol Square of Inconsistent Inconsistent calc / Residual / Residual / Density based on based on $\mathrm{kg} / \mathrm{m}^{3} \quad \mathrm{~kg} / \mathrm{m}^{3} \quad \mathrm{~m}^{3} / \mathrm{kg}$ Residual density apparent volume

$\begin{array}{llll}1273.2 & 0.7665 & -0.000001 & 0.588\end{array}$

$\begin{array}{llll}1274.1 & 0.7253 & -0.000001 & 0.526\end{array}$

$\begin{array}{llll}1285.2 & 0.4187 & 0.000000 & 0.175\end{array}$

$\begin{array}{llll}1301.2 & -0.0411 & 0.000000 & 0.002\end{array}$

$\begin{array}{llll}1301.4 & -0.1004 & 0.000000 & 0.010\end{array}$

$\begin{array}{llll}1311.9 & -0.4638 & 0.000000 & 0.215\end{array}$

$\begin{array}{llll}1326.9 & -0.8316 & 0.000001 & 0.692\end{array}$

$\begin{array}{llll}1327.1 & -0.8781 & 0.000001 & 0.771\end{array}$

$\begin{array}{llll}1370.2 & -1.7647 & 0.000001 & 3.114\end{array}$

$1386.2 \quad 1.4659-0.000001 \quad 2.149$

$\begin{array}{lrr}1395.9 & 16.5618 & -0.000010 \quad 274.294\end{array}$

$\begin{array}{llll}986.2 & -0.0225 & 0.000022 & 0.001\end{array}$

$\begin{array}{llll}986.8 & -0.0424 & 0.000021 & 0.002\end{array}$

$\begin{array}{llll}987.8 & -0.0304 & 0.000007 & 0.001\end{array}$

$\begin{array}{llll}990.0 & -0.1469 & 0.000018 & 0.022\end{array}$

$\begin{array}{llll}994.6 & -0.3370 & 0.000020 & 0.114\end{array}$

$\begin{array}{llll}1003.1 & -0.6551 & 0.000019 & 0.429\end{array}$

$\begin{array}{llll}988.3 & -0.1155 & 0.000012 & 0.013\end{array}$

$\begin{array}{llll}993.5 & -0.2628 & 0.000013 & 0.069\end{array}$

$\begin{array}{llll}998.6 & -0.4407 & 0.000015 & 0.194\end{array}$

$\begin{array}{llll}1003.8 & -0.5488 & 0.000014 & 0.301\end{array}$

$1009.1-0.6869-0.000014$

$\begin{array}{llll}1035.7 & -1.0131 & 0.000009 & 1.026\end{array}$

$\begin{array}{llll}1063.0 & -1.1222 & 0.000007 & 1.259\end{array}$

$\begin{array}{llll}1090.9 & -1.0353 & 0.000004 & 1.072\end{array}$

$\begin{array}{llll}1119.3 & -0.8441 & 0.000003 & 0.713\end{array}$

$\begin{array}{llll}1148.1 & 0.0956 & 0.000000 & 0.009\end{array}$

$1177.0 \quad 1.3715-0.000003 \quad 1.881$

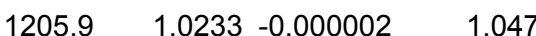

$1234.3 \quad 0.6529-0.000001 \quad 0.426$

$\begin{array}{llll}1262.1 & 0.7343 & -0.000001 & 0.539\end{array}$

$\begin{array}{llll}1288.6 & -0.2749 & 0.000000 & 0.076\end{array}$

$\begin{array}{llll}1313.3 & -0.9233 & 0.000001 & 0.853\end{array}$

$\begin{array}{llll}981.1 & -0.0106 & 0.000011 & 0.000\end{array}$

$\begin{array}{llll}981.6 & -0.0073 & 0.000004 & 0.000\end{array}$

$\begin{array}{llll}982.7 & -0.0577 & 0.000014 & 0.003\end{array}$

$\begin{array}{llll}984.7 & -0.0911 & 0.000011 & 0.008\end{array}$

$\begin{array}{llll}989.2 & -0.2264 & 0.000014 & 0.051\end{array}$

$\begin{array}{llll}997.6 & -0.4618 & 0.000014 & 0.213\end{array}$

$\begin{array}{llll}975.4 & 0.0055 & -0.000006 & 0.000\end{array}$

$\begin{array}{llll}975.9 & 0.0181 & -0.000009 & 0.000\end{array}$

$\begin{array}{llll}976.9 & -0.0448 & 0.000011 & 0.002\end{array}$

$\begin{array}{llll}978.9 & -0.0278 & 0.000004 & 0.001\end{array}$

$\begin{array}{llll}983.4 & -0.0971 & 0.000006 & 0.009\end{array}$
Apparent Density of molal water / volume / $\mathrm{kg} / \mathrm{m}^{3}$

988.0363 988.0363 988.0363 988.0363 988.0363 988.0363 988.0363 988.0363 988.0363 988.0363 988.0363 $31.9 \quad 985.6952$ $31.83 \quad 985.6952$ $31 \quad 985.6952$ $31.7 \quad 985.6952$

$31.877 \quad 985.6952$ $31.969 \quad 985.6952$ 983.1989 983.1989 983.1989 983.1989 983.1989 983.1989 983.1989 983.1989 983.1989 983.1989 983.1989 983.1989 983.1989 983.1989 983.1989 983.1989 $31.85 \quad 980.5548$ $31.42 \quad 980.5548$ $32.11 \quad 980.5548$ $31.95 \quad 980.5548$

32.15980 .5548 $32.291 \quad 980.5548$

$31.5 \quad 974.849$

$31.28 \quad 974.849$

$32.58 \quad 974.849$

$32.12 \quad 974.849$

$32.331 \quad 974.849$ 


$\begin{array}{rrrr}\text { molality } & \text { molarity } & \mathbf{t} /{ }^{\circ} \mathrm{C} \text { mass frac } & \text { Density } \\ & & & \text { exp } / \\ \mathrm{kg} / \mathrm{m}^{3}\end{array}$

Ref

App vol App vol

$$
\mathrm{m}^{3} / \mathrm{kg} \quad \mathrm{m}^{3} / \mathrm{kg}
$$

$\begin{array}{ll}0.000516 & 0.000509\end{array}$

$\begin{array}{ll}0.000513 & 0.000512\end{array}$

$\begin{array}{ll}0.000515 & 0.000513\end{array}$

$0.000518 \quad 0.000514$

$0.000518 \quad 0.000515$

$0.000519 \quad 0.000516$

$0.000523 \quad 0.000522$

$0.000527 \quad 0.000528$

0.0005330 .000535

$0.000540 \quad 0.000542$

$0.000547 \quad 0.000550$

$0.000554 \quad 0.000558$

$\begin{array}{ll}0.000565 & 0.000567\end{array}$

$0.000576 \quad 0.000576$

$\begin{array}{lll}0.000587 & 0.000586\end{array}$

$\begin{array}{ll}0.000599 & 0.000597\end{array}$

0.0006120 .000609

0.0004940 .000516

$0.000494 \quad 0.000516$

$0.000514 \quad 0.000516$

$\begin{array}{ll}0.000511 & 0.000517\end{array}$

$\begin{array}{ll}0.000514 & 0.000517\end{array}$

$0.000518 \quad 0.000519$

$0.000490 \quad 0.000526$

$0.000505 \quad 0.000526$

$\begin{array}{lll}0.000510 & 0.000527\end{array}$

$0.000514 \quad 0.000527$

$0.000518 \quad 0.000529$

$0.000520 \quad 0.000532$

0.0005190 .000533

$\begin{array}{ll}0.000520 & 0.000534\end{array}$

$0.000522 \quad 0.000535$

$0.000522 \quad 0.000536$

$\begin{array}{ll}0.000527 & 0.000542\end{array}$

$0.000534 \quad 0.000548$

$0.000544 \quad 0.000554$

$\begin{array}{ll}0.000554 & 0.000561\end{array}$

$\begin{array}{ll}0.000562 & 0.000568\end{array}$

0.0005710 .000576

$0.000583 \quad 0.000584$

$\begin{array}{ll}0.000595 & 0.000593\end{array}$

$0.000607 \quad 0.000602$

$0.000620 \quad 0.000612$

$0.000633 \quad 0.000624$
Density Density App Vol Square of Inconsistent Inconsistent calc / Residual / Residual / Density based on based on $\mathrm{kg} / \mathrm{m}^{3} \quad \mathrm{~kg} / \mathrm{m}^{3} \quad \mathrm{~m}^{3} / \mathrm{kg}$ Residual density apparent

$\begin{array}{llll}991.6 & -0.2264 & 0.000007 & 0.051\end{array}$

$\begin{array}{llll}976.7 & -0.0090 & 0.000001 & 0.000\end{array}$

$\begin{array}{llll}981.6 & -0.0486 & 0.000003 & 0.002\end{array}$

$\begin{array}{llll}986.6 & -0.1164 & 0.000004 & 0.014\end{array}$

$\begin{array}{llll}991.6 & -0.1121 & 0.000003 & 0.013\end{array}$

$\begin{array}{llll}996.6 & -0.1354 & 0.000003 & 0.018\end{array}$

$\begin{array}{llll}1022.2 & -0.0529 & 0.000001 & 0.003\end{array}$

$1048.3 \quad 0.2029-0.000001 \quad 0.041$

$\begin{array}{llll}1075.0 & 0.4072 & -0.000002 & 0.166\end{array}$

$\begin{array}{llll}1102.1 & 0.5621 & -0.000002 & 0.316\end{array}$

$\begin{array}{llll}1129.6 & 1.1028 & -0.000003 & 1.216\end{array}$

$1157.2 \quad 1.9037-0.000004 \quad 3.624$

$\begin{array}{llll}1184.7 & 1.0873 & -0.000002 & 1.182\end{array}$

$\begin{array}{llll}1211.9 & 0.0321 & 0.000000 & 0.001\end{array}$

$\begin{array}{llll}1238.3 & -0.6172 & 0.000001 & 0.381\end{array}$

$\begin{array}{llll}1263.6 & -2.1400 & 0.000002 & 4.579\end{array}$

$\begin{array}{llll}1287.3 & -3.4297 & 0.000003 & 11.763\end{array}$

$\begin{array}{llll}969.1 & 0.0217 & -0.000022 & 0.000\end{array}$

$\begin{array}{llll}969.6 & 0.0421 & -0.000022 & 0.002\end{array}$

$\begin{array}{llll}970.6 & 0.0062 & -0.000002 & 0.000\end{array}$

$\begin{array}{llll}972.6 & 0.0419-0.000005 & 0.002\end{array}$

$977.0-0.0567-0.000003$

$\begin{array}{llll}985.0 & 0.0349 & -0.000001 & 0.001\end{array}$

$\begin{array}{llll}962.9 & 0.0701 & -0.000036 & 0.005\end{array}$

$\begin{array}{llll}963.9 & 0.0805 & -0.000021 & 0.006\end{array}$

$\begin{array}{llll}965.8 & 0.1260 & -0.000016 & 0.016\end{array}$

$\begin{array}{llll}970.1 & 0.2202 & -0.000014 & 0.048\end{array}$

$\begin{array}{llll}978.0 & 0.3473 & -0.000011 & 0.121\end{array}$

$\begin{array}{llll}963.1 & 0.1121 & -0.000012 & 0.013\end{array}$

$\begin{array}{llll}967.8 & 0.2608 & -0.000014 & 0.068\end{array}$

$\begin{array}{llll}972.6 & 0.3828 & -0.000013 & 0.147\end{array}$

$\begin{array}{llll}977.4 & 0.4781 & -0.000013 & 0.229\end{array}$

$\begin{array}{llll}982.3 & 0.6471 & -0.000013 & 0.419\end{array}$

$1006.8 \quad 1.5090-0.000015$

$\begin{array}{llll}1031.9 & 2.1690 & -0.000014 & 4.705\end{array}$

$\begin{array}{llll}1057.6 & 2.1942 & -0.000010 \quad 4.814\end{array}$

$\begin{array}{llll}1083.7 & 1.9753 & -0.000007 & 3.902\end{array}$

$\begin{array}{llll}1110.2 & 2.0329 & -0.000005 & 4.132\end{array}$

$\begin{array}{llll}1136.8 & 2.2227 & -0.000005 & 4.940\end{array}$

$\begin{array}{llll}1163.4 & 0.4436 & -0.000001 & 0.197\end{array}$

$\begin{array}{llll}1189.7 & -1.7549 & 0.000003 & 3.080\end{array}$

$\begin{array}{llll}1215.4 & -3.5635 & 0.000005 & 12.699\end{array}$

$\begin{array}{llll}1240.1 & -6.2036 & 0.000007 & 38.485\end{array}$

$\begin{array}{llll}1263.4 & -8.7176 & 0.000009 & 75.996\end{array}$
Apparent Density of molal water / volume / $\quad \mathrm{kg} / \mathrm{m}^{3}$

$32.512 \quad 974.849$ 971.7978 971.7978 971.7978 971.7978 971.7978 971.7978 971.7978 971.7978 971.7978 971.7978 971.7978 971.7978 971.7978 971.7978 971.7978 971.7978 31.1968 .6203 $31.15 \quad 968.6203$ 32.42968 .6203 $32.21 \quad 968.6203$ $32.39 \quad 968.6203$ $32.656 \quad 968.6203$ $30.85 \quad 961.9004$ $31.84 \quad 961.9004$ $32.15 \quad 961.9004$ $32.377 \quad 961.9004$ $32.667 \quad 961.9004$ 958.3637 958.3637 958.3637 958.3637 958.3637 958.3637 958.3637 958.3637 958.3637 958.3637 958.3637 958.3637 958.3637 958.3637 958.3637 958.3637 
molality molarity

$t /{ }^{\circ} \mathrm{C}$ mass frac

Density

$\exp$

$\mathrm{kg} / \mathrm{m}^{3}$
Ref

App vol
$\exp /$
$\mathrm{m}^{3} / \mathrm{kg}$

App vol

Calc /

$\mathrm{m}^{3} / \mathrm{kg}$
Dens calc / Residual / Residual / Density based on $\mathrm{kg} / \mathrm{m}^{3}$ $\mathrm{kg} / \mathrm{m}^{3}$

$\mathrm{m}^{3} / \mathrm{kg}$

Density based on apparen

Average Res Std dev Res Avg - 4std

Avg +4 std

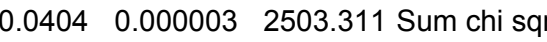
$2.2953 \quad 0.000010$

$-9.2217-0.000035$

$9.1408 \quad 0.000042$

Curve fit for the model App vol $=\left(w+c 2+c 3^{*} t{ }^{\circ} \mathrm{C}\right) /\left(c 0^{*} w+c 1\right) / e^{\wedge}\left(0.000001^{*}\left(t{ }^{\circ} \mathrm{C}+c 4\right)^{\wedge} 2\right)$

$\begin{array}{lr}\text { c0 } & 12.9926 \\ \text { c1 } & -23.57871 \\ \text { c2 } & -3.606972 \\ \text { c3 } & 0.007942 \\ \text { c4 } & -2427.104 \\ \text { Min T } & -10 \\ \text { Max T } & 100 \\ \text { Min w } & 0.001 \\ \text { Max w } & 0.801 \\ \text { Avg dens res } & -0.0404 \\ \text { Std dens res } & 2.2953 \\ \text { Avg app vol res *10^3 } & 0.003308 \\ \text { Std app vol res *10^3 } & 0.009635 \\ \text { No of points in corr } & 476 \\ \text { No of inconsistent poir } & 2\end{array}$

\section{Inconsistent data not used (Residual greater than average +/- $\mathbf{4}$ standard deviations)}

$\begin{array}{lllllll}0.03313 & 25 & 0.0021 & 998.06 & (82) & 0.000511 & 0.000451 \\ & 30 & 0.7011 & 1416.6 & (35) & 0.000579 & 0.000589\end{array}$

0.01657

$\begin{array}{lllll}0.0010 & 962.45 & (82) & 0.000471 & 0.000526\end{array}$

$998.2-0.1242 \quad 0.000060$

$1402.1 \quad 14.4725-0.000010$

962.4
$0.0526-0.000054$
0.015

0.003
Apparent Density of molal water / $\mathrm{cm}^{3} / \mathrm{mol} \quad \mathrm{kg} / \mathrm{m}^{3}$ 
Density of aqueous solution of $\mathrm{H} 3 \mathrm{PO} 4$

References (17) Christensen, J. H.; Reed, R. B. Density of Aqueous Solutions of Phosphoric Acid. Measurements at 25². Ind. Eng. Chem. 1955, 47, 1277-1279.

(25) Egan, E. P.; Luff, B. B. Density of Aqueous Solutions of Phosphoric Acid. Measurements at $15^{\circ}$ to $80^{\circ} \mathrm{C}$. Ind. Eng. Chem. 1955, 47 , 1280-1280.

(90) Perry, R. H.; Green, D. W. Perry's Chemical Engineers' Handbook; McGraw Hill: New York, 7th edition, 1997.

\begin{tabular}{|c|c|c|c|c|c|c|c|c|c|c|c|c|c|c|}
\hline \multirow[t]{40}{*}{ molality } & \multicolumn{2}{|c|}{$t^{\circ} \mathrm{C}$ mass frac } & $\begin{array}{r}\text { Density } \\
\text { exp } \mathrm{kg} / \mathrm{m}^{3}\end{array}$ & Ref & 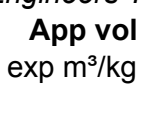 & $\begin{array}{r}\text { App vol } \\
\text { Calc } \\
\mathrm{m}^{3} / \mathrm{kg}\end{array}$ & $\begin{array}{r}\text { Density } \\
\text { alc } \mathrm{kg} / \mathrm{m}^{3}\end{array}$ & $\begin{array}{r}\text { Density } \\
\text { Residual } \\
\mathrm{kg} / \mathrm{m}^{3}\end{array}$ & $\begin{array}{r}\text { App Vol } \\
\text { Residual } \\
\mathrm{m}^{3} / \mathrm{kg}\end{array}$ & $\begin{array}{r}\text { Square of } \\
\text { Density } \\
\text { Residual }\end{array}$ & $\begin{array}{l}\text { Inconsistent } \\
\text { based on } \\
\text { density }\end{array}$ & $\begin{array}{c}\text { Inconsistent } \\
\text { based on } \\
\text { apparent } \\
\text { volume }\end{array}$ & $\begin{array}{r}\text { Delta density } \\
\qquad / \mathrm{kg} / \mathrm{m}^{3}\end{array}$ & $\begin{array}{r}\text { Density of } \\
\text { water } / \mathrm{kg} / \mathrm{m}^{3}\end{array}$ \\
\hline & 15.85 & 0.0446 & 1023.20 & (25) & 0.000469 & 0.000470 & 1023.17 & 0.0255 & -0.000001 & 0.001 & & & & 998.9673 \\
\hline & 15.85 & 0.0935 & 1051.40 & (25) & 0.000467 & 0.000472 & 1050.87 & 0.5288 & -0.000005 & 0.280 & & & & 998.9673 \\
\hline & 15.85 & 0.1921 & 1111.40 & (25) & 0.000474 & 0.000477 & 1110.67 & 0.7270 & -0.000003 & 0.529 & & & & 998.9673 \\
\hline & 15.85 & 0.2933 & 1179.10 & (25) & 0.000480 & 0.000482 & 1178.09 & 1.0069 & -0.000002 & 1.014 & & & & 998.9673 \\
\hline & 15.85 & 0.3915 & 1251.10 & (25) & 0.000486 & 0.000487 & 1250.05 & 1.0512 & -0.000002 & 1.105 & & & & 998.9673 \\
\hline & 15.85 & 0.4936 & 1332.50 & (25) & 0.000493 & 0.000493 & 1332.46 & 0.0356 & 0.000000 & 0.001 & & & & 998.9673 \\
\hline & 15.85 & 0.5954 & 1423.90 & (25) & 0.000499 & 0.000500 & 1423.18 & 0.7211 & -0.000001 & 0.520 & & & & 998.9673 \\
\hline & 15.85 & 0.7010 & 1528.00 & (25) & 0.000507 & 0.000507 & 1527.17 & 0.8321 & -0.000001 & 0.692 & & & & 998.9673 \\
\hline & 15.85 & 0.7997 & 1634.50 & (25) & 0.000514 & 0.000514 & 1634.16 & 0.3353 & 0.000000 & 0.112 & & & & 998.9673 \\
\hline & 15.85 & 0.8481 & 1690.30 & (25) & 0.000518 & 0.000518 & 1690.24 & 0.0588 & 0.000000 & 0.003 & & & & 998.9673 \\
\hline & 20 & 0.0200 & 1009.20 & (90) & 0.000456 & 0.000471 & 1008.91 & 0.2947 & -0.000014 & 0.087 & & & & 998.2041 \\
\hline & 20 & 0.0600 & 1030.90 & (90) & 0.000472 & 0.000472 & 1030.90 & 0.0021 & 0.000000 & 0.000 & & & & 998.2041 \\
\hline & 20 & 0.1400 & 1076.40 & (90) & 0.000482 & 0.000476 & 1077.37 & -0.9738 & 0.000006 & 0.948 & & & & 998.2041 \\
\hline & 20 & 0.2000 & 1113.40 & (90) & 0.000484 & 0.000479 & 1114.56 & -1.1639 & 0.000005 & 1.355 & & & & 998.2041 \\
\hline & 20 & 0.2600 & 1152.90 & (90) & 0.000485 & 0.000482 & 1153.91 & -1.0097 & 0.000003 & 1.020 & & & & 998.2041 \\
\hline & 20 & 0.3500 & 1216.00 & (90) & 0.000489 & 0.000487 & 1217.30 & -1.2990 & 0.000003 & 1.687 & & & & 998.2041 \\
\hline & 20 & 0.5000 & 1335.00 & (90) & 0.000496 & 0.000495 & 1335.94 & -0.9437 & 0.000001 & 0.891 & & & & 998.2041 \\
\hline & 25 & 0.0010 & 997.64 & (17) & 0.000400 & 0.000472 & 997.57 & 0.0715 & -0.000072 & 0.005 & & $!$ & 0.60 & 997.0449 \\
\hline & 25 & 0.0020 & 998.14 & (17) & 0.000450 & 0.000472 & 998.10 & 0.0425 & -0.000021 & 0.002 & & & 1.10 & 997.0449 \\
\hline & 25 & 0.0030 & 998.74 & (17) & 0.000434 & 0.000472 & 998.63 & 0.1130 & -0.000038 & 0.013 & & & 1.70 & 997.0449 \\
\hline & 25 & 0.0040 & 999.34 & (17) & 0.000426 & 0.000472 & 999.16 & 0.1830 & -0.000046 & 0.034 & & $!$ & 2.30 & 997.0449 \\
\hline & 25 & 0.0050 & 999.84 & (17) & 0.000441 & 0.000472 & 999.69 & 0.1526 & -0.000031 & 0.023 & & & 2.80 & 997.0449 \\
\hline & 25 & 0.0060 & 1000.44 & (17) & 0.000435 & 0.000472 & 1000.22 & 0.2217 & -0.000037 & 0.049 & & & 3.40 & 997.0449 \\
\hline & 25 & 0.0070 & 1000.94 & (17) & 0.000445 & 0.000472 & 1000.75 & 0.1903 & -0.000027 & 0.036 & & & 3.90 & 997.0449 \\
\hline & 25 & 0.0080 & 1001.54 & (17) & 0.000440 & 0.000472 & 1001.29 & 0.2584 & -0.000032 & 0.067 & & & 4.50 & 997.0449 \\
\hline & 25 & 0.0090 & 1002.04 & (17) & 0.000447 & 0.000472 & 1001.82 & 0.2261 & -0.000025 & 0.051 & & & 5.00 & 997.0449 \\
\hline & 25 & 0.0100 & 1002.64 & (17) & 0.000443 & 0.000472 & 1002.35 & 0.2933 & -0.000029 & 0.086 & & & 5.60 & 997.0449 \\
\hline & 25 & 0.0110 & 1003.14 & (17) & 0.000449 & 0.000472 & 1002.88 & 0.2600 & -0.000023 & 0.068 & & & 6.10 & 997.0449 \\
\hline & 25 & 0.0120 & 1003.74 & (17) & 0.000445 & 0.000472 & 1003.42 & 0.3262 & -0.000027 & 0.106 & & & 6.70 & 997.0449 \\
\hline & 25 & 0.0130 & 1004.24 & (17) & 0.000450 & 0.000472 & 1003.95 & 0.2919 & -0.000022 & 0.085 & & & 7.20 & 997.0449 \\
\hline & 25 & 0.0140 & 1004.74 & (17) & 0.000454 & 0.000472 & 1004.49 & 0.2572 & -0.000018 & 0.066 & & & 7.70 & 997.0449 \\
\hline & 25 & 0.0150 & 1005.34 & (17) & 0.000451 & 0.000472 & 1005.02 & 0.3219 & -0.000021 & 0.104 & & & 8.30 & 997.0449 \\
\hline & 25 & 0.0160 & 1005.84 & (17) & 0.000455 & 0.000472 & 1005.56 & 0.2862 & -0.000018 & 0.082 & & & 8.80 & 997.0449 \\
\hline & 25 & 0.0170 & 1006.44 & (17) & 0.000452 & 0.000472 & 1006.09 & 0.3500 & -0.000020 & 0.123 & & & 9.40 & 997.0449 \\
\hline & 25 & 0.0180 & 1006.94 & (17) & 0.000455 & 0.000472 & 1006.63 & 0.3133 & -0.000017 & 0.098 & & & 9.90 & 997.0449 \\
\hline & 25 & 0.0190 & 1007.54 & (17) & 0.000453 & 0.000472 & 1007.17 & 0.3762 & -0.000020 & 0.142 & & & 10.50 & 997.0449 \\
\hline & 25 & 0.0200 & 1008.04 & (17) & 0.000456 & 0.000472 & 1007.71 & 0.3385 & -0.000017 & 0.115 & & & 11.00 & 997.0449 \\
\hline & 25 & 0.0210 & 1008.54 & (17) & 0.000458 & 0.000472 & 1008.24 & 0.3004 & -0.000014 & 0.090 & & & 11.50 & 997.0449 \\
\hline & 25 & 0.0220 & 1009.14 & (17) & 0.000456 & 0.000472 & 1008.78 & 0.3618 & -0.000016 & 0.131 & & & 12.10 & 997.0449 \\
\hline
\end{tabular}


Density
Calc calc $\mathrm{kg} / \mathrm{m}^{3}$

$\begin{array}{llll}25 & 0.0230 & 1009.64 & (17) \\ 25 & 0.0240 & 1010.14 & (17) \\ 25 & 0.0250 & 1010.74 & (17) \\ 25 & 0.0260 & 1011.24 & (17) \\ 25 & 0.0270 & 1011.84 & (17) \\ 25 & 0.0280 & 1012.34 & (17) \\ 25 & 0.0290 & 1012.94 & (17) \\ 25 & 0.0300 & 1013.44 & (17) \\ 25 & 0.0310 & 1014.04 & (17) \\ 25 & 0.0320 & 1014.54 & (17) \\ 25 & 0.0330 & 1015.14 & (17) \\ 25 & 0.0340 & 1015.64 & (17) \\ 25 & 0.0350 & 1016.24 & (17) \\ 25 & 0.0360 & 1016.74 & (17) \\ 25 & 0.0370 & 1017.34 & (17) \\ 25 & 0.0380 & 1017.84 & (17) \\ 25 & 0.0390 & 1018.34 & (17) \\ 25 & 0.0400 & 1018.94 & (17) \\ 25 & 0.0410 & 1019.54 & (17) \\ 25 & 0.0420 & 1020.04 & (17) \\ 25 & 0.0430 & 1020.64 & (17) \\ 25 & 0.0440 & 1021.14 & (17) \\ 25 & 0.0450 & 1021.74 & (17) \\ 25 & 0.0460 & 1022.24 & (17) \\ 25 & 0.0470 & 1022.84 & (17) \\ 25 & 0.0480 & 1023.34 & (17) \\ 25 & 0.0490 & 1023.94 & (17) \\ 25 & 0.0500 & 1024.44 & (17) \\ 25 & 0.0510 & 1025.04 & (17) \\ 25 & 0.0520 & 1025.54 & (17) \\ 25 & 0.0530 & 1026.14 & (17) \\ 25 & 0.0540 & 1026.64 & (17) \\ 25 & 0.0550 & 1027.24 & (17) \\ 25 & 0.0560 & 1027.84 & (17) \\ 25 & 0.0570 & 1028.34 & (17) \\ 25 & 0.0580 & 1028.94 & (17) \\ 25 & 0.0590 & 1029.44 & (17) \\ 25 & 0.0600 & 1030.04 & (17) \\ 25 & 0.0700 & 1035.64 & (17) \\ 25 & 0.0800 & 1041.24 & (17) \\ 25 & 0.0900 & 1046.94 & (17) \\ 25 & 0.1000 & 1052.74 & (17) \\ 25 & 0.1100 & 1058.44 & (17) \\ 25 & 0.1200 & 1064.34 & (17) \\ 25 & 0.1300 & 1070.24 & (17) \\ & & & \end{array}$
$\mathrm{m}^{3} / \mathrm{kg}$

$\operatorname{exp~m^{3}/kg}$

$\begin{array}{lll}0.000459 & 0.000473 & 1009.32\end{array}$

$\begin{array}{lll}0.000461 & 0.000473 & 1009.86\end{array}$

$0.000459 \quad 0.000473$

$0.000461 \quad 0.000473$

$0.000460 \quad 0.000473$

$\begin{array}{ll}0.000462 & 0.000473\end{array}$

$0.000460 \quad 0.000473$

$0.000462 \quad 0.000473$

$0.000461 \quad 0.000473$

$0.000462 \quad 0.000473$

$0.000461 \quad 0.000473$

$0.000463 \quad 0.000473$

$0.000462 \quad 0.000473$

$\begin{array}{lll}0.000463 & 0.000473\end{array}$

$\begin{array}{ll}0.000462 & 0.000473\end{array}$

$0.000464 \quad 0.000473$

$0.000465 \quad 0.000473$

$0.000464 \quad 0.000473$

$0.000463 \quad 0.000473$

$0.000465 \quad 0.000473$

$0.000464 \quad 0.000473$

0.0004650 .000473

$0.000464 \quad 0.000473$

$0.000465 \quad 0.000474$

$0.000465 \quad 0.000474$

$0.000466 \quad 0.000474$

$0.000465 \quad 0.000474$

$0.000466 \quad 0.000474$

$0.000466 \quad 0.000474$

$0.000467 \quad 0.000474$

$0.000466 \quad 0.000474$

$0.000467 \quad 0.000474$

$\begin{array}{lll}0.000467 & 0.000474\end{array}$

$0.000466 \quad 0.000474$

$0.000467 \quad 0.000474$

$0.000467 \quad 0.000474$

$0.000468 \quad 0.000474$

$0.000467 \quad 0.000474$

$0.000469 \quad 0.000475$

$0.000471 \quad 0.000475$

0.0004720 .000476

$0.000472 \quad 0.000476$

$\begin{array}{ll}0.000474 & 0.000476\end{array}$

$0.000474 \quad 0.000477$

$\begin{array}{lll}0.000475 & 0.000477\end{array}$
1010.40

10.94

011.48

1012.03

012.57

1013.11

1013.65

014.20

1014.74

1015.28

015.83

1016.38

1016.92

017.47

1018.01

1018.56

019.11

1020.21

1020.76

1021.31

1021.86

1022.41

1022.96

1023.51

1024.06

1024.62

1025.17

1025.72

1026.28

1026.83

1027.39

1027.95

1028.50

1029.06

1029.62

1035.22

1040.87

1046.58

1052.34

1058.15

1064.02

1069.94
Density App Vol Square of Inconsistent Inconsistent Delta density Density of Residual Residual Density based on based on $\mathrm{kg} / \mathrm{m}^{3} \quad \mathrm{~m}^{3} / \mathrm{kg}$ Residual density $\begin{gathered}\text { apparent } \\ \text { volume }\end{gathered}$ $/ \mathrm{kg} / \mathrm{m}^{3}$ water $/ \mathrm{kg} / \mathrm{m}^{3}$ $0.3227-0.000014 \quad 0.104$ $0.2831-0.000012 \quad 0.080$ $0.3430-0.000013 \quad 0.118$ $0.3024-0.000011 \quad 0.091$ $0.3614-0.000013 \quad 0.131$ $0.3198-0.000011 \quad 0.102$ $0.3778-0.000013 \quad 0.143$ $0.3352-0.000011 \quad 0.112$ $0.3922-0.000012 \quad 0.154$ $0.3487-0.000011 \quad 0.122$ $0.4047-0.000012 \quad 0.164$ $0.3602-0.000010 \quad 0.130$ $0.4152-0.000011 \quad 0.172$ $0.3697-0.000010 \quad 0.137$ $0.4238-0.000011 \quad 0.180$ $0.3773-0.000010 \quad 0.142$ $0.3303-0.000008 \quad 0.109$ $0.3829-0.000009 \quad 0.147$ $0.4349-0.000010 \quad 0.189$ $0.3864-0.000009 \quad 0.149$ $0.4375-0.000010 \quad 0.191$ $0.3880-0.000008-0.151$ $0.4381-0.000009 \quad 0.192$ $0.3876-0.000008 \quad 0.150$ $\begin{array}{lll}0.4367 & -0.000009 & 0.191\end{array}$ $\begin{array}{lll}0.3852 & -0.000008 & 0.148\end{array}$ $0.4333-0.000008 \quad 0.188$ $\begin{array}{lll}0.3808 & -0.000007 & 0.145\end{array}$ $0.4278-0.000008 \quad 0.183$ $0.3744-0.000007 \quad 0.140$ $0.4204-0.000008 \quad 0.177$ $0.3659-0.000006 \quad 0.134$ $0.4110-0.000007 \quad 0.169$ $\begin{array}{lll}0.4555 & -0.000008 & 0.207\end{array}$ $0.3995-0.000007 \quad 0.160$ $0.4430-0.000007 \quad 0.196$ $0.3860-0.000006 \quad 0.149$ $0.4285-0.000007 \quad 0.184$ $0.4254-0.000006 \quad 0.181$ $0.3709-0.000004 \quad 0.138$ $0.3645-0.000004 \quad 0.133$ $0.4055-0.000004 \quad 0.164$ $0.2933-0.000002 \quad 0.086$ $0.3273-0.000002 \quad 0.107$ $\begin{array}{lll}0.3070 & -0.000002 & 0.094\end{array}$
$12.60 \quad 997.0449$

$13.10 \quad 997.0449$ $13.70 \quad 997.0449$

$14.20 \quad 997.0449$

$14.80 \quad 997.0449$

$15.30 \quad 997.0449$

$15.90 \quad 997.0449$

$16.40 \quad 997.0449$

$17.00 \quad 997.0449$

$17.50 \quad 997.0449$

$18.10 \quad 997.0449$

$18.60 \quad 997.0449$

$19.20 \quad 997.0449$

$19.70 \quad 997.0449$

$20.30 \quad 997.0449$

$20.80 \quad 997.0449$

$21.30 \quad 997.0449$

$21.90 \quad 997.0449$

$22.50 \quad 997.0449$

$23.00 \quad 997.0449$

$23.60 \quad 997.0449$

$24.10 \quad 997.0449$

$24.70 \quad 997.0449$

$25.20 \quad 997.0449$

$25.80 \quad 997.0449$

$26.30 \quad 997.0449$

$26.90 \quad 997.0449$

$27.40 \quad 997.0449$

$28.00 \quad 997.0449$

$28.50 \quad 997.0449$

$29.10 \quad 997.0449$

$29.60 \quad 997.0449$

$30.20 \quad 997.0449$

$30.80 \quad 997.0449$

$31.30 \quad 997.0449$

$31.90 \quad 997.0449$

$32.40 \quad 997.0449$

$33.00 \quad 997.0449$

$38.60 \quad 997.0449$

$44.20 \quad 997.0449$

$49.90 \quad 997.0449$

$55.70 \quad 997.0449$

$61.40 \quad 997.0449$

$67.30 \quad 997.0449$

$73.20 \quad 997.0449$ 
Density
Calc calc $\mathrm{kg} / \mathrm{m}^{3}$

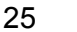

$0.1400 \quad 1076.24$

0.1500

$0.1600 \quad 1088.34$

$0.1700 \quad 1094.44$

$0.1800 \quad 1100.54$

$0.1900 \quad 1106.84$

$0.2000 \quad 1113.14$

$0.2100 \quad 1119.54$

$0.2200 \quad 1125.94$

$0.2300 \quad 1132.44$

$0.2400 \quad 1138.94$

$0.2500 \quad 1145.54$

$0.2600 \quad 1152.24$

$0.2700 \quad 1158.94$

$0.2800 \quad 1165.74$

$0.2900 \quad 1172.64$

$0.3000 \quad 1179.54$

$0.3100 \quad 1186.54$

$0.3200 \quad 1193.64$

$0.3300 \quad 1200.74$

$0.3400 \quad 1207.94$

$0.3500 \quad 1215.14$

$0.3600 \quad 1222.54$

$0.3700 \quad 1229.94$

$0.3800 \quad 1237.44$

$0.3900 \quad 1244.94$

$0.4000 \quad 1252.64$

$0.4100 \quad 1260.34$

$0.4200 \quad 1268.14$

$0.4300 \quad 1275.94$

$0.4400 \quad 1283.94$

$0.4500 \quad 1291.94$

$0.4600 \quad 1300.14$

$0.4700 \quad 1308.34$

$0.4800 \quad 1316.54$

$0.4900 \quad 1324.94$

$0.5000 \quad 1333.34$

$0.5100 \quad 1341.84$

$0.5200 \quad 1350.44$

$0.5300 \quad 1359.14$

$0.5400 \quad 1367.84$

$0.5500 \quad 1376.74$

$0.5600 \quad 1385.64$

$0.5700 \quad 1394.64$

$0.5800 \quad 1403.74$ $\mathrm{m}^{3} / \mathrm{kg}$

$\operatorname{exp~m^{3}/kg}$

$\begin{array}{lll}0.000476 & 0.000478 & 1075.91\end{array}$

$\begin{array}{lll}0.000477 & 0.000478 & 1081.94\end{array}$

$\begin{array}{lll}0.000477 & 0.000479 & 1088.03\end{array}$

$\begin{array}{lll}0.000478 & 0.000479 & 1094.18\end{array}$

$\begin{array}{lll}0.000479 & 0.000480 & 1100.38\end{array}$

$0.000479 \quad 0.000480 \quad 1106.64$

$\begin{array}{lll}0.000480 & 0.000481 & 1112.96\end{array}$

$\begin{array}{lll}0.000480 & 0.000481 & 1119.33\end{array}$

$\begin{array}{lll}0.000481 & 0.000482 & 1125.77\end{array}$

$\begin{array}{lll}0.000482 & 0.000482 & 1132.27\end{array}$

$\begin{array}{lll}0.000482 & 0.000483 & 1138.83\end{array}$

$\begin{array}{lll}0.000483 & 0.000483 & 1145.46\end{array}$

$\begin{array}{lll}0.000483 & 0.000484 & 1152.14\end{array}$

$\begin{array}{lll}0.000484 & 0.000484 & 1158.89\end{array}$

$\begin{array}{lll}0.000485 & 0.000485 & 1165.71\end{array}$

$0.000485 \quad 0.000485 \quad 1172.58$

$\begin{array}{lll}0.000486 & 0.000486 & 1179.53\end{array}$

$\begin{array}{lll}0.000486 & 0.000486 & 1186.54\end{array}$

$\begin{array}{lll}0.000487 & 0.000487 & 1193.62\end{array}$

$\begin{array}{llll}0.000487 & 0.000487 & 1200.77\end{array}$

$\begin{array}{lll}0.000488 & 0.000488 & 1207.98\end{array}$

$0.000489 \quad 0.000488 \quad 1215.27$

$\begin{array}{lll}0.000489 & 0.000489 & 1222.62\end{array}$

$\begin{array}{lll}0.000490 & 0.000489 & 1230.05\end{array}$

$\begin{array}{lll}0.000490 & 0.000490 & 1237.55\end{array}$

$\begin{array}{lll}0.000491 & 0.000491 & 1245.12\end{array}$

$\begin{array}{lll}0.000491 & 0.000491 & 1252.76\end{array}$

$0.000492 \quad 0.000492$

$0.000492 \quad 0.000492$

$0.000493 \quad 0.000493$

$0.000494 \quad 0.000493$

$0.000494 \quad 0.000494$

$0.000495 \quad 0.000495$

$0.000495 \quad 0.000495$

$0.000496 \quad 0.000496$

$0.000496 \quad 0.000496$

$0.000497 \quad 0.000497$

$0.000498 \quad 0.000498$

$0.000498 \quad 0.000498$

$\begin{array}{ll}0.000499 & 0.000499\end{array}$

$0.000499 \quad 0.000499$

$0.000500 \quad 0.000500$

$0.000501 \quad 0.000501$

$0.000501 \quad 0.000501$

$0.000502 \quad 0.000502$
1252.76
1260.48

1268.27

1276.14

1284.08

1292.10

1300.20

1308.38

1316.64

1324.98

1333.40

1341.90

1350.48

1359.15

1367.90

1376.74

1385.66

1394.67

1403.77
Density App Vol Square of Inconsistent Inconsistent Delta density Density of Residual Residual Density based on based on $/ \mathrm{kg} / \mathrm{m}^{3}$ water $/ \mathrm{kg} / \mathrm{m}^{3}$ $\mathrm{kg} / \mathrm{m}^{3} \quad \mathrm{~m}^{3} / \mathrm{kg}$ Residual density $\begin{gathered}\text { apparent } \\ \text { volume }\end{gathered}$

$0.3316-0.000002 \quad 0.110$

$0.3007-0.000002 \quad 0.090$

$\begin{array}{lll}0.3134 & -0.000002 & 0.098\end{array}$

$0.2693-0.000001 \quad 0.073$

$0.1676-0.000001 \quad 0.028$

$0.2077-0.000001 \quad 0.043$

$0.1890-0.000001 \quad 0.036$

$0.2107-0.000001 \quad 0.044$

$0.1723-0.000001 \quad 0.030$

$0.1730-0.000001 \quad 0.030$

$\begin{array}{lll}0.1121 & 0.000000 & 0.013\end{array}$

$\begin{array}{lll}0.0890 & 0.000000 & 0.008\end{array}$

$\begin{array}{lll}0.1029 & 0.000000 & 0.011\end{array}$

$\begin{array}{lll}0.0532 & 0.000000 & 0.003\end{array}$

$\begin{array}{lll}0.0391 & 0.000000 & 0.002\end{array}$

$\begin{array}{lll}0.0600 & 0.000000 & 0.004\end{array}$

$\begin{array}{lll}0.0151 & 0.000000 & 0.000\end{array}$

$\begin{array}{lll}0.0037 & 0.000000 & 0.000\end{array}$

$\begin{array}{lll}0.0250 & 0.000000 & 0.001\end{array}$

$\begin{array}{lll}-0.0217 & 0.000000 & 0.000\end{array}$

$\begin{array}{lll}-0.0370 & 0.000000 & 0.001\end{array}$

$\begin{array}{lll}-0.1219 & 0.000000 & 0.015\end{array}$

$\begin{array}{lll}-0.0770 & 0.000000 & 0.006\end{array}$

$\begin{array}{lll}-0.1030 & 0.000000 & 0.011\end{array}$

$\begin{array}{lll}-0.1008 & 0.000000 & 0.010\end{array}$

$\begin{array}{lll}-0.1711 & 0.000000 & 0.029\end{array}$

$\begin{array}{lll}-0.1147 & 0.000000 & 0.013\end{array}$

$\begin{array}{lll}-0.1323 & 0.000000 & 0.018\end{array}$

$\begin{array}{lll}-0.1248 & 0.000000 & 0.016\end{array}$

$\begin{array}{lll}-0.1928 & 0.000000 & 0.037\end{array}$

$\begin{array}{lll}-0.1373 & 0.000000 & 0.019\end{array}$

$\begin{array}{lll}-0.1589 & 0.000000 & 0.025\end{array}$

$\begin{array}{lll}-0.0584 & 0.000000 & 0.003\end{array}$

$\begin{array}{lll}-0.0368 & 0.000000 & 0.001\end{array}$

$\begin{array}{lll}-0.0946 & 0.000000 & 0.009\end{array}$

$\begin{array}{lll}-0.0328 & 0.000000 & 0.001\end{array}$

$\begin{array}{lll}-0.0522 & 0.000000 & 0.003\end{array}$

$\begin{array}{lll}-0.0534 & 0.000000 & 0.003\end{array}$

$\begin{array}{lll}-0.0373 & 0.000000 & 0.001\end{array}$

$\begin{array}{lll}-0.0048 & 0.000000 & 0.000\end{array}$

$\begin{array}{lll}-0.0565 & 0.000000 & 0.003\end{array}$

$\begin{array}{lll}0.0068 & 0.000000 & 0.000\end{array}$

$\begin{array}{lll}-0.0158 & 0.000000 & 0.000\end{array}$

$\begin{array}{lll}-0.0251 & 0.000000 & 0.001\end{array}$

$\begin{array}{lll}-0.0217 & 0.000000 & 0.000\end{array}$
$79.20 \quad 997.0449$

$85.20 \quad 997.0449$

$91.30 \quad 997.0449$

$97.40 \quad 997.0449$

$103.50 \quad 997.0449$

$109.80 \quad 997.0449$

$116.10 \quad 997.0449$

$122.50 \quad 997.0449$

$128.90 \quad 997.0449$

$135.40 \quad 997.0449$

$141.90 \quad 997.0449$

$148.50 \quad 997.0449$

$155.20 \quad 997.0449$

$161.90 \quad 997.0449$

$168.70 \quad 997.0449$

$175.60 \quad 997.0449$

$182.50 \quad 997.0449$

$189.50 \quad 997.0449$

$196.60 \quad 997.0449$

$203.70 \quad 997.0449$

$210.90 \quad 997.0449$

$218.10 \quad 997.0449$

$225.50 \quad 997.0449$

$232.90 \quad 997.0449$

$240.40 \quad 997.0449$

$247.90 \quad 997.0449$

$255.60 \quad 997.0449$

$263.30 \quad 997.0449$

$271.10 \quad 997.0449$

$278.90 \quad 997.0449$

$286.90 \quad 997.0449$

$294.90 \quad 997.0449$

$303.10 \quad 997.0449$

$311.30 \quad 997.0449$

$319.50 \quad 997.0449$

$327.90 \quad 997.0449$

$336.30 \quad 997.0449$

$344.80 \quad 997.0449$

$353.40 \quad 997.0449$

$362.10 \quad 997.0449$

$370.80 \quad 997.0449$

$379.70 \quad 997.0449$

$388.60 \quad 997.0449$

$397.60 \quad 997.0449$

$406.70 \quad 997.0449$ 

App vol Density
Calc calc kg/m $\mathrm{m}^{3}$ $\mathrm{m}^{3} / \mathrm{kg}$

$\begin{array}{rlll}25 & 0.5900 & 1412.94 & (17) \\ 25 & 0.6000 & 1422.24 & (17) \\ 25 & 0.6100 & 1431.54 & (17) \\ 25 & 0.6200 & 1441.04 & (17) \\ 25 & 0.6300 & 1450.54 & (17) \\ 25 & 0.6400 & 1460.24 & (17) \\ 25 & 0.6500 & 1469.94 & (17) \\ 25 & 0.6600 & 1479.84 & (17) \\ 25 & 0.6700 & 1489.74 & (17) \\ 25 & 0.6800 & 1499.84 & (17) \\ 25 & 0.6900 & 1509.94 & (17) \\ 25 & 0.7000 & 1520.14 & (17) \\ 25 & 0.7100 & 1530.44 & (17) \\ 25 & 0.7200 & 1540.84 & (17) \\ 25 & 0.7300 & 1551.34 & (17) \\ 25 & 0.7400 & 1561.94 & (17) \\ 25 & 0.7500 & 1572.64 & (17) \\ 25 & 0.7600 & 1583.34 & (17) \\ 25 & 0.7700 & 1594.24 & (17) \\ 25 & 0.7800 & 1605.14 & (17) \\ 25 & 0.7900 & 1616.24 & (17) \\ 25 & 0.8000 & 1627.44 & (17) \\ 25 & 0.8100 & 1638.64 & (17) \\ 25 & 0.8200 & 1649.94 & (17) \\ 25 & 0.8300 & 1661.34 & (17) \\ 25 & 0.8400 & 1672.84 & (17) \\ 25 & 0.8500 & 1684.44 & (17) \\ 25.18 & 0.0446 & 1021.30 & (25) \\ 25.18 & 0.0935 & 1048.90 & (25) \\ 25.18 & 0.1921 & 1107.20 & (25) \\ 25.18 & 0.2933 & 1174.20 & (25) \\ 25.18 & 0.3915 & 1246.10 & (25) \\ 25.18 & 0.4936 & 1327.20 & (25) \\ 25.18 & 0.5954 & 1417.60 & (25) \\ 25.18 & 0.7010 & 1521.30 & (25) \\ 25.18 & 0.7997 & 1627.10 & (25) \\ 25.18 & 0.8481 & 1682.30 & (25) \\ 40.82 & 0.0446 & 1015.90 & (25) \\ 40.82 & 0.0935 & 1042.80 & (25) \\ 40.82 & 0.1921 & 1100.90 & (25) \\ 40.82 & 0.2933 & 1166.70 & (25) \\ 40.82 & 0.3915 & 1237.10 & (25) \\ 40.82 & 0.4936 & 1318.30 & (25) \\ 40.82 & 0.5954 & 1407.60 & (25) \\ 40.82 & 0.7010 & 1509.60 & (25) \\ & & & \end{array}$

\section{$0.000503 \quad 0.000503$}

$\begin{array}{ll}0.000503 & 0.000503 \\ 0.000503 & 0.000503\end{array}$

$0.000504 \quad 0.000504$

0.0005050 .000505

0.0005050 .000505

$0.000506 \quad 0.000506$

$\begin{array}{lll}0.000507 & 0.000507\end{array}$

$\begin{array}{lll}0.000507 & 0.000507\end{array}$

0.0005080 .000508

$0.000509 \quad 0.000509$

$0.000509 \quad 0.000509$

$0.000510 \quad 0.000510$

$0.000511 \quad 0.000511$

$\begin{array}{ll}0.000511 & 0.000511\end{array}$

0.0005120 .000512

$0.000513 \quad 0.000513$

$\begin{array}{ll}0.000514 & 0.000514\end{array}$

$0.000514 \quad 0.000514$

$0.000515 \quad 0.000515$

$0.000516 \quad 0.000516$

$\begin{array}{ll}0.000517 & 0.000517\end{array}$

$\begin{array}{ll}0.000517 & 0.000517\end{array}$

$0.000518 \quad 0.000518$

$\begin{array}{lll}0.000519 & 0.000519\end{array}$

$0.000520 \quad 0.000520$

$0.000521 \quad 0.000520$

$0.000521 \quad 0.000521$

$0.000468 \quad 0.000474$

$\begin{array}{lll}0.000472 & 0.000476\end{array}$

$0.000483 \quad 0.000480$

$0.000487 \quad 0.000485$

$\begin{array}{lll}0.000491 & 0.000491\end{array}$

$\begin{array}{ll}0.000497 & 0.000497\end{array}$

$0.000503 \quad 0.000503$

$0.000510 \quad 0.000510$

$\begin{array}{ll}0.000517 & 0.000517\end{array}$

$\begin{array}{lll}0.000521 & 0.000521\end{array}$

$\begin{array}{lll}0.000474 & 0.000479\end{array}$

$\begin{array}{ll}0.000482 & 0.000481\end{array}$

0.0004890 .000486

$0.000493 \quad 0.000491$

$0.000498 \quad 0.000496$

$0.000502 \quad 0.000502$

$0.000508 \quad 0.000508$

$0.000515 \quad 0.000515$
1412.95

1422.23

1431.59

1441.04

1450.59

1460.22

1469.95

1479.78

1489.69

1499.70

1509.81

1520.01

1530.31

1540.71

1551.20

1561.79

1572.49

1583.28

1605.16

1616.25

1627.44

1638.73

1650.13

1661.62

673.22

1684.92

1021.04

1048.54

1107.90

1174.80

1246.18

1327.91

1417.84

1520.91

1626.96

1682.54

1015.67

1042.85

1101.52

1167.64

1238.17

1318.94

1407.83

1509.77
Density App
Residual Res $\mathrm{kg} / \mathrm{m}^{3}$ $\begin{array}{rrrr}\text { Residual Density based on } & \text { based on } \\ \text { density apparent }\end{array}$

Square of Inconsistent Inconsistent Delta density Density of $/ \mathrm{kg} / \mathrm{m}^{3}$ water $/ \mathrm{kg} / \mathrm{m}^{3}$ $\begin{array}{ll}0.0065 & 0.000000\end{array}$ $0.0198 \quad 0.000000$ $-0.04350 .000000$ $0.0027 \quad 0.000000$ $\begin{array}{ll}-0.0423 & 0.000000\end{array}$ $0.0209 \quad 0.000000$ $-0.0085 \quad 0.000000$ $0.0688 \quad 0.000000$ 0.05210 .000000 $0.1408 \quad 0.000000$ $\begin{array}{ll}0.1342 & 0.000000\end{array}$ $0.1317 \quad 0.000000$ $0.1325 \quad 0.000000$ $\begin{array}{ll}0.1363 & 0.000000\end{array}$ $0.1423 \quad 0.000000$ $0.1501 \quad 0.000000$ $0.1590 \quad 0.000000$ $0.0687 \quad 0.000000$ $0.0786 \quad 0.000000$ $\begin{array}{ll}-0.0116 & 0.000000\end{array}$ $-0.0025 \quad 0.000000$ $0.0058 \quad 0.000000$ $\begin{array}{ll}-0.0872 & 0.000000\end{array}$ $-0.1817 \quad 0.000000$ $\begin{array}{ll}-0.2780 & 0.000000\end{array}$ $-0.37620 .000000$ $\begin{array}{ll}-0.4765 & 0.000000\end{array}$ $0.2627-0.000006$ $0.3632-0.000004$ $\begin{array}{ll}-0.6976 & 0.000003\end{array}$ $\begin{array}{ll}-0.5982 & 0.000001\end{array}$ $-0.0760 \quad 0.000000$ $\begin{array}{ll}-0.7051 & 0.000001\end{array}$ $-0.2400 \quad 0.000000$ $0.3852 \quad 0.000000$ $\begin{array}{ll}0.1382 & 0.000000\end{array}$ $-0.24220 .000000$ $0.2344-0.000005$ $-0.0500 \quad 0.000000$ $-0.62190 .000003$ $\begin{array}{ll}-0.9361 & 0.000002\end{array}$ $\begin{array}{ll}-1.0704 & 0.000002\end{array}$ $-0.6377 \quad 0.000001$ $-0.2337 \quad 0.000000$ $-0.1651 \quad 0.000000$

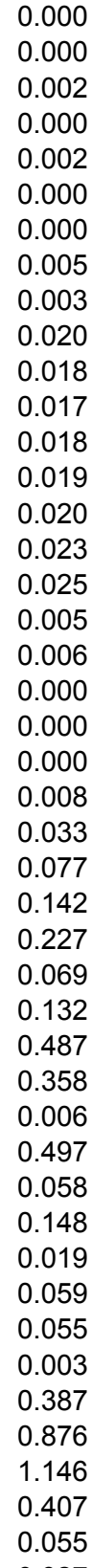

0.027
$415.90 \quad 997.0449$ $425.20 \quad 997.0449$ $434.50 \quad 997.0449$ $444.00 \quad 997.0449$ $453.50 \quad 997.0449$ $463.20 \quad 997.0449$ $472.90 \quad 997.0449$ $482.80 \quad 997.0449$ $492.70 \quad 997.0449$ $502.80 \quad 997.0449$ $512.90 \quad 997.0449$ $523.10 \quad 997.0449$ $533.40 \quad 997.0449$ $543.80 \quad 997.0449$ $554.30 \quad 997.0449$ $564.90 \quad 997.0449$ $575.60 \quad 997.0449$ $586.30 \quad 997.0449$ $597.20 \quad 997.0449$ $608.10 \quad 997.0449$ $619.20 \quad 997.0449$ $630.40 \quad 997.0449$ $641.60 \quad 997.0449$ $652.90 \quad 997.0449$ $664.30 \quad 997.0449$ $675.80 \quad 997.0449$ $687.40 \quad 997.0449$ 996.9986 996.9986 996.9986 996.9986 996.9986 996.9986 996.9986 996.9986 996.9986 996.9986 991.8998 991.8998 991.8998 991.8998 991.8998 991.8998 991.8998 991.8998 
molality molarity

$t{ }^{\circ} \mathrm{C}$ mass frac Density $\exp \mathrm{kg} / \mathrm{m}^{3}$

$\begin{array}{lrr}40.82 & 0.7997 & 1614.90 \\ 40.82 & 0.8481 & 1669.50 \\ 60.81 & 0.0446 & 1006.40 \\ 60.81 & 0.0935 & 1032.90 \\ 60.81 & 0.1921 & 1091.00 \\ 60.81 & 0.2933 & 1156.10 \\ 60.81 & 0.3915 & 1225.60 \\ 60.81 & 0.4936 & 1305.40 \\ 60.81 & 0.5954 & 1393.80 \\ 60.81 & 0.7010 & 1495.00 \\ 60.81 & 0.7997 & 1598.90 \\ 60.81 & 0.8481 & 1655.20 \\ 81.40 & 0.0446 & 994.50 \\ 81.40 & 0.0935 & 1021.00 \\ 81.40 & 0.1921 & 1077.60 \\ 81.40 & 0.2933 & 1141.50 \\ 81.40 & 0.3915 & 1211.60 \\ 81.40 & 0.4936 & 1291.10 \\ 81.40 & 0.5954 & 1378.80 \\ 81.40 & 0.7010 & 1479.40 \\ 81.40 & 0.7997 & 1583.20 \\ 81.40 & 0.8481 & 1637.80\end{array}$

Ref

$\operatorname{exp~m^{3}/kg}$

App v Cansity $\mathrm{m}^{3} / \mathrm{kg}$

$\begin{array}{lll}\text { (25) } \quad 0.000522 & 0.000522\end{array}$

$\begin{array}{lll}\text { (25) } & 0.000526 & 0.000526\end{array}$

$\begin{array}{lll}\text { (25) } & 0.000482 & 0.000486\end{array}$

0.0004890 .000488

$\begin{array}{ll}0.000492 & 0.000492\end{array}$

$0.000497 \quad 0.000497$

$0.000503 \quad 0.000502$

$\begin{array}{lll}0.000508 & 0.000508\end{array}$

$0.000514 \quad 0.000514$

$0.000520 \quad 0.000520$

$0.000527 \quad 0.000527$

$0.000530 \quad 0.000531$

$0.000482 \quad 0.000493$

$0.000490 \quad 0.000495$

$0.000499 \quad 0.000499$

$0.000505 \quad 0.000503$

$0.000507 \quad 0.000508$

$0.000512 \quad 0.000513$

$0.000518 \quad 0.000519$

$0.000525 \quad 0.000525$

$\begin{array}{ll}0.000532 & 0.000532 \\ 0.000535 & 0.000535\end{array}$

Std dev Res

Avg + 4std

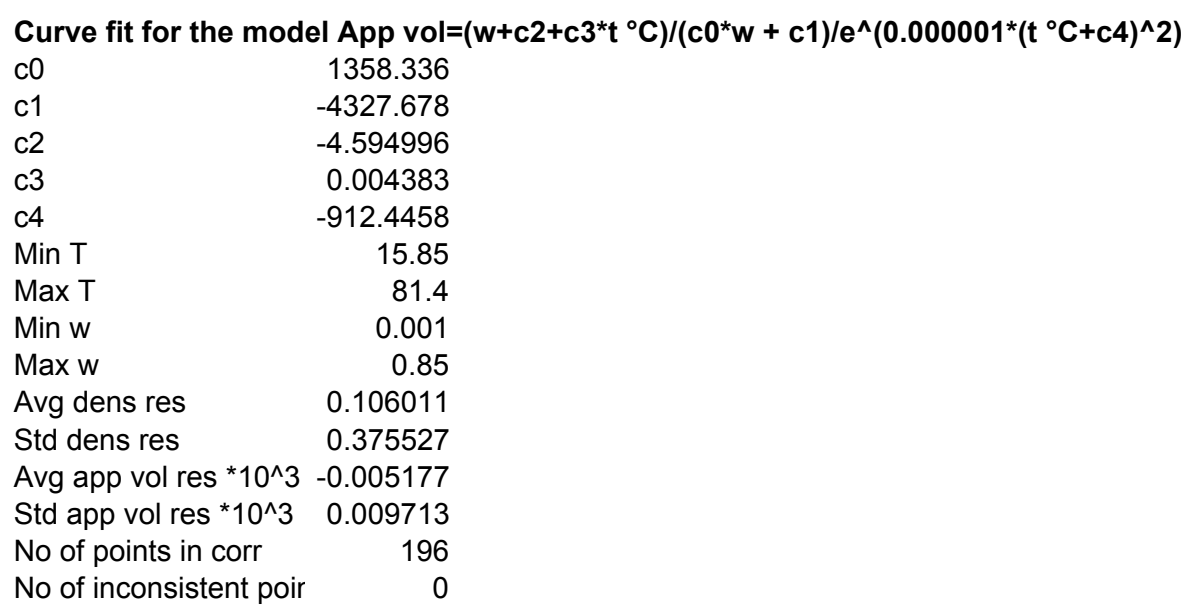

Density App Vol Square of Inconsistent Inconsistent Delta density

density Density of Residual Residual Density based on based on $/ \mathrm{kg} / \mathrm{m}^{3}$ water $/ \mathrm{kg} / \mathrm{m}^{3}$ $\mathrm{kg} / \mathrm{m}^{3} \quad \mathrm{~m}^{3} / \mathrm{kg}$ Residual density $\begin{gathered}\text { apparent } \\ \text { volume }\end{gathered}$

$\begin{array}{llll}1614.72 & 0.1822 & 0.000000 & 0.033\end{array}$

$\begin{array}{llll}1669.77 & -0.2696 & 0.000000 & 0.073\end{array}$

$\begin{array}{llll}1006.22 & 0.1810 & -0.000004 & 0.033\end{array}$

$\begin{array}{llll}1033.03 & -0.1322 & 0.000001 & 0.017\end{array}$

$\begin{array}{llll}1090.92 & 0.0831 & 0.000000 & 0.007\end{array}$

$\begin{array}{llll}225.84 & -0.2421 & 0.000000 & 0.059\end{array}$

$\begin{array}{llll}1494.79 & 0.1046 & 0.000000 & 0.011 \\ 15.2113 & 0.000000 & 0.045\end{array}$

$\begin{array}{llll}1599.13 & -0.2338 & 0.000000 & 0.055\end{array}$

$\begin{array}{llll}1653.99 & 1.2107 & -0.000001 & 1.466\end{array}$

$\begin{array}{llll}994.05 & 0.4499 & -0.000010 & 0.202\end{array}$

$\begin{array}{llll}1020.52 & 0.4804 & -0.000005 & 0.231\end{array}$

$\begin{array}{llll}1142.24 & -0.7445 & 0.000002 & 0.554\end{array}$

0.374

0.591

0.235

0.068

0.666

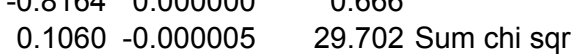

991.8998

991.8998

982.7804

982.7804

982.7804

982.7804

982.7804

982.7804

982.7804

982.7804

982.7804

982.7804

970.9207

970.9207

970.9207

970.9207

970.9207

970.9207

970.9207

970.9207

970.9207

970.9207

$-1.3961-0.000044$

1.60810 .000034

Inconsistent data not used (Residual greater than average $+/-4$ standard deviations) 
Density of aqueous solutions of $\mathrm{H} 2 \mathrm{SO} 4$

References (35) Haase, R.; Saurmann, P.-F.; Dücker, K.-H. Conductivity of Concentrated Electrolyte Solutions. V. Sulfuric Acid. Z. Physik. Chem. N. F.

1966, 48, 206-212.

(90) Perry, R. H.; Green, D. W. Perry's Chemical Engineers' Handbook; McGraw Hill: New York, 7th edition, 1997

(103) Przepiera, A.; Zielenkiewicz, A.; Apparent Molar Volumes of Aqueous Solution in the MnSO4 + H2SO4 System; Bull. Pol. Acad. Sci. Chem. , 2000, 48, 267-272

\begin{tabular}{|c|c|c|c|c|c|c|c|c|c|c|c|c|c|}
\hline \multirow[t]{36}{*}{ molality molarity } & \multicolumn{2}{|c|}{$\mathrm{t} /{ }^{\circ} \mathrm{C}$ mass frac } & $\begin{array}{r}\text { Density } \\
\exp / \\
\mathrm{kg} / \mathrm{m}^{3}\end{array}$ & Ref & $\begin{array}{r}\text { App vol } \\
\text { exp / } \\
\mathrm{m}^{3} / \mathrm{kg}\end{array}$ & $\begin{array}{r}\text { App vol } \\
\text { Calc / } \\
\mathrm{m}^{3} / \mathrm{kg}\end{array}$ & $\begin{array}{r}\text { Density } \\
\text { calc / } \\
\mathrm{kg} / \mathrm{m}^{3}\end{array}$ & $\begin{array}{r}\text { Density } \\
\text { Residual / } \\
\mathrm{kg} / \mathrm{m}^{3}\end{array}$ & $\begin{array}{r}\text { App Vol } \\
\text { Residual / } \\
\mathrm{m}^{3} / \mathrm{kg}\end{array}$ & $\begin{array}{r}\text { Square of } \\
\text { Density } \\
\text { Residual }\end{array}$ & $\begin{array}{l}\text { Inconsistent } \\
\text { based on } \\
\text { density }\end{array}$ & $\begin{array}{l}\text { Inconsistent } \\
\text { based on } \\
\text { apparent } \\
\text { volume }\end{array}$ & $\begin{array}{r}\text { Density of } \\
\text { water } / \mathrm{kg} / \mathrm{m}^{3}\end{array}$ \\
\hline & 0 & 0.0100 & 1007.40 & (90) & 0.000250 & 0.000313 & 1006.76 & 0.6405 & -0.000063 & 0.410 & & & 999.8395 \\
\hline & 0 & 0.0200 & 1014.70 & (90) & 0.000268 & 0.000315 & 1013.72 & 0.9755 & -0.000047 & 0.952 & & & 999.8395 \\
\hline & 0 & 0.0300 & 1021.90 & (90) & 0.000280 & 0.000318 & 1020.74 & 1.1648 & -0.000037 & 1.357 & & & 999.8395 \\
\hline & 0 & 0.0393 & 1028.60 & (35) & 0.000288 & 0.000320 & 1027.29 & 1.3105 & -0.000032 & 1.718 & & & 999.8395 \\
\hline & 0 & 0.0400 & 1029.10 & (90) & 0.000289 & 0.000320 & 1027.79 & 1.3080 & -0.000031 & 1.711 & & & 999.8395 \\
\hline & 0 & 0.0500 & 1036.40 & (90) & 0.000295 & 0.000323 & 1034.90 & 1.5045 & -0.000028 & 2.264 & & & 999.8395 \\
\hline & 0 & 0.0700 & 1051.10 & (35) & 0.000303 & 0.000327 & 1049.24 & 1.8557 & -0.000024 & 3.444 & & & 999.8395 \\
\hline & 0 & 0.1000 & 1073.50 & (90) & 0.000314 & 0.000334 & 1071.13 & 2.3695 & -0.000021 & 5.615 & & & 999.8395 \\
\hline & 0 & 0.1456 & 1108.20 & (35) & 0.000328 & 0.000345 & 1105.26 & 2.9381 & -0.000016 & 8.632 & & & 999.8395 \\
\hline & 0 & 0.1500 & 1111.60 & (90) & 0.000330 & 0.000346 & 1108.61 & 2.9878 & -0.000016 & 8.927 & & & 999.8395 \\
\hline & 0 & 0.1980 & 1149.50 & (35) & 0.000342 & 0.000357 & 1145.83 & 3.6651 & -0.000014 & 13.433 & & & 999.8395 \\
\hline & 0 & 0.2000 & 1151.00 & (90) & 0.000343 & 0.000357 & 1147.41 & 3.5868 & -0.000014 & 12.865 & & & 999.8395 \\
\hline & 0 & 0.2500 & 1191.40 & (90) & 0.000357 & 0.000368 & 1187.61 & 3.7880 & -0.000011 & 14.349 & & & 999.8395 \\
\hline & 0 & 0.2531 & 1193.80 & (35) & 0.000358 & 0.000368 & 1190.15 & 3.6477 & -0.000010 & 13.306 & & & 999.8395 \\
\hline & 0 & 0.2947 & 1228.20 & (35) & 0.000369 & 0.000377 & 1224.80 & 3.3979 & -0.000008 & 11.545 & & & 999.8395 \\
\hline & 0 & 0.3000 & 1232.60 & (90) & 0.000371 & 0.000378 & 1229.29 & 3.3067 & -0.000007 & 10.934 & & & 999.8395 \\
\hline & 0 & 0.3428 & 1268.50 & (35) & 0.000382 & 0.000386 & 1266.22 & 2.2810 & -0.000004 & 5.203 & & & 999.8395 \\
\hline & 0 & 0.3500 & 1274.60 & (90) & 0.000384 & 0.000388 & 1272.55 & 2.0516 & -0.000004 & 4.209 & & & 999.8395 \\
\hline & 0 & 0.3910 & 1310.00 & (35) & 0.000395 & 0.000396 & 1309.26 & 0.7387 & -0.000001 & 0.546 & & & 999.8395 \\
\hline & 0 & 0.4000 & 1317.90 & (90) & 0.000397 & 0.000397 & 1317.48 & 0.4235 & -0.000001 & 0.179 & & & 999.8395 \\
\hline & 0 & 0.4394 & 1353.30 & (35) & 0.000406 & 0.000405 & 1354.13 & -0.8284 & 0.000001 & 0.686 & & & 999.8395 \\
\hline & 0 & 0.4500 & 1363.00 & (90) & 0.000408 & 0.000407 & 1364.18 & -1.1849 & 0.000001 & 1.404 & & & 999.8395 \\
\hline & 0 & 0.4871 & 1398.50 & (35) & 0.000415 & 0.000413 & 1400.06 & -1.5618 & 0.000002 & 2.439 & & & 999.8395 \\
\hline & 0 & 0.5000 & 1411.00 & (90) & 0.000417 & 0.000415 & 1412.79 & -1.7905 & 0.000002 & 3.206 & & & 999.8395 \\
\hline & 0 & 0.5348 & 1446.20 & (35) & 0.000423 & 0.000422 & 1447.81 & -1.6065 & 0.000001 & 2.581 & & & 999.8395 \\
\hline & 0 & 0.5500 & 1461.90 & (90) & 0.000425 & 0.000424 & 1463.42 & -1.5201 & 0.000001 & 2.311 & & & 999.8395 \\
\hline & 0 & 0.5835 & 1497.40 & (35) & 0.000431 & 0.000430 & 1498.54 & -1.1428 & 0.000001 & 1.306 & & & 999.8395 \\
\hline & 0 & 0.6000 & 1515.40 & (90) & 0.000433 & 0.000432 & 1516.21 & -0.8123 & 0.000001 & 0.660 & & & 999.8395 \\
\hline & 0 & 0.6310 & 1549.90 & (35) & 0.000438 & 0.000437 & 1550.10 & -0.1953 & 0.000000 & 0.038 & & & 999.8395 \\
\hline & 0 & 0.6500 & 1571.40 & (90) & 0.000440 & 0.000441 & 1571.32 & 0.0816 & 0.000000 & 0.007 & & & 999.8395 \\
\hline & 0 & 0.6754 & 1600.80 & (35) & 0.000444 & 0.000445 & 1600.25 & 0.5491 & 0.000000 & 0.302 & & & 999.8395 \\
\hline & 0 & 0.7000 & 1629.30 & (90) & 0.000448 & 0.000448 & 1628.90 & 0.3962 & 0.000000 & 0.157 & & & 999.8395 \\
\hline & 0 & 0.7228 & 1656.30 & (35) & 0.000452 & 0.000452 & 1656.03 & 0.2654 & 0.000000 & 0.070 & & & 999.8395 \\
\hline & 0 & 0.7706 & 1713.50 & (35) & 0.000460 & 0.000459 & 1714.79 & -1.2919 & 0.000001 & 1.669 & & & 999.8395 \\
\hline & 5.96 & 0.0001 & 1000.01 & (90) & -0.000452 & 0.000320 & 999.98 & 0.0386 & -0.000771 & 0.001 & & ! & 999.9414 \\
\hline
\end{tabular}


molality

molarity

$\begin{array}{rrr}\mathbf{t} /{ }^{\circ} \mathrm{C} \text { mass frac } & \begin{array}{r}\text { Density } \\ \text { exp / } \\ \text { kg/m }\end{array} \\ & & \\ 5.96 & 0.0001 & 1000.06 \\ 5.96 & 0.0002 & 1000.14 \\ 5.96 & 0.0003 & 1000.23 \\ 5.96 & 0.0004 & 1000.31 \\ 5.96 & 0.0005 & 1000.39 \\ 5.96 & 0.0006 & 1000.47 \\ 5.96 & 0.0007 & 1000.55 \\ 5.96 & 0.0008 & 1000.63 \\ 5.96 & 0.0009 & 1000.71 \\ 5.96 & 0.0010 & 1000.79 \\ 5.96 & 0.0015 & 1001.17 \\ 5.96 & 0.0020 & 1001.55 \\ 5.96 & 0.0025 & 1001.93 \\ 5.96 & 0.0030 & 1002.30 \\ 5.96 & 0.0035 & 1002.66 \\ 5.96 & 0.0040 & 1003.03 \\ 5.96 & 0.0045 & 1003.39 \\ 5.96 & 0.0050 & 1003.75 \\ 10 & 0.0100 & 1006.80 \\ 10 & 0.0200 & 1013.80 \\ 10 & 0.0300 & 1020.60 \\ 10 & 0.0393 & 1026.90 \\ 10 & 0.0400 & 1027.50 \\ 10 & 0.0500 & 1034.40 \\ 10 & 0.0700 & 1048.50 \\ 10 & 0.1000 & 1070.00 \\ 10 & 0.1000 & 1070.00 \\ 10 & 0.1456 & 1103.60 \\ 10 & 0.1500 & 1106.90 \\ 10 & 0.1980 & 1143.80 \\ 10 & 0.2000 & 1145.30 \\ 10 & 0.2500 & 1184.80 \\ 10 & 0.2531 & 1187.20 \\ 10 & 0.2947 & 1221.10 \\ 10 & 0.3000 & 1225.50 \\ 10 & 0.3428 & 1261.20 \\ 10 & 0.3500 & 1267.20 \\ 10 & 0.3910 & 1302.50 \\ 10 & 0.4000 & 1310.30 \\ 10 & 0.4394 & 1345.60 \\ 10 & 0.4500 & 1355.30 \\ 10 & 0.4871 & 1390.40 \\ & & \end{array}$

exp /
Ref

$(90)$

(90) -0.0

$\begin{array}{lll}\text { (90) } \quad 0.000046 & 0.000320\end{array}$

(90) $\quad 0.000078 \quad 0.000320$

(90) $0.000099 \quad 0.000320$

$\begin{array}{lll}\text { (90) } \quad 0.000115 & 0.000320\end{array}$

(90) $\quad 0.000128 \quad 0.000320$

(90) $\quad 0.000138 \quad 0.000320$

$\begin{array}{lll}\text { (90) } \quad 0.000147 & 0.000320\end{array}$

(90) $\quad 0.000154 \quad 0.000320$

(90) $\quad 0.000180 \quad 0.000320$

(90) $\quad 0.000196 \quad 0.000320$

(90) $\quad 0.000208 \quad 0.000320$

(90) $\quad 0.000217 \quad 0.000320$

(90) $\quad 0.000224 \quad 0.000320$

(90) $\quad 0.000230 \quad 0.000320$

(90) $\quad 0.000236 \quad 0.000321$

$\begin{array}{lll}\text { (90) } & 0.000240 & 0.000321\end{array}$

$\begin{array}{lll}\text { (90) } \quad 0.000295 & 0.000328\end{array}$

(90) $\quad 0.000305 \quad 0.000330$

$\begin{array}{lll}\text { (90) } \quad 0.000317 & 0.000333\end{array}$

(35) $\quad 0.000326 \quad 0.000335$

(90) $\quad 0.000324 \quad 0.000335$

(90) $\quad 0.000329 \quad 0.000337$

(35)

(35)

(90)

(35)

(90)

(35)

(90)

(90)

(35)

(35)

(90)

(35)

(90)

(35)

(90)

(35)

(90)

(35) $\begin{array}{ll}0.000335 & 0.000342\end{array}$

$0.000343 \quad 0.000348$

$0.000343 \quad 0.000348$

$0.000353 \quad 0.000358$

$0.000354 \quad 0.000359$

$0.000364 \quad 0.000369$

$0.000364 \quad 0.000369$

$\begin{array}{ll}0.000375 & 0.000379\end{array}$

$0.000376 \quad 0.000380$

$0.000385 \quad 0.000387$

$\begin{array}{ll}0.000386 & 0.000388\end{array}$

0.0003950 .000396

$0.000397 \quad 0.000398$

$0.000406 \quad 0.000405$

$0.000408 \quad 0.000407$

$\begin{array}{ll}0.000415 & 0.000413\end{array}$

$0.000417 \quad 0.000415$

$0.000423 \quad 0.000421$
Density Density App Vol Square of Inconsistent Inconsistent Density of calc / Residual / Residual / Density based on based on water $/ \mathrm{kg} / \mathrm{m}^{3}$ $\mathrm{kg} / \mathrm{m}^{3} \quad \mathrm{~kg} / \mathrm{m}^{3} \quad \mathrm{~m}^{3} / \mathrm{kg}$ Residual density $\begin{gathered}\text { apparent } \\ \text { volume }\end{gathered}$

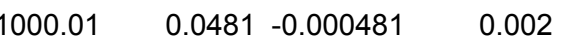

$\begin{array}{llll}1000.08 & 0.0659 & -0.000329 & 0.004\end{array}$

$\begin{array}{llll}1000.15 & 0.0820 & -0.000273 & 0.007\end{array}$

$\begin{array}{llll}1000.21 & 0.0968 & -0.000242 & 0.009\end{array}$

$1000.28 \quad 0.1103-0.000220 \quad 0.012$

$1000.35 \quad 0.1228-0.000205-0.015$

$1000.42 \quad 0.1345-0.000192 \quad 0.018$

$1000.49 \quad 0.1454-0.000182 \quad 0.021$

$\begin{array}{llll}1000.55 & 0.1558 & -0.000173 & 0.024\end{array}$

$1000.62 \quad 0.1659-0.000166 \quad 0.028$

$1000.96 \quad 0.2106-0.000140 \quad 0.044$

$\begin{array}{llll}1001.30 & 0.2482 & -0.000124 & 0.062\end{array}$

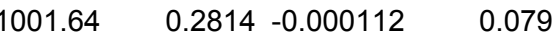

$1001.98 \quad 0.3113-0.000103 \quad 0.097$

$1002.33 \quad 0.3381-0.000096 \quad 0.114$

$\begin{array}{llll}1002.67 & 0.3624 & -0.000090 & 0.131\end{array}$

$\begin{array}{llll}1003.01 & 0.3843 & -0.000085 & 0.148\end{array}$

$1003.35 \quad 0.4041-0.000080 \quad 0.163$

$\begin{array}{llll}1006.47 & 0.3348 & -0.000033 & 0.112\end{array}$

$\begin{array}{llll}1013.28 & 0.5247 & -0.000026 & 0.275\end{array}$

$\begin{array}{llll}1020.13 & 0.4696 & -0.000015 & 0.220\end{array}$

$\begin{array}{llll}1026.54 & 0.3603 & -0.000009 & 0.130\end{array}$

$\begin{array}{llll}1027.03 & 0.4688 & -0.000011 & 0.220\end{array}$

$\begin{array}{llll}1033.98 & 0.4220 & -0.000008 & 0.178\end{array}$

$\begin{array}{llll}1048.01 & 0.4879 & -0.000006 & 0.238\end{array}$

$\begin{array}{llll}1069.42 & 0.5772 & -0.000005 & 0.333\end{array}$

$\begin{array}{llll}1069.42 & 0.5772 & -0.000005 & 0.333\end{array}$

$1102.83-0.7747-0.000004-0.600$

$\begin{array}{llll}1106.10 & 0.7952 & -0.000004 & 0.632\end{array}$

$\begin{array}{llll}1142.55 & 1.2459 & -0.000005 & 1.552\end{array}$

$1144.10 \quad 1.1998-0.000005 \quad 1.440$

$1183.49 \quad 1.3088-0.000004 \quad 1.713$

$\begin{array}{llll}1185.98 & 1.2186 & -0.000003 & 1.485\end{array}$

$\begin{array}{llll}1219.96 & 1.1388 & -0.000003 & 1.297\end{array}$

$1224.37 \quad 1.1328-0.000003 \quad 1.283$

$\begin{array}{llll}1260.61 & 0.5905 & -0.000001 & 0.349\end{array}$

$1266.82 \quad 0.3752-0.000001 \quad 0.141$

$\begin{array}{llll}1302.89 & -0.3936 & 0.000001 & 0.155\end{array}$

$\begin{array}{llll}1310.97 & -0.6690 & 0.000001 & 0.448\end{array}$

$\begin{array}{llll}1347.02 & -1.4173 & 0.000002 & 2.009\end{array}$

$\begin{array}{llll}1356.91 & -1.6142 & 0.000002 & 2.606\end{array}$

$\begin{array}{llll}1392.24 & -1.8427 & 0.000002 & 3.396\end{array}$
999.9414

999.9414

999.9414

999.9414

999.9414

999.9414

999.9414

999.9414

999.9414

999.9414

999.9414

999.9414

999.9414

999.9414

999.9414

999.9414

999.9414

999.9414

999.6996

999.6996

999.6996

999.6996

999.6996

999.6996

999.6996

999.6996

999.6996

999.6996

999.6996

999.6996

999.6996

999.6996

999.6996

999.6996

999.6996

999.6996

999.6996

999.6996

999.6996

999.6996

9996996

999.6996 
molality molarity

$\begin{array}{rrr}\mathbf{t} /{ }^{\circ} \mathrm{C} \text { mass frac } & \text { Density } \\ & & \text { exp } / \\ & & \mathrm{kg} / \mathrm{m}^{3} \\ 10 & 0.5000 & 1402.90 \\ 10 & 0.5348 & 1437.80 \\ 10 & 0.5500 & 1453.50 \\ 10 & 0.5835 & 1488.90 \\ 10 & 0.6000 & 1506.70 \\ 10 & 0.6310 & 1540.90 \\ 10 & 0.6500 & 1562.30 \\ 10 & 0.6754 & 1591.20 \\ 10 & 0.7000 & 1619.80 \\ 10 & 0.7228 & 1646.70 \\ 10 & 0.7706 & 1703.30 \\ 13 & 0.0005 & 999.81 \\ 13 & 0.0010 & 1000.19 \\ 13 & 0.0020 & 1000.91 \\ 13 & 0.0030 & 1001.62 \\ 13 & 0.0040 & 1002.33 \\ 13 & 0.0050 & 1003.02 \\ 13 & 0.0060 & 1003.72 \\ 13 & 0.0080 & 1005.09 \\ 13 & 0.0100 & 1006.45 \\ 13 & 0.0120 & 1007.81 \\ 13 & 0.0140 & 1009.16 \\ 13 & 0.0160 & 1010.51 \\ 13 & 0.0180 & 1011.86 \\ 13 & 0.0200 & 1013.21 \\ 13 & 0.0220 & 1014.56 \\ 13 & 0.0240 & 1015.90 \\ 18 & 0.0005 & 999.03 \\ 18 & 0.0010 & 999.40 \\ 18 & 0.0020 & 1000.12 \\ 18 & 0.0030 & 1000.82 \\ 18 & 0.0040 & 1001.51 \\ 18 & 0.0050 & 1002.20 \\ 18 & 0.0060 & 1002.88 \\ 18 & 0.0080 & 1004.23 \\ 18 & 0.0100 & 1005.57 \\ 18 & 0.0120 & 1006.91 \\ 18 & 0.0140 & 1008.25 \\ 18 & 0.0160 & 1009.58 \\ 18 & 0.0180 & 1010.92 \\ 18 & 0.0200 & 1012.25 \\ 18 & 0.0220 & 1013.59\end{array}$

Ref

$$
\begin{array}{rr}
\text { App vol } & \text { App vol } \\
\exp / & \text { Calc / } \\
\mathrm{m}^{3} / \mathrm{kg} & \mathrm{m}^{3} / \mathrm{kg}
\end{array}
$$

$\begin{array}{ll}0.000425 & 0.000423\end{array}$

$\begin{array}{ll}0.000430 & 0.000429\end{array}$

$0.000432 \quad 0.000431$

$\begin{array}{lll}0.000437 & 0.000437\end{array}$

$\begin{array}{lll}0.000439 & 0.000439\end{array}$

$0.000444 \quad 0.000444$

$\begin{array}{ll}0.000446 & 0.000447\end{array}$

$0.000450 \quad 0.000450$

$\begin{array}{lll}0.000453 & 0.000454\end{array}$

$\begin{array}{lll}0.000457 & 0.000457\end{array}$

$0.000464 \quad 0.000464$

$0.000134 \quad 0.000330$

$0.000192 \quad 0.000330$

$0.000233 \quad 0.000330$

$\begin{array}{lll}0.000253 & 0.000331\end{array}$

$\begin{array}{lll}0.000265 & 0.000331\end{array}$

$\begin{array}{ll}0.000273 & 0.000331\end{array}$

$0.000280 \quad 0.000331$

$0.000290 \quad 0.000332$

$\begin{array}{ll}0.000297 & 0.000332\end{array}$

$0.000303 \quad 0.000333$

$0.000308 \quad 0.000333$

$0.000312 \quad 0.000334$

$0.000315 \quad 0.000334$

$0.000318 \quad 0.000334$

$\begin{array}{ll}0.000320 & 0.000335\end{array}$

$0.000322 \quad 0.000335$

$0.000135 \quad 0.000337$

$\begin{array}{lll}0.000195 & 0.000337\end{array}$

$0.000239 \quad 0.000337$

$\begin{array}{lll}0.000260 & 0.000338\end{array}$

$\begin{array}{lll}(90) & 0.000272 & 0.000338 \\ (90) & 0.000282 & 0.000338\end{array}$

(90)

(90)

(90)

(90)

(90)

(90)

(90)

(90)

(90)

(90)

$0.000289 \quad 0.000338$

$0.000299 \quad 0.000339$

$\begin{array}{lll}0.000307 & 0.000339\end{array}$

$0.000312 \quad 0.000340$

$\begin{array}{ll}0.000317 & 0.000340\end{array}$

$\begin{array}{ll}0.000320 & 0.000340\end{array}$

$0.000323 \quad 0.000341$

$0.000326 \quad 0.000341$

$0.000328 \quad 0.000342$
Densit calc / Residual / Residual / Density based on based on water $/ \mathrm{kg} / \mathrm{m}^{3}$ $\mathrm{kg} / \mathrm{m}^{3} \quad \mathrm{~kg} / \mathrm{m}^{3} \quad \mathrm{~m}^{3} / \mathrm{kg}$ Residual density apparent

$\begin{array}{llll}1404.79 & -1.8851 & 0.000002 & 3.554\end{array}$

$\begin{array}{llll}1439.31 & -1.5115 & 0.000001 & 2.285\end{array}$

$\begin{array}{llll}1454.72 & -1.2178 & 0.000001 & 1.483\end{array}$

$\begin{array}{llll}1489.40 & -0.4999 & 0.000000 & 0.250\end{array}$

$\begin{array}{llll}1506.86 & -0.1615 & 0.000000 & 0.026\end{array}$

$\begin{array}{llll}1540.37 & 0.5281 & 0.000000 & 0.279\end{array}$

$\begin{array}{llll}1561.38 & 0.9204 & -0.000001 & 0.847\end{array}$

$\begin{array}{llll}1590.04 & 1.1588 & -0.000001 & 1.343\end{array}$

$\begin{array}{llll}1618.45 & 1.3480 & -0.000001 & 1.817\end{array}$

$\begin{array}{lllll}1645.38 & 1.3220 & -0.000001 & 1.748\end{array}$

$\begin{array}{llll}1703.78 & -0.4757 & 0.000000 & 0.226\end{array}$

$\begin{array}{llll}999.71 & 0.0979 & -0.000196 & 0.010\end{array}$

$\begin{array}{llll}1000.05 & 0.1378 & -0.000138 & 0.019\end{array}$

$\begin{array}{llll}1000.72 & 0.1942 & -0.000097 & 0.038\end{array}$

$\begin{array}{llll}1001.39 & 0.2342 & -0.000078 & 0.055\end{array}$

$\begin{array}{llll}1002.06 & 0.2658 & -0.000066 & 0.071\end{array}$

$\begin{array}{llll}1002.73 & 0.2909 & -0.000058 & 0.085\end{array}$

$\begin{array}{lllll}1003.40 & 0.3116 & -0.000052 & 0.097\end{array}$

$1004.75 \quad 0.3397-0.000042 \quad 0.115$

$\begin{array}{llll}1006.10 & 0.3539 & -0.000035 & 0.125\end{array}$

$\begin{array}{llll}1007.45 & 0.3594 & -0.000030 & 0.129\end{array}$

$\begin{array}{llll}1008.80 & 0.3602 & -0.000025 & 0.130\end{array}$

$\begin{array}{llll}1010.15 & 0.3581 & -0.000022 & 0.128\end{array}$

$\begin{array}{llll}1011.51 & 0.3532 & -0.000019 & 0.125\end{array}$

$101286-0.3456-0.000017-0.119$

$1014.22 \quad 0.3351-0.000015 \quad 0.112$

$\begin{array}{llll}1015.58 & 0.3218 & -0.000013 & 0.104\end{array}$

$\begin{array}{llll}998.93 & 0.1010 & -0.000202 & 0.010\end{array}$

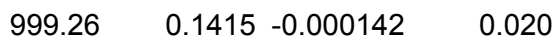

$\begin{array}{llll}999.92 & 0.1972 & -0.000099 & 0.039\end{array}$

1000.59

1001.25

1001.91

1002.58

1003.91

1005.24

1006.58

1007.92

1009.25

1010.59

1011.94

1013.28

$0.2345-0.000078$

0.055

0.069

0.080

0.089

0.100

0.106

0.109

0.110

0.108

0.104

0.099

$0.3231-0.000018$

0.093

999.6996

999.6996

999.6996

999.6996

999.6996

999.6996

999.6996

999.6996

999.6996

999.6996

999.6996

999.3771

999.3771

999.3771

999.3771

999.3771

999.3771

999.3771

999.3771

999.3771

999.3771

999.3771

999.3771

999.3771

999.3771

999.3771

999.3771

998.5956

998.5956

998.5956

998.5956

998.5956

998.5956

998.5956

998.5956

998.5956

998.5956

998.5956

998.5956

998.5956

998.5956

998.5956 
molality molarity

\begin{tabular}{|c|c|c|}
\hline \multicolumn{2}{|c|}{$\mathrm{t} /{ }^{\circ} \mathrm{C}$ mass frac } & $\begin{array}{r}\text { Density } \\
\exp / \\
\mathrm{kg} / \mathrm{m}^{3}\end{array}$ \\
\hline 18 & 0.0240 & 1014.92 \\
\hline 20 & 0.0100 & 1005.10 \\
\hline 20 & 0.0200 & 1011.80 \\
\hline 20 & 0.0300 & 1018.40 \\
\hline 20 & 0.0393 & 1024.60 \\
\hline 20 & 0.0400 & 1025.00 \\
\hline 20 & 0.0500 & 1031.70 \\
\hline 20 & 0.0700 & 1045.30 \\
\hline 20 & 0.1000 & 1066.10 \\
\hline 20 & 0.1000 & 1066.10 \\
\hline 20 & 0.1456 & 1098.80 \\
\hline 20 & 0.1500 & 1102.00 \\
\hline 20 & 0.1980 & 1138.00 \\
\hline 20 & 0.2000 & 1139.40 \\
\hline 20 & 0.2500 & 1178.30 \\
\hline 20 & 0.2531 & 1180.70 \\
\hline 20 & 0.2947 & 1214.20 \\
\hline 20 & 0.3000 & 1218.50 \\
\hline 20 & 0.3428 & 1253.90 \\
\hline 20 & 0.3500 & 1259.90 \\
\hline 20 & 0.3910 & 1295.00 \\
\hline 20 & 0.4000 & 1302.80 \\
\hline 20 & 0.4394 & 1337.90 \\
\hline 20 & 0.4500 & 1347.60 \\
\hline 20 & 0.4871 & 1382.60 \\
\hline 20 & 0.5000 & 1395.10 \\
\hline 20 & 0.5348 & 1429.70 \\
\hline 20 & 0.5500 & 1445.30 \\
\hline 20 & 0.5835 & 1480.50 \\
\hline 20 & 0.6000 & 1498.30 \\
\hline 20 & 0.6754 & 1582.10 \\
\hline 20 & 0.7228 & 1637.20 \\
\hline 20 & 0.7706 & 1693.50 \\
\hline 25 & 0.0393 & 1022.90 \\
\hline 25 & 0.0700 & 1043.40 \\
\hline 25 & 0.1000 & 1064.00 \\
\hline 25 & 0.1456 & 1096.20 \\
\hline 25 & 0.1980 & 1135.10 \\
\hline 25 & 0.2331 & 1163.88 \\
\hline 25 & 0.2531 & 1177.40 \\
\hline 25 & 0.2947 & 1210.70 \\
\hline 25 & 0.3428 & 1250.30 \\
\hline
\end{tabular}

Ref

$$
\begin{array}{rr}
\text { App vol } & \text { App vol } \\
\exp / & \text { Calc / } \\
\mathrm{m}^{3} / \mathrm{kg} & \mathrm{m}^{3} / \mathrm{kg}
\end{array}
$$

$\begin{array}{ll}0.000330 & 0.000342\end{array}$

$\begin{array}{ll}0.000314 & 0.000342\end{array}$

$0.000329 \quad 0.000344$

$0.000340 \quad 0.000346$

$\begin{array}{ll}0.000345 & 0.000348\end{array}$

$0.000347 \quad 0.000348$

$\begin{array}{ll}0.000351 & 0.000350\end{array}$

$\begin{array}{ll}0.000357 & 0.000354\end{array}$

$0.000364 \quad 0.000361$

$\begin{array}{ll}0.000364 & 0.000361\end{array}$

$0.000372 \quad 0.000370$

$0.000373 \quad 0.000370$

$0.000380 \quad 0.000380$

0.0003810 .000380

$0.000389 \quad 0.000389$

$0.000390 \quad 0.000390$

$\begin{array}{ll}0.000397 & 0.000397\end{array}$

$\begin{array}{lll}0.000398 & 0.000398\end{array}$

$\begin{array}{ll}0.000406 & 0.000405\end{array}$

$\begin{array}{ll}0.000407 & 0.000406\end{array}$

$0.000415 \quad 0.000413$

$0.000416 \quad 0.000415$

$\begin{array}{ll}0.000423 & 0.000421\end{array}$

$0.000425 \quad 0.000423$

$\begin{array}{lll}0.000430 & 0.000428\end{array}$

$0.000432 \quad 0.000430$

$0.000436 \quad 0.000436$

$0.000438 \quad 0.000438$

$\begin{array}{lll}0.000442 & 0.000443\end{array}$

$0.000445 \quad 0.000445$

$0.000454 \quad 0.000455$

$\begin{array}{ll}0.000461 & 0.000462\end{array}$

$\begin{array}{ll}0.000468 & 0.000468\end{array}$

$0.000358 \quad 0.000354$

$0.000366 \quad 0.000360$

0.0003720 .000366

$0.000380 \quad 0.000375$

$\begin{array}{lll}0.000387 & 0.000385\end{array}$

$\begin{array}{ll}0.000386 & 0.000391\end{array}$

$0.000396 \quad 0.000394$

$\begin{array}{ll}0.000402 & 0.000401\end{array}$

$\begin{array}{ll}0.000410 & 0.000409\end{array}$
Density Density App Vol Square of Inconsistent Inconsistent Density of calc / Residual / Residual / Density based on based on water $/ \mathrm{kg} / \mathrm{m}^{3}$ $\mathrm{kg} / \mathrm{m}^{3} \quad \mathrm{~kg} / \mathrm{m}^{3} \quad \mathrm{~m}^{3} / \mathrm{kg}$ Residual density apparent

$1014.63 \quad 0.2923-0.000012 \quad 0.085$

$1004.82 \quad 0.2756-0.000027 \quad 0.076$

$1011.49-0.3109-0.000015 \quad 0.097$

$1018.20 \quad 0.2014-0.000006 \quad 0.041$

$1024.47 \quad 0.1275-0.000003 \quad 0.016$

$1024.95-0.0464-0.000001 \quad 0.002$

$\begin{array}{llll}1031.75 & -0.0546 & 0.000001 & 0.003\end{array}$

$\begin{array}{llll}1045.50 & -0.1968 & 0.000003 & 0.039\end{array}$

$\begin{array}{llll}1066.47 & -0.3696 & 0.000003 & 0.137\end{array}$

$\begin{array}{llll}1066.47 & -0.3696 & 0.000003 & 0.137\end{array}$

$\begin{array}{llll}1099.21 & -0.4079 & 0.000002 & 0.166\end{array}$

$\begin{array}{llll}1102.42 & -0.4235 & 0.000002 & 0.179\end{array}$

$\begin{array}{llll}1138.18 & -0.1795 & 0.000001 & 0.032\end{array}$

$\begin{array}{llll}1139.70 & -0.2968 & 0.000001 & 0.088\end{array}$

$\begin{array}{llll}1178.38 & -0.0767 & 0.000000 & 0.006\end{array}$

$\begin{array}{llll}1180.82 & -0.1233 & 0.000000 & 0.015\end{array}$

$\begin{array}{llll}1214.22 & -0.0244 & 0.000000 & 0.001\end{array}$

$\begin{array}{llll}1218.56 & -0.0578 & 0.000000 & 0.003\end{array}$

$\begin{array}{llll}1254.22 & -0.3229 & 0.000001 & 0.104\end{array}$

$\begin{array}{llll}1260.34 & -0.4430 & 0.000001 & 0.196\end{array}$

$\begin{array}{llll}1295.88 & -0.8823 & 0.000001 & 0.779\end{array}$

$\begin{array}{llll}1303.84 & -1.0446 & 0.000002 & 1.091\end{array}$

$\begin{array}{llll}1339.41 & -1.5130 & 0.000002 & 2.289\end{array}$

$\begin{array}{llll}1349.19 & -1.5853 & 0.000002 & 2.513\end{array}$

$1384.10 \quad-1.4953 \quad 0.000002 \quad 2236$

$\begin{array}{llll}1396.50 & -1.3990 & 0.000001 & 1.957\end{array}$

$\begin{array}{llll}1430.67 & -0.9713 & 0.000001 & 0.943\end{array}$

$\begin{array}{llll}1445.93 & -0.6329 & 0.000001 & 0.401\end{array}$

$\begin{array}{llll}1480.32 & 0.1804 & 0.000000 & 0.033\end{array}$

$\begin{array}{llll}1497.65 & 0.6513 & 0.000000 & 0.424\end{array}$

$1580.35 \quad 1.7496-0.000001 \quad 3.061$

$\begin{array}{llll}1635.51 & 1.6868 & -0.000001 & 2.845\end{array}$

$\begin{array}{llll}1693.86 & -0.3557 & 0.000000 & 0.127\end{array}$

$\begin{array}{llll}1023.04 & -0.1433 & 0.000003 & 0.021\end{array}$

$\begin{array}{llll}1043.86 & -0.4574 & 0.000006 & 0.209\end{array}$

$\begin{array}{llll}1064.63 & -0.6260 & 0.000006 & 0.392\end{array}$

$\begin{array}{llll}1097.06 & -0.8572 & 0.000005 & 0.735\end{array}$

$\begin{array}{llll}1135.68 & -0.5829 & 0.000002 & 0.340\end{array}$

$\begin{array}{llll}1162.42 & 1.4614 & -0.000005 & 2.136\end{array}$

$\begin{array}{llll}1177.97 & -0.5740 & 0.000002 & 0.329\end{array}$

$\begin{array}{llll}1211.12 & -0.4188 & 0.000001 & 0.175\end{array}$

$\begin{array}{lll}-0.5345 & 0.000001 & 0.286\end{array}$
998.5956

998.2041

998.2041

998.2041

998.2041

998.2041

998.2041

998.2041

998.2041

998.2041

998.2041

998.2041

998.2041

998.2041

998.2041

998.2041

998.2041

998.2041

998.2041

998.2041

998.2041

998.2041

998.2041

998.2041

998.2041

998.2041

998.2041

998.2041

998.2041

998.2041

998.2041

998.2041

998.2041

997.0449

997.0449

997.0449

997.0449

997.0449

997.0449

997.0449

997.0449

997.0449 


\begin{tabular}{|c|c|c|c|c|c|c|c|c|c|c|c|c|c|c|}
\hline \multirow{2}{*}{$\begin{array}{r}\text { molality } \\
5.5776\end{array}$} & \multirow{2}{*}{ molarity } & \multicolumn{2}{|c|}{$\mathrm{t} /{ }^{\circ} \mathrm{C}$ mass frac } & \multirow{2}{*}{$\begin{array}{r}\text { Density } \\
\text { exp / } \\
\mathrm{kg} / \mathrm{m}^{3} \\
1258.33\end{array}$} & \multirow{2}{*}{$\begin{array}{c}\text { Ref } \\
\text { (103) }\end{array}$} & \multirow{2}{*}{$\begin{array}{r}\text { App vol } \\
\text { exp / } \\
\mathrm{m}^{3} / \mathrm{kg} \\
0.000414\end{array}$} & \multirow{2}{*}{$\begin{array}{r}\text { App vol } \\
\text { Calc / } \\
\mathrm{m}^{3} / \mathrm{kg} \\
0.000411\end{array}$} & \multirow{2}{*}{$\begin{array}{r}\text { Density } \\
\text { calc / } \\
\mathrm{kg} / \mathrm{m}^{3} \\
1259.97\end{array}$} & \multirow{2}{*}{$\begin{array}{r}\text { Density } \\
\text { Residual / } \\
\mathrm{kg} / \mathrm{m}^{3} \\
-1.6386\end{array}$} & \multirow{2}{*}{$\begin{array}{r}\text { App Vol } \\
\text { Residual / } \\
\mathrm{m}^{3} / \mathrm{kg} \\
0.000003\end{array}$} & \multirow{2}{*}{$\begin{array}{r}\text { Square of } \\
\text { Density } \\
\text { Residual } \\
2.685\end{array}$} & \multirow[t]{2}{*}{$\begin{array}{l}\text { Inconsistent } \\
\text { based on } \\
\text { density }\end{array}$} & $\begin{array}{c}\text { Inconsistent } \\
\text { based on } \\
\text { apparent } \\
\text { volume }\end{array}$ & $\begin{array}{r}\text { Density of } \\
\text { water } / \mathrm{kg} / \mathrm{m}^{3}\end{array}$ \\
\hline & & 25 & 0.3536 & & & & & & & & & & & 997.0449 \\
\hline & & 25 & 0.3910 & 1291.30 & $(35)$ & 0.000418 & 0.000417 & 1292.23 & -0.9283 & 0.000001 & 0.862 & & & 997.0449 \\
\hline 7.0913 & & 25 & 0.4102 & 1309.03 & (103) & 0.000420 & 0.000420 & 1309.19 & -0.1591 & 0.000000 & 0.025 & & & 997.0449 \\
\hline & & 25 & 0.4394 & 1334.00 & $(35)$ & 0.000426 & 0.000424 & 1335.51 & -1.5143 & 0.000002 & 2.293 & & & 997.0449 \\
\hline & & 25 & 0.4871 & 1378.70 & (35) & 0.000433 & 0.000432 & 1379.98 & -1.2814 & 0.000001 & 1.642 & & & 997.0449 \\
\hline & & 25 & 0.5348 & 1425.80 & (35) & 0.000439 & 0.000438 & 1426.37 & -0.5732 & 0.000001 & 0.329 & & & 997.0449 \\
\hline & & 25 & 0.5835 & 1476.20 & (35) & 0.000445 & 0.000445 & 1475.87 & 0.3287 & 0.000000 & 0.108 & & & 997.0449 \\
\hline & & 25 & 0.6310 & 1527.80 & (35) & 0.000451 & 0.000452 & 1526.38 & 1.4154 & -0.000001 & 2.003 & & & 997.0449 \\
\hline & & 25 & 0.6754 & 1577.60 & (35) & 0.000456 & 0.000458 & 1575.75 & 1.8514 & -0.000001 & 3.428 & & & 997.0449 \\
\hline & & 25 & 0.7228 & 1632.50 & (35) & 0.000463 & 0.000464 & 1630.91 & 1.5855 & -0.000001 & 2.514 & & & 997.0449 \\
\hline & & 25 & 0.7706 & 1688.50 & (35) & 0.000470 & 0.000470 & 1689.33 & -0.8298 & 0.000000 & 0.689 & & & 997.0449 \\
\hline & & 30 & 0.0100 & 1002.20 & (90) & 0.000348 & 0.000354 & 1002.13 & 0.0673 & -0.000007 & 0.005 & & & 995.6473 \\
\hline & & 30 & 0.0200 & 1008.70 & (90) & 0.000355 & 0.000356 & 1008.66 & 0.0374 & -0.000002 & 0.001 & & & 995.6473 \\
\hline & & 30 & 0.0300 & 1015.20 & (90) & 0.000360 & 0.000358 & 1015.24 & -0.0376 & 0.000001 & 0.001 & & & 995.6473 \\
\hline & & 30 & 0.0393 & 1021.10 & (35) & 0.000367 & 0.000360 & 1021.39 & -0.2867 & 0.000007 & 0.082 & & & 995.6473 \\
\hline & & 30 & 0.0400 & 1021.60 & (90) & 0.000366 & 0.000360 & 1021.86 & -0.2583 & 0.000006 & 0.067 & & & 995.6473 \\
\hline & & 30 & 0.0500 & 1028.10 & (90) & 0.000370 & 0.000362 & 1028.53 & -0.4252 & 0.000008 & 0.181 & & & 995.6473 \\
\hline & & 30 & 0.0700 & 1041.40 & (35) & 0.000374 & 0.000366 & 1042.00 & -0.6001 & 0.000008 & 0.360 & & & 995.6473 \\
\hline & & 30 & 0.1000 & 1061.70 & (35) & 0.000380 & 0.000372 & 1062.57 & -0.8746 & 0.000008 & 0.765 & & & 995.6473 \\
\hline & & 30 & 0.1000 & 1061.70 & (90) & 0.000380 & 0.000372 & 1062.57 & -0.8746 & 0.000008 & 0.765 & & & 995.6473 \\
\hline & & 30 & 0.1456 & 1093.60 & (35) & 0.000387 & 0.000380 & 1094.72 & -1.1153 & 0.000006 & 1.244 & & & 995.6473 \\
\hline & & 30 & 0.1500 & 1096.80 & (90) & 0.000387 & 0.000381 & 1097.87 & -1.0740 & 0.000006 & 1.153 & & & 995.6473 \\
\hline & & 30 & 0.1980 & 1132.10 & (35) & 0.000393 & 0.000389 & 1133.02 & -0.9165 & 0.000004 & 0.840 & & & 995.6473 \\
\hline & & 30 & 0.2000 & 1133.50 & (90) & 0.000394 & 0.000390 & 1134.51 & -1.0086 & 0.000004 & 1.017 & & & 995.6473 \\
\hline & & 30 & 0.2500 & 1171.80 & (90) & 0.000400 & 0.000398 & 1172.57 & -0.7710 & 0.000002 & 0.595 & & & 995.6473 \\
\hline & & 30 & 0.2531 & 1174.20 & (35) & 0.000401 & 0.000399 & 1174.98 & -0.7802 & 0.000002 & 0.609 & & & 995.6473 \\
\hline & & 30 & 0.2947 & 1207.30 & (35) & 0.000407 & 0.000406 & 1207.89 & -0.5898 & 0.000001 & 0.348 & & & 995.6473 \\
\hline & & 30 & 0.3000 & 1211.50 & (90) & 0.000408 & 0.000406 & 1212.16 & -0.6621 & 0.000002 & 0.438 & & & 995.6473 \\
\hline & & 30 & 0.3428 & 1246.60 & (35) & 0.000415 & 0.000413 & 1247.35 & -0.7493 & 0.000001 & 0.562 & & & 995.6473 \\
\hline & & 30 & 0.3500 & 1252.60 & (90) & 0.000416 & 0.000414 & 1253.39 & -0.7919 & 0.000001 & 0.627 & & & 995.6473 \\
\hline & & 30 & 0.3910 & 1287.50 & (35) & 0.000422 & 0.000421 & 1288.51 & -1.0071 & 0.000002 & 1.014 & & & 995.6473 \\
\hline & & 30 & 0.4000 & 1295.30 & (90) & 0.000423 & 0.000422 & 1296.38 & -1.0806 & 0.000002 & 1.168 & & & 995.6473 \\
\hline & & 30 & 0.4394 & 1330.30 & (35) & 0.000429 & 0.000428 & 1331.58 & -1.2808 & 0.000002 & 1.640 & & & 995.6473 \\
\hline & & 30 & 0.4500 & 1339.90 & (90) & 0.000431 & 0.000429 & 1341.26 & -1.3602 & 0.000002 & 1.850 & & & 995.6473 \\
\hline & & 30 & 0.4871 & 1374.80 & (35) & 0.000436 & 0.000435 & 1375.87 & -1.0679 & 0.000001 & 1.140 & & & 995.6473 \\
\hline & & 30 & 0.5000 & 1387.20 & (90) & 0.000437 & 0.000436 & 1388.18 & -0.9755 & 0.000001 & 0.952 & & & 995.6473 \\
\hline & & 30 & 0.5348 & 1421.80 & (35) & 0.000441 & 0.000441 & 1422.11 & -0.3139 & 0.000000 & 0.099 & & & 995.6473 \\
\hline & & 30 & 0.5500 & 1437.20 & (90) & 0.000443 & 0.000443 & 1437.29 & -0.0858 & 0.000000 & 0.007 & & & 995.6473 \\
\hline & & 30 & 0.5835 & 1472.10 & (35) & 0.000447 & 0.000448 & 1471.50 & 0.5953 & 0.000000 & 0.354 & & & 995.6473 \\
\hline & & 30 & 0.6000 & 1489.80 & (90) & 0.000449 & 0.000450 & 1488.77 & 1.0327 & -0.000001 & 1.066 & & & 995.6473 \\
\hline & & 30 & 0.6310 & 1523.60 & (35) & 0.000453 & 0.000454 & 1521.96 & 1.6389 & -0.000001 & 2.686 & & & 995.6473 \\
\hline & & 30 & 0.6500 & 1544.60 & (90) & 0.000455 & 0.000456 & 1542.81 & 1.7854 & -0.000001 & 3.188 & & & 995.6473 \\
\hline
\end{tabular}




\begin{tabular}{|c|c|c|c|c|}
\hline \multirow[t]{5}{*}{ molality } & molarity & $\mathrm{t} /{ }^{\circ} \mathrm{C}$ & ass frac & $\begin{array}{r}\text { Density } \\
\exp / \\
\mathrm{kg} / \mathrm{m}^{3}\end{array}$ \\
\hline & & 30 & 0.6754 & 1573.20 \\
\hline & & 30 & 0.7000 & 1601.40 \\
\hline & & 30 & 0.7228 & 1627.90 \\
\hline & & 30 & 0.7706 & 1683.60 \\
\hline 3.0991 & & 35 & 0.2331 & 1157.43 \\
\hline 5.5776 & & 35 & 0.3536 & 1250.99 \\
\hline 7.0913 & & 35 & 0.4102 & 1301.53 \\
\hline & & 40 & 0.0100 & 998.60 \\
\hline & & 40 & 0.0200 & 1005.00 \\
\hline & & 40 & 0.0300 & 1011.30 \\
\hline & & 40 & 0.0393 & 1017.10 \\
\hline & & 40 & 0.0400 & 1017.60 \\
\hline & & 40 & 0.0500 & 1024.00 \\
\hline & & 40 & 0.0700 & 1037.10 \\
\hline & & 40 & 0.1000 & 1057.00 \\
\hline & & 40 & 0.1000 & 1057.00 \\
\hline & & 40 & 0.1456 & 1088.30 \\
\hline & & 40 & 0.1500 & 1091.40 \\
\hline & & 40 & 0.1980 & 1126.10 \\
\hline & & 40 & 0.2000 & 1127.50 \\
\hline & & 40 & 0.2500 & 1165.30 \\
\hline & & 40 & 0.2531 & 1167.60 \\
\hline & & 40 & 0.2947 & 1200.40 \\
\hline & & 40 & 0.3000 & 1204.60 \\
\hline & & 40 & 0.3428 & 1239.40 \\
\hline & & 40 & 0.3500 & 1245.40 \\
\hline & & 40 & 0.3910 & 1280.20 \\
\hline & & 40 & 0.4000 & 1288.00 \\
\hline & & 40 & 0.4394 & 1322.90 \\
\hline & & 40 & 0.4500 & 1332.50 \\
\hline & & 40 & 0.4871 & 1367.20 \\
\hline & & 40 & 0.5000 & 1379.50 \\
\hline & & 40 & 0.5348 & 1413.90 \\
\hline & & 40 & 0.5500 & 1429.30 \\
\hline & & 40 & 0.5835 & 1464.00 \\
\hline & & 40 & 0.6000 & 1481.60 \\
\hline & & 40 & 0.6310 & 1515.10 \\
\hline & & 40 & 0.6500 & 1536.10 \\
\hline & & 40 & 0.6754 & 1561.50 \\
\hline & & 40 & 0.7000 & 1592.50 \\
\hline & & 40 & 0.7228 & 1618.80 \\
\hline & & 40 & 0.7706 & 1674.10 \\
\hline
\end{tabular}

Ref

$$
\begin{array}{rr}
\text { App vol } & \text { App vol } \\
\exp / & \text { Calc / } \\
\mathrm{m}^{3} / \mathrm{kg} & \mathrm{m}^{3} / \mathrm{kg}
\end{array}
$$

(35)

(90)

$\begin{array}{ll}0.000458 & 0.000460\end{array}$

0.0004620 .000463

$0.000465 \quad 0.000465$

$\begin{array}{ll}0.000472 & 0.000471\end{array}$

$0.000397 \quad 0.000400$

$\begin{array}{ll}0.000422 & 0.000418\end{array}$

$\begin{array}{ll}0.000427 & 0.000427\end{array}$

$\begin{array}{ccc}(103) & 0.000427 & 0.000427 \\ (90) & 0.000364 & 0.000366\end{array}$

$\begin{array}{lll}\text { (90) } & 0.000367 & 0.000368\end{array}$

(90) $\quad 0.000374 \quad 0.000369$

(35) $\quad 0.000380 \quad 0.000371$

$\begin{array}{lll}\text { (90) } \quad 0.000379 & 0.000371\end{array}$

$(90)$

(35)

(35)

(90)

(35)

(90)

(35)

(90)

(90)

(35)

(35)

(90)

(35)

(90)

(35)

(35)

$(35)$
$(90)$

(35)

(90)

(35)

(90)

(35)

(90)

(35)

(90)

(35)

(90)

(35)

(35)
$0.000382 \quad 0.000373$

0.0003850 .000377

$0.000390 \quad 0.000382$

$0.000390 \quad 0.000382$

$0.000397 \quad 0.000390$

$\begin{array}{ll}0.000397 & 0.000390\end{array}$

$\begin{array}{ll}0.000403 & 0.000398\end{array}$

$0.000403 \quad 0.000398$

0.0004090 .000406

$\begin{array}{ll}0.000410 & 0.000407\end{array}$

$\begin{array}{ll}0.000415 & 0.000413\end{array}$

$0.000416 \quad 0.000414$

$0.000421 \quad 0.000420$

$0.000422 \quad 0.000421$

$0.000428 \quad 0.000427$

$0.000429 \quad 0.000428$

$0.000434 \quad 0.000433$

$0.000436 \quad 0.000435$

$0.000440 \quad 0.000440$

$0.000442 \quad 0.000441$

$0.000446 \quad 0.000446$

$0.000447 \quad 0.000448$

$0.000451 \quad 0.000452$

$0.000453 \quad 0.000454$

$0.000457 \quad 0.000458$

$0.000459 \quad 0.000460$

0.0004640 .000463

$0.000465 \quad 0.000466$

$0.000468 \quad 0.000468$

$0.000475 \quad 0.000474$
Density Density App Vol Square of Inconsistent Inconsistent Density of calc / Residual / Residual / Density based on based on water $/ \mathrm{kg} / \mathrm{m}^{3}$ $\mathrm{kg} / \mathrm{m}^{3} \quad \mathrm{~kg} / \mathrm{m}^{3} \quad \mathrm{~m}^{3} / \mathrm{kg}$ Residual density apparent

$\begin{array}{llll}571.32 & 1.8783 & -0.000001 & 3.528\end{array}$

$1599.64 \quad 1.7561-0.000001 \quad 3.084$

$\begin{array}{llll}1626.55 & 1.3541 & -0.000001 & 1.834\end{array}$

$\begin{array}{llll}1685.10 & -1.4952 & 0.000001 & 2.236\end{array}$

$1156.53 \quad 0.9014-0.000003 \quad 0.813$

$\begin{array}{llll}1.82 .82 & -1.8250 & 0.000003 & 3.330\end{array}$

$\begin{array}{llll}1301.53 & -0.0021 & 0.000000 & 0.000\end{array}$

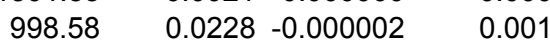

$\begin{array}{llll}0.0163 & -0.000001 & 0.000\end{array}$

$\begin{array}{llll}1011.44 & -0.1356 & 0.000004 & 0.018\end{array}$

$\begin{array}{llll}1017.47 & -0.3708 & 0.000009 & 0.138\end{array}$

$\begin{array}{llll}1017.93 & -0.3337 & 0.000008 & 0.111\end{array}$

$\begin{array}{llll}1024.48 & -0.4786 & 0.000009 & 0.229\end{array}$

$\begin{array}{llll}1037.71 & -0.6113 & 0.000008 & 0.374\end{array}$

$\begin{array}{llll}1057.93 & -0.9272 & 0.000008 & 0.860\end{array}$

$\begin{array}{llll}1057.93 & -0.9272 & 0.000008 & 0.860\end{array}$

$\begin{array}{llll}1089.54 & -1.2363 & 0.000007 & 1.529\end{array}$

$\begin{array}{llll}1092.64 & -1.2446 & 0.000007 & 1.549\end{array}$

$\begin{array}{llll}1127.25 & -1.1509 & 0.000005 & 1.325\end{array}$

$\begin{array}{llll}1128.72 & -1.2213 & 0.000005 & 1.492\end{array}$

$\begin{array}{llll}1166.26 & -0.9556 & 0.000003 & 0.913\end{array}$

$\begin{array}{lll}1168.63 & -1.0331 & 0.000003\end{array}$

$\begin{array}{llll}1201.13 & -0.7330 & 0.000002 & 0.537\end{array}$

$\begin{array}{llll}1205.36 & -0.7552 & 0.000002 & 0.570\end{array}$

$\begin{array}{lll}1240.16 & -0.7566 & 0.000001\end{array}$

$\begin{array}{llll}1246.14 & -0.7378 & 0.000001 & 0.544\end{array}$

$\begin{array}{llll}1280.93 & -0.7256 & 0.000001 & 0.527\end{array}$

$\begin{array}{lll}1288.73 & -0.7326 & 0.000001\end{array}$

$\begin{array}{llll}1323.67 & -0.7664 & 0.000001 & 0.587\end{array}$

$\begin{array}{llll}1333.28 & -0.7816 & 0.000001 & 0.611\end{array}$

$\begin{array}{lll}1367.69 & -0.4919 & 0.000001\end{array}$

$\begin{array}{llll}1379.94 & -0.4415 & 0.000000 & 0.195\end{array}$

$\begin{array}{lllll}1413.75 & 0.1463 & 0.000000 & 0.021\end{array}$

$0.4147 \quad 0.000000$

$1463.05 \quad 0.9499-0.000001 \quad 0.902$

$\begin{array}{llll}1480.30 & 1.2951 & -0.000001 & 1.677\end{array}$

$\begin{array}{llll}1513.52 & 1.5790 & -0.000001 & 2.493\end{array}$

$\begin{array}{llll}1534.41 & 1.6864 & -0.000001 & 2.844\end{array}$

$\begin{array}{llll}1563.01 & -1.5059 & 0.000001 & 2.268\end{array}$

$1591.45 \quad 1.0509-0.000001 \quad 1.104$

$\begin{array}{llll}1618.50 & 0.2999 & 0.000000 & 0.090\end{array}$

$\begin{array}{llll}1677.49 & -3.3886 & 0.000002 & 11.482\end{array}$
995.6473

995.6473

995.6473

995.6473

994.0319

994.0319

994.0319

992.2158

992.2158

992.2158

992.2158

992.2158

992.2158

992.2158

992.2158

992.2158

992.2158

992.2158

992.2158

992.2158

992.2158

992.2158

992.2158

992.2158

992.2158

992.2158

992.2158

992.2158

992.2158

992.2158

992.2158

992.2158

992.2158

992.2158

992.2158

992.2158

992.2158

9922158

992.2158

992.2158

9922158

992.2158 


\begin{tabular}{|c|c|c|c|c|}
\hline molality & molarity & $\mathrm{t} /{ }^{\circ} \mathrm{C}$ & ass frac & $\begin{array}{c}\text { Density } \\
\exp \\
\mathrm{kg} / \mathrm{m}^{3}\end{array}$ \\
\hline 3.0991 & & 45 & 0.2331 & 1153.08 \\
\hline 5.5776 & & 45 & 0.3536 & 1245.31 \\
\hline 7.0913 & & 45 & 0.4102 & 1294.82 \\
\hline & & 50 & 0.0100 & 994.40 \\
\hline & & 50 & 0.0200 & 1000.60 \\
\hline & & 50 & 0.0300 & 1006.70 \\
\hline & & 50 & 0.0393 & 1012.60 \\
\hline & & 50 & 0.0400 & 1012.90 \\
\hline & & 50 & 0.0500 & 1019.20 \\
\hline & & 50 & 0.0700 & 1032.10 \\
\hline & & 50 & 0.1000 & 1051.70 \\
\hline & & 50 & 0.1000 & 1051.70 \\
\hline & & 50 & 0.1456 & 1082.70 \\
\hline & & 50 & 0.1500 & 1085.70 \\
\hline & & 50 & 0.1980 & 1119.30 \\
\hline & & 50 & 0.2000 & 1121.50 \\
\hline & & 50 & 0.2500 & 1158.80 \\
\hline & & 50 & 0.2531 & 1161.10 \\
\hline & & 50 & 0.2947 & 1193.50 \\
\hline & & 50 & 0.3000 & 1197.70 \\
\hline & & 50 & 0.3428 & 1232.20 \\
\hline & & 50 & 0.3500 & 1238.30 \\
\hline & & 50 & 0.3910 & 1272.90 \\
\hline & & 50 & 0.4000 & 1280.60 \\
\hline & & 50 & 0.4394 & 1315.50 \\
\hline & & 50 & 0.4500 & 1325.10 \\
\hline & & 50 & 0.5000 & 1371.90 \\
\hline & & 50 & 0.5348 & 1406.10 \\
\hline & & 50 & 0.5500 & 1421.40 \\
\hline & & 50 & 0.5835 & 1456.00 \\
\hline & & 50 & 0.6000 & 1473.50 \\
\hline & & 50 & 0.6310 & 1506.90 \\
\hline & & 50 & 0.6500 & 1527.70 \\
\hline & & 50 & 0.6754 & 1555.90 \\
\hline & & 50 & 0.7000 & 1583.80 \\
\hline & & 50 & 0.7228 & 1610.00 \\
\hline & & 60 & 0.0100 & 989.50 \\
\hline & & 60 & 0.0200 & 995.60 \\
\hline & & 60 & 0.0300 & 1001.70 \\
\hline & & 60 & 0.0400 & 1007.80 \\
\hline & & 60 & 0.0500 & 1014.00 \\
\hline & & 60 & 0.1000 & 1046.00 \\
\hline
\end{tabular}

Ref

$$
\begin{array}{rr}
\text { App vol } & \text { App vol } \\
\exp / & \text { Calc / } \\
\mathrm{m}^{3} / \mathrm{kg} & \mathrm{m}^{3} / \mathrm{kg}
\end{array}
$$

$\begin{array}{ll}0.000398 & 0.000407\end{array}$

$\begin{array}{ll}0.000425 & 0.000425\end{array}$

$\begin{array}{ll}0.000431 & 0.000432\end{array}$

$0.000364 \quad 0.000376$

$\begin{array}{lll}0.000377 & 0.000378\end{array}$

$0.000387 \quad 0.000379$

$\begin{array}{lll}0.000387 & 0.000381\end{array}$

$\begin{array}{ll}0.000391 & 0.000381\end{array}$

$\begin{array}{lll}0.000393 & 0.000383\end{array}$

$0.000395 \quad 0.000386$

$\begin{array}{lll}0.000399 & 0.000391\end{array}$

$0.000399 \quad 0.000391$

$\begin{array}{lll}0.000404 & 0.000398\end{array}$

0.0004050 .000399

$0.000413 \quad 0.000406$

$0.000410 \quad 0.000406$

$0.000416 \quad 0.000413$

$\begin{array}{ll}0.000416 & 0.000414\end{array}$

$0.000421 \quad 0.000420$

$0.000422 \quad 0.000420$

$0.000427 \quad 0.000426$

$0.000428 \quad 0.000427$

$\begin{array}{ll}0.000433 & 0.000432\end{array}$

$0.000434 \quad 0.000433$

$0.000439 \quad 0.000438$

$\begin{array}{ll}0.000440 & 0.000440\end{array}$

$0.000446 \quad 0.000446$

0.0004490 .000450

$\begin{array}{ll}0.000451 & 0.000452\end{array}$

$0.000455 \quad 0.000455$

$0.000456 \quad 0.000457$

$\begin{array}{lll}0.000460 & 0.000461\end{array}$

0.0004620 .000463

$0.000465 \quad 0.000465$

$0.000468 \quad 0.000468$

$0.000471 \quad 0.000470$

$\begin{array}{ll}0.000369 & 0.000385\end{array}$

$0.000384 \quad 0.000387$

$\begin{array}{ll}0.000391 & 0.000388\end{array}$

$0.000396 \quad 0.000390$

$0.000399 \quad 0.000391$

$\begin{array}{lll}(90) & 0.000406 & 0.000399\end{array}$
Density Density App Vol Square of Inconsistent Inconsistent Density of calc / Residual / Residual / Density based on based on water $/ \mathrm{kg} / \mathrm{m}^{3}$ $\mathrm{kg} / \mathrm{m}^{3} \quad \mathrm{~kg} / \mathrm{m}^{3} \quad \mathrm{~m}^{3} / \mathrm{kg}$ Residual density apparent

$1150.16 \quad 2.9173 \quad-0.000009 \quad 8.510$

$\begin{array}{llll}1245.42 & -0.1139 & 0.000000 & 0.013\end{array}$

$1293.77 \quad 1.0527-0.000002 \quad 1.108$

$994.28 \quad 0.1152-0.000012 \quad 0.013$

$1000.58 \quad 0.0211-0.000001 \quad 0.000$

$\begin{array}{llll}1006.92 & -0.2193 & 0.000007 & 0.048\end{array}$

$1012.85-0.2516 \quad 0.000006-0.063$

$\begin{array}{llll}1013.31 & -0.4066 & 0.000010 & 0.165\end{array}$

$1019.74 \quad-0.5415 \quad 0.000010 \quad 0.293$

$\begin{array}{llll}1032.76 & -0.6565 & 0.000009 & 0.431\end{array}$

$\begin{array}{llll}1052.65 & -0.9528 & 0.000009 & 0.908\end{array}$

$\begin{array}{llll}1052.65 & -0.9528 & 0.000009 & 0.908\end{array}$

$\begin{array}{llll}1083.79 & -1.0939 & 0.000006 & 1.197\end{array}$

$\begin{array}{llll}1086.86 & -1.1583 & 0.000007 & 1.342\end{array}$

$\begin{array}{llll}1121.00 & -1.7020 & 0.000007 & 2.897\end{array}$

$1122.45 \quad-0.9538 \quad 0.000004 \quad 0.910$

$\begin{array}{llll}1159.54 & -0.7440 & 0.000002 & 0.554\end{array}$

$\begin{array}{llll}1161.90 & -0.7954 & 0.000002 & 0.633\end{array}$

$\begin{array}{llll}1194.06 & -0.5618 & 0.000001 & 0.316\end{array}$

$\begin{array}{llll}1198.24 & -0.5439 & 0.000001 & 0.296\end{array}$

$\begin{array}{lllll}1232.74 & -0.5446 & 0.000001 & 0.297\end{array}$

$\begin{array}{llll}1238.68 & -0.3794 & 0.000001 & 0.144\end{array}$

$\begin{array}{llll}1273.23 & -0.3285 & 0.000001 & 0.108\end{array}$

$\begin{array}{llll}1280.99 & -0.3892 & 0.000001 & 0.152\end{array}$

$\begin{array}{llll}1315.75 & -0.2495 & 0.000000 & 0.062\end{array}$

$\begin{array}{llll}1325.33 & -0.2266 & 0.000000 & 0.051\end{array}$

$\begin{array}{llll}1371.86 & 0.0392 & 0.000000 & 0.002\end{array}$

$\begin{array}{llll}1405.64 & 0.4559 & 0.000000 & 0.208\end{array}$

$1420.78 \quad 0.6201-0.000001 \quad 0.384$

$1454.99 \quad 1.0068-0.000001 \quad 1.014$

$1472.29-1.2067-0.000001 \quad 1.456$

$1505.64 \quad 1.2643-0.000001 \quad 1.598$

$1526.63 \quad 1.0655-0.000001 \quad 1.135$

$0.4944 \quad 0.000000$

$\begin{array}{llll}1584.07 & -0.2654 & 0.000000 & 0.070\end{array}$

$\begin{array}{llll}1611.36 & -1.3582 & 0.000001 & 1.845\end{array}$

$989.35-0.1548-0.000016$

$\begin{array}{lllll}995.54 & 0.0619 & -0.000003 & 0.004\end{array}$

$\begin{array}{llll}1001.78 & -0.0781 & 0.000003 & 0.006\end{array}$

$\begin{array}{llll}1008.07 & -0.2660 & 0.000007 & 0.071\end{array}$

$\begin{array}{llll}1014.40 & -0.4024 & 0.000008 & 0.162\end{array}$

$\begin{array}{lll}-0.8377 & 0.000008 & 0.702\end{array}$
990.2132

990.2132

990.2132

988.0363

988.0363

988.0363

988.0363

988.0363

988.0363

988.0363

988.0363

988.0363

988.0363

988.0363

988.0363

988.0363

988.0363

988.0363

988.0363

988.0363

988.0363

988.0363

988.0363

988.0363

988.0363

988.0363

988.0363

988.0363

988.0363

988.0363

988.0363

988.0363

988.0363

988.0363

988.0363

988.0363

983.1989

983.1989

983.1989

983.1989

983.1989

983.1989 
molality molarity

$\begin{array}{rrr}\text { t } /{ }^{\circ} \mathrm{C} \text { mass frac } & \text { Density } \\ & & \text { exp / } \\ \mathrm{kg} / \mathrm{m}^{3}\end{array}$

Ref

$$
\begin{array}{rr}
\text { App vol } & \text { App vol } \\
\exp / & \text { Calc / } \\
\mathrm{m}^{3} / \mathrm{kg} & \mathrm{m}^{3} / \mathrm{kg}
\end{array}
$$

$\begin{array}{ll}0.000410 & 0.000406\end{array}$

$0.000415 \quad 0.000413$

$\begin{array}{ll}0.000420 & 0.000419\end{array}$

$\begin{array}{ll}0.000426 & 0.000426\end{array}$

$0.000432 \quad 0.000432$

$0.000438 \quad 0.000438$

$0.000443 \quad 0.000444$

0.0004490 .000449

$0.000454 \quad 0.000455$

$0.000459 \quad 0.000460$

$0.000465 \quad 0.000465$

$\begin{array}{ll}0.000471 & 0.000470\end{array}$

$\begin{array}{ll}0.000387 & 0.000400\end{array}$

$0.000396 \quad 0.000402$

$0.000398 \quad 0.000403$

$\begin{array}{lll}0.000401 & 0.000404\end{array}$

$0.000405 \quad 0.000406$

$\begin{array}{ll}0.000412 & 0.000412\end{array}$

$0.000416 \quad 0.000418$

$\begin{array}{ll}0.000421 & 0.000423\end{array}$

$0.000425 \quad 0.000429$

$0.000431 \quad 0.000434$

$0.000437 \quad 0.000439$

0.0004420 .000444

$0.000448 \quad 0.000449$

$\begin{array}{lll}0.000453 & 0.000454\end{array}$

$\begin{array}{lll}0.000458 & 0.000458\end{array}$

$0.000464 \quad 0.000463$

$0.000469 \quad 0.000467$

$\begin{array}{ll}0.000380 & 0.000412\end{array}$

$0.000391 \quad 0.000413$

$0.000394 \quad 0.000414$

$0.000397 \quad 0.000415$

$\begin{array}{lll}0.000401 & 0.000416\end{array}$

$0.000409 \quad 0.000421$

$0.000414 \quad 0.000426$

$0.000420 \quad 0.000431$

$0.000425 \quad 0.000435$

$0.000431 \quad 0.000440$

$0.000438 \quad 0.000444$

$0.000444 \quad 0.000448$

$\begin{array}{lll}(90) & 0.000444 & 0.000448 \\ (90) & 0.000449 & 0.000452\end{array}$ calc / Residual / Residual / Density based on based on water $/ \mathrm{kg} / \mathrm{m}^{3}$ $\mathrm{kg} / \mathrm{m}^{3} \quad \mathrm{~kg} / \mathrm{m}^{3} \quad \mathrm{~m}^{3} / \mathrm{kg}$ Residual density apparent

$\begin{array}{llll}0.60 & -0.7978 & 0.000005 & 0.636\end{array}$

$\begin{array}{llll}1115.78 & -0.4844 & 0.000002 & 0.235\end{array}$

$\begin{array}{llll}1152.51 & -0.2091 & 0.000001 & 0.044\end{array}$

$\begin{array}{llll}1190.89 & 0.0059 & 0.000000 & 0.000\end{array}$

$\begin{array}{llll}1231.07 & 0.0254 & 0.000000 & 0.001\end{array}$

$\begin{array}{llll}1273.20 & 0.0007 & 0.000000 & 0.000\end{array}$

$\begin{array}{llll}1317.43 & 0.2667 & 0.000000 & 0.071\end{array}$

$\begin{array}{llll}1363.96 & 0.4401 & 0.000000 & 0.194\end{array}$

$\begin{array}{llll}1412.98 & 0.7167 & -0.000001 & 0.514\end{array}$

$\begin{array}{llll}1464.73 & 0.8681 & -0.000001 & 0.754\end{array}$

$\begin{array}{llll}1519.46 & 0.0384 & 0.000000 & 0.001\end{array}$

$\begin{array}{llll}1577.46 & -2.1606 & 0.000001 & 4.668\end{array}$

$\begin{array}{llll}977.77 & 0.1295 & -0.000014 & 0.017\end{array}$

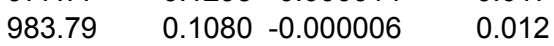

$\begin{array}{llll}989.86 & 0.1370 & -0.000005 & 0.019\end{array}$

$\begin{array}{llll}995.98 & 0.1158 & -0.000003 & 0.013\end{array}$

$\begin{array}{llll}1002.16 & 0.0436 & -0.000001 & 0.002\end{array}$

$\begin{array}{llll}1033.81 & -0.0103 & 0.000000 & 0.000\end{array}$

$\begin{array}{llll}1066.86 & 0.2366 & -0.000001 & 0.056\end{array}$

$1101.43 \quad 0.6702-0.000003 \quad 0.449$

$\begin{array}{lllll}1137.64 & 1.1646 & -0.000004 & 1.356\end{array}$

$\begin{array}{llll}1175.62 & 1.4807 & -0.000004 & 2.193\end{array}$

$\begin{array}{llll}1215.54 & 1.3644 & -0.000003 & 1.862\end{array}$

$\begin{array}{llll}1257.56 & 1.3442 & -0.000002 & 1.807\end{array}$

$\begin{array}{llll}1301.87 & 1.0288 & -0.000001 & 1.058\end{array}$

$\begin{array}{llll}1348.70 & 0.7038 & -0.000001 & 0.495\end{array}$

$\begin{array}{llll}1398.27 & 0.1288 & 0.000000 & 0.017\end{array}$

$\begin{array}{llll}1450.87 & -1.1678 & 0.000001 & 1.364\end{array}$

$\begin{array}{llll}1506.79 & -3.6931 & 0.000003 & 13.639\end{array}$

$\begin{array}{llll}964.20 & 0.3000 & -0.000032 & 0.090\end{array}$

$\begin{array}{llll}970.09 & 0.4122 & -0.000022 & 0.170\end{array}$

$\begin{array}{llll}976.03 & 0.5721 & -0.000020 & 0.327\end{array}$

$\begin{array}{llll}982.02 & 0.6790 & -0.000018 & 0.461\end{array}$

$\begin{array}{llll}988.07 & 0.7319 & -0.000015 & 0.536\end{array}$

$1019.14 \quad 1.2565-0.000012 \quad 1.579$

$\begin{array}{llll}1051.71 & 1.9947 & -0.000012 & 3.979\end{array}$

$1085.88 \quad 2.6189-0.000011 \quad 6.858$

$\begin{array}{llll}1121.81 & 3.1874 & -0.000010 & 10.159\end{array}$

$\begin{array}{llll}1159.66 & 3.3428 & -0.000008 & 11.174\end{array}$

$\begin{array}{llll}1199.59 & 3.1093 & -0.000006 & 9.668\end{array}$

$\begin{array}{llll}1241.81 & 2.7902 & -0.000005 & 7.785\end{array}$

$\begin{array}{lll}2.0640 & -0.000003 & 4.260\end{array}$
983.1989

983.1989

983.1989

983.1989

983.1989

983.1989

983.1989

983.1989

983.1989

983.1989

983.1989

983.1989

971.7978

971.7978

971.7978

971.7978

971.7978

971.7978

971.7978

971.7978

971.7978

971.7978

971.7978

971.7978

971.7978

971.7978

971.7978

971.7978

971.7978

958.3637

958.3637

958.3637

958.3637

958.3637

958.3637

958.3637

958.3637

958.3637

958.3637

958.3637

958.3637

958.3637 
molality molarity

$\begin{array}{lrrr}\text { t } /{ }^{\circ} \mathrm{C} \text { mass frac } & \begin{array}{r}\text { Density } \\ \exp / \\ \mathrm{kg} / \mathrm{m}^{3}\end{array} & \text { Ref } \\ 100 & 0.5000 & 1334.80 & (90) \\ 100 & 0.5500 & 1383.40 & (90) \\ 100 & 0.6000 & 1434.40 & (90)\end{array}$

Ref

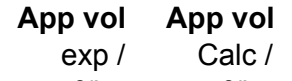

exp / Calc /

$\mathrm{m}^{3} / \mathrm{kg}$

$\mathrm{m}^{3} / \mathrm{kg}$

0.0004550 .000456

0.0004610 .000459

$0.000466 \quad 0.000463$

Average Res
Std dev Res

Avg - 4std

Avg + 4std

\section{Curve fit for the model App vol $=\left(w+c 2+c 3^{*} t{ }^{\circ} \mathrm{C}\right) /\left(\mathrm{c} 0^{*} \mathrm{w}+\mathrm{c} 1\right) / \mathrm{e}^{\wedge}\left(0.000001^{*}\left(\mathrm{t}{ }^{\circ} \mathrm{C}+\mathrm{c} 4\right)^{\wedge} 2\right)$}

c0

c1

c2

c3

\section{Min T}

$\operatorname{Max} T$

Min w

Max w 89.891385

224.47991

0.8228534

0.0068422

1571.49

Avg dens

Std dens res

Std app vol res *10^3 0.0663563

No of points in corr

No of inconsistent poin

332
6

Inconsistent data not used (Residual greater than average $+/$ - 4 standard deviations)

$\begin{array}{rrllllllllll}20 & 0.6500 & 1557.80 & (90) & 0.000448 & 0.000452 & 1551.82 & 5.9754 & -0.000004 & 35.706 & ! & 998.2041 \\ 20 & 0.7000 & 1615.10 & (90) & 0.000455 & 0.000459 & 1608.66 & 6.4428 & -0.000004 & 41.510 & ! & 9 \\ 50 & 0.7706 & 1664.70 & (35) & 0.000478 & 0.000475 & 1671.00 & -6.2962 & 0.000003 & 39.643 & ! & 9.2041 \\ 80 & 0.7000 & 1558.20 & (90) & 0.000476 & 0.000471 & 1566.40 & -8.1964 & 0.000005 & 67.181 & ! & 988.0363 \\ 100 & 0.6500 & 1487.30 & (90) & 0.000473 & 0.000467 & 1496.04 & -8.7402 & 0.000006 & 76.392 & ! & 971.7978 \\ 100 & 0.7000 & 1541.70 & (80) & 0.000479 & 0.000470 & 1557.81 & -16.1068 & 0.000010 & 259.429 & ! & 958.3637 \\ & & & & & & & 958.3637\end{array}$


Density of aqueous solutions of $\mathrm{KCl}$

References (20) Dedick, Eugene A.; Hershey, J. Peter; Sotolongo, Sara; Stade, David J.; Millero, Frank J. The PVT Properties of Concentrated Aqueous Electrolytes IX. The Volume Properties of $\mathrm{KCl}$ and $\mathrm{K} 2 \mathrm{SO} 4$ and their Mixtures with $\mathrm{NaCl}$ and $\mathrm{Na} 2 \mathrm{SO} 4$ as a Function of Temperature, J. Solution Chem. 1990, 19, 353-374

(28) Gates, J. A.; Wood, R. H. Densities of Aqueous Solutions of $\mathrm{NaCl}, \mathrm{MgCl} 2, \mathrm{KCl}, \mathrm{NaBr}, \mathrm{LiCl}$, and $\mathrm{CaCl} 2$ from 0.05 to $5.0 \mathrm{~mol} \mathrm{~kg}-1$ and 0.1013 to $40 \mathrm{MPa}$ at 298.15K. J. Chem. Eng. Data 1985, 30, 44-49.

(31) Gonçalves, F. Kestin, J. The Viscosity of $\mathrm{NaCl}$ and $\mathrm{KCl}$ solutions in the range $25-50{ }^{\circ} \mathrm{C}$, Ber. Bunsenges. Phys. Chem . 1977, 81, 11561161

(32) Gucker, F. T.; Stubley, D.; Hill, D. J. The Isentropic Compressibilities of Aqueous Solutions of Some Alkali Halides at 298.15K. J. Chem. Thermodynamics 1975, 7, 865-869.

(44) Isono, T. Measurements of Density, Viscosity, and Electrolytic Conductivity of Concentrated Aqueous Electrolyte Solutions. Rikagaku Kenkyusho Hokoku 1980, 56, 103-114.

(49) Kaminsky, M, Concentration and Temperature Dependence of the Viscosity of Aqueous Solutions of Strong Electrolytes. III. KCl, K2SO4, MgCl2, BeSO4, and MgSO4 Solutions. Z. Phys. Chem. N. F. 1957, 12, 206-231.

(52) Korosi, A.; Fabuss, B. M. Viscosities of Binary Aqueous Solutions of $\mathrm{NaCl}, \mathrm{KCl}, \mathrm{Na} 2 \mathrm{SO} 4$, and $\mathrm{MgSO} 4$ at Concentrations and Temperatures of Interest in Desalination Processes. J. Chem. Eng. Data 1968, 13, 548-552.

(62) Lengyel, S.; Tamás, J; Giber, J.; Holderith, J. Study of Viscosity of Aqueous Alkali Halide Solutions. Acta Chim. Acad. Sci. Hung. 1964, 40, 125-143.

(65) Maclnnes, D. A.; Dayhoff, M. O. The Partial Molal Volumes of Potassium Chloride, Potassium and Sodium lodides and lodine in Aqueous Solution at $25^{\circ} \mathrm{C}$. J. Am. Chem. Soc. 1952, 74, 1017-1020.

(73) Millero, F. J.; Ward, G. K.; Chetirkin, P. V. Relative Sound Velocities of Sea Salts at 25². J. Acoust. Soc. Am. 1977, 61, 1492-1498.

(79) Olofsson, Inger V.; Apparent molar heat capacities and volumes of aqueous $\mathrm{NaCl}, \mathrm{KCl}$, and $\mathrm{KNO}_{3}$ at $298.15 \mathrm{~K}$. Comparison of Picker flow calorimeter with other calorimeters, J. Chem. Thermodynamics, 1979, 11, 1005-1014

(80) Out, D. J. P.; Los, J. M. Viscosity of Aqueous Solutions of Univalent Electrolytes from 5 to $95^{\circ} \mathrm{C}$. J. Sol. Chem. 1980, 9, 19-35.

(81) Patterson, B. A.; Call, T. G.; Jardine, J. J.; Origlia-Luster, M. L.; Woolley, E. M.; Thermodynamics for lonization of Water at Temperatures from 278.15 K to 393.15 K and at the Pressure 0.35 MPa: Apparent Molar Volumes of Aqueous $\mathrm{KCl}, \mathrm{KOH}$, and $\mathrm{NaOH}$ and Apparent Molar Heat Capacities of Aqueous $\mathrm{HCl}, \mathrm{KCl}, \mathrm{KOH}$, and $\mathrm{NaOH}, \mathrm{J}$. Chem. Thermodynamics, 2001, 33,1237-1262

(90) Perry, R. H.; Green, D. W. Perry's Chemical Engineers' Handbook; McGraw Hill: New York, 7th edition, 1997.

(114) Saluja, P. P. S.; Lemire, R. J.; LeBlanc, J. C.; High-Temperature Thermodynamics of Aqueous Alkali-Metal Salts; J. Chem. Thermodynamics, 1992, 24, 181-203

(135) Zhang, H.-L.; Han, S-J. Viscosity and Density of Water + Sodium Chloride + Potassium Chloride Solutions at 298.15K. J. Chem. Eng. Data 1996, 41, 516-520

$\begin{array}{rrrrrrr}\text { molality molarity } \quad \mathbf{t} /{ }^{\circ} \mathrm{C} \text { mass frac } & \begin{array}{r}\text { Density } \\ \text { exp } / \\ \mathrm{kg} / \mathrm{m}^{3}\end{array} & \begin{array}{r}\text { Ref } \\ \end{array} & \begin{array}{r}\text { App vol } \\ \text { exp } / \\ \mathrm{m}^{3} / \mathrm{kg}\end{array} & \begin{array}{r}\text { App vol } \\ \mathrm{Calc} / \\ \mathrm{m}^{3} / \mathrm{kg}\end{array} \\ & 0 & 0.0100 & 1006.61 & (90) & 0.000327 & 0.000351 \\ & 0 & 0.0200 & 1013.35 & (90) & 0.000333 & 0.000353 \\ & 0 & 0.0400 & 1026.90 & (90) & 0.000341 & 0.000357 \\ & 0 & 0.0800 & 1054.31 & (90) & 0.000354 & 0.000364 \\ 0.01501 & 0 & 0.1200 & 1082.22 & (90) & 0.000366 & 0.000372 \\ 0.03002 & 0 & 0.1600 & 1110.68 & (90) & 0.000376 & 0.000379 \\ 0.04 & 0 & 0.2000 & 1139.73 & (90) & 0.000386 & 0.000387 \\ 0.06004 & 5 & 0.0011 & 1000.77 & (81) & 0.000283 & 0.000356 \\ 0.09 & 5 & 0.0022 & 1001.48 & (81) & 0.000320 & 0.000356 \\ & 5 & 0.0030 & 1001.93 & (80) & 0.000340 & 0.000356 \\ & 5 & 0.0045 & 1002.94 & (81) & 0.000335 & 0.000356 \\ & 5 & 0.0067 & 1004.38 & (80) & 0.000340 & 0.000357\end{array}$

\begin{tabular}{|c|c|c|c|c|c|}
\hline $\begin{array}{r}\text { Density } \\
\text { calc/ } \\
\mathrm{kg} / \mathrm{m}^{3}\end{array}$ & $\begin{array}{r}\text { Density } \\
\text { Residual / } \\
\mathrm{kg} / \mathrm{m}^{3}\end{array}$ & $\begin{array}{r}\text { App Vol } \\
\text { Residual / } \\
\mathrm{m}^{3} / \mathrm{kg}\end{array}$ & $\begin{array}{r}\text { Square of } \\
\text { Density } \\
\text { Res }\end{array}$ & $\begin{array}{l}\text { Inconsistent } \\
\text { based on } \\
\text { density }\end{array}$ & $\begin{array}{c}\text { Inconsistent } \\
\text { based on } \\
\text { apparent } \\
\text { volume }\end{array}$ \\
\hline 1006.4 & 0.2382 & -0.000024 & 0.057 & & \\
\hline 1013.0 & 0.3980 & -0.000019 & 0.158 & & \\
\hline 1026.3 & 0.6438 & -0.000015 & 0.414 & & \\
\hline 1053.4 & 0.8683 & -0.000010 & 0.754 & & \\
\hline 1081.4 & 0.8235 & -0.000006 & 0.678 & & \\
\hline 1110.1 & 0.5627 & -0.000003 & 0.317 & & \\
\hline 1139.6 & 0.1345 & -0.000001 & 0.018 & & \\
\hline 1000.7 & 0.0814 & -0.000073 & 0.007 & & \\
\hline 1001.4 & 0.0798 & -0.000036 & 0.006 & & \\
\hline 1001.9 & 0.0478 & -0.000016 & 0.002 & & \\
\hline 1002.8 & 0.0959 & -0.000021 & 0.009 & & \\
\hline 1004.3 & 0.1102 & -0.000016 & 0.012 & & \\
\hline
\end{tabular}

(Dens rel- Delta density Apparent Density of $\mathrm{cm}^{3} / \mathrm{mol}$

999.8395 999.8395
999.8395 999.8395 999.8395 999.8395 999.8395
999.8395 999.8395
999.8395 $21.1 \quad 999.9638$ $23.88 \quad 999.9638$ 999.9638 $24.97 \quad 999.9638$ 999.9638 


\begin{tabular}{|c|c|c|c|c|}
\hline molality & molarity & \multicolumn{2}{|c|}{$\mathrm{t} /{ }^{\circ} \mathrm{C}$ mass frac } & $\begin{array}{r}\text { Density } \\
\exp / \\
\mathrm{kg} / \mathrm{m}^{3}\end{array}$ \\
\hline 0.10001 & & 5 & 0.0074 & 1004.85 \\
\hline 0.12006 & & 5 & 0.0089 & 1005.86 \\
\hline 0.16 & & 5 & 0.0118 & 1007.77 \\
\hline 0.19998 & & 5 & 0.0147 & 1009.69 \\
\hline 0.25 & & 5 & 0.0183 & 1012.10 \\
\hline 0.25017 & & 5 & 0.0183 & 1012.10 \\
\hline 0.35002 & & 5 & 0.0254 & 1016.76 \\
\hline 0.4 & & 5 & 0.0290 & 1019.19 \\
\hline 0.45253 & & 5 & 0.0326 & 1021.65 \\
\hline 0.49966 & & 5 & 0.0359 & 1023.71 \\
\hline 0.49992 & & 5 & 0.0359 & 1023.76 \\
\hline 0.7 & & 5 & 0.0496 & 1033.00 \\
\hline 0.93692 & & 5 & 0.0653 & 1043.53 \\
\hline 1 & & 5 & 0.0694 & 1046.34 \\
\hline 1.39606 & & 5 & 0.0943 & 1063.27 \\
\hline 1.91739 & & 5 & 0.1251 & 1084.56 \\
\hline 2.39492 & & 5 & 0.1515 & 1103.19 \\
\hline 2.96122 & & 5 & 0.1808 & 1124.28 \\
\hline 3.44657 & & 5 & 0.2044 & 1141.52 \\
\hline 4.05968 & & 5 & 0.2323 & 1162.37 \\
\hline 0.5041 & & 10 & 0.0362 & 1023.50 \\
\hline 0.991 & & 10 & 0.0688 & 1045.10 \\
\hline 2.0476 & & 10 & 0.1324 & 1088.40 \\
\hline 2.4968 & & 10 & 0.1569 & 1105.60 \\
\hline 3.0013 & & 10 & 0.1828 & 1124.10 \\
\hline 3.5327 & & 10 & 0.2085 & 1142.90 \\
\hline 3.9618 & & 10 & 0.2280 & 1157.50 \\
\hline & 0.001 & 12.5 & 0.0001 & 999.49 \\
\hline & 0.0025 & 12.5 & 0.0002 & 999.57 \\
\hline & 0.005 & 12.5 & 0.0004 & 999.69 \\
\hline & 0.01 & 12.5 & 0.0007 & 999.93 \\
\hline & 0.02001 & 12.5 & 0.0015 & 1000.43 \\
\hline & 0.04003 & 12.5 & 0.0030 & 1001.42 \\
\hline & 0.05005 & 12.5 & 0.0037 & 1001.91 \\
\hline & 0.10009 & 12.5 & 0.0074 & 1004.35 \\
\hline & 0.20019 & 12.5 & 0.0148 & 1009.16 \\
\hline & 0.50052 & 12.5 & 0.0365 & 1023.39 \\
\hline & 0.001 & 15 & 0.0001 & 999.15 \\
\hline & 0.0025 & 15 & 0.0002 & 999.22 \\
\hline & 0.005 & 15 & 0.0004 & 999.35 \\
\hline & 0.01 & 15 & 0.0007 & 999.59 \\
\hline 0.01501 & & 15 & 0.0011 & 999.87 \\
\hline & 0.02001 & 15 & 0.0015 & 1000.08 \\
\hline 0.03002 & & 15 & 0.0022 & 1000.57 \\
\hline 0.04 & & 15 & 0.0030 & 1001.03 \\
\hline & 0.04003 & 15 & 0.0030 & 1001.06 \\
\hline 0.05 & & 15 & 0.0037 & 1001.51 \\
\hline & 0.05005 & 15 & 0.0037 & 1001.55 \\
\hline
\end{tabular}

\section{App vol App vol}

$\begin{array}{cc}\exp / & \text { Calc / } \\ \mathrm{m}^{3} / \mathrm{kg} & \mathrm{m}^{3} / \mathrm{kg}\end{array}$

$$
\mathrm{m}^{3} / \mathrm{k}
$$

$\mathrm{m}^{3} / \mathrm{kg}$

$\begin{array}{lll}\text { (81) } & 0.000344 & 0.000357 \\ \text { (81) } & 0.000339 & 0.000357\end{array}$

$0.000343 \quad 0.000358$

$\begin{array}{lll}0.000344 & 0.000358\end{array}$

$\begin{array}{lll}0.000345 & 0.000359\end{array}$

$\begin{array}{lll}0.000345 & 0.000359\end{array}$

$0.000351 \quad 0.000360$

$\begin{array}{ll}0.000349 & 0.000361 \\ 0.000350 & 0.000361\end{array}$

$\begin{array}{ll}0.000350 & 0.000361 \\ 0.000354 & 0.000362\end{array}$

$\begin{array}{ll}0.000353 & 0.000362\end{array}$

$\begin{array}{ll}0.000355 & 0.000365\end{array}$

$\begin{array}{lll}0.000361 & 0.000367\end{array}$

$0.000361 \quad 0.000368$

$0.000368 \quad 0.000373$

$\begin{array}{lll}0.000376 & 0.000379\end{array}$

$\begin{array}{lll}0.000382 & 0.000383\end{array}$

$\begin{array}{lll}0.000389 & 0.000389\end{array}$

$0.000393 \quad 0.000393$

$\begin{array}{ll}0.000399 & 0.000399\end{array}$

$0.000358 \quad 0.000368$

$\begin{array}{lll}0.000369 & 0.000374\end{array}$

$0.000385 \quad 0.000385$

$0.000390 \quad 0.000390$

$0.000395 \quad 0.000395$

$\begin{array}{lll}0.000399 & 0.000399\end{array}$

$0.000402 \quad 0.000403$

$\begin{array}{lll}0.000330 & 0.000364\end{array}$

$0.000303 \quad 0.000364$

$\begin{array}{lll}0.000330 & 0.000364\end{array}$

$0.000343 \quad 0.000364$

$0.000337 \quad 0.000364$

$0.000337 \quad 0.000364$

0.0003390 .000365

$0.000343 \quad 0.000365$

$0.000349 \quad 0.000367$

$0.000358 \quad 0.000370$

$0.000330 \quad 0.000366$

$0.000357 \quad 0.000367$

$0.000330 \quad 0.000367$

$0.000344 \quad 0.000367$

$0.000315 \quad 0.000367$

$\begin{array}{ll}0.000344 & 0.000367 \\ 0.000341 & 0.000367\end{array}$

$\begin{array}{lll}0.000352 & 0.000367\end{array}$

$\begin{array}{ll}0.000344 & 0.000367\end{array}$

$0.000352 \quad 0.000367$

$0.000344 \quad 0.000367$
Density Density App Vol Square of Inconsistent Inconsistent (Dens rel- Delta density Apparent Density of calc/ Residual / Residual / Density based on based on 1$)^{\star} 10^{\wedge} 4 \quad / \mathrm{kg} / \mathrm{m}^{3}$ molal volume water $/ \mathrm{kg} / \mathrm{m}^{3}$ $\mathrm{kg} / \mathrm{m}^{3} \quad \mathrm{~kg} / \mathrm{m}^{3} \quad \mathrm{~m}^{3} / \mathrm{kg} \quad$ Res density apparent

$\begin{array}{llll}1004.7 & 0.0997 & -0.000013 & 0.010\end{array}$

$\begin{array}{llll}1005.7 & 0.1595 & -0.000018 & 0.025\end{array}$

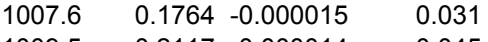

$\begin{array}{llll}1009.5 & 0.2117 & -0.000014 & 0.045\end{array}$

$\begin{array}{llll}1011.8 & 0.2652 & -0.000014 & 0.070\end{array}$

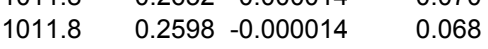

$\begin{array}{llll}1016.5 & 0.2524 & -0.000010 & 0.064\end{array}$

$\begin{array}{llll}1018.8 & 0.3665 & -0.000012 & 0.134\end{array}$

$\begin{array}{llll}1021.2 & 0.4063 & -0.000012 & 0.165\end{array}$

$\begin{array}{llll}1023.4 & 0.2981 & -0.000008 & 0.089\end{array}$

$\begin{array}{llll}1023.4 & 0.3363 & -0.000009 & 0.113\end{array}$

$\begin{array}{llll}1032.5 & 0.4930 & -0.000009 & 0.243\end{array}$

$\begin{array}{llll}1043.0 & 0.4877 & -0.000007 & 0.238\end{array}$

$\begin{array}{llll}1045.8 & 0.5310 & -0.000007 & 0.282 \\ 1062.8 & 0.4594 & -0.000004 & 0.211\end{array}$

$1103.0=0.1976-0.000001-0.039$

$\begin{array}{llll}1124.2 & 0.0866 & 0.000000 & 0.007\end{array}$

$\begin{array}{llll}1141.5 & 0.0122 & 0.000000 & 0.000\end{array}$

$\begin{array}{llll}1162.3 & 0.0268 & 0.000000 & 0.001\end{array}$

$\begin{array}{llll}1023.1 & 0.3660 & -0.000010 & 0.134\end{array}$

$\begin{array}{llll}1044.7 & 0.3711 & -0.000005 & 0.138\end{array}$

$\begin{array}{llll}1088.3 & 0.0734 & 0.000000 & 0.005\end{array}$

$\begin{array}{llll}1105.6 & -0.0035 & 0.000000 & 0.000\end{array}$

$\begin{array}{llll}1124.2 & -0.0772 & 0.000000 & 0.006\end{array}$

$\begin{array}{llll}1142.8 & 0.0572 & 0.000000 & 0.003\end{array}$

$\begin{array}{llll}1157.3 & 0.2218 & -0.000001 & 0.049\end{array}$

$\begin{array}{llll}999.5 & 0.0025 & -0.000034 & 0.000\end{array}$

$\begin{array}{llll}999.6 & 0.0113 & -0.000061 & 0.000\end{array}$

$\begin{array}{llll}999.7 & 0.0127 & -0.000034 & 0.000\end{array}$

$\begin{array}{llll}999.9 & 0.0155 & -0.000021 & 0.000\end{array}$

$\begin{array}{lllll}1000.4 & 0.0407 & -0.000027 & 0.002\end{array}$

$\begin{array}{llll}1001.3 & 0.0818 & -0.000027 & 0.007\end{array}$

$\begin{array}{llll}1001.8 & 0.0972 & -0.000026 & 0.009\end{array}$

$\begin{array}{llll}1004.2 & 0.1700 & -0.000023 & 0.029\end{array}$

$\begin{array}{llll}1008.9 & 0.2700 & -0.000018 & 0.073\end{array}$

$\begin{array}{llll}1022.9 & 0.4559 & -0.000012 & 0.208\end{array}$

$\begin{array}{llll}999.1 & 0.0027 & -0.000036 & 0.000\end{array}$

$\begin{array}{llll}999.2 & 0.0018 & -0.000009 & 0.000\end{array}$

$\begin{array}{llll}999.3 & 0.0135 & -0.000036 & 0.000\end{array}$

$\begin{array}{llll}999.6 & 0.0171 & -0.000023 & 0.000\end{array}$

$\begin{array}{llll}999.8 & 0.0575 & -0.000051 & 0.003\end{array}$

$\begin{array}{llll}1000.0 & 0.0339 & -0.000023 & 0.001 \\ 1000.5 & 0.0576 & -0.000026 & 0.003\end{array}$

$0.0454-0.000015$

$\begin{array}{llll}1001.0 & 0.0682 & -0.000023 & 0.005\end{array}$

$\begin{array}{llll}1001.5 & 0.0553 & -0.000015 & 0.003\end{array}$

$\begin{array}{llll}1001.5 & 0.0852 & -0.000023 & 0.007\end{array}$
$25.61 \quad 999.9638$

$25.3 \quad 999.9638$

999.9638

$25.65 \quad 999.9638$

999.9638

$25.72 \quad 999.9638$

$\begin{array}{cc}25.72 & 999.9638 \\ 26.134 & 999.9638\end{array}$

999.9638

21.69

$26.402 \quad 999.9638$

$26.327 \quad 999.9638$

999.9638
999.9638

999.9638

999638

$103.227 \quad 999.9638$

$124.313 \quad 999.9638$

$141.559 \quad 999.9638$

999.6996

999.6996

999.6996

999.6996

999.6996

999.6996

999.6996

999.4388

999.4388

999.4388

999.4388

999.4388

999.4388

999.4388

999.4388

999.4388

999.4388

999.0996

999.0996

999.0996

999.0996

$23.5 \quad 999.0996$

$25.43 \quad 999.0996$

999.0996

999.0996

999.0996

999.0996
$162.403 \quad 999.9638$ 


\begin{tabular}{|c|c|c|c|c|c|c|c|c|c|c|c|c|c|c|c|c|c|}
\hline molality & molarity & \multicolumn{2}{|c|}{$\mathrm{t} /{ }^{\circ} \mathrm{C}$ mass frac } & $\begin{array}{r}\text { Density } \\
\exp / \\
\mathrm{kg} / \mathrm{m}^{3}\end{array}$ & Ref & $\begin{array}{r}\text { App vol } \\
\text { exp } / \\
\mathrm{m}^{3} / \mathrm{kg}\end{array}$ & $\begin{array}{r}\text { App vol } \\
\text { Calc / } \\
\mathrm{m}^{3} / \mathrm{kg}\end{array}$ & $\begin{array}{r}\text { Density } \\
\text { calc / } \\
\mathrm{kg} / \mathrm{m}^{3}\end{array}$ & $\begin{array}{r}\text { Density } \\
\text { Residual / } \\
\mathrm{kg} / \mathrm{m}^{3}\end{array}$ & $\begin{array}{r}\text { App Vol } \\
\text { Residual / } \\
\mathrm{m}^{3} / \mathrm{kg}\end{array}$ & $\begin{array}{r}\text { Square of } \\
\text { Density } \\
\text { Res }\end{array}$ & $\begin{array}{l}\text { Inconsistent } \\
\text { based on } \\
\text { density }\end{array}$ & $\begin{array}{l}\text { Inconsistent } \\
\text { based on } \\
\text { apparent } \\
\text { volume }\end{array}$ & $\begin{array}{c}\text { (Dens rel - } \\
1)^{\star} 10^{\wedge} 4\end{array}$ & $\begin{array}{l}\text { Delta density } \\
\qquad / \mathrm{kg} / \mathrm{m}^{3} \mathrm{n}\end{array}$ & $\begin{array}{r}\text { Apparent } \\
\text { molal volume } \\
/ \mathrm{cm}^{3} / \mathrm{mol}\end{array}$ & $\begin{array}{r}\text { Density of } \\
\text { water } / \mathrm{kg} / \mathrm{m}^{3}\end{array}$ \\
\hline 0.06004 & & 15 & 0.0045 & 1001.99 & (81) & 0.000353 & 0.000367 & 1001.9 & 0.0625 & -0.000014 & 0.004 & & & & & 26.34 & 999.0996 \\
\hline 0.09 & & 15 & 0.0067 & 1003.41 & (80) & 0.000356 & 0.000368 & 1003.3 & 0.0794 & -0.000012 & 0.006 & & & & & & 999.0996 \\
\hline 0.1 & & 15 & 0.0074 & 1003.83 & (44) & 0.000364 & 0.000368 & 1003.8 & 0.0315 & -0.000004 & 0.001 & & & & & & 999.0996 \\
\hline 0.10001 & & 15 & 0.0074 & 1003.87 & (81) & 0.000359 & 0.000368 & 1003.8 & 0.0670 & -0.000009 & 0.004 & & & & & 26.75 & 999.0996 \\
\hline & 0.10009 & 15 & 0.0074 & 1003.97 & (49) & 0.000348 & 0.000368 & 1003.8 & 0.1461 & -0.000020 & 0.021 & & & 48.7 & & & 999.0996 \\
\hline 0.12006 & & 15 & 0.0089 & 1004.85 & (81) & 0.000356 & 0.000368 & 1004.7 & 0.1104 & -0.000012 & 0.012 & & & & & 26.52 & 999.0996 \\
\hline 0.16 & & 15 & 0.0118 & 1006.72 & (80) & 0.000358 & 0.000369 & 1006.6 & 0.1237 & -0.000010 & 0.015 & & & & & & 999.0996 \\
\hline 0.19998 & & 15 & 0.0147 & 1008.58 & (81) & 0.000360 & 0.000369 & 1008.5 & 0.1313 & -0.000009 & 0.017 & & & & & 26.86 & 999.0996 \\
\hline & 0.20019 & 15 & 0.0148 & 1008.75 & (49) & 0.000354 & 0.000369 & 1008.5 & 0.2319 & -0.000015 & 0.054 & & & 96.6 & & & 999.0996 \\
\hline 0.25 & & 15 & 0.0183 & 1010.94 & (80) & 0.000360 & 0.000370 & 1010.8 & 0.1769 & -0.000009 & 0.031 & & & & & & 999.0996 \\
\hline 0.25017 & & 15 & 0.0183 & 1010.92 & (81) & 0.000362 & 0.000370 & 1010.8 & 0.1472 & -0.000008 & 0.022 & & & & & 26.976 & 999.0996 \\
\hline 0.35002 & & 15 & 0.0254 & 1015.44 & (81) & 0.000368 & 0.000371 & 1015.4 & 0.0897 & -0.000003 & 0.008 & & & & & 27.401 & 999.0996 \\
\hline 0.4 & & 15 & 0.0290 & 1017.86 & (80) & 0.000364 & 0.000372 & 1017.6 & 0.2314 & -0.000008 & 0.054 & & & & & & 999.0996 \\
\hline 0.44404 & & 15 & 0.0320 & 1019.82 & (20) & 0.000366 & 0.000372 & 1019.6 & 0.1958 & -0.000006 & 0.038 & & & & 20.722 & & 999.0996 \\
\hline 0.49966 & & 15 & 0.0359 & 1022.29 & (81) & 0.000369 & 0.000373 & 1022.1 & 0.1544 & -0.000004 & 0.024 & & & & & 27.487 & 999.0996 \\
\hline 0.49992 & & 15 & 0.0359 & 1022.30 & (81) & 0.000369 & 0.000373 & 1022.1 & 0.1566 & -0.000004 & 0.025 & & & & & 27.483 & 999.0996 \\
\hline 0.5 & & 15 & 0.0359 & 1022.27 & (44) & 0.000370 & 0.000373 & 1022.2 & 0.1186 & -0.000003 & 0.014 & & & & & & 999.0996 \\
\hline & 0.50052 & 15 & 0.0365 & 1022.86 & (49) & 0.000364 & 0.000373 & 1022.5 & 0.3530 & -0.000009 & 0.125 & & & 237.8 & & & 999.0996 \\
\hline 0.7 & & 15 & 0.0496 & 1031.32 & (80) & 0.000370 & 0.000375 & 1031.1 & 0.2510 & -0.000005 & 0.063 & & & & & & 999.0996 \\
\hline 0.90026 & & 15 & 0.0629 & 1040.11 & (20) & 0.000373 & 0.000378 & 1039.8 & 0.2784 & -0.000004 & 0.078 & & & & 41.009 & & 999.0996 \\
\hline 1 & & 15 & 0.0694 & 1044.16 & (44) & 0.000378 & 0.000379 & 1044.1 & 0.0278 & 0.000000 & 0.001 & & & & & & 999.0996 \\
\hline 1 & & 15 & 0.0694 & 1044.35 & (80) & 0.000376 & 0.000379 & 1044.1 & 0.2178 & -0.000003 & 0.047 & & & & & & 999.0996 \\
\hline 1.37083 & & 15 & 0.0927 & 1059.90 & (20) & 0.000382 & 0.000383 & 1059.8 & 0.1192 & -0.000001 & 0.014 & & & & 60.796 & & 999.0996 \\
\hline 1.86235 & & 15 & 0.1219 & 1079.69 & (20) & 0.000388 & 0.000388 & 1079.7 & -0.0033 & 0.000000 & 0.000 & & & & 80.588 & & 999.0996 \\
\hline 2 & & 15 & 0.1298 & 1084.78 & (44) & 0.000392 & 0.000390 & 1085.1 & -0.3263 & 0.000002 & 0.106 & & & & & & 999.0996 \\
\hline 2.35904 & & 15 & 0.1496 & 1098.77 & (20) & 0.000394 & 0.000393 & 1098.9 & -0.1391 & 0.000001 & 0.019 & & & & 99.672 & & 999.0996 \\
\hline 2.87007 & & 15 & 0.1763 & 1117.58 & (20) & 0.000399 & 0.000398 & 1117.8 & -0.2050 & 0.000001 & 0.042 & & & & 118.484 & & 999.0996 \\
\hline 3 & & 15 & 0.1828 & 1121.97 & (44) & 0.000401 & 0.000399 & 1122.4 & -0.4793 & 0.000002 & 0.230 & & & & & & 999.0996 \\
\hline 3.40223 & & 15 & 0.2023 & 1136.30 & (20) & 0.000404 & 0.000403 & 1136.5 & -0.2359 & 0.000001 & 0.056 & & & & 137.199 & & 999.0996 \\
\hline 3.9512 & & 15 & 0.2275 & 1154.74 & (20) & 0.000408 & 0.000407 & 1155.0 & -0.2141 & 0.000001 & 0.046 & & & & 155.64 & & 999.0996 \\
\hline 4 & & 15 & 0.2297 & 1156.12 & (44) & 0.000409 & 0.000408 & 1156.5 & -0.4278 & 0.000001 & 0.183 & & & & & & 999.0996 \\
\hline 4.40156 & & 15 & 0.2471 & 1169.31 & (20) & 0.000411 & 0.000411 & 1169.4 & -0.0940 & 0.000000 & 0.009 & & & & 170.213 & & 999.0996 \\
\hline & 0.0025 & 20 & 0.0002 & 998.32 & (49) & 0.000358 & 0.000371 & 998.3 & 0.0025 & -0.000013 & 0.000 & & & 1.2 & & & 998.2041 \\
\hline & 0.005 & 20 & 0.0004 & 998.44 & (49) & 0.000358 & 0.000371 & 998.4 & 0.0049 & -0.000013 & 0.000 & & & 2.4 & & & 998.2041 \\
\hline & 0.01 & 20 & 0.0007 & 998.69 & (49) & 0.000345 & 0.000371 & 998.7 & 0.0199 & -0.000027 & 0.000 & & & 4.9 & & & 998.2041 \\
\hline & 0.02 & 20 & 0.0015 & 999.17 & (49) & 0.000351 & 0.000371 & 999.1 & 0.0299 & -0.000020 & 0.001 & & & 9.7 & & & 998.2041 \\
\hline & 0.04 & 20 & 0.0030 & 1000.14 & (49) & 0.000351 & 0.000372 & 1000.1 & 0.0607 & -0.000020 & 0.004 & & & 19.4 & & & 998.2041 \\
\hline 0.05 & & 20 & 0.0037 & 1000.57 & (44) & 0.000364 & 0.000372 & 1000.5 & 0.0289 & -0.000008 & 0.001 & & & & & & 998.2041 \\
\hline & 0.05 & 20 & 0.0037 & 1000.62 & (49) & 0.000353 & 0.000372 & 1000.5 & 0.0714 & -0.000019 & 0.005 & & & 24.2 & & & 998.2041 \\
\hline 0.0988 & & 20 & 0.0073 & 1002.90 & (62) & 0.000360 & 0.000372 & 1002.8 & 0.0888 & -0.000012 & 0.008 & & & & & & 998.2041 \\
\hline 0.1 & & 20 & 0.0074 & 1002.92 & (44) & 0.000365 & 0.000372 & 1002.9 & 0.0531 & -0.000007 & 0.003 & & & & & & 998.2041 \\
\hline & 0.1 & 20 & 0.0074 & 1003.02 & (49) & 0.000355 & 0.000372 & 1002.9 & 0.1279 & -0.000017 & 0.016 & & & 48.2 & & & 998.2041 \\
\hline & & 20 & 0.0100 & 1004.62 & (90) & 0.000362 & 0.000373 & 1004.5 & 0.1093 & -0.000011 & 0.012 & & & & & & 998.2041 \\
\hline & 0.2 & 20 & 0.0148 & 1007.75 & (49) & 0.000361 & 0.000374 & 1007.6 & 0.1954 & -0.000013 & 0.038 & & & 95.6 & & & 998.2041 \\
\hline & & 20 & 0.0200 & 1011.03 & (90) & 0.000366 & 0.000375 & 1010.9 & 0.1677 & -0.000008 & 0.028 & & & & & & 998.2041 \\
\hline 0.32974 & & 20 & 0.0240 & 1013.49 & (31) & 0.000372 & 0.000375 & 1013.4 & 0.0784 & -0.000003 & 0.006 & & & & & & 998.2041 \\
\hline 0.5 & & 20 & 0.0359 & 1021.16 & (44) & 0.000375 & 0.000377 & 1021.1 & 0.0817 & -0.000002 & 0.007 & & & & & & 998.2041 \\
\hline 0.5041 & & 20 & 0.0362 & 1021.50 & (62) & 0.000371 & 0.000377 & 1021.3 & 0.2386 & -0.000006 & 0.057 & & & & & & 998.2041 \\
\hline
\end{tabular}




\begin{tabular}{|c|c|c|c|c|c|c|c|}
\hline \multirow[t]{2}{*}{ molality } & \multirow{2}{*}{$\begin{array}{r}\text { molarity } \\
0.5\end{array}$} & \multicolumn{2}{|c|}{$\mathrm{t} /{ }^{\circ} \mathrm{C}$ mass frac } & $\begin{array}{r}\text { Density } \\
\exp / \\
\mathrm{kg} / \mathrm{m}^{3}\end{array}$ & Ref & $\begin{array}{r}\text { App vol } \\
\exp / \\
\mathrm{m}^{3} / \mathrm{kg}\end{array}$ & $\begin{array}{r}\text { App vol } \\
\text { Calc / } \\
\mathrm{m}^{3} / \mathrm{kg}\end{array}$ \\
\hline & & 20 & 0.0365 & 1021.72 & (49) & 0.000370 & 0.000377 \\
\hline \multirow[t]{2}{*}{0.51841} & & 20 & 0.0372 & 1021.96 & (31) & 0.000376 & 0.000378 \\
\hline & & 20 & 0.0400 & 1023.91 & (90) & 0.000373 & 0.000378 \\
\hline 0.991 & & 20 & 0.0688 & 1042.70 & (62) & 0.000380 & 0.000383 \\
\hline 1 & & 20 & 0.0694 & 1042.86 & (44) & 0.000384 & 0.000383 \\
\hline \multirow{2}{*}{1.02409} & & 20 & 0.0709 & 1043.89 & (31) & 0.000384 & 0.000383 \\
\hline & & 20 & 0.0800 & 1050.03 & (90) & 0.000384 & 0.000385 \\
\hline 1.5097 & & 20 & 0.1012 & 1064.10 & (62) & 0.000389 & 0.000389 \\
\hline \multirow[t]{2}{*}{1.54691} & & 20 & 0.1034 & 1065.44 & (31) & 0.000390 & 0.000389 \\
\hline & & 20 & 0.1200 & 1076.79 & (90) & 0.000393 & 0.000392 \\
\hline 2 & & 20 & 0.1298 & 1083.27 & (44) & 0.000396 & 0.000394 \\
\hline 2.0476 & & 20 & 0.1324 & 1085.30 & (62) & 0.000395 & 0.000394 \\
\hline 2.10665 & & 20 & 0.1357 & 1087.37 & (31) & 0.000397 & 0.000395 \\
\hline \multirow[t]{2}{*}{2.4968} & & 20 & 0.1569 & 1102.20 & (62) & 0.000399 & 0.000399 \\
\hline & & 20 & 0.1600 & 1104.34 & (90) & 0.000400 & 0.000399 \\
\hline 2.72517 & & 20 & 0.1689 & 1110.37 & (31) & 0.000403 & 0.000401 \\
\hline 3 & & 20 & 0.1828 & 1120.18 & (44) & 0.000405 & 0.000403 \\
\hline \multirow{2}{*}{3.0013} & & 20 & 0.1828 & 1120.50 & (62) & 0.000404 & 0.000403 \\
\hline & & 20 & 0.2000 & 1132.80 & (90) & 0.000407 & 0.000406 \\
\hline 3.42161 & & 20 & 0.2032 & 1134.90 & (31) & 0.000408 & 0.000407 \\
\hline 3.5327 & & 20 & 0.2085 & 1139.00 & (62) & 0.000408 & 0.000408 \\
\hline 3.93033 & & 20 & 0.2266 & 1151.93 & (31) & 0.000412 & 0.000411 \\
\hline 3.9618 & & 20 & 0.2280 & 1153.30 & (62) & 0.000411 & 0.000411 \\
\hline \multirow[t]{2}{*}{4} & & 20 & 0.2297 & 1154.10 & (44) & 0.000413 & 0.000412 \\
\hline & & 20 & 0.2400 & 1162.26 & (90) & 0.000413 & 0.000413 \\
\hline 4.55469 & & 20 & 0.2535 & 1171.94 & (31) & 0.000416 & 0.000416 \\
\hline 0.01013 & & 24.04 & 0.0008 & 997.99 & (114) & 0.000064 & 0.000375 \\
\hline 0.03034 & & 24.04 & 0.0023 & 998.95 & (114) & 0.000265 & 0.000375 \\
\hline 0.10983 & & 24.04 & 0.0081 & 1002.67 & (114) & 0.000340 & 0.000376 \\
\hline 0.19497 & & 24.04 & 0.0143 & 1006.62 & (114) & 0.000354 & 0.000377 \\
\hline 0.29443 & & 24.04 & 0.0215 & 1011.14 & (114) & 0.000363 & 0.000378 \\
\hline 0.41553 & & 24.04 & 0.0300 & 1016.60 & (114) & 0.000369 & 0.000380 \\
\hline \multirow[t]{6}{*}{0.57041} & & 24.04 & 0.0408 & 1023.51 & (114) & 0.000373 & 0.000381 \\
\hline & 0.0025 & 25 & 0.0002 & 997.16 & (49) & 0.000359 & 0.000375 \\
\hline & 0.00499 & 25 & 0.0004 & 997.28 & (49) & 0.000358 & 0.000375 \\
\hline & & 25 & 0.0006 & 997.49 & (65) & 0.000313 & 0.000375 \\
\hline & 0.00999 & 25 & 0.0007 & 997.52 & (49) & 0.000358 & 0.000375 \\
\hline & & 25 & 0.0010 & 997.70 & (65) & 0.000332 & 0.000375 \\
\hline \multirow[t]{3}{*}{0.01501} & & 25 & 0.0011 & 997.79 & (81) & 0.000331 & 0.000375 \\
\hline & & 25 & 0.0011 & 997.80 & (65) & 0.000335 & 0.000375 \\
\hline & 0.01998 & 25 & 0.0015 & 998.00 & (49) & 0.000358 & 0.000375 \\
\hline 0.0214 & & 25 & 0.0016 & 998.10 & (135) & 0.000337 & 0.000375 \\
\hline 0.03002 & & 25 & 0.0022 & 998.48 & (81) & 0.000355 & 0.000376 \\
\hline \multirow[t]{3}{*}{0.04} & & 25 & 0.0030 & 998.96 & (80) & 0.000356 & 0.000376 \\
\hline & 0.03995 & 25 & 0.0030 & 998.96 & (49) & 0.000358 & 0.000376 \\
\hline & 0.042077 & 25 & 0.0031 & 999.02 & (32) & 0.000370 & 0.000376 \\
\hline 0.04642 & & 25 & 0.0034 & 999.21 & (79) & 0.000371 & 0.000376 \\
\hline 0.05 & & 25 & 0.0037 & 999.38 & (44) & 0.000372 & 0.000376 \\
\hline
\end{tabular}

Density Density App Vol Square of Inconsistent Inconsistent (Dens rel- Delta density Apparent Density of Res density apparm $\begin{array}{llll}1021.9 & 0.0601 & -0.000002 & 0.004\end{array}$

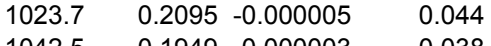

$\begin{array}{llll}1042.9 & -0.0286 & 0.000000 & 0.001\end{array}$

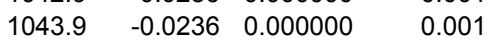

$1049.9 \quad 0.1115-0.000001 \quad 0.012$

$\begin{array}{llll}1064.1 & 0.0139 & 0.000000 & 0.000\end{array}$

$\begin{array}{llll}1065.6 & -0.1542 & 0.000001 & 0.024\end{array}$

$\begin{array}{llll}1076.9 & -0.0694 & 0.000000 & 0.005\end{array}$

$\begin{array}{llll}1083.5 & -0.2734 & 0.000002 & 0.075\end{array}$

$\begin{array}{llll}1085.4 & -0.0855 & 0.000001 & 0.007\end{array}$

$\begin{array}{llll}1087.7 & -0.2895 & 0.000002 & 0.084\end{array}$

$\begin{array}{llll}1102.4 & -0.1761 & 0.000001 & 0.031\end{array}$

$\begin{array}{llll}1104.5 & -0.1806 & 0.000001 & 0.033\end{array}$

$\begin{array}{llll}1110.7 & -0.3785 & 0.000002 & 0.143\end{array}$

$\begin{array}{llll}1120.6 & -0.4152 & 0.000002 & 0.172\end{array}$

$\begin{array}{llll}1120.6 & -0.1412 & 0.000001 & 0.020\end{array}$

$\begin{array}{llll}1132.9 & -0.0954 & 0.000000 & 0.009\end{array}$

$\begin{array}{llll}1135.2 & -0.3316 & 0.000001 & 0.110\end{array}$

$\begin{array}{llll}1139.0 & 0.0036 & 0.000000 & 0.000\end{array}$

$\begin{array}{llll}1152.2 & -0.2391 & 0.000001 & 0.057\end{array}$

$\begin{array}{llll}1153.2 & 0.1081 & 0.000000 & 0.012\end{array}$

$\begin{array}{llll}1154.4 & -0.3295 & 0.000001 & 0.109\end{array}$

$\begin{array}{llll}1162.0 & 0.2875 & -0.000001 & 0.083\end{array}$

$\begin{array}{llll}1171.9 & 0.0023 & 0.000000 & 0.000\end{array}$

$\begin{array}{lllll}997.8 & 0.2337 & -0.000311 & 0.055\end{array}$

$\begin{array}{llll}998.7 & 0.2480 & -0.000110 & 0.061\end{array}$

$\begin{array}{llll}1002.4 & 0.2897 & -0.000035 & 0.084\end{array}$

$\begin{array}{llll}1006.3 & 0.3339 & -0.000023 & 0.112\end{array}$

$\begin{array}{llll}1010.8 & 0.3279 & -0.000015 & 0.107\end{array}$

$\begin{array}{llll}1016.3 & 0.3398 & -0.000011 & 0.115\end{array}$

$\begin{array}{llll}1023.1 & 0.3577 & -0.000008 & 0.128\end{array}$

$\begin{array}{llll}997.2 & 0.0030 & -0.000016 & 0.000\end{array}$

$\begin{array}{lllll}997.3 & 0.0065 & -0.000017 & 0.000\end{array}$

$\begin{array}{llll}997.4 & 0.0398 & -0.000062 & 0.002\end{array}$

$\begin{array}{llll}997.5 & 0.0125 & -0.000017 & 0.000\end{array}$

$\begin{array}{llll}997.7 & 0.0422 & -0.000043 & 0.002\end{array}$

$\begin{array}{llll}997.7 & 0.0491 & -0.000044 & 0.002\end{array}$

$\begin{array}{lllll}997.8 & 0.0463 & -0.000041 & 0.002\end{array}$

$\begin{array}{lllll}998.0 & 0.0252 & -0.000017 & 0.001\end{array}$

$\begin{array}{llll}998.0 & 0.0605 & -0.000038 & 0.004\end{array}$

$\begin{array}{llll}998.4 & 0.0449 & -0.000020 & 0.002\end{array}$

$\begin{array}{llll}998.9 & 0.0577 & -0.000019 & 0.003\end{array}$

$\begin{array}{llll}998.9 & 0.0517 & -0.000017 & 0.003\end{array}$

$\begin{array}{llll}999.0 & 0.0174 & -0.000006 & 0.000\end{array}$

$\begin{array}{lllll}999.2 & 0.0151 & -0.000004 & 0.000\end{array}$

$\begin{array}{llll}999.4 & 0.0144 & -0.000004 & 0.000\end{array}$

\begin{tabular}{|c|c|c|}
\hline 235.6 & & $\begin{array}{l}998.2041 \\
998.2041 \\
998.2041 \\
998.2041 \\
998.2041 \\
998.2041 \\
998.2041 \\
998.2041 \\
998.2041 \\
998.2041 \\
998.2041 \\
998.2041 \\
998.2041 \\
998.2041 \\
998.2041 \\
998.2041 \\
998.2041 \\
998.2041 \\
998.2041 \\
998.2041 \\
998.2041 \\
998.2041 \\
998.2041 \\
998.2041 \\
998.2041 \\
998.2041 \\
997.2867 \\
997.2867 \\
997.2867 \\
997.2867 \\
997.2867 \\
997.2867 \\
997.2867\end{array}$ \\
\hline 1.2 & & 997.0449 \\
\hline 2.4 & & $\begin{array}{l}997.0449 \\
997.0449\end{array}$ \\
\hline 4.8 & 24.7 & $\begin{array}{l}997.0449 \\
997.0449 \\
997.0449 \\
997.0449\end{array}$ \\
\hline 9.6 & 26.5 & $\begin{array}{l}997.0449 \\
997.0449 \\
997.0449 \\
997.0449\end{array}$ \\
\hline 19.2 & 27.69 & $\begin{array}{l}997.0449 \\
997.0449 \\
997.0449 \\
997.0449\end{array}$ \\
\hline
\end{tabular}




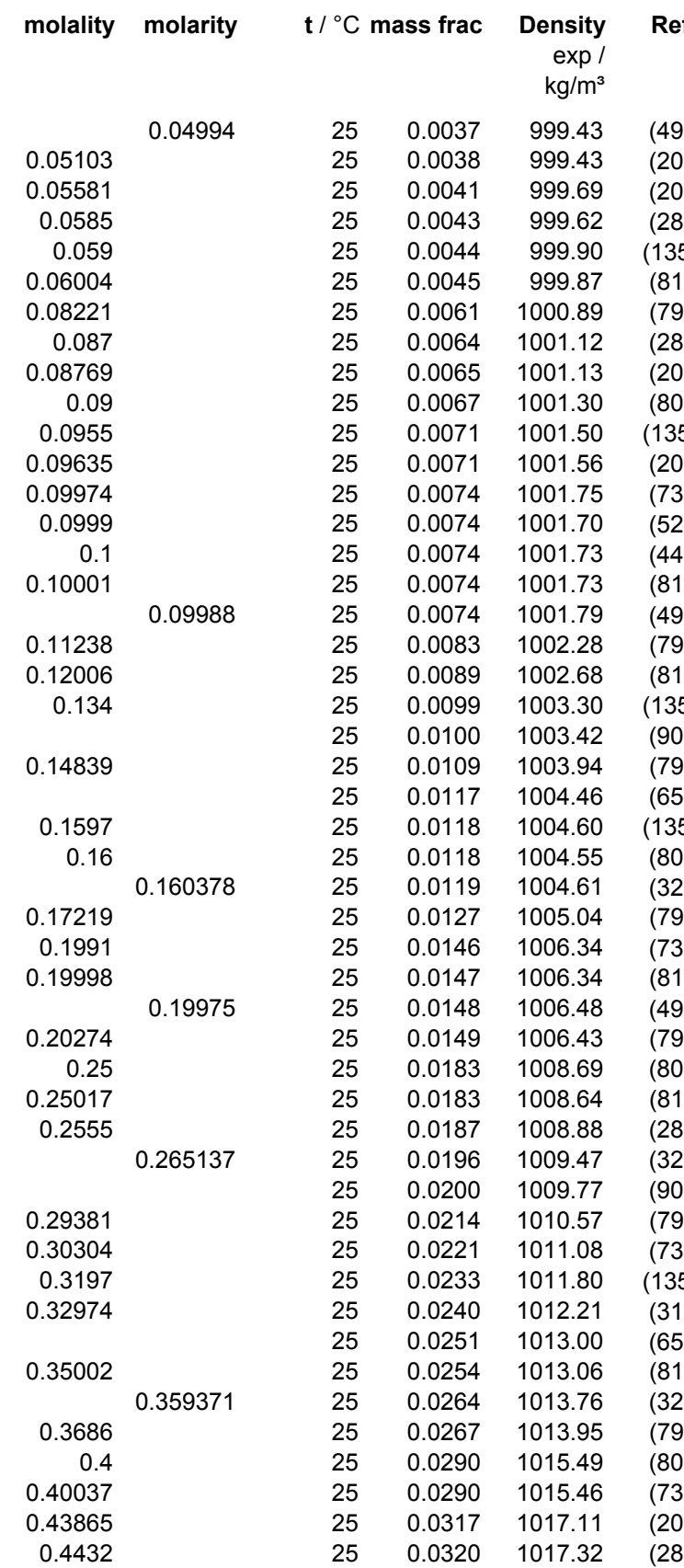

\section{App vol App vol}

exp / Calc /$$
\mathrm{m}^{3} / \mathrm{k}
$$

$\mathrm{m}^{3} / \mathrm{kg}$

$\begin{array}{ll}0.000361 & 0.000376 \\ 0.000371 & 0.000376\end{array}$

$0.000363 \quad 0.000376$

$\begin{array}{lll}0.000408 & 0.000376\end{array}$

$0.000349 \quad 0.000376$

$\begin{array}{lll}0.000368 & 0.000376\end{array}$

$\begin{array}{lll}0.000371 & 0.000376\end{array}$

$0.000370 \quad 0.000376$

$0.000373 \quad 0.000376$

$0.000363 \quad 0.000376$

$0.000372 \quad 0.000376$

0.0003690 .000376

$0.000365 \quad 0.000376$

$0.000373 \quad 0.000376$

$0.000369 \quad 0.000376$

$0.000369 \quad 0.000376$

$\begin{array}{lll}0.000364 & 0.000376\end{array}$

$0.000373 \quad 0.000377$

$0.000367 \quad 0.000377$

$\begin{array}{ll}0.000371 & 0.000377\end{array}$

$0.000366 \quad 0.000377$

$\begin{array}{lll}0.000373 & 0.000377\end{array}$

$\begin{array}{ll}0.000368 & 0.000377\end{array}$

$\begin{array}{lll}0.000362 & 0.000377\end{array}$

$\begin{array}{lll}0.000367 & 0.000377\end{array}$

$0.000368 \quad 0.000377$

$\begin{array}{ll}0.000374 & 0.000377\end{array}$

$\begin{array}{lll}0.000370 & 0.000378\end{array}$

$\begin{array}{lll}0.000372 & 0.000378\end{array}$

$\begin{array}{lll}0.000368 & 0.000378\end{array}$

$0.000375 \quad 0.000378$

$0.000370 \quad 0.000378$

$\begin{array}{lll}0.000373 & 0.000378\end{array}$

$\begin{array}{lll}0.000373 & 0.000378\end{array}$

$\begin{array}{ll}0.000372 & 0.000379\end{array}$

$\begin{array}{lll}0.000371 & 0.000379\end{array}$

$\begin{array}{ll}0.000377 & 0.000379\end{array}$

$0.000373 \quad 0.000379$

$\begin{array}{lll}0.000375 & 0.000379\end{array}$

$\begin{array}{lll}0.000377 & 0.000379\end{array}$

$\begin{array}{ll}0.000375 & 0.000379 \\ 0.000379 & 0.000380\end{array}$

$\begin{array}{lll}0.000377 & 0.000380\end{array}$

$\begin{array}{lll}0.000378 & 0.000380\end{array}$

$0.000374 \quad 0.000380$

$\begin{array}{lll}0.000375 & 0.000380\end{array}$

$\begin{array}{lll}0.000378 & 0.000381\end{array}$

$\begin{array}{lll}0.000378 & 0.000381\end{array}$
Density Density App Vol Square of Inconsistent Inconsistent (Dens rel- Delta density Apparent Density of calc / Residual / Residual / Density based on based on 1$)^{\star} 10^{\wedge} 4 \quad / \mathrm{kg} / \mathrm{m}^{3}$ molal volume water $/ \mathrm{kg} / \mathrm{m}^{3}$ $\mathrm{kg} / \mathrm{m}^{3} \quad \mathrm{~kg} / \mathrm{m}^{3} \quad \mathrm{~m}^{3} / \mathrm{kg} \quad$ Res density $\begin{gathered}\text { apparent } \\ \text { volume }\end{gathered}$

$999.4 \quad 0.0550-0.000015 \quad 0.003$ $\begin{array}{lll}0.0544 & -0.000013 & 0.003\end{array}$

$\begin{array}{llll}999.6 & 0.0544 & -0.000013 & 0.003 \\ 999.8 & -0.1381 & 0.000032 & 0.019\end{array}$

$\begin{array}{llll}999.8 & 0.1179 & -0.000027 & 0.014\end{array}$

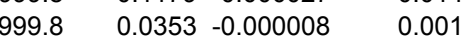

$000.9 \quad 0.0304-0.000005 \quad 0.001$

$\begin{array}{llll}1001.1 & 0.0401 & -0.000006 & 0.002\end{array}$

$\begin{array}{llll}1001.1 & 0.0233 & -0.000004 & 0.001\end{array}$

$\begin{array}{llll}001.2 & 0.0858 & -0.000013 & 0.007\end{array}$

$\begin{array}{llll}1001.5 & 0.0322 & -0.000005 & 0.001\end{array}$

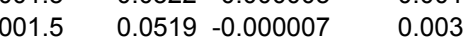

$\begin{array}{llll}001.7 & 0.0868 & -0.000012 & 0.008\end{array}$

$\begin{array}{llll}001.7 & 0.0294 & -0.000004 & 0.001\end{array}$

$\begin{array}{llll}1001.7 & 0.0548 & -0.000007 & 0.003\end{array}$

$\begin{array}{lllll}1001.7 & 0.0546 & -0.000007 & 0.003\end{array}$

$\begin{array}{llll}1001.7 & 0.0950 & -0.000013 & 0.009\end{array}$

$\begin{array}{llll}1002.2 & 0.0324 & -0.000004 & 0.001\end{array}$

$\begin{array}{llll}1002.6 & 0.0845 & -0.000009 & 0.007\end{array}$

$\begin{array}{llll}1003.2 & 0.0606 & -0.000006 & 0.004\end{array}$

$\begin{array}{llll}1003.3 & 0.1124 & -0.000011 & 0.013\end{array}$

$\begin{array}{llll}1003.9 & 0.0403 & -0.000004 & 0.002\end{array}$

$\begin{array}{llll}1004.4 & 0.1092 & -0.000009 & 0.012\end{array}$

$\begin{array}{llll}1004.4 & 0.1816 & -0.000015 & 0.033\end{array}$

$\begin{array}{llll}1004.4 & 0.1178 & -0.000010 & 0.014\end{array}$

$\begin{array}{llll}1004.5 & 0.1061 & -0.000009 & 0.011\end{array}$

$\begin{array}{llll}1005.0 & 0.0471 & -0.000004 & 0.002\end{array}$

$\begin{array}{llll}1006.2 & 0.1197 & -0.000008 & 0.014\end{array}$

$\begin{array}{llll}1006.3 & 0.0798 & -0.000005 & 0.006\end{array}$

$\begin{array}{llll}1006.3 & 0.1496 & -0.000010 & 0.022\end{array}$

$\begin{array}{llll}1006.4 & 0.0450 & -0.000003 & 0.002 \\ 1008.5 & 0.1520 & -0.000008 & 0.023\end{array}$

$1008.5-0.0913-0.000005$

$\begin{array}{llll}1008.8 & 0.0971 & -0.000005 & 0.009\end{array}$

$\begin{array}{llll}1009.4 & 0.1238 & -0.000006 & 0.015\end{array}$

$\begin{array}{llll}1009.6 & 0.1551 & -0.000008 & 0.024\end{array}$

$\begin{array}{llll}1010.5 & 0.0426 & -0.000002 & 0.002\end{array}$

$\begin{array}{llll}1010.9 & 0.1387 & -0.000006 & 0.019\end{array}$

$\begin{array}{llll}1011.7 & 0.1064 & -0.000004 & 0.011\end{array}$

$\begin{array}{llll}1012.1 & 0.0635 & -0.000003 & 0.004\end{array}$

$\begin{array}{llll}1012.9 & 0.1200 & -0.000005 & 0.014\end{array}$

$\begin{array}{llll}1013.1 & 0.0044 & 0.000000 & 0.000\end{array}$

$\begin{array}{llll}1013.7 & 0.0613 & -0.000002 & 0.004\end{array}$

$1015.3-0.1868-0.000006$

$\begin{array}{lll}1015.3 & 0.1402-0.000005 & 0.020\end{array}$

$\begin{array}{llll}1017.0 & 0.0782 & -0.000002 & 0.006\end{array}$

$\begin{array}{llll}1017.2 & 0.0842 & -0.000003 & 0.007\end{array}$

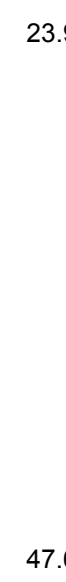

94.6

$\begin{array}{ccr} & 27.76 & 997.0449 \\ & 27.94 & 997.0449 \\ & & 997.0449 \\ 11.84 & 27.84 & 997.0449 \\ & & 997.0449 \\ & & 997.0449 \\ & 28.1 & 997.0449 \\ & & 997.0449 \\ & & 997.0449 \\ & & 997.0449 \\ & 28.283 & 997.0449 \\ & & 997.0449 \\ & 28.17 & 997.0449 \\ & & 997.0449 \\ 20.064 & & 997.0449 \\ 20.273 & & 997.0449 \\ & & 997.0449 \\ & & \end{array}$




\begin{tabular}{|c|c|c|c|c|c|}
\hline molality & molarity & \multicolumn{2}{|c|}{$\mathrm{t} /{ }^{\circ} \mathrm{C}$ mass frac } & $\begin{array}{r}\text { Density } \\
\exp / \\
\mathrm{kg} / \mathrm{m}^{3}\end{array}$ & Ref \\
\hline 0.45498 & & 25 & 0.0328 & 1017.72 & (20) \\
\hline 0.48243 & & 25 & 0.0347 & 1019.00 & (79) \\
\hline 0.4944 & & 25 & 0.0355 & 1019.70 & (135) \\
\hline 0.49966 & & 25 & 0.0359 & 1019.85 & (81) \\
\hline 0.49992 & & 25 & 0.0359 & 1019.80 & (81) \\
\hline 0.5 & & 25 & 0.0359 & 1019.77 & (44) \\
\hline 0.50389 & & 25 & 0.0362 & 1020.07 & (73) \\
\hline \multirow[t]{2}{*}{0.5041} & & 25 & 0.0362 & 1020.10 & (62) \\
\hline & 0.49933 & 25 & 0.0365 & 1020.30 & (49) \\
\hline \multirow[t]{2}{*}{0.51841} & & 25 & 0.0372 & 1020.61 & (31) \\
\hline & 0.509858 & 25 & 0.0372 & 1020.70 & (32) \\
\hline \multirow[t]{2}{*}{0.5331} & & 25 & 0.0382 & 1021.30 & (135) \\
\hline & & 25 & 0.0400 & 1022.55 & (90) \\
\hline \multirow[t]{2}{*}{0.5687} & & 25 & 0.0407 & 1022.82 & (79) \\
\hline & & 25 & 0.0428 & 1024.30 & (65) \\
\hline 0.6066 & & 25 & 0.0433 & 1024.45 & (79) \\
\hline \multirow{2}{*}{0.61488} & & 25 & 0.0438 & 1024.96 & (73) \\
\hline & 0.636132 & 25 & 0.0462 & 1026.48 & (32) \\
\hline 0.7 & & 25 & 0.0496 & 1028.72 & (80) \\
\hline 0.7069 & & 25 & 0.0501 & 1028.90 & (52) \\
\hline 0.72503 & & 25 & 0.0513 & 1029.76 & (73) \\
\hline 0.7618 & & 25 & 0.0537 & 1031.20 & (79) \\
\hline 0.8096 & & 25 & 0.0569 & 1033.24 & (79) \\
\hline 0.82477 & & 25 & 0.0579 & 1034.07 & (73) \\
\hline 0.89356 & & 25 & 0.0625 & 1036.70 & (20) \\
\hline 0.90321 & & 25 & 0.0631 & 1037.18 & (20) \\
\hline 0.92801 & & 25 & 0.0647 & 1038.47 & (73) \\
\hline 0.9414 & & 25 & 0.0656 & 1038.85 & (79) \\
\hline 0.9477 & & 25 & 0.0660 & 1039.30 & (135) \\
\hline 0.991 & & 25 & 0.0688 & 1041.20 & (62) \\
\hline 1 & & 25 & 0.0694 & 1041.35 & (44) \\
\hline 1 & & 25 & 0.0694 & 1041.52 & (80) \\
\hline 1.00491 & & 25 & 0.0697 & 1041.73 & (73) \\
\hline 1.0096 & & 25 & 0.0700 & 1041.74 & (79) \\
\hline \multirow[t]{3}{*}{1.018} & & 25 & 0.0705 & 1042.19 & (28) \\
\hline & 0.987727 & 25 & 0.0706 & 1042.40 & (32) \\
\hline & 0.98953 & 25 & 0.0708 & 1042.41 & (32) \\
\hline 1.02409 & & 25 & 0.0709 & 1042.37 & (31) \\
\hline \multirow[t]{2}{*}{1.1593} & & 25 & 0.0796 & 1047.98 & (79) \\
\hline & & 25 & 0.0800 & 1048.47 & (90) \\
\hline 1.2157 & & 25 & 0.0831 & 1050.32 & (79) \\
\hline 1.2173 & & 25 & 0.0832 & 1050.70 & (135) \\
\hline 1.2968 & & 25 & 0.0882 & 1053.90 & (135) \\
\hline 1.3584 & & 25 & 0.0920 & 1056.40 & (135) \\
\hline 1.37765 & & 25 & 0.0931 & 1056.74 & (20) \\
\hline 1.38366 & & 25 & 0.0935 & 1056.97 & (20) \\
\hline 1.4139 & & 25 & 0.0954 & 1058.40 & (52) \\
\hline 1.4195 & & 25 & 0.0957 & 1058.58 & (79) \\
\hline
\end{tabular}

App vol App vol

exp / Calc /

$\mathrm{m}^{3} / \mathrm{kg} \quad \mathrm{m}^{3} / \mathrm{kg}$

0.0003820 .000381 $0.000381 \quad 0.000381$ $0.000376 \quad 0.000381$ 0.0003790 .000381 $\begin{array}{lll}0.000380 & 0.000381\end{array}$ $\begin{array}{lll}0.000381 & 0.000381\end{array}$ $0.000378 \quad 0.000381$ $0.000377 \quad 0.000381$ $0.000377 \quad 0.000381$ $0.000381 \quad 0.000382$ $\begin{array}{ll}0.000379 & 0.000382\end{array}$ $0.000380 \quad 0.000382$ $\begin{array}{lll}0.000378 & 0.000382\end{array}$ 0.0003810 .000382 $0.000380 \quad 0.000382$ $0.000383 \quad 0.000383$ $0.000380 \quad 0.000383$ $0.000380 \quad 0.000383$ $0.000380 \quad 0.000384$ $0.000383 \quad 0.000384$ $0.000382 \quad 0.000384$ $0.000385 \quad 0.000384$ $\begin{array}{ll}0.000386 & 0.000385\end{array}$ $0.000383 \quad 0.000385$ 0.0003890 .000386 $0.000388 \quad 0.000386$ 0.0003850 .000386 $0.000387 \quad 0.000386$ 0.0003850 .000386 0.0003850 .000387 $0.000388 \quad 0.000387$ $\begin{array}{lll}0.000386 & 0.000387\end{array}$ $0.000386 \quad 0.000387$ $\begin{array}{lll}0.000388 & 0.000387\end{array}$ $\begin{array}{lll}0.000387 & 0.000387\end{array}$ $\begin{array}{lll}0.000385 & 0.000387\end{array}$ $\begin{array}{ll}0.000386 & 0.000387 \\ 0.000388 & 0.000387\end{array}$ $\begin{array}{ll}0.000388 & 0.000387\end{array}$ $0.000390 \quad 0.000389$ $\begin{array}{ll}0.000388 & 0.000389 \\ 0.000391 & 0.000389\end{array}$ $0.000387 \quad 0.000389$ $0.000389 \quad 0.000390$ $0.000390 \quad 0.000391$ $0.000395 \quad 0.000391$ $\begin{array}{ll}0.000395 & 0.000391\end{array}$ $0.000393 \quad 0.000391$ $0.000394 \quad 0.000392$
Density Density App Vol Square of Inconsistent Inconsistent (Dens rel- Delta density Apparent Density of

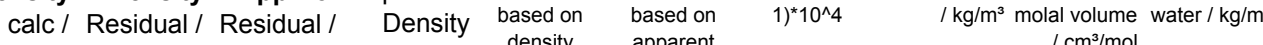
$\mathrm{kg} / \mathrm{m}^{3} \quad \mathrm{~kg} / \mathrm{m}^{3} \quad \mathrm{~m}^{3} / \mathrm{kg} \quad$ Res density apparent

$\begin{array}{llll}1017.8 & -0.0358 & 0.000001 & 0.001\end{array}$ $\begin{array}{llll}1019.0 & 0.0161 & 0.000000 & 0.000\end{array}$

$\begin{array}{llll}1019.5 & 0.1886 & -0.000005 & 0.036\end{array}$

$\begin{array}{llll}1019.7 & 0.1034 & -0.000003 & 0.011\end{array}$

$\begin{array}{lllll}1019.8 & 0.0484 & -0.000001 & 0.002\end{array}$

$\begin{array}{llll}1019.8 & 0.0102 & 0.000000 & 0.000\end{array}$

$\begin{array}{llll}1019.9 & 0.1377 & -0.000004 & 0.019\end{array}$

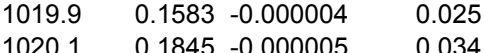

$1020.6 \quad 0.0343-0.000001-0.001$

$\begin{array}{llll}1020.6 & 0.1012 & -0.000003 & 0.010\end{array}$

$\begin{array}{llll}1021.2 & 0.0743 & -0.000002 & 0.006\end{array}$

$\begin{array}{llll}1022.4 & 0.1863 & -0.000004 & 0.035\end{array}$

$\begin{array}{llll}1022.8 & 0.0279 & -0.000001 & 0.001 \\ 1024.2 & 0.1307 & -0.000003 & 0.017\end{array}$

$1024.2-0.1307-0.0000$

$\begin{array}{llll}1024.5 & -0.0111 & 0.000000 & 0.000\end{array}$

$\begin{array}{llll}1024.8 & 0.1325 & -0.000003 & 0.018\end{array}$

$\begin{array}{llll}1026.4 & 0.1255 & -0.000003 & 0.016\end{array}$

$\begin{array}{llll}1028.5 & 0.1732 & -0.000003 & 0.030\end{array}$

$\begin{array}{llll}1028.8 & 0.0530 & -0.000001 & 0.003\end{array}$

$\begin{array}{llll}1029.6 & 0.1252 & -0.000002 & 0.016\end{array}$

$\begin{array}{llll}1031.2 & -0.0268 & 0.000000 & 0.001\end{array}$

$\begin{array}{llll}1033.3 & -0.0525 & 0.000001 & 0.003\end{array}$

$\begin{array}{llll}1033.9 & 0.1253 & -0.000002 & 0.016\end{array}$

$\begin{array}{llll}1036.9 & -0.1886 & 0.000003 & 0.036\end{array}$

$\begin{array}{llll}1037.3 & -0.1278 & 0.000002 & 0.016\end{array}$

$\begin{array}{llll}1038.4 & 0.1069 & -0.000002 & 0.011\end{array}$

$\begin{array}{llll}1038.9 & -0.0797 & 0.000001 & 0.006\end{array}$

$\begin{array}{llll}1039.2 & 0.0991 & -0.000001 & 0.010\end{array}$

$\begin{array}{llll}1041.0 & 0.1622 & -0.000002 & 0.026\end{array}$

$\begin{array}{llll}1041.4 & -0.0687 & 0.000001 & 0.005 \\ 1041.4 & 0.1013 & -0.000001 & 0.010\end{array}$

$\begin{array}{lll}1041.6 & 0.1037-0.000001 & 0.011\end{array}$

$\begin{array}{llll}1041.8 & -0.0892 & 0.000001 & 0.008\end{array}$

$\begin{array}{llll}1042.2 & 0.0125 & 0.000000 & 0.000\end{array}$

$\begin{array}{llll}1042.2 & 0.1508 & -0.000002 & 0.023\end{array}$

$\begin{array}{llll}1042.3 & 0.0756 & -0.000001 & 0.006\end{array}$

$\begin{array}{llll}1042.4 & -0.0665 & 0.000001 & 0.004\end{array}$

$\begin{array}{llll}1048.1 & -0.1233 & 0.000001 & 0.015\end{array}$

$\begin{array}{llll}1048.4 & 0.0701 & -0.000001 & 0.005\end{array}$

$\begin{array}{llll}1050.5 & -0.1350 & 0.000001 & 0.018\end{array}$

$\begin{array}{llll}1050.5 & 0.1831 & -0.000002 & 0.034\end{array}$

$\begin{array}{llll}1053.8 & 0.1007 & -0.000001 & 0.010 \\ 1056.3 & 0.0742 & -0.000001 & 0.006\end{array}$

$\begin{array}{llll}1057.1 & -0.3674 & 0.000004 & 0.135\end{array}$

$\begin{array}{lllll}1057.4 & -0.3877 & 0.000004 & 0.150\end{array}$

$\begin{array}{llll}1058.6 & -0.1896 & 0.000002 & 0.036\end{array}$

$\begin{array}{llll}1058.8 & -0.2418 & 0.000002 & 0.058\end{array}$

\begin{tabular}{|c|c|c|c|}
\hline & 20.678 & & 997.0449 \\
\hline & & 28.38 & 997.0449 \\
\hline & & & 997.0449 \\
\hline & & 28.222 & 997.0449 \\
\hline & & 28.332 & 997.0449 \\
\hline & & & 997.0449 \\
\hline & & & 997.0449 \\
\hline & & & 997.0449 \\
\hline 233.2 & & & 997.0449 \\
\hline & & & 997.0449 \\
\hline & & & 997.0449 \\
\hline & & & 997.0449 \\
\hline & & & 997.0449 \\
\hline & & 28.44 & 997.0449 \\
\hline & & & 997.0449 \\
\hline & & 28.54 & 997.0449 \\
\hline & & & 997.0449 \\
\hline & & & 997.0449 \\
\hline & & & 997.0449 \\
\hline & & & 997.0449 \\
\hline & & & 997.0449 \\
\hline & & 28.69 & 997.0449 \\
\hline & & 28.76 & 997.0449 \\
\hline & & & 997.0449 \\
\hline & 39.66 & & 997.0449 \\
\hline & 40.133 & & 997.0449 \\
\hline & & & 997.0449 \\
\hline & & 28.89 & 997.0449 \\
\hline & & & 997.0449 \\
\hline & & & 997.0449 \\
\hline & & & 997.0449 \\
\hline & & & 997.0449 \\
\hline & & & 997.0449 \\
\hline & & 28.95 & 997.0449 \\
\hline & 45.147 & & 997.0449 \\
\hline & & & 997.0449 \\
\hline & & & 997.0449 \\
\hline & & & 997.0449 \\
\hline & & 29.09 & 997.0449 \\
\hline & & & 997.0449 \\
\hline & & 29.14 & 997.0449 \\
\hline & & & 997.0449 \\
\hline & & & 997.0449 \\
\hline & & & 997.0449 \\
\hline & 59.7 & & 997.0449 \\
\hline & 59.925 & & 997.0449 \\
\hline & & & 997.0449 \\
\hline & & 29.36 & 997.0449 \\
\hline
\end{tabular}




\begin{tabular}{|c|c|c|c|c|c|c|c|c|c|c|c|c|c|c|c|c|c|}
\hline molality & molarity & \multicolumn{2}{|c|}{$\mathrm{t} /{ }^{\circ} \mathrm{C}$ mass frac } & $\begin{array}{r}\text { Density } \\
\exp / \\
\mathrm{kg} / \mathrm{m}^{3}\end{array}$ & Ref & $\begin{array}{r}\text { App vol } \\
\text { exp / } \\
\mathrm{m}^{3} / \mathrm{kg}\end{array}$ & $\begin{array}{c}\text { App vol } \\
\text { Calc / } \\
\mathrm{m}^{3} / \mathrm{kg}\end{array}$ & $\begin{array}{r}\text { Density } \\
\text { calc / } \\
\mathrm{kg} / \mathrm{m}^{3}\end{array}$ & $\begin{array}{r}\text { Density } \\
\text { Residual / } \\
\mathrm{kg} / \mathrm{m}^{3}\end{array}$ & $\begin{array}{r}\text { App Vol } \\
\text { Residual / } \\
\mathrm{m}^{3} / \mathrm{kg}\end{array}$ & $\begin{array}{r}\text { Square of } \\
\text { Density } \\
\text { Res }\end{array}$ & $\begin{array}{l}\text { Inconsistent } \\
\text { based on } \\
\text { density }\end{array}$ & $\begin{array}{l}\text { Inconsistent } \\
\text { based on } \\
\text { apparent } \\
\text { volume }\end{array}$ & $\begin{array}{c}\text { (Dens rel - } \\
1)^{\star} 10^{\wedge} 4\end{array}$ & $\begin{array}{l}\text { Delta density } \\
\qquad / \mathrm{kg} / \mathrm{m}^{3} \mathrm{~m}\end{array}$ & $\begin{array}{r}\text { Apparent } \\
\text { nolal volume } \\
/ \mathrm{cm}^{3} / \mathrm{mol}\end{array}$ & $\begin{array}{r}\text { Density of } \\
\text { water } / \mathrm{kg} / \mathrm{m}^{3}\end{array}$ \\
\hline & 1.433396 & 25 & 0.1006 & 1062.05 & (32) & 0.000393 & 0.000392 & 1062.1 & -0.0568 & 0.000001 & 0.003 & & & & & & 997.0449 \\
\hline & 1.452132 & 25 & 0.1019 & 1062.93 & (32) & 0.000393 & 0.000393 & 1062.9 & -0.0008 & 0.000000 & 0.000 & & & & & & 997.0449 \\
\hline 1.54691 & & 25 & 0.1034 & 1063.76 & (31) & 0.000395 & 0.000393 & 1064.0 & -0.2074 & 0.000002 & 0.043 & & & & & & 997.0449 \\
\hline 1.6048 & & 25 & 0.1069 & 1066.04 & (79) & 0.000396 & 0.000393 & 1066.3 & -0.2516 & 0.000002 & 0.063 & & & & & 29.49 & 997.0449 \\
\hline 1.7032 & & 25 & 0.1127 & 1070.10 & (135) & 0.000395 & 0.000394 & 1070.2 & -0.1016 & 0.000001 & 0.010 & & & & & & 997.0449 \\
\hline 1.7218 & & 25 & 0.1138 & 1070.90 & (135) & 0.000395 & 0.000395 & 1070.9 & -0.0375 & 0.000000 & 0.001 & & & & & & 997.0449 \\
\hline 1.7758 & & 25 & 0.1169 & 1073.20 & (135) & 0.000394 & 0.000395 & 1073.1 & 0.1332 & -0.000001 & 0.018 & & & & & & 997.0449 \\
\hline & & 25 & 0.1200 & 1075.06 & (90) & 0.000396 & 0.000396 & 1075.2 & -0.0954 & 0.000001 & 0.009 & & & & & & 997.0449 \\
\hline 1.84381 & & 25 & 0.1209 & 1075.25 & (20) & 0.000399 & 0.000396 & 1075.7 & -0.4833 & 0.000003 & 0.234 & & & & 78.205 & & 997.0449 \\
\hline 1.847 & & 25 & 0.1210 & 1075.50 & (79) & 0.000398 & 0.000396 & 1075.9 & -0.3597 & 0.000003 & 0.129 & & & & & 29.71 & 997.0449 \\
\hline 2 & & 25 & 0.1298 & 1081.43 & (44) & 0.000400 & 0.000397 & 1081.8 & -0.3636 & 0.000002 & 0.132 & & & & & & 997.0449 \\
\hline 2.0087 & & 25 & 0.1303 & 1081.78 & (79) & 0.000400 & 0.000398 & 1082.1 & -0.3523 & 0.000002 & 0.124 & & & & & 29.81 & 997.0449 \\
\hline & 1.892966 & 25 & 0.1304 & 1082.25 & (32) & 0.000397 & 0.000398 & 1082.2 & 0.0197 & 0.000000 & 0.000 & & & & & & 997.0449 \\
\hline 2.0476 & & 25 & 0.1324 & 1083.50 & (62) & 0.000399 & 0.000398 & 1083.6 & -0.1232 & 0.000001 & 0.015 & & & & & & 997.0449 \\
\hline & 1.956091 & 25 & 0.1344 & 1084.90 & (32) & 0.000399 & 0.000398 & 1085.0 & -0.0795 & 0.000001 & 0.006 & & & & & & 997.0449 \\
\hline 2.10665 & & 25 & 0.1357 & 1085.56 & (31) & 0.000400 & 0.000398 & 1085.9 & -0.3216 & 0.000002 & 0.103 & & & & & & 997.0449 \\
\hline 2.2846 & & 25 & 0.1455 & 1092.60 & (135) & 0.000400 & 0.000400 & 1092.6 & -0.0136 & 0.000000 & 0.000 & & & & & & 997.0449 \\
\hline 2.35 & & 25 & 0.1491 & 1094.87 & $(28)$ & 0.000402 & 0.000401 & 1095.1 & -0.1922 & 0.000001 & 0.037 & & & & 97.823 & & 997.0449 \\
\hline 2.36692 & & 25 & 0.1500 & 1095.03 & (20) & 0.000405 & 0.000401 & 1095.7 & -0.6648 & 0.000004 & 0.442 & & & & 97.981 & & 997.0449 \\
\hline 2.3745 & & 25 & 0.1504 & 1095.32 & (20) & 0.000405 & 0.000401 & 1096.0 & -0.6480 & 0.000004 & 0.420 & & & & 98.28 & & 997.0449 \\
\hline 2.424 & & 25 & 0.1531 & 1097.80 & (135) & 0.000402 & 0.000402 & 1097.8 & -0.0107 & 0.000000 & 0.000 & & & & & & 997.0449 \\
\hline 2.4968 & & 25 & 0.1569 & 1100.30 & $(62)$ & 0.000403 & 0.000402 & 1100.5 & -0.1984 & 0.000001 & 0.039 & & & & & & 997.0449 \\
\hline & & 25 & 0.1600 & 1102.45 & (90) & 0.000404 & 0.000403 & 1102.6 & -0.1784 & 0.000001 & 0.032 & & & & & & 997.0449 \\
\hline 2.72517 & & 25 & 0.1689 & 1108.45 & (31) & 0.000406 & 0.000404 & 1108.8 & -0.3644 & 0.000002 & 0.133 & & & & & & 997.0449 \\
\hline & 2.547544 & 25 & 0.1711 & 1110.39 & (32) & 0.000404 & 0.000405 & 1110.3 & 0.0418 & 0.000000 & 0.002 & & & & & & 997.0449 \\
\hline 2.7977 & & 25 & 0.1726 & 1111.40 & (135) & 0.000405 & 0.000405 & 1111.4 & -0.0194 & 0.000000 & 0.000 & & & & & & 997.0449 \\
\hline 2.88083 & & 25 & 0.1768 & 1113.67 & (20) & 0.000409 & 0.000406 & 1114.4 & -0.7112 & 0.000003 & 0.506 & & & & 116.628 & & 997.0449 \\
\hline 2.89358 & & 25 & 0.1774 & 1114.11 & (20) & 0.000409 & 0.000406 & 1114.8 & -0.7269 & 0.000003 & 0.528 & & & & 117.065 & & 997.0449 \\
\hline 3 & & 25 & 0.1828 & 1118.28 & (44) & 0.000408 & 0.000407 & 1118.6 & -0.3152 & 0.000001 & 0.099 & & & & & & 997.0449 \\
\hline 3.0013 & & 25 & 0.1828 & 1118.50 & (62) & 0.000407 & 0.000407 & 1118.6 & -0.1409 & 0.000001 & 0.020 & & & & & & 997.0449 \\
\hline 3.012 & & 25 & 0.1834 & 1118.74 & (28) & 0.000408 & 0.000407 & 1119.0 & -0.2728 & 0.000001 & 0.074 & & & & 121.699 & & 997.0449 \\
\hline & 2.754042 & 25 & 0.1835 & 1119.18 & (32) & 0.000406 & 0.000407 & 1119.1 & 0.0994 & 0.000000 & 0.010 & & & & & & 997.0449 \\
\hline & & 25 & 0.2000 & 1130.72 & (90) & 0.000410 & 0.000410 & 1130.8 & -0.0937 & 0.000000 & 0.009 & & & & & & 997.0449 \\
\hline 3.3554 & & 25 & 0.2001 & 1130.90 & (135) & 0.000410 & 0.000410 & 1130.9 & 0.0124 & 0.000000 & 0.000 & & & & & & 997.0449 \\
\hline 3.41002 & & 25 & 0.2027 & 1132.01 & (20) & 0.000413 & 0.000410 & 1132.7 & -0.7322 & 0.000003 & 0.536 & & & & 134.965 & & 997.0449 \\
\hline 3.4123 & & 25 & 0.2028 & 1132.18 & (20) & 0.000413 & 0.000410 & 1132.8 & -0.6364 & 0.000002 & 0.405 & & & & 135.138 & & 997.0449 \\
\hline 3.42161 & & 25 & 0.2032 & 1132.86 & (31) & 0.000411 & 0.000410 & 1133.1 & -0.2744 & 0.000001 & 0.075 & & & & & & 997.0449 \\
\hline 3.4934 & & 25 & 0.2066 & 1135.60 & (135) & 0.000411 & 0.000411 & 1135.6 & 0.0444 & 0.000000 & 0.002 & & & & & & 997.0449 \\
\hline 3.5327 & & 25 & 0.2085 & 1136.80 & (62) & 0.000412 & 0.000411 & 1136.9 & -0.0744 & 0.000000 & 0.006 & & & & & & 997.0449 \\
\hline 3.5347 & & 25 & 0.2086 & 1136.90 & (52) & 0.000411 & 0.000411 & 1136.9 & -0.0414 & 0.000000 & 0.002 & & & & & & 997.0449 \\
\hline 3.5485 & & 25 & 0.2092 & 1137.50 & (135) & 0.000411 & 0.000411 & 1137.4 & 0.0967 & 0.000000 & 0.009 & & & & & & 997.0449 \\
\hline & 3.225612 & 25 & 0.2111 & 1138.96 & (32) & 0.000411 & 0.000412 & 1138.8 & 0.1632 & -0.000001 & 0.027 & & & & & & 997.0449 \\
\hline 3.93033 & & 25 & 0.2266 & 1149.82 & (31) & 0.000415 & 0.000414 & 1150.0 & -0.1410 & 0.000000 & 0.020 & & & & & & 997.0449 \\
\hline 3.93177 & & 25 & 0.2267 & 1149.60 & (20) & 0.000416 & 0.000414 & 1150.0 & -0.4046 & 0.000001 & 0.164 & & & & 152.558 & & 997.0449 \\
\hline 3.9487 & & 25 & 0.2274 & 1150.80 & (135) & 0.000414 & 0.000415 & 1150.6 & 0.2455 & -0.000001 & 0.060 & & & & & & 997.0449 \\
\hline 3.95785 & & 25 & 0.2278 & 1150.44 & (20) & 0.000416 & 0.000415 & 1150.8 & -0.4058 & 0.000001 & 0.165 & & & & 153.399 & & 997.0449 \\
\hline 3.9618 & & 25 & 0.2280 & 1151.10 & (62) & 0.000414 & 0.000415 & 1151.0 & 0.1229 & 0.000000 & 0.015 & & & & & & 997.0449 \\
\hline 4 & & 25 & 0.2297 & 1152.03 & (44) & 0.000416 & 0.000415 & 1152.2 & -0.1767 & 0.000001 & 0.031 & & & & & & 997.0449 \\
\hline
\end{tabular}




\begin{tabular}{|c|c|c|c|c|c|c|c|c|c|c|c|c|c|c|c|c|}
\hline molality & molarity & \multicolumn{2}{|c|}{$\mathrm{t} /{ }^{\circ} \mathrm{C}$ mass frac } & $\begin{array}{r}\text { Density } \\
\exp / \\
\mathrm{kg} / \mathrm{m}^{3}\end{array}$ & Ref & $\begin{array}{r}\text { App vol } \\
\exp / \\
\mathrm{m}^{3} / \mathrm{kg}\end{array}$ & $\begin{array}{l}\text { App vol } \\
\text { Calc / } \\
\mathrm{m}^{3} / \mathrm{kg}\end{array}$ & $\begin{array}{r}\text { Density } \\
\text { calc / } \\
\mathrm{kg} / \mathrm{m}^{3}\end{array}$ & $\begin{array}{r}\text { Density } \\
\text { Residual / } \\
\mathrm{kg} / \mathrm{m}^{3}\end{array}$ & $\begin{array}{r}\text { App Vol } \\
\text { Residual / } \\
\mathrm{m}^{3} / \mathrm{kg}\end{array}$ & $\begin{array}{r}\text { Square of } \\
\text { Density } \\
\text { Res }\end{array}$ & $\begin{array}{l}\text { Inconsistent } \\
\text { based on } \\
\text { density }\end{array}$ & $\begin{array}{l}\text { Inconsistent } \\
\text { based on } \\
\text { apparent } \\
\text { volume }\end{array}$ & $\begin{array}{c}\text { (Dens rel - } \\
1)^{\star} 10^{\wedge} 4\end{array}$ & \multicolumn{2}{|c|}{ 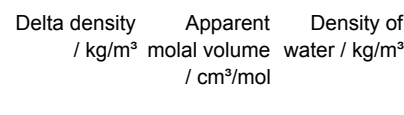 } \\
\hline & 3.577974 & 25 & 0.2312 & 1153.75 & (32) & 0.000414 & 0.000415 & 1153.3 & 0.4548 & -0.000001 & 0.207 & & & & & 997.0449 \\
\hline & & 25 & 0.2400 & 1159.95 & (90) & 0.000416 & 0.000417 & 1159.7 & 0.2491 & -0.000001 & 0.062 & & & & & 997.0449 \\
\hline 4.40004 & & 25 & 0.2470 & 1164.34 & (20) & 0.000420 & 0.000418 & 1164.8 & -0.4923 & 0.000001 & 0.242 & & & & 167.3 & 997.0449 \\
\hline 4.40058 & & 25 & 0.2470 & 1164.61 & (20) & 0.000419 & 0.000418 & 1164.9 & -0.2431 & 0.000001 & 0.059 & & & & 167.566 & 997.0449 \\
\hline 4.55469 & & 25 & 0.2535 & 1169.75 & (31) & 0.000419 & 0.000419 & 1169.6 & 0.1477 & 0.000000 & 0.022 & & & & & 997.0449 \\
\hline & 4.006439 & 25 & 0.2550 & 1171.34 & (32) & 0.000418 & 0.000420 & 1170.7 & 0.6184 & -0.000002 & 0.382 & & & & & 997.0449 \\
\hline 4.603 & & 25 & 0.2555 & 1171.70 & (135) & 0.000418 & 0.000420 & 1171.1 & 0.6223 & -0.000002 & 0.387 & & & & & 997.0449 \\
\hline 4.6934 & & 25 & 0.2592 & 1174.40 & (62) & 0.000419 & 0.000420 & 1173.8 & 0.5782 & -0.000002 & 0.334 & & & & & 997.0449 \\
\hline 4.8181 & & 25 & 0.2643 & 1178.20 & (135) & 0.000419 & 0.000421 & 1177.6 & 0.6280 & -0.000002 & 0.394 & & & & & 997.0449 \\
\hline & 0.001 & 30 & 0.0001 & 995.70 & (49) & 0.000334 & 0.000379 & 995.7 & 0.0033 & -0.000045 & 0.000 & & & 0.5 & & 995.6473 \\
\hline & 0.00249 & 30 & 0.0002 & 995.77 & (49) & 0.000358 & 0.000379 & 995.8 & 0.0039 & -0.000021 & 0.000 & & & 1.2 & & 995.6473 \\
\hline & 0.00499 & 30 & 0.0004 & 995.89 & (49) & 0.000359 & 0.000379 & 995.9 & 0.0073 & -0.000020 & 0.000 & & & 2.4 & & 995.6473 \\
\hline & 0.00997 & 30 & 0.0007 & 996.13 & (49) & 0.000359 & 0.000379 & 996.1 & 0.0150 & -0.000020 & 0.000 & & & 4.8 & & 995.6473 \\
\hline & 0.01995 & 30 & 0.0015 & 996.60 & (49) & 0.000359 & 0.000379 & 996.6 & 0.0298 & -0.000020 & 0.001 & & & 9.6 & & 995.6473 \\
\hline & 0.0399 & 30 & 0.0030 & 997.54 & (49) & 0.000366 & 0.000379 & 997.5 & 0.0405 & -0.000014 & 0.002 & & & 19 & & 995.6473 \\
\hline 0.05 & & 30 & 0.0037 & 997.96 & (44) & 0.000378 & 0.000379 & 998.0 & 0.0066 & -0.000002 & 0.000 & & & & & 995.6473 \\
\hline & 0.04987 & 30 & 0.0037 & 998.01 & (49) & 0.000367 & 0.000379 & 998.0 & 0.0463 & -0.000012 & 0.002 & & & 23.7 & & 995.6473 \\
\hline 0.1 & & 30 & 0.0074 & 1000.28 & (44) & 0.000376 & 0.000380 & 1000.2 & 0.0314 & -0.000004 & 0.001 & & & & & 995.6473 \\
\hline & 0.09973 & 30 & 0.0074 & 1000.35 & (49) & 0.000370 & 0.000380 & 1000.3 & 0.0779 & -0.000010 & 0.006 & & & 47.2 & & 995.6473 \\
\hline & 0.19946 & 30 & 0.0148 & 1005.00 & (49) & 0.000373 & 0.000381 & 1004.9 & 0.1246 & -0.000008 & 0.016 & & & 93.9 & & 995.6473 \\
\hline 0.32974 & & 30 & 0.0240 & 1010.71 & (31) & 0.000381 & 0.000383 & 1010.7 & 0.0555 & -0.000002 & 0.003 & & & & & 995.6473 \\
\hline 0.5 & & 30 & 0.0359 & 1018.19 & (44) & 0.000386 & 0.000385 & 1018.2 & -0.0306 & 0.000001 & 0.001 & & & & & 995.6473 \\
\hline 0.5041 & & 30 & 0.0362 & 1018.60 & (62) & 0.000380 & 0.000385 & 1018.4 & 0.1987 & -0.000005 & 0.039 & & & & & 995.6473 \\
\hline & 0.49856 & 30 & 0.0365 & 1018.72 & (49) & 0.000381 & 0.000385 & 1018.6 & 0.1462 & -0.000004 & 0.021 & & & 231.7 & & 995.6473 \\
\hline 0.51841 & & 30 & 0.0372 & 1019.05 & (31) & 0.000385 & 0.000385 & 1019.0 & 0.0186 & 0.000000 & 0.000 & & & & & 995.6473 \\
\hline 0.991 & & 30 & 0.0688 & 1039.50 & (62) & 0.000389 & 0.000390 & 1039.4 & 0.1322 & -0.000002 & 0.017 & & & & & 995.6473 \\
\hline 1 & & 30 & 0.0694 & 1039.66 & (44) & 0.000392 & 0.000390 & 1039.7 & -0.0863 & 0.000001 & 0.007 & & & & & 995.6473 \\
\hline 1.02409 & & 30 & 0.0709 & 1040.67 & (31) & 0.000392 & 0.000391 & 1040.8 & -0.0880 & 0.000001 & 0.008 & & & & & 995.6473 \\
\hline 1.5097 & & 30 & 0.1012 & 1060.60 & (62) & 0.000396 & 0.000396 & 1060.7 & -0.0701 & 0.000001 & 0.005 & & & & & 995.6473 \\
\hline 1.54691 & & 30 & 0.1034 & 1061.94 & (31) & 0.000398 & 0.000396 & 1062.2 & -0.2189 & 0.000002 & 0.048 & & & & & 995.6473 \\
\hline 2 & & 30 & 0.1298 & 1079.59 & (44) & 0.000403 & 0.000401 & 1079.9 & -0.2894 & 0.000002 & 0.084 & & & & & 995.6473 \\
\hline 2.0476 & & 30 & 0.1324 & 1081.60 & (62) & 0.000402 & 0.000401 & 1081.7 & -0.0982 & 0.000001 & 0.010 & & & & & 995.6473 \\
\hline 2.10665 & & 30 & 0.1357 & 1083.61 & (31) & 0.000404 & 0.000402 & 1083.9 & -0.3334 & 0.000002 & 0.111 & & & & & 995.6473 \\
\hline 2.4968 & & 30 & 0.1569 & 1098.30 & (62) & 0.000406 & 0.000405 & 1098.5 & -0.1755 & 0.000001 & 0.031 & & & & & 995.6473 \\
\hline 2.72517 & & 30 & 0.1689 & 1106.41 & (31) & 0.000409 & 0.000407 & 1106.7 & -0.3339 & 0.000002 & 0.111 & & & & & 995.6473 \\
\hline 3 & & 30 & 0.1828 & 1116.19 & (44) & 0.000411 & 0.000410 & 1116.5 & -0.2795 & 0.000001 & 0.078 & & & & & 995.6473 \\
\hline 3.0013 & & 30 & 0.1828 & 1116.40 & (62) & 0.000410 & 0.000410 & 1116.5 & -0.1149 & 0.000001 & 0.013 & & & & & 995.6473 \\
\hline 3.42161 & & 30 & 0.2032 & 1130.72 & (31) & 0.000414 & 0.000413 & 1130.9 & -0.2081 & 0.000001 & 0.043 & & & & & 995.6473 \\
\hline 3.5327 & & 30 & 0.2085 & 1134.60 & (62) & 0.000414 & 0.000414 & 1134.6 & -0.0476 & 0.000000 & 0.002 & & & & & 995.6473 \\
\hline 3.93033 & & 30 & 0.2266 & 1147.62 & (31) & 0.000417 & 0.000417 & 1147.7 & -0.0437 & 0.000000 & 0.002 & & & & & 995.6473 \\
\hline 3.9618 & & 30 & 0.2280 & 1148.80 & (62) & 0.000417 & 0.000418 & 1148.7 & 0.1256 & 0.000000 & 0.016 & & & & & 995.6473 \\
\hline 4 & & 30 & 0.2297 & 1149.79 & (44) & 0.000418 & 0.000418 & 1149.9 & -0.1074 & 0.000000 & 0.012 & & & & & 995.6473 \\
\hline 4.55469 & & 30 & 0.2535 & 1166.28 & (31) & 0.000425 & 0.000422 & 1167.2 & -0.9223 & 0.000003 & 0.851 & $!$ & & & & 995.6473 \\
\hline 4.6934 & & 30 & 0.2592 & 1171.90 & (62) & 0.000422 & 0.000423 & 1171.4 & 0.4997 & -0.000001 & 0.250 & & & & & 995.6473 \\
\hline & 0.00099 & 35 & 0.0001 & 994.08 & (49) & 0.000329 & 0.000382 & 994.1 & 0.0039 & -0.000053 & 0.000 & & & 0.5 & & 994.0319 \\
\hline & 0.00249 & 35 & 0.0002 & 994.15 & (49) & 0.000360 & 0.000382 & 994.1 & 0.0041 & -0.000022 & 0.000 & & & 1.2 & & 994.0319 \\
\hline & 0.00498 & 35 & 0.0004 & 994.27 & (49) & 0.000360 & 0.000382 & 994.3 & 0.0083 & -0.000022 & 0.000 & & & 2.4 & & 994.0319 \\
\hline & 0.00996 & 35 & 0.0007 & 994.50 & (49) & 0.000373 & 0.000382 & 994.5 & 0.0066 & -0.000009 & 0.000 & & & 4.7 & & 994.0319 \\
\hline
\end{tabular}




\begin{tabular}{|c|c|c|c|c|c|c|c|c|c|c|c|c|c|c|c|c|c|}
\hline molality & molarity & \multicolumn{2}{|c|}{$\mathrm{t} /{ }^{\circ} \mathrm{C}$ mass frac } & $\begin{array}{r}\text { Density } \\
\exp / \\
\mathrm{kg} / \mathrm{m}^{3}\end{array}$ & Ref & $\begin{array}{r}\text { App vol } \\
\exp / \\
\mathrm{m}^{3} / \mathrm{kg}\end{array}$ & $\begin{array}{c}\text { App vol } \\
\text { Calc / } \\
\mathrm{m}^{3} / \mathrm{kg}\end{array}$ & $\begin{array}{r}\text { Density } \\
\text { calc / } \\
\mathrm{kg} / \mathrm{m}^{3}\end{array}$ & $\begin{array}{r}\text { Density } \\
\text { Residual / } \\
\mathrm{kg} / \mathrm{m}^{3}\end{array}$ & $\begin{array}{r}\text { App Vol } \\
\text { Residual / } \\
\mathrm{m}^{3} / \mathrm{kg}\end{array}$ & $\begin{array}{r}\text { Square of } \\
\text { Density } \\
\text { Res }\end{array}$ & $\begin{array}{l}\text { Inconsistent } \\
\text { based on } \\
\text { density }\end{array}$ & $\begin{array}{l}\text { Inconsistent } \\
\text { based on } \\
\text { apparent } \\
\text { volume }\end{array}$ & $\begin{array}{c}\text { (Dens rel - } \\
1)^{\star} 10^{\wedge} 4\end{array}$ & $\begin{array}{l}\text { Delta density } \\
\qquad / \mathrm{kg} / \mathrm{m}^{3}\end{array}$ & $\begin{array}{r}\text { Apparent } \\
\text { molal volume } \\
/ \mathrm{cm}^{3} / \mathrm{mol}\end{array}$ & $\begin{array}{r}\text { Density of } \\
\text { water } / \mathrm{kg} / \mathrm{m}^{3}\end{array}$ \\
\hline 0.01501 & & 35 & 0.0011 & 994.79 & (81) & 0.000322 & 0.000382 & 994.7 & 0.0666 & -0.000060 & 0.004 & & & & & 24 & 994.0319 \\
\hline & 0.01992 & 35 & 0.0015 & 994.97 & (49) & 0.000373 & 0.000382 & 995.0 & 0.0134 & -0.000009 & 0.000 & & & 9.4 & & & 994.0319 \\
\hline 0.03002 & & 35 & 0.0022 & 995.44 & (81) & 0.000367 & 0.000382 & 995.4 & 0.0342 & -0.000015 & 0.001 & & & & & 27.35 & 994.0319 \\
\hline 0.04 & & 35 & 0.0030 & 995.93 & (80) & 0.000361 & 0.000382 & 995.9 & 0.0626 & -0.000021 & 0.004 & & & & & & 994.0319 \\
\hline & 0.03983 & 35 & 0.0030 & 995.90 & (49) & 0.000373 & 0.000382 & 995.9 & 0.0281 & -0.000009 & 0.001 & & & 18.8 & & & 994.0319 \\
\hline 0.05 & & 35 & 0.0037 & 996.35 & (44) & 0.000376 & 0.000383 & 996.3 & 0.0248 & -0.000007 & 0.001 & & & & & & 994.0319 \\
\hline & 0.04979 & 35 & 0.0037 & 996.37 & (49) & 0.000373 & 0.000383 & 996.3 & 0.0354 & -0.000010 & 0.001 & & & 23.5 & & & 994.0319 \\
\hline 0.05635 & & 35 & 0.0042 & 996.66 & (20) & 0.000372 & 0.000383 & 996.6 & 0.0422 & -0.000010 & 0.002 & & & & 2.626 & & 994.0319 \\
\hline 0.06004 & & 35 & 0.0045 & 996.83 & (81) & 0.000371 & 0.000383 & 996.8 & 0.0498 & -0.000011 & 0.002 & & & & & 27.69 & 994.0319 \\
\hline 0.07802 & & 35 & 0.0058 & 997.66 & (20) & 0.000374 & 0.000383 & 997.6 & 0.0513 & -0.000009 & 0.003 & & & & 3.625 & & 994.0319 \\
\hline 0.09 & & 35 & 0.0067 & 998.24 & (80) & 0.000370 & 0.000383 & 998.2 & 0.0881 & -0.000013 & 0.008 & & & & & & 994.0319 \\
\hline 0.1 & & 35 & 0.0074 & 998.65 & (44) & 0.000377 & 0.000383 & 998.6 & 0.0425 & -0.000006 & 0.002 & & & & & & 994.0319 \\
\hline 0.10001 & & 35 & 0.0074 & 998.64 & (81) & 0.000378 & 0.000383 & 998.6 & 0.0370 & -0.000005 & 0.001 & & & & & 28.19 & 994.0319 \\
\hline & 0.09957 & 35 & 0.0074 & 998.68 & (49) & 0.000376 & 0.000383 & 998.6 & 0.0560 & -0.000008 & 0.003 & & & 46.8 & & & 994.0319 \\
\hline 0.12006 & & 35 & 0.0089 & 999.60 & (81) & 0.000375 & 0.000383 & 999.5 & 0.0774 & -0.000009 & 0.006 & & & & & 27.93 & 994.0319 \\
\hline 0.16 & & 35 & 0.0118 & 1001.45 & (80) & 0.000374 & 0.000384 & 1001.3 & 0.1180 & -0.000010 & 0.014 & & & & & & 994.0319 \\
\hline 0.19998 & & 35 & 0.0147 & 1003.19 & (81) & 0.000381 & 0.000384 & 1003.1 & 0.0540 & -0.000004 & 0.003 & & & & & 28.38 & 994.0319 \\
\hline & 0.19913 & 35 & 0.0148 & 1003.29 & (49) & 0.000379 & 0.000384 & 1003.2 & 0.0807 & -0.000005 & 0.007 & & & 93.1 & & & 994.0319 \\
\hline 0.24738 & & 35 & 0.0181 & 1005.17 & (20) & 0.000390 & 0.000385 & 1005.3 & -0.1013 & 0.000006 & 0.010 & & & & 11.139 & & 994.0319 \\
\hline 0.25 & & 35 & 0.0183 & 1005.53 & (80) & 0.000377 & 0.000385 & 1005.4 & 0.1403 & -0.000008 & 0.020 & & & & & & 994.0319 \\
\hline 0.25017 & & 35 & 0.0183 & 1005.47 & (81) & 0.000381 & 0.000385 & 1005.4 & 0.0686 & -0.000004 & 0.005 & & & & & 28.42 & 994.0319 \\
\hline 0.35002 & & 35 & 0.0254 & 1009.84 & (81) & 0.000387 & 0.000386 & 1009.9 & -0.0230 & 0.000001 & 0.001 & & & & & 28.849 & 994.0319 \\
\hline 0.4 & & 35 & 0.0290 & 1012.24 & (80) & 0.000381 & 0.000387 & 1012.1 & 0.1641 & -0.000006 & 0.027 & & & & & & 994.0319 \\
\hline 0.43401 & & 35 & 0.0313 & 1013.65 & (20) & 0.000385 & 0.000387 & 1013.6 & 0.0722 & -0.000002 & 0.005 & & & & 19.619 & & 994.0319 \\
\hline 0.49966 & & 35 & 0.0359 & 1016.54 & (81) & 0.000386 & 0.000388 & 1016.5 & 0.0757 & -0.000002 & 0.006 & & & & & 28.759 & 994.0319 \\
\hline 0.49992 & & 35 & 0.0359 & 1016.48 & (81) & 0.000388 & 0.000388 & 1016.5 & 0.0021 & 0.000000 & 0.000 & & & & & 28.907 & 994.0319 \\
\hline 0.5 & & 35 & 0.0359 & 1016.45 & (44) & 0.000389 & 0.000388 & 1016.5 & -0.0307 & 0.000001 & 0.001 & & & & & & 994.0319 \\
\hline & 0.4977 & 35 & 0.0365 & 1016.89 & (49) & 0.000386 & 0.000388 & 1016.8 & 0.0648 & -0.000002 & 0.004 & & & 230 & & & 994.0319 \\
\hline 0.7 & & 35 & 0.0496 & 1025.31 & (80) & 0.000387 & 0.000390 & 1025.2 & 0.1439 & -0.000003 & 0.021 & & & & & & 994.0319 \\
\hline 0.91994 & & 35 & 0.0642 & 1034.52 & (20) & 0.000393 & 0.000392 & 1034.5 & -0.0092 & 0.000000 & 0.000 & & & & 40.489 & & 994.0319 \\
\hline 1 & & 35 & 0.0694 & 1037.84 & (44) & 0.000394 & 0.000393 & 1037.9 & -0.0508 & 0.000001 & 0.003 & & & & & & 994.0319 \\
\hline 1 & & 35 & 0.0694 & 1037.97 & (80) & 0.000392 & 0.000393 & 1037.9 & 0.0792 & -0.000001 & 0.006 & & & & & & 994.0319 \\
\hline 1.38222 & & 35 & 0.0934 & 1053.51 & (20) & 0.000398 & 0.000397 & 1053.6 & -0.0782 & 0.000001 & 0.006 & & & & 59.482 & & 994.0319 \\
\hline 1.8487 & & 35 & 0.1211 & 1071.82 & (20) & 0.000403 & 0.000402 & 1072.0 & -0.1964 & 0.000001 & 0.039 & & & & 77.784 & & 994.0319 \\
\hline 2 & & 35 & 0.1298 & 1077.56 & (44) & 0.000405 & 0.000403 & 1077.8 & -0.2579 & 0.000002 & 0.067 & & & & & & 994.0319 \\
\hline 2.3552 & & 35 & 0.1494 & 1090.99 & (20) & 0.000407 & 0.000407 & 1091.1 & -0.1416 & 0.000001 & 0.020 & & & & 96.961 & & 994.0319 \\
\hline 2.87947 & & 35 & 0.1767 & 1109.94 & (20) & 0.000412 & 0.000411 & 1110.0 & -0.0777 & 0.000000 & 0.006 & & & & 115.908 & & 994.0319 \\
\hline 3 & & 35 & 0.1828 & 1114.20 & (44) & 0.000412 & 0.000412 & 1114.2 & -0.0335 & 0.000000 & 0.001 & & & & & & 994.0319 \\
\hline 3.41915 & & 35 & 0.2031 & 1128.62 & (20) & 0.000415 & 0.000416 & 1128.5 & 0.0722 & 0.000000 & 0.005 & & & & 134.585 & & 994.0319 \\
\hline 3.94338 & & 35 & 0.2272 & 1145.99 & (20) & 0.000419 & 0.000420 & 1145.7 & 0.2846 & -0.000001 & 0.081 & & & & 151.961 & & 994.0319 \\
\hline 4 & & 35 & 0.2297 & 1147.53 & (44) & 0.000420 & 0.000420 & 1147.5 & 0.0150 & 0.000000 & 0.000 & & & & & & 994.0319 \\
\hline 4.40076 & & 35 & 0.2470 & 1160.60 & (20) & 0.000422 & 0.000423 & 1160.0 & 0.5509 & -0.000002 & 0.303 & & & & 166.569 & & 994.0319 \\
\hline 0.0988 & & 40 & 0.0073 & 996.80 & (62) & 0.000374 & 0.000386 & 996.7 & 0.0854 & -0.000012 & 0.007 & & & & & & 992.2158 \\
\hline 0.0999 & & 40 & 0.0074 & 996.80 & (52) & 0.000381 & 0.000386 & 996.8 & 0.0355 & -0.000005 & 0.001 & & & & & & 992.2158 \\
\hline & & 40 & 0.0100 & 998.47 & (90) & 0.000377 & 0.000386 & 998.4 & 0.0957 & -0.000010 & 0.009 & & & & & & 992.2158 \\
\hline & & 40 & 0.0200 & 1004.71 & (90) & 0.000381 & 0.000388 & 1004.6 & 0.1326 & -0.000007 & 0.018 & & & & & & 992.2158 \\
\hline 0.32974 & & 40 & 0.0240 & 1007.13 & (31) & 0.000386 & 0.000388 & 1007.1 & 0.0626 & -0.000003 & 0.004 & & & & & & 992.2158 \\
\hline 0.5041 & & 40 & 0.0362 & 1014.90 & (62) & 0.000386 & 0.000390 & 1014.7 & 0.1649 & -0.000004 & 0.027 & & & & & & 992.2158 \\
\hline
\end{tabular}




\begin{tabular}{|c|c|c|c|c|}
\hline molality & molarity & $\mathrm{t} /{ }^{\circ} \mathrm{C}$ & ass frac & $\begin{array}{r}\text { Density } \\
\exp / \\
\mathrm{kg} / \mathrm{m}^{3}\end{array}$ \\
\hline \multirow[t]{2}{*}{0.51841} & & 40 & 0.0372 & 1015.36 \\
\hline & & 40 & 0.0400 & 1017.27 \\
\hline 0.7069 & & 40 & 0.0501 & 1023.50 \\
\hline 0.991 & & 40 & 0.0688 & 1035.60 \\
\hline \multirow[t]{2}{*}{1.02409} & & 40 & 0.0709 & 1036.77 \\
\hline & & 40 & 0.0800 & 1042.78 \\
\hline 1.4139 & & 40 & 0.0954 & 1052.90 \\
\hline 1.5097 & & 40 & 0.1012 & 1056.50 \\
\hline \multirow[t]{2}{*}{1.54691} & & 40 & 0.1034 & 1057.84 \\
\hline & & 40 & 0.1200 & 1068.97 \\
\hline 2.0476 & & 40 & 0.1324 & 1077.30 \\
\hline 2.10665 & & 40 & 0.1357 & 1079.37 \\
\hline \multirow[t]{2}{*}{2.4968} & & 40 & 0.1569 & 1093.90 \\
\hline & & 40 & 0.1600 & 1096.00 \\
\hline 2.72517 & & 40 & 0.1689 & 1102.01 \\
\hline \multirow[t]{2}{*}{3.0013} & & 40 & 0.1828 & 1111.90 \\
\hline & & 40 & 0.2000 & 1123.99 \\
\hline 3.42161 & & 40 & 0.2032 & 1126.14 \\
\hline 3.5327 & & 40 & 0.2085 & 1130.10 \\
\hline 3.5347 & & 40 & 0.2086 & 1130.10 \\
\hline 3.93033 & & 40 & 0.2266 & 1142.93 \\
\hline \multirow[t]{2}{*}{3.9618} & & 40 & 0.2280 & 1144.20 \\
\hline & & 40 & 0.2400 & 1152.99 \\
\hline 4.55469 & & 40 & 0.2535 & 1162.72 \\
\hline \multirow[t]{11}{*}{4.6934} & & 40 & 0.2592 & 1167.30 \\
\hline & 0.00099 & 42.5 & 0.0001 & 991.29 \\
\hline & 0.00249 & 42.5 & 0.0002 & 991.36 \\
\hline & 0.00498 & 42.5 & 0.0004 & 991.47 \\
\hline & 0.00996 & 42.5 & 0.0007 & 991.70 \\
\hline & 0.01992 & 42.5 & 0.0015 & 992.17 \\
\hline & 0.03983 & 42.5 & 0.0030 & 993.10 \\
\hline & 0.04979 & 42.5 & 0.0037 & 993.56 \\
\hline & 0.09957 & 42.5 & 0.0075 & 995.87 \\
\hline & 0.19913 & 42.5 & 0.0148 & 1000.44 \\
\hline & 0.4977 & 42.5 & 0.0366 & 1013.99 \\
\hline 0.01501 & & 45 & 0.0011 & 990.96 \\
\hline 0.03002 & & 45 & 0.0022 & 991.64 \\
\hline 0.04 & & 45 & 0.0030 & 992.07 \\
\hline 0.05 & & 45 & 0.0037 & 992.50 \\
\hline 0.06004 & & 45 & 0.0045 & 993.00 \\
\hline 0.09 & & 45 & 0.0067 & 994.36 \\
\hline 0.1 & & 45 & 0.0074 & 994.81 \\
\hline 0.10001 & & 45 & 0.0074 & 994.79 \\
\hline 0.11084 & & 45 & 0.0082 & 995.30 \\
\hline 0.12006 & & 45 & 0.0089 & 995.73 \\
\hline 0.14024 & & 45 & 0.0103 & 996.63 \\
\hline 0.16 & & 45 & 0.0118 & 997.55 \\
\hline 0.19998 & & 45 & 0.0147 & 999.32 \\
\hline
\end{tabular}

Ref exp / Calc / $\begin{array}{ll}0.000390 & 0.000391\end{array}$ $0.000387 \quad 0.000391$ $\begin{array}{lll}0.000393 & 0.000393\end{array}$ $0.000394 \quad 0.000396$ $\begin{array}{lll}0.000397 & 0.000396\end{array}$ $\begin{array}{ll}0.000397 & 0.000397\end{array}$ $0.000399 \quad 0.000400$ $0.000402 \quad 0.000401$ $0.000403 \quad 0.000401$ $0.000405 \quad 0.000404$ $\begin{array}{lll}0.000407 & 0.000406\end{array}$ $0.000408 \quad 0.000407$ $\begin{array}{lll}0.000411 & 0.000410\end{array}$ $0.000411 \quad 0.000411$ $0.000413 \quad 0.000412$ $\begin{array}{lll}0.000415 & 0.000414\end{array}$ $\begin{array}{ll}0.000417 & 0.000417\end{array}$ $0.000418 \quad 0.000418$ $0.000418 \quad 0.000419$ $0.000418 \quad 0.000419$ $0.000421 \quad 0.000422$ $\begin{array}{lll}0.000421 & 0.000422\end{array}$ $\begin{array}{lll}0.000422 & 0.000424\end{array}$ $0.000425 \quad 0.000426$ $0.000425 \quad 0.000427$ $\begin{array}{lll}0.000331 & 0.000386\end{array}$ $0.000362 \quad 0.000386$ $\begin{array}{lll}0.000362 & 0.000386\end{array}$ $\begin{array}{lll}0.000376 & 0.000386\end{array}$ $\begin{array}{lll}0.000376 & 0.000386\end{array}$ $0.000376 \quad 0.000386$ 0.0003780 .000386 $0.000380 \quad 0.000387$ $0.000384 \quad 0.000388$ $\begin{array}{lll}0.000390 & 0.000392\end{array}$ $\begin{array}{lll}0.000330 & 0.000387\end{array}$ $\begin{array}{ll}0.000358 & 0.000387 \\ 0.000374 & 0.000387\end{array}$ $\begin{array}{lll}0.000374 & 0.000387\end{array}$ $\begin{array}{lll}0.000383 & 0.000387\end{array}$ $\begin{array}{lll}0.000374 & 0.000387\end{array}$ $\begin{array}{lll}0.000378 & 0.000388\end{array}$ 0.0003790 .000388 0.0003820 .000388 0.0003810 .000388 0.0003790 .000388 $\begin{array}{ll}0.000381 & 0.000388\end{array}$ $0.000380 \quad 0.000389$ $0.000383 \quad 0.000389$
Density Density App Vol Square of Inconsistent Inconsistent (Dens rel- Delta density Apparent Density of

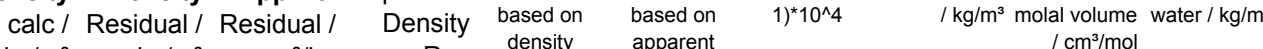
$\mathrm{kg} / \mathrm{m}^{3} \quad \mathrm{~kg} / \mathrm{m}^{3} \quad \mathrm{~m}^{3} / \mathrm{kg} \quad \operatorname{Res}$ density apparen

$\begin{array}{llll}1015.4 & 0.0012 & 0.000000 & 0.000\end{array}$ $\begin{array}{llll}1017.1 & 0.1521 & -0.000004 & 0.023\end{array}$

$\begin{array}{llll}1023.5 & 0.0036 & 0.000000 & 0.000\end{array}$

$\begin{array}{llll}1035.5 & 0.1075 & -0.000001 & 0.012\end{array}$

$\begin{array}{llll}1036.9 & -0.0991 & 0.000001 & 0.010\end{array}$

$\begin{array}{llll}1042.7 & 0.0413 & 0.000000 & 0.002\end{array}$

$\begin{array}{llll}1052.8 & 0.1300 & -0.000001 & 0.017\end{array}$

$\begin{array}{llll}1056.6 & -0.0904 & 0.000001 & 0.008 \\ 1058.1 & -0.2252 & 0.000002 & 0.051\end{array}$

$\begin{array}{lll}1058.1 & -0.2252 & 0.000002\end{array}$

$\begin{array}{llll}1077.4 & -0.1254 & 0.000001 & 0.016\end{array}$

$\begin{array}{llll}1079.7 & -0.2806 & 0.000002 & 0.079\end{array}$

$\begin{array}{llll}1094.1 & -0.1555 & 0.000001 & 0.024\end{array}$

$\begin{array}{llll}1096.2 & -0.1551 & 0.000001 & 0.024\end{array}$

$\begin{array}{llll}1102.3 & -0.2437 & 0.000001 & 0.059\end{array}$

$\begin{array}{llll}1111.9 & -0.0439 & 0.000000 & 0.002\end{array}$

$\begin{array}{llll}1124.0 & 0.0377 & 0.000000 & 0.001\end{array}$

$\begin{array}{llll}1126.2 & -0.1022 & 0.000000 & 0.010\end{array}$

$\begin{array}{llll}1129.9 & 0.1670 & -0.000001 & 0.028\end{array}$

$\begin{array}{llll}1130.0 & 0.1009 & 0.000000 & 0.010 \\ 1142.9 & 0.0786 & 0.000000 & 0.006\end{array}$

$1143.9-0.3453-0.000001$

$\begin{array}{lll}11525 & 0.5197-0.000002 & 0.270\end{array}$

$\begin{array}{llll}1162.3 & 0.4676 & -0.000001 & 0.219\end{array}$

$\begin{array}{llll}1166.4 & 0.8777 & -0.000002 & 0.770\end{array}$

$\begin{array}{llll}991.3 & 0.0040 & -0.000054 & 0.000\end{array}$

$\begin{array}{llll}991.4 & 0.0043 & -0.000023 & 0.000\end{array}$

$\begin{array}{llll}991.5 & 0.0086 & -0.000023 & 0.000\end{array}$

$\begin{array}{lll}991.7 & 0.0073-0.000010 & 0.000\end{array}$

$\begin{array}{llll}992.2 & 0.0148 & -0.000010 & 0.000\end{array}$

$\begin{array}{llll}993.1 & 0.0308 & -0.000010 & 0.001\end{array}$

$993.5-0.0289-0.000008-0.001$

$995.8-0.0527-0.000007$

$\begin{array}{lllll}1000.4 & 0.0637 & -0.000004 & 0.004\end{array}$

$\begin{array}{llll}1013.9 & 0.0442 & -0.000001 & 0.002\end{array}$

$\begin{array}{llll}990.9 & 0.0625 & -0.000057 & 0.004\end{array}$

$\begin{array}{llll}991.6 & 0.0648 & -0.000030 & 0.004\end{array}$

$\begin{array}{llll}992.0 & 0.0381 & -0.000013 & 0.001\end{array}$

$\begin{array}{llll}992.5 & 0.0145 & -0.000004 & 0.000\end{array}$

$\begin{array}{llll}992.9 & 0.0599 & -0.000014 & 0.004\end{array}$

$\begin{array}{lllll}994.3 & 0.0644 & -0.000010 & 0.004\end{array}$

$\begin{array}{llll}994.7 & 0.0629 & -0.000009 & 0.004\end{array}$

$\begin{array}{llll}994.7 & 0.0403 & -0.000006 & 0.002\end{array}$

$\begin{array}{llll}995.2 & 0.0603 & -0.000007 & 0.004\end{array}$

$\begin{array}{lllll}995.7 & 0.0835 & -0.000009 & 0.007\end{array}$

$\begin{array}{lllll}996.6 & 0.0708 & -0.000007 & 0.005\end{array}$

$\begin{array}{lllll}997.4 & 0.1031 & -0.000009 & 0.011\end{array}$

$\begin{array}{llll}999.2 & 0.0818 & -0.000006 & 0.007\end{array}$
992.2158

992.2158

992.2158

992.2158

992.2158

992.2158

992.2158

992.2158

992.2158

992.2158

992.2158

992.2158

992.2158

992.2158

992.2158

992.2158

992.2158

992.2158

992.2158

992.2158

992.2158

992.2158

992.2158

992.2158

992.2158

991.237

991.237

991.237

991.237

991.237

991.237

991.237

991.237

991.237

991.237

990.2132

$26.66 \quad 990.2132$

990.2132

990.2132

$27.87 \quad 990.2132$

990.2132

990.2132

$\begin{array}{lll}5.083 \quad 28.51 & 990.2132\end{array}$

$\begin{array}{lll}6.417 & 28.23 \quad 990.2132\end{array}$

990.2132

$28.59 \quad 990.2132$ 


\begin{tabular}{|c|c|c|c|c|c|c|c|}
\hline molality & molarity & \multicolumn{2}{|c|}{$t /{ }^{\circ} \mathrm{C}$ mass frac } & $\begin{array}{r}\text { Density } \\
\exp / \\
\mathrm{kg} / \mathrm{m}^{3}\end{array}$ & Ref & $\begin{array}{r}\text { App vol } \\
\exp / \\
m^{3} / \mathrm{kg}\end{array}$ & $\begin{array}{c}\text { App vol } \\
\text { Calc/ } \\
\mathrm{m}^{3} / \mathrm{kg}\end{array}$ \\
\hline 0.21499 & & 45 & 0.0158 & 1000.00 & (20) & 0.000384 & 0.000389 \\
\hline 0.25 & & 45 & 0.0183 & 1001.60 & (80) & 0.000382 & 0.000390 \\
\hline 0.25017 & & 45 & 0.0183 & 1001.57 & (81) & 0.000385 & 0.000390 \\
\hline 0.35002 & & 45 & 0.0254 & 1005.91 & (81) & 0.000390 & 0.000391 \\
\hline 0.4 & & 45 & 0.0290 & 1008.25 & (80) & 0.000386 & 0.000391 \\
\hline 0.43192 & & 45 & 0.0312 & 1009.61 & (20) & 0.000388 & 0.000392 \\
\hline 0.49966 & & 45 & 0.0359 & 1012.53 & (81) & 0.000390 & 0.000392 \\
\hline 0.49992 & & 45 & 0.0359 & 1012.45 & (81) & 0.000393 & 0.000392 \\
\hline 0.5 & & 45 & 0.0359 & 1012.47 & (44) & 0.000392 & 0.000392 \\
\hline 0.7 & & 45 & 0.0496 & 1021.23 & (80) & 0.000391 & 0.000395 \\
\hline 1 & & 45 & 0.0694 & 1033.71 & (44) & 0.000397 & 0.000398 \\
\hline 1 & & 45 & 0.0694 & 1033.79 & (80) & 0.000396 & 0.000398 \\
\hline 1.66876 & & 45 & 0.1106 & 1060.55 & (20) & 0.000405 & 0.000404 \\
\hline 2 & & 45 & 0.1298 & 1073.14 & (44) & 0.000408 & 0.000407 \\
\hline 2.10683 & & 45 & 0.1358 & 1077.30 & (20) & 0.000408 & 0.000408 \\
\hline 2.70941 & & 45 & 0.1681 & 1099.34 & (20) & 0.000413 & 0.000414 \\
\hline 3 & & 45 & 0.1828 & 1109.52 & (44) & 0.000416 & 0.000416 \\
\hline 3.02028 & & 45 & 0.1838 & 1110.28 & (20) & 0.000416 & 0.000416 \\
\hline 3.48132 & & 45 & 0.2061 & 1126.04 & (20) & 0.000419 & 0.000420 \\
\hline 3.98031 & & 45 & 0.2288 & 1142.46 & (20) & 0.000422 & 0.000424 \\
\hline 4 & & 45 & 0.2297 & 1142.70 & (44) & 0.000423 & 0.000424 \\
\hline 4.50107 & & 45 & 0.2513 & 1158.91 & (20) & 0.000425 & 0.000427 \\
\hline 0.01013 & & 48.75 & 0.0008 & 989.27 & (114) & 0.000093 & 0.000388 \\
\hline 0.03034 & & 48.75 & 0.0023 & 990.22 & (114) & 0.000275 & 0.000388 \\
\hline 0.10983 & & 48.75 & 0.0081 & 993.84 & (114) & 0.000355 & 0.000389 \\
\hline 0.19497 & & 48.75 & 0.0143 & 997.67 & (114) & 0.000369 & 0.000390 \\
\hline 0.29443 & & 48.75 & 0.0215 & 1002.09 & (114) & 0.000378 & 0.000391 \\
\hline 0.41553 & & 48.75 & 0.0300 & 1007.43 & (114) & 0.000382 & 0.000393 \\
\hline 0.57041 & & 48.75 & 0.0408 & 1014.15 & (114) & 0.000387 & 0.000394 \\
\hline 0.32974 & & 50 & 0.0240 & 1002.85 & (31) & 0.000389 & 0.000392 \\
\hline 0.51841 & & 50 & 0.0372 & 1011.01 & (31) & 0.000394 & 0.000394 \\
\hline 1.02409 & & 50 & 0.0709 & 1032.27 & (31) & 0.000401 & 0.000400 \\
\hline 1.54691 & & 50 & 0.1034 & 1053.23 & (31) & 0.000406 & 0.000405 \\
\hline 2.10665 & & 50 & 0.1357 & 1074.64 & (31) & 0.000411 & 0.000410 \\
\hline 2.72517 & & 50 & 0.1689 & 1097.18 & (31) & 0.000416 & 0.000415 \\
\hline 3.42161 & & 50 & 0.2032 & 1121.21 & (31) & 0.000421 & 0.000420 \\
\hline 3.93033 & & 50 & 0.2266 & 1137.95 & (31) & 0.000424 & 0.000424 \\
\hline 4.55469 & & 50 & 0.2535 & 1157.66 & (31) & 0.000427 & 0.000429 \\
\hline 0.00509 & & 55 & 0.0004 & 985.93 & (20) & 0.000377 & 0.000390 \\
\hline 0.01501 & & 55 & 0.0011 & 986.40 & (81) & 0.000362 & 0.000390 \\
\hline 0.01549 & & 55 & 0.0012 & 986.41 & (20) & 0.000381 & 0.000390 \\
\hline 0.03002 & & 55 & 0.0022 & 987.09 & (81) & 0.000373 & 0.000390 \\
\hline 0.04 & & 55 & 0.0030 & 987.53 & (80) & 0.000381 & 0.000390 \\
\hline 0.04933 & & 55 & 0.0037 & 987.98 & (20) & 0.000375 & 0.000390 \\
\hline 0.05 & & 55 & 0.0037 & 987.98 & (44) & 0.000383 & 0.000390 \\
\hline 0.06004 & & 55 & 0.0045 & 988.46 & (81) & 0.000379 & 0.000391 \\
\hline 0.09 & & 55 & 0.0067 & 989.81 & (80) & 0.000382 & 0.000391 \\
\hline 0.09271 & & 55 & 0.0069 & 989.92 & (20) & 0.000383 & 0.000391 \\
\hline
\end{tabular}

Density Density App Vol Square of Inconsistent Inconsistent (Dens rel- Delta density Apparent Density of calc/ Residual / Residual / Density $\begin{gathered}\text { based on } \\ \text { density }\end{gathered}$ $\mathrm{m}^{3} / \mathrm{kg} \quad$ Res density $\begin{gathered}\text { apparent } \\ \text { volume }\end{gathered}$

$999.9 \quad 0.0883-0.000006 \quad 0.008$ $\begin{array}{llll}1001.5 & 0.1319 & -0.000007 & 0.017\end{array}$ $\begin{array}{llll}1001.5 & 0.0932 & -0.000005 & 0.009\end{array}$ $\begin{array}{llll}1005.9 & 0.0179 & -0.000001 & 0.000\end{array}$ $\begin{array}{llll}1008.1 & 0.1553 & -0.000005 & 0.024\end{array}$ $\begin{array}{llll}009.5 & 0.1184 & -0.000004 & 0.014\end{array}$ $\begin{array}{llll}1012.4 & 0.0885 & -0.000002 & 0.008\end{array}$ $\begin{array}{llll}1012.5 & -0.0038 & 0.000000 & 0.000\end{array}$ $\begin{array}{llll}1012.5 & 0.0092 & 0.000000 & 0.000\end{array}$ $\begin{array}{llll}1021.1 & 0.1588 & -0.000003 & 0.025\end{array}$ $\begin{array}{llll}1033.7 & 0.0212 & 0.000000 & 0.000\end{array}$ $\begin{array}{llll}1033.7 & 0.1012 & -0.000001 & 0.010\end{array}$ $\begin{array}{llll}1060.6 & -0.0366 & 0.000000 & 0.001\end{array}$ $\begin{array}{llll}1073.3 & -0.1651 & 0.000001 & 0.027\end{array}$ $\begin{array}{llll}1077.3 & -0.0225 & 0.000000 & 0.001\end{array}$ $\begin{array}{llll}1099.3 & 0.0405 & 0.000000 & 0.002\end{array}$ $\begin{array}{llll}1109.5 & 0.0457 & 0.000000 & 0.002\end{array}$ $\begin{array}{llll}1110.2 & 0.1045 & 0.000000 & 0.011\end{array}$ $\begin{array}{lllll}1125.8 & 0.2724 & -0.000001 & 0.074\end{array}$ $\begin{array}{llll}1141.9 & 0.5225 & -0.000002 & 0.273\end{array}$ $\begin{array}{llll}1142.6 & 0.1343 & 0.000000 & 0.018\end{array}$ $\begin{array}{llll}1158.1 & 0.8142 & -0.000002 & 0.663\end{array}$ $\begin{array}{llll}989.1 & 0.2178 & -0.000295 & 0.047\end{array}$ $\begin{array}{llll}990.0 & 0.2505 & -0.000113 & 0.063\end{array}$ $\begin{array}{llll}993.6 & 0.2794 & -0.000035 & 0.078\end{array}$ $\begin{array}{lllll}997.4 & 0.2979 & -0.000021 & 0.089\end{array}$ $\begin{array}{llll}1001.8 & 0.2976 & -0.000014 & 0.089\end{array}$ $\begin{array}{llll}1007.1 & 0.3179 & -0.000010 & 0.101\end{array}$ $\begin{array}{llll}1013.8 & 0.3262 & -0.000008 & 0.106\end{array}$ $\begin{array}{llll}1002.8 & 0.0773 & -0.000003 & 0.006\end{array}$ $\begin{array}{llll}011.0 & 0.0077 & 0.000000 & 0.000\end{array}$ $\begin{array}{llll}032.4 & -0.0904 & 0.000001 & 0.008\end{array}$ $\begin{array}{llll}053.4 & -0.1890 & 0.000002 & 0.036\end{array}$ $\begin{array}{llll}1074.9 & -0.2383 & 0.000002 & 0.057\end{array}$ $\begin{array}{llll}097.4 & -0.1854 & 0.000001 & 0.034\end{array}$ $\begin{array}{llll}1121.3 & -0.0401 & 0.000000 & 0.002\end{array}$ $\begin{array}{llll}1137.8 & 0.1501 & -0.000001 & 0.023\end{array}$ $\begin{array}{llll}157.1 & 0.5148 & -0.000002 & 0.265\end{array}$ $\begin{array}{llll}985.9 & 0.0048 & -0.000013 & 0.000\end{array}$ $\begin{array}{llll}986.4 & 0.0303 & -0.000028 & 0.001\end{array}$ $\begin{array}{llll}986.4 & 0.0096 & -0.000009 & 0.000\end{array}$ $\begin{array}{lllll}987.1 & 0.0377 & -0.000017 & 0.001\end{array}$ $\begin{array}{llll}987.5 & 0.0283 & -0.000010 & 0.001\end{array}$ $\begin{array}{llll}987.9 & 0.0541 & -0.000015 & 0.003\end{array}$ $\begin{array}{lllll}988.0 & 0.0277 & -0.000008 & 0.001\end{array}$ $\begin{array}{llll}988.4 & 0.0524 & -0.000012 & 0.003\end{array}$ $\begin{array}{lllll}989.8 & 0.0596 & -0.000009 & 0.004\end{array}$ $\begin{array}{llll}989.9 & 0.0503 & -0.000007 & 0.003\end{array}$

\begin{tabular}{|c|c|c|}
\hline 9.783 & & 990.2132 \\
\hline & & 990.2132 \\
\hline & 28.67 & 990.2132 \\
\hline & 29.08 & 990.2132 \\
\hline & & 990.2132 \\
\hline 19.398 & & 990.2132 \\
\hline & 29.076 & 990.2132 \\
\hline & 29.263 & 990.2132 \\
\hline & & 990.2132 \\
\hline & & 990.2132 \\
\hline & & 990.2132 \\
\hline & & 990.2132 \\
\hline 70.334 & & 990.2132 \\
\hline & & 990.2132 \\
\hline 87.091 & & 990.2132 \\
\hline 109.124 & & 990.2132 \\
\hline & & 990.2132 \\
\hline 120.066 & & 990.2132 \\
\hline 135.824 & & 990.2132 \\
\hline 152.251 & & 990.2132 \\
\hline & & 990.2132 \\
\hline 168.695 & & 990.2132 \\
\hline & & 988.5963 \\
\hline & & 988.5963 \\
\hline & & 988.5963 \\
\hline & & 988.5963 \\
\hline & & 988.5963 \\
\hline & & 988.5963 \\
\hline & & 988.5963 \\
\hline & & 988.0363 \\
\hline & & 988.0363 \\
\hline & & 988.0363 \\
\hline & & 988.0363 \\
\hline & & 988.0363 \\
\hline & & 988.0363 \\
\hline & & 988.0363 \\
\hline & & 988.0363 \\
\hline & & 988.0363 \\
\hline 0.235 & & 985.6952 \\
\hline & 27 & 985.6952 \\
\hline 0.71 & & 985.6952 \\
\hline & 27.8 & 985.6952 \\
\hline & & 985.6952 \\
\hline 2.281 & & 985.6952 \\
\hline & & 985.6952 \\
\hline & 28.22 & 985.6952 \\
\hline & & 985.6952 \\
\hline 4.227 & & 985.6952 \\
\hline
\end{tabular}




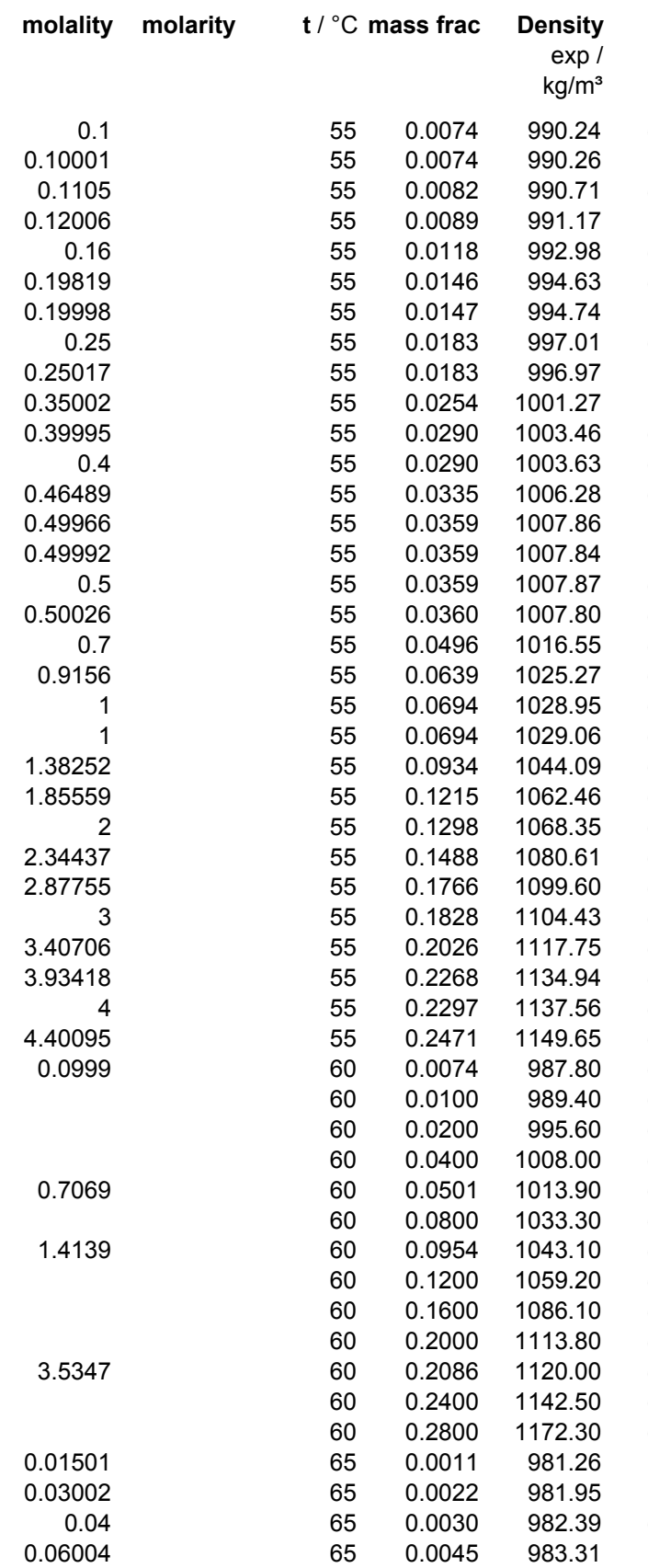

Ref

$\begin{array}{rr}\text { App vol } & \text { App vol } \\ \exp / & \text { Calc / } \\ \mathrm{m}^{3} / \mathrm{kg} & \mathrm{m}^{3} / \mathrm{kg}\end{array}$

(44) 0.0003850 .000391

$\begin{array}{lll}\text { (81) } & 0.000383 & 0.000391\end{array}$

$0.000387 \quad 0.000391$

$\begin{array}{lll}0.000383 & 0.000391\end{array}$

$\begin{array}{lll}0.000383 & 0.000392\end{array}$

$\begin{array}{lll}0.000389 & 0.000392\end{array}$

$\begin{array}{lll}0.000387 & 0.000392\end{array}$

$0.000385 \quad 0.000393$

0.0003880 .000393

$\begin{array}{lll}0.000394 & 0.000394\end{array}$

$\begin{array}{lll}0.000394 & 0.000394\end{array}$

$0.000388 \quad 0.000394$

$0.000395 \quad 0.000395$

$\begin{array}{ll}0.000393 & 0.000395 \\ 0.000394 & 0.000395\end{array}$

$\begin{array}{lll}0.000393 & 0.000395\end{array}$

$0.000396 \quad 0.000395$

$\begin{array}{ll}0.000394 & 0.000397\end{array}$

$\begin{array}{ll}0.000402 & 0.000400 \\ 0.000400 & 0.000400\end{array}$

$\begin{array}{ll}0.000400 & 0.000400 \\ 0.000398 & 0.000400\end{array}$

$\begin{array}{ll}0.000407 & 0.000404\end{array}$

$\begin{array}{ll}0.000407 & 0.000404 \\ 0.000411 & 0.000408\end{array}$

$\begin{array}{ll}0.000411 & 0.000408 \\ 0.000410 & 0.000410\end{array}$

$0.000416 \quad 0.000413$

$0.000420 \quad 0.000417$

$0.000418 \quad 0.000418$

$\begin{array}{lll}0.000423 & 0.000421\end{array}$

$\begin{array}{ll}0.000426 & 0.000425\end{array}$

$\begin{array}{lll}0.000425 & 0.000425\end{array}$

$0.000429 \quad 0.000428$

0.0003760 .000392

$0.000380 \quad 0.000392$

$\begin{array}{lll}0.000384 & 0.000394\end{array}$

$\begin{array}{ll}0.000391 & 0.000397\end{array}$

$\begin{array}{lll}0.000402 & 0.000398\end{array}$

$0.000401 \quad 0.000403$

$0.000405 \quad 0.000405$

$\begin{array}{lll}0.000409 & 0.000409\end{array}$

$\begin{array}{lll}0.000415 & 0.000415\end{array}$

$0.000421 \quad 0.000421$

$\begin{array}{lll}0.000421 & 0.000422\end{array}$

$\begin{array}{ll}0.000426 & 0.000427 \\ 0.000431 & 0.000434\end{array}$

$0.000362 \quad 0.00$

$\begin{array}{lll}0.000362 & 0.000392\end{array}$

$\begin{array}{ll}0.000370 & 0.000392 \\ 0.000379 & 0.000392\end{array}$

$0.000379 \quad 0.000392$
Density Density App Vol Square of Inconsistent Inconsistent (Dens rel- Delta density Apparent Density of calc / Residual / Residual / Density based on based on 1) $10^{\wedge} 4 \quad / \mathrm{kg} / \mathrm{m}^{3}$ molal volume water $/ \mathrm{kg} / \mathrm{m}^{3}$ $\mathrm{kg} / \mathrm{m}^{3} \quad \mathrm{~kg} / \mathrm{m}^{3} \quad \mathrm{~m}^{3} / \mathrm{kg} \quad$ Res density apparent

$\begin{array}{llll}990.2 & 0.0412 & -0.000006 & 0.002\end{array}$

$\begin{array}{llll}990.2 & 0.0566 & -0.000008 & 0.003\end{array}$

$\begin{array}{llll}990.7 & 0.0370 & -0.000005 & 0.001\end{array}$

$\begin{array}{llll}991.1 & 0.0734 & -0.000008 & 0.005\end{array}$

$\begin{array}{llll}992.9 & 0.0991 & -0.000009 & 0.010\end{array}$

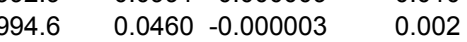

$994.7 \quad 0.0786-0.000005 \quad 0.006$

$\begin{array}{llll}996.9 & 0.1339 & -0.000007 & 0.018\end{array}$

$\begin{array}{llll}996.9 & 0.0840 & -0.000005 & 0.007\end{array}$

$\begin{array}{llll}1001.3 & -0.0093 & 0.000000 & 0.000\end{array}$

$\begin{array}{llll}1003.5 & 0.0046 & 0.000000 & 0.000\end{array}$

$\begin{array}{llll}1003.5 & 0.1691 & -0.000006 & 0.029\end{array}$

$\begin{array}{llll}1006.3 & 0.0011 & 0.000000 & 0.000\end{array}$

$\begin{array}{llll}1007.8 & 0.0697 & -0.000002 & 0.005\end{array}$

$\begin{array}{llll}1007.8 & 0.0415 & -0.000001 & 0.002\end{array}$

$\begin{array}{llll}1007.8 & 0.0699 & -0.000002 & 0.005\end{array}$

$\begin{array}{llll}1007.8 & -0.0121 & 0.000000 & 0.000\end{array}$

$1016.4 \quad 0.1908-0.000004 \quad 0.036$

$\begin{array}{llll}1025.4 & -0.1456 & 0.000002 & 0.021\end{array}$

$\begin{array}{llll}1028.9 & 0.0442 & -0.000001 & 0.002\end{array}$

$\begin{array}{llll}1062.9 & -0.4023 & 0.000003 & 0.162\end{array}$

$\begin{array}{llll}1068.3 & 0.0166 & 0.000000 & 0.000\end{array}$

$\begin{array}{llll}1081.1 & -0.4961 & 0.000003 & 0.246\end{array}$

$\begin{array}{llll}1100.1 & -0.5310 & 0.000002 & 0.282\end{array}$

$\begin{array}{llll}1104.4 & 0.0501 & 0.000000 & 0.003\end{array}$

$\begin{array}{llll}1118.2 & -0.4206 & 0.000002 & 0.177\end{array}$

$\begin{array}{llll}1135.3 & -0.3796 & 0.000001 & 0.144\end{array}$

$\begin{array}{llll}1137.4 & 0.1548 & -0.000001 & 0.024\end{array}$

$\begin{array}{llll}1149.9 & -0.2232 & 0.000001 & 0.050\end{array}$

$\begin{array}{llll}987.7 & 0.1132 & -0.000016 & 0.013\end{array}$

$\begin{array}{llll}989.3 & 0.1246 & -0.000013 & 0.016\end{array}$

$\begin{array}{llll}995.4 & 0.2019 & -0.000010 & 0.041\end{array}$

$\begin{array}{llll}1007.8 & 0.2172 & -0.000005 & 0.047\end{array}$

$\begin{array}{llll}1014.1 & -0.1856 & 0.000004 & 0.034\end{array}$

$\begin{array}{llll}1033.1 & 0.1851 & -0.000002 & 0.034\end{array}$

$\begin{array}{llll}1043.0 & 0.0554 & -0.000001 & 0.003\end{array}$

$\begin{array}{llll}1059.2 & -0.0075 & 0.000000 & 0.000\end{array}$

$\begin{array}{llll}1086.1 & 0.0295 & 0.000000 & 0.001\end{array}$

$\begin{array}{llll}1113.7 & 0.0882 & 0.000000 & 0.008\end{array}$

$\begin{array}{llll}1119.7 & 0.2670 & -0.000001 & 0.071\end{array}$

$\begin{array}{llll}1142.1 & 0.3637 & -0.000001 & 0.132 \\ 1171.3 & 0.9545 & -0.000002 & 0.911\end{array}$

$\begin{array}{llll}981.2 & 0.0318-0.000030 & 0.001\end{array}$

$\begin{array}{llll}981.9 & 0.0477-0.000022 & 0.002\end{array}$

$\begin{array}{lllll}982.4 & 0.0368 & -0.000013 & 0.001\end{array}$

$\begin{array}{llll}983.3 & 0.0564 & -0.000013 & 0.003\end{array}$
985.6952

985.6952

$5.011 \quad 985.6952$

$28.54 \quad 985.6952$

985.6952

8.931

$28.83 \quad 985.6952$

985.6952

$28.93 \quad 985.6952$

$17.768 \quad 29.382 \quad 985.6952$

985.6952

985.6952

20.587

$29.331 \quad 985.6952$

$29.389 \quad 985.6952$

985.6952

985.6952
985.6952

$\begin{array}{ll}22.104 & 985.6952 \\ & 985.6952\end{array}$

39.57

985.6952

985.6952

$58.396 \quad 985.6952$

$76.76 \quad 985.6952$

985.6952

985.6952

$113.909 \quad 985.6952$

985.6952

985.6952

132.053

$149.243 \quad 985.6952$

985.6952

985.6952

983.1989

983.1989

983.1989

983.1989

983.1989

983.1989

983.1989

983.1989

983.1989

983.1989

983.1989

983.1989

983.1989

\begin{tabular}{l}
$27 \quad 980.5548$ \\
\hline
\end{tabular}

$27.56 \quad 980.5548$

980.5548

$28.26 \quad 980.5548$ 
molality molarity

$\mathrm{t} /{ }^{\circ} \mathrm{C}$ mass frac Density

$\exp /$
$\mathrm{kg} / \mathrm{m}^{3}$

0.09

0.09998

0.10001

0.12006

0.16

0.19998
0.25

0.25017

0.35002

0.41494

0.49966
0.49992

0.7

0.79154

1.21081

2.0536

2.49408

3.42506

3.88956

4.40092

0.01013

0.03034

0.10983

0.19497

0.29443

0.41553

0.57041

0.01501

0.03002

0.04

0.09

0.10001

0.1001

0.12006

0.16

0.19998

0.25
25017

0.35002

0.4

0.40826

0.49966

0.49992

0.7
0.7083

$\begin{array}{rrr}65 & 0.0067 & 984.66 \\ 65 & 0.0074 & 985.12 \\ 65 & 0.0074 & 985.10 \\ 65 & 0.0089 & 986.02 \\ 65 & 0.0118 & 987.82 \\ 65 & 0.0147 & 989.59 \\ 65 & 0.0183 & 991.84 \\ 65 & 0.0183 & 991.80 \\ 65 & 0.0254 & 996.11 \\ 65 & 0.0290 & 998.45 \\ 65 & 0.0300 & 999.03 \\ 65 & 0.0359 & 1002.66 \\ 65 & 0.0359 & 1002.65 \\ 65 & 0.0496 & 1011.34 \\ 65 & 0.0557 & 1015.08 \\ 65 & 0.0694 & 1023.82 \\ 65 & 0.0828 & 1031.80 \\ 65 & 0.1328 & 1064.97 \\ 65 & 0.1568 & 1081.15 \\ 65 & 0.2034 & 1113.49 \\ 65 & 0.2248 & 1128.84 \\ 65 & 0.2471 & 1144.97 \\ 73.77 & 0.0008 & 976.27 \\ 73.77 & 0.0023 & 977.19 \\ 73.77 & 0.0081 & 980.78 \\ 73.77 & 0.0143 & 984.58 \\ 73.77 & 0.0215 & 988.98 \\ 73.77 & 0.0300 & 994.27 \\ 73.77 & 0.0408 & 1000.93 \\ 75 & 0.0011 & 975.56 \\ 75 & 0.0022 & 976.26 \\ 75 & 0.0030 & 976.70 \\ 75 & 0.0045 & 977.62 \\ 75 & 0.0067 & 978.98 \\ 75 & 0.0074 & 979.40 \\ 75 & 0.0074 & 979.50 \\ 75 & 0.0089 & 980.32 \\ 75 & 0.0118 & 982.14 \\ 75 & 0.0147 & 983.87 \\ 75 & 0.0183 & 986.16 \\ 75 & 0.0183 & 986.10 \\ 75 & 0.0254 & 990.40 \\ 75 & 0.0290 & 992.77 \\ 75 & 0.0295 & 993.01 \\ 75 & 0.0359 & 996.97 \\ 75 & 0.0359 & 996.97 \\ 75 & 0.0496 & 1005.65 \\ 75 & 0.0502 & 1006.20 \\ & & \\ & \end{array}$

\section{exp / App vol}

$\mathrm{m}^{3 / \mathrm{kg}} \quad \mathrm{m}^{3} / \mathrm{kg}$

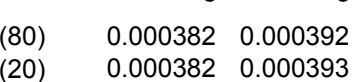

$\begin{array}{lll}\text { (81) } & 0.000384 & 0.000393\end{array}$

$0.000383 \quad 0.000393$

$0.000384 \quad 0.000393$

$\begin{array}{ll}0.000386 & 0.000394\end{array}$

$\begin{array}{ll}0.000386 & 0.000394 \\ 0.000389 & 0.000394\end{array}$

$\begin{array}{ll}0.000394 & 0.000395\end{array}$

$0.000389 \quad 0.000396$

$\begin{array}{lll}0.000391 & 0.000396\end{array}$

$0.000394 \quad 0.000397$

$\begin{array}{ll}0.000395 & 0.000397 \\ 0.000394 & 0.000399\end{array}$

$\begin{array}{ll}0.000394 & 0.000399 \\ 0.000397 & 0.000400\end{array}$

$0.000399 \quad 0.000402$

$\begin{array}{lll}0.000408 & 0.000404\end{array}$

0.0004110 .000411

$0.000415 \quad 0.000415$

$0.000421 \quad 0.000422$

$0.000424 \quad 0.000425$

$\begin{array}{lll}0.000427 & 0.000428\end{array}$

$\begin{array}{lll}0.000061 & 0.000392\end{array}$

$\begin{array}{ll}0.000276 & 0.000392\end{array}$

$\begin{array}{lll}0.000356 & 0.000393\end{array}$

0.0003710 .000394

$0.000379 \quad 0.000395$

$0.000384 \quad 0.000396$

$\begin{array}{ll}0.000389 & 0.000397\end{array}$

$\begin{array}{lll}0.000358 & 0.000392\end{array}$

$\begin{array}{ll}0.000364 & 0.000392 \\ 0.000372 & 0.000392\end{array}$

$\begin{array}{ll}0.000373 & 0.000392\end{array}$

$\begin{array}{ll}0.000376 & 0.000393\end{array}$

$\begin{array}{ll}0.000382 & 0.000393\end{array}$

$\begin{array}{lll}0.000368 & 0.000393\end{array}$

0.0003800 .000393

0.0003800 .000393

$\begin{array}{lll}0.000385 & 0.000394\end{array}$

$\begin{array}{ll}0.000386 & 0.000394\end{array}$

$0.000392 \quad 0.000395$

$0.000386 \quad 0.000396$

$\begin{array}{lll}0.000391 & 0.000396\end{array}$

$\begin{array}{ll}0.000392 & 0.000397\end{array}$

$0.000392 \quad 0.000397$

0.0003920 .000399

0.0003890 .000399
Density Density App Vol Square of Inconsistent Inconsistent (Dens rel- Delta density Apparent Density of calc/ Residual / Residual / Density based on based on 1$)^{*} 10^{\wedge} 4 \quad / \mathrm{kg} / \mathrm{m}^{3}$ molal volume water $/ \mathrm{kg} / \mathrm{m}^{3}$ $\mathrm{kg} / \mathrm{m}^{3} \quad \mathrm{~kg} / \mathrm{m}^{3} \quad \mathrm{~m}^{3} / \mathrm{kg} \quad \operatorname{Res}$ density $\begin{gathered}\text { apparent } \\ \text { volume }\end{gathered}$

$\begin{array}{llll}984.6 & 0.0681 & -0.000011 & 0.005\end{array}$

$\begin{array}{llll}985.0 & 0.0793 & -0.000011 & 0.006\end{array}$

$\begin{array}{llll}985.0 & 0.0614 & -0.000009 & 0.004\end{array}$

$\begin{array}{llll}985.9 & 0.0849 & -0.000010 & 0.007\end{array}$

$\begin{array}{llll}987.7 & 0.1110 & -0.000010 & 0.012\end{array}$

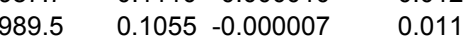

$\begin{array}{llll}991.7 & 0.1526 & -0.000008 & 0.023\end{array}$

$\begin{array}{llll}991.7 & 0.1005 & -0.000006 & 0.010\end{array}$

$\begin{array}{llll}996.1 & 0.0371 & -0.000001 & 0.001\end{array}$

$\begin{array}{llll}998.2 & 0.2041 & -0.000007 & 0.042\end{array}$

$\begin{array}{llll}998.9 & 0.1396 & -0.000005 & 0.019\end{array}$

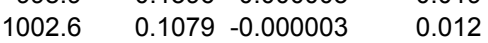

$\begin{array}{llll}1002.6 & 0.0799 & -0.000002 & 0.006\end{array}$

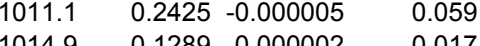

$1023.6-0.2151-0.000003$

$10322-0.3981-0.000005-0.158$

$\begin{array}{llll}1065.0 & 0.0042 & 0.000000 & 0.000\end{array}$

$\begin{array}{llll}1081.1 & -0.0002 & 0.000000 & 0.000\end{array}$

$\begin{array}{llll}1113.4 & 0.1114 & 0.000000 & 0.012\end{array}$

$\begin{array}{llll}1128.5 & 0.3070 & -0.000001 & 0.094\end{array}$

$\begin{array}{lll}1144.5 & 0.4238-0.000001 & 0.180\end{array}$

$\begin{array}{llll}976.0 & 0.2383 & -0.000331 & 0.057\end{array}$

$\begin{array}{llll}976.9 & 0.2508 & -0.000116 & 0.063\end{array}$

$\begin{array}{llll}980.5 & 0.2872 & -0.000037 & 0.082\end{array}$

$\begin{array}{llll}984.3 & 0.3173 & -0.000023 & 0.101\end{array}$

$\begin{array}{llll}988.6 & 0.3411 & -0.000016 & 0.116\end{array}$

$\begin{array}{llll}993.9 & 0.3639 & -0.000012 & 0.132\end{array}$

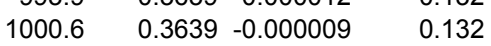

$\begin{array}{llll}975.5 & 0.0359 & -0.000034 & 0.001\end{array}$

$\begin{array}{llll}976.2 & 0.0601 & -0.000028 & 0.004\end{array}$

$\begin{array}{llll}976.6 & 0.0572 & -0.000020 & 0.003\end{array}$

$\begin{array}{llll}977.5 & 0.0823 & -0.000019 & 0.007\end{array}$

$\begin{array}{llll}978.9 & 0.1039 & -0.000016 & 0.011\end{array}$

$\begin{array}{llll}979.3 & 0.0734 & -0.000010 & 0.005\end{array}$

$\begin{array}{llll}979.3 & 0.1740 & -0.000024 & 0.030\end{array}$

$\begin{array}{llll}980.2 & 0.1093 & -0.000013 & 0.012\end{array}$

$0.1195-0.000008$

$986.0 \quad 0.2043-0.000011$

$0.1396-0.000008$

$0.1396-0.000008$

$0.0693-0.000003$

$\begin{array}{llll}992.5 & 0.2684 & -0.000009 & 0.072\end{array}$

$\begin{array}{llll}992.9 & 0.1535 & -0.000005 & 0.024\end{array}$

$\begin{array}{llll}996.8 & 0.1628 & -0.000005 & 0.027\end{array}$

$\begin{array}{llll}996.8 & 0.1563 & -0.000004 & 0.024\end{array}$

$\begin{array}{llll}1005.3 & 0.3161 & -0.000006 & 0.100\end{array}$

$\begin{array}{llll}1005.7 & 0.5159 & -0.000010 & 0.266\end{array}$

\begin{tabular}{|c|c|c|}
\hline & & 980.5548 \\
\hline \multirow[t]{9}{*}{4.562} & & 980.5548 \\
\hline & 28.63 & 980.5548 \\
\hline & 28.55 & 980.5548 \\
\hline & & 980.5548 \\
\hline & 28.8 & 980.5548 \\
\hline & & 980.5548 \\
\hline & 28.97 & 980.5548 \\
\hline & 29.354 & 980.5548 \\
\hline & & 980.5548 \\
\hline \multirow[t]{4}{*}{18.479} & & 980.5548 \\
\hline & 29.355 & 980.5548 \\
\hline & 29.413 & 980.5548 \\
\hline & & 980.5548 \\
\hline \multirow[t]{2}{*}{34.524} & & 980.5548 \\
\hline & & 980.5548 \\
\hline 51.241 & & 980.5548 \\
\hline 84.413 & & 980.5548 \\
\hline 00.591 & & 980.5548 \\
\hline 132.94 & & 980.5548 \\
\hline 48.288 & & 980.5548 \\
\hline \multirow[t]{22}{*}{64.417} & & 980.5548 \\
\hline & & 975.5797 \\
\hline & & 975.5797 \\
\hline & & 975.5797 \\
\hline & & 975.5797 \\
\hline & & 975.5797 \\
\hline & & 975.5797 \\
\hline & & 975.5797 \\
\hline & 26.7 & 974.849 \\
\hline & 27.12 & 974.849 \\
\hline & & 974.849 \\
\hline & 27.81 & 974.849 \\
\hline & & 974.849 \\
\hline & 28.51 & 974.849 \\
\hline & & 974.849 \\
\hline & 28.34 & 974.849 \\
\hline & & 974.849 \\
\hline & 28.73 & 974.849 \\
\hline & & 974.849 \\
\hline & 28.81 & 974.849 \\
\hline & 29.262 & 974.849 \\
\hline & & 974.849 \\
\hline \multirow[t]{4}{*}{18.164} & & 974.849 \\
\hline & 29.239 & 974.849 \\
\hline & 29.253 & 974.849 \\
\hline & & 974.849 \\
\hline
\end{tabular}




\begin{tabular}{|c|c|c|c|c|}
\hline molality & molarity & $\mathbf{t} /{ }^{\circ} \mathrm{C}$ & ass frac & $\begin{array}{r}\text { Density } \\
\exp / \\
\mathrm{kg} / \mathrm{m}^{3}\end{array}$ \\
\hline 0.81966 & & 75 & 0.0576 & 1010.46 \\
\hline 1 & & 75 & 0.0694 & 1018.13 \\
\hline 1.23664 & & 75 & 0.0844 & 1027.49 \\
\hline 1.4166 & & 75 & 0.0955 & 1034.80 \\
\hline 1.71254 & & 75 & 0.1132 & 1046.14 \\
\hline 2.10774 & & 75 & 0.1358 & 1061.04 \\
\hline 2.54781 & & 75 & 0.1596 & 1077.12 \\
\hline 3.01639 & & 75 & 0.1836 & 1093.70 \\
\hline 3.50044 & & 75 & 0.2070 & 1110.02 \\
\hline 3.5415 & & 75 & 0.2089 & 1111.80 \\
\hline 3.99611 & & 75 & 0.2295 & 1126.19 \\
\hline 4.50034 & & 75 & 0.2512 & 1142.00 \\
\hline & & 80 & 0.0100 & 978.00 \\
\hline & & 80 & 0.0200 & 984.20 \\
\hline & & 80 & 0.0400 & 996.60 \\
\hline & & 80 & 0.0800 & 1021.90 \\
\hline & & 80 & 0.1200 & 1047.80 \\
\hline & & 80 & 0.1600 & 1074.60 \\
\hline & & 80 & 0.2000 & 1102.40 \\
\hline & & 80 & 0.2400 & 1131.10 \\
\hline & & 80 & 0.2800 & 1160.90 \\
\hline 0.01501 & & 85 & 0.0011 & 969.31 \\
\hline 0.03002 & & 85 & 0.0022 & 970.00 \\
\hline 0.03254 & & 85 & 0.0024 & 970.12 \\
\hline 0.04 & & 85 & 0.0030 & 970.51 \\
\hline 0.06004 & & 85 & 0.0045 & 971.40 \\
\hline 0.06598 & & 85 & 0.0049 & 971.63 \\
\hline 0.09 & & 85 & 0.0067 & 972.78 \\
\hline 0.10001 & & 85 & 0.0074 & 973.18 \\
\hline 0.10045 & & 85 & 0.0074 & 973.20 \\
\hline 0.12006 & & 85 & 0.0089 & 974.09 \\
\hline 0.16 & & 85 & 0.0118 & 975.95 \\
\hline 0.19998 & & 85 & 0.0147 & 977.66 \\
\hline 0.20567 & & 85 & 0.0151 & 977.94 \\
\hline 0.25 & & 85 & 0.0183 & 979.98 \\
\hline 0.25017 & & 85 & 0.0183 & 979.89 \\
\hline 0.35002 & & 85 & 0.0254 & 984.20 \\
\hline 0.4 & & 85 & 0.0290 & 986.60 \\
\hline 0.49966 & & 85 & 0.0359 & 990.77 \\
\hline 0.49992 & & 85 & 0.0359 & 990.78 \\
\hline 0.7 & & 85 & 0.0496 & 999.50 \\
\hline 1 & & 85 & 0.0694 & 1012.00 \\
\hline 1.2306 & & 85 & 0.0840 & 1021.12 \\
\hline 1.66106 & & 85 & 0.1102 & 1038.09 \\
\hline 3.03015 & & 85 & 0.1843 & 1088.14 \\
\hline 3.50912 & & 85 & 0.2074 & 1104.33 \\
\hline 4.00293 & & 85 & 0.2298 & 1120.47 \\
\hline 4.50084 & & 85 & 0.2513 & 1136.10 \\
\hline
\end{tabular}

App vol Appuol

$\begin{array}{rr}\text { exp / } & \text { Calc / } \\ \mathrm{m}^{3} / \mathrm{kg} & \mathrm{m}^{3} / \mathrm{kg}\end{array}$

$\begin{array}{ll}0.000398 & 0.000400 \\ 0.000397 & 0.000401\end{array}$ $\begin{array}{ll}0.000397 & 0.000401\end{array}$ $0.000403 \quad 0.000404$ $\begin{array}{lll}0.000404 & 0.000405\end{array}$ $\begin{array}{lll}0.000408 & 0.000408\end{array}$ $\begin{array}{lll}0.000412 & 0.000411\end{array}$ $\begin{array}{ll}0.000416 & 0.000414\end{array}$ $0.000419 \quad 0.000418$ 0.0004220 .000421 $0.000421 \quad 0.000422$ $\begin{array}{lll}0.000425 & 0.000425\end{array}$ $\begin{array}{ll}0.000428 & 0.000428\end{array}$ $\begin{array}{lll}0.000376 & 0.000393\end{array}$ $0.000381 \quad 0.000394$ 0.0003890 .000397 $\begin{array}{lll}0.000398 & 0.000402\end{array}$ $\begin{array}{lll}0.000407 & 0.000408\end{array}$ $0.000414 \quad 0.000414$ $0.000419 \quad 0.000419$ $0.000425 \quad 0.000425$ $0.000430 \quad 0.000431$ $\begin{array}{lll}0.000373 & 0.000391\end{array}$ $\begin{array}{lll}0.000373 & 0.000391\end{array}$ $\begin{array}{ll}0.000375 & 0.000391\end{array}$ $0.000356 \quad 0.000391$ $0.000370 \quad 0.000391$ 0.0003790 .000391 $\begin{array}{lll}0.000370 & 0.000392\end{array}$ $\begin{array}{lll}0.000379 & 0.000392\end{array}$ $\begin{array}{lll}0.000379 & 0.000392\end{array}$ $0.000379 \quad 0.000392$ 0.0003750 .000392 $0.000383 \quad 0.000393$ $\begin{array}{lll}0.000381 & 0.000393\end{array}$ $\begin{array}{ll}0.000378 & 0.000393\end{array}$ $0.000384 \quad 0.000393$ $\begin{array}{ll}0.000390 & 0.000394\end{array}$ $0.000383 \quad 0.000395$ $0.000390 \quad 0.000396$ $0.000390 \quad 0.000396$ $\begin{array}{ll}0.000389 & 0.000397\end{array}$ $\begin{array}{lll}0.000395 & 0.000400\end{array}$ $\begin{array}{ll}0.000401 & 0.000402\end{array}$ $\begin{array}{lll}0.000405 & 0.000406\end{array}$ $\begin{array}{lll}0.000417 & 0.000416\end{array}$ $\begin{array}{ll}0.000421 & 0.000419\end{array}$ $\begin{array}{ll}0.000424 & 0.000422\end{array}$ $\begin{array}{ll}0.000427 & 0.000425\end{array}$
Density Density App Vol Square of Inconsistent Inconsistent (Dens rel- Delta density Apparent Density of calc/ Residual/ Residual / Density based on based on 1$)^{\star} 10^{\wedge} 4 \quad / \mathrm{kg} / \mathrm{m}^{3}$ molal volume water $/ \mathrm{kg} / \mathrm{m}^{3}$ $\mathrm{kg} / \mathrm{m}^{3} \quad \mathrm{~kg} / \mathrm{m}^{3} \quad \mathrm{~m}^{3} / \mathrm{kg} \quad$ Res density $\begin{gathered}\text { apparent } \\ \text { volume }\end{gathered}$

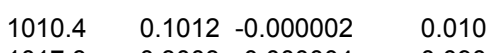

$\begin{array}{llll}1017.8 & 0.3003 & -0.000004 & 0.090\end{array}$

$\begin{array}{llll}1027.5 & 0.0329 & 0.000000 & 0.001\end{array}$

$\begin{array}{llll}1034.6 & 0.1557 & -0.000002 & 0.024\end{array}$

$\begin{array}{llll}1046.2 & -0.0828 & 0.000001 & 0.007\end{array}$

$\begin{array}{llll}1061.2 & -0.1854 & 0.000001 & 0.034\end{array}$

$\begin{array}{llll}1077.4 & -0.2348 & 0.000001 & 0.055\end{array}$

$\begin{array}{llll}1093.9 & -0.1652 & 0.000001 & 0.027\end{array}$

$\begin{array}{llll}1110.3 & -0.2444 & 0.000001 & 0.060\end{array}$

$\begin{array}{llll}1111.6 & 0.1761 & -0.000001 & 0.031\end{array}$

$\begin{array}{llll}1126.4 & -0.1894 & 0.000001 & 0.036\end{array}$

$\begin{array}{llll}1142.1 & -0.1256 & 0.000000 & 0.016\end{array}$

$\begin{array}{llll}977.8 & 0.1555 & -0.000016 & 0.024\end{array}$

$\begin{array}{llll}983.9 & 0.2598 & -0.000013 & 0.067 \\ 996.3 & 0.3195 & -0.000008 & 0.102\end{array}$

$1021.6 \quad 0.3351-0.000004$

$\begin{array}{llll}1047.7 & 0.1280 & -0.000001 & 0.016\end{array}$

$\begin{array}{llll}1074.6 & -0.0217 & 0.000000 & 0.000\end{array}$

$\begin{array}{llll}1102.4 & -0.0326 & 0.000000 & 0.001\end{array}$

$\begin{array}{llll}1131.1 & -0.0219 & 0.000000 & 0.000\end{array}$

$\begin{array}{llll}1160.7 & 0.1954 & -0.000001 & 0.038\end{array}$

$\begin{array}{llll}969.3 & 0.0188 & -0.000018 & 0.000\end{array}$

$\begin{array}{llll}970.0 & 0.0383 & -0.000018 & 0.001\end{array}$

$\begin{array}{llll}970.1 & 0.0374 & -0.000016 & 0.001\end{array}$

$\begin{array}{llll}970.4 & 0.0973 & -0.000035 & 0.009\end{array}$

$\begin{array}{lllll}971.3 & 0.0897 & -0.000021 & 0.008\end{array}$

$\begin{array}{llll}971.6 & 0.0568 & -0.000012 & 0.003\end{array}$

$\begin{array}{llll}972.6 & 0.1356 & -0.000022 & 0.018\end{array}$

$\begin{array}{llll}973.1 & 0.0884 & -0.000013 & 0.008\end{array}$

$\begin{array}{lllll}973.1 & 0.0908 & -0.000013 & 0.008\end{array}$

$\begin{array}{llll}974.0 & 0.1124 & -0.000013 & 0.013\end{array}$

$\begin{array}{llll}975.8 & 0.1977 & -0.000018 & 0.039\end{array}$

$\begin{array}{llll}977.5 & 0.1382 & -0.000010 & 0.019\end{array}$

$\begin{array}{llll}977.8 & 0.1694 & -0.000012 & 0.029\end{array}$

$\begin{array}{llll}979.7 & 0.2595 & -0.000015 & 0.067\end{array}$

$\begin{array}{llll}979.7 & 0.1604 & -0.000009 & 0.026\end{array}$

$\begin{array}{llll}984.1 & 0.1064 & -0.000004 & 0.011\end{array}$

$\begin{array}{llll}986.3 & 0.3347 & -0.000012 & 0.112\end{array}$

$\begin{array}{llll}990.6 & 0.2083 & -0.000006 & 0.043\end{array}$

$\begin{array}{llll}990.6 & 0.2052 & -0.000006 & 0.042\end{array}$

$\begin{array}{llll}999.1 & 0.3982 & -0.000008 & 0.159\end{array}$

$\begin{array}{llll}1011.6 & 0.3902 & -0.000005 & 0.152\end{array}$

$\begin{array}{llll}1021.0 & 0.1149 & -0.000001 & 0.013\end{array}$

$\begin{array}{llll}1038.1 & 0.0304 & 0.000000 & 0.001\end{array}$

$\begin{array}{llll}1088.4 & -0.2485 & 0.000001 & 0.062\end{array}$

$\begin{array}{llll}1104.7 & -0.3646 & 0.000001 & 0.133\end{array}$

$\begin{array}{llll}1120.9 & -0.3860 & 0.000001 & 0.149\end{array}$

$\begin{array}{llll}1136.5 & -0.4226 & 0.000001 & 0.179\end{array}$

\begin{tabular}{|c|c|c|}
\hline \multirow[t]{2}{*}{35.61} & & 974.849 \\
\hline & & 974.849 \\
\hline \multirow[t]{2}{*}{52.64} & & 974.849 \\
\hline & & 974.849 \\
\hline 71.291 & & 974.849 \\
\hline 86.195 & & 974.849 \\
\hline 102.268 & & 974.849 \\
\hline 118.855 & & 974.849 \\
\hline \multirow{2}{*}{135.17} & & 974.849 \\
\hline & & 974.849 \\
\hline 151.345 & & 974.849 \\
\hline \multirow[t]{12}{*}{167.15} & & 974.849 \\
\hline & & 971.7978 \\
\hline & & 971.7978 \\
\hline & & 971.7978 \\
\hline & & 971.7978 \\
\hline & & 971.7978 \\
\hline & & 971.7978 \\
\hline & & 971.7978 \\
\hline & & 971.7978 \\
\hline & & 971.7978 \\
\hline & 27.8 & 968.6203 \\
\hline & 27.79 & 968.6203 \\
\hline \multirow[t]{3}{*}{1.496} & & 968.6203 \\
\hline & & 968.6203 \\
\hline & 27.58 & 968.6203 \\
\hline \multirow[t]{3}{*}{3.01} & & 968.6203 \\
\hline & & 968.6203 \\
\hline & 28.26 & 968.6203 \\
\hline \multirow[t]{4}{*}{4.58} & & 968.6203 \\
\hline & 28.22 & 968.6203 \\
\hline & & 968.6203 \\
\hline & 28.54 & 968.6203 \\
\hline \multirow[t]{9}{*}{9.319} & & 968.6203 \\
\hline & & 968.6203 \\
\hline & 28.63 & 968.6203 \\
\hline & 29.06 & 968.6203 \\
\hline & & 968.6203 \\
\hline & 29.047 & 968.6203 \\
\hline & 29.054 & 968.6203 \\
\hline & & 968.6203 \\
\hline & & 968.6203 \\
\hline 52.502 & & 968.6203 \\
\hline 69.473 & & 968.6203 \\
\hline 119.516 & & 968.6203 \\
\hline 135.706 & & 968.6203 \\
\hline 151.848 & & 968.6203 \\
\hline 167.477 & & 968.6203 \\
\hline
\end{tabular}




\begin{tabular}{|c|c|c|c|c|c|c|c|}
\hline molality & molarity & \multicolumn{2}{|c|}{$\mathrm{t} /{ }^{\circ} \mathrm{C}$ mass frac } & $\begin{array}{r}\text { Density } \\
\exp / \\
\mathrm{kg} / \mathrm{m}^{3}\end{array}$ & Ref & $\begin{array}{r}\text { App vol } \\
\exp / \\
\mathrm{m}^{3} / \mathrm{kg}\end{array}$ & $\begin{array}{c}\text { App vol } \\
\text { Calc/ } \\
\mathrm{m}^{3} / \mathrm{kg}\end{array}$ \\
\hline 0.01501 & & 95 & 0.0011 & 962.61 & (81) & 0.000358 & 0.000389 \\
\hline 0.03002 & & 95 & 0.0022 & 963.30 & (81) & 0.000365 & 0.000389 \\
\hline 0.04 & & 95 & 0.0030 & 963.79 & (80) & 0.000354 & 0.000389 \\
\hline 0.06004 & & 95 & 0.0045 & 964.69 & (81) & 0.000364 & 0.000389 \\
\hline 0.09 & & 95 & 0.0067 & 966.07 & (80) & 0.000366 & 0.000389 \\
\hline 0.10001 & & 95 & 0.0074 & 966.50 & (81) & 0.000371 & 0.000389 \\
\hline 0.10244 & & 95 & 0.0076 & 966.60 & (20) & 0.000373 & 0.000389 \\
\hline 0.12006 & & 95 & 0.0089 & 967.40 & (81) & 0.000374 & 0.000390 \\
\hline 0.16 & & 95 & 0.0118 & 969.25 & (80) & 0.000371 & 0.000390 \\
\hline 0.18795 & & 95 & 0.0138 & 970.46 & (20) & 0.000376 & 0.000390 \\
\hline 0.19998 & & 95 & 0.0147 & 970.99 & (81) & 0.000377 & 0.000390 \\
\hline 0.25 & & 95 & 0.0183 & 973.30 & (80) & 0.000374 & 0.000391 \\
\hline 0.25017 & & 95 & 0.0183 & 973.23 & (81) & 0.000378 & 0.000391 \\
\hline 0.35002 & & 95 & 0.0254 & 977.55 & (81) & 0.000385 & 0.000392 \\
\hline 0.4 & & 95 & 0.0290 & 979.94 & (80) & 0.000379 & 0.000392 \\
\hline 0.40699 & & 95 & 0.0294 & 980.14 & (20) & 0.000383 & 0.000392 \\
\hline 0.49966 & & 95 & 0.0359 & 984.16 & (81) & 0.000385 & 0.000393 \\
\hline 0.49992 & & 95 & 0.0359 & 984.15 & (81) & 0.000385 & 0.000393 \\
\hline 0.7 & & 95 & 0.0496 & 992.90 & (80) & 0.000385 & 0.000395 \\
\hline 1 & & 95 & 0.0694 & 1005.44 & (80) & 0.000391 & 0.000398 \\
\hline 1.2073 & & 95 & 0.0826 & 1013.74 & (20) & 0.000396 & 0.000399 \\
\hline 1.67093 & & 95 & 0.1108 & 1032.09 & (20) & 0.000401 & 0.000403 \\
\hline 2.09748 & & 95 & 0.1352 & 1048.39 & (20) & 0.000405 & 0.000406 \\
\hline 2.55755 & & 95 & 0.1601 & 1065.21 & (20) & 0.000410 & 0.000410 \\
\hline 3.03769 & & 95 & 0.1847 & 1082.16 & (20) & 0.000414 & 0.000413 \\
\hline 3.49712 & & 95 & 0.2068 & 1097.85 & (20) & 0.000417 & 0.000416 \\
\hline 3.9927 & & 95 & 0.2294 & 1114.10 & (20) & 0.000420 & 0.000419 \\
\hline 4.50012 & & 95 & 0.2512 & 1130.09 & (20) & 0.000424 & 0.000422 \\
\hline 0.00975 & & 98.82 & 0.0007 & 959.91 & (114) & 0.000002 & 0.000388 \\
\hline 0.05091 & & 98.82 & 0.0038 & 961.83 & (114) & 0.000292 & 0.000388 \\
\hline 0.1045 & & 98.82 & 0.0077 & 964.25 & (114) & 0.000337 & 0.000388 \\
\hline 0.132 & & 98.82 & 0.0097 & 965.50 & (114) & 0.000345 & 0.000389 \\
\hline 0.2066 & & 98.82 & 0.0152 & 968.88 & (114) & 0.000357 & 0.000389 \\
\hline 0.4891 & & 98.82 & 0.0352 & 981.30 & (114) & 0.000375 & 0.000392 \\
\hline 0.9429 & & 98.82 & 0.0657 & 1000.77 & (114) & 0.000383 & 0.000396 \\
\hline 2.013 & & 98.82 & 0.1305 & 1042.96 & (114) & 0.000401 & 0.000404 \\
\hline 0.1004 & & 100 & 0.0074 & 963.20 & (52) & 0.000338 & 0.000388 \\
\hline & & 100 & 0.0100 & 964.60 & (90) & 0.000369 & 0.000388 \\
\hline & & 100 & 0.0200 & 970.80 & (90) & 0.000375 & 0.000390 \\
\hline 0.7104 & & 100 & 0.0503 & 990.00 & (52) & 0.000381 & 0.000393 \\
\hline 1.4207 & & 100 & 0.0958 & 1019.00 & (52) & 0.000395 & 0.000399 \\
\hline & & 100 & 0.1200 & 1035.00 & (90) & 0.000400 & 0.000402 \\
\hline & & 100 & 0.1600 & 1061.90 & (90) & 0.000408 & 0.000408 \\
\hline & & 100 & 0.2000 & 1089.70 & (90) & 0.000415 & 0.000413 \\
\hline 3.5517 & & 100 & 0.2094 & 1096.80 & (52) & 0.000414 & 0.000414 \\
\hline & & 100 & 0.2400 & 1118.50 & (90) & 0.000421 & 0.000418 \\
\hline & & 110 & 0.0379 & 973.30 & (90) & 0.000414 & 0.000388 \\
\hline & & 110 & 0.0745 & 997.80 & (90) & 0.000389 & 0.000393 \\
\hline
\end{tabular}

Density Density App Vol Square of Inconsistent Inconsistent (Dens rel- Delta density Apparent Density of

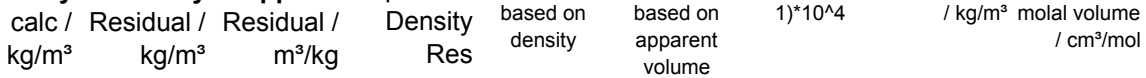

$962.6 \quad 0.0316-0.000031 \quad 0.001$ $\begin{array}{lllll}963.2 & 0.0497 & -0.000024 & 0.002\end{array}$

$\begin{array}{llll}963.7 & 0.0961 & -0.000035 & 0.009\end{array}$

$\begin{array}{llll}964.6 & 0.1028 & -0.000025 & 0.011\end{array}$

$\begin{array}{llll}965.9 & 0.1428 & -0.000023 & 0.020\end{array}$

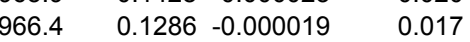

$\begin{array}{llll}966.5 & 0.1161 & -0.000016 & 0.013\end{array}$

$\begin{array}{llll}967.3 & 0.1315 & -0.000016 & 0.017\end{array}$

$\begin{array}{llll}969.0 & 0.2121 & -0.000019 & 0.045\end{array}$

$\begin{array}{lllll}970.3 & 0.1887 & -0.000014 & 0.036\end{array}$

$\begin{array}{llll}970.8 & 0.1815 & -0.000013 & 0.033\end{array}$

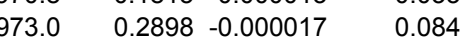

$\begin{array}{llll}973.0 & 0.2164 & -0.000012 & 0.047\end{array}$

$\begin{array}{llll}977.4 & 0.1634 & -0.000007 & 0.027\end{array}$

$\begin{array}{llll}979.6 & 0.3763 & -0.000014 & 0.142\end{array}$

$\begin{array}{llll}979.9 & 0.2743 & -0.000010 & 0.075\end{array}$

$\begin{array}{llll}983.9 & 0.2849 & -0.000008 & 0.081\end{array}$

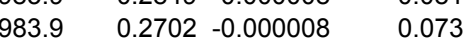

$\begin{array}{llll}992.4 & 0.4759 & -0.000010 & 0.227\end{array}$

$\begin{array}{llll}1005.0 & 0.4759 & -0.000007 & 0.227\end{array}$

$\begin{array}{llll}1013.4 & 0.2892 & -0.000003 & 0.084\end{array}$

$\begin{array}{llll}1031.9 & 0.1925 & -0.000002 & 0.037\end{array}$

$\begin{array}{llll}1048.3 & 0.1226 & -0.000001 & 0.015\end{array}$

$\begin{array}{llll}1065.3 & -0.0777 & 0.000000 & 0.006\end{array}$

$\begin{array}{llll}1082.4 & -0.2437 & 0.000001 & 0.059\end{array}$

$\begin{array}{llll}1098.2 & -0.3229 & 0.000001 & 0.104\end{array}$

$\begin{array}{llll}1114.6 & -0.4562 & 0.000002 & 0.208\end{array}$

$\begin{array}{llll}1130.7 & -0.5966 & 0.000002 & 0.356\end{array}$

$\begin{array}{llll}959.6 & 0.2583 & -0.000386 & 0.067\end{array}$

$\begin{array}{llll}961.5 & 0.3354 & -0.000096 & 0.112\end{array}$

$\begin{array}{lllll}963.9 & 0.3688 & -0.000051 & 0.136\end{array}$

$\begin{array}{llll}965.1 & 0.3923 & -0.000043 & 0.154\end{array}$

$\begin{array}{llll}968.4 & 0.4664 & -0.000033 & 0.218\end{array}$

$\begin{array}{llll}980.7 & 0.5619 & -0.000017 & 0.316\end{array}$

$\begin{array}{llll}999.9 & 0.8215 & -0.000012 & 0.675\end{array}$

$\begin{array}{llll}042.5 & 0.4633 & -0.000003 & 0.215\end{array}$

$\begin{array}{llll}962.9 & 0.3427 & -0.000050 & 0.117\end{array}$

$964.4 \quad 0.1813-0.000019-0.033$

$\begin{array}{llll}970.5 & 0.2731 & -0.000014 & 0.075\end{array}$

$\begin{array}{llll}989.4 & 0.6364 & -0.000013 & 0.405\end{array}$

$\begin{array}{lllll}018.6 & 0.4124 & -0.000004 & 0.170\end{array}$

$\begin{array}{llll}034.6 & 0.3646 & -0.000003 & 0.133\end{array}$

$\begin{array}{llll}061.9 & 0.0114 & 0.000000 & 0.000\end{array}$

$\begin{array}{llll}0.090 .1 & -0.4090 & 0.000002 & 0.167\end{array}$

$\begin{array}{llll}1096.9 & -0.0552 & 0.000000 & 0.003\end{array}$

$\begin{array}{llll}1119.3 & -0.8285 & 0.000003 & 0.686\end{array}$

$\begin{array}{llll}974.2 & -0.9453 & 0.000026 & 0.894\end{array}$

$\begin{array}{llll}997.5 & 0.2797 & -0.000004 & 0.078\end{array}$
$26.7 \quad 961.9004$

$27.2 \quad 961.9004$

961.9004

$27.16 \quad 961.9004$

961.9004

$27.65 \quad 961.9004$

4.697

$27.87 \quad 961.9004$

8.563

961.9004

961.900

961.9004

961.9004

$28.71 \quad 961.9004$

961.9004

$\begin{array}{lll}18.241 & & 961.9004 \\ & 28.702 & 961.9004\end{array}$

$\begin{array}{ll}28.702 & 961.9004 \\ 28.734 & 961.9004\end{array}$

961.9004

961.9004

51.836

70.192

86.488

103.314

120.257

135.948

152.199

168.194

961.9004

961.9004

961.9004

961.9004

961.9004

961.9004

961.9004

961.9004
$28.212 \quad 961.9004$

959.2088

959.2088

959.2088

959.2088

959.2088

959.2088

959.2088

959.2088

958.3637

958.3637

958.3637

958.3637

958.3637

958.3637

958.3637

958.3637

958.3637

958.3637

950.9471

950.9471 


$\begin{array}{lrrrr}\text { molality molarity } & \mathbf{t} /{ }^{\circ} \mathrm{C} \text { mass frac } & \begin{array}{r}\text { Density } \\ \text { exp } / \\ \mathrm{kg} / \mathrm{m}^{3}\end{array} & \text { Ref } \\ & & & & \\ & 110 & 0.1362 & 1038.80 & (90) \\ 0.1009 & 125 & 0.0075 & 944.40 & (52) \\ 1.4287 & 125 & 0.0963 & 1001.20 & (52) \\ 3.5718 & 125 & 0.2103 & 1080.60 & (52)\end{array}$

$\begin{array}{rr}\text { App vol } & \text { App vol } \\ \exp / & \text { Calc / } \\ \mathrm{m}^{3} / \mathrm{kg} & \mathrm{m}^{3} / \mathrm{kg}\end{array}$

Density Density App Vol Square of Inconsistent Inconsistent

(Dens rel -

Delta density Apparent Density of

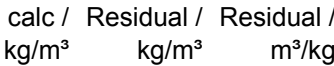

Density based on

based on $1)^{\star} 10^{\wedge} 4$

$/ \mathrm{kg} / \mathrm{m}^{3}$ molal volume

Density of $/ \mathrm{kg} / \mathrm{m}^{3}$

$\begin{array}{llllll}0.000399 & 0.000400 & 1038.6 & 0.2368 & -0.000002\end{array}$

$\begin{array}{llllll}0.000247 & 0.000377 & 943.5 & 0.8669 & -0.000130\end{array}$

0.056

950.9471

$\begin{array}{lllll}0.000377 & 0.000388 & 1000.2 & 0.9709 & -0.000010\end{array}$

0.752

938.9841

3.5718

$\begin{array}{lll}0.000401 & 0.000401 \quad 1080.7\end{array}$

$-0.0641-0.000000$

(3)

Average Res $0.0615-0.000010$

38.586 Sum Chi sqr

938.984

Curve fit for the model App vol $=\left(w+c 2+c 3^{*} t{ }^{\circ} \mathrm{C}\right) /\left(c 0^{*} w+c 1\right) / e^{\wedge}\left(0.000001^{*}\left(t{ }^{\circ} \mathrm{C}+c 4\right)^{\wedge} 2\right)$

Avg - 4std $\quad-0.8539-0.000124$

Avg + 4std $\quad 0.9769 \quad 0.000104$

$\begin{array}{lr}\text { c0 } & -0.467824 \\ \text { c1 } & 4.308005 \\ \text { c2 } & 2.378009 \\ \text { c3 } & 0.022044 \\ \text { c4 } & 2714.019 \\ \text { Min } & 0\end{array}$

125

Min w 7.42E-05

Maxw 0.28

Avg dens res $\quad 0.061545$

Std dens res $\quad 0.228851$

Avg app vol res *10^3 -0.009706

Std app vol res * $10^{\wedge} 3 \quad 0.028477$

No of points in corr

688

No of inconsistent poir

5

Inconsistent data not used (Residual greater than average + / $\mathbf{4}$ standard deviations)

$\begin{array}{lllllll}40 & 0.2800 & 1183.04 & (90) & 0.000427 & 0.000431\end{array}$

$\begin{array}{rrrrrr}40 & 0.2800 & 1183.04 & (90) & 0.000427 & 0.000431 \\ 100 & 0.0400 & 963.40 & (90) & 0.000907 & 0.000392\end{array}$

$\begin{array}{lllllll}100 & 0.0800 & 1088.80 & (90) & -0.000519 & 0.000397\end{array}$

0.7144

$\begin{array}{rrrrrr}100 & 0.2800 & 1148.30 & (90) & 0.000427 & 0.000424 \\ 125 & 0.0506 & 971.70 & (52) & 0.000356 & 0.000382\end{array}$

$1181.7 \quad 1.3399-0.000003$

$\begin{array}{lll}982.9 & -19.5044 & 0.000515\end{array}$

$1008.3 \quad 80.4821-0.000916$

$\begin{array}{rrr}1149.6 & -1.2781 & 0.000003\end{array}$

1.795

265.161

839.706

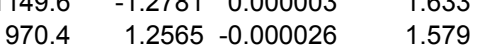

992.2158

958.3637

958.3637

958.3637

938.9841 
Density of aqueous solutions of $\mathbf{K 2 C O}$

References (29) Ginzburg, D. M.; Pikulina, N. S.; Litvin, V. P. Density of Potassium Carbonate Solutions. J. Appl. Chem. USSR 1964, 37, 2353-2357.

(41) Hitchcock, L. B.; Mcllhenny, J. S. Viscosity and Density of Pure Alkaline Solutions and their Mixtures. Ind. Engng. Chem. 1935, 27, 461466.

(73) Millero, F. J.; Ward, G. K.; Chetirkin, P. V. Relative Sound Velocities of Sea Salts at 25ㄷ. J. Acoust. Soc. Am. 1977, 61, $1492-1498$.

(90) Perry, R. H.; Green, D. W. Perry's Chemical Engineers' Handbook; McGraw Hill: New York, 7th edition, 1997.

\begin{tabular}{|c|c|c|c|c|c|c|c|c|c|c|c|c|c|c|}
\hline molality molarity & $\mathrm{t}^{\circ} \mathrm{C}$ & ass frac & $\begin{array}{r}\text { Density } \\
\exp \mathrm{kg} / \mathrm{m}^{3}\end{array}$ & Ref & 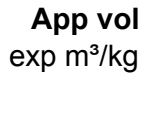 & $\begin{array}{r}\text { App vol } \\
\text { Calc } \\
\mathrm{m}^{3} / \mathrm{kg}\end{array}$ & $\begin{array}{r}\text { Density } \\
\text { salc } \mathrm{kg} / \mathrm{m}^{3}\end{array}$ & $\begin{array}{r}\text { Density } \\
\text { Residual } \\
\mathrm{kg} / \mathrm{m}^{3}\end{array}$ & $\begin{array}{r}\text { App Vol } \\
\text { Residual } \\
\mathrm{m}^{3} / \mathrm{kg}\end{array}$ & $\begin{array}{r}\text { Square of } \\
\text { Density } \\
\text { Residual }\end{array}$ & $\begin{array}{l}\text { Inconsistent } \\
\text { based on } \\
\text { density }\end{array}$ & $\begin{array}{c}\text { Inconsistent } \\
\text { based on } \\
\text { apparent } \\
\text { volume }\end{array}$ & Normality & $\begin{array}{r}\text { Density of } \\
\text { water } / \mathrm{kg} / \mathrm{m}^{3}\end{array}$ \\
\hline & 0 & 0.0100 & 1009.4 & (90) & 0.000053 & 0.000144 & 1008.47 & 0.9315 & -0.000092 & 0.868 & & $!$ & & 999.8395 \\
\hline & 0 & 0.0200 & 1018.9 & (90) & 0.000065 & 0.000147 & 1017.20 & 1.6967 & -0.000082 & 2.879 & & & & 999.8395 \\
\hline & 0 & 0.0400 & 1038.1 & (90) & 0.000079 & 0.000151 & 1034.99 & 3.1062 & -0.000072 & 9.648 & & & & 999.8395 \\
\hline & 0 & 0.0800 & 1076.8 & (90) & 0.000107 & 0.000160 & 1071.88 & 4.9215 & -0.000053 & 24.221 & & & & 999.8395 \\
\hline & 0 & 0.1200 & 1116.0 & (90) & 0.000133 & 0.000169 & 1110.53 & 5.4697 & -0.000037 & 29.918 & & & & 999.8395 \\
\hline & 0 & 0.1600 & 1156.2 & (90) & 0.000155 & 0.000179 & 1150.97 & 5.2345 & -0.000025 & 27.400 & & & & 999.8395 \\
\hline & 0 & 0.2000 & 1197.7 & (90) & 0.000174 & 0.000190 & 1193.17 & 4.5276 & -0.000016 & 20.499 & & & & 999.8395 \\
\hline & 0 & 0.2400 & 1240.5 & (90) & 0.000192 & 0.000201 & 1237.10 & 3.3984 & -0.000009 & 11.549 & & & & 999.8395 \\
\hline & 0 & 0.2800 & 1284.6 & (90) & 0.000208 & 0.000213 & 1282.65 & 1.9455 & -0.000004 & 3.785 & & & & 999.8395 \\
\hline & 0 & 0.3000 & 1307.1 & (90) & 0.000216 & 0.000219 & 1305.99 & 1.1081 & -0.000002 & 1.228 & & & & 999.8395 \\
\hline & 0 & 0.3500 & 1364.6 & (90) & 0.000236 & 0.000235 & 1365.74 & -1.1444 & 0.000002 & 1.310 & & & & 999.8395 \\
\hline & 0 & 0.4000 & 1424.4 & (90) & 0.000255 & 0.000252 & 1427.00 & -2.6018 & 0.000003 & 6.769 & & & & 999.8395 \\
\hline & 0 & 0.4500 & 1486.7 & (90) & 0.000272 & 0.000270 & 1488.88 & -2.1764 & 0.000002 & 4.737 & & & & 999.8395 \\
\hline & 0 & 0.5000 & 1551.7 & (90) & 0.000289 & 0.000290 & 1550.12 & 1.5839 & -0.000001 & 2.509 & & & & 999.8395 \\
\hline & 10 & 0.0100 & 1008.9 & (90) & 0.000088 & 0.000152 & 1008.25 & 0.6548 & -0.000064 & 0.429 & & & & 999.6996 \\
\hline & 10 & 0.0200 & 1018.2 & (90) & 0.000092 & 0.000155 & 1016.89 & 1.3057 & -0.000063 & 1.705 & & & & 999.6996 \\
\hline & 10 & 0.0400 & 1036.9 & (90) & 0.000103 & 0.000159 & 1034.51 & 2.3938 & -0.000056 & 5.730 & & & & 999.6996 \\
\hline & 10 & 0.0800 & 1074.6 & (90) & 0.000129 & 0.000168 & 1071.00 & 3.5968 & -0.000039 & 12.937 & & & & 999.6996 \\
\hline & 10 & 0.1200 & 1113.1 & (90) & 0.000151 & 0.000177 & 1109.22 & 3.8760 & -0.000026 & 15.023 & & & & 999.6996 \\
\hline & 10 & 0.1600 & 1153.0 & (90) & 0.000169 & 0.000187 & 1149.18 & 3.8184 & -0.000018 & 14.580 & & & & 999.6996 \\
\hline & 10 & 0.2000 & 1194.1 & (90) & 0.000186 & 0.000197 & 1190.86 & 3.2386 & -0.000011 & 10.489 & & & & 999.6996 \\
\hline & 10 & 0.2400 & 1236.6 & (90) & 0.000202 & 0.000208 & 1234.21 & 2.3884 & -0.000007 & 5.705 & & & & 999.6996 \\
\hline & 10 & 0.2800 & 1280.4 & (90) & 0.000217 & 0.000220 & 1279.13 & 1.2680 & -0.000003 & 1.608 & & & & 999.6996 \\
\hline & 10 & 0.3000 & 1302.8 & (90) & 0.000225 & 0.000226 & 1302.13 & 0.6669 & -0.000001 & 0.445 & & & & 999.6996 \\
\hline & 10 & 0.3500 & 1360.0 & (90) & 0.000243 & 0.000242 & 1360.99 & -0.9879 & 0.000002 & 0.976 & & & & 999.6996 \\
\hline & 10 & 0.4000 & 1419.5 & (90) & 0.000261 & 0.000259 & 1421.27 & -1.7729 & 0.000002 & 3.143 & & & & 999.6996 \\
\hline & 10 & 0.4500 & 1481.5 & (90) & 0.000277 & 0.000277 & 1482.12 & -0.6162 & 0.000001 & 0.380 & & & & 999.6996 \\
\hline & 10 & 0.5000 & 1546.2 & (90) & 0.000293 & 0.000296 & 1542.29 & 3.9068 & -0.000003 & 15.263 & & & & 999.6996 \\
\hline & 20 & 0.0100 & 1007.2 & (90) & 0.000107 & 0.000159 & 1006.67 & 0.5290 & -0.000052 & 0.280 & & & & 998.2041 \\
\hline & 20 & 0.0200 & 1016.3 & (90) & 0.000110 & 0.000161 & 1015.24 & 1.0604 & -0.000051 & 1.124 & & & & 998.2041 \\
\hline & 20 & 0.0400 & 1034.5 & (90) & 0.000123 & 0.000166 & 1032.69 & 1.8148 & -0.000042 & 3.294 & & & & 998.2041 \\
\hline 0.47395 & 20 & 0.0622 & 1052.6 & (41) & 0.000170 & 0.000170 & 1052.57 & 0.0337 & 0.000000 & 0.001 & & & 0.9479 & 998.2041 \\
\hline
\end{tabular}


molality molarity

$t^{\circ} \mathrm{C}$ mass frac Density $\exp \mathrm{kg} / \mathrm{m}^{3}$

\begin{tabular}{|c|c|c|c|c|}
\hline & & 20 & 0.0800 & 1071.5 \\
\hline \multirow{3}{*}{\multicolumn{2}{|c|}{0.91}} & 20 & 0.1143 & 1100.3 \\
\hline & & 20 & 0.1200 & 1109.6 \\
\hline & & 20 & 0.1600 & 1149.0 \\
\hline \multirow{2}{*}{\multicolumn{2}{|c|}{1.39}} & 20 & 0.1669 & 1151.4 \\
\hline & & 20 & 0.2000 & 1189.8 \\
\hline \multirow{2}{*}{\multicolumn{2}{|c|}{1.808}} & 20 & 0.2092 & 1194.5 \\
\hline & & 20 & 0.2400 & 1232.0 \\
\hline \multirow{2}{*}{\multicolumn{2}{|c|}{2.2105}} & 20 & 0.2474 & 1234.9 \\
\hline & & 20 & 0.2800 & 1275.6 \\
\hline \multirow{2}{*}{\multicolumn{2}{|c|}{2.634}} & 20 & 0.2852 & 1276.4 \\
\hline & & 20 & 0.3000 & 1297.9 \\
\hline \multirow{2}{*}{\multicolumn{2}{|c|}{3.0485}} & 20 & 0.3202 & 1315.9 \\
\hline & & 20 & 0.3500 & 1354.8 \\
\hline \multirow{4}{*}{\multicolumn{2}{|c|}{3.556}} & 20 & 0.3606 & 1363.1 \\
\hline & & 20 & 0.4000 & 1414.1 \\
\hline & & 20 & 0.4500 & 1475.9 \\
\hline & & 20 & 0.5000 & 1540.4 \\
\hline 0.30004 & & 25 & 0.0398 & 1032.1 \\
\hline 0.39988 & & 25 & 0.0524 & 1043.3 \\
\hline 0.49976 & & 25 & 0.0646 & 1054.3 \\
\hline 0.59989 & & 25 & 0.0766 & 1065.1 \\
\hline 0.69969 & & 25 & 0.0882 & 1075.8 \\
\hline 0.79944 & & 25 & 0.0995 & 1086.3 \\
\hline \multirow[t]{17}{*}{0.89994} & & 25 & 0.1106 & 1096.8 \\
\hline & 0.47235 & 30 & 0.0622 & 1049.0 \\
\hline & 0.9065 & 30 & 0.1143 & 1096.4 \\
\hline & 1.384 & 30 & 0.1668 & 1146.8 \\
\hline & 1.801 & 30 & 0.2092 & 1189.9 \\
\hline & 2.2015 & 30 & 0.2474 & 1229.9 \\
\hline & 2.623 & 30 & 0.2851 & 1271.7 \\
\hline & 3.0365 & 30 & 0.3202 & 1310.8 \\
\hline & \multirow[t]{9}{*}{3.542} & 30 & 0.3605 & 1357.8 \\
\hline & & 35 & 0.1604 & 1142.3 \\
\hline & & 35 & 0.2021 & 1184.2 \\
\hline & & 35 & 0.2439 & 1226.1 \\
\hline & & 35 & 0.2815 & 1266.1 \\
\hline & & 35 & 0.3599 & 1354.4 \\
\hline & & 35 & 0.3970 & 1397.6 \\
\hline & & 35 & 0.4395 & 1448.7 \\
\hline & & 35 & 0.4834 & 1505.3 \\
\hline
\end{tabular}

Ref $\operatorname{exp~m^{3}/kg}$ exp $\mathrm{m} / \mathrm{kg}$

\author{
(90)
}

(41)
0.0001890 .000182

$0.000164 \quad 0.000184$

$0.000180 \quad 0.000193$

$0.000203 \quad 0.000195$

$0.000195 \quad 0.000204$

$0.000215 \quad 0.000206$

$0.000210 \quad 0.000214$

$0.000226 \quad 0.000216$

$0.000224 \quad 0.000226$

$0.000236 \quad 0.000227$

$\begin{array}{lll}0.000231 & 0.000232\end{array}$

$0.000246 \quad 0.000238$

$0.000248 \quad 0.000247$

$0.000258 \quad 0.000251$

$0.000265 \quad 0.000264$

0.0002810 .000282

$0.000297 \quad 0.000301$

$0.000147 \quad 0.000168$

$0.000154 \quad 0.000171$

$0.000160 \quad 0.000174$

$0.000166 \quad 0.000176$

$0.000170 \quad 0.000179$

$\begin{array}{lll}0.000175 & 0.000182\end{array}$

$0.000178 \quad 0.000184$

$0.000184 \quad 0.000176$

$0.000197 \quad 0.000187$

$0.000211 \quad 0.000200$

$0.000221 \quad 0.000211$

$0.000231 \quad 0.000221$

$0.000240 \quad 0.000232$

$0.000250 \quad 0.000242$

$0.000261 \quad 0.000255$

$0.000192 \quad 0.000200$

$0.000207 \quad 0.000211$

$0.000225 \quad 0.000222$

$0.000238 \quad 0.000232$

0.0002620 .000256

$0.000274 \quad 0.000268$

$0.000288 \quad 0.000283$

$0.000299 \quad 0.000299$
Density
calc $\mathrm{kg} / \mathrm{m}^{3}$

1068.83

1101.18

1106.66

1146.20

1153.15

187.44

1197.15

1230.31

1238.42

1274.72

1280.62

1320.74

1355.63

1368.13

1415.22

1475.37

1534.90

1031.21

1042.33

1064.20

1074.91

1085.50

1096.03

1049.54

1097.68

1149.17

1192.83

1233.72

1275.40

1315.32

1362.32

1140.53

1183.02

1227.38

1268.71

1358.60

1402.44

1453.13

1505.35
Aensity App Vol Square of Inconsistent Inconsistent Normality Density of $\mathrm{kg} / \mathrm{m}^{3} \quad \mathrm{~m}^{3} / \mathrm{kg}$ Residual density apparent

$2.6725-0.000029 \quad 7.142$

$\begin{array}{lll}.8761 & 0.000006 & 0.767\end{array}$

$2.9368-0.000020 \quad 8.625$

$2.7956-0.000013 \quad 7.815$

$\begin{array}{lll}-1.7512 & 0.000008 & 3.067\end{array}$

$2.3637-0.000008 \quad 5.587$

$\begin{array}{lll}-2.6547 & 0.000009 & 7.048\end{array}$

$\begin{array}{lll}1.6919 & -0.000005 & 2.863\end{array}$

12.376

0.772

17.834

0.194

23.457

0.696

25.319

1.256

0.280

30.303

0.786

0.951

0.977

0.817

0.784

0.641

0.588

0.290

1.640

5.606

8.558

14.587

13.706

20.406

20.455

3.135

1.402

1.626

6.788

17.662

23.424

19.653

0.002
998.2041

1.82998 .2041

998.2041

998.2041

$2.78 \quad 998.2041$

998.2041

3.616998 .2041

998.2041

4.421998 .2041 998.2041

$5.268 \quad 998.2041$

998.2041

$6.097 \quad 998.2041$ 998.2041

7.112998 .2041

998.2041

998.2041

998.2041

997.0449

997.0449

997.0449

997.0449

997.0449

997.0449

997.0449

$0.9447 \quad 995.6473$

1.813995 .6473

$2.768 \quad 995.6473$

3.602995 .6473

$4.403 \quad 995.6473$

$5.246 \quad 995.6473$

6.073995 .6473

7.084995 .6473

994.0319

994.0319

994.0319

994.0319

994.0319

994.0319

994.0319

994.0319 
molality molarity

$t^{\circ} \mathrm{C}$ mass frac Density $\exp \mathrm{kg} / \mathrm{m}^{3}$

\begin{tabular}{|c|c|c|c|}
\hline & 35 & 0.5165 & 1542.2 \\
\hline & 35 & 0.5242 & 1554.7 \\
\hline & 40 & 0.0100 & 1001.0 \\
\hline & 40 & 0.0200 & 1009.8 \\
\hline & 40 & 0.0400 & 1027.6 \\
\hline 0.47085 & 40 & 0.0623 & 1045.3 \\
\hline & 40 & 0.0800 & 1064.0 \\
\hline 0.903 & 40 & 0.1143 & 1092.0 \\
\hline & 40 & 0.1200 & 1101.3 \\
\hline & 40 & 0.1600 & 1139.9 \\
\hline 1.3785 & 40 & 0.1668 & 1142.2 \\
\hline & 40 & 0.2000 & 1180.1 \\
\hline 1.7935 & 40 & 0.2092 & 1185.0 \\
\hline & 40 & 0.2400 & 1221.9 \\
\hline 2.1925 & 40 & 0.2474 & 1224.8 \\
\hline & 40 & 0.2800 & 1265.2 \\
\hline 2.6125 & 40 & 0.2851 & 1266.3 \\
\hline & 40 & 0.3000 & 1287.3 \\
\hline 3.0245 & 40 & 0.3202 & 1305.4 \\
\hline & 40 & 0.3500 & 1344.0 \\
\hline 3.5285 & 40 & 0.3606 & 1352.5 \\
\hline & 40 & 0.4000 & 1402.9 \\
\hline & 40 & 0.4500 & 1464.4 \\
\hline & 40 & 0.5000 & 1528.5 \\
\hline & 50 & 0.1604 & 1132.4 \\
\hline & 50 & 0.2021 & 1172.9 \\
\hline & 50 & 0.2411 & 1213.1 \\
\hline & 50 & 0.2819 & 1259.5 \\
\hline & 50 & 0.4395 & 1440.1 \\
\hline & 60 & 0.0100 & 991.9 \\
\hline & 60 & 0.0200 & 1000.5 \\
\hline & 60 & 0.0400 & 1018.0 \\
\hline & 60 & 0.0800 & 1053.8 \\
\hline & 60 & 0.1200 & 1090.6 \\
\hline & 60 & 0.1600 & 1129.0 \\
\hline & 60 & 0.2000 & 1169.0 \\
\hline & 60 & 0.2400 & 1210.6 \\
\hline & 60 & 0.2411 & 1209.5 \\
\hline & 60 & 0.2800 & 1253.8 \\
\hline & 60 & 0.3000 & 1275.9 \\
\hline & 60 & 0.3206 & 1294.6 \\
\hline
\end{tabular}

Ref

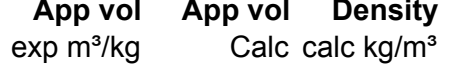
$\mathrm{m}^{3} / \mathrm{kg}$

(29)

(29)

(90)

(90)

(90)

(41)

(90)

(41)

(90)

(90)

(41)

(90)

(41)

(41)

(90)

(41)

$(41)$
$(90)$

(41)

(90)

(41)

(90)

(90)

(90)

(29)

(29)

(29)

(29)

(29)

(90)

(90)

(90)

(90)

(90)

(90)

(90)

(90)

(29)

(90)

(90) $\begin{array}{ll}0.000314 & 0.000312\end{array}$

$0.000314 \quad 0.000315$

$0.000123 \quad 0.000169$

$0.000130 \quad 0.000171$

$0.000140 \quad 0.000175$

$0.000186 \quad 0.000180$

$0.000158 \quad 0.000184$

$0.000202 \quad 0.000191$

$0.000176 \quad 0.000193$

$0.000192 \quad 0.000202$

$0.000214 \quad 0.000204$

$0.000206 \quad 0.000212$

$0.000224 \quad 0.000214$

$0.000218 \quad 0.000222$

$0.000234 \quad 0.000224$

0.0002310 .000233

$0.000243 \quad 0.000235$

$0.000238 \quad 0.000239$

$0.000253 \quad 0.000245$

$0.000254 \quad 0.000254$

$0.000263 \quad 0.000257$

$0.000270 \quad 0.000270$

$0.000286 \quad 0.000288$

$0.000301 \quad 0.000306$

$0.000208 \quad 0.000205$

$0.000223 \quad 0.000215$

$0.000233 \quad 0.000225$

$0.000238 \quad 0.000236$

$0.000289 \quad 0.000285$

$0.000125 \quad 0.000175$

$0.000138 \quad 0.000177$

$0.000148 \quad 0.000181$

$0.000165 \quad 0.000189$

$\begin{array}{ll}0.000182 & 0.000197\end{array}$

$0.000196 \quad 0.000206$

$0.000209 \quad 0.000216$

$0.000221 \quad 0.000226$

$0.000228 \quad 0.000226$

$0.000233 \quad 0.000236$

$0.000239 \quad 0.000242$

$0.000254 \quad 0.000248$
1544.06

1552.92

1000.54

1008.97

026.13

1045.70

1061.67

1093.46

1098.87

1137.76

1144.55
1178.32

1187.87

1220.52

1228.51

1264.26

1269.99
1286.66

1309.66

1344.05

1356.41

1402.95

1462.57

1521.82

1132.99

1175.09

1216.07

1260.55

1444.01

991.41

999.72

1016.64

1051.72

1088.46

1126.91

1167.06

1208.88

1210.06

1252.32

1274.61

1297.94
Density App Vol Square of Inconsistent Inconsistent Normality Density of Residual Residual Density based on based on $\mathrm{kg} / \mathrm{m}^{3} \quad \mathrm{~m}^{3} / \mathrm{kg}$ Residual density apparent

$\begin{array}{lll}-1.8565 & 0.000002 \quad 3.447\end{array}$

$1.7798-0.000001 \quad 3.168$

$0.4571-0.000046 \quad 0.209$

$0.8301-0.000041 \quad 0.689$

$\begin{array}{lll}1.4736 & -0.000035 & 2.172\end{array}$

$\begin{array}{lll}0.3991 & 0.000006 & 0.159\end{array}$

$2.3322-0.000026 \quad 5.439$

$\begin{array}{lll}-1.4624 & 0.000011 & 2.139\end{array}$

$2.4254-0.000017 \quad 5.882$

$2.1368-0.000010 \quad 4.566$

$\begin{array}{lll}2.3485 & 0.000011 \quad 5.516\end{array}$

$1.7751-0.000006 \quad 3.151$

$\begin{array}{lll}-2.8728 & 0.000010 \quad 8.253\end{array}$

$1.3822-0.000004 \quad 1.910$

$\begin{array}{lll}-3.7116 & 0.000010 & 13.776\end{array}$

$0.9438-0.000002 \quad 0.891$

$\begin{array}{lll}-3.6907 & 0.000008 & 13.621\end{array}$

$0.6368-0.000001 \quad 0.406$

$\begin{array}{lll}-4.2596 & 0.000008 & 18.144\end{array}$

$\begin{array}{lll}-0.0497 & 0.000000 & 0.002\end{array}$

$\begin{array}{lll}-3.9095 & 0.000006 & 15.285\end{array}$

$\begin{array}{lll}-0.0460 & 0.000000 & 0.002\end{array}$

$1.8298-0.000002 \quad 3.348$

$\begin{array}{lll}6.6832 & -0.000006 & 44.665\end{array}$

$\begin{array}{lll}-0.5902 & 0.000003 & 0.348\end{array}$

$\begin{array}{lll}-2.1852 & 0.000008 & 4.775\end{array}$

$\begin{array}{lll}-2.9744 & 0.000008 & 8.847\end{array}$

1.104

15.306

0.240

0.609

1.844

4.344

4.570

4.378

3.778

2.948

0.310

2.183

1.656

11.175
994.0319

994.0319

992.2158

992.2158

9922158

$0.9417 \quad 992.2158$ 992.2158

$1.806 \quad 992.2158$

992.2158 992.2158

$2.757 \quad 992.2158$

992.2158

$3.587 \quad 992.2158$ 992.2158

$4.385 \quad 992.2158$ 992.2158

$5.225 \quad 992.2158$

992.2158

$6.049 \quad 992.2158$ 992.2158

$7.057 \quad 992.2158$ 992.2158 992.2158

992.2158

988.0363

988.0363

988.0363

988.0363

988.0363

983.1989

983.1989

983.1989

983.1989

983.1989

983.1989

983.1989

983.1989

983.1989

983.1989

983.1989

983.1989 


\section{$t^{\circ} \mathrm{C}$ mass frac Density $\operatorname{exp~} \mathrm{kg} / \mathrm{m}^{3}$}

$\begin{array}{rrr}60 & 0.3500 & 1332.4 \\ 60 & 0.4000 & 1391.3 \\ 60 & 0.4500 & 1452.8 \\ 60 & 0.4804 & 1484.7 \\ 60 & 0.5000 & 1516.9 \\ 60 & 0.5242 & 1541.5 \\ 60 & 0.5404 & 1564.3 \\ 70 & 0.1604 & 1122.9 \\ 70 & 0.2021 & 1163.3 \\ 70 & 0.2411 & 1201.7 \\ 70 & 0.2819 & 1247.4 \\ 70 & 0.3607 & 1333.3 \\ 70 & 0.3970 & 1378.4 \\ 70 & 0.4395 & 1428.2 \\ 80 & 0.0100 & 980.3 \\ 80 & 0.0200 & 988.9 \\ 80 & 0.0400 & 1006.3 \\ 80 & 0.0800 & 1041.8 \\ 80 & 0.1200 & 1078.6 \\ 80 & 0.1600 & 1117.0 \\ 80 & 0.1604 & 1116.9 \\ 80 & 0.2000 & 1157.0 \\ 80 & 0.2400 & 1198.6 \\ 80 & 0.2411 & 1196.0 \\ 80 & 0.2800 & 1241.8 \\ 80 & 0.2819 & 1244.7 \\ 80 & 0.3000 & 1264.0 \\ 80 & 0.3500 & 1320.6 \\ 80 & 0.3599 & 1327.9 \\ 80 & 0.3607 & 1328.5 \\ 80 & 0.4000 & 1379.5 \\ 80 & 0.4500 & 1440.8 \\ 80 & 0.5000 & 1504.8 \\ 90 & 0.1604 & 1110.4 \\ 90 & 0.2021 & 1150.3 \\ 90 & 0.2439 & 1193.6 \\ 90 & 0.2815 & 1234.0 \\ 90 & 0.2819 & 1233.9 \\ 100 & 0.0100 & 967.0 \\ 100 & 0.0200 & 975.6 \\ 100 & 0.0800 & 1029.1\end{array}$

Ref $\operatorname{exp~} \mathrm{m}^{3} / \mathrm{kg}$ Calc calc $\mathrm{kg} / \mathrm{m}^{3}$ $\mathrm{m}^{3} / \mathrm{kg}$

\section{(90)}

$0.000255 \quad 0.000256$

$0.000271 \quad 0.000272$

$0.000287 \quad 0.000289$

$0.000302 \quad 0.000299$

$0.000301 \quad 0.000307$

$0.000314 \quad 0.000316$

$0.000318 \quad 0.000322$

$0.000199 \quad 0.000207$

$\begin{array}{ll}0.000216 & 0.000217\end{array}$

$0.000232 \quad 0.000227$

$0.000239 \quad 0.000237$

$0.000267 \quad 0.000259$

$0.000274 \quad 0.000270$

$0.000289 \quad 0.000284$

$0.000137 \quad 0.000177$

$0.000139 \quad 0.000179$

$0.000147 \quad 0.000183$

$0.000165 \quad 0.000190$

$0.000180 \quad 0.000199$

$0.000193 \quad 0.000207$

$0.000196 \quad 0.000207$

$0.000205 \quad 0.000216$

$0.000218 \quad 0.000226$

$0.000229 \quad 0.000226$

$0.000230 \quad 0.000236$

$0.000229 \quad 0.000236$

$0.000236 \quad 0.000241$

$0.000252 \quad 0.000255$

$0.000262 \quad 0.000258$

$0.000263 \quad 0.000258$

$0.000269 \quad 0.000270$

$0.000285 \quad 0.000286$

$0.000300 \quad 0.000303$

$\begin{array}{lll}0.000192 & 0.000207\end{array}$

0.0002120 .000216

$0.000224 \quad 0.000226$

$0.000235 \quad 0.000235$

$0.000236 \quad 0.000235$

$0.000112 \quad 0.000177$

$0.000122 \quad 0.000178$

$0.000147 \quad 0.000189$ $\begin{array}{r}1331.84 \\ 1390.81 \\ 1450.83 \\ 1487.42 \\ 1510.90 \\ 1539.59 \\ 1558.53 \\ 1121.14 \\ 1162.92 \\ 1203.68 \\ 1248.01 \\ 1338.06 \\ 1381.17 \\ 1432.49 \\ 979.91 \\ 988.12 \\ 1004.86 \\ 1039.57 \\ 1075.98 \\ 1114.15 \\ 1114.54 \\ 1154.08 \\ 1195.78 \\ 1196.95 \\ 1239.21 \\ 1241.31 \\ 1261.54 \\ 1319.07 \\ 1330.73 \\ 1331.67 \\ 1378.69 \\ 1439.81 \\ 1501.54 \\ 1107.52 \\ 1149.15 \\ 1192.87 \\ 1233.87 \\ 1234.32 \\ 966.39 \\ 974.52 \\ 1025.52 \\ \hline\end{array}$
Density App Vol Square of Inconsistent Inconsistent Normality Density of Residual Residual Density based on based on $\mathrm{kg} / \mathrm{m}^{3} \quad \mathrm{~m}^{3} / \mathrm{kg}$ Residual density apparent

$0.5646-0.000001 \quad 0.319$

0.244

$\begin{array}{rrr}1.9730 & -0.000002 & 3.893 \\ -2.7222 & 0.000003 & 7.410\end{array}$

$\begin{array}{rrr}-2.7222 & 0.000003 & 7.410 \\ 6.0044 & -0.000005 & 36.053\end{array}$

$1.9093-0.000002 \quad 3.646$

$\begin{array}{lll}5.7666 & -0.000004 \quad 33.254\end{array}$

$1.7598-0.000009 \quad 3.097$

$0.3826-0.000001 \quad 0.146$

$\begin{array}{lll}-1.9775 & 0.000006 & 3.911\end{array}$

$\begin{array}{lll}-0.6099 & 0.000001 & 0.372\end{array}$

$\begin{array}{lll}-4.7640 & 0.000007 & 22.696\end{array}$

$\begin{array}{lll}-2.7669 & 0.000004 & 7.656\end{array}$

$\begin{array}{lll}-4.2922 & 0.000005 & 18.423\end{array}$

$0.3892-0.000041 \quad 0.151$

$\begin{array}{lll}0.7757 & -0.000040 \quad 0.602\end{array}$

$1.4435-0.000036 \quad 2.084$

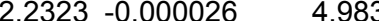

$2.6151-0.000019 \quad 6.839$

$2.8511-0.000014 \quad 8.129$

$2.3605-0.000012 \quad 5.572$

$2.9182-0.000011 \quad 8.516$

$2.8198-0.000008 \quad 7.951$

$\begin{array}{lll}-0.9516 & 0.000003 & 0.905\end{array}$

$2.5941-0.000006 \quad 6.729$

$3.3896-0.000008 \quad 11.490$

$2.4576-0.000005 \quad 6.040$

$1.5284-0.000003 \quad 2.336$

$\begin{array}{lll}-2.8257 & 0.000004 & 7.985\end{array}$

$\begin{array}{lll}-3.1709 & 0.000005 & 10.055\end{array}$

0.652

0.980

10.637

8.315

1.320

0.532

0.016

0.174

0.370

12.846 water $/ \mathrm{kg} / \mathrm{m}^{3}$

983.1989

983.1989

9831989

983.1989

983.1989

983.1989

983.1989

977.7696

977.7696

977.7696

977.7696

977.7696

977.7696

977.7696

971.7978

971.7978

971.7978

971.7978

971.7978

971.7978

971.7978

971.7978

971.7978

971.7978

971.7978

971.7978

971.7978

971.7978

971.7978

971.7978

971.7978

971.7978

971.7978

965.3201

965.3201

965.3201

965.3201

965.3201

958.3637

958.3637

958.3637 
molality molarity

$\begin{array}{llr}\mathbf{t}^{\circ} \mathbf{C} \text { mass frac } & \begin{array}{r}\text { Density } \\ \text { exp } \mathrm{kg} / \mathrm{m}^{3}\end{array} \\ & & \\ 100 & 0.1200 & 1066.3 \\ 100 & 0.1600 & 1104.9 \\ 100 & 0.2000 & 1145.1 \\ 100 & 0.2400 & 1186.9 \\ 100 & 0.2439 & 1189.8 \\ 100 & 0.2800 & 1230.1 \\ 100 & 0.2819 & 1229.4 \\ 100 & 0.3000 & 1252.2 \\ 100 & 0.3500 & 1308.9 \\ 100 & 0.3607 & 1317.2 \\ 100 & 0.4000 & 1367.8 \\ 100 & 0.4500 & 1429.0 \\ 100 & 0.4623 & 1440.8 \\ 100 & 0.5000 & 1492.8 \\ 100 & 0.5824 & 1590.4\end{array}$

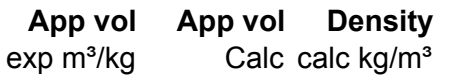

Calc
$\mathrm{m}^{3} / \mathrm{kg}$

(90)

(90)

$(90)$

(90)

(29)

(90)

(29)

(90)

(90)

$(29)$
$(90)$

$0.000206 \quad 0.000223$
0.0001630 .000197

$0.000179 \quad 0.000205$

$0.000193 \quad 0.000214$

$0.000211 \quad 0.000224$

$0.000220 \quad 0.000233$

$0.000227 \quad 0.000233$

$0.000227 \quad 0.000238$

$0.000245 \quad 0.000251$

$0.000255 \quad 0.000254$

$0.000263 \quad 0.000265$

$0.000280 \quad 0.000280$

$0.000288 \quad 0.000284$

$0.000296 \quad 0.000296$

$0.000331 \quad 0.000326$

Std dev Res

Avg - 4std

Avg +4 std
Density App Vol Square of Inconsistent Inconsistent Normality $\begin{array}{r}\text { Density of } \\ \text { based on }\end{array}$
water $/ \mathrm{kg} / \mathrm{m}^{3}$ $\mathrm{kg} / \mathrm{m}^{3} \quad \mathrm{~m}^{3} / \mathrm{kg}$ Residual density $\begin{gathered}\text { apparent } \\ \text { volume }\end{gathered}$

958.3637 958.3637 958.3637 958.3637 958.3637

958.3637 958.3637 958.3637

958.3637 958.3637 958.3637

958.3637 958.3637 958.3637

958.3637

Curve fit for the model App vol $=\left(w+c 2+c 3^{\star} t{ }^{\circ} \mathrm{C}\right) /\left(c 0^{\star} w+c 1\right) / e^{\wedge}\left(0.000001^{*}\left(t^{\circ} \mathrm{C}+c 4\right)^{\wedge} 2\right)$

$\begin{array}{lr}\text { c0 } & -1.431325 \\ \text { c1 } & 2.49168 \\ \text { c2 } & 1.102783 \\ \text { c3 } & 0.013116 \\ \text { c4 } & 2835.978 \\ \text { Min T } & 0 \\ \text { Max T } & 100 \\ \text { Min w } & 0.0100 \\ \text { Max w } & 0.5824 \\ \text { Avg dens res } & 0.5097 \\ \text { Std dens res } & 2.7583 \\ \text { Avg app vol res *10^3 } & -0.00978\end{array}$

Avg app vol res *10^3 -0.00978

Std app vol res *10^3 0.019844

No of points in corr

No of inconsistent poir

170
1

Inconsistent data not used (Residual greater than average +/- 4 standard deviations)

$\begin{array}{llllll}100 & 0.0400 & 995.1 & (90) & 0.000080 & 0.000182\end{array}$ 
Density of aqueous solutions of KNO3

References (7) Berchiesi, M. A.; Berchiesi, G.; Lobbia, G. G. Apparent Molal Volumes of Alkali Metal Nitrates at 30 C. J. Chem. Eng. Data 1974, 19, 326328

(22) Doan, T. H.; Sangster, J. Viscosities of Concentrated Aqueous Solutions of some 1:1, 2:1, and 3:1 Nitrates at $25^{\circ} \mathrm{C}$. J. Chem. Eng. Data 1981, 26, 141-144

(45) Isono, T. Densities, Viscosities, and Electrolytic Conductivity of Concentrated Aqueous Electrolyte Solutions at Several Temperatures. Alkaline-Earth Chlorides, $\mathrm{LaCl}_{3}, \mathrm{Na}_{2} \mathrm{SO}_{4}, \mathrm{NaNO}_{3}, \mathrm{NaBr}, \mathrm{KNO}_{3}, \mathrm{KBr}$, and $\mathrm{Cd}\left(\mathrm{NO}_{3}\right)_{2}$. J. Chem. Eng. Data 1984, 29, 45-52

(73) Millero, F. J.; Ward, G. K.; Chetirkin, P. V. Relative Sound Velocities of Sea Salts at $25^{\circ}$ C. J. Acoust. Soc. Am. 1977, 61, 1492-1498

(79) Olofsson, Inger $\mathrm{V}$;; Apparent molar heat capacities and volumes of aqueous $\mathrm{NaCl}, \mathrm{KCl}$, and $\mathrm{KNO}_{3}$ at $298.15 \mathrm{~K}$. Comparison of Picker flow calorimeter with other calorimeters, J. Chem. Thermodynamics, 1979, 11, 1005-1014

(82) Patterson, B. A.; Woolley, E. M.; Thermodynamics for lonization of Water at Temperatures from $278.15 \mathrm{~K}$ to $393.15 \mathrm{~K}$ and at the Pressure $0.35 \mathrm{MPa}$ : Apparent Molar Volumes and Apparent Molar Heat Capacities of Aqueous Solutions of Potassium and Sodium Nitrates and Nitric Acid, J. Chem. Thermodynamics, 2002, 34, $535-556$

(90) Perry, R. H.; Green, D. W. Perry's Chemical Engineers' Handbook; McGraw Hill: New York, 7th edition, 1997

(110) Roy, Mahendra Nath; Jha, Anupam; Choudhury, Ankan, Densities, Viscosities and Adiabatic Compressibilities of Some Mineral Salts in Water at Different Temperatures, J. Chem. Eng. Data 2004, 49, 291-296.

\begin{tabular}{|c|c|c|c|c|c|c|c|c|c|c|c|c|c|c|c|c|}
\hline molality & molarity & $\mathrm{t} /{ }^{\circ} \mathrm{C}$ & ass frac & $\begin{array}{r}\text { Density } \\
\exp / \\
\mathrm{kg} / \mathrm{m}^{3}\end{array}$ & Ref & $\begin{array}{r}\text { App vol } \\
\exp / \\
\mathrm{m}^{3} / \mathrm{kg}\end{array}$ & $\begin{array}{c}\text { App vol } \\
\text { Calc / } \\
\mathrm{m}^{3} / \mathrm{kg}\end{array}$ & $\begin{array}{r}\text { Density } \\
\text { calc / } \\
\mathrm{kg} / \mathrm{m}^{3}\end{array}$ & $\begin{array}{r}\text { Density } \\
\text { Residual / } \\
\mathrm{kg} / \mathrm{m}^{3}\end{array}$ & $\begin{array}{r}\text { App Vol } \\
\text { Residual / } \\
\mathrm{m}^{3} / \mathrm{kg}\end{array}$ & $\begin{array}{r}\text { Square of } \\
\text { Density Res }\end{array}$ & $\begin{array}{c}\text { Inconsistent } \\
\text { based on } \\
\text { density }\end{array}$ & $\begin{array}{l}\text { Inconsistent } \\
\text { based on } \\
\text { apparent } \\
\text { volume }\end{array}$ & $\begin{array}{l}\text { Apparent } \\
\text { molal volume } \\
1 \mathrm{~cm}^{3} / \mathrm{mol}\end{array}$ & $\begin{array}{r}\text { Delta } \\
\text { Density / } \\
\mathrm{kg} / \mathrm{m}^{3}\end{array}$ & $\begin{array}{r}\text { Density of } \\
\text { water / } \\
\mathrm{kg} / \mathrm{m}^{3}\end{array}$ \\
\hline & & 0 & 0.0100 & 1006.54 & (90) & 0.000334 & 0.000351 & 1006.4 & 0.1691 & -0.000017 & 0.029 & & & & & 999.8395 \\
\hline & & 0 & 0.0200 & 1013.26 & (90) & 0.000338 & 0.000353 & 1013.0 & 0.3089 & -0.000015 & 0.095 & & & & & 999.8395 \\
\hline & & 0 & 0.0400 & 1026.77 & (90) & 0.000344 & 0.000356 & 1026.3 & 0.5097 & -0.000012 & 0.260 & & & & & 999.8395 \\
\hline & & 0 & 0.0800 & 1054.19 & (90) & 0.000356 & 0.000363 & 1053.5 & 0.6994 & -0.000008 & 0.489 & & & & & 999.8395 \\
\hline & & 0 & 0.1200 & 1082.21 & (90) & 0.000366 & 0.000370 & 1081.6 & 0.6415 & -0.000005 & 0.412 & & & & & 999.8395 \\
\hline 0.0298 & & 5 & 0.0030 & 1001.95 & (82) & 0.000341 & 0.000358 & 1001.9 & 0.0531 & -0.000018 & 0.003 & & & 34.43 & & 999.9638 \\
\hline 0.05965 & & 5 & 0.0060 & 1003.94 & (82) & 0.000340 & 0.000359 & 1003.8 & 0.1126 & -0.000019 & 0.013 & & & 34.38 & & 999.9638 \\
\hline 0.11873 & & 5 & 0.0119 & 1007.81 & (82) & 0.000343 & 0.000360 & 1007.6 & 0.1962 & -0.000016 & 0.038 & & & 34.72 & & 999.9638 \\
\hline 0.24826 & & 5 & 0.0245 & 1016.21 & (82) & 0.000347 & 0.000362 & 1015.8 & 0.3724 & -0.000015 & 0.139 & & & 35.096 & & 999.9638 \\
\hline 0.49588 & & 5 & 0.0477 & 1031.64 & (82) & 0.000357 & 0.000366 & 1031.2 & 0.4545 & -0.000009 & 0.207 & & & 36.08 & & 999.9638 \\
\hline & & 10 & 0.0100 & 1006.15 & (90) & 0.000359 & 0.000367 & 1006.1 & 0.0823 & -0.000008 & 0.007 & & & & & 999.6996 \\
\hline & & 10 & 0.0200 & 1012.62 & (90) & 0.000362 & 0.000369 & 1012.5 & 0.1361 & -0.000007 & 0.019 & & & & & 999.6996 \\
\hline & & 10 & 0.0400 & 1025.66 & (90) & 0.000367 & 0.000372 & 1025.5 & 0.1972 & -0.000005 & 0.039 & & & & & 999.6996 \\
\hline & & 10 & 0.0800 & 1052.26 & (90) & 0.000376 & 0.000378 & 1052.0 & 0.2362 & -0.000003 & 0.056 & & & & & 999.6996 \\
\hline & & 10 & 0.1200 & 1079.63 & (90) & 0.000383 & 0.000385 & 1079.4 & 0.2081 & -0.000001 & 0.043 & & & & & 999.6996 \\
\hline 0.0298 & & 15 & 0.0030 & 1001.04 & (82) & 0.000354 & 0.000373 & 1001.0 & 0.0587 & -0.000019 & 0.003 & & & 35.77 & & 999.0996 \\
\hline 0.05 & & 15 & 0.0050 & 1002.34 & (45) & 0.000358 & 0.000374 & 1002.3 & 0.0810 & -0.000016 & 0.007 & & & & & 999.0996 \\
\hline 0.05965 & & 15 & 0.0060 & 1002.94 & (82) & 0.000362 & 0.000374 & 1002.9 & 0.0702 & -0.000012 & 0.005 & & & 36.61 & & 999.0996 \\
\hline 0.1 & & 15 & 0.0100 & 1005.46 & (45) & 0.000368 & 0.000374 & 1005.4 & 0.0613 & -0.000006 & 0.004 & & & & & 999.0996 \\
\hline 0.11873 & & 15 & 0.0119 & 1006.70 & (82) & 0.000364 & 0.000375 & 1006.6 & 0.1308 & -0.000011 & 0.017 & & & 36.78 & & 999.0996 \\
\hline 0.24826 & & 15 & 0.0245 & 1014.80 & (82) & 0.000368 & 0.000377 & 1014.6 & 0.2103 & -0.000008 & 0.044 & & & 37.235 & & 999.0996 \\
\hline 0.49588 & & 15 & 0.0477 & 1029.83 & (82) & 0.000375 & 0.000380 & 1029.6 & 0.2524 & -0.000005 & 0.064 & & & 37.936 & & 999.0996 \\
\hline 0.5 & & 15 & 0.0481 & 1029.92 & (45) & 0.000378 & 0.000380 & 1029.8 & 0.0928 & -0.000002 & 0.009 & & & & & 999.0996 \\
\hline 1 & & 15 & 0.0918 & 1058.62 & (45) & 0.000388 & 0.000387 & 1058.7 & -0.1189 & 0.000001 & 0.014 & & & & & 999.0996 \\
\hline 1.5 & & 15 & 0.1317 & 1085.53 & (45) & 0.000396 & 0.000393 & 1086.0 & -0.4638 & 0.000003 & 0.215 & & & & & 999.0996 \\
\hline 2 & & 15 & 0.1682 & 1111.35 & (45) & 0.000400 & 0.000398 & 1111.7 & -0.3824 & 0.000002 & 0.146 & & & & & 999.0996 \\
\hline 0.05 & & 20 & 0.0050 & 1001.36 & (45) & 0.000374 & 0.000380 & 1001.3 & 0.0316 & -0.000006 & 0.001 & & & & & 998.2041 \\
\hline & & 20 & 0.0100 & 1004.47 & (90) & 0.000377 & 0.000381 & 1004.4 & 0.0425 & -0.000004 & 0.002 & & & & & 998.204 \\
\hline
\end{tabular}




\begin{tabular}{|c|c|c|c|c|}
\hline molality & molarity & $\mathrm{t} /{ }^{\circ} \mathrm{C}$ & ass frac & $\begin{array}{r}\text { Density } \\
\exp / \\
\mathrm{kg} / \mathrm{m}^{3}\end{array}$ \\
\hline 0.1 & & 20 & 0.0100 & 1004.47 \\
\hline & & 20 & 0.0200 & 1010.75 \\
\hline & & 20 & 0.0400 & 1023.44 \\
\hline 0.5 & & 20 & 0.0481 & 1028.61 \\
\hline & & 20 & 0.0800 & 1049.40 \\
\hline 1 & & 20 & 0.0918 & 1057.00 \\
\hline & & 20 & 0.1200 & 1076.20 \\
\hline 1.5 & & 20 & 0.1317 & 1083.61 \\
\hline & & 20 & 0.1600 & 1103.92 \\
\hline 2 & & 20 & 0.1682 & 1109.36 \\
\hline & & 20 & 0.2000 & 1132.61 \\
\hline 2.5 & & 20 & 0.2018 & 1133.15 \\
\hline 3 & & 20 & 0.2327 & 1156.23 \\
\hline & & 20 & 0.2400 & 1162.33 \\
\hline 0.010347 & & 25 & 0.0010 & 997.69 \\
\hline 0.0298 & & 25 & 0.0030 & 998.92 \\
\hline 0.05 & & 25 & 0.0050 & 1000.16 \\
\hline 0.05965 & & 25 & 0.0060 & 1000.79 \\
\hline 0.06145 & & 25 & 0.0062 & 1000.88 \\
\hline 0.06959 & & 25 & 0.0070 & 1001.35 \\
\hline 0.07455 & & 25 & 0.0075 & 1001.67 \\
\hline 0.0866 & & 25 & 0.0087 & 1002.40 \\
\hline 0.1 & & 25 & 0.0100 & 1003.24 \\
\hline 0.100904 & & 25 & 0.0101 & 1003.28 \\
\hline 0.10443 & & 25 & 0.0104 & 1003.50 \\
\hline 0.11873 & & 25 & 0.0119 & 1004.43 \\
\hline 0.12041 & & 25 & 0.0120 & 1004.48 \\
\hline 0.14066 & & 25 & 0.0140 & 1005.71 \\
\hline 0.16696 & & 25 & 0.0166 & 1007.32 \\
\hline 0.19737 & & 25 & 0.0196 & 1009.16 \\
\hline 0.201495 & & 25 & 0.0200 & 1009.39 \\
\hline 0.24826 & & 25 & 0.0245 & 1012.35 \\
\hline 0.300997 & & 25 & 0.0295 & 1015.36 \\
\hline 0.33905 & & 25 & 0.0331 & 1017.65 \\
\hline 0.34078 & & 25 & 0.0333 & 1017.82 \\
\hline 0.39972 & & 25 & 0.0388 & 1021.21 \\
\hline 0.49588 & & 25 & 0.0477 & 1027.04 \\
\hline 0.498781 & & 25 & 0.0480 & 1027.00 \\
\hline 0.5 & & 25 & 0.0481 & 1027.20 \\
\hline 0.5 & & 25 & 0.0481 & 1027.11 \\
\hline 0.5593 & & 25 & 0.0535 & 1030.54 \\
\hline 0.599216 & & 25 & 0.0571 & 1032.80 \\
\hline 0.69984 & & 25 & 0.0661 & 1038.58 \\
\hline 0.7017 & & 25 & 0.0662 & 1038.70 \\
\hline 0.75 & & 25 & 0.0705 & 1041.60 \\
\hline 0.798286 & & 25 & 0.0747 & 1044.13 \\
\hline
\end{tabular}

App vol App vol

exp / Calc /

$\mathrm{m}^{3} / \mathrm{kg} \quad \mathrm{m}^{3} / \mathrm{kg}$

$\begin{array}{lll}0.000377 & 0.000381\end{array}$

$0.000380 \quad 0.000383$

$0.000384 \quad 0.000386$

$\begin{array}{ll}0.000386 & 0.000387\end{array}$

$\begin{array}{ll}0.000391 & 0.000391\end{array}$

$0.000395 \quad 0.000393$

$\begin{array}{lll}0.000397 & 0.000397\end{array}$

$0.000402 \quad 0.000399$

$0.000402 \quad 0.000402$

$\begin{array}{lll}0.000405 & 0.000404\end{array}$

$0.000407 \quad 0.000408$

$0.000410 \quad 0.000408$

$0.000413 \quad 0.000412$

$0.000378 \quad 0.000386$

$\begin{array}{lll}0.000375 & 0.000386\end{array}$

$0.000382 \quad 0.000387$

$0.000377 \quad 0.000387$

$\begin{array}{lll}0.000380 & 0.000387\end{array}$

$0.000385 \quad 0.000387$

$0.000383 \quad 0.000387$

0.0003850 .000387

$0.000384 \quad 0.000387$

$0.000385 \quad 0.000387$

$0.000385 \quad 0.000387$

$0.000381 \quad 0.000388$

$0.000386 \quad 0.000388$

$\begin{array}{ll}0.000386 & 0.000388\end{array}$

$0.000387 \quad 0.000388$

$0.000387 \quad 0.000389$

$0.000388 \quad 0.000389$

$0.000384 \quad 0.000389$

$0.000390 \quad 0.000390$

$0.000390 \quad 0.000391$

$0.000388 \quad 0.000391$

$0.000392 \quad 0.000391$

$\begin{array}{lll}0.000389 & 0.000393\end{array}$

$0.000394 \quad 0.000393$

$\begin{array}{ll}0.000391 & 0.000393\end{array}$

$0.000393 \quad 0.000393$

$0.000394 \quad 0.000393$

$0.000395 \quad 0.000394$

$0.000396 \quad 0.000395$

$0.000396 \quad 0.000395$

$0.000394 \quad 0.000396$

$\begin{array}{lll}0.000397 & 0.000396\end{array}$
Density Density App Vol calc / Residual / Residual / Density Res based on

$\mathrm{kg} / \mathrm{m}^{3} \quad \mathrm{~kg} / \mathrm{m}^{3} \quad \mathrm{~m}^{3} / \mathrm{kg}$

$1004.4 \quad 0.0368-0.000004$

$1010.7 \quad 0.0514-0.000003$

$\begin{array}{lll}1023.4 & 0.0531 & -0.000001\end{array}$

$\begin{array}{lll}1028.6 & 0.0158 & 0.000000\end{array}$

$\begin{array}{lll}1049.4 & 0.0354 & 0.000000\end{array}$

$\begin{array}{lll}057.2 & -0.1991 & 0.000002\end{array}$

$\begin{array}{lll}076.2 & 0.0216 & 0.000000\end{array}$

$\begin{array}{lll}1084.2 & -0.5649 & 0.000004\end{array}$

$1103.9 \quad 0.0479 \quad 0.000000$

$\begin{array}{lll}1109.7 & -0.2994 & 0.000001\end{array}$

$\begin{array}{lll}1132.5 & 0.1172 & 0.000000\end{array}$

$\begin{array}{lll}1133.8 & -0.6251 & 0.000002\end{array}$

$\begin{array}{lll}1156.6 & -0.4009 & 0.000001\end{array}$

$1162.1 \quad 0.2390-0.000001$

$997.7 \quad 0.0087-0.000008$

$\begin{array}{lll}998.9 & 0.0326 & -0.000011\end{array}$

$1000.1 \quad 0.0238-0.000005$

$1000.7 \quad 0.0595-0.000010$

$1000.8 \quad 0.0425-0.000007$

$1001.3 \quad 0.0122-0.000002$

$\begin{array}{lll}1001.6 & 0.0262 & -0.000003\end{array}$

$1002.4 \quad 0.0147-0.000002$

$1003.2 \quad 0.0315-0.000003$

$1003.3 \quad 0.0190-0.000002$

$\begin{array}{lll}1003.5 & 0.0214 & -0.000002\end{array}$

$1004.4 \quad 0.0799-0.000007$

$1004.5 \quad 0.0213-0.000002$

$1005.7 \quad 0.0205-0.000001$

$\begin{array}{lll}1007.3 & 0.0221 & -0.000001\end{array}$

$1009.1 \quad 0.0266-0.000001$

$\begin{array}{lll}1009.4 & 0.0060 & 0.000000\end{array}$

$10122 \quad 0.1378-0.000005$

$\begin{array}{lll}1015.4 & -0.0110 & 0.000000\end{array}$

$\begin{array}{lll}1017.6 & 0.0090 & 0.000000\end{array}$

$1017.7 \quad 0.0815-0.000002$

$\begin{array}{lll}1021.2 & -0.0269 & 0.000001\end{array}$

$1026.9 \quad 0.1633-0.000003$

$\begin{array}{lrr}1027.0 & -0.0450 & 0.000001\end{array}$

$1027.1 \quad 0.0790 \quad-0.000002$

$\begin{array}{lll}1027.1 & -0.0110 & 0.000000\end{array}$

$\begin{array}{lll}1030.6 & -0.0273 & 0.000000\end{array}$

$\begin{array}{lll}1032.9 & -0.0714 & 0.000001\end{array}$

$\begin{array}{lll}1038.6 & -0.0662 & 0.000001\end{array}$

$\begin{array}{lll}1038.7 & -0.0441 & 0.000001\end{array}$

$1041.5 \quad 0.1089-0.000001$

$\begin{array}{lll}1044.2 & -0.0869 & 0.000001\end{array}$

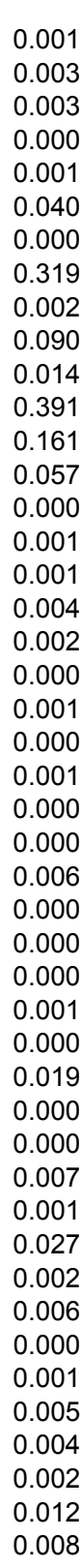

0.003

0.000

0.319

0.391

0.057

0.000

0.001

0.004

0.002

0.000

0.001

0.001

0.000

0.000

0.000

0.000

0.000

0.000

0.019

0.000

0.007

0.001

0.027

0.006

0.000

0.001

0.005

0.002

0.012

.008
Delta Density of Density / water / $\mathrm{kg} / \mathrm{m}^{3} \quad \mathrm{~kg} / \mathrm{m}^{3}$

998.2041 998.2041 998.2041 998.2041 998.2041 998.2041 998.2041 998.2041 998.2041

998.2041 998.2041 998.2041 998.2041 998.2041

$0.65 \quad 997.0449$ 997.0449 997.0449 997.0449 997.0449 997.0449 997.0449 997.0449 997.0449

$6.238 \quad 997.0449$ 997.0449 997.0449 997.0449 997.0449 997.0449 997.0449

39.12

39.16

38.809

39.46

39.25

39.363

39.82

40.01

$12.348 \quad 997.0449$ 997.0449

18.314997 .0449 997.0449 997.0449

$24.163 \quad 997.0449$ 997.0449

$29.96 \quad 997.0449$ 997.0449 997.0449 997.0449

$35.759 \quad 997.0449$

41.531997 .0449 997.0449 $47.086 \quad 997.0449$ 


\begin{tabular}{|c|c|c|c|c|}
\hline molality & molarity & $\mathbf{t} /{ }^{\circ} \mathrm{C}$ & ass frac & $\begin{array}{r}\text { Density } \\
\exp / \\
\mathrm{kg} / \mathrm{m}^{3}\end{array}$ \\
\hline 0.898592 & & 25 & 0.0833 & 1049.74 \\
\hline 0.9059 & & 25 & 0.0839 & 1050.21 \\
\hline 0.9864 & & 25 & 0.0907 & 1054.67 \\
\hline 0.999685 & & 25 & 0.0918 & 1055.31 \\
\hline 1 & & 25 & 0.0918 & 1055.50 \\
\hline 1 & & 25 & 0.0918 & 1055.22 \\
\hline 1.1649 & & 25 & 0.1054 & 1064.33 \\
\hline 1.2361 & & 25 & 0.1111 & 1068.21 \\
\hline 1.248 & & 25 & 0.1120 & 1068.80 \\
\hline 1.5 & & 25 & 0.1317 & 1082.30 \\
\hline 1.5 & & 25 & 0.1317 & 1081.60 \\
\hline 1.5869 & & 25 & 0.1383 & 1086.66 \\
\hline 1.6803 & & 25 & 0.1452 & 1091.47 \\
\hline 1.75 & & 25 & 0.1503 & 1095.10 \\
\hline 2 & & 25 & 0.1682 & 1107.60 \\
\hline 2 & & 25 & 0.1682 & 1107.15 \\
\hline 2.0732 & & 25 & 0.1733 & 1111.13 \\
\hline 2.25 & & 25 & 0.1853 & 1119.70 \\
\hline 2.4653 & & 25 & 0.1995 & 1130.11 \\
\hline 2.5 & & 25 & 0.2018 & 1131.60 \\
\hline 2.5 & & 25 & 0.2018 & 1130.82 \\
\hline 2.75 & & 25 & 0.2175 & 1143.20 \\
\hline 3 & & 25 & 0.2327 & 1154.40 \\
\hline 3 & & 25 & 0.2327 & 1153.82 \\
\hline 0.05 & & 30 & 0.0050 & 998.73 \\
\hline & 0.07935 & 30 & 0.0080 & 1000.55 \\
\hline & 0.09394 & 30 & 0.0095 & 1001.49 \\
\hline 0.1 & & 30 & 0.0100 & 1001.76 \\
\hline & 0.19776 & 30 & 0.0198 & 1007.81 \\
\hline & 0.39703 & 30 & 0.0394 & 1019.90 \\
\hline & 0.41281 & 30 & 0.0409 & 1020.90 \\
\hline 0.5 & & 30 & 0.0481 & 1025.40 \\
\hline & 0.51984 & 30 & 0.0511 & 1027.58 \\
\hline & 0.62315 & 30 & 0.0610 & 1033.52 \\
\hline 1 & & 30 & 0.0918 & 1053.26 \\
\hline & 1.0733 & 30 & 0.1023 & 1060.37 \\
\hline 1.5 & & 30 & 0.1317 & 1079.46 \\
\hline & 1.73791 & 30 & 0.1598 & 1099.59 \\
\hline 2 & & 30 & 0.1682 & 1104.77 \\
\hline 2.5 & & 30 & 0.2018 & 1128.27 \\
\hline 3 & & 30 & 0.2327 & 1151.14 \\
\hline 0.0298 & & 35 & 0.0030 & 995.87 \\
\hline 0.05 & & 35 & 0.0050 & 997.09 \\
\hline 0.05965 & & 35 & 0.0060 & 997.70 \\
\hline 0.1 & & 35 & 0.0100 & 1000.08 \\
\hline 0.11873 & & 35 & 0.0119 & 1001.28 \\
\hline
\end{tabular}

App vol App vol

exp / Calc/

$\mathrm{m}^{3} / \mathrm{kg} \quad \mathrm{m}^{3} / \mathrm{kg}$

$0.000398 \quad 0.000397$

$0.000398 \quad 0.000398$

$\begin{array}{lll}0.000399 & 0.000398\end{array}$

$\begin{array}{ll}0.000400 & 0.000399 \\ 0.000398 & 0.000399\end{array}$

$\begin{array}{ll}0.000398 & 0.000399\end{array}$

$\begin{array}{ll}0.000401 & 0.000400\end{array}$

$\begin{array}{ll}0.000401 & 0.000401\end{array}$

$0.000402 \quad 0.000401$

$0.000403 \quad 0.000404$

$0.000408 \quad 0.000404$

$0.000405 \quad 0.000405$

$0.000405 \quad 0.000406$

$0.000406 \quad 0.000406$

$0.000408 \quad 0.000409$

$\begin{array}{ll}0.000410 & 0.000409\end{array}$

$0.000409 \quad 0.000409$

$0.000410 \quad 0.000411$

$0.000411 \quad 0.000413$

$0.000412 \quad 0.000413$

$0.000415 \quad 0.000413$

$0.000414 \quad 0.000415$

$\begin{array}{ll}0.000416 & 0.000417\end{array}$

$\begin{array}{ll}0.000417 & 0.000417\end{array}$

$\begin{array}{ll}0.000388 & 0.000392\end{array}$

$\begin{array}{lll}0.000391 & 0.000393\end{array}$

$0.000387 \quad 0.000393$

$0.000392 \quad 0.000393$

$0.000393 \quad 0.000394$

$0.000398 \quad 0.000397$

$0.000397 \quad 0.000397$

$0.000399 \quad 0.000398$

$0.000394 \quad 0.000398$

$0.000401 \quad 0.000400$

$0.000406 \quad 0.000404$

$0.000405 \quad 0.000405$

$\begin{array}{lll}0.000412 & 0.000409\end{array}$

$\begin{array}{ll}0.000410 & 0.000412\end{array}$

$0.000415 \quad 0.000413$

$0.000419 \quad 0.000417$

$\begin{array}{lll}0.000421 & 0.000421\end{array}$

$\begin{array}{ll}0.000389 & 0.000397\end{array}$

$0.000393 \quad 0.000398$

$0.000388 \quad 0.000398$

$0.000398 \quad 0.000398$

$\begin{array}{ll}0.000392 & 0.000399\end{array}$
Density Density App Vol calc / Residual / Residual / Density Res based on

$\mathrm{kg} / \mathrm{m}^{3}$

$\mathrm{m}^{3} / \mathrm{kg}$

$\begin{array}{lll}1049.8 & -0.0878 & 0.000001\end{array}$

$\begin{array}{lll}050.2 & -0.0273 & 0.000000\end{array}$

$\begin{array}{lll}054.7 & -0.0272 & 0.000000\end{array}$

$\begin{array}{lll}055.4 & -0.1174 & 0.000001\end{array}$

$\begin{array}{llll}1055.4 & 0.0574 & -0.000001\end{array}$

$\begin{array}{lll}055.4 & -0.2226 & 0.000002\end{array}$

$\begin{array}{lll}1064.4 & -0.0923 & 0.000001\end{array}$

$\begin{array}{lll}1068.3 & -0.0389 & 0.000000\end{array}$

$\begin{array}{lll}1068.9 & -0.0864 & 0.000001\end{array}$

$1082.2 \quad 0.1372-0.000001$

$\begin{array}{lll}1082.2 & -0.5628 & 0.000004\end{array}$

$\begin{array}{lll}1086.7 & 0.0032 & 0.000000\end{array}$

$1091.4 \quad 0.0412 \quad 0.000000$

$1095.0 \quad 0.1348-0.000001$

$1107.4 \quad 0.1834-0.000001$

$\begin{array}{lll}1107.4 & -0.2666 & 0.000001\end{array}$

$\begin{array}{lll}1111.0 & 0.1314 & -0.000001\end{array}$

$1119.5 \quad 0.1684-0.000001$

$1129.7 \quad 0.4056-0.000002$

$\begin{array}{lll}1131.3 & 0.2762 & -0.000001\end{array}$

$\begin{array}{lll}1131.3 & -0.5038 & 0.000002\end{array}$

$1142.8 \quad 0.3938-0.000001$

$1154.0 \quad 0.4089-0.000001$

$\begin{array}{llll}1154.0 & -0.1711 & 0.000001\end{array}$

$\begin{array}{lll}998.7 & 0.0220 & -0.000004\end{array}$

$1000.5 \quad 0.0177-0.000002$

$1001.4 \quad 0.0616-0.000006$

$1001.8 \quad 0.0100-0.000001$

$\begin{array}{lll}1007.8 & 0.0195 & -0.000001\end{array}$

$\begin{array}{lll}1019.9 & -0.0243 & 0.000001\end{array}$

$\begin{array}{lll}1020.9 & 0.0202 & 0.000000\end{array}$

$\begin{array}{lll}1025.4 & -0.0324 & 0.000001\end{array}$

$1027.3 \quad 0.2351-0.000004$

$\begin{array}{lll}1033.6 & -0.0548 & 0.000001\end{array}$

$\begin{array}{lll}1053.5 & -0.2339 & 0.000002\end{array}$

$\begin{array}{lll}1060.4 & -0.0268 & 0.000000\end{array}$

$\begin{array}{lll}1080.0 & -0.5213 & 0.000003\end{array}$

$1099.2 \quad 0.3950-0.000002$

$\begin{array}{lll}1105.0 & -0.2569 & 0.000001\end{array}$

$\begin{array}{lll}1128.7 & -0.4777 & 0.000002\end{array}$

$\begin{array}{lll}1151.2 & -0.1084 & 0.000000\end{array}$

$\begin{array}{lll}995.8 & 0.0263 & -0.000009\end{array}$

$\begin{array}{llll}997.1 & 0.0257 & -0.000005\end{array}$

$\begin{array}{lll}997.6 & 0.0567 & -0.000010\end{array}$

$\begin{array}{lll}10001 & 0.0017 & 0.000000\end{array}$

$1001.2 \quad 0.0725-0.000006$

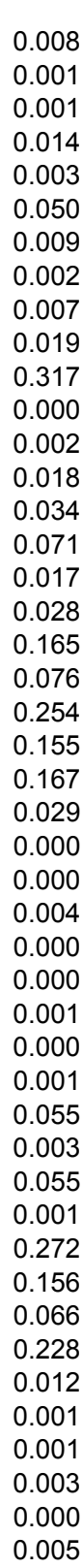

0.001

0.014

0.002

317

0.002

0.018

0.034

0.017

0.028

0.165

0.076

0.155

0.167

0.029

0.000

0.000

0.001

0.001

0.003

0.055

0.001

0.272

0.066

0.012

0.001

0.003

0.000

0.005 based on Apparent

apparent molal volume volume

$1 \mathrm{~cm}^{3} / \mathrm{mol}$

40.31

40.56

40.59

40.64

40.92

40.99

41.32

41.56 Density / water / $\mathrm{kg} / \mathrm{m}^{3} \quad \mathrm{~kg} / \mathrm{m}^{3}$

$52.7 \quad 997.0449$ 997.0449 997.0449

$58.263 \quad 997.0449$ 997.0449 997.0449 997.0449 997.0449 997.0449

997.0449

997.0449

997.0449

997.0449

997.0449

997.0449

997.0449

997.0449

997.0449

997.0449

997.0449

997.0449

997.0449

997.0449

997.0449

995.6473

995.6473

995.6473

995.6473

995.6473

995.6473

995.6473

995.6473

995.6473

995.6473

995.6473

995.6473

995.6473

995.6473

995.6473

995.6473

995.6473

994.0319

994.0319

94.0319

994.0319

994.0319 


\begin{tabular}{|c|c|c|c|c|}
\hline molality & molarity & $\mathrm{t} /{ }^{\circ} \mathrm{C}$ & ass frac & $\begin{array}{r}\text { Density } \\
\exp / \\
\mathrm{kg} / \mathrm{m}^{3}\end{array}$ \\
\hline 0.24826 & & 35 & 0.0245 & 1008.96 \\
\hline 0.49588 & & 35 & 0.0477 & 1023.39 \\
\hline 0.5 & & 35 & 0.0481 & 1023.50 \\
\hline 1 & & 35 & 0.0918 & 1051.14 \\
\hline 1.5 & & 35 & 0.1317 & 1077.13 \\
\hline 2 & & 35 & 0.1682 & 1102.29 \\
\hline 2.5 & & 35 & 0.2018 & 1125.68 \\
\hline 3 & & 35 & 0.2327 & 1148.42 \\
\hline & & 40 & 0.0100 & 998.25 \\
\hline & & 40 & 0.0200 & 1004.30 \\
\hline & & 40 & 0.0400 & 1016.52 \\
\hline & & 40 & 0.0800 & 1041.52 \\
\hline & & 40 & 0.1200 & 1067.40 \\
\hline & & 40 & 0.1600 & 1094.32 \\
\hline & & 40 & 0.2000 & 1122.40 \\
\hline & & 40 & 0.2400 & 1151.75 \\
\hline 0.0298 & & 45 & 0.0030 & 992.00 \\
\hline 0.05 & & 45 & 0.0050 & 993.20 \\
\hline 0.05965 & & 45 & 0.0060 & 993.80 \\
\hline 0.1 & & 45 & 0.0100 & 996.15 \\
\hline 0.11873 & & 45 & 0.0119 & 997.32 \\
\hline 0.24826 & & 45 & 0.0245 & 1004.93 \\
\hline 0.49588 & & 45 & 0.0477 & 1019.15 \\
\hline 0.5 & & 45 & 0.0481 & 1019.24 \\
\hline 1 & & 45 & 0.0918 & 1046.48 \\
\hline 1.5 & & 45 & 0.1317 & 1072.16 \\
\hline 2 & & 45 & 0.1682 & 1097.02 \\
\hline 2.5 & & 45 & 0.2018 & 1120.13 \\
\hline 3 & & 45 & 0.2327 & 1142.70 \\
\hline 0.0298 & & 55 & 0.0030 & 987.48 \\
\hline 0.05 & & 55 & 0.0050 & 988.64 \\
\hline 0.05965 & & 55 & 0.0060 & 989.25 \\
\hline 0.1 & & 55 & 0.0100 & 991.54 \\
\hline 0.11873 & & 55 & 0.0119 & 992.70 \\
\hline 0.24826 & & 55 & 0.0245 & 1000.23 \\
\hline 0.49588 & & 55 & 0.0477 & 1014.26 \\
\hline 0.5 & & 55 & 0.0481 & 1014.33 \\
\hline 1 & & 55 & 0.0918 & 1041.28 \\
\hline 1.5 & & 55 & 0.1317 & 1066.68 \\
\hline 2 & & 55 & 0.1682 & 1091.33 \\
\hline 2.5 & & 55 & 0.2018 & 1114.22 \\
\hline 3 & & 55 & 0.2327 & 1136.54 \\
\hline & & 60 & 0.0100 & 989.00 \\
\hline & & 60 & 0.0200 & 994.90 \\
\hline & & 60 & 0.0400 & 1006.80 \\
\hline & & 60 & 0.0800 & 1031.30 \\
\hline
\end{tabular}

App vol App vol

exp / Calc /

$\mathrm{m}^{3} / \mathrm{kg} \quad \mathrm{m}^{3} / \mathrm{kg}$

$0.000398 \quad 0.000400$

$0.000401 \quad 0.000403$

$0.000404 \quad 0.000403$

$0.000411 \quad 0.000408$

$0.000423 \quad 0.000421$

$0.000425 \quad 0.000425$

$0.000399 \quad 0.000403$

$0.000402 \quad 0.000404$

$0.000405 \quad 0.000407$

$0.000411 \quad 0.000411$

$0.000416 \quad 0.000416$

$0.000420 \quad 0.000420$

$\begin{array}{lll}0.000423 & 0.000425\end{array}$

$\begin{array}{ll}0.000426 & 0.000429\end{array}$

$\begin{array}{ll}0.000403 & 0.000407\end{array}$

$0.000406 \quad 0.000407$

$\begin{array}{ll}0.000401 & 0.000407\end{array}$

$0.000409 \quad 0.000408$

$\begin{array}{lll}0.000403 & 0.000408\end{array}$

$0.000406 \quad 0.000409$

$0.000409 \quad 0.000412$

$0.000412 \quad 0.000412$

$\begin{array}{ll}0.000419 & 0.000417\end{array}$

$0.000424 \quad 0.000421$

$0.000425 \quad 0.000425$

$0.000429 \quad 0.000428$

$0.000431 \quad 0.000431$

$0.000406 \quad 0.000414$

$0.000414 \quad 0.000415$

$0.000407 \quad 0.000415$

$0.000417 \quad 0.000415$

$0.000411 \quad 0.000415$

$0.000412 \quad 0.000417$

$\begin{array}{ll}0.000416 & 0.000419\end{array}$

$\begin{array}{lll}0.000419 & 0.000419\end{array}$

$0.000425 \quad 0.000423$

$0.000430 \quad 0.000427$

$0.000431 \quad 0.000430$

$0.000435 \quad 0.000434$

$0.000436 \quad 0.000436$

$0.000421 \quad 0.000418$

$0.000419 \quad 0.000419$

$0.000421 \quad 0.000421$

$0.000424 \quad 0.000425$
Density Density App Vol

Square of Inconsistent Inconsistent

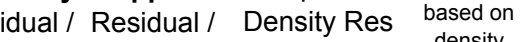

$\mathrm{kg} / \mathrm{m}^{3}$

$\mathrm{kg} / \mathrm{m}^{3}$

$\mathrm{m}^{3} / \mathrm{kg}$

$1008.9 \quad 0.0545-0.000002$

$023.3 \quad 0.0805-0.000002$

$\begin{array}{lll}023.5 & -0.0486 & 0.000001\end{array}$

$\begin{array}{lll}051.4 & -0.2323 & 0.000002\end{array}$

0.006

$\begin{array}{lll}1077.6 & -0.5190 & 0.000003\end{array}$

$\begin{array}{lll}102.5 & -0.2181 & 0.000001\end{array}$

$\begin{array}{lll}1126.1 & -0.3839 & 0.000002\end{array}$

$\begin{array}{lll}1148.4 & 0.0014 & 0.000000\end{array}$

$998.2 \quad 0.0452-0.000005$

$1004.2 \quad 0.0575-0.000003$

$1016.5 \quad 0.0537-0.000001$

$\begin{array}{lll}1041.5 & -0.0066 & 0.000000\end{array}$

$\begin{array}{lll}1067.4 & -0.0424 & 0.000000\end{array}$

$\begin{array}{lll}1094.3 & 0.0576 & 0.000000\end{array}$

$1122.0 \quad 0.3606-0.000001$

$1150.8 \quad 0.9200-0.000003$

$\begin{array}{llll}992.0 & 0.0121 & -0.000004\end{array}$

$\begin{array}{llll}993.2 & 0.0044 & -0.000001\end{array}$

$993.8 \quad 0.0344-0.000006$

$\begin{array}{llll}996.2 & -0.0102 & 0.000001\end{array}$

$\begin{array}{lll}997.3 \quad 0.0578 & -0.000005\end{array}$

$1004.8 \quad 0.0863-0.000003$

$1019.0 \quad 0.1289-0.000003$

$\begin{array}{lll}1019.3 & -0.0182 & 0.000000\end{array}$

$\begin{array}{lll}1046.7 & -0.1904 & 0.000002\end{array}$

$\begin{array}{lll}1072.6 & -0.4288 & 0.000003\end{array}$

$\begin{array}{lll}1097.1 & -0.1163 & 0.000001\end{array}$

$\begin{array}{lll}1120.4 & -0.2920 & 0.000001\end{array}$

$\begin{array}{lll}1142.5 & 0.1567 & -0.000001\end{array}$

$987.4 \quad 0.0259-0.000009$

$\begin{array}{lll}988.6 & 0.0043 & -0.000001\end{array}$

$989.20 .0445-0.000008$

$\begin{array}{llll}991.6 & -0.0191 & 0.000002\end{array}$

$992.6 \quad 0.0551-0.000005$

$1000.1 \quad 0.1018-0.000004$

$1014.1 \quad 0.1402-0.000003$

$\begin{array}{llll}1014.4 & -0.0204 & 0.000000\end{array}$

$\begin{array}{lll}1041.4 & -0.1511 & 0.000002\end{array}$

$\begin{array}{lll}1067.1 & -0.3892 & 0.000003\end{array}$

$\begin{array}{lll}1091.4 & -0.0513 & 0.000000\end{array}$

$\begin{array}{lll}1114.5 & -0.2508 & 0.000001\end{array}$

$\begin{array}{lll}1136.4 & 0.1095 & 0.000000\end{array}$

$\begin{array}{lll}989.0 & -0.0217 & 0.000002\end{array}$

$\begin{array}{lll}994.9 & 0.0049 & 0.000000\end{array}$

$\begin{array}{lll}1006.8 & 0.0036 & 0.000000\end{array}$

$\begin{array}{lll}1031.2 & 0.0611 & -0.000001\end{array}$

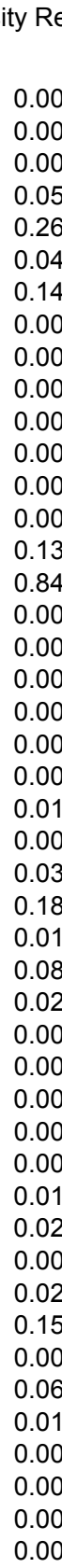

Apparent

Delta Density of / Density / water / $1 \mathrm{~cm}^{3} / \mathrm{mol} \quad \mathrm{kg} / \mathrm{m}^{3} \quad \mathrm{~kg} / \mathrm{m}^{3}$

\subsection{6}

40.588

$\mathrm{kg} / \mathrm{m}^{3} \quad \mathrm{~kg} / \mathrm{m}^{3}$

994.0319

994.0319

994.0319

994.0319

994.0319

994.0319

994.0319

994.0319

992.2158

992.2158

992.2158

992.2158

992.2158

992.2158

992.2158

992.2158

990.2132

990.2132

990.2132

990.2132

990.2132

990.2132

990.2132

990.2132

990.2132

990.2132

990.2132

990.2132

990.2132

985.6952

(985.6952

985.6952

985.6952

985.6952

985.6952

985.6952

985.6952

985.6952

985.6952

985.6952

985.6952

985.6952

983.1989

83.1989

983.1989

983.1989 


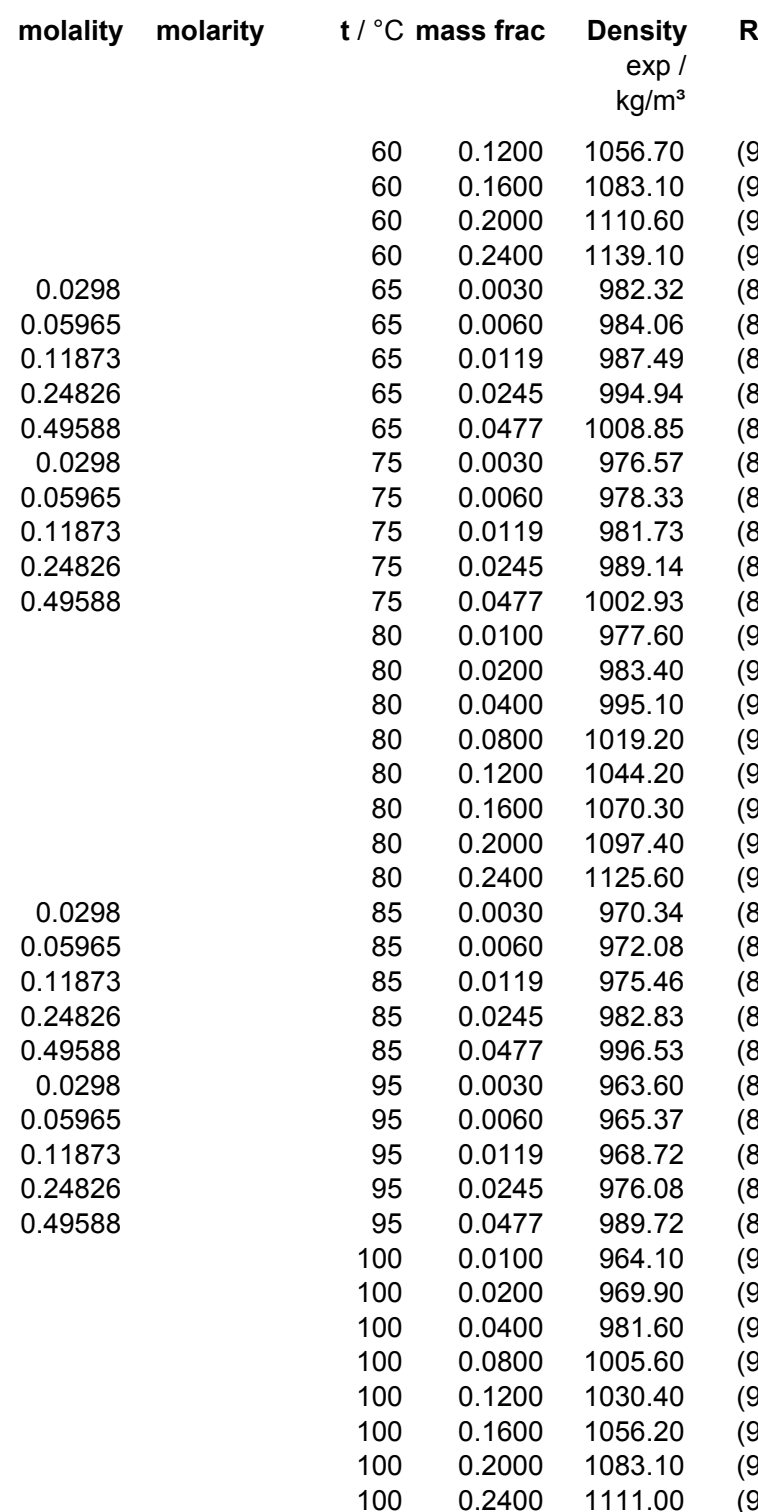

App vol App vol D

exp / Calc /

$$
\mathrm{m}^{3} / \mathrm{kg} \quad \mathrm{m}^{3} / \mathrm{kg}
$$

$0.000428 \quad 0.000428$

$\begin{array}{lll}0.000431 & 0.000432\end{array}$

$0.000434 \quad 0.000435$

$\begin{array}{lll}0.000437 & 0.000439\end{array}$

$0.000411 \quad 0.000420$

$0.000414 \quad 0.000421$

$0.000416 \quad 0.000421$

$\begin{array}{ll}0.000418 & 0.000422\end{array}$

$0.000421 \quad 0.000424$

$0.000425 \quad 0.000425$

$0.000416 \quad 0.000425$

$0.000420 \quad 0.000426$

$0.000420 \quad 0.000427$

$0.000424 \quad 0.000429$

$\begin{array}{ll}0.000418 & 0.000427\end{array}$

$0.000422 \quad 0.000428$

$0.000427 \quad 0.000430$

0.0004310 .000433

$0.000434 \quad 0.000435$

$0.000437 \quad 0.000438$

$0.000440 \quad 0.000441$

$0.000443 \quad 0.000443$

$0.000425 \quad 0.000428$

$0.000419 \quad 0.000429$

$0.000422 \quad 0.000429$

$0.000423 \quad 0.000430$

$0.000427 \quad 0.000431$

$\begin{array}{ll}0.000430 & 0.000430\end{array}$

$\begin{array}{ll}0.000416 & 0.000431\end{array}$

$0.000422 \quad 0.000431$

$0.000423 \quad 0.000432$

$0.000427 \quad 0.000433$

$0.000423 \quad 0.000431$

$0.000423 \quad 0.000432$

$0.000426 \quad 0.000433$

$0.000431 \quad 0.000435$

$\begin{array}{ll}0.000436 & 0.000437\end{array}$

$\begin{array}{ll}0.000439 & 0.000439\end{array}$

$0.000443 \quad 0.000441$

$0.000446 \quad 0.000443$
Density Density App Vol Square of Inconsistent
calc / Residual / Residual /
Density Res based on
based on

$\mathrm{kg} / \mathrm{m}^{3} \quad \mathrm{~kg} / \mathrm{m}^{3} \quad \mathrm{~m}^{3} / \mathrm{kg}$

$1056.6 \quad 0.1226-0.000001$

$1082.9 \quad 0.2332-0.000001$

$1110.2 \quad 0.4330-0.000002$

$1138.5 \quad 0.5577-0.000002$

$\begin{array}{llll}982.3 & 0.0270 & -0.000009\end{array}$

$984.0 \quad 0.0418-0.000007$

$\begin{array}{llll}987.4 & 0.0573 & -0.000005\end{array}$

$\begin{array}{lll}994.8 & 0.1172 & -0.000005\end{array}$

$1008.7 \quad 0.1853 \quad-0.000004$

$\begin{array}{lll}976.6 & 0.0000 & 0.000000\end{array}$

$\begin{array}{lll}978.3 & 0.0524 & -0.000009\end{array}$

$981.7 \quad 0.0689-0.000006$

$989.0 \quad 0.1521-0.000006$

$1002.7 \quad 0.2153-0.000004$

$977.5 \quad 0.0874-0.000009$

$983.3 \quad 0.1192-0.000006$

$995.0 \quad 0.1196-0.000003$

$1019.1 \quad 0.1432-0.000002$

$1044.1 \quad 0.1155-0.000001$

$1070.1-0.1742-0.000001$

$1097.2 \quad 0.1516-0.000001$

$\begin{array}{lll}1125.5 & 0.0740 \quad 0.000000\end{array}$

$970.3 \quad 0.0098-0.000003$

$972.0 \quad 0.0534-0.000009$

$\begin{array}{lll}975.4 & 0.0786 & -0.000007\end{array}$

$982.7 \quad 0.1635-0.000007$

$996.3 \quad 0.2242-0.000005$

$\begin{array}{llll}963.6 & 0.0000 & 0.000000\end{array}$

$965.3 \quad 0.0806-0.000014$

$968.6 \quad 0.0951-0.000009$

$\begin{array}{lll}975.9 & 0.2100 & -0.000009\end{array}$

$989.5 \quad 0.2646-0.000006$

$\begin{array}{llll}964.0 & 0.0821 & -0.000009\end{array}$

$969.7 \quad 0.1713-0.000009$

$981.3 \quad 0.2758-0.000007$

$1005.2 \quad 0.3630-0.000004$

$1030.2 \quad 0.2334-0.000002$

$\begin{array}{lll}1056.2 & 0.0165 & 0.000000\end{array}$

$\begin{array}{lll}1083.4 & -0.2648 & 0.000001\end{array}$

$\begin{array}{rrr}1111.8 & -0.7945 & 0.000003 \\ \text { verage Res } & 0.0328 & -0.000003\end{array}$

$\begin{array}{lll}\text { Std dev Res } & 0.219617 & 0.000005\end{array}$

Avg - 4std $\quad-0.84566 \quad-0.000021$

Avg + 4std $\quad 0.911279 \quad 0.000016$
0.054

0.187

0.311

0.001

0.002

0.003

0.014

0.034

0.000

0.003

0.005

0.023

0.008

0.014

0.014

0.021

0.013

0.030

0.023

0.005

0.000

0.003

0.006

0.027

0.050

0.000

0.006

0.009

0.044

0.070

0.007

0.029

0.076

0.132

0.054

0.000

0.070

0.631

10.1093 Sum Chi sqr
Delta Density of Density / water / $\mathrm{kg} / \mathrm{m}^{3} \quad \mathrm{~kg} / \mathrm{m}^{3}$ 983.1989 983.1989 983.1989 980.5548 980.5548 980.5548 980.5548 980.5548

974.849 974.849 974.849 974.849

974.849 971.7978 971.7978 971.7978 971.7978 971.7978 971.7978 971.7978 971.7978 968.6203 968.6203 968.6203 968.6203 968.6203 961.9004 961.9004 961.9004 961.9004 961.9004 958.3637 958.3637 958.3637 958.3637 958.3637 958.3637 958.3637 958.3637 
molality molarity

t $/{ }^{\circ} \mathrm{C}$ mass frac

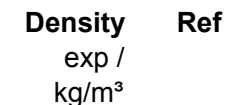

App vol App vol

exp/ Calc /

$\mathrm{kg} / \mathrm{m}^{3}$

$\left.01^{*}\left(t{ }^{\circ} \mathrm{C}+c 4\right)^{\wedge} 2\right)$

c0

$\mathrm{c} 1$
$\mathrm{c} 2$

c3

c4

$\operatorname{Min} T$

$\operatorname{Max} T$

Avax w

Avg dens res

Std dens res

Std app vol res *10^3 0.002757

No of points in corr

7.543551

26.38746

1.239547

0.011656

2213.955

206

Inconsistent data not used (Residual greater than average $+/-\mathbf{4}$ standard deviations)

1.25

$\begin{array}{rrrrrrr}0.01205 & 29.85 & 0.1122 & 1066.00 & (22) & 0.000425 & 0.000401 \\ 0.06026 & 29.85 & 0.0061 & 996.25 & (110) & 0.000545 & 0.000392 \\ 0.10847 & 29.85 & 0.0109 & 1001.59 & (110) & 0.000497 & 0.000392 \\ 0.16874 & 29.85 & 0.0170 & 1005.39 & (110) & 0.000464 & 0.000393 \\ 0.22899 & 29.85 & 0.0229 & 1009.09 & (110) & 0.000433 & 0.000394 \\ 0.01203 & 34.85 & 0.0012 & 994.55 & (110) & 0.000620 & 0.000395 \\ 0.06009 & 34.85 & 0.0061 & 996.67 & (110) & 0.000578 & 0.000397 \\ 0.10819 & 34.85 & 0.0109 & 999.11 & (110) & 0.000544 & 0.000398 \\ 0.16829 & 34.85 & 0.0170 & 1002.76 & (110) & 0.000493 & 0.000399 \\ 0.22839 & 34.85 & 0.0229 & 1006.25 & (110) & 0.000476 & 0.000400 \\ 0.01202 & 39.85 & 0.0012 & 992.68 & (110) & 0.000670 & 0.000402 \\ 0.01202 & 39.85 & 0.0012 & 992.68 & (110) & 0.000670 & 0.000402 \\ 0.02997 & 39.85 & 0.0030 & 994.61 & (110) & 0.000231 & 0.000402 \\ 0.10785 & 39.85 & 0.0109 & 997.05 & (110) & 0.000566 & 0.000403 \\ 0.16779 & 39.85 & 0.0170 & 1000.16 & (110) & 0.000539 & 0.000404 \\ 0.22776 & 39.85 & 0.0229 & 1003.72 & (110) & 0.000507 & 0.000405 \\ 0.01199 & 44.85 & 0.0012 & 990.67 & (110) & 0.000682 & 0.000406 \\ 0.05985 & 44.85 & 0.0061 & 992.51 & (110) & 0.000637 & 0.000407 \\ 0.1076 & 44.85 & 0.0109 & 994.90 & (110) & 0.000581 & 0.000408 \\ 0.16748 & 44.85 & 0.0170 & 997.97 & (110) & 0.000551 & 0.000408 \\ 0.22726 & 44.85 & 0.0229 & 1001.48 & (110) & 0.000517 & 0.000409 \\ 0.01198 & 49.85 & 0.0012 & 988.45 & (110) & 0.000723 & 0.000410 \\ 0.05976 & 49.85 & 0.0061 & 990.16 & (110) & 0.000668 & 0.000411 \\ 0.10744 & 49.85 & 0.0110 & 991.23 & (110) & 0.000721 & 0.000412 \\ 0.16729 & 49.85 & 0.0170 & 995.82 & (110) & 0.000550 & 0.000412 \\ 0.22712 & 49.85 & 0.0230 & 999.08 & (110) & 0.000528 & 0.000413\end{array}$

0.0010

0.2400

0.03281

\section{5}

Density Density App Vol Square of Inconsistent
calc / Residual / Residual / Density Res based on $\mathrm{kg} / \mathrm{m}^{3} \quad \mathrm{~kg} / \mathrm{m}^{3} \quad \mathrm{~m}^{3} / \mathrm{kg}$

$\begin{array}{rr}8.964 & \text { ! } \\ 0.034 & \\ 0.406 & \\ 0.613 & \\ 0.462 & \\ 0.445 & \\ 0.073 & \\ 1.190 & ! \\ 2.532 & ! \\ 2.575 & \text { ! } \\ 3.138 & \text { ! } \\ 0.105 & \\ 0.105 & \\ 0.267 & \\ 3.158 & ! \\ 5.302 & ! \\ 5.611 & \text { ! } \\ 0.109 & \\ 1.913 & ! \\ 3.522 & ! \\ 5.848 & ! \\ 6.266 & ! \\ 0.140 & \\ 2.365 & ! \\ 11.165 & ! \\ 5.443 & ! \\ 7.057 & \text { ! }\end{array}$

997.0449

995.6924 995.6924 995.6924 995.6924 995.6924 994.0833 994.0833 994.0833 994.0833 994.0833 992.273 992.273 992.273 992.273 992.273 992.273 990.2759 990.2759 990.2759 990.2759 990.2759 988.104 988.104 988.104 988.104 988.104 
Density of aqueous solutions of $\mathrm{KOH}$

References (4) Akerlof, G.; Bender, P. The Density of Aqueous Solutions of Potassium Hydroxide. J. Am. Chem. Soc. 1941, 63, 1085-1088.

(41) Hitchcock, L. B.; Mcllhenny, J. S. Viscosity and Density of Pure Alkaline Solutions and their Mixtures. Ind. Engng. Chem. 1935, 27, 461466

(69) Mashovets, V. P.; Dibrov, I. A.; Krumgal'z, B. S.; Mateeva, R. P. Density of Aqueous KOH Solutions at High Temperatures over a Wide Range of Concentrations. J. Appl. Chem. USSR 1965, 38, 2344-2347.

(81) Patterson, B. A.; Call, T. G.; Jardine, J. J.; Origlia-Luster, M. L.; Woolley, E. M.; Thermodynamics for lonization of Water at

Temperatures from $278.15 \mathrm{~K}$ to $393.15 \mathrm{~K}$ and at the Pressure $0.35 \mathrm{MPa}$ : Apparent Molar Volumes of Aqueous $\mathrm{KCl}, \mathrm{KOH}$, and $\mathrm{NaOH}$ and Apparent Molar Heat Capacities of Aqueous $\mathrm{HCl}, \mathrm{KCl}, \mathrm{KOH}$, and $\mathrm{NaOH}, \mathrm{J}$. Chem. Thermodynamics, 2001, 33,1237-1262

(90) Perry, R. H.; Green, D. W. Perry's Chemical Engineers' Handbook; McGraw Hill: New York, 7th edition, 1997.

(109) Roux, A. H.; Perron, G.; Desnoyers, J. E. Capacités calorifiques, volumes, expansibilités et compressibilités des solutions aqueuses concentrées de LiOH, $\mathrm{NaOH}$ et KOH. Can. J. Chem. 1984, 62, 878-885

(127) Tham, M. K.; Gubbins, K. E.; Walker Jr., R. D. Densities of Potassium Hydroxide Solutions J. Chem. Eng. Data 1967, 12, 525-526.

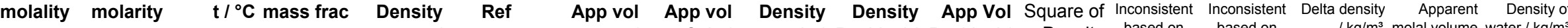

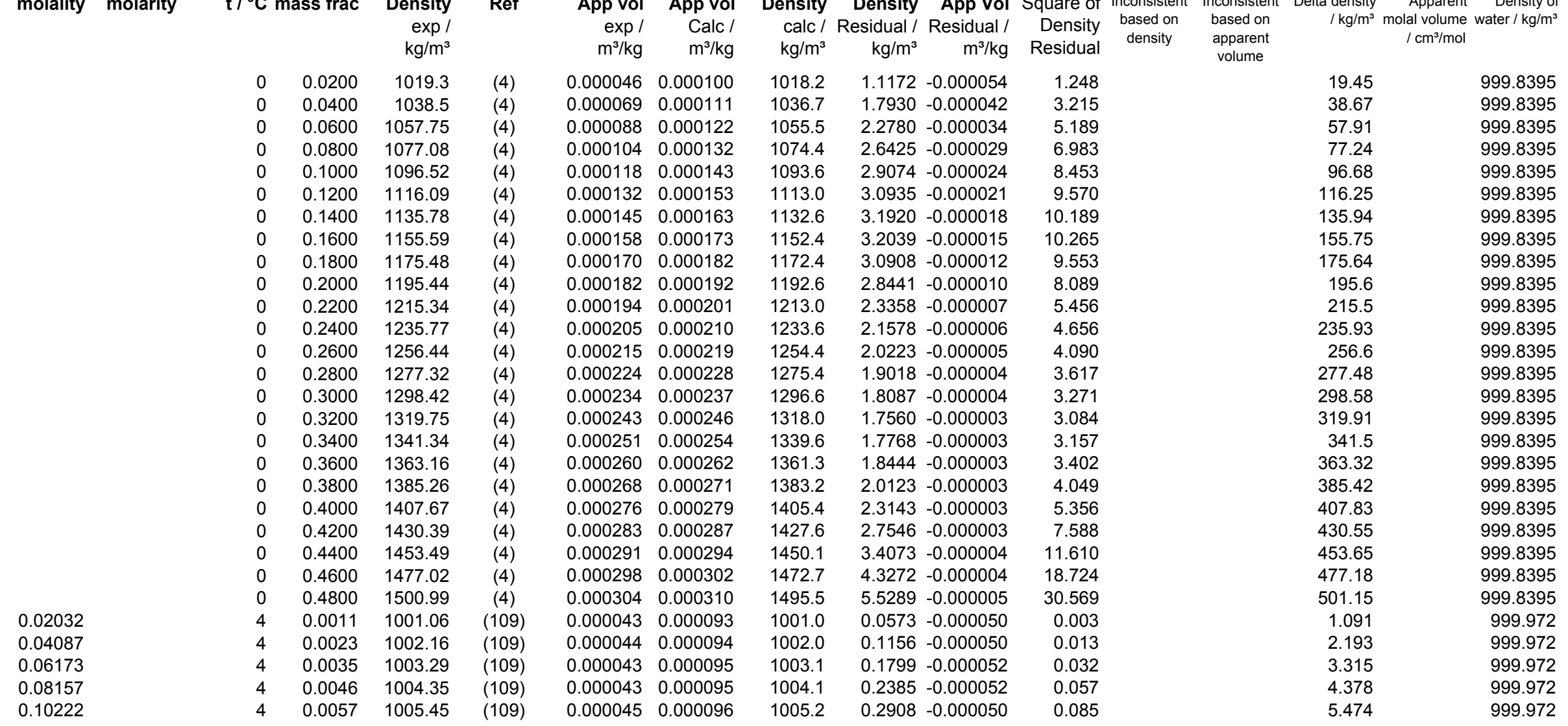




\begin{tabular}{|c|c|c|c|c|c|c|c|c|c|c|c|c|c|c|c|}
\hline molality molarity & \multicolumn{2}{|c|}{$\mathrm{t} /{ }^{\circ} \mathrm{C}$ mass frac } & $\begin{array}{r}\text { Density } \\
\exp / \\
\mathrm{kg} / \mathrm{m}^{3}\end{array}$ & Ref & $\begin{array}{r}\text { App vol } \\
\text { exp / } \\
\mathrm{m}^{3} / \mathrm{kg}\end{array}$ & $\begin{array}{r}\text { App vol } \\
\text { Calc / } \\
\mathrm{m}^{3} / \mathrm{kg}\end{array}$ & $\begin{array}{r}\text { Density } \\
\text { calc / } \\
\mathrm{kg} / \mathrm{m}^{3}\end{array}$ & $\begin{array}{r}\text { Density } \\
\text { Residual / } \\
\mathrm{kg} / \mathrm{m}^{3}\end{array}$ & $\begin{array}{r}\text { App Vol } \\
\text { Residual / } \\
\mathrm{m}^{3} / \mathrm{kg}\end{array}$ & $\begin{array}{r}\text { Square of } \\
\text { Density } \\
\text { Residual }\end{array}$ & $\begin{array}{l}\text { Inconsistent } \\
\text { based on } \\
\text { density }\end{array}$ & $\begin{array}{l}\text { Inconsistent } \\
\text { based on } \\
\text { apparent } \\
\text { volume }\end{array}$ & $\begin{array}{r}\text { Delta density } \\
\qquad / \mathrm{kg} / \mathrm{m}^{3}\end{array}$ & $\begin{array}{r}\text { Apparent } \\
\text { molal volume } \\
/ \mathrm{cm}^{3} / \mathrm{mol}\end{array}$ & $\begin{array}{r}\text { Density of } \\
\text { water } / \mathrm{kg} / \mathrm{m}^{3}\end{array}$ \\
\hline 0.2018 & 4 & 0.0112 & 1010.70 & (109) & 0.000052 & 0.000099 & 1010.2 & 0.5358 & -0.000047 & 0.287 & & & 10.728 & & 999.972 \\
\hline 0.3035 & 4 & 0.0167 & 1015.99 & (109) & 0.000059 & 0.000102 & 1015.2 & 0.7467 & -0.000043 & 0.557 & & & 16.014 & & 999.972 \\
\hline 0.5122 & 4 & 0.0279 & 1026.65 & (109) & 0.000070 & 0.000108 & 1025.5 & 1.1237 & -0.000038 & 1.263 & & & 26.679 & & 999.972 \\
\hline 1.0068 & 4 & 0.0535 & 1050.98 & (109) & 0.000092 & 0.000122 & 1049.2 & 1.7297 & -0.000029 & 2.992 & & & 51.004 & & 999.972 \\
\hline 1.0132 & 4 & 0.0538 & 1051.27 & (109) & 0.000093 & 0.000122 & 1049.5 & 1.7258 & -0.000029 & 2.978 & & & 51.301 & & 999.972 \\
\hline 2.019 & 4 & 0.1018 & 1097.23 & (109) & 0.000129 & 0.000147 & 1095.0 & 2.1891 & -0.000018 & 4.792 & & & 97.259 & & 999.972 \\
\hline 3.542 & 4 & 0.1658 & 1159.52 & (109) & 0.000170 & 0.000178 & 1157.7 & 1.8649 & -0.000008 & 3.478 & & & 159.551 & & 999.972 \\
\hline 4.972 & 4 & 0.2181 & 1211.63 & (109) & 0.000199 & 0.000203 & 1210.4 & 1.2191 & -0.000004 & 1.486 & & & 211.659 & & 999.972 \\
\hline 7.541 & 4 & 0.2973 & 1293.64 & (109) & 0.000236 & 0.000238 & 1292.9 & 0.7678 & -0.000002 & 0.589 & & & 293.673 & & 999.972 \\
\hline 10.254 & 4 & 0.3652 & 1367.01 & (109) & 0.000265 & 0.000266 & 1366.0 & 0.9944 & -0.000001 & 0.989 & & & 367.043 & & 999.972 \\
\hline 15.51 & 4 & 0.4653 & 1480.63 & (109) & 0.000302 & 0.000305 & 1477.7 & 2.9300 & -0.000003 & 8.585 & & & 480.659 & & 999.972 \\
\hline 0.015 & 5 & 0.0008 & 1000.78 & (81) & 0.000025 & 0.000094 & 1000.7 & 0.0582 & -0.000069 & 0.003 & & & & 1.4 & 999.9638 \\
\hline 0.03005 & 5 & 0.0017 & 1001.63 & (81) & 0.000012 & 0.000095 & 1001.5 & 0.1401 & -0.000083 & 0.020 & & & & 0.65 & 999.9638 \\
\hline 0.06006 & 5 & 0.0034 & 1003.23 & (81) & 0.000031 & 0.000095 & 1003.0 & 0.2191 & -0.000065 & 0.048 & & & & 1.72 & 999.9638 \\
\hline 0.12604 & 5 & 0.0070 & 1006.77 & (81) & 0.000038 & 0.000098 & 1006.3 & 0.4249 & -0.000060 & 0.181 & & & & 2.12 & 999.9638 \\
\hline 0.24963 & 5 & 0.0138 & 1013.18 & (81) & 0.000056 & 0.000101 & 1012.5 & 0.6468 & -0.000046 & 0.418 & & & & 3.12 & 999.9638 \\
\hline 0.49992 & 5 & 0.0273 & 1025.87 & (81) & 0.000075 & 0.000109 & 1024.9 & 0.9749 & -0.000034 & 0.950 & & & & 4.187 & 999.9638 \\
\hline 0.02032 & 10 & 0.0011 & 1000.78 & (109) & 0.000053 & 0.000099 & 1000.7 & 0.0523 & -0.000046 & 0.003 & & & 1.079 & & 999.6996 \\
\hline 0.04087 & 10 & 0.0023 & 1001.85 & (109) & 0.000062 & 0.000100 & 1001.8 & 0.0867 & -0.000038 & 0.008 & & & 2.15 & & 999.6996 \\
\hline 0.06173 & 10 & 0.0035 & 1002.94 & (109) & 0.000064 & 0.000100 & 1002.8 & 0.1282 & -0.000037 & 0.016 & & & 3.242 & & 999.6996 \\
\hline 0.08157 & 10 & 0.0046 & 1003.98 & (109) & 0.000065 & 0.000101 & 1003.8 & 0.1677 & -0.000037 & 0.028 & & & 4.279 & & 999.6996 \\
\hline 0.10222 & 10 & 0.0057 & 1005.06 & (109) & 0.000064 & 0.000102 & 1004.8 & 0.2171 & -0.000038 & 0.047 & & & 5.365 & & 999.6996 \\
\hline 0.2018 & 10 & 0.0112 & 1010.19 & (109) & 0.000073 & 0.000105 & 1009.8 & 0.3639 & -0.000032 & 0.132 & & & 10.487 & & 999.6996 \\
\hline 0.3035 & 10 & 0.0167 & 1015.35 & (109) & 0.000079 & 0.000108 & 1014.9 & 0.4890 & -0.000028 & 0.239 & & & 15.653 & & 999.6996 \\
\hline & 10 & 0.0200 & 1018.44 & (4) & 0.000080 & 0.000109 & 1017.8 & 0.6100 & -0.000029 & 0.372 & & & 18.74 & & 999.6996 \\
\hline 0.5122 & 10 & 0.0279 & 1025.79 & (109) & 0.000089 & 0.000114 & 1025.1 & 0.7117 & -0.000024 & 0.507 & & & 26.095 & & 999.6996 \\
\hline & 10 & 0.0400 & 1037.03 & $(4)$ & 0.000100 & 0.000120 & 1036.2 & 0.8573 & -0.000020 & 0.735 & & & 37.33 & & 999.6996 \\
\hline 1.0068 & 10 & 0.0535 & 1049.70 & (109) & 0.000109 & 0.000127 & 1048.6 & 1.0552 & -0.000018 & 1.113 & & & 50.002 & & 999.6996 \\
\hline 1.0132 & 10 & 0.0538 & 1049.99 & (109) & 0.000110 & 0.000127 & 1048.9 & 1.0422 & -0.000018 & 1.086 & & & 50.288 & & 999.6996 \\
\hline & 10 & 0.0600 & 1055.70 & (4) & 0.000116 & 0.000130 & 1054.7 & 0.9717 & -0.000015 & 0.944 & & & 56.00 & & 999.6996 \\
\hline & 10 & 0.0800 & 1074.51 & (4) & 0.000130 & 0.000141 & 1073.5 & 1.0131 & -0.000011 & 1.026 & & & 74.81 & & 999.6996 \\
\hline & 10 & 0.1000 & 1093.49 & (4) & 0.000142 & 0.000151 & 1092.5 & 1.0116 & -0.000008 & 1.023 & & & 93.79 & & 999.6996 \\
\hline 2.019 & 10 & 0.1018 & 1095.33 & (109) & 0.000142 & 0.000152 & 1094.2 & 1.1716 & -0.000010 & 1.373 & & & 95.628 & & 999.6996 \\
\hline & 10 & 0.1200 & 1112.65 & $(4)$ & 0.000154 & 0.000161 & 1111.7 & 0.9773 & -0.000007 & 0.955 & & & 112.95 & & 999.6996 \\
\hline & 10 & 0.1400 & 1131.99 & (4) & 0.000165 & 0.000170 & 1131.1 & 0.9104 & -0.000005 & 0.829 & & & 132.29 & & 999.6996 \\
\hline & 10 & 0.1600 & 1151.52 & $(4)$ & 0.000176 & 0.000180 & 1150.7 & 0.8214 & -0.000004 & 0.675 & & & 151.82 & & 999.6996 \\
\hline 3.542 & 10 & 0.1658 & 1157.11 & (109) & 0.000180 & 0.000183 & 1156.4 & 0.6888 & -0.000003 & 0.474 & & & 157.407 & & 999.6996 \\
\hline & 10 & 0.1800 & 1171.20 & (4) & 0.000187 & 0.000189 & 1170.5 & 0.6708 & -0.000003 & 0.450 & & & 171.50 & & 999.6996 \\
\hline & 10 & 0.2000 & 1191.04 & (4) & 0.000197 & 0.000198 & 1190.6 & 0.4693 & -0.000002 & 0.220 & & & 191.34 & & 999.6996 \\
\hline 4.972 & 10 & 0.2181 & 1208.91 & (109) & 0.000207 & 0.000207 & 1208.9 & -0.0022 & 0.000000 & 0.000 & & & 209.212 & & 999.6996 \\
\hline & 10 & 0.2200 & 1210.91 & (4) & 0.000207 & 0.000208 & 1210.8 & 0.0878 & 0.000000 & 0.008 & & & 211.21 & & 999.6996 \\
\hline & 10 & 0.2400 & 1231.16 & (4) & 0.000217 & 0.000216 & 1231.3 & -0.1227 & 0.000000 & 0.015 & & & 231.46 & & 999.6996 \\
\hline & 10 & 0.2600 & 1251.67 & (4) & 0.000226 & 0.000225 & 1252.0 & -0.2811 & 0.000001 & 0.079 & & & 251.97 & & 999.6996 \\
\hline
\end{tabular}




\begin{tabular}{|c|c|c|c|c|}
\hline \multirow[t]{2}{*}{ molality } & \multirow[t]{2}{*}{ molarity } & $\mathrm{t} /{ }^{\circ} \mathrm{C}$ & ass frac & $\begin{array}{r}\text { Density } \\
\exp / \\
\mathrm{kg} / \mathrm{m}^{3}\end{array}$ \\
\hline & & 10 & 0.2800 & 1272.41 \\
\hline \multirow[t]{5}{*}{7.541} & & 10 & 0.2973 & 1290.61 \\
\hline & & 10 & 0.3000 & 1293.40 \\
\hline & & 10 & 0.3200 & 1314.64 \\
\hline & & 10 & 0.3400 & 1336.13 \\
\hline & & 10 & 0.3600 & 1357.88 \\
\hline \multirow[t]{6}{*}{10.254} & & 10 & 0.3652 & 1363.70 \\
\hline & & 10 & 0.3800 & 1379.91 \\
\hline & & 10 & 0.4000 & 1402.21 \\
\hline & & 10 & 0.4200 & 1424.81 \\
\hline & & 10 & 0.4400 & 1447.73 \\
\hline & & 10 & 0.4600 & 1471.01 \\
\hline \multirow[t]{3}{*}{15.51} & & 10 & 0.4653 & 1477.00 \\
\hline & & 10 & 0.4800 & 1494.64 \\
\hline & & 10 & 0.5000 & 1518.83 \\
\hline 0.015 & & 15 & 0.0008 & 999.88 \\
\hline 0.03005 & & 15 & 0.0017 & 1000.70 \\
\hline 0.06006 & & 15 & 0.0034 & 1002.26 \\
\hline \multirow{2}{*}{0.12604} & & 15 & 0.0070 & 1005.68 \\
\hline & & 15 & 0.0100 & 1008.30 \\
\hline \multirow[t]{2}{*}{0.24963} & & 15 & 0.0138 & 1011.92 \\
\hline & & 15 & 0.0200 & 1017.50 \\
\hline \multirow[t]{21}{*}{0.49992} & & 15 & 0.0273 & 1024.27 \\
\hline & & 15 & 0.0400 & 1035.90 \\
\hline & & 15 & 0.0600 & 1054.40 \\
\hline & & 15 & 0.0800 & 1073.00 \\
\hline & & 15 & 0.1000 & 1091.80 \\
\hline & & 15 & 0.1500 & 1139.60 \\
\hline & & 15 & 0.2000 & 1188.40 \\
\hline & & 15 & 0.2500 & 1238.70 \\
\hline & & 15 & 0.3000 & 1290.50 \\
\hline & & 15 & 0.3500 & 1344.00 \\
\hline & & 15 & 0.4000 & 1399.10 \\
\hline & & 15 & 0.4500 & 1455.80 \\
\hline & & 15 & 0.5000 & 1514.30 \\
\hline & & 15 & 0.5170 & 1535.50 \\
\hline & & 20 & 0.0200 & 1016.43 \\
\hline & & 20 & 0.0400 & 1034.57 \\
\hline & & 20 & 0.0453 & 1039.00 \\
\hline & \multirow[t]{3}{*}{0.8385} & 20 & 0.0453 & 1038.50 \\
\hline & & 20 & 0.0600 & 1052.82 \\
\hline & & 20 & 0.0800 & 1071.26 \\
\hline & & 20 & 0.0856 & 1074.80 \\
\hline
\end{tabular}

Ref

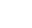

(4)

$(109)$
$(4)$

(4)

$(4)$

$(4)$
$(4)$

$(4)$
$(109)$

(4)

(4)

(4)

(4)

(4)

(109)

(4)

(4)

(81)

(81)

$(81)$
$(81)$

$(81)$
$(90)$

(81)

(90)

$(81)$
$(90)$

$(90)$
$(90)$

$(90)$
$(90)$

$(90)$
$(90)$

$(90)$
$(90)$

(90)

(90)

(90)

$(90)$
$(90)$

$(90)$
$(90)$

(90)

$(90)$
$(90)$

$(90)$
$(4)$

(4)

(69)

(41)

(4)

$(4)$
$(41)$
App vol App vol exp / Calc / $\mathrm{m}^{3} / \mathrm{kg}$ $\mathrm{m}^{3} / \mathrm{kg}$

$0.000235 \quad 0.000234$

$0.000242 \quad 0.000241$

$0.000243 \quad 0.000242$

$0.000251 \quad 0.000250$

$0.000260 \quad 0.000259$

$0.000267 \quad 0.000267$

$0.000269 \quad 0.000269$

$\begin{array}{ll}0.000275 & 0.000275\end{array}$

$0.000282 \quad 0.000282$

$0.000290 \quad 0.000290$

$0.000297 \quad 0.000297$

$0.000304 \quad 0.000305$

$0.000306 \quad 0.000307$

$0.000310 \quad 0.000312$

$\begin{array}{ll}0.000317 & 0.000319\end{array}$

$0.000068 \quad 0.000104$

$0.000051 \quad 0.000104$

$0.000060 \quad 0.000105$

0.0000690 .000107

$0.000088 \quad 0.000109$

$0.000083 \quad 0.000111$

$0.000096 \quad 0.000114$

$0.000099 \quad 0.000118$

0.0001120 .000125

0.000135

0.000145

$\begin{array}{ll}0.000204 & 0.000202\end{array}$

$0.000248 \quad 0.000245$

$0.000267 \quad 0.000265$

$0.000286 \quad 0.000284$

$0.000303 \quad 0.000303$

$0.000320 \quad 0.000320$

$0.000325 \quad 0.000326$

$0.000103 \quad 0.000119$

0.0001210 .000129

$0.000133 \quad 0.000132$

$0.000144 \quad 0.000132$

$0.000136 \quad 0.000139$

$0.000148 \quad 0.000149$

$0.000168 \quad 0.000152$
Density Density App Vol Square of Inconsistent Inconsistent Delta density Apparent Density of calc / Residual / Residual / Density based on based on $/ \mathrm{kg} / \mathrm{m}^{3}$ molal volume water $/ \mathrm{kg} / \mathrm{m}^{3}$ $\mathrm{kg} / \mathrm{m}^{3} \quad \mathrm{~kg} / \mathrm{m}^{3} \quad \mathrm{~m}^{3} / \mathrm{kg}$ Residual density $\begin{gathered}\text { apparent } \\ \text { volume }\end{gathered}$

$\begin{array}{llll}1272.8 & -0.4159 & 0.000001 & 0.173\end{array}$

$\begin{array}{llll}1291.1 & -0.4603 & 0.000001 & 0.212\end{array}$

$1293.9 \quad-0.5058 \quad 0.000001 \quad 0.256$

$\begin{array}{llll}1315.2 & -0.5490 & 0.000001 & 0.301\end{array}$

$\begin{array}{llll}1336.7 & -0.5435 & 0.000001 & 0.295\end{array}$

$\begin{array}{llll}1358.4 & -0.4774 & 0.000001 & 0.228\end{array}$

$\begin{array}{llll}1364.0 & -0.3431 & 0.000001 & 0.118\end{array}$

$\begin{array}{llll}1380.2 & -0.3284 & 0.000000 & 0.108\end{array}$

$1402.3 \quad-0.1041 \quad 0.000000 \quad 0.011$

$\begin{array}{llll}1424.6 & 0.2284 & 0.000000 & 0.052\end{array}$

$\begin{array}{llll}1447.0 & 0.6918 & -0.000001 & 0.479\end{array}$

$1469.7 \quad 1.3292-0.000001 \quad 1.767$

$\begin{array}{llll}1475.7 & 1.2711 & -0.000001 & 1.616\end{array}$

$1492.5 \quad 2.1340-0.000002 \quad 4.554$

$\begin{array}{llll}1515.5 & 3.3197 & -0.000003 & 11.021\end{array}$

$\begin{array}{llll}999.9 & 0.0304 & -0.000036 & 0.001\end{array}$

$\begin{array}{llll}1000.6 & 0.0896 & -0.000053 & 0.008\end{array}$

$\begin{array}{llll}1002.1 & 0.1517 & -0.000045 & 0.023\end{array}$

$1005.4 \quad 0.2711-0.000038 \quad 0.074$

$\begin{array}{llll}1008.1 & 0.2148 & -0.000021 & 0.046\end{array}$

$\begin{array}{llll}1011.5 & 0.3942 & -0.000028 & 0.155\end{array}$

$\begin{array}{llll}1017.1 & 0.3759 & -0.000018 & 0.141\end{array}$

$\begin{array}{llll}1023.7 & 0.5268 & -0.000018 & 0.278\end{array}$

$1035.4 \quad 0.5376-0.000013 \quad 0.289$

$\begin{array}{llll}1053.8 & 0.5850 & -0.000009 & 0.342\end{array}$

$\begin{array}{llll}1072.5 & 0.5176 & -0.000006 & 0.268\end{array}$

$\begin{array}{llll}1091.4 & 0.4352 & -0.000004 & 0.189\end{array}$

$\begin{array}{llll}1139.5 & 0.0866 & 0.000000 & 0.008\end{array}$

$\begin{array}{llll}1189.0 & -0.6082 & 0.000002 & 0.370\end{array}$

$\begin{array}{llll}1239.8 & -1.1453 & 0.000003 & 1.312\end{array}$

$\begin{array}{llll}1292.0 & -1.5151 & 0.000003 & 2.295\end{array}$

$\begin{array}{llll}1345.5 & -1.5025 & 0.000002 & 2.257\end{array}$

$\begin{array}{llll}1400.3 & -1.1860 & 0.000002 & 1.407\end{array}$

$\begin{array}{llll}1456.3 & -0.5372 & 0.000001 & 0.289\end{array}$

$\begin{array}{llll}1513.6 & 0.6795 & -0.000001 & 0.462\end{array}$

$\begin{array}{llll}1533.4 & 2.1299 & -0.000002 & 4.537\end{array}$

$\begin{array}{llll}1016.1 & 0.3126 & -0.000015 & 0.098\end{array}$

$\begin{array}{llll}1034.3 & 0.3200 & -0.000007 & 0.102\end{array}$

$\begin{array}{llll}1039.1 & -0.0954 & 0.000002 & 0.009\end{array}$

$\begin{array}{llll}1039.1 & -0.5986 & 0.000012 & 0.358\end{array}$

$\begin{array}{llll}1052.6 & 0.2215 & -0.000003 & 0.049\end{array}$

$\begin{array}{llll}1071.2 & 0.0963 & -0.000001 & 0.009\end{array}$

$\begin{array}{llll}1076.4 & -1.6000 & 0.000016 & 2.560\end{array}$

$\begin{array}{rrr}272.71 & & 999.6996 \\ 290.908 & & 999.6996 \\ 293.70 & & 999.6996 \\ 314.94 & & 999.6996 \\ 336.43 & & 999.6996 \\ 358.18 & & 999.6996 \\ 364.005 & & 999.6996 \\ 380.21 & & 999.6996 \\ 402.51 & & 999.6996 \\ 425.11 & & 999.6996 \\ 448.03 & & 999.6996 \\ 471.31 & & 999.6996 \\ 477.299 & & 999.6996 \\ 494.94 & & 999.6996 \\ 519.13 & & 999.6996 \\ & 3.8 & 999.0996 \\ & 2.87 & 999.0996 \\ & 3.38 & 999.0996 \\ & 3.87 & 999.0996 \\ & & 999.0996 \\ 5.651 & 999.0996 \\ 73.1 & & 999.0996 \\ & & 998.2041 \\ 36.4 & & 998.2041\end{array}$


molality molarity

$\mathrm{t} /{ }^{\circ} \mathrm{C}$ mass frac Density

\section{exp /}

$\mathrm{kg} / \mathrm{m}^{3}$

\begin{tabular}{|c|c|c|c|c|}
\hline & & 20 & 0.0879 & 1078.00 \\
\hline & & 20 & 0.1000 & 1089.90 \\
\hline & & 20 & 0.1200 & 1108.75 \\
\hline & \multirow[t]{2}{*}{2.495} & 20 & 0.1259 & 1111.90 \\
\hline & & 20 & 0.1400 & 1127.83 \\
\hline & \multirow[t]{4}{*}{3.246} & 20 & 0.1593 & 1143.50 \\
\hline & & 20 & 0.1600 & 1147.14 \\
\hline & & 20 & 0.1659 & 1153.00 \\
\hline & & 20 & 0.1800 & 1166.65 \\
\hline & \multirow[t]{3}{*}{3.964} & 20 & 0.1896 & 1173.10 \\
\hline & & 20 & 0.2000 & 1186.37 \\
\hline & & 20 & 0.2200 & 1206.20 \\
\hline & \multirow[t]{3}{*}{4.879} & 20 & 0.2263 & 1209.70 \\
\hline & & 20 & 0.2356 & 1222.00 \\
\hline & & 20 & 0.2400 & 1226.34 \\
\hline & \multirow[t]{3}{*}{5.606} & 20 & 0.2541 & 1237.90 \\
\hline & & 20 & 0.2600 & 1246.70 \\
\hline & & 20 & 0.2800 & 1267.32 \\
\hline & \multirow[t]{17}{*}{6.366} & 20 & 0.2819 & 1266.90 \\
\hline & & 20 & 0.2982 & 1286.00 \\
\hline & & 20 & 0.3000 & 1288.19 \\
\hline & & 20 & 0.3200 & 1309.33 \\
\hline & & 20 & 0.3400 & 1330.73 \\
\hline & & 20 & 0.3548 & 1346.00 \\
\hline & & 20 & 0.3600 & 1352.39 \\
\hline & & 20 & 0.3800 & 1374.33 \\
\hline & & 20 & 0.4000 & 1396.54 \\
\hline & & 20 & 0.4062 & 1403.00 \\
\hline & & 20 & 0.4200 & 1419.04 \\
\hline & & 20 & 0.4400 & 1441.84 \\
\hline & & 20 & 0.4531 & 1457.00 \\
\hline & & 20 & 0.4600 & 1464.97 \\
\hline & & 20 & 0.4800 & 1488.40 \\
\hline & & 20 & 0.4960 & 1507.00 \\
\hline & & 20 & 0.5000 & 1512.31 \\
\hline 0.015 & & 25 & 0.0008 & 997.82 \\
\hline 0.02032 & & 25 & 0.0011 & 998.08 \\
\hline 0.03005 & & 25 & 0.0017 & 998.63 \\
\hline 0.04087 & & 25 & 0.0023 & 999.12 \\
\hline 0.05172 & & 25 & 0.0029 & 999.66 \\
\hline 0.06006 & & 25 & 0.0034 & 1000.14 \\
\hline 0.06173 & & 25 & 0.0035 & 1000.16 \\
\hline 0.08157 & & 25 & 0.0046 & 1001.1 \\
\hline
\end{tabular}

Ref

App vol App vol D
$\exp / \quad$ Calc / $\mathrm{m}^{3} / \mathrm{kg} \quad \mathrm{m}^{3} / \mathrm{kg}$

$\begin{array}{ccc}\text { (69) } & 0.000158 & 0.000153 \\ \text { (4) } & 0.000159 & 0.000159\end{array}$

(4) $0.000169 \quad 0.000168$

(41)

(4)

$\begin{array}{ll}0.000188 & 0.000171\end{array}$

$\begin{array}{ll}0.000179 & 0.000177\end{array}$

$0.000203 \quad 0.000186$

$0.000189 \quad 0.000187$

$\begin{array}{lll}0.000191 & 0.000189\end{array}$

$0.000198 \quad 0.000196$

$0.000214 \quad 0.000200$

$0.000207 \quad 0.000205$

$0.000217 \quad 0.000213$

$0.000228 \quad 0.000216$

$0.000223 \quad 0.000220$

$0.000225 \quad 0.000222$

$0.000238 \quad 0.000228$

$0.000234 \quad 0.000230$

$0.000242 \quad 0.000239$

$0.000248 \quad 0.000239$

$0.000250 \quad 0.000246$

$0.000250 \quad 0.000247$

$0.000258 \quad 0.000255$

$0.000266 \quad 0.000263$

$0.000272 \quad 0.000268$

$0.000273 \quad 0.000270$

$0.000280 \quad 0.000278$

$0.000287 \quad 0.000286$

$0.000290 \quad 0.000288$

$0.000294 \quad 0.000293$

$0.000301 \quad 0.000300$

$0.000306 \quad 0.000305$

$0.000308 \quad 0.000307$

$0.000314 \quad 0.000314$

$0.000320 \quad 0.000320$

$0.000321 \quad 0.000321$

$0.000078 \quad 0.000113$

$0.000086 \quad 0.000113$

$0.000057 \quad 0.000114$

$0.000093 \quad 0.000114$

$0.000096 \quad 0.000114$

$0.000078 \quad 0.000114$

$0.000097 \quad 0.000114$

$0.000099 \quad 0.000115$
Density Density App Vol Square of Inconsistent Inconsistent Delta density Apparent Density of calc / Residual / Residual / Density based on based on $/ \mathrm{kg} / \mathrm{m}^{3}$ molal volume water $/ \mathrm{kg} / \mathrm{m}^{3}$ $\mathrm{kg} / \mathrm{m}^{3} \quad \mathrm{~kg} / \mathrm{m}^{3} \quad \mathrm{~m}^{3} / \mathrm{kg}$ Residual density $\begin{gathered}\text { apparent } \\ \text { volume }\end{gathered}$

$\begin{array}{llll}1078.6 & -0.5611 & 0.000005 & 0.315\end{array}$

$\begin{array}{llll}1090.0 & -0.0466 & 0.000000 & 0.002\end{array}$

$\begin{array}{llll}1109.0 & -0.1975 & 0.000001 & 0.039\end{array}$

$\begin{array}{llll}1114.6 & -2.7028 & 0.000017 & 7.305\end{array}$

$\begin{array}{llll}1128.2 & -0.3372 & 0.000002 & 0.114\end{array}$

$\begin{array}{rrrr}1146.9 & -3.4018 & 0.000016 & 11.572\end{array}$

$\begin{array}{llll}1147.6 & -0.4660 & 0.000002 & 0.217\end{array}$

$\begin{array}{llll}1153.4 & -0.3866 & 0.000002 & 0.149\end{array}$

$\begin{array}{llll}1167.3 & -0.6144 & 0.000003 & 0.378\end{array}$

$\begin{array}{llll}1176.8 & -3.6808 & 0.000014 & 13.549\end{array}$

$\begin{array}{rrrr}1187.1 & -0.7726 & 0.000003 & 0.597\end{array}$

$\begin{array}{llll}1207.2 & -1.0407 & 0.000003 & 1.083\end{array}$

$\begin{array}{llll}1213.6 & -3.9235 & 0.000012 & 15.394\end{array}$

$\begin{array}{llll}12231 & -1.0740 & 0.000003 & 1.153\end{array}$

$\begin{array}{llll}1227.6 & -1.2187 & 0.000003 & 1.485\end{array}$

$\begin{array}{llll}1242.0 & -4.1183 & 0.000011 & 16.961\end{array}$

$\begin{array}{llll}1248.1 & -1.3966 & 0.000003 & 1.951\end{array}$

$\begin{array}{llll}1268.9 & -1.5342 & 0.000003 & 2.354\end{array}$

$\begin{array}{rrrr}1270.9 & -3.9857 & 0.000009 & 15.886\end{array}$

$\begin{array}{llll}1287.9 & -1.9384 & 0.000004 & 3.757\end{array}$

$\begin{array}{llll}1289.8 & -1.6412 & 0.000003 & 2.693\end{array}$

$\begin{array}{llll}1311.0 & -1.6970 & 0.000003 & 2.880\end{array}$

$\begin{array}{llll}1332.4 & -1.7111 & 0.000003 & 2.928\end{array}$

$\begin{array}{llll}1348.4 & -2.4317 & 0.000004 & 5.913\end{array}$

$\begin{array}{llll}1354.1 & -1.6827 & 0.000003 & 2.832\end{array}$

$\begin{array}{llll}1375.9 & -1.5910 & 0.000002 & 2.531\end{array}$

$\begin{array}{llll}1398.0 & -1.4448 & 0.000002 & 2.088\end{array}$

$\begin{array}{llll}1404.9 & -1.8724 & 0.000002 & 3.506\end{array}$

$\begin{array}{llll}1420.3 & -1.2231 & 0.000001 & 1.496\end{array}$

$\begin{array}{llll}1442.8 & -0.9144 & 0.000001 & 0.836\end{array}$

$\begin{array}{llll}1457.6 & -0.6051 & 0.000001 & 0.366\end{array}$

$\begin{array}{llll}1465.5 & -0.4872 & 0.000000 & 0.237\end{array}$

$\begin{array}{llll}1488.4 & 0.0302 & 0.000000 & 0.001\end{array}$

$\begin{array}{llll}1506.9 & 0.1462 & 0.000000 & 0.021\end{array}$

$\begin{array}{llll}1511.5 & 0.8196 & -0.000001 & 0.672\end{array}$

$\begin{array}{llll}997.8 & 0.0293 & -0.000035 & 0.001\end{array}$

$\begin{array}{lllll}998.1 & 0.0307 & -0.000027 & 0.001\end{array}$

$\begin{array}{llll}998.5 & 0.0945 & -0.000056 & 0.009\end{array}$

$\begin{array}{llll}999.1 & 0.0476 & -0.000021 & 0.002\end{array}$

$\begin{array}{llll}999.6 & 0.0537 & -0.000019 & 0.003\end{array}$

$\begin{array}{llll}1000.0 & 0.1238 & -0.000037 & 0.015\end{array}$

$\begin{array}{llll}1000.1 & 0.0598 & -0.000017 & 0.004\end{array}$

$\begin{array}{llll}1001.1 & 0.0740 & -0.000016 & 0.005\end{array}$

\begin{tabular}{|c|c|c|}
\hline & & 998.2041 \\
\hline 91.7 & & 998.2041 \\
\hline 110.6 & & $\begin{array}{l}998.2041 \\
998.2041\end{array}$ \\
\hline 129.6 & & $\begin{array}{l}998.2041 \\
998.2041\end{array}$ \\
\hline 148.9 & & $\begin{array}{l}998.2041 \\
998.2041\end{array}$ \\
\hline 168.5 & & $\begin{array}{l}998.2041 \\
998.2041\end{array}$ \\
\hline 188.2 & & 998.2041 \\
\hline 208.0 & & $\begin{array}{l}998.2041 \\
998.2041 \\
998.2041\end{array}$ \\
\hline 228.1 & & $\begin{array}{l}998.2041 \\
998.2041\end{array}$ \\
\hline 248.5 & & 998.2041 \\
\hline 269.1 & & $\begin{array}{l}998.2041 \\
998.2041 \\
998.2041\end{array}$ \\
\hline 290.0 & & 998.2041 \\
\hline 311.1 & & 998.2041 \\
\hline 332.5 & & $\begin{array}{l}998.2041 \\
998.2041\end{array}$ \\
\hline 354.2 & & 998.2041 \\
\hline 376.1 & & 998.2041 \\
\hline 398.3 & & $\begin{array}{l}998.2041 \\
998.2041\end{array}$ \\
\hline 420.8 & & 998.2041 \\
\hline 443.6 & & $\begin{array}{l}998.2041 \\
998.2041\end{array}$ \\
\hline 466.8 & & 998.2041 \\
\hline 490.2 & & $\begin{array}{l}998.2041 \\
998.2041\end{array}$ \\
\hline 514.1 & & 998.2041 \\
\hline 1.039 & 4.38 & $\begin{array}{l}997.0449 \\
997.0449\end{array}$ \\
\hline & 3.21 & 997.0449 \\
\hline 2.074 & & 997.0449 \\
\hline 2.617 & & 997.0449 \\
\hline & 4.35 & 997.0449 \\
\hline $\begin{array}{l}3.118 \\
4.112\end{array}$ & & $\begin{array}{l}997.0449 \\
997.0449\end{array}$ \\
\hline
\end{tabular}


$\mathrm{t} /{ }^{\circ} \mathrm{C}$ mass frac Density

$$
\exp /
$$

$\exp /$
$\mathrm{kg} / \mathrm{m}^{3}$

0.1022

0.1056

0.12604

0.2084
0.24963

0.3035

0.3115

0.49992

0.5122

0.5199

1.0068

1.0132

1.014

1.0175

2.019

2.025

3.508

3.542

4.94

4.972

7.427

7.541

10.244

15.51

$\begin{array}{rrr}\text { molarity } & \mathbf{t} /{ }^{\circ} \mathbf{C} \text { mass frac } & \begin{array}{r}\text { Density } \\ \text { exp } /\end{array} \\ & & \mathrm{kg} / \mathrm{m}^{3} \\ & & 1002.19 \\ 25 & 0.0057 & 1002.36 \\ 25 & 0.0059 & 1003.47 \\ 25 & 0.0070 & 1003.4 \\ 25 & 0.0112 & 1007.12 \\ 25 & 0.0116 & 1007.43 \\ 25 & 0.0138 & 1009.57 \\ 25 & 0.0167 & 1012.10 \\ 25 & 0.0172 & 1012.48 \\ 25 & 0.0273 & 1021.65 \\ 25 & 0.0279 & 1022.15 \\ 25 & 0.0283 & 1022.53 \\ 25 & 0.0535 & 1045.27 \\ 25 & 0.0538 & 1045.57 \\ 25 & 0.0538 & 1045.60 \\ 25 & 0.0540 & 1045.77 \\ 25 & 0.1018 & 1089.77 \\ 25 & 0.1020 & 1090.00 \\ 25 & 0.1645 & 1149.28 \\ 25 & 0.1658 & 1150.50 \\ 25 & 0.2170 & 1200.83 \\ 25 & 0.2181 & 1201.67 \\ 25 & 0.2941 & 1279.44 \\ 25 & 0.2973 & 1282.68 \\ 25 & 0.3650 & 1355.04 \\ 25 & 0.3652 & 1355.50 \\ 25 & 0.4653 & 1467.72 \\ 30 & 0.0200 & 1013.53 \\ 30 & 0.0400 & 1031.36 \\ 30 & 0.0600 & 1049.34 \\ 30 & 0.0800 & 1067.51 \\ 30 & 0.1000 & 1085.91 \\ 30 & 0.1200 & 1104.55 \\ 30 & 0.1400 & 1123.44 \\ 30 & 0.1600 & 1142.57 \\ 30 & 0.1713 & 1153.60 \\ 30 & 0.1800 & 1161.95 \\ 30 & 0.2000 & 1181.57 \\ 30 & 0.2200 & 1201.34 \\ 30 & 0.2400 & 1221.44 \\ 30 & 0.2600 & 1241.66 \\ 30 & 0.2800 & 1262.16 \\ 30 & 0.3000 & 1282.92 \\ 30 & 0.3107 & 1292.30\end{array}$

Ref

(109) (109)

(81)

(109)

$(109)$
$(81)$

(109)

(109)

(81)

$(109)$
$(109)$

(109)

(109)

(109)

$(109)$
(109)

(109)

(109)

$(109)$
(109)

(109)

(109)

(109)

(109)

(109)

(109)

(4)

(4)

(4)

(4)

(4)

(4)

(4)

(4)

$(41)$
$(4)$

$(4)$
$(4)$

$(4)$
$(4)$

(4)

$(4)$
$(4)$

$(4)$
$(4)$

(4)
App vol App vol exp / Calc/ $\mathrm{m}^{3} / \mathrm{kg}$ $\mathrm{m}^{3} / \mathrm{kg}$

$0.000100 \quad 0.000116$

$0.000101 \quad 0.000116$

$0.000089 \quad 0.000116$

$0.000107 \quad 0.000118$

$0.000108 \quad 0.000119$

$0.000102 \quad 0.000120$

$0.000112 \quad 0.000121$

$\begin{array}{ll}0.000113 & 0.000121\end{array}$

$0.000118 \quad 0.000127$

$\begin{array}{ll}0.000121 & 0.000127\end{array}$

$\begin{array}{ll}0.000121 & 0.000127\end{array}$

$0.000138 \quad 0.000140$

$0.000138 \quad 0.000140$

$\begin{array}{ll}0.000138 & 0.000140\end{array}$

$0.000138 \quad 0.000140$

$0.000164 \quad 0.000163$

$0.000165 \quad 0.000163$

$0.000195 \quad 0.000192$

$0.000196 \quad 0.000192$

$0.000219 \quad 0.000215$

$0.000220 \quad 0.000215$

$0.000250 \quad 0.000247$

$0.000252 \quad 0.000248$

$0.000277 \quad 0.000274$

$0.000277 \quad 0.000274$

$0.000312 \quad 0.000310$

$0.000118 \quad 0.000127$

$0.000135 \quad 0.000137$

$0.000148 \quad 0.000147$

$0.000159 \quad 0.000156$

$0.000170 \quad 0.000166$

$0.000179 \quad 0.000175$

$0.000188 \quad 0.000184$

$0.000197 \quad 0.000193$

$0.000202 \quad 0.000198$

$0.000206 \quad 0.000202$

$0.000214 \quad 0.000210$

$\begin{array}{ll}0.000223 & 0.000219\end{array}$

$0.000231 \quad 0.000227$

$0.000239 \quad 0.000235$

$0.000247 \quad 0.000243$

$0.000255 \quad 0.000251$

$0.000262 \quad 0.000255$
Density Density App Vol Square of Inconsistent Inconsistent Delta density Apparent Density of calc / Residual / Residual / Density based on based on $/ \mathrm{kg} / \mathrm{m}^{3}$ molal volume water $/ \mathrm{kg} / \mathrm{m}^{3}$ $\mathrm{kg} / \mathrm{m}^{3} \quad \mathrm{~kg} / \mathrm{m}^{3} \quad \mathrm{~m}^{3} / \mathrm{kg}$ Residual density $\begin{gathered}\text { apparent } \\ \text { volume }\end{gathered}$

$1002.1 \quad 0.0918-0.000016 \quad 0.008$

$1002.3 \quad 0.0894-0.000015 \quad 0.008$

$1003.3 \quad 0.1933-0.000027 \quad 0.037$

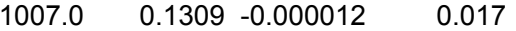

$1007.3 \quad 0.1213-0.000010 \quad 0.015$

$\begin{array}{llll}1009.3 & 0.2459 & -0.000017 & 0.060\end{array}$

$1011.9 \quad 0.1557-0.000009 \quad 0.024$

$1012.3 \quad 0.1558-0.000009 \quad 0.024$

$1021.4 \quad 0.2508-0.000009 \quad 0.063$

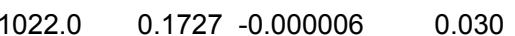

$1022.3 \quad 0.1823-0.000006 \quad 0.033$

$\begin{array}{llll}1045.1 & 0.1262 & -0.000002 & 0.016\end{array}$

$\begin{array}{llll}1045.4 & 0.1282 & -0.000002 & 0.016\end{array}$

$\begin{array}{llll}1045.5 & 0.1285 & -0.000002 & 0.017\end{array}$

$\begin{array}{llll}1045.6 & 0.1307 & -0.000002 & 0.017\end{array}$

$\begin{array}{llll}1089.9 & -0.1492 & 0.000001 & 0.022\end{array}$

$\begin{array}{llll}1090.2 & -0.1737 & 0.000001 & 0.030\end{array}$

$\begin{array}{llll}1150.0 & -0.7116 & 0.000003 & 0.506\end{array}$

$\begin{array}{llll}1151.3 & -0.7954 & 0.000004 & 0.633\end{array}$

$\begin{array}{llll}1202.1 & -1.2233 & 0.000004 & 1.497\end{array}$

$\begin{array}{llll}1203.2 & -1.4875 & 0.000005 & 2.213\end{array}$

$\begin{array}{llll}1281.3 & -1.8165 & 0.000004 & 3.300\end{array}$

$\begin{array}{llll}1284.6 & -1.9069 & 0.000004 & 3.636\end{array}$

$\begin{array}{llll}1357.0 & -1.9464 & 0.000003 & 3.788\end{array}$

$\begin{array}{llll}1357.2 & -1.7347 & 0.000003 & 3.009\end{array}$

$\begin{array}{llll}1469.1 & -1.3732 & 0.000001 & 1.886\end{array}$

$\begin{array}{llll}1013.3 & 0.1794 & -0.000009 & 0.032\end{array}$

$\begin{array}{llll}1031.3 & 0.0903 & -0.000002 & 0.008\end{array}$

$\begin{array}{llll}1049.4 & -0.0687 & 0.000001 & 0.005\end{array}$

$\begin{array}{llll}1067.8 & -0.2593 & 0.000003 & 0.067\end{array}$

$\begin{array}{llll}1086.4 & -0.4429 & 0.000004 & 0.196\end{array}$

$\begin{array}{llll}1105.2 & -0.6111 & 0.000004 & 0.373\end{array}$

$\begin{array}{llll}1124.2 & -0.7552 & 0.000004 & 0.570\end{array}$

$\begin{array}{llll}1143.5 & -0.8866 & 0.000004 & 0.786\end{array}$

$\begin{array}{llll}1154.4 & -0.8422 & 0.000004 & 0.709\end{array}$

$\begin{array}{llll}1162.9 & -0.9965 & 0.000004 & 0.993\end{array}$

$\begin{array}{llll}1182.7 & -1.0964 & 0.000004 & 1.202\end{array}$

$\begin{array}{llll}1202.6 & -1.2772 & 0.000004 & 1.631\end{array}$

$\begin{array}{llll}1222.8 & -1.3602 & 0.000004 & 1.850\end{array}$

$\begin{array}{llll}1243.2 & -1.5563 & 0.000004 & 2.422\end{array}$

$\begin{array}{llll}1263.9 & -1.7066 & 0.000004 & 2.913\end{array}$

$\begin{array}{llll}1284.7 & -1.8320 & 0.000004 & 3.356\end{array}$

14.187

$/ \mathrm{kg} / \mathrm{m}^{3}$ molal volume wat
\[ / \mathrm{cm}^{3} / \mathrm{mol} \]

5.147

5.312

10.074

10.387

15.051

15.439

25.109

25.486

48.225

48.521

48.558

48.721

92.728

92.959

152.24

153.454

203.785

204.626

282.393

285.633

357.995

358.453

470.673

17.88

35.71

53.69

71.86

90.26

108.90

127.79

146.92

166.30

185.92

205.69

225.79

246.01

266.51

287.27

$\begin{array}{lll}1296.1 & -3.7666 & 0.000007\end{array}$
997.0449

997.0449

$4.99 \quad 997.0449$

997.0449

997.0449

$5.74 \quad 997.0449$

997.0449

$6.611 \quad 997.0449$

997.0449
997.0449

997.0449

997.0449

997.0449

997.0449

997.0449

997.0449

997.0449

997.0449

997.0449

997.0449

997.0449

997.0449

997.0449

997.0449

997.0449

997.0449

995.6473

995.6473

995.6473

995.6473

995.6473

995.6473

995.6473

995.6473

995.6473

995.6473

995.6473

995.6473

995.6473

995.6473

995.6473

995.6473

995.6473 
$\mathrm{t} /{ }^{\circ} \mathrm{C}$ mass frac Density

\section{exp /}

$\mathrm{kg} / \mathrm{m}^{3}$

\begin{tabular}{|c|c|c|c|c|}
\hline & & 30 & 0.3200 & 1303.96 \\
\hline & & 30 & 0.3400 & 1325.26 \\
\hline & & 30 & 0.3600 & 1346.84 \\
\hline & & 30 & 0.3800 & 1368.69 \\
\hline & & 30 & 0.4000 & 1390.83 \\
\hline & & 30 & 0.4200 & 1413.25 \\
\hline & & 30 & 0.4400 & 1435.97 \\
\hline & & 30 & 0.4600 & 1459.01 \\
\hline & & 30 & 0.4800 & 1482.34 \\
\hline & & 30 & 0.5000 & 1506.12 \\
\hline 0.015 & & 35 & 0.0008 & 994.78 \\
\hline 0.03005 & & 35 & 0.0017 & 995.56 \\
\hline 0.06006 & & 35 & 0.0034 & 997.05 \\
\hline 0.12604 & & 35 & 0.0070 & 1000.30 \\
\hline 0.24963 & & 35 & 0.0138 & 1006.29 \\
\hline 0.49992 & & 35 & 0.0273 & 1018.15 \\
\hline 0.02032 & & 40 & 0.0011 & 993.24 \\
\hline 0.04087 & & 40 & 0.0023 & 994.25 \\
\hline 0.06173 & & 40 & 0.0035 & 995.28 \\
\hline 0.08157 & & 40 & 0.0046 & 996.25 \\
\hline 0.2018 & & 40 & 0.0112 & 1002.06 \\
\hline 0.3035 & & 40 & 0.0167 & 1006.92 \\
\hline & & 40 & 0.0200 & 1009.88 \\
\hline 0.5122 & & 40 & 0.0279 & 1016.77 \\
\hline & & 40 & 0.0400 & 1027.52 \\
\hline & 0.8316 & 40 & 0.0452 & 1031.30 \\
\hline 1.0068 & & 40 & 0.0535 & 1039.46 \\
\hline 1.0132 & & 40 & 0.0538 & 1039.72 \\
\hline & & 40 & 0.0600 & 1045.31 \\
\hline & & 40 & 0.0800 & 1063.32 \\
\hline & 1.6273 & 40 & 0.0856 & 1066.80 \\
\hline & & 40 & 0.1000 & 1081.56 \\
\hline 2.019 & & 40 & 0.1018 & 1083.27 \\
\hline & & 40 & 0.1200 & 1100.05 \\
\hline & 2.475 & 40 & 0.1259 & 1103.20 \\
\hline & & 40 & 0.1400 & 1118.80 \\
\hline & 3.22 & 40 & 0.1593 & 1134.40 \\
\hline & & 40 & 0.1600 & 1137.82 \\
\hline 3.542 & & 40 & 0.1658 & 1143.36 \\
\hline & & 40 & 0.1800 & 1157.09 \\
\hline & 3.932 & 40 & 0.1896 & 1163.60 \\
\hline & & 40 & 0.2000 & 1176.62 \\
\hline & & 40 & 0.2181 & 1194.37 \\
\hline
\end{tabular}

$(4)$
$(4)$

(4)

(4)

(4)

(4)

(4)

(4)

(4)

(4)

(81)

(81)

(81)

(81)

(81)

(81)

$(109)$

$(109)$
$(109)$

(109)

(109)

(109)

(4)

(109)

(4)

(41)

$(109)$
$(109)$

(4)

(4)

(41)

(4)

(109)

(4)

$(41)$
$(4)$

$(4)$
$(41)$
$(4)$

(4)

(109)

$(4)$
$(41)$

$(41)$
$(4)$

(109)
App vol App vol exp / Calc / $\mathrm{m}^{3} / \mathrm{kg}$ $\mathrm{m}^{3} / \mathrm{kg}$

$0.000262 \quad 0.000259$

$0.000270 \quad 0.000266$

$0.000277 \quad 0.000274$

$0.000284 \quad 0.000281$

$\begin{array}{lll}0.000291 & 0.000288\end{array}$

$0.000298 \quad 0.000296$

$0.000304 \quad 0.000303$

$\begin{array}{ll}0.000311 & 0.000310\end{array}$

$0.000317 \quad 0.000316$

$0.000324 \quad 0.000323$

$0.000111 \quad 0.000122$

$0.000091 \quad 0.000122$

(

$\begin{array}{ll}0.000119 & 0.000128\end{array}$

$0.000133 \quad 0.000135$

$0.000095 \quad 0.000126$

$0.000107 \quad 0.000127$

$0.000110 \quad 0.000127$

$0.000113 \quad 0.000128$

$0.000123 \quad 0.000131$

$0.000129 \quad 0.000134$

$0.000127 \quad 0.000135$

$0.000137 \quad 0.000139$

0.0001420 .000145

$0.000164 \quad 0.000147$

$0.000151 \quad 0.000151$

$0.000152 \quad 0.000151$

$0.000155 \quad 0.000154$

0.0001650 .000164

$0.000185 \quad 0.000166$

$0.000175 \quad 0.000173$

$0.000175 \quad 0.000173$

0.0001850 .000181

$0.000202 \quad 0.000184$

$0.000193 \quad 0.000190$

$0.000215 \quad 0.000198$

0.0002020 .000199

$0.000204 \quad 0.000201$

$0.000210 \quad 0.000207$

$0.000225 \quad 0.000211$

$0.000218 \quad 0.000216$

$0.000226 \quad 0.000223$
Density Density App Vol Square of Inconsistent Inconsistent Delta density Apparent Density of calc / Residual / Residual / Density based on based on $\mathrm{kg} / \mathrm{m}^{3} \quad \mathrm{~kg} / \mathrm{m}^{3} \quad \mathrm{~m}^{3} / \mathrm{kg}$ Residual density $\begin{gathered}\text { apparent } \\ \text { volume }\end{gathered}$

$\mathrm{kg} / \mathrm{m}^{3}$ molal volume water $/ \mathrm{kg} / \mathrm{m}^{3}$ $\begin{array}{llll}1305.9 & -1.9132 & 0.000004 & 3.660\end{array}$

$\begin{array}{llll}1327.2 & -1.9710 & 0.000003 & 3.885\end{array}$

$\begin{array}{llll}1348.8 & -1.9860 & 0.000003 & 3.944\end{array}$

$\begin{array}{llll}1370.7 & -1.9688 & 0.000003 & 3.876\end{array}$

$\begin{array}{llll}1392.7 & -1.8999 & 0.000002 & 3.610\end{array}$

$\begin{array}{llll}1415.0 & -1.7895 & 0.000002 & 3.202\end{array}$

$\begin{array}{llll}1437.6 & -1.6180 & 0.000002 & 2.618\end{array}$

$1460.4 \quad-1.3654 \quad 0.000001 \quad 1.864$

$\begin{array}{llll}1483.4 & -1.0618 & 0.000001 & 1.127\end{array}$

$\begin{array}{llll}1506.7 & -0.5471 & 0.000000 & 0.299\end{array}$

$\begin{array}{llll}994.8 & 0.0092 & -0.000011 & 0.000\end{array}$

$\begin{array}{llll}995.5 & 0.0519 & -0.000031 & 0.003\end{array}$

$\begin{array}{llll}997.0 & 0.0818 & -0.000024 & 0.007\end{array}$

$\begin{array}{llll}1000.2 & 0.1198 & -0.000017 & 0.014\end{array}$

$\begin{array}{llll}1006.2 & 0.1348 & -0.000010 & 0.018\end{array}$

$\begin{array}{llll}1018.1 & 0.0609 & -0.000002 & 0.004\end{array}$

$\begin{array}{llll}993.2 & 0.0344 & -0.000031 & 0.001\end{array}$

$\begin{array}{llll}994.2 & 0.0452 & -0.000020 & 0.002\end{array}$

$\begin{array}{llll}995.2 & 0.0605 & -0.000018 & 0.004\end{array}$

$\begin{array}{llll}996.2 & 0.0668 & -0.000015 & 0.004\end{array}$

$\begin{array}{llll}1002.0 & 0.0882 & -0.000008 & 0.008\end{array}$

$\begin{array}{llll}1006.8 & 0.0863 & -0.000005 & 0.007\end{array}$

$\begin{array}{llll}1009.7 & 0.1770 & -0.000009 & 0.031\end{array}$

$\begin{array}{llll}1016.7 & 0.0729 & -0.000003 & 0.005\end{array}$

$\begin{array}{llll}1027.4 & 0.1115 & -0.000003 & 0.012\end{array}$

$\begin{array}{llll}1032.1 & -0.7841 & 0.000016 & 0.615\end{array}$

$\begin{array}{llll}1039.5 & 0.0080 & 0.000000 & 0.000\end{array}$

$\begin{array}{llll}1039.7 & -0.0210 & 0.000000 & 0.000\end{array}$

$\begin{array}{llll}1045.3 & -0.0288 & 0.000000 & 0.001\end{array}$

$\begin{array}{llll}1063.5 & -0.1762 & 0.000002 & 0.031\end{array}$

$\begin{array}{llll}1068.6 & -1.8073 & 0.000019 & 3.267\end{array}$

$\begin{array}{llll}1081.9 & -0.3230 & 0.000003 & 0.104\end{array}$

$\begin{array}{llll}1083.5 & -0.2366 & 0.000002 & 0.056\end{array}$

$\begin{array}{llll}1100.5 & -0.4514 & 0.000003 & 0.204\end{array}$

$\begin{array}{llll}1106.0 & -2.8154 & 0.000018 & 7.926\end{array}$

$\begin{array}{llll}1119.3 & -0.5539 & 0.000003 & 0.307\end{array}$

$\begin{array}{llll}1137.7 & -3.3344 & 0.000016 & 11.118\end{array}$

$\begin{array}{llll}1138.4 & -0.6226 & 0.000003 & 0.388\end{array}$

$\begin{array}{llll}1144.0 & -0.6516 & 0.000003 & 0.425\end{array}$

$\begin{array}{llll}1157.8 & -0.6799 & 0.000003 & 0.462\end{array}$

$\begin{array}{lllr}1167.1 & -3.5312 & 0.000014 & 12.469\end{array}$

$\begin{array}{llll}1177.3 & -0.7180 & 0.000003 & 0.516\end{array}$

$\begin{array}{llll}1195.3 & -0.9039 & 0.000003 & 0.817\end{array}$

\begin{tabular}{|c|c|c|}
\hline 308.31 & & 995.6473 \\
\hline 329.61 & & 995.6473 \\
\hline 351.19 & & 995.6473 \\
\hline 373.04 & & 995.6473 \\
\hline 395.18 & & 995.6473 \\
\hline 417.60 & & 995.6473 \\
\hline 440.32 & & 995.6473 \\
\hline 463.36 & & 995.6473 \\
\hline 486.69 & & 995.6473 \\
\hline \multirow{7}{*}{510.47} & & 995.6473 \\
\hline & 6.21 & 994.0319 \\
\hline & 5.11 & 994.0319 \\
\hline & 5.53 & 994.0319 \\
\hline & 6.05 & 994.0319 \\
\hline & 6.655 & 994.0319 \\
\hline & 7.447 & 994.0319 \\
\hline 1.024 & & 992.2158 \\
\hline 2.034 & & 992.2158 \\
\hline 3.062 & & 992.2158 \\
\hline 4.03 & & 992.2158 \\
\hline 9.848 & & 992.2158 \\
\hline 14.708 & & 992.2158 \\
\hline 17.66 & & 992.2158 \\
\hline 24.555 & & 992.2158 \\
\hline \multirow[t]{2}{*}{35.30} & & 992.2158 \\
\hline & & 992.2158 \\
\hline 47.248 & & 992.2158 \\
\hline 47.508 & & 992.2158 \\
\hline 53.09 & & 992.2158 \\
\hline \multirow[t]{2}{*}{71.10} & & 992.2158 \\
\hline & & 992.2158 \\
\hline 89.34 & & 992.2158 \\
\hline 91.053 & & 992.2158 \\
\hline \multirow[t]{2}{*}{107.83} & & 992.2158 \\
\hline & & 992.2158 \\
\hline \multirow[t]{2}{*}{126.58} & & 992.2158 \\
\hline & & 992.2158 \\
\hline 145.60 & & 992.2158 \\
\hline 51.142 & & 992.2158 \\
\hline \multirow[t]{2}{*}{164.87} & & 992.2158 \\
\hline & & 992.2158 \\
\hline 4.40 & & 992.2158 \\
\hline & & 992.2158 \\
\hline
\end{tabular}


molality molarity

$\mathrm{t} /{ }^{\circ} \mathrm{C}$ mass frac Density

\section{$\exp$}

$\mathrm{kg} / \mathrm{m}^{3}$

\begin{tabular}{|c|c|c|c|c|}
\hline & & 40 & 0.2200 & 1196.33 \\
\hline & 4.84 & 40 & 0.2263 & 1199.90 \\
\hline & & 40 & 0.2400 & 1216.38 \\
\hline & 5.561 & 40 & 0.2541 & 1227.90 \\
\hline & & 40 & 0.2600 & 1236.54 \\
\hline & & 40 & 0.2800 & 1256.94 \\
\hline & 6.315 & 40 & 0.2819 & 1256.80 \\
\hline 7.541 & & 40 & 0.2973 & 1274.66 \\
\hline & & 40 & 0.3000 & 1277.61 \\
\hline & & 40 & 0.3200 & 1298.55 \\
\hline & & 40 & 0.3400 & 1319.77 \\
\hline & & 40 & 0.3600 & 1341.27 \\
\hline 10.254 & & 40 & 0.3652 & 1346.77 \\
\hline & & 40 & 0.3800 & 1363.06 \\
\hline & & 40 & 0.4000 & 1385.13 \\
\hline & & 40 & 0.4200 & 1407.50 \\
\hline & & 40 & 0.4400 & 1430.18 \\
\hline & & 40 & 0.4600 & 1453.18 \\
\hline & & 40 & 0.4800 & 1476.48 \\
\hline & & 40 & 0.5000 & 1500.25 \\
\hline 0.015 & & 45 & 0.0008 & 990.97 \\
\hline 0.03005 & & 45 & 0.0017 & 991.73 \\
\hline 0.06006 & & 45 & 0.0034 & 993.20 \\
\hline 0.12604 & & 45 & 0.0070 & 996.44 \\
\hline 0.24963 & & 45 & 0.0138 & 1002.36 \\
\hline 0.49992 & & 45 & 0.0273 & 1014.09 \\
\hline & & 50 & 0.0200 & 1005.57 \\
\hline & & 50 & 0.0400 & 1023.10 \\
\hline & & 50 & 0.0453 & 1027.00 \\
\hline & & 50 & 0.0600 & 1040.78 \\
\hline & & 50 & 0.0800 & 1058.67 \\
\hline & & 50 & 0.0879 & 1066.00 \\
\hline & & 50 & 0.1000 & 1076.81 \\
\hline & & 50 & 0.1200 & 1095.21 \\
\hline & & 50 & 0.1400 & 1113.87 \\
\hline & & 50 & 0.1600 & 1132.80 \\
\hline & & 50 & 0.1659 & 1138.00 \\
\hline & & 50 & 0.1800 & 1152.00 \\
\hline & & 50 & 0.2000 & 1171.46 \\
\hline & & 50 & 0.2200 & 1191.11 \\
\hline & & 50 & 0.2356 & 1207.00 \\
\hline & & 50 & 0.2400 & 1211.10 \\
\hline & & 50 & 02600 & 123123 \\
\hline
\end{tabular}

Ref

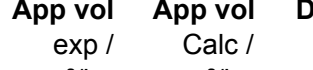$$
\mathrm{m}^{3} /
$$
$\mathrm{m}^{3} / \mathrm{kg}$

(4) $0.000226 \quad 0.000224$

(41)

$0.000237 \quad 0.000226$

$0.000234 \quad 0.000232$

$0.000247 \quad 0.000237$

$0.000242 \quad 0.000240$

$0.000250 \quad 0.000247$

$0.000255 \quad 0.000248$

$0.000257 \quad 0.000254$

$0.000257 \quad 0.000255$

$0.000265 \quad 0.000262$

$0.000272 \quad 0.000270$

$0.000279 \quad 0.000277$

$0.000281 \quad 0.000279$

$0.000286 \quad 0.000284$

$0.000293 \quad 0.000291$

$0.000300 \quad 0.000298$

$0.000306 \quad 0.000305$

$0.000313 \quad 0.000311$

$0.000319 \quad 0.000318$

$0.000325 \quad 0.000324$

$0.000093 \quad 0.000130$

$\begin{array}{lll}(81) & 0.000090 & 0.000130\end{array}$

$\begin{array}{ll}0.000090 & 0.000130 \\ 0.000106 & 0.000131\end{array}$

$0.000112 \quad 0.000133$

$\begin{array}{lll}81) & 0.000124 & 0.000136\end{array}$

(81)

$0.000138 \quad 0.000143$

$0.000130 \quad 0.000143$

$0.000145 \quad 0.000152$

$0.000164 \quad 0.000155$

$0.000157 \quad 0.000161$

$0.000168 \quad 0.000170$

$\begin{array}{ll}0.000170 & 0.000174\end{array}$

$0.000178 \quad 0.000179$

$0.000187 \quad 0.000187$

$0.000195 \quad 0.000196$

$0.000204 \quad 0.000204$

$0.000208 \quad 0.000207$

$0.000212 \quad 0.000212$

$0.000220 \quad 0.000220$

$0.000228 \quad 0.000228$

$0.000233 \quad 0.000234$

$0.000235 \quad 0.000236$

$0.000243 \quad 0.000243$
Density Density App Vol Square of Inconsistent Inconsistent Delta density Apparent Density of calc / Residual / Residual / Density based on based on $/ \mathrm{kg} / \mathrm{m}^{3}$ molal volume water $/ \mathrm{kg} / \mathrm{m}^{3}$ $\mathrm{kg} / \mathrm{m}^{3} \quad \mathrm{~kg} / \mathrm{m}^{3} \quad \mathrm{~m}^{3} / \mathrm{kg}$ Residual density apparent

$\begin{array}{llll}1197.1 & -0.8191 & 0.000003 & 0.671\end{array}$

$\begin{array}{lllr}1203.5 & -3.5629 & 0.000011 & 12.694\end{array}$

$\begin{array}{llll}1217.2 & -0.8255 & 0.000002 & 0.681\end{array}$

$\begin{array}{llll}1231.5 & -3.6020 & 0.000009 & 12.974\end{array}$

$\begin{array}{llll}1237.5 & -0.9693 & 0.000002 & 0.940\end{array}$

$\begin{array}{llll}1258.1 & -1.1228 & 0.000003 & 1.261\end{array}$

$\begin{array}{llll}1260.1 & -3.2563 & 0.000007 & 10.603\end{array}$

$\begin{array}{llll}1276.1 & -1.3985 & 0.000003 & 1.956\end{array}$

$\begin{array}{llll}1278.9 & -1.2580 & 0.000003 & 1.583\end{array}$

$\begin{array}{llll}1299.9 & -1.3772 & 0.000003 & 1.897\end{array}$

$\begin{array}{llll}1321.2 & -1.4725 & 0.000002 & 2.168\end{array}$

$\begin{array}{llll}1342.8 & -1.5458 & 0.000002 & 2.390\end{array}$

$\begin{array}{llll}1348.5 & -1.7148 & 0.000003 & 2.941\end{array}$

$\begin{array}{llll}1364.6 & -1.5893 & 0.000002 & 2.526\end{array}$

$\begin{array}{llll}1386.7 & -1.6149 & 0.000002 & 2.608\end{array}$

$\begin{array}{llll}1409.1 & -1.6046 & 0.000002 & 2.575\end{array}$

$\begin{array}{llll}1431.7 & -1.5504 & 0.000002 & 2.404\end{array}$

$\begin{array}{llll}1454.6 & -1.4440 & 0.000001 & 2.085\end{array}$

$\begin{array}{llll}1477.8 & -1.3074 & 0.000001 & 1.709\end{array}$

$\begin{array}{llll}1501.2 & -0.9722 & 0.000001 & 0.945\end{array}$

$\begin{array}{llll}990.9 & 0.0305 & -0.000037 & 0.001\end{array}$

$\begin{array}{llll}991.7 & 0.0669 & -0.000040 & 0.004\end{array}$

$\begin{array}{llll}993.1 & 0.0822 & -0.000025 & 0.007\end{array}$

$\begin{array}{llll}996.3 & 0.1480 & -0.000021 & 0.022\end{array}$

$\begin{array}{llll}1002.2 & 0.1690 & -0.000012 & 0.029\end{array}$

$\begin{array}{llll}1014.0 & 0.1222 & -0.000004 & 0.015\end{array}$

$\begin{array}{llll}1005.3 & 0.2632 & -0.000013 & 0.069\end{array}$

$\begin{array}{llll}1022.8 & 0.2993 & -0.000007 & 0.090\end{array}$

$\begin{array}{llll}1027.5 & -0.4713 & 0.000010 & 0.222\end{array}$

$\begin{array}{llll}1040.5 & 0.2551 & -0.000004 & 0.065\end{array}$

$\begin{array}{llll}1058.5 & 0.1877 & -0.000002 & 0.035\end{array}$

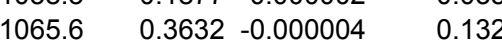

$1076.7 \quad 0.1338-0.000001 \quad 0.018$

$\begin{array}{llll}1095.1 & 0.1004 & -0.000001 & 0.010\end{array}$

$\begin{array}{llll}1113.8 & 0.0843 & 0.000000 & 0.007\end{array}$

$\begin{array}{llll}1132.7 & 0.0922 & 0.000000 & 0.009\end{array}$

$\begin{array}{llll}1138.3 & -0.3335 & 0.000002 & 0.111\end{array}$

$\begin{array}{llll}1151.9 & 0.1210 & -0.000001 & 0.015\end{array}$

$\begin{array}{llll}1171.3 & 0.1573 & -0.000001 & 0.025\end{array}$

$\begin{array}{llll}1191.0 & 0.1278 & 0.000000 & 0.016\end{array}$

$\begin{array}{llll}1206.5 & 0.4917 & -0.000001 & 0.242\end{array}$

$\begin{array}{llll}1210.9 & 0.1791 & -0.000001 & 0.032\end{array}$

$\begin{array}{llll}1231.1 & 0.1080 & 0.000000 & 0.012\end{array}$
$/ \mathrm{kg} / \mathrm{m}^{3}$ molal volume
\[ / \mathrm{cm}^{3} / \mathrm{mol} \]

\begin{tabular}{|c|c|c|}
\hline 204.11 & & 992.2158 \\
\hline & & 992.2158 \\
\hline 224.16 & & 992.2158 \\
\hline & & 992.2158 \\
\hline 244.32 & & 992.2158 \\
\hline 264.72 & & 992.2158 \\
\hline & & 992.2158 \\
\hline 282.446 & & 992.2158 \\
\hline 285.39 & & 992.2158 \\
\hline 306.33 & & 992.2158 \\
\hline 327.55 & & 992.2158 \\
\hline 349.05 & & 992.2158 \\
\hline 354.553 & & 992.2158 \\
\hline 370.84 & & 992.2158 \\
\hline 392.91 & & 992.2158 \\
\hline 415.28 & & 992.2158 \\
\hline 437.96 & & 992.2158 \\
\hline 460.96 & & 992.2158 \\
\hline 484.26 & & 992.2158 \\
\hline 508.03 & & 992.2158 \\
\hline & 5.22 & 990.2132 \\
\hline & 5.05 & 990.2132 \\
\hline & 5.97 & 990.2132 \\
\hline & 6.27 & 990.2132 \\
\hline & 6.96 & 990.2132 \\
\hline & 7.758 & 990.2132 \\
\hline 17.53 & & 988.0363 \\
\hline 35.06 & & 988.0363 \\
\hline & & 988.0363 \\
\hline 52.74 & & 988.0363 \\
\hline 70.63 & & 988.0363 \\
\hline & & 988.0363 \\
\hline 88.77 & & 988.0363 \\
\hline 107.17 & & 988.0363 \\
\hline 125.83 & & 988.0363 \\
\hline 144.76 & & 988.0363 \\
\hline & & 988.0363 \\
\hline 163.96 & & 988.0363 \\
\hline 183.42 & & 988.0363 \\
\hline 203.07 & & 988.0363 \\
\hline & & 988.0363 \\
\hline 223.06 & & 988.0363 \\
\hline
\end{tabular}


molality molarity

$\mathrm{t} /{ }^{\circ} \mathrm{C}$ mass frac Density

$$
\exp /
$$

$\mathrm{kg} / \mathrm{m}^{3}$

$\begin{array}{rrr}50 & 0.2800 & 1251.54 \\ 50 & 0.2982 & 1270.00 \\ 50 & 0.3000 & 1272.12 \\ 50 & 0.3200 & 1292.97 \\ 50 & 0.3400 & 1314.11 \\ 50 & 0.3548 & 1330.00 \\ 50 & 0.3600 & 1335.53 \\ 50 & 0.3800 & 1357.24 \\ 50 & 0.4000 & 1379.25 \\ 50 & 0.4062 & 1386.00 \\ 50 & 0.4200 & 1401.55 \\ 50 & 0.4400 & 1424.17 \\ 50 & 0.4531 & 1439.00 \\ 50 & 0.4600 & 1447.13 \\ 50 & 0.4800 & 1470.40 \\ 50 & 0.4960 & 1489.00 \\ 50 & 0.5000 & 1494.14 \\ 55 & 0.0008 & 986.43 \\ 55 & 0.0017 & 987.19 \\ 55 & 0.0034 & 988.64 \\ 55 & 0.0059 & 990.82 \\ 55 & 0.0070 & 991.85 \\ 55 & 0.0116 & 995.70 \\ 55 & 0.0138 & 997.73 \\ 55 & 0.0172 & 1000.59 \\ 55 & 0.0273 & 1009.37 \\ 55 & 0.0283 & 1010.31 \\ 55 & 0.0538 & 1032.64 \\ 55 & 0.0540 & 1032.81 \\ 55 & 0.1018 & 1075.86 \\ 55 & 0.1020 & 1076.11 \\ 55 & 0.1645 & 1134.20 \\ 55 & 0.2170 & 1185.13 \\ 55 & 0.2941 & 1262.95 \\ 55 & 0.3650 & 1337.68 \\ 55 & 0.4653 & 1449.30 \\ 59.9 & 0.0617 & 1038.10 \\ 59.9 & 0.1150 & 1086.30 \\ 59.9 & 0.2090 & 1175.80 \\ 59.9 & 0.2780 & 1244.80 \\ 59.9 & 0.3600 & 1329.40 \\ 59.9 & 0.4300 & 1408.00 \\ 59.9 & 0.4720 & 1456.00\end{array}$

$\begin{array}{lll}59.9 & 0.4720 & 1456.00\end{array}$

0.06006

0.10558

0.12604

0.2084

0.24963

0.3115

0.49992

1.014

1.0175

2.019

2.025

3.508
4.94

7.427

10.244

15.51
Ref

exp / Calc/

$\begin{array}{cc}\exp / & \text { Calc/ } \\ \mathrm{m}^{3} / \mathrm{kg} & \mathrm{m}^{3} / \mathrm{kg}\end{array}$

$0.000251 \quad 0.000251$

$0.000259 \quad 0.000258$

$0.000259 \quad 0.000258$

$0.000266 \quad 0.000266$

$0.000273 \quad 0.000273$

$\begin{array}{lll}0.000279 & 0.000278\end{array}$

$0.000281 \quad 0.000280$

$0.000288 \quad 0.000286$

$0.000294 \quad 0.000293$

$0.000297 \quad 0.000295$

$0.000301 \quad 0.000300$

$0.000308 \quad 0.000306$

$0.000312 \quad 0.000311$

$0.000314 \quad 0.000313$

$0.000320 \quad 0.000319$

$0.000326 \quad 0.000324$

$0.000326 \quad 0.000325$

$0.000112 \quad 0.000138$

$0.000100 \quad 0.000138$

$0.000113 \quad 0.000139$ $\begin{array}{lll}\text { (109) } \quad 0.000124 & 0.000140\end{array}$

$\begin{array}{lll}\text { (81) } & 0.000118 & 0.000141\end{array}$

(109)

(81)

(109)

(81)

(109)

$(109)$
$(109)$

(109)

(109)

(109)

(109)

(109)

(109)

(109)

(127)

(127)

(127)

(127)

$(127)$
$(127)$

(127)
$0.000132 \quad 0.000143$

$0.000135 \quad 0.000145$

$0.000142 \quad 0.000150$

$\begin{array}{ll}0.000142 & 0.000150\end{array}$

$0.000158 \quad 0.000162$

$0.000158 \quad 0.000162$

$0.000179 \quad 0.000183$

$0.000179 \quad 0.000183$

$0.000207 \quad 0.000209$

$0.000228 \quad 0.000229$

$0.000257 \quad 0.000258$

$0.000283 \quad 0.000282$

$0.000317 \quad 0.000315$

$0.000146 \quad 0.000168$

$0.000178 \quad 0.000191$

$0.000220 \quad 0.000228$

$0.000248 \quad 0.000254$

$0.000281 \quad 0.000282$

$0.000304 \quad 0.000305$

$0.000317 \quad 0.000318$
$0.000129 \quad 0.000144$
Density Density App Vol Square of Inconsistent Inconsistent Delta density Apparent $\begin{array}{r}\text { Density of } \\ \text { Anty }\end{array}$ calc / Residual / Residual / Density based on based on $\mathrm{kg} / \mathrm{m}^{3} \quad \mathrm{~kg} / \mathrm{m}^{3} \quad \mathrm{~m}^{3} / \mathrm{kg}$ Residual density $\begin{gathered}\text { apparent } \\ \text { volume }\end{gathered}$

$\begin{array}{llll}1251.6 & -0.0490 & 0.000000 & 0.002\end{array}$

$\begin{array}{llll}1270.4 & -0.4443 & 0.000001 & 0.197\end{array}$

$\begin{array}{llll}1272.3 & -0.2055 & 0.000000 & 0.042\end{array}$

$\begin{array}{llll}1293.3 & -0.3647 & 0.000001 & 0.133\end{array}$

$\begin{array}{llll}1314.6 & -0.5103 & 0.000001 & 0.260\end{array}$

$\begin{array}{llll}1330.5 & -0.5479 & 0.000001 & 0.300\end{array}$

$\begin{array}{llll}1336.2 & -0.6558 & 0.000001 & 0.430\end{array}$

$\begin{array}{llll}1358.0 & -0.7948 & 0.000001 & 0.632\end{array}$

$\begin{array}{llll}1380.2 & -0.9208 & 0.000001 & 0.848\end{array}$

$\begin{array}{llll}1387.1 & -1.0881 & 0.000001 & 1.184\end{array}$

$\begin{array}{llll}1402.6 & -1.0475 & 0.000001 & 1.097\end{array}$

$\begin{array}{llll}1425.3 & -1.1485 & 0.000001 & 1.319\end{array}$

$\begin{array}{llll}1440.4 & -1.3584 & 0.000001 & 1.845\end{array}$

$\begin{array}{llll}1448.3 & -1.2076 & 0.000001 & 1.458\end{array}$

$\begin{array}{llll}1471.7 & -1.2585 & 0.000001 & 1.584\end{array}$

$\begin{array}{llll}1490.5 & -1.5313 & 0.000001 & 2.345\end{array}$

$\begin{array}{llll}1495.3 & -1.1449 & 0.000001 & 1.311\end{array}$

$\begin{array}{llll}986.4 & 0.0208 & -0.000025 & 0.000\end{array}$

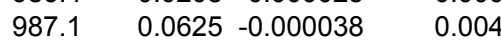

$\begin{array}{llll}988.6 & 0.0838 & -0.000026 & 0.007\end{array}$

$\begin{array}{llll}990.7 & 0.0909 & -0.000016 & 0.008\end{array}$

$\begin{array}{llll}991.7 & 0.1536 & -0.000022 & 0.024\end{array}$

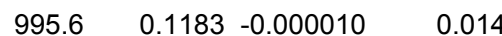

$\begin{array}{llll}997.5 & 0.2072 & -0.000015 & 0.043\end{array}$

$1000.4 \quad 0.1706-0.000010 \quad 0.029$

$\begin{array}{llll}1009.2 & 0.2091 & -0.000008 & 0.044\end{array}$

$\begin{array}{llll}10101 & 0.2283 & -0.000008 & 0.052\end{array}$

$\begin{array}{llll}1032.4 & 0.2373 & -0.000004 & 0.056\end{array}$

$\begin{array}{llll}1032.6 & 0.2440 & -0.000004 & 0.060\end{array}$

$\begin{array}{llll}1075.4 & 0.4325 & -0.000004 & 0.187\end{array}$

$\begin{array}{llll}1075.7 & 0.4308 & -0.000004 & 0.186\end{array}$

$\begin{array}{llll}1133.8 & 0.3623 & -0.000002 & 0.131\end{array}$

$\begin{array}{llll}1184.7 & 0.4016 & -0.000001 & 0.161\end{array}$

$\begin{array}{llll}1262.7 & 0.2043 & 0.000000 & 0.042\end{array}$

0.171

3.641

2.197

3.039

5.316

5.003

0.088

0.895

0.075
988.0363

988.0363

988.0363

988.0363

988.0363

988.0363

988.0363

988.0363

988.0363

988.0363

988.0363

988.0363

988.0363

988.0363

988.0363

988.0363

988.0363

$\begin{array}{lll}506.10 & & \\ & 6.3 & 985.6952\end{array}$

$5.61 \quad 985.6952$

$6.36 \quad 985.6952$

$5.12 \quad 985.6952$

10.007

$6.64 \quad 985.6952$

985.6952

\begin{tabular}{l}
$7.218 \quad 985.6952$ \\
\hline
\end{tabular}

985.6952
985.6952

$24.615 \quad 985.6952$

$46.949 \quad 985.6952$

$47.111 \quad 985.6952$

$90.41 \quad 985.6952$

$148.503 \quad 985.6952$

$199.438 \quad 985.6952$

277.256

$351.987 \quad 985.6952$

985.6952

983.2503

983.2503

983.2503

983.2503

983.2503

983.2503

983.2503 
molality molarity

$\begin{array}{rrrr}\mathbf{t} /{ }^{\circ} \mathbf{C} \text { mass frac } & \text { Density } & \text { Ref } \\ & & \text { exp } / & \\ & & \mathrm{kg} / \mathrm{m}^{3} & \\ 59.9 & 0.5037 & 1495.70 & (127) \\ 60 & 0.0200 & 1000.66 & (4) \\ 60 & 0.0400 & 1018.12 & (4) \\ 60 & 0.0600 & 1035.73 & (4) \\ 60 & 0.0800 & 1053.56 & (4) \\ 60 & 0.1000 & 1071.64 & (4) \\ 60 & 0.1200 & 1089.98 & (4) \\ 60 & 0.1400 & 1108.58 & (4) \\ 60 & 0.1600 & 1127.46 & (4) \\ 60 & 0.1800 & 1146.60 & (4) \\ 60 & 0.2000 & 1166.00 & (4) \\ 60 & 0.2200 & 1185.60 & (4) \\ 60 & 0.2400 & 1205.55 & (4) \\ 60 & 0.2600 & 1225.66 & (4) \\ 60 & 0.2800 & 1245.92 & (4) \\ 60 & 0.3000 & 1266.43 & (4) \\ 60 & 0.3200 & 1287.23 & (4) \\ 60 & 0.3400 & 1308.30 & (4) \\ 60 & 0.3600 & 1329.66 & (4) \\ 60 & 0.3800 & 1351.31 & (4) \\ 60 & 0.4000 & 1373.25 & (4) \\ 60 & 0.4200 & 1395.50 & (4) \\ 60 & 0.4400 & 1418.07 & (4) \\ 60 & 0.4600 & 1440.98 & (4) \\ 60 & 0.4800 & 1464.21 & (4) \\ 60 & 0.5000 & 1487.92 & (4) \\ 65 & 0.0008 & 981.30 & (81) \\ 65 & 0.0017 & 982.04 & (81) \\ 65 & 0.0034 & 983.49 & (81) \\ 65 & 0.0070 & 986.69 & (81) \\ 65 & 0.0138 & 992.52 & (81) \\ 65 & 0.0273 & 1004.13 & (81) \\ 70 & 0.0200 & 995.17 & (4) \\ 70 & 0.0400 & 1012.57 & (4) \\ 70 & 0.0600 & 1030.14 & (4) \\ 70 & 0.0800 & 1047.92 & (4) \\ 70 & 0.1000 & 1065.95 & (4) \\ 70 & 0.1200 & 1084.25 & (4) \\ 70 & 0.1400 & 1102.81 & (4) \\ 70 & 0.1600 & 1121.64 & (4) \\ 70 & 0.1800 & 1140.75 & (4) \\ 70 & 0.2000 & 1160.12 & (4) \\ 70 & 0.2200 & 1179.69 & (4)\end{array}$

App vol App vol Den $\begin{array}{cc}\exp / & \text { Calc } / \\ \mathrm{m}^{3} / \mathrm{kg} & \mathrm{m}^{3} / \mathrm{kg}\end{array}$

$0.000325 \quad 0.000327$

$0.000130 \quad 0.000150$

$0.000145 \quad 0.000159$

$0.000157 \quad 0.000168$

$0.000168 \quad 0.000176$

$0.000178 \quad 0.000185$

$0.000187 \quad 0.000193$

$0.000195 \quad 0.000201$

$0.000204 \quad 0.000209$

$\begin{array}{lll}0.000212 & 0.000217\end{array}$

$0.000220 \quad 0.000225$

$0.000228 \quad 0.000232$

$0.000235 \quad 0.000240$

$0.000243 \quad 0.000247$

$0.000251 \quad 0.000254$

$0.000259 \quad 0.000261$

$0.000266 \quad 0.000268$

$0.000274 \quad 0.000275$

$0.000281 \quad 0.000282$

$0.000288 \quad 0.000289$

$0.000295 \quad 0.000295$

$0.000302 \quad 0.000301$

$0.000308 \quad 0.000308$

$0.000315 \quad 0.000314$

$0.000321 \quad 0.000320$

$0.000327 \quad 0.000326$

$0.000096 \quad 0.000145$

$0.000101 \quad 0.000145$

$0.000114 \quad 0.000146$

$0.000117 \quad 0.000148$

$0.000130 \quad 0.000151$

$0.000142 \quad 0.000157$

0.0001290 .000157

$0.000144 \quad 0.000165$

$0.000156 \quad 0.000174$

$0.000167 \quad 0.000182$

$0.000177 \quad 0.000190$

$0.000186 \quad 0.000198$

$0.000194 \quad 0.000206$

$0.000203 \quad 0.000214$

$0.000211 \quad 0.000221$

$0.000219 \quad 0.000229$

$0.000227 \quad 0.000236$
App Vol Square of Inconsisten

$\mathrm{kg} / \mathrm{m}^{3}$ $\mathrm{m}^{3} / \mathrm{kg}$

Resid

1493.4

1000.3

1017.5

0.

$522-0.00000$

$0.4068-0.000020$

$0.5811-0.000014$

5.072

0.165

0.338

0.448

$0.7374-0.000008 \quad 0.544$

$\begin{array}{llll}1070.8 & 0.8116 & -0.000007 & 0.659\end{array}$

$\begin{array}{llll}1089.1 & 0.8978 & -0.000006 & 0.806\end{array}$

$\begin{array}{llll}1089.1 & 0.8978 & -0.000006 & 0.806\end{array}$

$\begin{array}{llll}1126.3 & 1.1102 & -0.000005 & 1.232\end{array}$

$\begin{array}{llll}1145.4 & 1.2279 & -0.000005 & 1.508\end{array}$

$\begin{array}{llll}1164.7 & 1.3408 & -0.000005 & 1.798\end{array}$

$\begin{array}{llll}1184.2 & 1.3848 & -0.000004 & 1.918\end{array}$

$\begin{array}{llll}1204.0 & 1.5051 & -0.000004 & 2.265\end{array}$

$1224.2 \quad 1.5072-0.000004 \quad 2.272$

$\begin{array}{llll}1244.5 & 1.3766 & -0.000003 & 1.895\end{array}$

$\begin{array}{llll}1265.2 & 1.2084 & -0.000003 & 1.460\end{array}$

$\begin{array}{llll}1286.2 & 1.0378-0.000002 & 1.077\end{array}$

$\begin{array}{llll}1307.5 & 0.8398 & -0.000001 & 0.705\end{array}$

$\begin{array}{llll}1329.0 & 0.6294 & -0.000001 & 0.396\end{array}$

$\begin{array}{llll}1350.9 & 0.4014 & -0.000001 & 0.161\end{array}$

$\begin{array}{llll}1373.1 & 0.1507 & 0.000000 & 0.023\end{array}$

$\begin{array}{llll}1395.6 & -0.1082 & 0.000000 & 0.012\end{array}$

$\begin{array}{llll}1418.4 & -0.3707 & 0.000000 & 0.137\end{array}$

$\begin{array}{llll}1441.6 & -0.6225 & 0.000001 & 0.387\end{array}$

$\begin{array}{llll}1465.1 & -0.8891 & 0.000001 & 0.790\end{array}$

$\begin{array}{llll}1488.9 & -1.0164 & 0.000001 & 1.033\end{array}$

$\begin{array}{llll}981.3 & 0.0399 & -0.000049 & 0.002\end{array}$

$\begin{array}{llll}982.0 & 0.0727 & -0.000045 & 0.005\end{array}$

$\begin{array}{llll}983.4 & 0.1045 & -0.000032 & 0.011\end{array}$

$\begin{array}{llll}986.5 & 0.2068 & -0.000030 & 0.043\end{array}$

$\begin{array}{llll}992.2 & 0.2845 & -0.000021 & 0.081\end{array}$

$\begin{array}{llll}1003.7 & 0.3918 & -0.000014 & 0.154\end{array}$

$\begin{array}{llll}994.6 & 0.5567 & -0.000028 & 0.310\end{array}$

$1011.7 \quad 0.8752-0.000021 \quad 0.766$

$\begin{array}{lll}1029.0 & 1.1212-0.000018 & 1.257\end{array}$

$\begin{array}{llll}1046.6 & 1.3300 & -0.000015 & 1.769\end{array}$

$\begin{array}{llll}1064.4 & 1.5369 & -0.000014 & 2.362\end{array}$

$\begin{array}{llll}1082.5 & 1.7570 & -0.000012 & 3.087\end{array}$

$\begin{array}{llll}1100.8 & 1.9754 & -0.000012 & 3.902\end{array}$

$\begin{array}{llll}1119.4 & 2.1969 & -0.000011 & 4.826\end{array}$

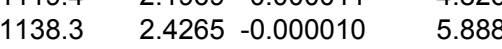

$\begin{array}{llll}1157.5 & 2.6386 & -0.000010 & 6.962\end{array}$

$\begin{array}{llll}1176.9 & 2.7679 & -0.000009 & 7.661\end{array}$ $\begin{array}{ccc}\text { based on } & \begin{array}{c}\text { Inconsistent } \\ \text { based on }\end{array} & \begin{array}{c}\text { Delta density } \\ / \mathrm{kg} / \mathrm{m}^{3}\end{array} \text { molal volume water } / \mathrm{kg} / \mathrm{m}^{3}\end{array}$ apparent
volume

$\begin{array}{rrr} & & \\ & & 983.2503 \\ 17.5 & & 983.1989 \\ 34.9 & & 983.1989 \\ 52.5 & & 983.1989 \\ 70.4 & & 983.1989 \\ 88.4 & & 983.1989 \\ 106.8 & & 983.1989 \\ 125.4 & & 983.1989 \\ 144.3 & & 983.1989 \\ 163.4 & & 983.1989 \\ 182.8 & & 983.1989 \\ 202.4 & & 983.1989 \\ 222.4 & & 983.1989 \\ 242.5 & & 983.1989 \\ 262.7 & & 983.1989 \\ 283.2 & & 983.1989 \\ 304.0 & & 983.1989 \\ 325.1 & & 983.1989 \\ 346.5 & & 983.1989 \\ 368.1 & & 983.1989 \\ 390.1 & & 983.1989 \\ 412.3 & & 983.1989 \\ 434.9 & & 983.1989 \\ 457.8 & & 983.1989 \\ 481.0 & & 983.1989 \\ 504.7 & & 983.1989 \\ & 5.37 & 980.5548 \\ & 5.64 & 980.5548 \\ & 6.39 & 980.5548 \\ & 6.59 & 980.5548 \\ & 7.284 & 980.5548 \\ 17.4 & 7.992 & 980.5548 \\ 34.8 & & 977.7696 \\ 52.4 & & 977.7696 \\ 70.2 & & 977.7696 \\ 88.2 & & 977.7696 \\ 106.5 & & 977.7696 \\ 125.0 & & 977.7696 \\ 143.9 & & 977.7696 \\ 163.0 & & 977.7696 \\ 182.4 & & 977.7696 \\ 201.9 & & 977.7696 \\ & & \end{array}$


molality molarity

$t /{ }^{\circ} \mathrm{C}$ mass frac Density

$\exp /$

$\mathrm{kg} / \mathrm{m}^{3}$

\begin{tabular}{|c|c|c|c|}
\hline & 70 & 0.2400 & 1199.61 \\
\hline & 70 & 0.2600 & 1219.70 \\
\hline & 70 & 0.2800 & 1239.94 \\
\hline & 70 & 0.3000 & 1260.42 \\
\hline & 70 & 0.3200 & 1281.17 \\
\hline & 70 & 0.3400 & 1302.20 \\
\hline & 70 & 0.3600 & 1323.52 \\
\hline & 70 & 0.3800 & 1345.12 \\
\hline & 70 & 0.4000 & 1367.01 \\
\hline & 70 & 0.4200 & 1389.20 \\
\hline & 70 & 0.4400 & 1411.70 \\
\hline & 70 & 0.4600 & 1434.53 \\
\hline & 70 & 0.4800 & 1457.67 \\
\hline & 70 & 0.5000 & 1481.27 \\
\hline 0.015 & 75 & 0.0008 & 975.59 \\
\hline 0.03005 & 75 & 0.0017 & 976.35 \\
\hline 0.06006 & 75 & 0.0034 & 977.77 \\
\hline 0.12604 & 75 & 0.0070 & 980.98 \\
\hline 0.24963 & 75 & 0.0138 & 986.79 \\
\hline 0.49992 & 75 & 0.0273 & 998.39 \\
\hline & 79.9 & 0.0617 & 1026.90 \\
\hline & 79.9 & 0.1150 & 1074.60 \\
\hline & 79.9 & 0.2780 & 1232.90 \\
\hline & 79.9 & 0.3600 & 1318.00 \\
\hline & 79.9 & 0.4300 & 1397.30 \\
\hline & 79.9 & 0.4720 & 1444.20 \\
\hline & 79.9 & 0.5037 & 1484.80 \\
\hline 0.015 & 85 & 0.0008 & 969.36 \\
\hline 0.03005 & 85 & 0.0017 & 970.12 \\
\hline 0.06006 & 85 & 0.0034 & 971.54 \\
\hline 0.12604 & 85 & 0.0070 & 974.74 \\
\hline 0.24963 & 85 & 0.0138 & 980.58 \\
\hline 0.49992 & 85 & 0.0273 & 992.17 \\
\hline 0.015 & 95 & 0.0008 & 962.66 \\
\hline 0.03005 & 95 & 0.0017 & 963.41 \\
\hline 0.06006 & 95 & 0.0034 & 964.84 \\
\hline 0.12604 & 95 & 0.0070 & 968.05 \\
\hline 0.24963 & 95 & 0.0138 & 973.91 \\
\hline 0.49992 & 95 & 0.0273 & 985.54 \\
\hline & 99.2 & 0.5540 & 1532.40 \\
\hline & 99.2 & 0.5946 & 1580.90 \\
\hline & 100 & 0.0453 & 999.00 \\
\hline & 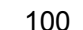 & 00879 & 103700 \\
\hline
\end{tabular}

Ref

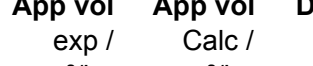

$\mathrm{m}^{3} / \mathrm{k}$

(4)

$0.000235 \quad 0.000243$

$0.000242 \quad 0.000250$

$0.000250 \quad 0.000257$

$\begin{array}{ll}0.000258 & 0.000264\end{array}$

$\begin{array}{ll}0.000266 & 0.000271\end{array}$

$\begin{array}{lll}0.000273 & 0.000277\end{array}$

$\begin{array}{ll}0.000281 & 0.000284\end{array}$

$0.000288 \quad 0.000290$

$\begin{array}{ll}0.000295 & 0.000297\end{array}$

$0.000302 \quad 0.000303$

$\begin{array}{ll}0.000308 & 0.000309\end{array}$

$0.000315 \quad 0.000315$

$\begin{array}{ll}0.000321 & 0.000321\end{array}$

$\begin{array}{ll}0.000327 & 0.000327\end{array}$

$\begin{array}{ll}0.000104 & 0.000152\end{array}$

$\begin{array}{ll}0.000091 & 0.000152\end{array}$

$0.000113 \quad 0.000153$

$\begin{array}{lll}\text { (81) } & 0.000114 & 0.000154\end{array}$

$\begin{array}{lll}(81) & 0.000114 & 0.000154 \\ (81) & 0.000127 & 0.000157\end{array}$

$\begin{array}{lll}\text { (81) } & 0.000139 & 0.000163\end{array}$

(127)

(127)

$0.000135 \quad 0.000180$

$\begin{array}{lll}0.000174 & 0.000201\end{array}$

$\begin{array}{ll}0.000245 & 0.000259\end{array}$

$0.000278 \quad 0.000286$

$0.000300 \quad 0.000307$

$\begin{array}{ll}0.000316 & 0.000319\end{array}$

$0.000323 \quad 0.000328$

$0.000091 \quad 0.000158$

$0.000085 \quad 0.000158$

$0.000108 \quad 0.000159$

$0.000109 \quad 0.000161$

$\begin{array}{ll}0.000121 & 0.000163\end{array}$

(81) $\quad 0.000134 \quad 0.000169$

(81) $\quad 0.000059 \quad 0.000164$

(81) $\quad 0.000069 \quad 0.000164$

(81) $\quad 0.000095 \quad 0.000165$

(81) $\quad 0.000100 \quad 0.000166$

(81) $\quad 0.000112 \quad 0.000169$

$\begin{array}{lll}(81) & 0.000126 & 0.000174\end{array}$

$\begin{array}{lll}\text { (127) } \quad 0.000338 & 0.000340\end{array}$

(127) $\quad 0.000353 \quad 0.000350$

(69) $\quad 0.000106 \quad 0.000184$

(69)
Density Density App Vol Square of Inconsistent Inconsistent Delta density Apparent Density of calc / Residual / Residual / Density based on based on $/ \mathrm{kg} / \mathrm{m}^{3}$ molal volume water $/ \mathrm{kg} / \mathrm{m}^{3}$ $\mathrm{kg} / \mathrm{m}^{3} \quad \mathrm{~kg} / \mathrm{m}^{3} \quad \mathrm{~m}^{3} / \mathrm{kg}$ Residual density apparent

$1196.7 \quad 2.9587-0.000009 \quad 8.754$

$1216.7 \quad 3.0253-0.000008 \quad 9.152$

$\begin{array}{llll}1237.0 & 2.9416 & -0.000007 & 8.653\end{array}$

$1257.6 \quad 2.7917-0.000006 \quad 7.794$

$1278.6 \quad 2.5993-0.000005 \quad 6.756$

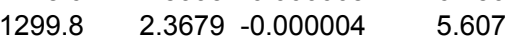

$1321.4 \quad 2.1011-0.000003 \quad 4.414$

$1343.3 \quad 1.7819-0.000003-3.175$

$\begin{array}{lll}1365.6 & 1.4136-0.000002 & 1.998\end{array}$

$\begin{array}{llll}1388.2 & 0.9989 & -0.000001 & 0.998\end{array}$

$\begin{array}{llll}1411.2 & 0.5405 & -0.000001 & 0.292\end{array}$

$\begin{array}{llll}1434.5 & 0.0509 & 0.000000 & 0.003\end{array}$

$\begin{array}{llll}1458.2 & -0.4976 & 0.000000 & 0.248\end{array}$

$\begin{array}{llll}1482.2 & -0.9631 & 0.000001 & 0.927\end{array}$

$\begin{array}{llll}975.5 & 0.0382 & -0.000048 & 0.001\end{array}$

$\begin{array}{llll}976.2 & 0.0984 & -0.000061 & 0.010\end{array}$

$\begin{array}{llll}977.6 & 0.1289 & -0.000040 & 0.017\end{array}$

$\begin{array}{lllll}980.7 & 0.2758 & -0.000041 & 0.076\end{array}$

$\begin{array}{llll}986.4 & 0.4038 & -0.000030 & 0.163\end{array}$

$\begin{array}{llll}997.7 & 0.6448 & -0.000024 & 0.416\end{array}$

$\begin{array}{llll}1024.0 & 2.9158 & -0.000045 & 8.502\end{array}$

$\begin{array}{llll}1071.0 & 3.6385 & -0.000027 & 13.239\end{array}$

$\begin{array}{llll}1227.0 & 5.8585 & -0.000014 & 34.322\end{array}$

$\begin{array}{llll}1313.5 & 4.5246 & -0.000007 & 20.472\end{array}$

$\begin{array}{llll}1392.0 & 5.3070 & -0.000006 & 28.165\end{array}$

$\begin{array}{llll}1441.4 & 2.8351 & -0.000003 & 8.038\end{array}$

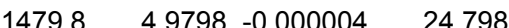

$\begin{array}{llll}969.3 & 0.0530 & -0.000067 & 0.003\end{array}$

$\begin{array}{llll}970.0 & 0.1164 & -0.000074 & 0.014\end{array}$

$\begin{array}{llll}971.4 & 0.1618 & -0.000051 & 0.026\end{array}$

$\begin{array}{llll}974.4 & 0.3447 & -0.000052 & 0.119\end{array}$

$\begin{array}{llll}980.0 & 0.5601 & -0.000042 & 0.314\end{array}$

$\begin{array}{llll}991.2 & 0.9254 & -0.000034 & 0.856\end{array}$

$\begin{array}{llll}962.6 & 0.0819 & -0.000105 & 0.007\end{array}$

$\begin{array}{llll}963.3 & 0.1487 & -0.000095 & 0.022\end{array}$

$\begin{array}{llll}964.6 & 0.2173 & -0.000070 & 0.047\end{array}$

$\begin{array}{llll}967.6 & 0.4388 & -0.000067 & 0.193\end{array}$

$\begin{array}{llll}973.2 & 0.7498 & -0.000057 & 0.562\end{array}$

$984.3 \quad 1.2863-0.000049 \quad 1.654$

$1530.4 \quad 1.9916-0.000002 \quad 3.966$

$\begin{array}{llll}1585.3 & -4.4170 & 0.000003 & 19.510\end{array}$

$\begin{array}{llll}995.5 & 3.4860 & -0.000077 & 12.152\end{array}$

$\begin{array}{llll}1031.7 & 5.3084 & -0.000056 & 28.179\end{array}$
977.7696

977.7696

977.7696

977.7696

977.7696

977.7696

977.7696

977.7696

977.7696

977.7696

977.7696

977.7696

$\begin{array}{ll}479.9 & 977.7696 \\ 503.5 & 977.7696\end{array}$

$\begin{array}{ll}479.9 & 977.7696 \\ 503.5 & 977.7696\end{array}$

$\begin{array}{ll}5.83 & 974.849 \\ 5.09 & 974.849\end{array}$

$6.32-974.849$

$6.37 \quad 974.849$

$7.14 \quad 974.849$

$7.816 \quad 974.849$

971.8601

971.8601

971.8601

971.8601

971.8601

971.8601

971.8601

$5.1 \quad 968.6203$

$4.76 \quad 968.6203$

$6.06-968.6203$

6.11968 .6203

$6.8 \quad 968.6203$

$7.543 \quad 968.6203$

$3.3 \quad 961.9004$

$3.87 \quad 961.9004$

$5.35 \quad 961.9004$

$5.59 \quad 961.9004$

$6.272 \quad 961.9004$

$7.058 \quad 961.9004$

958.9373

958.9373

958.3637

958.3637 
molality molarity

$\begin{array}{ccr}\mathbf{t} /{ }^{\circ} \mathbf{C} \text { mass frac } & \begin{array}{r}\text { Density } \\ \exp / \\ \mathrm{kg} / \mathrm{m}^{3}\end{array} \\ 100 & 0.2982 & 1234.00 \\ 100 & 0.3548 & 1294.00 \\ 100 & 0.4062 & 1349.00 \\ 100 & 0.4531 & 1403.00 \\ 100 & 0.4960 & 1452.00\end{array}$

Ref

(69)

$(69)$
$(69)$
$(69)$
$(69)$
$(69)$

App vol App vol

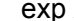

$\mathrm{m}^{3} / \mathrm{kg}$

0.00026

0.000270

0.0002810 .000286

$0.000300 \quad 0.000301$

$0.000314 \quad 0.000314$

1455

Average Res

Std dev Res

Avg - 4std

Avg +4 std
Density Density App Vol Square of Inconsistent Inconsistent Delta density Apparent Density of calc / Residual / Residual / Density based on based on

$\mathrm{kg} / \mathrm{m}^{3} \quad \mathrm{~kg} / \mathrm{m}^{3} \quad \mathrm{~m}^{3} / \mathrm{kg} \quad \begin{array}{r}\text { Residual density } \\ \text { apparent } \\ \text { volume }\end{array}$ $1 \mathrm{~cm} / \mathrm{mol}$

958.3637
958.3637
958.3637
958.3637
958.3637
12.696

12.104
1.218

0.053

10.416

978.626 Sum chi sqr

$0.1538-0.000010$

$\begin{array}{ll}1.5187 & 0.000019\end{array}$

$-5.9208-0.000085$

$6.2285 \quad 0.000065$

\section{Curve fit for the model App vol= $\left(w+c 2+c 3^{*} t{ }^{\circ} \mathrm{C}\right) /\left(c 0^{*} w+c 1\right) / e^{\wedge}\left(0.000001^{*}\left(t^{\circ} \mathrm{C}+c 4\right)^{\wedge} 2\right)$}

c0

c1 2

c3

c4

$\operatorname{Min} T$

$\operatorname{Max} T$

Min w

Max w

Avg dens res

1.518665

Std app vol res *10^3 0.018786

No of points in corr

No of inconsistent poir

194.8493

0.14542

0.00204

1180.944

100

0.000841

0.5946

421

Inconsistent data not used (Residual greater than average $+/ \mathbf{4}$ standard deviations)

$\begin{array}{llllll}0 & 0.5000 & 1525.67 & \text { (4) } & 0.000311 & 0.000317\end{array}$

10.21

$0.5000-1525.67$

$(109)$

$0.003656 \quad 0.000313$

$\begin{array}{ll}0.000301 & 0.000292 \\ 0.000212 & 0.000236\end{array}$

$\begin{array}{rrr}79.9 & 0.2090 & 1165.20\end{array}$

$(127)$

$\begin{array}{ll}0.000212 & 0.000236 \\ 0.000271 & 0.000288 \\ 0.000296 & 0.000308\end{array}$

99.2

$\begin{array}{ll}0.3600 & 1307.3 \\ 0.4300 & 1385.8\end{array}$

(127)

99.2

0.4397

(127)

$0.000296 \quad 0.000308$

$\begin{array}{lll}100 & 0.1659 & 1108.00\end{array}$

$\begin{array}{lll}100 & 0.2356 \quad 1174.00\end{array}$

$\begin{array}{lll}\text { (69) } \quad 0.000194 & 0.000227\end{array}$

$\begin{array}{lll}\text { (69) } \quad 0.000230 & 0.000250\end{array}$

1460.7 \#\#\#\#\#\#\# 0.003343 \#\#\#\#\#\#\#

$\begin{array}{llll}1404.0 & -7.0703 & 0.000009 & 49.989\end{array}$

$\begin{array}{llll}1158.6 & 6.6396 & -0.000024 & 44.085\end{array}$

$\begin{array}{llll}1296.9 & 10.3839 & -0.000017 & 107.826\end{array}$

$\begin{array}{llll}1376.1 & 9.6839 & -0.000012 & 93.779\end{array}$

$\begin{array}{llll}1387.5 & 9.3854 & -0.000011 & 88.086\end{array}$

$\begin{array}{llll}1101.3 & 6.7160 & -0.000033 & 45.105\end{array}$

$\begin{array}{llll}1167.5 & 6.5364 & -0.000020 & 42.725\end{array}$
525.83

446.423
999.8395

992.2158

995.6473

971.8601

958.9373

958.9373

958.9373

958.3637

958.3637 
Density of aqueous solutions of K2SO4

References (20) Dedick, Eugene A.; Hershey, J. Peter; Sotolongo, Sara; Stade, David J.; Millero, Frank J. The PVT Properties of Concentrated Aqueous Electrolytes IX. The Volume Properties of $\mathrm{KCl}$ and $\mathrm{K} 2 \mathrm{SO} 4$ and their Mixtures with $\mathrm{NaCl}$ and $\mathrm{Na} 2 \mathrm{SO} 4$ as a Function of Temperature, $\mathrm{J}$. Solution Chem. 1990, 19, 353-374

(49) Kaminsky, M, Concentration and Temperature Dependence of the Viscosity of Aqueous Solutions of Strong Electrolytes. III. $\mathrm{KCl}_{2} \mathrm{~K}_{2} \mathrm{SO}_{4}$ $\mathrm{MgCl}_{2}, \mathrm{BeSO}_{4}$, and $\mathrm{MgSO}_{4}$ Solutions. Z. Phys. Chem. N. F. 1957, 12, 206-231.

(72) Millero, F. J.; Knox, J. H. Apparent Molal Volumes of Aqueous $\mathrm{NaF}, \mathrm{Na}_{2} \mathrm{SO}_{4}, \mathrm{KCl}, \mathrm{K}_{2} \mathrm{SO}_{4}, \mathrm{MgCl}_{2}$, and $\mathrm{MgSO}_{4}$ Solutions at $0^{\circ}$ and $50^{\circ} \mathrm{C}$ J. Chem. Eng. Data 1973, 18, 407-411.

(78) Olofsson, I. V.; Spitzer, J. J.; Hepler, L. G. Apparent Molar Heat Capacities and Volumes of Aqueous Electrolytes at $25^{\circ} \mathrm{C}: \mathrm{Na}_{2} \mathrm{SO}_{4}$, $\mathrm{K}_{2} \mathrm{SO}_{4}, \mathrm{Na}_{2} \mathrm{~S}_{2} \mathrm{O}_{3}, \mathrm{Na}_{2} \mathrm{~S}_{2} \mathrm{O}_{8}, \mathrm{~K}_{2} \mathrm{~S}_{2} \mathrm{O}_{8}, \mathrm{~K}_{2} \mathrm{CrO}_{4}, \mathrm{Na}_{2} \mathrm{MoO}_{4}$, and $\mathrm{Na}_{2} \mathrm{WO}_{4}$. Can. J. Chem. 1978, 56, 1871-1873.

(88) Perron, G., Desnoyers, J. E., Millero, F. J., "Apparent molal volumes and heat capacities of some sulfates and carbonates in water at 25 ${ }^{\circ}$ C.", Can. J. Chem. , 1975, 53, 1134-1138

(90) Perry, R. H.; Green, D. W. Perry's Chemical Engineers' Handbook; McGraw Hill: New York, 7th edition, 1997.

(114) Saluja, P. P. S.; Lemire, R. J.; LeBlanc, J. C.; High-Temperature Thermodynamics of Aqueous Alkali-Metal Salts; J. Chem. Thermodynamics, 1992, 24, 181-203

\begin{tabular}{|c|c|c|c|c|}
\hline molality & molarity & t/ 2 & ass trac & $\begin{array}{r}\text { Density } \\
\exp / \\
\mathrm{kg} / \mathrm{m}^{3}\end{array}$ \\
\hline 0.003007 & & 0 & 0.0005 & 1000.29 \\
\hline 0.004334 & & 0 & 0.0008 & 1000.49 \\
\hline 0.005597 & & 0 & 0.0010 & 1000.68 \\
\hline 0.007897 & & 0 & 0.0014 & 1001.02 \\
\hline 0.010278 & & 0 & 0.0018 & 1001.38 \\
\hline 0.014715 & & 0 & 0.0026 & 1002.03 \\
\hline 0.01492 & & 0 & 0.0026 & 1002.11 \\
\hline 0.019454 & & 0 & 0.0034 & 1002.73 \\
\hline 0.027948 & & 0 & 0.0048 & 1003.98 \\
\hline 0.034366 & & 0 & 0.0060 & 1004.92 \\
\hline 0.036851 & & 0 & 0.0064 & 1005.29 \\
\hline 0.048361 & & 0 & 0.0084 & 1006.96 \\
\hline 0.054081 & & 0 & 0.0093 & 1007.79 \\
\hline 0.069961 & & 0 & 0.0120 & 1010.08 \\
\hline 0.099044 & & 0 & 0.0170 & 1014.25 \\
\hline 0.13033 & & 0 & 0.0222 & 1018.66 \\
\hline 0.15934 & & 0 & 0.0270 & 1022.78 \\
\hline 0.21831 & & 0 & 0.0366 & 1030.95 \\
\hline 0.28097 & & 0 & 0.0467 & 1039.48 \\
\hline 0.36472 & & 0 & 0.0598 & 1050.71 \\
\hline 0.04984 & & 5 & 0.0086 & 1007.14 \\
\hline 0.09856 & & 5 & 0.0169 & 1014.00 \\
\hline 0.1476 & & 5 & 0.0251 & 1020.82 \\
\hline 0.19721 & & 5 & 0.0332 & 1027.67 \\
\hline 0.2471 & & 5 & 0.0413 & 1034.33 \\
\hline 0.29668 & & 5 & 0.0492 & 1040.95 \\
\hline 0.34765 & & 5 & 0.0571 & 1047.64 \\
\hline 0.39755 & & 5 & 0.0648 & 1054.20 \\
\hline & 0.0025 & 12.5 & 0.0004 & 999.81 \\
\hline
\end{tabular}

Ref

\section{App vol}

App vol

$\exp / \quad$ Calc /

$\begin{array}{rrr}\mathrm{kg} / \mathrm{m}^{3} & \mathrm{~m}^{3} / \mathrm{kg} & \text { Residual } \\ 0.0154 & -0.000029 & 0.000\end{array}$

$0.0154-0.000029$

$0.0278-0.000029$

$0.0397-0.000029$

$\begin{array}{lll}0.0480 & -0.000027\end{array}$

$0.0620-0.000024$

$0.1142-0.000044$

$0.0798-0.000023$

$0.1037-0.000021$

$0.1184-0.000020$

$0.1231-0.000019$

$0.1447-0.000017$

$0.1712-0.000014$

$0.1869-0.000011$

$0.1671-0.000007$

$0.1970-0.000007$

$0.1419-0.000004$

0.000

0.001

0.002
0.002

0.004

0.013

0.006

0.011

0.014
0.015

0.021

0.023

0.029

0.035

0.028

0.039

$0.0286-0.000001-0.001$

$\begin{array}{lll}-0.1048 & 0.000002 & 0.011\end{array}$

$\begin{array}{lll}0.0654 & -0.000007 & 0.004\end{array}$

$\begin{array}{lll}0.0422 & -0.000002 & 0.002\end{array}$

$\begin{array}{rrr}0.0078 & 0.000000 & 0.000 \\ -0.0054 & 0.000000 & 0.000\end{array}$

$\begin{array}{lll}-0.1849 & 0.000004 & 0.034\end{array}$

$\begin{array}{lll}-0.2825 & 0.000005 & 0.080\end{array}$

$\begin{array}{lll}-0.4169 & 0.000007 & 0.174\end{array}$

$\begin{array}{lll}-0.4722 & 0.000007 & 0.223\end{array}$

$\begin{array}{lll}0.0165 & -0.000038 & 0.000 \\ 0.0332 & -0.000038 & 0.001\end{array}$ $\mathrm{kg} / \mathrm{m}^{3}$

0.4511

0.6501

0.8387

1.1835

1.5363

2.1919

2.2738

2.8944

4.1439

5.0833

5.4457

7.1219

7.9512

10.2435

14.4057

18.8196

22.9357

31.1124

39.6358

50.8675

7.175

14.033

20.856

27.709

34.362

40.982

47.678

54.235 relative Molal Density of density volume / water $/ \mathrm{kg} / \mathrm{m}^{3}$ $\mathrm{cm}^{3} /$ mole

999.8395

999.8395

999.8395

999.8395

999.8395

999.8395

999.8395

999.8395

999.8395

999.8395

999.8395

999.8395

999.8395

999.8395

999.8395

999.8395

999.8395

999.8395

999.8395

999.8395

999.9638

999.9638

999.9638

999.9638

999.9638

999.9638

999.9638

999.9638

999.4388

1.00074 


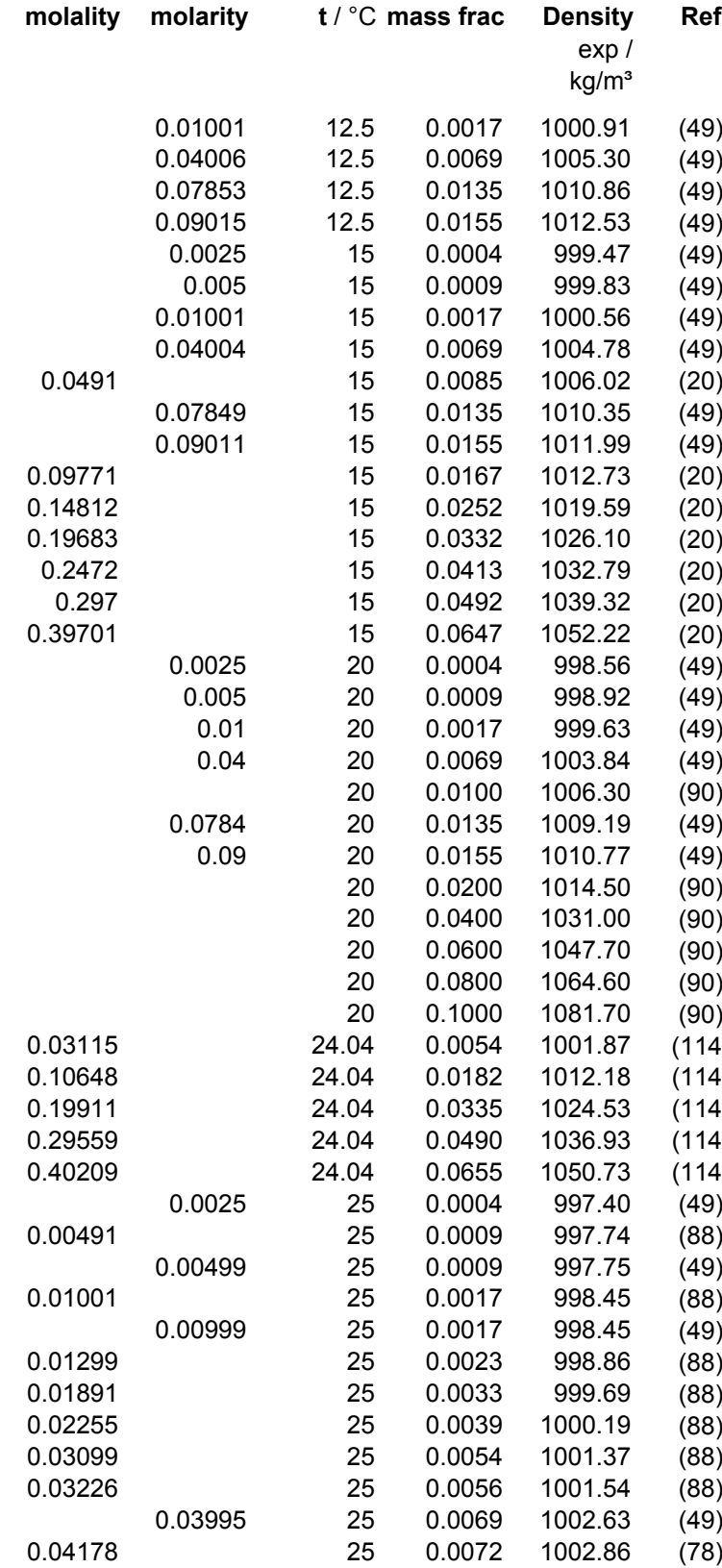

App vol App vol Density Density App Vol Square of Inconsistent Inconsistent

exp / Calc / calc/ Residual / Residual / Density based on based on

$0.000161 \quad 0.000191$

$0.000165 \quad 0.000194$

$0.000167 \quad 0.000194$

$0.000152 \quad 0.000193$

$0.000163 \quad 0.000193$

$0.000164 \quad 0.000193$

$0.000185 \quad 0.000195$

$\begin{array}{ll}0.000190 & 0.000195 \\ 0.000178 & 0.000197\end{array}$

0.0001790 .000198

$0.000196 \quad 0.000198$

$0.000202 \quad 0.000201$

$0.000207 \quad 0.000204$

$0.000210 \quad 0.000206$

0.0002140 .000209

$0.000220 \quad 0.000215$

$0.000175 \quad 0.000199$

$0.000175 \quad 0.000199$

$\begin{array}{ll}0.000181 & 0.000199\end{array}$

$\begin{array}{ll}0.000191 & 0.000201\end{array}$

$0.000196 \quad 0.000202$

$0.000196 \quad 0.000203$

$0.000199 \quad 0.000204$

$\begin{array}{ll}0.000197 & 0.000205\end{array}$

$0.000205 \quad 0.000212$

$\begin{array}{lll}0.000213 & 0.000219\end{array}$

0.0002210 .000226

$\begin{array}{ll}0.000229 & 0.000234 \\ 0.000154 & 0.000205\end{array}$

$\begin{array}{lll}0.000154 & 0.000205\end{array}$

$\begin{array}{ll}0.000193 & 0.000209 \\ 0.000208 & 0.000214\end{array}$

$0.000220 \quad 0.000219$

$\begin{array}{ll}0.000224 & 0.000225 \\ 0.000177 & 0.000204\end{array}$

$\begin{array}{lll}0.000189 & 0.000204\end{array}$

$0.000186 \quad 0.000204$

$\begin{array}{ll}0.000191 & 0.000204\end{array}$

$0.000193 \quad 0.000204$

$0.000195 \quad 0.000205$

$0.000194 \quad 0.000205$

$0.000196 \quad 0.000205$

$0.000197 \quad 0.000206$

$0.000197 \quad 0.000206$

0.0001990 .000206

$0.000198 \quad 0.000206$

$\mathrm{kg} / \mathrm{m}^{3} \quad \mathrm{~kg} / \mathrm{m}^{3} \quad \mathrm{~m}^{3} / \mathrm{kg}$ Residual density $\begin{gathered}\text { apparent } \\ \text { volume }\end{gathered}$

$\begin{array}{lll}1000.85 & 0.0556 & -0.000032 \\ 1005.08 & 0.2121 & -0.000030\end{array}$

1005.08

010.47

012.09

999.45
999.80

1000.51

1004.72

005.97

1010.08
1011.70

1012.70

1019.61

1026.21

1039.57

1052.61

998.90

999.60

1003.78
1006.24

1009.10

1010.70

1014.34

1030.71

1047.3

1064.11

1001.59

1011.88

1024.31

1036.98

1050.64

997.72

997.74

998.43

998.43

998.84

999.66

1000.16

1001.32

1001.50

1002.58
1002.80 $\begin{array}{lll}0.0179 & -0.000041 & 0.000\end{array}$

$0.0259-0.000030 \quad 0.001$

$0.0509-0.000029 \quad 0.003$

$\begin{array}{lll}0.0657 & -0.000009 & 0.004\end{array}$

$\begin{array}{lll}0.0468 & -0.000005 & 0.002\end{array}$

$\begin{array}{lll}0.2662 & -0.000019 & 0.071\end{array}$

$\begin{array}{lll}0.2891 & -0.000018 & 0.084\end{array}$

$0.0322-0.000002$

$\begin{array}{lll}0.0187 & 0.000001 & 0.000\end{array}$

$\begin{array}{lll}-0.1136 & 0.000003 & 0.013\end{array}$

$\begin{array}{lll}-0.1747 & 0.000004 & 0.031\end{array}$

$\begin{array}{lll}-0.2562 & 0.000005 & 0.066\end{array}$

$\begin{array}{lll}0.3913 & 0.000005 & 0.153\end{array}$

$\begin{array}{lll}0.0102 & -0.000023 & 0.000\end{array}$

$0.0205-0.000024 \quad 0.000$

$\begin{array}{lll}0.0314 & -0.000018 & 0.001\end{array}$

$\begin{array}{lll}0.0677 & -0.000010 & 0.005\end{array}$

$\begin{array}{lll}0.0984 & -0.000007 & 0.010\end{array}$

$0.0739-0.000005-0.005$

$\begin{array}{lll}0.1646 & -0.000008 & 0.027\end{array}$

$\begin{array}{lll}0.2900 & -0.000007 & 0.084\end{array}$

$0.4883-0.000005$

$\begin{array}{lll}0.6102 & -0.000005 & 0.372 \\ 0.2748 & -0.000051 & 0.076\end{array}$

$0.2985-0.000016$

$\begin{array}{lll}0.2267 & -0.000006 & 0.051\end{array}$

$\begin{array}{lll}0.0469 & 0.000001 & 0.002\end{array}$

$\begin{array}{lll}0.0940 & -0.000001 & 0.009\end{array}$

$\begin{array}{lll}0.0119 & -0.000027 & 0.000 \\ 0.0127 & -0.000015 & 0.000\end{array}$

$0.0154-0.000018$

$0.0229-0.000013$

$0.0199-0.000011 \quad 0.000$

$\begin{array}{lll}0.0214 & -0.000009 & 0.000\end{array}$

$\begin{array}{lll}0.0357 & -0.000011 & 0.001\end{array}$

$\begin{array}{lll}0.0360 & -0.000009 & 0.001\end{array}$

$\begin{array}{lll}0.0463 & -0.000009 & 0.002\end{array}$

$\begin{array}{lll}0.0497-0.000009 & 0.002\end{array}$

$\begin{array}{lll}0.0529 & -0.000008 & 0.003\end{array}$

$\begin{array}{lll}0.0573 & -0.000008 & 0.003\end{array}$ delta

density

$\mathrm{kg} / \mathrm{m}^{3}$

relative $\mathrm{m}^{3} / \mathrm{mole}$

\begin{tabular}{|c|c|c|}
\hline & $1.0014 !$ & 999.4388 \\
\hline & 1.00586 & 999.4388 \\
\hline & 1.01143 & 999.4388 \\
\hline & 1.0131 & 999.4388 \\
\hline & 1.00037 & 999.0996 \\
\hline & 1.00073 & 999.0996 \\
\hline & 1.00146 & 999.0996 \\
\hline & 1.00569 & 999.0996 \\
\hline \multirow[t]{3}{*}{6.916} & & 999.0996 \\
\hline & 1.01126 & 999.0996 \\
\hline & 1.0129 & 999.0996 \\
\hline 13.633 & & 999.0996 \\
\hline 20.49 & & 999.0996 \\
\hline 26.999 & & 999.0996 \\
\hline 33.693 & & 999.0996 \\
\hline 40.216 & & 999.0996 \\
\hline \multirow{19}{*}{53.12} & & 999.0996 \\
\hline & 1.00036 & 998.2041 \\
\hline & 1.00072 & 998.2041 \\
\hline & 1.00143 & 998.2041 \\
\hline & 1.00565 & 998.2041 \\
\hline & & 998.2041 \\
\hline & 1.01101 & 998.2041 \\
\hline & 1.01259 & 998.2041 \\
\hline & & 998.2041 \\
\hline & & 998.2041 \\
\hline & & 998.2041 \\
\hline & & 998.2041 \\
\hline & & 998.2041 \\
\hline & & 997.2867 \\
\hline & & 997.2867 \\
\hline & & 997.2867 \\
\hline & & 997.2867 \\
\hline & & 997.2867 \\
\hline & 1.00036 & 997.0449 \\
\hline \multirow[t]{2}{*}{0.692} & & 997.0449 \\
\hline & 1.00071 & 997.0449 \\
\hline \multirow[t]{2}{*}{1.407} & & 997.0449 \\
\hline & 1.00141 & 997.0449 \\
\hline 1.817 & & 997.0449 \\
\hline 2.648 & & 997.0449 \\
\hline 3.15 & & 997.0449 \\
\hline 4.322 & & 997.0449 \\
\hline 4.5 & & 997.0449 \\
\hline & 1.0056 & 997.0449 \\
\hline
\end{tabular}




$\begin{array}{rlrrrr}\text { molality } & \text { molarity } & \mathbf{t} /{ }^{\circ} \mathrm{C} \text { mass frac } & \text { Density } & \text { Ref } \\ & & & & \text { exp } / & \\ & & & & \mathrm{kg} / \mathrm{m}^{3} & \\ 0.04553 & & 25 & 0.0079 & 1003.36 & (88) \\ 0.04935 & & 25 & 0.0085 & 1003.89 & (20) \\ 0.05055 & & 25 & 0.0087 & 1004.04 & (78) \\ 0.05483 & & 25 & 0.0095 & 1004.62 & (78) \\ 0.06473 & & 25 & 0.0112 & 1005.98 & (78) \\ 0.06622 & & 25 & 0.0114 & 1006.21 & (88) \\ 0.0734 & & 25 & 0.0126 & 1007.14 & (78) \\ & 0.0783 & 25 & 0.0135 & 1007.96 & (49) \\ 0.07975 & & 25 & 0.0137 & 1008.06 & (78) \\ 0.08078 & & 25 & 0.0139 & 1008.19 & (78) \\ & 0.08989 & 25 & 0.0155 & 1009.51 & (49) \\ 0.09855 & & 25 & 0.0169 & 1010.58 & (20) \\ 0.10587 & & 25 & 0.0181 & 1011.57 & (78) \\ 0.10711 & & 25 & 0.0183 & 1011.76 & (78) \\ 0.11654 & & 25 & 0.0199 & 1013.02 & (88) \\ 0.11959 & & 25 & 0.0204 & 1013.43 & (78) \\ 0.13098 & & 25 & 0.0223 & 1014.97 & (88) \\ 0.13389 & & 25 & 0.0228 & 1015.31 & (78) \\ 0.1478 & & 25 & 0.0251 & 1017.20 & (20) \\ 0.16619 & & 25 & 0.0281 & 1019.65 & (78) \\ 0.19363 & & 25 & 0.0326 & 1023.27 & (88) \\ 0.19795 & & 25 & 0.0333 & 1023.88 & (88) \\ 0.2465 & & 25 & 0.0412 & 1030.18 & (20) \\ 0.26345 & & 25 & 0.0439 & 1032.45 & (88) \\ 0.29538 & & 25 & 0.0490 & 1036.54 & (20) \\ 0.33066 & & 25 & 0.0545 & 1041.12 & (88) \\ 0.34659 & & 25 & 0.0570 & 1043.09 & (20) \\ 0.39755 & & 25 & 0.0648 & 1049.54 & (20) \\ & 0.00249 & 35 & 0.0004 & 994.38 & (49) \\ 0.00498 & 35 & 0.0009 & 994.73 & (49) \\ 0.54892 & & 35 & 0.0017 & 995.41 & (49) \\ 0.59751 & & 35 & 0.0069 & 999.53 & (49) \\ 0.65018 & & 35 & 0.0943 & 1070.45 & (20) \\ & 0.00248 & 42.5 & 0.1018 & 1076.74 & (20) \\ 0.05091 & & 35 & 0.0088 & 1001.01 & (20) \\ & & 35 & 0.0135 & 1004.76 & (49) \\ 0.10041 & & & & 991.58 & (49) \\ 0.14747 & & 35 & 0.0155 & 1006.33 & (49) \\ 0.24422 & & 35 & 0.0172 & 1007.65 & (20) \\ 0.34405 & & 35 & 0.0251 & 1013.89 & (20) \\ 0.39666 & & 35 & 0.0408 & 1026.49 & (20) \\ 0.44506 & & 35 & 0.0566 & 1039.22 & (20) \\ 0.49574 & & 35 & 0.0647 & 1045.84 & (20) \\ & & 35 & 0.0720 & 1051.83 & (20) \\ & & 35 & 0.0795 & 1058.07 & (20) \\ & & & & \end{array}$

App vol App vol Density Density App Vol Square of Inconsistent Inconsistent

$\mathrm{m}^{3} / \mathrm{k}$

$\begin{array}{lll}0.000201 & 0.000206 \\ 0.000203 & 0.000207\end{array}$

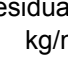

$\begin{array}{lll}0.0479 & -0.000006 & 0.002\end{array}$

$\begin{array}{llll}1003.84 & 0.0504-0.000006 & 0.003 \\ 1004.59 & 0.0334 & -0.000003 & 0.001\end{array}$

$\begin{array}{llll}1005.94 & 0.0405 & -0.000004 & 0.002\end{array}$

$\begin{array}{llll}1006.15 & 0.0626 & -0.000005 & 0.004\end{array}$

1007.13

1007.85

$0.0138-0.000001$

0.000

1007.99
1008.13

1009.45

1010.54

1011.54

1011.70
1012.98

1013.39

1014.92

1017.18

1019.65

1023.30
1023.87

1030.28

$0.1077-0.000008$

$0.0719-0.000005$

$0.0582-0.000004$

$0.0625-0.000004$

$0.0332-0.000002$

$0.0605-0.000003$

$0.0415-0.000002$

$0.0412-0.000002$

$0.0419-0.000002$

$\begin{array}{ll}-0.0095 & 0.000000\end{array}$

$0.0123 \quad 0.000000$

$0.0016 \quad 0.000000$

$\begin{array}{ll}0.0292 & 0.000001\end{array}$

$\begin{array}{ll}0.0036 & 0.000000 \\ -0.0907 & 0.000002\end{array}$

$\begin{array}{llll}1032.49 & -0.0477 & 0.000001\end{array}$

$\begin{array}{lll}1036.65 & -0.1115 & 0.000002\end{array}$

$\begin{array}{lll}1041.21 & -0.0931 & 0.000002\end{array}$

1043.25

1049.75

$\begin{array}{ll}-0.1657 & 0.000003\end{array}$

$-0.2085 \quad 0.000003$

$0.0055-0.000013$

$0.0111-0.000013$

999.49

1000.95

1004.71

$0.0128-0.000007$

$0.0341-0.000005$

$0.0581-0.000007$

$0.0479-0.000004$

$0.0484-0.000003$

007.61

1013.88

1026.55
1039.34

1045.96

1051.98

1058.21

1064.66

1070.48

1076.70
991.58

0.01160 .000000

$\begin{array}{ll}0.0634 & 0.000001\end{array}$

$\begin{array}{ll}-0.1184 & 0.000002\end{array}$

$\begin{array}{lll}-0.1450 & 0.000002\end{array}$

$\begin{array}{ll}-0.1340 & 0.000002\end{array}$

$-0.0652 \quad 0.000001$

$\begin{array}{ll}0.0293 & 0.000000\end{array}$

$0.0343 \quad 0.000000$

$\begin{array}{ll}0.0074 & -0.000017\end{array}$
0.012

0.005

0.003

0.004

0.001

0.001

0.004

0.002

0.002
0.002

0.000

0.000

0.000

0.001

0.000

0.008

0.002

0.012

0.009

0.027
0.043

0.000

0.000

0.000

0.001

0.003
0.002

0.002

0.002

0.000

0.004

0.016

0.014

0.021

0.018

0.001

0.001

0.000 delta

density

$\mathrm{kg} / \mathrm{m}^{3}$

6.846

9.165

13.533

1.0125

15.975

17.922

20.152

26.225

26.831

33.14

35.402

39.495

44.071

46.044

52.494

6.981

6.98

13.622

19.858

32.458

45.185

51.812

57.803

64.04

70.559

76.414

82.705
Molal Density of volume / water $/ \mathrm{kg} / \mathrm{m}^{3}$ $\mathrm{cm}^{3} / \mathrm{mole}$

997.0449 997.0449

$35.3 \quad 997.0449$

$35.46 \quad 997.0449$

$35.54 \quad 997.0449$ 997.0449

$36.06 \quad 997.0449$

997.0449

$35.41 \quad 997.0449$

$35.6 \quad 997.0449$

997.0449
997.0449

$36.25 \quad 997.0449$

36.01

997.0449

$36.35 \quad 997.0449$ 997.0449

$36.9 \quad 997.0449$

997.0449

$37.13 \quad 997.0449$

997.0449

997.0449

997.0449

997.0449

997.0449

997.0449

997.0449

997.0449

994.0319

994.0319

994.0319

994.0319

994.0319

994.0319

994.0319

994.0319

994.0319

994.0319

994.0319

994.0319

994.0319

994.0319

994.0319

994.0319

994.0319

1.00035

991.237 


\begin{tabular}{|c|c|c|c|c|}
\hline molality & molarity & $\mathrm{t} /{ }^{\circ} \mathrm{C}$ & ass frac & $\begin{array}{r}\text { Density } \\
\exp / \\
\mathrm{kg} / \mathrm{m}^{3}\end{array}$ \\
\hline & 0.00496 & 42.5 & 0.0009 & 991.92 \\
\hline & 0.00993 & 42.5 & 0.0017 & 992.60 \\
\hline & 0.03972 & 42.5 & 0.0069 & 996.70 \\
\hline & 0.07784 & 42.5 & 0.0135 & 1001.89 \\
\hline & 0.08936 & 42.5 & 0.0155 & 1003.46 \\
\hline 0.02002 & & 45 & 0.0035 & 992.96 \\
\hline 0.06143 & & 45 & 0.0106 & 998.56 \\
\hline 0.12751 & & 45 & 0.0217 & 1007.31 \\
\hline 0.18956 & & 45 & 0.0320 & 1015.40 \\
\hline 0.25584 & & 45 & 0.0427 & 1023.91 \\
\hline 0.32073 & & 45 & 0.0529 & 1032.16 \\
\hline 0.3855 & & 45 & 0.0630 & 1040.25 \\
\hline 0.45271 & & 45 & 0.0731 & 1048.57 \\
\hline 0.51582 & & 45 & 0.0825 & 1056.34 \\
\hline 0.58051 & & 45 & 0.0919 & 1064.12 \\
\hline 0.65002 & & 45 & 0.1018 & 1072.38 \\
\hline 0.01042 & & 48.75 & 0.0018 & 990.25 \\
\hline 0.03115 & & 48.75 & 0.0054 & 993.07 \\
\hline 0.10648 & & 48.75 & 0.0182 & 1003.11 \\
\hline 0.19911 & & 48.75 & 0.0335 & 1015.16 \\
\hline 0.29559 & & 48.75 & 0.0490 & 1027.08 \\
\hline 0.40209 & & 48.75 & 0.0655 & 1040.79 \\
\hline 0.001994 & & 50 & 0.0003 & 988.31 \\
\hline 0.003113 & & 50 & 0.0005 & 988.47 \\
\hline 0.004975 & & 50 & 0.0009 & 988.72 \\
\hline 0.007964 & & 50 & 0.0014 & 989.13 \\
\hline 0.012674 & & 50 & 0.0022 & 989.78 \\
\hline 0.018302 & & 50 & 0.0032 & 990.54 \\
\hline 0.027868 & & 50 & 0.0048 & 991.82 \\
\hline 0.035209 & & 50 & 0.0061 & 992.81 \\
\hline 0.04877 & & 50 & 0.0084 & 994.62 \\
\hline 0.067813 & & 50 & 0.0117 & 997.16 \\
\hline 0.096542 & & 50 & 0.0165 & 1000.97 \\
\hline 0.10892 & & 50 & 0.0186 & 1002.58 \\
\hline 0.13386 & & 50 & 0.0228 & 1005.87 \\
\hline 0.16999 & & 50 & 0.0288 & 1010.56 \\
\hline 0.20329 & & 50 & 0.0342 & 1014.88 \\
\hline 0.22523 & & 50 & 0.0378 & 1017.71 \\
\hline 0.25632 & & 50 & 0.0428 & 1021.64 \\
\hline 0.31119 & & 50 & 0.0514 & 1028.60 \\
\hline 0.3627 & & 50 & 0.0594 & 1035.01 \\
\hline 0.3688 & & 50 & 0.0604 & 1035.82 \\
\hline 0.52848 & & 50 & 0.0843 & 1055.32 \\
\hline 0.70706 & & 50 & 0.1097 & 1076.56 \\
\hline 0.05256 & & 55 & 0.0091 & 992.75 \\
\hline 0.09846 & & 55 & 0.0169 & 998.80 \\
\hline
\end{tabular}

App vol App vol Density Density App Vol Square of Inconsistent Inconsistent

$\mathrm{m}^{3} / \mathrm{kg} \quad \mathrm{m}^{3} / \mathrm{kg}$

0.0002110 .000216

0.0002110 .000217

$0.000213 \quad 0.000218$

$0.000216 \quad 0.000220$

$0.000217 \quad 0.000221$

$0.000206 \quad 0.000218$

$0.000221 \quad 0.000224$

$0.000227 \quad 0.000227$

$0.000231 \quad 0.000231$

$0.000235 \quad 0.000234$

$\begin{array}{ll}0.000238 & 0.000238 \\ 0.000241 & 0.000241\end{array}$

$0.000243 \quad 0.000245$

$\begin{array}{ll}0.000246 & 0.000248\end{array}$

$0.000249 \quad 0.000252$

$0.000082 \quad 0.000219$

$0.000168 \quad 0.000220$

$0.000208 \quad 0.000224$

$0.000222 \quad 0.000229$

$0.000238 \quad 0.000234$

$0.000237 \quad 0.000240$

$0.000198 \quad 0.000219$

$\begin{array}{lll}0.000199 & 0.000219\end{array}$

$\begin{array}{ll}0.000201 & 0.000219\end{array}$

$0.000203 \quad 0.000219$

$0.000205 \quad 0.000220$

$0.000207 \quad 0.000220$

$\begin{array}{ll}0.000213 & 0.000220 \\ 0.000214 & 0.000221\end{array}$

$0.000217 \quad 0.000221$

$\begin{array}{ll}0.000219 & 0.000222\end{array}$

0.0002210 .000224

$0.000224 \quad 0.000225$

0.0002250 .000226

$0.000228 \quad 0.000228$

$0.000230 \quad 0.000230$

0.0002310 .000231

0.0002340 .000232

$0.000236 \quad 0.000235$

0.0002390 .000238

$0.000239 \quad 0.000238$

$0.000247 \quad 0.000247$

$0.000253 \quad 0.000256$

$0.000220 \quad 0.000223$

$0.000225 \quad 0.000225$

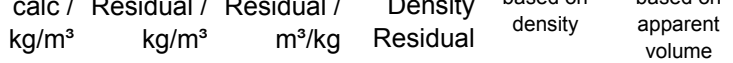

$991.92 \quad 0.0050-0.000006$

$\begin{array}{llll}9.66 & 0.0381 & -0.000006 & 0.001\end{array}$

$\begin{array}{llll}001.84 & 0.0552 & -0.000004 & 0.003\end{array}$

$\begin{array}{llll}003.40 & 0.0622 & -0.000004 & 0.004\end{array}$

$\begin{array}{llll}992.92 & 0.0422 & -0.000012 & 0.002 \\ 998.48 & 0.0767 & -0.000007 & 0.006\end{array}$

$\begin{array}{lll}907.25 & 0.0584 & -0.000003\end{array}$

$\begin{array}{llll}015.37 & 0.0245 & -0.000001 & 0.001\end{array}$

$\begin{array}{llll}023.93 & -0.0138 & 0.000000 & 0.000\end{array}$

$\begin{array}{llll}1032.17 & -0.0129 & 0.000000 & 0.000\end{array}$

$\begin{array}{llll}1040.29 & -0.0320 & 0.000000 & 0.001\end{array}$

$\begin{array}{llll}048.57 & -0.0024 & 0.000000 & 0.000\end{array}$

$\begin{array}{llll}1056.23 & 0.1038 & -0.000001 & 0.011\end{array}$

$\begin{array}{llll}1063.96 & 0.1553 & -0.000001 & 0.024\end{array}$

$\begin{array}{llll}072.13 & 0.2539 & -0.000002 & 0.064\end{array}$

$\begin{array}{llll}990.00 & 0.2430 & -0.000137 & 0.059\end{array}$

$\begin{array}{llll}992.79 & 0.2774 & -0.000052 & 0.077\end{array}$

$1002.82 \quad 0.2927-0.000016$

$\begin{array}{llll}1014.92 & 0.2394 & -0.000007 & 0.057\end{array}$

$1027.27-0.1945 \quad 0.000004=0.038$

$\begin{array}{llll}1040.59 & 0.2054 & -0.000003 & 0.042\end{array}$

$\begin{array}{lllll}988.31 & 0.0071 & -0.000021 & 0.000\end{array}$

$\begin{array}{llll}988.46 & 0.0105 & -0.000020 & 0.000\end{array}$

$\begin{array}{llll}988.71 & 0.0151 & -0.000018 & 0.000\end{array}$

$\begin{array}{llll}989.11 & 0.0216 & -0.000016 & 0.000\end{array}$

$\begin{array}{llll}989.74 & 0.0309 & -0.000014 & 0.001\end{array}$

$\begin{array}{llll}990.50 & 0.0387 & -0.000012 & 0.001\end{array}$

$\begin{array}{lllll}991.79 & 0.0368 & -0.000008 & 0.001\end{array}$

$\begin{array}{llll}992.77 & 0.0401 & -0.000007 & 0.002\end{array}$

$\begin{array}{llll}994.58 & 0.0333 & -0.000004 & 0.001\end{array}$

$\begin{array}{llll}997.12 & 0.0404 & -0.000003 & 0.002\end{array}$

$\begin{array}{llll}1000.93 & 0.0420 & -0.000003 & 0.002\end{array}$

$\begin{array}{llll}002.57 & 0.0158 & -0.000001 & 0.000\end{array}$

$\begin{array}{lllll}1005.85 & 0.0234 & -0.000001 & 0.001\end{array}$

$\begin{array}{llll}010.57 & -0.0092 & 0.000000 & 0.000\end{array}$

$\begin{array}{llll}1014.88 & 0.0022 & 0.000000 & 0.000\end{array}$

$\begin{array}{llll}1017.71 & -0.0012 & 0.000000 & 0.000\end{array}$

$\begin{array}{llll}1021.69 & -0.0493 & 0.000001 & 0.002\end{array}$

$\begin{array}{lllll}1028.65 & -0.0499 & 0.000001 & 0.002\end{array}$

$\begin{array}{llll}1035.10 & -0.0927 & 0.000001 & 0.009\end{array}$

$\begin{array}{lllll}1035.86 & -0.0415 & 0.000001 & 0.002\end{array}$

$\begin{array}{llll}055.35 & -0.0300 & 0.000000 & 0.001\end{array}$

$\begin{array}{llll}992.73 & 0.0273 & -0.000003 & 0.001\end{array}$

$\begin{array}{lrrr}998.80 & -0.0011 & 0.000000 & 0.000\end{array}$ delta

density

$\mathrm{kg} / \mathrm{m}^{3}$

relative

density volume / water $/ \mathrm{kg} / \mathrm{m}^{3}$ $\mathrm{cm}^{3} / \mathrm{mole}$

1.00069

1.00138

1.01075

1.01233

8.343

17.096

25.184

25.184

33.698

41.948

50.04

58.356

66.122

73.903

82.169

0.276

0.4304

0.6861

1.0954

1.7388

2.5036

3.7863

4.7737

6.5807

9.126

12.9375

14.5455

17.8327

22.5195

26.8477

29.671

33.6047

40.5636

46.9749

47.7854

67.2859

88.5197

7.059

13.108
991.237

991.237

991.237

991.237

991.237

990.2132

990.2132

990.2132

990.2132

990.2132

990.2132

990.2132

990.2132

990.2132

990.2132

988.5963

988.5963

988.5963

988.5963

988.5963

988.5963

988.0363

988.0363

988.0363

988.0363

988.0363

988.0363

988.0363

988.0363

988.0363

988.0363

988.0363

988.0363

988.0363

988.0363

988.0363

988.0363

988.0363

988.0363

988.0363

988.0363

988.0363

988.0363

985.6952

985.6952 


\begin{tabular}{|c|c|c|c|c|c|}
\hline molality & molarity & \multicolumn{2}{|c|}{$\mathrm{t} /{ }^{\circ} \mathrm{C}$ mass frac } & $\begin{array}{r}\text { Density } \\
\exp / \\
\mathrm{kg} / \mathrm{m}^{3}\end{array}$ & Ref \\
\hline 0.14613 & & 55 & 0.0248 & 1005.00 & (20) \\
\hline 0.25008 & & 55 & 0.0418 & 1018.27 & (20) \\
\hline 0.34613 & & 55 & 0.0569 & 1030.29 & (20) \\
\hline 0.39353 & & 55 & 0.0642 & 1036.14 & (20) \\
\hline 0.44485 & & 55 & 0.0719 & 1042.40 & (20) \\
\hline 0.49648 & & 55 & 0.0796 & 1048.70 & (20) \\
\hline 0.54761 & & 55 & 0.0871 & 1054.81 & (20) \\
\hline 0.65016 & & 55 & 0.1018 & 1066.95 & (20) \\
\hline 0.06426 & & 65 & 0.0111 & 989.18 & (20) \\
\hline 0.19267 & & 65 & 0.0325 & 1005.91 & (20) \\
\hline 0.24794 & & 65 & 0.0414 & 1012.94 & (20) \\
\hline 0.32068 & & 65 & 0.0529 & 1022.06 & (20) \\
\hline 0.38598 & & 65 & 0.0630 & 1030.16 & (20) \\
\hline 0.45192 & & 65 & 0.0730 & 1038.23 & (20) \\
\hline 0.51723 & & 65 & 0.0827 & 1046.12 & (20) \\
\hline 0.58483 & & 65 & 0.0925 & 1054.21 & (20) \\
\hline 0.64975 & & 65 & 0.1017 & 1061.89 & (20) \\
\hline 0.01042 & & 73.77 & 0.0018 & 977.17 & (114) \\
\hline 0.03115 & & 73.77 & 0.0054 & 979.94 & (114) \\
\hline 0.10648 & & 73.77 & 0.0182 & 989.91 & (114) \\
\hline 0.19911 & & 73.77 & 0.0335 & 1001.81 & (114) \\
\hline 0.29559 & & 73.77 & 0.0490 & 1013.46 & (114) \\
\hline 0.40209 & & 73.77 & 0.0655 & 1027.22 & (114) \\
\hline 0.03044 & & 75 & 0.0053 & 978.96 & (20) \\
\hline 0.0651 & & 75 & 0.0112 & 983.56 & (20) \\
\hline 0.0662 & & 75 & 0.0114 & 983.72 & (20) \\
\hline 0.12651 & & 75 & 0.0216 & 991.61 & (20) \\
\hline 0.12666 & & 75 & 0.0216 & 991.63 & (20) \\
\hline 0.25786 & & 75 & 0.0430 & 1008.40 & (20) \\
\hline 0.32113 & & 75 & 0.0530 & 1016.32 & (20) \\
\hline 0.32144 & & 75 & 0.0530 & 1016.40 & (20) \\
\hline 0.3866 & & 75 & 0.0631 & 1024.46 & (20) \\
\hline 0.3875 & & 75 & 0.0633 & 1024.55 & (20) \\
\hline 0.45013 & & 75 & 0.0727 & 1032.25 & (20) \\
\hline 0.51624 & & 75 & 0.0825 & 1040.19 & (20) \\
\hline 0.02788 & & 85 & 0.0048 & 972.39 & (20) \\
\hline 0.03223 & & 85 & 0.0056 & 972.98 & (20) \\
\hline 0.06563 & & 85 & 0.0113 & 977.41 & (20) \\
\hline 0.13054 & & 85 & 0.0222 & 985.89 & (20) \\
\hline 0.19073 & & 85 & 0.0322 & 993.61 & (20) \\
\hline 0.25913 & & 85 & 0.0432 & 1002.34 & (20) \\
\hline 0.38611 & & 85 & 0.0630 & 1018.08 & (20) \\
\hline 0.44989 & & 85 & 0.0727 & 1025.87 & (20) \\
\hline 0.51712 & & 85 & 0.0827 & 1034.02 & (20) \\
\hline 0.58604 & & 85 & 0.0927 & 1042.29 & (20) \\
\hline 0.64987 & & 85 & 0.1017 & 1049.76 & \\
\hline
\end{tabular}

App vol App vol Density Density App Vol Square of Inconsistent Inconsistent exp / Calc/$$
\text { exp/ }
$$$$
\begin{array}{rr}
\mathrm{m}^{3} / \mathrm{kg} & \mathrm{m}^{3} / \mathrm{kg} \\
0.000230 & 0.000228 \\
0.000237 & 0.000233
\end{array}
$$

$\begin{array}{ll}0.000237 & 0.000233\end{array}$

$\begin{array}{ll}0.000243 & 0.000238 \\ 0.000245 & 0.000240\end{array}$

$\begin{array}{ll}0.000247 & 0.000243\end{array}$

$\begin{array}{ll}0.000249 & 0.000246 \\ 0.000252 & 0.000248\end{array}$

$0.000255 \quad 0.000254$

$0.000217 \quad 0.000224$

$0.000229 \quad 0.000230$

$0.000233 \quad 0.000233$

$\begin{array}{ll}0.000237 & 0.000237 \\ 0.000241 & 0.000240\end{array}$

0.0002410 .000240

$\begin{array}{ll}0.000244 & 0.000243 \\ 0.000247 & 0.000247\end{array}$

$\begin{array}{ll}0.000249 & 0.000250\end{array}$

$0.000252 \quad 0.000253$

$0.000104 \quad 0.000220$

$0.000180 \quad 0.000221$

$0.000211 \quad 0.000225$

$0.000225 \quad 0.000230$

$0.000243 \quad 0.000234$

$0.000238 \quad 0.000240$

$\begin{array}{ll}0.000210 & 0.000221 \\ 0.000216 & 0.000223\end{array}$

$0.000215 \quad 0.000223$

$0.000222 \quad 0.000226$

0.0002220 .000226

$\begin{array}{ll}0.000232 & 0.000232 \\ 0.000236 & 0.000235\end{array}$

$0.000236 \quad 0.000235$

$0.000235 \quad 0.000235$

0.0002390 .000239

$\begin{array}{ll}0.000239 & 0.000239\end{array}$

0.0002420 .000242

$\begin{array}{ll}0.000245 & 0.000245 \\ 0.000205 & 0.000219\end{array}$

$0.000204 \quad 0.000219$

0.0002110 .000220

$0.000220 \quad 0.000223$

$0.000225 \quad 0.000226$

$0.000228 \quad 0.000229$

$0.000237 \quad 0.000235$

$0.000240 \quad 0.000238$

$\begin{array}{ll}0.000243 & 0.000242\end{array}$

$0.000245 \quad 0.000245$

$0.000248 \quad 0.000248$

calc / Residual / Residual / Density based on based on

$\mathrm{kg} / \mathrm{m}^{3} \quad \mathrm{~kg} / \mathrm{m}^{3} \quad \mathrm{~m}^{3} / \mathrm{kg}$ Residual density $\begin{gathered}\text { apparent } \\ \text { volume }\end{gathered}$

$\begin{array}{llll}1005.05 & -0.0582 & 0.000002 & 0.003\end{array}$

$\begin{array}{llll}018.46 & -0.1870 & 0.000004 & 0.035\end{array}$

$\begin{array}{llll}1030.57 & -0.2818 & 0.000005 & 0.079\end{array}$

$\begin{array}{llll}1036.45 & -0.3095 & 0.000004 & 0.096\end{array}$

$\begin{array}{llll}1042.74 & -0.3428 & 0.000004 & 0.118\end{array}$

$\begin{array}{llll}1049.00 & -0.3016 & 0.000003 & 0.091 \\ 1055.12 & -0.3100 & 0.000003 & 0.096\end{array}$

$\begin{array}{llll}1057.16 & -0.3100 & 0.000003\end{array}$

$\begin{array}{llll}989.10 & 0.0734 & -0.000007 & 0.005\end{array}$

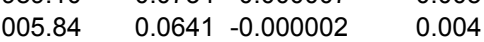

$\begin{array}{llll}012.91 & 0.0317 & -0.000001 & 0.001\end{array}$

$\begin{array}{llll}1022.08 & -0.0203 & 0.000000 & 0.000\end{array}$

$\begin{array}{llll}030.19 & -0.0278 & 0.000000 & 0.001\end{array}$

$\begin{array}{llll}1038.26 & -0.0290 & 0.000000 & 0.001\end{array}$

$\begin{array}{llll}1046.12 & -0.0061 & 0.000000 & 0.000\end{array}$

$\begin{array}{llll}1054.14 & 0.0671 & -0.000001 & 0.005\end{array}$

$\begin{array}{llll}061.72 & 0.1713 & -0.000001 & 0.029\end{array}$

$\begin{array}{llll}976.97 & 0.2013 & -0.000116 & 0.041\end{array}$

$\begin{array}{llll}979.73 & 0.2136 & -0.000041 & 0.046\end{array}$

$\begin{array}{lll}9 & 0.65-0.2609-0.000015 & 0.068\end{array}$

$\begin{array}{rrrr}1001.64 & 0.1709 & -0.000005 & 0.029 \\ 1013.89 & -0.4297 & 0.000009 & 0.185\end{array}$

$\begin{array}{llll}027.11 & 0.1039 & -0.000002 & 0.011\end{array}$

$\begin{array}{llll}978.90 & 0.0587 & -0.000012 & 0.003\end{array}$

$\begin{array}{lllll}983.48 & 0.0774 & -0.000007 & 0.006\end{array}$

$\begin{array}{lllll}983.63 & 0.0904 & -0.000008 & 0.008\end{array}$

$\begin{array}{llll}991.53 & 0.0798 & -0.000004 & 0.006\end{array}$

$\begin{array}{llll}991.55 & 0.0843 & -0.000004 & 0.007\end{array}$

$\begin{array}{llll}008.39 & 0.0075 & 0.000000 & 0.000\end{array}$

$\begin{array}{llll}016.35 & -0.0314 & 0.000001 & 0.001\end{array}$

$\begin{array}{llll}1016.39 & 0.0068 & 0.000000 & 0.000\end{array}$

$\begin{array}{llll}1024.47 & -0.0135 & 0.000000 & 0.000\end{array}$

$\begin{array}{llll}1024.58 & -0.0344 & 0.000001 & 0.001\end{array}$

$\begin{array}{llll}032.24 & 0.0118 & 0.000000 & 0.000\end{array}$

$\begin{array}{llll}1040.21 & -0.0271 & 0.000000 & 0.001\end{array}$

$\begin{array}{llll}972.33 & 0.0621 & -0.000014 & 0.004\end{array}$

$\begin{array}{llll}972.90 & 0.0806 & -0.000015 & 0.007\end{array}$

$\begin{array}{llll}977.31 & 0.0996 & -0.000009 & 0.010\end{array}$

$\begin{array}{llll}985.80 & 0.0845 & -0.000004 & 0.007\end{array}$

$\begin{array}{llll}993.58 & 0.0331 & -0.000001 & 0.001\end{array}$

$\begin{array}{lllll}1002.30 & 0.0443 & -0.000001 & 0.002\end{array}$

$\begin{array}{lllll}1018.17 & -0.0907 & 0.000001 & 0.008\end{array}$

$\begin{array}{llll}1025.99 & -0.1185 & 0.000002 & 0.014\end{array}$

$\begin{array}{llll}034.11 & -0.0868 & 0.000001 & 0.008\end{array}$

$\begin{array}{llll}042.31 & -0.0172 & 0.000000 & 0.000\end{array}$

$\begin{array}{llll}1049.79 & -0.0303 & 0.000000 & 0.001\end{array}$ delta

density

$\mathrm{kg} / \mathrm{m}^{3}$

19.3

32.575

44.592

50.444

56.704
63

63

81.253

81.253

25.354

32.388

41.507

49.607

57.672

65.564

73.656

81.34

4.11

8.712

8.712
8.87

16.757

16.781

33.55

41.47

41.547

49.61

49.7

57.406

65.337

3.768

4.363

8.792

17.266

24.99

33.724

49.461

57.247

65.4

73.672

81.144 relative Molal Density of volume / water $/ \mathrm{kg} / \mathrm{m}^{3}$ $\mathrm{cm}^{3} / \mathrm{mole}$

985.6952

985.6952

985.6952

985.6952

985.6952

985.6952

985.6952

985.6952

980.5548

980.5548

980.5548

980.5548

980.5548

980.5548

980.5548

980.5548

980.5548

975.5797

975.5797

975.5797

975.5797

975.5797

975.5797

974.849

974.849

974.849

974.849

974.849

974.849

974.849

974.849

974.849

974.849

974.849

974.849

968.6203

968.6203

968.6203

968.6203

968.6203

968.6203

968.6203

968.6203

968.6203

968.6203

968.6203 


\subsection{5}

0.01295

0.02577

0.03393

0.19078

0.25859

0.38658

0.51539

0.58329

0.65016

0.01042

0.03115

0.10648

0.19911

0.29559

0.40209

Curve fit for the model App vol $=\left(w+c 2+c 3^{*} t{ }^{\circ} \mathrm{C}\right) /\left(\mathrm{c} 0^{*} \mathrm{w}+\mathrm{c} 1\right) / \mathrm{e}^{\wedge}\left(0.000001^{\star}\left(\mathrm{t}^{\circ} \mathrm{C}+\mathrm{c} 4\right)^{\wedge} 2\right)$

$\begin{array}{lr}\text { Curve fit for the model App vol= } & -2.67 \mathrm{E}-05 \\ \text { c0 } & 3.04 \mathrm{E}-05 \\ \text { c1 } & 0.971179 \\ \text { c2 } & 0.019816 \\ \text { c3 } & 4366.045 \\ \text { c4 } & 0 \\ \text { Min T } & 98.67 \\ \text { Max T } & 0.000347 \\ \text { Min w } & 0.109699 \\ \text { Max w } & 0.037501 \\ \text { Avg dens res } & 0.155345 \\ \text { Std dens res } & -0.009149 \\ \text { Avg app vol res *10^3 } & 0.019272 \\ \text { Std app vol res *10^3 } & 230 \\ \text { No of points in corr } & 2 \\ \text { No of inconsistent poir } & 7\end{array}$

No of inconsistent poir

Inconsistent data not used (Residual greater than average $+/-4$ standard deviations)

$\begin{array}{lllllll}0.24662 & 15 & 0.0412 & 1045.76 & (20) & -0.000083 & 0.000206\end{array}$

0.51831

0.01015

0.01015

0.14377

0.56016

0.64975

$\mathrm{t} /{ }^{\circ} \mathrm{C}$ mass frac Den

$\exp /$

$\mathrm{kg} / \mathrm{m}^{3}$

$95 \quad 0.0023$

$95 \quad 0.0045$

$95 \quad 0.0059$

0.0431

0.0631

0.0824

0.0923
0.1018
0.0018

$\begin{array}{rr}95 & 0.1018 \\ 98.67 & 0.0018\end{array}$

$\begin{array}{ll}98.67 & 0.0054 \\ 98.67 & 0.0182\end{array}$

$98.67 \quad 0.0335$

$98.67 \quad 0.0490$

98.67

\subsection{6}

965.39

966.48

979.02
986.93

995.53

1011.43

1027.12

1035.28
1043.30

1043.30
960.99
$95 \quad 0.0322$

0.0655

$963.80 \quad(114)$
$973.92 \quad(114)$

$985.88 \quad(114)$

$997.65 \quad(114)$
Ref

App vol App vol

$\mathrm{m}^{3} / \mathrm{kg} \quad \mathrm{m}^{3} / \mathrm{kg}$

$0.000201 \quad 0.000215$

$\begin{array}{ll}0.000214 & 0.000219 \\ 0.000220 & 0.000222\end{array}$

$0.000225 \quad 0.000225$

$0.000233 \quad 0.000231$

$\begin{array}{lll}0.000239 & 0.000237\end{array}$

$\begin{array}{ll}0.000241 & 0.000240\end{array}$

$0.000243 \quad 0.000243$

$0.000043 \quad 0.000212$

$\begin{array}{ll}0.000145 & 0.000213 \\ 0.000184 & 0.000217\end{array}$

$0.000205 \quad 0.000221$

$0.000225 \quad 0.000225$

0.0002220 .000230
Average Res
Std dev Res
Avg - 4st
Avg + 4std

$\mathrm{kg} / \mathrm{m}^{3}$

963.62

966.41

978.90
986.87

995.52
1011.55

1027.27

1043.27

960.70
963.45

973.36

985.36

7.63

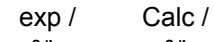

$\begin{array}{lll}\text { (20) } & 0.000197 & 0.000214\end{array}$

.$$
\begin{array}{r}
0.03 \\
0.15 \\
-0.58
\end{array}
$$

$\begin{array}{rr}0.0375 & -0.000009 \\ 0.1553 & 0.000019\end{array}$

dual / Residual /

$\mathrm{kg} / \mathrm{m}^{3} \quad \mathrm{~m}^{3} / \mathrm{kg}$ Residual density $\begin{gathered}\text { apparent } \\ \text { volume }\end{gathered}$

$\begin{array}{lll}0.0362 & -0.000017 & 0.001\end{array}$

$0.0667-0.000016$

0.004

0.006

$0.1164-0.000006$

$0.0669-0.000002$

0.014
0.004

0.000

$\begin{array}{ll}0.1253 & 0.000002\end{array}$

0.016

0.025

$0.0234 \quad 0.000000$

0.013

0.001

0.081
0.117

$0.3421-0.000068$

0.316

$0.5625-0.000033$

0.274

0.001

0.304

5.850 Sum chi sqr

0.65890 .000068 $\begin{array}{lll}35 & 0.0828 & 1059.60\end{array}$

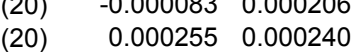

1032.89

$12.8677-0.000289$

$\begin{array}{ll}-0.001366 & 0.000218\end{array}$

1060.95

$-1.35390 .000015$

165.577

991.59

$2.7568-0.001584$

1.833
7.600

$\begin{array}{ll}0.0050 & 988.38\end{array}$

$\begin{array}{lll}(20) & -0.000579 & 0.000222\end{array}$

984.44

$3.9377-0.000802$

$1045.44-10.6008-0.0$

$\begin{array}{lll}75 & 0.0889 & 1056.04\end{array}$

$\begin{array}{lll}\text { (20) } & -0.000040 & 0.000227\end{array}$

1045.44

$10.6008-0.000108$

15.505

42.099

$\begin{array}{llll}(20) & 0.000241 & 0.000251 & 1055.95\end{array}$

$1.1172-0.000010$

1.248

delta

density

$\mathrm{kg} / \mathrm{m}^{3}$

1.759

3.491

4.581

17.115

25.033

33.631

49.529

65.215

73.375

81.395

$\begin{array}{lll}75 & 0.1017 & 1057.06\end{array}$ relative Molal Density of density volume / water $/ \mathrm{kg} / \mathrm{m}^{3}$ $\mathrm{cm}^{3} / \mathrm{mole}$

961.9004

961.9004

961.9004

961.9004

961.9004

961.9004

961.9004

961.9004

961.9004

961.9004

959.3157

959.3157

959.3157

959.3157

959.3157
999.0996

990.2132

980.5548

974.849

974.849

25.408

81.194

82.214 
Density of aqueous solutions of $\mathrm{LiCl}$

References (28) Gates, J. A.; Wood, R. H. Densities of Aqueous Solutions of $\mathrm{NaCl}, \mathrm{MgCl}_{2}, \mathrm{KCl}, \mathrm{NaBr}, \mathrm{LiCl}$, and $\mathrm{CaCl}_{2}$ from 0.05 to 5.0 mol kg ${ }^{-1}$ and 0.1013 to $40 \mathrm{MPa}$ at 298.15K. J. Chem. Eng. Data 1985, 30, 44-49.

(45) Isono, T. Densities, Viscosities, and Electrolytic Conductivity of Concentrated Aqueous Electrolyte Solutions at Several Temperatures. Alkaline-Earth Chlorides, $\mathrm{LaCl}_{3}, \mathrm{Na}_{2} \mathrm{SO}_{4}, \mathrm{NaNO}_{3}, \mathrm{NaBr}, \mathrm{KNO}_{3}, \mathrm{KBr}$, and $\mathrm{Cd}\left(\mathrm{NO}_{3}\right)_{2}$. J. Chem. Eng. Data 1984, 29 , 45-52.

(62) Lengyel, S.; Tamás, J; Giber, J.; Holderith, J. Study of Viscosity of Aqueous Alkali Halide Solutions. Acta Chim. Acad. Sci. Hung. 1964, $40,125-143$.

(73) Millero, F. J.; Ward, G. K.; Chetirkin, P. V. Relative Sound Velocities of Sea Salts at $25^{\circ}$ C. J. Acoust. Soc. Am. 1977, $61,1492-1498$.

(80) Out, D. J. P.; Los, J. M. Viscosity of Aqueous Solutions of Univalent Electrolytes from 5 to $95^{\circ} \mathrm{C}$. J. Sol. Chem. $1980,9,19-35$.

(130) Vaslow, F. The Apparent Molal Volumes of the Lithium and Sodium Halides. Critical Type Transitions in Aqueous Solution. J. Phys. Chem. 1969, 73, 3745-3750.

(133) Wimby, J. M.; Berntsson, T. S. Viscosity and Density of Aqueous Solutions of $\mathrm{LiBr}, \mathrm{LiCl}, \mathrm{ZnBr}_{2}, \mathrm{CaCl}_{2}$, and $\mathrm{LiNO}_{3}$. 1. Single Salt Solutions. J. Chem. Eng. Data 1994, 39, 68-72.

\begin{tabular}{|c|c|c|c|c|c|c|c|c|c|c|c|c|c|c|c|c|}
\hline molality & molarity & t $/{ }^{\circ} \mathrm{C}$ & ass frac & $\begin{array}{r}\text { Density } \\
\exp / \\
\mathrm{kg} / \mathrm{m}^{3}\end{array}$ & Ref & $\begin{array}{r}\text { App vol } \\
\exp / \\
\mathrm{m}^{3} / \mathrm{kg}\end{array}$ & $\begin{array}{r}\text { App vol } \\
\text { Calc / } \\
\mathrm{m}^{3} / \mathrm{kg}\end{array}$ & $\begin{array}{r}\text { Density } \\
\text { calc / } \\
\mathrm{kg} / \mathrm{m}^{3}\end{array}$ & $\begin{array}{r}\text { Density } \\
\text { Residual / } \\
\mathrm{kg} / \mathrm{m}^{3}\end{array}$ & $\begin{array}{r}\text { App Vol } \\
\text { Residual / } \\
\mathrm{m}^{3} / \mathrm{kg}\end{array}$ & $\begin{array}{r}\text { Square of } \\
\text { Density } \\
\text { Residual }\end{array}$ & $\begin{array}{l}\text { Inconsistent } \\
\text { based on } \\
\text { density }\end{array}$ & $\begin{array}{l}\text { Inconsistent } \\
\text { based on } \\
\text { apparent } \\
\text { volume }\end{array}$ & $\begin{array}{r}\text { Apparent } \\
\text { molar volume } \\
/ \mathrm{cm}^{3} / \mathrm{mol}\end{array}$ & $\begin{array}{l}\text { Delta density } \\
\mathrm{kg} / \mathrm{m}^{3}\end{array}$ & $\begin{array}{r}\text { Density of } \\
\text { water } / \mathrm{kg} / \mathrm{m}^{3}\end{array}$ \\
\hline 0.05 & & 5 & 0.0021 & 1001.3 & (130) & 0.000387 & 0.000463 & 1001.10 & 0.1605 & -0.000076 & 0.026 & & & 16.42 & & 999.9638 \\
\hline 0.1 & & 5 & 0.0042 & 1002.5 & (130) & 0.000391 & 0.000463 & 1002.24 & 0.3069 & -0.000072 & 0.094 & & & 16.57 & & 999.9638 \\
\hline 0.1189 & & 5 & 0.0050 & 1003.1 & $(80)$ & 0.000385 & 0.000463 & 1002.66 & 0.3970 & -0.000079 & 0.158 & & & & & 999.9638 \\
\hline 0.2 & & 5 & 0.0084 & 1005.1 & (130) & 0.000396 & 0.000464 & 1004.49 & 0.5770 & -0.000068 & 0.333 & & & 16.77 & & 999.9638 \\
\hline 0.2383 & & 5 & 0.0100 & 1006.1 & $(80)$ & 0.000393 & 0.000464 & 1005.36 & 0.7133 & -0.000071 & 0.509 & & & & & 999.9638 \\
\hline 0.3 & & 5 & 0.0126 & 1007.6 & (130) & 0.000399 & 0.000464 & 1006.74 & 0.8221 & -0.000065 & 0.676 & & & 16.93 & & 999.9638 \\
\hline 0.3581 & & 5 & 0.0150 & 1009.1 & $(80)$ & 0.000398 & 0.000464 & 1008.04 & 1.0071 & -0.000066 & 1.014 & & & & & 999.9638 \\
\hline 0.4 & & 5 & 0.0167 & 1010.0 & (130) & 0.000402 & 0.000464 & 1008.98 & 1.0502 & -0.000062 & 1.103 & & & 17.06 & & 999.9638 \\
\hline 0.4786 & & 5 & 0.0199 & 1012.0 & (80) & 0.000402 & 0.000464 & 1010.73 & 1.2620 & -0.000062 & 1.593 & & & & & 999.9638 \\
\hline 0.4786 & & 5 & 0.0199 & 1012.0 & (80) & 0.000402 & 0.000464 & 1010.73 & 1.2620 & -0.000062 & 1.593 & & & & & 999.9638 \\
\hline 0.5 & & 5 & 0.0208 & 1012.5 & (130) & 0.000405 & 0.000465 & 1011.20 & 1.2601 & -0.000059 & 1.588 & & & 17.18 & & 999.9638 \\
\hline 0.6 & & 5 & 0.0248 & 1014.9 & (130) & 0.000408 & 0.000465 & 1013.42 & 1.4549 & -0.000057 & 2.117 & & & 17.29 & & 999.9638 \\
\hline 0.7 & & 5 & 0.0288 & 1017.3 & (130) & 0.000410 & 0.000465 & 1015.62 & 1.6373 & -0.000055 & 2.681 & & & 17.39 & & 999.9638 \\
\hline 0.721 & & 5 & 0.0297 & 1017.8 & (80) & 0.000409 & 0.000465 & 1016.08 & 1.7303 & -0.000056 & 2.994 & & & & & 999.9638 \\
\hline 0.8 & & 5 & 0.0328 & 1019.6 & (130) & 0.000413 & 0.000466 & 1017.81 & 1.8023 & -0.000053 & 3.248 & & & 17.49 & & 999.9638 \\
\hline 0.9 & & 5 & 0.0368 & 1021.9 & (130) & 0.000415 & 0.000466 & 1019.99 & 1.9588 & -0.000051 & 3.837 & & & 17.58 & & 999.9638 \\
\hline 1 & & 5 & 0.0407 & 1024.3 & (130) & 0.000417 & 0.000466 & 1022.16 & 2.0998 & -0.000049 & 4.409 & & & 17.67 & & 999.9638 \\
\hline 1.2 & & 5 & 0.0484 & 1028.8 & (130) & 0.000421 & 0.000467 & 1026.46 & 2.3591 & -0.000046 & 5.565 & & & 17.83 & & 999.9638 \\
\hline 1.2128 & & 5 & 0.0489 & 1029.2 & $(80)$ & 0.000420 & 0.000467 & 1026.74 & 2.4421 & -0.000047 & 5.964 & & & & & 999.9638 \\
\hline 1.4 & & 5 & 0.0560 & 1033.3 & (130) & 0.000424 & 0.000467 & 1030.73 & 2.5634 & -0.000043 & 6.571 & & & 17.99 & & 999.9638 \\
\hline 1.6 & & 5 & 0.0635 & 1037.7 & (130) & 0.000428 & 0.000468 & 1034.95 & 2.7446 & -0.000040 & 7.533 & & & 18.13 & & 999.9638 \\
\hline 1.8 & & 5 & 0.0709 & 1042.0 & (130) & 0.000431 & 0.000468 & 1039.13 & 2.8962 & -0.000038 & 8.388 & & & 18.26 & & 999.9638 \\
\hline 2 & & 5 & 0.0782 & 1046.3 & (130) & 0.000434 & 0.000469 & 1043.26 & 3.0038 & -0.000035 & 9.023 & & & 18.39 & & 999.9638 \\
\hline 2.5 & & 5 & 0.0958 & 1056.6 & (130) & 0.000441 & 0.000470 & 1053.44 & 3.1662 & -0.000030 & 10.025 & & & 18.68 & & 999.9638 \\
\hline 3 & & 5 & 0.1128 & 1066.5 & (130) & 0.000447 & 0.000472 & 1063.37 & 3.1728 & -0.000025 & 10.067 & & & 18.94 & & 999.9638 \\
\hline 3.5 & & 5 & 0.1292 & 1076.2 & (130) & 0.000452 & 0.000473 & 1073.07 & 3.1362 & -0.000021 & 9.836 & & & 19.15 & & 999.9638 \\
\hline 0.05 & & 15 & 0.0021 & 1000.3 & (45) & 0.000414 & 0.000461 & 1000.24 & 0.0993 & -0.000047 & 0.010 & & & & & 999.0996 \\
\hline 0.1 & & 15 & 0.0042 & 1001.5 & (45) & 0.000423 & 0.000461 & 1001.38 & 0.1612 & -0.000038 & 0.026 & & & & & 999.0996 \\
\hline 0.1189 & & 15 & 0.0050 & 1002.1 & (80) & 0.000399 & 0.000461 & 1001.81 & 0.3118 & -0.000062 & 0.097 & & & & & 999.0996 \\
\hline 0.2383 & & 15 & 0.0100 & 1005.1 & (80) & 0.000407 & 0.000462 & 1004.51 & 0.5494 & -0.000054 & 0.302 & & & & & 999.0996 \\
\hline 0.3581 & & 15 & 0.0150 & 1008.0 & (80) & 0.000412 & 0.000462 & 1007.20 & 0.7653 & -0.000050 & 0.586 & & & & & 999.0996 \\
\hline
\end{tabular}


molality molarity

$\mathbf{t} /{ }^{\circ} \mathrm{C}$ mass frac Density

$\exp /$

$\mathrm{kg} / \mathrm{m}^{3}$

0.4786
0.4786
0.5
0.5
0.721
1
1.009
1.2128
2
2.5925
3
4
4.1814
5.7128
6
7.2417
8
8.8268
9.863
10
10.913
11.963
12.923
14.067
15.063
15.827
17.249
17.906
18.729
19.584

19.584

$\begin{array}{rrrrr} & 17 & 0.4539 & 1292.6 & (133) \\ & 18.84 & 0.3591 & 1221.7 & (133) \\ & 18.99 & 0.3932 & 1247.6 & (133) \\ 19.21 & 0.3001 & 1179.8 & (133) \\ & 19.29 & 0.1003 & 1056.8 & (133) \\ & 19.33 & 0.2022 & 1116.6 & (133) \\ 0.05 & 19.55 & 0.4156 & 1264.1 & (133) \\ 0.1 & 20 & 0.0021 & 999.4 & (45) \\ 0.5 & 20 & 0.0042 & 1000.7 & (45) \\ 0.5 & 20 & 0.0208 & 1010.3 & (45) \\ 1 & 20 & 0.0208 & 1010.3 & (45) \\ 1.009 & 20 & 0.0407 & 1021.4 & (45) \\ 2 & 20 & 0.0410 & 1022.1 & (62) \\ 2.5925 & 20 & 0.0782 & 1043.1 & (45) \\ 3 & 20 & 0.0990 & 1055.3 & (62) \\ 4 & 20 & 0.1128 & 1062.9 & (45) \\ & 20 & 0.1450 & 1081.6 & (45)\end{array}$

App vol App vol Density Density App Vol Square of Inconsistent Inconsistent Apparent Delta density Density of

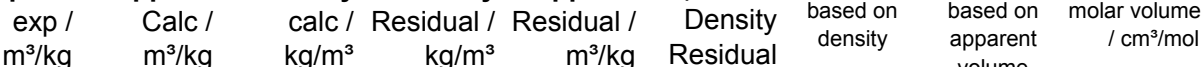
$\mathrm{m}^{3} / \mathrm{kg} \quad \mathrm{m}^{3} / \mathrm{kg} \quad \mathrm{kg} / \mathrm{m}^{3} \quad \mathrm{~kg} / \mathrm{m}^{3} \quad \mathrm{~m}^{3} / \mathrm{kg}$ Residual density apparen $1 \mathrm{~cm}^{3} / \mathrm{mol}$

999.0996 $\begin{array}{llllll}0.000416 & 0.000463 & 1009.90 & 0.9529 & -0.000047 & 0.908\end{array}$ $\begin{array}{lllllll}0.000416 & 0.000463 & 1009.90 & 0.9529 & -0.000047 & 0.908\end{array}$ $\begin{array}{lllllll}0.000423 & 0.000463 & 1010.37 & 0.8365 & -0.000039 & 0.700\end{array}$ $\begin{array}{llllll}0.000423 & 0.000463 & 1010.37 & 0.8365 & -0.000039 & 0.700\end{array}$ $\begin{array}{llllll}0.000422 & 0.000464 & 1015.26 & 1.2688 & -0.000041 & 1.610\end{array}$ $\begin{array}{llllll}0.000438 & 0.000465 & 1021.35 & 1.1192 & -0.000026 & 1.253\end{array}$ $\begin{array}{llllll}0.000426 & 0.000465 & 1021.55 & 1.6543 & -0.000039 & 2.737\end{array}$ $\begin{array}{llllll}0.000431 & 0.000466 & 1025.94 & 1.7640 & -0.000034 & 3.112\end{array}$ $\begin{array}{llllll}0.000449 & 0.000468 & 1042.47 & 1.6225 & -0.000019 & 2.633\end{array}$ $\begin{array}{llllll}0.000451 & 0.000470 & 1054.48 & 2.1169 & -0.000019 & 4.481\end{array}$ $\begin{array}{lllllll}0.000459 & 0.000471 & 1062.54 & 1.5554 & -0.000012 & 2.419\end{array}$ $\begin{array}{llllll}0.000467 & 0.000474 & 1081.67 & 1.1748 & -0.000007 & 1.380\end{array}$ $\begin{array}{llllll}0.000468 & 0.000474 & 1085.04 & 1.1631 & -0.000007 & 1.353\end{array}$ $\begin{array}{llllll}0.000473 & 0.000478 & 1112.40 & 1.1021 & -0.000005 & 1.215\end{array}$ $\begin{array}{llllll}0.000476 & 0.000479 & 1117.32 & 0.6400 & -0.000003 & 0.410\end{array}$ $\begin{array}{llllll}0.000481 & 0.000481 & 1137.89 & -0.0948 & 0.000000 & 0.009\end{array}$ $\begin{array}{llllll}0.000485 & 0.000483 & 1149.93 & -0.8451 & 0.000003 & 0.714\end{array}$ $\begin{array}{llllll}0.000485 & 0.000484 & 1162.61 & -0.5089 & 0.000001 & 0.259\end{array}$ $\begin{array}{llllll}0.000488 & 0.000486 & 1177.90 & -0.8030 & 0.000002 & 0.645\end{array}$ $\begin{array}{lllllll}0.000488 & 0.000486 & 1179.88 & -0.9971 & 0.000002 & 0.994\end{array}$ $\begin{array}{llllll}0.000489 & 0.000487 & 1192.76 & -0.8591 & 0.000002 & 0.738\end{array}$ $\begin{array}{llllll}0.000491 & 0.000489 & 1207.01 & -1.0103 & 0.000002 & 1.021\end{array}$ $\begin{array}{llllll}0.000490 & 0.000490 & 1219.54 & -0.1425 & 0.000000 & 0.020\end{array}$ $\begin{array}{lllllll}0.000492 & 0.000491 & 1233.89 & -0.2941 & 0.000001 & 0.086\end{array}$ $\begin{array}{llllll}0.000491 & 0.000492 & 1245.90 & 0.3970 & -0.000001 & 0.158\end{array}$ $\begin{array}{llllll}0.000493 & 0.000493 & 1254.82 & -0.0245 & 0.000000 & 0.001\end{array}$ $\begin{array}{lllllll}0.000494 & 0.000494 & 1270.80 & 0.5028 & -0.000001 & 0.253\end{array}$ $\begin{array}{lllllll}0.000495 & 0.000495 & 1277.91 & 0.0871 & 0.000000 & 0.008\end{array}$ $\begin{array}{llllll}0.000494 & 0.000496 & 1286.60 & 0.7970 & -0.000001 & 0.635\end{array}$ $\begin{array}{llllll}0.000494 & 0.000496 & 1295.38 & 1.4218 & -0.000002 & 2.021\end{array}$ $\begin{array}{llllll}0.000500 & 0.000497 & 1294.80 & -2.2156 & 0.000003 & 4.909\end{array}$ $\begin{array}{llllll}0.000492 & 0.000491 & 1222.20 & -0.4751 & 0.000001 & 0.226\end{array}$ $\begin{array}{llllll}0.000493 & 0.000493 & 1247.23 & 0.3562 & -0.000001 & 0.127\end{array}$ $\begin{array}{llllll}0.000488 & 0.000487 & 1180.53 & -0.6915 & 0.000002 & 0.478\end{array}$ $\begin{array}{llllll}0.000450 & 0.000470 & 1054.53 & 2.2307 & -0.000020 & 4.976\end{array}$ $\begin{array}{lllllll}0.000477 & 0.000479 & 1116.17 & 0.3997 & -0.000002 & 0.160\end{array}$ $\begin{array}{llllll}0.000495 & 0.000495 & 1263.96 & 0.1116 & 0.000000 & 0.012\end{array}$ $\begin{array}{llllll}0.000416 & 0.000460 & 999.35 & 0.0919 & -0.000044 & 0.008\end{array}$ $\begin{array}{llllll}0.000422 & 0.000460 & 1000.49 & 0.1611 & -0.000038 & 0.026\end{array}$ $\begin{array}{lllllll}0.000426 & 0.000462 & 1009.50 & 0.7468 & -0.000035 & 0.558\end{array}$ $\begin{array}{llllll}0.000426 & 0.000462 & 1009.50 & 0.7468 & -0.000035 & 0.558\end{array}$ $\begin{array}{llllll}0.000442 & 0.000464 & 1020.50 & 0.9207 & -0.000022 & 0.848\end{array}$ $\begin{array}{llllll}0.000431 & 0.000464 & 1020.69 & 1.4054 & -0.000033 & 1.975\end{array}$ $\begin{array}{llllll}0.000450 & 0.000467 & 1041.64 & 1.4334 & -0.000017 & 2.055\end{array}$ $\begin{array}{llllll}0.000454 & 0.000469 & 1053.65 & 1.6451 & -0.000015 & 2.706\end{array}$ $\begin{array}{llllll}0.000461 & 0.000471 & 1061.71 & 1.2054 & -0.000009 & 1.453\end{array}$ 0.564
999.0996 999.0996 999.0996 999.0996 999.0996 999.0996 999.0996 999.0996 999.0996 999.0996 999.0996 999.0996 999.0996 999.0996 999.0996 999.0996 999.0996 999.0996 999.0996 999.0996 999.0996 999.0996 999.0996 999.0996 999.0996 999.0996 999.0996 999.0996 999.0996 998.7749 998.4364 998.4072 998.3639 998.348 998.3401 998.2959 998.2041 998.2041 998.2041 998.2041 998.2041 998.2041 998.2041 998.2041 998.2041 998.2041 
molality molarity

$\mathbf{t} /{ }^{\circ} \mathrm{C}$ mass frac Density $\exp /$ $\mathrm{kg} / \mathrm{m}^{3}$

4.1814
5.7128
6
7.2417
8
8.8268
9.863
10
10.913
11.963
12.923
14.067
15.063
15.827
17.249
17.906
18.729
19.584

0.0502

0.0999

0.1
10782

0.1189
0.11689

0.21689

0.2383

0.2506

0.32518

0.3581

0.43406

0.4786

0.4786

0.5
0.5

0.5019

0.65179

0.721

0.76073

0.87037

0.97888

1.004

1.009

1.08789

1.2128

$\begin{array}{rrrr}20 & 0.1506 & 1084.7 & (62) \\ 20 & 0.1950 & 1112.0 & (62) \\ 20 & 0.2028 & 1116.3 & (45) \\ 20 & 0.2349 & 1136.4 & (62) \\ 20 & 0.2533 & 1147.7 & (45) \\ 20 & 0.2723 & 1160.7 & (62) \\ 20 & 0.2948 & 1175.7 & (62) \\ 20 & 0.2977 & 1177.5 & (45) \\ 20 & 0.3163 & 1190.4 & (62) \\ 20 & 0.3365 & 1204.7 & (62) \\ 20 & 0.3539 & 1217.8 & (62) \\ 20 & 0.3736 & 1231.9 & (62) \\ 20 & 0.3897 & 1244.5 & (62) \\ 20 & 0.4015 & 1253.0 & (62) \\ 20 & 0.4224 & 1269.4 & (62) \\ 20 & 0.4315 & 1276.2 & (62) \\ 20 & 0.4426 & 1285.6 & (62) \\ 20 & 0.4536 & 1294.9 & (62) \\ 20.17 & 0.4303 & 1274.8 & (133) \\ 20.19 & 0.2486 & 1145.6 & (133) \\ 25 & 0.0021 & 998.3 & (45) \\ 25 & 0.0021 & 998.3 & (28) \\ 25 & 0.0042 & 999.5 & (28) \\ 25 & 0.0042 & 999.5 & (45) \\ 25 & 0.0046 & 999.7 & (73) \\ 25 & 0.0050 & 1000.0 & (80) \\ 25 & 0.0091 & 1002.4 & (73) \\ 25 & 0.0100 & 1002.9 & (80) \\ 25 & 0.0105 & 1003.2 & (28) \\ 25 & 0.0136 & 1005.0 & (73) \\ 25 & 0.0150 & 1005.8 & (80) \\ 25 & 0.0181 & 1007.6 & (73) \\ 25 & 0.0199 & 1008.6 & (80) \\ 25 & 0.0199 & 1008.6 & (80) \\ 25 & 0.0208 & 1009.0 & (45) \\ 25 & 0.0208 & 1009.0 & (45) \\ 25 & 0.0208 & 1009.1 & (28) \\ 25 & 0.0269 & 1012.7 & (73) \\ 25 & 0.0297 & 1014.2 & (80) \\ 25 & 0.0312 & 1015.1 & (73) \\ 25 & 0.0356 & 1017.6 & (73) \\ 25 & 0.0398 & 1020.1 & (73) \\ 25 & 0.0407 & 1020.1 & (45) \\ 25 & 0.0408 & 1020.6 & (28) \\ 25 & 0.0410 & 1020.2 & (62) \\ 25 & 0.0441 & 1022.5 & (73) \\ 25 & 0.0489 & 1025.3 & (80) \\ & & & \end{array}$

Ref

exp / Calc /

$\mathrm{m}^{3} / \mathrm{kg} \quad \mathrm{m}^{3} / \mathrm{kg}$

$\begin{array}{ll}0.000471 & 0.000474 \\ 0.000476 & 0.000478\end{array}$

$\begin{array}{ll}0.000476 & 0.000478\end{array}$

0.0004790 .000479

$0.000483 \quad 0.000481$

$0.000487 \quad 0.000483$

$\begin{array}{ll}0.000487 & 0.000485\end{array}$

$0.000489 \quad 0.000486$

$0.000490 \quad 0.000487$

$\begin{array}{lll}0.000490 & 0.000488\end{array}$

$\begin{array}{ll}0.000491 & 0.000489\end{array}$

$\begin{array}{ll}0.000491 & 0.000491\end{array}$

$0.000493 \quad 0.000492$

$0.000493 \quad 0.000493$

$\begin{array}{ll}0.000494 & 0.000494\end{array}$

$\begin{array}{ll}0.000495 & 0.000496\end{array}$

0.0004960 .000496

$\begin{array}{lll}0.000496 & 0.000497\end{array}$

$0.000496 \quad 0.000498$

$\begin{array}{ll}0.000497 & 0.000496\end{array}$

$0.000483 \quad 0.000483$

$0.000416 \quad 0.000458$

$\begin{array}{lll}0.000408 & 0.000458\end{array}$

0.0004110 .000458

$0.000426 \quad 0.000458$

$\begin{array}{lll}0.000409 & 0.000458\end{array}$

$\begin{array}{lll}0.000408 & 0.000458\end{array}$

$0.000415 \quad 0.000459$

$\begin{array}{ll}0.000416 & 0.000459\end{array}$

$0.000420 \quad 0.000459$

$0.000419 \quad 0.000459$

$\begin{array}{ll}0.000420 & 0.000459\end{array}$

$0.000423 \quad 0.000460$

$0.000424 \quad 0.000460$

$0.000424 \quad 0.000460$

$0.000430 \quad 0.000460$

$0.000430 \quad 0.000460$

$0.000427 \quad 0.000460$

$0.000428 \quad 0.000461$

$0.000430 \quad 0.000461$

$\begin{array}{ll}0.000431 & 0.000461\end{array}$

$0.000433 \quad 0.000462$

$0.000435 \quad 0.000462$

$0.000446 \quad 0.000462$

$\begin{array}{lll}0.000436 & 0.000462\end{array}$

$0.000448 \quad 0.000462$

$\begin{array}{lll}0.000436 & 0.000463\end{array}$

$0.000438 \quad 0.000463$
Density Density App Vol Square of Inconsistent Inconsistent
based on molar volume $\begin{gathered}\text { Apparent } \\ \text { based on } \\ \mathrm{kg} / \mathrm{m}^{3}\end{gathered} \begin{array}{r}\text { Density of } \\ \text { water } / \mathrm{kg} / \mathrm{m}^{3}\end{array}$ calc / Residual / Residual / Density based on based on molar volume $\mathrm{kg} / \mathrm{m}^{3} \quad \mathrm{~kg} / \mathrm{m}^{3} \quad \mathrm{~m}^{3} / \mathrm{kg}$ Residual density $\begin{gathered}\text { apparen } \\ \text { volume }\end{gathered}$ $1 \mathrm{~cm}^{3} / \mathrm{mol}$

$084.19 \quad 0.5134-0.000003 \quad 0.264$

$1111.49 \quad 0.5065-0.000002 \quad 0.257$

$\begin{array}{llll}1116.40 & -0.1426 & 0.000001 & 0.020\end{array}$

$\begin{array}{lll}1116.91 & -0.5117 & 0.000002\end{array}$

$\begin{array}{llll}1161.52 & -0.8241 & 0.000002 & 0.679\end{array}$

$\begin{array}{llll}1176.74 & -1.0430 & 0.000003 & 1.088\end{array}$

$\begin{array}{llll}1178.71 & -1.2567 & 0.000003 & 1.579\end{array}$

$\begin{array}{llll}1191.52 & -1.1171 & 0.000002 & 1.248\end{array}$

$\begin{array}{llll}1205.68 & -0.9815 & 0.000002 & 0.963\end{array}$

$\begin{array}{llll}1218.13 & -0.3310 & 0.000001 & 0.110\end{array}$

$\begin{array}{llll}232.38 & -0.4803 & 0.000001 & 0.231\end{array}$

$\begin{array}{lll}0.2023 & 0.000000 & 0.041\end{array}$

$\begin{array}{lll}-0.1477 & 0.000000 & 0.022\end{array}$

$\begin{array}{lll}0.4151 & -0.000001 & 0.172\end{array}$

$\begin{array}{lll}0.1627 & 0.000000 & 0.026\end{array}$

$0.9525-0.000001 \quad 0.907$

$\begin{array}{lll}1.5608 & -0.000002 & 2.436\end{array}$

$\begin{array}{lll}-0.2198 & 0.000000 & 0.048\end{array}$

$\begin{array}{lll}-0.2056 & 0.000001 & 0.042\end{array}$

$\begin{array}{lll}0.0878 & -0.000042 \quad 0.008\end{array}$

$\begin{array}{lll}0.1061 & -0.000050 & 0.011\end{array}$

$\begin{array}{lll}0.2008 & -0.000048 \quad 0.040\end{array}$

$\begin{array}{lll}0.1337 & -0.000032 & 0.018\end{array}$

$0.2250-0.000049 \quad 0.051$

$0.2520-0.000050 \quad 0.064$

$0.4015-0.000044 \quad 0.161$

$\begin{array}{lll}0.4358 & -0.000043 \quad 0.190\end{array}$

$0.4079-0.000039 \quad 0.166$

$\begin{array}{lll}0.5507 & -0.000040 \quad 0.303\end{array}$

$\begin{array}{lll}0.5986 & -0.000040 \quad 0.358\end{array}$

$\begin{array}{lll}0.6814 & -0.000037 & 0.464\end{array}$

$0.7337-0.000036 \quad 0.538$

$0.7337-0.000036 \quad 0.538$

$0.6453-0.000031 \quad 0.416$

$\begin{array}{lll}0.6453 & -0.000031 & 0.416\end{array}$

$\begin{array}{lll}0.7057 & -0.000033 & 0.498\end{array}$

$\begin{array}{lll}0.8977 & -0.000033 & 0.806\end{array}$

$0.9570-0.000031 \quad 0.916$

$\begin{array}{lll}0.9809 & -0.000030 & 0.962\end{array}$

$\begin{array}{lll}1.0631 & -0.000029 & 1.130\end{array}$

$\begin{array}{lll}1.1343 & -0.000027 & 1.287\end{array}$

$\begin{array}{lll}0.6849 & -0.000016 & 0.469\end{array}$

$\begin{array}{lll}1.1068 & -0.000026 & 1.225\end{array}$

$\begin{array}{lll}0.6093 & -0.000014 & 0.371\end{array}$

$\begin{array}{lll}1.2086 & -0.000026 & 1.461\end{array}$

1.651
998.2041

998.2041

998.2041

998.2041

998.2041

998.2041

998.2041

998.2041

998.2041

998.2041

998.2041

998.2041

998.2041

998.2041

998.2041

998.2041

998.2041

998.2041

998.1689

998.1647

997.0449

$1.258 \quad 997.0449$

$2.49 \quad 997.0449$

997.0449

997.0449

997.0449

997.0449

997.0449

$6.126 \quad 997.0449$

997.0449

997.0449

997.0449

997.0449

997.0449

997.0449

997.0449

997.0449

997.0449

997.0449

997.0449

997.0449

997.0449

$23.544 \quad 997.0449$

997.0449

997.0449

997.0449
$12.078 \quad 997.0449$ 
molality molarity

$t /{ }^{\circ} \mathrm{C}$ mass frac Density $\exp /$ $\mathrm{kg} / \mathrm{m}^{3}$

2
2.015
2.5925
2.977
3
4
4.1814
4.974
5.7128
6
7.2417
8
8.8268
9.863
10
10.913
11.963
12.923
14.067
15.063
15.827
17.249
17.906
18.729
19.584

8.8268

11.963

12.923

15.063

5.827

17.906

19.584

$\begin{array}{rrrrr} & 26.97 & 0.3932 & 1243.8 & (133) \\ & 27.1 & 0.4539 & 1288.7 & (133) \\ 28.63 & 0.3591 & 1218.4 & (133) \\ & 29.5 & 0.1003 & 1053.9 & (133) \\ & 29.54 & 0.4156 & 1260.3 & (133) \\ & 29.64 & 0.3001 & 1176.6 & (133) \\ 0.05 & 29.65 & 0.2022 & 1113.5 & (133) \\ 0.1 & 30 & 0.0021 & 996.9 & (45) \\ 0.5 & 30 & 0.0042 & 998.1 & (45) \\ 0.5 & 30 & 0.0208 & 1007.6 & (45) \\ 1 & 30 & 0.0208 & 1007.6 & (45) \\ 1.009 & 30 & 0.0407 & 1018.7 & (45) \\ 2 & 30 & 0.0410 & 1019.3 & (62) \\ 2.5925 & 30 & 0.0782 & 1040.1 & (45) \\ 3 & 30 & 0.0990 & 1052.4 & (62) \\ 4 & 30 & 0.1128 & 1060.0 & (45) \\ 4.1814 & 30 & 0.1450 & 1078.7 & (45) \\ 5.7128 & 30 & 0.1506 & 1081.8 & (62) \\ 6 & 30 & 0.1950 & 1108.4 & (62) \\ 7.2417 & 30 & 0.2028 & 1113.3 & (45) \\ 8 & 30 & 0.2349 & 1133.2 & (62) \\ & 30 & 0.2533 & 1144.7 & (45)\end{array}$

$\begin{array}{rrrr}25 & 0.0782 & 1041.6 & (45) \\ 25 & 0.0787 & 1042.3 & (28) \\ 25 & 0.0990 & 1053.9 & (62) \\ 25 & 0.1121 & 1061.6 & (28) \\ 25 & 0.1128 & 1061.5 & (45) \\ 25 & 0.1450 & 1080.2 & (45) \\ 25 & 0.1506 & 1083.4 & (62) \\ 25 & 0.1741 & 1097.1 & (28) \\ 25 & 0.1950 & 1110.4 & (62) \\ 25 & 0.2028 & 1114.8 & (45) \\ 25 & 0.2349 & 1134.8 & (62) \\ 25 & 0.2533 & 1146.3 & (45) \\ 25 & 0.2723 & 1159.1 & (62) \\ 25 & 0.2948 & 1174.2 & (62) \\ 25 & 0.2977 & 1175.9 & (45) \\ 25 & 0.3163 & 1188.8 & (62) \\ 25 & 0.3365 & 1203.1 & (62) \\ 25 & 0.3539 & 1216.1 & (62) \\ 25 & 0.3736 & 1230.1 & (62) \\ 25 & 0.3897 & 1242.7 & (62) \\ 25 & 0.4015 & 1251.1 & (62) \\ 25 & 0.4224 & 1267.6 & (62) \\ 25 & 0.4315 & 1274.3 & (62) \\ 25 & 0.4426 & 1283.7 & (62) \\ 25 & 0.4536 & 1293.1 & (62) \\ 6.97 & 0.3932 & 1243.8 & (133) \\ 27.1 & 0.4539 & 1288.7 & (133) \\ 28.63 & 0.3591 & 1218.4 & (133) \\ 29.5 & 0.1003 & 1053.9 & (133) \\ 9.54 & 0.4156 & 1260.3 & (133) \\ 29.64 & 0.3001 & 1176.6 & (133) \\ 9.65 & 0.2022 & 1113.5 & (133) \\ 30 & 0.0021 & 996.9 & (45) \\ 30 & 0.0042 & 998.1 & (45) \\ 30 & 0.0208 & 1007.6 & (45) \\ 30 & 0.0208 & 1007.6 & (45) \\ 30 & 0.0407 & 1018.7 & (45) \\ 30 & 0.0410 & 1019.3 & (62) \\ 30 & 0.0782 & 1040.1 & (45) \\ 30 & 0.0990 & 1052.4 & (62) \\ 30 & 0.1128 & 1060.0 & (45) \\ 30 & 0.1450 & 1078.7 & (45) \\ 30 & 0.1506 & 1081.8 & (62) \\ 30 & 0.1950 & 1108.4 & (62) \\ 30 & 0.2028 & 1113.3 & (45) \\ 30 & 0.2349 & 1133.2 & (62) \\ 30 & 0.2533 & 1144.7 & (45) \\ & & & \end{array}$

Ref

$\begin{array}{ll}\exp / & \text { Calc / } \\ \mathrm{m}^{3} / \mathrm{kg} & \mathrm{m}^{3} / \mathrm{kg}\end{array}$

$$
\mathrm{m}^{3} / \mathrm{kg} \quad \mathrm{m}^{3} / \mathrm{kg}
$$

0.0004540 .000466

\subsection{0 .000466}

$0.000457 \quad 0.000468$

$\begin{array}{ll}0.000459 & 0.000470\end{array}$

$\begin{array}{ll}0.000463 & 0.000470\end{array}$

$\begin{array}{lll}0.000471 & 0.000473\end{array}$

$\begin{array}{lll}0.000472 & 0.000474\end{array}$

$0.000478 \quad 0.000476$

$\begin{array}{lll}0.000478 & 0.000478\end{array}$

$\begin{array}{ll}0.000480 & 0.000479\end{array}$

$\begin{array}{ll}0.000485 & 0.000482\end{array}$

$0.000487 \quad 0.000483$

$0.000488 \quad 0.000485$

$\begin{array}{ll}0.000490 & 0.000487\end{array}$

$0.000491 \quad 0.000487$

0.0004920 .000489

$0.000492 \quad 0.000490$

$0.000493 \quad 0.000492$

$0.000494 \quad 0.000493$

$0.000494 \quad 0.000494$

$0.000496 \quad 0.000495$

$\begin{array}{lll}0.000496 & 0.000497\end{array}$

$\begin{array}{ll}0.000497 & 0.000498\end{array}$

$0.000497 \quad 0.000498$

$0.000497 \quad 0.000499$

$0.000496 \quad 0.000495$

$0.000502 \quad 0.000500$

$0.000494 \quad 0.000493$

$0.000453 \quad 0.000468$

$0.000497 \quad 0.000497$

$\begin{array}{lll}0.000490 & 0.000487\end{array}$

0.0004790 .000478

$\begin{array}{lll}0.000408 & 0.000456\end{array}$

$0.000424 \quad 0.000456$

$0.000430 \quad 0.000458$

$0.000430 \quad 0.000458$

$\begin{array}{lll}0.000445 & 0.000461\end{array}$

$0.000436 \quad 0.000461$

$0.000455 \quad 0.000465$

$0.000457 \quad 0.000467$

$0.000464 \quad 0.000469$

$\begin{array}{ll}0.000471 & 0.000472\end{array}$

$\begin{array}{lll}0.000473 & 0.000473\end{array}$

$\begin{array}{ll}0.000480 & 0.000477\end{array}$

$0.000481 \quad 0.000478$

$0.000485 \quad 0.000481$

$0.000488 \quad 0.000483$

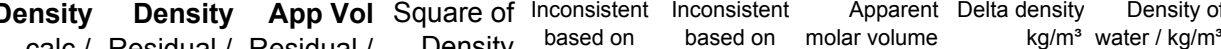

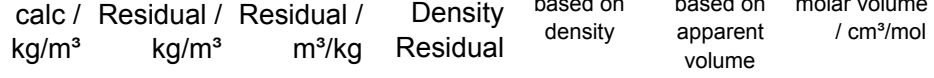

997.0449

$\begin{array}{llll}1.0665 & -0.000013 & 1.137\end{array}$

$1040.87 \quad 1.4723-0.000017 \quad 2.168$

$\begin{array}{llll}1052.59 & 1.3094 & -0.000012 & 1.714\end{array}$

$\begin{array}{llll}060.20 & 1.3489 & -0.000011 & 1.820\end{array}$

$\begin{array}{llll}1060.65 & 0.8774 & -0.000007 & 0.770\end{array}$

$1079.75 \quad 0.4286-0.000003 \quad 0.184$

$1083.12 \quad 0.2839-0.000002 \quad 0.081$

$\begin{array}{llll}1097.48 & -0.3864 & 0.000002 & 0.149\end{array}$

$\begin{array}{llll}110.38 & 0.0165 & 0.000000 & 0.000\end{array}$

$\begin{array}{llll}115.28 & -0.4621 & 0.000002 & 0.214\end{array}$

$\begin{array}{llll}1135.74 & -0.9368 & 0.000003 & 0.878\end{array}$

$\begin{array}{llll}1147.68 & -1.4208 & 0.000004 & 2.019\end{array}$

1.348

1.472

2.184

1.727

1.210

0.225

0.397

0.019

0.061

0.293

0.061

1.236

3.603

0.361

3.123

0.380

2.771

0.039

1.023

0.031

0.010

0.018

0.344

0.344

0.418

1.082

0.749

1.174

0.429

0.041

0.003

0.483

0.430

1.433

2.554
$45.3 \quad 997.0449$

997.0449

$64.506 \quad 997.0449$

997.0449

997.0449

997.0449

$100.045 \quad 997.0449$

997.0449

997.0449

997.0449

997.0449

997.0449

997.0449

997.0449

997.0449

997.0449

997.0449

997.0449

997.0449

997.0449

997.0449

997.0449

997.0449

997.0449

996.5215

996.4856

996.0527

995.7971

995.7852

995.7554

995.7524

995.6473

995.6473

995.6473

995.6473

995.6473

995.6473

995.6473

995.6473

995.6473

995.6473

995.6473

995.6473

995.6473

995.6473

995.6473 
molality molarity

$\mathrm{t} /{ }^{\circ} \mathrm{C}$ mass frac

Density

$\exp /$

$\mathrm{kg} / \mathrm{m}^{3}$

8.8268

9.863
10

10.913

11.963

12.923

14.067

15.827

17.249

17.906

18.729
19.584

0.05

0.1

0.1

0.1189

0.2

0.2383

0.3
0.3581

0.4

0.4786
0.4786

0.4786

0.5
0.5
0.5

0.5
0.6
0.7

0.7
0.721

0.721
0.8

0.9
1

1.009
1.2

1.2
2128

1.2128

1.4
1.6
1.8

2

2.5

2.5925

3
4

\section{0}

30
30
30

30
30

30
30

$\begin{array}{ll}30 & 0.3539\end{array}$

$\begin{array}{ll}30 & 0.3736 \\ 30 & 0.4015\end{array}$

$30 \quad 0.4224$

$30 \quad 0.4315$

$30 \quad 0.4426$

$30 \quad 0.4536$

$30.09 \quad 0.2486$

30.16

35
35

35
35
35

35
35

35
35

35

35

35

35
35
35

35
35
35

35
35

35

35
35

35
35

35
35

0.0489

$35 \quad 0.0635$

$35 \quad 0.0709$

0.0782

0.0782

0.0958
0.0990

0.1128

0.1450
1157.7

1172.7

1174.3

1187.3

1201.5

1214.4

1228.4

1249.3

1272.4

1281.7

1291.1

1143.5

$1271.2 \quad(133)$

$995.3 \quad(45)$

$996.5 \quad(45)$

$997.0 \quad(80)$

$998.9 \quad(130)$

$999.9 \quad(80)$

$1001.3 \quad(130)$

$1002.7 \quad(80)$

$\begin{array}{ll}1003.7 & (130) \\ 1005.6 & (80)\end{array}$

$1005.6 \quad(80)$

$1006.0 \quad(45)$

$1006.0 \quad(45)$

$1006.0 \quad(130)$

$1008.3 \quad(130)$

$1010.6 \quad(130)$

$1011.1 \quad(80)$

$\begin{array}{ll}1012.9 & (130) \\ 1015.2 & (130)\end{array}$

$1017.1 \quad$ (45)

$1017.4 \quad(130)$

$1017.6 \quad(62)$

$1021.8 \quad(130)$

$1022.1 \quad(80)$

$1026.2 \quad(130)$

$1034.7 \quad(130)$

$1038.6 \quad(45)$

$1038.8 \quad(130)$

$1049.0 \quad(130)$

$1050.7 \quad(62)$

1077.1
$(45)$
App vol App vol Density Density App Vol Square of Inconsistent Inconsistent $\begin{array}{r}\text { Apparent Delta density } \\ \mathrm{kg} / \mathrm{m}^{3} \mathrm{water} / \mathrm{kg} / \mathrm{m}^{3}\end{array}$

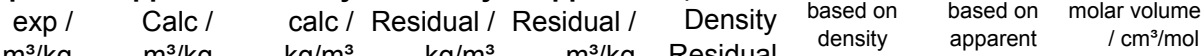
$\mathrm{m}^{3} / \mathrm{kg} \quad \mathrm{m}^{3} / \mathrm{kg} \quad \mathrm{kg} / \mathrm{m}^{3} \quad \mathrm{~kg} / \mathrm{m}^{3} \quad \mathrm{~m}^{3} / \mathrm{kg}$ Residual density apparen

$\begin{array}{llllll}0.000488 & 0.000485 & 1158.85 & -1.1475 & 0.000003 & 1.317\end{array}$

$\begin{array}{llllll}0.000490 & 0.000487 & 1173.94 & -1.2419 & 0.000003 & 1.542\end{array}$

$\begin{array}{llllll}0.000491 & 0.000487 & 1175.89 & -1.5783 & 0.000004 & 2.491\end{array}$

$\begin{array}{llllll}0.000492 & 0.000489 & 1188.58 & -1.2778 & 0.000003 & 1.633\end{array}$

$\begin{array}{llllll}0.000493 & 0.000491 & 1202.59 & -1.0936 & 0.000002 & 1.196\end{array}$

$\begin{array}{llllll}0.000493 & 0.000492 & 1214.90 & -0.4999 & 0.000001 & 0.250\end{array}$

$\begin{array}{llllll}0.000495 & 0.000494 & 1228.97 & -0.5707 & 0.000001 & 0.326\end{array}$

$\begin{array}{llllll}0.000497 & 0.000496 & 1249.45 & -0.1505 & 0.000000 & 0.023\end{array}$

$\begin{array}{llllll}0.000497 & 0.000498 & 1265.05 & 0.6531 & -0.000001 & 0.427\end{array}$

$\begin{array}{llllll}0.000498 & 0.000499 & 1271.99 & 0.4139 & -0.000001 & 0.171\end{array}$

$\begin{array}{llllll}0.000498 & 0.000500 & 1280.45 & 1.2469 & -0.000002 & 1.555\end{array}$

$\begin{array}{llllll}0.000498 & 0.000500 & 1288.99 & 2.1052 & -0.000003 & 4.432\end{array}$

$\begin{array}{llllll}0.000482 & 0.000483 & 1143.24 & 0.2954 & -0.000001 & 0.087\end{array}$

$\begin{array}{llllll}0.000498 & 0.000499 & 1270.98 & 0.2173 & 0.000000 & 0.047\end{array}$

$\begin{array}{llrlrl}0.000414 & 0.000454 & 995.19 & 0.0823 & -0.000039 & 0.007\end{array}$

$\begin{array}{llllll}0.000423 & 0.000454 & 996.34 & 0.1298 & -0.000031 & 0.017\end{array}$

$\begin{array}{llllll}0.000414 & 0.000454 & 996.34 & 0.1654 & -0.000039 & 0.027\end{array}$

$\begin{array}{llllll}0.000409 & 0.000454 & 996.77 & 0.2251 & -0.000045 & 0.051\end{array}$

$\begin{array}{llllll}0.000419 & 0.000454 & 998.64 & 0.2940 & -0.000035 & 0.086\end{array}$

$\begin{array}{llllll}0.000418 & 0.000455 & 999.51 & 0.3695 & -0.000037 & 0.137\end{array}$

$\begin{array}{llllll}0.000423 & 0.000455 & 1000.92 & 0.4065 & -0.000032 & 0.165\end{array}$

$\begin{array}{llllll}0.000422 & 0.000455 & 1002.24 & 0.4938 & -0.000033 & 0.244\end{array}$

$\begin{array}{llllll}0.000426 & 0.000456 & 1003.19 & 0.5038 & -0.000030 & 0.254\end{array}$

$\begin{array}{llllll}0.000427 & 0.000456 & 1004.96 & 0.5911 & -0.000029 & 0.349\end{array}$

$\begin{array}{llllll}0.000427 & 0.000456 & 1004.96 & 0.5911 & -0.000029 & 0.349\end{array}$

$\begin{array}{llllll}0.000431 & 0.000456 & 1005.44 & 0.5195 & -0.000025 & 0.270\end{array}$

$\begin{array}{llllll}0.000431 & 0.000456 & 1005.44 & 0.5195 & -0.000025 & 0.270\end{array}$

$\begin{array}{llllll}0.000428 & 0.000456 & 1005.44 & 0.5916 & -0.000028 & 0.350\end{array}$

$\begin{array}{lllllll}0.000430 & 0.000457 & 1007.68 & 0.6640 & -0.000026 & 0.441\end{array}$

$\begin{array}{lll}0.7347 & -0.000025 & 0.540\end{array}$

$\begin{array}{lll}0.000432 & 0.000457 & 1009.91\end{array}$

$\begin{array}{lll}0.000432 & 0.000457 & 1010.38\end{array}$

$\begin{array}{lll}0.000434 & 0.000458 & 1012.13\end{array}$

0.7507

0.564

0.630

0.722

$\begin{array}{lll}0.000445 & 0.000459 & 1016.53\end{array}$

$0.8499-0.000022$

0.318

$\begin{array}{lll}0.000437 & 0.000459 & 1016.53\end{array}$

$\begin{array}{lll}0.000438 & 0.000459 & 1016.72\end{array}$

$\begin{array}{lll}0.000440 & 0.000460 & 1020.87\end{array}$

0.8961

0.803

0.769

0.942

0.979

$\begin{array}{lll}0.000440 & 0.000460 & 1021.15 \\ 0.000443 & 0.000461 & 1025.17\end{array}$

$0.9705-0.000019$

$0.9897-0.000019$

1.045

$\begin{array}{lll}0.000446 & 0.000462 & 1029.42\end{array}$

$\begin{array}{lll}0.000448 & 0.000462 & 1033.63\end{array}$

1.117

1.165

$\begin{array}{lll}0.000454 & 0.000463 & 1037.79\end{array}$

$1.0795-0.000014$

0.624

$1.0361-0.000012-1.074$

1.048

$\begin{array}{lll}0.000456 & 0.000465 & 1048.00\end{array}$

$\begin{array}{lll}0.000458 & 0.000466 & 1049.85\end{array}$

1.048
0.716

$\begin{array}{lll}0.000463 & 0.000467 & 1057.93 \\ 0.000471 & 0.000471 & 1077.05\end{array}$

0.299

0.003
995.6473

995.6473

995.6473

995.6473

995.6473

995.6473

995.6473

995.6473

995.6473

995.6473

995.6473

995.6473

995.6201

995.5988

994.0319

994.0319

994.0319

994.0319

994.0319

994.0319

994.0319

994.0319

994.0319

994.0319

994.0319

994.0319

994.0319

994.0319

994.0319

994.0319

994.0319

994.0319

994.0319

994.0319

994.0319

994.0319

994.0319

994.0319

994.0319

994.0319

994.0319

994.0319

994.0319

994.0319

994.0319

994.0319

994.0319 
molality molarity

$\mathrm{t} /{ }^{\circ} \mathrm{C}$ mass frac Density

4.1814
5.7128
6
7.2417
8
8.8268
9.863
10
10.913
11.963
12.923
14.067
15.827
17.249
17.906
18.729
19.584

0.1
0.1189

0.2383

0.3581

0.4786

0.4786

0.5

0.5
0.721

1
.2128

2128

2
3
4

6

8
10

\section{$\exp /$
$\mathrm{kg} / \mathrm{m}^{3}$ \\ $\exp /$
$\mathrm{kg} / \mathrm{m}^{3}$}

$\begin{array}{rrrr}35 & 0.1506 & 1080.0 & (62) \\ 35 & 0.1950 & 1106.8 & (62) \\ 35 & 0.2028 & 1111.8 & (45) \\ 35 & 0.2349 & 1131.4 & (62) \\ 35 & 0.2533 & 1143.2 & (45) \\ 35 & 0.2723 & 1156.2 & (62) \\ 35 & 0.2948 & 1171.2 & (62) \\ 35 & 0.2977 & 1172.7 & (45) \\ 35 & 0.3163 & 1185.7 & (62) \\ 35 & 0.3365 & 1199.9 & (62) \\ 35 & 0.3539 & 1212.7 & (62) \\ 35 & 0.3736 & 1226.6 & (62) \\ 35 & 0.4015 & 1247.5 & (62) \\ 35 & 0.4224 & 1263.9 & (62) \\ 35 & 0.4315 & 1270.5 & (62) \\ 35 & 0.4426 & 1279.9 & (62) \\ 35 & 0.4536 & 1289.1 & (62) \\ 37.14 & 0.4539 & 1284.8 & (133) \\ 38.63 & 0.3591 & 1214.9 & (133) \\ 39.36 & 0.4156 & 1256.7 & (133) \\ 39.63 & 0.1003 & 1050.4 & (133) \\ 39.7 & 0.3932 & 1240.1 & (133) \\ 39.71 & 0.3001 & 1173.2 & (133) \\ 39.83 & 0.2022 & 1111.0 & (133) \\ 39.97 & 0.4303 & 1267.2 & (133) \\ 40.03 & 0.2486 & 1139.1 & (133) \\ 45 & 0.0021 & 991.5 & (45) \\ 45 & 0.0042 & 992.7 & (45) \\ 45 & 0.0050 & 993.2 & (80) \\ 45 & 0.0100 & 996.1 & (80) \\ 45 & 0.0150 & 998.9 & (80) \\ 45 & 0.0199 & 1001.7 & (80) \\ 45 & 0.0199 & 1001.7 & (80) \\ 45 & 0.0208 & 1002.2 & (45) \\ 45 & 0.0208 & 1002.2 & (45) \\ 45 & 0.0297 & 1007.3 & (80) \\ 45 & 0.0407 & 1013.4 & (45) \\ 45 & 0.0489 & 1018.4 & (80) \\ 45 & 0.0782 & 1034.9 & (45) \\ 45 & 0.1128 & 1054.9 & (45) \\ 45 & 0.1450 & 1073.6 & (45) \\ 45 & 0.2028 & 1108.5 & (45) \\ 45 & 0.2533 & 1139.8 & (45) \\ 45 & 0.2977 & 1169.3 & (45) \\ 47.28 & 0.4539 & 1280.7 & (133) \\ 48.58 & 0.3591 & 1211.2 & (133) \\ 49.32 & 0.4156 & 1252.6 & (133)\end{array}$

Ref

exp / Calc /

$$
\mathrm{m}^{3} / \mathrm{kg} \quad \mathrm{m}^{3} / \mathrm{kg}
$$

$0.000474 \quad 0.000472$

$\begin{array}{ll}0.000480 & 0.000477\end{array}$

$\begin{array}{ll}0.000481 & 0.000478\end{array}$

$\begin{array}{lll}0.000486 & 0.000481\end{array}$

$0.000488 \quad 0.000483$

$0.000488 \quad 0.000485$

$\begin{array}{ll}0.000490 & 0.000487 \\ 0.000491 & 0.000487\end{array}$

$\begin{array}{ll}0.000491 & 0.000487\end{array}$

$\begin{array}{lll}0.000492 & 0.000489\end{array}$

$\begin{array}{lll}0.000493 & 0.000491\end{array}$

$\begin{array}{ll}0.000494 & 0.000493\end{array}$

$\begin{array}{ll}0.000495 & 0.000494\end{array}$

$\begin{array}{lll}0.000497 & 0.000497\end{array}$

$\begin{array}{ll}0.000497 & 0.000499\end{array}$

$0.000499 \quad 0.000500$

$0.000498 \quad 0.000501$

$0.000498 \quad 0.000502$

$\begin{array}{ll}0.000504 & 0.000502\end{array}$

$0.000494 \quad 0.000493$

$\begin{array}{lll}0.000498 & 0.000499\end{array}$

$\begin{array}{lll}0.000453 & 0.000464\end{array}$

$\begin{array}{lll}0.000496 & 0.000497\end{array}$

$0.000490 \quad 0.000487$

$\begin{array}{ll}0.000475 & 0.000477\end{array}$

$0.000500 \quad 0.000500$

$0.000485 \quad 0.000482$

$0.000385 \quad 0.000448$

$\begin{array}{ll}0.000420 & 0.000448\end{array}$

$0.000408 \quad 0.000448$

$0.000416 \quad 0.000449$

$0.000421 \quad 0.000450$

$0.000426 \quad 0.000451$

$0.000426 \quad 0.000451$

$0.000428 \quad 0.000451$

$0.000428 \quad 0.000451$

$0.000431 \quad 0.000452$

$0.000443 \quad 0.000454$

$0.000439 \quad 0.000455$

$0.000452 \quad 0.000459$

$0.000461 \quad 0.000464$

$0.000469 \quad 0.000468$

$0.000479 \quad 0.000475$

$0.000487 \quad 0.000482$

$\begin{array}{lll}0.000490 & 0.000487\end{array}$

$0.000504 \quad 0.000503$

$\begin{array}{lll}0.000494 & 0.000493\end{array}$

$\begin{array}{lll}0.000498 & 0.000499\end{array}$
Density Density App Vol Square of Inconsistent Inconsistent Apparent Delta density Density of calc/ Residual/ Residual/ Density based on based on molar volume $\mathrm{kg} / \mathrm{m}^{3} \quad \mathrm{~kg} / \mathrm{m}^{3} \quad \mathrm{~m}^{3} / \mathrm{kg}$ Residual density $\begin{gathered}\text { apparen } \\ \text { volume }\end{gathered}$

$1 \mathrm{~cm}^{3} / \mathrm{mol}$

$\begin{array}{llll}0.40 .42 & -0.4159 & 0.000002 & 0.173\end{array}$

$\begin{array}{llll}1107.65 & -0.8492 & 0.000004 & 0.721\end{array}$

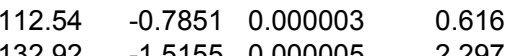

$\begin{array}{llll}1144.80 & -1.6504 & 0.000005 & 2.724\end{array}$

$\begin{array}{llll}1157.31 & -1.1060 & 0.000003 & 1.223\end{array}$

$\begin{array}{llll}1172.35 & -1.1515 & 0.000003 & 1.326\end{array}$

$\begin{array}{llll}1174.29 & -1.6109 & 0.000004 & 2.595\end{array}$

$\begin{array}{llll}1186.93 & -1.2314 & 0.000003 & 1.516\end{array}$

$\begin{array}{llll}1200.89 & -0.9857 & 0.000002 & 0.972\end{array}$

$\begin{array}{llll}1213.13 & -0.4316 & 0.000001 & 0.186\end{array}$

$\begin{array}{llll}1227.13 & -0.5264 & 0.000001 & 0.277\end{array}$

$\begin{array}{lll}0.0181 & 0.000000 & 0.000\end{array}$

$\begin{array}{lll}0.9271 & -0.000001 & 0.859\end{array}$

$\begin{array}{lll}0.6377 & -0.000001 & 0.407\end{array}$

$1.6338-0.000002 \quad 2.669$

$2.3586-0.000003-5.563$

$\begin{array}{lll}-1.2096 & 0.000002 & 1.463\end{array}$

$\begin{array}{lll}-0.5504 & 0.000001 & 0.303\end{array}$

$0.6465-0.000001 \quad 0.418$

1.659

$\begin{array}{lll}1.2881 & -0.000012 & 1.659 \\ 0.6080 & -0.000001 & 0.370\end{array}$

$\begin{array}{lll}-1.0671 & 0.000003 & 1.139\end{array}$

$\begin{array}{lll}0.3802 & -0.000002 & 0.145\end{array}$

$0.3692-0.000001-0.136$

$\begin{array}{lll}-1.0010 & 0.000003 & 1.002\end{array}$

$\begin{array}{lll}0.1300 & -0.000063 & 0.017\end{array}$

$\begin{array}{lll}0.1168 & -0.000028 & 0.014\end{array}$

$\begin{array}{lll}0.1980 & -0.000040 & 0.039\end{array}$

0.107

$\begin{array}{lll}0.3275 & -0.000033 & 0.107 \\ 0.4275 & -0.000029 & 0.183\end{array}$

$\begin{array}{lll}0.4275 & -0.000029 & 0.183 \\ 0.5013 & -0.000025 & 0.251\end{array}$

$\begin{array}{lll}0.5013 & -0.000025 & 0.251\end{array}$

$\begin{array}{lll}0.4756 & -0.000023 & 0.226\end{array}$

$\begin{array}{lll}0.4756 & -0.000023 & 0.226\end{array}$

$\begin{array}{lll}0.6359 & -0.000021 & 0.404\end{array}$

$\begin{array}{lll}0.4508 & -0.000011 & 0.203\end{array}$

$0.8128-0.000016 \quad 0.661$

$\begin{array}{lll}0.6259 & -0.000007 & 0.392\end{array}$

$\begin{array}{lll}0.3645 & -0.000003 & 0.133\end{array}$

$\begin{array}{lll}-0.1052 & 0.000001 & 0.011\end{array}$

$\begin{array}{lll}-0.7838 & 0.000003 & 0.614\end{array}$

2.695

$\begin{array}{lll}-1.6417 & 0.000005 & 2.695 \\ -1.5071 & 0.000004 & 2.271\end{array}$

$\begin{array}{lll}-0.6312 & 0.000001 & 0.398\end{array}$

$\begin{array}{lll}-0.4240 & 0.000001 & 0.180\end{array}$

$\begin{array}{lll}0.7725 & -0.000001 \quad 0.597\end{array}$
994.0319

994.0319

994.0319

994.0319

994.0319

994.0319

994.0319

994.0319

994.0319

994.0319

994.0319

994.0319

994.0319

994.0319

994.0319

994.0319

994.0319

993.2783

992.7325

992.4589

992.3567

992.3301

992.3263

992.2807

992.2272

992.2043

990.2132

990.2132

990.2132

990.2132

990.2132

990.2132

990.2132

990.2132

990.2132

990.2132

990.2132

990.2132

990.2132

990.2132

990.2132

990.2132

990.2132

990.2132

989.2415

988.6716

988.3422 
molality molarity

$\mathrm{t} /{ }^{\circ} \mathrm{C}$ mass frac

Density

$\exp /$

$\mathrm{kg} / \mathrm{m}^{3}$

Ref

exp / Calc /

$\mathrm{m}^{3} / \mathrm{kg} \quad \mathrm{m}^{3} / \mathrm{kg}$

$0.000451 \quad 0.000460$

$\begin{array}{llll}49.63 & 0.1003 & 1046.4 & \text { (133) }\end{array}$

0.05

0.1

0.1189

0.2383
0.3581

0.4786

0.4786

0.5
0.5

0.721

1.2128

2
3
4

4

8
10

0.1189
0.2383
0.3581
0.4786
0.4786
0.721
1.2128

$\begin{array}{rrrrr} & 67.49 & 0.4539 & 1272.3 & (133) \\ 69.24 & 0.4156 & 1244.6 & (133) \\ 69.64 & 0.3932 & 1228.3 & (133) \\ & 69.89 & 0.3001 & 1162.0 & (133) \\ & 69.89 & 0.4303 & 1255.0 & (133) \\ & 69.91 & 0.2486 & 1127.8 & (133) \\ 0.1189 & 69.96 & 0.2022 & 1099.5 & (133) \\ 0.2383 & 75 & 0.0050 & 977.9 & (80)\end{array}$

0.2383 $\begin{array}{llll}49.65 & 0.3932 & 1236.3 \quad(133)\end{array}$

$\begin{array}{llll}49.71 & 0.3001 & 1169.7 & (133)\end{array}$

$49.92 \quad 0.2022$

$49.94 \quad 0.4303$

$50 \quad 0.2486$

$1263.2 \quad(133)$

$0.0050 \quad 988.7$

$0.0100 \quad 991.6 \quad(80)$

$\begin{array}{lll}0.0150 & 994.5 & (80) \\ 0.0199 & 997.3 & (80) \\ 0.0199 & 997.3 & (80)\end{array}$

$0.0199 \quad 997.3 \quad(80)$

$0.0208 \quad 997.8 \quad(45)$

$\begin{array}{rrr}0.0208 & 997.8 & (45) \\ 0.0297 & 1002.9 & (80)\end{array}$

$0.0407 \quad 1009.0$

$0.0489 \quad 1014.1 \quad(80)$

$\begin{array}{lll}0.0782 & 1030.8 & (45) \\ 0.1128 & 1050.9 & (45)\end{array}$

$0.1450 \quad 1069.8 \quad(45)$

$0.2028 \quad 1104.8 \quad(45)$

$\begin{array}{lll}0.2533 & 1136.3 & (45) \\ 0.2977 & 1165.7 & (45)\end{array}$

$\begin{array}{rrrr}55 & 0.2977 & 1165.7 & (45) \\ 57.43 & 0.4539 & 1276.5 & (133)\end{array}$

$\begin{array}{rrrr}58.41 & 0.3591 & 1207.4 & (133) \\ 59.3 & 0.4156 & 1248.5 & (133)\end{array}$

$\begin{array}{llll}59.67 & 0.3932 & 1232.3 & (133)\end{array}$

$\begin{array}{llll}59.9 & 0.4303 & 1259.1 & (133)\end{array}$

$\begin{array}{llll}59.93 & 0.3001 & 1165.9 & \text { (133) }\end{array}$

0.2486
0.0050

0.0100

0.0150

0.0199

0.0199

0.0297

0.0489

$1131.7 \quad(133)$

$986.5 \quad(80)$

$989.4 \quad(80)$

$992.3 \quad(80)$

992.3

998.0

1009.2

(80)

(80)

$(133)$

$0.000495 \quad 0.000497$

0.0004890 .000486

$0.000473 \quad 0.000474$

$0.000500 \quad 0.000501$

$\begin{array}{ll}0.000380 & 0.000441\end{array}$

$0.000415 \quad 0.000441$

$\begin{array}{lll}0.000402 & 0.000441\end{array}$

$\begin{array}{lll}0.000411 & 0.000442\end{array}$

$0.000417 \quad 0.000443$

$\begin{array}{ll}0.000421 & 0.000444 \\ 0.000421 & 0.000444\end{array}$

$\begin{array}{ll}0.000422 & 0.000444\end{array}$

$\begin{array}{ll}0.000422 & 0.000444\end{array}$

$0.000426 \quad 0.000446$

$0.000438 \quad 0.000447$

$\begin{array}{lll}0.000434 & 0.000449\end{array}$

$0.000447 \quad 0.000454$

$0.000457 \quad 0.000459$

$0.000464 \quad 0.000464$

$0.000475 \quad 0.000472$

$0.000484 \quad 0.000479$

$0.000488 \quad 0.000485$

$0.000504 \quad 0.000504$

$0.000493 \quad 0.000492$

$0.000498 \quad 0.000499$

$0.000494 \quad 0.000496$

$0.000499 \quad 0.000501$

$0.000486 \quad 0.000484$

$\begin{array}{lll}0.000480 & 0.000477\end{array}$

$0.000394 \quad 0.000433$

$\begin{array}{ll}0.000403 & 0.000434\end{array}$

$0.000409 \quad 0.000435$

$0.000413 \quad 0.000436$

$\begin{array}{lll}0.000413 & 0.000436\end{array}$

$0.000419 \quad 0.000438$

$0.000427 \quad 0.000441$

$0.000503 \quad 0.000503$

$\begin{array}{lll}0.000496 & 0.000497\end{array}$

$\begin{array}{ll}0.000492 & 0.000494\end{array}$

$\begin{array}{ll}0.000483 & 0.000481\end{array}$

$0.000498 \quad 0.000499$

$\begin{array}{lll}0.000476 & 0.000473\end{array}$

$0.000463 \quad 0.000465$

$0.000386 \quad 0.000423$

$0.000394 \quad 0.000424$
$1135.5 \quad$ (133)

$\begin{array}{lll}0.0021 & 987.0 & (45) \\ 0.0042 & 988.2 & (45)\end{array}$

$0.000483 \quad 0.000480$

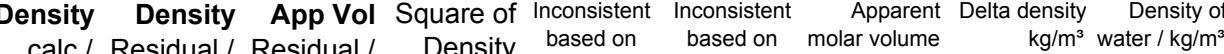
$\begin{array}{lrrr}\text { calc / Residual / Residual / Density based on } & \text { based on } & \text { molar volume } \\ \text { apparent } & / \mathrm{cm}^{3} / \mathrm{mol}\end{array}$ $\mathrm{kg} / \mathrm{m}^{3} \quad \mathrm{~kg} / \mathrm{m}^{3} \quad \mathrm{~m}^{3} / \mathrm{kg}$ Residual density $\begin{gathered}\text { apparent } \\ \text { volume }\end{gathered}$

$045.40 \quad 1.0148-0.000009-1.030$

$1235.44 \quad 0.8382-0.000001 \quad 0.703$

$\begin{array}{llll}1170.67 & -0.9844 & 0.000002 & 0.969\end{array}$

$\begin{array}{lll}0.1939 & -0.000001 & 0.038\end{array}$

$\begin{array}{rrrr}1136.62 & -1.0908 & 0.000003 & 1.190\end{array}$

$\begin{array}{llll}986.88 & 0.1246 & -0.000060 & 0.016\end{array}$

$\begin{array}{llll}88.05 & 0.1082 & -0.000026 & 0.012\end{array}$

$\begin{array}{lllll}988.50 & 0.1944 & -0.000040 & 0.038\end{array}$

$\begin{array}{lllll}991.29 & 0.3030 & -0.000031 & 0.092\end{array}$

$\begin{array}{llll}994.07 & 0.3929 & -0.000027 & 0.154\end{array}$

$\begin{array}{llll}996.84 & 0.4571 & -0.000023 & 0.209\end{array}$

$\begin{array}{llll}996.84 & 0.4571 & -0.000023 & 0.209\end{array}$

$\begin{array}{llll}997.33 & 0.4663 & -0.000023 & 0.217\end{array}$

$\begin{array}{llll}997.33 & 0.4663 & -0.000023 & 0.217\end{array}$

$\begin{array}{llll}0.37 & 0.5749 & -0.000019 & 0.330\end{array}$

$0.3883-0.000009-0.151$

$\begin{array}{lll}0.7360 & -0.000015 & 0.542\end{array}$

$\begin{array}{lll}0.5546 & -0.000007 & 0.308\end{array}$

$\begin{array}{lll}0.2987 & -0.000002 & 0.089\end{array}$

$\begin{array}{lll}-0.0975 & 0.000001 & 0.009\end{array}$

$\begin{array}{lll}-0.7056 & 0.000003 & 0.498\end{array}$

2.118

1.769

0.063

0.079

0.798

1.051

0.868

0.728

1.165

0.035

0.089

0.146

0.195

0.195

0.291

0.491

0.008

1.260

1.187

0.646

1.126

0.952

0.302

0.032

0.083
988.2031

988.1941

988.1671

988.0724

988.0634

988.0363

985.6952

985.6952

985.6952

985.6952

985.6952

985.6952

985.6952

985.6952

985.6952

985.6952

985.6952

985.6952

985.6952

985.6952

985.6952

985.6952

985.6952

985.6952

984.5009

984.0091

983.5574

983.3683

983.2503

9832349

983.2194

980.5548

980.5548

980.5548

980.5548

980.5548

980.5548

980.5548

979.1851

978.2018

977.9747

977.8324

977.8324

977.821

977.7924

974.849

974.849 
molality molarity

$t /{ }^{\circ} \mathrm{C}$ mass frac Density

0.3581
0.4786

0.4786

0.721

1.2128

0.1189

0.2383

0.3581

0.4786
0.4786

0.721
1.2128

1.2128

0.1189

0.2383

0.3581

0.4786

0.4786

0.721
1.2128 $\exp /$ $\mathrm{kg} / \mathrm{m}^{3}$

Ref

983.8

986.7

986.7

992.5

1003.9

971.7

974.8

977.7

980.7

980.7

986.5

998.1

965.1

968.1

971.1

974.1

974.1

980.1

991.8
(80)

$(80)$
$(80)$

$(80)$
$(80)$
$(80)$

$(80)$
$(80)$

$(80)$
$(80)$

(80)

$(80)$
$(80)$

(80)

(80)

$(80)$
$(80)$

$(80)$
$(80)$

(80)

(80)
App vol App vol

$\begin{array}{cc}\exp / & \text { Calc } / \\ \mathrm{m}^{3} / \mathrm{kg} & \mathrm{m}^{3} / \mathrm{kg}\end{array}$

$0.000400 \quad 0.000425$

$0.000404 \quad 0.000426$

$0.000404 \quad 0.000426$

$\begin{array}{ll}0.000410 & 0.000428\end{array}$

$0.000418 \quad 0.000432$

$0.000374 \quad 0.000411$

$0.000383 \quad 0.000412$

$0.000389 \quad 0.000414$

$0.000394 \quad 0.000415$

$0.000394 \quad 0.000415$

$\begin{array}{lll}0.000400 & 0.000417\end{array}$

$0.000409 \quad 0.000422$

$\begin{array}{lll}0.000361 & 0.000398\end{array}$

$0.000372 \quad 0.000399$

$0.000378 \quad 0.000400$

$0.000383 \quad 0.000402$

$0.000383 \quad 0.000402$

$0.000390 \quad 0.000404$

$0.000398 \quad 0.000409$

Average Res

Std dev Res

Avg - 4std

$\mathrm{Avg}+4 \mathrm{std}$

Curve fit for the model App vol $=\left(w+c 2+c 3^{\star} t{ }^{\circ} \mathrm{C}\right) /\left(\mathrm{c} 0^{\star} w+c 1\right) / \mathrm{e}^{\wedge}\left(0.000001^{*}\left(\mathrm{t}^{\circ} \mathrm{C}+\mathrm{c} 4\right)^{\wedge} 2\right)$

c1

c3 -0.00623

c4 -2131.59

Min

Max T

Min w $\quad 0.0021$

5

Max w $\quad 0.4539$

0.3421

Std dens res $\quad 0.93$

Avg app vol res *10^3 $\quad-0.01654$

Std app vol res *10^3 0.019592

No of points in corr

No of inconsistent poir

332
0

Inconsistent data not used (Residual greater than average $+/-4$ standard deviations) 
Density of aqueous solutions of $\mathrm{Li2SO} 4$

References (12) Cartón, A.; Sobrón, F.; Bolado, S.; Gerbolés, J. I. Density, Viscosity, and Electrical Conductivity of Aqueous Solutions of Lithium Sulfate. J. Chem. Eng. Data 1995, 40, 987-991.

(48) Kaminsky, M. Concentration and Temperature Dependence of the Viscosity of Aqueous Solutions of Strong Electrolytes. II. NaCl, Li2SO4, FeCl2, and CeCl3 Solutions. Z. Physik. Chem. N. F, $1956,8,173-191$.

(85) Pearce, J. N.; Eckstrom, H. C. Vapor Pressures and Partial Molal Volumes of Aqueous Solutions of the Alkali Sulfates at $25^{\circ} \mathrm{C} . \mathrm{J}$. Am. Chem. Soc. 1937, 59, 2689-2691.

(134) Wirth, H. E.; Lo Surdo, A. Temperature Dependence of Volume Changes of Aqueous Solutions of Ammonium Chloride and Ammonium Nitrate at $25^{\circ} \mathrm{C}$. J. Chem. Eng. Data 1968, 13, 226-231.

\begin{tabular}{|c|c|c|c|c|c|c|c|c|c|c|c|c|c|}
\hline molality & molarity & \multicolumn{2}{|c|}{$\mathrm{t} /{ }^{\circ} \mathrm{C}$ mass frac } & $\begin{array}{r}\text { Density } \\
\exp / \\
\mathrm{kg} / \mathrm{m}^{3}\end{array}$ & Ref & $\begin{array}{r}\text { App vol } \\
\exp / \\
\mathrm{m}^{3} / \mathrm{kg}\end{array}$ & $\begin{array}{r}\text { App vol } \\
\text { Calc / } \\
\mathrm{m}^{3} / \mathrm{kg}\end{array}$ & $\begin{array}{r}\text { Density } \\
\text { calc / } \\
\mathrm{kg} / \mathrm{m}^{3}\end{array}$ & $\begin{array}{r}\text { Density } \\
\text { Residual / } \\
\mathrm{kg} / \mathrm{m}^{3}\end{array}$ & $\begin{array}{r}\text { App Vol } \\
\text { Residual / } \\
\mathrm{m}^{3} / \mathrm{kg}\end{array}$ & $\begin{array}{r}\text { Square of } \\
\text { Density } \\
\text { Residual }\end{array}$ & $\begin{array}{l}\text { Inconsistent } \\
\text { based on } \\
\text { density }\end{array}$ & $\begin{array}{l}\text { Inconsistent } \\
\text { based on } \\
\text { apparent } \\
\text { volume }\end{array}$ \\
\hline 1.3334 & & 0 & 0.1279 & 1116.29 & $(134)$ & 0.000184 & 0.000185 & 1116.10 & 0.1847 & -0.000001 & 0.034 & & \\
\hline 2.9853 & & 5 & 0.2471 & 1229.08 & $(12)$ & 0.000246 & 0.000246 & 1229.05 & 0.0253 & 0.000000 & 0.001 & & \\
\hline 3.066 & & 5 & 0.2521 & 1234.54 & (12) & 0.000246 & 0.000248 & 1233.94 & 0.5971 & -0.000002 & 0.357 & & \\
\hline 3.1248 & & 5 & 0.2557 & 1238.10 & (12) & 0.000248 & 0.000249 & 1237.47 & 0.6299 & -0.000002 & 0.397 & & \\
\hline 3.1992 & & 5 & 0.2602 & 1242.26 & $(12)$ & 0.000250 & 0.000251 & 1241.89 & 0.3680 & -0.000001 & 0.135 & & \\
\hline 1.3334 & & 5.12 & 0.1279 & 1115.19 & $(134)$ & 0.000192 & 0.000191 & 1115.34 & -0.1473 & 0.000001 & 0.022 & & \\
\hline 0.4057 & & 10 & 0.0427 & 1037.18 & $(12)$ & 0.000154 & 0.000154 & 1037.16 & 0.0217 & 0.000000 & 0.000 & & \\
\hline 0.7952 & & 10 & 0.0804 & 1071.19 & (12) & 0.000170 & 0.000173 & 1070.91 & 0.2822 & -0.000003 & 0.080 & & \\
\hline 1.1924 & & 10 & 0.1159 & 1103.09 & (12) & 0.000191 & 0.000190 & 1103.28 & -0.1899 & 0.000001 & 0.036 & & \\
\hline 1.5788 & & 10 & 0.1479 & 1132.37 & (12) & 0.000208 & 0.000205 & 1132.95 & -0.5823 & 0.000003 & 0.339 & & \\
\hline 1.9562 & & 10 & 0.1770 & 1160.00 & (12) & 0.000219 & 0.000218 & 1160.34 & -0.3387 & 0.000001 & 0.115 & & \\
\hline 2.603 & & 10 & 0.2225 & 1202.86 & (12) & 0.000241 & 0.000238 & 1203.95 & -1.0902 & 0.000003 & 1.189 & & \\
\hline 2.9853 & & 10 & 0.2471 & 1227.65 & (12) & 0.000249 & 0.000248 & 1227.93 & -0.2831 & 0.000001 & 0.080 & & \\
\hline 3.066 & & 10 & 0.2521 & 1233.09 & (12) & 0.000249 & 0.000250 & 1232.84 & 0.2525 & -0.000001 & 0.064 & & \\
\hline 3.1248 & & 10 & 0.2557 & 1237.05 & (12) & 0.000250 & 0.000251 & 1236.38 & 0.6725 & -0.000002 & 0.452 & & \\
\hline 3.1992 & & 10 & 0.2602 & 1241.27 & (12) & 0.000252 & 0.000253 & 1240.82 & 0.4534 & -0.000001 & 0.206 & & \\
\hline 2.9853 & & 15 & 0.2471 & 1226.13 & (12) & 0.000251 & 0.000250 & 1226.61 & -0.4830 & 0.000001 & 0.233 & & \\
\hline 3.066 & & 15 & 0.2521 & 1231.65 & (12) & 0.000251 & 0.000252 & 1231.54 & 0.1136 & 0.000000 & 0.013 & & \\
\hline 3.1248 & & 15 & 0.2557 & 1235.29 & (12) & 0.000252 & 0.000253 & 1235.09 & 0.1990 & -0.000001 & 0.040 & & \\
\hline 3.1992 & & 15 & 0.2602 & 1239.96 & (12) & 0.000254 & 0.000255 & 1239.55 & 0.4101 & -0.000001 & 0.168 & & \\
\hline 1.3334 & & 15.04 & 0.1279 & 1112.68 & (134) & 0.000202 & 0.000200 & 1112.97 & -0.2960 & 0.000002 & 0.088 & & \\
\hline & 0.00085 & 15.1 & 0.0001 & 999.16 & $(48)^{\prime}$ & 0.000145 & 0.000139 & 999.16 & -0.0005 & 0.000005 & 0.000 & & \\
\hline & 0.00172 & 15.1 & 0.0002 & 999.25 & (48) & 0.000102 & 0.000140 & 999.25 & 0.0071 & -0.000038 & 0.000 & & \\
\hline & 0.00423 & 15.1 & 0.0005 & 999.49 & (48) & 0.000119 & 0.000140 & 999.48 & 0.0095 & -0.000020 & 0.000 & & \\
\hline & 0.00858 & 15.1 & 0.0009 & 999.91 & (48) & 0.000121 & 0.000140 & 999.90 & 0.0178 & -0.000019 & 0.000 & & \\
\hline & 0.01694 & 15.1 & 0.0019 & 1000.71 & (48) & 0.000126 & 0.000140 & 1000.69 & 0.0273 & -0.000015 & 0.001 & & \\
\hline & 0.0429 & 15.1 & 0.0047 & 1003.19 & (48) & 0.000130 & 0.000142 & 1003.13 & 0.0582 & -0.000012 & 0.003 & & \\
\hline & 0.08579 & 15.1 & 0.0094 & 1007.25 & (48) & 0.000135 & 0.000144 & 1007.16 & 0.0897 & -0.000009 & 0.008 & & \\
\hline & 0.17348 & 15.1 & 0.0188 & 1015.42 & (48) & 0.000144 & 0.000149 & 1015.32 & 0.1002 & -0.000005 & 0.010 & & \\
\hline & 0.00084 & 20 & 0.0001 & 998.28 & (48) & 0.000136 & 0.000146 & 998.28 & 0.0010 & -0.000010 & 0.000 & & \\
\hline
\end{tabular}

Relative Density of density water $/ \mathrm{kg} / \mathrm{m}^{3}$

999.8395 999.9638 999.9638 999.9638 999.9638 999.9618 999.6996 999.6996 999.6996 999.6996 999.6996 999.6996 999.6996 999.6996 999.6996 999.6996 999.0996 999.0996 999.0996 999.0996 999.0936

$1.00008 \quad 999.0845$ 1.00017999 .0845 1.00041999 .0845 1.00083999 .0845 1.00163999 .0845 1.00411999 .0845 1.00817999 .0845 1.01635999 .0845 1.00008998 .2041 
molality molarity

\section{$\mathrm{t} /{ }^{\circ} \mathrm{C}$ mass frac Density}

0.00171

0.00423

0.00857

0.01692

0.04285

0.08571

0.4057

0.7952

1.1924

1.5788

1.9562

2.603

2.9853

3.066

3.1248

3.1992

1.3334

0.00171

0.00422

0.00856

0.0169

0.0428

0.08559

0.1

0.17309

0.2

0.4
4057

0.6

0.7952

0.8

1.1924

1.5

1.5788

1.9562

2.603 $\exp /$

$\mathrm{kg} / \mathrm{m}^{3}$

$\begin{array}{rrrr}\mathbf{t} /{ }^{\circ} \mathrm{C} \text { mass frac } & \text { Density } & \text { Ref } \\ & & \exp / & \\ & & \mathrm{kg} / \mathrm{m}^{3} & \\ 20 & 0.0002 & 998.37 & (48) \\ 20 & 0.0005 & 998.61 & (48) \\ 20 & 0.0009 & 999.03 & (48) \\ 20 & 0.0019 & 999.83 & (48) \\ 20 & 0.0047 & 1002.30 & (48) \\ 20 & 0.0094 & 1006.34 & (48) \\ 20 & 0.0188 & 1014.47 & (48) \\ 20 & 0.0427 & 1035.09 & (12) \\ 20 & 0.0804 & 1068.70 & (12) \\ 20 & 0.1159 & 1100.32 & (12) \\ 20 & 0.1479 & 1129.42 & (12) \\ 20 & 0.1770 & 1156.94 & (12) \\ 20 & 0.2225 & 1199.70 & (12) \\ 20 & 0.2471 & 1224.68 & (12) \\ 20 & 0.2521 & 1230.23 & (12) \\ 20 & 0.2557 & 1233.66 & (12) \\ 20 & 0.2602 & 1238.32 & (12) \\ 24.6 & 0.1279 & 1109.74 & (134) \\ 25 & 0.0002 & 997.21 & (48) \\ 25 & 0.0005 & 997.45 & (48) \\ 25 & 0.0009 & 997.87 & (48) \\ 25 & 0.0019 & 998.67 & (48) \\ 25 & 0.0047 & 1001.12 & (48) \\ 25 & 0.0094 & 1005.15 & (48) \\ 25 & 0.0109 & 1006.48 & (85) \\ 25 & 0.0188 & 1013.26 & (48) \\ 25 & 0.0215 & 1015.63 & (85) \\ 25 & 0.0421 & 1033.37 & (85) \\ 25 & 0.0427 & 1033.72 & (12) \\ 25 & 0.0619 & 1050.53 & (85) \\ 25 & 0.0804 & 1067.20 & (12) \\ 25 & 0.0808 & 1067.22 & (85) \\ 25 & 0.0991 & 1083.42 & (85) \\ 25 & 0.1159 & 1098.74 & (12) \\ 25 & 0.1416 & 1122.18 & (85) \\ 25 & 0.1479 & 1127.79 & (12) \\ 25 & 0.1770 & 1155.28 & (12) \\ 25 & 0.1803 & 1158.51 & (85) \\ 25 & 0.2156 & 1192.68 & (85) \\ 25 & 0.2225 & 1198.09 & (12) \\ & & & \end{array}$

\section{App vol App vol}

$\begin{array}{cc}\exp / & \text { Calc / } \\ \mathrm{m}^{3} / \mathrm{kg} & \mathrm{m}^{3} / \mathrm{kg}\end{array}$

$0.000098 \quad 0.000146$

$0.000120 \quad 0.000146$

$0.000121 \quad 0.000146$

$\begin{array}{ll}0.000126 & 0.000147\end{array}$

$0.000132 \quad 0.000148$

$0.000137 \quad 0.000150$

$\begin{array}{ll}0.000146 & 0.000155\end{array}$

$0.000166 \quad 0.000166$

$0.000180 \quad 0.000183$

$0.000200 \quad 0.000198$

$0.000215 \quad 0.000212$

$0.000225 \quad 0.000224$

$\begin{array}{ll}0.000246 & 0.000242\end{array}$

$0.000252 \quad 0.000251$

$0.000252 \quad 0.000253$

$0.000254 \quad 0.000254$

$0.000255 \quad 0.000256$

$0.000207 \quad 0.000206$

$0.000099 \quad 0.000152$

$0.000119 \quad 0.000152$

$0.000121 \quad 0.000152$

$0.000126 \quad 0.000153$

$0.000134 \quad 0.000154$

$0.000139 \quad 0.000156$

$0.000139 \quad 0.000157$

$0.000149 \quad 0.000160$

$0.000150 \quad 0.000162$

$\begin{array}{lll}0.000166 & 0.000171\end{array}$

$\begin{array}{ll}0.000170 & 0.000171\end{array}$

$0.000178 \quad 0.000179$

$\begin{array}{ll}0.000183 & 0.000187\end{array}$

$0.000187 \quad 0.000187$

$0.000196 \quad 0.000195$

$0.000202 \quad 0.000202$

$0.000213 \quad 0.000212$

$0.000217 \quad 0.000215$

$0.000227 \quad 0.000226$

$0.000227 \quad 0.000227$

$0.000240 \quad 0.000240$

$\begin{array}{lll}0.000247 & 0.000243\end{array}$
Density Density App Vol Square of Inconsistent Inconsistent calc / Residual/ Residual / Density based on based on $\mathrm{kg} / \mathrm{m}^{3} \quad \mathrm{~kg} / \mathrm{m}^{3} \quad \mathrm{~m}^{3} / \mathrm{kg}$ Residual density apparent $\mathrm{kg} / \mathrm{m}^{3} \quad \mathrm{~m}^{3} / \mathrm{kg}$ Residual volume

$\begin{array}{llll}998.60 & 0.0120 & -0.000026 & 0.000\end{array}$

$\begin{array}{llll}999.01 & 0.0240 & -0.000025 & 0.001\end{array}$

$999.79 \quad 0.0395-0.000021 \quad 0.002$

$\begin{array}{llll}0.0787 & -0.000017 & 0.006\end{array}$

$1006.21 \quad 0.1279-0.000013 \quad 0.016$

$1014.31 \quad 0.1639-0.000008 \quad 0.027$

$\begin{array}{llll}035.08 & 0.0136 & 0.000000 & 0.000\end{array}$

$\begin{array}{llll}068.41 & 0.2932 & -0.000003 & 0.086\end{array}$

$\begin{array}{llll}1100.48 & -0.1636 & 0.000001 & 0.027\end{array}$

$\begin{array}{llll}1129.98 & -0.5643 & 0.000003 & 0.318\end{array}$

$\begin{array}{llll}157.30 & -0.3621 & 0.000002 & 0.131\end{array}$

$\begin{array}{llll}1200.99 & -1.2949 & 0.000004 & 1.677\end{array}$

$\begin{array}{llll}125.13 & -0.4485 & 0.000001 & 0.201\end{array}$

$\begin{array}{llll}230.07 & 0.1565 & 0.000000 & 0.025\end{array}$

$\begin{array}{llll}1233.64 & 0.0153 & 0.000000 & 0.000\end{array}$

$\begin{array}{llll}238.13 & 0.1944 & 0.000000 & 0.038\end{array}$

$\begin{array}{llll}109.83 & -0.0995 & 0.000001 & 0.010\end{array}$

$\begin{array}{llll}997.20 & 0.0100 & -0.000053 & 0.000\end{array}$

$\begin{array}{lll}997.44 & 0.0152 & -0.000033\end{array}$

$\begin{array}{llll}997.84 & 0.0294 & -0.000031 & 0.001\end{array}$

$\begin{array}{llll}998.62 & 0.0501 & -0.000027 & 0.003\end{array}$

$1001.03 \quad 0.0953-0.000020 \quad 0.009$

$0.1620-0.000017$

$1006.28 \quad 0.1980-0.000018 \quad 0.039$

$1013.03 \quad 0.2286-0.000012 \quad 0.052$

$\begin{array}{llll}1015.37 & 0.2560 & -0.000012 & 0.066\end{array}$

$1033.16 \quad 0.2087-0.000005 \quad 0.044$

$1033.66 \quad 0.0634-0.000001 \quad 0.004$

$\begin{array}{llll}1050.43 & 0.1035 & -0.000002 & 0.011\end{array}$

$1066.81 \quad 0.3932-0.000004 \quad 0.155$

$\begin{array}{llll}1067.20 & 0.0151 & 0.000000 & 0.000\end{array}$

$\begin{array}{llll}083.51 & -0.0844 & 0.000001 & 0.007\end{array}$

$\begin{array}{llll}1098.76 & -0.0238 & 0.000000 & 0.001\end{array}$

$\begin{array}{llll}1122.33 & -0.1414 & 0.000001 & 0.020\end{array}$

$\begin{array}{llll}1128.20 & -0.4136 & 0.000002 & 0.171\end{array}$

$\begin{array}{llll}1155.51 & -0.2294 & 0.000001 & 0.053\end{array}$

$\begin{array}{llll}1158.59 & -0.0767 & 0.000000 & 0.006\end{array}$

$\begin{array}{llll}1192.55 & 0.1316 & 0.000000 & 0.017\end{array}$

$\begin{array}{llll}1199.28 & -1.1872 & 0.000004 & 1.409\end{array}$
Relative Density of density water $/ \mathrm{kg} / \mathrm{m}^{3}$

1.00017998 .2041

1.00041998 .2041

1.00083998 .2041

1.00163998 .2041

1.0041998 .2041

1.00815998 .2041

1.0163998 .2041

998.2041

998.2041

998.2041

998.2041

998.2041

998.2041

998.2041

998.2041

998.2041

998.2041

997.1467

$1.00017 \quad 997.0449$

1.00041997 .0449

1.00083997 .0449

$1.00163 \quad 997.0449$

1.00409997 .0449

$1.00813 \quad 997.0449$ 997.0449

1.01626997 .0449 997.0449

997.0449

997.0449

997.0449

997.0449

997.0449

997.0449

997.0449

997.0449

997.0449

997.0449

997.0449

997.0449

997.0449 
molality molarity

2.9853
3.066
3.0944
3.1248
3.1992
3.1248
3.1992

3.1992

0.00084

0.00171

0.00854

0.0169

0.04276

0.08545

0.17278

0.4057

0.7952

1.1924

1.5788

1.9562

2.603

2.9853

3.066

3.1248

3.1992

3.1248

3.1992

1.3334

0.00084

0.0017

0.00421

0.01684

0.04266

0.08531

0.1725

2.9853

3.066

3.1248

3.1248

3.066

3.1248 $\mathrm{t} /{ }^{\circ} \mathrm{C}$ mass frac Density exp / $\exp /$
$\mathrm{kg} / \mathrm{m}^{3}$

223.05

$.2571 \quad 1223.05$

$0.2538 \quad 1231.00$

$0.2557 \quad 1232.11$

$0.2602 \quad 1236.80$

$0.2557 \quad 1231.40$

$0.2602 \quad 1235.96$

$0.0001 \quad 995.73$

$0.0002 \quad 995.82$

$0.0009 \quad 996.47$

$0.0019 \quad 997.27$

$0.0047 \quad 999.73$

$0.0094 \quad 1003.73$

$0.0188 \quad 1011.77$

$0.0427 \quad 1032.20$

$0.0804 \quad 1065.59$

$0.1159 \quad 1097.05$

$0.1479 \quad 1126.05$

$0.1770 \quad 1153.55$

$0.2225 \quad 1196.32$

$0.2471 \quad 1221.34$

$0.2521 \quad 1226.98$

$0.2557 \quad 1230.40$

$0.2602 \quad 1234.95$

$0.2557 \quad 1229.66$

$0.2602 \quad 1233.28$

$0.1279 \quad 1106.04$

$0.0001 \quad 994.11$

$0.0002 \quad 994.20$

$0.0005 \quad 994.44$

$0.0019 \quad 995.66$

$0.0047 \quad 998.11$

$0.0094 \quad 1002.10$

$0.0188 \quad 1010.11$

$0.2471 \quad 1219.51$

$0.2521 \quad 1225.13$

$0.2557 \quad 1228.58$

$0.2557 \quad 1227.82$

$0.2521 \quad 1223.98$

$0.2557 \quad 1227.07$

(n)

Ref

exp / Calc /

$$
\mathrm{m}^{3} / \mathrm{kg} \quad \mathrm{m}^{3} / \mathrm{kg}
$$

$0.000253 \quad 0.000252$

$0.000253 \quad 0.000253$

$0.000252 \quad 0.000254$

$0.000255 \quad 0.000255$

$0.000256 \quad 0.000256$

$0.000255 \quad 0.000255$

0.0002560 .000256

$\begin{array}{lll}0.000138 & 0.000157\end{array}$

$\begin{array}{ll}0.000100 & 0.000157\end{array}$

$0.000120 \quad 0.000158$

$0.000127 \quad 0.000158$

$0.000132 \quad 0.000159$

$0.000140 \quad 0.000161$

$\begin{array}{ll}0.000152 & 0.000165\end{array}$

$0.000171 \quad 0.000175$

$0.000184 \quad 0.000191$

$0.000203 \quad 0.000205$

$0.000218 \quad 0.000217$

$0.000228 \quad 0.000228$

$\begin{array}{lll}0.000247 & 0.000244\end{array}$

$0.000253 \quad 0.000252$

$0.000253 \quad 0.000254$

0.0002550 .000255

$0.000256 \quad 0.000257$

$0.000255 \quad 0.000255$

0.0002590 .000257

$0.000209 \quad 0.000211$

$\begin{array}{lll}0.000140 & 0.000162\end{array}$

$0.000096 \quad 0.000162$

$0.000120 \quad 0.000162$

$0.000120 \quad 0.000163$

0.0001320 .000164

$0.000140 \quad 0.000166$

$0.000153 \quad 0.000170$

$0.000253 \quad 0.000252$

$0.000253 \quad 0.000254$

$0.000255 \quad 0.000255$

$0.000255 \quad 0.000255$

$0.000253 \quad 0.000254$

$0.000255 \quad 0.000255$
Density Density App Vol Square of Inconsistent Inconsistent calc / Residual / Residual / Density based on based on $\mathrm{kg} / \mathrm{m}^{3} \quad \mathrm{~kg} / \mathrm{m}^{3} \quad \mathrm{~m}^{3} / \mathrm{kg}$ Residual density apparent

$1223.50 \quad-0.4542 \quad 0.000001 \quad 0.206$

$1228.47 \quad 0.2270-0.000001 \quad 0.052$

$\begin{array}{llll}1230.21 & 0.7900 & -0.000002 & 0.624\end{array}$

$\begin{array}{llll}1232.06 & 0.0475 & 0.000000 & 0.002\end{array}$

$1236.57 \quad 0.2325-0.000001 \quad 0.054$

$\begin{array}{llll}1231.40 & 0.0044 & 0.000000 & 0.000\end{array}$

$\begin{array}{llll}1235.91 & 0.0492 & 0.000000 & 0.002\end{array}$

$\begin{array}{llll}995.73 & 0.0018 & -0.000019 & 0.000\end{array}$

$\begin{array}{llll}995.81 & 0.0107 & -0.000057 & 0.000\end{array}$

$\begin{array}{lllll}996.44 & 0.0348 & -0.000037 & 0.001\end{array}$

$\begin{array}{llll}997.21 & 0.0572 & -0.000031 & 0.003\end{array}$

$999.60 \quad 0.1268-0.000027 \quad 0.016$

$1003.53 \quad 0.1998-0.000021 \quad 0.040$

$\begin{array}{llll}1011.51 & 0.2536 & -0.000013 & 0.064\end{array}$

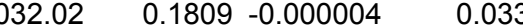

$\begin{array}{lllll}1065.01 & 0.5824 & -0.000006 & 0.339\end{array}$

$\begin{array}{llll}1126.26 & -0.2060 & 0.000001 & 0.042\end{array}$

$\begin{array}{llll}6.26 & -0.2060 & 0.000001 & 0.042\end{array}$

$\begin{array}{llll}153.56 & -0.0134 & 0.000000 & 0.000\end{array}$

$\begin{array}{llll}197.43 & -1.1065 & 0.000003 & 1.224\end{array}$

$\begin{array}{llll}1226.75 & 0.2277 & -0.000001 & 0.052\end{array}$

$\begin{array}{llll}1230.36 & 0.0384 & 0.000000 & 0.001\end{array}$

$\begin{array}{llll}234.89 & 0.0574 & 0.000000 & 0.003\end{array}$

$\begin{array}{llll}1229.65 & 0.0092 & 0.000000 & 0.000\end{array}$

$\begin{array}{llll}1234.19 & -0.9127 & 0.000002 & 0.833\end{array}$

$\begin{array}{llll}105.71 & 0.3311 & -0.000002 & 0.110\end{array}$

$\begin{array}{llll}994.11 & 0.0020 & -0.000022 & 0.000\end{array}$

$\begin{array}{llll}994.19 & 0.0122 & -0.000066 & 0.000\end{array}$

$994.42 \quad 0.0193-0.000042 \quad 0.000$

$\begin{array}{llll}995.58 & 0.0783 & -0.000042 & 0.006\end{array}$

$\begin{array}{lllll}997.96 & 0.1498 & -0.000032 & 0.022\end{array}$

$\begin{array}{llll}1001.86 & 0.2397 & -0.000026 & 0.057\end{array}$

$\begin{array}{llll}1009.79 & 0.3108 & -0.000016 & 0.097\end{array}$

$\begin{array}{llll}1219.90 & -0.3908 & 0.000001 & 0.153\end{array}$

$\begin{array}{llll}1224.92 & 0.2065 & -0.000001 & 0.043\end{array}$

$\begin{array}{llll}1228.55 & 0.0261 & 0.000000 & 0.001\end{array}$

$\begin{array}{llll}1227.80 & 0.0171 & 0.000000 & 0.000\end{array}$

$\begin{array}{llll}1223.78 & 0.2020 & -0.000001 & 0.041\end{array}$

$\begin{array}{llll}1227.04 & 0.0334 & 0.000000 & 0.001\end{array}$
Relative Density of density water $/ \mathrm{kg} / \mathrm{m}^{3}$

997.0449

997.0449

997.0449

997.0449

997.0449

996.5132

996.5132

$1.00008 \quad 995.6473$

$1.00017 \quad 995.6473$

1.00083995 .6473

1.00163995 .6473

1.0041995 .6473

1.00812995 .6473

1.01619995 .6473

995.6473

995.6473

995.6473

995.6473

995.6473

995.6473

995.6473

995.6473

995.6473

995.6473

995.0262

995.0262

994.0559

$1.00008 \quad 994.0319$

1.00017994 .0319

1.00041994 .0319

$1.00164 \quad 994.0319$

1.0041994 .0319

1.00812994 .0319

1.01617994 .0319 994.0319 994.0319

994.0319

993.3287

992.9653

992.5943 
molality molarity

$\mathbf{t} /{ }^{\circ} \mathrm{C}$ mass frac Density
$\exp /$
$\mathrm{kg} / \mathrm{m}^{3}$

$t /{ }^{\circ} \mathrm{C}$ mass frac Density
$\exp /$
$\mathrm{kg} / \mathrm{m}^{3}$

$t /{ }^{\circ} \mathrm{C}$ mass frac Density
$\exp /$
$\mathrm{kg} / \mathrm{m}^{3}$

0.4057

0.7952

1.1924

1.5788

1.9562

2.603

2.9853

3.066

3.1248

3.1248

3.066

2.9853

3.066

1.3334

1.3334

3.066

2.9853

3.066

2.9853

2.9853

2.9853

2.9853

2.9853

2.9853

2.9853

2.9853

2.9853

Ref

$\begin{array}{llll}40 & 0.0427 & 1028.62 & (12) \\ 40 & 0.0804 & 1061.89 & (12) \\ 40 & 0.1159 & 1093.27 & (12)\end{array}$

$\begin{array}{lll}0.1159 & 1093.27 & (12) \\ 0.1479 & 1122.31 & (12)\end{array}$

$0.1770 \quad 1150.11 \quad$ (12)

$\begin{array}{lll}0.2225 & 1192.81 \quad(12)\end{array}$

$\begin{array}{lll}0.2471 & 1217.67 & (12)\end{array}$

$0.2521 \quad 1223.23$

$0.2557 \quad 1226.71 \quad(12)$

$0.2557 \quad 1226.30 \quad(12)$

$0.0001 \quad 991.33 \quad(48)$

$0.0002 \quad 991.41 \quad(48)$

0.00169

0.0042

0.00851

0.0168

0.04255

0.08508

0.171999

(1)

4

$\begin{array}{lll}42.5 & 0.0005 & 991.65 \\ 42.5 & 0.0009 & 992.06 \\ 42.5 & 0.0019 & 992.86\end{array}$

$\begin{array}{lll}42.5 & 0.0019 & 992.86 \\ 42.5 & 0.0047 & 995.31\end{array}$

$\begin{array}{lll}42.5 & 0.0094 & 999.30\end{array}$

$\begin{array}{lll}42.5 & 0.0188 & 1007.27\end{array}$

$\begin{array}{ll}0.2521 & 1221.94\end{array}$

$0.2471 \quad 1215.59$

$0.2521 \quad 1221.31$

$\begin{array}{llll}45.02 & 0.1279 & 1101.95 & (134)\end{array}$

$\begin{array}{llll}45.12 & 0.1279 & 1101.94 & (134)\end{array}$

$\begin{array}{llll}0.2521 & 1220.47 \quad(12)\end{array}$

$0.2471 \quad 1213.36 \quad(12)$

$0.2521 \quad 1219.11 \quad(12)$

$0.2471 \quad 1213.12 \quad$ (12)

$0.2471 \quad 1212.62 \quad(12)$

$0.2471 \quad 1212.39 \quad(12)$

$0.2471 \quad 1211.92 \quad$ (12)

$0.2471 \quad 1211.67 \quad(12)$

$0.2471 \quad 1210.05 \quad(12)$

$0.2471 \quad 1209.13 \quad(12)$

$0.2471 \quad 1207.96 \quad(12)$

$\begin{array}{llll}65 & 0.2471 & 1206.97 & (12)\end{array}$

\section{App vol App vol Der}

$\begin{array}{cc}\exp / & \text { Calc / } \\ \mathrm{m}^{3} / \mathrm{kg} & \mathrm{m}^{3} / \mathrm{kg}\end{array}$

Density

App Vol Square of Inconsistent Inconsistent / / Residual / Residual / Density based on based on $\mathrm{kg} / \mathrm{m}^{3} \quad \mathrm{~kg} / \mathrm{m}^{3} \quad \mathrm{~m}^{3} / \mathrm{kg}$ Residual density apparent

$0.000172 \quad 0.000183$

$0.000185 \quad 0.000196$

1028.17

1060.88

1092.58

$0.000204 \quad 0.000209$

$0.000218 \quad 0.000220$

$0.000226 \quad 0.000230$

1121.92

1149.27

1193.38

1217.94

$0.000253 \quad 0.000252$

$0.000253 \quad 0.000253$

1223.00

1226.65

$0.000254 \quad 0.000255$

$0.000254 \quad 0.000254$

$0.000034 \quad 0.000168$

$0.000094 \quad 0.000168$

$0.000099 \quad 0.000168$

0.0001220 .000168

$0.000121 \quad 0.000169$

$0.000130 \quad 0.000170$

$0.000140 \quad 0.000172$

$0.000154 \quad 0.000175$

$0.000253 \quad 0.000253$

$0.000252 \quad 0.000251$

$0.000252 \quad 0.000253$

0.0002090 .000215

0.0002090 .000215

$0.000252 \quad 0.000252$

$0.000252 \quad 0.000250$

$0.000251 \quad 0.000252$

$0.000251 \quad 0.000250$

$0.000251 \quad 0.000250$

$0.000250 \quad 0.000250$

$0.000250 \quad 0.000250$

$0.000249 \quad 0.000249$

$0.000249 \quad 0.000248$

$0.000248 \quad 0.000248$

$\begin{array}{lll}0.000246 & 0.000247\end{array}$

$\begin{array}{lll}0.000246 & 0.000246 & 1206.78\end{array}$

Average Res

Std dev Re

Avg - 4std

Avg + 4std
$0.4538-0.000010$

$1.0078-0.000011$

$0.6918-0.000005$

$0.3879-0.000002$

$0.8410-0.000004$

$\begin{array}{ll}0.5663 & 0.000002\end{array}$

$\begin{array}{ll}-0.2729 & 0.000001\end{array}$

$0.2349-0.000001$

$0.0622 \quad 0.000000$

$0.0448 \quad 0.000000$

$0.0122-0.000134$

$0.0137-0.000074$

$0.0315-0.000069$

$0.0433-0.000047$

$0.0876-0.000048$

$0.1839-0.000040$

$0.2978-0.000032$

$0.4071-0.000021$

$0.1474 \quad 0.000000$

$\begin{array}{ll}-0.2995 & 0.000001\end{array}$

$0.3373-0.000001$

$0.8765-0.000006$

$0.9081-0.000006$

$0.3317-0.000001$

$-0.3845 \quad 0.000001$

$0.2501-0.000001$

$\begin{array}{ll}-0.1847 & 0.000001\end{array}$

$\begin{array}{ll}-0.2414 & 0.000001\end{array}$

$\begin{array}{ll}-0.0245 & 0.000000\end{array}$

$\begin{array}{ll}-0.0440 & 0.000000\end{array}$

$0.1600 \quad 0.000000$

$\begin{array}{ll}-0.0768 & 0.000000\end{array}$

$-0.0570 \quad 0.000000$

$0.2093-0.000001$

$0.1946-0.000001$

$0.0457-0.000010$

$0.3577 \quad 0.000019$

$-1.3851-0.000087$

$\begin{array}{ll}1.4764 & 0.000067\end{array}$

0.206
1.016
0.479
0.150
0.707
0.321
0.074
0.055
0.004
0.002
0.000
0.000

0.000

0.001

0.002

0.008

0.034

0.089

0.166

0.022

0.090

0.114

0.768

0.825

0.110

0.148

0.063

0.034

0.058

0.001

0.002

0.026

0.006

0.003

0.044

0.038

18.726 Sum chi sqr
Relative Density of

density water $/ \mathrm{kg} / \mathrm{m}^{3}$

992.2158

992.2158

992.2158

992.2158

992.2158

992.2158

992.2158

992.2158

992.2158

991.8298

$1.00009 \quad 991.237$

$1.00017 \quad 991.237$

$1.00042 \quad 991.237$

$1.00083 \quad 991.237$

$1.00164 \quad 991.237$

$1.00411 \quad 991.237$

$1.00813 \quad 991.237$

$1.01617 \quad 991.237$

991.0358

990.2132

990.2132

990.2049

990.163

989.3628

988.0363

988.0363

987.5809

987.119

986.6508

986.1761

985.6952

984.2156

983.1989

981.6297

980.5548 
molality molarity

$t /{ }^{\circ} \mathrm{C}$ mass frac

\section{Density \\ Ref}

$\exp /$ $\begin{array}{rr}\text { App vol } & \text { App vol } \\ \exp / & \text { Calc / } \\ \mathrm{m}^{3} / \mathrm{kg} & \mathrm{m}^{3} / \mathrm{kg}\end{array}$
Density Density App Vol Square of Inconsistent Inconsistent / Residual / Residual / Density based on $\mathrm{kg} / \mathrm{m}^{3}$

apparen $\mathrm{kg} / \mathrm{m}^{3}$
Residual
Relative Density of

density water $/ \mathrm{kg} / \mathrm{m}^{3}$

Curve fit for the model App vol $=\left(w+c 2+c 3^{*} t{ }^{\circ} \mathrm{C}\right) /\left(\mathrm{c0} 0^{*} \mathrm{w}+\mathrm{c} 1\right) / \mathrm{e}^{\wedge}\left(0.000001^{*}\left(\mathrm{t}^{\circ} \mathrm{C}+\mathrm{c} 4\right)^{\wedge} 2\right)$

c0

0.001473

0.002693

$\begin{array}{ll}\mathrm{c} 2 & 0.176989 \\ \mathrm{c} 3 & 0.004132\end{array}$

$\begin{array}{ll}\text { c2 } & 0.176989 \\ \text { c3 } & 0.004132\end{array}$

c4 3640.732

Min T

$\operatorname{Max} T$

65

Min w

Max w $\quad 0.2602$

Avg dens res $\quad 0.0457$

Std dens res $\quad 0.3577$

Avg app vol res *10^3 -0.010136

Std app vol res *10^3 0.019234

No of points in corr

145

No of inconsistent poir

Inconsistent data not used (Residual greater than average +/- 4 standard deviations) 0.00084

$25 \quad 0.0001$

997.9

(48)

$-0.0077680 .000152$

997.12

$0.7293-0.007920$

0.532 
Density of aqueous solutions of $\mathbf{M g C l} 2$

References (9) Call, T. G.; Ballerat-Busserolles, K.; Origlia, M. L.; Ford, T. D.; Woolley, E. M. Apparent Molar Volumes and Heat Capacities of Aqueous Magnesium Chloride and Cadmium Chloride at Temperatures from $278.15 \mathrm{~K}$ to $393.15 \mathrm{~K}$ at the Pressure $0.35 \mathrm{MPa}$ : a Comparison of lonIon Interactions. J. Chem. Thermodynamics 2000, 32, 1525-1538.

(13) Chen, C.-T. A.; Chen, J. H.; Millero, F. J. Densities of $\mathrm{NaCl}, \mathrm{MgCl}_{2}, \mathrm{Na}_{2} \mathrm{SO}_{4}$, and $\mathrm{MgSO}_{4}$ Aqueous Solutions at 1 atm. from 0 to $50^{\circ} \mathrm{C}$ and from 0.001 to 1.5 m. J. Chem. Eng. Data 1980, 25, 307-310.

(28) Gates, J. A.; Wood, R. H. Densities of Aqueous Solutions of $\mathrm{NaCl}, \mathrm{MgCl} 2, \mathrm{KCl}, \mathrm{NaBr}$, LiCl, and $\mathrm{CaCl} 2$ from 0.05 to $5.0 \mathrm{~mol} \mathrm{kg-1}$ and 0.1013 to $40 \mathrm{MPa}$ at 298.15K. J. Chem. Eng. Data 1985, 30,44-49.

(45) Isono, T. Densities, Viscosities, and Electrolytic Conductivity of Concentrated Aqueous Electrolyte Solutions at Several Temperatures. Alkaline-Earth Chlorides, $\mathrm{LaCl}_{3}, \mathrm{Na}_{2} \mathrm{SO}_{4}, \mathrm{NaNO}_{3}, \mathrm{NaBr}, \mathrm{KNO}_{3}, \mathrm{KBr}$, and $\mathrm{Cd}\left(\mathrm{NO}_{3}\right)_{2}$. J. Chem. Eng. Data 1984, $29,45-52$.

(49) Kaminsky, M, Concentration and Temperature Dependence of the Viscosity of Aqueous Solutions of Strong Electrolytes. III. KCl, K2SO4, $\mathrm{MgCl} 2$, BeSO4, and MgSO4 Solutions. Z. Phys. Chem. N. F. 1957, 12, 206-231.

(72) Millero, F. J.; Knox, J. H. Apparent Molal Volumes of Aqueous $\mathrm{NaF}, \mathrm{Na}_{2} \mathrm{SO}_{4}, \mathrm{KCl}_{2} \mathrm{~K}_{2} \mathrm{SO}_{4}, \mathrm{MgCl}_{2}$, and $\mathrm{MgSO}_{4}$ Solutions at $0^{\circ}$ and $50^{\circ} \mathrm{C}$. J. Chem. Eng. Data 1973, 18, 407-411.

(87) Perron, G.; Desnoyers, J. E. Apparent Molal Volumes and Heat Capacities of Alkaline Earth Chlorides in Water at $25^{\circ} \mathrm{C}$. Can. J. Chem. 1974, 52, 3738-3741.

(90) Perry, R. H.; Green, D. W. Perry's Chemical Engineers' Handbook; McGraw Hill: New York, 7th edition, 1997.

(113) Saluja, P. P. S.; LeBlanc, J. C. Apparent Molar Heat Capacities and Volumes of Aqueous Solutions of $\mathrm{MgCl}_{2}, \mathrm{CaCl}_{2}$, and $\mathrm{SrCl}_{2}$ at Elevated Temperatures. J. Chem. Eng. Data 1987, 32, 72-76.

(129) Vasilev, Y. A.; Fedyainov, N. V.; Kurenkov, V. V. Specific Heats and Specific Volumes of Isopiestic Aqueous Solutions of BerylliumSubgroup Metal Chlorides. Russ. J. Phys. Chem. 1973, 47, 2799-2803

\begin{tabular}{|c|c|c|c|c|c|c|c|c|c|c|c|c|c|c|c|c|c|}
\hline molality & molarity & $\mathrm{t} /{ }^{\circ} \mathrm{C}$ & iass frac & $\begin{array}{r}\text { Density } \\
\exp / \\
\mathrm{kg} / \mathrm{m}^{3}\end{array}$ & Ref & $\begin{array}{r}\text { App vol } \\
\text { exp } / \\
\mathrm{m}^{3} / \mathrm{kg}\end{array}$ & $\begin{array}{r}\text { App vol } \\
\text { Calc } / \\
\mathrm{m}^{3} / \mathrm{kg}\end{array}$ & $\begin{array}{r}\text { Density } \\
\text { calc / } \\
\mathrm{kg} / \mathrm{m}^{3}\end{array}$ & $\begin{array}{r}\text { Density } \\
\text { Residual / } \\
\mathrm{kg} / \mathrm{m}^{3}\end{array}$ & $\begin{array}{r}\text { App Vol } \\
\text { Residual / } \\
\mathrm{m}^{3} / \mathrm{kg}\end{array}$ & $\begin{array}{r}\text { Square of } \\
\text { Density } \\
\text { Residual }\end{array}$ & $\begin{array}{l}\text { Inconsistent } \\
\text { based on } \\
\text { density }\end{array}$ & $\begin{array}{l}\text { Inconsistent } \\
\text { based on } \\
\text { apparent } \\
\text { volume }\end{array}$ & $\begin{array}{l}\text { Apparent } \\
\text { molal volume } \\
/ \mathrm{cm}^{3} / \mathrm{mol}\end{array}$ & $\begin{array}{r}\text { (Relative } \\
\text { density - } \\
1)^{\star} 10^{\wedge} 4\end{array}$ & $\begin{array}{l}\text { Delta density } \\
\qquad / \mathrm{kg} / \mathrm{m}^{3}\end{array}$ & $\begin{array}{r}\text { Density of } \\
\text { water at } t^{\circ} \mathrm{C} / \\
\mathrm{kg} / \mathrm{m}^{3}\end{array}$ \\
\hline 0.00469 & & 0 & 0.0004 & 1000.23 & (13) & 0.000124 & 0.000167 & 1000.21 & 0.0190 & -0.000042 & 0.000 & & & & & 0.391 & 999.8395 \\
\hline .008785 & & 0 & 0.0008 & 1000.58 & (72) & 0.000117 & 0.000167 & 1000.54 & 0.0419 & -0.000050 & 0.002 & & & & & 0.7386 & 999.8395 \\
\hline 0.00978 & & 0 & 0.0009 & 1000.66 & (13) & 0.000117 & 0.000167 & 1000.62 & 0.0464 & -0.000050 & 0.002 & & & & & 0.822 & 999.8395 \\
\hline .010774 & & 0 & 0.0010 & 1000.75 & (72) & 0.000117 & 0.000167 & 1000.69 & 0.0512 & -0.000050 & 0.003 & & & & & 0.9056 & 999.8395 \\
\hline .015048 & & 0 & 0.0014 & 1001.10 & (72) & 0.000119 & 0.000167 & 1001.03 & 0.0687 & -0.000048 & 0.005 & & & & & 1.2617 & 999.8395 \\
\hline .018179 & & 0 & 0.0017 & 1001.36 & (72) & 0.000120 & 0.000167 & 1001.28 & 0.0821 & -0.000047 & 0.007 & & & & & 1.5231 & 999.8395 \\
\hline 0.01972 & & 0 & 0.0019 & 1001.48 & (13) & 0.000124 & 0.000167 & 1001.40 & 0.0820 & -0.000044 & 0.007 & & & & & 1.645 & 999.8395 \\
\hline .025081 & & 0 & 0.0024 & 1001.93 & (72) & 0.000123 & 0.000167 & 1001.83 & 0.1062 & -0.000044 & 0.011 & & & & & 2.0935 & 999.8395 \\
\hline .026659 & & 0 & 0.0025 & 1002.06 & (72) & 0.000123 & 0.000167 & 1001.95 & 0.1126 & -0.000044 & 0.013 & & & & & 2.2248 & 999.8395 \\
\hline .031383 & & 0 & 0.0030 & 1002.45 & (72) & 0.000125 & 0.000168 & 1002.33 & 0.1275 & -0.000043 & 0.016 & & & & & 2.6133 & 999.8395 \\
\hline 0.04137 & & 0 & 0.0039 & 1003.26 & (13) & 0.000130 & 0.000168 & 1003.11 & 0.1480 & -0.000037 & 0.022 & & & & & 3.423 & 999.8395 \\
\hline .043333 & & 0 & 0.0041 & 1003.43 & (72) & 0.000129 & 0.000168 & 1003.27 & 0.1626 & -0.000039 & 0.026 & & & & & 3.5927 & 999.8395 \\
\hline 0.04851 & & 0 & 0.0046 & 1003.86 & (13) & 0.000129 & 0.000168 & 1003.68 & 0.1793 & -0.000039 & 0.032 & & & & & 4.018 & 999.8395 \\
\hline .056311 & & 0 & 0.0053 & 1004.49 & (72) & 0.000131 & 0.000168 & 1004.29 & 0.1997 & -0.000037 & 0.040 & & & & & 4.6538 & 999.8395 \\
\hline 0.0789 & & 0 & 0.0075 & 1006.32 & (13) & 0.000137 & 0.000169 & 1006.07 & 0.2450 & -0.000032 & 0.060 & & & & & 6.478 & 999.8395 \\
\hline 0.07966 & & 0 & 0.0075 & 1006.40 & (72) & 0.000134 & 0.000169 & 1006.13 & 0.2678 & -0.000035 & 0.072 & & & & & 6.5606 & 999.8395 \\
\hline 0.09627 & & 0 & 0.0091 & 1007.75 & (13) & 0.000136 & 0.000170 & 1007.44 & 0.3112 & -0.000034 & 0.097 & & & & & 7.909 & 999.8395 \\
\hline 0.09649 & & 0 & 0.0091 & 1007.77 & (72) & 0.000135 & 0.000170 & 1007.45 & 0.3201 & -0.000035 & 0.102 & & & & & 7.9352 & 999.8395 \\
\hline 0.1372 & & 0 & 0.0129 & 1011.06 & (72) & 0.000140 & 0.000171 & 1010.64 & 0.4130 & -0.000031 & 0.171 & & & & & 11.2162 & 999.8395 \\
\hline 0.17359 & & 0 & 0.0163 & 1013.96 & (72) & 0.000144 & 0.000172 & 1013.48 & 0.4790 & -0.000029 & 0.229 & & & & & 14.1197 & 999.8395 \\
\hline 0.195 & & 0 & 0.0182 & 1015.65 & (13) & 0.000146 & 0.000173 & 1015.14 & 0.5064 & -0.000027 & 0.256 & & & & & 15.811 & 999.8395 \\
\hline 0.19525 & & 0 & 0.0183 & 1015.70 & (13) & 0.000145 & 0.000173 & 1015.16 & 0.5320 & -0.000028 & 0.283 & & & & & 15.856 & 999.8395 \\
\hline & & 0 & 0.0200 & 1016.80 & (90) & 0.000166 & 0.000174 & 1016.64 & 0.1570 & -0.000008 & 0.025 & & & & & & 999.8395 \\
\hline 0.25072 & & 0 & 0.0233 & 1020.06 & (72) & 0.000150 & 0.000175 & 1019.46 & 0.6077 & -0.000025 & 0.369 & & & & & 20.2238 & 999.8395 \\
\hline & & 0 & & 102257 & & & & & & & & & & & & & \\
\hline
\end{tabular}




\begin{tabular}{|c|c|c|c|c|}
\hline molality & molarity & \multicolumn{2}{|c|}{$\mathrm{t} /{ }^{\circ} \mathrm{C}$ mass frac } & $\begin{array}{r}\text { Density } \\
\exp / \\
\mathrm{kg} / \mathrm{m}^{3}\end{array}$ \\
\hline 0.34124 & & 0 & 0.0315 & 1027.12 \\
\hline 0.37485 & & 0 & 0.0345 & 1029.71 \\
\hline 0.40811 & & 0 & 0.0374 & 1032.27 \\
\hline \multirow[t]{2}{*}{0.42814} & & 0 & 0.0392 & 1033.79 \\
\hline & & 0 & 0.0400 & 1033.80 \\
\hline 0.50904 & & 0 & 0.0462 & 1039.96 \\
\hline 0.55193 & & 0 & 0.0499 & 1043.18 \\
\hline 0.62437 & & 0 & 0.0561 & 1048.61 \\
\hline 0.63404 & & 0 & 0.0569 & 1049.33 \\
\hline 0.70544 & & 0 & 0.0629 & 1054.56 \\
\hline 0.79758 & & 0 & 0.0706 & 1061.39 \\
\hline \multirow[t]{2}{*}{0.82932} & & 0 & 0.0732 & 1063.66 \\
\hline & & 0 & 0.0800 & 1068.30 \\
\hline 0.9741 & & 0 & 0.0849 & 1074.09 \\
\hline 1.01833 & & 0 & 0.0884 & 1077.25 \\
\hline \multirow[t]{6}{*}{1.0184} & & 0 & 0.0884 & 1077.25 \\
\hline & & 0 & 0.1200 & 1103.50 \\
\hline & & 0 & 0.1600 & 1139.50 \\
\hline & & 0 & 0.2000 & 1176.40 \\
\hline & & 0 & 0.2500 & 1224.60 \\
\hline & & 0 & 0.3000 & 1275.40 \\
\hline 0.00469 & & 5 & 0.0004 & 1000.35 \\
\hline 0.00837 & & 5 & 0.0008 & 1000.68 \\
\hline 0.00978 & & 5 & 0.0009 & 1000.78 \\
\hline 0.01646 & & 5 & 0.0016 & 1001.35 \\
\hline 0.01972 & & 5 & 0.0019 & 1001.58 \\
\hline 0.03278 & & 5 & 0.0031 & 1002.65 \\
\hline 0.04137 & & 5 & 0.0039 & 1003.34 \\
\hline 0.04851 & & 5 & 0.0046 & 1003.93 \\
\hline 0.06562 & & 5 & 0.0062 & 1005.29 \\
\hline 0.0789 & & 5 & 0.0075 & 1006.35 \\
\hline 0.09627 & & 5 & 0.0091 & 1007.77 \\
\hline 0.13064 & & 5 & 0.0123 & 1010.51 \\
\hline 0.195 & & 5 & 0.0182 & 1015.59 \\
\hline 0.19525 & & 5 & 0.0183 & 1015.59 \\
\hline 0.26767 & & 5 & 0.0249 & 1021.14 \\
\hline 0.28278 & & 5 & 0.0262 & 1022.39 \\
\hline 0.37485 & & 5 & 0.0345 & 1029.45 \\
\hline 0.40811 & & 5 & 0.0374 & 1031.98 \\
\hline 0.52476 & & 5 & 0.0476 & 1040.48 \\
\hline 0.55193 & & 5 & 0.0499 & 1042.79 \\
\hline 0.63404 & & 5 & 0.0569 & 1048.86 \\
\hline 0.76535 & & 5 & 0.0679 & 1058.26 \\
\hline \multirow[t]{2}{*}{0.99851} & & 5 & 0.0868 & 1074.55 \\
\hline & 0.0025 & 15 & 0.0002 & 999.31 \\
\hline \multirow[t]{2}{*}{0.00469} & & 15 & 0.0004 & 999.48 \\
\hline & 0.005 & 15 & 0.0005 & 999.51 \\
\hline 0.00837 & & 15 & 0.0008 & 999.82 \\
\hline
\end{tabular}

Ref

App

exp / App vol D $\mathrm{m}^{3} / \mathrm{kg}$

0.0

$\begin{array}{ll}0.000156 & 0.000178\end{array}$

$\begin{array}{ll}0.000156 & 0.000178 \\ 0.000158 & 0.000179\end{array}$ $\begin{array}{ll}0.000160 & 0.000180 \\ 0.000162 & 0.000180\end{array}$

0.0001620 .000180

$\begin{array}{ll}0.000179 & 0.000181\end{array}$

$\begin{array}{ll}0.000166 & 0.000183\end{array}$

$\begin{array}{ll}0.000168 & 0.000184 \\ 0.000171 & 0.000187\end{array}$

$\begin{array}{ll}0.000172 & 0.000187\end{array}$

$\begin{array}{ll}0.000176 & 0.000189\end{array}$

$\begin{array}{ll}0.000178 & 0.000192\end{array}$

$0.000180 \quad 0.000193$

$\begin{array}{ll}0.000199 & 0.000195\end{array}$

$\begin{array}{ll}0.000186 & 0.000197 \\ 0.000187 & 0.000199\end{array}$

$\begin{array}{ll}0.000187 & 0.000199 \\ 0.000187 & 0.000199\end{array}$

$0.000217 \quad 0.000210$

$\begin{array}{ll}0.000217 & 0.000210 \\ 0.000234 & 0.000226\end{array}$

$\begin{array}{ll}0.000234 & 0.000226 \\ 0.000250 & 0.000242\end{array}$

$\begin{array}{ll}0.000266 & 0.000263 \\ 0.000280 & 0.000285\end{array}$

$\begin{array}{ll}0.000280 & 0.000285 \\ 0.000140 & 0.000172\end{array}$

$\begin{array}{ll}0.000140 & 0.000172\end{array}$

$\begin{array}{ll}0.000095 & 0.000172\end{array}$

$\begin{array}{ll}0.000124 & 0.000172 \\ 0.000118 & 0.000173\end{array}$

$\begin{array}{ll}0.000118 & 0.000173 \\ 0.000140 & 0.000173\end{array}$

$\begin{array}{ll}0.000141 & 0.000173\end{array}$

$\begin{array}{ll}0.000141 & 0.000173\end{array}$

$\begin{array}{ll}0.000141 & 0.000174\end{array}$

$0.000147 \quad 0.000174$

$\begin{array}{ll}0.000149 & 0.000175 \\ 0.000147 & 0.000175\end{array}$

$\begin{array}{ll}0.000147 & 0.000175 \\ 0.000151 & 0.000176\end{array}$

$\begin{array}{ll}0.000156 & 0.000178\end{array}$

$\begin{array}{lll}0.000157 & 0.000178\end{array}$

$\begin{array}{lll}0.000166 & 0.000181\end{array}$

$0.000163 \quad 0.000181$

0.0001690 .000184

$\begin{array}{ll}0.000171 & 0.000185\end{array}$

$0.000182 \quad 0.000189$

$\begin{array}{ll}0.000177 & 0.000189\end{array}$

0.0001810 .000192

0.0001890 .000196

$0.000200 \quad 0.000203$

0.0001190 .000181

$0.000155 \quad 0.000181$

$\begin{array}{lll}0.000140 & 0.000181\end{array}$

$0.000093 \quad 0.000181$
Density Density App
calc/ Residual / Residu

Apparent volume

$1 \mathrm{~cm}^{3} / \mathrm{mol}$

1026.40

1028.96

$\mathrm{kg} / \mathrm{m}^{3}$

$\mathrm{m}^{3} / \mathrm{kg}$

$0.7525-0.00002$

0.516

$\begin{array}{llll}1031.49 & 0.7874 & -0.000020 & 0.620\end{array}$

$\begin{array}{llll}1033.00 & 0.7853 & -0.000019 & 0.617\end{array}$

$\begin{array}{llll}033.72 & 0.0846 & -0.000002 & 0.007\end{array}$

$\begin{array}{llll}039.09 & 0.8716 & -0.000017 & 0.760\end{array}$

$\begin{array}{llll}042.29 & 0.8928 & -0.000016 & 0.797\end{array}$

$\begin{array}{llll}1047.66 & 0.9449 & -0.000015 & 0.893\end{array}$

$\begin{array}{llll}1048.37 & 0.9537 & -0.000015 & 0.910\end{array}$

$\begin{array}{llll}053.62 & 0.9392 & -0.000013 & 0.882\end{array}$

$\begin{array}{llll}1060.32 & 1.0697 & -0.000013 & 1.144\end{array}$

$\begin{array}{llll}1062.61 & 1.0501 & -0.000013 & 1.103\end{array}$

$\begin{array}{llll}1068.63 & -0.3286 & 0.000004 & 0.108\end{array}$

$\begin{array}{llll}1072.95 & 1.1375 & -0.000012 & 1.294\end{array}$

$\begin{array}{llll}1076.08 & 1.1707 & -0.000011 & 1.371\end{array}$

$\begin{array}{llll}076.08 & 1.1708 & -0.000011 & 1.371\end{array}$

$\begin{array}{llll}1104.48 & -0.9835 & 0.000007 & 0.967\end{array}$

$\begin{array}{llll}1141.15 & -1.6492 & 0.000008 & 2.720\end{array}$

$\begin{array}{llll}1178.45 & -2.0530 & 0.000007 & 4.215\end{array}$

$\begin{array}{llll}1225.64 & -1.0392 & 0.000003 & 1.080\end{array}$

$\begin{array}{llll}1272.92 & 2.4785 & -0.000005 & 6.143\end{array}$

$\begin{array}{llll}1000.33 & 0.0144 & -0.000032 & 0.000\end{array}$

$\begin{array}{llll}1000.62 & 0.0613 & -0.000077 & 0.004\end{array}$

$\begin{array}{llll}1000.73 & 0.0454 & -0.000049 & 0.002\end{array}$

$\begin{array}{llll}1001.26 & 0.0853 & -0.000054 & 0.007\end{array}$

$\begin{array}{llll}001.52 & 0.0621 & -0.000033 & 0.004\end{array}$

$\begin{array}{lll}0.1020 & -0.000033 & 0.010\end{array}$

$\begin{array}{lll}0.1272 & -0.000032 & 0.016\end{array}$

$\begin{array}{lll}0.1532 & -0.000033 & 0.023\end{array}$

$\begin{array}{lll}0.1704 & -0.000027 & 0.029\end{array}$

$\begin{array}{lll}0.1954 & -0.000026 & 0.038\end{array}$

$\begin{array}{lll}0.2565 & -0.000028 & 0.066\end{array}$

$\begin{array}{lll}0.3207 & -0.000026 & 0.103\end{array}$

$\begin{array}{lll}0.4183 & -0.000022 & 0.175\end{array}$

$\begin{array}{lll}0.3990 & -0.000021 & 0.159\end{array}$

$\begin{array}{lll}0.3912 & -0.000015 & 0.153\end{array}$

$\begin{array}{lll}0.4858 & -0.000018 & 0.236\end{array}$

$\begin{array}{lll}0.5484 & -0.000015 & 0.301\end{array}$

$\begin{array}{lll}0.5723 & -0.000014 & 0.327\end{array}$

$\begin{array}{lll}0.3548 & -0.000007 & 0.126\end{array}$

$\begin{array}{lll}0.6523 & -0.000012 & 0.425\end{array}$

$\begin{array}{lll}0.6679 & -0.000011 & 0.446\end{array}$

$\begin{array}{lll}0.5156 & -0.000007 & 0.266\end{array}$

$\begin{array}{lll}0.2173 & -0.000002 & 0.047\end{array}$

$\begin{array}{lll}0.0149 & -0.000062 & 0.000\end{array}$

$\begin{array}{lll}0.0117 & -0.000026 & 0.000\end{array}$

$\begin{array}{lll}0.0198 & -0.000042 & 0.000\end{array}$

$\begin{array}{llll}999.75 & 0.0704 & -0.000088 & 0.005\end{array}$
11.26
Relative Delta density Density of density - $\quad / \mathrm{kg} / \mathrm{m}^{3}$ water at $\mathrm{t}^{\circ} \mathrm{C} /$

27.2805999 .8395 $29.875 \quad 999.8395$ $32.434 \quad 999.8395$ $33.9473 \quad 999.8395$ 999.8395 $40.1183 \quad 999.8395$ $43.342 \quad 999.8395$ $48.7665 \quad 999.8395$ $49.489 \quad 999.8395$ $54.7187 \quad 999.8395$

$61.551 \quad 999.8395$

$63.823 \quad 999.8395$ 999.8395

74.252999 .8395 $77.4084 \quad 999.8395$ $77.4134 \quad 999.8395$ 999.8395 999.8395 999.8395 999.8395 999.8395

$0.384 \quad 999.9638$ 999.9638

$0.816 \quad 999.9638$ 999.9638

1.615999 .9638 999.9638

3.381999 .9638

$3.967 \quad 999.9638$ 999.9638

$6.388 \quad 999.9638$

$7.805 \quad 999.9638$ 999.9638

$15.624 \quad 999.9638$

15.624999 .9638 999.9638

22.428999 .9638

$29.484 \quad 999.9638$

$32.016 \quad 999.9638$ 999.9638

$42.831 \quad 999.9638$

$48.895 \quad 999.9638$ 999.9638 999.9638

$0.377 \quad 999.0996$ 999.0996 999.0996 


\begin{tabular}{|c|c|c|c|c|c|}
\hline molality & molarity & \multicolumn{2}{|c|}{$\mathrm{t} /{ }^{\circ} \mathrm{C}$ mass frac } & $\begin{array}{r}\text { Density } \\
\exp / \\
\mathrm{kg} / \mathrm{m}^{3}\end{array}$ & $\operatorname{Ref}$ \\
\hline 0.00978 & \multirow{4}{*}{0.01} & 15 & 0.0009 & 999.89 & \\
\hline & & 15 & 0.0010 & 999.92 & \\
\hline 0.01646 & & 15 & 0.0016 & 1000.47 & \\
\hline \multirow[t]{3}{*}{0.01972} & & 15 & 0.0019 & 1000.69 & \\
\hline & 0.02002 & 15 & 0.0019 & 1000.73 & \\
\hline & 0.03002 & 15 & 0.0029 & 1001.53 & \\
\hline \multirow[t]{2}{*}{0.03278} & & 15 & 0.0031 & 1001.75 & \\
\hline & 0.04004 & 15 & 0.0038 & 1002.33 & \\
\hline 0.04137 & & 15 & 0.0039 & 1002.42 & \\
\hline 0.04851 & & 15 & 0.0046 & 1002.99 & \\
\hline 0.05 & & 15 & 0.0047 & 1003.10 & \\
\hline 0.06562 & & 15 & 0.0062 & 1004.32 & \\
\hline \multirow[t]{2}{*}{0.0789} & & 15 & 0.0075 & 1005.37 & \\
\hline & 0.09008 & 15 & 0.0085 & 1006.28 & \\
\hline 0.09627 & & 15 & 0.0091 & 1006.76 & \\
\hline 0.1 & & 15 & 0.0094 & 1006.99 & \\
\hline \multirow[t]{2}{*}{0.13064} & & 15 & 0.0123 & 1009.36 & \\
\hline & 0.16014 & 15 & 0.0151 & 1011.72 & \\
\hline 0.19525 & & 15 & 0.0183 & 1014.43 & \\
\hline 0.26767 & & 15 & 0.0249 & 1019.85 & \\
\hline 0.37485 & & 15 & 0.0345 & 1028.09 & \\
\hline 0.40811 & & 15 & 0.0374 & 1030.56 & \\
\hline 0.5 & & 15 & 0.0454 & 1037.20 & \\
\hline 0.52476 & & 15 & 0.0476 & 1038.92 & \\
\hline 0.55193 & & 15 & 0.0499 & 1041.21 & \\
\hline 0.63404 & & 15 & 0.0569 & 1047.20 & \\
\hline 0.76535 & & 15 & 0.0679 & 1056.46 & \\
\hline 0.9741 & & 15 & 0.0849 & 1071.41 & \\
\hline 0.99851 & & 15 & 0.0868 & 1072.58 & \\
\hline 1 & & 15 & 0.0869 & 1072.67 & \\
\hline 2 & & 15 & 0.1600 & 1138.28 & \\
\hline 3 & & 15 & 0.2222 & 1194.63 & \\
\hline \multirow[t]{7}{*}{4} & & 15 & 0.2758 & 1247.60 & \\
\hline & 0.0025 & 20 & 0.0002 & 998.40 & \\
\hline & 0.005 & 20 & 0.0005 & 998.61 & \\
\hline & 0.01 & 20 & 0.0010 & 999.01 & \\
\hline & 0.02 & 20 & 0.0019 & 999.82 & \\
\hline & 0.03 & 20 & 0.0029 & 1000.62 & \\
\hline & 0.04 & 20 & 0.0038 & 1001.41 & \\
\hline \multirow[t]{2}{*}{0.05} & & 20 & 0.0047 & 1002.17 & \\
\hline & 0.09 & 20 & 0.0085 & 1005.35 & \\
\hline \multirow[t]{4}{*}{0.1} & & 20 & 0.0094 & 1006.05 & \\
\hline & 0.16 & 20 & 0.0151 & 1010.68 & \\
\hline & & 20 & 0.0200 & 1014.60 & \\
\hline & & 20 & 0.0400 & 1031.10 & \\
\hline \multirow[t]{2}{*}{0.5} & & 20 & 0.0454 & 1036.09 & \\
\hline & & 20 & 0.0800 & 1064.60 & \\
\hline 1 & & 20 & 0.0869 & 1071.59 & \\
\hline
\end{tabular}

\section{App vol App vol D} $\begin{array}{cc}\exp / & \text { Calc / } \\ \mathrm{m}^{3} / \mathrm{kg} & \mathrm{m}^{3} / \mathrm{kg}\end{array}$ $\mathrm{m}^{3} / \mathrm{kg} \quad \mathrm{m}^{3} / \mathrm{kg}$ $\begin{array}{ll}0.000151 & 0.000181 \\ 0.000140 & 0.000181\end{array}$ $0.000124 \quad 0.000182$ 0.0001510 .000182 $0.000146 \quad 0.000182$ $\begin{array}{lll}0.000151 & 0.000182\end{array}$ $\begin{array}{ll}0.000151 & 0.000182\end{array}$ 0.0001540 .000182 $0.000156 \quad 0.000182$ $\begin{array}{lll}0.000157 & 0.000183\end{array}$ $0.000158 \quad 0.000183$ $0.000164 \quad 0.000183$ $\begin{array}{ll}0.000163 & 0.000184\end{array}$ $\begin{array}{lll}0.000163 & 0.000184\end{array}$ 0.0001620 .000184 $0.000169 \quad 0.000184$ $0.000172 \quad 0.000185$ $\begin{array}{lll}0.000173 & 0.000186\end{array}$ $0.000172 \quad 0.000187$ $\begin{array}{lll}0.000182 & 0.000189\end{array}$ 0.0001820 .000192 $0.000184 \quad 0.000193$ 0.0001920 .000196 $\begin{array}{lll}0.000195 & 0.000197\end{array}$ $0.000190 \quad 0.000198$ $0.000193 \quad 0.000200$ 0.0002010 .000204 $0.000205 \quad 0.000210$ $0.000211 \quad 0.000210$ 0.0002110 .000210 0.0002360 .000236 $0.000264 \quad 0.000260$ $\begin{array}{lll}0.000278 & 0.000281\end{array}$ $0.000162 \quad 0.000185$ $\begin{array}{ll}0.000141 & 0.000185\end{array}$ $0.000151 \quad 0.000185$ 0.0001510 .000185 0.0001550 .000186 $0.000159 \quad 0.000186$ $0.000165 \quad 0.000186$ $\begin{array}{ll}0.000166 & 0.000187\end{array}$ 0.0001730 .000188 $\begin{array}{ll}0.000181 & 0.000190\end{array}$ $0.000192 \quad 0.000191$ $0.000203 \quad 0.000198$ $0.000196 \quad 0.000199$ $\begin{array}{ll}0.000221 & 0.000211\end{array}$ $\begin{array}{ll}0.000213 & 0.000213\end{array}$ volume

$\begin{array}{llll}1005.22 & 0.1540 & -0.000020 & 0.024\end{array}$ $\begin{array}{llll}1006.10 & 0.1835 & -0.000021 & 0.034\end{array}$ $\begin{array}{llll}1006.56 & 0.2036 & -0.000022 & 0.041 \\ 1006.85 & 0.1415 & -0.000015 & 0.020\end{array}$ $\begin{array}{ll}0.1415 & -0.000015 \\ 0.1587 & -0.000013\end{array}$ $1014.15-0.2835-0.00$ $\begin{array}{lll}1019.65 & 0.1982 & -0.000008\end{array}$ $\begin{array}{llll}1027.71 & 0.3861 & -0.000011 & 0.149\end{array}$ $\begin{array}{llll}1030.19 & 0.3755 & -0.000009 & 0.141\end{array}$ $\begin{array}{llll}1036.99 & 0.2071 & -0.000004 & 0.043\end{array}$ $\begin{array}{llll}1038.81 & 0.1083 & -0.000002 & 0.012\end{array}$ $\begin{array}{llll}1040.81 & 0.4017 & -0.000007 & 0.161\end{array}$ $\begin{array}{llll}046.79 & 0.4135 & -0.000007 & 0.171\end{array}$ $\begin{array}{llll}1056.24 & 0.2199 & -0.000003 & 0.048\end{array}$ $\begin{array}{llll}1070.97 & 0.4353 & -0.000004 & 0.189\end{array}$ $\begin{array}{llll}1072.67 & -0.0894 & 0.000001 & 0.008\end{array}$ $\begin{array}{llll}1072.78 & -0.1057 & 0.000001 & 0.011\end{array}$ $\begin{array}{llll}1138.19 & 0.0862 & 0.000000 & 0.007\end{array}$ $\begin{array}{llll}1195.85 & -1.2206 & 0.000004 & 1.490\end{array}$ $\begin{array}{llll}1246.38 & 1.2223 & -0.000003 & 1.494\end{array}$ $\begin{array}{llll}998.40 & 0.0055 & -0.000023 & 0.000\end{array}$ $\begin{array}{llll}998.59 & 0.0211 & -0.000044 & 0.000\end{array}$ $\begin{array}{llll}998.98 & 0.0323 & -0.000034 & 0.001\end{array}$ $\begin{array}{llll}999.76 & 0.0652 & -0.000034 & 0.004\end{array}$ $\begin{array}{lllll}1000.53 & 0.0887 & -0.000031 & 0.008\end{array}$ $\begin{array}{llll}1001.31 & 0.1029 & -0.000027 & 0.011\end{array}$ $\begin{array}{llll}1002.07 & 0.1006 & -0.000021 & 0.010\end{array}$ $\begin{array}{llll}1005.17 & 0.1824 & -0.000021 & 0.033\end{array}$ $\begin{array}{llll}1005.91 & 0.1363 & -0.000014 & 0.019\end{array}$ $\begin{array}{llll}1010.55 & 0.1269 & -0.000008 & 0.016\end{array}$ $\begin{array}{llll}1014.63 & -0.0251 & 0.000001 & 0.001 \\ 1031.32 & -0.2207 & 0.000005 & 0.049\end{array}$ $\begin{array}{lll}1031.32 & -0.2207 & 0.000005 \\ 1035.91 & 0.1769 & -0.000004\end{array}$ $\begin{array}{llll}1065.51 & -0.9081 & 0.000010 & 0.825\end{array}$ $\begin{array}{llll}1071.54 & 0.0464 & 0.000000 & 0.002\end{array}$
Apparent

15.5

(Relative Delta density Density of density - $\quad / \mathrm{kg} / \mathrm{m}^{3}$ water at $\mathrm{t}^{\circ} \mathrm{C} /$

\begin{tabular}{|c|c|c|c|}
\hline & & 0.79 & 999.0996 \\
\hline & 8.2 & & 999.0996 \\
\hline 11.84 & & & 999.0996 \\
\hline & & 1.593 & 999.0996 \\
\hline & 16.3 & & 999.0996 \\
\hline & 24.3 & & 999.0996 \\
\hline 14.37 & & & 999.0996 \\
\hline & 32.3 & & 999.0996 \\
\hline & & 3.319 & 999.0996 \\
\hline & & 3.886 & 999.0996 \\
\hline & & & 999.0996 \\
\hline 15.57 & & & 999.0996 \\
\hline & & 6.275 & 999.0996 \\
\hline & 71.9 & & 999.0996 \\
\hline & & 7.665 & 999.0996 \\
\hline & & & 999.0996 \\
\hline 16.42 & & & 999.0996 \\
\hline & 126.3 & & 999.0996 \\
\hline & & 15.334 & 999.0996 \\
\hline 17.29 & & & 999.0996 \\
\hline & & 28.994 & 999.0996 \\
\hline & & 31.464 & 999.0996 \\
\hline & & & 999.0996 \\
\hline 18.54 & & & 999.0996 \\
\hline & & 42.109 & 999.0996 \\
\hline & & 48.105 & 999.0996 \\
\hline 19.12 & & & 999.0996 \\
\hline & & 72.309 & 999.0996 \\
\hline 20.1 & & & 999.0996 \\
\hline & & & 999.0996 \\
\hline & & & 999.0996 \\
\hline & & & 999.0996 \\
\hline & & & 999.0996 \\
\hline & 2 & & 998.2041 \\
\hline & 4.1 & & 998.2041 \\
\hline & 8.1 & & 998.2041 \\
\hline & 16.2 & & 998.2041 \\
\hline & 24.2 & & 998.2041 \\
\hline & 32.1 & & 998.2041 \\
\hline & & & 998.2041 \\
\hline & 71.6 & & 998.2041 \\
\hline & & & 998.2041 \\
\hline & 125 & & 998.2041 \\
\hline & & & 998.2041 \\
\hline & & & 998.2041 \\
\hline & & & 998.2041 \\
\hline & & & 998.2041 \\
\hline & & & 998.2041 \\
\hline
\end{tabular}




\begin{tabular}{|c|c|c|c|c|c|}
\hline \multirow[t]{2}{*}{ molality } & \multirow[t]{2}{*}{ molarity } & \multicolumn{2}{|c|}{$\mathrm{t} /{ }^{\circ} \mathrm{C}$ mass frac } & \multicolumn{2}{|l|}{$\begin{array}{r}\text { Density } \\
\exp / \\
\mathrm{kg} / \mathrm{m}^{3}\end{array}$} \\
\hline & & 20 & 0.1200 & 1098.90 & \\
\hline 2 & & 20 & 0.1600 & 1136.82 & \\
\hline & & 20 & 0.1600 & 1134.20 & \\
\hline & & 20 & 0.2000 & 1170.60 & \\
\hline 3 & & 20 & 0.2222 & 1193.03 & \\
\hline & & 20 & 0.2500 & 1218.40 & \\
\hline 4 & & 20 & 0.2758 & 1245.89 & \\
\hline & & 20 & 0.3000 & 1268.80 & \\
\hline 0.10844 & & 24.04 & 0.0102 & 1005.76 & \\
\hline 0.19711 & & 24.04 & 0.0184 & 1012.59 & \\
\hline 0.28126 & & 24.04 & 0.0261 & 1018.98 & \\
\hline 0.38842 & & 24.04 & 0.0357 & 1027.03 & \\
\hline 0.52775 & & 24.04 & 0.0478 & 1037.30 & \\
\hline & 0.0025 & 25 & 0.0002 & 997.24 & \\
\hline 0.00386 & & 25 & 0.0004 & 997.36 & \\
\hline 0.00469 & & 25 & 0.0004 & 997.42 & \\
\hline & 0.00499 & 25 & 0.0005 & 997.44 & \\
\hline 0.00837 & & 25 & 0.0008 & 997.75 & \\
\hline 0.00978 & & 25 & 0.0009 & 997.83 & \\
\hline 0.00989 & & 25 & 0.0009 & 997.84 & \\
\hline 0.00989 & & 25 & 0.0009 & 997.85 & \\
\hline & 0.00998 & 25 & 0.0010 & 997.85 & \\
\hline 0.01332 & & 25 & 0.0013 & 998.11 & \\
\hline 0.01646 & & 25 & 0.0016 & 998.40 & \\
\hline 0.01972 & & 25 & 0.0019 & 998.62 & \\
\hline & 0.01998 & 25 & 0.0019 & 998.64 & \\
\hline 0.0232 & & 25 & 0.0022 & 998.89 & \\
\hline & 0.02996 & 25 & 0.0029 & 999.44 & \\
\hline 0.0312 & & 25 & 0.0030 & 999.52 & \\
\hline 0.03278 & & 25 & 0.0031 & 999.66 & \\
\hline 0.03312 & & 25 & 0.0031 & 999.67 & \\
\hline & 0.03995 & 25 & 0.0038 & 1000.23 & \\
\hline 0.04851 & & 25 & 0.0046 & 1000.90 & \\
\hline 0.05 & & 25 & 0.0047 & 1001.00 & \\
\hline 0.05038 & & 25 & 0.0048 & 1001.04 & \\
\hline 0.05038 & & 25 & 0.0048 & 1001.04 & \\
\hline 0.06562 & & 25 & 0.0062 & 1002.20 & \\
\hline 0.0661 & & 25 & 0.0063 & 1002.26 & \\
\hline 0.0789 & & 25 & 0.0075 & 1003.26 & \\
\hline & 0.08989 & 25 & 0.0085 & 1004.12 & \\
\hline 0.0912 & & 25 & 0.0086 & 1004.21 & \\
\hline 0.0997 & & 25 & 0.0094 & 1004.89 & \\
\hline 0.0997 & & 25 & 0.0094 & 1004.89 & \\
\hline 0.1 & & 25 & 0.0094 & 1004.87 & \\
\hline 0.13064 & & 25 & 0.0123 & 1007.22 & \\
\hline 0.13161 & & 25 & 0.0124 & 1007.33 & \\
\hline & 0.1598 & 25 & 0.0151 & 1009.51 & \\
\hline 0.195 & & 25 & 0.0182 & 1012.23 & \\
\hline
\end{tabular}

App vol App vol De $\begin{array}{cc}\exp / & \text { Calc } / \\ \mathrm{m}^{3} / \mathrm{kg} & \mathrm{m}^{3} / \mathrm{kg}\end{array}$

Density
calc/

Density App Vol Square of Inconsistent Inconsistent

Apparent

(Relative Delta density Density of $\mathrm{m}^{3} / \mathrm{kg} \quad \mathrm{kg} / \mathrm{m}^{3} \quad \mathrm{~kg} / \mathrm{m}^{3} \quad \mathrm{~m}^{3} / \mathrm{kg} \quad$ Residual density $\begin{gathered}\text { apparent } \\ \text { volume }\end{gathered}$

$\begin{array}{llllll}0.000237 & 0.000224 & 1100.70 & -1.7952 & 0.000012 & 3.223\end{array}$

$\begin{array}{llllll}0.000238 & 0.000239 & 1136.76 & 0.0647 & 0.000000 & 0.004\end{array}$

$\begin{array}{llllll}0.000251 & 0.000239 & 1136.78 & -2.5812 & 0.000013 & 6.662\end{array}$

$\begin{array}{llllll}0.000264 & 0.000253 & 1173.63 & -3.0298 & 0.000011 & 9.179\end{array}$

$\begin{array}{llllll}0.000265 & 0.000261 & 1194.33 & -1.3031 & 0.000004 & 1.698\end{array}$

$\begin{array}{llllll}0.000278 & 0.000272 & 1220.49 & -2.0881 & 0.000006 & 4.360\end{array}$

$\begin{array}{llllll}0.000280 & 0.000282 & 1244.90 & 0.9914 & -0.000002 & 0.983\end{array}$

$\begin{array}{llllll}0.000290 & 0.000292 & 1267.80 & 0.9959 & -0.000002 & 0.992\end{array}$

$\begin{array}{llllll}0.000176 & 0.000190 & 1005.61 & 0.1473 & -0.000014 & 0.022\end{array}$

$\begin{array}{llllll}0.000180 & 0.000193 & 1012.35 & 0.2461 & -0.000013 & 0.061\end{array}$

$\begin{array}{llllll}0.000184 & 0.000195 & 1018.68 & 0.3072 & -0.000011 & 0.094\end{array}$

$\begin{array}{llllll}0.000188 & 0.000198 & 1026.65 & 0.3787 & -0.000010 & 0.143\end{array}$

$\begin{array}{lllllll}0.000194 & 0.000202 & 1036.89 & 0.4102 & -0.000008 & 0.168\end{array}$

$\begin{array}{llllll}0.000163 & 0.000188 & 997.24 & 0.0060 & -0.000025 & 0.000\end{array}$

$\begin{array}{llllll}0.000152 & 0.000188 & 997.34 & 0.0132 & -0.000036 & 0.000\end{array}$

$\begin{array}{llllll}0.000165 & 0.000188 & 997.41 & 0.0102 & -0.000023 & 0.000\end{array}$

$\begin{array}{llllll}0.000161 & 0.000188 & 997.43 & 0.0128 & -0.000027 & 0.000\end{array}$

$0.000108 \quad 0.000188$

$0.000155 \quad 0.000188$

$\begin{array}{lll}997.69 & 0.0632 & -0.000080\end{array}$

$\begin{array}{lll}997.80 & 0.0308 & -0.000033\end{array}$

0.000

$0.000151 \quad 0.000188$

997.81

$0.0344-0.000037$

0.001

$\begin{array}{ll}0.000146 & 0.000188 \\ 0.000151 & 0.000188\end{array}$

997.82

$0.0394-0.000042$

0.001

$\begin{array}{ll}0.000158 & 0.000188\end{array}$

$0.000131 \quad 0.000188$

$0.000158 \quad 0.000188$

098.07

$0.0356-0.000038$

0.001

$\begin{array}{lll}0.0902 & -0.000058 & 0.008 \\ 0.0561 & -0.000030 & 0.003\end{array}$

$\begin{array}{lll}0.0504 & -0.000027 & 0.003\end{array}$

$\begin{array}{ll}0.000161 & 0.000189\end{array}$

998.59

$0.0613-0.000028$

0.004

$0.000162 \quad 0.000189$

998.83

$0.0772-0.000027$

0.006

$0.000164 \quad 0.000189$

0.0001590 .000189

999.45

$\begin{array}{ll}0.0728 & -0.000025\end{array}$

$0.0921-0.000030$

$0.0792-0.000025$

0.005

0.008

$\begin{array}{ll}0.000164 & 0.000189\end{array}$

999.60

$0.0939-0.000025$

0.006

0.015

$\begin{array}{ll}0.000163 & 0.000189 \\ 0.000167 & 0.000189\end{array}$

1000.78

$0.1210-0.000026$

0.012

$\begin{array}{ll}0.000165 & 0.000189\end{array}$

$\begin{array}{ll}0.000164 & 0.000189\end{array}$

000.89

$0.1078-0.000023$

$\begin{array}{lll}0.1175 & -0.000025 & 0.014\end{array}$

$\begin{array}{lll}0.1225 & -0.000026 & 0.015\end{array}$

$\begin{array}{lll}0.1100 & -0.000018 & 0.012\end{array}$

$\begin{array}{lll}0.1322 & -0.000021 & 0.017\end{array}$

$\begin{array}{llllll}0.000169 & 0.000190 & 1002.13 & 0.1322 & -0.000021 & 0.017 \\ 0.000170 & 0.000190 & 1003.11 & 0.1503 & -0.000020 & 0.023\end{array}$

$\begin{array}{lllllll}0.000173 & 0.000190 & 1003.98 & 0.1465 & -0.000017 & 0.021\end{array}$

$\begin{array}{lllllll}0.000171 & 0.000191 & 1004.05 & 0.1670 & -0.000019 & 0.028\end{array}$

$\begin{array}{lllllll}0.000170 & 0.000191 & 1004.70 & 0.1939 & -0.000020 & 0.038\end{array}$

$\begin{array}{lllllll}0.000170 & 0.000191 & 1004.70 & 0.1989 & -0.000021 & 0.040\end{array}$

$\begin{array}{llllll}0.000175 & 0.000191 & 1004.72 & 0.1511 & -0.000016 & 0.023\end{array}$

$\begin{array}{llllll}0.000179 & 0.000192 & 1007.05 & 0.1622 & -0.000013 & 0.026\end{array}$

$\begin{array}{llllll}0.000175 & 0.000192 & 1007.13 & 0.2045 & -0.000016 & 0.042\end{array}$

$\begin{array}{lll}0.000181 & 0.000193\end{array}$

$0.1701-0.000011$

0.042

0.088

$/ \mathrm{cm}^{3} / \mathrm{mol}$

density -
$1)^{*} 10^{\wedge} 4$

$\mathrm{kg} / \mathrm{m}^{3}$

998.2041

998.2041

998.2041

998.2041

998.2041

998.2041

998.2041

998.2041

997.2867

997.2867

17.14

997.2867

997.2867

997.2867

17.94

18.51

997.0449

0.311997 .0449

$0.372 \quad 997.0449$

997.0449

10.32

997.0449

$0.785 \quad 997.0449$

$0.797 \quad 997.0449$

$0.802 \quad 997.0449$

997.0449

8.1

1.065997 .0449 997.0449

12.43

$1.576 \quad 997.0449$ 997.0449

$1.849 \quad 997.0449$

997.0449

$2.476 \quad 997.0449$ 997.0449

15.16

$2.63 \quad 997.0449$ 997.0449

31.9

$3.854 \quad 997.0449$

997.0449

$3.994 \quad 997.0449$

$3.999 \quad 997.0449$ 997.0449

$5.214 \quad 997.0449$

$6.212 \quad 997.0449$

997.0449

$\begin{array}{ll}7.169 & 997.0449\end{array}$

$7.845 \quad 997.0449$

$7.85 \quad 997.0449$ 997.0449 997.0449

$10.287 \quad 997.0449$ 997.0449

15.185997 .0449 


\section{molality molarity}

t $/{ }^{\circ} \mathrm{C}$ mass frac exp $\mathrm{kg} / \mathrm{m}^{3}$

0.19525

0.1973

0.19893

0.19893

0.2518

0.2633

0.26767

0.28278

0.34096

0.37485

0.4636

0.4893

0.49487

0.49487

0.5
0.5008

0.5008

0.5247

0.55193
0.63404

0.63404
0.71531

0.7405

0.76535

0.79758

0.9778

0.99097

0.99097

0.9917

0.99851

1.0851

1.2621

1.351

1.47531

1.47531

1.7281

2.262

2.4934

2.952

3.5081

4
6479

5.1139

0.00249

0.00498

0.00997

0.01995

$\begin{array}{cccc}25 & 0.0183 & 1012.22 & (13) \\ 25 & 0.0184 & 1012.36 & (87) \\ 25 & 0.0186 & 1012.52 & (13) \\ 25 & 0.0186 & 1012.52 & (13) \\ 25 & 0.0234 & 1016.53 & (28) \\ 25 & 0.0245 & 1017.36 & (87) \\ 25 & 0.0249 & 1017.58 & (9) \\ 25 & 0.0262 & 1018.86 & (13) \\ 25 & 0.0314 & 1023.19 & (87) \\ 25 & 0.0345 & 1025.75 & (13) \\ 25 & 0.0423 & 1031.68 & (129) \\ 25 & 0.0445 & 1034.17 & (28) \\ 25 & 0.0450 & 1034.62 & (13) \\ 25 & 0.0450 & 1034.62 & (13) \\ 25 & 0.0454 & 1034.79 & (45) \\ 25 & 0.0455 & 1034.38 & (129) \\ 25 & 0.0476 & 1036.50 & (9) \\ 25 & 0.0499 & 1038.76 & (13) \\ 25 & 0.0569 & 1044.69 & (13) \\ 25 & 0.0638 & 1050.55 & (13) \\ 25 & 0.0659 & 1051.23 & (129) \\ 25 & 0.0679 & 1053.89 & (9) \\ 25 & 0.0706 & 1056.37 & (13) \\ 25 & 0.0852 & 1067.50 & (129) \\ 25 & 0.0862 & 1069.90 & (13) \\ 25 & 0.0862 & 1069.89 & (13) \\ 25 & 0.0863 & 1069.69 & (28) \\ 25 & 0.0868 & 1069.92 & (9) \\ 25 & 0.0869 & 1070.40 & (45) \\ 25 & 0.0936 & 1074.70 & (129) \\ 25 & 0.1073 & 1086.40 & (129) \\ 25 & 0.1140 & 1092.19 & (129) \\ 25 & 0.1232 & 1102.46 & (13) \\ 25 & 0.1232 & 1102.47 & (13) \\ 25 & 0.1413 & 1116.02 & (129) \\ 25 & 0.1600 & 1135.26 & (45) \\ 25 & 0.1772 & 1151.44 & (28) \\ 25 & 0.1919 & 1161.46 & (129) \\ 25 & 0.2194 & 1192.06 & (28) \\ 25 & 0.2222 & 1191.36 & (45) \\ 25 & 0.2504 & 1217.10 & (129) \\ 25 & 0.2758 & 1244.19 & (45) \\ 25 & 0.3068 & 1274.07 & (129) \\ 25 & 0.3275 & 1294.63 & (129) \\ 30 & 0.0002 & 995.85 & (49) \\ 30 & 0.0005 & 996.05 & (49) \\ 30 & 0.0010 & 996.44 & (49) \\ 30 & 0.0019 & 997.24 & (49) \\ & & & \end{array}$

App vol App vol De

$$
\begin{array}{cc}
\exp / & \text { Calc / } \\
\mathrm{m}^{3} / \mathrm{kg} & \mathrm{m}^{3} / \mathrm{kg}
\end{array}
$$

$\mathrm{m}^{3} / \mathrm{kg} \quad \mathrm{m}^{3} / \mathrm{kg}$

$\begin{array}{ll}0.000180 & 0.000194\end{array}$

$0.000178 \quad 0.000194$

$\begin{array}{ll}0.000178 & 0.000194\end{array}$

$\begin{array}{ll}0.000182 & 0.000195\end{array}$

$0.000184 \quad 0.000195$

$0.000188 \quad 0.000196$

$0.000184 \quad 0.000196$

$0.000188 \quad 0.000198$

0.0001890 .000199

$0.000207 \quad 0.000201$

$0.000194 \quad 0.000202$

$0.000194 \quad 0.000202$

$\begin{array}{ll}0.000194 & 0.000202 \\ 0.000198 & 0.000202\end{array}$

$\begin{array}{ll}0.000198 & 0.000202\end{array}$

$0.000201 \quad 0.000203$

$0.000196 \quad 0.000204$

$0.000200 \quad 0.000206$

$\begin{array}{lll}0.000202 & 0.000208\end{array}$

$\begin{array}{ll}0.000218 & 0.000209\end{array}$

$0.000207 \quad 0.000209$

$0.000205 \quad 0.000210$

$0.000226 \quad 0.000215$

$0.000211 \quad 0.000215$

$\begin{array}{ll}0.000211 & 0.000215 \\ 0.000214 & 0.000215\end{array}$

$0.000214 \quad 0.000215$

$\begin{array}{ll}0.000212 & 0.000216\end{array}$

$\begin{array}{ll}0.000212 & 0.000216 \\ 0.000229 & 0.000218\end{array}$

$\begin{array}{ll}0.000229 & 0.000218 \\ 0.000234 & 0.000222\end{array}$

$0.000236 \quad 0.000224$

$0.000224 \quad 0.000228$

$0.000224 \quad 0.000228$

$\begin{array}{ll}0.000246 & 0.000234\end{array}$

$0.000240 \quad 0.000240$

$0.000244 \quad 0.000246$

$\begin{array}{ll}0.000263 & 0.000252\end{array}$

$0.000255 \quad 0.000261$

$\begin{array}{ll}0.000267 & 0.000263 \\ 0.000279 & 0.000273\end{array}$

$\begin{array}{ll}0.000279 & 0.000273 \\ 0.000281 & 0.000283\end{array}$

$0.000292 \quad 0.000295$

0.0002990 .000303

$\begin{array}{lll}0.000161 & 0.000191\end{array}$

$\begin{array}{lll}0.000161 & 0.000191\end{array}$

$\begin{array}{ll}0.000162 & 0.000191\end{array}$

0.0001620 .000191
Density Density App Vol Square of Inconsistent Inconsistent
calc / Residual / Residual / Density based on based on $\mathrm{kg} / \mathrm{m}^{3} \quad \mathrm{~m}^{3} / \mathrm{kg}$ Rensity density apparent molal volume volume

\subsection{5}

1012.11

0.2655

$\mathrm{m}^{3} / \mathrm{kg}$

Residual

$0.2547-0.000013$

0.071

$\begin{array}{llll}1012.23 & 0.2906 & -0.000015 & 0.084\end{array}$

$\begin{array}{llll}1016.21 & 0.3181 & -0.000013 & 0.101\end{array}$

$\begin{array}{llll}1017.07 & 0.2853 & -0.000011 & 0.081\end{array}$

$\begin{array}{llll}1017.40 & 0.1844 & -0.000007 & 0.034\end{array}$

$\begin{array}{llll}1018.53 & 0.3313 & -0.000012 & 0.110\end{array}$

$\begin{array}{llll}1022.87 & 0.3165 & -0.000010 & 0.100\end{array}$

$\begin{array}{llll}025.38 & 0.3609 & -0.000010 & 0.130\end{array}$

$\begin{array}{llll}1031.93 & -0.2453 & 0.000005 & 0.060\end{array}$

$\begin{array}{llll}1033.81 & 0.3587 & -0.000008 & 0.129\end{array}$

$\begin{array}{llll}1034.21 & 0.4026 & -0.000008 & 0.162\end{array}$

$\begin{array}{llll}1034.21 & 0.4056 & -0.000008 & 0.165\end{array}$

$\begin{array}{llll}1034.59 & 0.2009 & -0.000004 & 0.040\end{array}$

$\begin{array}{llll}1034.65 & -0.2675 & 0.000005 & 0.072\end{array}$

$\begin{array}{llll}1036.39 & 0.1055 & -0.000002 & 0.011\end{array}$

$\begin{array}{llll}1038.37 & 0.3933 & -0.000007 & 0.155\end{array}$

$\begin{array}{llll}1044.30 & 0.3841 & -0.000006 & 0.148\end{array}$

$\begin{array}{llll}1050.12 & 0.4237 & -0.000006 & 0.179\end{array}$

$\begin{array}{llll}1051.92 & -0.6871 & 0.000009 & 0.472\end{array}$

$\begin{array}{llll}1053.68 & 0.2051 & -0.000003 & 0.042\end{array}$

$\begin{array}{lll}1068.55 & -1.0549 & 0.000011\end{array}$

$\begin{array}{rrrr}1069.47 & 0.4319 & -0.000004 & 0.187\end{array}$

$\begin{array}{llll}1069.47 & 0.4299 & -0.000004 & 0.185\end{array}$

$\begin{array}{llll}1069.52 & 0.1725 & -0.000002 & 0.030\end{array}$

$\begin{array}{llll}1069.99 & -0.0665 & 0.000001 & 0.004\end{array}$

$\begin{array}{llll}1070.09 & 0.3118 & -0.000003 & 0.097\end{array}$

$\begin{array}{llll}1075.93 & -1.2295 & 0.000011 & 1.512\end{array}$

$\begin{array}{llll}1087.89 & -1.4940 & 0.000012 & 2.232\end{array}$

$\begin{array}{llll}1093.81 & -1.6198 & 0.000012 & 2.624\end{array}$

$\begin{array}{llll}1101.98 & 0.4850 & -0.000003 & 0.235\end{array}$

$\begin{array}{llll}1101.98 & 0.4890 & -0.000003 & 0.239\end{array}$

$\begin{array}{llll}1118.22 & -2.1977 & 0.000012 & 4.830\end{array}$

$\begin{array}{llll}1135.14 & 0.1213 & -0.000001 & 0.015\end{array}$

$\begin{array}{llll}1150.92 & 0.5178 & -0.000002 & 0.268\end{array}$

$\begin{array}{llll}1150.92 & 0.5178 & -0.000002 & 0.268 \\ 1190.09 & 1.9711 & 0.000011 & 8.845\end{array}$

$\begin{array}{lll}1.9711 & -0.000006\end{array}$

$\begin{array}{llll}1192.69 & -1.3253 & 0.000004 & 1.756\end{array}$

$\begin{array}{llll}1219.24 & -2.1424 & 0.000006 & 4.590\end{array}$

$\begin{array}{llll}1243.34 & 0.8525 & -0.000002 & 0.727\end{array}$

$\begin{array}{lll}1272.78 & 1.2851 & -0.000003\end{array}$

$\begin{array}{llll}292.45 & 2.1818 & -0.000004 & 4.760 \\ 995.84 & 0.0070 & -0.000030 & 0.000\end{array}$

$996.03-0.0141-0.000030$

$\begin{array}{llll}996.42 & 0.0275 & -0.000029 & 0.001\end{array}$

$\begin{array}{llll}997.19 & 0.0548 & -0.000029 & 0.003\end{array}$
Apparen

$1 \mathrm{~cm}^{3} / \mathrm{mol}$

17.94

19.11

20.58

Relative Delta density Density of

density - $\quad / \mathrm{kg} / \mathrm{m}^{3}$ water at $\mathrm{t}^{\circ} \mathrm{C} /$

$15.172 \quad 997.0449$

$15.316 \quad 997.0449$

$15.476 \quad 997.0449$

$15.475 \quad 997.0449$

$19.483 \quad 997.0449$

$20.313 \quad 997.0449$

997.0449

$21.818 \quad 997.0449$

$26.142 \quad 997.0449$

28.701997 .0449

997.0449

$37.121 \quad 997.0449$

$37.572 \quad 997.0449$

$37.575 \quad 997.0449$

997.0449

997.0449

41719997.

$47.644 \quad 997.0449$

$53.503 \quad 997.0449$ 997.0449

$59.328 \quad 997$.

$72.85 \quad 997.0449$

997.0449
997.0449

997.0449

997.0449
997.0449

997.0449

997.0449

$105.418 \quad 997.0449$

105.422997. 997.0449 997.0449

$154.391 \quad 997.0449$ 997.0449

195.012997 .0449

997.0449

997.0449

997.0449

997.0449

997.0449

995.6473

995.6473

995.6473

995.6473 
App vol App vol De $\begin{array}{cc}\exp / & \text { Calc / } \\ \mathrm{m}^{3} / \mathrm{kg} & \mathrm{m}^{3} / \mathrm{kg}\end{array}$ $\begin{array}{rr}\mathrm{m}^{3} / \mathrm{kg} & \mathrm{m}^{3} / \mathrm{kg} \\ 0.000165 & 0.000191\end{array}$ $\begin{array}{ll}0.000167 & 0.000192 \\ 0.000170 & 0.000192\end{array}$ $\begin{array}{ll}0.000170 & 0.000192 \\ 0.000175 & 0.000193\end{array}$ $\begin{array}{ll}0.000175 & 0.000193 \\ 0.000176 & 0.000193\end{array}$ $\begin{array}{ll}0.000182 & 0.000195\end{array}$ $\begin{array}{ll}0.000182 & 0.000195 \\ 0.000199 & 0.000204 \\ 0.000217 & 0.000217\end{array}$ $\begin{array}{ll}0.000199 & 0.000204 \\ 0.000217 & 0.000217\end{array}$ $0.000240 \quad 0.000241$ $\begin{array}{lll}0.000267 & 0.000263\end{array}$ $\begin{array}{lll}0.000281 & 0.000283\end{array}$ $0.000162 \quad 0.000193$ $\begin{array}{lll}0.000186 & 0.000193\end{array}$ $\begin{array}{ll}0.000162 & 0.000193 \\ 0.000136 & 0.000193\end{array}$ $0.000162 \quad 0.000193$ $0.000145 \quad 0.000193$ $0.000167 \quad 0.000193$ 0.0001690 .000193 $\begin{array}{lll}0.000162 & 0.000193\end{array}$ $0.000170 \quad 0.000194$ $0.000172 \quad 0.000194$ $\begin{array}{lll}0.000168 & 0.000194\end{array}$ $\begin{array}{lll}0.000177 & 0.000194\end{array}$ $\begin{array}{ll}0.000171 & 0.000195\end{array}$ 0.0002520 .000195 $\begin{array}{lll}0.000176 & 0.000195\end{array}$ $\begin{array}{ll}0.000182 & 0.000196\end{array}$ $\begin{array}{lll}0.000183 & 0.000197\end{array}$ $\begin{array}{lll}0.000191 & 0.000200\end{array}$ $\begin{array}{ll}0.000187 & 0.000200\end{array}$ $\begin{array}{ll}0.000191 & 0.000203 \\ 0.000193 & 0.000204\end{array}$ 0.0001990 .000206 $0.000203 \quad 0.000207$ $\begin{array}{lll}0.000199 & 0.000207\end{array}$ $0.000208 \quad 0.000213$ $\begin{array}{ll}0.000218 & 0.000219 \\ 0.000217 & 0.000219\end{array}$ $0.000217 \quad 0.000219$ $\begin{array}{lll}0.000240 & 0.000242\end{array}$ $\begin{array}{lll}0.000267 & 0.000263\end{array}$ $0.000281 \quad 0.000282$ $0.000199 \quad 0.000200$ $0.000207 \quad 0.000206$ $0.000240 \quad 0.00023$ $\begin{array}{ll}0.000240 & 0.000230 \\ 0.000254 & 0.000243\end{array}$ $0.000266 \quad 0.000256$
Density Density App Vol Square of Inconsistent Inconsistent

Apparent
molal volume $\mathrm{kg} / \mathrm{m}^{3} \mathrm{~kg} / \mathrm{m}^{3} \mathrm{~m}^{3} / \mathrm{kg}$ Density density apparent $/ \mathrm{cm}^{3} / \mathrm{mol}$ 997.95 998.72 999.48 1002.55 1003.29 1007.89 1033.05
1068.43 1133.36 1190.92 1241.71 994.22 994.3 994.42 994.67 995.29 996.33 996.33
996.54 996.54
997.09
$0.0735-0.000026$

$0.0920-0.000024$

$0.1016-0.000021$

$0.1559-0.000018$

$0.1608-0.000017$

$0.2031-0.000013$

$\begin{array}{ll}0.2160 & -0.000001 \\ -1.2734 & 0.0000004\end{array}$

$0.6933-0.000002$

$0.0071-0.000030$

$0.0031-0.000007$

$0.0143-0.000030$

$0.0444-0.000056$

$0.0487-0.000026$

$0.0685-0.000024$

$0.0987-0.000032$

$\begin{array}{lll}0.0882 & -0.000023\end{array}$

$0.1215-0.000026$

$0.1105-0.000018$

$0.2163-0.000024$

$\begin{array}{ll}-0.5337 & 0.000057\end{array}$

$0.1849-0.000020$

$0.2183-0.000014$

$0.2394-0.000009$

$0.3667-0.000014$

$0.4310-0.000012$

$0.4194-0.000011$

$0.3394-0.000007$

$0.1924-0.000004$

$0.4473-0.000008$

$\begin{array}{ll}0.1020 & -0.000001 \\ 0.1649 & -0.000002\end{array}$

$0.3737-0.000002$

$\begin{array}{ll}0.3737 & 0.000004\end{array}$

$0.5664-0.000001$

$0.0175-0.00000$

$\begin{array}{ll}0.0405 & 0.000001\end{array}$

$\begin{array}{ll}-1.3988 & 0.000010\end{array}$

$-2.22240 .000011$

$2.7901 \quad 0.000010$
0.005

0.008

0.010

0.024

0.041

0.055

0.000

0.047

1.622

0.000

0.000

0.000

0.001

0.006

0.002

0.005

0.010
0.008

0.010

0.015

0.012

0.047

0.285

0.034

0.032

0.048

0.057
0.134

0.186

0.176

0.115

0.037

0.200

0.115
0.010

0.027

0.140

1.437

0.321

0.000

0.002

1.957

4.939

7.785
Relative Delta density Density of density - $\quad / \mathrm{kg} / \mathrm{m}^{3}$ water at $\mathrm{t}^{\circ} \mathrm{C} /$

23.9

31.8

70.9

125

995.6473

995.6473

995.6473

995.6473

995.6473

995.6473

995.6473

995.6473

995.6473

995.6473

995.6473

994.0319

0.362994 .0319

994.0319

994.0319

994.0319

994.0319

994.0319

994.0319

994.0319

$31.7 \quad 3.805 \quad 994.0319$

994.0319

994.0319

$7.547 \quad 994.0319$

994.0319

994.0319

994.0319

994.0319

18.14

124.9

21.689994 .0319

$28.558 \quad 994.0319$

$30.988 \quad 994.0319$

994.0319

994.0319

$41.473 \quad 994.0319$

994.0319

994.0319

9940319

994.0319

994.0319

992.2158

992.2158

992.2158

992.2158

992.2158

992.2158 
molality molarity

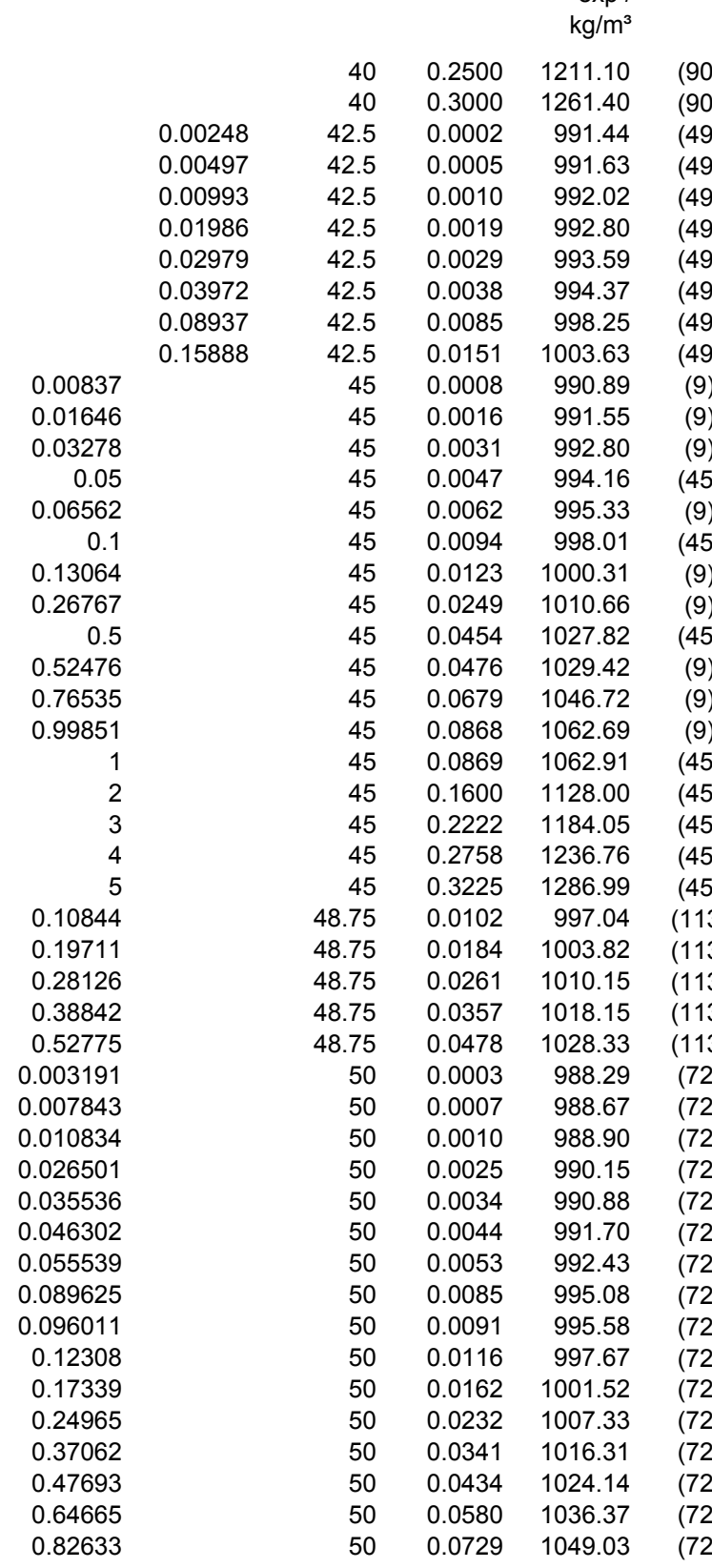
App vol App vol Density Density App Vol Square of Inconsistent Inconsistent Density Density Inconsistent
based on morent
molal volume $\mathrm{m}^{3} / \mathrm{kg} \quad \mathrm{m}^{3} / \mathrm{kg}$ $\mathrm{kg} / \mathrm{m}^{3}$ $\mathrm{kg} / \mathrm{m}^{3}$ $\mathrm{m}^{3} / \mathrm{k}$ Residual

5.751
$\begin{array}{lll}0.000279 & 0.000273\end{array}$ $\begin{array}{ll}0.000291 & 0.000290 \\ 0.000162 & 0.000195\end{array}$ 1261. $0.000164 \quad 0.000195$ $\begin{array}{lll}0.000173 & 0.000195\end{array}$ $\begin{array}{lll}0.000173 & 0.000195\end{array}$ 0.0001730 .000196 0.0001730 .000196 $\begin{array}{lll}0.000178 & 0.000197\end{array}$ $0.000183 \quad 0.000199$ $\begin{array}{ll}0.000149 & 0.000196\end{array}$ $\begin{array}{lll}0.000142 & 0.000196\end{array}$ 0.0001640 .000196 $0.000164 \quad 0.000197$ $0.000174 \quad 0.000197$ $\begin{array}{lll}0.000173 & 0.000198\end{array}$ 0.0001810 .000199 $0.000188 \quad 0.000202$ $0.000197 \quad 0.000208$ $0.000202 \quad 0.000209$ $0.000207 \quad 0.000215$ $0.000217 \quad 0.000220$ $0.000215 \quad 0.000220$ $0.000239 \quad 0.000243$ $0.000266 \quad 0.000263$ $0.000280 \quad 0.000281$ $\begin{array}{lll}0.000288 & 0.000297\end{array}$ $0.000173 \quad 0.000199$ $0.000179 \quad 0.000201$ 0.0001840 .000203 $0.000188 \quad 0.000206$ $0.000195 \quad 0.000209$ $0.000146 \quad 0.000196$ $0.000149 \quad 0.000197$ $\begin{array}{lll}0.000151 & 0.000197\end{array}$ $0.000155 \quad 0.000197$ $\begin{array}{ll}0.000151 & 0.000197 \\ 0.000159 & 0.000198\end{array}$ $\begin{array}{ll}0.000161 & 0.000198\end{array}$ $0.000165 \quad 0.000199$ $0.000165 \quad 0.000199$ 0.0001690 .000199 $0.000173 \quad 0.000201$ $0.000177 \quad 0.000203$ $\begin{array}{lll}0.000186 & 0.000206\end{array}$ $\begin{array}{ll}0.000191 & 0.000208\end{array}$ $\begin{array}{ll}0.000198 & 0.000212\end{array}$ $\begin{array}{ll}0.000205 & 0.000217\end{array}$

$-0.2237 \quad 0.000000$

$\begin{array}{lll}991.43 & 0.0077 & -0.000033\end{array}$

991.62

$0.0148-0.000031$

0.050

0.000

$\begin{array}{llll}992.00 & 0.0205 & -0.000022 & 0.000\end{array}$

$\begin{array}{llll}992.76 & 0.0415 & -0.000022 & 0.002\end{array}$

$\begin{array}{llll}993.52 & 0.0631 & -0.000022 & 0.004\end{array}$

$\begin{array}{llll}994.28 & 0.0851 & -0.000023 & 0.007\end{array}$

$\begin{array}{llll}998.08 & 0.1637 & -0.000019 & 0.027\end{array}$

$\begin{array}{llll}1003.38 & 0.2515 & -0.000017 & 0.063\end{array}$

$\begin{array}{llll}990.85 & 0.0362 & -0.000046 & 0.001\end{array}$

$\begin{array}{llll}991.46 & 0.0834 & -0.000054 & 0.007\end{array}$

$\begin{array}{llll}992.70 & 0.0993 & -0.000032 & 0.010\end{array}$

$\begin{array}{llll}994.01 & 0.1546 & -0.000033 & 0.024\end{array}$

$\begin{array}{llll}995.19 & 0.1425 & -0.000023 & 0.020 \\ 997.78 & 0.2317 & -0.000025 & 0.054\end{array}$

$1000.08-0.2255-0.000018$

$\begin{array}{llll}1010.29 & 0.3737 & -0.000015 & 0.140\end{array}$

$\begin{array}{llll}1027.27 & 0.5490 & -0.000011 & 0.301\end{array}$

$\begin{array}{llll}1029.06 & 0.3588 & -0.000007 & 0.129\end{array}$

$\begin{array}{llll}1046.16 & 0.5526 & -0.000007 & 0.305\end{array}$

$\begin{array}{llll}1062.33 & 0.3661 & -0.000004 & 0.134\end{array}$

$\begin{array}{llll}1062.43 & 0.4812 & -0.000005 & 0.232\end{array}$

$\begin{array}{llll}1127.22 & 0.7764 & -0.000004 & 0.603\end{array}$

$\begin{array}{llll}1185.05 & -0.9989 & 0.000003 & 0.998\end{array}$

$\begin{array}{lllll}236.45 & 0.3064 & -0.000001 & 0.094\end{array}$

$\begin{array}{rrrr}282.03 & 4.9641 & -0.000009 & 24.642 \\ 096.78 & 0.2636 & -0.000026 & 0.069\end{array}$

0.069

$\begin{array}{llll}1009.64 & 0.5129 & -0.000019 & 0.263\end{array}$

$\begin{array}{llll}1017.50 & 0.6477 & -0.000018 & 0.420\end{array}$

$\begin{array}{llll}1027.59 & 0.7454 & -0.000015 & 0.556\end{array}$

$\begin{array}{llll}988.28 & 0.0149 & -0.000050 & 0.000\end{array}$

$\begin{array}{llll}988.63 & 0.0345 & -0.000047 & 0.001\end{array}$

$\begin{array}{llll}988.86 & 0.0460 & -0.000046 & 0.002\end{array}$

$\begin{array}{llll}990.04 & 0.1039 & -0.000042 & 0.011\end{array}$

$\begin{array}{llll}990.73 & 0.1536 & -0.000046 & 0.024\end{array}$

$\begin{array}{llll}991.54 & 0.1647 & -0.000038 & 0.027\end{array}$

$\begin{array}{llll}992.24 & 0.1916 & -0.000037 & 0.037\end{array}$

$\begin{array}{llll}994.80 & 0.2786 & -0.000033 & 0.078\end{array}$

$\begin{array}{llll}995.28 & 0.3029 & -0.000034 & 0.092\end{array}$

$\begin{array}{llll}997.31 & 0.3535 & -0.000031 & 0.125\end{array}$

$\begin{array}{llll}001.07 & 0.4536 & -0.000028 & 0.206\end{array}$

$\begin{array}{lll}0.5946 & -0.000025 & 0.354\end{array}$

$\begin{array}{llll}1015.62 & 0.6858 & -0.000019 & 0.470\end{array}$

$\begin{array}{llll}1023.35 & 0.7939 & -0.000017 & 0.630\end{array}$

$\begin{array}{llll}1035.50 & 0.8688 & -0.000014 & 0.755\end{array}$

$\begin{array}{lll}0.8688 & -0.000014 & 0.755 \\ 0.8922 & -0.000011 & 0.796\end{array}$
Apparent

(Relative Delta density Density of density - $\quad / \mathrm{kg} / \mathrm{m}^{3}$ water at $\mathrm{t}^{\circ} \mathrm{C} /$ volume

992.2158 992.2158 991.237 991.237 991.237 991.237 991.237 991.237 991.237 991.237 990.2132 990.2132 990.2132 990.2132 990.2132 990.2132 990.2132 990.2132 990.2132 990.2132 990.2132 990.2132 990.2132 990.2132 990.2132 990.2132 990.2132 988.5963 988.5963 988.5963 988.5963 988.5963

$0.2568 \quad 988.0363$ $0.629 \quad 988.0363$ 0.8671988 .0363 $2.1108 \quad 988.0363$ 2.8435988 .0363 3.6677988 .0363 $4.3914 \quad 988.0363$ $7.0445 \quad 988.0363$ $7.5486 \quad 988.0363$ $9.6294 \quad 988.0363$ 13.4882988 .0363 $19.2903 \quad 988.0363$ $28.2719 \quad 988.0363$ $36.1026 \quad 988.0363$ $48.3318 \quad 988.0363$ 60.9902988 .0363 


\section{molality molarity}

$\mathrm{t} /{ }^{\circ} \mathrm{C}$ mass frac Density

$\exp /$

0.99421

0.00837

0.01646

0.03278

0.05
0.1

0.1

0.13064

0.26767

52476

0.76535

0.99851

1
2
3

0.00837

0.01646

0.03278

0.06562

0.13064

0.26767

0.52476

0.76535

0.99851

0.10844

0.19711

0.28126

0.38842

0.52775

0.00837

0.01646

0.03278

0.06562

0.13064

0.26767

0.52476

0.76535

0.99851
Ref

App $\begin{array}{cc}\text { App vol } & \text { App vol } \\ \text { exp / } & \text { Calc / } \\ \mathrm{m}^{3} / \mathrm{kg} & \mathrm{m}^{3} / \mathrm{kg}\end{array}$

Density
calc /

Density App Vol Square of Inconsistent Inconsistent

Apparent $\mathrm{m}^{3} / \mathrm{kg}$ $\mathrm{kg} / \mathrm{m}^{3}$

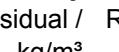
$\mathrm{m}^{3 /}$ Square of of Inconsistent
based on ased on molal volum volume

$/ \mathrm{cm}^{3} / \mathrm{mol}$

Relative Delta density Density of 1)*10^4 $\quad / \mathrm{kg} / \mathrm{m}^{3}$ water at $\mathrm{t}^{\circ} \mathrm{C} /$

$\begin{array}{rrrc}50 & 0.0865 & 1060.63 & (72) \\ 55 & 0.0008 & 986.38 & (9) \\ 55 & 0.0016 & 987.02 & (9) \\ 55 & 0.0031 & 988.29 & (9) \\ 55 & 0.0047 & 989.66 & (45) \\ 55 & 0.0062 & 990.81 & (9) \\ 55 & 0.0094 & 993.54 & (45) \\ 55 & 0.0123 & 995.84 & (9) \\ 55 & 0.0249 & 1006.20 & (9) \\ 55 & 0.0454 & 1023.43 & (45) \\ 55 & 0.0476 & 1025.00 & (9) \\ 55 & 0.0679 & 1042.36 & (9) \\ 55 & 0.0868 & 1058.36 & (9) \\ 55 & 0.0869 & 1058.63 & (45) \\ 55 & 0.1600 & 1123.86 & (45) \\ 55 & 0.2222 & 1180.02 & (45) \\ 55 & 0.2758 & 1232.77 & (45) \\ 60 & 0.0200 & 999.50 & (90) \\ 60 & 0.0400 & 1015.90 & (90) \\ 60 & 0.0800 & 1049.30 & (90) \\ 60 & 0.1200 & 1083.60 & (90) \\ 60 & 0.1600 & 1118.80 & (90) \\ 60 & 0.2000 & 1155.20 & (90) \\ 60 & 0.2500 & 1203.10 & (90) \\ 60 & 0.3000 & 1253.50 & (90) \\ 65 & 0.0008 & 981.24 & (9) \\ 65 & 0.0016 & 981.87 & (9) \\ 65 & 0.0031 & 983.16 & (9) \\ 65 & 0.0062 & 985.71 & (9) \\ 65 & 0.0123 & 990.76 & (9) \\ 65 & 0.0249 & 1001.14 & (9) \\ 65 & 0.0476 & 1020.04 & (9) \\ 65 & 0.0679 & 1037.49 & (9) \\ 65 & 0.0868 & 1053.53 & (9) \\ 73.77 & 0.0102 & 984.15 & (113) \\ 73.77 & 0.0184 & 991.02 & (113) \\ 73.77 & 0.0261 & 997.44 & (113) \\ 73.77 & 0.0357 & 1005.50 & (113) \\ 73.77 & 0.0478 & 1015.80 & (113) \\ 75 & 0.0008 & 975.51 & (9) \\ 75 & 0.0016 & 976.17 & (9) \\ 75 & 0.0031 & 977.49 & (9) \\ 75 & 0.0062 & 980.05 & (9) \\ 75 & 0.0123 & 985.17 & (9) \\ 75 & 0.0249 & 995.62 & (9) \\ 75 & 0.0476 & 1014.66 & (9) \\ 75 & 0.0679 & 1032.23 & (9) \\ 75 & 0.0868 & 1048.38 & (9)\end{array}$

$\begin{array}{ll}0.000211 & 0.000220 \\ 0.000129 & 0.000197\end{array}$ $0.000147 \quad 0.000197$ 1059.73
986.33

0.812 $0.0529-0.000068$

0.812 $\begin{array}{ll}0.000159 & 0.000198\end{array}$ 986.94 $0.0760-0.000050$ $0.000157 \quad 0.000198$ $\begin{array}{ll}0.000170 & 0.000198\end{array}$ $\begin{array}{ll}0.000165 & 0.000199\end{array}$ $\begin{array}{rrrrrr}0.000174 & 0.000200 & 995.51 & 0.3222 & -0.000026 & 0.104 \\ 0.000183 & 0.000203 & 1005.68 & 0.5169 & -0.000021 & 0.267\end{array}$ 988.17 $0.1180-0.000039$

$0.1917-0.00004$

$990.64 \quad 0.1713-0.000028 \quad 0.029$

$\begin{array}{llll}993.22 & 0.3171 & -0.000034 & 0.101\end{array}$ $\begin{array}{lll}0.000191 & 0.000209 & 1022.60\end{array}$

$0.5169-0.000021$ $0.000197 \quad 0.000210$ 1024.38 $0.8333-0.000018$ $0.6191-0.000012$ $\begin{array}{lll}0.9216 & -0.000012 & 0.849\end{array}$ $\begin{array}{lll}0.7863 & -0.000008 & 0.618\end{array}$ $0.000203 \quad 0.000215$ $0.000212 \quad 0.000220$ 57.57 1057.57 $0.9551-0.000010$ $1.3288-0.000007$ $0.000235 \quad 0.000241$

$\begin{array}{lll}0.000263 & 0.000260\end{array}$

1180.69

$-0.6693 \quad 0.000002$

$\begin{array}{ll}0.1069 & 0.000000\end{array}$

$0.000277 \quad 0.000278$

$\begin{array}{ll}0.000188 & 0.000202 \\ 0.000199 & 0.000207\end{array}$

232.66

999.21
1015.54

$0.2883-0.000014$

$0.3605-0.000009$

$0.1609-0.000002$

$\begin{array}{ll}0.000216 & 0.000218\end{array}$

1049.14

$\begin{array}{ll}0.000232 & 0.000229 \\ 0.000247 & 0.000240\end{array}$

1083.99

$\begin{array}{ll}-0.3850 & 0.000003\end{array}$

$-1.2459 \quad 0.000006$

$\begin{array}{ll}0.000260 & 0.000252\end{array}$

$\begin{array}{ll}0.000273 & 0.000267\end{array}$

1120.05

$\begin{array}{ll}-2.0666 & 0.000008\end{array}$

$\begin{array}{ll}-2.1933 & 0.000006\end{array}$

$0.000286 \quad 0.000283$

205.29

981.19

$\begin{array}{ll}0.000146 & 0.000197\end{array}$

981.79

$\begin{array}{ll}0.000152 & 0.000198\end{array}$

$\begin{array}{ll}0.000161 & 0.000198\end{array}$

$0.000165 \quad 0.000200$

983.02

$0.0520-0.000068$

$0.0773-0.000051$

$0.1373-0.000046$

$\begin{array}{llll}985.48 & 0.2274 & -0.000038\end{array}$

990.34

$0.000176 \quad 0.000203$

1000.47

$0.4196-0.000035$

$0.6759-0.000027$

$0.9181-0.000019$

$1.3335-0.000018$

$\begin{array}{lll}0.000196 & 0.000214\end{array}$

1036.16

$0.000206 \quad 0.000219$

$1.2460-0.000013$

$\begin{array}{lll}0.4639 & -0.000047\end{array}$

$\begin{array}{lll}0.7597 & -0.000042\end{array}$

$0.9926-0.000038$

$\begin{array}{ll}1.2507 & -0.000035\end{array}$

$1.5063-0.000031$

$0.0345-0.000046$

$\begin{array}{lll}0.0810 & -0.000054\end{array}$

$0.1775-0.000060$

$\begin{array}{lll}0.2896 & -0.000049\end{array}$

$\begin{array}{lll}0.5646 & -0.000047\end{array}$

$\begin{array}{ll}1.3192 & -0.000027\end{array}$

$1.8604-0.000026$

$1.8707-0.000020$ 0.006 0.014

0.014

0.267

12.26

14.03
15.12

15.12

16.22

16.53

17.41

18.77

19.29

20.21

1.766

0.448

0.011

0.083

0.130

0.026
0.148

1.552

4.271

4.810

1.585

0.003
0.006

0.019

0.052

0.176

0.457

0.843
1.778

1.552

0.215

0.577

0.985

1.564

0.001
0.007

0.031

0.084

0.319

0.826

1.740

3.461
3.499 $\begin{array}{ll}0.000186 & 0.000212 \\ 0.000197 & 0.000217\end{array}$

$\begin{array}{lll}0.000197 & 0.000217\end{array}$
(1040.51
12.3

13.9

4.47

5.72

16.75

8.12

18.65

19.63

14.42

15.07

15.6

16.16

16.84

1432

13.5

13.02

14.15

14.4

15.68

17.15

17.74

18.77
$72.59 \quad 988.0363$

985.6952

985.6952

985.6952

985.6952

985.6952

985.6952

985.6952

985.6952

985.6952

985.6952

985.6952

985.6952

985.6952

985.6952

985.6952

983.1989

983.1989

983.1989

983.1989

983.198

983.1989

983.1989

983.1989

980.5548

980.5548

980.5548

980.5548

980.5548

980.5548

980.5548

980.5548

980.5548

975.5797

975.5797

975.5797

975.5797

975.5797

974.849

974.849

974.849

974.849

974.849

974.849

974.849

974.849

974.849 
molality molarity

$\begin{array}{rrrr}\mathbf{t} /{ }^{\circ} \mathrm{C} \text { mass frac } & \begin{array}{r}\text { Density } \\ \text { exp } /\end{array} & \text { Ref } \\ & & \mathrm{kg} / \mathrm{m}^{3} & \\ & & \\ 80 & 0.0200 & 988.30 & (90) \\ 80 & 0.0400 & 1005.00 & (90) \\ 80 & 0.0800 & 1038.80 & (90) \\ 80 & 0.1200 & 1073.50 & (90) \\ 80 & 0.1600 & 1109.20 & (90) \\ 80 & 0.2000 & 1146.00 & (90) \\ 80 & 0.2500 & 1194.20 & (90) \\ 80 & 0.3000 & 1245.10 & (90) \\ 85 & 0.0008 & 969.30 & (9) \\ 85 & 0.0016 & 969.96 & (9) \\ 85 & 0.0031 & 971.29 & (9) \\ 85 & 0.0062 & 973.90 & (9) \\ 85 & 0.0123 & 979.03 & (9) \\ 85 & 0.0249 & 989.59 & (9) \\ 85 & 0.0476 & 1008.81 & (9) \\ 85 & 0.0679 & 1026.51 & (9) \\ 85 & 0.0868 & 1042.79 & (9) \\ 95 & 0.0008 & 962.59 & (9) \\ 95 & 0.0016 & 963.27 & (9) \\ 95 & 0.0031 & 964.61 & (9) \\ 95 & 0.0062 & 967.27 & (9) \\ 95 & 0.0123 & 972.44 & (9) \\ 95 & 0.0249 & 983.13 & (9) \\ 95 & 0.0476 & 1002.53 & (9) \\ 95 & 0.0679 & 1020.38 & (9) \\ 95 & 0.0868 & 1036.80 & (9) \\ 98.67 & 0.0102 & 968.13 & (113) \\ 98.67 & 0.0184 & 975.15 & (113) \\ 98.67 & 0.0261 & 981.70 & (113) \\ 98.67 & 0.0357 & 990.03 & (113) \\ 98.67 & 0.0478 & 1000.62 & (113) \\ 100 & 0.0200 & 975.30 & (90) \\ 100 & 0.0400 & 992.30 & (90) \\ 100 & 0.0800 & 1026.90 & (90) \\ 100 & 0.1200 & 1062.20 & (90) \\ 100 & 0.1600 & 1098.40 & (90) \\ 100 & 0.2000 & 1135.90 & (90) \\ 100 & 0.2500 & 1184.70 & (90) \\ 100 & 0.3000 & 1236.00 & (90) \\ & & & \end{array}$

App vol App vol De

$$
\begin{array}{rr}
\exp / & \text { Calc / } \\
\mathrm{m}^{3} / \mathrm{kg} & \mathrm{m}^{3} / \mathrm{kg}
\end{array}
$$

$\begin{array}{ll}0.000170 & 0.000199\end{array}$

$\begin{array}{ll}0.000179 & 0.000204 \\ 0.000199 & 0.000214\end{array}$

$\begin{array}{ll}0.000217 & 0.000223\end{array}$

$\begin{array}{ll}0.000232 & 0.000234\end{array}$

$\begin{array}{ll}0.000247 & 0.000244\end{array}$

$\begin{array}{ll}0.000262 & 0.000258\end{array}$

$\begin{array}{ll}0.000276 & 0.000272 \\ 0.000118 & 0.000194\end{array}$

$\begin{array}{ll}0.000118 & 0.000194 \\ 0.000119 & 0.000194\end{array}$

$\begin{array}{ll}0.000120 & 0.000194\end{array}$

$\begin{array}{lll}0.000131 & 0.000195\end{array}$

$\begin{array}{lll}0.000139 & 0.000196\end{array}$

$\begin{array}{lll}0.000152 & 0.000199\end{array}$

$0.000168 \quad 0.000204$

$\begin{array}{lll}0.000175 & 0.000209\end{array}$

$0.000187 \quad 0.000213$

$0.000099 \quad 0.000191$

$0.000098 \quad 0.000191$

$0.000103 \quad 0.000191$

$0.000109 \quad 0.000192$

$0.000122 \quad 0.000193$

$0.000137 \quad 0.000196$

$0.000154 \quad 0.000201$

$0.000162 \quad 0.000205$

$0.000175 \quad 0.000209$

$\begin{array}{lll}0.000114 & 0.000191\end{array}$

$0.000124 \quad 0.000193$

0.0001310 .000195

$\begin{array}{ll}0.000136 & 0.000197\end{array}$

$\begin{array}{ll}0.000143 & 0.000199 \\ 0.000137 & 0.000193\end{array}$

$\begin{array}{ll}0.000137 & 0.000193 \\ 0.000151 & 0.000197\end{array}$

$\begin{array}{ll}0.000151 & 0.000197 \\ 0.000173 & 0.000206\end{array}$

$\begin{array}{ll}0.000193 & 0.000214\end{array}$

$0.000212 \quad 0.000224$

$\begin{array}{ll}0.000228 & 0.000233 \\ 0.000246 & 0.000245\end{array}$

$\begin{array}{ll}0.000246 & 0.000245 \\ 0.000262 & 0.000258\end{array}$

$0.000262 \quad 0.000258$
Average
Avg - 4st

Avg $+4 s$

2)

$\begin{array}{lr}\text { Curve fit for the model App vol= } \\ \text { c0 } \\ \text { c1 } \\ \text { c2 } & -0.000515 \\ \text { c3 } & 0.001344 \\ & 0.583581 \\ & 0.008583\end{array}$

Density App Vol Square of Inconsistent Inconsistent Apparent

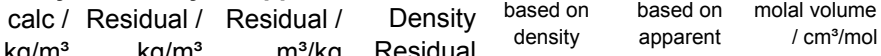
volume

$/ \mathrm{cm}^{3} / \mathrm{mol}$

Relative Delta density Density of density - $\quad / \mathrm{kg} / \mathrm{m}^{3}$ water at $\mathrm{t}^{\circ} \mathrm{C} /$

$\begin{array}{llll}87.73 & 0.5749 & -0.000029 & 0.331\end{array}$

$\begin{array}{llll}004.00 & 1.0050 & -0.000025 & 1.010\end{array}$

$\begin{array}{llll}1.2244 & -0.000014 & 1.499 \\ 1072.56 & 0.9421 & -0.000007 & 0.888\end{array}$

$\begin{array}{llll}108.95 & 0.2528 & -0.000001 & 0.064\end{array}$

$\begin{array}{llll}1146.73 & -0.7313 & 0.000003 & 0.535\end{array}$

$\begin{array}{llll}195.87 & -1.6688 & 0.000005 & 2.785\end{array}$

$\begin{array}{lll}94.00 & -1.9031 & 0.000004\end{array}$

$\begin{array}{llll}969.25 & 0.0569 & -0.000076 & 0.003 \\ 969.85 & 0.1105 & -0.000075 & 0.012\end{array}$

$\begin{array}{lll}971.07-0.2191 & -0.000075\end{array}$

$\begin{array}{llll}973.52 & 0.3738 & -0.000063 & 0.140\end{array}$

$\begin{array}{llll}978.35 & 0.6729 & -0.000057 & 0.453\end{array}$

$\begin{array}{llll}988.45 & 1.1446 & -0.000047 & 1.310\end{array}$

$\begin{array}{llll}1007.06 & 1.7550 & -0.000036 & 3.080\end{array}$

\begin{tabular}{llll}
1040.26 & 2.4111 & -0.000034 & 5.813 \\
\hline & 2.5307 & -0.000027 & 6.405
\end{tabular}

$\begin{array}{llll}962.53 & 0.0680 & -0.000092 & 0.005\end{array}$

$\begin{array}{llll}963.13 & 0.1350 & -0.000093 & 0.018\end{array}$

\begin{tabular}{llll}
966.80 & 0.4791 & -0.000083 & 0.230 \\
\hline
\end{tabular}

$\begin{array}{llll}971.62 & 0.8216 & -0.000071 & 0.675\end{array}$

$\begin{array}{llll}1000.31 & 2.2197 & -0.000047 & 4.927\end{array}$

$\begin{array}{llll}1017.36 & 3.0160 & -0.000043 & 9.096\end{array}$

$\begin{array}{llll}1033.56 & 3.2465 & -0.000035 & 10.540\end{array}$

$\begin{array}{llll}967.39 & 0.7431 & -0.000078 & 0.552 \\ 073.93 & 1.2154 & -0.000069 & 1.477\end{array}$

$\begin{array}{llll}980.10 & 1.5962 & -0.000064 & 2.548\end{array}$

$\begin{array}{llll}987.90 & 2.1350 & -0.000061 & 4.558\end{array}$

$\begin{array}{llll}997.93 & 2.6862 & -0.000056 & 7.216\end{array}$

$\begin{array}{lll}2.6862 & -0.000056\end{array}$

$\begin{array}{llll}990.50 & 1.8027 & -0.000046 & 3.250\end{array}$

$\begin{array}{llll}1024.14 & 2.7550 & -0.000033 & 7.590\end{array}$

$2.8436-0.000021 \quad 8.086$

$\begin{array}{lll}2.2262 & -0.000012 & 4.956\end{array}$

$\begin{array}{lll}1.2715 & -0.000005 & 1.617\end{array}$

$\begin{array}{rrr}-0.3229 & 0.000001 & 0.104 \\ -1.9895 & 0.000004 & 3.958\end{array}$

$0.2345-0.000021 \quad 311.531$ Sum chi sqr

$\begin{array}{ll}0.8519 & 0.000021\end{array}$

$-3.1730-0.000104$

$\begin{array}{ll}3.6419 & 0.000062\end{array}$
971.7978

971.7978

971.7978

971.7978

971.7978

971.7978

971.7978

971.7978

968.6203

968.6203

968.6203

968.6203

968.6203

968.6203

968.6203

968.6203

968.6203

961.9004

961.9004

961.9004

961.9004

961.9004

961.9004

961.9004

961.9004

961.9004

959.3157

959.3157

959.3157

959.3157

959.3157

958.3637

958.3637

958.3637

958.3637

958.3637

958.3637

958.3637

958.3637 


$\begin{array}{lr}\text { molality } & \text { molarity } \\ & \mathbf{t} /{ }^{\circ} \mathrm{C} \\ & \\ \text { C4 } & 3843.612 \\ \text { Min T } & 0 \\ \text { Max T } & 100 \\ \text { Min w } & 0.0002 \\ \text { Max w } & 0.3275 \\ \text { Avg dens res } & 0.2345 \\ \text { Std dens res } & 0.8519 \\ \text { Avg app vol res *10^3 } & -0.021224 \\ \text { Std app vol res *1033 } & 0.020814 \\ \text { No of points in corr } & 400 \\ \text { No of inconsistent poir } & 6 \\ & \end{array}$

Inconsistent data not used (Residual greater than average + / $\mathbf{4}$ standard deviations)

$\begin{array}{llllllllllll}5 & 15 & 0.3225 & 1298.03 & (45) & 0.000286 & 0.000300 & 1290.47 & 7.5650 & -0.000014 & 57.229 \\ 5 & 20 & 0.3225 & 1296.26 & (45) & 0.000288 & 0.000301 & 1289.13 & 7.1331 & -0.000013 & 50.881 \\ 5 & 25 & 0.3225 & 1294.50 & (45) & 0.000288 & 0.000301 & 1287.75 & 6.7451 & -0.000013 & 45.497 \\ 5 & 30 & 0.3225 & 1292.66 & (45) & 0.000289 & 0.000301 & 1286.36 & 6.3032 & -0.000012 & 39.731 \\ 5 & 35 & 0.3225 & 1290.82 & (45) & 0.000289 & 0.000300 & 1284.94 & 5.8839 & -0.000011 & 34.620 \\ 5 & 55 & 0.323 & 1288.0 & (45) & 0.000276 & 0.000293 & 1279.00 & 9.0376 & -0.000017 & 81.679\end{array}$


Density of aqueous solutions of MgSO4

References (6) Asmus, A., "The viscosities of aqueous solutions of strong electrolytes of high valence type", Ann. der Physik, ser 5, 1939, 35, 1-22

(13) Chen, C.-T. A.; Chen, J. H.; Millero, F. J. Densities of $\mathrm{NaCl}, \mathrm{MgCl} 2, \mathrm{Na} 2 \mathrm{SO} 4$, and MgSO4 Aqueous Solutions at 1 atm. from 0 to $50^{\circ} \mathrm{C}$ and from 0.001 to $1.5 \mathrm{~m}$. J. Chem. Eng. Data 1980, 25, 307-310.

(27) Fabuss, B. M.; Korosi, A.; Huq, A. K. M. S.; Densities of Binary and Ternary Aqueous Solutions of NaCl, Na2SO4, and MgSO4, of Sea Waters, and Sea Water Concentrates, J. Chem. Eng. Data, 1966, 3, 325-331

(45) Isono, T. Densities, Viscosities, and Electrolytic Conductivity of Concentrated Aqueous Electrolyte Solutions at Several Temperatures. Alkaline-Earth Chlorides, $\mathrm{LaCl}_{3}, \mathrm{Na}_{2} \mathrm{SO}_{4}, \mathrm{NaNO}_{3}, \mathrm{NaBr}, \mathrm{KNO}_{3}, \mathrm{KBr}$, and $\mathrm{Cd}\left(\mathrm{NO}_{3}\right)_{2}$. J. Chem. Eng. Data 1984, 29, 45-52.

(49) Kaminsky, M, Concentration and Temperature Dependence of the Viscosity of Aqueous Solutions of Strong Electrolytes. III. KCl, K2SO4, $\mathrm{MgCl} 2$, BeSO4, and MgSO4 Solutions. Z. Phys. Chem. N. F. 1957, 12, 206-231.

(52) Korosi, A.; Fabuss, B. M. Viscosities of Binary Aqueous Solutions of $\mathrm{NaCl}, \mathrm{KCl}, \mathrm{Na2SO} 4$, and MgSO4 at Concentrations and Temperatures of Interest in Desalination Processes. J. Chem. Eng. Data 1968, 13, 548-552.

(72) Millero, F. J.; Knox, J. H. Apparent Molal Volumes of Aqueous $\mathrm{NaF}, \mathrm{Na} 2 \mathrm{SO} 4, \mathrm{KCl}, \mathrm{K} 2 \mathrm{SO} 4, \mathrm{MgCl} 2$, and $\mathrm{MgSO} 4 \mathrm{Solutions}$ at $0^{\circ}$ and $50^{\circ} \mathrm{C}$. J. Chem. Eng. Data 1973, 18, 407-411.

(74) Motin, M. A., Temperature and Concentration Dependence of Apparent Molar Volumes and Viscosities of $\mathrm{NaCl}, \mathrm{NH} 4 \mathrm{Cl}, \mathrm{CuCl} 2$, and MgSO4 in Pure Water and Water + Urea Mixtures, J. Chem. Eng. Data, 2004, 49, 94-98

(88) Perron, G., Desnoyers, J. E., Millero, F. J., "Apparent molal volumes and heat capacities of some sulfates and carbonates in water at 25 ${ }^{\circ}$ C.", Can. J. Chem. , 1975, 53, 1134-1138

(90) Perry, R. H.; Green, D. W. Perry's Chemical Engineers' Handbook; McGraw Hill: New York, 7th edition, 1997.

\begin{tabular}{|c|c|c|c|c|c|c|c|c|c|c|c|c|c|c|c|c|}
\hline molality & molarity & $\mathbf{t} /{ }^{\circ} \mathrm{C}$ & ass frac & $\begin{array}{r}\text { Density } \\
\exp / \mathrm{kg} / \mathrm{m}^{3}\end{array}$ & Ref & $\begin{array}{r}\text { App vol } \\
\exp / \\
\mathrm{m}^{3} / \mathrm{kg}\end{array}$ & $\begin{array}{r}\text { App vol } \\
\text { Calc } / \\
\mathrm{m}^{3} / \mathrm{kg}\end{array}$ & $\begin{array}{r}\text { Density } \\
\mathrm{calc} / \\
\mathrm{kg} / \mathrm{m}^{3}\end{array}$ & $\begin{array}{r}\text { Density } \\
\text { Residual / } \\
\mathrm{kg} / \mathrm{m}^{3}\end{array}$ & $\begin{array}{r}\text { App Vol } \\
\text { Residual / } \\
\mathrm{m}^{3} / \mathrm{kg}\end{array}$ & $\begin{array}{r}\text { Square of } \\
\text { Density } \\
\text { Residual }\end{array}$ & $\begin{array}{l}\text { Inconsistent } \\
\text { based on } \\
\text { density }\end{array}$ & $\begin{array}{l}\text { Inconsistent } \\
\text { based on } \\
\text { apparent } \\
\text { volume }\end{array}$ & $\begin{array}{r}\text { Delta density } \begin{array}{r}\text { Apparent } \\
/ \mathrm{kg} / \mathrm{m}^{3} \mathrm{molal} \text { volume } \\
\\
1 \mathrm{~cm}^{3} / \mathrm{mol}\end{array}\end{array}$ & $\begin{array}{r}\text { Relative } \\
\text { density }\end{array}$ & $\begin{array}{r}\text { Density of } \\
\text { water } / \mathrm{kg} / \mathrm{m}^{3}\end{array}$ \\
\hline 0.00099 & & 0 & 0.0001 & 999.97 & (13) & -0.000083 & -0.000034 & 999.96 & 0.0058 & -0.000049 & 0.000 & & & 0.129 & & 999.8395 \\
\hline 0.00517 & & 0 & 0.0006 & 1000.52 & (72) & -0.000096 & -0.000033 & 1000.48 & 0.0392 & -0.000063 & 0.002 & & & 0.6822 & & 999.8395 \\
\hline 0.00549 & & 0 & 0.0007 & 1000.57 & (13) & -0.000105 & -0.000033 & 1000.52 & 0.0472 & -0.000071 & 0.002 & & & 0.730 & & 999.8395 \\
\hline .007469 & & 0 & 0.0009 & 1000.82 & (72) & -0.000090 & -0.000033 & 1000.77 & 0.0512 & -0.000057 & 0.003 & & & 0.98 & & 999.8395 \\
\hline 0.01177 & & 0 & 0.0014 & 1001.38 & (13) & -0.000086 & -0.000033 & 1001.30 & 0.0748 & -0.000053 & 0.006 & & & 1.538 & & 999.8395 \\
\hline .014391 & & 0 & 0.0017 & 1001.72 & (72) & -0.000086 & -0.000033 & 1001.63 & 0.0918 & -0.000053 & 0.008 & & & 1.8805 & & 999.8395 \\
\hline 0.01909 & & 0 & 0.0023 & 1002.32 & (72) & -0.000078 & -0.000032 & 1002.21 & 0.1065 & -0.000046 & 0.011 & & & 2.4784 & & 999.8395 \\
\hline .027767 & & 0 & 0.0033 & 1003.42 & (72) & -0.000070 & -0.000032 & 1003.29 & 0.1305 & -0.000039 & 0.017 & & & 3.5784 & & 999.8395 \\
\hline 0.02786 & & 0 & 0.0033 & 1003.42 & (13) & -0.000069 & -0.000032 & 1003.30 & 0.1245 & -0.000037 & 0.016 & & & 3.584 & & 999.8395 \\
\hline .032379 & & 0 & 0.0039 & 1004.00 & (72) & -0.000068 & -0.000031 & 1003.86 & 0.1448 & -0.000037 & 0.021 & & & 4.1641 & & 999.8395 \\
\hline .036607 & & 0 & 0.0044 & 1004.53 & (72) & -0.000064 & -0.000031 & 1004.38 & 0.1481 & -0.000033 & 0.022 & & & 4.6909 & & 999.8395 \\
\hline 0.03855 & & 0 & 0.0046 & 1004.78 & (13) & -0.000065 & -0.000031 & 1004.62 & 0.1607 & -0.000034 & 0.026 & & & 4.944 & & 999.8395 \\
\hline .044662 & & 0 & 0.0053 & 1005.55 & (72) & -0.000062 & -0.000030 & 1005.38 & 0.1734 & -0.000032 & 0.030 & & & 5.7127 & & 999.8395 \\
\hline .053878 & & 0 & 0.0064 & 1006.71 & (72) & -0.000059 & -0.000030 & 1006.52 & 0.1931 & -0.000030 & 0.037 & & & 6.8711 & & 999.8395 \\
\hline .063246 & & 0 & 0.0076 & 1007.88 & (72) & -0.000056 & -0.000029 & 1007.67 & 0.2095 & -0.000027 & 0.044 & & & 8.0435 & & 999.8395 \\
\hline .069471 & & 0 & 0.0083 & 1008.70 & (72) & -0.000059 & -0.000028 & 1008.44 & 0.2621 & -0.000031 & 0.069 & & & 8.8634 & & 999.8395 \\
\hline 0.07019 & & 0 & 0.0084 & 1008.75 & (13) & -0.000054 & -0.000028 & 1008.53 & 0.2181 & -0.000026 & 0.048 & & & 8.908 & & 999.8395 \\
\hline .089133 & & 0 & 0.0106 & 1011.10 & (72) & -0.000049 & -0.000027 & 1010.86 & 0.2423 & -0.000022 & 0.059 & & & 11.2628 & & 999.8395 \\
\hline .100205 & & 0 & 0.0119 & 1012.46 & (72) & -0.000046 & -0.000026 & 1012.22 & 0.2449 & -0.000020 & 0.060 & & & 12.6247 & & 999.8395 \\
\hline 0.11478 & & 0 & 0.0136 & 1014.27 & (72) & -0.000044 & -0.000025 & 1014.01 & 0.2631 & -0.000019 & 0.069 & & & 14.4291 & & 999.8395 \\
\hline 0.11669 & & 0 & 0.0139 & 1014.52 & (13) & -0.000044 & -0.000025 & 1014.24 & 0.2782 & -0.000020 & 0.077 & & & 14.678 & & 999.8395 \\
\hline 0.14239 & & 0 & 0.0169 & 1017.66 & (72) & -0.000039 & -0.000023 & 1017.38 & 0.2856 & -0.000016 & 0.082 & & & 17.8252 & & 999.8395 \\
\hline & & 0 & 0.020 & 1021.00 & (90) & -0.000036 & -0.000021 & 1020.68 & 0.3174 & -0.000015 & 0.101 & & & & & 999.8395 \\
\hline
\end{tabular}




$\begin{array}{rrrr}\text { molality } & \text { molarity } & \mathbf{t} /{ }^{\circ} \mathrm{C} \text { mass frac } & \text { Density } \\ & & & \text { exp } / \mathrm{kg} / \mathrm{m}^{3} \\ & & & \\ 0.18277 & 0 & 0.0215 & 1022.57 \\ 0.24491 & 0 & 0.0286 & 1030.05 \\ & 0 & 0.0400 & 1042.30 \\ 0.36328 & 0 & 0.0419 & 1044.12 \\ 0.49772 & 0 & 0.0565 & 1059.81 \\ 0.71312 & 0 & 0.0791 & 1084.41 \\ & 0 & 0.0800 & 1085.80 \\ 0.89006 & 0 & 0.0968 & 1104.07 \\ 0.89033 & 0 & 0.0968 & 1104.10 \\ & 0 & 0.1200 & 1130.90 \\ & 0 & 0.1600 & 1177.70 \\ 0.00099 & 0 & 0.2000 & 1226.40 \\ 0.00549 & 5 & 0.0001 & 1000.09 \\ 0.01177 & 5 & 0.0007 & 1000.68 \\ 0.02231 & 5 & 0.0014 & 1001.48 \\ 0.02786 & 5 & 0.0027 & 1002.81 \\ 0.03855 & 5 & 0.0033 & 1003.51 \\ 0.07019 & 5 & 0.0046 & 1004.85 \\ 0.11669 & 5 & 0.0084 & 1008.76 \\ 0.1201 & 5 & 0.0139 & 1014.47 \\ 0.21211 & 5 & 0.0143 & 1014.87 \\ 0.23758 & 5 & 0.0249 & 1025.98 \\ 1.48251 & 5 & 0.0278 & 1029.06 \\ & 5 & 0.1514 & 1166.25 \\ 0.00099 & 15 & 0.0001 & 999.16 \\ & 15 & 0.0001 & 999.23 \\ 0.15 & 15 & 0.0001 & 999.23 \\ 0.1201 & 15 & 0.0003 & 999.42 \\ 0.21211 & 15 & 0.0006 & 999.73 \\ 0.00549 & 15 & 0.0007 & 999.80 \\ 0.01177 & 15 & 0.0012 & 1000.36 \\ 0.02231 & 15 & 0.0014 & 1000.59 \\ 0.02786 & 15 & 0.0024 & 1001.61 \\ 0.03855 & 15 & 0.0027 & 1001.89 \\ 0.05 & 15 & 0.0033 & 1002.59 \\ 0.07019 & 15 & 0.0046 & 1003.89 \\ & 15 & 0.0048 & 1004.10 \\ & 15 & 0.0060 & 1005.37 \\ & 15 & 0.0060 & 1005.32 \\ & 15 & 0.0084 & 1007.73 \\ & 15 & 0.0119 & 1011.40 \\ & 15 & 0.0119 & 1011.44 \\ & 15 & 0.0139 & 1013.34 \\ & 15 & 0.0143 & 1013.74 \\ & & 0.0236 & 1023.48 \\ & 0.0249 & 1024.66 \\ & & & \\ & & & \\ & & & \end{array}$

Ref

\section{Ap}

App vol App vol Den

$\begin{array}{cc}\exp / & \text { Calc / } \\ \mathrm{m}^{3} / \mathrm{kg} & \mathrm{m}^{3} / \mathrm{kg}\end{array}$

Density Density App Vol Square of Inconsistent
calc / Residual / Residual / Density based on

Inconsisten

$-0.000032-0.000020$ $-0.000024-0.000016$ $-0.000018-0.000009$ $-0.000012-0.000007$ $\begin{array}{lll}0.000014 & 0.000016 & 10 \\ 0.000010 & 0.000016 & 108\end{array}$ $\begin{array}{lll}1085.26 & 0.5400-0.00006 & 0.292 \\ -0.2097-0.000002 & 0.044\end{array}$ $\begin{array}{lll}0.000034 & 0.000040 & 1129.98\end{array}$ $\begin{array}{llllll}0.000076 & 0.000087 & 1223.20 & 3.2027 & -0.000011 & 10.257\end{array}$ $\begin{array}{llllll}-0.000099 & -0.000026 & 1000.09 & 0.0087 & -0.000073 & 0.000\end{array}$

$\begin{array}{llllll}-0.000085 & -0.000026 & 1000.64 & 0.0392 & -0.000059 & 0.002\end{array}$ $-0.00006-0.000025-1001.42$ $\begin{array}{lll}-0.000061 & -0.000025 & 1001.42 \\ -0.000061 & -0.000024 & 1002.72\end{array}$ $\begin{array}{lll}-0.000059 & -0.000024 & 1003.40\end{array}$ $\begin{array}{lll}-0.000052 & -0.000023 & 1004.71\end{array}$ $\begin{array}{lll}-0.000041 & -0.000021 & 1008.59\end{array}$ $\begin{array}{lll}-0.000032 & -0.000018 & 1014.26\end{array}$ $\begin{array}{lll}-0.000031 & -0.000017 & 1014.68\end{array}$ $\begin{array}{lll}-0.000018 & -0.000011 & 1025.79\end{array}$ $\begin{array}{lll}-0.000017 & -0.000009 & 1028.84\end{array}$ $\begin{array}{lll}0.000058 & 0.000062 & 1165.49\end{array}$ $\begin{array}{llll}0.000006 & -0.000012 \quad 999.16\end{array}$ $-0.000117-0.000012$ $-0.000077-0.000012$ $-0.000060-0.000012$ $-0.000044-0.000012$ $-0.000057-0.000012$ $-0.000044-0.000012$ $-0.000051-0.000012$ $-0.000039-0.000011$ $-0.000040-0.000011$ $-0.000040-0.000011$ $-0.000034-0.000010$ $-0.000035-0.000010$ $-0.000042-0.000009$ $-0.000032-0.000009$ $-0.000022-0.000008$ $-0.000022-0.000006$ $-0.000023-0.000006$ $-0.000015-0.000005$ $-0.000013-0.000005$ $\begin{array}{rr}-0.000010 & 0.000000\end{array}$ $\begin{array}{ll}-0.000002 & 0.000001\end{array}$ $\begin{array}{lll}0.0997 & -0.000037 & 0.010\end{array}$

$\begin{array}{lll}0.1166 & -0.000035 & 0.014\end{array}$

$\begin{array}{lll}0.1343 & -0.000029 & 0.018\end{array}$

$\begin{array}{lll}0.1693 & -0.000020 & 0.029\end{array}$

$\begin{array}{lll}0.2049 & -0.000014 & 0.042\end{array}$

$\begin{array}{lll}0.1925-0.000013 & 0.037\end{array}$

$0.1915-0.000007 \quad 0.037$

$\begin{array}{lll}0.2200 & -0.000007 & 0.048\end{array}$

$\begin{array}{lll}0.7623 & -0.000004 & 0.581\end{array}$

$\begin{array}{lll}-0.0011 & 0.000019 & 0.000\end{array}$

$\begin{array}{lll}0.0125 & -0.000105 & 0.000\end{array}$

$\begin{array}{lll}0.0078 & -0.000064 & 0.000\end{array}$

$\begin{array}{llll}999.40 & 0.0144 & -0.000048 & 0.000\end{array}$

$\begin{array}{lllll}999.71 & 0.0190 & -0.000031 & 0.000\end{array}$

$999.77-0.0297-0.000045 \quad 0.001$

$\begin{array}{lll}0.0384 & -0.000032 & 0.001\end{array}$

$\begin{array}{lll}0.0558 & -0.000039 & 0.003\end{array}$

$\begin{array}{lll}0.0684 & -0.000028 & 0.005\end{array}$

$\begin{array}{lll}0.0792 & -0.000029 & 0.006\end{array}$

0.010

0.012

$0.1103-0.000024$

0.015

0.041

0.019

0.014

0.040

0.042

0.019

0.016

0.066

0.006

$0.1283-0.000009$

$0.2563-0.000010$

$0.0801-0.000003$ based on volume $\mathrm{kg} / \mathrm{m}^{3}$ $\mathrm{kg} / \mathrm{m}^{3}$ $\mathrm{m}^{3} / \mathrm{kg}$ Residual 1022.29 $0.2781-0.000012$ 0.077 1041.87 1084.22 0.069 $\begin{array}{lll}0.4309 & -0.000010 & 0.186 \\ 0.2190 & -0.000005 & 0.048\end{array}$ $\begin{array}{lll}0.2190 & -0.000005 & 0.048 \\ 0.1776 & -0.000003 & 0.032\end{array}$ $\begin{array}{lll}0.1832 & -0.000002 & 0.034 \\ 0.5400 & -0.000006 & 0.292\end{array}$ $0.5400-0.000006 \quad 0.292$ $0.2097-0.000002$ $0.2113-0.000002$ $0.9208-0.000006$

0.044 0.045 0.848

$\begin{array}{lll}0.0625 & -0.000044 & 0.004\end{array}$ molal volume

22.7279

30.2141

44.2783

59.9735

84.567

104.2264

104.2576

0.131

0.717

1.515

2.851

3.551

4.883

8.797

14.503

14.905

26.013

29.092

166.290

0.133

0.698

1.488

2.792

3.486

4.793

8.629

1024.58

14.242

14.641

25.561

Relative Density of density water $/ \mathrm{kg} / \mathrm{m}^{3}$

999.8395 999.8395 999.8395 999.8395 999.8395 999.8395 999.8395 999.8395 999.8395 999.8395 999.8395 999.8395 999.9638 999.9638 999.9638 999.9638 999.9638 999.9638 999.9638 999.9638 999.9638 999.9638 999.9638 999.9638

$1.00006 \quad 999.0996$ 999.0996 1.00013999 .0996 1.00032999 .0996 1.00063999 .0996 999.0996

1.00126999 .0996 999.0996

1.00251999 .0996 999.0996 999.0996 999.0996

1.005999 .0996 999.0996

1.00623999 .0996 999.0996 999.0996 999.0996 $1.0244 \quad 999.0996$
999.0996 


\begin{tabular}{|c|c|c|c|c|}
\hline \multirow{2}{*}{$\begin{array}{l}\text { molality } \\
0.23758\end{array}$} & molarity & \multicolumn{2}{|c|}{$\mathrm{t} /{ }^{\circ} \mathrm{C}$ mass frac } & $\begin{array}{r}\text { Density } \\
\exp / \mathrm{kg} / \mathrm{m}^{3}\end{array}$ \\
\hline & & 15 & 0.0278 & 1027.67 \\
\hline 0.39375 & & 15 & 0.0453 & 1045.79 \\
\hline 0.5 & & 15 & 0.0568 & 1057.75 \\
\hline 0.64325 & & 15 & 0.0719 & 1073.98 \\
\hline 0.88167 & & 15 & 0.0960 & 1100.18 \\
\hline 1 & & 15 & 0.1074 & 1112.59 \\
\hline 1.2232 & & 15 & 0.1283 & 1137.60 \\
\hline 1.5 & & 15 & 0.1530 & 1164.41 \\
\hline 2 & & 15 & 0.1940 & 1212.95 \\
\hline \multirow[t]{11}{*}{2.5} & & 15 & 0.2313 & 1260.71 \\
\hline & & 18 & 0.0001 & 998.66 \\
\hline & & 18 & 0.0001 & 998.73 \\
\hline & & 18 & 0.0003 & 998.92 \\
\hline & & 18 & 0.0006 & 999.22 \\
\hline & & 18 & 0.0012 & 999.84 \\
\hline & & 18 & 0.0024 & 1001.08 \\
\hline & & 18 & 0.0048 & 1003.55 \\
\hline & & 18 & 0.0060 & 1004.77 \\
\hline & & 18 & 0.0119 & 1010.82 \\
\hline & & 18 & 0.0236 & 1022.71 \\
\hline 0.05 & & 20 & 0.0060 & 1004.38 \\
\hline \multirow[t]{3}{*}{0.1} & & 20 & 0.0119 & 1010.40 \\
\hline & & 20 & 0.020 & 1018.60 \\
\hline & & 20 & 0.0400 & 1039.20 \\
\hline \multirow[t]{2}{*}{0.5} & & 20 & 0.0568 & 1056.47 \\
\hline & & 20 & 0.0800 & 1081.60 \\
\hline \multirow[t]{2}{*}{1} & & 20 & 0.1074 & 1111.16 \\
\hline & & 20 & 0.1200 & 1125.60 \\
\hline \multirow[t]{2}{*}{1.5} & & 20 & 0.1530 & 1162.82 \\
\hline & & 20 & 0.1600 & 1171.70 \\
\hline \multirow[t]{2}{*}{2} & & 20 & 0.1940 & 1211.10 \\
\hline & & 20 & 0.2000 & 1219.80 \\
\hline \multirow[t]{5}{*}{2.5} & & 20 & 0.2313 & 1258.76 \\
\hline & & 20 & 0.2600 & 1296.10 \\
\hline & 0.0005 & 25 & 0.0001 & 997.10 \\
\hline & 0.000587 & 25 & 0.0001 & 997.12 \\
\hline & 0.000979 & 25 & 0.0001 & 997.17 \\
\hline \multirow[t]{6}{*}{0.00099} & & 25 & 0.0001 & 997.17 \\
\hline & 0.001 & 25 & 0.0001 & 997.16 \\
\hline & 0.001442 & 25 & 0.0002 & 997.24 \\
\hline & 0.001949 & 25 & 0.0002 & 997.31 \\
\hline & 0.0025 & 25 & 0.0003 & 997.35 \\
\hline & 0.00258 & 25 & 0.0003 & 997.39 \\
\hline \multirow[t]{3}{*}{0.00277} & & 25 & 0.0003 & 997.39 \\
\hline & 0.003388 & 25 & 0.0004 & 997.51 \\
\hline & 0.004225 & 25 & 0.0005 & 997.62 \\
\hline
\end{tabular}

Ref

\section{App}

App vol App vol Density Density App Vol Square of Inconsistent Inconsistent Delta density Apparent exp / Calc / calc / Residual / Residual / Density based on based on / $/ \mathrm{kg} / \mathrm{m}^{3}$ molal volum $\mathrm{m}^{3} / \mathrm{kg} \quad \mathrm{m}^{3} / \mathrm{kg}$ calc / Residual / Residual / Density based on based on
apparent
volume $\begin{array}{llllll}0.000000 & 0.000003 & 1027.59 & 0.0808 & -0.000003 & 0.007\end{array}$ $\mathrm{kg} / \mathrm{m}$ $\mathrm{m}^{3} / \mathrm{kg}$ 0.007 $\begin{array}{llllll}0.000013 & 0.000012 & 1045.86 & -0.0659 & 0.000001 & 0.004\end{array}$ $\begin{array}{llllll}0.000023 & 0.000018 & 1058.07 & -0.3210 & 0.000005 & 0.103\end{array}$ $\begin{array}{llllll}0.000030 & 0.000026 & 1074.28 & -0.2929 & 0.000004 & 0.086\end{array}$ $\begin{array}{llllll}0.000043 & 0.000039 & 1100.59 & -0.4135 & 0.000004 & 0.171\end{array}$ $\begin{array}{llllll}0.000051 & 0.000045 & 1113.35 & -0.7584 & 0.000006 & 0.575\end{array}$ $\begin{array}{llllll}0.000051 & 0.000056 & 1136.89 & 0.7165 & -0.000004 & 0.513\end{array}$ $\begin{array}{llllll}0.000072 & 0.000068 & 1165.14 & -0.7338 & 0.000004 & 0.538\end{array}$ $\begin{array}{llllll}0.000091 & 0.000089 & 1213.68 & -0.7262 & 0.000003 & 0.527\end{array}$ $\begin{array}{llllll}0.000103 & 0.000107 & 1259.16 & 1.5457 & -0.000004 & 2.389\end{array}$ $\begin{array}{llllll}0.000006 & -0.000009 & 998.66 & -0.0009 & 0.000015 & 0.000\end{array}$ $\begin{array}{llllll}-0.000077 & -0.000009 & 998.72 & 0.0082 & -0.000068 & 0.000\end{array}$

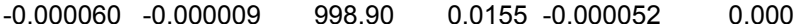
$\begin{array}{llllll}-0.000044 & -0.000009 & 999.20 & 0.0212 & -0.000035 & 0.000\end{array}$ $\begin{array}{lllllll}-0.000035 & -0.000008 & 999.81 & 0.0327 & -0.000027 & 0.001\end{array}$ $\begin{array}{llllll}-0.000031 & -0.000008 & 1001.03 & 0.0571 & -0.000024 & 0.003\end{array}$ $\begin{array}{llllll}-0.000027 & -0.000006 & 1003.45 & 0.1004 & -0.000021 & 0.010\end{array}$ $\begin{array}{llllll}-0.000024 & -0.000006 & 1004.66 & 0.1094 & -0.000018 & 0.012\end{array}$ $\begin{array}{llllll}-0.000014 & -0.000002 & 1010.68 & 0.1365 & -0.000011 & 0.019\end{array}$ $\begin{array}{llllll}0.000000 & 0.000004 & 1022.62 & 0.0869 & -0.000004 & 0.008\end{array}$ $\begin{array}{llllll}-0.000028 & -0.000003 & 1004.23 & 0.1472 & -0.000024 & 0.022\end{array}$ $\begin{array}{llllll}-0.000015 & 0.000000 & 1010.22 & 0.1758 & -0.000014 & 0.031\end{array}$ $\begin{array}{llllll}-0.000001 & 0.000004 & 1018.49 & 0.1054 & -0.000005 & 0.011\end{array}$ $\begin{array}{llllll}0.000014 & 0.000014 & 1039.18 & 0.0187 & 0.000000 & 0.000\end{array}$ $\begin{array}{llllll}0.000029 & 0.000023 & 1056.84 & -0.3692 & 0.000006 & 0.136\end{array}$ $\begin{array}{lllllll}0.000036 & 0.000035 & 1081.76 & -0.1633 & 0.000002 & 0.027\end{array}$ $\begin{array}{llllll}0.000054 & 0.000048 & 1111.93 & -0.7682 & 0.000006 & 0.590\end{array}$ $\begin{array}{llllll}0.000057 & 0.000054 & 1125.99 & -0.3870 & 0.000003 & 0.150\end{array}$ $\begin{array}{lllllll}0.000075 & 0.000070 & 1163.67 & -0.8548 & 0.000004 & 0.731\end{array}$ $\begin{array}{llllll}0.000075 & 0.000074 & 1171.89 & -0.1856 & 0.000001 & 0.034\end{array}$ $\begin{array}{llllll}0.000094 & 0.000090 & 1212.29 & -1.1881 & 0.000004 & 1.412\end{array}$ $\begin{array}{lllllll}0.000092 & 0.000093 & 1219.49 & 0.3133 & -0.000001 & 0.098\end{array}$ $\begin{array}{lllllll}0.000105 & 0.000108 & 1257.97 & 0.7853 & -0.000002 & 0.617\end{array}$ $\begin{array}{llllll}0.000116 & 0.000121 & 1294.13 & 1.9740 & -0.000005 & 3.897\end{array}$ $\begin{array}{llllll}0.000006 & -0.000001 & 997.11 & -0.0004 & 0.000007 & 0.000\end{array}$ $\begin{array}{llllll}-0.000129 & -0.000001 & 997.12 & 0.0090 & -0.000128 & 0.000\end{array}$ $\begin{array}{llllll}-0.000100 & -0.000001 & 997.16 & 0.0116 & -0.000099 & 0.000\end{array}$ $\begin{array}{llllll}-0.000077 & -0.000001 & 997.16 & 0.0091 & -0.000076 & 0.000\end{array}$ $\begin{array}{lllllll}0.000006 & -0.000001 & 997.17 & -0.0009 & 0.000007 & 0.000\end{array}$ $\begin{array}{llllll}-0.000149 & -0.000001 & 997.22 & 0.0257 & -0.000148 & 0.001\end{array}$ $\begin{array}{llllll}-0.000148 & -0.000001 & 997.28 & 0.0344 & -0.000147 & 0.001\end{array}$ $\begin{array}{llllll}-0.000027 & -0.000001 & 997.35 & 0.0079 & -0.000026 & 0.000\end{array}$ $\begin{array}{llllll}-0.000124 & -0.000001 & 997.36 & 0.0381 & -0.000123 & 0.001\end{array}$ $\begin{array}{llllll}-0.000035 & -0.000001 & 997.38 & 0.0112 & -0.000034 & 0.000\end{array}$ $\begin{array}{llllll}-0.000149 & -0.000001 & 997.45 & 0.0604 & -0.000149 & 0.004 \\ -0.000137 & -0.000001 & 997.55 & 0.0693 & -0.000137 & 0.005\end{array}$
Relative Density of density water $/ \mathrm{kg} / \mathrm{m}^{3}$

28.575
46.690

74.884
101.076

999.0996 999.0996 999.0996 999.0996 999.0996 999.0996 999.0996 999.0996 999.0996 999.0996 $1.00006 \quad 998.5956$ 1.00013998 .5956 1.00032998 .5956 $1.00063 \quad 998.5956$ 1.00125998 .5956 1.00249998 .5956 1.00496998 .5956 1.00618998 .5956 1.01224998 .5956 $1.02415 \quad 998.5956$ 998.2041 998.2041 998.2041 998.2041 998.2041 998.2041 998.2041 998.2041 998.2041 998.2041 998.2041 998.2041 998.2041 998.2041

$1.00006 \quad 997.0449$ $1.00008 \quad 997.0449$ $1.00013 \quad 997.0449$ 997.0449

.00012997 .0449 $1.0002 \quad 997.0449$ $1.00027 \quad 997.0449$ 1.00031997 .0449 $1.00035 \quad 997.0449$ 997.0449 
molality molarity

\section{Density
$\exp / \mathrm{kg} / \mathrm{m}^{3} \mathrm{C}$ mass frac}

$\begin{array}{rrrrrr} & 0.005 & 25 & 0.0006 & 997.66 & (49) \\ & 0.005171 & 25 & 0.0006 & 997.75 & (6) \\ 0.00549 & & 25 & 0.0007 & 997.73 & (13) \\ 0.00972 & & 25 & 0.0012 & 998.20 & (27) \\ 0.00997 & & 25 & 0.0012 & 998.28 & (88) \\ & 0.01 & 25 & 0.0012 & 998.28 & (49) \\ 0.01177 & & 25 & 0.0014 & 998.50 & (13) \\ 0.01731 & & 25 & 0.0021 & 999.17 & (88) \\ & 0.02 & 25 & 0.0024 & 999.51 & (49) \\ 0.02231 & & 25 & 0.0027 & 999.79 & (13) \\ 0.02472 & & 25 & 0.0030 & 1000.07 & (88) \\ 0.025 & & 25 & 0.0030 & 1000.10 & (52) \\ 0.02786 & & 25 & 0.0033 & 1000.47 & (13) \\ 0.03855 & & 25 & 0.0046 & 1001.77 & (13) \\ 0.03855 & & 25 & 0.0046 & 1001.77 & (13) \\ & 0.04 & 25 & 0.0048 & 1001.95 & (49) \\ 0.0497 & & 25 & 0.0059 & 1003.09 & (88) \\ 0.05 & & 25 & 0.0060 & 1003.20 & (4) \\ & 0.05 & 25 & 0.0060 & 1003.16 & (49) \\ 0.07019 & & 25 & 0.0084 & 1005.57 & (13) \\ 0.07019 & & 25 & 0.0084 & 1005.57 & (13) \\ 0.09722 & & 25 & 0.0116 & 1008.60 & (27) \\ 0.09903 & & 25 & 0.0118 & 1008.96 & (88) \\ 0.1 & & 25 & 0.0119 & 1009.16 & (45) \\ 0.11669 & 0.1 & 25 & 0.0119 & 1009.14 & (49) \\ 0.1201 & & 25 & 0.0139 & 1011.09 & (13) \\ 0.1201 & & 25 & 0.0143 & 1011.50 & (13) \\ 0.14931 & & 25 & 0.0143 & 1011.51 & (13) \\ 0.177 & & 25 & 0.0177 & 1014.88 & (88) \\ 0.19444 & & 25 & 0.0209 & 1018.20 & (52) \\ 0.19904 & & 25 & 0.0229 & 1020.10 & (27) \\ & & 25 & 0.0234 & 1020.67 & (88) \\ 0.21211 & 0.2 & 25 & 0.0236 & 1020.84 & (49) \\ 0.21211 & & 25 & 0.0249 & 1022.29 & (13) \\ 0.23758 & & 25 & 0.0249 & 1022.30 & (13) \\ 0.24722 & & 25 & 0.0278 & 1025.23 & (13) \\ 0.29165 & & 25 & 0.0289 & 1026.34 & (88) \\ 0.3639 & & 25 & 0.0339 & 1031.50 & (27) \\ 0.39375 & & 25 & 0.0420 & 1038.80 & (52) \\ 0.39375 & & 25 & 0.0453 & 1043.18 & (13) \\ 0.5 & & 25 & 0.0453 & 1043.18 & (13) \\ 0.64325 & & 25 & 0.0568 & 1055.02 & (4)) \\ 0.88167 & & 25 & 0.0719 & 1071.13 & (13) \\ 0.88167 & & 25 & 0.0960 & 1097.09 & (13) \\ 0.8848 & & 25 & 0.0960 & 1097.09 & (13) \\ & & & 0.0963 & 1097.50 & (52)\end{array}$

App vol App vol Density Density App Vol Square of Inconsistent Inconsistent Delta density Apparent exp / Calc /$$
\begin{array}{rr}
\mathrm{m}^{3} / \mathrm{kg} & \mathrm{m}^{3} / \mathrm{kg} \\
-0.000027 & -0.000001
\end{array}
$$

$\begin{array}{ll}-0.000027 & -0.000001 \\ -0.000138 & -0.000001\end{array}$

$\begin{array}{ll}-0.000138 & -0.000001 \\ -0.000044 & -0.000001\end{array}$

$0.000010-0.000001$

$-0.000031 \quad 0.000000$

$\begin{array}{ll}-0.000027 & 0.000000\end{array}$

$-0.000032 \quad 0.000000$

$-0.000025 \quad 0.000000$

$\begin{array}{ll}-0.000023 & 0.000000\end{array}$

$\begin{array}{ll}-0.000024 & 0.000000\end{array}$

$\begin{array}{ll}-0.000021 & 0.000000\end{array}$

$\begin{array}{ll}-0.000018 & 0.000000\end{array}$

$\begin{array}{ll}-0.000025 & 0.000001\end{array}$

$\begin{array}{ll}-0.000021 & 0.000001\end{array}$

$\begin{array}{ll}-0.000022 & 0.000001\end{array}$

$\begin{array}{rr}-0.000019 & 0.000001 \\ -0.000013 & 0.000002\end{array}$

$\begin{array}{ll}-0.000013 & 0.000002 \\ -0.000026 & 0.000002 \\ -0.000015 & 0.000002\end{array}$

$\begin{array}{ll}-0.000015 & 0.000002\end{array}$

$-0.000012 \quad 0.000003$

$\begin{array}{ll}-0.000012 & 0.000003\end{array}$

$0.000010 \quad 0.000005$

$\begin{array}{ll}-0.000002 & 0.000005 \\ -0.000009 & 0.000005\end{array}$

$\begin{array}{ll}-0.000009 & 0.000005 \\ -0.000005 & 0.000005\end{array}$

$\begin{array}{ll}-0.000003 & 0.000006\end{array}$

$\begin{array}{ll}-0.000003 & 0.000006\end{array}$

$-0.000003 \quad 0.000006$

$0.000005 \quad 0.000008$

$0.000004 \quad 0.000009$

$0.000012 \quad 0.000010$

$\begin{array}{ll}0.000011 & 0.000011 \\ 0.000012 & 0.000011\end{array}$

$\begin{array}{ll}0.000008 & 0.000011 \\ 0.000008 & 0.000011\end{array}$

$0.000008 \quad 0.000011$

$\begin{array}{ll}0.000011 & 0.000013\end{array}$

$0.000012 \quad 0.000013$

$0.000015 \quad 0.000016$

$0.000042 \quad 0.000020$

$\begin{array}{ll}0.000023 & 0.00002 \\ 0.000023 & 0.000021\end{array}$

$0.000023 \quad 0.000021$

$\begin{array}{ll}0.000038 & 0.000034\end{array}$

$\begin{array}{ll}0.000038 & 0.000034 \\ 0.000050 & 0.000046\end{array}$

$0.000050 \quad 0.000046$

$0.000049 \quad 0.000046$

Density App Vol Square of Inconsistent
calc / Residual / Residual / Density based on

Ridual / Residual / Density based on based on

$\mathrm{kg} / \mathrm{m}^{3} \quad \mathrm{~kg} / \mathrm{m}^{3} \quad \mathrm{~m}^{3} / \mathrm{kg} \quad$ Residual density $\begin{gathered}\text { apparent } \\ \text { volume }\end{gathered}$

$\begin{array}{llll}997.65 & 0.0158 & -0.000026 & 0.000\end{array}$

$\begin{array}{llll}997.67 & 0.0850 & -0.000137 & 0.007\end{array}$

$997.70=0.0286-0.000043-0.001$

$\begin{array}{llll}998.21 & -0.0121 & 0.000010 & 0.000\end{array}$

$\begin{array}{llll}998.24 & 0.0368 & -0.000031 & 0.001\end{array}$

$\begin{array}{llll}998.25 & 0.0320 & -0.000027 & 0.001\end{array}$

$\begin{array}{llll}998.46 & 0.0448 & -0.000032 & 0.002\end{array}$

$\begin{array}{llll}999.12 & 0.0523 & -0.000025 & 0.003\end{array}$

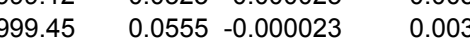

$0.0649-0.000024$

$0.0642-0.000022$

$0.0557-0.000019$

1001.67

1001.67

$0.0841-0.000025$

$0.1047-0.000023$

$0.1057-0.000023$

$0.0970-0.000020$

$0.0910-0.000015$

$0.1653-0.000027$

$0.1050-0.000017$

$0.1277-0.000015$

$0.1297-0.000015$

$-0.0586 \quad 0.000005$

$0.0843-0.000007$

$0.1712-0.000014$

$0.1158-0.000010$

$0.1246-0.000009$

$0.1316-0.000009$

$0.1356-0.000009$

$0.0520-0.000003$

$0.1114-0.000005$

$\begin{array}{ll}-0.0380 & 0.000002\end{array}$

$\begin{array}{lll}-0.0060 & 0.000000\end{array}$

$\begin{array}{ll}-0.0222 & 0.000001\end{array}$

$0.0808-0.000003$

$0.0878-0.000003$

$0.0437-0.000001$

$0.0276-0.000001$

$0.0168 \quad 0.000000$

$\begin{array}{ll}-1.0300 & 0.000023\end{array}$

$\begin{array}{ll}-0.0805 & 0.000002\end{array}$

$\begin{array}{ll}-0.0745 & 0.000002\end{array}$

$\begin{array}{lll}-0.3378 & 0.000005\end{array}$

1071.43

1097.57
1097.57

$-0.2950 \quad 0.000004$

$-0.48230 .000004$

$\begin{array}{ll}-0.4763 & 0.000004 \\ -0.4084 & 0.000004\end{array}$
0.003
0.004

0.004

0.003

0.007

0.011

0.011

0.009

0.008
0.027

0.011

0.016

0.017

0.003

0.007

0.029

0.013

0.016

0.017

0.018

0.003

0.012

0.001

0.000

0.000

0.007

0.008
0.002

0.002

0.001

0.000

1.061

0.006
0.006

0.006

0.087
0.233

0.227

0.167
$1 \mathrm{~kg} / \mathrm{m}^{3}$ molal volume $1 \mathrm{~cm}^{3} / \mathrm{mol}$

Relative Density of density water $/ \mathrm{kg} / \mathrm{m}^{3}$

1.00062997 .0449

1.00071997 .0449 997.0449 997.0449 997.0449

$1.00124 \quad 997.0449$ 997.0449 997.0449

$1.00247 \quad 997.0449$ 997.0449 997.0449 997.0449 997.0449 997.0449

$1.00492 \quad 997.0449$ 997.0449 997.0449 997.0449 997.0449 997.0449
997.0449

$1.01213 \quad 997.0449$ 997.0449 997.0449 997.0449 997.0449 997.0449 997.0449 997.0449

25.246

25.253

28.188

29.297

46.132

46.138

74.089

100.043

100.049
$1.02387 \quad 997.0449$ 997.0449 997.0449 997.0449 997.0449 997.0449 997.0449 997.0449 997.0449 997.0449 997.0449 997.0449 997.0449 
$\mathrm{t} /{ }^{\circ} \mathrm{C}$ mass frac Density

$\exp / \mathrm{kg} / \mathrm{m}^{3}$

1.2332
1.2332
1.35795
1.35795
1.424
1.424
1.48251
1.48251
1.5
2
2.5

0.05

0.1

0.5

1

1.5

2

25

0.00099

0.00549

0.01177

0.02231

$\begin{array}{rrr}25 & 0.1074 & 1109.26 \\ 25 & 0.1293 & 1134.26 \\ 25 & 0.1293 & 1134.27 \\ 25 & 0.1405 & 1147.12 \\ 25 & 0.1405 & 1147.13 \\ 25 & 0.1463 & 1153.88 \\ 25 & 0.1463 & 1153.89 \\ 25 & 0.1514 & 1159.78 \\ 25 & 0.1514 & 1159.79 \\ 25 & 0.1530 & 1160.97 \\ 25 & 0.1940 & 1209.14 \\ 25 & 0.2313 & 1256.82 \\ 30 & 0.0001 & 995.71 \\ 30 & 0.0001 & 995.78 \\ 30 & 0.0003 & 995.96 \\ 30 & 0.0006 & 996.26 \\ 30 & 0.0012 & 996.88 \\ 30 & 0.0024 & 998.10 \\ 30 & 0.0048 & 1000.52 \\ 30 & 0.0060 & 1001.76 \\ 30 & 0.0060 & 1001.71 \\ 30 & 0.0119 & 1007.69 \\ 30 & 0.0119 & 1007.62 \\ 30 & 0.020 & 1015.80 \\ 30 & 0.0236 & 1019.17 \\ 30 & 0.0400 & 1036.20 \\ 30 & 0.0568 & 1053.37 \\ 30 & 0.0800 & 1078.20 \\ 30 & 0.1074 & 1107.46 \\ 30 & 0.1200 & 1122.00 \\ 30 & 0.1530 & 1159.04 \\ 30 & 0.1600 & 1167.90 \\ 30 & 0.1940 & 1207.17 \\ 30 & 0.2000 & 1215.90 \\ 30 & 0.2313 & 1254.81 \\ 30 & 0.2600 & 1292.20 \\ 35 & 0.0001 & 994.09 \\ 35 & 0.0001 & 994.15 \\ 35 & 0.0001 & 994.15 \\ 35 & 0.0003 & 994.34 \\ 35 & 0.0006 & 994.65 \\ 35 & 0.0007 & 994.71 \\ 35 & 0.0012 & 995.25 \\ 35 & 0.0014 & 995.48 \\ 35 & 0.0024 & 996.47 \\ 35 & 0.0027 & 996.75 \\ & & \end{array}$

Ref

\section{App}

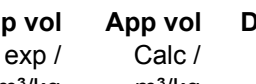

$$
\begin{gathered}
\exp / \\
\mathrm{m}^{3} / \mathrm{kg}
\end{gathered}
$$$$
\mathrm{m}^{3} / \mathrm{kg}
$$

$0.000059 \quad 0.000051$

$0.000064 \quad 0.000061$

$\begin{array}{ll}0.000064 & 0.000061\end{array}$

$0.000069 \quad 0.000066$

$0.000069 \quad 0.000066$

$0.000071 \quad 0.000069$

0.0000740 .000072

$\begin{array}{ll}0.000074 & 0.000072\end{array}$

$\begin{array}{ll}0.000077 & 0.000072\end{array}$

$\begin{array}{ll}0.000096 & 0.000091\end{array}$

$\begin{array}{ll}0.000107 & 0.000108\end{array}$

$0.000006 \quad 0.000004$

$\begin{array}{ll}-0.000077 & 0.000004\end{array}$

$\begin{array}{ll}-0.000027 & 0.000004\end{array}$

$\begin{array}{ll}-0.000027 & 0.000004 \\ -0.000027 & 0.000004\end{array}$

$\begin{array}{ll}-0.000019 & 0.000005 \\ -0.000013 & 0.000006\end{array}$

$-0.000013 \quad 0.000006$

$\begin{array}{lll}-0.000020 & 0.000007\end{array}$

$\begin{array}{ll}-0.0000005 & 0.000010 \\ -0.000004 & 0.000010\end{array}$

$0.000004 \quad 0.000010$

$0.000008 \quad 0.000013$

$0.000021 \quad 0.000015$

$0.000022 \quad 0.000023$

$0.000035 \quad 0.000030$

$\begin{array}{ll}0.000043 & 0.00004\end{array}$

$\begin{array}{ll}0.000061 & 0.000054\end{array}$

0.0000790 .000074

$\begin{array}{lll}0.000079 & 0.000077\end{array}$

$0.000097 \quad 0.000092$

$0.000095 \quad 0.000094$

$\begin{array}{lll}0.000108 & 0.000107\end{array}$

$0.000118 \quad 0.000119$

$0.000006 \quad 0.000008$

$\begin{array}{ll}0.000029 & 0.000008\end{array}$

$0.000006 \quad 0.000008$

$\begin{array}{ll}-0.000027 & 0.000009\end{array}$

$\begin{array}{ll}-0.000027 & 0.000009\end{array}$

$\begin{array}{ll}-0.000028 & 0.000009\end{array}$

$\begin{array}{ll}-0.000019 & 0.000009\end{array}$

$\begin{array}{ll}-0.000025 & 0.000009\end{array}$

$\begin{array}{ll}-0.000015 & 0.000009\end{array}$

$-0.000018 \quad 0.000010$
Density Density App Vol Square of Inconsistent Inconsistent Delta density Apparent calc / Residual / Residual / Density based on based on $/ \mathrm{kg} / \mathrm{m}^{3}$ molal volum $\mathrm{kg} / \mathrm{m}^{3} \quad \mathrm{~kg} / \mathrm{m}^{3} \quad \mathrm{~m}^{3} / \mathrm{kg}$ Residual density apparent volume

$/ \mathrm{kg} / \mathrm{m}^{3}$ molal volume

Relative Density of $\begin{array}{llll}110.27 & -1.0083 & 0.000008 & 1.017\end{array}$ $\begin{array}{llll}1134.77 & -0.5051 & 0.000003 & 0.255\end{array}$ $\begin{array}{llll}1134.77 & -0.5021 & 0.000003 & 0.252\end{array}$ $\begin{array}{llll}1147.60 & -0.4749 & 0.000003 & 0.226\end{array}$ $\begin{array}{llll}1147.60 & -0.4709 & 0.000003 & 0.222\end{array}$ $1000.43 \quad 0.0903-0.000019 \quad 0.008$

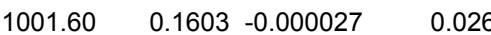
$\begin{array}{llll}1001.62 & 0.0940 & -0.000016 & 0.009\end{array}$ $\begin{array}{llll}1019.33 & -0.1535 & 0.000006 & 0.024\end{array}$ $\begin{array}{llll}1036.16 & 0.0430 & -0.000001 & 0.002\end{array}$ $\begin{array}{llll}1053.65 & -0.2816 & 0.000004 & 0.079\end{array}$ $\begin{array}{llll}1078.39 & -0.1871 & 0.000002 & 0.035\end{array}$ $\begin{array}{llll}1160.06 & -1.0206 & 0.000005 & 1.042\end{array}$ $\begin{array}{llll}1168.28 & -0.3824 & 0.000002 & 0.146\end{array}$ $\begin{array}{llll}1208.84 & -1.6714 & 0.000006 & 2.794\end{array}$ $\begin{array}{llll}1154.31 & -0.4363 & 0.000002 & 0.190\end{array}$ $\begin{array}{llll}1154.31 & -0.4363 & 0.000002 & 0.190\end{array}$ $\begin{array}{llll}160.22 & -0.4309 & 0.000002 & 0.186\end{array}$ $\begin{array}{llll}160.22 & -0.4229 & 0.000002 & 0.179\end{array}$ $\begin{array}{llll}161.97 & -1.0024 & 0.000005 & 1.005\end{array}$ $\begin{array}{llll}210.67 & -1.5287 & 0.000005 & 2.337\end{array}$ $\begin{array}{llll}256.55 & 0.2675 & -0.000001 & 0.072\end{array}$ $\begin{array}{llll}995.71 & -0.0001 & 0.000002 & 0.000\end{array}$ $\begin{array}{llll}995.77 & 0.0097 & -0.000081 & 0.000\end{array}$ $\begin{array}{llll}995.95 & 0.0093 & -0.000031 & 0.000\end{array}$ $\begin{array}{lllll}996.25 & 0.0187 & -0.000031 & 0.000\end{array}$ $\begin{array}{llll}996.84 & 0.0379 & -0.000032 & 0.001\end{array}$ $\begin{array}{llll}998.04 & 0.0573 & -0.000024 & 0.003\end{array}$ $\begin{array}{llll}007.52 & 0.1721 & -0.000014 & 0.030\end{array}$ $\begin{array}{llll}1007.55 & 0.0726 & -0.000006 & 0.005\end{array}$ $\begin{array}{llll}1015.69 & 0.1087 & -0.000005 & 0.012\end{array}$ $\begin{array}{llll}1108.39 & -0.9334 & 0.000007 & 0.871\end{array}$ $\begin{array}{llll}1122.41 & -0.4059 & 0.000003 & 0.165\end{array}$ $\begin{array}{llll}1216.09 & -0.1862 & 0.000001 & 0.035\end{array}$ $\begin{array}{llll}254.92 & -0.1105 & 0.000000 & 0.012\end{array}$ $\begin{array}{llll}291.56 & 0.6433 & -0.000001 & 0.414\end{array}$ $\begin{array}{llll}994.09 & 0.0001 & -0.000002 & 0.000\end{array}$ $\begin{array}{llll}994.15 & -0.0025 & 0.000021 & 0.000\end{array}$ $\begin{array}{llll}994.15 & 0.0003 & -0.000002 & 0.000\end{array}$ $\begin{array}{lllll}994.33 & 0.0107 & -0.000036 & 0.000\end{array}$ $\begin{array}{llll}994.63 & 0.0214 & -0.000036 & 0.000\end{array}$ $\begin{array}{lllll}994.68 & 0.0237 & -0.000036 & 0.001\end{array}$ $\begin{array}{llll}995.22 & 0.0332 & -0.000028 & 0.001\end{array}$ $\begin{array}{llll}995.43 & 0.0483 & -0.000034 & 0.002\end{array}$ $\begin{array}{llll}996.41 & 0.0579 & -0.000024 & 0.003 \\ 996.68 & 0.0739 & -0.000028 & 0.005\end{array}$

150.082

156.831

162.740

$\begin{array}{llll}996.68 & 0.0739 & -0.000028\end{array}$

0.005
137.220

137.223

150.078

156.843

162.748

997.0449 997.0449 997.0449 997.0449 997.0449 997.0449 997.0449 997.0449 997.0449 997.0449 997.0449 997.0449

$1.00006 \quad 995.6473$ 1.00013 1.00031995 .6473 1.00062995 .6473 $1.00124 \quad 995.6473$ $1.00246 \quad 995.6473$

$1.00489 \quad 995.6473$ 995.6473 $1.00609 \quad 995.6473$ 995.6473

$1.01203 \quad 995.6473$ 995.6473

$1.02363 \quad 995.6473$ 995.6473 995.6473 995.6473 995.6473 995.6473 995.6473 995.6473 995.6473 995.6473 995.6473 995.6473 994.0319
994.0319 1.00012994 .0319 1.00031994 .0319

1.00062994 .0319 994.0319 $1.00123 \quad 994.0319$ 994.0319

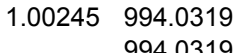




\begin{tabular}{|c|c|c|c|c|}
\hline molality & molarity & $\mathrm{t} /{ }^{\circ} \mathrm{C}$ & ass frac & $\begin{array}{l}\text { Density } \\
\mathrm{xp} / \mathrm{kg} / \mathrm{m}^{3}\end{array}$ \\
\hline 0.02786 & & 35 & 0.0033 & 997.43 \\
\hline 0.03855 & & 35 & 0.0046 & 998.74 \\
\hline & & 35 & 0.0048 & 998.86 \\
\hline 0.05 & & 35 & 0.0060 & 1000.14 \\
\hline & & 35 & 0.0060 & 1000.05 \\
\hline 0.07019 & & 35 & 0.0084 & 1002.48 \\
\hline 0.1 & & 35 & 0.0119 & 1006.05 \\
\hline & & 35 & 0.0119 & 1005.89 \\
\hline 0.11669 & & 35 & 0.0139 & 1007.99 \\
\hline 0.1201 & & 35 & 0.0143 & 1008.35 \\
\hline & & 35 & 0.0236 & 1017.26 \\
\hline 0.23758 & & 35 & 0.0278 & 1022.03 \\
\hline 0.39375 & & 35 & 0.0453 & 1039.80 \\
\hline 0.5 & & 35 & 0.0568 & 1051.60 \\
\hline 0.64325 & & 35 & 0.0719 & 1067.58 \\
\hline 0.88167 & & 35 & 0.0960 & 1093.36 \\
\hline 1 & & 35 & 0.1074 & 1105.55 \\
\hline 1.2332 & & 35 & 0.1293 & 1130.31 \\
\hline 1.35795 & & 35 & 0.1405 & 1143.14 \\
\hline 1.424 & & 35 & 0.1463 & 1149.86 \\
\hline 1.48251 & & 35 & 0.1514 & 1155.69 \\
\hline 1.5 & & 35 & 0.1530 & 1157.05 \\
\hline 2 & & 35 & 0.1940 & 1205.12 \\
\hline 2.5 & & 35 & 0.2313 & 1252.87 \\
\hline 0.025 & & 40 & 0.0030 & 995.20 \\
\hline & & 40 & 0.020 & 1012.30 \\
\hline 0.177 & & 40 & 0.0209 & 1013.10 \\
\hline & & 40 & 0.0400 & 1032.60 \\
\hline 0.3639 & & 40 & 0.0420 & 1033.40 \\
\hline & & 40 & 0.0800 & 1074.30 \\
\hline 0.8848 & & 40 & 0.0963 & 1091.60 \\
\hline & & 40 & 0.1200 & 1117.90 \\
\hline & & 40 & 0.1600 & 1163.70 \\
\hline & & 40 & 0.2000 & 1211.70 \\
\hline & & 40 & 0.2600 & 1287.90 \\
\hline & & 42.5 & 0.0001 & 991.30 \\
\hline & & 42.5 & 0.0001 & 991.36 \\
\hline & & 42.5 & 0.0003 & 991.54 \\
\hline & & 42.5 & 0.0006 & 991.84 \\
\hline & & 42.5 & 0.0012 & 992.45 \\
\hline & & 42.5 & 0.0024 & 993.64 \\
\hline & & 42.5 & 0.0048 & 995.99 \\
\hline & & 42.5 & 0.0060 & 997.15 \\
\hline & & 42.5 & 0.0119 & 1002.88 \\
\hline & & 42.5 & 0.0236 & 1013.97 \\
\hline 0.00972 & & 45 & 0.0012 & 991.20 \\
\hline
\end{tabular}

App vol App vol Density Density App Vol Square of Inconsistent Inconsistent Delta density Apparent exp / Calc / Density based on based on $/ \mathrm{kg} / \mathrm{m}^{3}$ molal volum exp/ Calc/ $\mathrm{m}^{3} / \mathrm{kg}$ $\mathrm{m}^{3} / \mathrm{kg}$

$-0.000020 \quad 0.000010$ $-0.000020 \quad 0.000010$ $-0.000006 \quad 0.000011$ $-0.000021 \quad 0.000011$ $\begin{array}{ll}-0.000002 & 0.000011\end{array}$ $-0.000006 \quad 0.000012$ $\begin{array}{ll}-0.000004 & 0.000014\end{array}$ $0.000012 \quad 0.000014$ $0.000000 \quad 0.000015$ $0.000003 \quad 0.000015$ $0.000032 \quad 0.000019$ $0.000015 \quad 0.000021$ $\begin{array}{ll}0.000027 & 0.000029\end{array}$ $0.000036 \quad 0.000034$ $0.000042 \quad 0.000040$ $\begin{array}{ll}0.000053 & 0.000051\end{array}$ $0.000062 \quad 0.000056$ $0.000068 \quad 0.000065$ $\begin{array}{ll}0.000072 & 0.000070\end{array}$ $0.000074 \quad 0.000072$ $\begin{array}{ll}0.000077 & 0.000074\end{array}$ $0.000079 \quad 0.000075$ $\begin{array}{ll}0.000098 & 0.000092 \\ 0.000108 & 0.000107\end{array}$ $0.000108 \quad 0.000107$ $\begin{array}{ll}0.000001 & 0.000014\end{array}$ $0.000008 \quad 0.000021$ $\begin{array}{ll}0.000012 & 0.000021\end{array}$ $0.000022 \quad 0.000030$ $0.000051 \quad 0.000030$ $0.000045 \quad 0.000046$ $0.000055 \quad 0.000053$ $\begin{array}{ll}0.000064 & 0.000063 \\ 0.000080 & 0.000079\end{array}$ $\begin{array}{ll}0.000095 & 0.000094\end{array}$ $0.000118 \quad 0.000117$ $0.000006 \quad 0.000014$ $0.000006 \quad 0.000014$ $\begin{array}{ll}-0.000027 & 0.000015\end{array}$ $\begin{array}{ll}-0.000011 & 0.000015\end{array}$ $\begin{array}{ll}-0.000011 & 0.000015\end{array}$ $\begin{array}{ll}-0.000002 & 0.000015\end{array}$ $0.000006 \quad 0.000016$ $\begin{array}{ll}0.000011 & 0.000017\end{array}$ $0.000027 \quad 0.000019$ $\begin{array}{ll}0.000050 & 0.000024 \\ 0.000150 & 0.000017\end{array}$

\section{$\mathrm{kg} / \mathrm{m}^{3}$} $\mathrm{kg} / \mathrm{m}^{3}$ $\mathrm{m}^{3} / \mathrm{kg}$ Desiduty$$
\text { dual denside }
$$
$\begin{array}{llll}997.33 & 0.0982 & -0.000030 & 0.010\end{array}$ $\begin{array}{llll}998.60 & 0.1394 & -0.000030 & 0.019\end{array}$ $\begin{array}{llll}998.78 & 0.0815 & -0.000017 & 0.007\end{array}$ $\begin{array}{llll}999.95 & 0.1915 & -0.000032 & 0.037\end{array}$ $\begin{array}{llll}999.97 & 0.0803 & -0.000013 & 0.006\end{array}$ $\begin{array}{llll}1002.33 & 0.1555 & -0.000018 & 0.024\end{array}$ $\begin{array}{llll}1005.83 & 0.2177 & -0.000018 & 0.047\end{array}$ $\begin{array}{llll}1005.87 & 0.0242 & -0.000002 & 0.001\end{array}$ $\begin{array}{llll}1007.79 & 0.2028 & -0.000014 & 0.041\end{array}$ $\begin{array}{llll}1008.19 & 0.1645 & -0.000011 & 0.027\end{array}$ $\begin{array}{llll}1017.58 & -0.3149 & 0.000013 & 0.099\end{array}$ $\begin{array}{llll}1021.85 & 0.1748 & -0.000006 & 0.031\end{array}$ $\begin{array}{llll}1039.75 & 0.0565 & -0.000001 & 0.003\end{array}$ $\begin{array}{llll}1051.74 & -0.1409 & 0.000002 & 0.020\end{array}$ $\begin{array}{llll}1067.69 & -0.1105 & 0.000001 & 0.012\end{array}$ $\begin{array}{llll}1093.68 & -0.3157 & 0.000003 & 0.100\end{array}$ $\begin{array}{llll}1106.32 & -0.7730 & 0.000006 & 0.598\end{array}$ $\begin{array}{llll}1130.76 & -0.4485 & 0.000003 & 0.201\end{array}$ $\begin{array}{llll}1143.58 & -0.4379 & 0.000002 & 0.192\end{array}$ $\begin{array}{llll}1150.29 & -0.4274 & 0.000002 & 0.183\end{array}$ $\begin{array}{llll}1156.20 & -0.5056 & 0.000002 & 0.256\end{array}$ $\begin{array}{llll}1157.96 & -0.9079 & 0.000004 & 0.824\end{array}$ $\begin{array}{llll}1206.82 & -1.7042 & 0.000006 & 2.904\end{array}$ $\begin{array}{llll}1253.10 & -0.2259 & 0.000001 & 0.051\end{array}$ $\begin{array}{llll}995.16 & 0.0392 & -0.000013 & 0.002\end{array}$ $\begin{array}{llll}1012.03 & 0.2670 & -0.000013 & 0.071\end{array}$ $\begin{array}{llll}1012.90 & 0.2022 & -0.000009 & 0.041\end{array}$ $\begin{array}{llll}1032.30 & 0.3038 & -0.000007 & 0.092\end{array}$ $1034.31 \quad-0.9144 \quad 0.000020 \quad 0.836$ $\begin{array}{llll}1074.21 & 0.0931 & -0.000001 & 0.009\end{array}$ $\begin{array}{llll}1091.79 & -0.1884 & 0.000002 & 0.035\end{array}$ $\begin{array}{llll}1118.05 & -0.1453 & 0.000001 & 0.021\end{array}$ $\begin{array}{llll}1163.91 & -0.2140 & 0.000001 & 0.046\end{array}$ $\begin{array}{llll}1211.92 & -0.2214 & 0.000001 & 0.049\end{array}$ $\begin{array}{llll}1288.19 & -0.2947 & 0.000001 & 0.087\end{array}$ $\begin{array}{llll}991.30 & 0.0005 & -0.000008 & 0.000\end{array}$ $\begin{array}{llll}991.35 & 0.0010 & -0.000008 & 0.000\end{array}$ $\begin{array}{llll}991.53 & 0.0124 & -0.000042 & 0.000\end{array}$ $\begin{array}{llll}991.83 & 0.0150 & -0.000025 & 0.000\end{array}$ $\begin{array}{llll}992.42 & 0.0303 & -0.000025 & 0.001\end{array}$ $\begin{array}{llll}993.59 & 0.0420 & -0.000018 & 0.002\end{array}$ $\begin{array}{llll}995.95 & 0.0495 & -0.000010 & 0.002\end{array}$ $\begin{array}{llll}997.12 & 0.0353 & -0.000006 & 0.001\end{array}$ $\begin{array}{llll}1002.97 & -0.0872 & 0.000007 & 0.008\end{array}$ $\begin{array}{llll}1014.59 & -0.6237 & 0.000026 & 0.389\end{array}$ $\begin{array}{llll}991.35 & -0.1527 & 0.000133 & 0.023\end{array}$
$/ \mathrm{kg} / \mathrm{m}^{3}$ molal volume

Relative Density of 3.399 994.0319 994.0319 $1.00486 \quad 994.0319$ 994.0319 $1.00605 \quad 994.0319$ 994.0319 994.0319

$1.01193 \quad 994.0319$ 994.0319

13.960

14.321

27.997

45.770

73.548

99.33

136.281

149.108

155.833

161.662 
molality molarity

$t /{ }^{\circ} \mathrm{C}$ mass frac Density

$\exp / \mathrm{kg} / \mathrm{m}^{3}$

\begin{tabular}{|c|c|c|c|}
\hline 0.05 & 45 & 0.0060 & 996.31 \\
\hline 0.09722 & 45 & 0.0116 & 1001.60 \\
\hline 0.1 & 45 & 0.0119 & 1002.21 \\
\hline 0.19444 & 45 & 0.0229 & 1012.90 \\
\hline 0.29165 & 45 & 0.0339 & 1024.20 \\
\hline 0.5 & 45 & 0.0568 & 1047.58 \\
\hline 1 & 45 & 0.1074 & 1101.38 \\
\hline 1.5 & 45 & 0.1530 & 1152.67 \\
\hline 2 & 45 & 0.1940 & 1200.59 \\
\hline 2.5 & 45 & 0.2313 & 1248.60 \\
\hline .002439 & 50 & 0.0003 & 988.34 \\
\hline .006453. & 50 & 0.0008 & 988.83 \\
\hline 013103 & 50 & 0.0016 & 989.64 \\
\hline .018164. & 50 & 0.0022 & 990.25 \\
\hline .026523. & 50 & 0.0032 & 991.25 \\
\hline 0.0401 & 50 & 0.0048 & 992.87 \\
\hline .064372 & 50 & 0.0077 & 995.74 \\
\hline \multirow[t]{2}{*}{0.10706} & 50 & 0.0127 & 1000.76 \\
\hline & 50 & 0.020 & 1008.10 \\
\hline 0.17204 & 50 & 0.0203 & 1008.31 \\
\hline \multirow{2}{*}{0.24497} & 50 & 0.0286 & 1016.67 \\
\hline & 50 & 0.0400 & 1028.30 \\
\hline 0.35275 & 50 & 0.0407 & 1028.88 \\
\hline 0.44699 & 50 & 0.0511 & 1039.37 \\
\hline 0.58829 & 50 & 0.0661 & 1054.91 \\
\hline \multirow[t]{2}{*}{0.72061} & 50 & 0.0798 & 1069.24 \\
\hline & 50 & 0.0800 & 1070.00 \\
\hline \multirow[t]{5}{*}{0.88872} & 50 & 0.0966 & 1087.17 \\
\hline & 50 & 0.1200 & 1113.50 \\
\hline & 50 & 0.1600 & 1159.20 \\
\hline & 50 & 0.2000 & 1207.20 \\
\hline & 50 & 0.2600 & 1283.60 \\
\hline 0.05 & 55 & 0.0060 & 991.78 \\
\hline 0.1 & 55 & 0.0119 & 997.65 \\
\hline 0.5 & 55 & 0.0568 & 1042.88 \\
\hline 1 & 55 & 0.1074 & 1096.66 \\
\hline 1.5 & 55 & 0.1530 & 1147.93 \\
\hline 2 & 55 & 0.1940 & 1195.85 \\
\hline 2.5 & 55 & 0.2313 & 1243.81 \\
\hline \multirow[t]{2}{*}{0.025} & 60 & 0.0030 & 986.30 \\
\hline & 60 & 0.020 & 1003.20 \\
\hline \multirow[t]{2}{*}{0.177} & 60 & 0.0209 & 1003.90 \\
\hline & 60 & 0.0400 & 1023.40 \\
\hline \multirow[t]{2}{*}{0.3639} & 60 & 0.0420 & 1024.20 \\
\hline & 60 & 0.0800 & 1065.00 \\
\hline 0.8848 & 60 & 0.0963 & 1082.20 \\
\hline
\end{tabular}

App vc

App vol App vol Density Density App Vol Square of Inconsistent Inconsistent Delta density Apparent

exp / Calc / calc/ Residual / Residual / Density based on based on $/ \mathrm{kg} / \mathrm{m}^{3} \mathrm{molal}$ volum

$\mathrm{m}^{3} / \mathrm{kg} \quad \mathrm{m}^{3} / \mathrm{kg}$

calc/

$\mathrm{kg} / \mathrm{m}^{3}$

$\mathrm{m}^{3} / \mathrm{kg}$

$1 \mathrm{~cm}^{3} / \mathrm{mol}$

$\begin{array}{ll}0.000023 & 0.000019 \\ 0.000017 & 0.000021\end{array}$

$996.06 \quad 0.2475-0.000042 \quad 0.061$

.061

$0.0412-0.000004 \quad 0.002$

$\begin{array}{lllllll}-0.000006 & 0.000021 & 1001.88 & 0.3285 & -0.000028 & 0.108\end{array}$

$\begin{array}{llllll}0.000021 & 0.000026 & 1012.79 & 0.1091 & -0.000005 & 0.012 \\ 0.000022 & 0.000030 & 1023.91 & 0.2903 & -0.000008 & 0.084\end{array}$

$\begin{array}{llllll}0.000036 & 0.000039 & 1047.37 & 0.2118 & -0.000003 & 0.045\end{array}$

$\begin{array}{lllllll}0.000061 & 0.000059 & 1101.65 & -0.2749 & 0.000002 & 0.076\end{array}$

$\begin{array}{llllll}0.000079 & 0.000077 & 1153.24 & -0.5666 & 0.000003 & 0.321\end{array}$

$\begin{array}{llllll}0.000098 & 0.000092 & 1202.27 & -1.6833 & 0.000006 & 2.834\end{array}$

$\begin{array}{llllll}0.000106 & 0.000106 & 1248.92 & -0.3192 & 0.000001 & 0.102\end{array}$

$\begin{array}{llllll}-0.000048 & 0.000020 & 988.32 & 0.0195 & -0.000068 & 0.000\end{array}$

$\begin{array}{llllll}-0.000040 & 0.000020 & 988.79 & 0.0454 & -0.000060 & 0.002\end{array}$

$\begin{array}{llllll}-0.000029 & 0.000020 & 989.56 & 0.0763 & -0.000049 & 0.006\end{array}$

$\begin{array}{llllll}-0.000024 & 0.000020 & 990.15 & 0.0948 & -0.000044 & 0.009\end{array}$

990.15

$0.1220-0.000039$

$0.1630-0.000034$

$\begin{array}{ll}-0.000013 & 0.000021 \\ -0.000007 & 0.000023\end{array}$

995.52

$0.2225-0.000029$

0.015

0.015
0.027

0.050

0.090

$0.000001 \quad 0.000025$

000.46

$0.3008-0.000024$

0.208

$0.000005 \quad 0.000027$

1007.93

0.000017

1016.26

$0.4555-0.000022$

$0.3768-0.000018$

$0.4099-0.000014$

0.142

0.168

$0.000021 \quad 0.000035$

1028.47

$-0.000014$

0.335

$\begin{array}{llllll}0.000026 & 0.000035 & 1028.47 & 0.4079 & -0.000009 & 0.166\end{array}$

$\begin{array}{llllll}0.000033 & 0.000039 & 1039.03 & 0.3398 & -0.000006 & 0.115\end{array}$

$\begin{array}{llllll}0.000042 & 0.000045 & 1054.69 & 0.2216 & -0.000003 & 0.049\end{array}$

0.007

$\begin{array}{llllll}0.000043 & 0.000050 & 1069.34 & 0.6593 & -0.000007 & 0.435\end{array}$

$\begin{array}{llllll}0.000057 & 0.000056 & 1087.26 & -0.0852 & 0.000001 & 0.007\end{array}$

$\begin{array}{llllll}0.000062 & 0.000065 & 1113.02 & 0.4820 & -0.000003 & 0.232\end{array}$

0.098

$\begin{array}{llllll}0.000078 & 0.000080 & 1158.89 & 0.3128 & -0.000001 & 0.098\end{array}$

$\begin{array}{llllll}0.000093 & 0.000094 & 1207.09 & 0.1067 & 0.000000 & 0.011\end{array}$

$\begin{array}{llllll}0.000116 & 0.000115 & 1284.13 & -0.5316 & 0.000001 & 0.283\end{array}$

$\begin{array}{llllll}-0.000026 & 0.000025 & 991.48 & 0.2979 & -0.000051 & 0.089\end{array}$

$\begin{array}{llllll}-0.000008 & 0.000027 & 997.24 & 0.4086 & -0.000035 & 0.167\end{array}$

$\begin{array}{llllll}0.000035 & 0.000043 & 1042.34 & 0.5407 & -0.000009 & 0.292\end{array}$

$\begin{array}{llllll}0.000059 & 0.000061 & 1096.36 & 0.3034 & -0.000002 & 0.092\end{array}$

$\begin{array}{llllll}0.000077 & 0.000077 & 1147.89 & 0.0354 & 0.000000 & 0.001\end{array}$

$\begin{array}{lllllll}0.000096 & 0.000091 & 1197.10 & -1.2460 & 0.000004 & 1.552\end{array}$

$\begin{array}{lllllll}0.000104 & 0.000104 & 1244.10 & -0.2867 & 0.000001 & 0.082\end{array}$

$\begin{array}{llllll}-0.000049 & 0.000026 & 986.08 & 0.2191 & -0.000075 & 0.048\end{array}$

$\begin{array}{lllllll}0.000003 & 0.000032 & 1002.61 & 0.5868 & -0.000029 & 0.344\end{array}$

$\begin{array}{llllll}0.000012 & 0.000033 & 1003.46 & 0.4384 & -0.000021 & 0.192\end{array}$

$\begin{array}{llllll}0.000018 & 0.000039 & 1022.52 & 0.8821 & -0.000021 & 0.778\end{array}$

$\begin{array}{llllll}0.000047 & 0.000040 & 1024.50 & -0.3035 & 0.000007 & 0.092\end{array}$

$\begin{array}{llllll}0.000041 & 0.000053 & 1063.87 & 1.1319 & -0.000012 & 1.281\end{array}$

$\begin{array}{llllll}0.000050 & 0.000059 & 1081.29 & 0.9095 & -0.000008 & 0.827\end{array}$
Relative Density of
density $w a t e r / \mathrm{kg} / \mathrm{m}^{3}$

990.2132

990.2132

990.2132

990.2132

990.2132

990.2132

990.2132

990.2132

990.2132

990.2132

988.0363

988.0363

988.0363

988.0363

988.0363

988.0363

988.0363

988.0363

988.0363

988.0363

988.0363

988.0363

988.0363

988.0363

988.0363

988.0363

988.0363

988.0363

988.0363

988.0363

988.0363

985.6952

985.6952

985.6952

985.6952

985.6952

985.6952

985.6952

983.1989

983.1989

983.1989

983.1989

983.1989

983.1989

983.1989 
molality molarity

$t /{ }^{\circ} \mathrm{C}$ mass frac Density $\exp / \mathrm{kg} / \mathrm{m}^{3}$

$\begin{array}{rrr}60 & 0.1200 & 1108.30 \\ 65 & 0.0012 & 981.70 \\ 65 & 0.0116 & 992.00 \\ 65 & 0.0229 & 1003.20 \\ 65 & 0.0339 & 1014.50 \\ 75 & 0.0012 & 976.20 \\ 75 & 0.0030 & 978.20 \\ 75 & 0.0116 & 986.40 \\ 75 & 0.0209 & 996.40 \\ 75 & 0.0229 & 997.60 \\ 75 & 0.0339 & 1008.90 \\ 75 & 0.0409 & 1015.70 \\ 75 & 0.0964 & 1074.80 \\ 80 & 0.0200 & 991.60 \\ 80 & 0.0400 & 1011.80 \\ 80 & 0.0800 & 1053.40 \\ 80 & 0.1200 & 1096.80 \\ 100 & 0.0012 & 959.80 \\ 100 & 0.0030 & 961.80 \\ 100 & 0.0116 & 970.20 \\ 100 & 0.0210 & 980.20 \\ 100 & 0.0229 & 981.60 \\ 100 & 0.0339 & 992.70 \\ 100 & 0.0410 & 999.60 \\ 125 & 0.0012 & 940.50 \\ 125 & 0.0030 & 943.00 \\ 125 & 0.0116 & 951.00 \\ 125 & 0.0210 & 961.30 \\ 125 & 0.0229 & 962.40 \\ 125 & 0.0339 & 973.70 \\ 125 & 0.0413 & 981.00 \\ 125 & 0.0972 & 1040.80\end{array}$

0.19444

0.29165

0.00972

$$
0.025
$$

0.09722

0.1773

0.19444

0.29165

0.3546

0.8865

0.00972

0.0251

0.09722

0.1778

0.19444

0.29165

0.3556

0.00972

0.0253

0.0972

0.1778

0.19444

0.894

\section{App}

App vol

$$
\begin{array}{cc}
\exp / & \text { Calc } / \\
\mathrm{m}^{3} / \mathrm{kg} & \mathrm{m}^{3} / \mathrm{kg}
\end{array}
$$$$
\mathrm{m}^{3} / \mathrm{kg}
$$

$\begin{array}{ll}0.000060 & 0.000067\end{array}$

$\begin{array}{ll}0.000002 & 0.000028\end{array}$

$\begin{array}{ll}0.000003 & 0.000032\end{array}$

$0.000013 \quad 0.000035$

$0.000014 \quad 0.000039$

$\begin{array}{ll}0.000189 & 0.000032\end{array}$

$-0.000145 \quad 0.000033$

$\begin{array}{lll}-0.000013 & 0.000035\end{array}$

$\begin{array}{ll}-0.000036 & 0.000038\end{array}$

$0.000003 \quad 0.000039$

$0.000005 \quad 0.000042$

$0.000018 \quad 0.000044$

0.000370 .000060

$0.000012 \quad 0.000045$

$0.000033 \quad 0.000056$

$\begin{array}{ll}0.000052 & 0.000067\end{array}$

$-0.000293 \quad 0.000038$

$\begin{array}{ll}-0.000194 & 0.000039\end{array}$

$\begin{array}{ll}-0.000057 & 0.000041\end{array}$

$\begin{array}{ll}-0.000066 & 0.000043\end{array}$

$-0.000037 \quad 0.000043$

$\begin{array}{ll}0.000021 & 0.000046\end{array}$

$\begin{array}{lll}-0.000404 & 0.000040\end{array}$

$\begin{array}{ll}-0.000404 & 0.000040 \\ -0.000429 & 0.000041\end{array}$

$\begin{array}{ll}-0.000098 & 0.000042\end{array}$

$\begin{array}{ll}-0.000115 & 0.000044\end{array}$

$\begin{array}{ll}-0.000068 & 0.000045\end{array}$

$\begin{array}{ll}-0.000054 & 0.000047\end{array}$

$\begin{array}{ll}-0.000040 & 0.000048\end{array}$

$\begin{array}{ll}-0.000007 & 0.000058\end{array}$

977.52

Average Res

Std dev Res

Avg +4 std
Density App Vol Square of Inconsistent

kg/m ${ }^{3} \mathrm{~kg} / \mathrm{m}^{3}$ Residual/ Density by

$\mathrm{kg} / \mathrm{m}^{3}$

$\mathrm{m}^{3} / \mathrm{kg}$

esidual

based on

ta density Apparent olal volume

$0.9022-0.000006$

$0.0295-0.000026$

0.814

0.001

$\begin{array}{lll}0.3291 & -0.000029 & 0.108 \\ 0.5081 & -0.000022 & 0.258\end{array}$

$\begin{array}{lll}0.5081 & -0.000022 & 0.258 \\ 0.8821 & -0.000025 & 0.778\end{array}$

$\begin{array}{lll}0.2461 & -0.000221 & 0.061\end{array}$

$\begin{array}{llll}977.69 & 0.5109 & -0.000178 & 0.261\end{array}$

$\begin{array}{llll}985.86 & 0.5389 & -0.000048 & 0.290\end{array}$

$\begin{array}{llll}994.87 & 1.5333 & -0.000074 & 2.351\end{array}$

$\begin{array}{llll}996.79 & 0.8134 & -0.000036 & 0.662\end{array}$

$\begin{array}{llll}1007.63 & 1.2748 & -0.000037 & 1.625\end{array}$

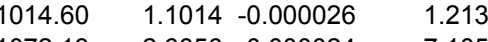

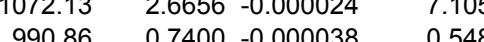

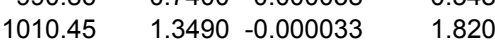

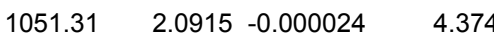

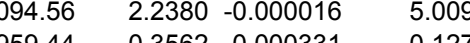

0.127

0.420

1.131

4.781

3.095

4.946

4.683

0.211

1.603

2.158

9.415

5.647

10.483

12.078

46.414

197.423 Sum chi sqr

$0.8128-0.000065$

$0.1689-0.000026$

$0.7547 \quad 0.000053$

$\begin{array}{ll}0.8501 & -0.000236\end{array}$

$\begin{array}{lll}3.1879 & 0.000185\end{array}$
Relative Density of density water $/ \mathrm{kg} / \mathrm{m}^{3}$

983.1989 980.5548 980.5548 980.5548 980.5548 974.849

974.849

974849

974.849

974.849 974.849 974.849

974.849 971.7978 971.7978 971.7978 971.7978 958.3637 958.3637 958.3637 958.3637 958.3637 958.3637 958.3637 938.9841 938.9841 938.9841 938.9841 938.9841

938.9841 938.9841 938.9841

Curve fit for the model App vol $=\left(w+c 2+c 3^{*} t{ }^{\circ} \mathrm{C}\right) /\left(c 0^{*} w+c 1\right) / e^{\wedge}\left(0.000001^{*}\left(t{ }^{\circ} \mathrm{C}+c 4\right)^{\wedge} 2\right)$

$\begin{array}{lr}\text { c0 } & 3.94 \mathrm{E}-07 \\ \text { c1 } & 1.44 \mathrm{E}-06 \\ \text { c2 } & -0.053722 \\ \text { c3 } & 0.002062 \\ \text { c4 } & 4563.25 \\ \text { Min T } & 0\end{array}$


molality molarity

\author{
$\mathbf{t} /{ }^{\circ} \mathrm{C}$ mass frac Density \\ $\exp / \mathrm{kg} / \mathrm{m}^{3}$
}

\section{App}

$\begin{array}{rr}\text { App vol } & \text { App vol } \\ \exp / & \text { Calc } / \\ \mathrm{m}^{3} / \mathrm{kg} & \mathrm{m}^{3} / \mathrm{kg}\end{array}$
Density

calc / Residual / Residual / Density based on

$\mathrm{kg} / \mathrm{m}^{3}$
125

0.000

0.260

$\begin{array}{lr}\text { Avg dens res } & 0.16889 \\ \text { Std dens res } & 0.754745 \\ \text { Avg } & \end{array}$

$\begin{array}{lr}\text { Avg dens res } & 0.16889 \\ \text { Std dens res } & 0.754745 \\ \text { Avg } & \end{array}$

Avg app vol res *10^3 -0.02575

Std app vol res *10^3 0.052589

No of points in corr

No of inconsistent poir

Inconsistent data not used (Residual greater than average + /- $\mathbf{4}$ standard deviations)

$\begin{array}{rrrrrrrrrrr} & 0 & 0.2600 & 1303.2 & (90) & 0.000105 & 0.000121 & 1296.12 & 7.0756 & -0.000016 & 50.064 \\ 0.0625 & 35 & 0.0075 & 993.60 & (74) & 0.001064 & 0.000012 & 1001.42 & -7.8194 & 0.001052 & 61.143 \\ 0.125 & 35 & 0.0148 & 993.00 & (74) & 0.001077 & 0.000015 & 1008.76 & -15.7630 & 0.001062 & 248.471 \\ 0.25 & 35 & 0.0292 & 991.66 & (74) & 0.001088 & 0.000022 & 1023.29 & -31.6242 & 0.001067 & 1000.093 \\ 0.5 & 35 & 0.0568 & 988.56 & (74) & 0.001104 & 0.000034 & 1051.74 & -63.1763 & 0.001070 & 3991.243 \\ 1 & 35 & 0.1074 & 982.36 & (74) & 0.001117 & 0.000056 & 1106.32 & -123.9678 & 0.001062 & 15368.024 \\ 0.0625 & 40 & 0.0075 & 991.74 & (74) & 0.001073 & 0.000016 & 999.56 & -7.8286 & 0.001058 & 61.288 \\ 0.125 & 40 & 0.0148 & 991.08 & (74) & 0.001086 & 0.000019 & 1006.86 & -15.7821 & 0.001067 & 249.073 \\ 0.25 & 40 & 0.0292 & 989.65 & (74) & 0.001097 & 0.000025 & 1021.31 & -31.6662 & 0.001072 & 1002.745 \\ 0.5 & 40 & 0.0568 & 986.41 & (74) & 0.001112 & 0.000037 & 1049.64 & -63.2308 & 0.001076 & 3998.138 \\ 1 & 40 & 0.1074 & 979.91 & (74) & 0.001126 & 0.000058 & 1104.07 & -124.1587 & 0.001068 & 15415.385 \\ 0.0625 & 50 & 0.0075 & 987.54 & (74) & 0.001081 & 0.000022 & 995.30 & -7.7674 & 0.001058 & 60.333 \\ 0.125 & 50 & 0.0148 & 986.85 & (74) & 0.001094 & 0.000025 & 1002.53 & -15.6738 & 0.001069 & 245.669 \\ 0.25 & 50 & 0.0292 & 985.38 & (74) & 0.001106 & 0.000031 & 1016.84 & -31.4614 & 0.001075 & 989.821 \\ 0.5 & 50 & 0.0568 & 982.01 & (74) & 0.001121 & 0.000041 & 1044.93 & -62.9176 & 0.001080 & 3958.620 \\ 1 & 50 & 0.1074 & 975.34 & (74) & 0.001135 & 0.000060 & 1099.08 & -123.7413 & 0.001074 & 15311.905 \\ 0.889 & 100 & 0.0967 & 1059.1 & (52) & 0.000017 & 0.000061 & 1054.38 & 4.7191 & -0.000044 & 22.270\end{array}$

999.8395 994.0319 994.0319 994.0319 994.0319 994.0319 992.2158 992.2158 992.2158 992.2158 992.2158 988.0363 988.0363 988.0363 988.0363 988.0363 958.3637 
Density of aqueous solutions of $\mathbf{M n C l} 2$

References (37) Herrington, Thelma M.; Roffey, Michael G.; Smith, David P.; Densities of Aqueous Electrolytes $\mathrm{MnCl}_{2}, \mathrm{CoCl}_{2}, \mathrm{NiCl}_{2}, \mathrm{ZnCl} 2$ and $\mathrm{CdCl}_{2}$ from 25 to $72^{\circ} \mathrm{C}$ at $1 \mathrm{~atm}, \mathrm{~J}$. Chem. Eng. Data, 1986, 31, 221-225

(40) Herz, W. Internal Friction of Salt Solutions (C. A. Trans. from German). Z. Anorg. Chemie 1914, 89, 393-396.

(92) Phang, S. The Density, Viscosity, and Transference Number of Aqueous Manganese Chloride at 298.15 K. Aust. J. Chem. 1980, 33, 413417.

(97) Pogue, R. F.; Atkinson, G. Solution Thermodynamics of First-Row Transition Elements. 2. Apparent Molal Volumes of Aqueous MnCl2 $\mathrm{Mn}(\mathrm{ClO} 4) 2, \mathrm{CoCl}$, $\mathrm{Co}(\mathrm{ClO} 4) 2, \mathrm{FeCl} 2$, and $\mathrm{Fe}(\mathrm{ClO} 4) 2$, from 15 to $55^{\circ} \mathrm{C}$. J. Chem. Eng. Data 1989, 34, 227-232.

(104) Rard, J. A.; Miller, D. G. Densities and Apparent Molal Volumes of Aqueous Manganese, Cadmium, and Zinc Chlorides at $25^{\circ} \mathrm{C}$. J. Chem. Eng. Data 1984, 29, 151-156.

(128) Torók, T. I.; Berecz, E. Volumetric Properties and Electrolytic Conductances of Aqueous Ternary Mixtures of Hydrogen Chloride and some Transition Metal Chlorides at $25^{\circ} \mathrm{C}$. J. Sol. Chem . 1989, 18, 1117-1131.

\begin{tabular}{|c|c|c|c|c|c|c|c|c|c|c|c|c|c|c|c|}
\hline molality & molarity & \multicolumn{2}{|c|}{$\mathrm{t} /{ }^{\circ} \mathrm{C}$ mass frac } & $\begin{array}{r}\text { Density } \\
\exp / \\
\mathrm{kg} / \mathrm{m}^{3}\end{array}$ & Ref & $\begin{array}{r}\text { App vol } \\
\exp / \\
\mathrm{m}^{3} / \mathrm{kg}\end{array}$ & $\begin{array}{l}\text { App vol } \\
\text { Calc } / \mathrm{m}^{3} / \mathrm{kg}\end{array}$ & $\begin{array}{r}\text { Density } \\
\text { calc / } \\
\mathrm{kg} / \mathrm{m}^{3}\end{array}$ & $\begin{array}{r}\text { Density } \\
\text { Residual / } \\
\mathrm{kg} / \mathrm{m}^{3}\end{array}$ & $\begin{array}{r}\text { App Vol } \\
\text { Residual / } \\
\mathrm{m}^{3} / \mathrm{kg}\end{array}$ & $\begin{array}{r}\text { Square of } \\
\text { Density } \\
\text { Residual }\end{array}$ & $\begin{array}{l}\text { Inconsistent } \\
\text { based on } \\
\text { density }\end{array}$ & $\begin{array}{c}\text { Inconsistent } \\
\text { based on } \\
\text { apparent } \\
\text { volume }\end{array}$ & $\begin{array}{r}\text { Relative } \\
\text { density / } \\
1000 \text { (rhor-1) }\end{array}$ & $\begin{array}{r}\text { Density of } \\
\text { water } / \mathrm{kg} / \mathrm{m}^{3}\end{array}$ \\
\hline 0.00971 & & 15 & 0.0012 & 1000.14 & (97) & 0.000149 & 0.000181 & 1000.10 & 0.0389 & -0.000032 & 0.002 & & & 1.04 & 999.0996 \\
\hline 0.01972 & & 15 & 0.0025 & 1001.21 & (97) & 0.000150 & 0.000181 & 1001.13 & 0.0776 & -0.000031 & 0.006 & & & 2.11 & 999.0996 \\
\hline 0.02927 & & 15 & 0.0037 & 1002.23 & (97) & 0.000150 & 0.000181 & 1002.11 & 0.1146 & -0.000031 & 0.013 & & & 3.13 & 999.0996 \\
\hline 0.04979 & & 15 & 0.0062 & 1004.39 & (97) & 0.000153 & 0.000181 & 1004.22 & 0.1749 & -0.000028 & 0.031 & & & 5.3 & 999.0996 \\
\hline 0.07421 & & 15 & 0.0093 & 1006.98 & (97) & 0.000154 & 0.000182 & 1006.72 & 0.2589 & -0.000028 & 0.067 & & & 7.89 & 999.0996 \\
\hline 0.1005 & & 15 & 0.0125 & 1009.74 & (97) & 0.000156 & 0.000182 & 1009.41 & 0.3267 & -0.000026 & 0.107 & & & 10.65 & 999.0996 \\
\hline 0.2028 & & 15 & 0.0249 & 1020.37 & (97) & 0.000162 & 0.000184 & 1019.82 & 0.5456 & -0.000021 & 0.298 & & & 21.29 & 999.0996 \\
\hline 0.4018 & & 15 & 0.0481 & 1040.72 & (97) & 0.000169 & 0.000186 & 1039.83 & 0.8923 & -0.000017 & 0.796 & & & 41.66 & 999.0996 \\
\hline 0.5992 & & 15 & 0.0701 & 1060.47 & (97) & 0.000175 & 0.000189 & 1059.36 & 1.1184 & -0.000014 & 1.251 & & & 61.43 & 999.0996 \\
\hline 0.7945 & & 15 & 0.0909 & 1079.62 & (97) & 0.000180 & 0.000191 & 1078.37 & 1.2478 & -0.000012 & 1.557 & & & 80.59 & 999.0996 \\
\hline 0.9978 & & 15 & 0.1116 & 1099.14 & (97) & 0.000184 & 0.000194 & 1097.85 & 1.2936 & -0.000010 & 1.673 & & & 100.13 & 999.0996 \\
\hline 1.4979 & & 15 & 0.1586 & 1145.62 & (97) & 0.000194 & 0.000200 & 1144.43 & 1.1870 & -0.000006 & 1.409 & & & 146.65 & 999.0996 \\
\hline 1.9854 & & 15 & 0.1999 & 1189.05 & (97) & 0.000201 & 0.000204 & 1188.10 & 0.9456 & -0.000003 & 0.894 & & & 190.12 & 999.0996 \\
\hline 2.4993 & & 15 & 0.2393 & 1233.00 & (97) & 0.000207 & 0.000209 & 1232.38 & 0.6190 & -0.000002 & 0.383 & & & 234.11 & 999.0996 \\
\hline 3.0071 & & 15 & 0.2745 & 1274.78 & (97) & 0.000212 & 0.000213 & 1274.45 & 0.3305 & -0.000001 & 0.109 & & & 275.93 & 999.0996 \\
\hline 3.4336 & & 15 & 0.3017 & 1308.65 & (97) & 0.000216 & 0.000216 & 1308.56 & 0.0903 & 0.000000 & 0.008 & & & 309.83 & 999.0996 \\
\hline 0.5292 & & 25 & 0.0624 & 1050.50 & (37) & 0.000186 & 0.000190 & 1050.18 & 0.3164 & -0.000005 & 0.100 & & & & 997.0449 \\
\hline 0.8038 & & 25 & 0.0919 & 1077.08 & (37) & 0.000192 & 0.000194 & 1076.83 & 0.2524 & -0.000002 & 0.064 & & & & 997.0449 \\
\hline 1.0684 & & 25 & 0.1185 & 1102.01 & (37) & 0.000197 & 0.000198 & 1101.92 & 0.0863 & -0.000001 & 0.007 & & & & 997.0449 \\
\hline 1.3397 & & 25 & 0.1443 & 1126.91 & (37) & 0.000202 & 0.000201 & 1127.08 & -0.1741 & 0.000001 & 0.030 & & & & 997.0449 \\
\hline 1.6251 & & 25 & 0.1698 & 1152.43 & (37) & 0.000206 & 0.000204 & 1152.95 & -0.5170 & 0.000002 & 0.267 & & & & 997.0449 \\
\hline 1.9287 & & 25 & 0.1953 & 1178.89 & (37) & 0.000211 & 0.000208 & 1179.80 & -0.9094 & 0.000003 & 0.827 & & & & 997.0449 \\
\hline 2.2401 & & 25 & 0.2199 & 1205.36 & (37) & 0.000215 & 0.000211 & 1206.66 & -1.2986 & 0.000004 & 1.686 & & & & 997.0449 \\
\hline 2.5142 & & 25 & 0.2404 & 1228.14 & (37) & 0.000218 & 0.000213 & 1229.75 & -1.6068 & 0.000004 & 2.582 & & & & 997.0449 \\
\hline 2.8098 & & 25 & 0.2612 & 1252.24 & (37) & 0.000221 & 0.000216 & 1254.08 & -1.8438 & 0.000004 & 3.400 & & & & 997.0449 \\
\hline 3.1178 & & 25 & 0.2818 & 1276.88 & (37) & 0.000223 & 0.000219 & 1278.84 & -1.9610 & 0.000004 & 3.846 & & & & 997.0449 \\
\hline & 0.82 & 25 & 0.0956 & 1079.60 & (40) & 0.000201 & 0.000195 & 1080.28 & -0.6762 & 0.000006 & 0.457 & & & & 997.0449 \\
\hline & 1.64 & 25 & 0.1778 & 1160.70 & (40) & 0.000208 & 0.000205 & 1161.28 & -0.5801 & 0.000002 & 0.336 & & & & 997.0449 \\
\hline & 2.465 & 25 & 0.2502 & 1239.70 & (40) & 0.000218 & 0.000215 & 1241.16 & -1.4618 & 0.000004 & 2.137 & & & & 997.0449 \\
\hline & 3.285 & 25 & 0.3138 & 1317.40 & (40) & 0.000226 & 0.000223 & 1319.00 & -1.6020 & 0.000003 & 2.567 & & & & 997.0449 \\
\hline 0.1009 & & 25 & 0.0125 & 1007.65 & (92) & 0.000161 & 0.000184 & 1007.36 & 0.2867 & -0.000023 & 0.082 & & & & 997.0449 \\
\hline 0.1033 & & 25 & 0.0128 & 1007.91 & (92) & 0.000160 & 0.000184 & 1007.61 & 0.3023 & -0.000023 & 0.091 & & & & 997.0449 \\
\hline
\end{tabular}




\begin{tabular}{|c|c|c|c|c|c|}
\hline molality & molarity & \multicolumn{2}{|c|}{$t /{ }^{\circ} \mathrm{C}$ mass frac } & $\begin{array}{r}\text { Density } \\
\exp / \\
\mathrm{kg} / \mathrm{m}^{3}\end{array}$ & Ref \\
\hline 0.2359 & & 25 & 0.0288 & 1021.45 & (92) \\
\hline 0.4924 & & 25 & 0.0583 & 1047.41 & (92) \\
\hline 0.9873 & & 25 & 0.1105 & 1095.03 & (92) \\
\hline 1.61 & & 25 & 0.1685 & 1151.69 & (92) \\
\hline 2.016 & & 25 & 0.2024 & 1187.13 & (92) \\
\hline 2.594 & & 25 & 0.2461 & 1235.69 & (92) \\
\hline 3.083 & & 25 & 0.2795 & 1277.04 & (92) \\
\hline 3.851 & & 25 & 0.3264 & 1336.53 & (92) \\
\hline 4.444 & & 25 & 0.3587 & 1380.10 & (92) \\
\hline 5.16 & & 25 & 0.3937 & 1429.99 & (92) \\
\hline 5.271 & & 25 & 0.3988 & 1437.38 & (92) \\
\hline 5.763 & & 25 & 0.4204 & 1469.63 & (92) \\
\hline 0.00971 & & 25 & 0.0012 & 998.07 & (97) \\
\hline 0.01972 & & 25 & 0.0025 & 999.13 & (97) \\
\hline 0.02927 & & 25 & 0.0037 & 1000.14 & (97) \\
\hline 0.04979 & & 25 & 0.0062 & 1002.28 & (97) \\
\hline 0.07421 & & 25 & 0.0093 & 1004.84 & (97) \\
\hline 0.1005 & & 25 & 0.0125 & 1007.57 & (97) \\
\hline 0.2028 & & 25 & 0.0249 & 1018.09 & (97) \\
\hline 0.4018 & & 25 & 0.0481 & 1038.24 & (97) \\
\hline 0.5992 & & 25 & 0.0701 & 1057.78 & (97) \\
\hline 0.7945 & & 25 & 0.0909 & 1076.75 & (97) \\
\hline 0.9978 & & 25 & 0.1116 & 1096.08 & (97) \\
\hline 1.4979 & & 25 & 0.1586 & 1142.17 & (97) \\
\hline 1.9854 & & 25 & 0.1999 & 1185.27 & (97) \\
\hline 2.4993 & & 25 & 0.2393 & 1228.89 & (97) \\
\hline 3.0071 & & 25 & 0.2745 & 1270.37 & (97) \\
\hline 3.4336 & & 25 & 0.3017 & 1303.95 & (97) \\
\hline 0.044493 & & 25 & 0.0056 & 1001.75 & (104) \\
\hline 0.090013 & & 25 & 0.0112 & 1006.51 & (104) \\
\hline 0.16003 & & 25 & 0.0197 & 1013.75 & (104) \\
\hline 0.24995 & & 25 & 0.0305 & 1022.96 & (104) \\
\hline 0.36 & & 25 & 0.0433 & 1034.09 & (104) \\
\hline 0.48896 & & 25 & 0.0580 & 1046.95 & (104) \\
\hline 0.63661 & & 25 & 0.0742 & 1061.46 & (104) \\
\hline 0.80898 & & 25 & 0.0924 & 1078.15 & (104) \\
\hline 1.0001 & & 25 & 0.1118 & 1096.34 & (104) \\
\hline 1.2102 & & 25 & 0.1322 & 1115.94 & (104) \\
\hline 1.4407 & & 25 & 0.1535 & 1137.04 & (104) \\
\hline 1.6897 & & 25 & 0.1754 & 1159.36 & (104) \\
\hline 1.9607 & & 25 & 0.1979 & 1183.12 & (104) \\
\hline 2.2507 & & 25 & 0.2207 & 1207.98 & (104) \\
\hline 2.5608 & & 25 & 0.2437 & 1234.01 & (104) \\
\hline 2.8911 & & 25 & 0.2668 & 1261.02 & (104) \\
\hline 3.2733 & & 25 & 0.2917 & 1291.40 & (104) \\
\hline
\end{tabular}

App vol App vol

$\exp /$ Calc $/ \mathrm{m}^{3} / \mathrm{kg}$ $\mathrm{m}^{3} / \mathrm{kg}$

$0.000172 \quad 0.000186$

$0.000176 \quad 0.000190$

$0.000191 \quad 0.000196$

$0.000204 \quad 0.000204$

$0.000209 \quad 0.000208$

$0.000216 \quad 0.000214$

$0.000216 \quad 0.000218$

$0.000223 \quad 0.000224$

$0.000227 \quad 0.000229$

$0.000232 \quad 0.000233$

$0.000233 \quad 0.000234$

.000157

0.000158

0.000162

0.000162

.000164

0.000170

0.000176

0.000182

0.000186

0.000191

0.000199

0.000212

0.000217

0.000221

0.000157

0.000161

0.000166

0.000170

0.000174

0.000178

0.000182

0.000186

0.000190

0.000194

0.000198

0.000202

0.000206

0.000209

0.000213

0.00021

0.000219

0.000236

0.000182

0.000182

0.000183

0.000184

0.000185

0.000188

0.000191

0.000194

0.000203

0.000208

0.000213

0.000221

0.000183

0.000185

0.000186

0.000188

0.000190

0.000194

0.000197

0.000199

0.000202

$0.000208 \quad 1182.59$

$0.000211 \quad 1207.56$

$0.000214 \quad 1233.62$

Density Density

$\mathrm{kg} / \mathrm{m}^{3}$

$\mathrm{kg} / \mathrm{m}^{3}$

0.4201

0.8444

0.7391

0.0962

$-0.2680$

$-0.6836$

0.9662

1.1024

1.1849

1.0889

0.9643

0.6303

0.0301

0.0601

0.0884

0.1316

0.1992

0.2511

0.3989

0.6331

0.7503

0.8134

0.7993

0.6792

0.5263

0.3830

0.3626

0.3354

0.1440

0.2557

0.3813

0.5169

0.6356

0.7232

0.7807

0.8257

0.8409

0.7947

0.7333

0.6432

0.5290

0.4190
0.3883

0.3425

0.2863
App Vol Square of Inconsistent Inconsistent Residual / Density based on based on $\mathrm{m}^{3} / \mathrm{kg}$ Residual density $\begin{gathered}\text { apparent } \\ \text { volume }\end{gathered}$

$-0.000014 \quad 0.177$

$-0.000013 \quad 0.713$

$-0.000006 \quad 0.546$

$0.000000 \quad 0.009$

$0.000001 \quad 0.072$

$0.000002 \quad 0.467$

$\begin{array}{ll}0.000002 & 0.933\end{array}$

$-0.000002 \quad 1.215$

1.404

$-0.000001 \quad 1.186$

$-0.000001 \quad 0.930$
-0.000001

$-0.000001 \quad 0.397$

0.001

$-0.000024 \quad 0.004$

$\begin{array}{ll}-0.000024 & 0.008\end{array}$

$\begin{array}{ll}-0.000021 & 0.017\end{array}$

$0.000021 \quad 0.040$

0.063

$-0.000015 \quad 0.159$

$-0.000012 \quad 0.401$

$-0.000010 \quad 0.563$

0.662

$-0.000006 \quad 0.639$

$-0.000003 \quad 0.461$

$-0.000002 \quad 0.277$

$\begin{array}{ll}-0.000001 & 0.147\end{array}$

$-0.000001 \quad 0.131$

$-0.000001 \quad 0.113$

$-0.000026 \quad 0.021$

$-0.000023 \quad 0.065$

$0.000019 \quad 0.145$

$\begin{array}{ll}-0.000016 & 0.267\end{array}$

$-0.000014 \quad 0.404$

$\begin{array}{ll}-0.000011 & 0.523\end{array}$

0.610

$-0.000008 \quad 0.682$

$\begin{array}{ll}-0.000006 & 0.707\end{array}$

$-0.000005 \quad 0.632$

0.538

$-0.000003 \quad 0.414$

$\begin{array}{ll}-0.000002 & 0.280\end{array}$

$-0.000001 \quad 0.176$

$-0.000001 \quad 0.151$

0.117

0.082
Relative Density of 1000(rhor-1)

997.0449

997.0449

997.0449

997.0449

997.0449

997.0449

997.0449

997.0449

997.0449

997.0449

997.0449

997.0449

$1.03 \quad 997.0449$

$2.09 \quad 997.0449$

3.1997 .0449

$\begin{array}{ll}5.25 & 997.0449\end{array}$

$7.82 \quad 997.0449$

$10.56 \quad 997.0449$

21.11997 .0449

$41.32 \quad 997.0449$

$60.92 \quad 997.0449$

$79.94 \quad 997.0449$

$99.33 \quad 997.0449$

$145.56 \quad 997.0449$

$188.78 \quad 997.0449$

$232.53 \quad 997.0449$

$274.14 \quad 997.0449$

$307.81 \quad 997.0449$

997.0449

997.0449

997.0449

997.0449

997.0449

997.0449

997.0449

997.0449

997.0449

997.0449

997.0449

997.0449

997.0449

997.0449

997.0449

997.0449

997.0449 


\begin{tabular}{|c|c|c|c|c|c|}
\hline molality & molarity & \multicolumn{2}{|c|}{$\mathrm{t} /{ }^{\circ} \mathrm{C}$ mass frac } & $\begin{array}{r}\text { Density } \\
\exp / \\
\mathrm{kg} / \mathrm{m}^{3}\end{array}$ & Ref \\
\hline 3.6024 & & 25 & 0.3119 & 1316.87 & (104) \\
\hline 3.9183 & & 25 & 0.3303 & 1340.80 & (104) \\
\hline 4.4054 & & 25 & 0.3567 & 1376.35 & (104) \\
\hline 4.8581 & & 25 & 0.3794 & 1408.29 & (104) \\
\hline 5.4244 & & 25 & 0.4057 & 1446.65 & (104) \\
\hline 6.0292 & & 25 & 0.4314 & 1485.62 & (104) \\
\hline 1.8478 & & 25 & 0.1887 & 1173.28 & (128) \\
\hline 2.37 & & 25 & 0.2297 & 1218.13 & (128) \\
\hline 3.334 & & 25 & 0.2956 & 1296.17 & (128) \\
\hline 5.5964 & & 25 & 0.4132 & 1457.95 & (128) \\
\hline 6.11 & & 25 & 0.4347 & 1490.44 & (128) \\
\hline 0.5292 & & 35 & 0.0624 & 1047.22 & (37) \\
\hline 0.8038 & & 35 & 0.0919 & 1073.68 & (37) \\
\hline 1.0684 & & 35 & 0.1185 & 1098.49 & (37) \\
\hline 1.3397 & & 35 & 0.1443 & 1123.27 & (37) \\
\hline 1.6251 & & 35 & 0.1698 & 1148.68 & (37) \\
\hline 1.9287 & & 35 & 0.1953 & 1175.03 & (37) \\
\hline 2.2401 & & 35 & 0.2199 & 1201.37 & (37) \\
\hline 2.5142 & & 35 & 0.2404 & 1224.05 & (37) \\
\hline 2.8098 & & 35 & 0.2612 & 1248.03 & (37) \\
\hline 3.1178 & & 35 & 0.2818 & 1272.54 & (37) \\
\hline 0.00971 & & 35 & 0.0012 & 995.05 & (97) \\
\hline 0.01972 & & 35 & 0.0025 & 996.10 & (97) \\
\hline 0.02927 & & 35 & 0.0037 & 997.10 & (97) \\
\hline 0.04979 & & 35 & 0.0062 & 999.24 & (97) \\
\hline 0.07421 & & 35 & 0.0093 & 1001.77 & (97) \\
\hline 0.1005 & & 35 & 0.0125 & 1004.48 & (97) \\
\hline 0.2028 & & 35 & 0.0249 & 1014.93 & (97) \\
\hline 0.4018 & & 35 & 0.0481 & 1034.90 & (97) \\
\hline 0.5992 & & 35 & 0.0701 & 1054.27 & (97) \\
\hline 0.7945 & & 35 & 0.0909 & 1073.12 & (97) \\
\hline 0.9978 & & 35 & 0.1116 & 1092.28 & (97) \\
\hline 1.4979 & & 35 & 0.1586 & 1137.99 & (97) \\
\hline 1.9854 & & 35 & 0.1999 & 1180.73 & (97) \\
\hline 2.4993 & & 35 & 0.2393 & 1224.02 & (97) \\
\hline 3.0071 & & 35 & 0.2745 & 1265.11 & (97) \\
\hline 3.4336 & & 35 & 0.3017 & 1298.38 & (97) \\
\hline 0.5292 & & 45 & 0.0624 & 1043.26 & (37) \\
\hline 0.8038 & & 45 & 0.0919 & 1069.66 & (37) \\
\hline 1.0684 & & 45 & 0.1185 & 1094.41 & (37) \\
\hline 1.3397 & & 45 & 0.1443 & 1119.14 & (37) \\
\hline 1.6251 & & 45 & 0.1698 & 1144.49 & (37) \\
\hline 1.9287 & & 45 & 0.1953 & 1170.77 & (37) \\
\hline 2.2401 & & 45 & 0.2199 & 1197.03 & (37) \\
\hline 2.5142 & & 45 & 0.2404 & 1219.63 & (37) \\
\hline
\end{tabular}

App vol App vol

$\exp /$ Calc $/ \mathrm{m}^{3} / \mathrm{kg}$ $\mathrm{m}^{3} / \mathrm{kg}$

$0.000222 \quad 0.000223$

0.000224

0.000228

0.000231

0.000235

0.000238

0.000204

0.000211

0.000220

0.000236

0.000239

0.000188

0.000194

0.000199

0.000204

0.000208

0.000213

0.000216

0.000219

0.00022

0.000225

0.000166

0.00016

0.000162

0.000164

0.000168

0.000174

0.000181

0.000186

0.000190

0.000195

0.000204

0.000210

0.00021

0.00022

0.00022

0.00018

0.000193

0.000199

0.000203

0.000208

0.000212

0.00021

$0.000220 \quad 0.000218$ 0.000225 0.000228 0.000231 0.000238 0.000207 0.000212 0.000220 0.000238 0.000191 0.000195 0.000199 0.000203 0.000206 0.000210

0.000214

0.000216

0.000219

0.000182

0.000182

0.000183

0.000183

0.000183

0.000186

0.000189

0.000192

0.000195

0.000198

0.000205

0.000211

0.000221

0.000225

0.000191

0.000195

0.000199

0.000203

0.000207

0.000215
Density Density calc / Residual / $\mathrm{kg} / \mathrm{m}^{3}$ $\mathrm{kg} / \mathrm{m}^{3}$
$1316.60 \quad 0.2689$

1340.46

1376.14

0.3375

0.2065

0.1411

$1446.70-0.0505$

$1486.15 \quad-0.5319$

1172.71

0.5708

0.4660

0.3060

1295.86

1458.10

1491.29

1046.99
1073.50

1098.45

1123.44

1149.10
1175.71

1175.71

1202.30

1225.13
1249.18

1273.61
995.03

995.03

996.05

997.03

999.12

1001.61

1014.62

1034.47

1053.81

1072.61

1091.85

1137.74
1180.60

1223.90

1264.90

1298.03

1043.05

1069.47

1094.30

1119.15

1171.06

1197.43

1220.04

$-0.1464$

$-0.8537$

0.2333
0.1804

0.0422

$-0.1656$

-0.4151
-0.6781

$-0.9287$

$-1.0823$

$-1.1469$

-1.0710
0.0196

0.0491

0.0768

0.1191
0.1560

0.1973

0.3052

0.4310
0.4637

0.4637

0.4343

0.2506

0.1286

0.1164

0.2139

0.3541

0.2100
0.1930

0.1083

$-0.0127$

-0.1570
-0.2917

$-0.2917$

$-0.4127$
App Vol Square of Inconsistent Inconsistent apparent $\mathrm{m}^{3} / \mathrm{kg}$ Residual

$0.000000 \quad 0.072$

$\begin{array}{ll}0.000001 & 0.114\end{array}$

$0.000000 \quad 0.043$

$0.000000 \quad 0.020$

$0.000000 \quad 0.003$

$0.000001 \quad 0.283$

$\begin{array}{ll}0.000002 & 0.326\end{array}$

$\begin{array}{ll}-0.000001 & 0.217\end{array}$

$0.000001 \quad 0.094$

$0.000000 \quad 0.021$

$0.000001 \quad 0.729$

$-0.000003 \quad 0.054$

$-0.000002 \quad 0.033$

$0.000000 \quad 0.002$

$0.000001 \quad 0.027$

$0.000002 \quad 0.172$

$0.000003 \quad 0.460$

$0.000003 \quad 0.863$

$0.000003 \quad 1.171$

$0.000003 \quad 1.315$

$0.000002 \quad 1.147$

0.000

$-0.000020 \quad 0.002$

$-0.000021 \quad 0.006$
-0.000019

$\begin{array}{ll}-0.000019 & 0.014\end{array}$

$\begin{array}{ll}-0.000017 & 0.024\end{array}$

$\begin{array}{ll}-0.000016 & 0.039\end{array}$

$-0.000012 \quad 0.093$
-0.000008

$-0.000008 \quad 0.186$

$-0.000006 \quad 0.215$

0.000005

0.189

0.063

0.017

0.014

0.046

0.125

0.044

0.037

0.012

0.000

0.025

0.085

0.157

0.170
Relative Density of 1000(rhor-1)

997.0449

997.0449

997.0449

997.0449

997.0449

997.0449

997.0449

997.0449

997.0449

997.0449

997.0449

994.0319

994.0319

994.0319

994.0319

994.0319

994.0319

994.0319

994.0319

994.0319

994.0319

$1.02 \quad 994.0319$

$2.08 \quad 994.0319$

$3.09 \quad 994.0319$

$5.24 \quad 994.0319$

$7.78 \quad 994.0319$

$10.51 \quad 994.0319$

$21.02 \quad 994.0319$

41.11994 .0319

$60.6 \quad 994.0319$

$79.56 \quad 994.0319$

$98.84 \quad 994.0319$

144.82994 .0319

$187.82 \quad 994.0319$

$231.37 \quad 994.0319$

$272.71 \quad 994.0319$

$306.18 \quad 994.0319$

990.2132

990.2132

990.2132

990.2132

990.2132

990.2132

990.2132

990.2132 


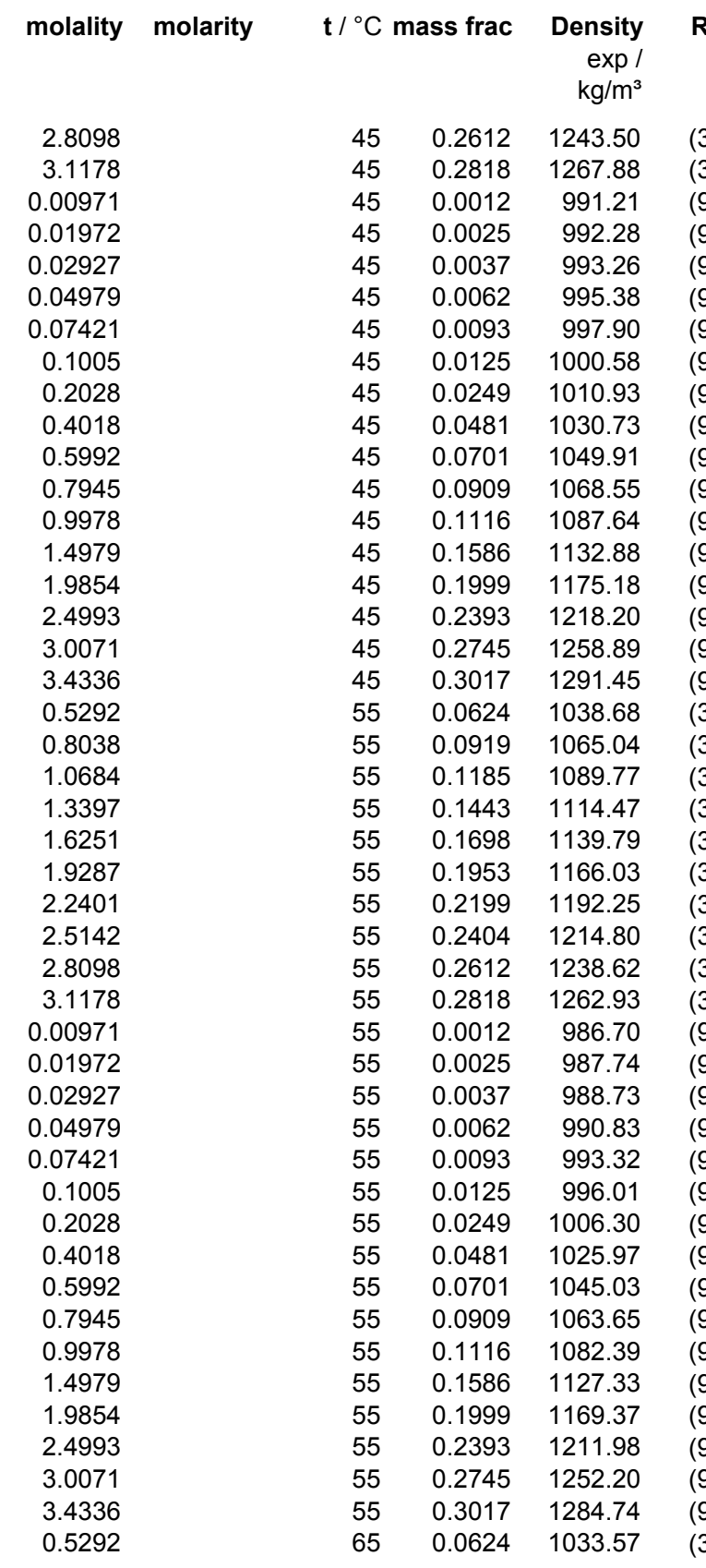

App vol App vol

$\exp /$ Calc $/ \mathrm{m}^{3} / \mathrm{kg}$ $\mathrm{m}^{3} / \mathrm{kg}$

$0.000222 \quad 0.000222$

$0.000225 \quad 0.000225$

$0.000175 \quad 0.000181$

$\begin{array}{ll}0.000159 & 0.000181\end{array}$

$0.000165 \quad 0.000181$

$0.000168 \quad 0.000182$

$0.000169 \quad 0.000182$

$0.000172 \quad 0.000183$

$0.000178 \quad 0.000185$

$0.000185 \quad 0.000188$

$\begin{array}{ll}0.000191 & 0.000192\end{array}$

$0.000195 \quad 0.000195$

$0.000199 \quad 0.000198$

$0.000208 \quad 0.000206$

$0.000215 \quad 0.000212$

$0.000220 \quad 0.000218$

$0.000225 \quad 0.000224$

$\begin{array}{ll}0.000229 & 0.000228\end{array}$

$0.000186 \quad 0.000188$

$0.000192 \quad 0.000193$

$\begin{array}{ll}0.000197 & 0.000198\end{array}$

$0.000202 \quad 0.000202$

$0.000207 \quad 0.000207$

$0.000211 \quad 0.000211$

$0.000215 \quad 0.000215$

$0.000218 \quad 0.000219$

$0.000221 \quad 0.000222$

$0.000224 \quad 0.000226$

$\begin{array}{ll}0.000167 & 0.000178\end{array}$

$0.000168 \quad 0.000178$

$0.000166 \quad 0.000178$

$0.000170 \quad 0.000179$

$0.000172 \quad 0.000179$

$0.000174 \quad 0.000180$

$0.000180 \quad 0.000182$

$\begin{array}{ll}0.000187 & 0.000186\end{array}$

$0.000193 \quad 0.000190$

$0.000196 \quad 0.000193$

$0.000202 \quad 0.000197$

$0.000211 \quad 0.000205$

$0.000217 \quad 0.000212$

$\begin{array}{ll}0.000223 & 0.000219 \\ 0.000228 & 0.000225\end{array}$

$\begin{array}{ll}0.000232 & 0.000229\end{array}$

$\begin{array}{ll}0.000182 & 0.000184\end{array}$
Density Density $\mathrm{kg} / \mathrm{m}^{3} \quad \mathrm{~kg} / \mathrm{m}^{3}$

$1243.84-0.3357$ (267.99 991.21 992.23

993.20

995.30

997.78

1000.45
1010.77

1030.57

1049.85

1068.58
1087.73

1087.73

1175.92

1218.83

1259.38

1038.50

1064.87

1089.63

1114.39

1166.04

1192.23

1214.68

1262.20

986.69

987.71

988.69

990.78

993.26

995.93

1006.25

1026.03
1045.29

1063.98

1083.08

1128.54

1170.86

1213.47
1253.67

1286.06

1033.43
App Vol Square of Inconsistent Inconsistent

Residual / Density based on based on apparent
volume

$$
\mathrm{m}^{3} / \mathrm{kg} \text { Residual }
$$

$0.000001 \quad 0.113$

$0.000000 \quad 0.012$

$-0.000006 \quad 0.000$

$\begin{array}{ll}-0.000022 & 0.003\end{array}$

$-0.000016 \quad 0.004$

$\begin{array}{ll}-0.000014 & 0.007\end{array}$

$\begin{array}{ll}-0.000013 & 0.014\end{array}$

$-0.000011 \quad 0.018$

$-0.000006 \quad 0.026$

$-0.000003 \quad 0.028$

$\begin{array}{rr}-0.000001 \quad 0.004 \\ 0.000000 & 0.001\end{array}$

$0.000000 \quad 0.001$

$0.000001 \quad 0.009$

$0.000002 \quad 0.232$

$0.000003 \quad 0.549$

$0.000002 \quad 0.393$

$0.000001 \quad 0.244$

$0.000001 \quad 0.432$

$\begin{array}{ll}-0.000003 & 0.033\end{array}$

$\begin{array}{ll}-0.000002 & 0.031\end{array}$

$\begin{array}{ll}-0.000001 & 0.019\end{array}$

$0.000000 \quad 0.006$

$0.000000 \quad 0.000$

$0.000000 \quad 0.000$

$0.000000 \quad 0.000$

$0.000000 \quad 0.015$

$\begin{array}{ll}-0.000001 & 0.123\end{array}$

$\begin{array}{ll}-0.000002 & 0.538\end{array}$

$-0.000010 \quad 0.000$

$-0.000010 \quad 0.001$

$\begin{array}{ll}-0.000013 & 0.002\end{array}$

$\begin{array}{ll}-0.000009 & 0.003\end{array}$

$\begin{array}{ll}-0.000007 & 0.004\end{array}$

$-0.000006 \quad 0.006$

$-0.000002 \quad 0.002$

$0.000001 \quad 0.004$

$0.000003 \quad 0.063$

$0.000003 \quad 0.109$

$0.000005 \quad 0.478$

$0.000006 \quad 1.468$

$0.000005 \quad 2.228$

$0.000004 \quad 2.219$

$0.000003 \quad 2.175$

$0.000003 \quad 1.756$

$\begin{array}{ll}-0.000002 & 0.020\end{array}$
Relative Density of density / water $/ \mathrm{kg} / \mathrm{m}^{3}$

990.2132

990.2132

$1.01 \quad 990.2132$

$2.09 \quad 990.2132$

$3.08 \quad 990.2132$

$5.22 \quad 990.2132$

$\begin{array}{ll}7.76 & 990.2132\end{array}$

$10.47 \quad 990.2132$

20.92990 .2132

$40.92 \quad 990.2132$

$60.29 \quad 990.2132$

$79.11 \quad 990.2132$

$98.39 \quad 990.2132$

$144.08 \quad 990.2132$

$186.79 \quad 990.2132$

$230.24 \quad 990.2132$

$271.33 \quad 990.2132$

304.21990 .2132

985.6952

985.6952

985.6952

985.6952

985.6952

985.6952

985.6952

985.6952

985.6952

985.6952

$1.02 \quad 985.6952$

2.07985 .6952

$3.08 \quad 985.6952$

$5.21 \quad 985.6952$

$7.74 \quad 985.6952$

$10.46 \quad 985.6952$

20.9985 .6952

$40.86 \quad 985.6952$

60.2985 .6952

$79.09 \quad 985.6952$

98.1985 .6952

143.69985 .6952

$186.34 \quad 985.6952$

229.57985 .6952

$270.37 \quad 985.6952$

303.38985 .6952

980.5548 


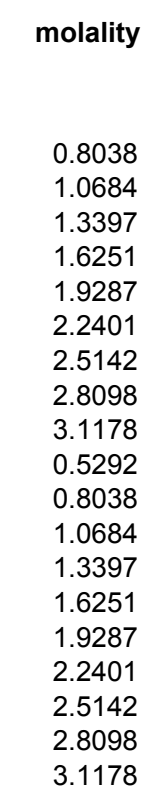

$\begin{array}{ccrc}\mathbf{t} /{ }^{\circ} \mathrm{C} \text { mass frac } & \begin{array}{r}\text { Density } \\ \text { exp / } \\ \mathrm{kg} / \mathrm{m}^{3}\end{array} & \text { Ref } \\ & & \\ 65 & 0.0919 & 1059.95 & (37) \\ 65 & 0.1185 & 1084.70 & (37) \\ 65 & 0.1443 & 1109.41 & (37) \\ 65 & 0.1698 & 1134.74 & (37) \\ 65 & 0.1953 & 1160.97 & (37) \\ 65 & 0.2199 & 1187.17 & (37) \\ 65 & 0.2404 & 1209.68 & (37) \\ 65 & 0.2612 & 1233.44 & (37) \\ 65 & 0.2818 & 1257.67 & (37) \\ 75 & 0.0624 & 1027.78 & (37) \\ 75 & 0.0919 & 1054.18 & (37) \\ 75 & 0.1185 & 1078.96 & (37) \\ 75 & 0.1443 & 1103.73 & (37) \\ 75 & 0.1698 & 1129.12 & (37) \\ 75 & 0.1953 & 1155.41 & (37) \\ 75 & 0.2199 & 1181.66 & (37) \\ 75 & 0.2404 & 1204.20 & (37) \\ 75 & 0.2612 & 1227.94 & (37) \\ 75 & 0.2818 & 1252.10 & (37)\end{array}$

App vol App vol Density Density

$\exp /$ Calc $/ \mathrm{m}^{3} / \mathrm{kg}$

$\mathrm{m}^{3} / \mathrm{kg}$

0.000188

0.000188

0.000194
0.000199

0.000204

0.000208

0.000213

0.000216

0.000219

0.000222

0.000180

0.000185

0.000191

0.000196

0.000200

0.000205

0.000209

0.000213

0.000216

0.000220

0.000190

$\mathrm{kg} / \mathrm{m}^{3} \quad \mathrm{~kg} / \mathrm{m}^{3}$

0.000195

1059.80

0.1497

$0.000200 \quad 1109.28$

0.1340

0.000204

1134.60

0.1431

App Vol Square of Inconsistent Inconsisten

Residual / Density based on based on

$\mathrm{m}^{3} / \mathrm{kg}$

Density density

apparent
volume

$-0.000001$

0.022

$-0.000001$

0.022

$-0.000001$

0.018

$-0.000001$

0.020

0.035

$-0.000001 \quad 0.091$

$-0.000001 \quad 0.226$

$-0.000002 \quad 0.609$

$-0.000003 \quad 1.550$

$0.000002 \quad 0.019$

$0.000002 \quad 0.032$

$0.000001 \quad 0.039$

$0.000001 \quad 0.034$

$0.000001 \quad 0.017$

$0.000000 \quad 0.000$

$\begin{array}{ll}-0.000001 & 0.030\end{array}$

$\begin{array}{ll}-0.000001 & 0.177\end{array}$

$-0.000002 \quad 0.591$

$-0.000003 \quad 1.557$

$-0.000005$

0.000009

$-0.000040$

0.000030

72.096 Sum chi sqr
Relative Density of density $/$ water $/ \mathrm{kg} / \mathrm{m}^{3}$

980.5548

980.5548

980.5548

980.5548

980.5548

980.5548

980.5548

980.5548

980.5548

974.849

974.849

974.849

974.849

974.849

974.849

974.849

974.849

974.849

974.849

Curve fit for the model App vol $=\left(w+c 2+c 3^{*} t{ }^{\circ} \mathrm{C}\right) /\left(\mathrm{c} 0^{\star} \mathrm{w}+\mathrm{c} 1\right) / \mathrm{e}^{\wedge}\left(0.000001^{*}\left(\mathrm{t}^{\circ} \mathrm{C}+\mathrm{c} 4\right)^{\wedge} 2\right)$

$\begin{array}{rrr}\text { Avg - 4std } & -2.3876 & -0.000040 \\ \text { Avg + 4std } & 2.5590 & 0.000030\end{array}$

$\begin{array}{lr}\text { c0 } 1 & 1.87 \mathrm{E}-06 \\ \mathrm{c} 2 & 4.55 \mathrm{E}-05 \\ \mathrm{c} 3 & 1.575826 \\ \mathrm{c} 4 & -0.01078 \\ \text { Min T } & -4369.86 \\ \text { Max T } & 15 \\ \text { Min w } & 75 \\ \text { Max w } & 0.0012 \\ \text { Avg dens res } & 0.4347 \\ \text { Std dens res } & 0.0857 \\ \text { Avg app vor } & 0.618321\end{array}$

Std dens res $\quad 0.618321$

Avg app vol res *10^3 $\quad-0.00496$

Std app vol res *10^3 0.008667

No of points in corr

No of inconsistent poir

0

Inconsistent data not used (Residual greater than average $+/-4$ standard deviations) 
Density of aqueous solutions of MnSO4

Ref (6) Asmus, E, The viscosities of aqueous solutions of strong..., Ann der Physik, 1939, 35, 1-22

(7) Bakeev, M. I.; Zharmenov, A. A.; Andamasov, R. S.; Baikenova, N. A.; Abdygalimova, S. Sh. Electrical Conductivity and Viscosity of the Binary Systems MeSO4-H2O (Me=Mn2+, Fe2+, Co2+, Zn2+, Cd2+) at 25-75 Degree and the Structure of Electrolyte Solutions. Izv Nats. Akad. Nauk Resp. Kaz., Ser. Khim. 1994, 6, 25-30.

(19) Deckwer, W. D., Density, viscosity, vapor pressure, and hydrogen solubility of aqueous MnSO4 solutions, J. Chem. Eng. Data, 1980, $25,75-76$

(40) Herz, W. Internal Friction of Salt Solutions (C. A. Trans. from German). Z. Anorg. Chemie 1914, 89, 393-396

(75) National Research Council, International Critical Tables of Physical and Numerical Data, Physics, Chemistry, and Technology., McGrawHill, New York, 1928

(103) Przepiera, A.; Zielenkiewicz, A.; Apparent Molar Volumes of Aqueous Solution in the MnSO4 + H2SO4 System; Bull. Pol. Acad. Sci. Chem. , 2000, 48, 267-272

(117) Schmelzer, N.; Einfeldt, J. Density Measurements in Some Aqueous and Non-Aqueous Electrolyte Solutions at $25^{\circ} \mathrm{C}$. Wiss. Z. Uni. Rostock 1989, 38, 81-82

molality

$\begin{array}{rrr}\mathbf{t} /{ }^{\circ} \mathrm{C} \text { mass frac } & \begin{array}{r}\text { Density } \\ \text { exp } / \\ \mathrm{kg} / \mathrm{m}^{3}\end{array} \\ & & 1010.0 \\ 0 & 0.0100 & 1020.2 \\ 0 & 0.0200 & 1020 \\ 0 & 0.0400 & 1040.9 \\ 0 & 0.0600 & 1062.0 \\ 0 & 0.0800 & 1083.6 \\ 0 & 0.1000 & 1105.7 \\ 0 & 0.1200 & 1128.2 \\ 0 & 0.1400 & 1151.1 \\ 0 & 0.1600 & 1174.4 \\ 0 & 0.1800 & 1198.2 \\ 10 & 0.0100 & 1009.8 \\ 10 & 0.0200 & 1019.7 \\ 10 & 0.0400 & 1039.9 \\ 10 & 0.0600 & 1060.6 \\ 10 & 0.0800 & 1081.9 \\ 10 & 0.1000 & 1103.6 \\ 10 & 0.1200 & 1125.9 \\ 10 & 0.1400 & 1148.8 \\ 10 & 0.1600 & 1172.3 \\ 10 & 0.1800 & 1196.5 \\ 15 & 0.0100 & 1008.9 \\ 15 & 0.0200 & 1018.8 \\ 15 & 0.0400 & 1038.9 \\ 15 & 0.0600 & 1059.5 \\ 15 & 0.0800 & 1080.7 \\ 15 & 0.1000 & 1102.5 \\ 15 & 0.1200 & 1124.8 \\ 15 & 0.1400 & 1147.8 \\ 15 & 0.1600 & 1171.4 \\ & & \end{array}$

Ref

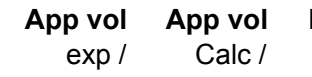

exp / Calc I $\mathrm{m}^{3} / \mathrm{kg} \quad \mathrm{m}^{3} / \mathrm{kg}$

Density App Vol Square of Inconsistent Inconsistent calc / Residual / Residual / Density based on based on $\mathrm{kg} / \mathrm{m}^{3} \quad \mathrm{~kg} / \mathrm{m}^{3} \quad \mathrm{~m}^{3} / \mathrm{kg}$ Residual density apparent

$\begin{array}{lllllll}(75) & -0.000006 & 0.000022 & 1009.72 & 0.2818 & -0.000028 & 0.079\end{array}$

$\begin{array}{lllllll}\text { (75) } & 0.000002 & 0.000025 & 1019.72 & 0.4789 & -0.000023 & 0.229\end{array}$

$\begin{array}{lllllll}(75) & 0.000014 & 0.000032 & 1040.11 & 0.7943 & -0.000018 & 0.631\end{array}$

$\begin{array}{llllllll}(75) & 0.000024 & 0.000039 & 1061.01 & 0.9928 & -0.000015 & 0.986\end{array}$

$\begin{array}{lllllll}(75) & 0.000034 & 0.000046 & 1082.44 & 1.1606 & -0.000012 & 1.347\end{array}$

$\begin{array}{lllllll}(75) & 0.000043 & 0.000053 & 1104.42 & 1.2835 & -0.000011 & 1.647\end{array}$

$\begin{array}{lllllll}\text { (75) } & 0.000052 & 0.000060 & 1126.95 & 1.2472 & -0.000008 & 1.555\end{array}$

$\begin{array}{lllllll}75) & 0.000061 & 0.000067 & 1150.06 & 1.0368 & -0.000006 & 1.075\end{array}$

$\begin{array}{lllllll}(75) & 0.000071 & 0.000074 & 1173.76 & 0.6376 & -0.000003 & 0.407\end{array}$

$\begin{array}{lllllll}(75) & 0.000080 & 0.000081 & 1198.07 & 0.1345 & -0.000001 & 0.018\end{array}$

$\begin{array}{lllllll}\text { (75) } & 0.000000 & 0.000027 & 1009.52 & 0.2811 & -0.000028 & 0.079\end{array}$

$\begin{array}{lllllll}(75) & 0.000019 & 0.000031 & 1019.46 & 0.2354 & -0.000011 & 0.055\end{array}$

$\begin{array}{llllll}0.000034 & 0.000037 & 1039.74 & 0.1568 & -0.000004 & 0.025\end{array}$

$\begin{array}{llllll}0.000043 & 0.000044 & 1060.55 & 0.0493 & -0.000001 & 0.002\end{array}$

$\begin{array}{llllll}0.000050 & 0.000050 & 1081.90 & -0.0030 & 0.000000 & 0.000\end{array}$

$\begin{array}{llllll}0.000059 & 0.000057 & 1103.82 & -0.2163 & 0.000002 & 0.047\end{array}$

$\begin{array}{llllll}0.000066 & 0.000063 & 1126.31 & -0.4072 & 0.000003 & 0.166\end{array}$

$\begin{array}{llllll}0.000073 & 0.000070 & 1149.39 & -0.5928 & 0.000003 & 0.351\end{array}$

$\begin{array}{llllll}0.000080 & 0.000076 & 1173.09 & -0.7908 & 0.000004 & 0.625\end{array}$

$\begin{array}{lllllll}0.000086 & 0.000083 & 1197.42 & -0.9191 & 0.000004 & 0.845\end{array}$

$\begin{array}{llllll}0.000029 & 0.000030 & 1008.89 & 0.0128 & -0.000001 & 0.000\end{array}$

$\begin{array}{llllll}0.000033 & 0.000033 & 1018.80 & -0.0026 & 0.000000 & 0.000\end{array}$

$\begin{array}{llllll}0.000042 & 0.000039 & 1039.02 & -0.1245 & 0.000003 & 0.015\end{array}$

$\begin{array}{llllll}0.000050 & 0.000046 & 1059.78 & -0.2813 & 0.000004 & 0.079\end{array}$

$\begin{array}{llllll}0.000056 & 0.000052 & 1081.09 & -0.3898 & 0.000004 & 0.152\end{array}$

$\begin{array}{llllll}0.000062 & 0.000058 & 1102.97 & -0.4673 & 0.000004 & 0.218\end{array}$

$\begin{array}{llllll}0.000069 & 0.000065 & 1125.43 & -0.6314 & 0.000004 & 0.399\end{array}$

$\begin{array}{lllllll}0.000075 & 0.000071 & 1148.50 & -0.7006 & 0.000004 & 0.491\end{array}$

$\begin{array}{llllll}0.000081 & 0.000077 & 1172.19 & -0.7937 & 0.000004 & 0.630\end{array}$
Relative Density of density water $/ \mathrm{kg} / \mathrm{m}^{3}$

999.8395

999.8395

999.8395

999.8395

999.8395

999.8395

999.8395

999.8395

999.8395

999.8395

999.6996

999.6996

999.6996

999.6996

999.6996

999.6996

999.6996

999.6996

999.6996

999.6996

999.0996

999.0996

999.0996

999.0996

999.0996

999.0996

999.0996

999.0996

999.0996 


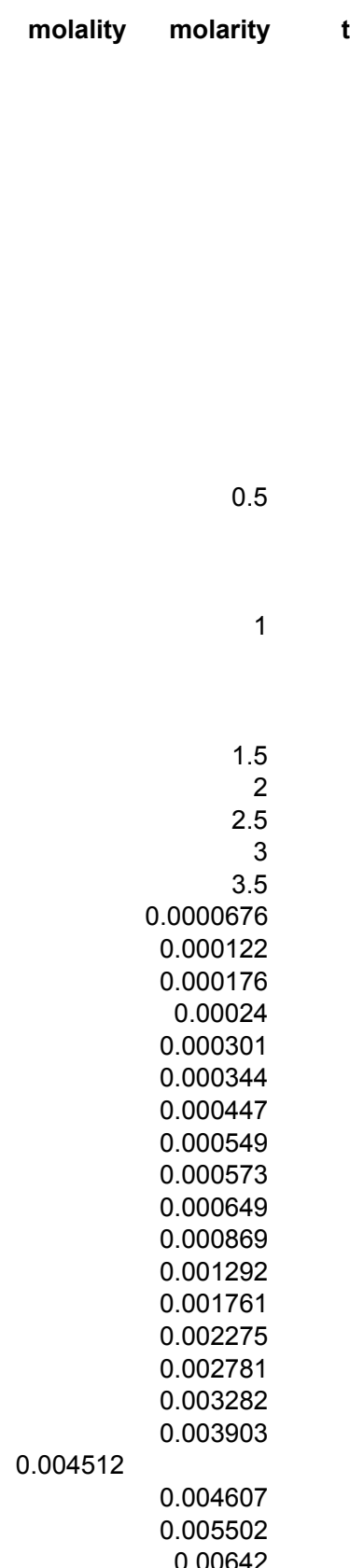

$\begin{array}{rrr}\mathbf{t} /{ }^{\circ} \mathrm{C} \text { mass frac } & \text { Density } \\ & & \text { exp } / \\ & & \mathrm{kg} / \mathrm{m}^{3} \\ & & 1195.6 \\ 15 & 0.1800 & 175 \\ 15 & 0.2000 & 1220.5 \\ 15 & 0.2200 & 1246.1 \\ 15 & 0.2400 & 1272.5 \\ 15 & 0.2600 & 1299.7 \\ 15 & 0.2800 & 1327.7 \\ 15 & 0.3000 & 1356.5 \\ 20 & 0.0100 & 1008.0 \\ 20 & 0.0200 & 1017.8 \\ 20 & 0.0400 & 1037.8 \\ 20 & 0.0600 & 1058.3 \\ 20 & 0.0706 & 1069.0 \\ 20 & 0.0800 & 1079.4 \\ 20 & 0.1000 & 1101.2 \\ 20 & 0.1200 & 1123.6 \\ 20 & 0.1327 & 1138.0 \\ 20 & 0.1400 & 1146.7 \\ 20 & 0.1600 & 1170.5 \\ 20 & 0.1800 & 1195.0 \\ 20 & 0.1877 & 1207.0 \\ 20 & 0.2380 & 1269.0 \\ 20 & 0.2838 & 1330.0 \\ 20 & 0.3250 & 1394.0 \\ 20 & 0.3640 & 1452.0 \\ 25 & 0.0000 & 997.1 \\ 25 & 0.0000 & 997.1 \\ 25 & 0.0000 & 997.1 \\ 25 & 0.0000 & 997.1 \\ 25 & 0.0000 & 997.1 \\ 25 & 0.0001 & 997.1 \\ 25 & 0.0001 & 997.1 \\ 25 & 0.0001 & 997.1 \\ 25 & 0.0001 & 997.1 \\ 25 & 0.0001 & 997.1 \\ 25 & 0.0001 & 997.2 \\ 25 & 0.0002 & 997.2 \\ 25 & 0.0003 & 997.3 \\ 25 & 0.0003 & 997.4 \\ 25 & 0.0004 & 997.5 \\ 25 & 0.0005 & 997.5 \\ 25 & 0.0006 & 997.6 \\ 25 & 0.0007 & 997.7 \\ 25 & 0.0007 & 997.7 \\ 25 & 0.0008 & 997.9 \\ 25 & 0.0010 & 998.0 \\ & & \end{array}$

Ref

$\begin{array}{rr}\text { App vol } & \text { App vol } \\ \exp / & \text { Calc / } \\ \mathrm{m}^{3} / \mathrm{kg} & \mathrm{m}^{3} / \mathrm{kg}\end{array}$

Density
calc /

alc / Density App Vol Square of Inconsistent Inconsistent / Residual/ Residual/ Density based on based on

$0.000087 \quad 0.000083$ $0.000093 \quad 0.000090$ $\begin{array}{ll}0.000099 & 0.000096 \\ 0.000105 & 0.000102\end{array}$ $\begin{array}{ll}0.000111 & 0.000108\end{array}$ $0.000116 \quad 0.000114$ 0.0001220 .000121 $\begin{array}{ll}0.000028 & 0.000032 \\ 0.000037 & 0.000035\end{array}$ $\begin{array}{ll}0.000037 & 0.000035 \\ 0.000046 & 0.000041\end{array}$ $\begin{array}{ll}0.000054 & 0.000048\end{array}$ 0.0000620 .000051 $\begin{array}{ll}0.000060 & 0.000054\end{array}$ 0.0000650 .000060 $\begin{array}{ll}0.000070 & 0.000066\end{array}$ $\begin{array}{ll}0.000074 & 0.000070\end{array}$ $\begin{array}{ll}0.000075 & 0.000072\end{array}$ $0.000080 \quad 0.000078$ $\begin{array}{ll}0.000085 & 0.000084\end{array}$ $\begin{array}{ll}0.000078 & 0.000086\end{array}$ $0.000104 \quad 0.000101$ $0.000121 \quad 0.000115$ $0.000127 \quad 0.000127$ $\begin{array}{ll}0.000142 & 0.000139\end{array}$ (6) $\quad-0.000173 \quad 0.000031$ $\begin{array}{lll}\text { 6) } & -0.000083 & 0.000031\end{array}$ $\begin{array}{ll}0.000025 & 0.000031\end{array}$ $\begin{array}{ll}-0.000073 & 0.000031\end{array}$ $\begin{array}{lll}\text { (6) } & -0.000053 & 0.000031\end{array}$ (6) $\quad-0.000056 \quad 0.000031$ (6) $\quad 0.000010 \quad 0.000031$ (6) $\quad-0.000034 \quad 0.000031$ (6) $\quad-0.000026 \quad 0.000031$ (6) $\quad-0.000017 \quad 0.000031$ (6) $\quad-0.000011 \quad 0.000031$ (6) $\quad-0.000002 \quad 0.000031$ (6) $\quad 0.000010 \quad 0.000031$ (6) $\quad 0.000007 \quad 0.000032$ (6) $\quad 0.000031 \quad 0.000032$ (6) $\quad 0.000016 \quad 0.000032$ (6) $-0.000017 \quad 0.000032$ $\begin{array}{lll}(117) & -0.000009 & 0.000032\end{array}$ (6) $\quad 0.000024 \quad 0.000032$ (6) $\quad 0.000021 \quad 0.000032$ (6) $\quad 0.000025 \quad 0.000032$ $\mathrm{kg} / \mathrm{m}^{3} \quad \mathrm{~kg} / \mathrm{m}^{3} \quad \mathrm{~m}^{3} / \mathrm{kg}$ Residual density $\begin{gathered}\text { apparent } \\ \text { volume }\end{gathered}$

$1196.53 \quad-0.9301 \quad 0.000004 \quad 0.865$

$\begin{array}{lllll}221.53 & -1.0300 & 0.000003 & 1.061\end{array}$

$\begin{array}{llll}247.21 & -1.1140 & 0.000003 & 1.241\end{array}$

$\begin{array}{llll}1273.60 & -1.1033 & 0.000003 & 1.217\end{array}$

$\begin{array}{llll}300.72 & -1.0199 & 0.000002 & 1.040\end{array}$

$\begin{array}{llll}328.59 & -0.8861 & 0.000002 & 0.785\end{array}$

$\begin{array}{llll}357.23 & -0.7251 & 0.000001 & 0.526\end{array}$

$\begin{array}{lllll}1007.96 & 0.0405 & -0.000004 & 0.002\end{array}$

$\begin{array}{llll}1017.84 & -0.0437 & 0.000002 & 0.002\end{array}$

$\begin{array}{llll}1038.01 & -0.2073 & 0.000005 & 0.043\end{array}$

$\begin{array}{llll}1058.71 & -0.4118 & 0.000006 & 0.170\end{array}$

$\begin{array}{llll}1069.94 & -0.9388 & 0.000012 & 0.881\end{array}$

$\begin{array}{llll}1079.97 & -0.5749 & 0.000006 & 0.331\end{array}$

$\begin{array}{llll}1101.81 & -0.6148 & 0.000005 & 0.378\end{array}$

$\begin{array}{llll}1124.25 & -0.6504 & 0.000004 & 0.423\end{array}$

$\begin{array}{llll}138.80 & -0.8018 & 0.000005 & 0.643\end{array}$

$\begin{array}{llll}1147.30 & -0.6011 & 0.000003 & 0.361\end{array}$

$\begin{array}{llll}170.99 & -0.4872 & 0.000002 & 0.237\end{array}$

$\begin{array}{llll}195.33 & -0.3296 & 0.000001 & 0.109\end{array}$

$\begin{array}{llll}204.82 & 2.1754 & -0.000008 & 4.732\end{array}$

$\begin{array}{llll}1269.81 & -0.8129 & 0.000002 & 0.661\end{array}$

$\begin{array}{llll}1333.12 & -3.1204 & 0.000006 & 9.737\end{array}$

$\begin{array}{lllll}393.49 & 0.5106 & -0.000001 & 0.261\end{array}$

$\begin{array}{llll}1454.11 & -2.1087 & 0.000003 & 4.447\end{array}$

$\begin{array}{llll}997.05 & 0.0021 & -0.000204 & 0.000\end{array}$

$\begin{array}{llll}997.06 & 0.0021 & -0.000114 & 0.000\end{array}$

$\begin{array}{lllll}997.07 & 0.0002 & -0.000007 & 0.000\end{array}$

$\begin{array}{llll}997.08 & 0.0038 & -0.000105 & 0.000\end{array}$

$\begin{array}{llll}997.09 & 0.0038 & -0.000085 & 0.000\end{array}$

$\begin{array}{llll}997.10 & 0.0045 & -0.000087 & 0.000\end{array}$

$\begin{array}{lllll}997.11 & 0.0014 & -0.000021 & 0.000\end{array}$

$\begin{array}{llll}997.13 & 0.0054 & -0.000066 & 0.000\end{array}$

$\begin{array}{llll}997.13 & 0.0049 & -0.000057 & 0.000\end{array}$

$\begin{array}{llll}997.17 & 0.0055 & -0.000042 & 0.000\end{array}$

$\begin{array}{llll}997.23 & 0.0065 & -0.000033 & 0.000\end{array}$

$\begin{array}{lllll}997.30 & 0.0057 & -0.000021 & 0.000\end{array}$

$\begin{array}{llll}997.38 & 0.0083 & -0.000024 & 0.000\end{array}$

$\begin{array}{llll}997.45 & 0.0001 & 0.000000 & 0.000\end{array}$

$\begin{array}{llll}997.52 & 0.0076 & -0.000015 & 0.000\end{array}$

$\begin{array}{llll}997.62 & 0.0085 & -0.000014 & 0.000\end{array}$

$\begin{array}{llll}997.70 & 0.0272 & -0.000040 & 0.001\end{array}$

$\begin{array}{llll}997.72 & 0.0053 & -0.000008 & 0.000\end{array}$

$\begin{array}{llll}997.85 & 0.0090 & -0.000011 & 0.000\end{array}$

$\begin{array}{llll}997.98 & 0.0064 & -0.000007 & 0.000\end{array}$ $\begin{array}{llll}997.14 & 0.0048 & -0.000049 & 0.000\end{array}$
Relative Density of density water $/ \mathrm{kg} / \mathrm{m}^{3}$

999.0996

999.0996

999.0996

999.0996

999.0996

999.0996

999.0996

998.2041

998.2041

998.2041

998.2041

998.2041

998.2041

998.2041

998.2041

998.2041

998.2041

998.2041

998.2041

998.2041

998.2041

998.2041

998.2041

998.2041

1.000012997 .0449

1.00002997 .0449

1.000026997 .0449

1.000039997 .0449

1.000048997 .0449

1.000055997 .0449

1.000067997 .0449

1.000086997 .0449

1.000089997 .0449

1.0001997 .0449

1.000133997 .0449

1.000196997 .0449

$1.000264 \quad 997.0449$

1.000342997 .0449

1.000408997 .0449

1.000489997 .0449

1.000581997 .0449 997.0449

1.000681997 .0449

$1.000816 \quad 997.0449$

1.000948997 .0449 


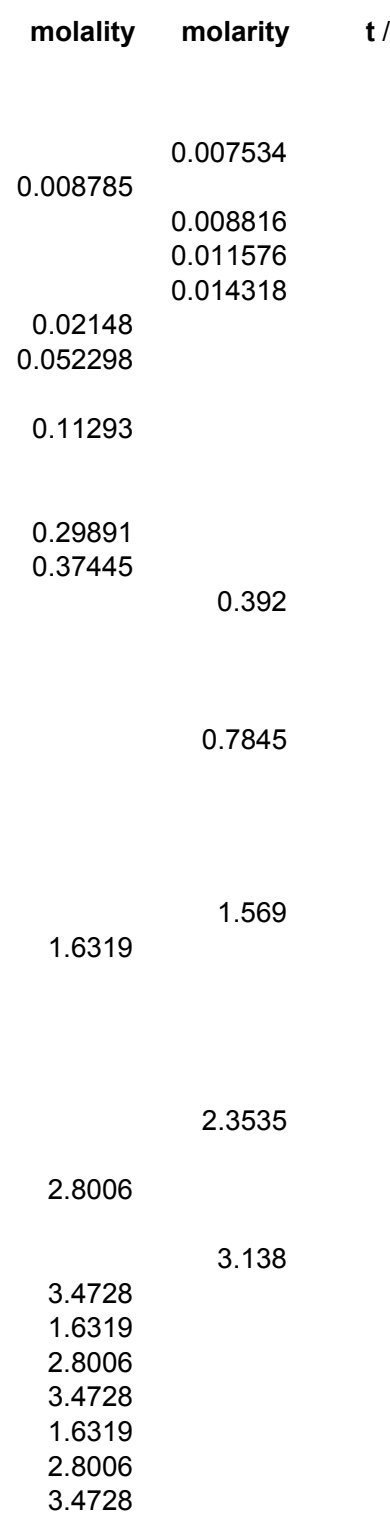

$\begin{array}{ccrc}\mathbf{t} /{ }^{\circ} \mathrm{C} \text { mass frac } & \begin{array}{r}\text { Density } \\ \text { exp } /\end{array} & \text { Ref } \\ & & \mathrm{kg} / \mathrm{m}^{3} & \\ & & & \\ 25 & 0.0011 & 998.2 & (6) \\ 25 & 0.0013 & 998.4 & (117) \\ 25 & 0.0013 & 998.3 & (6) \\ 25 & 0.0018 & 998.7 & (6) \\ 25 & 0.0022 & 999.1 & (6) \\ 25 & 0.0032 & 1000.3 & (117) \\ 25 & 0.0078 & 1004.8 & (117) \\ 25 & 0.0100 & 1006.8 & (75) \\ 25 & 0.0168 & 1013.8 & (117) \\ 25 & 0.0200 & 1016.5 & (75) \\ 25 & 0.0400 & 1036.4 & (75) \\ 25 & 0.0432 & 1041.5 & (117) \\ 25 & 0.0535 & 1053.3 & (117) \\ 25 & 0.0562 & 1052.4 & (40) \\ 25 & 0.0600 & 1056.9 & (75) \\ 25 & 0.0800 & 1078.0 & (75) \\ 25 & 0.1000 & 1099.8 & (75) \\ 25 & 0.1070 & 1107.3 & (40) \\ 25 & 0.1200 & 1122.4 & (75) \\ 25 & 0.1400 & 1145.8 & (75) \\ 25 & 0.1600 & 1169.9 & (75) \\ 25 & 0.1800 & 1194.8 & (75) \\ 25 & 0.1952 & 1214.0 & (40) \\ 25 & 0.1977 & 1216.4 & (103) \\ 25 & 0.2000 & 1220.3 & (75) \\ 25 & 0.2200 & 1246.4 & (75) \\ 25 & 0.2400 & 1273.1 & (75) \\ 25 & 0.2600 & 1300.4 & (75) \\ 25 & 0.2704 & 1314.4 & (40) \\ 25 & 0.2800 & 1328.3 & (75) \\ 25 & 0.2972 & 1351.3 & (103) \\ 25 & 0.3000 & 1356.8 & (75) \\ 25 & 0.3361 & 1409.9 & (40) \\ 25 & 0.3440 & 1425.3 & (103) \\ 35 & 0.1977 & 1212.5 & (103) \\ 35 & 0.2972 & 1346.4 & (103) \\ 35 & 0.3440 & 1420.5 & (103) \\ 45 & 0.1977 & 1208.0 & (103) \\ 45 & 0.2972 & 1341.7 & (103) \\ 45 & 0.3440 & 1416.0 & (103) \\ & & & \end{array}$

$\begin{array}{cc}\text { App vol } & \text { App vol } \\ \exp / & \text { Calc / }\end{array}$

$\mathrm{m}^{3} / \mathrm{kg} \quad \mathrm{m}^{3} / \mathrm{kg}$

$0.000023 \quad 0.000032$

$\begin{array}{ll}-0.000009 & 0.000032\end{array}$

$\begin{array}{ll}0.000026 & 0.000032\end{array}$

$0.000028 \quad 0.000032$

$0.000027 \quad 0.000032$

$0.000006 \quad 0.000032$

$0.000014 \quad 0.000034$

$0.000031 \quad 0.000034$

$0.000014 \quad 0.000036$

$0.000043 \quad 0.000037$

$\begin{array}{ll}0.000051 & 0.000043\end{array}$

$\begin{array}{ll}0.000013 & 0.000044\end{array}$

$\begin{array}{ll}0.000003 & 0.000047\end{array}$

$0.000065 \quad 0.000048$

$0.000056 \quad 0.000049$

$0.000061 \quad 0.000055$

$0.000066 \quad 0.000061$

$0.000069 \quad 0.000063$

$0.000069 \quad 0.000067$

$\begin{array}{ll}0.000073 & 0.000073\end{array}$

$\begin{array}{ll}0.000077 & 0.000079\end{array}$

0.0000810 .000084

$0.000085 \quad 0.000089$

$0.000088 \quad 0.000090$

0.0000850 .000090

$0.000091 \quad 0.000096$

$\begin{array}{ll}0.000097 & 0.000102\end{array}$

$0.000103 \quad 0.000108$

$\begin{array}{ll}0.000107 & 0.000111\end{array}$

$0.000110 \quad 0.000113$

$0.000118 \quad 0.000118$

$\begin{array}{ll}0.000117 & 0.000119\end{array}$

0.0001290 .000130

$0.000127 \quad 0.000132$

0.0000890 .000090

$0.000120 \quad 0.000117$

$0.000128 \quad 0.000129$

0.0000890 .000090

$0.000120 \quad 0.000115$

$0.000127 \quad 0.000126 \quad 1416.50$

Average Res

Std dev Res

Avg - 4std

Avg + 4std
Density Density App Vol Square of Inconsistent Inconsistent calc/ Residual / Residual / Density based on based on $\mathrm{kg} / \mathrm{m}^{3} \quad \mathrm{~kg} / \mathrm{m}^{3} \quad \mathrm{~m}^{3} / \mathrm{kg} \quad$ Residual density $\begin{gathered}\text { apparent } \\ \text { volume }\end{gathered}$

$\begin{array}{llll}998.15 & 0.0101 & -0.000009 & 0.000\end{array}$

$\begin{array}{llll}998.33 & 0.0545 & -0.000041 & 0.003\end{array}$

$\begin{array}{llll}998.33 & 0.0072 & -0.000005 & 0.000\end{array}$

$\begin{array}{llll}998.74 & 0.0077 & -0.000004 & 0.000\end{array}$

$\begin{array}{llll}999.14 & 0.0109 & -0.000005 & 0.000\end{array}$

$\begin{array}{llll}0.00 .17 & 0.0859 & -0.000027 & 0.007\end{array}$

$\begin{array}{llll}004.65 & 0.1584 & -0.000020 & 0.025\end{array}$

$\begin{array}{llll}0.3802 & -0.000022 & 0.145 \\ 1016.62 & -0.1200 & 0.000006 & 0.014\end{array}$

$\begin{array}{llll}036.72 & -0.3242 & 0.000008 & 0.105\end{array}$

$\begin{array}{llll}039.98 & 1.4731 & -0.000031 & 2.170\end{array}$

$\begin{array}{llll}1.450 .62 & 2.6509 & -0.000045 & 7.027\end{array}$

$\begin{array}{llll}3.46 & -1.0551 & 0.000017 & 1.113\end{array}$

$\begin{array}{llll}057.38 & -0.4753 & 0.000007 & 0.226\end{array}$

$\begin{array}{llll}108.14 & -0.5928 & 0.000005 & 0.351 \\ 1122.80 & -0.3983 & 0.000006 & 0.710\end{array}$

$\begin{array}{llll}1122.80 & -0.3983 & 0.000003 & 0.159\end{array}$

$\begin{array}{llll}145.83 & -0.0290 & 0.000000 & 0.001\end{array}$

$\begin{array}{llll}1 & 0.9472 & -0.000004 & 0.897 \\ 1212.76 & 1.2388 & -0.000004 & 1.535\end{array}$

$\begin{array}{llll}1215.98 & 0.4293 & -0.000001 & 0.184\end{array}$

$1218.89 \quad 1.4086-0.000005 \quad 1.984$

$\begin{array}{llll} & 1.9573 & -0.000005 & 3.831\end{array}$

$\begin{array}{lll}1.9937-0.000005 & 3.975\end{array}$

$\begin{array}{llll}1326.46 & 1.8357 & -0.000004 & 3.370\end{array}$

$\begin{array}{llll}351.26 & 0.0132 & 0.000000 & 0.000\end{array}$

$\begin{array}{lllll}355.34 & 1.4553 & -0.000003 & 2.118\end{array}$

$\begin{array}{rrrr}1212.32 & 0.1663 & -0.000001 & 0.028\end{array}$

$\begin{array}{llll}348.28 & -1.8655 & 0.000003 & 3.480\end{array}$

$\begin{array}{lll}0.8405 & -0.000001 & 0.706\end{array}$

$\begin{array}{lll}0.1870 & -0.000001 & 0.035\end{array}$

$\begin{array}{lll}-2.6683 & 0.000005 & 7.120\end{array}$

$\begin{array}{lll}-0.4681 & 0.000001 & 0.219\end{array}$

$0.0183-0.000011 \quad 101.409$ Sum chi sqr

$\begin{array}{ll}0.9471 & 0.000029\end{array}$

$-3.7703-0.000126$

$3.8068 \quad 0.000104$
Relative Density of density water $/ \mathrm{kg} / \mathrm{m}^{3}$

1.001115997 .0449

997.0449

$1.0013 \quad 997.0449$

1.001705997 .0449

1.00211997 .0449

997.0449

997.0449

997.0449

997.0449

997.0449

997.0449

997.0449

997.0449

997.0449

997.0449

997.0449

997.0449

997.0449

997.0449

997.0449

997.0449

997.0449

997.0449

997.0449

997.0449

997.0449

997.0449

997.0449

997.0449

997.0449

997.0449

997.0449

997.0449

997.0449

994.0319

994.0319

994.0319

990.2132

990.2132

990.2132 
molality

molarity
Ref

$$
\begin{array}{r}
\exp / \\
\mathrm{kg} / \mathrm{m}^{3}
\end{array}
$$

$\begin{array}{rr}\text { App vol } & \text { App vol } \\ \exp / & \text { Calc / } \\ \mathrm{m}^{3} / \mathrm{kg} & \mathrm{m}^{3} / \mathrm{kg}\end{array}$

\section{Density} $\mathrm{kg} / \mathrm{m}^{3}$
Density App Vol Square of Inconsistent Inconsistent Ridual / Residual / Density based on based on $\mathrm{kg} / \mathrm{m}^{3}$

$\mathrm{m}^{3} / \mathrm{kg}$ Residual
Relative Density of density water $/ \mathrm{kg} / \mathrm{m}^{3}$

Curve fit for the model App vol $=\left(w+c 2+c 3^{*} t{ }^{\circ} \mathrm{C}\right) /\left(c 0^{*} w+c 1\right) / e^{\wedge}\left(0.000001^{*}\left(t^{\circ} \mathrm{C}+c 4\right)^{\wedge} 2\right)$

$\begin{array}{lr}\text { c0 } 1 & 0.003245 \\ \text { c2 } & 0.057246 \\ \text { c3 } & 0.051358 \\ \text { c4 } & 0.002145 \\ \text { Min T } & 3287.822 \\ \text { Max T } & 0 \\ \text { Min w } & 45 \\ \text { Max w } & 0.0000 \\ \text { Avg dens res } & 0.3640 \\ \text { Std dens res } & 0.0183 \\ \text { Avg app vol res *10^3 } & 0.947147 \\ \text { Std app vol res *10^3 } & -0.01108 \\ \text { No of points in corr } & 0.02885 \\ \text { No of inconsistent point } & 114 \\ & 36\end{array}$

Inconsistent data not used (Residual greater than average +/- 4 standard deviations)

\begin{tabular}{|c|c|c|c|c|c|c|c|c|c|c|c|c|c|}
\hline & 25 & 0.0005 & 998.6 & (7) & -0.002121 & 0.000032 & 997.53 & 1.0720 & -0.002152 & 1.149 & & $!$ & 997.0449 \\
\hline 0.004199 & 25 & 0.0006 & 997.1 & (117) & 0.000884 & 0.000032 & 997.66 & -0.5372 & 0.000852 & 0.289 & & ! & 997.0449 \\
\hline 0.006039 & 25 & 0.0009 & 997.1 & (117) & 0.000986 & 0.000032 & 997.93 & -0.8653 & 0.000955 & 0.749 & & ! & 997.0449 \\
\hline \multirow[t]{4}{*}{0.007229} & 25 & 0.0011 & 997.2 & (117) & 0.000906 & 0.000032 & 998.10 & -0.9488 & 0.000874 & 0.900 & & ! & 997.0449 \\
\hline & 25 & 0.0030 & 999.6 & (7) & 0.000148 & 0.000032 & 999.95 & -0.3481 & 0.000116 & 0.121 & & ! & 997.0449 \\
\hline & 25 & 0.0200 & 1020.8 & (7) & -0.000163 & 0.000037 & 1016.64 & 4.1602 & -0.000200 & 17.307 & ! & $!$ & 997.0449 \\
\hline & 25 & 0.0701 & 1081.2 & (7) & -0.000110 & 0.000052 & 1068.06 & 13.1398 & -0.000162 & 172.655 & $!$ & $!$ & 997.0449 \\
\hline 0.65005 & 25 & 0.0894 & 1097.4 & (117) & -0.000023 & 0.000058 & 1088.75 & 8.6136 & -0.000081 & 74.194 & $!$ & & 997.0449 \\
\hline \multirow[t]{3}{*}{0.82535} & 25 & 0.1108 & 1124.0 & (117) & -0.000020 & 0.000064 & 1112.43 & 11.5959 & -0.000084 & 134.465 & $!$ & & 997.0449 \\
\hline & 25 & 0.1201 & 1145.4 & $(7)$ & -0.000079 & 0.000067 & 1122.86 & 22.5449 & -0.000146 & 508.274 & $!$ & ! & 997.0449 \\
\hline & 25 & 0.1700 & 1211.0 & (7) & -0.000039 & 0.000082 & 1181.64 & 29.3568 & -0.000121 & 861.822 & ! & & 997.0449 \\
\hline \multirow[t]{15}{*}{1.49488} & 25 & 0.1842 & 1249.2 & (117) & -0.000096 & 0.000086 & 1199.00 & 50.2005 & -0.000182 & 2520.091 & ! & $!$ & 997.0449 \\
\hline & 25 & 0.2203 & 1287.5 & $(7)$ & -0.000024 & 0.000096 & 1245.04 & 42.4617 & -0.000120 & 1802.998 & ! & & 997.0449 \\
\hline & 25 & 0.2785 & 1375.8 & (7) & 0.000012 & 0.000113 & 1324.33 & 51.4682 & -0.000101 & 2648.975 & ! & & 997.0449 \\
\hline & 25 & 0.2913 & 1395.9 & (7) & 0.000019 & 0.000117 & 1342.61 & 53.2935 & -0.000098 & 2840.196 & ! & & 997.0449 \\
\hline & 25 & 0.3159 & 1434.4 & (7) & 0.000035 & 0.000124 & 1378.90 & 55.5043 & -0.000089 & 3080.727 & ! & & 997.0449 \\
\hline & 50 & 0.0005 & 989.6 & (7) & -0.002186 & 0.000040 & 988.51 & 1.0891 & -0.002227 & 1.186 & & ! & 988.0363 \\
\hline & 50 & 0.0030 & 992.3 & (7) & -0.000437 & 0.000041 & 990.89 & 1.4113 & -0.000478 & 1.992 & & ! & 988.0363 \\
\hline & 50 & 0.0200 & 1009.5 & (7) & -0.000063 & 0.000045 & 1007.30 & 2.1984 & -0.000108 & 4.833 & & & 988.0363 \\
\hline & 50 & 0.0701 & 1072.2 & (7) & -0.000121 & 0.000058 & 1058.01 & 14.1879 & -0.000178 & 201.297 & ! & ! & 988.0363 \\
\hline & 50 & 0.1201 & 1132.0 & (7) & -0.000060 & 0.000070 & 1112.32 & 19.6831 & -0.000130 & 387.425 & ! & ! & 988.0363 \\
\hline & 50 & 0.1700 & 1197.5 & (7) & -0.000029 & 0.000082 & 1170.91 & 26.5874 & -0.000112 & 706.891 & ! & & 988.0363 \\
\hline & 50 & 0.2203 & 1272.0 & (7) & -0.000014 & 0.000095 & 1234.52 & 37.4799 & -0.000108 & 1404.740 & ! & & 988.0363 \\
\hline & 50 & 0.2785 & 1362.0 & (7) & 0.000014 & 0.000109 & 1314.75 & 47.2533 & -0.000095 & 2232.872 & ! & & 988.0363 \\
\hline & 50 & 0.2913 & 1380.8 & (7) & 0.000024 & 0.000112 & 1333.35 & 47.4518 & -0.000088 & 2251.670 & ! & & 988.0363 \\
\hline & 50 & 0.3159 & 1423.4 & (7) & 0.000032 & 0.000118 & 1370.42 & 52.9834 & -0.000086 & 2807.246 & ! & & 988.0363 \\
\hline
\end{tabular}


molality molarity

\begin{tabular}{|c|c|c|c|}
\hline \multicolumn{2}{|c|}{$\mathrm{t} /{ }^{\circ} \mathrm{C}$ mass frac } & $\begin{array}{r}\text { Density } \\
\exp / \\
\mathrm{kg} / \mathrm{m}^{3}\end{array}$ & Ref \\
\hline 75 & 0.0005 & 977.1 & (7) \\
\hline 75 & 0.0030 & 978.3 & (7) \\
\hline 75 & 0.0200 & 996.9 & (7) \\
\hline 75 & 0.0701 & 1055.0 & (7) \\
\hline 75 & 0.1201 & 1117.1 & (7) \\
\hline 75 & 0.1700 & 1182.4 & (7) \\
\hline 75 & 0.2203 & 1257.2 & (7) \\
\hline 75 & 0.2785 & 1346.7 & (7) \\
\hline 75 & 0.2913 & 1363.3 & (7) \\
\hline 75 & 0.3159 & 1407.0 & (7) \\
\hline
\end{tabular}

App vol App vol

exp / Calc/

$\mathrm{m}^{3} / \mathrm{kg} \quad \mathrm{m}^{3} / \mathrm{kg}$

$-0.003701 \quad 0.000046$

$-0.000180 \quad 0.000046$

$\begin{array}{ll}-0.000108 & 0.000050\end{array}$

$\begin{array}{ll}-0.000085 & 0.000060\end{array}$

$\begin{array}{ll}-0.000062 & 0.000071\end{array}$

$-0.000033 \quad 0.000081$

$\begin{array}{ll}-0.000020 & 0.000092 \\ 0.000009 & 0.000104\end{array}$

$\begin{array}{ll}0.000009 & 0.000104 \\ 0.000022 & 0.000106\end{array}$

$0.000022 \quad 0.000106$

$0.000028 \quad 0.000111$
Density Density App Vol Square of Inconsistent Inconsistent calc / Residual / Residual / Density based on based on $\mathrm{kg} / \mathrm{m}^{3} \quad \mathrm{~kg} / \mathrm{m}^{3} \quad \mathrm{~m}^{3} / \mathrm{kg}$ Residual density apparent $\begin{array}{llll}975.31 & 1.7850 & -0.003746 & 3.186\end{array}$ $\begin{array}{llll}977.65 & 0.6500 & -0.000227 & 0.422\end{array}$ $\begin{array}{llll}993.78 & 3.1199 & -0.000157 & 9.734\end{array}$ $\begin{array}{llll}1043.76 & 11.2439 & -0.000146 & 126.425\end{array}$ $\begin{array}{llll}1097.52 & 19.5787 & -0.000133 & 383.324\end{array}$ $\begin{array}{llll}1155.84 & 26.5586 & -0.000114 & 705.358\end{array}$ $\begin{array}{llll}1219.53 & 37.6660 & -0.000112 & 1418.726\end{array}$

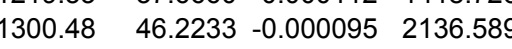
$1319.35 \quad 43.9540 \quad-0.000084 \quad 1931.953$ $\begin{array}{llll}1357.07 & 49.9337 & -0.000083 & 2493.373\end{array}$
Relative Density of density water $/ \mathrm{kg} / \mathrm{m}^{3}$

974.849

974.849

974.849

974.849

974.849

974.849

974.849

974.849

974.849

974.849 
Density of aqueous solutions of $\mathrm{NaBr}$

References (24) Doménech, J.; Rivera, S. Viscosity B-Coefficient for Sodium Bromide in Formamide-Water Mixtures. Z. Physik. Chem. N. F. 1983, 136, 153-161.

(28) Gates, J. A.; Wood, R. H. Densities of Aqueous Solutions of $\mathrm{NaCl}, \mathrm{MgCl}_{2}, \mathrm{KCl}, \mathrm{NaBr}$, $\mathrm{LiCl}$, and $\mathrm{CaCl}_{2}$ from 0.05 to $5.0 \mathrm{~mol} \mathrm{~kg}^{-1}$ and 0.1013 to $40 \mathrm{MPa}$ at 298.15K. J. Chem. Eng. Data 1985, 30,44-49.

(32) Gucker, F. T.; Stubley, D.; Hill, D. J. The Isentropic Compressibilities of Aqueous Solutions of Some Alkali Halides at 298.15K. J. Chem. Thermodynamics 1975, 7, 865-869.

(45) Isono, T. Densities, Viscosities, and Electrolytic Conductivity of Concentrated Aqueous Electrolyte Solutions at Several Temperatures. Alkaline-Earth Chlorides, $\mathrm{LaCl}_{3}, \mathrm{Na}_{2} \mathrm{SO}_{4}, \mathrm{NaNO}_{3}, \mathrm{NaBr}, \mathrm{KNO}_{3}, \mathrm{KBr}$, and $\mathrm{Cd}\left(\mathrm{NO}_{3}\right)_{2}$. J. Chem. Eng. Data 1984, $29,45-52$.

(73) Millero, F. J.; Ward, G. K.; Chetirkin, P. V. Relative Sound Velocities of Sea Salts at $25^{\circ}$ C. J. Acoust. Soc. Am. 1977, 61, 1492-1498.

(90) Perry, R. H.; Green, D. W. Perry's Chemical Engineers' Handbook; McGraw Hill: New York, 7th edition, 1997.

(118) Scott, A. F.; Durham, E. J.; Studies in the solubilities of the soluble electrolytes, J. Phys. Chem., 1930, 34, 1424-1438

(119) Scott, A. F.; Obenhaus, V. M.; Wilson, R. W. The Compressibility Coefficients of Solutions of Eight Alkali Halides. J. Phys. Chem. 1934, 38, 931-940.

(130) Vaslow, F. The Apparent Molal Volumes of the Lithium and Sodium Halides. Critical Type Transitions in Aqueous Solution. J. Phys. Chem. 1969, 73, 3745-3750.

molality

0.05
0.1
0.5
1
3
4
6
8




0.05
0.1
0.5
1
2
3
4
6
8

$\begin{array}{crr}\mathbf{t} /{ }^{\circ} \mathrm{C} \text { mass frac } & \begin{array}{r}\text { Density } \\ \text { exp } / \\ \mathrm{kg} / \mathrm{m}^{3}\end{array} \\ 15 & 0.0051 & 1003.09 \\ 15 & 0.0102 & 1007.14 \\ 15 & 0.0489 & 1038.27 \\ 15 & 0.0933 & 1076.03 \\ 15 & 0.2359 & 1214.33 \\ 15 & 0.2916 & 1277.12 \\ 15 & 0.3817 & 1392.05 \\ 15 & 0.4515 & 1494.20 \\ 17 & 0.0400 & 1029.80 \\ 17 & 0.0800 & 1063.10 \\ 17 & 0.1000 & 1080.30 \\ 17 & 0.1200 & 1098.10 \\ 17 & 0.2000 & 1174.50 \\ 20 & 0.0051 & 1002.16 \\ 20 & 0.0102 & 1006.17 \\ 20 & 0.0489 & 1037.04 \\ 20 & 0.0933 & 1074.50 \\ 20 & 0.1707 & 1145.45 \\ 20 & 0.2359 & 1211.84 \\ 20 & 0.2916 & 1274.28 \\ 20 & 0.3817 & 1388.66 \\ 20 & 0.4515 & 1490.45\end{array}$

\section{Ref}

\section{App vol}

App vol

$\mathrm{m}^{3} / \mathrm{kg} \quad \mathrm{m}^{3} / \mathrm{kg}$

$0.000223 \quad 0.000232$

0.0002160 .000232

$0.000229 \quad 0.000236$

$0.000234 \quad 0.000239$

$0.000249 \quad 0.000251$

$0.000254 \quad 0.000255$

$\begin{array}{lll}0.000261 & 0.000262\end{array}$

$\begin{array}{ll}0.000266 & 0.000267\end{array}$

$0.000247 \quad 0.000236$

$0.000244 \quad 0.000240$

$0.000246 \quad 0.000241$

$0.000247 \quad 0.000243$

$0.000252 \quad 0.000249$

$0.000229 \quad 0.000236$

$0.000223 \quad 0.000236$

$0.000235 \quad 0.000239$

$0.000239 \quad 0.000243$

$0.000247 \quad 0.000249$

$0.000253 \quad 0.000254$

$0.000257 \quad 0.000258$

$0.000264 \quad 0.000264$

$0.000269 \quad 0.000269$ calc / Residual / Residual / Density based on density apparen $\mathrm{kg} / \mathrm{m}^{3} \quad \mathrm{~kg} / \mathrm{m}^{3} \quad \mathrm{~m}^{3} / \mathrm{kg}$ Residual density $\begin{gathered}\text { apparent } \\ \text { volume }\end{gathered}$

$\begin{array}{llll}1003.04 & 0.0464 & -0.000009 & 0.002\end{array}$

$1006.97 \quad 0.1662-0.000016 \quad 0.028$

$\begin{array}{llll}0.97 .93 & 0.3444 & -0.000007 & 0.119\end{array}$

$1075.43 \quad 0.5959-0.000006 \quad 0.355$

$1213.62 \quad 0.7062-0.000002 \quad 0.499$

$\begin{array}{llll}1276.41 & 0.7130 & -0.000001 & 0.508\end{array}$

$1391.22 \quad 0.8343-0.000001 \quad 0.696$

$\begin{array}{llll}1493.66 & 0.5445 & -0.000001 & 0.296\end{array}$

$\begin{array}{llll}1030.26 & -0.4554 & 0.000011 & 0.207\end{array}$

$\begin{array}{llll}1063.49 & -0.3862 & 0.000004 & 0.149\end{array}$

$\begin{array}{llll}1080.81 & -0.5054 & 0.000004 & 0.255\end{array}$

$\begin{array}{llll}1098.62 & -0.5214 & 0.000004 & 0.272\end{array}$

$\begin{array}{llll}1175.32 & -0.8169 & 0.000003 & 0.667\end{array}$

$\begin{array}{llll}1002.13 & 0.0326 & -0.000006 & 0.001\end{array}$

$1006.04 \quad 0.1332-0.000013 \quad 0.018$

$1036.83 \quad 0.2144-0.000004 \quad 0.046$

$\begin{array}{llll}1074.14 & 0.3630 & -0.000003 & 0.132\end{array}$

$\begin{array}{llll}1145.10 & 0.3455 & -0.000002 & 0.119\end{array}$

$1211.60 \quad 0.2362-0.000001 \quad 0.056$

$\begin{array}{llll}1274.06 & 0.2196 & 0.000000 & 0.048\end{array}$

$\begin{array}{llll}1388.27 & 0.3854 & -0.000001 & 0.149\end{array}$

$\begin{array}{llll}1490.19 & 0.2631 & 0.000000 & 0.069\end{array}$
Apparent Density of molal water $/ \mathrm{kg} / \mathrm{m}^{3}$ volume / $\mathrm{cm}^{3} / \mathrm{mol}$

999.0996 999.0996 999.0996 999.0996 999.0996 999.0996 999.0996 999.0996 998.7749 998.7749 998.7749 998.7749 998.7749 998.2041 998.2041 998.2041 998.2041 998.2041 998.2041 998.2041 998.2041 998.2041 
molality molarity

$\mathbf{t} /{ }^{\circ} \mathrm{C}$ mass frac Density

$\exp /$
$\mathrm{kg} / \mathrm{m}^{3}$

0.05

0.05

0.05

0.1

0.1

0.1003

0.10079

0.2

0.20023

0.2507

0.29966

0.3

0.39954

0.4
9932

0.49932
0.5

0.5
0.5
5017

0.5017

0.59956

0.6

0.69869

0.8
0.80033

0.89927

1.00023

1.004

1.2

1.4

1.6

1.8

2
.006
25

$0.0051 \quad 1001.01$

$0.0051 \quad 1000.97$

$0.0051 \quad 1000.98$

$0.0082 \quad 1003.40$

$0.0102 \quad 1004.95$

$0.0102 \quad 1004.89$

$0.0102 \quad 1004.94$

$0.0103 \quad 1004.98$

$0.0113 \quad 1005.80$

$0.0153 \quad 1008.70$

$0.0202 \quad 1012.65$

$0.0202 \quad 1012.70$

$0.0251 \quad 1016.59$

$0.0299 \quad 1020.36$

$0.0299 \quad 1020.35$

$0.0395 \quad 1027.99$

$0.0395 \quad 1027.99$

$0.0489 \quad 1035.54$

$0.0489 \quad 1035.58$

$0.0489 \quad 1035.58$

$0.0491 \quad 1035.73$

$0.0581 \quad 1043.08$

$0.0581 \quad 1043.10$

$0.0653 \quad 1049.00$

$0.0671 \quad 1050.48$

$0.0672 \quad 1050.57$

$0.0761 \quad 1057.99$

$0.0761 \quad 1058.00$

$0.0847 \quad 1065.29$

$0.0848 \quad 1065.36$

$0.0933 \quad 1072.76$

$0.0933 \quad 1072.68$

$0.0933 \quad 1072.65$

$0.0936 \quad 1073.06$

$0.1099 \quad 1087.15$

$0.1299 \quad 1105.05$

$0.1414 \quad 1115.52 \quad(130)$

$0.1563 \quad 1129.42 \quad(130)$

$0.1707 \quad 1143.24$

$0.1711 \quad 1143.70$
Ref

exp / Calc/

$\mathrm{m}^{3} / \mathrm{kg} \quad \mathrm{m}^{3} / \mathrm{kg}$

$0.000228 \quad 0.000239$

$0.000235 \quad 0.000239$

$0.000233 \quad 0.000239$

$0.000230 \quad 0.000239$

$0.000228 \quad 0.000239$

$\begin{array}{ll}0.000234 & 0.000239\end{array}$

$0.000231 \quad 0.000239$

$0.000231 \quad 0.000239$

$0.000227 \quad 0.000239$

$0.000245 \quad 0.000240$

$0.000236 \quad 0.000240$

$0.000235 \quad 0.000240$

$\begin{array}{ll}0.000236 & 0.000241\end{array}$

$0.000237 \quad 0.000241$

$0.000238 \quad 0.000241$

$0.000238 \quad 0.000242$

$0.000239 \quad 0.000242$

$0.000240 \quad 0.000242$

$0.000240 \quad 0.000242$

$0.000240 \quad 0.000242$

$0.000240 \quad 0.000242$

$0.000241 \quad 0.000243$

$0.000241 \quad 0.000243$

$0.000242 \quad 0.000244$

$0.000242 \quad 0.000244$

0.0002420 .000244

$0.000243 \quad 0.000245$

$0.000244 \quad 0.000245$

$0.000244 \quad 0.000245$

$0.000244 \quad 0.000245$

$0.000244 \quad 0.000246$

$0.000245 \quad 0.000246$

$0.000245 \quad 0.000246$

$0.000244 \quad 0.000246$

$\begin{array}{ll}0.000247 & 0.000247\end{array}$

$0.000248 \quad 0.000248$

$\begin{array}{ll}0.000248 & 0.000249\end{array}$

$0.000249 \quad 0.000250$

$0.000251 \quad 0.000251$

$0.000251 \quad 0.000252$

$0.000251 \quad 0.000252$
Dens calc/ Residual / Residual / Density based on based on $\mathrm{kg} / \mathrm{m}^{3} \quad \mathrm{~kg} / \mathrm{m}^{3} \quad \mathrm{~m}^{3} / \mathrm{kg}$ Residual density $\begin{gathered}\text { apparen } \\ \text { volume }\end{gathered}$

$1000.95 \quad 0.0577 \quad-0.000011 \quad 0.003$

$1000.95 \quad 0.0223-0.000004 \quad 0.000$

$\begin{array}{llll}000.95 & 0.0318 & -0.000006 & 0.001\end{array}$

$\begin{array}{llll}1003.32 & 0.0792 & -0.000010 & 0.006\end{array}$

$\begin{array}{llll}1004.84 & 0.1133 & -0.000011 & 0.013\end{array}$

$\begin{array}{llll}004.84 & 0.0520 & -0.000005 & 0.003\end{array}$

$\begin{array}{llll}1004.86 & 0.0823 & -0.000008 & 0.007\end{array}$

$\begin{array}{llll}1004.90 & 0.0819 & -0.000008 & 0.007\end{array}$

$\begin{array}{llll}1005.66 & 0.1397 & -0.000012 & 0.020\end{array}$

$\begin{array}{llll}1008.78 & -0.0830 & 0.000005 & 0.007\end{array}$

$\begin{array}{llll}1012.57 & 0.0787 & -0.000004 & 0.006\end{array}$

$1012.59 \quad 0.1081-0.000005 \quad 0.012$

$1016.48 \quad 0.1143-0.000004 \quad 0.013$

$1020.23-0.1284-0.000004$

$\begin{array}{llll}1020.26 & 0.0944 & -0.000003 & 0.009\end{array}$

$\begin{array}{llll}1027.85 & 0.1371 & -0.000003 & 0.019\end{array}$

$1027.89 \quad 0.1052-0.000003 \quad 0.011$

$1035.41 \quad 0.1258-0.000002 \quad 0.016$

$\begin{array}{llll}1035.47 & 0.1145 & -0.000002 & 0.013\end{array}$

$\begin{array}{llll}1035.47 & 0.1097 & -0.000002 & 0.012\end{array}$

$\begin{array}{llll}1035.59 & 0.1312 & -0.000002 & 0.017\end{array}$

$\begin{array}{llll}1042.96 & 0.1219 & -0.000002 & 0.015\end{array}$

$\begin{array}{llll}1042.99 & 0.1102 & -0.000002 & 0.012\end{array}$

$1048.88 \quad 0.1238-0.000002 \quad 0.015$

$1050.37 \quad 0.1124-0.000002 \quad 0.013$

$\begin{array}{llll}1050.47 & 0.1021 & -0.000001 & 0.010\end{array}$

$1057.89 \quad 0.1013-0.000001 \quad 0.010$

$\begin{array}{llll}1057.91 & 0.0872 & -0.000001 & 0.008\end{array}$

$\begin{array}{llll}1065.21 & 0.0824 & -0.000001 & 0.007\end{array}$

$\begin{array}{llll}1065.26 & 0.0950 & -0.000001 & 0.009\end{array}$

$1072.58 \quad 0.1758-0.000002 \quad 0.031$

$\begin{array}{llll}2.58 & 0.0931 & -0.000001 & 0.009\end{array}$

$\begin{array}{llll}072.60 & 0.0490 & 0.000000 & 0.002\end{array}$

$\begin{array}{llll}072.88 & 0.1801 & -0.000002 & 0.032\end{array}$

$\begin{array}{llll}1087.08 & 0.0634 & 0.000000 & 0.004\end{array}$

$\begin{array}{llll} & 0.0379 & 0.000000 & 0.001\end{array}$

$\begin{array}{llll}105.02 & 0.0276 & 0.000000 & 0.001\end{array}$

$0.0097 \quad 0.000000$

$\begin{array}{llll}1129.44 & -0.0177 & 0.000000 & 0.000\end{array}$

$\begin{array}{llll}1143.19 & 0.0513 & 0.000000 & 0.003\end{array}$

1143.60

$0.0973 \quad 0.000000$

0.009
Apparent Density of molal water $/ \mathrm{kg} / \mathrm{m}^{3}$ volume

23.43997 .0449

997.0449

$23.95 \quad 997.0449$

997.0449

997.0449

24.11997 .0449

23.81997 .0449

997.0449

997.0449

997.0449

$24.32 \quad 997.0449$

997.0449

$24.3 \quad 997.0449$

997.0449

$24.48 \quad 997.0449$

997.0449

24.61997 .0449

997.0449

997.0449

$24.73 \quad 997.0449$

24.69997 .0449 997.0449

$24.84 \quad 997.0449$

997.0449

997.0449

$24.95 \quad 997.0449$

$25.04 \quad 997.0449$

997.0449

997.0449

$25.13 \quad 997.0449$ 997.0449

$25.21 \quad 997.0449$

997.0449

$25.13 \quad 997.0449$

25.38997 .0449

$25.53 \quad 997.0449$

997.0449

25.67997 .0449

$25.8 \quad 997.0449$

997.0449

$25.86 \quad 997.0449$ 


\section{molality molarity}

3

$\begin{array}{rr}3.013 & \\ & 2.933968 \\ 4 & \\ & 4.20558 \\ 4.972 & \\ 6 & \\ & 5.53142 \\ 8 & \\ & 6.765459 \\ 0.05 & \end{array}$

0.1

0.1098

0.1497

0.5

1

3

0.05

0.1

0.5

1.06

2
2.548

4.654

9.448
9.91
0.05

05

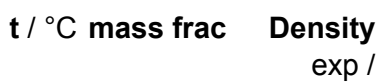

$\exp /$
$\mathrm{kg} / \mathrm{m}^{3}$

25

$0.2359 \quad 1209.32$

$0.2367 \quad 1210.25$

$0.2472 \quad 1221.23$

$0.2916 \quad 1271.44$

$0.3289 \quad 1315.82$

$0.3385 \quad 1327.72$

$0.3817 \quad 1385.25$

$0.4027 \quad 1413.48$

$0.4515 \quad 1486.63$

$0.4629 \quad 1503.82$

$0.0051 \quad 999.53$

$0.0102 \quad 1003.49$

$0.0112 \quad 1004.40$

$0.0153 \quad 1007.40$

$0.0489 \quad 1033.91$

$0.0933 \quad 1070.87$

$0.1707 \quad 1140.97$

$0.2359 \quad 1206.66$

$0.2916 \quad 1268.50$

$0.3817 \quad 1381.77$

$0.4515 \quad 1482.75$

$0.0051 \quad 997.90$

$0.0102 \quad 1001.83$

$0.0489 \quad 1032.07$

$0.0933 \quad 1068.83$

$0.0983 \quad 1073.92$

$0.1707 \quad 1138.58$

$0.2077 \quad 1174.43$

$0.2359 \quad 1203.95$

$0.2916 \quad 1265.54$

$0.3238 \quad 1302.94$

$0.3817 \quad 1378.33$

$0.4515 \quad 1478.91$

$0.4929 \quad 1544.00$

$0.5049 \quad 1565.50$

$0.0051 \quad 996.06$

$0.0102 \quad 999.97$

$\begin{array}{ll}0.0489 & 1030.07\end{array}$

$0.0933 \quad 1066.64$

$0.1707 \quad 1136.07$

$0.2359 \quad 1201.15$

Ref

exp / Calc /

$$
\begin{array}{cc}
\exp / & \text { Calc / } \\
\mathrm{m}^{3} / \mathrm{kg} & \mathrm{m}^{3} / \mathrm{kg}
\end{array}
$$

Dens

Density App Vol Square of Inconsistent Inconsistent calc / Residual / Residual / Density based on based on $\mathrm{kg} / \mathrm{m}^{3} \quad \mathrm{~kg} / \mathrm{m}^{3} \quad \mathrm{~m}^{3} / \mathrm{kg}$ Residual density apparen

$0.000257 \quad 0.000257$ $0.000256 \quad 0.000257$

$0.000258 \quad 0.000257$

$0.000261 \quad 0.000260$

$0.000264 \quad 0.000263$

$0.000265 \quad 0.000264$

$0.000267 \quad 0.000267$

$0.000269 \quad 0.000268$

$0.000271 \quad 0.000271$

$0.000273 \quad 0.000272$

$0.000242 \quad 0.000242$

$0.000234 \quad 0.000243$

$0.000226 \quad 0.000243$

$0.000238 \quad 0.000243$

$0.000245 \quad 0.000245$

$0.000248 \quad 0.000249$

$0.000255 \quad 0.000254$

$0.000260 \quad 0.000259$

$0.000263 \quad 0.000263$

$0.000269 \quad 0.000269$

$0.000274 \quad 0.000274$

$0.000244 \quad 0.000245$

$0.000237 \quad 0.000246$

$0.000248 \quad 0.000248$

$0.000251 \quad 0.000252$

$0.000245 \quad 0.000252$

$0.000258 \quad 0.000257$

0.0002620 .000260

$0.000262 \quad 0.000261$

$0.000266 \quad 0.000265$

0.0002690 .000267

$0.000271 \quad 0.000271$

$0.000276 \quad 0.000275$

0.0002790 .000278

$\begin{array}{ll}0.000279 & 0.000279\end{array}$

$0.000248 \quad 0.000248$

$0.000241 \quad 0.000248$

$0.000251 \quad 0.000251$

$0.000254 \quad 0.000254$

$0.000260 \quad 0.000259$

$0.000265 \quad 0.000264$ $\begin{array}{llll}1209.35 & -0.0321 & 0.000000 & 0.001\end{array}$

$\begin{array}{llll}1210.18 & 0.0677 & 0.000000 & 0.005\end{array}$

$\begin{array}{llll}1221.55 & -0.3160 & 0.000001 & 0.100\end{array}$

$\begin{array}{llll}1271.50 & -0.0569 & 0.000000 & 0.003\end{array}$

$\begin{array}{llll}1316.41 & -0.5835 & 0.000001 & 0.340\end{array}$

$\begin{array}{llll}1328.40 & -0.6758 & 0.000001 & 0.457\end{array}$

$\begin{array}{llll}1385.15 & 0.1002 & 0.000000 & 0.010\end{array}$

$\begin{array}{llll}1414.25 & -0.7671 & 0.000001 & 0.589\end{array}$

$\begin{array}{llll}1486.57 & 0.0591 & 0.000000 & 0.003\end{array}$

$\begin{array}{llll}1504.46 & -0.6402 & 0.000001 & 0.410\end{array}$

$\begin{array}{llll}999.53 & 0.0000 & 0.000000 & 0.000\end{array}$

$\begin{array}{llll}1003.40 & 0.0910 & -0.000009 & 0.008\end{array}$

$\begin{array}{llll}1004.21 & 0.1853 & -0.000016 & 0.034\end{array}$

$\begin{array}{llll}1007.32 & 0.0755 & -0.000005 & 0.006\end{array}$

$1033.87 \quad 0.0387-0.000001 \quad 0.002$

$\begin{array}{llll}1070.80 & 0.0679 & -0.000001 & 0.005\end{array}$

$\begin{array}{lll}141.06 & -0.0853 & 0.000000\end{array}$

$\begin{array}{llll}1206.90 & -0.2363 & 0.000001 & 0.056\end{array}$

$\begin{array}{llll}1268.74 & -0.2442 & 0.000001 & 0.060\end{array}$

$\begin{array}{llll}1381.87 & -0.0990 & 0.000000 & 0.010\end{array}$

$\begin{array}{llll}1482.83 & -0.0847 & 0.000000 & 0.007\end{array}$

$\begin{array}{llll}997.89 & 0.0051 & -0.000001 & 0.000\end{array}$

$\begin{array}{llll}1001.74 & 0.0855 & -0.000008 & 0.007\end{array}$

$\begin{array}{llll}1032.06 & 0.0061 & 0.000000 & 0.000\end{array}$

$\begin{array}{llll}1068.81 & 0.0178 & 0.000000 & 0.000\end{array}$

$\begin{array}{llll}073.14 & 0.7810 & -0.000007 & 0.610\end{array}$

$\begin{array}{llll}1138.73 & -0.1461 & 0.000001 & 0.021\end{array}$

$\begin{array}{llll}1175.15 & -0.7225 & 0.000003 & 0.522\end{array}$

$\begin{array}{llll}1204.26 & -0.3084 & 0.000001 & 0.095\end{array}$

$\begin{array}{llll}1265.82 & -0.2845 & 0.000001 & 0.081\end{array}$

$\begin{array}{llll}1304.12 & -1.1779 & 0.000002 & 1.387\end{array}$

$\begin{array}{llll}378.45 & -0.1239 & 0.000000 & 0.015\end{array}$

$\begin{array}{llll}1479.00 & -0.0889 & 0.000000 & 0.008\end{array}$

$\begin{array}{lll}1545.33 & -1.3273 & 0.000001\end{array}$

$\begin{array}{llll}1565.46 & 0.0377 & 0.000000\end{array}$

$\begin{array}{lll}996.06 & 0.0004 & 0.000000\end{array}$

$\begin{array}{llll}999.89 & 0.0799 & -0.000008\end{array}$

$\begin{array}{lll}1030.06 & 0.0093 & 0.000000\end{array}$

$0.0081 \quad 0.000000$

$\begin{array}{lll}1136.22 & -0.1488 & 0.000001\end{array}$

1201.46

$\begin{array}{ll}-0.3063 & 0.000001\end{array}$

0.001

0.000

0.006

0.000

0.000

0.022

0.094
1.762
Apparent Density of molal water $/ \mathrm{kg} / \mathrm{m}^{3}$

volume /
$\mathrm{cm}^{3} / \mathrm{mol}$

997.0449

$26.38 \quad 997.0449$

997.0449

997.0449

997.0449

$27.26 \quad 997.0449$

997.0449

997.0449

997.0449

997.0449

995.6473

995.6473

995.6473

995.6473

995.6473

995.6473

995.6473

995.6473

995.6473

995.6473

995.6473

994.0319

994.0319

994.0319

994.0319

994.0319

994.0319

994.0319

994.0319

994.0319

994.0319

994.0319

994.0319

994.0319

994.0319

992.2158

992.2158

992.2158

992.2158

992.2158

992.2158 


\section{molality molarity}

$\mathbf{t} /{ }^{\circ} \mathrm{C}$ mass frac Density

4
6
8
0.05
0.1
0.5
1
2
3
4
6
8
10.81
0.05
0.1
0.5
1
2
3
4
6
8
11.32
0.05
0.1
0.5
1
2
3
4
6
11.45
11.79
$\exp$

$\mathrm{kg} / \mathrm{m}^{3}$

$\begin{array}{rrr}\mathbf{t} /{ }^{\circ} \mathrm{C} \text { mass frac } & \begin{array}{r}\text { Density } \\ \text { exp } / \\ \mathrm{kg} / \mathrm{m}^{3}\end{array} \\ & & 1262.53 \\ 40 & 0.2916 & 1374.89 \\ 40 & 0.3817 & 135.03 \\ 40 & 0.4515 & 1475.04 \\ 45 & 0.0051 & 994.04 \\ 45 & 0.0102 & 997.93 \\ 45 & 0.0489 & 1027.89 \\ 45 & 0.0933 & 1064.33 \\ 45 & 0.1707 & 1133.47 \\ 45 & 0.2359 & 1198.28 \\ 45 & 0.2916 & 1259.48 \\ 45 & 0.3817 & 1371.46 \\ 45 & 0.4515 & 1471.17 \\ 45.21 & 0.5266 & 1595.00 \\ 50 & 0.0051 & 991.85 \\ 50 & 0.0102 & 995.72 \\ 50 & 0.0489 & 1025.55 \\ 50 & 0.0933 & 1061.87 \\ 50 & 0.1707 & 1130.79 \\ 50 & 0.2359 & 1195.38 \\ 50 & 0.2916 & 1256.41 \\ 50 & 0.3817 & 1367.89 \\ 50 & 0.4515 & 1467.32 \\ 50.21 & 0.5381 & 1610.80 \\ 55 & 0.0051 & 989.50 \\ 55 & 0.0102 & 993.35 \\ 55 & 0.0489 & 1023.11 \\ 55 & 0.0933 & 1059.32 \\ 55 & 0.1707 & 1128.03 \\ 55 & 0.2359 & 1192.42 \\ 55 & 0.2916 & 1253.27 \\ 55 & 0.3817 & 1364.46 \\ 55 & 0.4515 & 1463.47 \\ 60.17 & 0.5409 & 1607.30 \\ 75.44 & 0.5441 & 1598.60 \\ 91.95 & 0.5482 & 1591.50 \\ & & \end{array}$

Ref

A

0

App vol App vol Der

$\begin{array}{cc}\exp / & \text { Calc / } \\ \mathrm{m}^{3} / \mathrm{kg} & \mathrm{m}^{3} / \mathrm{kg}\end{array}$

Density

Density App Vol Square of Inconsistent Inconsistent calc / Residual / Residual / Density based on based on $\mathrm{kg} / \mathrm{m}^{3} \quad \mathrm{~kg} / \mathrm{m}^{3} \quad \mathrm{~m}^{3} / \mathrm{kg}$ Residual density apparent

$0.000268 \quad 0.000267$

$\begin{array}{ll}0.000273 & 0.000273\end{array}$

$0.000277 \quad 0.000277$

1262.76

1374.92

1475.08

994.04

$0.000243 \quad 0.000251$

997.85

1027.88

$\begin{array}{ll}0.000253 & 0.000254\end{array}$

$\begin{array}{ll}0.000256 & 0.000257\end{array}$

$0.000262 \quad 0.000262$

$0.000266 \quad 0.000266$

0.0002690 .000269

$0.000274 \quad 0.000275$

0.0002790 .000279

$0.000283 \quad 0.000283$

$0.000252 \quad 0.000253$

$0.000245 \quad 0.000253$

$0.000256 \quad 0.000256$

$0.000258 \quad 0.000259$

$0.000263 \quad 0.000264$

$\begin{array}{ll}0.000268 & 0.000268\end{array}$

$0.000271 \quad 0.000271$

$0.000276 \quad 0.000276$

$0.000280 \quad 0.000280$

$0.000285 \quad 0.000285$

$0.000252 \quad 0.000255$

$0.000247 \quad 0.000256$

$0.000256 \quad 0.000258$

$0.000259 \quad 0.000261$

$0.000264 \quad 0.000266$

$0.000269 \quad 0.000270$

$0.000272 \quad 0.000273$

$\begin{array}{ll}0.000277 & 0.000278\end{array}$

0.0002810 .000282

$\begin{array}{lll}0.000287 & 0.000287\end{array}$

$0.000290 \quad 0.000290$

$0.000291 \quad 0.000291$

\begin{abstract}
Average Res
\end{abstract}
Std dev Res

Avg - 4std

Avg + 4std
$-0.2255 \quad 0.000000$

$\begin{array}{ll}-0.0315 & 0.000000\end{array}$

$\begin{array}{ll}-0.0499 & 0.000000\end{array}$

$\begin{array}{ll}0.0016 & 0.000000\end{array}$

$0.0797-0.000008$

$0.0138 \quad 0.000000$

$0.0541-0.000001$

$\begin{array}{ll}-0.0782 & 0.000000\end{array}$

$\begin{array}{ll}-0.2244 & 0.000001\end{array}$

$\begin{array}{ll}-0.0714 & 0.000000\end{array}$

$0.1746 \quad 0.000000$

$0.0801 \quad 0.000000$

$0.5752 \quad 0.000000$

$0.0067-0.000001$

$0.0829-0.000008$

$0.0276-0.000001$

$0.1137-0.000001$

$0.0637 \quad 0.000000$

$\begin{array}{ll}-0.0348 & 0.000000\end{array}$

0.18610 .000000

$0.3337 \quad 0.000000$

$0.2814 \quad 0.000000$

$0.2341 \quad 0.000000$

$0.0155-0.000003$

$0.0893-0.000009$

$0.1003-0.000002$

$0.2367-0.000002$

$0.2228-0.000001$

$0.4876-0.000001$

$0.7174-0.000001$

$0.5367-0.000001$

$\begin{array}{ll}0.4813 & 0.000000\end{array}$

$\begin{array}{ll}-0.4673 & 0.000000\end{array}$

$\begin{array}{ll}-0.2324 & 0.000000\end{array}$

$0.0309-0.000002$

$0.3213 \quad 0.000004$

$-1.2543-0.000017$

$\begin{array}{lll}1.3161 & 0.000014\end{array}$
$0.2668-0.000001$
0.051

0.001

0.002

0.000

0.006

0.000

0.003

0.006

0.050

0.005

0.030

0.006

0.331

0.000

0.007

0.001

0.013

0.004

0.001

0.035

0.111

0.079

0.055

0.000

0.008

0.010

0.056

0.071

0.050

0.238

0.515

0.288

0.232

0.218

0.054

14.38 Sum chi sqr
Apparent Density of molal water $/ \mathrm{kg} / \mathrm{m}^{3}$ volume /

992.2158

992.2158

992.2158

990.2132

990.2132

990.2132

990.2132

990.2132

990.2132

990.2132

990.2132

990.2132

990.1252

988.0363

988.0363

988.0363

988.0363

988.0363

988.0363

988.0363

988.0363

988.0363

987.9412

985.6952

985.6952

985.6952

985.6952

985.6952

985.6952

985.6952

985.6952

985.6952

983.1114

974.5856

964.0005

Curve fit for the model App vol $=\left(w+c 2+c 3^{*} t{ }^{\circ} \mathrm{C}\right) /\left(\mathrm{c} 0^{*} \mathrm{w}+\mathrm{c} 1\right) / \mathrm{e}^{\wedge}\left(0.000001^{*}\left(\mathrm{t}{ }^{\circ} \mathrm{C}+\mathrm{c} 4\right)^{\wedge} 2\right)$ 
molality molarity

$\mathrm{t} /{ }^{\circ} \mathrm{C}$ mass frac Density

$\exp /$

$\mathrm{kg} / \mathrm{m}^{3}$
Ref

$\begin{array}{rr}\exp / & \text { Calc / } \\ \mathrm{m}^{3} / \mathrm{kg} & \mathrm{m}^{3} / \mathrm{kg}\end{array}$

De
Density Density App Vol Square of Inconsistent Inconsistent calc / Residual / Residual / Density based on based on $\mathrm{kg} / \mathrm{m}^{3} \quad \mathrm{~kg} / \mathrm{m}^{3} \quad \mathrm{~m}^{3} / \mathrm{kg}$ Residual density apparent
Apparent Density of molal water $/ \mathrm{kg} / \mathrm{m}^{3}$ volume / volume
$\mathrm{cm}^{3} / \mathrm{mol}$

$\begin{array}{lr}\text { c0 } & 109.7728 \\ \text { c1 } & 513.0387 \\ \text { c2 } & 1.545407 \\ \text { c3 } & 0.011019 \\ \text { c4 } & 1618.137 \\ \text { Min T } & 15 \\ \text { Max T } & 91.95 \\ \text { Min w } & 0.0051 \\ \text { Max w } & 0.548161 \\ \text { Avg dens res } & 0.030904 \\ \text { Std dens res } & 0.321297 \\ \text { Avg app vol res *10^33 } & -0.001816 \\ \text { Std app vol res *10^3 } & 0.003864 \\ \text { No of points in corr } & 139 \\ \text { No of inconsistent poir } & 20\end{array}$

Inconsistent data not used (Residual greater than average + /- $\mathbf{4}$ standard deviations) 7.78 2

$$
\begin{array}{r} 
\\
\\
\\
\\
0.0104 \\
0.0503 \\
0.0104 \\
0.0502 \\
0.08 \\
0.0104 \\
0.0501 \\
0.0799 \\
0.1097 \\
0.1494 \\
0.0103 \\
0.05 \\
0.0797 \\
0.1095 \\
0.1492
\end{array}
$$

$\begin{array}{rrr}0 & 0.4446 & 1495.70 \\ 15 & 0.1707 & 1047.45 \\ 17 & 0.0100 & 1006.00 \\ 17 & 0.0200 & 1013.90 \\ 17 & 0.3000 & 1284.10 \\ 17 & 0.4000 & 1413.80 \\ 25 & 0.0011 & 998.00 \\ 25 & 0.0052 & 1001.10 \\ 30 & 0.0011 & 996.50 \\ 30 & 0.0052 & 999.90 \\ 30 & 0.0082 & 1002.10 \\ 35 & 0.0011 & 1003.20 \\ 35 & 0.0051 & 1005.90 \\ 35 & 0.0082 & 1008.10 \\ 35 & 0.0112 & 1011.00 \\ 35 & 0.0152 & 1013.10 \\ 40 & 0.0011 & 1001.40 \\ 40 & 0.0051 & 1005.20 \\ 40 & 0.0081 & 1007.30 \\ 40 & 0.0112 & 1010.00 \\ 40 & 0.0152 & 1012.90\end{array}$

$\begin{array}{lrl}(118) & 0.000254 & 0.000258 \\ (45) & 0.000730 & 0.000246 \\ (90) & 0.000282 & 0.000234 \\ (90) & 0.000254 & 0.000235 \\ (90) & 0.000260 & 0.000257 \\ (90) & 0.000266 & 0.000264 \\ (24) & 0.000108 & 0.000239 \\ (24) & 0.000217 & 0.000239 \\ (24) & 0.000204 & 0.000242 \\ (24) & 0.000177 & 0.000242 \\ (24) & 0.000217 & 0.000242 \\ (24) & -0.007613 & 0.000245 \\ (24) & -0.001310 & 0.000245 \\ (24) & -0.000715 & 0.000245 \\ (24) & -0.000506 & 0.000246 \\ (24) & -0.000242 & 0.000246 \\ (24) & -0.007726 & 0.000248 \\ (24) & -0.001536 & 0.000248 \\ (24) & -0.000846 & 0.000248 \\ (24) & -0.000583 & 0.000248 \\ (24) & -0.000350 & 0.000249\end{array}$

$\begin{array}{rrrrr}1491.84 & 3.8564 & -0.000004 & 14.872 & ! \\ 1146.78 & -99.3255 & 0.000484 & 9865.554 & ! \\ 1006.49 & -0.4887 & 0.000048 & 0.239 & \\ 1014.31 & -0.4053 & 0.000020 & 0.164 & ! \\ 1285.47 & -1.3692 & 0.000003 & 1.875 & ! \\ 1415.58 & -1.7830 & 0.000002 & 3.179 & ! \\ 997.86 & 0.1397 & -0.000131 & 0.020 & \\ 1000.99 & 0.1129 & -0.000022 & 0.013 & \\ 996.46 & 0.0403 & -0.000038 & 0.002 & \\ 999.57 & 0.3339 & -0.000065 & 0.112 & \\ 1001.89 & 0.2088 & -0.000025 & 0.044 & ! \\ 994.83 & 8.3652 & -0.007857 & 69.977 & ! \\ 997.90 & 8.0002 & -0.001555 & 64.004 & ! \\ 1000.20 & 7.9007 & -0.000961 & 62.421 & ! \\ 1002.49 & 8.5079 & -0.000752 & 72.384 & ! \\ 1005.56 & 7.5393 & -0.000488 & 56.841 & ! \\ 993.01 & 8.3916 & -0.007973 & 70.418 & ! \\ 996.06 & 9.1406 & -0.001784 & 83.550 & ! \\ 998.34 & 8.9580 & -0.001094 & 80.245 & ! \\ 1000.63 & 9.3730 & -0.000831 & 87.853 & ! \\ 1003.67 & 9.2258 & -0.000599 & 85.115 & !\end{array}$


Density of aqueous solutions of $\mathrm{NaCl}$

References (13) Chen, C.-T. A.; Chen, J. H.; Millero, F. J. Densities of $\mathrm{NaCl}, \mathrm{MgCl}_{2}, \mathrm{Na}_{2} \mathrm{SO}_{4}$, and $\mathrm{MgSO}_{4} \mathrm{Aqueous}$ Solutions at 1 atm. from 0 to $50^{\circ} \mathrm{C}$ and from 0.001 to $1.5 \mathrm{~m}$. J. Chem. Eng. Data 1980, $25,307-310$.

(20) Dedick, Eugene A.; Hershey, J. Peter; Sotolongo, Sara; Stade, David J.; Millero, Frank J. The PVT Properties of Concentrated Aqueous Electrolytes IX. The Volume Properties of $\mathrm{KCl}$ and $\mathrm{K} 2 \mathrm{SO} 4$ and their Mixtures with $\mathrm{NaCl}$ and $\mathrm{Na} 2 \mathrm{SO} 4$ as a Function of Temperature, J. Solution Chem . 1990, 19, 353-374

(27) Fabuss, B. M.; Korosi, A.; Huq, A. K. M. S.; Densities of Binary and Ternary Aqueous Solutions of NaCl, Na2SO4, and MgSO4, of Sea Waters, and Sea Water Concentrates, J. Chem. Eng. Data, 1966, 3, 325-331

(28) Gates, J. A.; Wood, R. H. Densities of Aqueous Solutions of $\mathrm{NaCl}, \mathrm{MgCl} 2, \mathrm{KCl}, \mathrm{NaBr}, \mathrm{LiCl}$, and $\mathrm{CaCl} 2$ from 0.05 to $5.0 \mathrm{~mol} \mathrm{kg-1} \mathrm{and}$ 0.1013 to $40 \mathrm{MPa}$ at 298.15K. J. Chem. Eng. Data 1985, 30, 44-49.

(31) Gonçalves, F. Kestin, J. The Viscosity of $\mathrm{NaCl}$ and $\mathrm{KCl}$ solutions in the range $25-50{ }^{\circ} \mathrm{C}$, Ber. Bunsenges. Phys. Chem . 1977, 81, 11561161

(32) Gucker, F. T.; Stubley, D.; Hill, D. J. The Isentropic Compressibilities of Aqueous Solutions of Some Alkali Halides at 298.15K. J. Chem. Thermodynamics 1975, 7, 865-869.

(44) Isono, T. Measurements of Density, Viscosity, and Electrolytic Conductivity of Concentrated Aqueous Electrolyte Solutions. Rikagaku Kenkyusho Hokoku 1980, 56, 103-114.

(48) Kaminsky, M. Concentration and Temperature Dependence of the Viscosity of Aqueous Solutions of Strong Electrolytes. II. NaCl, Li2SO4, FeCl2, and CeCl3 Solutions. Z. Physik. Chem. N. F, 1956 , 8, 173-191.

(52) Korosi, A.; Fabuss, B. M. Viscosities of Binary Aqueous Solutions of $\mathrm{NaCl}, \mathrm{KCl}, \mathrm{Na2SO} 4$, and $\mathrm{MgSO} 4$ at Concentrations and Temperatures of Interest in Desalination Processes. J. Chem. Eng. Data 1968, 13, 548-552.

(62) Lengyel, S.; Tamás, J; Giber, J.; Holderith, J. Study of Viscosity of Aqueous Alkali Halide Solutions. Acta Chim. Acad. Sci. Hung. 1964, 40, 125-143.

(67) Manohar, S.; Puchalska, D.; Atkinson, G. Pressure-Volume-Temperature Properties of Aqueous Mized Electrolyte Solutions: $\mathrm{NaCl}+$ $\mathrm{BaCl} 2$ from $25 \mathrm{C}$ to $140^{\circ} \mathrm{C}$ J. Chem. Eng. Data 1994, 39, 150-154

(73) Millero, F. J.; Ward, G. K.; Chetirkin, P. V. Relative Sound Velocities of Sea Salts at $25^{\circ}$ C. J. Acoust. Soc. Am. 1977, $61,1492-1498$.

(74) Motin, M. A., Temperature and Concentration Dependence of Apparent Molar Volumes and Viscosities of $\mathrm{NaCl}, \mathrm{NH} 4 \mathrm{Cl}, \mathrm{CuCl} 2$, and MgSO4 in Pure Water and Water + Urea Mixtures, J. Chem. Eng. Data, 2004, 49, 94-98

(79) Olofsson, Inger $\mathrm{V}$.; Apparent molar heat capacities and volumes of aqueous $\mathrm{NaCl}, \mathrm{KCl}$, and $\mathrm{KNO}_{3}$ at $298.15 \mathrm{~K}$. Comparison of Picker flow calorimeter with other calorimeters, J. Chem. Thermodynamics, 1979, 11, 1005-1014

(80) Out, D. J. P.; Los, J. M. Viscosity of Aqueous Solutions of Univalent Electrolytes from 5 to $95^{\circ} \mathrm{C}$. J. Sol. Chem. 1980, 9, 19-35.

(89) Perron, G.; Fortier, J.. L.; Desnoyers, J. E.; Apparent Molar Heat Capacities and Volumes of Aqueous NaCl from 0.01 to $3 \mathrm{~mol} k \mathrm{~kg}-1$ in the Temperature Range 274.65 to 318.15 K., J. Chem. Thermodynamics, 1975, 7, 1177-1184

(90) Perry, R. H.; Green, D. W. Perry's Chemical Engineers' Handbook; McGraw Hill: New York, 7th edition, 1997.

(135) Zhang, H.-L.; Han, S-J. Viscosity and Density of Water + Sodium Chloride + Potassium Chloride Solutions at 298.15K. J. Chem. Eng. Data 1996, $41,516-520$

molality molarity

\section{$\mathrm{t} /{ }^{\circ} \mathrm{C}$ mass frac}

$\begin{aligned} \begin{array}{r}\text { Density } \\ \text { exp / }\end{array} & \text { Ref } \\ \mathrm{kg} / \mathrm{m}^{3} & \\ 1000.31 & (13) \\ 1001.70 & (13) \\ 1007.27 & (13) \\ 1007.47 & (90) \\ 1015.09 & (90) \\ 1021.68 & (13) \\ 1030.38 & (90) \\ 1042.13 & (13) \\ 1061.21 & (90)\end{aligned}$

App vol App vol exp / Calc/ $\mathrm{m}^{3} / \mathrm{kg} \quad \mathrm{m}^{3} / \mathrm{kg}$ $0.000218 \quad 0.000264$ 0.0002250 .000265 $0.000234 \quad 0.000267$ $0.000243 \quad 0.000267$ $0.000249 \quad 0.000270$ $0.000250 \quad 0.000272$ $0.000259 \quad 0.000275$ $0.000265 \quad 0.000279$ $0.000277 \quad 0.000286$

\author{
Density
} capp Incol Square of Instent Inconsistent calc/ Residual/ Residual/ Density based on based on $\mathrm{kg} / \mathrm{m}^{3} \quad \mathrm{~m}^{3} / \mathrm{kg}$ Residual volume

$\begin{array}{llll}1000.3 & 0.0277 & -0.000046 & 0.001\end{array}$

$\begin{array}{llll}1001.6 & 0.0950 & -0.000039 & 0.009\end{array}$

$1006.9-0.3219-0.000033-0.104$

$\begin{array}{llll}1007.2 & 0.2459 & -0.000024 & 0.060\end{array}$

$\begin{array}{llll}1014.7 & 0.4286 & -0.000021 & 0.184\end{array}$

$\begin{array}{llll}1021.0 & 0.6628 & -0.000022 & 0.439\end{array}$

$\begin{array}{llll}1029.7 & 0.6870 & -0.000016 & 0.472\end{array}$

$\begin{array}{llll}1041.2 & 0.8933 & -0.000015 & 0.798\end{array}$

$1060.4 \quad 0.8333-0.000009$

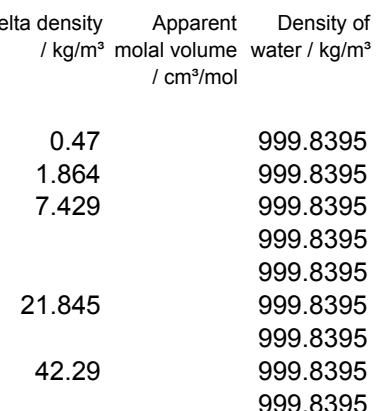


molality molarity

$t /{ }^{\circ} \mathrm{C}$ mass frac Density R

$$
\exp
$$

$\mathrm{kg} / \mathrm{m}^{3}$

$\begin{array}{lll}0 & 0.1200 & 1092.44\end{array}$

$0.1600 \quad 1124.19$

$0.2000 \quad 1156.63$

$\begin{array}{ll}0.2400 & 1189.99 \\ 0.2600 & 1207.09\end{array}$

0.06845

0.0934

0.12426

0.25724

0.33312

0.48319

0.50786

0.65563

0.84115

1.4309

1.8001

0.01029

0.02677

0.0412

0.06845

0.0934

0.09485

0.1242

0.1663

0.19849

0.2
5724

0.25724

0.32

0.33312
0.48319

0.5
5012

0.5019

0.50786

0.65563

0.72467

0.8
-84115

0.9471

0.9992

1.0012

1.2

1.49986
1.95

0.0986 $\begin{array}{lll}0 & 0.2600 & 1207.09 \\ 5 & 0.0040 & 1002.98\end{array}$

$\begin{array}{lll}5 & 0.0054 & 1004.08\end{array}$

$\begin{array}{lll}1.5 & 0.0072 & 1005.44 \\ 1.5 & 0.0148 & 1011.24\end{array}$

$\begin{array}{lll}0.0191 & 1014.47\end{array}$

$\begin{array}{ll}0.0275 & 1020.85\end{array}$

$\begin{array}{lll}0.0288 & 1021.87\end{array}$

$0.0369 \quad 1027.99$

0.04691035 .58

$0.0772 \quad 1058.80$

$0.0952 \quad 1072.76$

$0.0006 \quad 1000.42$

$0.0054 \quad 1004.04$

$0.0055 \quad 1004.12$

$0.0058 \quad 1004.29$

$0.0072 \quad 1005.37$

$0.0115 \quad 1008.59$

$0.0116 \quad 1008.57$

$0.0148 \quad 1011.06$

$0.0184 \quad 1013.66$

$0.0191 \quad 1014.27$

$\begin{array}{ll}0.0275 & 1020.53\end{array}$

$0.0284 \quad 1021.17$

$0.0285 \quad 1021.31$

$0.0285 \quad 1021.32$

$0.0288 \quad 1021.55$

$0.0369 \quad 1027.61$

$0.0406 \quad 1030.43$

$0.0447 \quad 1033.41$

$0.0469 \quad 1035.09$

$0.0524 \quad 1039.30$

$0.0552 \quad 1041.38$

$0.0553 \quad 1041.48$

$0.0655 \quad 1049.19$

$0.0806 \quad 1060.68$

$\begin{array}{ll}0.1023 & 1076.71 \\ 0.0057 & 1003.90\end{array}$

$\begin{array}{lll}0.0100 & 1007.07\end{array}$

$\begin{array}{lll}10 & 0.0200 & 1014.42\end{array}$
$0.0040 \quad 1002.96$

$0.0096 \quad 1007.22$
Ref

$$
\text { exp }
$$

exp / App vol

$\begin{array}{cc}\exp / & \text { Calc / } \\ \mathrm{m}^{3} / \mathrm{kg} & \mathrm{m}^{3} / \mathrm{kg}\end{array}$

$\mathrm{kg}$

$\begin{array}{lll}(90) & 0.000294 & 0.000298 \\ (90) & 0.000309 & 0.000309\end{array}$

$\begin{array}{lll}(90) & 0.000309 & 0.000309 \\ (90) & 0.000322 & 0.000320\end{array}$

$0.000334 \quad 0.000332$

$0.000340 \quad 0.000338$

$\begin{array}{lll}0.000236 & 0.000268\end{array}$

$\begin{array}{ll}0.000237 & 0.000269\end{array}$

0.0002390 .000269

$\begin{array}{ll}0.000245 & 0.000271 \\ 0.000249 & 0.000272\end{array}$

$\begin{array}{ll}0.000249 & 0.000272 \\ 0.000254 & 0.000275\end{array}$

$\begin{array}{ll}0.000255 & 0.000275\end{array}$

$\begin{array}{ll}0.000260 & 0.000277\end{array}$

$0.000265 \quad 0.000280$

0.0002790 .000288

$\begin{array}{lll}0.000287 & 0.000293\end{array}$

$\begin{array}{lll}0.000243 & 0.000274\end{array}$

$\begin{array}{lll}0.000248 & 0.000274\end{array}$

$\begin{array}{ll}0.000244 & 0.000275\end{array}$

$\begin{array}{lll}0.000251 & 0.000275\end{array}$

$0.000252 \quad 0.000275$

$0.000250 \quad 0.000275$

$0.000259 \quad 0.000275$

$0.000254 \quad 0.000276$

$\begin{array}{ll}0.000251 & 0.000276\end{array}$

$0.000254 \quad 0.000277$

$\begin{array}{ll}0.000261 & 0.000277 \\ 0.000259 & 0.000278\end{array}$

$0.000264 \quad 0.000279$

$\begin{array}{ll}0.000262 & 0.000279\end{array}$

$\begin{array}{lll}0.000266 & 0.000281\end{array}$

$\begin{array}{lll}0.000269 & 0.000282\end{array}$

$\begin{array}{lll}0.000266 & 0.000282\end{array}$

$\begin{array}{lll}0.000266 & 0.000282\end{array}$

$\begin{array}{ll}0.000267 & 0.000282\end{array}$

$\begin{array}{ll}0.000271 & 0.000284\end{array}$

$\begin{array}{ll}0.000272 & 0.000285\end{array}$

$\begin{array}{ll}0.000275 & 0.000286 \\ 0.000276 & 0.000287\end{array}$

$\begin{array}{lll}0.000276 & 0.000287 \\ 0.000278 & 0.000288\end{array}$

$0.000279 \quad 0.000289$

$\begin{array}{lll}0.000279 & 0.000289\end{array}$

$\begin{array}{ll}0.000284 & 0.000292\end{array}$

$0.000290 \quad 0.000296$

$\begin{array}{ll}0.000303 & 0.000302\end{array}$

$0.000270 \quad 0.000284$

$\begin{array}{lll}\text { (62) } & 0.000270 & 0.000284 \\ \text { (90) } & 0.000268 & 0.000285\end{array}$

(90)

$\begin{array}{ll}0.000275 & 0.000288\end{array}$ $\mathrm{kg} / \mathrm{m}^{3} \quad \mathrm{~kg} / \mathrm{m}^{3} \quad \mathrm{~m}^{3} / \mathrm{kg}$ Residual density apparent

$\begin{array}{llll}1091.9 & 0.5714 & -0.000004 & 0.326\end{array}$

$\begin{array}{llll}1124.1 & 0.0523 & 0.000000 & 0.003\end{array}$

$\begin{array}{llll}1157.1 & -0.5131 & 0.000002 & 0.263\end{array}$

$\begin{array}{llll}1190.8 & -0.8429 & 0.000002 & 0.710 \\ 1207.9 & -0.8248 & 0.000002 & 0.680\end{array}$

$1002.8 \quad 0.1299-0.000032$

$\begin{array}{llll}1003.9 & 0.1718 & -0.000031 & 0.030\end{array}$

$\begin{array}{llll}1005.2 & 0.2208 & -0.000030 & 0.049\end{array}$

$\begin{array}{llll}1010.8 & 0.4025 & -0.000027 & 0.162\end{array}$

$\begin{array}{llll}0.4589 & -0.000023 & 0.211 \\ 1020.2 & 0.6059 & -0.000021 & 0.367\end{array}$

$1027.3-0.6721-0.000017$

$\begin{array}{llll}1034.8 & 0.7456 & -0.000015 & 0.556\end{array}$

$\begin{array}{llll}1058.0 & 0.7848 & -0.000009 & 0.616\end{array}$

$\begin{array}{llll}1058.0 & 0.7848 & -0.000009 & 0.616\end{array}$

$1000.4 \quad 0.0185-0.000031$

$\begin{array}{llll}1001.1 & 0.0411 & -0.000026 & 0.002\end{array}$

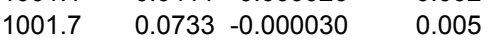

$\begin{array}{llll}1002.9 & 0.0967 & -0.000024 & 0.009\end{array}$

$\begin{array}{llll}1003.9 & 0.1263 & -0.000023 & 0.016\end{array}$

$\begin{array}{llll}1004.0 & 0.1432 & -0.000026 & 0.021\end{array}$

$\begin{array}{llll}1004.2 & 0.0985 & -0.000017 & 0.010\end{array}$

$\begin{array}{llll}1005.2 & 0.1614 & -0.000022 & 0.026\end{array}$

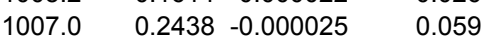

$\begin{array}{llll}1008.3 & 0.2705 & -0.000023 & 0.073\end{array}$

$\begin{array}{llll}1008.4 & 0.1825 & -0.000016 & 0.033\end{array}$

$\begin{array}{llll}1010.8 & 0.2868 & -0.000019 & 0.082\end{array}$

$\begin{array}{llll}1013.4 & 0.2790 & -0.000015 & 0.078\end{array}$

$\begin{array}{llll}1013.9 & 0.3436 & -0.000017 & 0.118\end{array}$

$\begin{array}{llll}1020.1 & 0.4334 & -0.000015 & 0.188\end{array}$

$\begin{array}{llll}1020.8 & 0.3832 & -0.000013 & 0.147\end{array}$

$\begin{array}{llll}1020.8 & 0.4750 & -0.000016 & 0.226 \\ 1020.9 & 0.4594 & -0.000015 & 0.211\end{array}$

$1027.1-0.5025-0.000013-0.253$

$\begin{array}{llll}1029.9 & 0.5425 & -0.000013 & 0.294\end{array}$

$\begin{array}{lllll}1032.9 & 0.5019 & -0.000011 & 0.252\end{array}$

$\begin{array}{llll}1034.5 & 0.5414 & -0.000011 & 0.293\end{array}$

$\begin{array}{llll}1038.8 & 0.5500 & -0.000010 & 0.302\end{array}$

$\begin{array}{llll}1040.8 & 0.5778 & -0.000010 & 0.334\end{array}$

$\begin{array}{llll}1040.9 & 0.5911 & -0.000010 & 0.349\end{array}$

$\begin{array}{llll}1048.6 & 0.5422 & -0.000008 & 0.294\end{array}$

$\begin{array}{lrrr}1060.1 & 0.5444 & -0.000006 & 0.296 \\ 1076.9 & -0.2000 & 0.000002 & 0.040\end{array}$

$\begin{array}{llll}1003.8 & 0.0830 & -0.000014 & 0.007\end{array}$

$\begin{array}{llll}1006.9 & 0.1733 & -0.000017 & 0.030\end{array}$

$\begin{array}{llll}1014.1 & 0.2758 & -0.000013 & 0.076\end{array}$
999.8395

999.8395

999.8395

999.8395

999.8395

999.9214

999.9214

999.9214

999.9214

999.9214

999.9214

999.9214

999.9214

999.9214

999.9214

999.9214

999.9638

999.9638

999.9638

999.9638

999.9638

999.9638

999.9638

999.9638

999.9638

999.9638

999.9638

999.9638

999.9638

999.9638

999.9638

999.9638

999.9638

999.9638

999.9638

999.9638

999.9638

999.9638

999.9638

999.9638

999.9638

999.9638

999.9638

60.719

999.9638

999.6996

999.6996
999.6996 
molality molarity

$t /{ }^{\circ} \mathrm{C}$ mass frac Density

0.4971

0.9865

1.993

2.9296

3.4865

3.9873

5.4952

5.8023

0.01029

0.02677

0.0412

0.05

0.06845

0.0934

0.09485

0.1
0.1

0.12426

0.1663

0.19656

0.19849

0.2

0.25724

0.32

0.48319 $\exp$

$\mathrm{kg} / \mathrm{m}^{3}$

$\begin{array}{rrrr} & & \text { exp } / & \\ 10 & 0.0282 & 1020.50 & (62) \\ 10 & 0.0400 & 1029.20 & (90) \\ 10 & 0.0545 & 1040.00 & (62) \\ 10 & 0.0800 & 1059.07 & (90) \\ 10 & 0.1043 & 1077.50 & (62) \\ 10 & 0.1200 & 1089.46 & (90) \\ 10 & 0.1462 & 1109.70 & (62) \\ 10 & 0.1600 & 1120.56 & (90) \\ 10 & 0.1693 & 1127.90 & (62) \\ 10 & 0.1890 & 1143.60 & (62) \\ 10 & 0.2000 & 1152.54 & (90) \\ 10 & 0.2400 & 1185.57 & (90) \\ 10 & 0.2431 & 1188.20 & (62) \\ 10 & 0.2532 & 1196.80 & (62) \\ 10 & 0.2600 & 1202.54 & (90) \\ 12.5 & 0.0001 & 999.48 & (48) \\ 12.5 & 0.0001 & 999.53 & (48) \\ 12.5 & 0.0003 & 999.66 & (48) \\ 12.5 & 0.0006 & 999.88 & (48) \\ 12.5 & 0.0012 & 1000.31 & (48) \\ 12.5 & 0.0116 & 1008.01 & (48) \\ 12.5 & 0.0231 & 1016.38 & (48) \\ 12.5 & 0.0287 & 1020.51 & (48) \\ 15 & 0.0001 & 999.14 & (48) \\ 15 & 0.0001 & 999.19 & (48) \\ 15 & 0.0003 & 999.32 & (48) \\ 15 & 0.0006 & 999.53 & (48) \\ 15 & 0.0006 & 999.54 & (13) \\ 15 & 0.0012 & 999.96 & (48) \\ 15 & 0.0016 & 1000.24 & (89) \\ 15 & 0.0024 & 1000.85 & (13) \\ 15 & 0.0029 & 1001.11 & (44) \\ 15 & 0.0040 & 1002.00 & (89) \\ 15 & 0.0054 & 1003.05 & (89) \\ 15 & 0.0055 & 1003.10 & (13) \\ 15 & 0.0058 & 1003.21 & (44) \\ 15 & 0.0058 & 1003.29 & (80) \\ 15 & 0.0072 & 1004.33 & (89) \\ 15 & 0.0096 & 1006.09 & (13) \\ 15 & 0.0114 & 1007.32 & (89) \\ 15 & 0.0115 & 1007.41 & (13) \\ 15 & 0.0116 & 1007.43 & (80) \\ 15 & 0.0116 & 1007.59 & (48) \\ 15 & 0.0148 & 1009.47 & (89) \\ 15 & 0.0184 & 1012.35 & (80) \\ 15 & 0.0191 & 1012.93 & (89) \\ 15 & 0.0231 & 1015.88 & (48) \\ 15 & 0.0275 & 1019.00 & (89) \\ & & & \end{array}$

Ref$$
\begin{array}{r}
\exp / \\
\mathrm{m}^{3} / \mathrm{kg}
\end{array}
$$

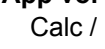

Calc /

$\mathrm{m}^{3} / \mathrm{kg}$

Density

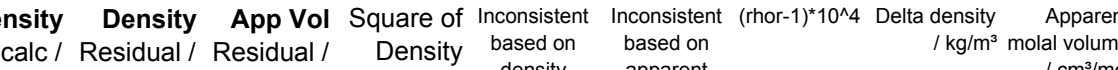

Density of

$0.000278 \quad 0.000290$

$0.000284 \quad 0.000293$

0.0002890 .000297

$\begin{array}{ll}0.000299 & 0.000304 \\ 0.000308 & 0.000310\end{array}$

$\begin{array}{ll}0.000314 & 0.000314\end{array}$

$0.000322 \quad 0.000321$

$0.000326 \quad 0.000325$

$0.000329 \quad 0.000328$

$0.000334 \quad 0.000333$

$\begin{array}{ll}0.000337 & 0.000336\end{array}$

$\begin{array}{ll}0.000347 & 0.000347\end{array}$

$0.000347 \quad 0.000347$

$\begin{array}{ll}0.000350 & 0.000350\end{array}$

$\begin{array}{ll}0.000351 & 0.000352 \\ 0.000249 & 0.000287\end{array}$

$0.000249 \quad 0.000287$

$\begin{array}{ll}0.000253 & 0.000287\end{array}$

$0.000255 \quad 0.000287$

$0.000255 \quad 0.000287$

$\begin{array}{ll}0.000269 & 0.000290 \\ 0.000278 & 0.000293\end{array}$

$\begin{array}{ll}0.000281 & 0.000294\end{array}$

$0.000267 \quad 0.000291$

$\begin{array}{ll}0.000258 & 0.000291\end{array}$

$0.000260 \quad 0.000291$

$\begin{array}{ll}0.000260 & 0.000291 \\ 0.000273 & 0.000291\end{array}$

$\begin{array}{ll}0.000273 & 0.000291\end{array}$

$\begin{array}{ll}0.000262 & 0.000291\end{array}$

$\begin{array}{ll}0.000267 & 0.000291 \\ 0.000272 & 0.000291\end{array}$

$\begin{array}{ll}0.000311 & 0.000291\end{array}$

$0.000273 \quad 0.000292$

$0.000275 \quad 0.000292$

$\begin{array}{ll}0.000276 & 0.000292\end{array}$

$\begin{array}{ll}0.000295 & 0.000292\end{array}$

0.0002810 .000292

$0.000277 \quad 0.000292$

0.0002790 .000293

$\begin{array}{ll}0.000281 & 0.000294\end{array}$

$\begin{array}{ll}0.000281 & 0.000294\end{array}$

$0.000285 \quad 0.000294$

$0.000276 \quad 0.000294$

$0.000306 \quad 0.000294$

$0.000287 \quad 0.000295$

$0.000285 \quad 0.000295$

$0.000284 \quad 0.000297$

$0.000289 \quad 0.000298$

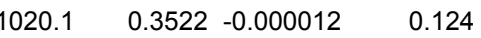

$\begin{array}{llll}1028.8 & 0.4104 & -0.000010 & 0.168\end{array}$

$\begin{array}{llll}039.5 & 0.4598 & -0.000008 & 0.211 \\ 058.7 & 0.3949 & -0.000004 & 0.156\end{array}$

$\begin{array}{llll}1077.2 & 0.3949 & -0.000004 & 0.156 \\ 1089.3 & 0.1231 & -0.000002 & 0.073\end{array}$

$\begin{array}{llll}1089.3 & 0.1231 & -0.000001 & 0.015\end{array}$

$\begin{array}{llll}1109.8 & -0.1166 & 0.000001 & 0.014\end{array}$

$\begin{array}{llll}1120.7 & -0.1877 & 0.000001 & 0.035\end{array}$

$\begin{array}{llll}1128.1 & -0.2307 & 0.000001 & 0.053\end{array}$

$\begin{array}{llll}1144.0 & -0.3602 & 0.000001 & 0.130\end{array}$

$\begin{array}{llll}1144.0 & -0.3602 & 0.000001 & 0.130\end{array}$

$\begin{array}{llll}1185.7 & -0.0933 & 0.000000 & 0.009\end{array}$

$\begin{array}{llll}1188.2 & -0.0197 & 0.000000 & 0.000\end{array}$

$\begin{array}{llll}1196.6 & 0.1524 & 0.000000 & 0.023\end{array}$

$\begin{array}{llll}1202.3 & 0.2480 & -0.000001 & 0.062\end{array}$

$\begin{array}{llll}999.5 & 0.0022 & -0.000037 & 0.000\end{array}$

$\begin{array}{llll}999.5 & 0.0044 & -0.000037 & 0.000\end{array}$

$\begin{array}{lllll}999.6 & 0.0099 & -0.000034 & 0.000\end{array}$

$\begin{array}{llll}999.9 & 0.0189 & -0.000032 & 0.000\end{array}$

$\begin{array}{llll}000.3 & 0.0380 & -0.000032 & 0.001\end{array}$

$\begin{array}{lllll}1007.8 & 0.2442 & -0.000021 & 0.060\end{array}$

$\begin{array}{llll}1016.0 & 0.3554 & -0.000015 & 0.126\end{array}$

$\begin{array}{lll}1020.1 & 0.3848-0.000013 & 0.148\end{array}$

$\begin{array}{llll}999.1 & 0.0014 & -0.000024 & 0.000\end{array}$

$\begin{array}{llll}999.2 & 0.0038 & -0.000033 & 0.000\end{array}$

$\begin{array}{llll}999.3 & 0.0090 & -0.000031 & 0.000\end{array}$

$\begin{array}{llll}99.5 & 0.0181 & -0.000031 & 0.000 \\ 99.5 & 0.0108 & -0.000018 & 0.000\end{array}$

$1000.2=0.0369-0.000024$

$\begin{array}{llll}1000.8 & 0.0465 & -0.000019 & 0.002\end{array}$

$\begin{array}{llll}1001.9 & 0.0761 & -0.000019 & 0.006\end{array}$

$\begin{array}{llll}1003.0 & 0.0906 & -0.000017 & 0.008\end{array}$

$\begin{array}{llll}1003.0 & 0.0879 & -0.000016 & 0.008\end{array}$

$\begin{array}{llll}1003.2 & -0.0175 & 0.000003 & 0.000\end{array}$

$\begin{array}{llll}1003.2 & 0.0625 & -0.000011 & 0.004\end{array}$

$\begin{array}{llll}1004.2 & 0.1094 & -0.000015 & 0.012\end{array}$

$\begin{array}{llll}1005.9 & 0.1385 & -0.000014 & 0.019\end{array}$

$\begin{array}{llll}1007.2 & 0.1408 & -0.000012 & 0.020\end{array}$

$\begin{array}{llll}1007.3 & 0.1490 & -0.000013 & 0.022\end{array}$

$\begin{array}{llll}1007.3 & 0.1057 & -0.000009 & 0.011\end{array}$

$\begin{array}{llll}1007.4 & 0.2111 & -0.000018 & 0.045\end{array}$

$\begin{array}{rrrr}1009.7 & -0.1807 & 0.000012 & 0.033 \\ 10122 & 0.1505 & -0.000008 & 0.023\end{array}$

$\begin{array}{lll}1012.7 & 0.1978-0.000010-0.039\end{array}$

$\begin{array}{llll}1015.6 & 0.2959 & -0.000012 & 0.088\end{array}$

$\begin{array}{llll}1018.8 & 0.2451 & -0.000009 & 0.060\end{array}$ $\begin{array}{llll}1001.2 & -0.0575 & 0.000020 & 0.003\end{array}$

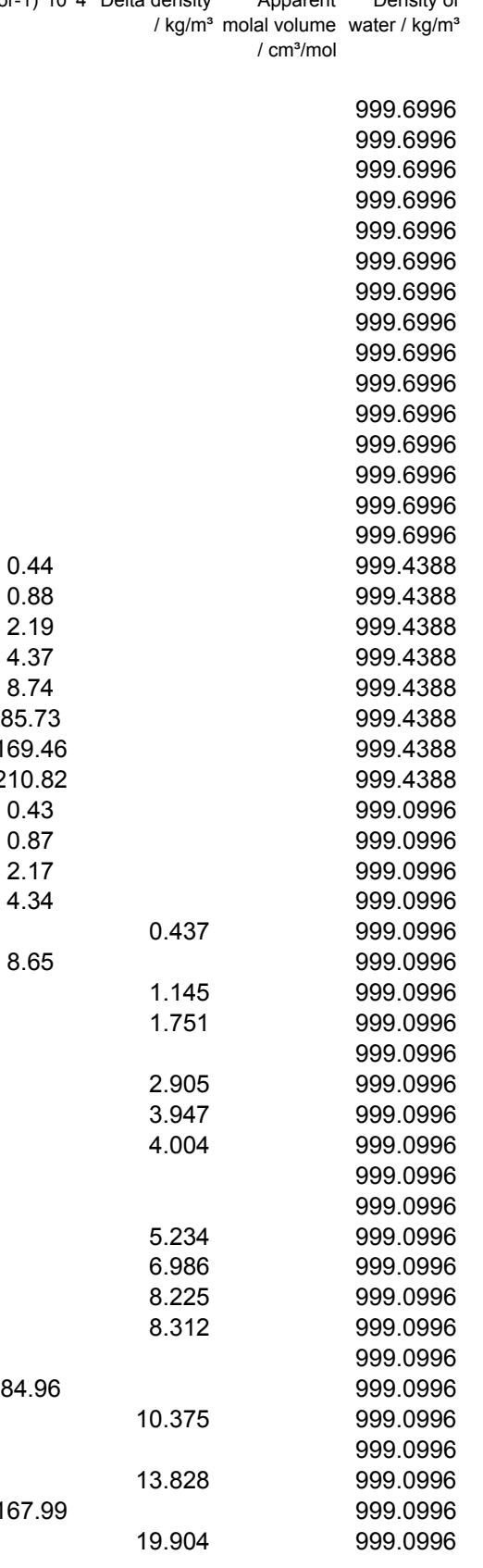


molality molarity

0.5012

0.5019

0.50786

0.65563

0.72467

0.8
-8115

0.9471

0.9992

1
1.0012

1.2
4309

1.4309

2.5333

3
142

0.05
0.0986

0.1

0.4971

0.5

0.9865

1.993

2

2.9296
3

3.4865

3.9873

5.4952

5.8023

0.001

0.002

0.005

0.00784

0.00992

0.01011

$\begin{array}{lrrr} & & \mathrm{kg} / \mathrm{m}^{3} & \\ 15 & 0.0284 & 1019.62 & (44) \\ 15 & 0.0284 & 1019.62 & (80) \\ 15 & 0.0285 & 1019.71 & (13) \\ 15 & 0.0285 & 1019.73 & (13) \\ 15 & 0.0287 & 1019.98 & (48) \\ 15 & 0.0288 & 1019.98 & (89) \\ 15 & 0.0369 & 1025.86 & (89) \\ 15 & 0.0406 & 1028.56 & (13) \\ 15 & 0.0447 & 1031.48 & (80) \\ 15 & 0.0469 & 1033.08 & (89) \\ 15 & 0.0524 & 1037.22 & (89) \\ 15 & 0.0552 & 1039.19 & (13) \\ 15 & 0.0552 & 1039.16 & (44) \\ 15 & 0.0553 & 1039.27 & (13) \\ 15 & 0.0655 & 1046.81 & (80) \\ 15 & 0.0772 & 1055.42 & (89) \\ 15 & 0.0806 & 1057.95 & (13) \\ 15 & 0.0952 & 1068.85 & (89) \\ 15 & 0.1047 & 1075.90 & (44) \\ 15 & 0.1290 & 1094.32 & (89) \\ 15 & 0.1492 & 1109.76 & (44) \\ 15 & 0.1498 & 1110.47 & (89) \\ 15 & 0.1895 & 1141.59 & (44) \\ 15 & 0.2261 & 1171.05 & (44) \\ 20 & 0.0029 & 1000.21 & (44) \\ 20 & 0.0057 & 1002.30 & (62) \\ 20 & 0.0058 & 1002.28 & (44) \\ 20 & 0.0282 & 1018.30 & (62) \\ 20 & 0.0284 & 1018.39 & (44) \\ 20 & 0.0545 & 1037.30 & (62) \\ 20 & 0.0552 & 1037.66 & (44) \\ 20 & 0.1043 & 1073.90 & (62) \\ 20 & 0.1047 & 1074.07 & (44) \\ 20 & 0.1462 & 1105.60 & (62) \\ 20 & 0.1492 & 1107.61 & (44) \\ 20 & 0.1693 & 1123.50 & (62) \\ 20 & 0.1890 & 1139.00 & (62) \\ 20 & 0.1895 & 1139.34 & (44) \\ 20 & 0.2261 & 1168.59 & (44) \\ 20 & 0.2431 & 1183.00 & (62) \\ 20 & 0.2532 & 1191.50 & (62) \\ 25 & 0.0001 & 997.09 & (48) \\ 25 & 0.0001 & 997.13 & (48) \\ 25 & 0.0003 & 997.26 & (48) \\ 25 & 0.0005 & 997.34 & (31) \\ 25 & 0.0006 & 997.46 & (13) \\ 25 & 0.0006 & 997.45 & (48) \\ 25 & 0.0006 & 997.46 & (73) \\ & & & \end{array}$

Ref$$
\text { exp / }
$$$$
\begin{gathered}
\exp / \\
\mathrm{m}^{3} / \mathrm{kg}
\end{gathered}
$$

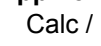

Calc /
$\mathrm{m}^{3} / \mathrm{kg}$

8

0.0002910 .000298

$0.000290 \quad 0.000298$

$\begin{array}{ll}0.000290 & 0.000298 \\ 0.000287 & 0.000298\end{array}$

$\begin{array}{lll}0.000290 & 0.000298\end{array}$

$\begin{array}{lll}0.000293 & 0.000300\end{array}$

$\begin{array}{lll}0.000295 & 0.000301\end{array}$

$\begin{array}{ll}0.000297 & 0.000302 \\ 0.000298 & 0.000303\end{array}$

$\begin{array}{ll}0.000298 & 0.000303 \\ 0.000300 & 0.000304\end{array}$

$\begin{array}{ll}0.000301 & 0.000305\end{array}$

$\begin{array}{ll}0.000302 & 0.000305\end{array}$

$\begin{array}{ll}0.000301 & 0.000305\end{array}$

$0.000305 \quad 0.000307$

$\begin{array}{ll}0.000309 & 0.000310\end{array}$

$\begin{array}{lll}0.000310 & 0.000311\end{array}$

$\begin{array}{ll}0.000315 & 0.000315\end{array}$

$\begin{array}{ll}0.000318 & 0.000317\end{array}$

$\begin{array}{ll}0.000326 & 0.000324 \\ 0.000332 & 0.000329\end{array}$

$\begin{array}{ll}0.000331 & 0.000329\end{array}$

$\begin{array}{ll}0.000342 & 0.000339\end{array}$

$\begin{array}{ll}0.000351 & 0.000349\end{array}$

$\begin{array}{ll}0.000312 & 0.000298\end{array}$

$\begin{array}{ll}0.000287 & 0.000299 \\ 0.000301 & 0.000299\end{array}$

$\begin{array}{ll}0.000301 & 0.000299 \\ 0.000302 & 0.000305\end{array}$

$\begin{array}{lll}0.000302 & 0.000305\end{array}$

$\begin{array}{lll}0.000309 & 0.000311\end{array}$

$\begin{array}{ll}0.000312 & 0.000311 \\ 0.000325 & 0.000324\end{array}$

$\begin{array}{lll}0.000326 & 0.000324\end{array}$

$\begin{array}{lll}0.000336 & 0.000334\end{array}$

$\begin{array}{lll}0.000338 & 0.000335\end{array}$

$\begin{array}{ll}0.000342 & 0.000340\end{array}$

$\begin{array}{ll}0.000347 & 0.000345 \\ 0.000347 & 0.000345\end{array}$

$\begin{array}{ll}0.000356 & 0.000355\end{array}$

$\begin{array}{lll}0.000358 & 0.000359\end{array}$

$0.000360 \quad 0.000361$

$\begin{array}{lll}0.000284 & 0.000304\end{array}$

$0.000284 \quad 0.000304$

$\begin{array}{ll}0.000281 & 0.000304\end{array}$

$\begin{array}{ll}0.000355 & 0.000304\end{array}$

$\begin{array}{ll}0.000285 & 0.000304\end{array}$

$\begin{array}{ll}0.000300 & 0.000304 \\ 0.000288 & 0.000304\end{array}$

$\begin{array}{ll}0.000288 & 0.000304\end{array}$
Density Density App Vol Square of Inconsistent Inconsistent (rhor-1)*10^4 Delta density Apparent Density of calc/ Residual / Residual / Density based on based on $1 / \mathrm{kg} / \mathrm{m}^{3}$ molal volume $\mathrm{kg} / \mathrm{m}^{3} \quad \mathrm{~kg} / \mathrm{m}^{3} \quad \mathrm{~m}^{3} / \mathrm{kg}$ Residual density apparen

$\begin{array}{llll}1019.4 & 0.1904 & -0.000006 & 0.036\end{array}$

$1019.4 \quad 0.1904-0.000006 \quad 0.036$

$\begin{array}{llll}1019.5 & 0.2322 & -0.000008 & 0.054\end{array}$

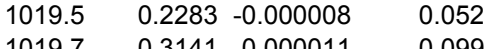

1019.7

0.056

$\begin{array}{lll}1019.7 & 0.2366-0.000008 & 0.056\end{array}$

$\begin{array}{llll}1028.3 & 0.2402 & -0.000006 & 0.058\end{array}$

$\begin{array}{lllll}1031.3 & 0.2177 & -0.000005 & 0.047\end{array}$

$\begin{array}{llll}1032.9 & 0.2151 & -0.000004 & 0.046\end{array}$

$1039.0=0.2149-0.000004$

$\begin{array}{llll}1039.0 & 0.1575 & -0.000003 & 0.025\end{array}$

$\begin{array}{llll}1039.0 & 0.2191 & -0.000004 & 0.048\end{array}$

$\begin{array}{llll}1046.6 & 0.1840 & -0.000003 & 0.034\end{array}$

$\begin{array}{llll}1055.3 & 0.1338 & -0.000002 & 0.018\end{array}$

$\begin{array}{llll}1057.8 & 0.1060 & -0.000001 & 0.011\end{array}$

$\begin{array}{llll}1068.8 & 0.0267 & 0.000000 & 0.001\end{array}$

$\begin{array}{llll}1076.0 & -0.0905 & 0.000001 & 0.008\end{array}$

$\begin{array}{llll}1094.6 & -0.2832 & 0.000002 & 0.080\end{array}$

$\begin{array}{llll}1110.3 & -0.5266 & 0.000003 & 0.277\end{array}$

$\begin{array}{llll}1110.8 & -0.2847 & 0.000002 & 0.081\end{array}$

$\begin{array}{llll}1142.1 & -0.5142 & 0.000002 & 0.264\end{array}$

$\begin{array}{llll}1171.6 & -0.5947 & 0.000002 & 0.354\end{array}$

$\begin{array}{llll}1000.3 & -0.0403 & 0.000014 & 0.002\end{array}$

$\begin{array}{llll}1002.2 & 0.0683 & -0.000012 & 0.005\end{array}$

$\begin{array}{llll}1002.3 & -0.0087 & 0.000001 & 0.000\end{array}$

$\begin{array}{llll}1018.2 & 0.0939 & -0.000003 & 0.009\end{array}$

$\begin{array}{llll}1018.3 & 0.0694 & -0.000002 & 0.005\end{array}$

$\begin{array}{llll}1037.2 & 0.1245 & -0.000002 & 0.015\end{array}$

$\begin{array}{llll}1037.7 & -0.0289 & 0.000000 & 0.001\end{array}$

$\begin{array}{llll}1074.0 & -0.1475 & 0.000001 & 0.022\end{array}$

$\begin{array}{llll}1074.3 & -0.2242 & 0.000002 & 0.050 \\ 1105.9 & -0.3353 & 0.000002 & 0.112\end{array}$

$\begin{array}{lll}1108.2 & -0.6329 & 0.000003\end{array}$

$\begin{array}{llll}1123.9 & -0.3624 & 0.000002 & 0.131\end{array}$

$\begin{array}{llll}1139.4 & -0.3617 & 0.000001 & 0.131\end{array}$

$\begin{array}{llll}1139.7 & -0.4073 & 0.000002 & 0.166\end{array}$

$\begin{array}{llll}1169.0 & -0.4174 & 0.000001 & 0.174\end{array}$

$\begin{array}{llll}1182.7 & 0.2760 & -0.000001 & 0.076\end{array}$

$\begin{array}{llll}1191.0 & 0.5137 & -0.000001 & 0.264\end{array}$

$\begin{array}{llll}997.1 & 0.0012 & -0.000020 & 0.000\end{array}$

$\begin{array}{llll}997.1 & 0.0023 & -0.000020 & 0.000\end{array}$

$\begin{array}{llll}997.2 & 0.0068 & -0.000023 & 0.000\end{array}$

$\begin{array}{llll}997.4 & -0.0232 & 0.000051 & 0.001\end{array}$

$\begin{array}{llll}997.4 & 0.0113 & -0.000020 & 0.000\end{array}$

$\begin{array}{llll}997.5 & 0.0096 & -0.000016 & 0.000\end{array}$

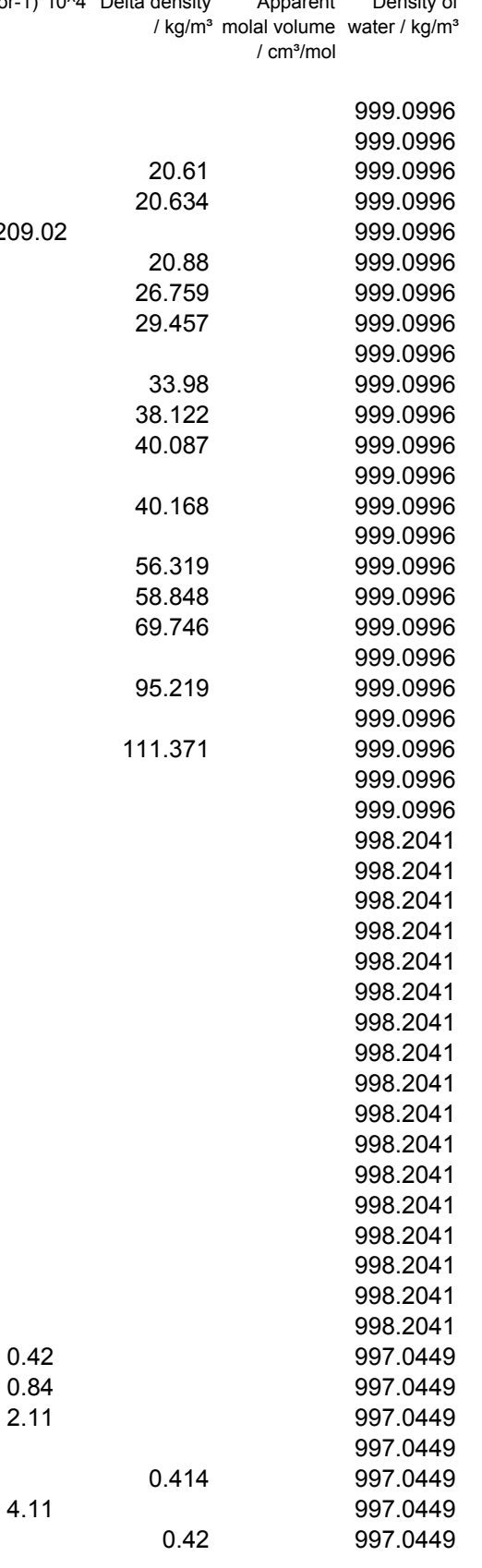


molality molarity

$\mathrm{t} /{ }^{\circ} \mathrm{C}$ mass

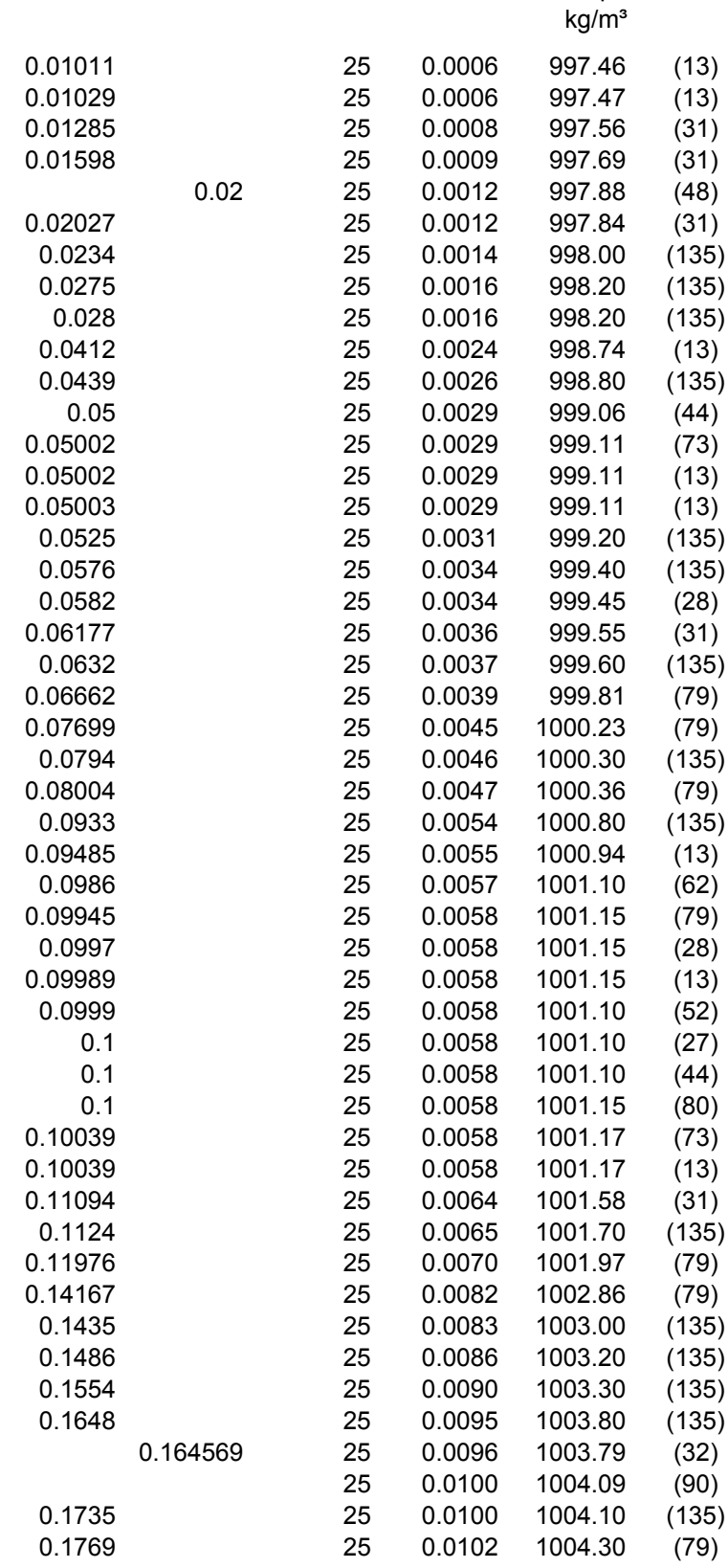

App vol App vol $\begin{array}{cc}\exp / & \text { Calc / } \\ \mathrm{m}^{3} / \mathrm{kg} & \mathrm{m}^{3} / \mathrm{kg}\end{array}$ $0.000288 \quad 0.000304$ $0.000290 \quad 0.000304$ $0.000313 \quad 0.000304$ $0.000308 \quad 0.000304$ $0.000284 \quad 0.000304$ $0.000328 \quad 0.000304$ $0.000300 \quad 0.000304$ $0.000280 \quad 0.000304$ $0.000293 \quad 0.000304$ $0.000294 \quad 0.000305$ $0.000314 \quad 0.000305$ $0.000309 \quad 0.000305$ 0.0002910 .000305 0.0002910 .000305 $0.000292 \quad 0.000305$ $0.000296 \quad 0.000305$ $0.000298 \quad 0.000305$ $0.000290 \quad 0.000305$ $0.000304 \quad 0.000305$ $0.000306 \quad 0.000305$ $0.000287 \quad 0.000305$ $0.000290 \quad 0.000305$ $0.000296 \quad 0.000305$ $0.000290 \quad 0.000305$ $0.000309 \quad 0.000305$ $0.000295 \quad 0.000305$ $0.000294 \quad 0.000305$ $0.000292 \quad 0.000305$ $0.000294 \quad 0.000305$ $0.000295 \quad 0.000305$ $0.000303 \quad 0.000305$ $0.000304 \quad 0.000305$ $0.000304 \quad 0.000305$ $0.000295 \quad 0.000305$ $0.000294 \quad 0.000305$ $0.000294 \quad 0.000305$ $0.000298 \quad 0.000306$ 0.0002890 .000306 $0.000293 \quad 0.000306$ $0.000294 \quad 0.000306$ $0.000287 \quad 0.000306$ $0.000288 \quad 0.000306$ $0.000308 \quad 0.000306$ $0.000295 \quad 0.000306$ $0.000300 \quad 0.000306$ $0.000299 \quad 0.000306$ $0.000301 \quad 0.000306$ $0.000295 \quad 0.000306$ calc / Residual / Residual / Density based on based on $\mathrm{kg} / \mathrm{m}^{3} \quad \mathrm{~kg} / \mathrm{m}^{3} \quad \mathrm{~m}^{3} / \mathrm{kg}$ Residual density apparen

$\begin{array}{llll}997.5 & 0.0096 & -0.000016 & 0.000\end{array}$

$\begin{array}{llll}997.5 & 0.0083 & -0.000014 & 0.000\end{array}$

$\begin{array}{llll}997.6 & -0.0064 & 0.000009 & 0.000\end{array}$

$\begin{array}{llll}997.7 & -0.0034 & 0.000004 & 0.000\end{array}$

$\begin{array}{llll}997.9 & 0.0233 & -0.000020 & 0.001\end{array}$

$\begin{array}{llll}997.9 & -0.0274 & 0.000023 & 0.001\end{array}$

$\begin{array}{llll}998.0 & 0.0058 & -0.000004 & 0.000\end{array}$

$\begin{array}{llll}998.2 & 0.0396 & -0.000025 & 0.002\end{array}$

$\begin{array}{llll}998.2 & 0.0193 & -0.000012 & 0.000\end{array}$

$\begin{array}{lllll}998.7 & 0.0256 & -0.000011 & 0.001\end{array}$

$\begin{array}{llll}998.8 & -0.0246 & 0.000010 & 0.001\end{array}$

$\begin{array}{llll}999.1 & -0.0114 & 0.000004 & 0.000\end{array}$

$\begin{array}{lllll}999.1 & 0.0407 & -0.000014 & 0.002\end{array}$

$\begin{array}{llll}999.1 & 0.0407 & -0.000014 & 0.002\end{array}$

$\begin{array}{llll}999.1 & 0.0383 & -0.000013 & 0.001\end{array}$

$\begin{array}{llll}999.2 & 0.0275 & -0.000009 & 0.001\end{array}$

$\begin{array}{llll}999.4 & 0.0213 & -0.000006 & 0.000\end{array}$

$\begin{array}{llll}999.4 & 0.0489 & -0.000014 & 0.002\end{array}$

$\begin{array}{llll}999.5 & 0.0027 & -0.000001 & 0.000\end{array}$

$\begin{array}{llll}999.6 & -0.0051 & 0.000001 & 0.000\end{array}$

$\begin{array}{llll}999.7 & 0.0685 & -0.000018 & 0.005\end{array}$

$\begin{array}{llll}1000.2 & 0.0684 & -0.000015 & 0.005\end{array}$

$\begin{array}{llll}1000.3 & 0.0406 & -0.000009 & 0.002\end{array}$

$\begin{array}{llll}1000.3 & 0.0705 & -0.000015 & 0.005\end{array}$

$\begin{array}{llll}1000.8 & -0.0201 & 0.000004 & 0.000\end{array}$

$\begin{array}{llll}1000.9 & 0.0563 & -0.000010 & 0.003\end{array}$

$\begin{array}{llll}1001.0 & 0.0662 & -0.000012 & 0.004\end{array}$

$1001.1-0.0803-0.000014$

$\begin{array}{llll}1001.1 & 0.0688 & -0.000012 & 0.005\end{array}$

$\begin{array}{llll}1001.1 & 0.0601 & -0.000010 & 0.004\end{array}$

$\begin{array}{llll}1001.1 & 0.0138 & -0.000002 & 0.000\end{array}$

$\begin{array}{llll}1001.1 & 0.0098 & -0.000002 & 0.000\end{array}$

$\begin{array}{llll}1001.1 & 0.0098 & -0.000002 & 0.000\end{array}$

$1001.1 \quad 0.0598-0.000010$

$\begin{array}{llll}1001.1 & 0.0680 & -0.000012 & 0.005\end{array}$

$\begin{array}{llll}1001.1 & 0.0680 & -0.000012 & 0.005\end{array}$

$\begin{array}{llll}1001.5 & 0.0491 & -0.000008 & 0.002\end{array}$

$\begin{array}{llll}1001.6 & 0.1103 & -0.000017 & 0.012\end{array}$

$\begin{array}{llll}1001.9 & 0.0879 & -0.000013 & 0.008\end{array}$

$\begin{array}{llll}1002.8 & 0.0981 & -0.000012 & 0.010\end{array}$

$\begin{array}{llll}1002.8 & 0.1596 & -0.000019 & 0.025\end{array}$

$\begin{array}{llll}1003.0 & 0.1548 & -0.000018 & 0.024\end{array}$

$\begin{array}{llll}1003.3 & -0.0182 & 0.000002 & 0.000\end{array}$

$\begin{array}{llll}1003.7 & 0.1047 & -0.000011 & 0.011\end{array}$

$\begin{array}{llll}1003.7 & 0.0633 & -0.000007 & 0.004\end{array}$

$\begin{array}{llll}1004.0 & 0.0725 & -0.000007 & 0.005\end{array}$

$\begin{array}{llll}1004.0 & 0.0559 & -0.000006 & 0.003\end{array}$

$\begin{array}{llll}1004.2 & 0.1170 & -0.000011 & 0.014\end{array}$
Density of

0.42

907.0449

997.0449

997.0449

997.0449

997.0449

997.0449

997.0449

997.0449

997.0449

1.696

997.0449

2.068

997.0449

$\begin{array}{ll}2.068 & 997.0449 \\ 2.066 & 997.0449\end{array}$

997.0449

2.407

997.0449

997.0449

997.0449

997.0449

$16.79 \quad 997.0449$

$16.94 \quad 997.0449$

997.0449

997.0449

$3.894 \quad 997.0449$

997.0449

4.102

$17.04 \quad 997.0449$

997.0449

997.0449

997.0449

997.0449

997.0449

997.0449

4.129

997.0449

997.0449

997.0449

997.0449

$17.13 \quad 997.0449$

$17.19 \quad 997.0449$

997.0449

997.0449

997.0449

997.0449

997.0449

997.0449

997.0449

$17.25 \quad 997.0449$ 
molality molarity

$t /{ }^{\circ} \mathrm{C}$ mass

Density

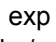

$\mathrm{kg} / \mathrm{m}^{3}$

0.17842

0.19749

0.19849

0.2

0.20009

0.20009

0.2187
0.23631

0.23631

0.288

0.29972

0.29972

0.298016

0.3395

0.3495

0.40009

0.40009

0.4057

0.42086

0.4476

0.4478

0.4708

0.4971

0.49946

0.49946

0.5

0.5
0.5

0.5
0.5
0.5012

0.5019

0.5614

0.56874

0.5997

0.59971

0.6504

0.652

0.69917

0.69917

0.7023

0.7069

0.7198

$\begin{array}{llll}25 & 0.0103 & 1004.36 & (79) \\ 25 & 0.0114 & 1005.12 & (79) \\ 25 & 0.0115 & 1005.14 & (13) \\ 25 & 0.0116 & 1005.19 & (80) \\ 25 & 0.0116 & 1005.21 & (73) \\ 25 & 0.0116 & 1005.21 & (13) \\ 25 & 0.0116 & 1005.29 & (48) \\ 25 & 0.0126 & 1005.90 & (135) \\ 25 & 0.0136 & 1006.63 & (31) \\ 25 & 0.0144 & 1007.30 & (135) \\ 25 & 0.0166 & 1008.77 & (28) \\ 25 & 0.0172 & 1009.19 & (73) \\ 25 & 0.0172 & 1009.19 & (13) \\ 25 & 0.0173 & 1009.21 & (32) \\ 25 & 0.0184 & 1009.98 & (80) \\ 25 & 0.0195 & 1010.80 & (135) \\ 25 & 0.0200 & 1011.12 & (90) \\ 25 & 0.0200 & 1011.20 & (135) \\ 25 & 0.0228 & 1013.16 & (73) \\ 25 & 0.0228 & 1013.16 & (13) \\ 25 & 0.0231 & 1013.37 & (48) \\ 25 & 0.0232 & 1013.50 & (135) \\ 25 & 0.0240 & 1014.02 & (79) \\ 25 & 0.0255 & 1015.00 & (135) \\ 25 & 0.0255 & 1014.90 & (135) \\ 25 & 0.0268 & 1015.98 & (28) \\ 25 & 0.0282 & 1016.90 & (62) \\ 25 & 0.0284 & 1017.06 & (73) \\ 25 & 0.0284 & 1017.06 & (13) \\ 25 & 0.0284 & 1016.99 & (44) \\ 25 & 0.0284 & 1017.09 & (67) \\ 25 & 0.0284 & 1017.08 & (80) \\ 25 & 0.0285 & 1017.14 & (13) \\ 25 & 0.0285 & 1017.16 & (13) \\ 25 & 0.0287 & 1017.37 & (48) \\ 25 & 0.0300 & 1018.20 & (32) \\ 25 & 0.0318 & 1019.60 & (135) \\ 25 & 0.0322 & 1019.70 & (31) \\ 25 & 0.0339 & 1020.95 & (73) \\ 25 & 0.0339 & 1020.95 & (13) \\ 25 & 0.0366 & 1022.94 & (79) \\ 25 & 0.0367 & 1023.00 & (79) \\ 25 & 0.0393 & 1024.78 & (73) \\ 25 & 0.0393 & 1024.78 & (13) \\ 25 & 0.0394 & 1025.00 & (135) \\ 25 & 0.0397 & 1025.30 & (52) \\ 25 & 0.0400 & 1025.30 & (90) \\ 25 & 0.0404 & 1025.70 & (135) \\ & & & \end{array}$

Ref

$$
\text { exp }
$$

exp / App vol

$\mathrm{m}^{3} / \mathrm{kg} \quad \mathrm{m}^{3} / \mathrm{kg}$

$\begin{array}{ll}0.000295 & 0.000307\end{array}$

$\begin{array}{ll}0.000297 & 0.000307\end{array}$

$\begin{array}{ll}0.000298 & 0.000307 \\ 0.000300 & 0.000307\end{array}$

$\begin{array}{ll}0.000300 & 0.000307 \\ 0.000298 & 0.000307\end{array}$

$0.000298 \quad 0.000307$

$\begin{array}{lll}0.000296 & 0.000307\end{array}$

$0.000303 \quad 0.000307$

$0.000302 \quad 0.000307$

$\begin{array}{ll}0.000294 & 0.000307\end{array}$

0.0002990 .000308

$\begin{array}{ll}0.000302 & 0.000308 \\ 0.000302 & 0.000308\end{array}$

0.0003020 .000308

$0.000303 \quad 0.000308$

$\begin{array}{ll}0.000301 & 0.000309\end{array}$

$0.000305 \quad 0.000309$

$\begin{array}{lll}0.000302 & 0.000309\end{array}$

$0.000305 \quad 0.000310$

$0.000305 \quad 0.000310$

$0.000303 \quad 0.000310$

$0.000300 \quad 0.000310$

$0.000304 \quad 0.000310$

$0.000307 \quad 0.000310$

$0.000311 \quad 0.000310$

$0.000305 \quad 0.000310$

$\begin{array}{ll}0.000309 & 0.000311 \\ 0.000307 & 0.000311\end{array}$

$0.000307 \quad 0.000311$

$0.000310 \quad 0.000311$

$0.000307 \quad 0.000311$

$0.000307 \quad 0.000311$

$0.000307 \quad 0.000311$

$0.000307 \quad 0.000311$

$0.000305 \quad 0.000311$

$\begin{array}{ll}0.000308 & 0.000311\end{array}$

$0.000305 \quad 0.000312$

$0.000310 \quad 0.000312$

$\begin{array}{ll}0.000309 & 0.000312\end{array}$

$0.000310 \quad 0.000313$

$0.000310 \quad 0.000313$

$\begin{array}{ll}0.000311 & 0.000313\end{array}$

$0.000311 \quad 0.000313$

$0.000309 \quad 0.000314$

$0.000306 \quad 0.000314$

$0.000312 \quad 0.000314$

$0.000309 \quad 0.000314$ $\mathrm{kg} / \mathrm{m}^{3} \quad \mathrm{~kg} / \mathrm{m}^{3} \quad \mathrm{~m}^{3} / \mathrm{kg}$ Residual density apparent

$\begin{array}{llll}1004.2 & 0.1164 & -0.000011 & 0.014\end{array}$

$\begin{array}{llll}1005.0 & 0.1142 & -0.000010 & 0.013\end{array}$

$\begin{array}{llll}1005.0 & 0.0962 & -0.000008 & 0.009\end{array}$

$\begin{array}{llll}0.05 .1 & 0.0850 & -0.000007 & 0.007 \\ 1005.1 & 0.0982 & -0.000008 & 0.010\end{array}$

$0.0982-0.000008$

$\begin{array}{llll}005.2 & 0.1319 & -0.000011 & 0.017\end{array}$

$\begin{array}{llll}005.9 & 0.0475 & -0.000004 & 0.002\end{array}$

$\begin{array}{lllll}1006.6 & 0.0747 & -0.000005 & 0.006\end{array}$

$\begin{array}{llll}1006.6 & 0.0747 & -0.000005 & 0.006 \\ 1007.1 & 0.2029 & -0.000014 & 0.041\end{array}$

$1009.1-0.1114-0.000006$

$\begin{array}{lll}1009.1 & 0.1114-0.000006 & 0.012 \\ 1009.1 & 0.1114-0.000006 & 0.012\end{array}$

$\begin{array}{llll}009.1 & 0.1043 & -0.000006 & 0.011 \\ 009.9 & 0.0970 & -0.000005 & 0.009\end{array}$

$\begin{array}{llll}1010.7 & 0.1447 & -0.000007 & 0.021\end{array}$

$\begin{array}{lll}1011.0 & 0.0810-0.000004 & 0.007\end{array}$

$1011.1-0.1491-0.000007-0.022$

$\begin{array}{llll}1013.0 & 0.1131 & -0.000005 & 0.013\end{array}$

$\begin{array}{llll}1013.0 & 0.1131 & -0.000005 & 0.013\end{array}$

$\begin{array}{llll}1013.2 & 0.1670 & -0.000007 & 0.028\end{array}$

$\begin{array}{llll}1013.3 & 0.2313 & -0.000010 & 0.053\end{array}$

$\begin{array}{lll}0.1547-0.000006 & 0.024\end{array}$

$10149-0.0240-0.000001$

$\begin{array}{llll}1015.8 & 0.1549 & -0.000006 & 0.024\end{array}$

$\begin{array}{llll}1016.9 & 0.0444 & -0.000002 & 0.002\end{array}$

$\begin{array}{llll}1016.9 & 0.1090 & -0.000004 & 0.012\end{array}$

$\begin{array}{llll}1016.9 & 0.1090 & -0.000004 & 0.012\end{array}$

$\begin{array}{llll}1017.0 & 0.0210 & -0.000001 & 0.000\end{array}$

$\begin{array}{llll}1017.0 & 0.1229 & -0.000004 & 0.015\end{array}$

$\begin{array}{llll}1017.0 & 0.1110 & -0.000004 & 0.012\end{array}$

$\begin{array}{llll}1017.0 & 0.1260 & -0.000004 & 0.016 \\ 1017.0 & 0.1146 & -0.000004 & 0.013\end{array}$

$1017.2-0.1662-0.000006$

$\begin{array}{llll}1018.1 & 0.1139 & -0.000004 & 0.013\end{array}$

$\begin{array}{llll}1019.4 & 0.2358 & -0.000007 & 0.056\end{array}$

$\begin{array}{llll}1019.6 & 0.0502 & -0.000002 & 0.003\end{array}$

$\begin{array}{llll}1020.9 & 0.0989 & -0.000003 & 0.010\end{array}$

$\begin{array}{llll}1020.9 & 0.0989 & -0.000003 & 0.010\end{array}$

$\begin{array}{llll}1022.8 & 0.1208 & -0.000003 & 0.015\end{array}$

$\begin{array}{llll}1022.9 & 0.1219 & -0.000003 & 0.015\end{array}$

$\begin{array}{lllll}1024.7 & 0.0867 & -0.000002 & 0.008\end{array}$

$\begin{array}{llll}1024.7 & 0.0867 & -0.000002 & 0.008\end{array}$

$\begin{array}{llll}1024.8 & 0.1813 & -0.000004 & 0.033\end{array}$

$1025.0-0.3042-0.000007$

$\begin{array}{llll}1025.2 & 0.0714 & -0.000002 & 0.005\end{array}$

$\begin{array}{llll}1025.5 & 0.2078 & -0.000005 & 0.043\end{array}$
$/ \mathrm{kg} / \mathrm{m}^{3}$ molal volume water

\begin{tabular}{|c|c|c|c|}
\hline 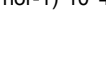 & $/ \mathrm{kg} / \mathrm{m}^{3}$ & $\begin{array}{l}\text { nolal volum } \\
1 / \mathrm{cm}^{3} / \mathrm{m}\end{array}$ & water $/ \mathrm{kg} / \mathrm{m}^{3}$ \\
\hline & & 17.26 & 997.0449 \\
\hline & & 17.35 & 997.0449 \\
\hline & 8.096 & & 997.0449 \\
\hline & & & 997.0449 \\
\hline & 8.162 & & 997.0449 \\
\hline & 8.162 & & 997.0449 \\
\hline 82.68 & & & 997.0449 \\
\hline & & & 997.0449 \\
\hline & & & 997.0449 \\
\hline & & & 997.0449 \\
\hline & 11.728 & & 997.0449 \\
\hline & 12.145 & & 997.0449 \\
\hline & 12.145 & & 997.0449 \\
\hline & & & 997.0449 \\
\hline & & & 997.0449 \\
\hline & & & 997.0449 \\
\hline & & & 997.0449 \\
\hline & & & 997.0449 \\
\hline & 16.116 & & 997.0449 \\
\hline & 16.116 & & 997.0449 \\
\hline 163.74 & & & 997.0449 \\
\hline & & & 997.0449 \\
\hline & & 17.74 & 997.0449 \\
\hline & & & 997.0449 \\
\hline & & & 997.0449 \\
\hline & 18.936 & & 997.0449 \\
\hline & & & 997.0449 \\
\hline & 20.012 & & 997.0449 \\
\hline & 20.012 & & 997.0449 \\
\hline & & & 997.0449 \\
\hline & 20.047 & & 997.0449 \\
\hline & & & 997.0449 \\
\hline & 20.097 & & 997.0449 \\
\hline & 20.113 & & 997.0449 \\
\hline 203.85 & & & 997.0449 \\
\hline & & & 997.0449 \\
\hline & & & 997.0449 \\
\hline & & & 997.0449 \\
\hline & 23.907 & & 997.0449 \\
\hline & 23.907 & & 997.0449 \\
\hline & & 18.1 & 997.0449 \\
\hline & & 18.1 & 997.0449 \\
\hline & 27.74 & & 997.0449 \\
\hline & 27.74 & & 997.0449 \\
\hline & & & 997.0449 \\
\hline & & & 997.0449 \\
\hline & & & 997.0449 \\
\hline & & & 997.0449 \\
\hline
\end{tabular}

Page 6 
molality molarity

0.72467

0.79957

0.79957

0.85382

0.8636
0.90125

0.90125

0.9208

0.9807

0.9865

0.9992

1
1

1.0001

1.0001

1.0012

1.0034

1.021

0.997147

1.1888

1.2

1.3174
1.4138

1.47458

1.49986

1.5088

1.522627

1.585

1.6654

1.6728

1.8995

1.9384

1.95

1.961

1.993

1.994

2.001

2.0889

2.3156

2.3666

25

$\mathrm{t} /{ }^{\circ} \mathrm{C}$ mass frac Density

$\exp /$
$\mathrm{kg} / \mathrm{m}^{3}$

$\begin{array}{ll}0.0446 \quad 1028.62 \\ 0.0446 & 1028.62\end{array}$

$\begin{array}{lll}25 & 0.0446 & 1028.62 \\ 25 & 0.0447 & 1028.65\end{array}$

$\begin{array}{lll}25 & 0.0447 & 1028.65 \\ 25 & 0.0475 & 1030.71\end{array}$

$\begin{array}{llll}25 & 0.0480 & 1031.20 & (135)\end{array}$

$\begin{array}{lll}25 & 0.0500 & 1032.48\end{array}$

$\begin{array}{llll}25 & 0.0500 & 1032.48 & (13) \\ 25 & 0.0511 & 1033.31 & (79)\end{array}$

$\begin{array}{llll}25 & 0.0542 & 1035.50 & (135)\end{array}$

$\begin{array}{lll}25 & 0.0545 & 1035.70\end{array}$

\begin{tabular}{llll} 
& 0.0552 & 1036.17 & $(13)$ \\
\hline & 0.0552 & 1036.10 & $(27)$
\end{tabular}

$\begin{array}{llll} & 0.0552 & 1036.07 & (44)\end{array}$

$\begin{array}{lll}0.0552 & 1036.14 & (67)\end{array}$

$\begin{array}{lll}0.0552 & 1036.20\end{array}$

$0.0552 \quad 1036.20 \quad(13)$

$\begin{array}{lll}0.0553 & 1036.26 & (13) \\ 0.0554 & 1036.40\end{array}$

$\begin{array}{llll} & 0.0554 & 1036.40 & (135) \\ & 0.0562 & 1036.97 & (32)\end{array}$

$\begin{array}{lll}0.0563 & 1037.12 \quad(28)\end{array}$

$\begin{array}{lll}0.0650 & 1043.38 \quad(79)\end{array}$

$\begin{array}{lll}0.0655 & 1043.65 \quad(80)\end{array}$

$\begin{array}{lll}0.0715 & 1048.00 & (135) \\ 0.0763 & 1051.40\end{array}$

$0.0763 \quad 1051.40$

$0.0793 \quad 1053.53$

$0.0806 \quad 1054.56$

$0.0810 \quad 1054.97$

$\begin{array}{lll}0.0848 & 1057.80 & (135) \\ 0.0887 & 1060.70 & (135)\end{array}$

$0.0891 \quad 1060.90$

$\begin{array}{lll}0.0999 & 1068.90 \quad(135)\end{array}$

$\begin{array}{lll}0.1018 & 1070.20 \quad(79)\end{array}$

$\begin{array}{lll}0.1023 & 1070.17 \quad(20)\end{array}$

$\begin{array}{lll}0.1028 & 1070.90 \quad(135)\end{array}$

$\begin{array}{lll}0.1043 & 1072.00 & (62)\end{array}$

$\begin{array}{lll}0.1044 & 1072.08 \quad \text { (28) }\end{array}$

$\begin{array}{lll}0.1047 & 1072.10 \quad(27) \\ 0.1047 & 1072.11 & (44)\end{array}$

$\begin{array}{lll}0.1047 & 1072.30 & (135)\end{array}$

$\begin{array}{llll}0.1088 & 1075.40 \quad(79)\end{array}$

$0.1192 \quad 1083.10 \quad(135)$

$\begin{array}{lll}0.1200 & 1083.65 & (90)\end{array}$

$0.1215 \quad 1084.85$

$0.1275 \quad 1089.20$
App exp / Calc/ $\mathrm{m}^{3} / \mathrm{kg}$

Density Density App Vol Square of Inconsistent Inconsistent

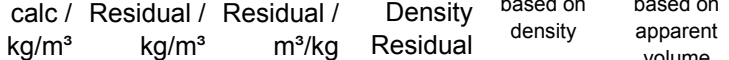

$0.000308 \quad 0.000314$

0.0003110 .000314

$\begin{array}{lll}0.000313 & 0.000315\end{array}$

0.0003130 .000315

$\begin{array}{ll}0.000313 & 0.000315 \\ 0.000314 & 0.000315\end{array}$

$\begin{array}{ll}0.000314 & 0.000315\end{array}$

0.0003120 .000316

$\begin{array}{ll}0.000315 & 0.000316 \\ 0.000315 & 0.000316\end{array}$

$\begin{array}{ll}0.000314 & 0.000316\end{array}$

$\begin{array}{ll}0.000316 & 0.000317\end{array}$

$\begin{array}{ll}0.000316 & 0.000317\end{array}$

$\begin{array}{ll}0.000317 & 0.000317\end{array}$

$0.000318 \quad 0.000317$

$\begin{array}{ll}0.000319 & 0.000317 \\ 0.000318 & 0.000317\end{array}$

$\begin{array}{lll}0.000317 & 0.000317\end{array}$

$\begin{array}{ll}0.000317 & 0.000317\end{array}$

$0.000316 \quad 0.000317$

$\begin{array}{ll}0.000315 & 0.000317\end{array}$

$\begin{array}{ll}0.000316 & 0.000318 \\ 0.000315 & 0.000318\end{array}$

$0.000317 \quad 0.000320$

$0.000320 \quad 0.000320$

$0.000321 \quad 0.000321$

$0.000324 \quad 0.000322$

$\begin{array}{ll}0.000325 & 0.000323 \\ 0.000324 & 0.000323\end{array}$

$\begin{array}{ll}0.000324 & 0.000324\end{array}$

$0.000323 \quad 0.000324$

$0.000324 \quad 0.000324$

$0.000324 \quad 0.000325$

$\begin{array}{ll}0.000324 & 0.000325 \\ 0.000325 & 0.000326\end{array}$

$0.000328 \quad 0.000328$

$0.000329 \quad 0.000329$

0.0003330 .000329

$0.000330 \quad 0.000329$

$\begin{array}{ll}0.000331 & 0.000329\end{array}$

0.0003320 .000329

$\begin{array}{ll}0.000332 & 0.000329\end{array}$

$\begin{array}{ll}0.000331 & 0.000329\end{array}$

$0.000331 \quad 0.000330$

$0.000334 \quad 0.000333$

$0.000335 \quad 0.000333$

$\begin{array}{lll}\text { (79) } \quad 0.000335 & 0.000333\end{array}$

$0.000337 \quad 0.000335$ $\begin{array}{llll}1025.7 & 0.2310 & -0.000005 & 0.053\end{array}$

$\begin{array}{llll}1025.7 & 0.1055 & -0.000002 & 0.011\end{array}$

$\begin{array}{llll}1028.6 & 0.0723 & -0.000002 & 0.005\end{array}$

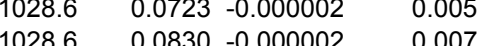

$\begin{array}{llll}1030.6 & 0.0900 & -0.000002 & 0.008\end{array}$

$\begin{array}{llll}1031.0 & 0.2078 & -0.000004 & 0.043\end{array}$

$\begin{array}{llll}1032.4 & 0.0575 & -0.000001 & 0.003\end{array}$

$\begin{array}{lllll}1032.4 & 0.0575 & -0.000001 & 0.003\end{array}$

$\begin{array}{llll}1033.2 & 0.1477 & -0.000003 & 0.022 \\ 1035.4 & 0.0729 & -0.000001 & 0.005\end{array}$

$\begin{array}{llll}1036.1 & 0.0468 & -0.000001 & 0.002\end{array}$

$\begin{array}{llll}1036.2 & -0.0542 & 0.000001 & 0.003\end{array}$

$\begin{array}{llll}1036.2 & -0.0163 & 0.000000 & 0.000\end{array}$

$\begin{array}{llll}1036.2 & 0.0429 & -0.000001 & 0.002\end{array}$

$\begin{array}{llll}1036.2 & 0.0429 & -0.000001 & 0.002\end{array}$

$\begin{array}{llll}1036.2 & 0.0605 & -0.000001 & 0.004\end{array}$

$\begin{array}{llll}1036.3 & 0.1178 & -0.000002 & 0.014\end{array}$

$\begin{array}{llll}1036.9 & 0.1043 & -0.000002 & 0.011 \\ 1036.9 & 0.1786 & -0.000003 & 0.032\end{array}$

$\begin{array}{lll}1043.2 & 0.1633-0.000002 & 0.027\end{array}$

$\begin{array}{llll}1043.6 & 0.0222 & 0.000000 & 0.000\end{array}$

$\begin{array}{llll}1048.0 & 0.0374 & 0.000000 & 0.001\end{array}$

$\begin{array}{llll}1051.5 & -0.0935 & 0.000001 & 0.009\end{array}$

$\begin{array}{llll}1053.7 & -0.1765 & 0.000002 & 0.031\end{array}$

$\begin{array}{llll}1054.2 & -0.0698 & 0.000001 & 0.005\end{array}$

$\begin{array}{llll}1054.6 & -0.0591 & 0.000001 & 0.003\end{array}$

$\begin{array}{llll}1054.9 & 0.0199 & 0.000000 & 0.000\end{array}$

$\begin{array}{llll}1057.2 & 0.0331 & 0.000000 & 0.001\end{array}$

$\begin{array}{llll}1057.7 & 0.0844 & -0.000001 & 0.007\end{array}$

$\begin{array}{llll}1060.6 & 0.1110 & -0.000001 & 0.012 \\ 1060.9 & 0.0461 & 0.000000 & 0.002\end{array}$

$\begin{array}{llll}1068.9 & 0.0013 & 0.000000\end{array}$

$\begin{array}{llll}1070.3 & -0.0648 & 0.000001 & 0.004\end{array}$

$\begin{array}{llll}1070.7 & -0.5023 & 0.000004 & 0.252\end{array}$

$\begin{array}{llll}1071.1 & -0.1576 & 0.000001 & 0.025\end{array}$

$\begin{array}{llll}1072.2 & -0.1770 & 0.000001 & 0.031 \\ 1072.2 & -0.1291 & 0.000001 & 0.017\end{array}$

$\begin{array}{llll}1072.4 & -0.3115 & 0.000003 & 0.097\end{array}$

$\begin{array}{llll}1072.5 & -0.1564 & 0.000001 & 0.024\end{array}$

$\begin{array}{llll}1075.5 & -0.1123 & 0.000001 & 0.013\end{array}$

$\begin{array}{llll}1083.3 & -0.2133 & 0.000002 & 0.045\end{array}$

$\begin{array}{llll}1083.9 & -0.2660 & 0.000002 & 0.071\end{array}$

$\begin{array}{llll}1085.0 & -0.1981 & 0.000001 & 0.039\end{array}$

$\begin{array}{llll}1089.6 & -0.3591 & 0.000002 & 0.129\end{array}$ $\begin{array}{llll}1036.2 & -0.0842 & 0.000001 & 0.007\end{array}$

$\begin{array}{llll}1072.4 & -0.3215 & 0.000003 & 0.017\end{array}$
997.0449

997.0449

997.0449

997.0449

997.0449

997.0449

997.0449

997.0449

35.435

35.435

$18.33 \quad 997.0449$

997.0449

997.0449

39.126

997.0449

997.0449

997.0449

39.093

997.0449

39.156

39.156

997.0449

997.0449

997.0449

997.0449

997.0449

40.078

-1
-107.0449

$18.55 \quad 997.0449$

997.0449

997.0449

997.0449

997.0449

997.0449

57.52

$18.9-997.0449$

997.0449

997.0449

997.0449

997.0449

997.0449

$19.24 \quad 997.0449$

73.125

997.0449

997.0449

997.0449

75.038

997.0449

997.0449

997.0449

997.0449

$19.36 \quad 997.0449$

997.0449

$19.57 \quad 997.0449$

997.0449 
molality molarity

2.51393
2.7186

2.8116

2.9296

2.9405

3
3.023

2.866916

3.09392

3.2249

3.2579

3.432

3.4865

3.5345

3.293967

3.6736

3.6813

3.9873

3.729894

4.2179

4.3397

4.5

4.6368

4.171523

4.9288

4.991

4.534427

5.116

5.24324

5.2593

5.346

5.4952

5.5426

5.8023

5.82267

5.8345

6.0216

0.00784

0.01285

0.01598

0.02027

25 \\ Density Ref
exp /}

$\exp /$
$\mathrm{kg} / \mathrm{m}^{3}$

$\begin{array}{lll}25 & 0.1492 & 1105.44 \\ 25 & 0.1501 & 1106.65\end{array}$

$\begin{array}{lll}25 & 0.1513 & 1107.55 \\ 25 & 0.1531 & 1108.49\end{array}$

$\begin{array}{lll}25 & 0.1586 & 1113.00\end{array}$

$\begin{array}{llll}25 & 0.1599 & 1114.10 & (135)\end{array}$

$\begin{array}{llll}25 & 0.1600 & 1114.01 & \text { (90) }\end{array}$

$\begin{array}{llll}25 & 0.1671 & 1119.60 & (135)\end{array}$

\begin{tabular}{llll}
0.1693 & 1121.20 & $(62)$ \\
\hline & 0.1712 & 1122.60 & $(52)$
\end{tabular}

$\begin{array}{llll}25 & 0.1714 & 1123.08\end{array}$

$25 \quad 0.1767 \quad 1127.10 \quad(135)$

$\begin{array}{llll} & 0.1771 & 1127.50 & (135)\end{array}$

$\begin{array}{lll}0.1890 & 1136.60 \quad(62)\end{array}$

$\begin{array}{lll}0.1895 & 1136.98 & (44) \\ 0.1914 & 1138.82 & (32)\end{array}$

$0.1978 \quad 1143.70$

$\begin{array}{lll}0.2000 & 1145.33 \quad(90)\end{array}$

$\begin{array}{ccc}0.2000 & 1145.33 & (90) \\ 0.2023 & 1147.30 & (135)\end{array}$

$\begin{array}{lll}0.2082 & 1152.00 \quad(67)\end{array}$

$\begin{array}{lll}0.2111 & 1154.66\end{array}$

$\begin{array}{lll}0.2132 & 1156.20 \quad(135)\end{array}$

$0.2236 \quad 1164.70$

$\begin{array}{lll}0.2258 & 1166.26 & (28)\end{array}$

$\begin{array}{lll}0.2261 & 1166.07 \quad(44)\end{array}$

$\begin{array}{rrr}0.2270 & 1167.43 & (32) \\ 0.2302 & 1169.80 & (135)\end{array}$

$0.2346 \quad 1172.90$

$\begin{array}{lll}0.2351 & 1173.90 \quad(135)\end{array}$

$\begin{array}{lll}0.2381 & 1176.20 \quad(135)\end{array}$

$\begin{array}{lll}0.2400 & 1177.76 \quad(90)\end{array}$

$\begin{array}{rrr}0.2431 & 1180.30 & (62) \\ 0.2447 & 1181.60 & (135)\end{array}$

$0.2532 \quad 1188.80$

$0.2539 \quad 1188.88$

$\begin{array}{lll}0.2543 & 1189.70 \quad(135)\end{array}$

$\begin{array}{lll}0.2600 & 1194.43 \quad(90)\end{array}$

$\begin{array}{rrr}0.2603 & 1194.90 & (135) \\ 0.0005 & 995.94 & (31) \\ 0.0008 & 996.15 & (31)\end{array}$

0.0008

0.00090960 .15

0.0012

996.28
996.46

$(31)$
$(31)$
$(31)$
App vol App vol

$\begin{array}{cc}\exp / & \text { Calc } / \\ \mathrm{m}^{3} / \mathrm{kg} & \mathrm{m}^{3} / \mathrm{kg}\end{array}$

$3 / \mathrm{kg}$
35

$\begin{array}{ll}0.000336 & 0.000335 \\ 0.000338 & 0.000335\end{array}$

0.0003390 .000337

$\begin{array}{ll}0.000339 & 0.000338 \\ 0.000342 & 0.000340\end{array}$

$\begin{array}{ll}0.000342 & 0.000340\end{array}$

$0.000344 \quad 0.000340$

0.0003410 .000340

$0.000342 \quad 0.000341$

$\begin{array}{ll}0.000344 & 0.000341 \\ 0.000344 & 0.000343\end{array}$

$\begin{array}{lll}0.000344 & 0.000343\end{array}$

$0.000344 \quad 0.000343$

$0.000345 \quad 0.000343$

$\begin{array}{ll}0.000346 & 0.000345 \\ 0.000347 & 0.000345\end{array}$

$0.000348 \quad 0.000346$

$0.000346 \quad 0.000346$

$\begin{array}{ll}0.000348 & 0.000347\end{array}$

$0.000348 \quad 0.000347$

$0.000351 \quad 0.000350$

$\begin{array}{ll}0.000351 & 0.000350 \\ 0.000351 & 0.000351\end{array}$

$\begin{array}{lll}0.000353 & 0.000352\end{array}$

$0.000354 \quad 0.000353$

$0.000354 \quad 0.000353$

$0.000355 \quad 0.000355$

$\begin{array}{ll}0.000355 & 0.000355 \\ 0.000355 & 0.000356\end{array}$

$0.000357 \quad 0.000359$

0.0003590 .000359

$0.000360 \quad 0.000359$

$0.000358 \quad 0.000359$

$\begin{array}{ll}0.000359 & 0.000360 \\ 0.000362 & 0.000361\end{array}$

$\begin{array}{ll}0.000360 & 0.000361\end{array}$

$\begin{array}{lll}0.000361 & 0.000362\end{array}$

0.0003620 .000363

0.0003620 .000363

$\begin{array}{ll}0.000363 & 0.000364\end{array}$

$0.00364-0.000366$

$\begin{array}{ll}0.000364 & 0.000366\end{array}$

$0.000365 \quad 0.000368$

$0.000365 \quad 0.000368$

$0.000360 \quad 0.000310$

$0.000329 \quad 0.000310$

0.0003210 .000310

$0.000312 \quad 0.000310$
Density Density App Vol Square of Inconsistent Inconsistent (rhor-1)* ${ }^{\star} 10^{\wedge} 4$ Delta density Apparent Density of calc/ Residual / Residual / Density based on based on $\mathrm{kg} / \mathrm{m}^{3} \quad \mathrm{~kg} / \mathrm{m}^{3} \quad \mathrm{~m}^{3} / \mathrm{kg}$ Residual density $\begin{gathered}\text { apparent } \\ \text { volume }\end{gathered}$

$\begin{array}{llll}1089.6 & -0.2022 & 0.000001 & 0.041\end{array}$

$\begin{array}{llll}1090.0 & -0.3974 & 0.000003 & 0.158\end{array}$

$\begin{array}{llll}1096.9 & -0.2527 & 0.000002 & 0.064\end{array}$

$\begin{array}{llll}1099.9 & -0.1766 & 0.000001 & 0.031\end{array}$

$\begin{array}{llll}1103.8 & -0.3810 & 0.000002 & 0.145\end{array}$

$\begin{array}{llll}1104.1 & -0.3359 & 0.000002 & 0.113\end{array}$

$\begin{array}{llll}1106.1 & -0.6285 & 0.000003 & 0.395\end{array}$

$\begin{array}{llll}1106.8 & -0.1623 & 0.000001 & 0.026\end{array}$

$\begin{array}{llll}1107.7 & -0.1386 & 0.000001 & 0.019\end{array}$

$\begin{array}{llll}109.1 & -0.6116 & 0.000003 & 0.374 \\ 113.3 & -0.2965 & 0.000002 & 0.088\end{array}$

$1114.3-0.2470 \quad 0.000001$

$\begin{array}{llll}1114.4 & -0.3780 & 0.000002 & 0.143\end{array}$

$\begin{array}{llll}1119.8 & -0.2499 & 0.000001 & 0.062\end{array}$

$\begin{array}{llll}1121.6 & -0.3539 & 0.000002 & 0.125\end{array}$

$\begin{array}{llll}1123.1 & -0.4517 & 0.000002 & 0.204\end{array}$

$\begin{array}{llll}1123.2 & -0.1390 & 0.000001 & 0.019\end{array}$

$\begin{array}{llll}1127.4 & -0.2625 & 0.000001 & 0.069\end{array}$

$\begin{array}{llll}1127.6 & -0.0998 & 0.000000 & 0.010\end{array}$

$\begin{array}{llll}1136.9 & -0.3235 & 0.000001 & 0.105\end{array}$

$\begin{array}{llll}1137.3 & -0.3260 & 0.000001 & 0.106\end{array}$

$\begin{array}{llll}1138.8 & -0.0072 & 0.000000 & 0.000\end{array}$

$\begin{array}{llll}1143.8 & -0.1126 & 0.000000 & 0.013\end{array}$

$\begin{array}{llll}1145.6 & -0.2497 & 0.000001 & 0.062\end{array}$

$\begin{array}{llll}1147.4 & -0.1046 & 0.000000 & 0.011\end{array}$

$\begin{array}{llll}1152.1 & -0.0856 & 0.000000 & 0.007\end{array}$

$\begin{array}{llll}1154.4 & 0.2704 & -0.000001 & 0.073\end{array}$

$\begin{array}{llll}1156.0 & 0.1665 & -0.000001 & 0.028\end{array}$

$\begin{array}{llll}1164.3 & 0.3657 & -0.000001 & 0.134\end{array}$

$\begin{array}{llll}1166.1 & 0.1850 & -0.000001 & 0.034\end{array}$

$\begin{array}{llll}1166.3 & -0.2618 & 0.000001 & 0.069\end{array}$

$\begin{array}{llll}1167.0 & 0.4039 & -0.000001 & 0.163\end{array}$

$\begin{array}{llll}1169.6 & 0.2358 & -0.000001 & 0.056 \\ 1173.1 & -0.1787 & 0.000001 & 0.032\end{array}$

$1173.5-0.3800-0.000001$

$\begin{array}{lll}11759 & 0.3064-0.000001 & 0.094\end{array}$

$\begin{array}{llll}1177.5 & 0.3032 & -0.000001 & 0.092\end{array}$

$\begin{array}{llll}1179.9 & 0.3563 & -0.000001 & 0.127\end{array}$

$\begin{array}{llll}1181.2 & 0.3786 & -0.000001 & 0.143\end{array}$

$\begin{array}{llll}1188.1 & 0.6552 & -0.000002 & 0.429\end{array}$

$\begin{array}{llll}1188.7 & 0.1976 & -0.000001 & 0.039\end{array}$

$\begin{array}{lllll}1189.0 & 0.7057 & -0.000002 & 0.498\end{array}$

$\begin{array}{llll}1193.6 & 0.7910 & -0.000002 & 0.626\end{array}$

$\begin{array}{llll}1193.9 & 1.0082 & -0.000003 & 1.017\end{array}$

$\begin{array}{llll}996.0 & -0.0227 & 0.000050 & 0.001\end{array}$

$\begin{array}{llll}996.2 & -0.0142 & 0.000019 & 0.000\end{array}$

$\begin{array}{llll}996.3 & -0.0101 & 0.000011 & 0.000\end{array}$

$\begin{array}{llll}996.5 & -0.0025 & 0.000002 & 0.000\end{array}$
$/ \mathrm{kg} / \mathrm{m}^{3}$ molal volume water $/ \mathrm{kg} / \mathrm{m}^{3}$

92.312

997.0449
997.0449

997.0449

$19.83 \quad 997.0449$

997.0449

997.0449

997.0449

997.0449

997.0449

997.0449

997.0449

997.0449

997.0449

997.0449

997.0449

997.0449

997.0449

997.0449

997.0449

997.0449

997.0449

997.0449

997.0449

997.0449

997.0449

997.0449

997.0449

997.0449

997.0449

997.0449

997.0449

997.0449

997.0449

997.0449

997.0449

997.0449

997.0449

997.0449

997.0449

997.0449

997.0449

997.0449

995.6473

995.6473

995.6473

995.6473 
molality molarity

$t /{ }^{\circ} \mathrm{C}$ mass frac

Density

$\exp$

$\mathrm{kg} / \mathrm{m}^{3}$

0.05
0.06177

0.0986

0.1

0.11094

0.23631

0.4971
0.5

0.56874

0.85382

0.9865

1.47458

1.993

2

2.51393

9296

3.09392

3.486

3.9873

4
5

5.24324

5.4952

5.8023

5.82267

0.02677

0.0412

0.05

0.0625

0.06845

0.0934

0.09485

0.1

0.1

0.12426
0.125

1663

0.19656

0.25

$\begin{array}{rrrr}30 & 0.0029 & 997.65 & (44) \\ 30 & 0.0036 & 998.14 & (31) \\ 30 & 0.0057 & 999.60 & (62) \\ 30 & 0.0058 & 999.68 & (44) \\ 30 & 0.0064 & 1000.13 & (31) \\ 30 & 0.0136 & 1005.13 & (31) \\ 30 & 0.0282 & 1015.30 & (62) \\ 30 & 0.0284 & 1015.43 & (44) \\ 30 & 0.0322 & 1018.08 & (31) \\ 30 & 0.0475 & 1028.97 & (31) \\ 30 & 0.0545 & 1033.90 & (62) \\ 30 & 0.0552 & 1034.32 & (44) \\ 30 & 0.0793 & 1051.67 & (31) \\ 30 & 0.1043 & 1069.90 & (62) \\ 30 & 0.1047 & 1070.09 & (44) \\ 30 & 0.1281 & 1087.47 & (31) \\ 30 & 0.1462 & 1101.20 & (62) \\ 30 & 0.1492 & 1103.22 & (44) \\ 30 & 0.1531 & 1106.23 & (31) \\ 30 & 0.1693 & 1118.80 & (62) \\ 30 & 0.1890 & 1134.20 & (62) \\ 30 & 0.1895 & 1134.60 & (44) \\ 30 & 0.2261 & 1163.51 & (44) \\ 30 & 0.2346 & 1170.30 & (31) \\ 30 & 0.2431 & 1177.70 & (62) \\ 30 & 0.2532 & 1186.10 & (62) \\ 30 & 0.2539 & 1186.16 & (31) \\ 35 & 0.0001 & 994.07 & (48) \\ 35 & 0.0001 & 994.11 & (48) \\ 35 & 0.0003 & 994.24 & (48) \\ 35 & 0.0006 & 994.44 & (48) \\ 35 & 0.0012 & 994.85 & (48) \\ 35 & 0.0016 & 995.12 & (89) \\ 35 & 0.0024 & 995.70 & (13) \\ 35 & 0.0029 & 996.03 & (44) \\ 35 & 0.0036 & 996.60 & (74) \\ 35 & 0.0040 & 996.80 & (89) \\ 35 & 0.0054 & 997.80 & (89) \\ 35 & 0.0055 & 997.85 & (13) \\ 35 & 0.0058 & 998.02 & (44) \\ 35 & 0.0058 & 998.07 & (80) \\ 35 & 0.0072 & 999.03 & (89) \\ 35 & 0.0073 & 999.10 & (74) \\ 35 & 0.0096 & 1000.70 & (13) \\ 35 & 0.0114 & 1001.92 & (89) \\ 35 & 0.0116 & 1002.02 & (80) \\ 35 & 0.0116 & 1002.11 & (48) \\ 35 & 0.0144 & 1003.99 & (74) \\ & & & \end{array}$

Ref$$
\begin{gathered}
\text { App vo } \\
\text { exp }
\end{gathered}
$$$$
\begin{gathered}
\exp / \\
\mathrm{m}^{3} / \mathrm{kg}
\end{gathered}
$$

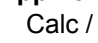

Calc /
$\mathrm{m}^{3} / \mathrm{kg}$

$\mathrm{g}$

(9)

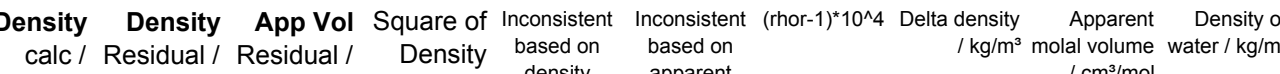
$\mathrm{kg} / \mathrm{m}^{3} \quad \mathrm{~kg} / \mathrm{m}^{3} \quad \mathrm{~m}^{3} / \mathrm{kg}$ Residual density apparent

$997.7-0.0061-0.000002$

$\begin{array}{llllll}0.000307 & 0.000310 & 998.1 & 0.0121 & -0.000003 & 0.000\end{array}$

$\begin{array}{llllll}0.000311 & 0.000311 & 999.6 & -0.0014 & 0.000000 & 0.000\end{array}$

$\begin{array}{llllll}0.000307 & 0.000311 & 999.7 & 0.0227 & -0.000004 & 0.001\end{array}$

$\begin{array}{llllll}0.000306 & 0.000311 & 1000.1 & 0.0358 & -0.000006 & 0.001\end{array}$

$\begin{array}{llllll}0.000309 & 0.000313 & 1005.1 & 0.0549 & -0.000004 & 0.003\end{array}$

$\begin{array}{llllll}0.000316 & 0.000316 & 1015.3 & 0.0132 & 0.000000 & 0.000\end{array}$

$\begin{array}{llllll}0.000315 & 0.000316 & 1015.4 & 0.0308 & -0.000001 & 0.001\end{array}$

$\begin{array}{lllllll}0.000316 & 0.000317 & 1018.1 & 0.0230 & -0.000001 & 0.001\end{array}$

$\begin{array}{llllll}0.000320 & 0.000321 & 1028.9 & 0.0357 & -0.000001 & 0.001 \\ 0.000323 & 0.000322 & 1033.9 & -0.0178 & 0.000000 & 0.000\end{array}$

$\begin{array}{llllll}0.000324 & 0.000323 & 1034.4 & -0.1021 & 0.000002 & 0.010\end{array}$

$\begin{array}{llllll}0.000330 & 0.000328 & 1051.8 & -0.1592 & 0.000002 & 0.025\end{array}$

$\begin{array}{llllll}0.000336 & 0.000334 & 1070.2 & -0.2510 & 0.000002 & 0.063\end{array}$

$\begin{array}{lllllll}0.000337 & 0.000334 & 1070.4 & -0.3036 & 0.000003 & 0.092\end{array}$

$\begin{array}{llllll}0.000342 & 0.000340 & 1087.9 & -0.3920 & 0.000003 & 0.154\end{array}$

$\begin{array}{llllll}0.000346 & 0.000344 & 1101.5 & -0.3114 & 0.000002 & 0.097\end{array}$

$\begin{array}{lllllll}0.000348 & 0.000345 & 1103.8 & -0.5619 & 0.000003 & 0.316\end{array}$

$\begin{array}{llllll}0.000349 & 0.000346 & 1106.8 & -0.5626 & 0.000003 & 0.317\end{array}$

$\begin{array}{llllll}0.000351 & 0.000350 & 1119.2 & -0.3543 & 0.000002 & 0.126\end{array}$

$\begin{array}{lllllll}0.000355 & 0.000354 & 1134.4 & -0.2159 & 0.000001 & 0.047\end{array}$

$\begin{array}{llllll}0.000355 & 0.000354 & 1134.8 & -0.1958 & 0.000001 & 0.038\end{array}$

$\begin{array}{llllll}0.000364 & 0.000363 & 1163.6 & -0.1205 & 0.000000 & 0.015\end{array}$

$\begin{array}{llllll}0.000365 & 0.000365 & 1170.3 & -0.0355 & 0.000000 & 0.001\end{array}$

$\begin{array}{llllll}0.000366 & 0.000367 & 1177.2 & 0.5410 & -0.000002 & 0.293\end{array}$

$\begin{array}{llllll}0.000368 & 0.000370 & 1185.3 & 0.7882 & -0.000002 & 0.621\end{array}$

$\begin{array}{rrrrrr}0.000369 & 0.000370 & 1185.8 & 0.3137 & -0.000001 & 0.098 \\ 0.000302 & 0.000314 & 994.1 & 0.0007 & -0.000012 & 0.000\end{array}$

$\begin{array}{llllll}0.000294 & 0.000314 & 994.1 & 0.0024 & -0.000021 & 0.000\end{array}$

$\begin{array}{llllll}0.000295 & 0.000315 & 994.2 & 0.0055 & -0.000019 & 0.000\end{array}$

$\begin{array}{llllll}0.000302 & 0.000315 & 994.4 & 0.0071 & -0.000012 & 0.000\end{array}$

$\begin{array}{llllll}0.000298 & 0.000315 & 994.8 & 0.0193 & -0.000017 & 0.000\end{array}$

$\begin{array}{llllll}0.000301 & 0.000315 & 995.1 & 0.0210 & -0.000014 & 0.000\end{array}$

$\begin{array}{llllll}0.000304 & 0.000315 & 995.7 & 0.0272 & -0.000011 & 0.001\end{array}$

$0.000313 \quad 0.000315$

$0.000294 \quad 0.000315$

$0.000304 \quad 0.000315$

$\begin{array}{llll}996.0 & 0.0051 & -0.000002 & 0.000\end{array}$

$\begin{array}{llll}996.5 & 0.0758 & -0.000021 & 0.006\end{array}$

$\begin{array}{llll}996.8 & 0.0444 & -0.000011 & 0.002\end{array}$

$997.7 \quad 0.0510-0.000009 \quad 0.003$

$\begin{array}{llll}997.8 & 0.0425 & -0.000008 & 0.002\end{array}$

$998.0-0.0095-0.000002-0.000$

$0.000314 \quad 0.000316$

$\begin{array}{ll}0.000305 & 0.000316\end{array}$

$0.000308 \quad 0.000316$

$0.000303 \quad 0.000316$

999.0

$\begin{array}{lll}999.0 & 0.0958 & -0.000013\end{array}$

$\begin{array}{llll}1000.6 & 0.0728 & -0.000008\end{array}$

0.004

$\begin{array}{lll}0.000309 & 0.000317\end{array}$

$\begin{array}{lll}0.000312 & 0.000317\end{array}$

1001.8

$0.0937-0.000008$

0.009
0.005

0.009

$\begin{array}{lll}0.0605 & -0.000005 & 0.004\end{array}$

$\begin{array}{llll}1002.0 & 0.1031 & -0.000009 & 0.011\end{array}$

$0.000313 \quad 0.000318$

\begin{tabular}{|c|c|c|c|}
\hline \multicolumn{4}{|c|}{$1 \mathrm{~cm}^{3} / \mathrm{mol}$} \\
\hline & & & 995.6473 \\
\hline & & & 995.6473 \\
\hline & & & 995.6473 \\
\hline & & & 995.6473 \\
\hline & & & 995.6473 \\
\hline & & & 995.6473 \\
\hline & & & 995.6473 \\
\hline & & & 995.6473 \\
\hline & & & 995.6473 \\
\hline & & & 995.6473 \\
\hline & & & 995.6473 \\
\hline & & & 995.6473 \\
\hline & & & 995.6473 \\
\hline & & & 995.6473 \\
\hline & & & 995.6473 \\
\hline & & & 995.6473 \\
\hline & & & 995.6473 \\
\hline & & & 995.6473 \\
\hline & & & 995.6473 \\
\hline & & & 995.6473 \\
\hline & & & 995.6473 \\
\hline & & & 995.6473 \\
\hline & & & 995.6473 \\
\hline & & & 995.6473 \\
\hline & & & 995.6473 \\
\hline & & & 995.6473 \\
\hline & & & 995.6473 \\
\hline 0.41 & & & 994.0319 \\
\hline 0.83 & & & 994.0319 \\
\hline 2.07 & & & 994.0319 \\
\hline 4.1 & & & 994.0319 \\
\hline 8.25 & & & 994.0319 \\
\hline & 1.089 & & 994.0319 \\
\hline & 1.67 & & 994.0319 \\
\hline & & & 994.0319 \\
\hline & & 17.2 & 994.0319 \\
\hline & 2.771 & & 994.0319 \\
\hline & 3.768 & & 994.0319 \\
\hline & 3.817 & & 994.0319 \\
\hline & & & 994.0319 \\
\hline & & & 994.0319 \\
\hline & 4.998 & & 994.0319 \\
\hline & & 17.7 & 994.0319 \\
\hline & 6.673 & & 994.0319 \\
\hline & 7.886 & & 994.0319 \\
\hline & & & 994.0319 \\
\hline 81.3 & & & 994.0319 \\
\hline & & 18.3 & 994.0319 \\
\hline
\end{tabular}


molality molarity

0.25724

0.32
0.33312

0.48319

0.5

0.5

0.5
0.5

0.5019

0.50786

0.65563

0.8

0.84115
0.9471

0.9471

1
1

1.2

1.4309

1.8001

2
2.5

2.5333

3.0142

4

5

0.01719

0.05351

0.0625

0.0986

0.0999

0.125

0.25

0.4971

0.5

0.55893

0.7069

0.9865

1.13947

1.4138

\section{$\mathrm{t} /{ }^{\circ} \mathrm{C}$ mass frac Density Ref}

$\exp /$
$\mathrm{kg} / \mathrm{m}^{3}$

35

35
35
35

$0.0231 \quad 1010.06$

$0.0275 \quad 1013.07$

$0.0284 \quad 1013.66$

$0.0284 \quad 1013.73$

$0.0284 \quad 1013.51$

$0.0284 \quad 1013.69$

$0.0285 \quad 1013.77$

0.0287

0.0288

$0.0469 \quad 1026.61$

$0.0524 \quad 1030.57$

$0.0552 \quad 1032.44$

$0.0552 \quad 1032.40$

$0.0552 \quad 1032.56$

$0.0655 \quad 1039.82$

$0.0772 \quad 1048.12$

$0.0952 \quad 1061.12$

$\begin{array}{ll}0.1047 & 1067.93 \\ 0.1275 & 1085.03\end{array}$

$0.1275 \quad 1085.03$

$0.1492 \quad 1100.92$

$0.1498 \quad 1101.57$

$0.2082 \quad 1147.12$

$0.2261 \quad 1160.92$

$0.0010 \quad 992.88$

$0.0031 \quad 994.37$

$\begin{array}{ll}0.0036 & 994.74 \\ 0.0057 & 996.10\end{array}$

996.10

$0.0058 \quad 996.10$

$0.0100 \quad 999.04$

$0.0100 \quad 999.08$

$0.0144 \quad 1002.06$

$0.0200 \quad 1005.93$

$0.0282 \quad 1011.60$

$0.0284 \quad 1011.43$

$0.0316 \quad 1013.96$

$0.0397 \quad 1019.70$

$0.0400 \quad 1019.77$

$0.0545 \quad 1029.90$

$\begin{array}{ll}0.0624 & 1035.43\end{array}$

$\begin{array}{lll}40 & 0.0763 & 1045.30\end{array}$
Ref

\author{
$\exp$
}

exp / App vol

Calc /

Density App Vol Square of Inconsistent Inconsistent (rhor- -1$)^{\star} 10^{\wedge} 4$ Delta density Apparen

Density of

$\begin{array}{lll}(89) & 0.000312 & 0.000318 \\ (80) & 0.000315 & 0.000319\end{array}$

$0.000314 \quad 0.000319$

$0.000314 \quad 0.000320$

$\begin{array}{ll}0.000318 & 0.000321\end{array}$

$0.000317 \quad 0.000321$

$0.000325 \quad 0.000321$

$0.000319 \quad 0.000321$

$0.000319 \quad 0.000321$

$\begin{array}{ll}0.000318 & 0.000321\end{array}$

$\begin{array}{ll}0.000321 & 0.000323\end{array}$

$0.000324 \quad 0.000325$

$\begin{array}{ll}0.000325 & 0.000325 \\ 0.000326 & 0.000326\end{array}$

$\begin{array}{ll}0.000326 & 0.000326 \\ 0.000328 & 0.000327\end{array}$

$\begin{array}{ll}0.000329 & 0.000327\end{array}$

$\begin{array}{lll}0.000326 & 0.000327\end{array}$

$\begin{array}{ll}0.000330 & 0.000329 \\ 0.000333 & 0.000332\end{array}$

$\begin{array}{ll}0.000338 & 0.000336\end{array}$

0.0003410 .000338

$0.000344 \quad 0.000344$

$0.000346 \quad 0.000344$

$\begin{array}{lll}0.000351 & 0.000349\end{array}$

$\begin{array}{ll}0.000350 & 0.000349\end{array}$

$0.000358 \quad 0.000358$

$\begin{array}{lll}0.000361 & 0.000362\end{array}$

$\begin{array}{lll}0.000366 & 0.000367\end{array}$

$\begin{array}{ll}0.000336 & 0.000319 \\ 0.000307 & 0.000319\end{array}$

$0.000305 \quad 0.000319$

$0.000322 \quad 0.000320$

$\begin{array}{ll}0.000331 & 0.000320\end{array}$

$\begin{array}{ll}0.000310 & 0.000320\end{array}$

$0.000317 \quad 0.000321$

$\begin{array}{ll}0.000315 & 0.000321 \\ 0.000320 & 0.000322\end{array}$

$\begin{array}{ll}0.000321 & 0.000323\end{array}$

$0.000324 \quad 0.000325$

$\begin{array}{ll}0.000334 & 0.000325\end{array}$

$\begin{array}{lll}0.000325 & 0.000326\end{array}$

$\begin{array}{ll}0.000323 & 0.000327 \\ 0.000327 & 0.000327\end{array}$

$\begin{array}{lll}0.000331 & 0.000331\end{array}$

$0.000334 \quad 0.000332$

$\begin{array}{ll}0.000337 & 0.000336\end{array}$ $\mathrm{kg} / \mathrm{m}^{3} \quad \mathrm{~kg} / \mathrm{m}^{3} \quad \begin{aligned} \text { Residual / Density based on based on } \\ \text { density apparent }\end{aligned}$

$\mathrm{kg} / \mathrm{m}^{3} \quad \mathrm{~m}^{3} / \mathrm{kg}$ Residual volume

$\begin{array}{llll}1004.2 & 0.0812 & -0.000005 & 0.007\end{array}$

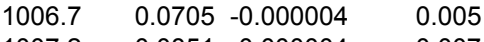

$\begin{array}{llll}1007.2 & 0.0851 & -0.000004 & 0.007 \\ 1009.9 & 0.1305 & -0.000006 & 0.017\end{array}$

$\begin{array}{llll}1013.0 & 0.0831 & -0.000003 & 0.007\end{array}$

$\begin{array}{llll}1013.6 & 0.0292 & -0.000001 & 0.001\end{array}$

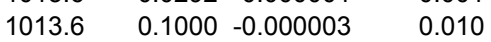

$\begin{array}{llll}1013.6 & -0.1228 & 0.000004 & 0.015\end{array}$

$\begin{array}{llll}1013.6 & 0.0592 & -0.000002 & 0.004\end{array}$

$\begin{array}{llll}1013.7 & 0.0680 & -0.000002 & 0.005 \\ 1013.9 & 0.1405 & -0.000005 & 0.020\end{array}$

$\begin{array}{llll}1013.9 & 0.0758 & -0.000003 & 0.006\end{array}$

$\begin{array}{llll}1019.6 & 0.0587 & -0.000002 & 0.003\end{array}$

$\begin{array}{llll}1025.0 & 0.0267 & -0.000001 & 0.001\end{array}$

$\begin{array}{llll}1026.6 & 0.0210 & 0.000000 & 0.000\end{array}$

$\begin{array}{llll}1030.5 & 0.0223 & 0.000000 & 0.000\end{array}$

$\begin{array}{llll}1032.5 & -0.0364 & 0.000001 & 0.001\end{array}$

$\begin{array}{llll}1032.5 & -0.1109 & 0.000002 & 0.012\end{array}$

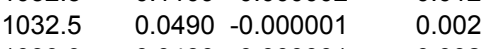

$\begin{array}{llll}1039.9 & -0.0480 & 0.000001 & 0.002\end{array}$

$\begin{array}{llll}1048.2 & -0.1068 & 0.000001 & 0.011 \\ 1061.3 & -0.1815 & 0.000002 & 0.033\end{array}$

$\begin{array}{llll}1068.2 & -0.2966 & 0.000002\end{array}$

$\begin{array}{llll}1085.1 & -0.0838 & 0.000001 & 0.007\end{array}$

$\begin{array}{llll}1086.2 & -0.3737 & 0.000002 & 0.140\end{array}$

$\begin{array}{llll}1101.4 & -0.4768 & 0.000003 & 0.227\end{array}$

$\begin{array}{rrrr}1101.9 & -0.2796 & 0.000002 & 0.078 \\ 1132.2 & 0.0824 & 0.000000 & 0.007\end{array}$

$1146.8 \quad 0.2954-0.000001$

$\begin{array}{llll}1160.9 & 0.0085 & 0.000000 & 0.000\end{array}$

$\begin{array}{llll}992.9 & -0.0170 & 0.000017 & 0.000\end{array}$

$\begin{array}{llll}994.3 & 0.0365 & -0.000012 & 0.001\end{array}$

$\begin{array}{llll}994.7 & 0.0535 & -0.000015 & 0.003\end{array}$

$\begin{array}{llll}996.1 & -0.0115 & 0.000002 & 0.000\end{array}$

$\begin{array}{llll}996.2 & -0.0627 & 0.000011 & 0.004\end{array}$

$\begin{array}{lllll}997.1 & 0.0758 & -0.000011 & 0.006\end{array}$

$\begin{array}{llll}999.0 & 0.0351 & -0.000004 & 0.001\end{array}$

$\begin{array}{llll}999.0 & 0.0542 & -0.000005 & 0.003\end{array}$

$\begin{array}{llll}1002.0 & 0.0264 & -0.000002 & 0.001\end{array}$

$\begin{array}{llll}1005.9 & 0.0446 & -0.000002 & 0.002\end{array}$

$\begin{array}{llll}1011.6 & 0.0309 & -0.000001 & 0.001\end{array}$

$\begin{array}{llll}1011.7 & -0.2549 & 0.000009 & 0.065\end{array}$

$\begin{array}{llll}1013.9 & 0.0332 & -0.000001 & 0.001\end{array}$

$\begin{array}{rrrr}1019.5 & 0.1746 & -0.000004 & 0.030 \\ 1019.8 & 0.0171 & 0.000000 & 0.000\end{array}$

$\begin{array}{llll}1029.9 & -0.0384 & 0.000001 & 0.001\end{array}$

$\begin{array}{llll}1035.5 & -0.1149 & 0.000002 & 0.013\end{array}$

$\begin{array}{llll}1045.4 & -0.1422 & 0.000002 & 0.020\end{array}$

\begin{tabular}{|c|c|c|c|}
\hline & \multirow[t]{2}{*}{10.256} & & $\begin{array}{l}994.0319 \\
994.0319\end{array}$ \\
\hline & & & 994.0319 \\
\hline \multirow{7}{*}{161.21} & 13.224 & & 994.0319 \\
\hline & & & 994.0319 \\
\hline & 19.035 & & 994.0319 \\
\hline & & & 994.0319 \\
\hline & 19.699 & & 994.0319 \\
\hline & & 19 & 994.0319 \\
\hline & & & 994.0319 \\
\hline \multirow{39}{*}{200.9} & 19.74 & & 994.0319 \\
\hline & & & 994.0319 \\
\hline & 19.977 & & 994.0319 \\
\hline & 25.61 & & 994.0319 \\
\hline & & & 994.0319 \\
\hline & 32.578 & & 994.0319 \\
\hline & 36.537 & & 994.0319 \\
\hline & 38.413 & & 994.0319 \\
\hline & & & 994.0319 \\
\hline & 38.528 & & 994.0319 \\
\hline & & & 994.0319 \\
\hline & 54.088 & & 994.0319 \\
\hline & 67.084 & & 994.0319 \\
\hline & & & 994.0319 \\
\hline & 91.001 & & 994.0319 \\
\hline & 91.814 & & 994.0319 \\
\hline & & & 994.0319 \\
\hline & 107.539 & & 994.0319 \\
\hline & & & 994.0319 \\
\hline & 153.09 & & 994.0319 \\
\hline & & & 994.0319 \\
\hline & & & 992.2158 \\
\hline & & & 992.2158 \\
\hline & & 17.8 & 992.2158 \\
\hline & & & 992.2158 \\
\hline & & & 992.2158 \\
\hline & & 18.1 & 992.2158 \\
\hline & & & 992.2158 \\
\hline & & & 992.2158 \\
\hline & & 18.7 & 992.2158 \\
\hline & & & 992.2158 \\
\hline & & & 992.2158 \\
\hline & & 19.5 & 992.2158 \\
\hline & & & 992.2158 \\
\hline & & & 992.2158 \\
\hline & & & 992.2158 \\
\hline & & & 992.2158 \\
\hline & & & 992.2158 \\
\hline & & & 992.2158 \\
\hline
\end{tabular}


molality molarity

$t /{ }^{\circ} \mathrm{C}$ mass frac Density

1.993

2.35546

2.9296

3.4865

3.5345

3.87979
3.9873

5.45241

5.4952

5.8023

0.02677

0.05

0.06845

0.0934

0.1

0.1

0.12426

0.19656

0.2

0.25724

0.32
.33312

0.48319

0.5

0.5
0.50786

0.65563

0.8
-84115

0.84115

1
1 $\exp /$
$\mathrm{kg} / \mathrm{m}^{3}$

\begin{tabular}{|c|c|c|}
\hline & & $\begin{array}{r}\exp / \\
\mathrm{kg} / \mathrm{m}^{3}\end{array}$ \\
\hline 40 & 0.0800 & 1047.98 \\
\hline 40 & 0.1043 & 1065.50 \\
\hline 40 & 0.1200 & 1076.99 \\
\hline 40 & 0.1210 & 1077.57 \\
\hline 40 & 0.1462 & 1096.40 \\
\hline 40 & 0.1600 & 1106.88 \\
\hline 40 & 0.1693 & 1113.90 \\
\hline 40 & 0.1712 & 1115.40 \\
\hline 40 & 0.1848 & 1125.59 \\
\hline 40 & 0.1890 & 1129.10 \\
\hline 40 & 0.2000 & 1137.74 \\
\hline 40 & 0.2400 & 1169.71 \\
\hline 40 & 0.2417 & 1170.69 \\
\hline 40 & 0.2431 & 1172.20 \\
\hline 40 & 0.2532 & 1179.90 \\
\hline 40 & 0.2600 & 1186.14 \\
\hline 42.5 & 0.0001 & 991.28 \\
\hline 42.5 & 0.0001 & 991.32 \\
\hline 42.5 & 0.0003 & 991.44 \\
\hline 42.5 & 0.0006 & 991.64 \\
\hline 42.5 & 0.0012 & 992.05 \\
\hline 42.5 & 0.0116 & 999.29 \\
\hline 42.5 & 0.0231 & 1007.20 \\
\hline 42.5 & 0.0287 & 1011.12 \\
\hline 45 & 0.0016 & 991.28 \\
\hline 45 & 0.0029 & 992.15 \\
\hline 45 & 0.0040 & 992.95 \\
\hline 45 & 0.0054 & 993.92 \\
\hline 45 & 0.0058 & 994.10 \\
\hline 45 & 0.0058 & 994.14 \\
\hline 45 & 0.0058 & 994.18 \\
\hline 45 & 0.0072 & 995.15 \\
\hline 45 & 0.0114 & 998.01 \\
\hline 45 & 0.0116 & 998.09 \\
\hline 45 & 0.0148 & 1000.35 \\
\hline 45 & 0.0184 & 1002.73 \\
\hline 45 & 0.0191 & 1003.29 \\
\hline 45 & 0.0275 & 1009.03 \\
\hline 45 & 0.0284 & 1009.61 \\
\hline 45 & 0.0284 & 1009.63 \\
\hline 45 & 0.0284 & 1009.60 \\
\hline 45 & 0.0288 & 1009.96 \\
\hline 45 & 0.0369 & 1015.53 \\
\hline 45 & 0.0447 & 1020.83 \\
\hline 45 & 0.0469 & 1022.52 \\
\hline 45 & 0.0524 & 1026.43 \\
\hline 45 & 0.0552 & 1028.00 \\
\hline 45 & 0.0552 & 1028.14 \\
\hline
\end{tabular}

Ref

$$
\text { exp }
$$

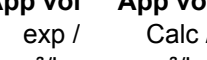

$\begin{array}{cc}\exp / & \text { Calc / } \\ \mathrm{m}^{3} / \mathrm{kg} & \mathrm{m}^{3} / \mathrm{kg}\end{array}$

$0.000337 \quad 0.000336$

$\begin{array}{ll}0.000343 & 0.000342\end{array}$

$0.000347 \quad 0.000345$

$\begin{array}{ll}0.000348 & 0.000345\end{array}$

$\begin{array}{ll}0.000353 & 0.000351 \\ 0.000355 & 0.000354\end{array}$

$\begin{array}{ll}0.000355 & 0.000354 \\ 0.000357 & 0.000356\end{array}$

$\begin{array}{lll}0.000358 & 0.000357\end{array}$

0.0003620 .000360

$\begin{array}{ll}0.000361 & 0.000361 \\ 0.000363 & 0.000363\end{array}$

$\begin{array}{lll}0.000371 & 0.000372\end{array}$

$\begin{array}{lll}0.000372 & 0.000373\end{array}$

$\begin{array}{lll}0.000371 & 0.000373\end{array}$

$\begin{array}{ll}0.000375 & 0.000375 \\ 0.000374 & 0.000377\end{array}$

$\begin{array}{ll}0.000374 & 0.000377 \\ 0.000303 & 0.000320\end{array}$

$\begin{array}{ll}0.000303 & 0.000320\end{array}$

$\begin{array}{ll}0.000300 & 0.000321\end{array}$

$\begin{array}{lll}0.000303 & 0.000321\end{array}$

$0.000300 \quad 0.00032$

$0.000310 \quad 0.000323$

$\begin{array}{lll}0.000316 & 0.000325\end{array}$

$\begin{array}{ll}0.000318 & 0.000327\end{array}$

$\begin{array}{ll}0.000316 & 0.000322\end{array}$

$\begin{array}{lll}0.000333 & 0.000323\end{array}$

$\begin{array}{ll}0.000312 & 0.000323 \\ 0.000315 & 0.000323\end{array}$

$0.000330 \quad 0.000323$

$\begin{array}{ll}0.0003323 & 0.000323\end{array}$

$\begin{array}{lll}0.000316 & 0.000323\end{array}$

$\begin{array}{ll}0.000315 & 0.000324\end{array}$

$\begin{array}{ll}0.000316 & 0.000325 \\ 0.000320 & 0.000325\end{array}$

$0.000320 \quad 0.000325$

$\begin{array}{lll}0.000319 & 0.000325\end{array}$

$0.000323 \quad 0.000326$

$\begin{array}{ll}0.000321 & 0.000326 \\ 0.000324 & 0.000328\end{array}$

$\begin{array}{ll}0.000327 & 0.000328\end{array}$

$\begin{array}{lll}0.000326 & 0.000328\end{array}$

$\begin{array}{ll}0.000327 & 0.000328\end{array}$

$\begin{array}{ll}0.000325 & 0.000328\end{array}$

$0.000328 \quad 0.000330$

$\begin{array}{ll}0.000332 & 0.000332 \\ 0.000329 & 0.000332\end{array}$

$\begin{array}{lll}0.000331 & 0.000333\end{array}$

$\begin{array}{ll}0.000338 & 0.000334\end{array}$

$\begin{array}{ll}0.000335 & 0.000334\end{array}$
Density Density App Vol Square of Inconsistent Inconsistent (rhor- -$)^{\star} 10^{\wedge} 4$ Delta density Apparent

$/ \mathrm{kg} / \mathrm{m}^{3}$ molal volume water $/ \mathrm{kg} / \mathrm{m}^{3}$

$\mathrm{kg} / \mathrm{m}^{3} \quad \mathrm{~kg} / \mathrm{m}^{3} \quad \mathrm{~m}^{3} / \mathrm{kg}$ Residual density $\begin{gathered}\text { apparen } \\ \text { volume }\end{gathered}$

$\begin{array}{llll}1048.1 & -0.1012 & 0.000001 & 0.010\end{array}$

$\begin{array}{llll}1065.7 & -0.1936 & 0.000002 & 0.037\end{array}$

$\begin{array}{llll}1077.2 & -0.2065 & 0.000001 & 0.043\end{array}$

$\begin{array}{llll}1077.9 & -0.3662 & 0.000003 & 0.134 \\ 1096.7 & -0.2784 & 0.000002 & 0.077\end{array}$

$\begin{array}{llll}1107.1 & -0.2100 & 0.000001\end{array}$

$\begin{array}{llll}1114.1 & -0.2275 & 0.000001 & 0.052\end{array}$

$\begin{array}{llll}1115.6 & -0.1990 & 0.000001 & 0.040\end{array}$

$\begin{array}{llll}1126.0 & -0.4455 & 0.000002 & 0.199\end{array}$

$\begin{array}{llll}1129.2 & -0.1328 & 0.000001 & 0.018\end{array}$

$\begin{array}{llll}1137.7 & -0.0069 & 0.000000 & 0.000\end{array}$

$\begin{array}{llll}1169.1 & 0.5640 & -0.000002 & 0.318\end{array}$

$\begin{array}{llll}1170.5 & 0.2325 & -0.000001 & 0.054\end{array}$

$\begin{array}{llll}1171.6 & 0.6011 & -0.000002 & 0.361\end{array}$

$\begin{array}{llll}1179.7 & 0.2091 & -0.000001 & 0.044\end{array}$

$\begin{array}{llll}1185.1 & 1.0248 & -0.000003 & 1.050\end{array}$

$\begin{array}{llll}991.3 & 0.0010 & -0.000017 & 0.000\end{array}$

$\begin{array}{llll}991.3 & 0.0020 & -0.000017 & 0.000\end{array}$

$\begin{array}{lllll}991.4 & 0.0060 & -0.000021 & 0.000\end{array}$

$\begin{array}{lllll}991.6 & 0.0100 & -0.000017 & 0.000\end{array}$

$\begin{array}{lllll}992.0 & 0.0242 & -0.000021 & 0.001\end{array}$

$\begin{array}{llll}999.1 & 0.1532 & -0.000013 & 0.023\end{array}$

$\begin{array}{llll}1007.0 & 0.2270 & -0.000010 & 0.052\end{array}$

$\begin{array}{llll}1010.9 & 0.2506 & -0.000009 & 0.063\end{array}$

$\begin{array}{lllll}991.3 & 0.0101 & -0.000007 & 0.000\end{array}$

$\begin{array}{llll}992.2 & -0.0301 & 0.000010 & 0.001\end{array}$

$\begin{array}{llll}992.9 & 0.0432 & -0.000011 & 0.002\end{array}$

$\begin{array}{lll}993.9 & 0.0428 & -0.000008\end{array}$

$\begin{array}{llll}994.1 & -0.0397 & 0.000007 & 0.002\end{array}$

$\begin{array}{llll}994.1 & 0.0003 & 0.000000 & 0.000\end{array}$

$\begin{array}{llll}994.1 & 0.0403 & -0.000007 & 0.002\end{array}$

$\begin{array}{llll}995.1 & 0.0643 & -0.000009 & 0.004\end{array}$

$\begin{array}{llll}997.9 & 0.1015 & -0.000009 & 0.010\end{array}$

$\begin{array}{lll}9 & 0.0527 & -0.000005\end{array}$

$\begin{array}{lllll}1000.3 & 0.0957 & -0.000006 & 0.009\end{array}$

$\begin{array}{llll}1002.7 & 0.0532 & -0.000003 & 0.003\end{array}$

$\begin{array}{llll}1003.2 & 0.1096 & -0.000006 & 0.012\end{array}$

$\begin{array}{llll}1008.9 & 0.1134 & -0.000004 & 0.013\end{array}$

$\begin{array}{llll}1009.6 & 0.0502 & -0.000002 & 0.003\end{array}$

$\begin{array}{llll}1009.6 & 0.0655 & -0.000002 & 0.004\end{array}$

$\begin{array}{llll}1009.6 & 0.0402 & -0.000001 & 0.002\end{array}$

$\begin{array}{llll}1009.9 & 0.1010 & -0.000003 & 0.010\end{array}$

$\begin{array}{llll}1015.4 & 0.0933 & -0.000002 & 0.009\end{array}$

$\begin{array}{llll}1020.8 & -0.0011 & 0.000000 & 0.000\end{array}$

$\begin{array}{llll}1022.4 & 0.1634 & -0.000003 & 0.027\end{array}$

$\begin{array}{llll}1026.3 & 0.1594 & -0.000003 & 0.025\end{array}$

$\begin{array}{llll}1028.2 & -0.2087 & 0.000004 & 0.044\end{array}$

$\begin{array}{llll}1028.2 & -0.0687 & 0.000001 & 0.005\end{array}$
992.2158

992.2158

992.2158

992.2158

992.2158

992.2158

992.2158

992.2158

992.2158

992.2158

992.2158

992.2158

992.2158

992.2158

992.2158

992.2158

0.41

0.82

2.06

4.1
8.24

81.22

161.03

200.58

991.237

991.237

991.237

991.237

991.237

991.237

991.237

991.237

990.2132

990.2132

990.2132

990.2132

990.2132

990.2132

990.2132

990.2132

4.939

7.792

990.2132

990.2132

990.2132

990.2132

990.2132

990.2132

990.2132

990.2132

990.2132

990.2132

990.2132

990.2132

990.2132

990.2132

990.2132 
molality molarity

$t /{ }^{\circ} \mathrm{C}$ mass frac Density $\exp$

1

1.2
1.4309

1.8001

2

2.5
2.5333

3
4

4.5

0.01719

0.05351

0.0625

0.125

0.1723

0.25
0.5

0.55893

1.13947

2.35546

3.87979

5.45241

0.05
0.1

0.1
0.1
0.2

0.32

0.5
0.5

0.5

0.8
1
1

1.2

1.95
2

25

3

4.5

0.0999

0.7069 $\mathrm{kg} / \mathrm{m}^{3}$

$\begin{array}{rrrr} & & \mathrm{kg} / \mathrm{m}^{3} & \\ 45 & 0.0552 & 1028.18 & (67) \\ 45 & 0.0655 & 1035.42 & (80) \\ 45 & 0.0772 & 1043.77 & (89) \\ 45 & 0.0952 & 1056.70 & (89) \\ 45 & 0.1047 & 1063.20 & (27) \\ 45 & 0.1047 & 1063.32 & (44) \\ 45 & 0.1275 & 1079.90 & (27) \\ 45 & 0.1275 & 1079.92 & (67) \\ 45 & 0.1290 & 1081.10 & (89) \\ 45 & 0.1492 & 1095.99 & (44) \\ 45 & 0.1895 & 1126.98 & (44) \\ 45 & 0.2082 & 1141.33 & (67) \\ 45 & 0.2261 & 1155.52 & (44) \\ 50 & 0.0010 & 988.68 & (31) \\ 50 & 0.0031 & 990.16 & (31) \\ 50 & 0.0036 & 990.52 & (74) \\ 50 & 0.0073 & 992.96 & (74) \\ 50 & 0.0100 & 994.81 & (31) \\ 50 & 0.0144 & 997.69 & (74) \\ 50 & 0.0284 & 1006.86 & (74) \\ 50 & 0.0316 & 1009.57 & (31) \\ 50 & 0.0624 & 1030.84 & (31) \\ 50 & 0.1210 & 1072.58 & (31) \\ 50 & 0.1848 & 1120.30 & (31) \\ 50 & 0.2417 & 1165.12 & (31) \\ 55 & 0.0029 & 987.63 & (44) \\ 55 & 0.0058 & 989.57 & (44) \\ 55 & 0.0058 & 989.61 & (80) \\ 55 & 0.0116 & 993.48 & (80) \\ 55 & 0.0184 & 998.09 & (80) \\ 55 & 0.0284 & 1004.95 & (44) \\ 55 & 0.0284 & 1004.96 & (67) \\ 55 & 0.0284 & 1004.90 & (80) \\ 55 & 0.0447 & 1016.05 & (80) \\ 55 & 0.0552 & 1023.33 & (44) \\ 55 & 0.0552 & 1023.34 & (67) \\ 55 & 0.0655 & 1030.52 & (80) \\ 55 & 0.1023 & 1056.63 & (20) \\ 55 & 0.1047 & 1058.25 & (44) \\ 55 & 0.1275 & 1074.85 & (67) \\ 55 & 0.1492 & 1090.78 & (44) \\ 55 & 0.1895 & 1121.55 & (44) \\ 55 & 0.2082 & 1135.93 & (67) \\ 55 & 0.2261 & 1149.90 & (44) \\ 60 & 0.0058 & 987.10 & (52) \\ 60 & 0.0100 & 990.00 & (90) \\ 60 & 0.0200 & 996.70 & (90) \\ 60 & 0.0397 & 1010.40 & (52) \\ & & & \end{array}$

Ref$$
\text { exp / }
$$$$
\begin{aligned}
& \exp / \\
& \mathrm{m}^{3} / \mathrm{kg}
\end{aligned}
$$

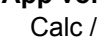

Calc /
$\mathrm{m}^{3} / \mathrm{kg}$

g

$\begin{array}{ll}0.000335 & 0.000334\end{array}$

$\begin{array}{ll}0.000337 & 0.000336\end{array}$

$\begin{array}{ll}0.000338 & 0.000339 \\ 0.000342 & 0.000343\end{array}$

$\begin{array}{ll}0.000342 & 0.000343 \\ 0.000347 & 0.000345\end{array}$

$0.000346 \quad 0.000345$

$0.000352 \quad 0.000350$

0.0003520 .000350

$\begin{array}{ll}0.000352 & 0.000350 \\ 0.000357 & 0.000354\end{array}$

$\begin{array}{ll}0.000363 & 0.000363\end{array}$

$\begin{array}{ll}0.000368 & 0.000367\end{array}$

$\begin{array}{ll}0.000371 & 0.000371\end{array}$

$0.000356 \quad 0.000325$

$\begin{array}{ll}0.000316 & 0.000326 \\ 0.000315 & 0.000326\end{array}$

$\begin{array}{ll}0.000315 & 0.000326 \\ 0.000320 & 0.000327\end{array}$

$\begin{array}{ll}0.000321 & 0.000327\end{array}$

0.0003320 .000328

$0.000346 \quad 0.000331$

$0.000330 \quad 0.000332$

0.0003390 .000338

$0.000353 \quad 0.000350$

$\begin{array}{ll}0.000366 & 0.000364\end{array}$

$0.000376 \quad 0.000376$

$\begin{array}{ll}0.000332 & 0.000328 \\ 0.000331 & 0.000329\end{array}$

$\begin{array}{ll}0.000331 & 0.000329 \\ 0.000324 & 0.000329\end{array}$

$0.000326 \quad 0.000330$

$\begin{array}{lll}0.000328 & 0.000331\end{array}$

$\begin{array}{ll}0.000330 & 0.000333 \\ 0.000329 & 0.000333\end{array}$

$0.000332 \quad 0.000333$

$\begin{array}{ll}0.000336 & 0.000337\end{array}$

0.0003390 .000339

$\begin{array}{ll}0.000339 & 0.000339\end{array}$

$\begin{array}{lll}0.000341 & 0.000341\end{array}$

$\begin{array}{ll}0.000349 & 0.000348 \\ 0.000350 & 0.000349\end{array}$

$0.000354 \quad 0.000353$

$\begin{array}{lll}0.000359 & 0.000358\end{array}$

$\begin{array}{lll}0.000366 & 0.000366\end{array}$

$0.000370 \quad 0.000370$

$\begin{array}{ll}0.000374 & 0.000374 \\ 0.000325 & 0.000330\end{array}$

$\begin{array}{ll}0.000318 & 0.000331\end{array}$

$\begin{array}{ll}0.000328 & 0.000333\end{array}$

$\begin{array}{ll}0.000327 & 0.000337\end{array}$
Density App Vol Square of Inconsistent
based on $\begin{gathered}\text { Inconsistent } \\ \text { based on }\end{gathered}$ calc / Residual / Residual / Density based on based on $\mathrm{kg} / \mathrm{m}^{3} \quad \mathrm{~kg} / \mathrm{m}^{3} \quad \mathrm{~m}^{3} / \mathrm{kg} \quad$ Residual density $\begin{gathered}\text { apparent } \\ \text { volume }\end{gathered}$

$\begin{array}{llll}1028.2 & -0.0315 & 0.000001 & 0.001\end{array}$

$\begin{array}{llll}1035.5 & -0.0591 & 0.000001 & 0.003\end{array}$

$\begin{array}{llll}1043.7 & 0.0265 & 0.000000 & 0.001\end{array}$

$\begin{array}{llll}1056.7 & 0.0345 & 0.000000 & 0.001 \\ 1063.5 & -0.3230 & 0.000003 & 0.104\end{array}$

$1063.5-0.32$

$\begin{array}{lll}1080.2 & -0.3415-0.00002 & 0.041\end{array}$

$\begin{array}{llll}1080.2 & -0.3223 & 0.000002 & 0.104\end{array}$

$\begin{array}{llll}1081.3 & -0.2334 & 0.000002 & 0.054\end{array}$

$\begin{array}{llll}1096.4 & -0.3783 & 0.000002 & 0.143\end{array}$

$\begin{array}{llll}1126.9 & 0.0360 & 0.000000 & 0.001\end{array}$

$\begin{array}{llll}1141.4 & -0.1122 & 0.000000 & 0.013\end{array}$

$\begin{array}{llll}1155.4 & 0.0840 & 0.000000 & 0.007\end{array}$

$\begin{array}{llll}988.7 & -0.0296 & 0.000030 & 0.001\end{array}$

$\begin{array}{llll}990.1 & 0.0305 & -0.000010 & 0.001\end{array}$

$\begin{array}{llll}990.5 & 0.0394 & -0.000011 & 0.002\end{array}$

$\begin{array}{llll}992.9 & 0.0475 & -0.000007 & 0.002\end{array}$

$\begin{array}{lllll}994.7 & 0.0626 & -0.000006 & 0.004\end{array}$

$\begin{array}{llll}997.7 & -0.0551 & 0.000004 & 0.003\end{array}$

$\begin{array}{llll}1007.3 & -0.4208 & 0.000015 & 0.177\end{array}$

$\begin{array}{llll}1009.5 & 0.0663 & -0.000002 & 0.004\end{array}$

$\begin{array}{llll}1030.9 & -0.0575 & 0.000001 & 0.003\end{array}$

$\begin{array}{llll}1072.9 & -0.3272 & 0.000002 & 0.107\end{array}$

$\begin{array}{llll}1120.7 & -0.3815 & 0.000002 & 0.146\end{array}$

$\begin{array}{llll}1164.9 & 0.1980 & -0.000001 & 0.039\end{array}$

$\begin{array}{llll}987.6 & -0.0123 & 0.000004 & 0.000\end{array}$

$\begin{array}{llll}989.6 & -0.0123 & 0.000002 & 0.000 \\ 989.6 & 0.0277 & -0.000005 & 0.001\end{array}$

$993.4-0.0385-0.000003$

$998.0=0.0541-0.000003-0.003$

$\begin{array}{lllll}004.9 & 0.0962 & -0.000003 & 0.009\end{array}$

$\begin{array}{llll}1004.9 & 0.1095 & -0.000004 & 0.012\end{array}$

$\begin{array}{llll}1004.9 & 0.0462 & -0.000002 & 0.002\end{array}$

$\begin{array}{llll}1016.0 & 0.0271 & -0.000001 & 0.001\end{array}$

$\begin{array}{llll}1023.3 & -0.0066 & 0.000000 & 0.000\end{array}$

$\begin{array}{llll}1023.3 & 0.0006 & 0.000000 & 0.000\end{array}$

$\begin{array}{llll}1030.5 & -0.0266 & 0.000000 & 0.001\end{array}$

$\begin{array}{llll}1056.7 & -0.0582 & 0.000001 & 0.003\end{array}$

$\begin{array}{llll}1058.4 & -0.1312 & 0.000001 & 0.017\end{array}$

$\begin{array}{llll}1075.0 & -0.1428 & 0.000001 & 0.020\end{array}$

$\begin{array}{llll}1091.0 & -0.2534 & 0.000001 & 0.064\end{array}$

$\begin{array}{llll}1121.5 & 0.0649 & 0.000000 & 0.004\end{array}$

$\begin{array}{llll}1135.9 & -0.0128 & 0.000000 & 0.000\end{array}$

$\begin{array}{rrrr}1149.9 & -0.0146 & 0.000000 & 0.000 \\ 987.1 & 0.0333 & -0.000006 & 0.001\end{array}$

$989.9 \quad 0.1268-0.000013-$

$\begin{array}{llll}996.6 & 0.1003 & -0.000005 & 0.010\end{array}$

$\begin{array}{llll}1010.0 & 0.4131 & -0.000010 & 0.171\end{array}$

\begin{tabular}{|c|c|c|}
\hline \\
\hline \multirow[t]{2}{*}{37.964} & & 990.2132 \\
\hline & & 990.2132 \\
\hline \multirow{5}{*}{$\begin{array}{l}53.555 \\
66.489\end{array}$} & & 990.2132 \\
\hline & & 990.2132 \\
\hline & & 990.2132 \\
\hline & & 990.2132 \\
\hline & & 990.2132 \\
\hline 89.706 & & 990.2132 \\
\hline \multirow[t]{3}{*}{90.887} & & 990.2132 \\
\hline & & 990.2132 \\
\hline & & 990.2132 \\
\hline \multirow[t]{20}{*}{151.114} & & 990.2132 \\
\hline & & 990.2132 \\
\hline & & 988.0363 \\
\hline & & 988.0363 \\
\hline & 18.4 & 988.0363 \\
\hline & 18.7 & 988.0363 \\
\hline & & 988.0363 \\
\hline & 19.4 & 988.0363 \\
\hline & 20.2 & 988.0363 \\
\hline & & 988.0363 \\
\hline & & 988.0363 \\
\hline & & 988.0363 \\
\hline & & 988.0363 \\
\hline & & 988.0363 \\
\hline & & 985.6952 \\
\hline & & 985.6952 \\
\hline & & 985.6952 \\
\hline & & 985.6952 \\
\hline & & 985.6952 \\
\hline & & 985.6952 \\
\hline \multirow[t]{4}{*}{19.268} & & 985.6952 \\
\hline & & 985.6952 \\
\hline & & 985.6952 \\
\hline & & 985.6952 \\
\hline \multirow[t]{2}{*}{37.642} & & 985.6952 \\
\hline & & 985.6952 \\
\hline \multirow[t]{2}{*}{70.934} & & 985.6952 \\
\hline & & 985.6952 \\
\hline \multirow[t]{3}{*}{89.156} & & 985.6952 \\
\hline & & 985.6952 \\
\hline & & 985.6952 \\
\hline \multirow[t]{6}{*}{150.234} & & 985.6952 \\
\hline & & 985.6952 \\
\hline & & 983.1989 \\
\hline & & 983.1989 \\
\hline & & 983.1989 \\
\hline & & 983.1989 \\
\hline
\end{tabular}


molality molarity

$t /{ }^{\circ} \mathrm{C}$ mass frac Density $\exp$

1.4138

3.5345

0.1
0.1
0.2
0.32
0.5
0.8
1
1.2
2
2.5
0.0999
0.1
0.1
0.2
0.32
0.5
0.7069
0.8
1
1.2
1.4138
2
2.5
3.5345

3.5345

0.5 $\mathrm{kg} / \mathrm{m}^{3}$

$\begin{array}{rrrr} & & \mathrm{kg} / \mathrm{m}^{3} & \\ & & \\ 60 & 0.0400 & 1010.30 & (90) \\ 60 & 0.0763 & 1035.60 & (52) \\ 60 & 0.0800 & 1038.10 & (90) \\ 60 & 0.1200 & 1066.70 & (90) \\ 60 & 0.1600 & 1096.20 & (90) \\ 60 & 0.1712 & 1104.70 & (52) \\ 60 & 0.2000 & 1126.80 & (90) \\ 60 & 0.2400 & 1158.40 & (90) \\ 60 & 0.2600 & 1174.70 & (90) \\ 65 & 0.0058 & 984.50 & (27) \\ 65 & 0.0058 & 984.45 & (80) \\ 65 & 0.0116 & 988.30 & (80) \\ 65 & 0.0184 & 992.88 & (80) \\ 65 & 0.0284 & 999.67 & (80) \\ 65 & 0.0447 & 1010.77 & (80) \\ 65 & 0.0552 & 1017.80 & (27) \\ 65 & 0.0655 & 1025.17 & (80) \\ 65 & 0.1047 & 1052.60 & (27) \\ 65 & 0.1275 & 1069.20 & (27) \\ 75 & 0.0058 & 978.60 & (52) \\ 75 & 0.0058 & 978.90 & (27) \\ 75 & 0.0058 & 978.76 & (80) \\ 75 & 0.0116 & 982.60 & (80) \\ 75 & 0.0184 & 987.18 & (80) \\ 75 & 0.0284 & 993.95 & (80) \\ 75 & 0.0397 & 1001.60 & (52) \\ 75 & 0.0447 & 1005.03 & (80) \\ 75 & 0.0552 & 1012.30 & (27) \\ 75 & 0.0655 & 1019.40 & (80) \\ 75 & 0.0763 & 1027.20 & (52) \\ 75 & 0.1047 & 1047.00 & (27) \\ 75 & 0.1275 & 1063.30 & (27) \\ 75 & 0.1712 & 1096.90 & (52) \\ 80 & 0.0100 & 978.50 & (90) \\ 80 & 0.0200 & 985.20 & (90) \\ 80 & 0.0284 & 990.92 & (67) \\ 80 & 0.0400 & 998.80 & (90) \\ 80 & 0.0552 & 1009.07 & (67) \\ 80 & 0.0800 & 1026.40 & (90) \\ 80 & 0.1200 & 1054.90 & (90) \\ 80 & 0.1275 & 1060.18 & (67) \\ 80 & 0.1600 & 1084.20 & (90) \\ 80 & 0.2000 & 1114.60 & (90) \\ 80 & 0.2082 & 1120.63 & (67) \\ 80 & 0.2400 & 1146.30 & (90) \\ 80 & 0.2600 & 1162.60 & (90) \\ 85 & 0.0058 & 972.55 & (80) \\ 85 & 0.0116 & 976.40 & (80) \\ & & & \end{array}$

Ref

$\exp /$
$\mathrm{m}^{3} / \mathrm{kg}$

$\exp /$
$\mathrm{m}^{3} / \mathrm{kg}$

Calc /
$\mathrm{m}^{3} / \mathrm{kg}$

$\begin{array}{ll}0.000343 & 0.000344\end{array}$

$0.000345 \quad 0.000345$

$0.000354 \quad 0.000353$

$0.000362 \quad 0.000361$

$0.000364 \quad 0.000363$

0.0003690 .000369

$\begin{array}{lll}0.000376 & 0.000377\end{array}$

$\begin{array}{ll}0.000379 & 0.000381 \\ 0.000316 & 0.000332\end{array}$

$0.000325 \quad 0.000332$

$0.000328 \quad 0.000333$

$0.000330 \quad 0.000334$

$0.000333 \quad 0.000336$

$\begin{array}{ll}0.000337 & 0.000339 \\ 0.000344 & 0.000341\end{array}$

$0.000343 \quad 0.000343$

$\begin{array}{ll}0.000353 & 0.000351\end{array}$

$0.000357 \quad 0.000355$

$0.000348 \quad 0.000333$

$0.000295 \quad 0.000333$

$0.000320 \quad 0.000333$

$0.000325 \quad 0.000334$

$\begin{array}{ll}0.000328 & 0.000335\end{array}$

$0.000331 \quad 0.000337$

0.0003350 .000339

$\begin{array}{ll}0.000336 & 0.000340 \\ 0.000338 & 0.000342\end{array}$

$0.000342 \quad 0.000344$

$\begin{array}{ll}0.000341 & 0.000346\end{array}$

$0.000350 \quad 0.000351$

$\begin{array}{ll}0.000356 & 0.000355 \\ 0.000359 & 0.000363\end{array}$

$0.000324 \quad 0.000334$

0.0003290 .000336

$0.000330 \quad 0.000337$

0.0003340 .000339

$\begin{array}{ll}0.000341 & 0.000342 \\ 0.000345 & 0.000346\end{array}$

$0.000353 \quad 0.000353$

$\begin{array}{ll}0.000356 & 0.000354\end{array}$

0.0003620 .000360

$\begin{array}{ll}0.000370 & 0.000367\end{array}$

$\begin{array}{ll}0.000373 & 0.000369 \\ 0.000376 & 0.000375\end{array}$

$0.000379-0.000378$

$\begin{array}{lll}0.000314 & 0.000333\end{array}$

$\begin{array}{lll}0.000320 & 0.000334\end{array}$

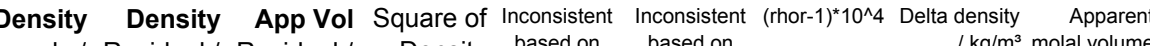

$\mathrm{kg} / \mathrm{m}^{3} \quad \mathrm{~kg} / \mathrm{m}^{3} \quad \mathrm{~m}^{3} / \mathrm{kg}$ Residual density $\begin{gathered}\text { apparent } \\ \text { volume }\end{gathered}$

$\begin{array}{llll}1010.2 & 0.0897 & -0.000002 & 0.008\end{array}$

$\begin{array}{llll}1035.5 & 0.1317 & -0.000002 & 0.017\end{array}$

$\begin{array}{llll}1038.1 & 0.0336 & 0.000000 & 0.001\end{array}$

$\begin{array}{llll}1066.8 & -0.0767 & 0.000001 & 0.006 \\ 1096.3 & -0.1478 & 0.000001 & 0.022\end{array}$

$\begin{array}{llll}1104.8 & -0.0836 & 0.000000 & 0.007\end{array}$

$\begin{array}{llll}1126.8 & 0.0177 & 0.000000 & 0.000\end{array}$

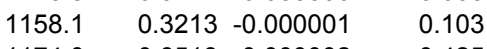

$\begin{array}{llll}1174.0 & 0.6518 & -0.000002 & 0.425\end{array}$

$\begin{array}{llll}984.4 & 0.0864 & -0.000015 & 0.007\end{array}$

$\begin{array}{llll}984.4 & 0.0364 & -0.000006 & 0.001\end{array}$

$\begin{array}{llll}988.2 & 0.0545 & -0.000005 & 0.003\end{array}$

$\begin{array}{llll}992.8 & 0.0716 & -0.000004 & 0.005\end{array}$

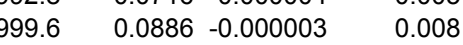

$\begin{array}{llll}1010.7 & 0.0879 & -0.000002 & 0.008\end{array}$

$\begin{array}{llll}1018.0 & -0.1547 & 0.000003 & 0.024\end{array}$

$\begin{array}{llll}1025.1 & 0.0431 & -0.000001 & 0.002\end{array}$

$\begin{array}{llll}1052.8 & -0.2431 & 0.000002 & 0.059\end{array}$

$\begin{array}{llll}1069.4 & -0.2070 & 0.000001 & 0.043\end{array}$

$\begin{array}{llll}978.7 & -0.0853 & 0.000015 & 0.007\end{array}$

$\begin{array}{llll}978.7 & 0.2109 & -0.000038 & 0.044\end{array}$

$\begin{array}{llll}978.7 & 0.0709-0.000013 & 0.005\end{array}$

$\begin{array}{lllll}982.5 & 0.0967 & -0.000009 & 0.009\end{array}$

$\begin{array}{llll}987.0 & 0.1338 & -0.000007 & 0.018\end{array}$

$\begin{array}{llll}993.8 & 0.1584 & -0.000006 & 0.025\end{array}$

$\begin{array}{llll}1001.4 & 0.1558 & -0.000004 & 0.024\end{array}$

$\begin{array}{llll}1004.9 & 0.1771 & -0.000004 & 0.031\end{array}$

$\begin{array}{llll}1012.1 & 0.1964 & -0.000003 & 0.039\end{array}$

$\begin{array}{llll}1019.3 & 0.1428 & -0.000002 & 0.020\end{array}$

$\begin{array}{llll}1026.8 & 0.4010 & -0.000005 & 0.161\end{array}$

$\begin{array}{llll}1046.9 & 0.0679 & -0.000001 & 0.005\end{array}$

$\begin{array}{llll}1063.5 & -0.1953 & 0.000001 & 0.038\end{array}$

$\begin{array}{llll}1096.1 & 0.8183 & -0.000004 & 0.670\end{array}$

$\begin{array}{llll}978.4 & 0.0919 & -0.000010 & 0.008\end{array}$

$\begin{array}{lllll}985.1 & 0.1249 & -0.000006 & 0.016\end{array}$

$\begin{array}{lllll}990.7 & 0.2058 & -0.000007 & 0.042\end{array}$

$\begin{array}{llll}998.6 & 0.2191 & -0.000005 & 0.048\end{array}$

$\begin{array}{llll}1009.0 & 0.0609 & -0.000001 & 0.004\end{array}$

$\begin{array}{llll}1026.3 & 0.1097 & -0.000001 & 0.012\end{array}$

$\begin{array}{llll}1055.0 & -0.0511 & 0.000000 & 0.003\end{array}$

$\begin{array}{llll}1060.4 & -0.2343 & 0.000002 & 0.055\end{array}$

$\begin{array}{llll}1084.6 & -0.3870 & 0.000002 & 0.150\end{array}$

$\begin{array}{llll}1115.2 & -0.6201 & 0.000002 & 0.385\end{array}$

$\begin{array}{llll}1121.6 & -1.0224 & 0.000004 & 1.045\end{array}$

$\begin{array}{llll}1146.9 & -0.5705 & 0.000002 & 0.325\end{array}$

$\begin{array}{llll}1163.1 & -0.4828 & 0.000001 & 0.233\end{array}$

$\begin{array}{llll}972.4 & 0.1002 & -0.000018 & 0.010\end{array}$

$\begin{array}{llll}976.3 & 0.1457 & -0.000013 & 0.021\end{array}$
983.1989

983.1989

983.1989

983.1989

983.1989

983.1989

983.1989

983.1989

980.5548

980.5548

980.5548

980.5548

980.5548

980.5548

980.5548

980.5548

980.5548

980.5548

974.849

974.849

974.849

974.849

974.849

974.849

974.849

974.849

974.849

974.849

974.849

974.849

974.849

974.849

971.7978

971.7978

971.7978

971.7978

971.7978

971.7978

971.7978

971.7978

971.7978

971.7978

971.7978

971.7978

968.6203 
molality molarity

$\mathrm{t} /{ }^{\circ} \mathrm{C}$ mass frac Density

0.32

0.5

0.5
0.8
1.2

1.2
0.1

0.2

0.32

0.8

1.2

0.5

0.7069

1
1
1.4138

2

3.5345

4.5
0.5
1
2.5
4.5
0.0999
0.7069
1
1.4138
2
2.5
3.5345
0.5
1
2.5
4.5 $\exp$ $\mathrm{kg} / \mathrm{m}^{3}$

$\begin{array}{rrr} & & \begin{array}{r}\text { exp / } \\ \mathrm{kg} / \mathrm{m}^{3}\end{array} \\ 85 & 0.0184 & 980.98 \\ 85 & 0.0284 & 987.76 \\ 85 & 0.0447 & 998.86 \\ 85 & 0.0655 & 1013.24 \\ 95 & 0.0058 & 965.84 \\ 95 & 0.0116 & 969.70 \\ 95 & 0.0184 & 974.29 \\ 95 & 0.0284 & 981.09 \\ 95 & 0.0447 & 992.23 \\ 95 & 0.0655 & 1006.67 \\ 95 & 0.1023 & 1032.45 \\ 100 & 0.0058 & 962.30 \\ 100 & 0.0058 & 962.70 \\ 100 & 0.0100 & 965.10 \\ 100 & 0.0200 & 971.90 \\ 100 & 0.0284 & 977.65 \\ 100 & 0.0397 & 985.50 \\ 100 & 0.0400 & 985.50 \\ 100 & 0.0552 & 996.30 \\ 100 & 0.0552 & 995.97 \\ 100 & 0.0763 & 1011.30 \\ 100 & 0.0800 & 1013.40 \\ 100 & 0.1047 & 1031.30 \\ 100 & 0.1200 & 1042.00 \\ 100 & 0.1275 & 1047.70 \\ 100 & 0.1275 & 1047.14 \\ 100 & 0.1600 & 1071.30 \\ 100 & 0.1712 & 1081.30 \\ 100 & 0.2000 & 1101.70 \\ 100 & 0.2082 & 1108.22 \\ 120 & 0.0284 & 962.67 \\ 120 & 0.0552 & 981.20 \\ 120 & 0.1275 & 1032.98 \\ 120 & 0.2082 & 1094.25 \\ 125 & 0.0058 & 943.40 \\ 125 & 0.0397 & 967.10 \\ 125 & 0.0552 & 978.10 \\ 125 & 0.0763 & 993.50 \\ 125 & 0.1047 & 1013.30 \\ 125 & 0.1275 & 1029.90 \\ 125 & 0.1712 & 1064.40 \\ 140 & 0.0284 & 946.16 \\ 140 & 0.0552 & 965.09 \\ 140 & 0.1275 & 1017.76 \\ 140 & 0.2082 & 1079.91 \\ & & \end{array}$

Ref

$\mathrm{m}^{3} / \mathrm{k}$

$(80)$
$(80)$

$(80)$

$\begin{array}{ll}0.000324 & 0.000335\end{array}$

$0.000328 \quad 0.000336$

$0.000333 \quad 0.000339$

$\begin{array}{ll}0.000339 & 0.000343 \\ 0.000310 & 0.000331\end{array}$

$\begin{array}{lll}(80) & 0.000310 & 0.000331 \\ (80) & 0.000316 & 0.000332\end{array}$

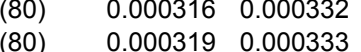

$\begin{array}{lll}(80) & 0.000323 & 0.000334\end{array}$

$\begin{array}{ll}0.000328 & 0.000337\end{array}$

$0.000334 \quad 0.000340$

$\begin{array}{ll}0.000345 & 0.000346\end{array}$

$\begin{array}{ll}0.000308 & 0.000329\end{array}$

$\begin{array}{ll}0.000235 & 0.000329\end{array}$

$\begin{array}{ll}0.000315 & 0.000330\end{array}$

$\begin{array}{ll}0.000317 & 0.000332\end{array}$

$\begin{array}{ll}0.000318 & 0.000333\end{array}$

$\begin{array}{lll}0.000319 & 0.000335 \\ 0.000325 & 0.000335\end{array}$

$\begin{array}{ll}0.000324 & 0.000337\end{array}$

$\begin{array}{ll}0.000330 & 0.000337\end{array}$

$0.000328 \quad 0.000340$

$\begin{array}{lll}0.000335 & 0.000341\end{array}$

$0.000338 \quad 0.000345$

$\begin{array}{ll}0.000346 & 0.000347\end{array}$

$\begin{array}{ll}0.000346 & 0.000348\end{array}$

$\begin{array}{lll}0.000350 & 0.000348\end{array}$

$\begin{array}{ll}0.000356 & 0.000354 \\ 0.000351 & 0.000355\end{array}$

$\begin{array}{lll}0.000351 & 0.000355 \\ 0.000365 & 0.000360\end{array}$

$\begin{array}{ll}0.000366 & 0.000361\end{array}$

$\begin{array}{ll}0.000300 & 0.000324\end{array}$

$\begin{array}{ll}0.000314 & 0.000328\end{array}$

$\begin{array}{lll}0.000336 & 0.000338\end{array}$

$\begin{array}{lll}0.000357 & 0.000349\end{array}$

$\begin{array}{ll}0.000206 & 0.000318\end{array}$

$\begin{array}{ll}0.000285 & 0.000323\end{array}$

$0.000294 \quad 0.000325$

$0.000299 \quad 0.000328$

$\begin{array}{lll}0.000319 & 0.000332\end{array}$

$0.000328 \quad 0.000335$

$\begin{array}{lll}0.000332 & 0.000341\end{array}$

$0.000270 \quad 0.000312$

$0.000288 \quad 0.000315$

$\begin{array}{ll}0.000316 & 0.000324 \\ 0.000341 & 0.000334\end{array}$

Average Res

Std dev Res

Avg - 4std
$/ \mathrm{kg} / \mathrm{m}^{3}$ molal volume water $/ \mathrm{kg} / \mathrm{m}^{3}$

$\mathrm{kg} / \mathrm{m}^{3} \quad \mathrm{~m}^{3} / \mathrm{kg}$ Residual volume

$\begin{array}{llll}980.8 & 0.1931 & -0.000011 & 0.037\end{array}$

$\begin{array}{lllll}987.5 & 0.2407 & -0.000009 & 0.058\end{array}$

$\begin{array}{llll}998.6 & 0.2947 & -0.000007 & 0.087\end{array}$

$\begin{array}{llll}9 & 0.2784 & -0.000004 & 0.077\end{array}$

$\begin{array}{llll}965.7 & 0.1140 & -0.000021 & 0.013\end{array}$

$\begin{array}{llll}969.5 & 0.1726 & -0.000016 & 0.030\end{array}$

$\begin{array}{llll}974.1 & 0.2324 & -0.000013 & 0.054\end{array}$

$\begin{array}{llll}980.8 & 0.3011 & -0.000011 & 0.091\end{array}$

$\begin{array}{llll}991.8 & 0.3904 & -0.000009 & 0.152\end{array}$

$\begin{array}{llll}1006.3 & 0.4157 & -0.000006 & 0.173 \\ 1032.3 & 0.1220 & -0.000001 & 0.015\end{array}$

$962.2-0.1145-0.000021-0.013$

$\begin{array}{llll}962.2 & 0.5107 & -0.000095 & 0.261\end{array}$

$\begin{array}{llll}965.0 & 0.1392 & -0.000015 & 0.019\end{array}$

$\begin{array}{llll}971.6 & 0.2797 & -0.000015 & 0.078\end{array}$

$\begin{array}{llll}977.3 & 0.3965 & -0.000015 & 0.157\end{array}$

$\begin{array}{llll}984.9 & 0.5938 & -0.000015 & 0.353\end{array}$

$\begin{array}{llll}985.1 & 0.3717 & -0.000010 & 0.138\end{array}$

$\begin{array}{llll}995.6 & 0.7233 & -0.000013 & 0.523\end{array}$

$\begin{array}{llll}995.6 & 0.3900 & -0.000007 & 0.152\end{array}$

$\begin{array}{llll}1010.3 & 0.9813 & -0.000013 & 0.963 \\ 1012.9 & 0.4808 & -0.000006 & 0.231\end{array}$

$1030.6-0.7232-0.000007$

$\begin{array}{llll}1041.8 & 0.2219 & -0.000002 & 0.049\end{array}$

$\begin{array}{llll}1047.3 & 0.4025 & -0.000003 & 0.162\end{array}$

$\begin{array}{llll}1047.3 & -0.1568 & 0.000001 & 0.025\end{array}$

$\begin{array}{rrrr}1071.7 & -0.4475 & 0.000002 & 0.200 \\ 1080.3 & 0.9549 & -0.000005 & 0.912\end{array}$

$\begin{array}{llll}11029 & -1.1709 & 0.000005 & 1.371\end{array}$

$\begin{array}{llll}1109.4 & -1.2008 & 0.000005 & 1.442\end{array}$

$\begin{array}{llll}962.0 & 0.6237 & -0.000024 & 0.389\end{array}$

$\begin{array}{llll}980.5 & 0.7185 & -0.000014 & 0.516\end{array}$

$\begin{array}{llll}1032.8 & 0.1913 & -0.000001 & 0.037\end{array}$

$\begin{array}{llll}1096.2 & -1.9276 & 0.000008 & 3.715\end{array}$

$965.7-0.5792-0.000112$

$.6516-0.000031$

2.013
2.728

2.728
4.590

$\begin{array}{llll}1011.9 & 1.3897 & -0.000013 & 1.931\end{array}$

$\begin{array}{llll}1028.9 & 0.9675 & -0.000007 & 0.936\end{array}$

$\begin{array}{llll}1062.7 & 1.6632 & -0.000009 & 2.766\end{array}$

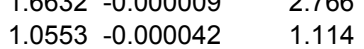

$\begin{array}{lll}1.3862 & -0.000027 & 1.922\end{array}$

$\begin{array}{lll}1.0177 & -0.000008 \quad 1.036\end{array}$

2.742

$0.0713-0.000006$

73.159 Sum Chi sq

$0.3335 \quad 0.000011$

$\begin{array}{ll}-1.2626 & -0.000051\end{array}$
968.6203

968.6203

968.6203

961.9004

961.9004

961.9004

961.9004

961.9004

961.9004

$70.554 \quad 961.9004$

958.3637

958.3637

958.3637

958.3637

$19.29 \quad 958.3637$

958.3637

958.3637

958.3637

958.3637

958.3637

958.3637

958.3637

958.3637

958.3637

958.3637

958.3637

958.3637

958.3637

149.857

19.588

38.118

89.901

151.163

943.0825

943.0825

943.0825

943.0825

938.9841

938.9841

938.9841

938.9841

938.9841

938.9841

938.9841

20.138

926.026

926.026

91.737

926.026

153.884 
molality molarity

$t /{ }^{\circ} \mathrm{C}$ mass frac

Density

$\exp /$
$\mathrm{kg} / \mathrm{m}^{3}$

Ref

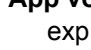

$\exp /$
$\mathrm{m}^{3} / \mathrm{kg}$

Calc /
$\mathrm{m}^{3} / \mathrm{kg}$

Density

calc $/$
$\mathrm{kg} / \mathrm{m}^{3}$

Residual /

$\mathrm{kg} / \mathrm{m}^{3}$

App Vol

Vol Square of Inconsisten

$\mathrm{m}^{3} / \mathrm{kg}$

Density

Avg $+4 s t d$

1.40530 .000039

Curve fit for the model App vol $=\left(w+c 2+c 3^{*} t{ }^{\circ} \mathrm{C}\right) /\left(\mathrm{c} 0^{*} \mathrm{w}+\mathrm{c} 1\right) / \mathrm{e}^{\wedge}\left(0.000001^{*}\left(\mathrm{t}{ }^{\circ} \mathrm{C}+\mathrm{c} 4\right)^{\wedge} 2\right)$

$\mathrm{cO}$

c1

c2

c3

$\mathrm{c} 4$

Min T

$\operatorname{Max} T$

Min w

$-0.004329$

0.064714

0.014624

3315.634

Max w

Avg dens res

0
140

140

0.0713

Avg app vol res *10^3 -0.00568

Std app vol res *10^3 $\quad 0.01129$

No of points in corr

No of inconsistent poir

Inconsistent data not used (Residual greater than average + /- 4 standard deviations)

\begin{tabular}{|c|c|c|c|c|c|c|}
\hline 1.965444 & 25 & 0.1097 & 1047.07 & (32) & 0.000566 & 0.000331 \\
\hline 1 & 35 & 0.0552 & 1030.91 & (74) & 0.000354 & 0.000327 \\
\hline 1 & 40 & 0.0552 & 1028.47 & (74) & 0.000364 & 0.000331 \\
\hline \multirow[t]{4}{*}{1} & 50 & 0.0552 & 1023.73 & (74) & 0.000373 & 0.000337 \\
\hline & 100 & 0.2400 & 1133.1 & (90) & 0.000373 & 0.000366 \\
\hline & 100 & 0.2600 & 1149.2 & (90) & 0.000377 & 0.000369 \\
\hline & 125 & 0.0058 & 943.60 & (27) & 0.000168 & 0.000318 \\
\hline
\end{tabular}

$\begin{array}{rrr}1076.2 & -29.1232 & 0.000236 \\ 1032.5 & -1.5976 & 0.000027\end{array}$

$\begin{array}{llll}1030.4 & -1.9680 & 0.000034\end{array}$

$-2.1100 \quad 0.000036$

$\begin{array}{lll}1135.2 & -2.0922 & 0.000007\end{array}$

$\begin{array}{lll}1135.2 & -2.0922 & 0.000007 \\ 1151.8 & -2.6159 & 0.000008\end{array}$

$\begin{array}{rrr}1151.8 & -2.6159 & 0.000008 \\ 942.8 & 0.7754 & -0.000150\end{array}$

848.162

4.377

6.843

0.601

Inconsistent (rhor-1)*10^4 Delta density Apparent

$0.000168 \quad 0.000318$ based on

apparen
$1 \mathrm{~cm}^{3} / \mathrm{mol}$

Density o

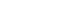

Data from Lyle, T. R., Hosking, R., "The temperature variations ...", Phil Mag Ser 1902, 6, 487 was excluded because many data points are inconsistent 
Density of aqueous solutions of $\mathrm{NaClO} 3$

References (11) Campbell, A. N.; Kartzmark, E. M.; Oliver, B. G. The Electrolytic Conductances of Sodium Chlorate and of Lithium Chlorate in Water and in Water-Dioxane. Can. J. Chem. 1966, 44, 925-934.

(90) Perry, R. H.; Green, D. W. Perry's Chemical Engineers' Handbook; McGraw Hill: New York, 7th edition, 1997.

(108) Roux, A.; Musbally, G. M.; Perron, G.; Singh, P. P.; Woolley, E. M.; Hepler, L. G. Apparent Molal Heat Capacities and Volumes of Aqueous Electrolytes at $25^{\circ} \mathrm{C}$ : $\mathrm{NaClO} 3, \mathrm{NaClO} 4, \mathrm{NaNO} 3, \mathrm{NaBrO} 3, \mathrm{NaIO} 3, \mathrm{KClO} 3, \mathrm{KBrO} 3, \mathrm{KIO} 3, \mathrm{NH} 4 \mathrm{NO} 3, \mathrm{NH} 4 \mathrm{Cl}$, and $\mathrm{NH} 4 \mathrm{ClO} 4$. Can J. Chem. 1978, 56, 24-28.

molality molarity

\begin{tabular}{|c|c|c|c|}
\hline molarity & $\mathbf{t} / \mathrm{C}^{\circ}$ & ass trac & $\begin{array}{r}\text { Density } \\
\exp \\
\mathrm{kg} / \mathrm{m}^{3}\end{array}$ \\
\hline & 18 & 0.0100 & 1005.30 \\
\hline & 18 & 0.0200 & 1012.10 \\
\hline & 18 & 0.0400 & 1025.80 \\
\hline & 18 & 0.0600 & 1039.70 \\
\hline & 18 & 0.0800 & 1053.80 \\
\hline & 18 & 0.1000 & 1068.10 \\
\hline & 18 & 0.1200 & 1082.70 \\
\hline & 18 & 0.1400 & 1097.70 \\
\hline & 18 & 0.1600 & 1113.10 \\
\hline & 18 & 0.1800 & 1128.80 \\
\hline & 18 & 0.2000 & 1144.90 \\
\hline & 18 & 0.2200 & 1161.40 \\
\hline & 18 & 0.2400 & 1178.20 \\
\hline & 18 & 0.2600 & 1195.30 \\
\hline & 18 & 0.2800 & 1212.80 \\
\hline & 18 & 0.3000 & 1230.70 \\
\hline & 18 & 0.3200 & 1249.10 \\
\hline & 18 & 0.3400 & 1268.00 \\
\hline & 18 & 0.4000 & 1333.00 \\
\hline & 18 & 0.4100 & 1344.00 \\
\hline & 18 & 0.4200 & 1354.00 \\
\hline & 18 & 0.4300 & 1365.00 \\
\hline & 18 & 0.4400 & 1376.00 \\
\hline & 18 & 0.4500 & 1387.00 \\
\hline & 18 & 0.4600 & 1399.00 \\
\hline & 18 & 0.4700 & 1410.00 \\
\hline & 18 & 0.4800 & 1422.00 \\
\hline & 18 & 0.4900 & 1434.00 \\
\hline & 18 & 0.5000 & 1446.00 \\
\hline 0.005 & 25 & 0.0005 & 997.60 \\
\hline 0.01 & 25 & 0.0011 & 997.90 \\
\hline 0.02433 & 25 & 0.0026 & 999.00 \\
\hline
\end{tabular}

Ref

(90)

(90)

$(90)$
$(90)$

$(90)$
$(90)$

$(90)$
$(90)$
$(90)$

(90)

(90)

$(90)$
$(90)$

(90)

(90)

$(90)$
$(90)$

$(90)$

(90)

$(90)$
$(90)$

(90)

$(90)$
$(90)$

(90)

$(90)$
$(90)$

(90)

(90)

(90)

(90)

(90)

(90)

(90)

(90)

(11)

(11)

\section{App vol App vol}

$\begin{array}{cc}\exp / & \text { Calc } / \\ \mathrm{m}^{3} / \mathrm{kg} & \mathrm{m}^{3} / \mathrm{kg}\end{array}$

$0.000334 \quad 0.000346$

$0.000333 \quad 0.000347$

$0.000337 \quad 0.000349$

$0.000342 \quad 0.000351$

$0.000346 \quad 0.000352$

$0.000350 \quad 0.000354$

$0.000353 \quad 0.000356$

$0.000356 \quad 0.000357$

$0.000358 \quad 0.000359$

$0.000360 \quad 0.000361$

0.0003620 .000362

$0.000363 \quad 0.000364$

$0.000365 \quad 0.000365$

$0.000368 \quad 0.000366$

$0.000370 \quad 0.000368$

$0.000372 \quad 0.000369$

$0.000374 \quad 0.000371$

$0.000376 \quad 0.000372$

$\begin{array}{lll}0.000373 & 0.000376\end{array}$

$0.000374 \quad 0.000376$

$\begin{array}{ll}0.000376 & 0.000377\end{array}$

$\begin{array}{lll}0.000376 & 0.000377\end{array}$

$0.000377 \quad 0.000378$

$0.000378 \quad 0.000379$

$\begin{array}{lll}0.000378 & 0.000379\end{array}$

$0.000380 \quad 0.000380$

$0.000380 \quad 0.000380$

$0.000381 \quad 0.000381$

0.0003820 .000382

$-0.000043 \quad 0.000346$

$0.000197 \quad 0.000346$

$0.000246 \quad 0.000346$
Density Density App Vol Square of Inconsistent Inconsistent Density based on based on $\mathrm{kg} / \mathrm{m}^{3} \mathrm{~kg} / \mathrm{m}^{3} \quad \mathrm{~m}^{3} / \mathrm{kg}$ Residual density apparent

$1005.2 \quad 0.1271-0.000013 \quad 0.016$

$\begin{array}{llll}1011.8 & 0.2815 & -0.000014 & 0.079\end{array}$

$1025.3 \quad 0.4802-0.000011 \quad 0.231$

$\begin{array}{llll}1039.1 & 0.5897 & -0.000009 & 0.348\end{array}$

$1053.2 \quad 0.5984-0.000007 \quad 0.358$

$\begin{array}{llll}1067.6 & 0.4946 & -0.000004 & 0.245\end{array}$

$1082.3 \quad 0.3656-0.000003 \quad 0.134$

$\begin{array}{llll}1097.4 & 0.2984 & -0.000002 & 0.089\end{array}$

$\begin{array}{llll}1112.8 & 0.2793 & -0.000001 & 0.078\end{array}$

$\begin{array}{llll}1128.6 & 0.1939 & -0.000001 & 0.038\end{array}$

$\begin{array}{llll}1144.8 & 0.1271 & 0.000000 & 0.016\end{array}$

$\begin{array}{llll}1161.3 & 0.0632 & 0.000000 & 0.004\end{array}$

$\begin{array}{llll}1178.3 & -0.1146 & 0.000000 & 0.013\end{array}$

$\begin{array}{llll}1195.7 & -0.4239 & 0.000001 & 0.180\end{array}$

$\begin{array}{llll}1213.6 & -0.7830 & 0.000002 & 0.613\end{array}$

$\begin{array}{llll}1231.9 & -1.2116 & 0.000003 & 1.468\end{array}$

$\begin{array}{llll}1250.7 & -1.6300 & 0.000003 & 2.657\end{array}$

$1270.1 \quad-2.0600 \quad 0.000004 \quad 4.244$

$\begin{array}{llll}1331.4 & 1.6443 & -0.000002 & 2.704\end{array}$

$1342.1 \quad 1.9128-0.000003 \quad 3.659$

$\begin{array}{llll}1353.0 & 1.0248 & -0.000001 & 1.050\end{array}$

$\begin{array}{llll}1364.0 & 0.9768 & -0.000001 & 0.954\end{array}$

$\begin{array}{llll}1375.2 & 0.7648 & -0.000001 & 0.585\end{array}$

$\begin{array}{llll}1386.6 & 0.3852 & 0.000000 & 0.148\end{array}$

$\begin{array}{lllll}1398.2 & 0.8337 & -0.000001 & 0.695\end{array}$

$\begin{array}{llll}1409.9 & 0.1064 & 0.000000 & 0.011\end{array}$

$\begin{array}{llll}1421.8 & 0.1988 & 0.000000 & 0.040\end{array}$

$\begin{array}{llll}1433.9 & 0.1068 & 0.000000 & 0.011\end{array}$

$\begin{array}{llll}1446.2 & -0.1744 & 0.000000 & 0.030\end{array}$

$\begin{array}{llll}997.4 & 0.2063 & -0.000389 & 0.043\end{array}$

$\begin{array}{llll}997.7 & 0.1576 & -0.000148 & 0.025\end{array}$

$\begin{array}{llll}998.7 & 0.2586 & -0.000100 & 0.067\end{array}$
Apparent Density of molal water $/ \mathrm{kg} / \mathrm{m}^{3}$ volume / $\mathrm{cm}^{3} / \mathrm{mol}$

998.5956

998.5956

998.5956

998.5956

998.5956

998.5956

998.5956

998.5956

998.5956

998.5956

998.5956

998.5956

998.5956

998.5956

998.5956

998.5956

998.5956

998.5956

998.5956

998.5956

998.5956

998.5956

998.5956

998.5956

998.5956

998.5956

998.5956

998.5956

998.5956

997.0449

997.0449

997.0449 
molality molarity

$t /{ }^{\circ} \mathrm{C}$ mass frac Density

exp /

$\exp /$
$\mathrm{kg} / \mathrm{m}^{3}$

0.050379

0.12012

0.20006

0.3004

0.42522

0.50573

0.7371

0.88968

1.16574

2.0202

2.9217

3.5062

4.3207

4.9154

5.5812

7.2695

7.7911

\subsection{3}

0.1007

0.3027

0.5045

0.7015

0.905

1.5818

2.5246

3.5329

4.4592

5.2282

5.7954

6.7685

0.00499

0.00997

0.02426

0.0501

0.1003

0.3017

0.5033

0.6987

0.9014
Ref

$\begin{array}{cc}\exp / & \text { Calc / } \\ \mathrm{m}^{3} / \mathrm{kg} & \mathrm{m}^{3} / \mathrm{kg}\end{array}$

0.0003370 .000346

$0.000318 \quad 0.000346$

$0.000324 \quad 0.000347$

$0.000343 \quad 0.000347$

$\begin{array}{lll}0.000342 & 0.000348\end{array}$

$0.000343 \quad 0.000349$

$0.000341 \quad 0.000349$

$0.000346 \quad 0.000350$

$0.000346 \quad 0.000350$

$0.000346 \quad 0.000351$

$0.000365 \quad 0.000351$

$0.000348 \quad 0.000353$

$0.000350 \quad 0.000353$

$\begin{array}{lll}0.000351 & 0.000354\end{array}$

$0.000352 \quad 0.000355$

$0.000354 \quad 0.000357$

$0.000355 \quad 0.000361$

$\begin{array}{ll}0.000361 & 0.000363\end{array}$

0.0003650 .000368

$0.000367 \quad 0.000368$

$\begin{array}{ll}0.000370 & 0.000371\end{array}$

$\begin{array}{lll}0.000373 & 0.000374\end{array}$

$\begin{array}{ll}0.000374 & 0.000374\end{array}$

$\begin{array}{ll}0.000377 & 0.000377\end{array}$

$\begin{array}{lll}0.000378 & 0.000378\end{array}$

0.0003790 .000379

$0.000383 \quad 0.000382$

$\begin{array}{lll}0.000385 & 0.000383\end{array}$

0.0003850 .000384

$0.000389 \quad 0.000384$

$0.000389 \quad 0.000388$

0.0001190 .000344

$0.000088 \quad 0.000345$

$0.000278 \quad 0.000345$

$0.000352 \quad 0.000345$

$0.000340 \quad 0.000346$

$\begin{array}{lll}0.000352 & 0.000348\end{array}$

$0.000357 \quad 0.000351$

$\begin{array}{lll}0.000360 & 0.000353\end{array}$

$\begin{array}{lll}0.000363 & 0.000356\end{array}$
Density Density App Vol Square of Inconsistent Inconsistent calc / Residual / Residual / Density based on based on $\mathrm{kg} / \mathrm{m}^{3} \quad \mathrm{~kg} / \mathrm{m}^{3} \quad \mathrm{~m}^{3} / \mathrm{kg}$ Residual density apparent

$\begin{array}{llll}1000.5 & 0.0489 & -0.000009 & 0.002\end{array}$

$\begin{array}{llll}1000.6 & 0.1490 & -0.000028 & 0.022\end{array}$

$1004.1 \quad 0.2426-0.000023 \quad 0.059$

$\begin{array}{llll}1005.3 & 0.0513 & -0.000004 & 0.003\end{array}$

$1010.8 \quad 0.1325-0.000006 \quad 0.018$

$\begin{array}{llll}1017.6 & 0.1856 & -0.000006 & 0.034\end{array}$

$\begin{array}{llll}1018.1 & 0.2474 & -0.000008 & 0.061\end{array}$

$1026.0 \quad 0.1979-0.000004-0.039$

$1028.6 \quad 0.2090-0.000004 \quad 0.044$

$\begin{array}{lllll}1031.3 & 0.2488 & -0.000005 & 0.062\end{array}$

$\begin{array}{llll}1032.0 & -0.7793 & 0.000014 & 0.607\end{array}$

$1045.4 \quad 0.3793-0.000005 \quad 0.144$

$\begin{array}{llll}1046.4 & 0.2701 & -0.000003 & 0.073\end{array}$

$1056.1 \quad 0.3138-0.000003 \quad 0.098$

$\begin{array}{llll}1059.3 & 0.3280 & -0.000003 & 0.108\end{array}$

$\begin{array}{llll}1073.4 & 0.3382 & -0.000003 & 0.114\end{array}$

$1104.8 \quad 1.0341-0.000006 \quad 1.069$

$\begin{array}{llll}1124.0 & 0.3530 & -0.000002 & 0.125\end{array}$

$\begin{array}{llll}1167.2 & 0.7016 & -0.000002 & 0.492\end{array}$

$\begin{array}{llll}1173.2 & 0.2809 & -0.000001 & 0.079\end{array}$

$\begin{array}{llll}1203.1 & 0.2356 & -0.000001 & 0.056\end{array}$

$\begin{array}{llll}1232.9 & 0.4634 & -0.000001 & 0.215\end{array}$

$\begin{array}{lllll}1242.3 & 0.0329 & 0.000000 & 0.001\end{array}$

$\begin{array}{llll}1269.4 & -0.0624 & 0.000000 & 0.004\end{array}$

$\begin{array}{llll}1292.6 & 0.1713 & 0.000000 & 0.029\end{array}$

$\begin{array}{llll}1298.2 & -0.3656 & 0.000001 & 0.134\end{array}$

$\begin{array}{llll}1341.9 & -0.6039 & 0.000001 & 0.365\end{array}$

$\begin{array}{llll}1365.0 & -1.1672 & 0.000001 & 1.362\end{array}$

$\begin{array}{llll}1377.9 & -0.6149 & 0.000001 & 0.378\end{array}$

$\begin{array}{llll}1384.0 & -3.6526 & 0.000004 & 13.342\end{array}$

$\begin{array}{llll}1439.6 & -1.5015 & 0.000001 & 2.255\end{array}$

$\begin{array}{llll}994.4 & 0.1189 & -0.000225 & 0.014\end{array}$

$\begin{array}{llll}994.7 & 0.2706 & -0.000256 & 0.073\end{array}$

$\begin{array}{llll}995.7 & 0.1711 & -0.000067 & 0.029\end{array}$

$\begin{array}{llll}997.5 & -0.0354 & 0.000007 & 0.001\end{array}$

$\begin{array}{llll}1001.0 & 0.0622 & -0.000006 & 0.004\end{array}$

$\begin{array}{llll}1015.0 & -0.1235 & 0.000004 & 0.015\end{array}$

$\begin{array}{llll}1028.9 & -0.3205 & 0.000006 & 0.103\end{array}$

$\begin{array}{llll}1042.3 & -0.4988 & 0.000006 & 0.249\end{array}$

$\begin{array}{llll}1056.1 & -0.6890 & 0.000007 & 0.475\end{array}$
Apparent Density of molal water $/ \mathrm{kg} / \mathrm{m}^{3}$ volume /

$\mathrm{cm}^{3} / \mathrm{mol}$

$35.86 \quad 997.0449$ 997.0449

997.0449

$36.49 \quad 997.0449$

$36.35 \quad 997.0449$

$36.51 \quad 997.0449$ 997.0449

$36.8 \quad 997.0449$

$36.86 \quad 997.0449$

$36.86 \quad 997.0449$ 997.0449

997.0449

$37.22 \quad 997.0449$

$37.38 \quad 997.0449$ 997.0449

$37.69 \quad 997.0449$ 997.0449

$38.46 \quad 997.0449$ 997.0449

$39.09 \quad 997.0449$

39.42997 .0449 997.0449

$39.84 \quad 997.0449$

$40.09 \quad 997.0449$ 997.0449

$40.37 \quad 997.0449$ 997.0449

$40.94 \quad 997.0449$ 997.0449

$41.36 \quad 997.0449$ 997.0449 994.0319 994.0319 994.0319 994.0319 994.0319 994.0319 994.0319 994.0319 994.0319 
molality molarity

1.5758

2.5114

3.5137

4.4335

5.1998

5.7647

6.7303 $\mathbf{t} /{ }^{\circ} \mathrm{C}$ mass frac Density

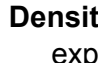

$\exp /$
$\mathrm{kg} / \mathrm{m}^{3}$

$0.1523 \quad 1101.60$

$0.2301 \quad 1161.80$

$0.3049 \quad 1226.70$

$0.3669 \quad 1286.30$

$0.4149 \quad 1334.00$

$0.4479 \quad 1370.00$

$0.5010 \quad 1430.00$
Ref

.

\section{Den}

exp / Calc

$\begin{array}{cc}\exp / & \text { Calc / } \\ \mathrm{m}^{3} / \mathrm{kg} & \mathrm{m}^{3} / \mathrm{kg}\end{array}$

$\begin{array}{ll}0.000361 & 0.000363\end{array}$

$0.000375 \quad 0.000371$

$0.000380 \quad 0.000378$

0.0003830 .000384

$0.000388 \quad 0.000388$

$0.000390 \quad 0.000391$

$0.000394 \quad 0.000395$ calc / Resic

$\mathrm{kg} / \mathrm{m}^{3}$

1101.2

1162.9

1227.5

1285.6

1333.9

1368.8

1428.5

Average Res

Std dev Res

Avg - 4stc

Avg + 4std
Density App V

$$
\mathrm{kg} / \mathrm{m}^{3}
$$

$\mathrm{m}^{3} / \mathrm{k}$

$0.3596-0.000002$

$\begin{array}{ll}-1.1131 & 0.000004\end{array}$

$-0.8136 \quad 0.000002$

$0.6794-0.000001$

$0.1249 \quad 0.000000$

$1.1701-0.000001$

$1.5075-0.000001$

$0.0610-0.000017$

$0.7929 \quad 0.000060$

$-3.1108-0.000258$

$3.2327 \quad 0.000225$

\section{Curve fit for the model App vol $=\left(w+c 2+c 3^{*} t{ }^{\circ} \mathrm{C}\right) /\left(\mathrm{c} 0^{*} \mathrm{w}+\mathrm{c} 1\right) / \mathrm{e}^{\wedge}\left(0.000001^{*}\left(\mathrm{t}{ }^{\circ} \mathrm{C}+\mathrm{c} 4\right)^{\wedge} 2\right)$}

$\begin{array}{lr}\text { c0 } & 0.014762 \\ \text { c1 } 2 & 0.024913 \\ \text { c3 } & 1.292409 \\ \text { c4 } & -0.007617 \\ \text { Min T } & -3454.295 \\ \text { Max T } & 18 \\ \text { Min w } & 35 \\ \text { Max w } & 0.000533 \\ \text { Avg dens res } & 0.500971 \\ \text { Std dens res } & 0.060966 \\ \text { Avg app vol res *10^3 } & 0.0 .0192944 \\ \text { Std app vol res *10^3 } & 0.060387 \\ \text { No of points in corr } & 79 \\ \text { No of inconsistent poir } & 1\end{array}$

Inconsistent data not used (Residual greater than average $+/ \mathbf{4}$ standard deviations)

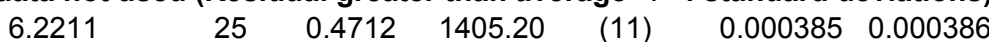

Apparent Density of molal water $/ \mathrm{kg} / \mathrm{m}^{3}$ volume / $\mathrm{cm}^{3} / \mathrm{mol}$

994.0319 994.0319

994.0319

994.0319

994.0319

994.0319

1369

2.273

994.0319 
Density of aqueous solutions of $\mathrm{Na} 2 \mathrm{CO} 3$

References (38) Hershey, J. P.; Sotolongo, S.; Millero, F. J. Densities and Compressibilities of Aqueous Sodium Carbonate and Bicarbonate from 0 to $45^{\circ}$ C. J. Sol. Chem. 1983, $12,233-254$

(41) Hitchcock, L. B.; Mcllhenny, J. S. Viscosity and Density of Pure Alkaline Solutions and their Mixtures. Ind. Engng. Chem. 1935, 27, 461466

(73) Millero, F. J.; Ward, G. K.; Chetirkin, P. V. Relative Sound Velocities of Sea Salts at 25ㄷ. J. Acoust. Soc. Am. 1977, 61, $1492-1498$.

(88) Perron, G., Desnoyers, J. E., Millero, F. J., Apparent molal volumes and heat capacities of some sulfates and carbonates in water at 25 ${ }^{\circ} \mathrm{C}$, Can. J. Chem. , 1975, 53, 1134-1138

molality

0.00974
0.01938
0.02221
0.04425
0.04798
0.06729
0.06737
0.0906
0.12763
0.13143
0.13197
0.18106
0.24773
0.37697
0.49229
0.62869
0.05519
0.05527
0.11524
0.11534
0.22457
0.22529
0.30377
0.30384
0.41673
0.4174
0.56892
0.57107
0.68423
0.68476

\begin{abstract}
$\mathrm{t} /{ }^{\circ} \mathrm{C}$ mass frac Density Ref
\end{abstract}

$$
\exp /
$$$$
\mathrm{kg} / \mathrm{m}^{3}
$$

0
0
0

$0.0010 \quad 1001.02 \quad$ (38)

$0.0020 \quad 1002.17$

$0.0023 \quad 1002.51$

$0.0047 \quad 1005.12$

$0.0051 \quad 1005.57$

$0.0071 \quad 1007.82$

$0.0071 \quad 1007.83$

$0.0095 \quad 1010.53$

$0.0133 \quad 1014.80$

$0.0137 \quad 1015.20$

$0.0138 \quad 1015.29$

$0.0188 \quad 1020.86$

$0.0256 \quad 1028.30$

$0.0384 \quad 1042.42$

$0.0496 \quad 1054.70$

$0.0625 \quad 1068.86$

$0.0058 \quad 1006.41$

$0.0058 \quad 1006.41$

$0.0121 \quad 1013.29$

$0.0121 \quad 1013.28$

$0.0232 \quad 1025.50$

$0.0233 \quad 1025.59$

$0.0312 \quad 1034.19$

$0.0312 \quad 1034.14$

$0.0423 \quad 1046.33$

$0.0424 \quad 1046.39$

$0.0569 \quad 1062.23$

$0.0571 \quad 1062.45$

$0.0676 \quad 1073.96$

$0.0677 \quad 1074.01$ $\begin{array}{rr}\text { App vol } & \text { App vol } \\ \exp / & \text { Calc / }\end{array}$ $\mathrm{m}^{3} / \mathrm{kg} \quad \mathrm{m}^{3} / \mathrm{kg}$

$-0.000144-0.000089$

(38) $\quad-0.000134-0.000088$

(38) $\quad-0.000135-0.000088$

(38) $\quad-0.000126-0.000086$

(38) $\quad-0.000126-0.000085$

(38) $\quad-0.000119-0.000083$

(38) $\quad-0.000119-0.000083$

(38) $\quad-0.000112-0.000080$

(38) $\quad-0.000104-0.000076$

(38) $\quad-0.000102-0.000076$

(38) $\quad-0.000103-0.000076$

(38) $\quad-0.000094-0.000071$

(38) $\quad-0.000082-0.000064$

(38) $\quad-0.000063-0.000051$

(38) $\quad-0.000049-0.000040$

(38) $\quad-0.000034-0.000027$

(38) $\quad-0.000101-0.000071$

(38) $\quad-0.000100-0.000071$

(38) $\quad-0.000090-0.000065$

(38) $\quad-0.000088-0.000065$

(38) $\quad-0.000071-0.000054$

(38) $\quad-0.000071-0.000054$

(38) $\quad-0.000061-0.000046$

(38) $\quad-0.000059-0.000046$

(38) $\quad-0.000048-0.000035$

(38) $\quad-0.000047-0.000035$

(38) $\quad-0.000031-0.000022$

(38) $\quad-0.000031-0.000022$

(38) $\quad-0.000019-0.000012$

(38)
$-0.000019-0.000012$
Density Density App Vol Square of Inconsistent Inconsistent Delta density Density of calc / Residual / Residual / Density based on based on $/ \mathrm{kg} / \mathrm{m}^{3}$ water $/ \mathrm{kg} / \mathrm{m}^{3}$ $\mathrm{kg} / \mathrm{m}^{3} \quad \mathrm{~kg} / \mathrm{m}^{3} \quad \mathrm{~m}^{3} / \mathrm{kg}$ Residual density apparent $\mathrm{kg} / \mathrm{m}^{3} \quad \mathrm{~m}^{3} / \mathrm{kg}$ Residual volume

$1001.0 \quad 0.0565-0.000055 \quad 0.003$

$1002.1 \quad 0.0935-0.000045 \quad 0.009$

$1002.4 \quad 0.1107-0.000047 \quad 0.012$

$\begin{array}{llll}1004.9 & 0.1917 & -0.000041 & 0.037\end{array}$

$1005.4 \quad 0.2113-0.000041 \quad 0.045$

$\begin{array}{llll}1007.6 & 0.2579 & -0.000036 & 0.067\end{array}$

$\begin{array}{llll}1007.6 & 0.2578 & -0.000036 & 0.066\end{array}$

$\begin{array}{llll}1010.2 & 0.3050 & -0.000031 & 0.093\end{array}$

$\begin{array}{llll}1014.4 & 0.3827 & -0.000028 & 0.146\end{array}$

$1014.8 \quad 0.3621-0.000026 \quad 0.131$

$\begin{array}{llll}1014.9 & 0.3872 & -0.000027 & 0.150\end{array}$

$\begin{array}{llll}1020.4 & 0.4517 & -0.000023 & 0.204\end{array}$

$\begin{array}{llll}1027.8 & 0.4869 & -0.000018 & 0.237\end{array}$

$\begin{array}{llll}1041.9 & 0.5187 & -0.000012 & 0.269\end{array}$

$\begin{array}{llll}1054.2 & 0.5073 & -0.000009 & 0.257\end{array}$

$\begin{array}{llll}1068.4 & 0.4679 & -0.000007 & 0.219\end{array}$

$\begin{array}{llll}1006.2 & 0.1745 & -0.000030 & 0.030\end{array}$

$\begin{array}{llll}1006.2 & 0.1705 & -0.000029 & 0.029\end{array}$

$\begin{array}{llll}1013.0 & 0.3112 & -0.000025 & 0.097\end{array}$

$\begin{array}{lllll}1013.0 & 0.2890 & -0.000023 & 0.084\end{array}$

$\begin{array}{llll}1025.1 & 0.4227 & -0.000017 & 0.179\end{array}$

$\begin{array}{llll}1025.2 & 0.4268 & -0.000017 & 0.182\end{array}$

$\begin{array}{llll}1033.7 & 0.4921 & -0.000015 & 0.242\end{array}$

$\begin{array}{llll}1033.7 & 0.4366 & -0.000013 & 0.191\end{array}$

$\begin{array}{llll}1045.8 & 0.5606 & -0.000012 & 0.314\end{array}$

$\begin{array}{llll}1045.8 & 0.5527 & -0.000012 & 0.305\end{array}$

$\begin{array}{llll}1061.7 & 0.5787 & -0.000009 & 0.335\end{array}$

$\begin{array}{llll}1061.9 & 0.5754 & -0.000009 & 0.331\end{array}$

$\begin{array}{llll}1073.4 & 0.5518 & -0.000007 & 0.304\end{array}$

$\begin{array}{llll}1073.5 & 0.5533 & -0.000007 & 0.306\end{array}$
1.181999 .8395

$2.329 \quad 999.8395$

2.672999 .8395

5.284999 .8395

5.731999 .8395

7.985999 .8395

$7.994 \quad 999.8395$

10.686999 .8395

$14.956 \quad 999.8395$

15.364999 .8395

$15.45 \quad 999.8395$

$21.023 \quad 999.8395$

$28.459 \quad 999.8395$

$42.579 \quad 999.8395$

$54.858 \quad 999.8395$

69.025999 .8395

$6.444 \quad 999.9638$

6.449999 .9638

$13.33 \quad 999.9638$

13.319999 .9638

25.54999 .9638

$25.623 \quad 999.9638$

34.224999 .9638

$34.176 \quad 999.9638$

$46.367 \quad 999.9638$

$46.43 \quad 999.9638$

$62.27 \quad 999.9638$

$62.488 \quad 999.9638$

$73.995 \quad 999.9638$

$74.05 \quad 999.9638$ 
molality molarity

\begin{tabular}{|c|c|}
\hline 0.83972 & \\
\hline 0.84138 & \\
\hline 0.95454 & \\
\hline 0.06234 & \\
\hline 0.11948 & \\
\hline 0.23971 & \\
\hline 0.32078 & \\
\hline 0.43994 & \\
\hline 0.60125 & \\
\hline 0.72112 & \\
\hline 0.8833 & \\
\hline & 0.4822 \\
\hline & 0.9725 \\
\hline & 1.425 \\
\hline & 1.9225 \\
\hline & 2.412 \\
\hline & 2.842 \\
\hline & 3.31 \\
\hline & 3.809 \\
\hline 0.004 & \\
\hline 0.00404 & \\
\hline 0.00646 & \\
\hline 0.00653 & \\
\hline 0.00667 & \\
\hline 0.00856 & \\
\hline 0.00858 & \\
\hline 0.01094 & \\
\hline 0.01151 & \\
\hline 0.0185 & \\
\hline 0.01976 & \\
\hline 0.02229 & \\
\hline 0.02384 & \\
\hline 0.02781 & \\
\hline 0.03965 & \\
\hline 0.03972 & \\
\hline 0.04332 & \\
\hline 0.04881 & \\
\hline 0.05626 & \\
\hline 0.06512 & \\
\hline 0.06523 & \\
\hline
\end{tabular}

$\mathrm{t} /{ }^{\circ} \mathrm{C}$ mass frac Density

$\exp /$

$\exp /$
$\mathrm{kg} / \mathrm{m}^{3}$

(10.0

\begin{tabular}{rr}
0.0817 & 1089.40 \\
0.0819 & 1089.48 \\
0.0919 & 1100.31 \\
0.0066 & 1006.13 \\
0.0125 & 1012.44 \\
0.0248 & 1025.41 \\
0.0329 & 1033.98 \\
0.0446 & 1046.34 \\
0.0599 & 1062.70 \\
0.0710 & 1074.55 \\
0.0856 & 1090.25 \\
0.0488 & 1048.10 \\
0.0941 & 1095.40 \\
0.1328 & 1137.50 \\
0.1720 & 1184.80 \\
0.2089 & 1224.00 \\
0.2392 & 1259.10 \\
0.2710 & 1294.60 \\
0.3034 & 1330.80 \\
0.0004 & 997.49 \\
0.0004 & 997.49 \\
0.0007 & 997.77 \\
0.0007 & 997.77 \\
0.0007 & 997.79 \\
0.0009 & 998.00 \\
0.0009 & 998.00 \\
0.0012 & 998.26 \\
0.0012 & 998.33 \\
0.0020 & 999.10 \\
0.0021 & 999.24 \\
0.0024 & 999.52 \\
0.0025 & 999.69 \\
0.0029 & 1000.13 \\
0.0042 & 1001.41 \\
0.0042 & 1001.41 \\
0.0046 & 1001.82 \\
0.0051 & 1002.42 \\
0.0059 & 1003.22 \\
0.0069 & 1004.17 \\
0.0069 & 1004.17 \\
& \\
\hline
\end{tabular}

Ref

$\begin{array}{rr}\exp / & \text { Calc / } \\ \mathrm{m}^{3} / \mathrm{kg} & \mathrm{m}^{3} / \mathrm{kg}\end{array}$

$$
\mathrm{m}^{3} / \mathrm{kg} \quad \mathrm{m}^{3} / \mathrm{kg}
$$

(38) $\quad-0.000005 \quad 0.000001$

$\begin{array}{lll}\text { (38) } & -0.000004 & 0.000001\end{array}$

$\begin{array}{lll}\text { (38) } & 0.000007 & 0.000010\end{array}$

$\begin{array}{lll}\text { (38) } & -0.000065 & -0.000047\end{array}$

(38) $\quad-0.000054-0.000042$

(38) $\quad-0.000036-0.000030$

(38) $\quad-0.000026-0.000023$

(38) $\quad-0.000013-0.000013$

(38) $\quad 0.000001 \quad 0.000000$

(38) 0.0000110 .000010

(38) $\quad 0.000023 \quad 0.000022$

(41) $\quad 0.000024 \quad 0.000000$

(41) $0.000057 \quad 0.000036$

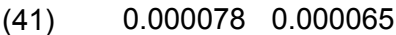

$\begin{array}{lll}\text { (41) } & 0.000084 & 0.000092\end{array}$

$\begin{array}{lll}\text { (41) } \quad 0.000117 & 0.000117\end{array}$

(41) $\quad 0.000134 \quad 0.000136$

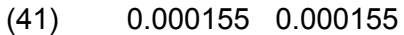

(41) $\quad 0.000176 \quad 0.000173$

(38) $\quad-0.000065-0.000032$

(88) $\quad-0.000054-0.000032$

(38) $\quad-0.000061-0.000031$

(88) $\quad-0.000049-0.000031$

(38) $-0.000056-0.000031$

(38) $\quad-0.000055-0.000031$

(88) $\quad-0.000053-0.000031$

(38) $\quad-0.000055-0.000031$

(38) $\quad-0.000057-0.000031$

(38) $\quad-0.000052-0.000030$

(38) $\quad-0.000051-0.000030$

(38) $\quad-0.000051-0.000030$

(38) $\quad-0.000049-0.000030$

(38) $\quad-0.000049-0.000029$

(38) $\quad-0.000041-0.000028$

$\begin{array}{lll}\text { (88) } & -0.000040 & -0.000028\end{array}$

(38) $\quad-0.000042-0.000028$

(38) $\quad-0.000042-0.000027$

(38) $\quad-0.000038-0.000027$

(38) $\quad-0.000035-0.000026$

(88) $\quad-0.000034-0.000026$
Density Density App Vol Square of Inconsistent Inconsistent Delta density Density of calc / Residual / Residual / Density based on based on $/ \mathrm{kg} / \mathrm{m}^{3}$ water $/ \mathrm{kg} / \mathrm{m}^{3}$ $\mathrm{kg} / \mathrm{m}^{3} \quad \mathrm{~kg} / \mathrm{m}^{3} \quad \mathrm{~m}^{3} / \mathrm{kg}$ Residual density apparent

$1088.9 \quad 0.5225-0.000005 \quad 0.273$

$1089.0 \quad 0.4416-0.000005 \quad 0.195$

$1100.0 \quad 0.2663-0.000002 \quad 0.071$

$\begin{array}{llll}1006.0 & 0.1211 & -0.000018 & 0.015\end{array}$

$\begin{array}{llll}1012.3 & 0.1587 & -0.000012 & 0.025\end{array}$

$1025.3 \quad 0.1413-0.000005 \quad 0.020$

$1033.9 \quad 0.0984-0.000003 \quad 0.010$

$\begin{array}{llll}1046.3 & 0.0294 & -0.000001 & 0.001\end{array}$

$\begin{array}{llll}1062.7 & -0.0378 & 0.000001 & 0.001\end{array}$

$\begin{array}{llll}1074.7 & -0.1106 & 0.000001 & 0.012\end{array}$

$\begin{array}{llll}1090.4 & -0.1647 & 0.000002 & 0.027\end{array}$

$\begin{array}{llll}1049.4 & -1.2746 & 0.000024 & 1.625\end{array}$

$\begin{array}{llll}1097.8 & -2.3714 & 0.000021 & 5.623\end{array}$

$\begin{array}{llll}1139.7 & -2.2190 & 0.000013 & 4.924\end{array}$

$1182.9 \quad 1.9233-0.000008 \quad 3.699$

$\begin{array}{llll}1224.1 & -0.0871 & 0.000000 & 0.008\end{array}$

$1258.5 \quad 0.6092-0.000002 \quad 0.371$

$\begin{array}{llll}1294.9 & -0.3196 & 0.000001 & 0.102\end{array}$

$\begin{array}{llll}1332.6 & -1.7555 & 0.000003 & 3.082\end{array}$

$\begin{array}{lllll}997.5 & 0.0140 & -0.000033 & 0.000\end{array}$

$\begin{array}{llll}997.5 & 0.0096 & -0.000023 & 0.000\end{array}$

$\begin{array}{llll}997.7 & 0.0200 & -0.000029 & 0.000\end{array}$

$\begin{array}{lllll}997.8 & 0.0124 & -0.000018 & 0.000\end{array}$

$\begin{array}{llll}997.8 & 0.0171 & -0.000024 & 0.000\end{array}$

$\begin{array}{llll}998.0 & 0.0213 & -0.000024 & 0.000\end{array}$

$\begin{array}{lllll}998.0 & 0.0202 & -0.000022 & 0.000\end{array}$

$\begin{array}{llll}998.2 & 0.0283 & -0.000024 & 0.001\end{array}$

$\begin{array}{llll}998.3 & 0.0322 & -0.000027 & 0.001\end{array}$

$\begin{array}{llll}999.1 & 0.0420 & -0.000022 & 0.002\end{array}$

$\begin{array}{llll}999.2 & 0.0431 & -0.000021 & 0.002\end{array}$

$\begin{array}{llll}999.5 & 0.0492 & -0.000021 & 0.002\end{array}$

$\begin{array}{llll}999.6 & 0.0479 & -0.000019 & 0.002\end{array}$

$\begin{array}{llll}1000.1 & 0.0569 & -0.000019 & 0.003\end{array}$

$\begin{array}{llll}1001.4 & 0.0553 & -0.000013 & 0.003\end{array}$

$\begin{array}{lllll}1001.4 & 0.0477 & -0.000011 & 0.002\end{array}$

$\begin{array}{llll}1001.8 & 0.0650 & -0.000014 & 0.004\end{array}$

$\begin{array}{llll}1002.3 & 0.0740 & -0.000014 & 0.005\end{array}$

$\begin{array}{llll}1003.1 & 0.0689 & -0.000012 & 0.005\end{array}$

$\begin{array}{llll}1004.1 & 0.0648 & -0.000009 & 0.004\end{array}$

$\begin{array}{llll}1004.1 & 0.0529 & -0.000008 & 0.003\end{array}$
89.439999 .9638

$89.521 \quad 999.9638$

$100.342 \quad 999.9638$

7.035999 .0996

13.343999 .0996

26.315999 .0996

$34.878 \quad 999.0996$

47.241999 .0996

$63.604 \quad 999.0996$

$75.453 \quad 999.0996$

$91.153 \quad 999.0996$

998.2041

998.2041

998.2041

998.2041

998.2041

998.2041

998.2041

998.2041

$0.45 \quad 997.0449$

$0.45 \quad 997.0449$

$0.724 \quad 997.0449$

$0.724 \quad 997.0449$

$0.744 \quad 997.0449$

$0.954 \quad 997.0449$

$0.955 \quad 997.0449$

$1.22 \quad 997.0449$

$1.286 \quad 997.0449$

$2.056 \quad 997.0449$

$2.194 \quad 997.0449$

$2.475 \quad 997.0449$

2.642997 .0449

3.082997 .0449

4.364997 .0449

$4.364 \quad 997.0449$

4.771997 .0449

$5.374 \quad 997.0449$

$6.174 \quad 997.0449$

$\begin{array}{ll}7.126 & 997.0449\end{array}$

$7.126 \quad 997.0449$ 
molality molarity

\section{$\mathrm{t} /{ }^{\circ} \mathrm{C}$ mass frac Density}

$\exp /$

$\exp /$
$\mathrm{kg} / \mathrm{m}^{3}$

0.09175

0.09705

0.12738

0.15489

0.15515

0.17942

0.19736

0.2001

0.24096

0.24137

0.24814

0.29549

0.29598

0.29998

0.37363

0.39969

0.43177

0.43249

0.48684

0.49011

0.49927

0.58212

0.58309

0.59974

0.63524

0.69957

0.71082

0.78884

0.79952

0.87488

0.89963

0.98336

0.98394

0.98578

1.00387

1.9685
2.615

$\begin{array}{ll}0.0096 & 1007.04 \\ 0.0102 & 1007.62 \\ 0.0133 & 1010.86 \\ 0.0162 & 1013.76 \\ 0.0162 & 1013.22 \\ 0.0187 & 1016.34 \\ 0.0205 & 1018.24 \\ 0.0208 & 1018.22 \\ 0.0249 & 1022.78 \\ 0.0249 & 1022.78 \\ 0.0256 & 1023.52 \\ 0.0304 & 1028.42 \\ 0.0304 & 1028.42 \\ 0.0308 & 1028.42 \\ 0.0381 & 1036.36 \\ 0.0406 & 1038.43 \\ 0.0438 & 1042.28 \\ 0.0438 & 1042.28 \\ 0.0491 & 1047.80 \\ 0.0494 & 1048.12 \\ 0.0503 & 1048.28 \\ 0.0581 & 1057.22 \\ 0.0582 & 1057.22 \\ 0.0598 & 1058.06 \\ 0.0631 & 1062.42 \\ 0.0690 & 1067.63 \\ 0.0701 & 1069.72 \\ 0.0772 & 1077.18 \\ 0.0781 & 1077.08 \\ 0.0849 & 1085.36 \\ 0.0871 & 1086.39 \\ 0.0944 & 1095.46 \\ 0.0944 & 1095.45 \\ 0.0946 & 1095.67 \\ 0.0962 & 1099.66 \\ 0.0506 & 1047.20 \\ 0.1375 & 1137.30 \\ 0.1764 & 1182.60 \\ 0.2241 & 1236.70 \\ 0.2498 & 1267.30 \\ & \\ 0\end{array}$

Ref

$\begin{array}{cc}\exp / & \text { Calc / } \\ \mathrm{m}^{3} / \mathrm{kg} & \mathrm{m}^{3} / \mathrm{kg}\end{array}$

$-0.000031-0.000024$

$-0.000031-0.000023$

$-0.000026-0.000020$

$-0.000021-0.000018$

$0.000013-0.000018$

$-0.000017-0.000016$

$-0.000016-0.000014$

$-0.000001-0.000014$

$-0.000010-0.000011$

$-0.000009-0.000011$

$-0.000009-0.000010$

$-0.000005-0.000006$

$-0.000003-0.000006$

$0.000010-0.000006$

$0.000004 \quad 0.000000$

0.0000190 .000002

$\begin{array}{lll}0.000008 & 0.000005\end{array}$

$0.000010 \quad 0.000005$

0.0000130 .000009

$0.000013 \quad 0.000009$

$0.000028 \quad 0.000010$

$0.000021 \quad 0.000016$

$0.000022 \quad 0.000016$

$0.000035 \quad 0.000017$

$0.000025 \quad 0.000020$

$0.000042 \quad 0.000024$

$0.000030 \quad 0.000025$

$0.000036 \quad 0.000031$

0.0000490 .000031

$\begin{array}{lll}0.000041 & 0.000036\end{array}$

$0.000055 \quad 0.000038$

$0.000048 \quad 0.000043$

$0.000049 \quad 0.000043$

$0.000049 \quad 0.000044$

$0.000030 \quad 0.000045$

$0.000026 \quad 0.000018$

$0.000094 \quad 0.000079$

$0.000104 \quad 0.000104$

$0.000131 \quad 0.000133$

$0.000143 \quad 0.000147$
Density Density App Vol Square of Inconsistent Inconsistent Delta density Density of calc / Residual / Residual / Density based on based on $/ \mathrm{kg} / \mathrm{m}^{3}$ water $/ \mathrm{kg} / \mathrm{m}^{3}$ $\mathrm{kg} / \mathrm{m}^{3} \quad \mathrm{~kg} / \mathrm{m}^{3} \quad \mathrm{~m}^{3} / \mathrm{kg}$ Residual density apparent

$1007.5 \quad 0.0769-0.000007$

$\begin{array}{llll}1010.8 & 0.0742 & -0.000005 & 0.006\end{array}$

$\begin{array}{lllll}1013.7 & 0.0494 & -0.000003 & 0.002\end{array}$

$\begin{array}{llll}1013.7 & -0.5182 & 0.000031 & 0.269\end{array}$

$1016.3 \quad 0.0283-0.000001 \quad 0.001$

$\begin{array}{llll}1018.2 & 0.0355 & -0.000002 & 0.001\end{array}$

$\begin{array}{llll}1018.5 & -0.2773 & 0.000013 & 0.077\end{array}$

$\begin{array}{llll}1022.8 & -0.0110 & 0.000000 & 0.000\end{array}$

$\begin{array}{llll}1022.8 & -0.0529 & 0.000002 & 0.003\end{array}$

$\begin{array}{llll}1023.5 & -0.0253 & 0.000001 & 0.001\end{array}$

$\begin{array}{llll}1028.5 & -0.0555 & 0.000002 & 0.003\end{array}$

$\begin{array}{llll}1028.5 & -0.1083 & 0.000003 & 0.012\end{array}$

$1028.9 \quad-0.5191 \quad 0.000016 \quad 0.269$

$\begin{array}{llll}1036.5 & -0.1639 & 0.000004 & 0.027\end{array}$

$\begin{array}{llll}1039.2 & -0.7624 & 0.000017 & 0.581\end{array}$

$\begin{array}{llll}1042.5 & -0.1729 & 0.000004 & 0.030\end{array}$

$\begin{array}{llll}1042.5 & -0.2489 & 0.000005 & 0.062\end{array}$

$\begin{array}{llll}1048.0 & -0.2192 & 0.000004 & 0.048\end{array}$

$\begin{array}{llll}1048.3 & -0.2219 & 0.000004 & 0.049\end{array}$

$\begin{array}{llll}1049.3 & -0.9867 & 0.000018 & 0.974\end{array}$

$\begin{array}{llll}1057.5 & -0.3045 & 0.000005 & 0.093\end{array}$

$\begin{array}{llll}1057.6 & -0.4015 & 0.000006 & 0.161\end{array}$

$\begin{array}{llll}1059.3 & -1.2076 & 0.000018 & 1.458\end{array}$

$\begin{array}{llll}1062.8 & -0.3429 & 0.000005 & 0.118\end{array}$

$\begin{array}{llll}1069.0 & -1.4162 & 0.000018 & 2.006\end{array}$

$\begin{array}{llll}1070.1 & -0.4136 & 0.000005 & 0.171\end{array}$

$\begin{array}{llll}1077.7 & -0.4841 & 0.000005 & 0.234\end{array}$

$\begin{array}{llll}1078.7 & -1.6024 & 0.000018 & 2.568\end{array}$

$\begin{array}{llll}1085.8 & -0.4882 & 0.000005 & 0.238\end{array}$

$\begin{array}{llll}1088.2 & -1.7931 & 0.000017 & 3.215\end{array}$

$\begin{array}{llll}1096.0 & -0.5571 & 0.000005 & 0.310\end{array}$

$\begin{array}{llll}1096.1 & -0.6170 & 0.000005 & 0.381\end{array}$

$\begin{array}{llll}1096.2 & -0.5680 & 0.000005 & 0.323\end{array}$

$\begin{array}{llll}1097.9 & 1.7456 & -0.000015 & 3.047\end{array}$

$\begin{array}{llll}1047.7 & -0.4685 & 0.000008 & 0.219\end{array}$

$\begin{array}{llll}1140.0 & -2.6843 & 0.000015 & 7.205\end{array}$

$\begin{array}{llll}1182.7 & -0.0773 & 0.000000 & 0.006\end{array}$

$\begin{array}{llll}1236.1 & 0.5670 & -0.000002 & 0.321\end{array}$

$\begin{array}{llll}1265.5 & 1.7775 & -0.000004 & 3.160\end{array}$
9.994997 .0449

10.572997 .0449

13.814997 .0449

16.718997 .0449

$16.178 \quad 997.0449$

$19.297 \quad 997.0449$

$21.199 \quad 997.0449$ 997.0449

$25.734 \quad 997.0449$

$25.735 \quad 997.0449$

26.471997 .0449

$31.373 \quad 997.0449$

31.371997 .0449 997.0449

$39.32 \quad 997.0449$ 997.0449

$45.238 \quad 997.0449$

$45.235 \quad 997.0449$

$50.754 \quad 997.0449$

$51.08 \quad 997.0449$ 997.0449

$60.176 \quad 997.0449$

$60.175 \quad 997.0449$ 997.0449

$65.375 \quad 997.0449$ 997.0449

$72.68 \quad 997.0449$

80.131997 .0449 997.0449

$88.315 \quad 997.0449$ 997.0449

$98.414 \quad 997.0449$

$98.408 \quad 997.0449$

$98.628 \quad 997.0449$

102.62997 .0449 995.6473

995.6473

995.6473

995.6473

995.6473 
molality molarity

$\mathbf{t} /{ }^{\circ} \mathrm{C}$ mass frac Density
$\exp /$
$\mathrm{kg} / \mathrm{m}^{3}$

$t /{ }^{\circ} \mathrm{C}$ mass frac Density
$\exp /$
$\mathrm{kg} / \mathrm{m}^{3}$

$t /{ }^{\circ} \mathrm{C}$ mass frac Density
$\exp /$
$\mathrm{kg} / \mathrm{m}^{3}$

0.04367
0.15058
0.26374
0.33415
0.59236
0.76027
0.88907
0.97945
1.00752

0.47915

0.965

1.414

1.907

2.393

2.8195

3.285

3.7665

$0.3082 \quad 1336.20$

$\begin{array}{rr}0.0046 & 998.77 \\ 0.0157 & 1010.02\end{array}$

$0.0272 \quad 1021.65$

$0.0342 \quad 1028.79$

$0.0591 \quad 1054.30$

$0.0746 \quad 1070.38$

$0.0861 \quad 1082.44$

$0.0940 \quad 1090.76$

$0.0965 \quad 1093.27$

$0.0488 \quad 1040.70$

$0.0941 \quad 1087.20$

$0.1329 \quad 1128.10$

$0.1725 \quad 1171.70$

$0.2090 \quad 1213.70$

$0.2712 \quad 1283.80$

$0.3025 \quad 1319.90$
0.04369

0.15064

0.19707

0.26384

0.29544

0.33428

0.39365

0.49172

0.59067

0.59258

0.68899

0.76056

0.78743

0.88603

0.88973

0.97926

1.00771

1.0079

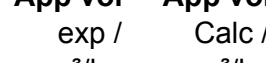

$\mathrm{m}^{3} / \mathrm{kg} \quad \mathrm{m}^{3} / \mathrm{kg}$

0.0001620 .000165

$0.000174 \quad 0.000178$

$0.2394 \quad 1248.30$

$0.0046 \quad 994.86$

$0.0157 \quad 1005.98$

$0.0205 \quad 1011.36$

$0.0272 \quad 1017.47$

$0.0304 \quad 1021.56$

$0.0342 \quad 1024.50$

$0.0401 \quad 1031.58$

$0.0495 \quad 1041.42$

$0.0589 \quad 1051.20$

$0.0591 \quad 1049.68$

$0.0681 \quad 1060.78$

$0.0746 \quad 1065.59$

$0.0770 \quad 1070.22$

$0.0858 \quad 1079.53$

$0.0862 \quad 1077.58$

$0.0940 \quad 1085.75$

$0.0965 \quad 1090.88$

$0.0965 \quad 1088.32$ $\begin{array}{lll}(38) & -0.000029 & -0.000009 \\ (38) & -0.000008 & -0.000001\end{array}$

(38) $\quad-0.000008-0.000001$

$0.000006 \quad 0.000008$

$0.000012 \quad 0.000013$

$0.000033 \quad 0.000031$

$0.000044 \quad 0.000042$

$0.000052 \quad 0.000050$

$0.000057 \quad 0.000056$

$0.000060 \quad 0.000057$

$0.000046 \quad 0.000031$

0.0000720 .000061

$0.000094 \quad 0.000086$

$0.000113 \quad 0.000109$

$0.000128 \quad 0.000129$

$0.000144 \quad 0.000145$

$0.000164 \quad 0.000161$

0.0001810 .000176

$-0.000014 \quad 0.000007$

$0.000003 \quad 0.000015$

$-0.000022 \quad 0.000018$

$\begin{array}{lll}0.000015 & 0.000023\end{array}$

$\begin{array}{ll}-0.000011 & 0.000025\end{array}$

0.0000220 .000028

$\begin{array}{ll}-0.000001 & 0.000032\end{array}$

$0.000007 \quad 0.000038$

$0.000015 \quad 0.000044$

$0.000042 \quad 0.000044$

$0.000023 \quad 0.000050$

$0.000052 \quad 0.000054$

$0.000030 \quad 0.000056$

$0.000037 \quad 0.000061$

$0.000060 \quad 0.000062$

$0.000065 \quad 0.000066$

$0.000044 \quad 0.000068$

$\begin{array}{lll}0.000067 & 0.000068\end{array}$
Density Density App Vol Square of Inconsistent Inconsistent Delta density Density of calc / Residual / Residual / Density based on based on $/ \mathrm{kg} / \mathrm{m}^{3}$ water $/ \mathrm{kg} / \mathrm{m}^{3}$ $\mathrm{kg} / \mathrm{m}^{3} \quad \mathrm{~kg} / \mathrm{m}^{3} \quad \mathrm{~m}^{3} / \mathrm{kg}$ Residual density $\begin{gathered}\text { apparent } \\ \text { volume }\end{gathered}$

$1304.8 \quad 1.4405-0.000003 \quad 2.075$

$1334.0 \quad 2.2399-0.000004 \quad 5.017$

$998.7 \quad 0.0896-0.000019 \quad 0.008$

$1009.9 \quad 0.1105-0.000007 \quad 0.012$

$\begin{array}{llll}1021.6 & 0.0625 & -0.000002 & 0.004\end{array}$

$\begin{array}{llll}1028.8 & 0.0317 & -0.000001 & 0.001\end{array}$

$\begin{array}{llll}1054.4 & -0.0763 & 0.000001 & 0.006\end{array}$

$\begin{array}{llll}1070.5 & -0.1165 & 0.000001 & 0.014\end{array}$

$\begin{array}{llll}1082.6 & -0.1507 & 0.000001 & 0.023\end{array}$

$\begin{array}{llll}1090.9 & -0.1853 & 0.000002 & 0.034\end{array}$

$\begin{array}{llll}1093.5 & -0.2510 & 0.000002 & 0.063\end{array}$

$\begin{array}{llll}1041.5 & -0.7778 & 0.000015 & 0.605\end{array}$

$\begin{array}{llll}1088.4 & -1.1710 & 0.000011 & 1.371\end{array}$

$\begin{array}{llll}1129.5 & -1.4188 & 0.000008 & 2.013\end{array}$

$\begin{array}{llll}1172.6 & -0.9204 & 0.000004 & 0.847\end{array}$

$1213.2 \quad 0.4535-0.000001 \quad 0.206$

$\begin{array}{llll}1247.9 & 0.3988 & -0.000001 & 0.159\end{array}$

$\begin{array}{llll}1284.9 & -1.1359 & 0.000003 & 1.290\end{array}$

$\begin{array}{llll}1322.2 & -2.2526 & 0.000004 & 5.074\end{array}$

$\begin{array}{llll}994.8 & 0.0962 & -0.000021 & 0.009\end{array}$

$\begin{array}{llll}1005.8 & 0.1868 & -0.000012 & 0.035\end{array}$

$\begin{array}{llll}1010.5 & 0.8410 & -0.000040 & 0.707\end{array}$

$\begin{array}{llll}1017.3 & 0.2086 & -0.000007 & 0.044\end{array}$

$\begin{array}{llll}1020.4 & 1.1291 & -0.000036 & 1.275\end{array}$

$\begin{array}{llll}1024.3 & 0.1908 & -0.000005 & 0.036\end{array}$

$\begin{array}{llll}1030.2 & 1.3885 & -0.000033 & 1.928\end{array}$

$\begin{array}{llll}1039.8 & 1.6330 & -0.000030 & 2.667\end{array}$

$\begin{array}{llll}1049.3 & 1.8626 & -0.000029 & 3.469\end{array}$

$\begin{array}{llll}1049.5 & 0.1646 & -0.000003 & 0.027\end{array}$

$1058.7 \quad 2.0841-0.000027 \quad 4.343$

$\begin{array}{llll}1065.4 & 0.1664 & -0.000002 & 0.028\end{array}$

$1067.9 \quad 2.2887-0.000026 \quad 5.238$

$1077.1 \quad 2.4751-0.000025 \quad 6.126$

$\begin{array}{llll}1077.4 & 0.1831 & -0.000002 & 0.034\end{array}$

$1085.6 \quad 0.1773-0.000002 \quad 0.031$

$\begin{array}{llll}1088.1 & 2.7286 & -0.000024 & 7.445\end{array}$

$1088.2 \quad 0.1515-0.000001 \quad 0.023$

$\begin{array}{lll}\text { Average Res } & 0.0608 & -0.000008\end{array}$

995.6473

995.6473

$4.734 \quad 994.0319$

$15.988 \quad 994.0319$

27.623994 .0319

$34.757 \quad 994.0319$

$60.264 \quad 994.0319$

$76.348 \quad 994.0319$

88.411994 .0319

$96.729 \quad 994.0319$

99.235994 .0319

992.2158

992.2158

992.2158

992.2158

992.2158

992.2158

992.2158

992.2158

4.651990 .2132

$15.764 \quad 990.2132$

$21.148 \quad 990.2132$

$27.26 \quad 990.2132$

$31.349 \quad 990.2132$

$34.285 \quad 990.2132$

$41.362 \quad 990.2132$

$51.207 \quad 990.2132$

$60.986 \quad 990.2132$

$59.471 \quad 990.2132$

$70.563 \quad 990.2132$

$75.374 \quad 990.2132$

$80.005 \quad 990.2132$

$89.317 \quad 990.2132$

$87.365 \quad 990.2132$

$95.534 \quad 990.2132$

$100.662 \quad 990.2132$

$98.102 \quad 990.2132$ 
molality molarity

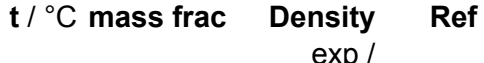

$\exp /$

$\mathrm{kg} / \mathrm{m}^{3}$
Ref
$\exp /$
$\mathrm{m}^{3} / \mathrm{kg}$

\section{App vol App vol \\ Calc /}

Calc /
$\mathrm{m}^{3} / \mathrm{kg}$
Density Density App Vol Square of Inconsistent Incon calc / R $\mathrm{kg} / \mathrm{m}^{3}$

$\mathrm{kg} / \mathrm{m}^{3}$

Residual

$\mathrm{m}^{3} / \mathrm{kg}$

Avg +4 std

3.44340 .000055

Curve fit for the model App vol $=\left(w+c 2+c 3^{\star} t{ }^{\circ} \mathrm{C}\right) /\left(\mathrm{c} 0^{\star} w+c 1\right) / e^{\wedge}\left(0.000001^{\star}\left(t^{\circ} \mathrm{C}+c 4\right)^{\wedge} 2\right)$

c0

c1

c1 0.014217

c2 -0.091456

c3 $\quad 0.002134$

c4 3342.364

Min T 0

Max T $\quad 45$

Min w $\quad 0.0004$

Max w $\quad 0.3082$

Avg dens res $\quad 0.060811$

Std dens res $\quad 0.84564$

Avg app vol res *10^3 -0.008186

Std app vol res *10^3 $\quad 0.0159$

No of points in corr

No of inconsistent poir

147

Inconsistent data not used (Residual greater than average $+/-4$ standard deviations) Perry's data not used 
Density of aqueous solutions of $\mathrm{NaF}$

References (55) Krishnamurty, B. Ultrasonic Studies in Electrolytes: Part I - Alkali Halides. Ind. Research (India) 1950, 9B, 215-219.

(72) Millero, F. J.; Knox, J. H. Apparent Molal Volumes of Aqueous NaF, Na2SO4, KCl, K2SO4, MgCl2, and $\mathrm{MgSO} 4 \mathrm{Solutions}$ at $0^{\circ}$ and $50^{\circ} \mathrm{C}$. J. Chem. Eng. Data 1973, 18, 407-411.

(73) Millero, F. J.; Ward, G. K.; Chetirkin, P. V. Relative Sound Velocities of Sea Salts at 25 C. J. Acoust. Soc. Am. 1977, 61, $1492-1498$.

(86) Pedersen, T. G.; Dethlefsen, C.; Hvidt, A. Volumetric Properties of Aqueous Solutions of Alkali Halides Carlsberg Res. Commun. 1984, 49, 445-455.

(114) Saluja, P. P. S.; Lemire, R. J.; LeBlanc, J. C.; High-Temperature Thermodynamics of Aqueous Alkali-Metal Salts; J. Chem. Thermodynamics, $1992,24,181-203$

$\begin{array}{rrrrr}\text { molality } & \text { molarity } & \mathbf{t} /{ }^{\circ} \mathrm{C} \text { mass frac } & \begin{array}{r}\text { Density } \\ \text { exp } / \mathrm{kg} / \mathrm{m}^{3}\end{array} & \\ & & & & \\ 0.00972 & & & & \\ 0.029258 & 0 & 0.0004 & 1000.31 & (72) \\ 0.090096 & 0 & 0.0012 & 1001.25 & (72) \\ 0.153786 & 0 & 0.0038 & 1004.15 & (72) \\ 0.243098 & 0 & 0.0064 & 1007.14 & (72) \\ 0.268629 & 0 & 0.0101 & 1011.30 & (72) \\ 0.360255 & 0 & 0.0112 & 1012.47 & (72) \\ 0.360703 & 0 & 0.0149 & 1016.67 & (72) \\ 0.497018 & 0 & 0.0149 & 1016.70 & (72) \\ 0.01 & 0 & 0.0204 & 1022.86 & (72) \\ 0.03299 & 24.04 & 0.0004 & 997.97 & (114) \\ 0.11127 & 24.04 & 0.0014 & 998.98 & (114) \\ 0.19158 & 24.04 & 0.0047 & 1002.40 & (114) \\ 0.30593 & 24.04 & 0.0080 & 1005.88 & (114) \\ 0.43944 & 24.04 & 0.0127 & 1010.77 & (114) \\ & 24.04 & 0.0181 & 1016.41 & (114) \\ & 25 & 0.0010 & 998.11 & (86) \\ & 25 & 0.0011 & 998.23 & (86) \\ & 25 & 0.0012 & 998.35 & (86) \\ & 25 & 0.0013 & 998.47 & (86) \\ 0.29634 & 25 & 0.0023 & 999.49 & (86) \\ & 25 & 0.0030 & 1000.18 & (86) \\ 0.10084 & 25 & 0.0034 & 1000.66 & (86) \\ & 25 & 0.0040 & 1001.28 & (86) \\ 0.20120 & 25 & 0.0042 & 1001.47 & (73) \\ & 25 & 0.0066 & 1003.95 & (86) \\ & 25 & 0.0076 & 1005.01 & (86) \\ & 25 & 0.0084 & 1005.78 & (73) \\ & 25 & 0.0086 & 1006.03 & (86) \\ & 25 & 0.0096 & 1007.09 & (86) \\ & 25 & 0.0111 & 1008.67 & (86) \\ & 25 & 0.0122 & 1009.73 & (86) \\ & 25 & 0.0123 & 1009.84 & (73) \\ & & & & \\ & & & & \\ & & & \end{array}$

\begin{tabular}{|c|c|c|c|c|c|c|c|c|c|}
\hline $\begin{array}{l}\text { App vol } \\
\exp / \\
\mathrm{m}^{3} / \mathrm{kg}\end{array}$ & $\begin{array}{r}\text { App vol } \\
\text { Calc } / \\
\mathrm{m}^{3} / \mathrm{kg}\end{array}$ & $\begin{array}{r}\text { Density } \\
\text { calc / } \\
\mathrm{kg} / \mathrm{m}^{3}\end{array}$ & $\begin{array}{r}\text { Density } \\
\text { Residual / } \\
\mathrm{kg} / \mathrm{m}^{3}\end{array}$ & $\begin{array}{r}\text { App Vol } \\
\text { Residual / } \\
\mathrm{m}^{3} / \mathrm{kg}\end{array}$ & $\begin{array}{r}\text { Square of } \\
\text { Density } \\
\text { Residual }\end{array}$ & $\begin{array}{l}\text { Inconsistent } \\
\text { based on } \\
\text { density }\end{array}$ & $\begin{array}{c}\text { Inconsistent } \\
\text { based on } \\
\text { apparent } \\
\text { volume }\end{array}$ & $\begin{array}{l}\text { Delta density } \\
\qquad / \mathrm{kg} / \mathrm{m}^{3}\end{array}$ & $\begin{array}{r}\text { Density of } \\
\text { water } / \mathrm{kg} / \mathrm{m}^{3}\end{array}$ \\
\hline-0.000152 & -0.000137 & 1000.3 & 0.0064 & -0.000016 & 0.000 & & & 0.47000 & 999.8395 \\
\hline-0.000148 & -0.000133 & 1001.2 & 0.0195 & -0.000016 & 0.000 & & & 1.4109 & 999.8395 \\
\hline-0.000138 & -0.000120 & 1004.1 & 0.0669 & -0.000018 & 0.004 & & & 4.3064 & 999.8395 \\
\hline-0.000129 & -0.000108 & 1007.0 & 0.1355 & -0.000021 & 0.018 & & & 7.2963 & 999.8395 \\
\hline-0.000122 & -0.000093 & 1011.0 & 0.2988 & -0.000029 & 0.089 & & & 11.4628 & 999.8395 \\
\hline-0.000119 & -0.000089 & 1012.1 & 0.3410 & -0.000030 & 0.116 & & & 12.631 & 999.8395 \\
\hline-0.000111 & -0.000075 & 1016.1 & 0.5583 & -0.000036 & 0.312 & & & 16.8286 & 999.8395 \\
\hline-0.000112 & -0.000075 & 1016.1 & 0.5715 & -0.000037 & 0.327 & & & 16.861 & 999.8395 \\
\hline-0.000101 & -0.000056 & 1021.9 & 0.9717 & -0.000045 & 0.944 & & & 23.0247 & 999.8395 \\
\hline-0.000631 & -0.000096 & 997.7 & 0.2236 & -0.000535 & 0.050 & & $!$ & & 997.2867 \\
\hline-0.000223 & -0.000092 & 998.8 & 0.1811 & -0.000131 & 0.033 & & & & 997.2867 \\
\hline-0.000097 & -0.000080 & 1002.3 & 0.0815 & -0.000017 & 0.007 & & & & 997.2867 \\
\hline-0.000071 & -0.000069 & 1005.9 & 0.0143 & -0.000002 & 0.000 & & & & 997.2867 \\
\hline-0.000052 & -0.000054 & 1010.8 & -0.0317 & 0.000002 & 0.001 & & & & 997.2867 \\
\hline-0.000039 & -0.000039 & 1016.4 & -0.0111 & 0.000001 & 0.000 & & & & 997.2867 \\
\hline-0.000060 & -0.000092 & 998.1 & -0.0324 & 0.000032 & 0.001 & & & 1.066 & 997.0449 \\
\hline-0.000057 & -0.000092 & 998.3 & -0.0382 & 0.000034 & 0.001 & & & 1.183 & 997.0449 \\
\hline-0.000056 & -0.000091 & 998.4 & -0.0429 & 0.000035 & 0.002 & & & 1.301 & 997.0449 \\
\hline-0.000058 & -0.000091 & 998.5 & -0.0438 & 0.000033 & 0.002 & & & 1.425 & 997.0449 \\
\hline-0.000052 & -0.000087 & 999.6 & -0.0820 & 0.000035 & 0.007 & & & 2.448 & 997.0449 \\
\hline-0.000049 & -0.000085 & 1000.3 & -0.1064 & 0.000036 & 0.011 & & & 3.139 & 997.0449 \\
\hline-0.000049 & -0.000083 & 1000.8 & -0.1188 & 0.000034 & 0.014 & & & 3.612 & 997.0449 \\
\hline-0.000047 & -0.000081 & 1001.4 & -0.1384 & 0.000034 & 0.019 & & & 4.24 & 997.0449 \\
\hline-0.000048 & -0.000080 & 1001.6 & -0.13 & 0.000 & 0.018 & & & & 997.0449 \\
\hline-0.000042 & -0.000072 & 1004.2 & -0.2036 & 0.0000 & 0.041 & & & 6.91 & 997.0449 \\
\hline-0.000039 & -0.000069 & 1005.2 & -0.2288 & 0.000030 & 0.052 & & & 7.966 & 997.0449 \\
\hline-0.000037 & -0.000066 & 1006.0 & -0.2504 & 0.000030 & 0.063 & & & & 997.0449 \\
\hline-0.000039 & -0.000066 & 1006.3 & -0.2315 & 0.000027 & 0.054 & & & 8.988 & 997.0449 \\
\hline-0.000037 & -0.000062 & 1007.3 & -0.2462 & 0.000025 & 0.061 & & & 10.045 & 997.0449 \\
\hline-0.000034 & -0.000058 & 1008.9 & -0.2654 & 0.000023 & 0.070 & & & 11.629 & 997.0449 \\
\hline-0.000033 & -0.000055 & 1010.0 & -0.2705 & 0.000022 & 0.073 & & & 12.682 & 997.0449 \\
\hline-0.000031 & -0.000054 & 1010.1 & -0.2939 & 0.000023 & 0.086 & & & & 997.0449 \\
\hline
\end{tabular}


molality molarity $\exp / \mathrm{kg} / \mathrm{m}^{3}$

\begin{tabular}{|c|c|c|c|c|}
\hline & 25 & 0.0137 & 1011.31 & (86) \\
\hline & 25 & 0.0147 & 1012.35 & (86) \\
\hline & 25 & 0.0158 & 1013.53 & (86) \\
\hline & 25 & 0.0167 & 1014.43 & (86) \\
\hline & 25 & 0.0181 & 1015.92 & (86) \\
\hline & 25 & 0.0203 & 1018.15 & (86) \\
\hline \multirow[t]{4}{*}{0.49991} & 25 & 0.0206 & 1018.40 & (73) \\
\hline & 25 & 0.0215 & 1019.49 & (86) \\
\hline & 25 & 0.0227 & 1020.69 & (86) \\
\hline & 25 & 0.0238 & 1021.87 & (86) \\
\hline \multirow{7}{*}{0.59012} & 25 & 0.0242 & 1022.15 & (73) \\
\hline & 25 & 0.0250 & 1023.05 & (86) \\
\hline & 25 & 0.0261 & 1024.23 & (86) \\
\hline & 25 & 0.0272 & 1025.37 & (86) \\
\hline & 25 & 0.0273 & 1025.42 & (86) \\
\hline & 25 & 0.0281 & 1026.26 & (86) \\
\hline & 25 & 0.0284 & 1026.60 & (86) \\
\hline \multirow[t]{5}{*}{0.70804} & 25 & 0.0289 & 1027.02 & (73) \\
\hline & 25 & 0.0289 & 1027.14 & (86) \\
\hline & 25 & 0.0295 & 1027.78 & (86) \\
\hline & 25 & 0.0307 & 1028.95 & (86) \\
\hline & 25 & 0.0318 & 1030.11 & (86) \\
\hline \multirow[t]{6}{*}{0.79744} & 25 & 0.0324 & 1030.69 & (73) \\
\hline & 25 & 0.0329 & 1031.28 & (86) \\
\hline & 25 & 0.0341 & 1032.46 & (86) \\
\hline & 25 & 0.0352 & 1033.63 & (86) \\
\hline & 25 & 0.0363 & 1034.79 & (86) \\
\hline & 25 & 0.0374 & 1035.94 & (86) \\
\hline 0.03299 & 48.75 & 0.0014 & 990.27 & (114) \\
\hline 0.11127 & 48.75 & 0.0047 & 993.55 & (114) \\
\hline 0.19158 & 48.75 & 0.0080 & 996.88 & (114) \\
\hline 0.30593 & 48.75 & 0.0127 & 1001.70 & (114) \\
\hline 0.43944 & 48.75 & 0.0181 & 1007.22 & (114) \\
\hline 0.010102 & 50 & 0.0004 & 988.47 & $(72)$ \\
\hline 0.0196 & 50 & 0.0008 & 988.88 & (72) \\
\hline 0.039394 & 50 & 0.0017 & 989.72 & (72) \\
\hline 0.079954 & 50 & 0.0033 & 991.44 & (72) \\
\hline 0.143533 & 50 & 0.0060 & 994.12 & (72) \\
\hline 0.24328 & 50 & 0.0101 & 998.26 & (72) \\
\hline 0.337712 & 50 & 0.0140 & 1002.18 & (72) \\
\hline 0.430678 & 50 & 0.0178 & 1005.99 & (72) \\
\hline 0.571046 & 50 & 0.0234 & 1011.71 & (72) \\
\hline 0.803873 & 50 & 0.0327 & 1021.03 & (72) \\
\hline
\end{tabular}

Ref
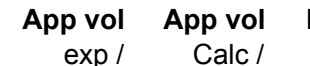

$\mathrm{m}^{3} / \mathrm{kg} \quad \mathrm{m}^{3} / \mathrm{k}$

$-0.000031-0.000051$

$-0.000029-0.000048$

$-0.000029-0.000045$

$-0.000026-0.000042$

$-0.000027-0.000039$

$-0.000023-0.000033$

$-0.000020-0.000033$

$-0.000022-0.000030$

$-0.000021-0.000027$

$-0.000019-0.000025$

$-0.000016-0.000024$

$-0.000018-0.000022$

$-0.000017-0.000020$

$-0.000015-0.000017$

$-0.000015-0.000017$

$-0.000014-0.000016$

$-0.000014-0.000015$

$-0.000011-0.000014$

$-0.000012-0.000014$

$-0.000012-0.000013$

$-0.000011-0.000010$

$-0.000010-0.000008$

$-0.000008-0.000007$

$-0.000008-0.000006$

$-0.000007-0.000004$

$\begin{array}{lll}-0.000006 & -0.000002\end{array}$

$-0.000005 \quad 0.000000$

$-0.000003 \quad 0.000003$

$-0.000221-0.000062$

$-0.000073-0.000053$

$-0.000042-0.000044$

$-0.000032-0.000033$

$-0.000021-0.000022$

$-0.000036-0.000063$

$-0.000034-0.000062$

$-0.000031-0.000060$

$-0.000027-0.000055$

$-0.000022-0.000048$

$-0.000013-0.000038$

$-0.000010-0.000030$

$-0.000005-0.000022$

$0.000001-0.000012$

$0.000010 \quad 0.000003$
Density Density App Vol Square of Inconsistent
based on $\begin{gathered}\text { Inconsistent } \\ \text { based on }\end{gathered}$ $\begin{gathered}\text { Delta density } \\ / \mathrm{kg} / \mathrm{m}^{3} \text { water } / \mathrm{kg} / \mathrm{m}^{3}\end{gathered}$ calc/ Residual/ Residual/ Density based on based on $\mathrm{kg} / \mathrm{m}^{3} \quad \mathrm{~kg} / \mathrm{m}^{3} \quad \mathrm{~m}^{3} / \mathrm{kg}$ Residual density $\begin{gathered}\text { apparent } \\ \text { volume }\end{gathered}$

$\begin{array}{llll}1011.6 & -0.2787 & 0.000020 & 0.078\end{array}$

$\begin{array}{llll}1012.6 & -0.2803 & 0.000019 & 0.079\end{array}$

$\begin{array}{llll}1013.8 & -0.2484 & 0.000015 & 0.062\end{array}$

$\begin{array}{llll}1014.7 & -0.2867 & 0.000017 & 0.082\end{array}$

$\begin{array}{llll}1016.2 & -0.2263 & 0.000012 & 0.051\end{array}$

$\begin{array}{llll}018.4 & -0.2121 & 0.000010 & 0.045\end{array}$

$\begin{array}{llll}018.7 & -0.2665 & 0.000012 & 0.071\end{array}$

$\begin{array}{llll}1019.7 & -0.1812 & 0.000008 & 0.033\end{array}$

$\begin{array}{llll}020.8 & -0.1619 & 0.000007 & 0.026\end{array}$

$\begin{array}{llll}1022.0 & -0.1422 & 0.000006 & 0.020\end{array}$

$\begin{array}{llll}1022.4 & -0.2067 & 0.000008 & 0.043\end{array}$

$\begin{array}{llll}1023.2 & -0.1123 & 0.000004 & 0.013\end{array}$

$\begin{array}{llll}1024.3 & -0.0884 & 0.000003 & 0.008\end{array}$

$\begin{array}{llll}1025.4 & -0.0733 & 0.000003 & 0.005\end{array}$

$\begin{array}{llll}1025.5 & -0.0618 & 0.000002 & 0.004\end{array}$

$\begin{array}{llll}1026.3 & -0.0530 & 0.000002 & 0.003\end{array}$

$\begin{array}{llll}026.6 & -0.0324 & 0.000001 & 0.001\end{array}$

$\begin{array}{llll}027.1 & -0.0890 & 0.000003 & 0.008\end{array}$

$\begin{array}{llll}1027.2 & -0.0479 & 0.000002 & 0.002\end{array}$

$\begin{array}{llll}1027.8 & -0.0034 & 0.000000 & 0.000\end{array}$

$\begin{array}{llll}1028.9 & 0.0292 & -0.000001 & 0.001\end{array}$

$\begin{array}{llll}1030.1 & 0.0561 & -0.000002 & 0.003\end{array}$

$\begin{array}{llll}030.7 & 0.0261 & -0.000001 & 0.001\end{array}$

$\begin{array}{llll}031.2 & 0.0862 & -0.000002 & 0.007\end{array}$

$\begin{array}{llll}1032.3 & 0.1224 & -0.000003 & 0.015\end{array}$

$\begin{array}{llll}033.5 & 0.1578 & -0.000004 & 0.025\end{array}$

$\begin{array}{llll}1034.6 & 0.1951 & -0.000005 & 0.038\end{array}$

$\begin{array}{llll}1035.7 & 0.2133 & -0.000005 & 0.045\end{array}$

$\begin{array}{llll}990.0 & 0.2155 & -0.000159 & 0.046\end{array}$

$\begin{array}{llll}993.5 & 0.0946 & -0.000021 & 0.009\end{array}$

$\begin{array}{llll}996.9 & -0.0179 & 0.000002 & 0.000\end{array}$

$\begin{array}{rrrr}1001.7 & -0.0226 & 0.000002 & 0.001\end{array}$

$\begin{array}{llll}1007.2 & -0.0245 & 0.000001 & 0.001\end{array}$

$\begin{array}{llll}988.5 & -0.0115 & 0.000028 & 0.000\end{array}$

$\begin{array}{llll}988.9 & -0.0225 & 0.000028 & 0.001\end{array}$

$\begin{array}{llll}989.8 & -0.0471 & 0.000029 & 0.002\end{array}$

$\begin{array}{llll}991.5 & -0.0913 & 0.000028 & 0.008\end{array}$

$\begin{array}{llll}994.3 & -0.1558 & 0.000026 & 0.024\end{array}$

$\begin{array}{llll}998.5 & -0.2513 & 0.000025 & 0.063\end{array}$

$\begin{array}{llll}1002.5 & -0.2830 & 0.000020 & 0.080\end{array}$

$\begin{array}{llll}1006.3 & -0.3086 & 0.000017 & 0.095\end{array}$

$\begin{array}{llll}1012.0 & -0.2950 & 0.000012 & 0.087\end{array}$

$\begin{array}{llll}1021.3 & -0.2523 & 0.000007 & 0.064\end{array}$
$14.261 \quad 997.0449$

$15.308 \quad 997.0449$

16.481997 .0449

17.389997 .0449

$18.879 \quad 997.0449$

$21.108 \quad 997.0449$ 997.0449

$22.449 \quad 997.0449$

$23.641 \quad 997.0449$

$24.823 \quad 997.0449$ 997.0449

26.01997 .0449

$27.189 \quad 997.0449$

$28.326 \quad 997.0449$

$28.372 \quad 997.0449$

$29.213 \quad 997.0449$

$29.557 \quad 997.0449$ 997.0449

$30.095 \quad 997.0449$

30.731997 .0449

$31.907 \quad 997.0449$

33.068997 .0449 997.0449

$34.239 \quad 997.0449$

$35.418 \quad 997.0449$

$36.588 \quad 997.0449$

$37.744 \quad 997.0449$

$38.894 \quad 997.0449$ 988.5963 988.5963 988.5963 988.5963 988.5963

$0.4338 \quad 988.0363$

$0.8406 \quad 988.0363$

1.684988 .0363

3.407988 .0363

6.0842988 .0363

$10.2267 \quad 988.0363$

$14.1444 \quad 988.0363$

$17.9545 \quad 988.0363$

$23.6743 \quad 988.0363$

32.9935988 .0363 
molality molarity

$\mathrm{t} /{ }^{\circ} \mathrm{C}$ mass frac Density

0.03299
0.11127
0.19158
0.30593
0.43944
0.03299
0.11127
0.19158
0.30593
0.43944
$\exp / \mathrm{kg} / \mathrm{m}^{3}$ $\begin{array}{lll}73.77 & 0.0014 & 977.19\end{array}$

$\begin{array}{lll}73.77 & 0.0047 & 980.47\end{array}$

$\begin{array}{lll}73.77 & 0.0080 & 983.94\end{array}$

$\begin{array}{lll}73.77 & 0.0127 & 988.66\end{array}$

$\begin{array}{lll}73.77 & 0.0181 & 994.10\end{array}$

$\begin{array}{lll}98.67 & 0.0014 & 961.05\end{array}$

$\begin{array}{lll}98.67 & 0.0014 & 961.05 \\ 98.67 & 0.0047 & 964.44\end{array}$

$\begin{array}{lll}98.67 & 0.0080 & 967.77\end{array}$

$\begin{array}{lll}98.67 & 0.0127 & 972.62\end{array}$

$\begin{array}{lll}98.67 & 0.0181 & 978.31\end{array}$
Ref

(114)

(114)

(114)

(114)

(114)

(114)

(114)

(114)

\section{App vol App vol}

exp / Calc /

$\mathrm{m}^{3} / \mathrm{kg} \quad \mathrm{m}^{3} / \mathrm{kg}$

$-0.000194-0.000040$

$-0.000073-0.000033$

$\begin{array}{lll}-0.000067 & -0.000027\end{array}$

$-0.000045-0.000019$

$-0.000029-0.000010$

$-0.000317-0.000025$

$-0.000150-0.000019$

$-0.000098-0.000015$

$-0.000082-0.000008$

$-0.000075-0.000002$ calc/

$\mathrm{kg} / \mathrm{m}^{3}$

977.0

980.3

983.6

988.3

993.8

960.7

963.9

967.1

977.1

Average Res

Std dev Res

Avg - 4std

Avg + 4std
Density

$\mathrm{kg} / \mathrm{m}^{3}$

0.2031

0.2031
0.1792

$\begin{array}{ll}0.3091-0.000040 \\ 0.3231 & -0.000026\end{array}$

$0.3231-0.000026$

$\begin{array}{ll}0.3433 & -0.000019\end{array}$

$\begin{array}{ll}0.3731 & -0.000292\end{array}$

$0.5624-0.000130$

$\begin{array}{ll}0.6244 & -0.000084\end{array}$

$\begin{array}{ll}0.8774 & -0.000073\end{array}$

$\begin{array}{ll}1.2621 & -0.000073\end{array}$

$0.0249-0.000014$

$\begin{array}{ll}0.2943 & 0.000077\end{array}$

$\begin{array}{ll}-1.1523 & -0.000320\end{array}$

$\begin{array}{ll}1.2021 & 0.000293\end{array}$

$\mathrm{m}^{3} / \mathrm{kg}$
00154
00040
00040
00026
00019
00292
000084
000073
00073
000074
000320
000293

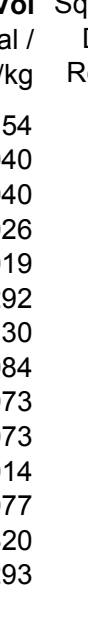

Curve fit for the model App vol $=\left(w+c 2+c 3^{*} t{ }^{\circ} \mathrm{C}\right) /\left(\mathrm{c} 0^{*} w+c 1\right) / e^{\wedge}\left(0.000001^{*}\left(t{ }^{\circ} \mathrm{C}+\mathrm{c} 4\right)^{\wedge} 2\right)$

$\begin{array}{lr}\text { c0 } & 2.82 \mathrm{E}-06 \\ \mathrm{c} 1 & 2.18 \mathrm{E}-07 \\ \mathrm{c} 2 & -0.04148 \\ \mathrm{c} 3 & 0.000218 \\ \mathrm{c} 4 & 4586.88 \\ \text { Min T } & 0 \\ \text { Max T } & 98.67 \\ \text { Min w } & 0.000408 \\ \text { Max w } & 0.03744 \\ \text { Avg dens res } & 0.024891 \\ \text { Std dens res } & 0.294304 \\ \text { Avg app vol res *10^3 } & -0.01375 \\ \text { Std app vol res *10^3 } & 0.07657 \\ \text { No of points in corr } & 85 \\ \text { No of inconsistent poir } & 6\end{array}$

Inconsistent data not used (Residual greater than average $+/-\mathbf{4}$ standard deviations)

\begin{tabular}{|c|c|c|c|c|c|c|c|c|c|c|c|c|}
\hline 28 & 0.0042 & 1003.00 & (55) & -0.000614 & -0.000077 & 1000.7 & 2.2575 & -0.000537 & 5.097 & $!$ & $!$ & 996.2335 \\
\hline 0.2 & 0.0083 & 1008.00 & (55) & -0.000403 & -0.000063 & 1005.1 & 2.8656 & -0.000339 & 8.212 & $!$ & $!$ & 996.2335 \\
\hline 28 & 0.0124 & 1013.00 & (55) & -0.000332 & -0.000051 & 1009.4 & 3.5749 & -0.000281 & 12.780 & ! & & 996.2335 \\
\hline 28 & 0.0165 & 1015.00 & (55) & -0.000118 & -0.000040 & 1013.7 & 1.3223 & -0.000078 & 1.748 & $!$ & & 996.2335 \\
\hline 28 & 0.0206 & 1021.00 & (55) & -0.000180 & -0.000030 & 1017.8 & 3.2068 & -0.000150 & 10.283 & $!$ & & 996.2335 \\
\hline 28 & 0.0405 & 1036.00 & (55) & 0.000053 & 0.000009 & 1037.9 & -1.9346 & 0.000044 & 3.743 & $i$ & & 996.2335 \\
\hline
\end{tabular}


Density of aqueous solutions of $\mathrm{NaHCO} 3$

References (38) Hershey, J. P.; Sotolongo, S.; Millero, F. J. Densities and Compressibilities of Aqueous Sodium Carbonate and Bicarbonate from 0 to $45^{\circ}$ C. J. Sol. Chem. 1983, $12,233-254$

(73) Millero, F. J.; Ward, G. K.; Chetirkin, P. V. Relative Sound Velocities of Sea Salts at 25C. J. Acoust. Soc. Am. 1977, 61, 1492-1498.

(88) Perron, G., Desnoyers, J. E., Millero, F. J., Apparent molal volumes and heat capacities of some sulfates and carbonates in water at 25 ${ }^{\circ} \mathrm{C}$, Can. J. Chem. , 1975, 53, 1134-1138

(106) Rashkovskaya, E. A.; Chernen'kaya, E. I. Densities of Solutions of $\mathrm{NH} 4 \mathrm{HCO}, \mathrm{NaHCO} 3, \mathrm{NH} 4 \mathrm{Cl}$ and Ammonia in the $20-100^{\circ} \mathrm{Range.} \mathrm{J.}$ Appl. Chem. USSR 1967, 40, 301-308.

\begin{tabular}{|c|c|c|c|c|c|c|c|c|c|c|c|c|c|c|c|}
\hline molality & molarity & $\mathrm{t} /{ }^{\circ} \mathrm{C}$ & ass frac & $\begin{array}{r}\text { Density } \\
\exp / \\
\mathrm{kg} / \mathrm{m}^{3}\end{array}$ & Ref & $\begin{array}{r}\text { App vol } \\
\exp / \\
m^{3} / \mathrm{kg}\end{array}$ & $\begin{array}{r}\text { App vol } \\
\text { Calc / } \\
\mathrm{m}^{3} / \mathrm{kg}\end{array}$ & $\begin{array}{r}\text { Density } \\
\text { calc / } \\
\mathrm{kg} / \mathrm{m}^{3}\end{array}$ & $\begin{array}{r}\text { Density } \\
\text { Residual / } \\
\mathrm{kg} / \mathrm{m}^{3}\end{array}$ & $\begin{array}{r}\text { App Vol } \\
\text { Residual / } \\
\mathrm{m}^{3} / \mathrm{kg}\end{array}$ & $\begin{array}{r}\text { Square of } \\
\text { Density } \\
\text { Residual }\end{array}$ & $\begin{array}{c}\text { Inconsistent } \\
\text { based on } \\
\text { density }\end{array}$ & $\begin{array}{l}\text { Inconsistent } \\
\text { based on } \\
\text { apparent } \\
\text { volume }\end{array}$ & $\begin{array}{l}\text { Delta density / } \\
\mathrm{kg} / \mathrm{m}^{3}\end{array}$ & $\begin{array}{r}\text { Density of } \\
\text { water } / \mathrm{kg} / \mathrm{m}^{3}\end{array}$ \\
\hline 0.00434 & & 0 & 0.0004 & 1000.1 & (38) & 0.000207 & 0.000236 & 1000.1 & 0.0106 & -0.000029 & 0.000 & & & 0.289 & 999.8395 \\
\hline 0.0167 & & 0 & 0.0014 & 1000.9 & (38) & 0.000210 & 0.000237 & 1000.9 & 0.0375 & -0.000027 & 0.001 & & & 1.108 & 999.8395 \\
\hline 0.03491 & & 0 & 0.0029 & 1002.1 & (38) & 0.000212 & 0.000237 & 1002.1 & 0.0726 & -0.000025 & 0.005 & & & 2.308 & 999.8395 \\
\hline 0.06027 & & 0 & 0.0050 & 1003.8 & (38) & 0.000215 & 0.000238 & 1003.7 & 0.1168 & -0.000023 & 0.014 & & & 3.97 & 999.8395 \\
\hline 0.09949 & & 0 & 0.0083 & 1006.3 & (38) & 0.000220 & 0.000239 & 1006.2 & 0.1649 & -0.000020 & 0.027 & & & 6.51 & 999.8395 \\
\hline 0.1333 & & 0 & 0.0111 & 1008.5 & (38) & 0.000223 & 0.000240 & 1008.3 & 0.1975 & -0.000018 & 0.039 & & & 8.681 & 999.8395 \\
\hline 0.18649 & & 0 & 0.0154 & 1011.9 & (38) & 0.000227 & 0.000242 & 1011.7 & 0.2347 & -0.000015 & 0.055 & & & 12.064 & 999.8395 \\
\hline 0.26204 & & 0 & 0.0215 & 1016.7 & (38) & 0.000232 & 0.000244 & 1016.4 & 0.2678 & -0.000012 & 0.072 & & & 16.811 & 999.8395 \\
\hline 0.36248 & & 0 & 0.0296 & 1022.9 & (38) & 0.000238 & 0.000248 & 1022.6 & 0.2950 & -0.000010 & 0.087 & & & 23.035 & 999.8395 \\
\hline 0.47636 & & 0 & 0.0385 & 1029.8 & (38) & 0.000244 & 0.000251 & 1029.5 & 0.2846 & -0.000007 & 0.081 & & & 29.954 & 999.8395 \\
\hline 0.59586 & & 0 & 0.0477 & 1036.9 & (38) & 0.000250 & 0.000255 & 1036.7 & 0.2533 & -0.000005 & 0.064 & & & 37.084 & 999.8395 \\
\hline 0.74565 & & 0 & 0.0589 & 1045.7 & (38) & 0.000256 & 0.000259 & 1045.5 & 0.2079 & -0.000003 & 0.043 & & & 45.856 & 999.8395 \\
\hline 0.05186 & & 5 & 0.0043 & 1003.3 & (38) & 0.000233 & 0.000252 & 1003.2 & 0.0842 & -0.000019 & 0.007 & & & 3.339 & 999.9638 \\
\hline 0.18933 & & 5 & 0.0157 & 1011.9 & (38) & 0.000248 & 0.000256 & 1011.7 & 0.1375 & -0.000009 & 0.019 & & & 11.916 & 999.9638 \\
\hline 0.29355 & & 5 & 0.0241 & 1018.2 & (38) & 0.000257 & 0.000260 & 1018.1 & 0.0609 & -0.000002 & 0.004 & & & 18.201 & 999.9638 \\
\hline 0.39653 & & 5 & 0.0322 & 1024.3 & (38) & 0.000262 & 0.000263 & 1024.3 & 0.0180 & -0.000001 & 0.000 & & & 24.359 & 999.9638 \\
\hline 0.5958 & & 5 & 0.0477 & 1036.0 & (38) & 0.000271 & 0.000269 & 1036.1 & -0.0804 & 0.000002 & 0.006 & & & 36.02 & 999.9638 \\
\hline 0.70303 & & 5 & 0.0558 & 1042.1 & (38) & 0.000274 & 0.000272 & 1042.3 & -0.1171 & 0.000002 & 0.014 & & & 42.181 & 999.9638 \\
\hline 0.79821 & & 5 & 0.0628 & 1047.5 & (38) & 0.000277 & 0.000275 & 1047.7 & -0.1532 & 0.000002 & 0.023 & & & 47.57 & 999.9638 \\
\hline 0.8976 & & 5 & 0.0701 & 1053.0 & (38) & 0.000281 & 0.000278 & 1053.3 & -0.2551 & 0.000003 & 0.065 & & & 53.057 & 999.9638 \\
\hline 1.0003 & & 5 & 0.0775 & 1058.7 & (38) & 0.000284 & 0.000281 & 1059.0 & -0.2797 & 0.000003 & 0.078 & & & 58.726 & 999.9638 \\
\hline 1.00034 & & 5 & 0.0775 & 1058.7 & (38) & 0.000284 & 0.000281 & 1059.0 & -0.2319 & 0.000003 & 0.054 & & & 58.776 & 999.9638 \\
\hline 0.05528 & & 15 & 0.0046 & 1002.5 & (38) & 0.000268 & 0.000275 & 1002.5 & 0.0336 & -0.000007 & 0.001 & & & 3.393 & 999.0996 \\
\hline 0.1202 & & 15 & 0.0100 & 1006.4 & (38) & 0.000275 & 0.000277 & 1006.4 & 0.0187 & -0.000002 & 0.000 & & & 7.292 & 999.0996 \\
\hline 0.23922 & & 15 & 0.0197 & 1013.4 & (38) & 0.000284 & 0.000281 & 1013.5 & -0.0571 & 0.000003 & 0.003 & & & 14.304 & 999.0996 \\
\hline 0.31752 & & 15 & 0.0260 & 1018.0 & (38) & 0.000287 & 0.000283 & 1018.1 & -0.1002 & 0.000004 & 0.010 & & & 18.862 & 999.0996 \\
\hline 0.4566 & & 15 & 0.0369 & 1025.9 & (38) & 0.000292 & 0.000288 & 1026.1 & -0.1853 & 0.000005 & 0.034 & & & 26.829 & 999.0996 \\
\hline 0.60394 & & 15 & 0.0483 & 1034.3 & (38) & 0.000296 & 0.000292 & 1034.5 & -0.2209 & 0.000004 & 0.049 & & & 35.156 & 999.0996 \\
\hline 0.72429 & & 15 & 0.0574 & 1041.0 & (38) & 0.000299 & 0.000296 & 1041.2 & -0.2022 & 0.000003 & 0.041 & & & 41.878 & 999.0996 \\
\hline 0.88532 & & 15 & 0.0692 & 1049.9 & (38) & 0.000302 & 0.000300 & 1050.0 & -0.1000 & 0.000001 & 0.010 & & & 50.771 & 999.0996 \\
\hline 1.00844 & & 15 & 0.0781 & 1056.6 & (38) & 0.000304 & 0.000304 & 1056.6 & 0.0067 & 0.000000 & 0.000 & & & 57.462 & 999.0996 \\
\hline 0.00298 & & 25 & 0.0003 & 997.2 & (38) & 0.000276 & 0.000290 & 997.2 & 0.0036 & -0.000015 & 0.000 & & & 0.181 & 997.0449 \\
\hline 0.00468 & & 25 & 0.0004 & 997.3 & (38) & 0.000276 & 0.000290 & 997.3 & 0.0055 & -0.000014 & 0.000 & & & 0.284 & 997044 \\
\hline
\end{tabular}




$\begin{array}{rrrrr} & & & \mathrm{kg} / \mathrm{m}^{3} & \\ 0.00811 & 25 & 0.0007 & 997.5 & (88) \\ 0.01405 & 25 & 0.0012 & 997.9 & (88) \\ 0.01769 & 25 & 0.0015 & 998.1 & (38) \\ 0.02314 & 25 & 0.0019 & 998.4 & (88) \\ 0.03495 & 25 & 0.0029 & 999.1 & (88) \\ 0.03688 & 25 & 0.0031 & 999.3 & (38) \\ 0.04072 & 25 & 0.0034 & 999.5 & (38) \\ 0.06435 & 25 & 0.0054 & 1000.9 & (88) \\ 0.08565 & 25 & 0.0071 & 1002.2 & (38) \\ 0.10029 & 25 & 0.0084 & 1003.0 & (38) \\ 0.10029 & 25 & 0.0084 & 1003.04 & (73) \\ 0.12723 & 25 & 0.0106 & 1004.6 & (88) \\ 0.17697 & 25 & 0.0146 & 1007.5 & (38) \\ 0.188 & 25 & 0.0155 & 1008.2 & (88) \\ 0.19854 & 25 & 0.0164 & 1008.7 & (38) \\ 0.19854 & 25 & 0.0164 & 1008.76 & (73) \\ 0.24972 & 25 & 0.0205 & 1011.7 & (38) \\ 0.27007 & 25 & 0.0222 & 1012.9 & (88) \\ 0.29909 & 25 & 0.0245 & 1014.5 & (38) \\ 0.29909 & 25 & 0.0245 & 1014.52 & (73) \\ 0.3189 & 25 & 0.0261 & 1015.7 & (88) \\ 0.37438 & 25 & 0.0305 & 1018.8 & (38) \\ 0.39916 & 25 & 0.0324 & 1020.2 & (38) \\ 0.39916 & 25 & 0.0324 & 1020.28 & (73) \\ 0.48897 & 25 & 0.0395 & 1025.2 & (38) \\ 0.5285 & 25 & 0.0425 & 1027.5 & (88) \\ 0.60053 & 25 & 0.0480 & 1031.4 & (38) \\ 0.60053 & 25 & 0.0480 & 1031.38 & (73) \\ 0.63853 & 25 & 0.0509 & 1033.5 & (38) \\ 0.69427 & 25 & 0.0551 & 1036.5 & (38) \\ 0.69427 & 25 & 0.0551 & 1036.52 & (73) \\ 0.7740 & 25 & 0.0611 & 1040.9 & (88) \\ 0.79985 & 25 & 0.0630 & 1042.2 & (38) \\ 0.79985 & 25 & 0.0630 & 1042.27 & (73) \\ 0.8074 & 25 & 0.0635 & 1042.6 & (38) \\ 0.97909 & 25 & 0.0760 & 1051.9 & (38) \\ 0.05817 & 35 & 0.0049 & 997.5 & (38) \\ 0.1178 & 35 & 0.0098 & 1000.9 & (38) \\ 0.23556 & 0.0194 & 1007.6 & (38) \\ 0.31109 & 0.0255 & 1011.8 & (38) \\ 0.43769 & 0.0481 & 1018.8 & (38) \\ 0.60204 & 35.7 & (38) \\ 0.71868 & 25 & & & (38)\end{array}$
exp / Calc / calc/ Residual / Residual / Density based on based on $\mathrm{m}^{3} / \mathrm{kg} \quad \mathrm{m}^{3} / \mathrm{kg} \quad \mathrm{kg} / \mathrm{m}^{3} \quad \mathrm{~kg} / \mathrm{m}^{3} \quad \mathrm{~m}^{3} / \mathrm{kg}$ Residual density apparent $0.000279 \quad 0.000290$ 0.0002890 .000291 $0.000280 \quad 0.000291$ $0.000281 \quad 0.000291$ $0.000281 \quad 0.000291$ $0.000283 \quad 0.000291$ $0.000282 \quad 0.000291$ $0.000285 \quad 0.000292$ $0.000287 \quad 0.000293$ $0.000288 \quad 0.000293$ $0.000285 \quad 0.000293$ $0.000289 \quad 0.000294$ $0.000294 \quad 0.000295$ $0.000292 \quad 0.000296$ $0.000294 \quad 0.000296$ $0.000293 \quad 0.000296$ $0.000297 \quad 0.000298$ $\begin{array}{lll}0.000295 & 0.000298\end{array}$ $0.000299 \quad 0.000299$ $0.000298 \quad 0.000299$ $0.000296 \quad 0.000300$ $0.000302 \quad 0.000301$ $0.000303 \quad 0.000302$ 0.0002990 .000302 $0.000305 \quad 0.000305$ $0.000304 \quad 0.000306$ $0.000308 \quad 0.000308$ $0.000308 \quad 0.000308$ $0.000309 \quad 0.000309$ $0.000310 \quad 0.000311$ $0.000310 \quad 0.000311$ $\begin{array}{ll}0.000311 & 0.000313\end{array}$ $0.000312 \quad 0.000314$ $0.000312 \quad 0.000314$ $0.000313 \quad 0.000314$ $0.000315 \quad 0.000319$ $0.000295 \quad 0.000303$ $0.000301 \quad 0.000305$ $0.000309 \quad 0.000308$ 0.0003110 .000310 $\begin{array}{ll}0.000316 & 0.000314\end{array}$ $0.000321 \quad 0.000319$ $\begin{array}{lll}0.000323 & 0.000322\end{array}$

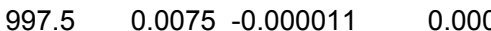

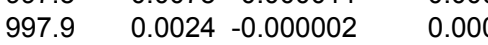

$\begin{array}{llll}998.1 & 0.0161 & -0.000011 & 0.000\end{array}$

$\begin{array}{llll}998.4 & 0.0196 & -0.000010 & 0.000\end{array}$

$\begin{array}{llll}999.1 & 0.0292 & -0.000010 & 0.001\end{array}$

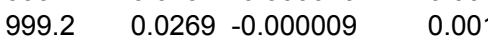

$\begin{array}{llll}999.5 & 0.0315 & -0.000009 & 0.001\end{array}$

$\begin{array}{llll}1000.9 & 0.0386 & -0.000007 & 0.001\end{array}$

$\begin{array}{llll}1002.1 & 0.0433 & -0.000006 & 0.002\end{array}$

$\begin{array}{llll}1003.0 & 0.0406 & -0.000005 & 0.002\end{array}$

$\begin{array}{llll}1003.0 & 0.0647 & -0.000008 & 0.004\end{array}$

$\begin{array}{llll}1004.6 & 0.0552 & -0.000005 & 0.003\end{array}$

$\begin{array}{llll}1007.5 & 0.0273 & -0.000002 & 0.001\end{array}$

$\begin{array}{llll}1008.1 & 0.0596 & -0.000004 & 0.004\end{array}$

$\begin{array}{llll}1008.7 & 0.0284 & -0.000002 & 0.001\end{array}$

$\begin{array}{llll}1008.7 & 0.0525 & -0.000003 & 0.003\end{array}$

$\begin{array}{llll}1011.7 & 0.0107 & -0.000001 & 0.000\end{array}$

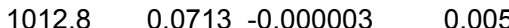

$\begin{array}{llll}1014.5 & 0.0253 & -0.000001 & 0.001\end{array}$

$\begin{array}{llll}1015.6 & 0.0874 & -0.000003 & 0.008\end{array}$

$\begin{array}{llll}1018.8 & -0.0237 & 0.000001 & 0.001\end{array}$

$\begin{array}{llll}1020.2 & -0.0189 & 0.000001 & 0.000\end{array}$

$1020.2 \quad 0.1052-0.000003 \quad 0.01$

$\begin{array}{llll}1025.2 & 0.0026 & 0.000000 & 0.000\end{array}$

$\begin{array}{llll}1027.4 & 0.0958 & -0.000002 & 0.009\end{array}$

$\begin{array}{llll}1031.4 & -0.0111 & 0.000000 & 0.000\end{array}$

$\begin{array}{llll}1031.4 & 0.0140 & 0.000000 & 0.000\end{array}$

$\begin{array}{llll}1033.4 & 0.0157 & 0.000000 & 0.000\end{array}$

$\begin{array}{llll}1036.5 & 0.0296 & 0.000000 & 0.001\end{array}$

$\begin{array}{llll}1036.5 & 0.0527 & -0.000001 & 0.003\end{array}$

$\begin{array}{llll}1040.8 & 0.1470 & -0.000002 & 0.022\end{array}$

$\begin{array}{llll}1042.1 & 0.1109 & -0.000002 & 0.012\end{array}$

$\begin{array}{llll}1042.1 & 0.1390 & -0.000002 & 0.019\end{array}$

$\begin{array}{llll}1042.5 & 0.1032 & -0.000001 & 0.011\end{array}$

$\begin{array}{llll}1051.5 & 0.3673 & -0.000004 & 0.135\end{array}$

$\begin{array}{llll}997.4 & 0.0385 & -0.000008 & 0.001\end{array}$

$\begin{array}{llll}1000.9 & 0.0314 & -0.000003 & 0.001\end{array}$

$\begin{array}{llll}1007.6 & -0.0224 & 0.000001 & 0.001\end{array}$

$\begin{array}{llll}1011.9 & -0.0288 & 0.000001 & 0.001\end{array}$

$\begin{array}{llll}1018.9 & -0.0792 & 0.000002 & 0.006\end{array}$

$\begin{array}{llll}1027.8 & -0.1148 & 0.000002 & 0.013\end{array}$

$\begin{array}{llll}1034.1 & -0.0878 & 0.000001 & 0.008\end{array}$ $\begin{array}{llll}1014.5 & 0.0022 & 0.000000 & 0.000\end{array}$
$0.49 \quad 997.0449$

$0.838 \quad 997.0449$

1.068997 .0449

$1.395 \quad 997.0449$

$2.105 \quad 997.0449$

2.217997 .0449

2.449997 .0449

3.853997 .0449

$5.113 \quad 997.0449$

5.971997 .0449 997.0449

$7.565 \quad 997.0449$

$10.438 \quad 997.0449$

$11.111 \quad 997.0449$

11.691997 .0449 997.0449

$14.629 \quad 997.0449$

$15.859 \quad 997.0449$

17.452997 .0449 997.0449

$18.665 \quad 997.0449$

$21.707 \quad 997.0449$

23.111997 .0449 997.0449

$28.163 \quad 997.0449$

$30.452 \quad 997.044$

34.31997 .0449 997.0449

$36.413 \quad 997.0449$

39.452997 .0449 997.0449

$43.856 \quad 997.0449$

45.197997 .0449 997.0449

$45.591 \quad 997.0449$

54.871997 .0449

3.429994 .0319

$\begin{array}{ll}6.87 & 994.0319\end{array}$

$13.544 \quad 994.0319$

$17.796 \quad 994.0319$

$24.784 \quad 994.0319$

$33.701 \quad 994.0319$

$39.956 \quad 994.0319$ 
molality molarity

$t /{ }^{\circ} \mathrm{C}$ mass frac Density

\section{$\exp /$}

$\mathrm{kg} / \mathrm{m}^{3}$

0.88105

1.00324

0.05837

0.11598

0.23638

0.23823

0.31888

0.4355

0.4373

0.60109

0.71888

0.87795

0.88221

1.00082

1.00182

$\begin{array}{lrrl}35 & 0.0689 & 1042.6 \\ 35 & 0.0777 & 1049.0 & (38) \\ 45 & 0.0049 & 993.6 & (38) \\ 45 & 0.0096 & 996.9 & (38) \\ 45 & 0.0195 & 1003.6 & (38) \\ 45 & 0.0196 & 1003.7 & (38) \\ 45 & 0.0261 & 1008.1 & (38) \\ 45 & 0.0353 & 1014.5 & (38) \\ 45 & 0.0354 & 1014.6 & (38) \\ 45 & 0.0481 & 1023.4 \\ 45 & 0.0570 & 1029.6 & (38) \\ 45 & 0.0687 & 1038.1 \\ 45 & 0.0690 & 1038.3 \\ 45 & 0.0776 & 1044.5 & (38) \\ 45 & 0.0776 & 1044.5\end{array}$

Ref

$\begin{array}{cc}\text { App vol } & \text { App vol } \\ \exp / & \text { Calc / }\end{array}$

Density Density App Vol Square of Inconsistent Inconsistent Delta density / Density of $\mathrm{m}^{3} / \mathrm{kg} \quad \mathrm{m}^{3} / \mathrm{kg}$ calc/ Inare of Inconsistent Inconsistent Delta density / $\begin{array}{r}\text { Density of } \\ \text { based on } \\ \mathrm{kg} / \mathrm{m}^{3}\end{array}$ water $/ \mathrm{kg} / \mathrm{m}^{3}$

(38) $\quad 0.000326 \quad 0.000326$ $0.000327 \quad 0.000330$ $\mathrm{kg} / \mathrm{m}^{3}$ $\mathrm{kg} / \mathrm{m}^{3}$ $\mathrm{m}^{3} / \mathrm{kg}$

$\begin{array}{rrr}\text { Density } & \text { based on } & \text { based on } \\ \text { apparent } \\ \text { volume }\end{array}$

$48.587 \quad 994.0319$

$\begin{array}{llll}1042.6 & 0.0460 & -0.000001 & 0.002\end{array}$

$\begin{array}{llll}1048.8 & 0.1980 & -0.000002 & 0.039\end{array}$

$\begin{array}{llllll}0.000296 & 0.000309 & 993.6 & 0.0624 & -0.000013 & 0.004\end{array}$

$\begin{array}{llllll}0.000307 & 0.000311 & 996.9 & 0.0402 & -0.000004 & 0.002\end{array}$

$\begin{array}{llllll}0.000318 & 0.000314 & 1003.7 & -0.0780 & 0.000004 & 0.006\end{array}$

$\begin{array}{llllll}0.000317 & 0.000314 & 1003.8 & -0.0567 & 0.000003 & 0.003\end{array}$

$\begin{array}{llllll}0.000322 & 0.000316 & 1008.3 & -0.1474 & 0.000006 & 0.022 \\ 0.000326 & 0.000320 & 1014.7 & -0.2253 & 0.000006 & 0.051\end{array}$

$\begin{array}{llllll}0.000326 & 0.000320 & 1014.8 & -0.2234 & 0.000006 & 0.050\end{array}$

$\begin{array}{llllll}0.000329 & 0.000324 & 1023.6 & -0.2275 & 0.000005 & 0.052\end{array}$

$\begin{array}{llllll}0.000331 & 0.000327 & 1029.9 & -0.2089 & 0.000003 & 0.044\end{array}$

0.0003320 .000332

$\begin{array}{llll}1038.1 & -0.0235 & 0.000000 & 0.001\end{array}$

$\begin{array}{llll}1038.3 & -0.0228 & 0.000000 & 0.001\end{array}$

$\begin{array}{llllll}0.000332 & 0.000332 & 1038.3 & -0.0228 & 0.000000 & 0.001 \\ 0.000334 & 0.000335 & 1044.3 & 0.1125 & -0.000001 & 0.013\end{array}$

$\begin{array}{llll}1044.4 & 0.1142 & -0.000001 & 0.013\end{array}$

55.002994 .0319

$3.427 \quad 990.2132$

$\begin{array}{ll}6.7 & 990.2132\end{array}$

$13.387 \quad 990.2132$

13.512990 .2132

$17.917 \quad 990.2132$

$24.253 \quad 990.2132$

$24.353 \quad 990.2132$

$33.182 \quad 990.2132$

39.429990 .2132

$47.862 \quad 990.2132$

48.081990 .2132

$54.241 \quad 990.2132$

$\begin{array}{ll}54.293 & 990.2132\end{array}$

1.501 Sum chi sqr

998.2041

998.2041

998.2041

998.2041

998.2041 
molality molarity

\begin{tabular}{|c|c|c|c|c|c|}
\hline \multicolumn{2}{|c|}{$\mathrm{t} /{ }^{\circ} \mathrm{C}$ mass frac } & $\begin{array}{r}\text { Density } \\
\exp / \\
\mathrm{kg} / \mathrm{m}^{3}\end{array}$ & Ref & $\begin{array}{r}\text { App vol } \\
\text { exp / } \\
\mathrm{m}^{3} / \mathrm{kg}\end{array}$ & $\begin{array}{r}\text { App vol } \\
\text { Calc / } \\
\mathrm{m}^{3} / \mathrm{kg}\end{array}$ \\
\hline 20 & 0.0600 & 1042.8 & (106) & 0.000288 & 0.000305 \\
\hline 20 & 0.0700 & 1050.1 & (106) & 0.000295 & 0.000309 \\
\hline 20 & 0.0800 & 1056.7 & (106) & 0.000309 & 0.000313 \\
\hline 20 & 0.0880 & 1062.8 & (106) & 0.000310 & 0.000317 \\
\hline 25 & 0.0100 & 1005.6 & (106) & 0.000150 & 0.000294 \\
\hline 25 & 0.0200 & 1013.3 & (106) & 0.000198 & 0.000297 \\
\hline 25 & 0.0300 & 1021.1 & (106) & 0.000215 & 0.000301 \\
\hline 25 & 0.0400 & 1027.8 & (106) & 0.000253 & 0.000305 \\
\hline 25 & 0.0500 & 1035.1 & (106) & 0.000265 & 0.000309 \\
\hline 25 & 0.0600 & 1042.0 & (106) & 0.000282 & 0.000313 \\
\hline 25 & 0.0700 & 1049.3 & (106) & 0.000289 & 0.000317 \\
\hline 25 & 0.0800 & 1056.0 & (106) & 0.000303 & 0.000321 \\
\hline 25 & 0.0900 & 1062.4 & (106) & 0.000317 & 0.000325 \\
\hline 25 & 0.0955 & 1065.6 & (106) & 0.000327 & 0.000327 \\
\hline 30 & 0.0100 & 1004.3 & (106) & 0.000139 & 0.000300 \\
\hline 30 & 0.0200 & 1012.2 & (106) & 0.000183 & 0.000303 \\
\hline 30 & 0.0300 & 1020.1 & (106) & 0.000202 & 0.000307 \\
\hline 30 & 0.0400 & 1027.0 & (106) & 0.000238 & 0.000311 \\
\hline 30 & 0.0500 & 1034.2 & (106) & 0.000256 & 0.000315 \\
\hline 30 & 0.0600 & 1041.2 & (106) & 0.000272 & 0.000318 \\
\hline 30 & 0.0700 & 1048.5 & (106) & 0.000281 & 0.000322 \\
\hline 30 & 0.0800 & 1055.2 & (106) & 0.000296 & 0.000326 \\
\hline 30 & 0.0900 & 1061.7 & (106) & 0.000310 & 0.000330 \\
\hline 30 & 0.1000 & 1068.1 & (106) & 0.000323 & 0.000334 \\
\hline 30 & 0.1020 & 1068.5 & (106) & 0.000333 & 0.000335 \\
\hline 35 & 0.0100 & 1003.0 & (106) & 0.000107 & 0.000305 \\
\hline 35 & 0.0200 & 1011.1 & (106) & 0.000157 & 0.000308 \\
\hline 35 & 0.0300 & 1019.1 & (106) & 0.000181 & 0.000312 \\
\hline 35 & 0.0400 & 1026.1 & (106) & 0.000220 & 0.000316 \\
\hline 35 & 0.0500 & 1033.4 & (106) & 0.000240 & 0.000319 \\
\hline 35 & 0.0600 & 1040.4 & (106) & 0.000259 & 0.000323 \\
\hline 35 & 0.0700 & 1047.8 & (106) & 0.000269 & 0.000327 \\
\hline 35 & 0.0800 & 1054.5 & (106) & 0.000285 & 0.000331 \\
\hline 35 & 0.0900 & 1061.0 & (106) & 0.000300 & 0.000335 \\
\hline 35 & 0.1000 & 1067.4 & (106) & 0.000315 & 0.000338 \\
\hline 35 & 0.1075 & 1071.2 & (106) & 0.000332 & 0.000341 \\
\hline 40 & 0.0100 & 1001.7 & (106) & 0.000054 & 0.000308 \\
\hline 40 & 0.0200 & 1010.0 & (106) & 0.000121 & 0.000312 \\
\hline 40 & 0.0300 & 1018.0 & (106) & 0.000157 & 0.000315 \\
\hline 40 & 0.0400 & 1025.2 & (106) & 0.000197 & 0.000319 \\
\hline 40 & 0.0500 & 1032.6 & (106) & 0.000220 & 0.000323 \\
\hline 40 & 0.0600 & 1039.6 & (106) & 0.000242 & 0.000326 \\
\hline 40 & 0.0700 & 1046.9 & (106) & 0.000256 & 0.000330 \\
\hline
\end{tabular}

Density Density App Vol Square of Inconsistent Inconsistent Delta density / Density of calc / Residual / Residual / Density based on based on $\mathrm{kg} / \mathrm{m}^{3} \quad \mathrm{~kg} / \mathrm{m}^{3} \quad \mathrm{~m}^{3} / \mathrm{kg}$ Residual density apparent

$\begin{array}{llll}1041.6 & 1.1527 & -0.000018 \quad 1.329\end{array}$

$1049.0 \quad 1.1481-0.000015 \quad 1.318$

$1056.3 \quad 0.4335-0.000005 \quad 0.188$

$1062.1-0.6771-0.000007 \quad 0.458$

$1004.1-1.4548-0.000144 \quad 2.116$

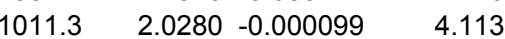

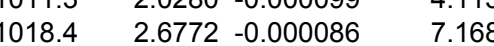

$1025.6-2.2051-0.000052-4.862$

$1032.8 \quad 2.3143-0.000043 \quad 5.356$

$1040.0-2.0079-0.000031-4.032$

$1.5606-0.000018$

4.363
2.435

2.435

$0.7266-0.000007-0.528$

$\begin{array}{llll}1065.7 & -0.0533 & 0.000000\end{array}$

$1002.7 \quad 1.6193-0.000161$

$1009.7 \quad 2.4599-0.000120$

$3.2769-0.000105$

$1023.9 \quad 3.0729-0.000073$

$1031.0 \quad 3.1505-0.000059$

$1038.2 \quad 3.0127-0.000046$

$1045.3 \quad 3.1625-0.000041$

$1052.5 \quad 2.7031-0.000030$

$\begin{array}{lll}1059.7 & 2.0380 & -0.000020\end{array}$

$1066.8 \quad 1.2707-0.000011$

$1068.3 \quad 0.2373-0.000002$

$1001.0 \quad 1.9893-0.000198$

$1008.0 \quad 3.0845-0.000151$

$1015.0 \quad 4.0560-0.000131$

$1022.1 \quad 4.0063-0.000095$

$1029.24 .2381-0.000080$

$1036.24 .1541-0.000064$

$1043.3 \quad 4.4573-0.000058$

$1050.4 \quad 4.0510-0.000046$

$1057.6 \quad 3.4383-0.000034$

$1064.7 \quad 2.7228-0.000024$

$1070.0 \quad 1.1866-0.000010$

$\begin{array}{rrr}999.2 & 2.5488 & -0.000255\end{array}$

$1006.1 \quad 3.8869-0.000191$

$1013.1 \quad 4.9008-0.000158$

$1020.1 \quad 5.0930-0.000122$

$1027.15 .4659-0.000103$

$1034.2 \quad 5.4223-0.000084$

$1041.2 \quad 5.6651-0.000074$
0.003
2.622

6.051

10.738

9.442

9.926

9.076

7.307
4.153

1.615

0.056

3.957

9.514
16.451

16.050

17.961

17.256

19.868

16.410

11.822

7.414

1.408

6.496
15.108

24.018

25.938

29.876
29.401

32.093 $\mathrm{kg} / \mathrm{m}^{3}$ water $/ \mathrm{kg} / \mathrm{m}^{3}$

998.2041

998.204

998.2041

998.2041

997.0449

997.0449

997.0449

997.0449

997.0449

997.0449

997.0449

997.0449

997.0449

997.0449

995.6473

995.6473

995.6473

995.6473

995.6473

995.6473

995.6473

995.6473

995.6473

995.6473

995.6473

994.0319

994.0319

994.0319

994.0319

994.0319

994.0319

994.0319

994.0319

994.0319

994.0319

994.0319

992.2158

992.2158

992.2158

992.2158

992.2158

992.2158

992.2158 
molality molarity

\begin{tabular}{|c|c|c|c|c|c|}
\hline \multicolumn{2}{|c|}{$\mathrm{t} /{ }^{\circ} \mathrm{C}$ mass frac } & $\begin{array}{r}\text { Density } \\
\exp / \\
\mathrm{kg} / \mathrm{m}^{3}\end{array}$ & Ref & $\begin{array}{r}\text { App vol } \\
\exp / \\
\mathrm{m}^{3} / \mathrm{kg}\end{array}$ & $\begin{array}{r}\text { App vol } \\
\text { Calc / } \\
\mathrm{m}^{3} / \mathrm{kg}\end{array}$ \\
\hline 40 & 0.0800 & 1053.8 & (106) & 0.000272 & 0.000334 \\
\hline 40 & 0.0900 & 1060.3 & (106) & 0.000289 & 0.000338 \\
\hline 40 & 0.1000 & 1066.8 & (106) & 0.000303 & 0.000342 \\
\hline 40 & 0.1130 & 1074.0 & (106) & 0.000329 & 0.000347 \\
\hline 45 & 0.0100 & 1000.3 & (106) & -0.000008 & 0.000311 \\
\hline 45 & 0.0200 & 1008.9 & (106) & 0.000075 & 0.000314 \\
\hline 45 & 0.0300 & 1017.1 & (106) & 0.000120 & 0.000318 \\
\hline 45 & 0.0400 & 1024.4 & (106) & 0.000167 & 0.000321 \\
\hline 45 & 0.0500 & 1031.7 & (106) & 0.000198 & 0.000325 \\
\hline 45 & 0.0600 & 1038.8 & (106) & 0.000223 & 0.000329 \\
\hline 45 & 0.0700 & 1046.2 & (106) & 0.000238 & 0.000332 \\
\hline 45 & 0.0800 & 1053.1 & (106) & 0.000256 & 0.000336 \\
\hline 45 & 0.0900 & 1059.7 & (106) & 0.000274 & 0.000340 \\
\hline 45 & 0.1000 & 1066.1 & (106) & 0.000291 & 0.000343 \\
\hline 45 & 0.1200 & 1077.0 & (106) & 0.000332 & 0.000351 \\
\hline 50 & 0.0100 & 998.7 & (106) & -0.000069 & 0.000312 \\
\hline 50 & 0.0200 & 1007.8 & (106) & 0.000020 & 0.000316 \\
\hline 50 & 0.0300 & 1016.1 & (106) & 0.000080 & 0.000319 \\
\hline 50 & 0.0400 & 1023.5 & (106) & 0.000135 & 0.000323 \\
\hline 50 & 0.0500 & 1030.9 & (106) & 0.000170 & 0.000326 \\
\hline 50 & 0.0600 & 1037.9 & (106) & 0.000202 & 0.000330 \\
\hline 50 & 0.0700 & 1045.4 & (106) & 0.000219 & 0.000333 \\
\hline 50 & 0.0800 & 1052.4 & (106) & 0.000238 & 0.000337 \\
\hline 50 & 0.0900 & 1059.0 & (106) & 0.000259 & 0.000341 \\
\hline 50 & 0.1000 & 1065.5 & (106) & 0.000276 & 0.000344 \\
\hline 50 & 0.1270 & 1080.0 & (106) & 0.000334 & 0.000355 \\
\hline 55 & 0.0100 & 997.2 & (106) & -0.000156 & 0.000313 \\
\hline 55 & 0.0200 & 1006.7 & (106) & -0.000044 & 0.000316 \\
\hline 55 & 0.0300 & 1015.1 & (106) & 0.000035 & 0.000320 \\
\hline 55 & 0.0400 & 1022.7 & (106) & 0.000097 & 0.000323 \\
\hline 55 & 0.0500 & 1030.0 & (106) & 0.000142 & 0.000327 \\
\hline 55 & 0.0600 & 1037.2 & (106) & 0.000175 & 0.000330 \\
\hline 55 & 0.0700 & 1044.6 & (106) & 0.000197 & 0.000334 \\
\hline 55 & 0.0800 & 1051.7 & (106) & 0.000219 & 0.000337 \\
\hline 55 & 0.0900 & 1058.4 & (106) & 0.000240 & 0.000341 \\
\hline 55 & 0.1000 & 1064.8 & (106) & 0.000261 & 0.000345 \\
\hline 55 & 0.1340 & 1082.9 & (106) & 0.000335 & 0.000357 \\
\hline 60 & 0.0100 & 995.6 & (106) & -0.000250 & 0.000313 \\
\hline 60 & 0.0200 & 1005.6 & (106) & -0.000116 & 0.000316 \\
\hline 60 & 0.0300 & 1014.1 & (106) & -0.000016 & 0.000319 \\
\hline 60 & 0.0400 & 1021.8 & (106) & 0.000057 & 0.000323 \\
\hline 60 & 0.0500 & 1029.2 & (106) & 0.000108 & 0.000326 \\
\hline 60 & & & & 0.000147 & 0.000330 \\
\hline
\end{tabular}

Density Density App Vol Square of Inconsistent Inconsistent Delta density / Density of calc / Residual / Residual / Density based on based on $\mathrm{kg} / \mathrm{m}^{3} \quad \mathrm{~kg} / \mathrm{m}^{3} \quad \mathrm{~m}^{3} / \mathrm{kg}$ Residual density apparent

$1048.3 \quad 5.4973-0.000062 \quad 30.221$ $1055.4 \quad 4.9222-0.000049$ $1062.5 \quad 4.3432-0.000038$ $1071.7-2.3405-0.000018$

$997.13 .1845-0.000319$ $1004.0 \quad 4.8550-0.000240$ $1011.0 \quad 6.1004-0.000198$ $1018.0 \quad 6.4230-0.000154$

$1025.0 \quad 6.7253-0.000127$ $1032.0 \quad 6.8100-0.000106$ $1039.0 \quad 7.1798-0.000094$ $1046.1 \quad 7.0376-0.000080$

$6.5866-0.000066$ $1060.2 \quad 5.9301-0.000052$ $\begin{array}{rrr}1074.3 & 2.7147 & -0.000020\end{array}$ $994.9 \quad 3.7854-0.000381$ $5.9788-0.000296$ $1008.8 \quad 7.3457-0.000239$ $1015.7 \quad 7.7886-0.000187$ $1022.7 \quad 8.2097-0.000156$ $1029.7 \quad 8.2117-0.000128$ $1036.7 \quad 8.6971-0.000115$ $1043.7 \quad 8.6689-0.000099$ $1050.8 \quad 8.2299-0.000082$ $1057.8 \quad 7.6835-0.000068$ $3.1475-0.000021$ $9926 \quad 4.6422-0.000469$ $999.5 \quad 7.2498-0.000360$ $1006.4 \quad 8.7295-0.000285$ $1013.3-9.3834-0.000226$ $1020.3 \quad 9.7139-0.000185$ $1027.3 \quad 9.9233-0.000155$ $1034.3 \quad 10.3143-0.000136$ $1041.3 \quad 10.3894-0.000119$ $1048.3 \quad 10.0517-0.000101$ $1055.4 \quad 9.4042-0.000084$ $1079.4 \quad 3.5140-0.000022$ $990.1 \quad 5.5469-0.000563$

$996.9 \quad 8.6611-0.000432$ $1003.9 \quad 10.2456-0.000335$ $1010.8 \quad 11.0025-0.000266$ $1017.8 \quad 11.4338-0.000218$

$1024.8 \quad 11.6420-0.000183 \quad 135.535$
24.228
18.863

5.478

10.141

23.571

37.215
41.255

41.255

46.376

51.549

49.528

43.384

35.166

7.369

14.330

35.745

53.960

60.662

67.400

67.432

75.640

75.149

67.732

59.036

9.907

21.550

52.559

76.204

88.049

94.360

98.473

106.384

107.940

101.037

88.439

12.348

30.768

75.015

104.972

121.054 $\mathrm{kg} / \mathrm{m}^{3}$ water $/ \mathrm{kg} / \mathrm{m}^{3}$

992.2158

992.2158

992.2158

992.2158

990.2132

990.2132

990.2132

990.2132

990.2132

990.2132

990.2132

990.2132

990.2132

990.2132

990.2132

988.0363

988.0363

988.0363

988.0363

988.0363

988.0363

988.0363

988.0363

988.0363

988.0363

988.0363

985.6952

985.6952

985.6952

985.6952

985.6952

985.6952

985.6952

985.6952

985.6952

985.6952

985.6952

983.1989

983.1989

983.1989

983.1989

983.1989

983.1989 
molality molarity

\begin{tabular}{|c|c|c|c|c|c|}
\hline $1{ }^{\circ} \mathrm{C}$ & is frac & $\begin{array}{r}\text { Density } \\
\exp / \\
\mathrm{kg} / \mathrm{m}^{3}\end{array}$ & Ref & $\begin{array}{c}\text { App vol } \\
\exp / \\
\mathrm{m}^{3} / \mathrm{kg}\end{array}$ & $\begin{array}{c}\text { App vol } \\
\text { Calc/ } \\
\mathrm{m}^{3} / \mathrm{kg}\end{array}$ \\
\hline 60 & 0.0700 & 1043.8 & (106) & 0.000174 & 0.000333 \\
\hline 60 & 0.0800 & 1051.0 & (106) & 0.000197 & 0.000337 \\
\hline 60 & 0.0900 & 1057.7 & (106) & 0.000221 & 0.000340 \\
\hline 60 & 0.1000 & 1064.2 & (106) & 0.000243 & 0.000344 \\
\hline 60 & 0.1410 & 1085.9 & (106) & 0.000335 & 0.000359 \\
\hline 65 & 0.0100 & 993.9 & (106) & -0.000350 & 0.000312 \\
\hline 65 & 0.0200 & 1004.5 & (106) & -0.000196 & 0.000315 \\
\hline 65 & 0.0300 & 1013.1 & (106) & -0.000072 & 0.000318 \\
\hline 65 & 0.0400 & 1020.9 & (106) & 0.000012 & 0.000322 \\
\hline 65 & 0.0500 & 1028.4 & (106) & 0.000071 & 0.000325 \\
\hline 65 & 0.0600 & 1035.6 & (106) & 0.000116 & 0.000328 \\
\hline 65 & 0.0700 & 1043.1 & (106) & 0.000146 & 0.000332 \\
\hline 65 & 0.0800 & 1050.3 & (106) & 0.000173 & 0.000335 \\
\hline 65 & 0.0900 & 1057.1 & (106) & 0.000199 & 0.000339 \\
\hline 65 & 0.1000 & 1063.6 & (106) & 0.000224 & 0.000342 \\
\hline 70 & 0.0100 & 992.2 & (106) & -0.000465 & 0.000311 \\
\hline 70 & 0.0200 & 1003.4 & (106) & -0.000283 & 0.000314 \\
\hline 70 & 0.0300 & 1012.1 & (106) & -0.000134 & 0.000317 \\
\hline 70 & 0.0400 & 1020.0 & (106) & -0.000036 & 0.000320 \\
\hline 70 & 0.0500 & 1027.5 & (106) & 0.000033 & 0.000323 \\
\hline 70 & 0.0600 & 1034.7 & (106) & 0.000085 & 0.000327 \\
\hline 70 & 0.0700 & 1047.3 & (106) & 0.000053 & 0.000330 \\
\hline 70 & 0.0800 & 1049.6 & (106) & 0.000148 & 0.000333 \\
\hline 70 & 0.0900 & 1056.4 & (106) & 0.000177 & 0.000337 \\
\hline 70 & 0.1000 & 1063.0 & (106) & 0.000203 & 0.000340 \\
\hline 75 & 0.0100 & 990.5 & (106) & -0.000595 & 0.000308 \\
\hline 75 & 0.0200 & 1002.2 & (106) & -0.000374 & 0.000311 \\
\hline 75 & 0.0300 & 1011.1 & (106) & -0.000200 & 0.000315 \\
\hline 75 & 0.0400 & 1019.2 & (106) & -0.000090 & 0.000318 \\
\hline 75 & 0.0500 & 1026.7 & (106) & -0.000010 & 0.000321 \\
\hline 75 & 0.0600 & 1033.9 & (106) & 0.000049 & 0.000324 \\
\hline 75 & 0.0700 & 1046.5 & (106) & 0.000022 & 0.000327 \\
\hline 75 & 0.0800 & 1049.0 & (106) & 0.000119 & 0.000331 \\
\hline 75 & 0.0900 & 1055.8 & (106) & 0.000152 & 0.000334 \\
\hline 75 & 0.1000 & 1062.4 & (106) & 0.000180 & 0.000337 \\
\hline 80 & 0.0100 & 988.9 & (106) & -0.000751 & 0.000306 \\
\hline 80 & 0.0200 & 1001.1 & (106) & -0.000477 & 0.000309 \\
\hline 80 & 0.0300 & 1010.1 & (106) & -0.000272 & 0.000312 \\
\hline 80 & 0.0400 & 1018.3 & (106) & -0.000146 & 0.000315 \\
\hline 80 & 0.0500 & 1025.8 & (106) & -0.000054 & 0.000318 \\
\hline 80 & 0.0600 & 1033.1 & (106) & 0.000011 & 0.000321 \\
\hline 80 & 0.0700 & 1045.7 & (106) & -0.000010 & 0.000324 \\
\hline 80 & 0.0800 & 1048.3 & (106) & 0.000090 & 0.000327 \\
\hline
\end{tabular}

Density Density App Vol Square of Inconsistent Inconsistent Delta density / Density of calc / Residual / Residual / Density based on based on $\mathrm{kg} / \mathrm{m}^{3} \quad \mathrm{~kg} / \mathrm{m}^{3} \quad \mathrm{~m}^{3} / \mathrm{kg}$ Residual density $\begin{gathered}\text { apparent } \\ \text { volume }\end{gathered}$

$1031.8 \quad 12.0294-0.000160 \quad 144.706$

$\begin{array}{llll}1038.8 & 12.1986 & -0.000140 & 148.807\end{array}$

$1045.8 \quad 11.8525-0.000119 \quad 140.482$

$1052.9 \quad 11.2939-0.000101 \quad 127.553$

$1081.9 \quad 3.9921-0.000024 \quad 15.937$

$987.4 \quad 6.4927-0.000662 \quad 42.156$

$994.3 \quad 10.2068-0.000511 \quad 104.179$

$10012 \quad 11.8891-0.000391-141.351$

$1008.2 \quad 12.7416-0.000309 \quad 162.349$

$1015.1 \quad 13.2663-0.000254 \quad 175.996$

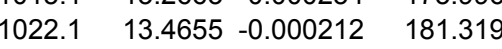

$\begin{array}{llll}1029.2 & 13.9414 & -0.000186 & 194.362\end{array}$

$\begin{array}{llll}1036.2 & 14.0965 & -0.000162 & 198.711\end{array}$

$\begin{array}{llll}1043.3 & 13.8334 & -0.000139 & 191.363\end{array}$

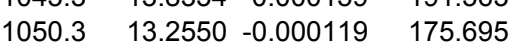

$\begin{array}{llll}984.6 & 7.5740 & -0.000775 & 57.365\end{array}$

$\begin{array}{llll}991.5 & 11.8819 & -0.000597 & 141.179\end{array}$

$\begin{array}{llll}998.4 & 13.6558 & -0.000450 & 186.482\end{array}$

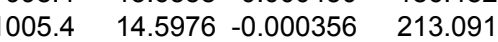

$\begin{array}{llll}1012.4 & 15.1091 & -0.000290 & 228.286\end{array}$

$\begin{array}{llll}1019.4 & 15.2925 & -0.000242 & 233.860\end{array}$

$\begin{array}{llll}1026.5 & 20.8498 & -0.000277 & 434.715\end{array}$

$\begin{array}{llll}1033.5 & 16.0835 & -0.000185 & 258.679\end{array}$

$\begin{array}{llll}1040.6 & 15.7960 & -0.000160 & 249.514\end{array}$

$\begin{array}{rrrr}1047.7 & 15.2900 & -0.000137 & 233.784\end{array}$

$\begin{array}{llll}981.7 & 8.7856 & -0.000904 & 77.187\end{array}$

$\begin{array}{llll}988.6 & 13.5822 & -0.000685 & 184.477\end{array}$

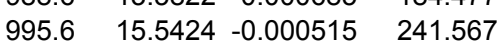

$\begin{array}{llll}1002.5 & 16.6679 & -0.000408 & 277.820\end{array}$

$\begin{array}{llll}1009.5 & 17.1605 & -0.000331 & 294.483\end{array}$

$\begin{array}{llll}1016.6 & 17.3221 & -0.000275 & 300.054\end{array}$

$\begin{array}{llll}1023.6 & 22.8547 & -0.000305 & 522.339\end{array}$

$\begin{array}{lllll}1030.7 & 18.2607 & -0.000211 & 333.452\end{array}$

$\begin{array}{llll}1037.9 & 17.9422 & -0.000182 & 321.923\end{array}$

$\begin{array}{llll}1045.0 & 17.4019 & -0.000157 & 302.826\end{array}$

$\begin{array}{llll}978.7 & 10.2234 & -0.001056 & 104.519\end{array}$

$\begin{array}{llll}985.6 & 15.5043 & -0.000786 & 240.382\end{array}$

$\begin{array}{llll}992.6 & 17.5461 & -0.000583 & 307.865\end{array}$

$9995-18.7505-0.000461 \quad 351.581$

$\begin{array}{rrrr}1006.6 & 19.2192 & -0.000372 & 369.376\end{array}$

$\begin{array}{llll}1013.6 & 19.4539 & -0.000310 & 378.453\end{array}$

$\begin{array}{llll}1020.7 & 24.9565 & -0.000334 & 622.829\end{array}$

$\begin{array}{llll}1027.9 & 20.4293 & -0.000237 & 417.355\end{array}$ $\mathrm{kg} / \mathrm{m}^{3}$ water $/ \mathrm{kg} / \mathrm{m}^{3}$

983.1989

983.1989

983.1989

983.1989

980.5548

980.5548

980.5548

980.5548

980.5548

980.5548

980.5548

980.5548

980.5548

980.5548

977.7696

977.7696

977.7696

977.7696

977.7696

977.7696

977.7696

977.7696

977.7696

977.7696

974.849

974.849

974.849

974.849

974.849

974.849

974.849

974.849

974.849

974.849

971.7978

971.7978

971.7978

971.7978

971.7978

971.7978

971.7978

971.7978 
molality molarity

\begin{tabular}{|c|c|c|c|c|c|}
\hline $\mathbf{t} /{ }^{\circ} \mathrm{C}$ & ass frac & $\begin{array}{r}\text { Density } \\
\exp / \\
\mathrm{kg} / \mathrm{m}^{3}\end{array}$ & Ref & $\begin{array}{r}\text { App vol } \\
\exp / \\
\mathrm{m}^{3} / \mathrm{kg}\end{array}$ & $\begin{array}{r}\text { App vol } \\
\text { Calc / } \\
\mathrm{m}^{3} / \mathrm{kg}\end{array}$ \\
\hline 80 & 0.0900 & 1055.2 & (106) & 0.000125 & 0.000331 \\
\hline 80 & 0.1000 & 1061.9 & (106) & 0.000156 & 0.000334 \\
\hline 85 & 0.0100 & 987.1 & (106) & -0.000900 & 0.000303 \\
\hline 85 & 0.0200 & 999.9 & (106) & -0.000582 & 0.000306 \\
\hline 85 & 0.0300 & 999.1 & (106) & -0.000017 & 0.000308 \\
\hline 85 & 0.0400 & 1017.4 & (106) & -0.000205 & 0.000311 \\
\hline 85 & 0.0500 & 1025.0 & (106) & -0.000103 & 0.000314 \\
\hline 85 & 0.0600 & 1032.3 & (106) & -0.000029 & 0.000317 \\
\hline 85 & 0.0700 & 1039.9 & (106) & 0.000021 & 0.000321 \\
\hline 85 & 0.0800 & 1047.6 & (106) & 0.000059 & 0.000324 \\
\hline 85 & 0.0900 & 1054.7 & (106) & 0.000096 & 0.000327 \\
\hline 85 & 0.1000 & 1061.3 & (106) & 0.000131 & 0.000330 \\
\hline 90 & 0.0100 & 985.4 & (106) & -0.001075 & 0.000299 \\
\hline 90 & 0.0200 & 998.8 & (106) & -0.000700 & 0.000302 \\
\hline 90 & 0.0300 & 998.0 & (106) & -0.000095 & 0.000305 \\
\hline 90 & 0.0400 & 1016.5 & (106) & -0.000268 & 0.000308 \\
\hline 90 & 0.0500 & 1024.2 & (106) & -0.000155 & 0.000311 \\
\hline 90 & 0.0600 & 1031.5 & (106) & -0.000072 & 0.000313 \\
\hline 90 & 0.0700 & 1039.2 & (106) & -0.000016 & 0.000316 \\
\hline 90 & 0.0800 & 1047.0 & (106) & 0.000026 & 0.000320 \\
\hline 90 & 0.0900 & 1054.1 & (106) & 0.000066 & 0.000323 \\
\hline 90 & 0.1000 & 1060.8 & (106) & 0.000104 & 0.000326 \\
\hline 95 & 0.0100 & 988.7 & (106) & -0.001778 & 0.000295 \\
\hline 95 & 0.0200 & 997.7 & (106) & -0.000826 & 0.000298 \\
\hline 95 & 0.0300 & 997.0 & (106) & -0.000180 & 0.000301 \\
\hline 95 & 0.0400 & 1015.6 & (106) & -0.000335 & 0.000303 \\
\hline 95 & 0.0500 & 1023.4 & (106) & -0.000210 & 0.000306 \\
\hline 95 & 0.0600 & 1030.7 & (106) & -0.000117 & 0.000309 \\
\hline 95 & 0.0700 & 1038.4 & (106) & -0.000055 & 0.000312 \\
\hline 95 & 0.0800 & 1046.3 & (106) & -0.000009 & 0.000315 \\
\hline 95 & 0.0900 & 1053.6 & (106) & 0.000034 & 0.000318 \\
\hline 95 & 0.1000 & 1060.2 & (106) & 0.000076 & 0.000321 \\
\hline 100 & 0.0100 & 981.9 & (106) & -0.001458 & 0.000291 \\
\hline 100 & 0.0200 & 996.5 & (106) & -0.000953 & 0.000293 \\
\hline 100 & 0.0300 & 996.0 & (106) & -0.000271 & 0.000296 \\
\hline 100 & 0.0400 & 1014.7 & (106) & -0.000405 & 0.000299 \\
\hline 100 & 0.0500 & 1022.5 & (106) & -0.000266 & 0.000302 \\
\hline 100 & 0.0600 & 1029.1 & (106) & -0.000152 & 0.000304 \\
\hline 100 & 0.0700 & 1037.6 & (106) & -0.000095 & 0.000307 \\
\hline 100 & 0.0800 & 1045.7 & (106) & -0.000046 & 0.000310 \\
\hline 100 & 0.0900 & 1053.1 & (106) & 0.000000 & 0.000313 \\
\hline 100 & 0.1000 & 1059.7 & (106) & 0.000046 & 0.000316 \\
\hline
\end{tabular}

Density Density App Vol Square of Inconsistent Inconsistent Delta density / Density of calc / Residual / Residual / Density based on based on $\mathrm{kg} / \mathrm{m}^{3} \quad \mathrm{~kg} / \mathrm{m}^{3} \quad \mathrm{~m}^{3} / \mathrm{kg}$ Residual density apparent

$\begin{array}{llll}1035.0 & 20.1742 & -0.000205 & 406.999\end{array}$ $\begin{array}{cccc}1042.2 & 19.6937 & -0.000178 & 387.844\end{array}$

$975.5 \quad 11.5839-0.001203 \quad 134.186$

$982.5 \quad 17.4451-0.000888 \quad 304.330$

$989.4 \quad 9.6646-0.000326 \quad 93.404$

$996.5 \quad 20.9438-0.000516 \quad 438.643$

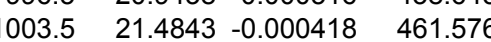

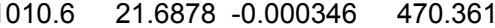

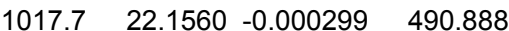

$1024.9 \quad 22.6908-0.000264 \quad 514.875$

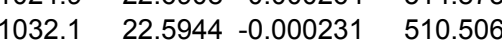

$\begin{array}{llll}1039.3 & 21.9688 & -0.000199 & 482.629\end{array}$

$\begin{array}{llll}972.2 & 13.1639 & -0.001374 & 173.288\end{array}$

$\begin{array}{llll}979.2 & 19.6022 & -0.001002 & 384.247\end{array}$

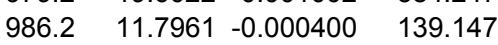

$993.3 \quad 23.2467 \quad-0.000576 \quad 540.410$

$\begin{array}{llll}1000.3 & 23.8556 & -0.000466 & 569.088\end{array}$

$\begin{array}{llll}1007.5 & 24.0242 & -0.000385 & 577.160\end{array}$

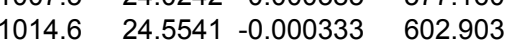

$1021.9 \quad 25.1472-0.000294 \quad 632.380$

$\begin{array}{llll}1029.1 & 25.0053 & -0.000256 & 625.263\end{array}$

$\begin{array}{llll}1036.4 & 24.4304 & -0.000222 & 596.845\end{array}$

$\begin{array}{llll}968.8 & 19.8609 & -0.002073 & 394.456\end{array}$

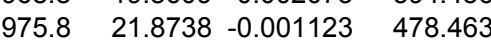

$\begin{array}{llll}982.9 & 14.1393 & -0.000481 & 199.919\end{array}$

$\begin{array}{llll}989.9 & 25.6585 & -0.000638 & 658.359\end{array}$

$\begin{array}{llll}997.1 & 26.3328 & -0.000516 & 693.416\end{array}$

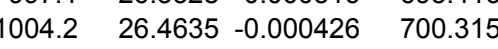

$\begin{array}{llll}1011.4 & 26.9520 & -0.000367 & 726.412\end{array}$

$\begin{array}{llll}1018.7 & 27.6001 & -0.000324 & 761.766\end{array}$

$\begin{array}{llll}1026.0 & 27.6094 & -0.000284 & 762.281\end{array}$

$\begin{array}{llll}1033.3 & 26.8819 & -0.000245 & 722.635\end{array}$

$\begin{array}{llll}965.3 & 16.5729 & -0.001748 & 274.662\end{array}$

$\begin{array}{llll}9723 & 24.1582 & -0.001247 & 583.621\end{array}$

$\begin{array}{lllll}979.4 & 16.5932 & -0.000567 & 275.334\end{array}$

$\begin{array}{llll}986.5 & 28.1788 & -0.000704 & 794.043\end{array}$

$\begin{array}{lllll}993.7 & 28.8161 & -0.000567 & 830.370\end{array}$

$\begin{array}{llll}1000.9 & 28.2065 & -0.000456 & 795.607\end{array}$

$\begin{array}{llll}1008.1 & 29.4512 & -0.000402 & 867.375\end{array}$

$\begin{array}{llll}1015.4 & 30.2517 & -0.000356 & 915.168\end{array}$

$\begin{array}{llll}1022.8 & 30.3096 & -0.000313 & 918.674\end{array}$

$\begin{array}{llll}1030.2 & 29.5266 & -0.000270 & 871.821\end{array}$ $\mathrm{kg} / \mathrm{m}^{3}$ water $/ \mathrm{kg} / \mathrm{m}^{3}$

971.7978

968.6203

968.6203

968.6203

968.6203

968.6203

968.6203

968.6203

968.6203

968.6203

968.6203

965.3201

965.3201

965.3201

965.3201

965.3201

965.3201

965.3201

965.3201

965.3201

965.3201

961.9004

961.9004

961.9004

961.9004

961.9004

961.9004

961.9004

961.9004

961.9004

961.9004

958.3637

958.3637

958.3637

958.3637

958.3637

958.3637

958.3637

958.3637

958.3637

958.3637 
Density of aqueous solutions of $\mathrm{NaH2PO4}$

References (71) Mason, C. M., Culvern, J. B., Electrical conductivity of orthophosphoric acid and of sodium and potassium dihydrogen phosphates at 25 ${ }^{\circ} \mathrm{C}, \mathrm{J}$ Am Chem Soc, 1949, 71, 2387-2393

(122) Sohnel, P.; Novotny, P.; Solc, Z.; Densities of aqueous solutions of 18 inorganic substances, J Chem Eng Data, 1984, 29, 379-382

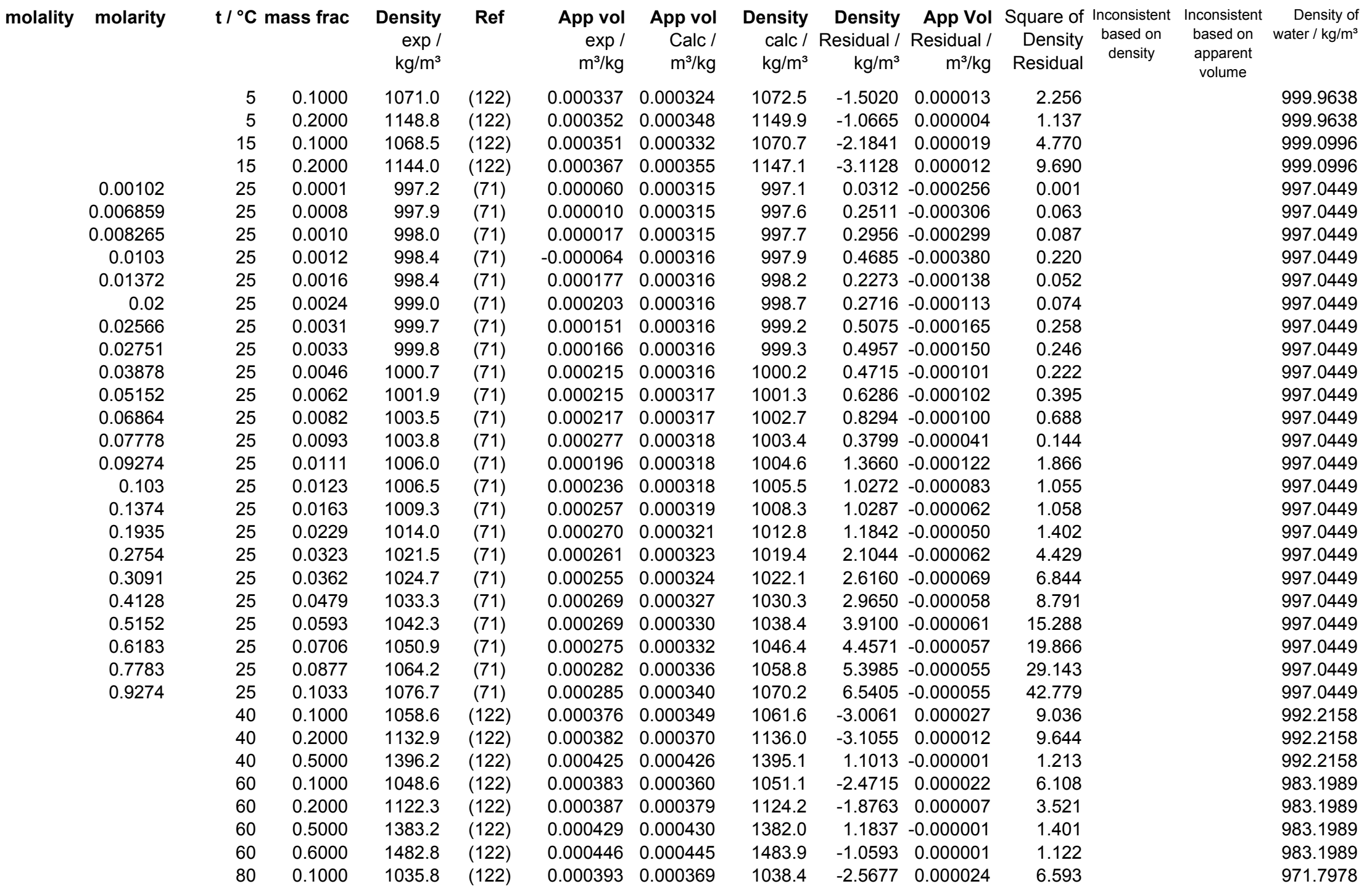


molality molarity

$\begin{array}{ccrc}\mathbf{t} /{ }^{\circ} \mathbf{C} \text { mass frac } & \begin{array}{r}\text { Density } \\ \text { exp / } \\ \mathrm{kg} / \mathrm{m}^{3}\end{array} & \text { Ref } \\ & & \\ 80 & 0.2000 & 1108.7 & (122) \\ 80 & 0.5000 & 1372.2 & (122) \\ 80 & 0.6000 & 1469.9 & (122)\end{array}$

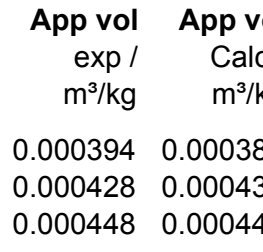

Std dev Res

Avg - 4std

Avg + 4std
Density Density App Vol Square of Inconsistent Inconsistent calc / Residual / Residua / $\mathrm{kg} / \mathrm{m}^{3}$

$\mathrm{m}^{3} / \mathrm{kg}$

$\begin{array}{ll}-1.7509 & 0.000007\end{array}$

$3.8770-0.000004$

$-2.0205 \quad 0.000002$

$0.4709-0.000072$

$2.3551 \quad 0.000099$

$-8.9494-0.000469$

9.89120 .000325

\section{Curve fit for the model App vol $=\left(w+c 2+c 3^{*} t{ }^{\circ} \mathrm{C}\right) /\left(\mathrm{c} 0^{*} \mathrm{w}+\mathrm{c} 1\right) / \mathrm{e}^{\wedge}\left(0.000001^{*}\left(\mathrm{t}{ }^{\circ} \mathrm{C}+\mathrm{c} 4\right)^{\wedge} 2\right)$}

$\mathrm{cO}$

c1

c2

c3

$$
\text { c4 }
$$

Min T

$\operatorname{Max} T$

Min w

Max w 208.7729

641.0473

0.788927

0.004552

1198.407

\section{Avg dens res}

Std dens res

Avg app vol res *10^3 -0.072159

Std app vol res *10^3 0.099282

No of points in corr

No of inconsistent poir

38

Inconsistent data not used (Residual greater than average $+/-4$ standard deviations) Data at high concentration for Mason seems inconsistent with Sohnel

$\begin{array}{lllllll}1.367 & 25 & 0.1474 & 1112.9 & (71) & 0.000294 & 0.000350 \\ 2.061 & 25 & 0.2118 & 1167.6 & (71) & 0.000311 & 0.000364 \\ 3.885 & 25 & 0.3571 & 1305.3 & (71) & 0.000340 & 0.000394\end{array}$

$1102.9 \quad 10.0254-0.000055 \quad 100.508$

$\begin{array}{llll}1152.5 & 15.0758 & -0.000053 & 227.279\end{array}$

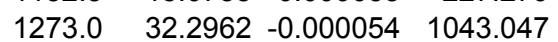

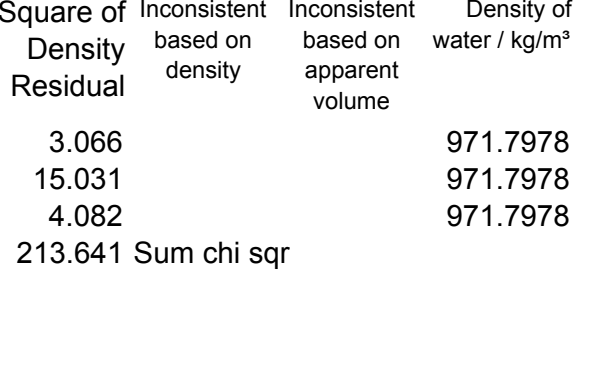


Density of aqueous solutions of Na2HPO4

References (122) Sohnel, P.; Novotny, P.; Solc, Z.; Densities of aqueous solutions of 18 inorganic substances, J Chem Eng Data, 1984, 29, 379-382

\begin{tabular}{|c|c|c|c|c|c|c|c|c|c|c|c|c|c|c|}
\hline \multirow[t]{17}{*}{ molality } & \multirow[t]{17}{*}{ molarity } & \multicolumn{2}{|c|}{$\mathrm{t} /{ }^{\circ} \mathrm{C}$ mass frac } & $\begin{array}{r}\text { Density } \\
\exp / \\
\mathrm{kg} / \mathrm{m}^{3}\end{array}$ & Ref & $\begin{array}{r}\text { App vol } \\
\text { exp / } \\
\mathrm{m}^{3} / \mathrm{kg}\end{array}$ & $\begin{array}{r}\text { App vol } \\
\text { Calc / } \\
\mathrm{m}^{3} / \mathrm{kg}\end{array}$ & $\begin{array}{r}\text { Density } \\
\text { calc / } \\
\mathrm{kg} / \mathrm{m}^{3}\end{array}$ & $\begin{array}{r}\text { Density } \\
\text { Residual / } \\
\mathrm{kg} / \mathrm{m}^{3}\end{array}$ & $\begin{array}{r}\text { App Vol } \\
\text { Residual / } \\
\mathrm{m}^{3} / \mathrm{kg}\end{array}$ & $\begin{array}{r}\text { Square of } \\
\text { Density } \\
\text { Residual }\end{array}$ & $\begin{array}{l}\text { Inconsistent } \\
\text { based on } \\
\text { density }\end{array}$ & $\begin{array}{l}\text { Inconsistent } \\
\text { based on } \\
\text { apparent } \\
\text { volume }\end{array}$ & $\begin{array}{r}\text { Density of } \\
\text { water } / \mathrm{kg} / \mathrm{m}^{3}\end{array}$ \\
\hline & & 40 & 0.0500 & 1043.1 & (122) & 0.000025 & 0.000022 & 1043.3 & -0.1650 & 0.000003 & 0.027 & & & 992.2158 \\
\hline & & 40 & 0.1000 & 1097.6 & (122) & 0.000040 & 0.000045 & 1097.0 & 0.5517 & -0.000005 & 0.304 & & & 992.2158 \\
\hline & & 40 & 0.2000 & 1215.4 & (122) & 0.000082 & 0.000085 & 1214.8 & 0.6312 & -0.000002 & 0.398 & & & 992.2158 \\
\hline & & 40 & 0.3000 & 1350.4 & (122) & 0.000117 & 0.000118 & 1349.9 & 0.4517 & -0.000001 & 0.204 & & & 992.2158 \\
\hline & & 60 & 0.0500 & 1033.7 & (122) & 0.000023 & 0.000016 & 1034.1 & -0.3745 & 0.000007 & 0.140 & & & 983.1989 \\
\hline & & 60 & 0.1000 & 1087.1 & (122) & 0.000045 & 0.000041 & 1087.6 & -0.5188 & 0.000004 & 0.269 & & & 983.1989 \\
\hline & & 60 & 0.2000 & 1203.6 & (122) & 0.000086 & 0.000082 & 1204.6 & -1.0246 & 0.000004 & 1.050 & & & 983.1989 \\
\hline & & 60 & 0.3000 & 1337.6 & (122) & 0.000119 & 0.000117 & 1338.7 & -1.0807 & 0.000002 & 1.168 & & & 983.1989 \\
\hline & & 80 & 0.0500 & 1022.5 & (122) & 0.000009 & 0.000011 & 1022.4 & 0.1133 & -0.000002 & 0.013 & & & 971.7978 \\
\hline & & 80 & 0.1000 & 1076.0 & (122) & 0.000032 & 0.000036 & 1075.6 & 0.4167 & -0.000004 & 0.174 & & & 971.7978 \\
\hline & & 80 & 0.2000 & 1191.9 & (122) & 0.000079 & 0.000080 & 1191.7 & 0.2270 & -0.000001 & 0.052 & & & 971.7978 \\
\hline & & 80 & 0.3000 & 1325.1 & (122) & 0.000114 & 0.000116 & 1324.4 & 0.6672 & -0.000001 & 0.445 & & & 971.7978 \\
\hline & & & & & & & & rage Res & -0.0087 & 0.000000 & 4.245 & Sum chi sqr & & \\
\hline & & & & & & & & dev Res & 0.6211 & 0.000004 & & & & \\
\hline & & & & & & & & $v g-4 s t d$ & -2.4932 & -0.000014 & & & & \\
\hline & & & & & & & & $\mathrm{vg}+4 s t d$ & 2.4757 & 0.000015 & & & & \\
\hline
\end{tabular}

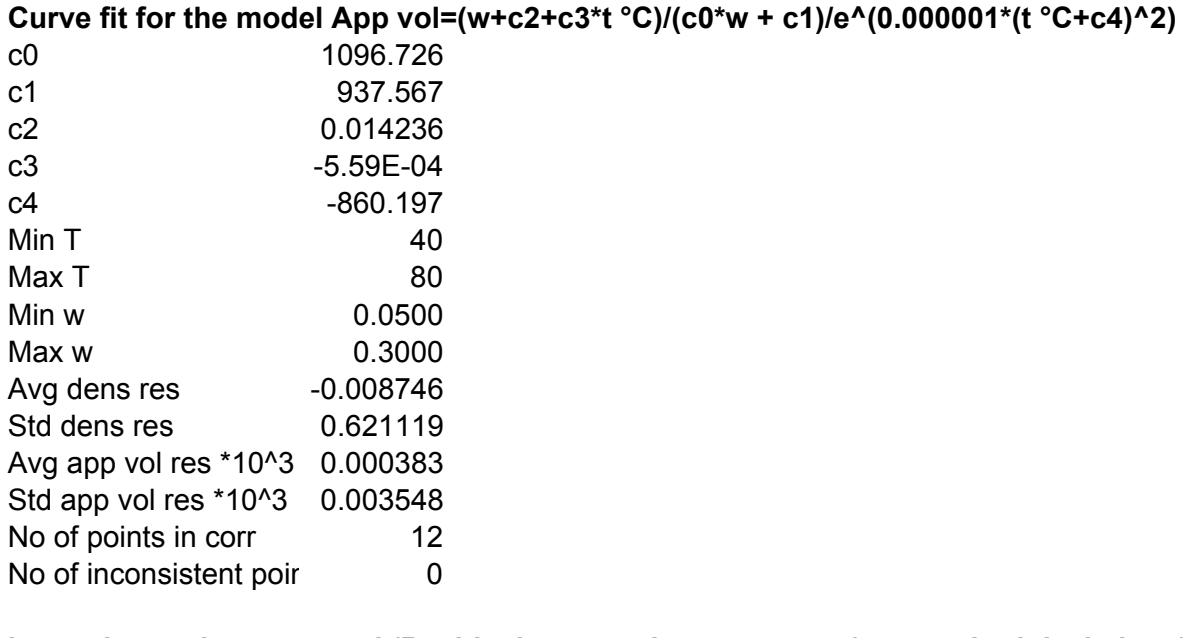

Inconsistent data not used (Residual greater than average $+/-4$ standard deviations) 
Density of aqueous solutions of NaHSO3

References (16) Choudary, N. V.; Jasra, R. V. Densities of Aqueous Solutions of Sodium Bisulfite and Sodium 2-Methalallyl Sulfate. J. Chem. Eng. Data 1994, 39, 181-183.

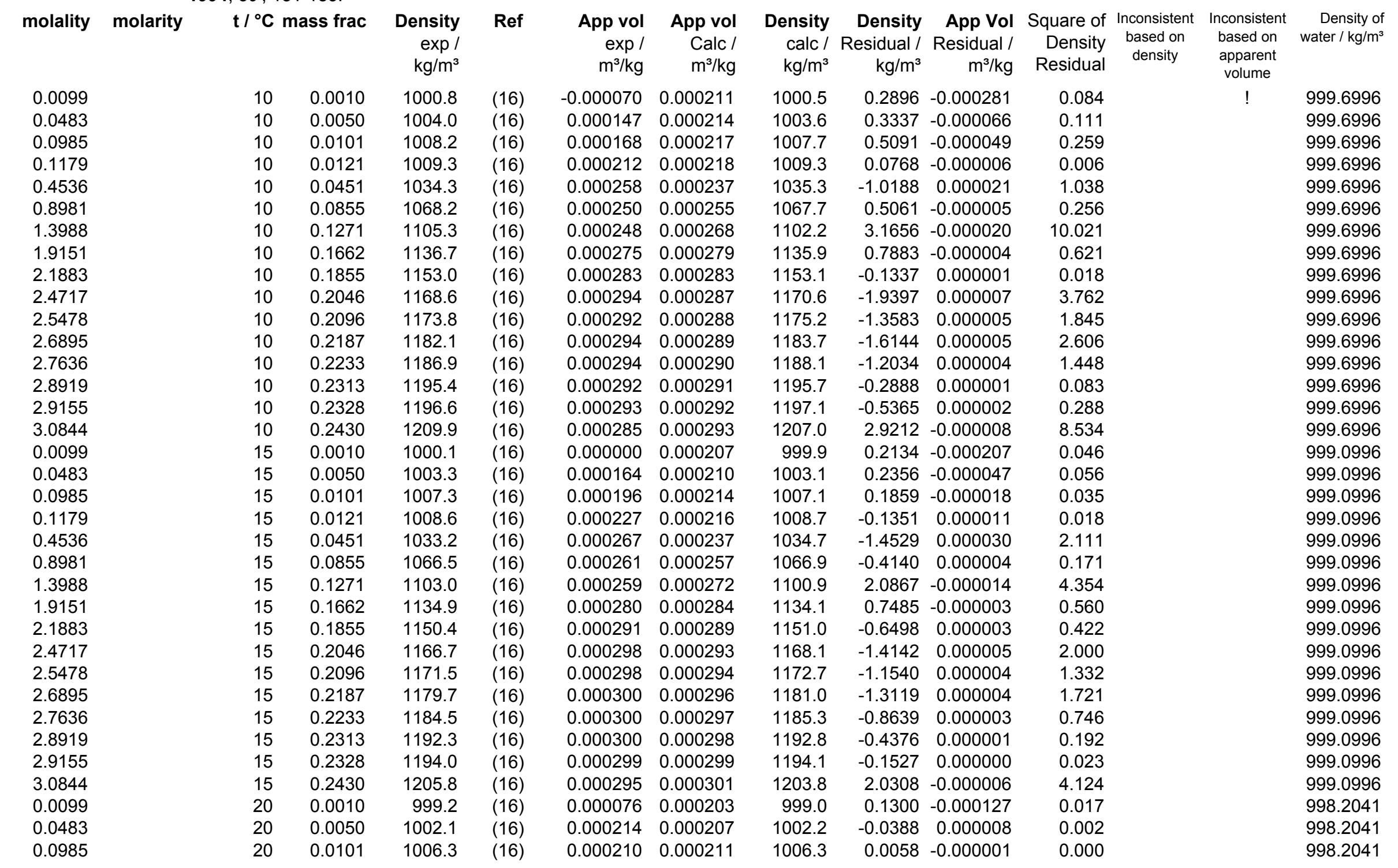


molality molarity

0.1179
0.4536
0.8981
1.3988
1.9151
2.1883
2.4717
2.5478
2.6895
2.7636
2.8919
2.9155
3.0844
0.0099
0.0483
0.0985
0.1179
0.4536
0.8981
1.3988
1.9151
2.1883
2.4717
2.5478
2.6895
2.7636
2.8919
2.9155
3.0844
0.0099
0.0985
0.1179
0.4536
0.8981
1.9151
2.1883
2.4717
2.5478
2.6895

\section{$\mathrm{t} /{ }^{\circ} \mathrm{C}$ mass frac}

$\exp /$

$\mathrm{kg} / \mathrm{m}^{3}$

1007.5

1032.2

1065.0

1099.9

1132.1

1147.7

1164.5

1168.7

1177.6

1182.1

1190.2

1191.8

1203.6

997.8

1000.9

1004.7

1006.1

1031.1

1062.6

1096.2

1129.8

1145.6

1163.1

1167.5

1175.8

1179.9

1187.1

1188.3

1197.8

996.8

1003.6

1004.9

1031.5

1063.7

1127.9

1143.6

1159.1

1163.1

1171.1
Ref

App vol App vol

exp / Calc /

Density

Density App Vol Square of Inconsistent Inconsistent

Density of $\begin{array}{rrrr}\mathrm{kg} / \mathrm{m}^{3} & \mathrm{~kg} / \mathrm{m}^{3} & \mathrm{~m}^{3} / \mathrm{kg} & \text { Residual density apparent }\end{array}$

(16) $\quad 0.000240 \quad 0.000213$

$\begin{array}{lll}\text { (16) } & 0.000271 & 0.000237\end{array}$

(16) $\quad 0.000267 \quad 0.000259$

$0.000273 \quad 0.000276$

$0.000289 \quad 0.000289$

$0.000298 \quad 0.000294$

$0.000303 \quad 0.000299$

$0.000304 \quad 0.000300$

$0.000304 \quad 0.000303$

$0.000304 \quad 0.000304$

$0.000303 \quad 0.000305$

$0.000303 \quad 0.000306$

$0.000298 \quad 0.000308$

$0.000248 \quad 0.000198$

$0.000237 \quad 0.000202$

$0.000254 \quad 0.000207$

$0.000259 \quad 0.000209$

$0.000268 \quad 0.000236$

0.0002790 .000260

$0.000289 \quad 0.000280$

$0.000294 \quad 0.000294$

0.0003020 .000300

$0.000303 \quad 0.000306$

$0.000304 \quad 0.000307$

$0.000306 \quad 0.000309$

$0.000307 \quad 0.000310$

$0.000309 \quad 0.000312$

0.0003090 .000313

0.0003110 .000315

$\begin{array}{ll}-0.000111 & 0.000192\end{array}$

$0.000218 \quad 0.000202$

$\begin{array}{lll}0.000241 & 0.000204\end{array}$

$0.000230 \quad 0.000234$

$0.000252 \quad 0.000261$

$0.000296 \quad 0.000299$

$0.000304 \quad 0.000306$

$\begin{array}{ll}0.000312 & 0.000312\end{array}$

$0.000314 \quad 0.000313$

$0.000316 \quad 0.000316$ $\begin{array}{ll}-0.3329 & 0.000027\end{array}$

$\begin{array}{ll}-1.6280 & 0.000034\end{array}$

$-0.77390 .000008$

$0.5125-0.000003$

$0.0494 \quad 0.000000$

$\begin{array}{ll}-0.9128 & 0.000004\end{array}$

$-0.9283 \quad 0.000003$

$\begin{array}{lll}-1.1434 & 0.000004\end{array}$

$\begin{array}{ll}-0.4142 & 0.000001\end{array}$

$-0.1542 \quad 0.000000$

$0.7422-0.000002$

$0.9453-0.000003$

$3.3233-0.000009$

$-0.05130 .000050$

$\begin{array}{ll}-0.1766 & 0.000035\end{array}$

$\begin{array}{ll}-0.4848 & 0.000047\end{array}$

$\begin{array}{ll}-0.6232 & 0.000051\end{array}$

$-1.57450 .000033$

$\begin{array}{ll}-1.7648 & 0.000018\end{array}$

$\begin{array}{ll}-1.3731 & 0.000009\end{array}$

0.09010 .000000

$\begin{array}{ll}-0.3562 & 0.000001\end{array}$

$0.7136-0.000003$

$0.7512-0.000003$

$1.0819-0.000004$

$1.0757-0.000003$

$1.1655-0.000004$

$1.1104-0.000003$

$1.3881-0.000004$

$0.3091-0.000302$

$-0.1677 \quad 0.000016$

$\begin{array}{ll}-0.4550 & 0.000037\end{array}$

$0.1735-0.000004$

$0.8982-0.000009$

$0.7309-0.000003$

$0.4635-0.000002$

$-0.0935 \quad 0.000000$

$\begin{array}{ll}-0.3026 & 0.000001\end{array}$

$\begin{array}{ll}-0.0962 & 0.000000\end{array}$
0.111

2.650

0.599

0.263

0.002

0.833

0.862

1.307

0.172

0.024

0.551

0.894

11.044

0.003

0.031

0.235

0.388

2.479

3.115

1.885

0.008

0.127

0.509

0.564

1.171

1.157

1.358

1.233

1.927

0.096

0.028

0.207

0.030

0.807

0.534

0.215

0.009

0.092

0.009
998.2041

998.2041

998.2041

998.2041

998.2041

998.2041

998.2041

998.2041

998.2041

998.2041

998.2041

998.2041

998.2041

997.0449

997.0449

997.0449

997.0449

997.0449

997.0449

997.0449

997.0449

997.0449

997.0449

997.0449

997.0449

997.0449

997.0449

997.0449

997.0449

995.6473

995.6473

995.6473

995.6473

995.6473

995.6473

995.6473

995.6473

995.6473

995.6473 


\begin{tabular}{|c|c|c|c|c|c|c|c|c|c|c|c|c|c|c|}
\hline molality & molarity & $\mathrm{t} /{ }^{\circ} \mathrm{C}$ & ass frac & $\begin{array}{r}\text { Density } \\
\exp / \\
\mathrm{kg} / \mathrm{m}^{3}\end{array}$ & Ref & $\begin{array}{r}\text { App vol } \\
\text { exp / } \\
\mathrm{m}^{3} / \mathrm{kg}\end{array}$ & $\begin{array}{r}\text { App vol } \\
\text { Calc / } \\
\mathrm{m}^{3} / \mathrm{kg}\end{array}$ & $\begin{array}{r}\text { Density } \\
\text { calc / } \\
\mathrm{kg} / \mathrm{m}^{3}\end{array}$ & $\begin{array}{r}\text { Density } \\
\text { Residual / } \\
\mathrm{kg} / \mathrm{m}^{3}\end{array}$ & $\begin{array}{r}\text { App Vol } \\
\text { Residual / } \\
\mathrm{m}^{3} / \mathrm{kg}\end{array}$ & $\begin{array}{r}\text { Square of } \\
\text { Density } \\
\text { Residual }\end{array}$ & $\begin{array}{l}\text { Inconsistent } \\
\text { based on } \\
\text { density }\end{array}$ & $\begin{array}{c}\text { Inconsistent } \\
\text { based on } \\
\text { apparent } \\
\text { volume }\end{array}$ & $\begin{array}{r}\text { Density of } \\
\text { water } / \mathrm{kg} / \mathrm{m}^{3}\end{array}$ \\
\hline 2.7636 & & 30 & 0.2233 & 1175.3 & (16) & 0.000317 & 0.000317 & 1175.2 & 0.0721 & 0.000000 & 0.005 & & & 995.6473 \\
\hline 2.8919 & & 30 & 0.2313 & 1181.5 & (16) & 0.000321 & 0.000319 & 1182.2 & -0.6867 & 0.000002 & 0.472 & & & 995.6473 \\
\hline 2.9155 & & 30 & 0.2328 & 1182.5 & (16) & 0.000322 & 0.000320 & 1183.4 & -0.8715 & 0.000003 & 0.760 & & & 995.6473 \\
\hline 3.0844 & & 30 & 0.2430 & 1191.2 & (16) & 0.000326 & 0.000322 & 1192.4 & -1.1312 & 0.000003 & 1.280 & & & 995.6473 \\
\hline 0.0099 & & 40 & 0.0010 & 993.3 & (16) & -0.000068 & 0.000177 & 993.1 & 0.2485 & -0.000245 & 0.062 & & & 992.2158 \\
\hline 0.0483 & & 40 & 0.0050 & 996.4 & (16) & 0.000161 & 0.000182 & 996.3 & 0.1051 & -0.000021 & 0.011 & & & 992.2158 \\
\hline 0.1179 & & 40 & 0.0121 & 1003.6 & (16) & 0.000068 & 0.000192 & 1002.1 & 1.5135 & -0.000124 & 2.291 & & & 992.2158 \\
\hline 0.4536 & & 40 & 0.0451 & 1028.1 & (16) & 0.000228 & 0.000228 & 1028.1 & 0.0220 & 0.000000 & 0.000 & & & 992.2158 \\
\hline 0.8981 & & 40 & 0.0855 & 1059.7 & (16) & 0.000256 & 0.000262 & 1059.2 & 0.5586 & -0.000006 & 0.312 & & & 992.2158 \\
\hline 1.9151 & & 40 & 0.1662 & 1123.9 & (16) & 0.000297 & 0.000308 & 1121.6 & 2.3548 & -0.000011 & 5.545 & & & 992.2158 \\
\hline 2.4717 & & 40 & 0.2046 & 1153.8 & (16) & 0.000318 & 0.000324 & 1152.2 & 1.6395 & -0.000006 & 2.688 & & & 992.2158 \\
\hline 2.5478 & & 40 & 0.2096 & 1157.4 & (16) & 0.000321 & 0.000326 & 1156.2 & 1.2284 & -0.000004 & 1.509 & & & 992.2158 \\
\hline 2.6895 & & 40 & 0.2187 & 1164.7 & (16) & 0.000325 & 0.000329 & 1163.6 & 1.0677 & -0.000004 & 1.140 & & & 992.2158 \\
\hline 2.7636 & & 40 & 0.2233 & 1167.3 & (16) & 0.000331 & 0.000331 & 1167.4 & -0.1594 & 0.000001 & 0.025 & & & 992.2158 \\
\hline 2.8919 & & 40 & 0.2313 & 1172.3 & (16) & 0.000339 & 0.000333 & 1174.0 & -1.7136 & 0.000005 & 2.936 & & & 992.2158 \\
\hline 2.9155 & & 40 & 0.2328 & 1173.6 & (16) & 0.000339 & 0.000334 & 1175.2 & -1.5913 & 0.000005 & 2.532 & & & 992.2158 \\
\hline 3.0844 & & 40 & 0.2430 & 1180.4 & (16) & 0.000347 & 0.000337 & 1183.7 & -3.2559 & 0.000010 & 10.601 & & & 992.2158 \\
\hline \multicolumn{9}{|c|}{ Average Res } & -0.0085 & -0.000012 & 118.524 & Sum chi sqr & & \\
\hline \multicolumn{9}{|c|}{ Std dev Res } & 1.1480 & 0.000059 & & & & \\
\hline \multicolumn{9}{|c|}{ Avg - 4std } & -4.6003 & -0.000250 & & & & \\
\hline \multicolumn{9}{|c|}{ Avg + 4std } & 4.5833 & 0.000225 & & & & \\
\hline
\end{tabular}

\author{
Curve fit for the model App vol $=\left(w+c 2+c 3^{*} t{ }^{\circ} \mathrm{C}\right) /\left(\mathrm{c} 00^{*} w+c 1\right) / \mathrm{e}^{\wedge}\left(0.000001^{*}\left(\mathrm{t}^{\circ} \mathrm{C}+\mathrm{c} 4\right)^{\wedge} 2\right)$ \\ 6.14E-06 \\ $\begin{array}{rr}\mathrm{c} 1 & 1.3 \mathrm{E}-06 \\ \mathrm{c} 2 & 0.136346\end{array}$ \\ c3 -0.001462 \\ c4 -4472.457 \\ Min T \\ $\operatorname{Max} T$ \\ 10
40 \\ Min w $\quad 0.0010$ \\ Max w $\quad 0.2430$ \\ Avg dens res $\quad-0.0085$ \\ Std dens res $\quad 1.1480$ \\ Avg app vol res *10^3 $\quad-0.012283$ \\ Std app vol res *10^3 0.059418 \\ No of points in corr \\ No of inconsistent poin \\ 91
1
}




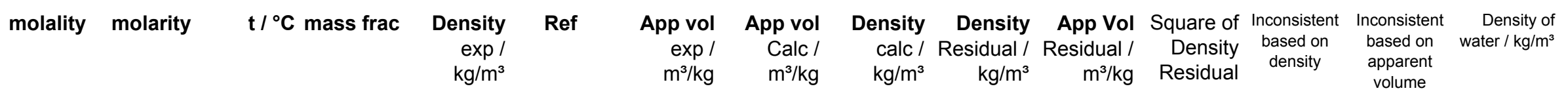

Inconsistent data not used (Residual greater than average +/- 4 standard deviations)
0.0483
$30 \quad 0.0050$
998.1
(16)
\#DIV/0! 0.000196 \#DIV/0!
\#DIV/0!
\#DIV/O! \#DIV/O!
\#DIV/0! \#DIV/0!

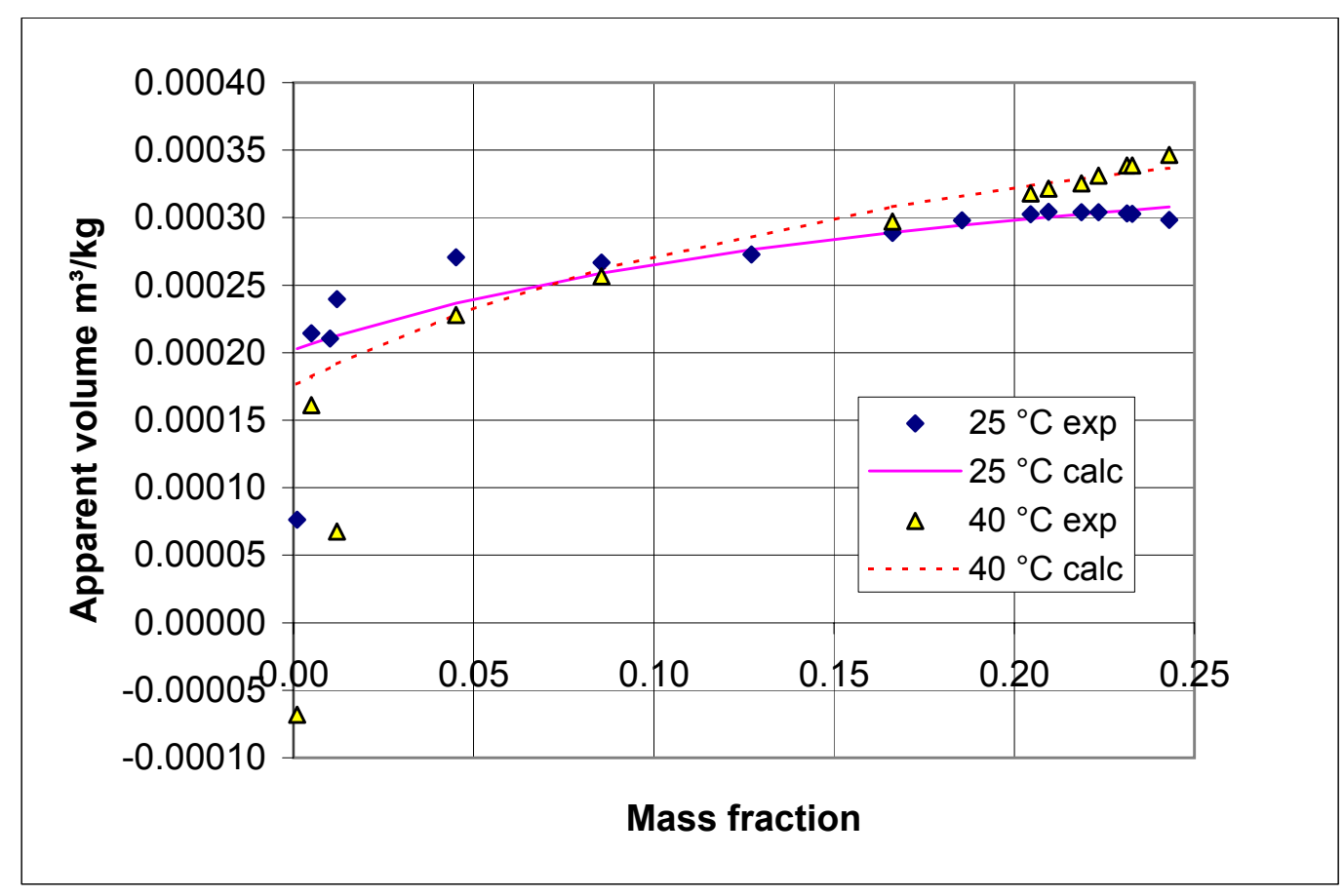


Density of aqueous solutions of $\mathrm{Nal}$

References (62) Lengyel, S.; Tamás, J; Giber, J.; Holderith, J. Study of Viscosity of Aqueous Alkali Halide Solutions. Acta Chim. Acad. Sci. Hung. 1964, 40, 125-143.

(65) Maclnnes, D. A.; Dayhoff, M. O. The Partial Molal Volumes of Potassium Chloride, Potassium and Sodium lodides and lodine in Aqueous Solution at $25^{\circ} \mathrm{C}$. J. Am. Chem. Soc. 1952, 74, 1017-1020.

(73) Millero, F. J.; Ward, G. K.; Chetirkin, P. V. Relative Sound Velocities of Sea Salts at $25^{\circ}$ C. J. Acoust. Soc. Am. 1977, 61, $1492-1498$.

(112) Saluja, P. P. S., LeBlanc, J. C., Hume, H. B., Apparent molar heat capacities and volumes of aqueous solutions of several 1:1 electrolytes at elevated temperatures, Can. J. Chem., 1986, 64, 926-931

(118) Scott, A. F.; Durham, E. J.; Studies in the solubilities of the soluble electrolytes, J. Phys. Chem., 1930, 34, 1424-1438

(119) Scott, A. F.; Obenhaus, V. M.; Wilson, R. W. The Compressibility Coefficients of Solutions of Eight Alkali Halides. J. Phys. Chem. 1934, $38,931-940$.

(130) Vaslow, F. The Apparent Molal Volumes of the Lithium and Sodium Halides. Critical Type Transitions in Aqueous Solution. J. Phys. Chem. 1969, 73, 3745-3750.

\begin{tabular}{|c|c|c|c|c|c|c|c|c|c|c|c|c|c|}
\hline molality & molarity & \multicolumn{2}{|c|}{$\mathrm{t} /{ }^{\circ} \mathrm{C}$ mass frac } & $\begin{array}{r}\text { Density } \\
\exp / \\
\mathrm{kg} / \mathrm{m}^{3}\end{array}$ & Ref & $\begin{array}{r}\text { App vol } \\
\exp / \\
\mathrm{m}^{3} / \mathrm{kg}\end{array}$ & $\begin{array}{r}\text { App vol } \\
\text { Calc / } \\
\mathrm{m}^{3} / \mathrm{kg}\end{array}$ & $\begin{array}{r}\text { Density } \\
\text { calc / } \\
\mathrm{kg} / \mathrm{m}^{3}\end{array}$ & $\begin{array}{r}\text { Density } \\
\text { Residual / } \\
\mathrm{kg} / \mathrm{m}^{3}\end{array}$ & $\begin{array}{r}\text { App Vol } \\
\text { Residual / } \\
\mathrm{m}^{3} / \mathrm{kg}\end{array}$ & $\begin{array}{r}\text { Square of } \\
\text { Density } \\
\text { Residual }\end{array}$ & $\begin{array}{c}\text { Inconsistent } \\
\text { based on } \\
\text { density }\end{array}$ & $\begin{array}{c}\text { Inconsistent } \\
\text { based on } \\
\text { apparent } \\
\text { volume }\end{array}$ \\
\hline 2.507 & & 10 & 0.2732 & 1263.80 & (62) & 0.000235 & 0.000239 & 1261.9 & 1.8597 & -0.000004 & 3.459 & & \\
\hline 2.968 & & 10 & 0.3079 & 1306.70 & (62) & 0.000237 & 0.000241 & 1304.8 & 1.8855 & -0.000004 & 3.555 & & \\
\hline 5.107 & & 10 & 0.4336 & 1487.20 & (62) & 0.000244 & 0.000245 & 1486.0 & 1.1671 & -0.000001 & 1.362 & & \\
\hline 8.095 & & 10 & 0.5482 & 1698.60 & (62) & 0.000250 & 0.000249 & 1699.0 & -0.4331 & 0.000000 & 0.188 & & \\
\hline 8.473 & & 10 & 0.5595 & 1722.00 & (62) & 0.000250 & 0.000250 & 1723.2 & -1.2138 & 0.000001 & 1.473 & & \\
\hline 11.286 & & 10 & 0.6285 & 1889.90 & (62) & 0.000251 & 0.000252 & 1887.1 & 2.8350 & -0.000001 & 8.037 & & \\
\hline 2.507 & & 20 & 0.2732 & 1258.20 & (62) & 0.000244 & 0.000245 & 1257.7 & 0.5141 & -0.000001 & 0.264 & & \\
\hline 2.968 & & 20 & 0.3079 & 1300.50 & (62) & 0.000246 & 0.000246 & 1300.1 & 0.4067 & -0.000001 & 0.165 & & \\
\hline 5.107 & & 20 & 0.4336 & 1479.50 & (62) & 0.000250 & 0.000250 & 1479.3 & 0.2270 & 0.000000 & 0.052 & & \\
\hline 8.095 & & 20 & 0.5482 & 1688.80 & (62) & 0.000255 & 0.000254 & 1689.7 & -0.9244 & 0.000001 & 0.855 & & \\
\hline 8.473 & & 20 & 0.5595 & 1712.20 & (62) & 0.000255 & 0.000254 & 1713.6 & -1.4046 & 0.000001 & 1.973 & & \\
\hline 11.286 & & 20 & 0.6285 & 1875.80 & (62) & 0.000256 & 0.000256 & 1875.4 & 0.4429 & 0.000000 & 0.196 & & \\
\hline 2.507 & & 25 & 0.2732 & 1255.10 & (62) & 0.000248 & 0.000248 & 1255.2 & -0.0698 & 0.000000 & 0.005 & & \\
\hline 2.968 & & 25 & 0.3079 & 1297.30 & (62) & 0.000249 & 0.000249 & 1297.4 & -0.0519 & 0.000000 & 0.003 & & \\
\hline 5.107 & & 25 & 0.4336 & 1474.90 & (62) & 0.000254 & 0.000253 & 1475.6 & -0.6603 & 0.000001 & 0.436 & & \\
\hline 8.095 & & 25 & 0.5482 & 1684.20 & (62) & 0.000257 & 0.000256 & 1684.8 & -0.6226 & 0.000000 & 0.388 & & \\
\hline 8.473 & & 25 & 0.5595 & 1707.50 & (62) & 0.000257 & 0.000256 & 1708.6 & -1.0642 & 0.000001 & 1.132 & & \\
\hline 11.286 & & 25 & 0.6285 & 1871.00 & (62) & 0.000258 & 0.000258 & 1869.4 & 1.6434 & -0.000001 & 2.701 & & \\
\hline 1.1472 & & 30 & 0.1467 & 1119.50 & (62) & 0.000247 & 0.000247 & 1119.6 & -0.1177 & 0.000001 & 0.014 & & \\
\hline 1.1472 & & 30 & 0.1467 & 1119.50 & (62) & 0.000247 & 0.000247 & 1119.6 & -0.1177 & 0.000001 & 0.014 & & \\
\hline 2.507 & & 30 & 0.2732 & 1252.30 & (62) & 0.000251 & 0.000251 & 1252.4 & -0.1359 & 0.000000 & 0.018 & & \\
\hline 2.968 & & 30 & 0.3079 & 1294.20 & (62) & 0.000252 & 0.000252 & 1294.4 & -0.1988 & 0.000000 & 0.040 & & \\
\hline 5.107 & & 30 & 0.4336 & 1470.90 & (62) & 0.000256 & 0.000255 & 1471.7 & -0.7698 & 0.000001 & 0.593 & & \\
\hline 8.095 & & 30 & 0.5482 & 1679.70 & (62) & 0.000258 & 0.000258 & 1679.8 & -0.0997 & 0.000000 & 0.010 & & \\
\hline 8.473 & & 30 & 0.5595 & 1702.70 & (62) & 0.000259 & 0.000259 & 1703.4 & -0.7102 & 0.000000 & 0.504 & & \\
\hline
\end{tabular}

$\begin{gathered}\text { Apparent } \\ \text { molal } \\ \text { volume } / \\ \text { water } / \mathrm{kg} / \mathrm{m}^{3}\end{gathered}$
$\mathrm{~cm}^{3} / \mathrm{mol}$

999.6996
999.6996
999.6996
999.6996
999.6996
999.6996
998.2041
998.2041
998.2041
998.2041
998.2041
998.2041
997.0449
997.0449
997.0449
997.0449
997.0449
997.0449
995.6473
995.6473
995.6473
995.6473
995.6473
995.6473
995.6473


molality molarity

$\mathrm{t} /{ }^{\circ} \mathrm{C}$ mass frac Density

11.286

1.1472

2.507

2.968

5.107

8.095

8.473

0.100677

0.200029

0.300304

0.399862

0.499648

0.599737

0.698274

0.799093

0.899269

0.99457

0.04911

0.09791

0.20015

0.49648

0.99059

2.01

0.09847

0.20365

0.51703

0.05266

0.09723

0.20453

1.02118

0.0485

0.1051

0.2136 $\exp /$

$\exp /$
$\mathrm{kg} / \mathrm{m}^{3}$

Ref

exp / Calc /

$\mathrm{m}^{3} / \mathrm{kg} \quad \mathrm{m}^{3} / \mathrm{kg}$

0.0002590 .000260

$0.000253 \quad 0.000252$

$0.000256 \quad 0.000255$

$0.000257 \quad 0.000256$

$0.000260 \quad 0.000259$

$\begin{array}{ll}0.000262 & 0.000262\end{array}$

$\begin{array}{ll}0.4336 & 1463.00 \\ 0.5482 & 1669.80\end{array}$

$0.5595 \quad 1692.70$

$0.0066 \quad 1002.09$

$0.0103 \quad 1004.97$

$0.0131 \quad 1007.13$

$0.0183 \quad 1011.21$

$0.0221 \quad 1014.20$

$0.0288 \quad 1019.44$

$0.0295 \quad 1020.06$

$0.0149 \quad 1008.52$

$0.0291 \quad 1019.70$

$0.0431 \quad 1030.88$

$0.0566 \quad 1041.89$

$0.0697 \quad 1052.82$

$0.0825 \quad 1063.70$

$0.0948 \quad 1074.31$

$0.1070 \quad 1085.08$

$0.1188 \quad 1095.69$

$0.1298 \quad 1105.70$

$0.0073 \quad 993.97$

$0.0145 \quad 999.39$

$0.0291 \quad 1010.64$

$0.0693 \quad 1042.59$

$0.1293 \quad 1094.13$

$0.2316 \quad 1194.34$

$0.0145 \quad 986.11$

$0.0296 \quad 997.42 \quad(112)$

$0.0719 \quad 1030.61$

$0.0078 \quad 980.61 \quad(112)$

$0.0144 \quad 985.45 \quad(112)$

$0.0298 \quad 997.02 \quad(112)$

$0.1328 \quad 1081.35 \quad(112)$

$0.0072 \quad 963.67 \quad(112)$

$0.0155 \quad 969.76 \quad(112)$

$0.0310 \quad 981.26 \quad(112)$
0.0002620 .000262

$0.000233 \quad 0.000238$

$0.000234 \quad 0.000239$

$0.000235 \quad 0.000239$

$0.000236 \quad 0.000239$

$0.000237 \quad 0.000239$

$0.000237 \quad 0.000239$

$0.000237 \quad 0.000239$

$0.000235 \quad 0.000239$

$0.000238 \quad 0.000239$

$0.000239 \quad 0.000240$

$0.000240 \quad 0.000240$

$0.000241 \quad 0.000241$

$0.000241 \quad 0.000241$

$0.000242 \quad 0.000242$

$\begin{array}{ll}0.000242 & 0.000242\end{array}$

$0.000243 \quad 0.000243$

$0.000243 \quad 0.000243$

0.0002520 .000253

$0.000251 \quad 0.000253$

$0.000251 \quad 0.000253$

$0.000254 \quad 0.000255$

$0.000256 \quad 0.000256$

$0.000259 \quad 0.000258$

$0.000259 \quad 0.000265$

$0.000261 \quad 0.000265$

0.0002620 .000266

$0.000257 \quad 0.000265$

$0.000259 \quad 0.000266$

$0.000260 \quad 0.000266$

$0.000265 \quad 0.000267$

$0.000251 \quad 0.000275$

$0.000255 \quad 0.000275$

$0.000260 \quad 0.000275$
Density Density App Vol Square of Inconsistent Inconsistent calc / Residual / Residual / Density based on based on $\mathrm{kg} / \mathrm{m}^{3} \quad \mathrm{~kg} / \mathrm{m}^{3} \quad \mathrm{~m}^{3} / \mathrm{kg}$ Residual density apparent

$\begin{array}{llll}1863.3 & 2.7997 & -0.000001 & 7.838\end{array}$

$\begin{array}{llll}1114.9 & -0.1204 & 0.000001 & 0.015\end{array}$

$1246.4 \quad-0.3051 \quad 0.000001 \quad 0.093$

$\begin{array}{llll}1287.9 & -0.5486 & 0.000001 & 0.301\end{array}$

$\begin{array}{llll}1463.4 & -0.4482 & 0.000000 & 0.201\end{array}$

$\begin{array}{llll}1669.5 & 0.3191 & 0.000000 & 0.102\end{array}$

$\begin{array}{llll}1692.9 & -0.1516 & 0.000000 & 0.023\end{array}$

$1002.1 \quad 0.0376-0.000006 \quad 0.001$

$1004.9 \quad 0.0443-0.000004 \quad 0.002$

$1007.1 \quad 0.0437-0.000003 \quad 0.002$

$\begin{array}{llll}1011.2 & 0.0558 & -0.000003 & 0.003\end{array}$

$\begin{array}{llll}1014.1 & 0.0480 & -0.000002 & 0.002\end{array}$

$\begin{array}{llll}1019.4 & 0.0568 & -0.000002 & 0.003\end{array}$

$\begin{array}{llll}1020.0 & 0.0651 & -0.000002 & 0.004\end{array}$

$\begin{array}{llll}1008.5 & 0.0503 & -0.000003 & 0.003\end{array}$

$\begin{array}{llll}1019.6 & 0.0523 & -0.000002 & 0.003\end{array}$

$\begin{array}{llll}1030.8 & 0.0461 & -0.000001 & 0.002\end{array}$

$\begin{array}{llll}1041.8 & 0.0436 & -0.000001 & 0.002\end{array}$

$\begin{array}{llll}1052.8 & 0.0282 & 0.000000 & 0.001\end{array}$

$\begin{array}{llll}1063.7 & 0.0211 & 0.000000 & 0.000\end{array}$

$\begin{array}{llll}1074.3 & 0.0012 & 0.000000 & 0.000\end{array}$

$\begin{array}{llll}1085.1 & -0.0155 & 0.000000 & 0.000\end{array}$

$\begin{array}{llll}1095.7 & -0.0349 & 0.000000 & 0.001\end{array}$

$\begin{array}{llll}1105.8 & -0.0564 & 0.000000 & 0.003\end{array}$

$\begin{array}{llll}994.0 & 0.0078 & -0.000001 & 0.000\end{array}$

$\begin{array}{llll}999.4 & 0.0309 & -0.000002 & 0.001\end{array}$

$1010.6-0.0594-0.000002$

$\begin{array}{llll}1042.6 & 0.0185 & 0.000000 & 0.000\end{array}$

$\begin{array}{llll}1094.2 & -0.0675 & 0.000000 & 0.005\end{array}$

$1194.4 \quad-0.0814 \quad 0.000000$

$\begin{array}{llll}986.0 & 0.0821-0.000006 & 0.007\end{array}$

$\begin{array}{llll}997.3 & 0.1288 & -0.000004 & 0.017\end{array}$

$\begin{array}{llll}1030.3 & 0.3278-0.000004 & 0.107\end{array}$

$\begin{array}{llll}980.6 & 0.0603 & -0.000008 & 0.004\end{array}$

$\begin{array}{lllll}985.4 & 0.0964 & -0.000007 & 0.009\end{array}$

$\begin{array}{llll}996.8 & 0.1817 & -0.000006 & 0.033\end{array}$

$1081.0 \quad 0.3627-0.000002 \quad 0.132$

$\begin{array}{llll}963.5 & 0.1555 & -0.000023 & 0.024\end{array}$

$\begin{array}{llll}969.5 & 0.2825 & -0.000019 & 0.080\end{array}$

$\begin{array}{llll}980.8 & 0.4498 & -0.000015 & 0.202\end{array}$
Apparent Density of

molal water $/ \mathrm{kg} / \mathrm{m}^{3}$ volume /

$\mathrm{cm}^{3} / \mathrm{mo}$

995.6473

992.2158

992.2158

992.2158

992.2158

992.2158

992.2158

997.0449

997.0449

997.0449

997.0449

997.0449

997.0449

997.0449

997.0449

997.0449

997.0449

997.0449

997.0449

997.0449

997.0449

997.0449

997.0449

997.0449

$37.75 \quad 988.5163$

37.62988 .5163

$37.7 \quad 988.5163$

38.12988 .5163

38.45988 .5163

$38.79 \quad 988.5163$

$38.88 \quad 975.3963$

$39.13 \quad 975.3963$

$39.24 \quad 975.3963$

$38.59 \quad 974.8609$

38.77974 .8609

38.92974 .8609

$39.72 \quad 974.8609$

37.69958 .3924

$38.27 \quad 958.3924$

$38.93 \quad 958.3924$ 


molality

0.5267
1.003
10.67
13.15
15.12
17.98
19.81
20.05
1.077
1.96
4.525
10.348
0.05
0.1
0.2
0.3
0.4
0.5
0.6
0.7
0.8
0.9
1
1.2
1.4
1.6
1.8

$\begin{array}{rrrr}\mathbf{t} /{ }^{\circ} \mathrm{C} \text { mass frac } & \begin{array}{r}\text { Density } \\ \text { exp / } \\ \mathrm{kg} / \mathrm{m}^{3}\end{array} \\ & & \text { Ref } \\ 99.96 & 0.0732 & 1013.86 & (112) \\ 99.96 & 0.1307 & 1061.48 & (112) \\ 0 & 0.6153 & 1861.20 & (118) \\ 35 & 0.6635 & 1951.20 & (118) \\ 50.02 & 0.6939 & 2016.90 & (118) \\ 63.13 & 0.7294 & 2106.80 & (118) \\ 75.4 & 0.7481 & 2154.40 & (118) \\ 92.23 & 0.7504 & 2142.50 & (118) \\ 35 & 0.1390 & 1109.98 & (119) \\ 35 & 0.2271 & 1197.58 & (119) \\ 35 & 0.4042 & 1421.73 & (119) \\ 35 & 0.6081 & 1806.44 & (119) \\ 25 & 0.0074 & 1002.75 & (130) \\ 25 & 0.0148 & 1008.42 & (130) \\ 25 & 0.0291 & 1019.69 & (130) \\ 25 & 0.0430 & 1030.84 & (130) \\ 25 & 0.0566 & 1041.91 & (130) \\ 25 & 0.0697 & 1052.87 & (130) \\ 25 & 0.0825 & 1063.75 & (130) \\ 25 & 0.0950 & 1074.53 & (130) \\ 25 & 0.1071 & 1085.22 & (130) \\ 25 & 0.1189 & 1095.83 & (130) \\ 25 & 0.1304 & 1106.35 & (130) \\ 25 & 0.1525 & 1127.14 & (130) \\ 25 & 0.1735 & 1147.60 & (130) \\ 25 & 0.1935 & 1167.75 & (130) \\ 25 & 0.2125 & 1187.57 & (130)\end{array}$

\section{App vol App vol D}

$\begin{array}{cc}\exp / & \text { Calc / } \\ \mathrm{m}^{3} / \mathrm{kg} & \mathrm{m}^{3} / \mathrm{kg}\end{array}$

$0.000263 \quad 0.000275$

$0.000268 \quad 0.000275$

$0.000248 \quad 0.000247$

$0.000262 \quad 0.000263$

$0.000268 \quad 0.000268$

$0.000273 \quad 0.000272$

$0.000275 \quad 0.000275$

$0.000277 \quad 0.000277$

$\begin{array}{ll}0.000250 & 0.000249\end{array}$

$0.000253 \quad 0.000252$

$\begin{array}{ll}0.000257 & 0.000257\end{array}$

$0.000262 \quad 0.000262$

$0.000236 \quad 0.000239$

$0.000237 \quad 0.000239$

$0.000238 \quad 0.000239$

$0.000239 \quad 0.000240$

$0.000240 \quad 0.000240$

$0.000240 \quad 0.000241$

$0.000241 \quad 0.000241$

$0.000241 \quad 0.000242$

$0.000242 \quad 0.000242$

$0.000242 \quad 0.000243$

$0.000243 \quad 0.000243$

$0.000244 \quad 0.000244$

$0.000244 \quad 0.000245$

$0.000245 \quad 0.000245$

$0.000246 \quad 0.000246$
Density Density App Vol Square of Inconsistent Inconsistent calc / Residual/ Residual/ Density based on based on $\mathrm{kg} / \mathrm{m}^{3}$

1013.0

1060.4

1863.9

1949.4

2016.5

2109.2

2155.3

2141.

1110.1

1198.0

1422.3

1807.1

1002.7

1008.4

1019.6

1030.8

1041.9

1052.8

1063.7

1074.5

1085.2

1095.8

1106.3

1127.1

1147.6

1167.7

1187.5

Average Res

Std dev Res

Avg - 4std

Avg + 4std

$$
\mathrm{kg} / \mathrm{m}^{3}
$$$$
\mathrm{m}^{3} / \mathrm{kg}
$$

$0.8695-0.000012$

$1.0426-0.000007$

$-2.72750 .000001$

$1.8090-0.000001$

$0.4422 \quad 0.000000$

$\begin{array}{ll}-2.4498 & 0.000001\end{array}$

$-0.92920 .000000$

$\begin{array}{ll}1.3828 & 0.000000\end{array}$

$\begin{array}{ll}-0.1687 & 0.000001\end{array}$

$\begin{array}{ll}-0.4529 & 0.000001\end{array}$

$\begin{array}{ll}-0.5294 & 0.000001\end{array}$

$0.6189 \quad 0.000000$

$0.0204-0.000003$

$0.0251-0.000002$

$0.0409-0.000001$

$0.0431-0.000001$

$0.0441-0.000001$

$0.0417-0.000001$

$0.0383 \quad 0.000000$

0.03640 .000000

$0.0300 \quad 0.000000$

0.02790 .000000

0.02210 .000000

$0.0230 \quad 0.000000$

$0.0266 \quad 0.000000$

$0.0361 \quad 0.000000$

$0.0350 \quad 0.000000$

$0.0508-0.000002$

$0.8044 \quad 0.000004$

$-3.1666-0.000018$

$3.2682 \quad 0.000014$ volume

0.756

087

7.439

3.272
0.196

0.863

1.912

0.028

0.205

0.280

0.383

0.000

0.001

0.002

0.002

0.002

0.002

0.001

0.001

0.001

0.000

0.001

0.001

59.114 Sum chi sqr
Apparent Density of molal water $/ \mathrm{kg} / \mathrm{m}^{3}$ volume /

39.49958 .3924

40.21958 .3924

999.8395

994.0319

988.0272

981.5606

974.6096

963.8096

994.0319

994.0319

994.0319

994.0319

35.35997 .0449

$35.55 \quad 997.0449$

$35.68 \quad 997.0449$

35.82997 .0449

$35.93 \quad 997.0449$

$36.03 \quad 997.0449$

36.12997 .0449

36.2997 .0449

$36.28 \quad 997.0449$

$36.35 \quad 997.0449$

36.42997 .0449

$36.54 \quad 997.0449$

36.65997 .0449

36.75997 .0449

$36.85 \quad 997.0449$

$\begin{array}{lr}\text { Curve fit for the model App vol } \\ \text { c0 } \\ \text { c1 } \\ \text { C2 } \\ \text { C3 } \\ \text { c4 } & 1858.1478 \\ \text { Min T } & 1.738657 \\ \text { Max T } & 0.0105 \\ & 1203.275 \\ & 0 \\ & 99.96\end{array}$


molality molarity

$t /{ }^{\circ} \mathrm{C}$ mass frac

Density

$\exp /$

$\mathrm{kg} / \mathrm{m}^{3}$

Ref

App vol
$\exp /$
$\mathrm{m}^{3} / \mathrm{kg}$

App vo
Calc
$\mathrm{m}^{3} / \mathrm{kg}$

D

D
Density App Vol Square of Inconsistent Inconsistent
$\mathrm{m}^{3} / \mathrm{kg}$
Residual
based on
based on
apparen

$\mathrm{kg} / \mathrm{m}^{3}$

$\mathrm{kg} / \mathrm{m}^{3}$

Apparent Density of

molal water $/ \mathrm{kg} / \mathrm{m}^{3}$

volume /

$\begin{array}{lr}\text { Min w } & 0.0066 \\ \text { Max w } & 0.7504 \\ \text { Avg dens res } & 0.0508 \\ \text { Std dens res } & 0.8044 \\ \text { Avg app vol res *10^3 } & -0.001712 \\ \text { Std app vol res *10^3 } & 0.004 \\ \text { No of points in corr } & 92 \\ \text { No of inconsistent poir } & 3\end{array}$

Inconsistent data not used (Residual greater than average +/ $\mathbf{4}$ standard deviations)

$\begin{array}{rrrrcrr}11.286 & 40 & 0.6285 & 1856.50 & (62) & 0.000261 & 0.000264 \\ 19 & 67.93 & 0.7402 & 2155.30 & (118) & 0.000268 & 0.000273\end{array}$

67.93

70.87

$0.7402 \quad 2155.30$

(118)

$0.000268 \quad 0.000273$

$1851.1 \quad 5.3956-0.000002$

2138.4

$\begin{array}{rrrr}2168.7 & -11.0345 & 0.000003 & 121.760\end{array}$ 
Density of aqueous solutions of $\mathrm{Na2MoO4}$

References (78) Olofsson, I. V.; Spitzer, J. J.; Hepler, L. G.; Apparent molar heat capacities and volumes of aqueous electrolytes at $25^{\circ} \mathrm{C}$ : $\mathrm{Na}_{2} \mathrm{SO}_{4}$, $\mathrm{K}_{2} \mathrm{SO}_{4}, \mathrm{Na}_{2} \mathrm{~S}_{2} \mathrm{O}_{3}, \mathrm{Na}_{2} \mathrm{~S}_{2} \mathrm{O}_{8}, \mathrm{~K}_{2} \mathrm{~S}_{2} \mathrm{O}_{8}, \mathrm{~K}_{2} \mathrm{CrO}_{4}, \mathrm{Na}_{2} \mathrm{MoO}_{4}$, and $\mathrm{Na}_{2} \mathrm{WO}_{4}$, Can. J. Chem. , 1978, 56, 1871-1873

(122) Sohnel, P.; Novotny, P; Solc, Z.; Densities of aqueous solutions of 18 inorganic substances, J Chem Eng Data, 1984, 29, 379-382

molality molarity

\section{$t /{ }^{\circ} \mathrm{C}$ mass frac Density

$$
\exp / \mathrm{kg} / \mathrm{m}^{3}
$$

0.02986
0.04337
0.04812
0.06478
0.07729
0.09278
0.11
0.12477
0.14357
0.16223

$\begin{array}{llll}25 & 0.0061 & 1002.3 & (78) \\ 25 & 0.0089 & 1004.7 & (78) \\ 25 & 0.0098 & 1005.5 & (78) \\ 25 & 0.0132 & 1008.4 & (78) \\ 25 & 0.0157 & 1010.5 & (78) \\ 25 & 0.0187 & 1013.2 & (78) \\ 25 & 0.0221 & 1016.2 & (78) \\ 25 & 0.0250 & 1018.7 & (78) \\ 25 & 0.0287 & 1021.9 & (78) \\ 25 & 0.0323 & 1025.1 & (78) \\ 25 & 0.0500 & 1040.6 & (122) \\ 25 & 0.1000 & 1087.1 & (122) \\ 25 & 0.2000 & 1191.3 & (122) \\ 25 & 0.3000 & 1311.5 & (122) \\ 25 & 0.3500 & 1379.3 & (122) \\ 40 & 0.0500 & 1035.4 & (122) \\ 40 & 0.1000 & 1080.9 & (122) \\ 40 & 0.2000 & 1184.6 & (122) \\ 40 & 0.3000 & 1304.7 & (122) \\ 40 & 0.3500 & 1372.0 & (122) \\ 60 & 0.0500 & 1026.3 & (122) \\ 60 & 0.1000 & 1071.9 & (122) \\ 60 & 0.2000 & 1174.4 & (122) \\ 60 & 0.3000 & 1292.9 & (122) \\ 60 & 0.3500 & 1360.6 & (122) \\ 80 & 0.0500 & 1014.3 & (122) \\ 80 & 0.1000 & 1060.5 & (122) \\ 80 & 0.2000 & 1162.3 & (122) \\ 80 & 0.3000 & 1281.0 & (122) \\ 80 & 0.3500 & 1347.2 & (122)\end{array}$

\section{App vol}

App vol

$$
\mathrm{m}^{3} / \mathrm{kg} \quad \mathrm{m}^{3} / \mathrm{kg}
$$

0.0001450 .000163

$0.000145 \quad 0.000164$

$0.000145 \quad 0.000164$

$0.000148 \quad 0.000164$

$0.000149 \quad 0.000164$

$0.000151 \quad 0.000165$

$0.000151 \quad 0.000165$

$0.000152 \quad 0.000165$

$0.000153 \quad 0.000166$

0.0001550 .000166

$0.000163 \quad 0.000168$

$0.000172 \quad 0.000174$

0.0001850 .000187

0.0002010 .000201

$0.000209 \quad 0.000209$

$\begin{array}{lll}0.000167 & 0.000169\end{array}$

$\begin{array}{ll}0.000181 & 0.000175\end{array}$

$0.000189 \quad 0.000189$

$0.000203 \quad 0.000203$

$0.000211 \quad 0.000211$

$0.000163 \quad 0.000168$

$0.000175 \quad 0.000175$

$0.000189 \quad 0.000189$

$0.000205 \quad 0.000204$

$\begin{array}{lll}0.000211 & 0.000212\end{array}$

$0.000167 \quad 0.000165$

$0.000168 \quad 0.000171$

$0.000186 \quad 0.000186$

$0.000201 \quad 0.000201$

$0.000210 \quad 0.000210$
Density Density App Vol Square of Inconsistent Inconsistent calc / Residual / Residual / Density based on based on $\mathrm{kg} / \mathrm{m}^{3} \quad \mathrm{~kg} / \mathrm{m}^{3} \quad \mathrm{~m}^{3} / \mathrm{kg}$ Residual density apparent

\section{$1002.2 \quad 0.1144-0.000019 \quad 0.013$}

$\begin{array}{llll}1004.5 & 0.1701 & -0.000019 & 0.029\end{array}$

$1005.3 \quad 0.1817-0.000018 \quad 0.033$

$1008.1 \quad 0.2100-0.000016 \quad 0.044$

$1010.3 \quad 0.2516-0.000016 \quad 0.063$

$1012.9 \quad 0.2683-0.000014 \quad 0.072$

$1015.8 \quad 0.3154-0.000014 \quad 0.099$

$1018.3 \quad 0.3431-0.000013 \quad 0.118$

$1021.5 \quad 0.3894-0.000013 \quad 0.152$

$\begin{array}{llll}1024.7 & 0.3957 & -0.000012 & 0.157\end{array}$

$\begin{array}{llll}1040.3 & 0.2706 & -0.000005 & 0.073\end{array}$

$1086.8 \quad 0.2607-0.000002 \quad 0.068$

$\begin{array}{llll}1190.8 & 0.5155 & -0.000002 & 0.266\end{array}$

$\begin{array}{llll}1311.7 & -0.1632 & 0.000000 & 0.027\end{array}$

$\begin{array}{llll}1379.4 & -0.1426 & 0.000000 & 0.020\end{array}$

$\begin{array}{llll}1035.3 & 0.1130 & -0.000002 & 0.013\end{array}$

$\begin{array}{llll}1081.5 & -0.6454 & 0.000006 & 0.416\end{array}$

$\begin{array}{llll}1184.8 & -0.2315 & 0.000001 & 0.054\end{array}$

$\begin{array}{llll}1304.7 & -0.0457 & 0.000000 & 0.002\end{array}$

$\begin{array}{llll}1371.9 & 0.1246 & 0.000000 & 0.016\end{array}$

$\begin{array}{llll}1026.0 & 0.2920 & -0.000006 & 0.085\end{array}$

$\begin{array}{llll}1072.0 & -0.0743 & 0.000001 & 0.006\end{array}$

$\begin{array}{llll}1174.6 & -0.1563 & 0.000001 & 0.024\end{array}$

$\begin{array}{llll}1293.5 & -0.6316 & 0.000001 & 0.399\end{array}$

$1360.1 \quad 0.5375-0.000001 \quad 0.289$

$\begin{array}{llll}1014.4 & -0.1077 & 0.000002 & 0.012\end{array}$

$\begin{array}{llll}1060.2 & 0.3256 & -0.000003 & 0.106\end{array}$

$\begin{array}{llll}1162.4 & -0.0575 & 0.000000 & 0.003\end{array}$

$\begin{array}{llll}1280.9 & 0.0692 & 0.000000 & 0.005\end{array}$

$\begin{array}{llll}1347.3 & -0.0557 & 0.000000 & 0.003\end{array}$

Average Res $0.0946-0.000005$

Std dev Res $\quad 0.2875 \quad 0.000008$

Avg - 4std $\quad-1.0556-0.000036$

$\begin{array}{lll}\text { Avg }+4 s t d \quad 1.2447 & 0.000025\end{array}$
Apparent Density of molal water $/ \mathrm{kg} / \mathrm{m}^{3}$ volume /

$\mathrm{cm}^{3} / \mathrm{mol}$

$29.8 \quad 997.0449$ $29.78 \quad 997.0449$ $29.95 \quad 997.0449$ 30.57997 .0449 $30.62 \quad 997.0449$

$31.06 \quad 997.0449$ $31.17 \quad 997.0449$ $31.36 \quad 997.0449$

$31.49 \quad 997.0449$

31.85997 .0449 997.0449 997.0449 997.0449 997.0449 997.0449 992.2158 992.2158 992.2158 992.2158 992.2158 983.1989 983.1989 983.1989 983.1989 983.1989 971.7978 971.7978 971.7978 971.7978 971.7978 
molality molarity $\mathbf{t} /{ }^{\circ} \mathbf{C}$ mass frac $\begin{array}{r}\text { Density } \\ \exp / \mathrm{kg} / \mathrm{m}^{3}\end{array}$
Ref

App vol
$\exp /$
$\mathrm{m}^{3} / \mathrm{kg}$

App vol Den
Calc /

(

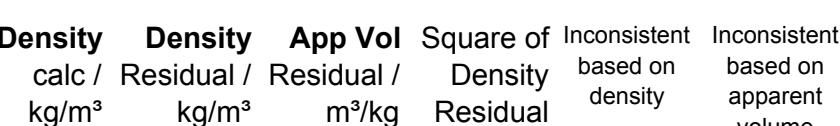

Apparent Density of molal water $/ \mathrm{kg} / \mathrm{m}^{3}$ volume /
$\mathrm{cm}^{3} / \mathrm{mol}$

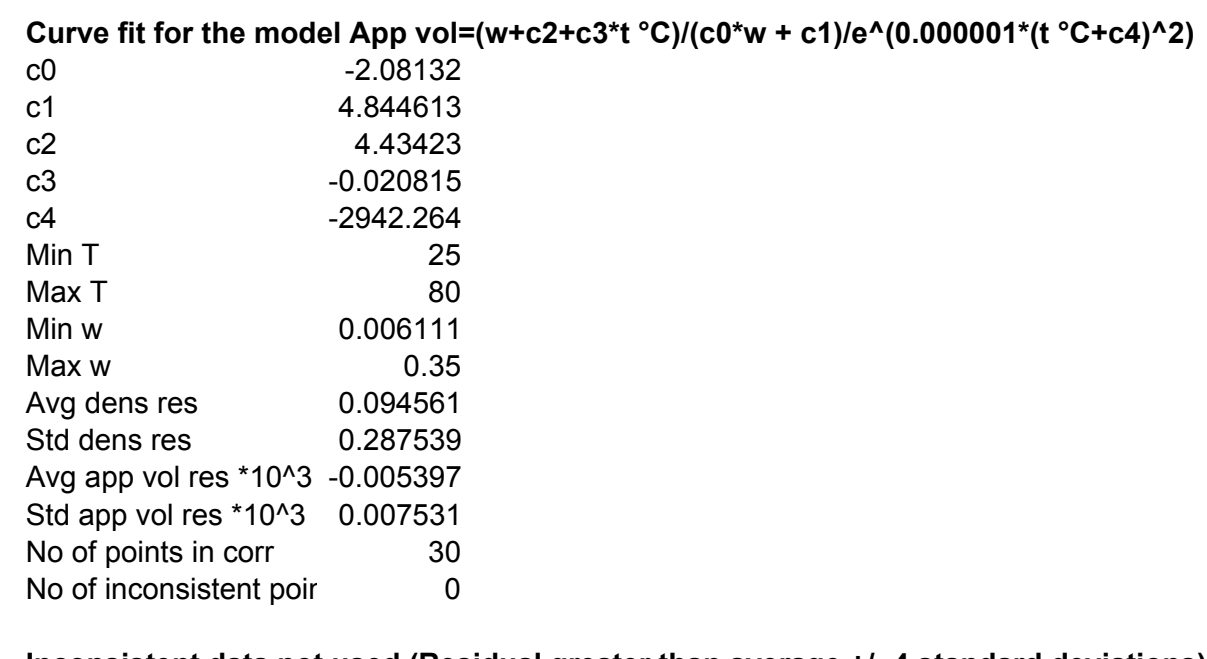

Inconsistent data not used (Residual greater than average +/- 4 standard deviations) 
Density of aqueous solutions of $\mathrm{NaNO} 2$

References (33) Gunther, P.; Perschke, W.; Comparison of some physical constants of thyocyanate, azide and nitrite solutions, J. Chem. Soc., 1930, 100-104

(90) Perry, R. H.; Green, D. W. Perry's Chemical Engineers' Handbook; McGraw Hill: New York, 7th edition, 1997.

\begin{tabular}{|c|c|c|c|c|c|c|c|c|c|c|c|c|c|c|}
\hline molality & molarity & $\mathrm{t} /{ }^{\circ} \mathrm{C}$ & ass frac & $\begin{array}{r}\text { Density } \\
\exp / \\
\mathrm{kg} / \mathrm{m}^{3}\end{array}$ & Ref & $\begin{array}{r}\text { App vol } \\
\text { exp / } \\
\mathrm{m}^{3} / \mathrm{kg}\end{array}$ & $\begin{array}{r}\text { App vol } \\
\text { Calc / } \\
\mathrm{m}^{3} / \mathrm{kg}\end{array}$ & $\begin{array}{r}\text { Density } \\
\text { calc / } \\
\mathrm{kg} / \mathrm{m}^{3}\end{array}$ & $\begin{array}{r}\text { Density } \\
\text { Residual / } \\
\mathrm{kg} / \mathrm{m}^{3}\end{array}$ & $\begin{array}{r}\text { App Vol } \\
\text { Residual / } \\
\mathrm{m}^{3} / \mathrm{kg}\end{array}$ & $\begin{array}{r}\text { Square of } \\
\text { Density } \\
\text { Residual }\end{array}$ & $\begin{array}{l}\text { Inconsistent } \\
\text { based on } \\
\text { density }\end{array}$ & $\begin{array}{l}\text { Inconsistent } \\
\text { based on } \\
\text { apparent } \\
\text { volume }\end{array}$ & $\begin{array}{r}\text { Density of } \\
\text { water } / \mathrm{kg} / \mathrm{m}^{3}\end{array}$ \\
\hline & & 15 & 0.0100 & 1005.8 & (90) & 0.000334 & 0.000339 & 1005.8 & 0.0494 & -0.000005 & 0.002 & & & 999.0996 \\
\hline & & 15 & 0.0200 & 1012.5 & (90) & 0.000339 & 0.000341 & 1012.4 & 0.0599 & -0.000003 & 0.004 & & & 999.0996 \\
\hline & & 15 & 0.0400 & 1026.0 & (90) & 0.000345 & 0.000346 & 1025.9 & 0.0653 & -0.000002 & 0.004 & & & 999.0996 \\
\hline & & 15 & 0.0800 & 1053.5 & (90) & 0.000355 & 0.000356 & 1053.4 & 0.1085 & -0.000001 & 0.012 & & & 999.0996 \\
\hline & & 15 & 0.1200 & 1081.6 & (90) & 0.000365 & 0.000366 & 1081.5 & 0.1199 & -0.000001 & 0.014 & & & 999.0996 \\
\hline & & 15 & 0.1600 & 1110.3 & (90) & 0.000374 & 0.000375 & 1110.2 & 0.0898 & 0.000000 & 0.008 & & & 999.0996 \\
\hline & & 15 & 0.2000 & 1139.4 & (90) & 0.000385 & 0.000384 & 1139.6 & -0.1911 & 0.000001 & 0.037 & & & 999.0996 \\
\hline & 0.5 & 20 & 0.0338 & 1021.0 & (33) & 0.000340 & 0.000343 & 1020.9 & 0.1215 & -0.000003 & 0.015 & & & 998.2041 \\
\hline & 1 & 20 & 0.0662 & 1042.7 & (33) & 0.000356 & 0.000351 & 1043.0 & -0.3409 & 0.000005 & 0.116 & & & 998.2041 \\
\hline & 2 & 20 & 0.1271 & 1085.7 & (33) & 0.000367 & 0.000365 & 1085.9 & -0.2107 & 0.000001 & 0.044 & & & 998.2041 \\
\hline & 3 & 20 & 0.1836 & 1127.3 & (33) & 0.000377 & 0.000378 & 1127.1 & 0.2267 & -0.000001 & 0.051 & & & 998.2041 \\
\hline & & & & & & & & age Res & 0.0089 & -0.000001 & 0.308 & Sum chi sq & & \\
\hline & & & & & & & & dev Res & 0.1752 & 0.000003 & & & & \\
\hline & & & & & & & & $v g-4 s t d$ & -0.6918 & -0.000011 & & & & \\
\hline & & & & & & & & $a+4 s t d$ & 0.7097 & 0.000009 & & & & \\
\hline
\end{tabular}

\section{Curve fit for the model App vol $=\left(w+c 2+c 3^{\star} t{ }^{\circ} \mathrm{C}\right) /\left(\mathrm{c} 0^{*} \mathrm{w}+\mathrm{c} 1\right) / \mathrm{e}^{\wedge}\left(0.000001^{*}\left(\mathrm{t}^{\circ} \mathrm{C}+\mathrm{c} 4\right)^{\wedge} 2\right)$}

$\begin{array}{lr}\text { c0 } 1 & 78.36507 \\ \text { c2 } & 297.9973 \\ \text { c3 } & 0.962457 \\ \text { c4 } & 0.0022 \\ \text { Min T } & 1500 \\ \text { Max T } & 15 \\ \text { Min w } & 20 \\ \text { Max w } & 0.0100 \\ \text { Avg dens res } & 0.2000 \\ \text { Std dens res } & 0.0089 \\ \text { Avg app vol } & 0.1752\end{array}$

Avg app vol res *10^3 -0.000857

Std app vol res *10^3 0.002587

No of points in corr

No of inconsistent poir

Inconsistent data not used (Residual greater than average $+/-4$ standard deviations) 
Density of aqueous solutions of NaNO3

References (8) Berchiesi, M. A.; Berchiesi, G.; Lobbia, G. G. Apparent Molal Volumes of Alkali Metal Nitrates at 30C. J. Chem. Eng. Data 1974, 19, 326328.

(22) Doan, T. H.; Sangster, J. Viscosities of Concentrated Aqueous Solutions of some 1:1, 2:1, and 3:1 Nitrates at $25^{\circ} \mathrm{C} . \mathrm{J}$. Chem. Eng. Data 1981, $26,141-144$.

(45) Isono, T. Densities, Viscosities, and Electrolytic Conductivity of Concentrated Aqueous Electrolyte Solutions at Several Temperatures. Alkaline-Earth Chlorides, $\mathrm{LaCl}_{3}, \mathrm{Na}_{2} \mathrm{SO}_{4}, \mathrm{NaNO}_{3}, \mathrm{NaBr}, \mathrm{KNO}_{3}, \mathrm{KBr}$, and $\mathrm{Cd}\left(\mathrm{NO}_{3}\right)_{2}$. J. Chem. Eng. Data 1984, $29,45-52$.

(50) Kartzmark, E. M. Conductances, Densities, and Viscosities of Solutions of Sodium Nitrate in Water and in Dioxane-Water at $25^{\circ} \mathrm{C}$. Can. J. Chem. 1972, 50, 2845-2850.

(73) Millero, F. J.; Ward, G. K.; Chetirkin, P. V. Relative Sound Velocities of Sea Salts at 25C. J. Acoust. Soc. Am. 1977, 61, 1492-1498.

(82) Patterson, B. A.; Woolley, E. M.; Thermodynamics for lonization of Water at Temperatures from $278.15 \mathrm{~K}$ to $393.15 \mathrm{~K}$ and at the Pressure $0.35 \mathrm{MPa}$ : Apparent Molar Volumes and Apparent Molar Heat Capacities of Aqueous Solutions of Potassium and Sodium Nitrates and Nitric Acid, J. Chem. Thermodynamics, 2002, 34, 535 - 556

(90) Perry, R. H.; Green, D. W. Perry's Chemical Engineers' Handbook; McGraw Hill: New York, 7th edition, 1997.

(102) Puchkov, L. V.; Matashkin, V. G. Densities of $\mathrm{LiNO}_{3}-\mathrm{H}_{2} \mathrm{O}$ and $\mathrm{NaNO}_{3}-\mathrm{H}_{2} \mathrm{O}$ Solutions at Temperatures in the Range 25-300 ${ }^{\circ} \mathrm{C}$. J. Appl. Chem. USSR 1970, 43, 1848-1851.

(110) Roy, Mahendra Nath; Jha, Anupam; Choudhury, Ankan, Densities, Viscosities and Adiabatic Compressibilities of Some Mineral Salts in Water at Different Temperatures, J. Chem. Eng. Data 2004, 49, 291-296.

\begin{tabular}{|c|c|c|c|c|c|c|c|c|c|c|c|c|}
\hline \multirow[t]{18}{*}{ molality molarity } & \multicolumn{2}{|c|}{$\mathbf{t} /{ }^{\circ} \mathrm{C}$ mass frac } & $\begin{array}{r}\text { Density } \\
\operatorname{exp~} \mathrm{kg} / \mathrm{m}^{3}\end{array}$ & Ref & $\begin{array}{r}\text { App vol } \\
\text { exp } / \\
m^{3} / \mathrm{kg}\end{array}$ & $\begin{array}{r}\text { App vol } \\
\text { Calc / } \\
\mathrm{m}^{3} / \mathrm{kg}\end{array}$ & $\begin{array}{r}\text { Density } \\
\text { calc / } \\
\mathrm{kg} / \mathrm{m}^{3}\end{array}$ & $\begin{array}{r}\text { Density } \\
\text { Residual / } \\
\mathrm{kg} / \mathrm{m}^{3}\end{array}$ & $\begin{array}{r}\text { App Vol } \\
\text { Residual / } \\
\mathrm{m}^{3} / \mathrm{kg}\end{array}$ & $\begin{array}{r}\text { Square of } \\
\text { Density Res }\end{array}$ & $\begin{array}{c}\text { Inconsistent } \\
\text { based on } \\
\text { density }\end{array}$ & $\begin{array}{l}\text { Inconsistent } \\
\text { based on } \\
\text { apparent } \\
\text { volume }\end{array}$ \\
\hline & 0 & 0.0100 & 1007.10 & (90) & 0.000279 & 0.000303 & 1006.9 & 0.2403 & -0.000024 & 0.058 & & \\
\hline & 0 & 0.0200 & 1014.40 & (90) & 0.000282 & 0.000305 & 1013.9 & 0.4673 & -0.000023 & 0.218 & & \\
\hline & 0 & 0.0400 & 1029.00 & (90) & 0.000292 & 0.000310 & 1028.2 & 0.7593 & -0.000018 & 0.577 & & \\
\hline & 0 & 0.0499 & 1036.30 & $(102)$ & 0.000295 & 0.000312 & 1035.4 & 0.8953 & -0.000017 & 0.802 & & \\
\hline & 0 & 0.0800 & 1058.70 & (90) & 0.000305 & 0.000318 & 1057.5 & 1.1708 & -0.000013 & 1.371 & & \\
\hline & 0 & 0.0993 & 1073.30 & (102) & 0.000311 & 0.000322 & 1072.0 & 1.3041 & -0.000011 & 1.701 & & \\
\hline & 0 & 0.1200 & 1089.10 & $(90)$ & 0.000317 & 0.000326 & 1087.8 & 1.3348 & -0.000009 & 1.782 & & \\
\hline & 0 & 0.1600 & 1120.30 & (90) & 0.000328 & 0.000334 & 1119.0 & 1.2871 & -0.000006 & 1.657 & & \\
\hline & 0 & 0.2000 & 1152.60 & $(90)$ & 0.000337 & 0.000342 & 1151.3 & 1.2581 & -0.000005 & 1.583 & & \\
\hline & 0 & 0.2007 & 1153.20 & (102) & 0.000337 & 0.000342 & 1151.9 & 1.2823 & -0.000005 & 1.644 & & \\
\hline & 0 & 0.2400 & 1186.00 & $(90)$ & 0.000346 & 0.000350 & 1184.8 & 1.1730 & -0.000003 & 1.376 & & \\
\hline & 0 & 0.2800 & 1220.40 & $(90)$ & 0.000355 & 0.000357 & 1219.5 & 0.8505 & -0.000002 & 0.723 & & \\
\hline & 0 & 0.2974 & 1235.70 & $(102)$ & 0.000358 & 0.000360 & 1235.1 & 0.6380 & -0.000001 & 0.407 & & \\
\hline & 0 & 0.3000 & 1238.00 & $(90)$ & 0.000359 & 0.000360 & 1237.4 & 0.5980 & -0.000001 & 0.358 & & \\
\hline & 0 & 0.3500 & 1283.40 & $(90)$ & 0.000369 & 0.000369 & 1283.6 & -0.1601 & 0.000000 & 0.026 & & \\
\hline & 0 & 0.3970 & 1328.70 & (102) & 0.000377 & 0.000376 & 1329.1 & -0.3798 & 0.000001 & 0.144 & & \\
\hline & 0 & 0.4000 & 1331.60 & $(90)$ & 0.000377 & 0.000377 & 1332.1 & -0.4597 & 0.000001 & 0.211 & & \\
\hline 0.01509 & 5 & 0.0013 & 1000.89 & (82) & 0.000279 & 0.000310 & 1000.8 & 0.0393 & -0.000031 & 0.002 & & \\
\hline 0.03017 & 5 & 0.0026 & 1001.80 & (82) & 0.000283 & 0.000310 & 1001.7 & 0.0702 & -0.000027 & 0.005 & & \\
\hline 0.06039 & 5 & 0.0051 & 1003.61 & (82) & 0.000288 & 0.000310 & 1003.5 & 0.1156 & -0.000022 & 0.013 & & \\
\hline 0.12079 & 5 & 0.0102 & 1007.17 & (82) & 0.000296 & 0.000312 & 1007.0 & 0.1581 & -0.000015 & 0.025 & & \\
\hline 0.2514 & 5 & 0.0209 & 1014.88 & (82) & 0.000297 & 0.000314 & 1014.5 & 0.3568 & -0.000017 & 0.127 & & \\
\hline
\end{tabular}

Apparent Density of molal water / volume / $\mathrm{kg} / \mathrm{m}^{3}$ 999.8395 999.8395 999.8395 999.8395 999.8395 999.8395 999.8395 999.8395 999.8395 999.8395 999.8395 999.8395 999.8395 999.8395 999.8395 999.8395 999.8395

23.72999 .9638 $24.02 \quad 999.9638$ $24.48 \quad 999.9638$ 25.18999 .9638 $25.272 \quad 999.9638$ 


\begin{tabular}{|c|c|c|c|c|}
\hline \multirow{2}{*}{$\begin{array}{r}\text { molality } \\
0.501\end{array}$} & \multirow{2}{*}{ molarity } & \multicolumn{2}{|c|}{$\mathrm{t} /{ }^{\circ} \mathrm{C}$ mass frac } & \multirow{2}{*}{$\begin{array}{r}\begin{array}{r}\text { Density } \\
\text { exp } \mathrm{kg} / \mathrm{m}^{3}\end{array} \\
1029.08\end{array}$} \\
\hline & & 5 & 0.0408 & \\
\hline 0.01509 & & 15 & 0.0013 & 999.99 \\
\hline 0.03017 & & 15 & 0.0026 & 1000.86 \\
\hline 0.05 & & 15 & 0.0042 & 1002.09 \\
\hline 0.06039 & & 15 & 0.0051 & 1002.59 \\
\hline 0.1 & & 15 & 0.0084 & 1004.99 \\
\hline 0.12079 & & 15 & 0.0102 & 1006.04 \\
\hline 0.2514 & & 15 & 0.0209 & 1013.45 \\
\hline 0.5 & & 15 & 0.0408 & 1027.47 \\
\hline 0.501 & & 15 & 0.0408 & 1027.15 \\
\hline 1 & & 15 & 0.0783 & 1054.28 \\
\hline 2 & & 15 & 0.1453 & 1103.85 \\
\hline 3 & & 15 & 0.2032 & 1148.94 \\
\hline 3.959 & & 15 & 0.2518 & 1188.07 \\
\hline 6 & & 15 & 0.3377 & 1261.22 \\
\hline 0.05 & & 20 & 0.0042 & 1001.09 \\
\hline \multirow[t]{4}{*}{0.1} & & 20 & 0.0084 & 1003.96 \\
\hline & & 20 & 0.0100 & 1004.90 \\
\hline & & 20 & 0.0200 & 1011.70 \\
\hline & & 20 & 0.0400 & 1025.40 \\
\hline 0.5 & & 20 & 0.0408 & 1026.10 \\
\hline \multirow[t]{3}{*}{1} & & 20 & 0.0783 & 1052.46 \\
\hline & & 20 & 0.0800 & 1053.20 \\
\hline & & 20 & 0.1200 & 1081.90 \\
\hline \multirow[t]{3}{*}{2} & & 20 & 0.1453 & 1101.47 \\
\hline & & 20 & 0.1600 & 1111.80 \\
\hline & & 20 & 0.2000 & 1142.90 \\
\hline \multirow[t]{2}{*}{3} & & 20 & 0.2032 & 1146.29 \\
\hline & & 20 & 0.2400 & 1175.20 \\
\hline \multirow[t]{3}{*}{3.959} & & 20 & 0.2518 & 1185.15 \\
\hline & & 20 & 0.2800 & 1208.50 \\
\hline & & 20 & 0.3000 & 1225.60 \\
\hline \multirow[t]{4}{*}{6} & & 20 & 0.3377 & 1257.87 \\
\hline & & 20 & 0.3500 & 1270.10 \\
\hline & & 20 & 0.4000 & 1317.50 \\
\hline & & 20 & 0.4500 & 1368.30 \\
\hline 0.01509 & & 25 & 0.0013 & 997.90 \\
\hline 0.03017 & & 25 & 0.0026 & 998.75 \\
\hline 0.05 & & 25 & 0.0042 & 999.87 \\
\hline 0.05176 & & 25 & 0.0044 & 999.99 \\
\hline \multirow[t]{2}{*}{0.06039} & & 25 & 0.0051 & 1000.43 \\
\hline & 0.09617 & 25 & 0.0082 & 1002.50 \\
\hline
\end{tabular}

Ref

App vol App vol D

$$
\begin{array}{cc}
\exp / & \text { Calc } / \\
\mathrm{m}^{3} / \mathrm{kg} & \mathrm{m}^{3} / \mathrm{kg}
\end{array}
$$

$0.000307 \quad 0.000318$

$0.000308 \quad 0.000326$

$0.000313 \quad 0.000326$

$\begin{array}{ll}0.000295 & 0.000327\end{array}$

$0.000318 \quad 0.000327$

$\begin{array}{ll}0.000305 & 0.000327\end{array}$

$0.000321 \quad 0.000328$

$0.000324 \quad 0.000330$

$0.000323 \quad 0.000334$

$0.000332 \quad 0.000334$

0.0003320 .000341

$\begin{array}{lll}0.000347 & 0.000352\end{array}$

$0.000358 \quad 0.000362$

0.0003690 .000369

$0.000385 \quad 0.000382$

$\begin{array}{lll}0.000319 & 0.000334\end{array}$

$0.000320 \quad 0.000335$

$0.000334 \quad 0.000335$

$\begin{array}{ll}0.000334 & 0.000337\end{array}$

$\begin{array}{lll}0.000338 & 0.000341\end{array}$

$0.000334 \quad 0.000341$

$\begin{array}{ll}0.000343 & 0.000347\end{array}$

$0.000348 \quad 0.000348$

$0.000356 \quad 0.000354$

$0.000355 \quad 0.000358$

0.0003620 .000361

$\begin{array}{ll}0.000368 & 0.000367\end{array}$

$\begin{array}{ll}0.000365 & 0.000367\end{array}$

$0.000373 \quad 0.000373$

$0.000374 \quad 0.000375$

$0.000379 \quad 0.000379$

0.0003820 .000381

0.0003890 .000387

$\begin{array}{ll}0.000389 & 0.000388\end{array}$

$0.000395 \quad 0.000395$

$0.000400 \quad 0.000401$

$\begin{array}{ll}0.000329 & 0.000341\end{array}$

$0.000335 \quad 0.000341$

0.0003330 .000341

$0.000329 \quad 0.000341$

$0.000338 \quad 0.000341$

$\begin{array}{ll}0.000334 & 0.000342\end{array}$
Density Density App Vol

Square of Inconsistent Inconsistent

$\mathrm{kg} / \mathrm{m}^{3} \quad \mathrm{~kg} / \mathrm{m}^{3} \quad \mathrm{~m}^{3} / \mathrm{kg}$ density apparent

$1028.6 \quad 0.4671-0.000011$

$1000.0 \quad 0.0233-0.000018$

$1000.8 \quad 0.0330-0.000013$

$1002.0 \quad 0.1341-0.000032$

$1002.5 \quad 0.0470-0.000009$

$1004.8 \quad 0.1927-0.000023$

$1006.0 \quad 0.0667-0.000006$

$1013.3 \quad 0.1333-0.000006$

$1027.0 \quad 0.4599-0.000011$

$1027.10 .0866-0.000002$

$1053.5 \quad 0.7308-0.000008$

$1102.9 \quad 0.9031-0.000005$

$1148.0 \quad 0.9045-0.000003$

$\begin{array}{lll}1187.8 & 0.2983 & -0.000001\end{array}$

$\begin{array}{lll}1262.8 & -1.5522 & 0.000003\end{array}$

$1001.0 \quad 0.0629-0.000015$

$1003.8 \quad 0.1245-0.000015$

$1004.9 \quad 0.0099-0.000001$

$1011.6 \quad 0.0710-0.000003$

$1025.3 \quad 0.1313-0.000003$

$1025.8 \quad 0.3052-0.000007$

$1052.0 \quad 0.4181-0.000005$

$1053.2 \quad-0.0230 \quad 0.000000$

$\begin{array}{lll}1082.1 & -0.2293 & 0.000002\end{array}$

$1100.9 \quad 0.5421-0.000003$

$\begin{array}{lll}1112.1 & -0.2550 & 0.000001\end{array}$

$1143.1 \quad-0.1728 \quad 0.000001$

$1145.6 \quad 0.7044-0.000003$

$1175.3 \quad-0.0613 \quad 0.000000$

$1185.0 \quad 0.1791-0.000001$

$1208.7 \quad-0.2057 \quad 0.000001$

$\begin{array}{lll}1225.9 & -0.3276 & 0.000001\end{array}$

$\begin{array}{lll}1259.4 & -1.5124 & 0.000003\end{array}$

$\begin{array}{lll}1270.5 & -0.4394 & 0.000001\end{array}$

$\begin{array}{lll}1317.5 & -0.0453 & 0.000000\end{array}$

$1367.2 \quad 1.1209-0.000001$

$997.9 \quad 0.0145-0.000011$

$998.7 \quad 0.0158-0.000006$

$\begin{array}{llll}999.8 & 0.0340 & -0.000008\end{array}$

$999.9 \quad 0.0560-0.000013$

$1000.4 \quad 0.0166-0.000003$

$1002.4 \quad 0.0690-0.000008$
0.218

0.001

0.001

0.018

0.002

0.004

0.018

0.212

0.007

0.534

0.816

0.818

0.089

2.409

0.004

0.015

0.000

0.005

0.017

0.093

0.175

0.001

0.053

0.294

0.065

0.030

0.496

0.004

0.032

0.042

0.107

2.287

0.193

0.002

1.257

0.000

0.000

0.001

0.003

0.000

0.005
Apparent Density of molal water / $\begin{array}{ll}\text { volume / } & \mathrm{kg} / \mathrm{m}^{3} / \mathrm{mol}\end{array}$

$26.119 \quad 999.9638$

$26.17 \quad 999.0996$

$26.64 \quad 999.0996$ 999.0996

$27 \quad 999.0996$ 999.0996

$27.31 \quad 999.0996$

$27.51 \quad 999.0996$

999.0996

$28.188 \quad 999.0996$ 999.0996 999.0996

999.0996 999.0996 999.0996

998.2041

998.2041

998.2041

998.2041

998.2041

998.2041

998.2041

998.2041

998.2041

998.2041

998.2041

998.2041

998.2041

998.2041

998.2041

998.2041

998.2041

998.2041

998.2041

998.2041

998.2041

$28 \quad 997.0449$

$28.46 \quad 997.0449$

997.0449

997.0449

$28.75 \quad 997.0449$

997.0449 
$t /{ }^{\circ} \mathrm{C}$ mass frac Density $\operatorname{exp~} \mathrm{kg} / \mathrm{m}^{3}$

0.1

0.101107

0.12079

0.200333

0.2514

0.298821

0.400891

0.498377

0.5

501

0.598955

0.699736

0.798943

0.896968

1

1.000043

0.9782

1.5
2

2

2.5

1.9511

3

3.5

3.959

3.7502

5

5.1299

6.0413

6.9868

$\begin{array}{ll}0.0084 & 1002.72 \\ 0.0085 & 1002.75 \\ 0.0102 & 1003.76 \\ 0.0167 & 1008.23 \\ 0.0209 & 1010.93 \\ 0.0248 & 1013.61 \\ 0.0277 & 1015.50 \\ 0.0330 & 1018.94 \\ 0.0406 & 1024.32 \\ 0.0408 & 1023.90 \\ 0.0408 & 1024.54 \\ 0.0408 & 1024.28 \\ 0.0484 & 1029.64 \\ 0.0499 & 1031.00 \\ 0.0561 & 1034.89 \\ 0.0636 & 1040.06 \\ 0.0708 & 1045.02 \\ 0.0783 & 1050.00 \\ 0.0783 & 1050.60 \\ 0.0783 & 1050.26 \\ 0.0791 & 1050.80 \\ 0.0993 & 1065.00 \\ 0.1131 & 1073.70 \\ 0.1453 & 1098.00 \\ 0.1453 & 1099.06 \\ 0.1504 & 1102.40 \\ 0.1752 & 1120.10 \\ 0.2007 & 1140.00 \\ 0.2032 & 1140.90 \\ 0.2032 & 1143.62 \\ 0.2109 & 1149.00 \\ 0.2293 & 1162.10 \\ 0.2518 & 1182.10 \\ 0.2537 & 1182.00 \\ 0.2670 & 1193.90 \\ 0.2974 & 1219.00 \\ 0.2982 & 1219.10 \\ 0.3377 & 1254.40 \\ 0.3454 & 1262.50 \\ 0.3930 & 1306.40 \\ 0.3970 & 1312.00 \\ 0.4395 & 1351.30 \\ & \\ 0\end{array}$

Ref

App vol App vol
$\exp / \quad$ Calc $\mathrm{m}^{3} / \mathrm{kg} \quad \mathrm{m}^{3} / \mathrm{kg}$

0.0003290 .000342

$0.000333 \quad 0.000342$

$0.000342 \quad 0.000342$

$0.000338 \quad 0.000344$

$0.000345 \quad 0.000344$

$\begin{array}{ll}0.000341 & 0.000345\end{array}$

$0.000346 \quad 0.000345$

$0.000349 \quad 0.000346$

$\begin{array}{lll}0.000346 & 0.000348\end{array}$

$\begin{array}{lll}0.000358 & 0.000348\end{array}$

$0.000343 \quad 0.000348$

$\begin{array}{lll}0.000350 & 0.000348\end{array}$

$0.000348 \quad 0.000349$

$\begin{array}{lll}0.000341 & 0.000349\end{array}$

$0.000350 \quad 0.000350$

$\begin{array}{ll}0.000351 & 0.000351\end{array}$

$0.000353 \quad 0.000353$

$0.000357 \quad 0.000354$

$0.000350 \quad 0.000354$

$0.000354 \quad 0.000354$

$\begin{array}{lll}0.000355 & 0.000354\end{array}$

$\begin{array}{lll}0.000358 & 0.000357\end{array}$

$0.000370 \quad 0.000359$

$0.000368 \quad 0.000364$

$\begin{array}{lll}0.000362 & 0.000364\end{array}$

$\begin{array}{lll}0.000366 & 0.000365\end{array}$

$\begin{array}{lll}0.000374 & 0.000369\end{array}$

$0.000376 \quad 0.000372$

$0.000381 \quad 0.000373$

$\begin{array}{lll}0.000370 & 0.000373\end{array}$

$0.000374 \quad 0.000374$

0.0003820 .000376

$0.000379 \quad 0.000380$

$0.000384 \quad 0.000380$

$0.000384 \quad 0.000382$

0.0003890 .000386

$0.000390 \quad 0.000386$

$0.000394 \quad 0.000391$

$0.000392 \quad 0.000392$

$0.000399 \quad 0.000398$

$\begin{array}{ll}0.000396 & 0.000398\end{array}$

$0.000405 \quad 0.000403$
Density Density App Vol

Square of Inconsistent Inconsistent

$\mathrm{kg} / \mathrm{m}^{3} \quad \mathrm{~kg} / \mathrm{m}^{3} \quad \mathrm{~m}^{3} / \mathrm{kg}$ density apparent

$\begin{array}{llll}1002.6 & 0.1072 & -0.000013 & 0.011\end{array}$

$1002.7 \quad 0.0759-0.000009$

$\begin{array}{lll}1003.8 & -0.0012 & 0.000000\end{array}$

$1008.1 \quad 0.0874-0.000005$

$\begin{array}{lll}1010.9 & -0.0064 & 0.000000\end{array}$

$1013.5 \quad 0.0935-0.000004$

$\begin{array}{lll}1015.5 & -0.0181 & 0.000001\end{array}$

$\begin{array}{lll}1019.0 & -0.0900 & 0.000003\end{array}$

$1024.2 \quad 0.0761-0.000002$

$1024.3 \quad-0.4303 \quad 0.000010$

$\begin{array}{lll}1024.3 & 0.2097 & -0.000005\end{array}$

$\begin{array}{lll}1024.4 & -0.1062 & 0.000002\end{array}$

$1029.6 \quad 0.0691-0.000001$

$1030.6 \quad 0.4300-0.000008$

$1034.9 \quad 0.0339-0.000001$

$1040.0 \quad 0.0516-0.000001$

$\begin{array}{lll}1045.1 & -0.0309 & 0.000000\end{array}$

$\begin{array}{lll}1050.3 & -0.3002 & 0.000003\end{array}$

$1050.3 \quad 0.2998-0.000003$

$1050.3 \quad-0.0423 \quad 0.000000$

$1050.9 \quad-0.0522 \quad 0.000001$

$\begin{array}{lll}1065.2 & -0.1529 & 0.000001\end{array}$

$\begin{array}{lll}1075.1 & -1.3604 & 0.000010\end{array}$

$\begin{array}{lll}1098.7 & -0.7038 & 0.000004\end{array}$

$1098.7 \quad 0.3562-0.000002$

$\begin{array}{lll}1102.5 & -0.1378 & 0.000001\end{array}$

$\begin{array}{lll}1121.3 & -1.2126 & 0.000006\end{array}$

$\begin{array}{lll}1141.0 & -1.0167 & 0.000004\end{array}$

$\begin{array}{lll}1143.0 & -2.0597 & 0.000008\end{array}$

$1143.0 \quad 0.6603-0.000002$

$\begin{array}{lll}1149.0 & -0.0376 & 0.000000\end{array}$

$\begin{array}{lll}1163.7 & -1.6104 & 0.000005\end{array}$

$\begin{array}{lll}1182.0 & 0.0789 & 0.000000\end{array}$

$\begin{array}{lll}1183.6 & -1.6238 & 0.000005\end{array}$

$\begin{array}{lll}1194.6 & -0.7277 & 0.000002\end{array}$

$\begin{array}{lll}1220.4 & -1.4289 & 0.000003\end{array}$

$\begin{array}{lll}1221.1 & -2.0463 & 0.000005\end{array}$

$\begin{array}{llll}1255.9 & -1.4981 & 0.000003\end{array}$

$\begin{array}{lll}1262.8 & -0.2712 & 0.000000\end{array}$

$\begin{array}{lll}1307.1 & -0.6630 & 0.000001\end{array}$

$1310.8 \quad 1.1631-0.000002$

$\begin{array}{lll}1352.5 & -1.1527 & 0.000001\end{array}$
0.006

0.008

0.000

0.009

0.000

0.008

0.006

0.185

0.044

0.011

0.005

0.185

0.001

0.003

0.001

0.090

0.090

0.002

0.003

0.023

1.851

0.495

0.127

0.019

1.470

1.034

4.242

0.436

0.001

2.593

0.006

2.637

0.530

2.042

4.187

2.244

0.074

0.440

1.353

1.329
Apparent Density of molal water / volume / $\mathrm{kg} / \mathrm{m}^{3}$ 997.0449 997.0449

$29.11 \quad 997.0449$ 997.0449

$29.283 \quad 997.0449$ 997.0449 997.0449 997.0449 997.0449 997.0449 997.0449

$29.755 \quad 997.0449$ 997.0449 997.0449 997.0449 997.0449 997.0449 997.0449 997.0449 997.0449 997.0449

997.0449 997.0449 997.0449 997.0449 997.0449 997.0449 997.0449 997.0449 997.0449 997.0449 997.0449 997.0449 997.0449 997.0449 997.0449 997.0449 997.0449 997.0449 997.0449 997.0449 997.0449 
$t /{ }^{\circ} \mathrm{C}$ mass frac Density

$\operatorname{exp~} \mathrm{kg} / \mathrm{m}^{3}$

\begin{tabular}{|c|c|c|c|c|}
\hline & & 25 & 0.4682 & 1383.00 \\
\hline & 0.16869 & 29.85 & 0.0143 & 1004.74 \\
\hline & 0.22894 & 29.85 & 0.0193 & 1008.31 \\
\hline 0.05 & & 30 & 0.0042 & 998.42 \\
\hline \multirow[t]{3}{*}{0.1} & & 30 & 0.0084 & 1001.23 \\
\hline & 0.19705 & 30 & 0.0166 & 1006.57 \\
\hline & 0.34304 & 30 & 0.0287 & 1014.61 \\
\hline \multirow[t]{3}{*}{0.5} & & 30 & 0.0408 & 1022.80 \\
\hline & 0.57912 & 30 & 0.0479 & 1027.44 \\
\hline & 0.90402 & 30 & 0.0735 & 1044.95 \\
\hline \multirow[t]{2}{*}{1} & & 30 & 0.0783 & 1048.54 \\
\hline & 1.83007 & 30 & 0.1422 & 1093.68 \\
\hline 2 & & 30 & 0.1453 & 1096.56 \\
\hline \multirow[t]{2}{*}{3} & & 30 & 0.2032 & 1140.76 \\
\hline & 2.84293 & 30 & 0.2109 & 1145.70 \\
\hline 3.959 & & 30 & 0.2518 & 1178.97 \\
\hline 6 & & 30 & 0.3377 & 1250.95 \\
\hline 0.01509 & & 35 & 0.0013 & 994.87 \\
\hline 0.03017 & & 35 & 0.0026 & 995.67 \\
\hline 0.05 & & 35 & 0.0042 & 996.77 \\
\hline 0.06039 & & 35 & 0.0051 & 997.32 \\
\hline 0.1 & & 35 & 0.0084 & 999.55 \\
\hline 0.12079 & & 35 & 0.0102 & 1000.56 \\
\hline 0.2514 & & 35 & 0.0209 & 1007.55 \\
\hline 0.5 & & 35 & 0.0408 & 1020.87 \\
\hline 0.501 & & 35 & 0.0408 & 1020.62 \\
\hline 1 & & 35 & 0.0783 & 1046.34 \\
\hline 2 & & 35 & 0.1453 & 1093.94 \\
\hline 3 & & 35 & 0.2032 & 1137.87 \\
\hline \multirow{13}{*}{$\begin{array}{r}3.959 \\
6\end{array}$} & & 35 & 0.2518 & 1175.85 \\
\hline & & 35 & 0.3377 & 1247.49 \\
\hline & & 40 & 0.0100 & 998.60 \\
\hline & & 40 & 0.0200 & 1005.00 \\
\hline & & 40 & 0.0400 & 1018.00 \\
\hline & & 40 & 0.0800 & 1044.70 \\
\hline & & 40 & 0.1200 & 1072.40 \\
\hline & & 40 & 0.1600 & 1101.30 \\
\hline & & 40 & 0.2000 & 1131.40 \\
\hline & & 40 & 0.2400 & 1162.90 \\
\hline & & 40 & 0.2800 & 1195.50 \\
\hline & & 40 & 0.3000 & 1212.20 \\
\hline & & 40 & 0.3500 & 1256.00 \\
\hline
\end{tabular}

Ref

App vol App vol Der

$\mathrm{m}^{3} / \mathrm{kg} \quad \mathrm{m}^{3} / \mathrm{kg}$

0.0004050 .000407

$\begin{array}{ll}0.000371 & 0.000350\end{array}$

$0.000353 \quad 0.000350$

0.0003450 .000348

$0.000340 \quad 0.000349$

$0.000349 \quad 0.000350$

$0.000351 \quad 0.000352$

$0.000350 \quad 0.000354$

$0.000356 \quad 0.000355$

$\begin{array}{lll}\text { (8) } & 0.000360 & 0.000359\end{array}$

$\begin{array}{ccc}(8) & 0.000360 & 0.000359 \\ (45) & 0.000358 & 0.000360\end{array}$

(8) $\quad 0.000371 \quad 0.000369$

$\begin{array}{lllll}(4) & 0.000371 & 0.00036\end{array}$

$\begin{array}{lll}\text { (45) } & 0.000376 & 0.000378\end{array}$

(8) $\quad 0.000381 \quad 0.000379$

(45) $0.000384 \quad 0.000384$

(45) $\quad 0.000397 \quad 0.000395$

$\begin{array}{lll}\text { (82) } \quad 0.000343 & 0.000354\end{array}$

$\begin{array}{lll}\text { (82) } & 0.000358 & 0.000354\end{array}$

(45) $\quad 0.000353 \quad 0.000354$

$\begin{array}{lll}\text { (82) } & 0.000357 & 0.000355\end{array}$

$\begin{array}{lll}\text { (45) } & 0.000347 & 0.000355\end{array}$

$\begin{array}{lll}\text { (82) } & 0.000360 & 0.000355\end{array}$

$\begin{array}{lll}\text { (82) } & 0.000361 & 0.000357\end{array}$

(45) $\quad 0.000357 \quad 0.000360$

(82) $\quad 0.000364 \quad 0.000360$

$\begin{array}{lll}\text { (45) } \quad 0.000364 & 0.000366\end{array}$

$\begin{array}{lll}(45) & 0.000374 & 0.000375\end{array}$

(45) $\quad 0.000380 \quad 0.000382$

$\begin{array}{lll}\text { (45) } \quad 0.000388 & 0.000388\end{array}$

(45) $\quad 0.000401 \quad 0.000398$

(90) $\quad 0.000364 \quad 0.000361$

$\begin{array}{lll}\text { (90) } & 0.000367 & 0.000363\end{array}$

(90) $\quad 0.000370 \quad 0.000366$

(90) $\quad 0.000375 \quad 0.000371$

(90) $\quad 0.000380 \quad 0.000376$

(90)

(90)

(90)

(90)

(90)

(90)

$0.000384 \quad 0.000381$

$0.000388 \quad 0.000386$

$\begin{array}{ll}0.000391 & 0.000391\end{array}$

$0.000396 \quad 0.000396$

$0.000398 \quad 0.000398$

$0.000403 \quad 0.000403$

Density Density App Vol

Square of Inconsistent Inconsistent / Residual / Residual / Density Res based on based on $\mathrm{kg} / \mathrm{m}^{3} \quad \mathrm{~kg} / \mathrm{m}^{3} \quad \mathrm{~m}^{3} / \mathrm{kg}$ density apparent

Apparent Density of $1381.8 \quad 1.2157 \quad-0.000001 \quad 1.478$ $\begin{array}{lll}1005.0 & -0.3029 & 0.000021\end{array}$

0.092

$\begin{array}{llll}1008.4 & -0.0535 & 0.000003 & 0.003\end{array}$

$\begin{array}{llll}998.4 & 0.0121 & -0.000003 & 0.000\end{array}$

$\begin{array}{llll}1001.2 & 0.0753 & -0.000009 & 0.006\end{array}$

$\begin{array}{llll}1006.6 & 0.0132 & -0.000001 & 0.000\end{array}$

$\begin{array}{llll}1014.6 & 0.0274 & -0.000001 & 0.001\end{array}$

$\begin{array}{llll}1022.6 & 0.1578 & -0.000004 & 0.025\end{array}$

$\begin{array}{llll}1027.5 & -0.0266 & 0.000001 & 0.001\end{array}$

$\begin{array}{llll}1045.0 & -0.0658 & 0.000001 & 0.004\end{array}$

$\begin{array}{llll}1048.3 & 0.1905 & -0.000002 & 0.036\end{array}$

$\begin{array}{llll}1094.0 & -0.3589 & 0.000002 & 0.129\end{array}$

$1096.3 \quad 0.2600-0.000001 \quad 0.068$

$\begin{array}{llll}1140.2 & 0.5777 & -0.000002 & 0.334\end{array}$

$\begin{array}{llll}1146.2 & -0.5246 & 0.000002 & 0.275\end{array}$

$\begin{array}{llll}1178.9 & 0.0240 & 0.000000 & 0.001\end{array}$

$\begin{array}{llll}1252.3 & -1.3911 & 0.000003 & 1.935\end{array}$

$994.9-0.0145-0.000011 \quad 0.000$

$\begin{array}{llll}995.7 & -0.0105 & 0.000004 & 0.000\end{array}$

$\begin{array}{lllll}996.8 & 0.0064 & -0.000002 & 0.000\end{array}$

$\begin{array}{llll}997.3 & -0.0137 & 0.000003 & 0.000\end{array}$

$\begin{array}{llll}999.5 & 0.0682 & -0.000008 & 0.005\end{array}$

$\begin{array}{llll}1000.6 & -0.0471 & 0.000005 & 0.002\end{array}$

$\begin{array}{llll}1007.6 & -0.0853 & 0.000004 & 0.007\end{array}$

$\begin{array}{llll}1020.8 & 0.1189 & -0.000003 & 0.014\end{array}$

$\begin{array}{llll}1020.8 & -0.1841 & 0.000004 & 0.034\end{array}$

$\begin{array}{llll}1046.2 & 0.1294 & -0.000002 & 0.017\end{array}$

$\begin{array}{llll}1093.7 & 0.2036 & -0.000001 & 0.041\end{array}$

$\begin{array}{llll}1137.3 & 0.5973 & -0.000002 & 0.357\end{array}$

$\begin{array}{llll}1175.8 & 0.0858 & 0.000000 & 0.007\end{array}$

$\begin{array}{llll}1248.7 & -1.2378 & 0.000002 & 1.532\end{array}$

$\begin{array}{llll}998.6 & -0.0218 & 0.000002 & 0.000\end{array}$

$\begin{array}{llll}1005.1 & -0.0820 & 0.000004 & 0.007\end{array}$

$\begin{array}{llll}1018.2 & -0.1690 & 0.000004 & 0.029\end{array}$

$\begin{array}{llll}1045.0 & -0.3363 & 0.000004 & 0.113\end{array}$

$\begin{array}{llll}1072.9 & -0.4839 & 0.000004 & 0.234\end{array}$

$\begin{array}{llll}1101.8 & -0.4829 & 0.000002 & 0.233\end{array}$

$\begin{array}{llll}1131.8 & -0.4106 & 0.000002 & 0.169\end{array}$

$\begin{array}{llll}1163.1 & -0.1508 & 0.000000 & 0.023\end{array}$

$\begin{array}{llll}1195.6 & -0.0948 & 0.000000 & 0.009\end{array}$

$\begin{array}{llll}1212.4 & -0.1866 & 0.000000 & 0.035\end{array}$

$\begin{array}{llll}1256.0 & 0.0106 & 0.000000 & 0.000\end{array}$

volume / Water /
$\mathrm{kg} / \mathrm{m}^{3}$

997.0449

995.6924

995.6924

995.6473

995.6473

995.6473

995.6473

995.6473

995.6473

995.6473

995.6473

995.6473

995.6473

995.6473

995.6473

995.6473

995.6473

$29.12 \quad 994.0319$

$30.46 \quad 994.0319$

994.0319

$30.37 \quad 994.0319$

994.0319

$30.6 \quad 994.0319$

$30.688 \quad 994.0319$

994.0319

$30.969 \quad 994.0319$

994.0319

994.0319

994.0319

994.0319

994.0319

992.2158

992.2158

992.2158

992.2158

992.2158

992.2158

992.2158

992.2158

992.2158

992.2158

992.2158 


\begin{tabular}{|c|c|c|c|c|}
\hline \multirow[t]{3}{*}{ molality } & \multirow[t]{3}{*}{ molarity } & \multicolumn{2}{|c|}{$\mathrm{t} /{ }^{\circ} \mathrm{C}$ mass frac } & \multirow{2}{*}{$\begin{array}{r}\text { Density } \\
\text { exp kg/m } \\
1302.70\end{array}$} \\
\hline & & 40 & 0.4000 & \\
\hline & & 40 & 0.4500 & 1352.80 \\
\hline 0.01509 & & 45 & 0.0013 & 991.04 \\
\hline 0.03017 & & 45 & 0.0026 & 991.81 \\
\hline 0.05 & & 45 & 0.0042 & 992.89 \\
\hline 0.06039 & & 45 & 0.0051 & 993.38 \\
\hline 0.1 & & 45 & 0.0084 & 995.61 \\
\hline 0.12079 & & 45 & 0.0102 & 996.59 \\
\hline 0.2514 & & 45 & 0.0209 & 1003.42 \\
\hline 0.5 & & 45 & 0.0408 & 1016.52 \\
\hline 0.501 & & 45 & 0.0408 & 1016.27 \\
\hline 1 & & 45 & 0.0783 & 1041.56 \\
\hline 2 & & 45 & 0.1453 & 1088.49 \\
\hline 3 & & 45 & 0.2032 & 1131.81 \\
\hline 3.959 & & 45 & 0.2518 & 1169.37 \\
\hline \multirow[t]{7}{*}{6} & & 45 & 0.3377 & 1240.38 \\
\hline & & 50 & 0.0499 & 1020.00 \\
\hline & & 50 & 0.0993 & 1052.00 \\
\hline & & 50 & 0.2007 & 1126.00 \\
\hline & & 50 & 0.2974 & 1202.00 \\
\hline & & 50 & 0.3970 & 1292.00 \\
\hline & & 50 & 0.4682 & 1363.00 \\
\hline 0.01509 & & 55 & 0.0013 & 986.50 \\
\hline 0.03017 & & 55 & 0.0026 & 987.30 \\
\hline 0.05 & & 55 & 0.0042 & 988.32 \\
\hline 0.06039 & & 55 & 0.0051 & 988.89 \\
\hline 0.1 & & 55 & 0.0084 & 991.00 \\
\hline 0.12079 & & 55 & 0.0102 & 992.02 \\
\hline 0.2514 & & 55 & 0.0209 & 998.75 \\
\hline 0.5 & & 55 & 0.0408 & 1011.60 \\
\hline 0.501 & & 55 & 0.0408 & 1011.38 \\
\hline 1 & & 55 & 0.0783 & 1036.28 \\
\hline 2 & & 55 & 0.1453 & 1082.65 \\
\hline 3 & & 55 & 0.2032 & 1125.46 \\
\hline 3.959 & & 55 & 0.2518 & 1162.67 \\
\hline \multirow[t]{7}{*}{6} & & 55 & 0.3377 & 1233.13 \\
\hline & & 60 & 0.0100 & 989.40 \\
\hline & & 60 & 0.0200 & 995.60 \\
\hline & & 60 & 0.0400 & 1008.20 \\
\hline & & 60 & 0.0800 & 1034.00 \\
\hline & & 60 & 0.1200 & 1060.90 \\
\hline & & 60 & 0.1600 & 1089.20 \\
\hline
\end{tabular}

App vol App vol

/ Calc /

$\mathrm{m}^{3} / \mathrm{kg} \quad \mathrm{m}^{3} / \mathrm{kg}$

$\begin{array}{ll}0.000407 & 0.000408\end{array}$

$\begin{array}{lll}0.000411 & 0.000413\end{array}$

$0.000348 \quad 0.000366$

0.0003750 .000366

$0.000367 \quad 0.000366$

$\begin{array}{ll}0.000380 & 0.000366\end{array}$

$\begin{array}{lll}0.000360 & 0.000367\end{array}$

$\begin{array}{ll}0.000374 & 0.000367\end{array}$

$\begin{array}{lll}0.000375 & 0.000368\end{array}$

$\begin{array}{lll}0.000369 & 0.000371\end{array}$

$0.000376 \quad 0.000371$

$0.000374 \quad 0.000376$

$\begin{array}{ll}0.000382 & 0.000384\end{array}$

$0.000388 \quad 0.000391$

$0.000395 \quad 0.000396$

$\begin{array}{ll}0.000407 & 0.000405\end{array}$

$0.000377 \quad 0.000377$

$0.000392 \quad 0.000383$

$0.000394 \quad 0.000394$

$0.000406 \quad 0.000404$

$0.000412 \quad 0.000413$

$\begin{array}{ll}0.000417 & 0.000419\end{array}$

$0.000368 \quad 0.000376$

$\begin{array}{lll}0.000371 & 0.000376\end{array}$

$0.000378 \quad 0.000376$

$\begin{array}{ll}0.000374 & 0.000377\end{array}$

$\begin{array}{ll}0.000370 & 0.000377\end{array}$

$\begin{array}{ll}0.000378 & 0.000377\end{array}$

$0.000381 \quad 0.000378$

$\begin{array}{lll}0.000377 & 0.000381\end{array}$

$0.000384 \quad 0.000381$

0.0003820 .000385

$\begin{array}{lll}0.000389 & 0.000392\end{array}$

$0.000394 \quad 0.000398$

0.0004010 .000403

0.0004120 .000410

$\begin{array}{lll}0.000380 & 0.000382\end{array}$

$0.000384 \quad 0.000383$

$0.000387 \quad 0.000385$

$0.000392 \quad 0.000389$

$0.000396 \quad 0.000393$

$0.000398 \quad 0.000397$
Density Density App Vol

Square of Inconsistent Inconsistent

$\mathrm{kg} / \mathrm{m}^{3} \quad \mathrm{~kg} / \mathrm{m}^{3} \quad \mathrm{~m}^{3} / \mathrm{kg}$ density apparent

$1302.10 .6049-0.000001 \quad 0.366$

$1351.0 \quad 1.8421-0.000002$

$991.0 \quad 0.0220-0.000017$

$\begin{array}{lll}991.8 & -0.0237 & 0.000009\end{array}$

$\begin{array}{lll}992.9 & -0.0015 & 0.000000\end{array}$

$\begin{array}{lll}993.4 & -0.0694 & 0.000014\end{array}$

$\begin{array}{llll}995.6 & 0.0531 & -0.000006\end{array}$

$\begin{array}{lll}996.7 & -0.0667 & 0.000007\end{array}$

$\begin{array}{lll}1003.5 & -0.1292 & 0.000006\end{array}$

$1016.4 \quad 0.0951-0.000002$

$\begin{array}{lll}1016.5 & -0.2019 & 0.000005\end{array}$

$1041.4 \quad 0.1285-0.000002$

$1088.2 \quad 0.2983-0.000002$

$1131.10 .6944-0.000003$

$1169.10 .2349-0.000001$

$1241.4 \quad-0.9988 \quad 0.000002$

$\begin{array}{llll}1020.0 & 0.0322 & -0.000001\end{array}$

$\begin{array}{llll}1053.0 & -1.0335 & 0.000009\end{array}$

$\begin{array}{lll}1126.0 & -0.0129 & 0.000000\end{array}$

$\begin{array}{lll}1203.0 & -1.0456 & 0.000002\end{array}$

$1291.5 \quad 0.4789-0.000001$

$1361.5 \quad 1.4817-0.000002$

$\begin{array}{llll}986.5 & 0.0096 & -0.000008\end{array}$

$\begin{array}{llll}987.3 & 0.0128 & -0.000005\end{array}$

$\begin{array}{llll}988.3 & -0.0059 & 0.000001\end{array}$

$\begin{array}{llll}988.9 & 0.0142 & -0.000003\end{array}$

$990.9 \quad 0.0559-0.000007$

$\begin{array}{lll}992.0 & -0.0103 & 0.000001\end{array}$

$\begin{array}{lll}998.8 & -0.0458 & 0.000002\end{array}$

$1011.5 \quad 0.1440-0.000003$

$\begin{array}{llll}1011.5 & -0.1300 & 0.000003\end{array}$

$\begin{array}{llll}1036.1 & 0.2148 & -0.000003\end{array}$

$1082.2 \quad 0.4844-0.000003$

$1124.6 \quad 0.8827-0.000003$

$\begin{array}{llll}1162.2 & 0.4544 & -0.000001\end{array}$

$1233.9 \quad-0.7867 \quad 0.000002$

$\begin{array}{llll}989.4 & 0.0200 & -0.000002\end{array}$

$\begin{array}{lll}995.6 & -0.0175 & 0.000001\end{array}$

$\begin{array}{lll}1008.3 & -0.0662 & 0.000002\end{array}$

$\begin{array}{llll}1034.3 & -0.2869 & 0.000003\end{array}$

$\begin{array}{lll}1061.3 & -0.4312 & 0.000003\end{array}$

$\begin{array}{lll}1089.5 & -0.2750 & 0.000001\end{array}$
3.393

0.000

0.001

0.000

0.005

0.003

0.004

0.017

0.009

0.041

0.017

0.089

0.482

0.055

0.998

0.001

1.068

0.000

1.093

0.229

2.196

0.000

0.000

0.000

0.000

0.003

0.000

0.002

0.021

0.017

0.046

0.235

0.779

0.206

0.619

0.000

0.000

0.004

0.082

0.186

0.076
Apparent Density of molal water / volume / $\mathrm{kg} / \mathrm{m}^{3}$ 992.2158 992.2158

$29.6 \quad 990.2132$

$31.9 \quad 990.2132$ 990.2132

$32.3 \quad 990.2132$ 990.2132

$31.75 \quad 990.2132$

$31.833 \quad 990.2132$ 990.2132

$31.943 \quad 990.2132$ 990.2132 990.2132 990.2132 990.2132 990.2132 988.0363 988.0363 988.0363 988.0363 988.0363 988.0363

$31.31 \quad 985.6952$

$31.54 \quad 985.6952$ 985.6952

$31.76 \quad 985.6952$ 985.6952

$32.14 \quad 985.6952$

$32.346 \quad 985.6952$ 985.6952

$32.62 \quad 985.6952$ 985.6952 985.6952 985.6952 985.6952 985.6952 983.1989 983.1989 983.1989 983.1989 983.1989 983.1989 
$t /{ }^{\circ} \mathrm{C}$ mass frac Density

0.01509

0.03017

0.06039

0.12079

0.2514

0.501

0.01509

0.03017

0.06039

0.12079

0.2514

0.501

0.01509

0.03017

0.06039

0.12079

0.2514

0.501

0.01509

0.03017

0.06039

0.12079

\section{exp $\mathrm{kg} / \mathrm{m}^{3}$}

$\begin{array}{rrr}60 & 0.2000 & 1118.70 \\ 60 & 0.2400 & 1149.60 \\ 60 & 0.2800 & 1181.60 \\ 60 & 0.3000 & 1198.00 \\ 60 & 0.3500 & 1241.30 \\ 60 & 0.4000 & 1287.50 \\ 60 & 0.4500 & 1337.10 \\ 65 & 0.0013 & 981.35 \\ 65 & 0.0026 & 982.13 \\ 65 & 0.0051 & 983.71 \\ 65 & 0.0102 & 986.79 \\ 65 & 0.0209 & 993.46 \\ 65 & 0.0408 & 1005.91 \\ 75 & 0.0013 & 975.63 \\ 75 & 0.0026 & 976.42 \\ 75 & 0.0051 & 977.97 \\ 75 & 0.0102 & 981.02 \\ 75 & 0.0209 & 987.61 \\ 75 & 0.0408 & 999.97 \\ 80 & 0.0100 & 977.90 \\ 80 & 0.0200 & 984.00 \\ 80 & 0.0400 & 996.40 \\ 80 & 0.0800 & 1021.80 \\ 80 & 0.1200 & 1048.10 \\ 80 & 0.1600 & 1075.70 \\ 80 & 0.2000 & 1104.80 \\ 80 & 0.2400 & 1135.10 \\ 80 & 0.2800 & 1166.70 \\ 80 & 0.3000 & 1183.00 \\ 80 & 0.3500 & 1225.80 \\ 80 & 0.4000 & 1271.50 \\ 80 & 0.4500 & 1320.60 \\ 85 & 0.0013 & 969.41 \\ 85 & 0.0026 & 970.18 \\ 85 & 0.0051 & 971.72 \\ 85 & 0.0102 & 974.75 \\ 85 & 0.0209 & 981.30 \\ 85 & 0.0408 & 993.54 \\ 95 & 0.0013 & 962.67 \\ 95 & 0.0026 & 963.46 \\ 95 & 0.0051 & 964.99 \\ 95 & 0.0102 & 968.01\end{array}$

Ref

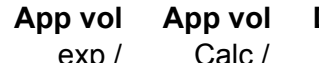

Calc

$\mathrm{m}^{3} / \mathrm{kg} \quad \mathrm{m}^{3} / \mathrm{kg}$

0.0004010 .000401

$\begin{array}{lll}0.000404 & 0.000404\end{array}$

$0.000407 \quad 0.000408$

$0.000409 \quad 0.000409$

$\begin{array}{ll}0.000413 & 0.000414\end{array}$

$0.000416 \quad 0.000417$

$0.000419 \quad 0.000421$

$0.000378 \quad 0.000385$

$0.000379 \quad 0.000385$

$0.000380 \quad 0.000385$

$0.000386 \quad 0.000386$

$0.000387 \quad 0.000387$

$0.000390 \quad 0.000389$

$\begin{array}{ll}0.000384 & 0.000393\end{array}$

$0.000382 \quad 0.000393$

$0.000385 \quad 0.000393$

$0.000390 \quad 0.000394$

$0.000392 \quad 0.000394$

$0.000395 \quad 0.000396$

$0.000387 \quad 0.000397$

$0.000391 \quad 0.000398$

$0.000394 \quad 0.000399$

$0.000400 \quad 0.000402$

$0.000405 \quad 0.000405$

$0.000408 \quad 0.000408$

$0.000410 \quad 0.000411$

$0.000412 \quad 0.000413$

$0.000415 \quad 0.000416$

$0.000417 \quad 0.000417$

$0.000420 \quad 0.000420$

$0.000423 \quad 0.000423$

$0.000425 \quad 0.000426$

$0.000376 \quad 0.000399$

$0.000384 \quad 0.000399$

$0.000387 \quad 0.000400$

$0.000393 \quad 0.000400$

$0.000395 \quad 0.000401$

$0.000398 \quad 0.000402$

$0.000392 \quad 0.000405$

$\begin{array}{ll}0.000381 & 0.000405\end{array}$

0.0003880 .000405

$0.000394 \quad 0.000405$
Density Density App Vol

Square of Inconsistent Inconsistent

$\mathrm{kg} / \mathrm{m}^{3} \quad \mathrm{~kg} / \mathrm{m}^{3} \quad \mathrm{~m}^{3} / \mathrm{kg}$ density apparent

$\begin{array}{llll}1118.8 & -0.1013 & 0.000000 & 0.010\end{array}$

$1149.4 \quad 0.1998-0.000001$

0.040

$1181.4 \quad 0.2296-0.000001$

$\begin{array}{lll}1197.9 & 0.0967 & 0.000000\end{array}$

$1240.9 \quad 0.3500-0.000001$

$0.8526-0.000001$

$1335.3 \quad 1.8243-0.000002$

$\begin{array}{llll}981.3 & 0.0091 & -0.000007\end{array}$

$982.1 \quad 0.0159-0.000006$

$983.7 \quad 0.0290-0.000006$

$\begin{array}{lll}986.8 & 0.0028 & 0.000000\end{array}$

$\begin{array}{lll}993.5 & 0.0060 & 0.000000\end{array}$

$\begin{array}{lll}1006.0 & -0.0555 & 0.000001\end{array}$

$975.6 \quad 0.0112-0.000009$

$976.4 \quad 0.0259-0.000011$

$977.9 \quad 0.0409-0.000008$

$981.0 \quad 0.0299-0.000003$

$987.6 \quad 0.0491-0.000002$

$999.9 \quad 0.0576-0.000001$

$977.8 \quad 0.0955-0.000010$

$983.9 \quad 0.1295-0.000007$

$996.2 \quad 0.2152-0.000005$

$1021.6 \quad 0.2261-0.000003$

$\begin{array}{lll}1048.0 & 0.0602 & 0.000000\end{array}$

$\begin{array}{lll}1075.7 & 0.0359 & 0.000000\end{array}$

$\begin{array}{lll}1104.5 & 0.2640 & -0.000001\end{array}$

$1134.8 \quad 0.3467-0.000001$

$1166.4 \quad 0.2766-0.000001$

$\begin{array}{lll}1182.8 & 0.1603 & 0.000000\end{array}$

$\begin{array}{lll}1225.7 & 0.0953 & 0.000000\end{array}$

$\begin{array}{llll}1271.4 & 0.1021 & 0.000000\end{array}$

$\begin{array}{ccc}1320.2 & 0.3696 & 0.000000\end{array}$

$969.4 \quad 0.0275-0.000023$

$970.1 \quad 0.0365-0.000015$

$\begin{array}{llll}971.7 & 0.0604 & -0.000013\end{array}$

$\begin{array}{llll}974.7 & 0.0631 & -0.000007\end{array}$

$981.2 \quad 0.1170-0.000006$

$993.4 \quad 0.1539-0.000004$

$\begin{array}{llll}962.7 & 0.0154 & -0.000013\end{array}$

$963.4 \quad 0.0567-0.000024$

$964.9 \quad 0.0829-0.000017$

$\begin{array}{llll}967.9 & 0.1117 & -0.000012\end{array}$
0.053

0.009

0.123

0.727

3.328

0.000

0.000

0.001

0.000

0.000

0.003

0.000

0.001

0.002

0.001

0.002

0.003

0.009

0.017

0.046

0.051

0.004

0.001

0.070

0.120

0.077

0.026

0.009

0.010

0.137

0.001

0.004

0.004

0.014

0.024

0.000

0.003

0.007

0.012
Apparent Density of volume / $\mathrm{kg} / \mathrm{m}^{3}$

983.1989 983.1989

983.1989

983.1989

983.1989

983.1989

983.1989

32.1

980.5548

$32.19 \quad 980.5548$

$32.26 \quad 980.5548$

$32.78 \quad 980.5548$

$32.872 \quad 980.5548$

$33.18 \quad 980.5548$

$32.6 \quad 974.849$

$32.49 \quad 974.849$

32.7974 .849

$33.19 \quad 974.849$

$33.324 \quad 974.849$

$33.553 \quad 974.849$

971.7978

971.7978

971.7978

971.7978

971.7978

971.7978

971.7978

971.7978

971.7978

971.7978

971.7978

971.7978

971.7978

968.6203

3266

32.9

968.6203

968.6203

968.6203

$33.568 \quad 968.6203$

$33.858 \quad 968.6203$

$33.3 \quad 961.9004$

$32.38 \quad 961.9004$

$32.94 \quad 961.9004$

$33.45 \quad 961.9004$ 


\begin{tabular}{|c|c|c|c|c|c|c|c|c|c|c|c|c|c|}
\hline \multirow{2}{*}{$\begin{array}{c}\text { molality } \\
0.2514\end{array}$} & \multirow{2}{*}{ molarity } & $\mathrm{t} /{ }^{\circ} \mathrm{C}$ & ass frac & $\begin{array}{r}\text { Density } \\
\text { exp } \mathrm{kg} / \mathrm{m}^{3}\end{array}$ & Ref & \multirow{2}{*}{$\begin{array}{r}\text { App vol } \\
\text { exp / } \\
\mathrm{m}^{3} / \mathrm{kg} \\
0.000395\end{array}$} & \multirow{2}{*}{$\begin{array}{r}\text { App vol } \\
\text { Calc / } \\
\mathrm{m}^{3} / \mathrm{kg} \\
0.000406\end{array}$} & \multirow{2}{*}{$\begin{array}{r}\text { Density } \\
\text { calc / } \\
\mathrm{kg} / \mathrm{m}^{3} \\
974.3\end{array}$} & \multirow{2}{*}{$\begin{array}{r}\text { Density } \\
\text { Residual / } \\
\mathrm{kg} / \mathrm{m}^{3} \\
0.2121\end{array}$} & \multirow{2}{*}{$\begin{array}{r}\text { App Vol } \\
\text { Residual / } \\
\mathrm{m}^{3} / \mathrm{kg} \\
-0.000011\end{array}$} & \multirow{2}{*}{$\begin{array}{r}\begin{array}{r}\text { Square of } \\
\text { Density Res }\end{array} \\
0.045\end{array}$} & \multirow{2}{*}{$\begin{array}{l}\text { Inconsistent } \\
\text { based on } \\
\text { density }\end{array}$} & $\begin{array}{l}\text { Inconsistent } \\
\text { based on } \\
\text { apparent } \\
\text { volume }\end{array}$ \\
\hline & & 95 & 0.0209 & 974.54 & (82) & & & & & & & & \\
\hline 0.501 & & 95 & 0.0408 & 986.74 & (82) & 0.000399 & 0.000407 & 986.4 & 0.3225 & -0.000008 & 0.104 & & \\
\hline & & 100 & 0.0100 & 964.40 & (90) & 0.000390 & 0.000408 & 964.2 & 0.1600 & -0.000017 & 0.026 & & \\
\hline & & 100 & 0.0200 & 970.40 & (90) & 0.000396 & 0.000408 & 970.2 & 0.2212 & -0.000012 & 0.049 & & \\
\hline & & 100 & 0.0400 & 982.60 & $(90)$ & 0.000400 & 0.000409 & 982.2 & 0.3515 & -0.000009 & 0.124 & & \\
\hline & & 100 & 0.0499 & 989.00 & (102) & 0.000396 & 0.000410 & 988.3 & 0.6800 & -0.000014 & 0.462 & & \\
\hline & & 100 & 0.0800 & 1007.80 & $(90)$ & 0.000404 & 0.000411 & 1007.2 & 0.6105 & -0.000008 & 0.373 & & \\
\hline & & 100 & 0.1200 & 1034.00 & (90) & 0.000407 & 0.000413 & 1033.3 & 0.7331 & -0.000006 & 0.537 & & \\
\hline & & 100 & 0.1600 & 1061.40 & (90) & 0.000410 & 0.000415 & 1060.6 & 0.8315 & -0.000005 & 0.691 & & \\
\hline & & 100 & 0.2000 & 1090.10 & $(90)$ & 0.000413 & 0.000417 & 1089.2 & 0.9093 & -0.000004 & 0.827 & & \\
\hline & & 100 & 0.2007 & 1091.00 & (102) & 0.000411 & 0.000417 & 1089.7 & 1.2960 & -0.000005 & 1.680 & & \\
\hline & & 100 & 0.2400 & 1120.00 & $(90)$ & 0.000416 & 0.000419 & 1119.2 & 0.7602 & -0.000003 & 0.578 & & \\
\hline & & 100 & 0.2800 & 1151.30 & $(90)$ & 0.000419 & 0.000420 & 1150.8 & 0.4669 & -0.000001 & 0.218 & & \\
\hline & & 100 & 0.2974 & 1167.00 & (102) & 0.000416 & 0.000421 & 1165.1 & 1.9094 & -0.000005 & 3.646 & & \\
\hline & & 100 & 0.3000 & 1167.40 & $(90)$ & 0.000421 & 0.000421 & 1167.2 & 0.1511 & 0.000000 & 0.023 & & \\
\hline & & 100 & 0.3500 & 1210.00 & (90) & 0.000423 & 0.000423 & 1210.2 & -0.2364 & 0.000000 & 0.056 & & \\
\hline & & 100 & 0.3970 & 1252.00 & (102) & 0.000427 & 0.000425 & 1253.4 & -1.4006 & 0.000002 & 1.962 & & \\
\hline & & 100 & 0.4000 & 1255.50 & $(90)^{\prime}$ & 0.000426 & 0.000425 & 1256.3 & -0.7531 & 0.000001 & 0.567 & & \\
\hline & & 100 & 0.4500 & 1304.40 & $(90)$ & 0.000428 & 0.000427 & 1305.6 & -1.2463 & 0.000002 & 1.553 & & \\
\hline & & 100 & 0.4682 & 1323.00 & (102) & 0.000429 & 0.000427 & 1324.5 & -1.5402 & 0.000002 & 2.372 & & \\
\hline & & & & & & & \multicolumn{2}{|c|}{ Average Res } & 0.0494 & -0.000003 & 93.4162 & Sum Chi sqr & \\
\hline & & & & & & & \multicolumn{2}{|c|}{ Std dev Res } & 0.6081 & 0.000007 & & & \\
\hline & & & & & & & \multicolumn{2}{|c|}{ Avg - 4std } & -2.3828 & -0.000032 & & & \\
\hline & & & & & & & \multicolumn{2}{|c|}{$A v g+4 s t d$} & 2.4816 & 0.000026 & & & \\
\hline
\end{tabular}

Apparent Density of molal water / $\begin{aligned} & \text { volume / } \\ & \mathrm{cm}^{3} / \mathrm{mol}\end{aligned} \mathrm{kg} / \mathrm{m}^{3}$ $33.594 \quad 961.9004$ $33.911 \quad 961.9004$ 958.3637 958.3637 958.3637 958.3637 958.3637 958.3637 958.3637 958.3637 958.3637 958.3637 958.3637 958.3637 958.3637 958.3637 958.3637 958.3637 958.3637 958.3637

Curve fit for the model App vol= $\left(w+c 2+c 3^{\star} t{ }^{\circ} \mathrm{C}\right) /\left(\mathrm{c0} 0^{*} \mathrm{w}+\mathrm{c} 1\right) / \mathrm{e}^{\wedge}\left(0.000001^{\star}\left(\mathrm{t}^{\circ} \mathrm{C}+\mathrm{c} 4\right)^{\wedge} 2\right)$

$\begin{array}{lr}\text { c0 } & 49.20868 \\ \text { c1 } 2 & 94.73699 \\ \text { c3 } & 0.779268 \\ \text { c4 } & 0.007545 \\ \text { Min T } & 1819.194 \\ \text { Max T } & 0 \\ \text { Min w } & 100 \\ \text { Max w } & 0.0013 \\ \text { Avg dens res } & 0.4682 \\ \text { Std dens res } & 0.049383 \\ \text { Avg app vol res } & 0.608053\end{array}$

Avg app vol res *10^3 -0.003057 Std app vol res *10^3 0.007157

No of points in corr

252

No of inconsistent poir 
molality molarity

$t /{ }^{\circ} \mathrm{C}$ mass frac Density

$\operatorname{exp~} \mathrm{kg} / \mathrm{m}^{3}$
Ref $\begin{array}{rr}\text { App vol } & \text { App vol } \\ \exp / & \text { Calc / }\end{array}$

$\mathrm{m}^{3} / \mathrm{kg} \quad \mathrm{m}^{3} / \mathrm{kg}$
Dens

Density App Vo

calc / Residual / Residual /

$\mathrm{kg} / \mathrm{m}^{3}$

ual / D

Square of Inconsistent
based on
density Inconsisten parent volume
Apparent Density of molal water / volume / $\mathrm{kg} / \mathrm{m}^{3}$

Inconsistent data not used (Residual greater than average +/- $\mathbf{4}$ standard deviations)$$
8
$$

4.5

0.10845

0.01203

0.06016

0.10807

0.16813

0.01202

0.06012

0.10791

0.16772

0.22779

0.012

0.06

0.10777
0.16729

0.22731

8 $\begin{array}{llllll}15 & 0.4047 & 1318.96 & (45) & 0.000401 & 0.000391\end{array}$

$\begin{array}{llllll}20 & 0.4047 & 1315.32 & (45) & 0.000405 & 0.000395\end{array}$

$\begin{array}{llllll}25 & 0.0061 & 1000.80 & (50) & 0.000385 & 0.000342\end{array}$

$\begin{array}{lll}25 & 0.2767 & 1200.10\end{array}$

$25 \quad 0.4047 \quad 1311.74$

$25 \quad 0.4776 \quad 1386.40$

$29.85 \quad 0.0010 \quad 996.18$

29.85

29.85

$0.0051 \quad 998.54$

$0.0092 \quad 1001.23$

$0.4047 \quad 1308.08$

34.85

34.85

34.85

$0.0010 \quad 994.44$

$0.0051 \quad 996.37$

$0.0092 \quad 998.79$

$0.0143 \quad 1001.99$

34.85

35

$0.0193 \quad 1005.74$

$0.4047 \quad 1304.42$

39.85

39.85
39.85

0.0010

0.0051

0.0092

992.59

$(22)$
$(45)$

$(45)$
$(110)$

$\begin{array}{lll}0.000390 & 0.000383\end{array}$

0.0004080 .000399

$\begin{array}{ll}0.000413 & 0.000408\end{array}$

$\begin{array}{ll}0.000526 & 0.000347\end{array}$

$\begin{array}{lll}(110) & 0.000446 & 0.000348\end{array}$

$\begin{array}{lll}\text { (110) } & 0.000401 & 0.000349\end{array}$

(45)

$0.000412 \quad 0.000403$

$\begin{array}{lll}\text { (110) } & 0.000655 & 0.000354\end{array}$

$\begin{array}{lll}\text { (110) } & 0.000556 & 0.000354\end{array}$

(110)

$0.000490 \quad 0.000355$

$\begin{array}{lll}(110) & 0.000449 & 0.000356\end{array}$

$\begin{array}{lll}\text { (110) } & 0.000402 \quad 0.000357\end{array}$

(45)

$0.000415 \quad 0.000406$

$0.000695 \quad 0.000360$

$0.000598 \quad 0.000360$

$0.000500 \quad 0.000361$

39.85

(110)

$\begin{array}{lll}0.000481 & 0.000362\end{array}$

$0.0193 \quad 1003.27 \quad(110)$

$0.0010 \quad 990.57 \quad(110)$

$0.000435 \quad 0.000362$

$0.000719 \quad 0.000366$

44.85

0.0051

992.21

(110)

$0.000627 \quad 0.000366$

44.85

$0.0092 \quad 994.46 \quad(110)$

$0.0143 \quad 997.22 \quad(110)$

$0.000549 \quad 0.000367$

$\begin{array}{ll}0.000517 & 0.000367\end{array}$

44.85

0.0193

1001.15

(110)

$\begin{array}{ll}0.4047 & 1296.85 \\ 0.4047 & 1289.21\end{array}$

$0.0993 \quad 1023.00$
$(45)$
$(45)$

$(45)$
$(102)$

$\begin{array}{rrr}1326.0 & -6.9926 & 0.000010 \\ 1322.1 & -6.8207 & 0.000010 \\ 1001.1 & -0.2646 & 0.000043 \\ 1202.8 & -2.6529 & 0.000007 \\ 1318.3 & -6.5435 & 0.000009 \\ 1391.6 & -5.1646 & 0.000006 \\ 996.4 & -0.1825 & 0.000179 \\ 999.0 & -0.5004 & 0.000098 \\ 1001.7 & -0.4825 & 0.000052 \\ 1314.4 & -6.3207 & 0.000009 \\ 994.7 & -0.3064 & 0.000301 \\ 997.4 & -1.0284 & 0.000202 \\ 1000.0 & -1.2440 & 0.000135 \\ 1003.3 & -1.3409 & 0.000094 \\ 1006.6 & -0.8770 & 0.000045 \\ 1310.5 & -6.0865 & 0.000009 \\ 992.9 & -0.3401 & 0.000335 \\ 995.6 & -1.2093 & 0.000238 \\ 998.2 & -1.2766 & 0.000139 \\ 1001.4 & -1.6966 & 0.000119 \\ 1004.7 & -1.4175 & 0.000073 \\ 990.9 & -0.3569 & 0.000353 \\ 993.5 & -1.3211 & 0.000261 \\ 996.1 & -1.6597 & 0.000182 \\ 999.3 & -2.1216 & 0.000149 \\ 1002.6 & -1.4225 & 0.000073 \\ 1302.7 & -5.8724 & 0.000009 \\ 1295.0 & -5.7712 & 0.000009 \\ 1019.6 & 3.3752 & -0.000033\end{array}$

$0.000424 \quad 0.000416$

1019.6

$\begin{aligned} 48.896 & ! \\ 46.521 & ! \\ 0.070 & \\ 7.038 & ! \\ 42.817 & ! \\ 26.673 & ! \\ 0.033 & \\ 0.250 & \\ 0.233 & \\ 39.951 & ! \\ 0.094 & \\ 1.058 & \\ 1.548 & \\ 1.798 & \\ 0.769 & \\ 37.046 & ! \\ 0.116 & \\ 1.462 & \\ 1.630 & \\ 2.878 & \\ 2.009 & \\ 0.127 & \\ 1.745 & \\ 2.754 & \\ 4.501 & \\ 2.023 & \\ 34.485 & ! \\ 33.306 & ! \\ 11.392 & !\end{aligned}$

999.0996 998.2041 997.0449 997.0449 997.0449 997.0449 995.6924 995.6924 995.6924 995.6473 994.0833 994.0833 994.0833 994.0833 994.0833 994.0319 992.273 992.273 992.273 992.273 992.273 990.2759 990.2759 990.2759 990.2759 990.2759 990.2132 985.6952 958.3637 
Density of aqueous solutions of $\mathrm{NaOH}$

References (3) Akerlof, G.; Kegeles, G.; The density of aqueous solutions of sodium hydroxide, J. Am. Chem., 1939, 61, 1027-1032

(39) Hershey, J. P.; Sotolongo, S.; Millero, F. J. Densities and Compressiblities of Aqueous $\mathrm{HCl}$ and $\mathrm{NaOH}$ from 0 to $45^{\circ} \mathrm{C}$. The Effect of Pressure on the lonization of Water. J. Sol. Chem. 1984, 13, 825-848.

(41) Hitchcock, L. B.; Mcllhenny, J. S. Viscosity and Density of Pure Alkaline Solutions and their Mixtures. Ind. Engng. Chem. 1935, 27, 461466

(54) Krey, J.; Vapour Pressure and Density of the System Water-Sodium Hydroxide. Z. Physik. Chem. N. F. 1972, 81, 252-273.

(66) Maksimova, I. N.; Yushkevich, V. F. Electrical Conductivity of Sodium Hydroxide Solutions at High Temperatures. Z. Fiz. Khim. 1963, 37, 903-907.

(73) Millero, F. J.; Ward, G. K.; Chetirkin, P. V. Relative Sound Velocities of Sea Salts at $25^{\circ}$ C. J. Acoust. Soc. Am. 1977, $61,1492-1498$.

(81) Patterson, B. A.; Call, T. G.; Jardine, J. J.; Origlia-Luster, M. L.; Woolley, E. M.; Thermodynamics for lonization of Water at Temperatures from $278.15 \mathrm{~K}$ to $393.15 \mathrm{~K}$ and at the Pressure $0.35 \mathrm{MPa}$ : Apparent Molar Volumes of Aqueous $\mathrm{KCl}, \mathrm{KOH}$, and $\mathrm{NaOH}$ and Apparent Molar Heat Capacities of Aqueous $\mathrm{HCl}, \mathrm{KCl}, \mathrm{KOH}$, and $\mathrm{NaOH}, \mathrm{J}$. Chem. Thermodynamics, 2001, 33,1237-1262

(88) Perron, G., Desnoyers, J. E., Millero, F. J., Apparent molal volumes and heat capacities of some sulfates and carbonates in water at 25 ${ }^{\circ} \mathrm{C}$, Can. J. Chem. , 1975, 53, 1134-1138

(90) Perry, R. H.; Green, D. W. Perry's Chemical Engineers' Handbook; McGraw Hill: New York, 7th edition, 1997.

(109) Roux, A. H.; Perron, G.; Desnoyers, J. E. Capacités calorifiques, volumes, expansibilités et compressibilités des solutions aqueuses concentrées de LiOH, $\mathrm{NaOH}$ et KOH. Can. J. Chem. 1984, 62, 878-885

\begin{tabular}{|c|c|c|c|c|c|c|c|c|c|c|c|c|c|c|c|}
\hline molality molarity & $\mathrm{t} /{ }^{\circ} \mathrm{C}$ & ass frac & $\begin{array}{r}\text { Density } \\
\exp / \\
\mathrm{kg} / \mathrm{m}^{3}\end{array}$ & Ref & $\begin{array}{c}\text { App vol } \\
\exp / \\
\mathrm{m}^{3} / \mathrm{kg}\end{array}$ & $\begin{array}{r}\text { App vol } \\
\text { Calc / } \\
\mathrm{m}^{3} / \mathrm{kg}\end{array}$ & $\begin{array}{r}\text { Density } \\
\mathrm{calc} / \\
\mathrm{kg} / \mathrm{m}^{3}\end{array}$ & $\begin{array}{r}\text { Density } \\
\text { Residual / } \\
\mathrm{kg} / \mathrm{m}^{3}\end{array}$ & $\begin{array}{r}\text { App Vol } \\
\text { Residual } / \\
\mathrm{m}^{3} / \mathrm{kg}\end{array}$ & $\begin{array}{r}\text { Square of } \\
\text { Density } \\
\text { Res }\end{array}$ & $\begin{array}{l}\text { Inconsistent } \\
\text { based on } \\
\text { density }\end{array}$ & $\begin{array}{l}\text { Inconsistent } \\
\text { based on } \\
\text { apparent } \\
\text { volume }\end{array}$ & $\begin{array}{r}\text { Apparent } \\
\text { molal volume } \\
/ \mathrm{cm}^{3} / \mathrm{mol}\end{array}$ & $\begin{array}{l}\text { Delta density } \\
\qquad / \mathrm{kg} / \mathrm{m}^{3}\end{array}$ & $\begin{array}{r}\text { Density of } \\
\text { water } / \mathrm{kg} / \mathrm{m}^{3}\end{array}$ \\
\hline & 0 & 0.0100 & 1012.40 & (90) & -0.000241 & -0.000098 & 1010.94 & 1.4633 & -0.000143 & 2.14 & & $!$ & & & 999.8395 \\
\hline & 0 & 0.0200 & 1023.76 & (3) & -0.000168 & -0.000087 & 1022.07 & 1.6918 & -0.000081 & 2.86 & & & & & 999.8395 \\
\hline & 0 & 0.0200 & 1024.40 & $(90)$ & -0.000199 & -0.000087 & 1022.07 & 2.3318 & -0.000111 & 5.44 & & & & & 999.8395 \\
\hline & 0 & 0.0400 & 1047.08 & $(3)$ & -0.000128 & -0.000067 & 1044.42 & 2.6565 & -0.000061 & 7.06 & & & & & 999.8395 \\
\hline & 0 & 0.0400 & 1048.20 & (90) & -0.000153 & -0.000067 & 1044.42 & 3.7765 & -0.000086 & 14.26 & & & & & 999.8395 \\
\hline & 0 & 0.0600 & 1070.19 & (3) & -0.000096 & -0.000047 & 1066.88 & 3.3066 & -0.000048 & 10.93 & & & & & 999.8395 \\
\hline & 0 & 0.0800 & 1093.21 & (3) & -0.000068 & -0.000028 & 1089.42 & 3.7854 & -0.000040 & 14.33 & & & & & 999.8395 \\
\hline & 0 & 0.0800 & 1094.30 & (90) & -0.000079 & -0.000028 & 1089.42 & 4.8754 & -0.000051 & 23.77 & & & & & 999.8395 \\
\hline & 0 & 0.1000 & 1116.14 & (3) & -0.000042 & -0.000009 & 1112.02 & 4.1174 & -0.000033 & 16.95 & & & & & 999.8395 \\
\hline & 0 & 0.1000 & 1117.44 & (54) & -0.000052 & -0.000009 & 1112.02 & 5.4169 & -0.000044 & 29.34 & & & & 117.60 & 999.8395 \\
\hline & 0 & 0.1200 & 1139.00 & (3) & -0.000018 & 0.000010 & 1134.65 & 4.3485 & -0.000028 & 18.91 & & & & & 999.8395 \\
\hline & 0 & 0.1200 & 1139.90 & (90) & -0.000024 & 0.000010 & 1134.65 & 5.2485 & -0.000034 & 27.55 & & & & & 999.8395 \\
\hline & 0 & 0.1400 & 1161.73 & (3) & 0.000005 & 0.000028 & 1157.28 & 4.4455 & -0.000024 & 19.76 & & & & & 999.8395 \\
\hline & 0 & 0.1600 & 1184.33 & (3) & 0.000026 & 0.000046 & 1179.89 & 4.4366 & -0.000020 & 19.68 & & & & & 999.8395 \\
\hline & 0 & 0.1600 & 1184.90 & (90) & 0.000024 & 0.000046 & 1179.89 & 5.0066 & -0.000022 & 25.07 & & & & & 999.8395 \\
\hline & 0 & 0.1800 & 1206.61 & (3) & 0.000048 & 0.000064 & 1202.45 & 4.1609 & -0.000016 & 17.31 & & & & & 999.8395 \\
\hline & 0 & 0.2000 & 1228.63 & (3) & 0.000069 & 0.000081 & 1224.92 & 3.7088 & -0.000012 & 13.76 & & & & & 999.8395 \\
\hline & 0 & 0.2000 & 1229.64 & (54) & 0.000066 & 0.000081 & 1224.92 & 4.7183 & -0.000016 & 22.26 & & & & 229.80 & 999.8395 \\
\hline & 0 & 0.2000 & 1229.60 & (90) & 0.000066 & 0.000081 & 1224.92 & 4.6788 & -0.000016 & 21.89 & & & & & 999.8395 \\
\hline & 0 & 0.2200 & 1250.23 & (3) & 0.000090 & 0.000098 & 1247.28 & 2.9513 & -0.000009 & 8.71 & & & & & 999.8395 \\
\hline & 0 & 0.2400 & 1271.33 & (3) & 0.000110 & 0.000115 & 1269.49 & 1.8405 & -0.000005 & 3.39 & & & & & 999.8395 \\
\hline & 0 & 0.2400 & 1274.10 & (90) & 0.000103 & 0.000115 & 1269.49 & 4.6105 & -0.000012 & 21.26 & & & & & 999.8395 \\
\hline & 0 & 0.2600 & 1291.80 & (3) & 0.000131 & 0.000131 & 1291.52 & 0.2790 & -0.000001 & 0.08 & & & & & 999.8395 \\
\hline & 0 & 0.2800 & 1311.50 & (3) & 0.000151 & 0.000148 & 1313.34 & -1.8397 & 0.000004 & 3.38 & & & & & 999.8395 \\
\hline
\end{tabular}




$\begin{array}{rrrr}\text { molality } & \text { molarity } & \mathbf{t} /{ }^{\circ} \mathrm{C} \text { mass frac } & \text { Density } \\ & & & \text { exp } / \\ \mathrm{kg} / \mathrm{m}^{3}\end{array}$

Ref

$\begin{array}{rr}\text { App vol } & \text { App vol } \\ \exp / & \text { Calc / } \\ \mathrm{m}^{3} / \mathrm{kg} & \mathrm{m}^{3} / \mathrm{kg}\end{array}$

Density Density App Vol Square of Inconsistent Inconsistent

Apparent Delta density Density of $\mathrm{m}^{3} / \mathrm{kg} \quad \mathrm{m}^{3} / \mathrm{kg}$ $\mathrm{kg} / \mathrm{m}^{3}$

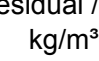

\begin{tabular}{|c|c|c|}
\hline & & 999.8395 \\
\hline & & 999.8395 \\
\hline & & 999.8395 \\
\hline & & 999.8395 \\
\hline & & 999.8395 \\
\hline & & 999.8395 \\
\hline & & 999.8395 \\
\hline & & 999.8395 \\
\hline & & 999.8395 \\
\hline & & 999.8395 \\
\hline & & 999.8395 \\
\hline & & 999.8395 \\
\hline & & 999.8395 \\
\hline & & 999.8395 \\
\hline & & 999.8395 \\
\hline & & 999.8395 \\
\hline & & 999.8395 \\
\hline & & 999.8395 \\
\hline & & 999.8395 \\
\hline & 0.992 & 999.972 \\
\hline & 1.485 & 999.972 \\
\hline & 1.97 & 999.972 \\
\hline & 2.461 & 999.972 \\
\hline & 2.935 & 999.972 \\
\hline & 3.901 & 999.972 \\
\hline & 4.889 & 999.972 \\
\hline & 14.39 & 999.972 \\
\hline & 23.63 & 999.972 \\
\hline & 45.82 & 999.972 \\
\hline & 45.947 & 999.972 \\
\hline & 86.901 & 999.972 \\
\hline & 124.54 & 999.972 \\
\hline & 191.208 & 999.972 \\
\hline & 262.516 & 999.972 \\
\hline & 340.774 & 999.972 \\
\hline-6.2 & & 999.9638 \\
\hline & 4.711 & 999.9638 \\
\hline-8.3 & & 999.9638 \\
\hline-7.53 & & 999.9638 \\
\hline & 9.387 & 999.9638 \\
\hline & 13.93 & 999.9638 \\
\hline-6.99 & & 999.9638 \\
\hline & 22.703 & 999.9638 \\
\hline & & 9999638 \\
\hline
\end{tabular}




\begin{tabular}{|c|c|c|c|c|c|c|c|c|c|c|c|}
\hline molality & molarity & \multicolumn{2}{|c|}{$\mathbf{t} /{ }^{\circ} \mathrm{C}$ mass frac } & $\begin{array}{r}\text { Density } \\
\exp / \\
\mathrm{kg} / \mathrm{m}^{3}\end{array}$ & Ref & $\begin{array}{r}\text { App vol } \\
\exp / \\
m^{3} / \mathrm{kg}\end{array}$ & $\begin{array}{r}\text { App vol } \\
\text { Calc / } \\
\mathrm{m}^{3} / \mathrm{kg}\end{array}$ & $\begin{array}{r}\text { Density } \\
\mathrm{calc} / \\
\mathrm{kg} / \mathrm{m}^{3}\end{array}$ & $\begin{array}{r}\text { Density } \\
\text { Residual / } \\
\mathrm{kg} / \mathrm{m}^{3}\end{array}$ & $\begin{array}{r}\text { App Vol } \\
\text { Residual / } \\
\mathrm{m}^{3} / \mathrm{kg}\end{array}$ & $\begin{array}{r}\text { Square of } \\
\text { Density } \\
\text { Res }\end{array}$ \\
\hline 0.6 & & 5 & 0.0234 & 1027.38 & (39) & -0.000139 & -0.000080 & 1025.93 & 1.4473 & -0.000059 & 2.09 \\
\hline 0.71076 & & 5 & 0.0276 & 1031.89 & (39) & -0.000119 & -0.000076 & 1030.61 & 1.2780 & -0.000043 & 1.63 \\
\hline 0.78379 & & 5 & 0.0304 & 1035.51 & (39) & -0.000129 & -0.000073 & 1033.68 & 1.8283 & -0.000056 & 3.34 \\
\hline 0.87099 & & 5 & 0.0337 & 1039.33 & (39) & -0.000125 & -0.000070 & 1037.32 & 2.0103 & -0.000055 & 4.04 \\
\hline 0.99676 & & 5 & 0.0383 & 1044.81 & (39) & -0.000120 & -0.000065 & 1042.54 & 2.2774 & -0.000055 & 5.19 \\
\hline 0.02035 & & 10 & 0.0008 & 1000.66 & (109) & -0.000175 & -0.000099 & 1000.59 & 0.0615 & -0.000075 & 0.00 \\
\hline 0.03045 & & 10 & 0.0012 & 1001.13 & (109) & -0.000175 & -0.000099 & 1001.04 & 0.0929 & -0.000076 & 0.01 \\
\hline 0.04054 & & 10 & 0.0016 & 1001.60 & (109) & -0.000175 & -0.000098 & 1001.48 & 0.1241 & -0.000076 & 0.02 \\
\hline 0.05078 & & 10 & 0.0020 & 1002.08 & (109) & -0.000173 & -0.000098 & 1001.93 & 0.1531 & -0.000075 & 0.02 \\
\hline 0.06058 & & 10 & 0.0024 & 1002.54 & (109) & -0.000172 & -0.000098 & 1002.36 & 0.1796 & -0.000074 & 0.03 \\
\hline 0.08075 & & 10 & 0.0032 & 1003.47 & (109) & -0.000167 & -0.000097 & 1003.24 & 0.2275 & -0.000070 & 0.05 \\
\hline 0.10144 & & 10 & 0.0040 & 1004.43 & (109) & -0.000165 & -0.000096 & 1004.15 & 0.2809 & -0.000069 & 0.08 \\
\hline 0.3042 & & 10 & 0.0120 & 1013.67 & (109) & -0.000146 & -0.000088 & 1012.95 & 0.7232 & -0.000059 & 0.52 \\
\hline \multirow{2}{*}{\multicolumn{2}{|c|}{0.5067}} & 10 & 0.0199 & 1022.68 & (109) & -0.000131 & -0.000080 & 1021.61 & 1.0616 & -0.000051 & 1.13 \\
\hline & & 10 & 0.0200 & 1022.42 & (3) & -0.000111 & -0.000080 & 1021.76 & 0.6554 & -0.000031 & 0.43 \\
\hline 1.0126 & & 10 & 0.0389 & 1044.37 & (109) & -0.000099 & -0.000061 & 1042.76 & 1.6100 & -0.000038 & 2.59 \\
\hline \multirow{3}{*}{\multicolumn{2}{|c|}{1.0158}} & 10 & 0.0390 & 1044.49 & (109) & -0.000098 & -0.000061 & 1042.89 & 1.6035 & -0.000038 & 2.57 \\
\hline & & 10 & 0.0400 & 1044.89 & (3) & -0.000081 & -0.000060 & 1043.95 & 0.9354 & -0.000021 & 0.88 \\
\hline & & 10 & 0.0600 & 1067.26 & (3) & -0.000055 & -0.000040 & 1066.25 & 1.0116 & -0.000015 & 1.02 \\
\hline \multirow{4}{*}{\multicolumn{2}{|c|}{2.02}} & 10 & 0.0748 & 1084.73 & (109) & -0.000049 & -0.000026 & 1082.75 & 1.9860 & -0.000023 & 3.94 \\
\hline & & 10 & 0.0800 & 1089.63 & (3) & -0.000032 & -0.000021 & 1088.62 & 1.0065 & -0.000011 & 1.01 \\
\hline & & 10 & 0.1000 & 1111.20 & (3) & -0.000003 & -0.000002 & 1111.06 & 0.1438 & -0.000001 & 0.02 \\
\hline & & 10 & 0.1000 & 1113.00 & (54) & -0.000018 & -0.000002 & 1111.06 & 1.9435 & -0.000016 & 3.78 \\
\hline \multirow{4}{*}{\multicolumn{2}{|c|}{3.026}} & 10 & 0.1080 & 1121.90 & (109) & -0.000009 & 0.000005 & 1120.00 & 1.8988 & -0.000014 & 3.61 \\
\hline & & 10 & 0.1200 & 1134.45 & (3) & 0.000010 & 0.000016 & 1133.52 & 0.9286 & -0.000006 & 0.86 \\
\hline & & 10 & 0.1400 & 1156.86 & (3) & 0.000030 & 0.000034 & 1155.99 & 0.8668 & -0.000005 & 0.75 \\
\hline & & 10 & 0.1600 & 1179.25 & (3) & 0.000048 & 0.000052 & 1178.44 & 0.8056 & -0.000004 & 0.65 \\
\hline \multirow{5}{*}{\multicolumn{2}{|c|}{5.027}} & 10 & 0.1674 & 1188.11 & (109) & 0.000053 & 0.000059 & 1186.75 & 1.3602 & -0.000006 & 1.85 \\
\hline & & 10 & 0.1800 & 1201.55 & (3) & 0.000067 & 0.000069 & 1200.85 & 0.7033 & -0.000003 & 0.49 \\
\hline & & 10 & 0.2000 & 1223.71 & (3) & 0.000085 & 0.000087 & 1223.17 & 0.5389 & -0.000002 & 0.29 \\
\hline & & 10 & 0.2000 & 1223.70 & (54) & 0.000085 & 0.000087 & 1223.17 & 0.5285 & -0.000002 & 0.28 \\
\hline & & 10 & 0.2200 & 1245.67 & (3) & 0.000102 & 0.000103 & 1245.39 & 0.2827 & -0.000001 & 0.08 \\
\hline \multirow{6}{*}{\multicolumn{2}{|c|}{7.532}} & 10 & 0.2315 & 1259.09 & (109) & 0.000110 & 0.000113 & 1258.12 & 0.9711 & -0.000003 & 0.94 \\
\hline & & 10 & 0.2400 & 1267.31 & (3) & 0.000120 & 0.000120 & 1267.46 & -0.1546 & 0.000000 & 0.02 \\
\hline & & 10 & 0.2600 & 1288.55 & (3) & 0.000138 & 0.000136 & 1289.37 & -0.8211 & 0.000002 & 0.67 \\
\hline & & 10 & 0.2800 & 1309.29 & (3) & 0.000156 & 0.000152 & 1311.07 & -1.7848 & 0.000004 & 3.19 \\
\hline & & 10 & 0.3000 & 1329.40 & (3) & 0.000173 & 0.000167 & 1332.54 & -3.1428 & 0.000006 & 9.88 \\
\hline & & 10 & 0.3000 & 1332.20 & (54) & 0.000168 & 0.000167 & 1332.54 & -0.3432 & 0.000001 & 0.12 \\
\hline \multirow{6}{*}{\multicolumn{2}{|c|}{10.88}} & 10 & 0.3032 & 1337.02 & (109) & 0.000168 & 0.000170 & 1335.97 & 1.0467 & -0.000002 & 1.10 \\
\hline & & 10 & 0.3200 & 1349.96 & (3) & 0.000189 & 0.000183 & 1353.74 & -3.7819 & 0.000006 & 14.30 \\
\hline & & 10 & 0.3400 & 1372.43 & (3) & 0.000201 & 0.000198 & 1374.64 & -2.2088 & 0.000003 & 4.88 \\
\hline & & 10 & 0.3600 & 1396.54 & (3) & 0.000211 & 0.000213 & 1395.20 & 1.3400 & -0.000002 & 1.80 \\
\hline & & 10 & 0.3800 & 1416.29 & (3) & 0.000226 & 0.000227 & 1415.39 & 0.8982 & -0.000001 & 0.81 \\
\hline & & 10 & 0.4000 & 1437.44 & (3) & 0.000239 & 0.000241 & 1435.18 & 2.2589 & -0.000003 & 5.10 \\
\hline
\end{tabular}

\begin{tabular}{|c|c|c|c|}
\hline $\begin{array}{l}\text { consisten } \\
\text { based on }\end{array}$ & $\begin{array}{r}\text { Apparent } \\
\text { molal volume }\end{array}$ & $\begin{array}{l}\text { Delta density } \\
\qquad / \mathrm{kg} / \mathrm{m}^{3}\end{array}$ & $\begin{array}{r}\text { Density of } \\
\text { water } / \mathrm{kg} / \mathrm{m}^{3}\end{array}$ \\
\hline & & 27.417 & 999.9638 \\
\hline & & 31.928 & 999.9638 \\
\hline & & 35.545 & 999.9638 \\
\hline & & 39.369 & 999.9638 \\
\hline & & 44.851 & 999.9638 \\
\hline & & 0.956 & 999.6996 \\
\hline & & 1.431 & 999.6996 \\
\hline & & 1.905 & 999.6996 \\
\hline & & 2.383 & 999.6996 \\
\hline & & 2.839 & 999.6996 \\
\hline & & 3.77 & 999.6996 \\
\hline & & 4.728 & 999.6996 \\
\hline & & 13.969 & 999.6996 \\
\hline & & 22.976 & 999.6996 \\
\hline & & & 999.6996 \\
\hline & & 44.669 & 999.6996 \\
\hline & & 44.794 & 999.6996 \\
\hline & & & 999.6996 \\
\hline & & & 999.6996 \\
\hline & & 85.035 & 999.6996 \\
\hline & & & 999.6996 \\
\hline & & & 999.6996 \\
\hline & & 113.30 & 999.6996 \\
\hline & & 122.199 & 999.6996 \\
\hline & & & 999.6996 \\
\hline & & & 999.6996 \\
\hline & & & 999.6996 \\
\hline & & 188.408 & 999.6996 \\
\hline & & & 999.6996 \\
\hline & & & 999.6996 \\
\hline & & 224.00 & 999.6996 \\
\hline & & & 999.6996 \\
\hline & & 259.387 & 999.6996 \\
\hline & & & 999.6996 \\
\hline & & & 999.6996 \\
\hline & & & 999.6996 \\
\hline & & & 999.6996 \\
\hline & & 332.50 & 999.6996 \\
\hline & & 337.32 & 999.6996 \\
\hline & & & 999.6996 \\
\hline & & & 999.6996 \\
\hline & & & 999.6996 \\
\hline & & & 999.6996 \\
\hline & & & 999.6996 \\
\hline
\end{tabular}




\begin{tabular}{|c|c|c|c|c|}
\hline \multirow[t]{7}{*}{ molality } & \multirow[t]{7}{*}{ molarity } & \multicolumn{2}{|c|}{$\mathrm{t} /{ }^{\circ} \mathrm{C}$ mass frac } & $\begin{array}{r}\text { Density } \\
\exp / \\
\mathrm{kg} / \mathrm{m}^{3}\end{array}$ \\
\hline & & 10 & 0.4200 & 1457.83 \\
\hline & & 10 & 0.4400 & 1477.32 \\
\hline & & 10 & 0.4600 & 1495.68 \\
\hline & & 10 & 0.4800 & 1512.72 \\
\hline & & 10 & 0.5000 & 1528.14 \\
\hline & & 10 & 0.5200 & 1541.74 \\
\hline 0.07496 & & 15 & 0.0030 & 1002.51 \\
\hline 0.0998 & & 15 & 0.0040 & 1003.59 \\
\hline 0.10003 & & 15 & 0.0040 & 1003.74 \\
\hline 0.15009 & & 15 & 0.0060 & 1005.93 \\
\hline \multirow[t]{2}{*}{0.2022} & & 15 & 0.0080 & 1008.16 \\
\hline & & 15 & 0.0100 & 1010.65 \\
\hline 0.2941 & & 15 & 0.0116 & 1012.23 \\
\hline 0.30073 & & 15 & 0.0119 & 1012.72 \\
\hline 0.39 & & 15 & 0.0154 & 1016.45 \\
\hline 0.4842 & & 15 & 0.0190 & 1020.56 \\
\hline \multirow[t]{2}{*}{0.50002} & & 15 & 0.0196 & 1021.46 \\
\hline & & 15 & 0.0200 & 1021.98 \\
\hline 0.5875 & & 15 & 0.0230 & 1025.03 \\
\hline 0.7021 & & 15 & 0.0273 & 1029.96 \\
\hline 0.7829 & & 15 & 0.0304 & 1033.41 \\
\hline 0.8704 & & 15 & 0.0336 & 1037.12 \\
\hline \multirow[t]{22}{*}{1.0036} & & 15 & 0.0386 & 1042.74 \\
\hline & & 15 & 0.0400 & 1044.41 \\
\hline & & 15 & 0.0800 & 1088.87 \\
\hline & & 15 & 0.1200 & 1133.27 \\
\hline & & 15 & 0.1600 & 1177.61 \\
\hline & & 15 & 0.2000 & 1221.83 \\
\hline & & 15 & 0.2400 & 1265.82 \\
\hline & & 15 & 0.2800 & 1309.40 \\
\hline & & 15 & 0.3200 & 1352.00 \\
\hline & & 15 & 0.3600 & 1393.30 \\
\hline & & 15 & 0.4000 & 1433.40 \\
\hline & & 15 & 0.4400 & 1472.00 \\
\hline & & 15 & 0.4800 & 1510.20 \\
\hline & & 15 & 0.5000 & 1529.00 \\
\hline & & 20 & 0.0005 & 998.70 \\
\hline & & 20 & 0.0099 & 1008.60 \\
\hline & & 20 & 0.0100 & 1009.50 \\
\hline & & 20 & 0.0200 & 1020.22 \\
\hline & & 20 & 0.0200 & 1020.70 \\
\hline & & 20 & 0.0400 & 1042.07 \\
\hline & & 20 & 0.0400 & 1042.80 \\
\hline & 1.1 & 20 & 0.0424 & 1045.40 \\
\hline
\end{tabular}

Ref

$\begin{array}{rr}\text { App vol } & \text { App vol } \\ \exp / & \text { Calc / } \\ \mathrm{m}^{3} / \mathrm{kg} & \mathrm{m}^{3} / \mathrm{kg}\end{array}$

Density

Density App Vol Square of Inconsistent Inconsistent

0.0002520 .000256

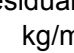

$\mathrm{kg} / \mathrm{m}^{3}$

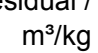

$\begin{array}{ll}0.000265 & 0.000269\end{array}$

1454.53

$3.8998-0.000004$

$3.8743-0.000004$

$3.0598-0.000003$

$1.1866-0.000001$

$\begin{array}{ll}-1.9165 & 0.000002\end{array}$

$0.1302-0.000043$

$0.1371-0.000034$

$0.2754-0.000069$

$0.2936-0.000049$

$0.2658-0.000033$

$0.5814-0.000057$

$0.3747-0.000031$

$0.5765-0.000047$

$0.4843-0.000031$

$0.5874-0.000030$

$0.8234-0.000040$

$0.9087-0.000044$

$0.6967-0.000029$

$0.8159-0.000028$

$0.8993-0.000028$

$\begin{array}{lll}0.9892 & -0.000027\end{array}$

$1.1340-0.000027$

$1.2422-0.000029$

$1.2195-0.000013$

$0.9038-0.000006$

$0.4950-0.000002$

$0.1501-0.000001$

$-0.00850 .000000$

$0.0844 \quad 0.000000$
0.1136

$\begin{array}{lll}0.1136 & 0.000000\end{array}$

$0.0205 \quad 0.000000$

$0.1682 \quad 0.000000$

$0.5176-0.000001$

$2.4222-0.000002$

$3.8837-0.000003$

$\begin{array}{lll}-0.0493 & 0.000099\end{array}$

$-0.4142 \quad 0.000041$

$0.3764-0.000037$

$0.1434-0.000007$

$0.6234-0.000030$

$\begin{array}{ll}-0.0040 & 0.000000\end{array}$

$0.7260-0.000017$

$\begin{array}{lll}0.6452 & -0.000014\end{array}$

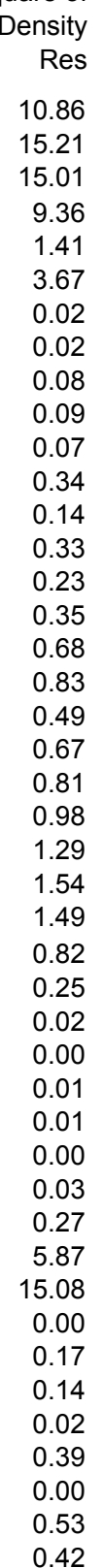

sistent Apparent Delta density Density of $/ \mathrm{cm}^{3} / \mathrm{mol}$ volume

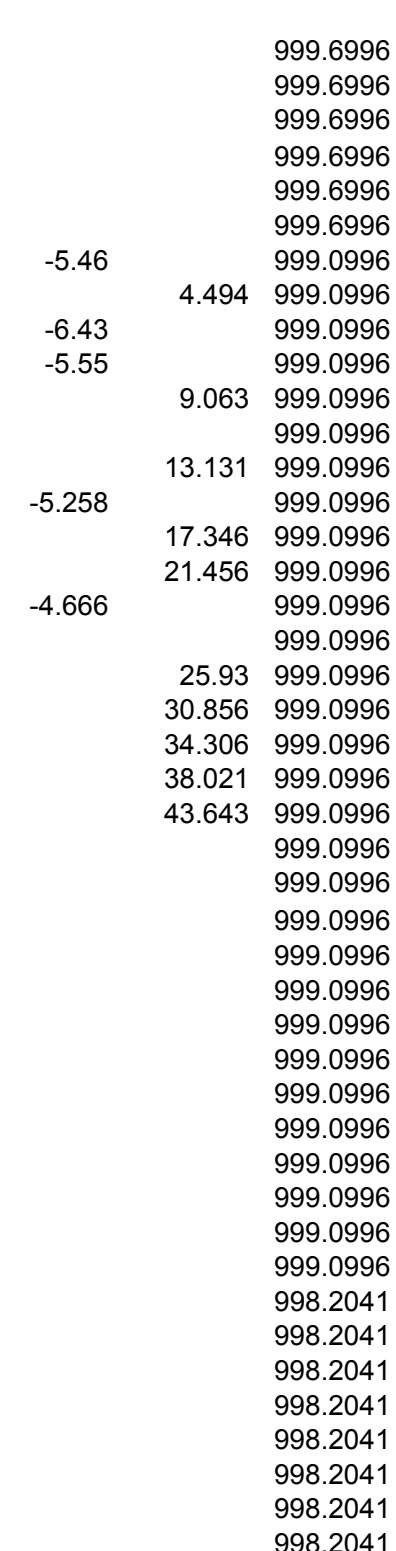


molality

\begin{tabular}{|c|c|c|c|}
\hline \multirow[t]{3}{*}{ nolarity } & \multicolumn{2}{|c|}{$\mathrm{t} /{ }^{\circ} \mathrm{C}$ mass frac } & $\begin{array}{r}\text { Density } \\
\exp / \\
\mathrm{kg} / \mathrm{m}^{3}\end{array}$ \\
\hline & 20 & 0.0476 & 1048.20 \\
\hline & 20 & 0.0600 & 1063.90 \\
\hline \multirow[t]{6}{*}{1.888} & 20 & 0.0702 & 1075.80 \\
\hline & 20 & 0.0800 & 1085.77 \\
\hline & 20 & 0.0800 & 1086.90 \\
\hline & 20 & 0.0910 & 1093.80 \\
\hline & 20 & 0.1000 & 1107.73 \\
\hline & 20 & 0.1000 & 1108.10 \\
\hline \multirow[t]{4}{*}{2.99} & 20 & 0.1071 & 1116.50 \\
\hline & 20 & 0.1200 & 1129.79 \\
\hline & 20 & 0.1200 & 1130.90 \\
\hline & 20 & 0.1400 & 1151.92 \\
\hline \multirow[t]{4}{*}{4.677} & 20 & 0.1594 & 1173.80 \\
\hline & 20 & 0.1600 & 1174.09 \\
\hline & 20 & 0.1600 & 1175.10 \\
\hline & 20 & 0.1800 & 1196.28 \\
\hline 5.8 & 20 & 0.1919 & 1209.00 \\
\hline \multirow[t]{7}{*}{6.032} & 20 & 0.1984 & 1216.20 \\
\hline & 20 & 0.2000 & 1218.84 \\
\hline & 20 & 0.2000 & 1217.80 \\
\hline & 20 & 0.2000 & 1219.10 \\
\hline & 20 & 0.2200 & 1240.49 \\
\hline & 20 & 0.2400 & 1262.37 \\
\hline & 20 & 0.2400 & 1262.90 \\
\hline \multirow[t]{20}{*}{8.309} & 20 & 0.2592 & 1282.40 \\
\hline & 20 & 0.2600 & 1284.02 \\
\hline & 20 & 0.2800 & 1305.30 \\
\hline & 20 & 0.2800 & 1306.40 \\
\hline & 20 & 0.3000 & 1326.12 \\
\hline & 20 & 0.3000 & 1325.70 \\
\hline & 20 & 0.3200 & 1346.37 \\
\hline & 20 & 0.3200 & 1349.00 \\
\hline & 20 & 0.3400 & 1365.90 \\
\hline & 20 & 0.3600 & 1387.48 \\
\hline & 20 & 0.3600 & 1390.00 \\
\hline & 20 & 0.3800 & 1409.06 \\
\hline & 20 & 0.4000 & 1432.15 \\
\hline & 20 & 0.4000 & 1429.20 \\
\hline & 20 & 0.4000 & 1430.00 \\
\hline & 20 & 0.4200 & 1450.47 \\
\hline & 20 & 0.4400 & 1469.98 \\
\hline & 20 & 0.4400 & 1468.50 \\
\hline & 20 & 0.4600 & 1488.47 \\
\hline & 20 & 0.4800 & 1505.69 \\
\hline
\end{tabular}

Ref

$\begin{array}{rr}\text { App vol } & \text { App vol } \\ \exp / & \text { Calc / } \\ \mathrm{m}^{3} / \mathrm{kg} & \mathrm{m}^{3} / \mathrm{kg}\end{array}$

(66) $\quad-0.000002-0.000045$

$-0.000029-0.000033$

$-0.000028-0.000024$

$-0.000008-0.000014$

$-0.000020-0.000014$

$0.000040-0.000004$

$\begin{array}{lll}0.000011 & 0.000004\end{array}$

$\begin{array}{ll}0.000008 & 0.000004\end{array}$

$0.000011 \quad 0.000011$

$0.000029 \quad 0.000022$

$0.000022 \quad 0.000022$

$0.000047 \quad 0.000040$

$0.000061 \quad 0.000057$

$0.000064 \quad 0.000058$

$0.000059 \quad 0.000058$

$0.000080 \quad 0.000075$

$\begin{array}{ll}0.000091 & 0.000085\end{array}$

$0.000097 \quad 0.000090$

$0.000095 \quad 0.000092$

$0.000099 \quad 0.000092$

$0.000094 \quad 0.000092$

$0.000112 \quad 0.000108$

$0.000128 \quad 0.000124$

$0.000127 \quad 0.000124$

$0.000145 \quad 0.000140$

$0.000144 \quad 0.000140$

$0.000160 \quad 0.000156$

$0.000158 \quad 0.000156$

$\begin{array}{lll}0.000176 & 0.000171\end{array}$

$0.000177 \quad 0.000171$

$\begin{array}{lll}0.000192 & 0.000187\end{array}$

$0.000188 \quad 0.000187$

$0.000209 \quad 0.000201$

$0.000221 \quad 0.000216$

$0.000217 \quad 0.000216$

$0.000233 \quad 0.000230$

$0.000243 \quad 0.000244$

$0.000247 \quad 0.000244$

$0.000246 \quad 0.000244$

$0.000258 \quad 0.000258$

$\begin{array}{lll}0.000271 & 0.000272\end{array}$

$\begin{array}{lll}0.000273 & 0.000272\end{array}$

$0.000284 \quad 0.000285$

$0.000298 \quad 0.000298$
Apparent Delta density Density of calc / Residual / Residual / Density based on based on molal volume $\mathrm{kg} / \mathrm{m}^{3} \quad \mathrm{~kg} / \mathrm{m}^{3} \quad \mathrm{~m}^{3} / \mathrm{kg} \quad$ Res density $\begin{gathered}\text { apparent } \\ \text { volume }\end{gathered}$ $1 \mathrm{~cm}^{3} / \mathrm{mol}$

$/ \mathrm{kg} / \mathrm{m}^{3}$ water $/ \mathrm{kg} / \mathrm{m}^{3}$ $1050.46 \quad-2.2614 \quad 0.000043$ 5.11 0.08 $\begin{array}{lll}0.18 & -0.2756 & 0.000004\end{array}$

$1075.47 \quad 0.3260-0.000004$ $\begin{array}{lll}1086.36 & -0.5899 & 0.000006\end{array}$ $1086.36 \quad 0.5401-0.000006$ $\begin{array}{lll}1098.59 & -4.7883 & 0.000044\end{array}$ $\begin{array}{lll}1108.60 & -0.8741 & 0.000007\end{array}$ $\begin{array}{lll}-0.5000 & 0.000004\end{array}$ $\begin{array}{cc}-0.5000 & 0.000004 \\ -0.0252 & 0.000000\end{array}$

0.11

0.35

0.29

22.93

0.76

0.25

$\begin{array}{lll}1116.53 & -0.0252 & 0.000\end{array}$

$\begin{array}{llll}1130.88 & -1.0943 & 0.000007 & 1.20\end{array}$

0.00

$0.0157 \quad 0.000000$

$\begin{array}{ll}-1.2555 & 0.000007\end{array}$

$\begin{array}{ll}-0.9488 & 0.000004\end{array}$

1.58

0.90

1.85

$\begin{array}{llll}1175.45 & -0.3515 & 0.000002 & 0.12\end{array}$

$\begin{array}{lll}1197.69 & -1.4054 & 0.000005\end{array}$

$\begin{array}{lll}1210.86 & -1.8617 & 0.000007\end{array}$

$\begin{array}{lll}1218.05 & -1.8511 & 0.000006\end{array}$

3.47

3.43

1.02

$\begin{array}{llll}1219.85 & -2.0450 & 0.000007 & 4.18\end{array}$

$\begin{array}{lll}1219.85 & -0.7491 & 0.000003\end{array}$

0.56

0.56
2.03

2.19

0.90

5.32

2.58

3.66

0.66

6.04

8.26

11.01

0.47

21.29

12.54

1.04

4.49

1.44

3.06

0.91

0.03

0.57

0.52

0.66

0.01
998.2041

998.2041

998.2041

998.2041

998.2041

998.2041

998.2041

$109.90 \quad 998.2041$

998.2041

998.2041

998.2041

998.2041

998.2041

998.2041

998.2041

998.2041

998.2041

998.2041

998.2041

$219.60 \quad 998.2041$

998.2041

998.2041

998.2041

998.2041

998.2041

998.2041

998.2041

998.2041

998.2041

$327.50 \quad 998.2041$

998.2041

998.2041

998.2041

998.2041

998.2041

998.2041

431.00

998.2041

998.2041

998.2041

998.2041

998.2041

998.2041 
molality molarity

$t /{ }^{\circ} \mathrm{C}$ mass frac

\section{exp}

$\mathrm{kg} / \mathrm{m}^{3}$

20

$0.4800 \quad 1506.50$

$0.4896 \quad 1512.00$

$0.5000 \quad 1521.42$

$0.5000 \quad 1524.00$

$\begin{array}{ll}0.5000 & 1525.30\end{array}$

$\begin{array}{rr}0.5200 & 1535.41 \\ 0.0015 & 998.77\end{array}$

$\begin{array}{ll}0.0015 & 998.77 \\ 0.0015 & 998.77\end{array}$

$0.0027 \quad 1000.10$

$\begin{array}{ll}0.0027 & 1000.10\end{array}$

$0.0030 \quad 1000.35$

$0.0038 \quad 1001.34$

$0.0038 \quad 1001.34$

$0.0040 \quad 1001.53$

$0.0040 \quad 1001.49$

$0.0040 \quad 1001.51$

$0.0058 \quad 1003.45$

$0.0058 \quad 1003.45$

$0.0060 \quad 1003.69$

$0.0076 \quad 1005.49$

$0.0076 \quad 1005.49$

$0.0079 \quad 1005.86$

$0.0079 \quad 1005.89$

$0.0119 \quad 1010.18$

$0.0119 \quad 1010.20$

$0.0119 \quad 1010.26$

$0.0155 \quad 1014.21$

$0.0155 \quad 1014.21$

$0.0158 \quad 1014.47$

$0.0158 \quad 1014.44$

$0.0194 \quad 1018.39$

$0.0194 \quad 1018.39$

$0.0196 \quad 1018.64$

$0.0196 \quad 1018.67$

$0.0196 \quad 1018.72$

$0.0197 \quad 1018.78$

$0.0234 \quad 1022.83$

$0.0234 \quad 1022.86$

$0.0272 \quad 1027.03$

$0.0272 \quad 1027.00$

$0.0310 \quad 1031.13$

$0.0310 \quad 1031.11$

$0.0348 \quad 1035.19$

$0.0348 \quad 1035.21$
Ref

$\begin{array}{cc}\text { App vol } & \text { App vol } \\ \exp / & \text { Calc / } \\ \mathrm{m}^{3} / \mathrm{kg} & \mathrm{m}^{3} / \mathrm{kg}\end{array}$

Density App Vol Square of Inconsistent
calc / Residual / Residual / Density based on

nconsistent

Apparent Delta density Density of

$$
\mathrm{m}^{3} / \mathrm{kg} \quad \mathrm{m}^{3} / \mathrm{kg}
$$

$\mathrm{kg} / \mathrm{m}^{3}$

$\mathrm{kg} / \mathrm{m}^{3} \quad \mathrm{~m}^{3} / \mathrm{kg}$

$0.9111-0.000001$

$\begin{array}{ll}-2.0056 & 0.000002\end{array}$

$\begin{array}{ll}-1.5605 & 0.000001\end{array}$

$1.0237-0.000001$

$2.3195-0.000002$

$\begin{array}{ll}-4.3967 & 0.000004\end{array}$

$0.0592-0.000039$

$0.0592-0.000039$

$0.1083-0.000040$

$0.1083-0.000040$

$0.0645-0.000022$

$0.1169-0.000030$

$0.1169-0.000030$

$0.1601-0.000040$

$0.1111-0.000028$

$0.1322-0.000033$

$0.1549-0.000027$

$0.1549-0.000027$

$0.1656-0.000028$

$0.1715-0.000022$

$0.1715-0.000022$

$0.1835-0.000023$

$0.2095-0.000026$

$0.2328-0.000019$

$0.2558-0.000021$

$0.2996-0.000025$

$0.2695-0.000017$

$0.2674-0.000017$

$0.2893-0.000018$

$0.2639-0.000016$

$0.2820-0.000014$

$0.2804-0.000014$

$0.2817-0.000014$

$0.3108-0.000015$

$0.3377-0.000017$

$0.2830-0.000014$

$0.2851-0.000012$

$0.3131-0.000013$

$0.3074-0.000011$

$0.2778-0.000010$

$0.2809-0.000009$

$0.2596-0.000008$

$0.2323-0.000006$

$0.2553-0.000007$
0.83

4.02

2.44

1.05

5.38
9.33

0.00

0.00

0.01

0.01

0.01

0.01

0.03

0.01

0.02

0.02

0.02

0.03

0.03

0.03

0.03

0.04

0.05

0.07

0.09

0.07

0.07

0.08

0.07

0.08

0.08

0.08

0.10

0.11

0.08

0.08

0.10

0.09

0.08

0.08

0.07

0.05

0.07
998.2041

998.2041

998.2041

$525.80 \quad 998.2041$

998.2041

998.2041

$\begin{array}{ll}1.73 & 997.0449\end{array}$

$1.73 \quad 997.0449$

$3.059 \quad 997.0449$

3.059997 .0449

997.0449

$4.292 \quad 997.0449$

4.292997 .0449 997.0449

$4.444 \quad 997.0449$

997.0449

$\begin{array}{ll}6.407 & 997.0449\end{array}$

$6.407 \quad 997.0449$ 997.0449

$8.449 \quad 997.0449$

8.449997 .0449

8.819997 .0449 997.0449

13.132997 .0449

997.0449

$-4.05$ 997.0449

$17.163 \quad 997.0449$

$17.163 \quad 997.0449$

997.0449

$17.4 \quad 997.0449$

21.342997 .0449

$21.342 \quad 997.0449$

$21.596 \quad 997.0449$

997.0449

$-3.42$ 997.0449

$21.735 \quad 997.0449$

$25.787 \quad 997.0449$

997.0449

997.0449

$29.956 \quad 997.0449$

997.0449

$34.064 \quad 997.0449$

38.142997 .0449

997.0449 


\begin{tabular}{|c|c|c|c|c|}
\hline molality & molarity & $\mathrm{t} /{ }^{\circ} \mathrm{C}$ & ass frac & $\begin{array}{l}\text { Density } \\
\exp \\
\mathrm{kg} / \mathrm{m}^{3}\end{array}$ \\
\hline 0.9996 & & 25 & 0.0384 & 1039.24 \\
\hline 1.0006 & & 25 & 0.0385 & 1039.29 \\
\hline 1.0006 & & 25 & 0.0385 & 1039.29 \\
\hline 2.032 & & 25 & 0.0752 & 1079.30 \\
\hline 3.098 & & 25 & 0.1103 & 1117.65 \\
\hline 5.256 & & 25 & 0.1737 & 1187.23 \\
\hline 7.422 & & 25 & 0.2289 & 1247.70 \\
\hline \multirow[t]{37}{*}{10.88} & & 25 & 0.3032 & 1327.77 \\
\hline & & 30 & 0.0200 & 1017.26 \\
\hline & 0.9983 & 30 & 0.0385 & 1037.20 \\
\hline & & 30 & 0.0400 & 1038.68 \\
\hline & 1.085 & 30 & 0.0417 & 1040.70 \\
\hline & & 30 & 0.0600 & 1060.10 \\
\hline & & 30 & 0.0800 & 1081.61 \\
\hline & & 30 & 0.1000 & 1103.24 \\
\hline & & 30 & 0.1000 & 1103.25 \\
\hline & 3.018 & 30 & 0.1085 & 1112.80 \\
\hline & & 30 & 0.1200 & 1124.99 \\
\hline & & 30 & 0.1400 & 1146.86 \\
\hline & 4.585 & 30 & 0.1573 & 1166.10 \\
\hline & & 30 & 0.1600 & 1168.82 \\
\hline & & 30 & 0.1800 & 1190.84 \\
\hline & 5.977 & 30 & 0.1975 & 1210.20 \\
\hline & 6.023 & 30 & 0.1988 & 1211.60 \\
\hline & & 30 & 0.2000 & 1212.88 \\
\hline & & 30 & 0.2000 & 1212.05 \\
\hline & & 30 & 0.2200 & 1234.88 \\
\hline & & 30 & 0.2400 & 1256.79 \\
\hline & 8.274 & 30 & 0.2591 & 1277.10 \\
\hline & & 30 & 0.2600 & 1278.52 \\
\hline & & 30 & 0.2800 & 1300.00 \\
\hline & & 30 & 0.3000 & 1321.12 \\
\hline & & 30 & 0.3000 & 1319.55 \\
\hline & & 30 & 0.3200 & 1341.77 \\
\hline & & 30 & 0.3400 & 1361.82 \\
\hline & & 30 & 0.3600 & 1380.50 \\
\hline & & 30 & 0.3800 & 1401.98 \\
\hline & & 30 & 0.4000 & 1422.98 \\
\hline & & 30 & 0.4000 & 1422.35 \\
\hline & & 30 & 0.4200 & 1443.34 \\
\hline & & 30 & 0.4400 & 1462.92 \\
\hline & & 30 & 0.4600 & 1481.53 \\
\hline & & 30 & 0.4800 & 1498.93 \\
\hline & & 30 & 0.5000 & 1514.95 \\
\hline
\end{tabular}

Ref

$\begin{array}{cc}\text { App vol } & \text { App vol } \\ \exp / & \text { Calc / } \\ \mathrm{m}^{3} / \mathrm{kg} & \mathrm{m}^{3} / \mathrm{kg}\end{array}$

Density Density App Vol Square of Inconsistent Inconsistent

Apparent Delta density Density of calc / Residual / Residual / Density based on based on molal volume $/ \mathrm{kg} / \mathrm{m}^{3}$ water $/ \mathrm{kg} / \mathrm{m}^{3}$ $\mathrm{kg} / \mathrm{m}^{3} \quad \mathrm{~kg} / \mathrm{m}^{3} \quad \mathrm{~m}^{3} / \mathrm{kg}$

$$
\text { Res }
$$

(109) $\quad-0.000056-0.000050$

(88) $\quad-0.000056-0.000050$

1039.00

$0.2417-0.000006$

$0.2501-0.000006$

$0.2501-0.000006$

$\begin{array}{ll}-0.1444 & 0.000002\end{array}$

$\begin{array}{ll}-0.6407 & 0.000005\end{array}$

$\begin{array}{ll}-1.4430 & 0.000006\end{array}$

$\begin{array}{ll}-1.7563 & 0.000005\end{array}$

$\begin{array}{ll}-1.7562 & 0.000003\end{array}$

$\begin{array}{ll}-0.0468 & 0.000002\end{array}$

$-0.2509 \quad 0.000006$

$\begin{array}{ll}-0.4119 & 0.000010\end{array}$

$-0.2485 \quad 0.000006$

$\begin{array}{ll}-0.8829 & 0.000013\end{array}$

$\begin{array}{ll}-1.3492 & 0.000014\end{array}$

$\begin{array}{ll}-1.7590 & 0.000014\end{array}$

$\begin{array}{ll}-1.7518 & 0.000014\end{array}$

$\begin{array}{ll}-1.5526 & 0.000012\end{array}$

$\begin{array}{ll}-2.0896 & 0.000014\end{array}$

$\begin{array}{ll}-2.3171 & 0.000013\end{array}$

$\begin{array}{lll}-2.1477 & 0.000010\end{array}$

$\begin{array}{lll}-2.4466 & 0.000011\end{array}$

$\begin{array}{ll}-2.4822 & 0.000010\end{array}$

$\begin{array}{ll}-2.4165 & 0.000008\end{array}$

$\begin{array}{lll}-2.4334 & 0.000008\end{array}$

$\begin{array}{lll}-2.4373 & 0.000008\end{array}$

$\begin{array}{ll}-3.2701 & 0.000011\end{array}$

$\begin{array}{lll}-2.3443 & 0.000007\end{array}$

$-2.2247 \quad 0.000006$

$\begin{array}{lll}-2.6230 & 0.000006\end{array}$

$\begin{array}{ll}-2.1396 & 0.000005\end{array}$

$\begin{array}{ll}-2.1292 & 0.000004\end{array}$

$\begin{array}{ll}-2.2733 & 0.000004\end{array}$

$\begin{array}{ll}-3.8461 & 0.000007\end{array}$

$\begin{array}{lll}-2.6515 & 0.000005\end{array}$

$\begin{array}{ll}-3.3627 & 0.000005\end{array}$

$\begin{array}{ll}-5.1459 & 0.000007\end{array}$

$\begin{array}{lll}-3.8001 & 0.000005\end{array}$

$\begin{array}{lll}-2.5740 & 0.000003\end{array}$

$\begin{array}{ll}-3.2067 & 0.000004\end{array}$

$\begin{array}{lll}-1.5968 & 0.000002\end{array}$

$\begin{array}{ll}-0.9781 & 0.000001\end{array}$

$\begin{array}{ll}-0.8777 & 0.000001\end{array}$

$\begin{array}{ll}-1.5063 & 0.000001\end{array}$

$\begin{array}{ll}-3.0051 & 0.000003\end{array}$
0.06

0.06

0.06

0.02

0.41
2.08

08

(2.08

. 08

0.00

0.06

0.17

0.06

0.78

1.82

3.09

3.07

2.41

4.37

5.37

4.61

5.99

6.16

5.84

5.92

5.94

10.69

5.50

6.88

4.58

4.53

5.17

14.79

7.03

11.31

26.48

14.44

6.63

10.28

2.55

0.96

0.77

2.27
$42.196 \quad 997.0449$ $42245 \quad 997.0449$ $82.253 \quad 997.0449$ 120.61997 .0449 $190.188 \quad 997.0449$ $250.656 \quad 997.0449$ $330.727 \quad 997.0449$ 995.6473 995.6473 995.6473 995.6473 995.6473 995.6473 995.6473

$107.60 \quad 995.6473$ 995.6473 995.6473 995.6473 995.6473 995.6473 995.6473 995.6473 995.6473 995.6473

$216.40 \quad 995.6473$ 995.6473 995.6473 995.6473 995.6473 995.6473 995.6473

$323.90 \quad 995.6473$ 995.6473 995.6473 995.6473 995.6473 995.6473

$426.70 \quad 995.6473$ 995.6473 995.6473 995.6473 995.6473 995.6473 


\begin{tabular}{|c|c|c|c|c|}
\hline \multirow[t]{3}{*}{ molality } & \multirow[t]{3}{*}{ molarity } & $\mathrm{t} /{ }^{\circ} \mathrm{C}$ & ass frac & $\begin{array}{r}\text { Density } \\
\exp / \\
\mathrm{kg} / \mathrm{m}^{3}\end{array}$ \\
\hline & & 30 & 0.5000 & 1516.75 \\
\hline & & 30 & 0.5200 & 1529.31 \\
\hline 0.07496 & & 35 & 0.0030 & 997.28 \\
\hline 0.09934 & & 35 & 0.0040 & 998.36 \\
\hline 0.10003 & & 35 & 0.0040 & 998.43 \\
\hline 0.15009 & & 35 & 0.0060 & 1000.55 \\
\hline 0.19896 & & 35 & 0.0079 & 1002.62 \\
\hline 0.29978 & & 35 & 0.0118 & 1006.88 \\
\hline 0.30073 & & 35 & 0.0119 & 1006.96 \\
\hline 0.38521 & & 35 & 0.0152 & 1010.44 \\
\hline 0.49461 & & 35 & 0.0194 & 1014.97 \\
\hline 0.50002 & & 35 & 0.0196 & 1015.22 \\
\hline 0.5804 & & 35 & 0.0227 & 1018.50 \\
\hline 0.68698 & & 35 & 0.0267 & 1022.84 \\
\hline 0.78077 & & 35 & 0.0303 & 1026.64 \\
\hline 0.87904 & & 35 & 0.0340 & 1030.59 \\
\hline 0.99742 & & 35 & 0.0384 & 1035.32 \\
\hline 0.03727 & & 40 & 0.0015 & 993.83 \\
\hline 0.06069 & & 40 & 0.0024 & 994.84 \\
\hline 0.08169 & & 40 & 0.0033 & 995.74 \\
\hline 0.09978 & & 40 & 0.0040 & 996.51 \\
\hline 0.1 & & 40 & 0.0040 & 996.53 \\
\hline \multirow[t]{3}{*}{0.10144} & & 40 & 0.0040 & 996.58 \\
\hline & & 40 & 0.0099 & 1002.60 \\
\hline & & 40 & 0.0100 & 1003.30 \\
\hline 0.3042 & & 40 & 0.0120 & 1005.12 \\
\hline \multirow[t]{4}{*}{0.5067} & & 40 & 0.0199 & 1013.47 \\
\hline & & 40 & 0.0200 & 1013.60 \\
\hline & & 40 & 0.0200 & 1013.90 \\
\hline & 0.9751 & 40 & 0.0378 & 1031.80 \\
\hline 1.0094 & & 40 & 0.0388 & 1033.62 \\
\hline 1.0126 & & 40 & 0.0389 & 1033.81 \\
\hline \multirow[t]{5}{*}{1.0158} & & 40 & 0.0390 & 1033.88 \\
\hline & & 40 & 0.0400 & 1034.73 \\
\hline & & 40 & 0.0400 & 1035.20 \\
\hline & & 40 & 0.0476 & 1042.20 \\
\hline & & 40 & 0.0600 & 1055.87 \\
\hline \multirow[t]{7}{*}{2.02} & & 40 & 0.0748 & 1072.09 \\
\hline & 2.01 & 40 & 0.0751 & 1071.10 \\
\hline & & 40 & 0.0800 & 1077.11 \\
\hline & & 40 & 0.0800 & 1078.00 \\
\hline & & 40 & 0.0910 & 1087.80 \\
\hline & & 40 & 0.1000 & 1098.49 \\
\hline & & 40 & 0.1000 & 1098.12 \\
\hline
\end{tabular}

Ref

$\begin{array}{cc}\text { App vol App vol } \\ \exp / & \text { Calc / }\end{array}$

$\begin{array}{cc}\exp / & \text { Calc / } \\ \mathrm{m}^{3} / \mathrm{kg} & \mathrm{m}^{3} / \mathrm{kg}\end{array}$

0.0003140 .000313

$0.000330 \quad 0.000326$

$-0.000089-0.000078$

$\begin{array}{lll}-0.000096 & -0.000077\end{array}$

$\begin{array}{lll}-0.000107 & -0.000077\end{array}$

$-0.000093-0.000075$

$-0.000086-0.000073$

$-0.000077-0.000069$

$-0.000080-0.000069$

$-0.000071-0.000066$

$-0.000064-0.000062$

$-0.000065-0.000062$

$-0.000059-0.000059$

$-0.000054-0.000055$

$-0.000049-0.000051$

$-0.000045-0.000048$

$-0.000040-0.000044$

$-0.000094-0.000076$

$-0.000091-0.000075$

$-0.000086-0.000074$

$-0.000084-0.000073$

$-0.000087-0.000073$

$\begin{array}{lll}-0.000086 & -0.000073\end{array}$

$\begin{array}{lll}-0.000047 & -0.000067\end{array}$

$-0.000106-0.000067$

(109) $\quad-0.000069-0.000065$

$-0.000056-0.000058$

$-0.000055-0.000058$

$-0.000070-0.000058$

$-0.000015-0.000041$

$-0.000033-0.000040$

$-0.000034-0.000040$

$-0.000032-0.000039$

$\begin{array}{lll}-0.000027 & -0.000038\end{array}$

$-0.000038-0.000038$

$-0.000008-0.000031$

$-0.000005-0.000020$

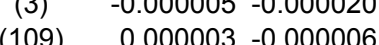

$\begin{array}{crr}(109) & 0.000003 & -0.000006 \\ (41) & 0.000019 & -0.000006\end{array}$

$0.000019-0.000006$

$0.000005-0.000001$

$\begin{array}{lll}0.000035 & 0.000009\end{array}$

$0.000033 \quad 0.000017$

$\begin{array}{ll}0.000036 & 0.000017\end{array}$
Density Density App Vol Square of Inconsistent Inconsistent

Apparent Delta density Density of ( based on molal volume $\quad / \mathrm{kg} / \mathrm{m}^{3}$ water $/ \mathrm{kg} / \mathrm{m}^{3}$ $\mathrm{kg} / \mathrm{m}^{3} \quad \mathrm{~kg} / \mathrm{m}^{3} \quad \mathrm{~m}^{3} / \mathrm{kg} \quad$ Res volume $/ \mathrm{cm}^{3} / \mathrm{mol}$

1.46

31.66

$\begin{array}{lll}534.94 & -5.6263 & 0.000005\end{array}$

$0.0331-0.000011$

$0.0771-0.000020$

$0.1196-0.000030$

0.00

0.01

0.01
0.01

$000.45 \quad 0.1050-0.000018$

$0.0995-0.000013$

$0.0954-0.000008$

$0.1355-0.000011$

0.01

0.01

0.02

0.01

$\begin{array}{lll}0.0440 & -0.000002 & 0.00\end{array}$

0.00

0.00

0.00

0.00

$\begin{array}{ll}-0.0305 & 0.000001\end{array}$

$\begin{array}{ll}-0.0698 & 0.000002\end{array}$

0.01

0.03

$\begin{array}{ll}-0.1123 & 0.000003 \\ -0.1587 & 0.000004\end{array}$

$0.0278-0.000019$

0.00

$94.80-0.0385-0.000016$

$0.0406-0.000013$

$0.0424-0.000011$

0.00

0.00

$\begin{array}{llll}996.47 & 0.0561 & -0.000014\end{array}$

$0.0500-0.000012$

0.00

0.00

$\begin{array}{llll}1002.81 & -0.2073 & 0.000021\end{array}$

$0.3855-0.000038$

$0.0410-0.000003$

$\begin{array}{ll}-0.0352 & 0.000002\end{array}$

0.15

0.00

0.00

0.00

$\begin{array}{lll}1013.65 & -0.0471 & 0.000002\end{array}$

$0.2529-0.000012$

$\begin{array}{ll}-1.0276 & 0.000026\end{array}$

0.06

1.06

0.09

0.06

0.08

0.23

0.00

1.51

1.00

0.68

4.60

2.30

0.39

7.98

3.85

5.46
$521.10 \quad 995.6473$ 995.6473 994.0319

$4.33 \quad 994.0319$ 994.0319

$-3.7 \quad 994.0319$

$\begin{array}{rr}12.844 \quad 994.0319 \\ & 994.0319\end{array}$

$16.413 \quad 994.0319$

20.942994 .0319

994.0319

$24.466 \quad 994.0319$

28.81994 .0319

$32.606 \quad 994.0319$

$36.556 \quad 994.0319$

41.284994 .0319

1.618992 .2158

$2.626 \quad 992.2158$

$3.521 \quad 992.2158$

4.291992 .2158

$4.314 \quad 992.2158$

$4.369 \quad 992.2158$

992.2158

992.2158

$12.906 \quad 992.2158$

$21.25 \quad 992.2158$

992.2158

992.2158

992.2158

$41.405 \quad 992.2158$

$41.59 \quad 992.2158$

$41.665 \quad 992.2158$

992.2158

992.2158

992.2158

992.2158

$79.876 \quad 992.2158$

992.2158

992.2158

992.2158

992.2158

992.2158

$105.90 \quad 992.2158$ 


\begin{tabular}{|c|c|c|c|c|c|c|c|c|c|c|c|}
\hline molality & molarity & \multicolumn{2}{|c|}{$\mathrm{t} /{ }^{\circ} \mathrm{C}$ mass frac } & $\begin{array}{r}\text { Density } \\
\exp / \\
\mathrm{kg} / \mathrm{m}^{3}\end{array}$ & Ref & $\begin{array}{r}\text { App vol } \\
\exp / \\
\mathrm{m}^{3} / \mathrm{kg}\end{array}$ & $\begin{array}{c}\text { App vol } \\
\text { Calc / } \\
\mathrm{m}^{3} / \mathrm{kg}\end{array}$ & $\begin{array}{r}\text { Density } \\
\mathrm{calc} / \\
\mathrm{kg} / \mathrm{m}^{3}\end{array}$ & $\begin{array}{r}\text { Density } \\
\text { Residual / } \\
\mathrm{kg} / \mathrm{m}^{3}\end{array}$ & $\begin{array}{r}\text { App Vol } \\
\text { Residual / } \\
\mathrm{m}^{3} / \mathrm{kg}\end{array}$ & $\begin{array}{r}\text { Square of } \\
\text { Density } \\
\text { Res }\end{array}$ \\
\hline \multirow[t]{8}{*}{3.026} & & 40 & 0.1080 & 1107.99 & (109) & 0.000032 & 0.000024 & 1109.16 & -1.1685 & 0.000009 & 1.37 \\
\hline & \multirow[t]{3}{*}{3.063} & 40 & 0.1105 & 1108.40 & (41) & 0.000052 & 0.000026 & 1111.96 & -3.5628 & 0.000026 & 12.69 \\
\hline & & 40 & 0.1200 & 1120.00 & (3) & 0.000050 & 0.000034 & 1122.32 & -2.3230 & 0.000015 & 5.40 \\
\hline & & 40 & 0.1200 & 1121.00 & (90) & 0.000043 & 0.000034 & 1122.32 & -1.3230 & 0.000009 & 1.75 \\
\hline & \multirow[t]{4}{*}{3.949} & 40 & 0.1387 & 1138.70 & (41) & 0.000073 & 0.000050 & 1142.81 & -4.1052 & 0.000023 & 16.85 \\
\hline & & 40 & 0.1400 & 1141.64 & (3) & 0.000066 & 0.000052 & 1144.22 & -2.5778 & 0.000014 & 6.64 \\
\hline & & 40 & 0.1600 & 1163.40 & (3) & 0.000081 & 0.000068 & 1166.11 & -2.7130 & 0.000012 & 7.36 \\
\hline & & 40 & 0.1600 & 1164.50 & (90) & 0.000076 & 0.000068 & 1166.11 & -1.6130 & 0.000007 & 2.60 \\
\hline \multirow[t]{9}{*}{5.027} & & 40 & 0.1674 & 1172.13 & (109) & 0.000084 & 0.000075 & 1174.22 & -2.0845 & 0.000009 & 4.35 \\
\hline & & 40 & 0.1800 & 1185.23 & (3) & 0.000096 & 0.000085 & 1187.98 & -2.7542 & 0.000011 & 7.59 \\
\hline & & 40 & 0.2000 & 1207.11 & (3) & 0.000111 & 0.000102 & 1209.81 & -2.6958 & 0.000009 & 7.27 \\
\hline & & 40 & 0.2000 & 1206.32 & (54) & 0.000113 & 0.000102 & 1209.81 & -3.4901 & 0.000012 & 12.18 \\
\hline & & 40 & 0.2000 & 1207.90 & (90) & 0.000108 & 0.000102 & 1209.81 & -1.9058 & 0.000007 & 3.63 \\
\hline & & 40 & 0.2026 & 1205.10 & (66) & 0.000129 & 0.000104 & 1212.64 & -7.5377 & 0.000025 & 56.82 \\
\hline & 6.161 & 40 & 0.2036 & 1210.50 & (41) & 0.000115 & 0.000104 & 1213.69 & -3.1949 & 0.000011 & 10.21 \\
\hline & 6.376 & 40 & 0.2095 & 1217.50 & (41) & 0.000118 & 0.000109 & 1220.11 & -2.6068 & 0.000008 & 6.80 \\
\hline & & 40 & 0.2200 & 1228.98 & (3) & 0.000125 & 0.000118 & 1231.55 & -2.5718 & 0.000008 & 6.61 \\
\hline \multirow[t]{9}{*}{7.532} & & 40 & 0.2315 & 1241.67 & (109) & 0.000133 & 0.000127 & 1244.03 & -2.3598 & 0.000007 & 5.57 \\
\hline & & 40 & 0.2400 & 1250.78 & (3) & 0.000140 & 0.000133 & 1253.20 & -2.4151 & 0.000006 & 5.83 \\
\hline & & 40 & 0.2400 & 1251.20 & (90) & 0.000139 & 0.000133 & 1253.20 & -1.9951 & 0.000005 & 3.98 \\
\hline & 7.883 & 40 & 0.2497 & 1262.60 & (41) & 0.000144 & 0.000141 & 1263.67 & -1.0697 & 0.000003 & 1.14 \\
\hline & & 40 & 0.2600 & 1272.44 & (3) & 0.000154 & 0.000149 & 1274.71 & -2.2681 & 0.000005 & 5.14 \\
\hline & & 40 & 0.2800 & 1293.89 & (3) & 0.000169 & 0.000164 & 1296.06 & -2.1725 & 0.000005 & 4.72 \\
\hline & & 40 & 0.2800 & 1294.20 & (90) & 0.000168 & 0.000164 & 1296.06 & -1.8625 & 0.000004 & 3.47 \\
\hline & & 40 & 0.3000 & 1315.03 & (3) & 0.000183 & 0.000179 & 1317.23 & -2.1995 & 0.000004 & 4.84 \\
\hline & & 40 & 0.3000 & 1313.32 & (54) & 0.000186 & 0.000179 & 1317.23 & -3.9138 & 0.000008 & 15.32 \\
\hline \multirow[t]{18}{*}{10.88} & & 40 & 0.3032 & 1318.25 & (109) & 0.000186 & 0.000181 & 1320.62 & -2.3643 & 0.000004 & 5.59 \\
\hline & & 40 & 0.3200 & 1335.76 & (3) & 0.000198 & 0.000194 & 1338.18 & -2.4200 & 0.000004 & 5.86 \\
\hline & & 40 & 0.3200 & 1336.20 & (90) & 0.000197 & 0.000194 & 1338.18 & -1.9800 & 0.000003 & 3.92 \\
\hline & & 40 & 0.3298 & 1338.50 & (66) & 0.000217 & 0.000201 & 1348.36 & -9.8577 & 0.000017 & 97.17 \\
\hline & & 40 & 0.3400 & 1355.95 & (3) & 0.000213 & 0.000208 & 1358.88 & -2.9343 & 0.000005 & 8.61 \\
\hline & & 40 & 0.3600 & 1375.47 & (3) & 0.000228 & 0.000222 & 1379.31 & -3.8428 & 0.000006 & 14.77 \\
\hline & & 40 & 0.3600 & 1376.80 & (90) & 0.000226 & 0.000222 & 1379.31 & -2.5128 & 0.000004 & 6.31 \\
\hline & & 40 & 0.3800 & 1394.85 & (3) & 0.000242 & 0.000236 & 1399.44 & -4.5855 & 0.000006 & 21.03 \\
\hline & & 40 & 0.4000 & 1415.85 & (3) & 0.000254 & 0.000250 & 1419.22 & -3.3726 & 0.000004 & 11.37 \\
\hline & & 40 & 0.4000 & 1415.52 & (54) & 0.000254 & 0.000250 & 1419.22 & -3.7069 & 0.000005 & 13.74 \\
\hline & & 40 & 0.4000 & 1416.40 & (90) & 0.000253 & 0.000250 & 1419.22 & -2.8226 & 0.000004 & 7.97 \\
\hline & & 40 & 0.4200 & 1436.25 & (3) & 0.000266 & 0.000263 & 1438.64 & -2.3944 & 0.000003 & 5.73 \\
\hline & & 40 & 0.4400 & 1455.91 & (3) & 0.000278 & 0.000276 & 1457.67 & -1.7614 & 0.000002 & 3.10 \\
\hline & & 40 & 0.4400 & 1454.50 & (90) & 0.000280 & 0.000276 & 1457.67 & -3.1714 & 0.000003 & 10.06 \\
\hline & & 40 & 0.4600 & 1474.65 & (3) & 0.000291 & 0.000289 & 1476.27 & -1.6246 & 0.000002 & 2.64 \\
\hline & & 40 & 0.4800 & 1492.26 & (3) & 0.000304 & 0.000302 & 1494.43 & -2.1653 & 0.000002 & 4.69 \\
\hline & & 40 & 0.4800 & 1492.20 & (90) & 0.000304 & 0.000302 & 1494.43 & -2.2253 & 0.000002 & 4.95 \\
\hline & & 40 & 0.4896 & 1506.30 & (66) & 0.000305 & 0.000308 & 1502.97 & 3.3313 & -0.000003 & 11.10 \\
\hline
\end{tabular}
992.2158 992.2158 992.2158 992.2158 992.2158 992.2158 992.2158

$179.916 \quad 992.2158$ 992.2158 992.2158

$214.10 \quad 992.2158$ 992.2158 992.2158 992.2158 992.2158 992.2158

$249.45 \quad 992.2158$ 992.2158 992.2158 992.2158 992.2158 992.2158 992.2158 992.2158 $321.10 \quad 992.2158$ $326.036 \quad 992.2158$ 992.2158 992.2158 992.2158 992.2158 992.2158 992.2158 992.2158 992.2158

$423.30 \quad 992.2158$ 992.2158 992.2158 992.2158 992.2158 992.2158 992.2158 992.2158 992.2158 


\begin{tabular}{|c|c|c|c|c|}
\hline \multirow[t]{5}{*}{ molality } & \multirow[t]{5}{*}{ molarity } & $\mathrm{t} /{ }^{\circ} \mathrm{C}$ & ass frac & $\begin{array}{r}\text { Density } \\
\exp / \\
\mathrm{kg} / \mathrm{m}^{3}\end{array}$ \\
\hline & & 40 & 0.5000 & 1508.55 \\
\hline & & 40 & 0.5000 & 1509.42 \\
\hline & & 40 & 0.5000 & 1510.90 \\
\hline & & 40 & 0.5200 & 1523.26 \\
\hline 0.05109 & & 45 & 0.0020 & 992.46 \\
\hline 0.07496 & & 45 & 0.0030 & 993.40 \\
\hline 0.10003 & & 45 & 0.0040 & 994.55 \\
\hline 0.1033 & & 45 & 0.0041 & 994.72 \\
\hline 0.15009 & & 45 & 0.0060 & 996.66 \\
\hline 0.2004 & & 45 & 0.0080 & 998.58 \\
\hline 0.2991 & & 45 & 0.0118 & 1002.97 \\
\hline 0.30073 & & 45 & 0.0119 & 1002.93 \\
\hline 0.3933 & & 45 & 0.0155 & 1006.87 \\
\hline 0.4988 & & 45 & 0.0196 & 1011.19 \\
\hline 0.50002 & & 45 & 0.0196 & 1011.05 \\
\hline 0.5979 & & 45 & 0.0234 & 1015.21 \\
\hline 0.7045 & & 45 & 0.0274 & 1019.49 \\
\hline 0.8159 & & 45 & 0.0316 & 1023.93 \\
\hline 0.907 & & 45 & 0.0350 & 1027.54 \\
\hline 0.9839 & & 45 & 0.0379 & 1030.58 \\
\hline & & 50 & 0.0200 & 1009.29 \\
\hline & & 50 & 0.0400 & 1030.21 \\
\hline & & 50 & 0.0600 & 1051.16 \\
\hline & & 50 & 0.0800 & 1072.21 \\
\hline & & 50 & 0.1000 & 1093.40 \\
\hline & & 50 & 0.1000 & 1092.84 \\
\hline & & 50 & 0.1200 & 1114.72 \\
\hline & & 50 & 0.1400 & 1136.18 \\
\hline & & 50 & 0.1600 & 1157.74 \\
\hline & & 50 & 0.1800 & 1179.42 \\
\hline & & 50 & 0.2000 & 1201.14 \\
\hline & & 50 & 0.2000 & 1200.54 \\
\hline & & 50 & 0.2200 & 1222.86 \\
\hline & & 50 & 0.2400 & 1244.52 \\
\hline & & 50 & 0.2600 & 1266.06 \\
\hline & & 50 & 0.2800 & 1287.40 \\
\hline & & 50 & 0.3000 & 1308.45 \\
\hline & & 50 & 0.3000 & 1307.14 \\
\hline & & 50 & 0.3200 & 1329.10 \\
\hline & & 50 & 0.3400 & 1349.24 \\
\hline & & 50 & 0.3600 & 1368.73 \\
\hline & & 50 & 0.3800 & 1387.53 \\
\hline & & 50 & 0.4000 & 1408.53 \\
\hline & & 50 & 0.4000 & 1408.74 \\
\hline
\end{tabular}

Ref

$\begin{array}{rr}\text { App vol } & \text { App vol } \\ \exp / & \text { Calc } / \\ \mathrm{m}^{3} / \mathrm{kg} & \mathrm{m}^{3} / \mathrm{kg}\end{array}$

Density

Density App Vol Square of Inconsistent Inconsistent

Apparent Delta density Density of

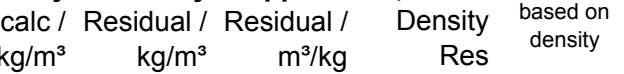
based on molal volume $/ \mathrm{kg} / \mathrm{m}^{3}$ water $/ \mathrm{kg} / \mathrm{m}^{3}$

(1)

$0.000318 \quad 0.000315$ 1512.10 $\mathrm{kg} / \mathrm{m}^{3} / \mathrm{kg}$ $1 \mathrm{~cm}^{3} / \mathrm{mol}$

$\begin{array}{ll}0.000317 & 0.000315\end{array}$

12.10

$-3.5456 \quad 0.000003$

12.57
7.18

$\begin{array}{llllll}0.000316 & 0.000315 & 1512.10 & -1.1956 & 0.000001 & 1.43\end{array}$

$\begin{array}{lll}0.000332 & 0.000327 & 1529.26\end{array}$

1.43

$\begin{array}{ll}-5.9984 & 0.000005\end{array}$

35.98

$-0.000075-0.000070$

992.38

$0.0832-0.000041$

$0.0135-0.000005$

$-0.000096-0.000069$

093.39

$0.1026-0.000026$

$0.1325-0.000033$

$\begin{array}{ll}-0.000102 & -0.000069 \\ -0.000084 & -0.000068\end{array}$

$-0.000054-0.000066$

$\begin{array}{lll}0.0990 & -0.000017\end{array}$

$-0.0932 \quad 0.000012$

$0.1798-0.000015$

$-0.000077-0.000062$

1002.79

$0.0650-0.000005$

$0.1670-0.000011$

$0.1322-0.000007$

$\begin{array}{lll}-0.0530 & 0.000003\end{array}$

$0.0902-0.000004$

$0.0400-0.000001$

$\begin{array}{ll}-0.0154 & 0.000000\end{array}$

$\begin{array}{ll}-0.0592 & 0.000002\end{array}$

$\begin{array}{ll}-0.0941 & 0.000002\end{array}$

$0.0622-0.000003$

$\begin{array}{ll}-0.3385 & 0.000008\end{array}$

$-0.8212 \quad 0.000012$

$-1.29720 .000014$

$\begin{array}{ll}-1.7073 & 0.000014\end{array}$

$\begin{array}{ll}-2.2710 & 0.000019\end{array}$

-2.0409 0.000014

$-2.2667 \quad 0.000013$

$-2.40250 .000011$

$\begin{array}{ll}-2.4051 \quad 0.000010 \\ -2.3305 & 0.000008\end{array}$

$\begin{array}{ll}-2.3305 & 0.000008\end{array}$

$\begin{array}{ll}-2.9342 \quad 0.000010 \\ -2.1940 & 0.000007\end{array}$

$\begin{array}{ll}-2.1940 & 0.000007\end{array}$

$\begin{array}{lll}-2.0299 & 0.000005\end{array}$

$\begin{array}{ll}-1.8721 & 0.000004\end{array}$

$-1.77370 .000004$

$-1.79750 .000003$

$\begin{array}{lll}-3.1112 & 0.000006\end{array}$

$\begin{array}{ll}-2.0257 & 0.000004\end{array}$

$\begin{array}{ll}-2.5401 & 0.000004\end{array}$

$-3.4523 \quad 0.000005$

$\begin{array}{ll}-4.7739 & 0.000007\end{array}$

$\begin{array}{ll}-3.5861 & 0.000005\end{array}$

$\begin{array}{ll}-3.3798 & 0.000004\end{array}$
0.01
0.00

0.01

0.02

0.01

0.01

0.00
0.03
0.02

0.02

0.00

0.01

0.00

0.00

0.01
0.00

0.11

0.67

1.68

2.91

5.16

4.17

5.14

5.77

5.78

5.43

8.61

4.81

4.12

3.50

3.15

3.23

9.68

4.10

6.45

11.92

22.79

12.86

11.42
992.2158

$517.20 \quad 992.2158$

992.2158

992.2158

$2.25 \quad 990.2132$

990.2132

990.2132

990.2132

8.364990 .2132

12.761990 .2132

990.2132

$20.975 \quad 990.2132$

990.2132

$24.992 \quad 990.2132$

29.278990 .2132

33.721990 .2132

$37.331 \quad 990.2132$

$40.363 \quad 990.2132$

988.0363

988.0363

988.0363

988.0363

988.0363

$104.80 \quad 988.0363$

988.0363

988.0363

988.0363

988.0363

988.0363

$212.50 \quad 988.0363$

988.0363

988.0363

988.0363

988.0363

988.0363

319.10988 .0363

988.0363

988.0363

988.0363

988.0363

988.0363

$420.70 \quad 988.0363$
$4.506 \quad 990.2132$

$16.659 \quad 990.2132$ 


molality








0.07496
0.10003
0.1005
0.15009
0.2028
0.3007
0.30073
0.50002
0.5063
1.0146
2.027
3.026
5.045
7.494
10.893

$\begin{array}{rrr}\mathbf{t} /{ }^{\circ} \mathrm{C} \text { mass frac } & \begin{array}{r}\text { Density } \\ \text { exp } / \\ \end{array} \\ & & \mathrm{kg} / \mathrm{m}^{3} \\ 50 & 0.4200 & 1431.17 \\ 50 & 0.4400 & 1448.81 \\ 50 & 0.4600 & 1467.72 \\ 50 & 0.4800 & 1485.59 \\ 50 & 0.5000 & 1502.19 \\ 50 & 0.5000 & 1502.14 \\ 50 & 0.5200 & 1517.27 \\ 55 & 0.0030 & 988.85 \\ 55 & 0.0040 & 989.97 \\ 55 & 0.0040 & 989.91 \\ 55 & 0.0060 & 992.10 \\ 55 & 0.0080 & 994.15 \\ 55 & 0.0119 & 998.16 \\ 55 & 0.0119 & 998.26 \\ 55 & 0.0196 & 1006.29 \\ 55 & 0.0198 & 1006.47 \\ 55 & 0.0390 & 1026.45 \\ 55 & 0.0750 & 1064.25 \\ 55 & 0.1080 & 1099.16 \\ 55 & 0.1679 & 1163.34 \\ 55 & 0.2306 & 1230.75 \\ 55 & 0.3035 & 1307.86 \\ 60 & 0.0099 & 993.60 \\ 60 & 0.0100 & 994.10 \\ 60 & 0.0200 & 1004.34 \\ 60 & 0.0200 & 1004.50 \\ 60 & 0.0400 & 1025.11 \\ 60 & 0.0400 & 1025.40 \\ 60 & 0.0476 & 1033.20 \\ 60 & 0.0600 & 1045.92 \\ 60 & 0.0800 & 1066.82 \\ 60 & 0.0800 & 1067.60 \\ 60 & 0.0910 & 1078.80 \\ 60 & 0.1000 & 1087.87 \\ 60 & 0.1000 & 1087.50 \\ 60 & 0.1200 & 1109.06 \\ 60 & 0.1200 & 1110.10 \\ 60 & 0.1400 & 1130.40 \\ 60 & 0.1600 & 1151.85 \\ 60 & 0.1600 & 1153.10 \\ 60 & 0.1800 & 1173.40 \\ 60 & 0.2000 & 1195.01 \\ 60 & 0.2000 & 1195.00 \\ 60 & 0.2000 & 1196.00\end{array}$

Ref

$\begin{array}{rr}\text { App vol } & \text { App vol } \\ \exp / & \text { Calc / } \\ \mathrm{m}^{3} / \mathrm{kg} & \mathrm{m}^{3} / \mathrm{kg}\end{array}$

$\begin{aligned} \exp / & \text { Calc } / \\ \mathrm{m}^{3} / \mathrm{kg} & \mathrm{m}^{3} / \mathrm{kg}\end{aligned}$

0.0002660 .000265

$0.000281 \quad 0.000279$

$0.000293 \quad 0.000291$

$0.000306 \quad 0.000304$

0.0003190 .000316

$0.000319 \quad 0.000316$

$0.000333 \quad 0.000329$

(81)

(81)

$-0.000068-0.000063$

$-0.000085-0.000062$

$-0.000065-0.000062$

(81)

(109)

(109)

$-0.000082-0.000060$

$-0.000058-0.000058$

$\begin{array}{lll}-0.000060 & -0.000055\end{array}$

$-0.000044-0.000047$

(109)

$(109)$
$(109)$

(109)

(109)

(109)

(109)

(66)

(90)

$(3)$
$(90)$

$(90)$
$(3)$

(90)

(66)

(3)

(3)

(90)

(66)

(3)

$(54)$
$(3)$

(3)

$(90)$
$(3)$

$(3)$
$(3)$

(90)

(3)

(3)

$(54)$
$(90)$

$-0.000041-0.000047$

$-0.000018-0.000029$

$0.000016 \quad 0.000003$

$0.000045 \quad 0.000032$

$0.000092 \quad 0.000083$

$0.000139 \quad 0.000132$

$\begin{array}{lll}0.000191 & 0.000187\end{array}$

$-0.000058-0.000053$

$-0.000098-0.000053$

$-0.000053-0.000044$

$-0.000061-0.000044$

$-0.000022-0.000025$

$-0.000029-0.000025$

$-0.000017-0.000018$

$0.000001-0.000007$

$0.000021 \quad 0.000011$

$0.000012 \quad 0.000011$

$0.000027 \quad 0.000021$

$0.000038 \quad 0.000028$

$0.000042 \quad 0.000028$

$0.000055 \quad 0.000045$

$\begin{array}{ll}0.000048 & 0.000045\end{array}$

$\begin{array}{lll}0.000071 & 0.000062\end{array}$

$\begin{array}{lll}0.000086 & 0.000079\end{array}$

$\begin{array}{lll}0.000080 & 0.000079\end{array}$

$0.000101 \quad 0.000095$

$\begin{array}{ll}0.000116 & 0.000111\end{array}$

$0.000116 \quad 0.000111$

$0.000112 \quad 0.000111$

Density Density App Vol Square of Inconsistent In
calc / Residual / Residual / Density based on

Apparent Delta density Density of $\mathrm{kg} / \mathrm{m}^{3} \quad \mathrm{~kg} / \mathrm{m}^{3} \quad \mathrm{~m}^{3} / \mathrm{kg}$

Res

0.18

3.57

2.86

4.47

11.27

11.63

31.85

0.00

0.01

0.00

0.02

0.00

0.00

0.00

0.00

0.02

0.02

0.20

1.14

2.52

4.34

4.66

5.11

0.00

0.20

0.04

0.13

0.01

0.03

0.00

0.24

0.77

0.01

0.41

1.43

2.46

2.07

0.16

2.47
2.60

0.13

2.42

1.42

1.99

2.03

0.18 nconsistent

based on molal volume $/ \mathrm{kg} / \mathrm{m}^{3}$ water $/ \mathrm{kg} / \mathrm{m}^{3}$ volume $1 \mathrm{~cm}^{3} / \mathrm{mo}$

\begin{tabular}{|c|c|c|}
\hline & & 988.0363 \\
\hline & & 988.0363 \\
\hline & & 988.0363 \\
\hline & & 988.0363 \\
\hline & & 988.0363 \\
\hline & 514.10 & 988.0363 \\
\hline & & 988.0363 \\
\hline-2.71 & & 985.6952 \\
\hline-3.38 & & 985.6952 \\
\hline & 4.218 & 985.6952 \\
\hline-3.29 & & 985.6952 \\
\hline & 8.459 & 985.6952 \\
\hline & 12.468 & 985.6952 \\
\hline-2.4 & & 985.6952 \\
\hline-1.774 & & 985.6952 \\
\hline & 20.776 & 985.6952 \\
\hline & 40.754 & 985.6952 \\
\hline & 78.552 & 985.6952 \\
\hline & 113.46 & 985.6952 \\
\hline & 177.641 & 985.6952 \\
\hline & 245.059 & 985.6952 \\
\hline & 322.162 & 985.6952 \\
\hline & & 983.1989 \\
\hline & & 983.1989 \\
\hline & & 983.1989 \\
\hline & & 983.1989 \\
\hline & & 983.1989 \\
\hline & & 983.1989 \\
\hline & & 983.1989 \\
\hline & & 983.1989 \\
\hline & & 983.1989 \\
\hline & & 983.1989 \\
\hline & & 983.1989 \\
\hline & & 983.1989 \\
\hline & 104.30 & 983.1989 \\
\hline & & 983.1989 \\
\hline & & 983.1989 \\
\hline & & 983.1989 \\
\hline & & 983.1989 \\
\hline & & 983.1989 \\
\hline & & 983.1989 \\
\hline & & 983.1989 \\
\hline & 211.80 & 983.1989 \\
\hline & & 983.1989 \\
\hline
\end{tabular}




\begin{tabular}{|c|c|c|c|c|}
\hline \multirow[t]{32}{*}{ molality } & molarity & $\mathrm{t} /{ }^{\circ} \mathrm{C}$ mass frac & ass frac & $\begin{array}{r}\text { Density } \\
\exp / \\
\mathrm{kg} / \mathrm{m}^{3}\end{array}$ \\
\hline & & 60 & 0.2026 & 1195.90 \\
\hline & & 60 & 0.2200 & 1216.62 \\
\hline & & 60 & 0.2400 & 1238.20 \\
\hline & & 60 & 0.2400 & 1238.80 \\
\hline & & 60 & 0.2600 & 1259.65 \\
\hline & & 60 & 0.2800 & 1280.93 \\
\hline & & 60 & 0.2800 & 1281.40 \\
\hline & & 60 & 0.3000 & 1301.92 \\
\hline & & 60 & 0.3000 & 1301.10 \\
\hline & & 60 & 0.3200 & 1322.53 \\
\hline & & 60 & 0.3200 & 1323.20 \\
\hline & & 60 & 0.3298 & 1329.50 \\
\hline & & 60 & 0.3400 & 1342.26 \\
\hline & & 60 & 0.3600 & 1362.16 \\
\hline & & 60 & 0.3600 & 1363.40 \\
\hline & & 60 & 0.3800 & 1380.89 \\
\hline & & 60 & 0.4000 & 1400.78 \\
\hline & & 60 & 0.4000 & 1402.10 \\
\hline & & 60 & 0.4000 & 1402.70 \\
\hline & & 60 & 0.4200 & 1421.38 \\
\hline & & 60 & 0.4400 & 1441.32 \\
\hline & & 60 & 0.4400 & 1440.50 \\
\hline & & 60 & 0.4600 & 1460.70 \\
\hline & & 60 & 0.4800 & 1478.64 \\
\hline & & 60 & 0.4800 & 1478.10 \\
\hline & & 60 & 0.4896 & 1497.30 \\
\hline & & 60 & 0.5000 & 1495.61 \\
\hline & & 60 & 0.5000 & 1495.20 \\
\hline & & 60 & 0.5000 & 1496.70 \\
\hline & & 60 & 0.5200 & 1511.16 \\
\hline & & 60 & 0.6000 & 1578.60 \\
\hline 0.07496 & & 65 & 0.0030 & 983.65 \\
\hline 0.10003 & & 65 & 0.0040 & 984.75 \\
\hline 0.15009 & & 65 & 0.0060 & 986.93 \\
\hline 0.30073 & & 65 & 0.0119 & 993.09 \\
\hline 0.50002 & & 65 & 0.0196 & 1001.05 \\
\hline & & 70 & 0.0200 & 998.76 \\
\hline & & 70 & 0.0400 & 1019.39 \\
\hline & & 70 & 0.0600 & 1040.06 \\
\hline & & 70 & 0.0800 & 1060.85 \\
\hline & & 70 & 0.1000 & 1081.79 \\
\hline & & 70 & 0.1000 & 1081.87 \\
\hline & & 70 & 0.1200 & 1102.89 \\
\hline & & 70 & 0.1400 & 1124.16 \\
\hline
\end{tabular}

Ref

$\begin{array}{rr}\text { App vol } & \text { App vol } \\ \exp / & \text { Calc / } \\ \mathrm{m}^{3} / \mathrm{kg} & \mathrm{m}^{3} / \mathrm{kg}\end{array}$

$$
\begin{array}{cc}
\exp / & \text { Calc / } \\
\mathrm{m}^{3} / \mathrm{kg} & \mathrm{m}^{3} / \mathrm{kg}
\end{array}
$$

(66)
983.1989 983.1989 983.1989 983.1989 983.1989 983.1989 983.1989 983.1989

$317.90 \quad 983.1989$ 983.1989 983.1989 983.1989 983.1989 983.1989 983.1989 983.1989 983.1989

$418.90 \quad 983.1989$ 983.1989 983.1989 983.1989 983.1989 983.1989 983.1989 983.1989 983.1989 983.1989

$512.00 \quad 983.1989$ 983.1989 983.1989 $595.40 \quad 983.1989$ 980.5548 980.5548 980.5548 980.5548 980.5548 977.7696 977.7696 977.7696 977.7696 977.7696

$104.10 \quad 977.7696$ 977.7696 977.7696 


\begin{tabular}{|c|c|c|c|c|}
\hline \multirow[t]{26}{*}{ molality } & \multirow[t]{26}{*}{ molarity } & $\mathbf{t} /{ }^{\circ} \mathrm{C}$ & ass frac & $\begin{array}{c}\text { Density } \\
\exp \\
\mathrm{kg} / \mathrm{m}^{3}\end{array}$ \\
\hline & & 70 & 0.1600 & 1145.55 \\
\hline & & 70 & 0.1800 & 1167.06 \\
\hline & & 70 & 0.2000 & 1188.66 \\
\hline & & 70 & 0.2000 & 1189.27 \\
\hline & & 70 & 0.2200 & 1210.29 \\
\hline & & 70 & 0.2400 & 1231.89 \\
\hline & & 70 & 0.2600 & 1253.42 \\
\hline & & 70 & 0.2800 & 1274.79 \\
\hline & & 70 & 0.3000 & 1295.92 \\
\hline & & 70 & 0.3000 & 1294.97 \\
\hline & & 70 & 0.3200 & 1316.72 \\
\hline & & 70 & 0.3400 & 1337.05 \\
\hline & & 70 & 0.3600 & 1356.82 \\
\hline & & 70 & 0.3800 & 1375.87 \\
\hline & & 70 & 0.4000 & 1394.03 \\
\hline & & 70 & 0.4000 & 1395.37 \\
\hline & & 70 & 0.4200 & 1413.18 \\
\hline & & 70 & 0.4400 & 1433.36 \\
\hline & & 70 & 0.4600 & 1452.84 \\
\hline & & 70 & 0.4800 & 1471.37 \\
\hline & & 70 & 0.5000 & 1488.82 \\
\hline & & 70 & 0.5000 & 1488.17 \\
\hline & & 70 & 0.5200 & 1504.92 \\
\hline & & 70 & 0.6000 & 1572.17 \\
\hline & & 70 & 0.7000 & 1647.57 \\
\hline 0.07496 & & 75 & 0.0030 & 977.93 \\
\hline 0.10003 & & 75 & 0.0040 & 979.06 \\
\hline 0.15009 & & 75 & 0.0060 & 981.21 \\
\hline 0.30073 & & 75 & 0.0119 & 987.34 \\
\hline 0.50002 & & 75 & 0.0196 & 995.28 \\
\hline & & 80 & 0.0005 & 972.30 \\
\hline & & 80 & 0.0099 & 982.20 \\
\hline & & 80 & 0.0100 & 982.40 \\
\hline & & 80 & 0.0200 & 992.90 \\
\hline & & 80 & 0.0400 & 1013.90 \\
\hline & & 80 & 0.0476 & 1021.80 \\
\hline & & 80 & 0.0800 & 1056.00 \\
\hline & & 80 & 0.0910 & 1067.40 \\
\hline & & 80 & 0.1000 & 1076.10 \\
\hline & & 80 & 0.1200 & 1098.30 \\
\hline & & 80 & 0.1600 & 1140.80 \\
\hline & & 80 & 0.2000 & 1183.40 \\
\hline & & 80 & 0.2000 & 1183.30 \\
\hline & & 80 & 0.2026 & 1184.50 \\
\hline
\end{tabular}

Ref

$\begin{array}{rr}\text { App vol } & \text { App vol } \\ \exp / & \text { Calc / } \\ \mathrm{m}^{3} / \mathrm{kg} & \mathrm{m}^{3} / \mathrm{kg}\end{array}$

\section{$\begin{array}{cc}\exp / & \text { Calc / } \\ \mathrm{m}^{3} / \mathrm{kg} & \mathrm{m}^{3} / \mathrm{kg}\end{array}$}

$\begin{array}{ll}0.000087 & 0.000084\end{array}$

$\begin{array}{ll}0.000101 & 0.000100\end{array}$

$\begin{array}{ll}0.000115 & 0.000115\end{array}$

$\begin{array}{ll}0.000113 & 0.000115\end{array}$

$0.000130 \quad 0.000131$

$0.000144 \quad 0.000146$

$0.000158 \quad 0.000160$

$\begin{array}{ll}0.000172 & 0.000175\end{array}$

$\begin{array}{ll}0.000186 & 0.000189\end{array}$

0.0001880 .000189

$0.000200 \quad 0.000203$

$\begin{array}{ll}0.000214 & 0.000217\end{array}$

0.0002290 .000230

$0.000244 \quad 0.000244$

$0.000259 \quad 0.000257$

$\begin{array}{lll}0.000258 & 0.000257\end{array}$

0.0002720 .000270

$0.000284 \quad 0.000282$

$0.000296 \quad 0.000295$

$0.000308 \quad 0.000307$

$0.000321 \quad 0.000319$

$0.000321 \quad 0.000319$

$0.000334 \quad 0.000331$

$0.000378 \quad 0.000376$

$0.000429 \quad 0.000429$

$-0.000054-0.000049$

$-0.000081-0.000048$

$-0.000089-0.000046$

$-0.000066-0.000041$

$-0.000048-0.000034$

$-0.000034-0.000048$

$-0.000072-0.000039$

$-0.000082-0.000039$

$-0.000064-0.000030$

$-0.000039-0.000012$

$-0.000029-0.000005$

$\begin{array}{ll}0.000003 & 0.000023\end{array}$

$0.000016 \quad 0.000032$

$0.000032 \quad 0.000040$

$0.000041 \quad 0.000056$

$0.000076 \quad 0.000088$

$0.000109 \quad 0.000119$

$0.000109 \quad 0.000119$

$0.000117 \quad 0.000121$
Density Density App Vol Square of Inconsistent Inconsistent calc / Residual / Residual / Density based on Apparent Delta density Density of $\mathrm{kg} / \mathrm{m}^{3} \quad \mathrm{~kg} / \mathrm{m}^{3} \quad \mathrm{~m}^{3} / \mathrm{kg}$ $\begin{array}{ll}-0.6033 & 0.000003\end{array}$ $\begin{array}{ll}-0.3924 & 0.000002\end{array}$

Res

0.36

0.15

$\begin{array}{lll}187.45 & -0.3924 & 0.000002 \\ 188.74 & -0.0817 & 0.000000\end{array}$

0.01

$0.5279-0.000002$

0.28

0.08

0.47

$0.6871-0.000002$

1.19

$1.0909-0.00$

$1.4359-0.000003$

$1.6663-0.000003$

2.06

2.78

0.51

$\begin{array}{lll}1.7170 & -0.000003 \quad 2.95\end{array}$

$\begin{array}{ll}1.4732-0.000002 & 2.17\end{array}$

$0.8705-0.000001 \quad 0.76$

$\begin{array}{lll}-0.2253 & 0.000000 & 0.05\end{array}$

3.84

0.38

5.87

2.41

1.10

1.30

1.30

3.73

6.67

13.43

10.22

0.36

0.00

0.02

0.06

0.09

0.08

0.00

0.10

0.17

0.46

1.24

1.36

2.96

2.68

0.85

4.61

6.39

8.46

7.90

1.61
$/ \mathrm{kg} / \mathrm{m}^{3}$ water $/ \mathrm{kg} / \mathrm{m}^{3}$ apparen $1 \mathrm{~cm}^{3} / \mathrm{mo}$

\begin{tabular}{|c|c|c|}
\hline & & 977.7696 \\
\hline & & 977.7696 \\
\hline & & 977.7696 \\
\hline & 211.50 & 977.7696 \\
\hline & & 977.7696 \\
\hline & & 977.7696 \\
\hline & & 977.7696 \\
\hline & & 977.7696 \\
\hline & & 977.7696 \\
\hline & 317.20 & 977.7696 \\
\hline & & 977.7696 \\
\hline & & 977.7696 \\
\hline & & 977.7696 \\
\hline & & 977.7696 \\
\hline & & 977.7696 \\
\hline & 417.60 & 977.7696 \\
\hline & & 977.7696 \\
\hline & & 977.7696 \\
\hline & & 977.7696 \\
\hline & & 977.7696 \\
\hline & & 977.7696 \\
\hline & 510.40 & 977.7696 \\
\hline & & 977.7696 \\
\hline & 594.40 & 977.7696 \\
\hline & 669.80 & 977.7696 \\
\hline-2.16 & & 974.849 \\
\hline-3.22 & & 974.849 \\
\hline-3.57 & & 974.849 \\
\hline-2.651 & & 974.849 \\
\hline-1.928 & & 974.849 \\
\hline & & 971.7978 \\
\hline & & 971.7978 \\
\hline & & 971.7978 \\
\hline & & 971.7978 \\
\hline & & 971.7978 \\
\hline & & 971.7978 \\
\hline & & 971.7978 \\
\hline & & 971.7978 \\
\hline & 104.30 & 971.7978 \\
\hline & & 971.7978 \\
\hline & & 971.7978 \\
\hline & 211.60 & 971.7978 \\
\hline & & 971.7978 \\
\hline
\end{tabular}




\begin{tabular}{|c|c|c|c|c|}
\hline \multirow[t]{15}{*}{ molality } & \multirow[t]{15}{*}{ molarity } & $\mathrm{t} /{ }^{\circ} \mathrm{C}$ mass frac & ass frac & $\begin{array}{r}\text { Density } \\
\exp / \\
\mathrm{kg} / \mathrm{m}^{3}\end{array}$ \\
\hline & & 80 & 0.2400 & 1225.90 \\
\hline & & 80 & 0.2800 & 1268.20 \\
\hline & & 80 & 0.3000 & 1288.80 \\
\hline & & 80 & 0.3200 & 1309.70 \\
\hline & & 80 & 0.3298 & 1318.10 \\
\hline & & 80 & 0.3600 & 1349.80 \\
\hline & & 80 & 0.4000 & 1388.70 \\
\hline & & 80 & 0.4000 & 1388.90 \\
\hline & & 80 & 0.4400 & 1426.60 \\
\hline & & 80 & 0.4800 & 1464.10 \\
\hline & & 80 & 0.5000 & 1481.30 \\
\hline & & 80 & 0.5000 & 1482.70 \\
\hline & & 80 & 0.6000 & 1566.00 \\
\hline & & 80 & 0.7000 & 1643.70 \\
\hline 0.07496 & & 85 & 0.0030 & 971.75 \\
\hline 0.10003 & & 85 & 0.0040 & 972.83 \\
\hline 0.15009 & & 85 & 0.0060 & 975.02 \\
\hline 0.30073 & & 85 & 0.0119 & 981.14 \\
\hline 0.50002 & & 85 & 0.0196 & 989.05 \\
\hline & & 90 & 0.1000 & 1070.12 \\
\hline & & 90 & 0.2000 & 1177.42 \\
\hline & & 90 & 0.3000 & 1282.52 \\
\hline & & 90 & 0.4000 & 1382.02 \\
\hline & & 90 & 0.5000 & 1474.42 \\
\hline & & 90 & 0.6000 & 1559.82 \\
\hline & & 90 & 0.7000 & 1639.72 \\
\hline 0.07496 & & 95 & 0.0030 & 965.04 \\
\hline 0.10003 & & 95 & 0.0040 & 966.13 \\
\hline 0.15009 & & 95 & 0.0060 & 968.33 \\
\hline 0.30073 & & 95 & 0.0119 & 974.49 \\
\hline 0.50002 & & 95 & 0.0196 & 982.41 \\
\hline & & 100 & 0.0099 & 968.70 \\
\hline & & 100 & 0.0100 & 969.30 \\
\hline & & 100 & 0.0200 & 979.70 \\
\hline & & 100 & 0.0400 & 1000.90 \\
\hline & & 100 & 0.0476 & 1008.40 \\
\hline & & 100 & 0.0800 & 1043.20 \\
\hline & & 100 & 0.0910 & 1053.90 \\
\hline & & 100 & 0.1000 & 1063.76 \\
\hline & & 100 & 0.1200 & 1085.50 \\
\hline & & 100 & 0.1600 & 1127.70 \\
\hline & & 100 & 0.2000 & 1171.16 \\
\hline & & 100 & 0.2000 & 1170.00 \\
\hline & & 100 & 0.2026 & 1171.10 \\
\hline
\end{tabular}

Ref

$\begin{array}{rr}\text { App vol } & \text { App vol } \\ \exp / & \text { Calc / } \\ \mathrm{m}^{3} / \mathrm{kg} & \mathrm{m}^{3} / \mathrm{kg}\end{array}$

$$
\begin{array}{rr}
\exp / & \text { Calc / } \\
\mathrm{m}^{3} / \mathrm{kg} & \mathrm{m}^{3} / \mathrm{kg}
\end{array}
$$

(90) $\quad 0.000140 \quad 0.000149$

$\begin{array}{ll}0.000170 & 0.000178\end{array}$

0.0001850 .000192

0.0001990 .000206

$0.000209 \quad 0.000213$

0.0002290 .000233

$0.000257 \quad 0.000259$

$0.000256 \quad 0.000259$

$\begin{array}{ll}0.000283 & 0.000284\end{array}$

$0.000308 \quad 0.000308$

$0.000321 \quad 0.000320$

$0.000320 \quad 0.000320$

$0.000378 \quad 0.000376$

$0.000428 \quad 0.000428$

$-0.000082-0.000042$

$\begin{array}{lll}(81) & -0.000088 & -0.000041 \\ (81) & -0.000103 & -0.000040\end{array}$

(81)

(81)

(54)

(54)

(54)

(54)

(54)

(54)

(54)

(81)

(81)

$(81)$
$(81)$

$(81)$
$(81)$

(66)

(90)

(90)

(90)

(66)

(90)

(66)

(54)

$(90)$
$(90)$

(90)

$(54)$
$(90)$

(66)

$-0.000076-0.000034$

$\begin{array}{lll}-0.000055 & -0.000027\end{array}$

$0093-0.000036$

-0.000104 -0.000035

$-0.000091-0.000028$

$-0.000081-0.000026$

(1000000

Density Density App Vol Square of Inconsistent Inconsistent

Apparent Delta density Density of calc/Residual/ Residual / Density based on

based on molal volume $/ \mathrm{kg} / \mathrm{m}^{3}$ water $/ \mathrm{kg} / \mathrm{m}^{3}$ $\mathrm{kg} / \mathrm{m}^{3}$ $\mathrm{kg} / \mathrm{m}^{3} \quad \mathrm{~m}^{3} / \mathrm{kg}$

Res

10.60

13.20

$3.6329-0.000008$

11.64

$285.39 \quad 3.4124-0.000007$

13.12

$3.6225-0.000007$

13.12
3.75

$\begin{array}{llll}1316.16 & 1.9367 & -0.000003 & 3.75 \\ 1346.99 & 2.8124 & -0.000004 & 7.91\end{array}$

$\begin{array}{ll}1.5939-0.000002 & 2.54\end{array}$

$\begin{array}{rrrr}1387.10 & 1.7961 & -0.000002 & 3.23 \\ 1426.23 & 0.3709 & 0.000000 & 0.14\end{array}$

2.54
3.23

$\begin{array}{llll}1464.17 & -0.0651 & 0.000000 & 0.00\end{array}$

$\begin{array}{lll}-1.3279 & 0.000001 & 1.76\end{array}$

$482.63 \quad 0.07$

0.01

$\begin{array}{lll}1568.90 & -2.9025 & 0.000002\end{array}$

$\begin{array}{ll}0.5797 & 0.000000\end{array}$

8.42
0.34

$971.64-0.1108-0.000039-0.01$

$\begin{array}{lll}972.65 & 0.1741-0.000046 & 0.03\end{array}$

$\begin{array}{llll}974.66 & 0.3585 & -0.000063 & 0.13\end{array}$

$\begin{array}{llll}980.66 & 0.4786 & -0.000042 & 0.23\end{array}$

0.29

$2.6935-0.000024$

7.25

$5.7107-0.000021 \quad 32.61$

$6.5193-0.000013 \quad 42.50$

$\begin{array}{llllll}0.000255 & 0.000261 & 1377.73 & 4.2880 & -0.000006 & 18.39\end{array}$

$\begin{array}{llllll}0.000321 & 0.000321 & 1474.06 & 0.3562 & 0.000000 & 0.13\end{array}$

$\begin{array}{lllll}0.000378 & 0.000376 & 1562.08 & -2.2613 & 0.000002\end{array}$

$\begin{array}{rrr}0.000427 & 0.000428 & 1639.04\end{array}$

$\begin{array}{rr}-2.2613 & 0.000002 \\ 0.6829 & 0.000000\end{array}$

964.88

965.88

$0.1598-0.000057$

$0.2568-0.000069$

$0.4745-0.000085$

967.86

$\begin{array}{lll}-0.000067 & -0.000021 & 981.53\end{array}$

$0.7121-0.000063$

$0.8773-0.000046$

968.19

$0.5109-0.000055$

$1.0115-0.000108$

$\begin{array}{lll}-0.000093 & -0.000017 & 978.25\end{array}$

$\begin{array}{rrr}-0.000044 & 0.000007 & 1005.94\end{array}$

$1.4478-0.000076$

$2.6095-0.000065$

$2.4580-0.000051$

$4.4312-0.000051$

$3.9163-0.000039$

$\begin{array}{lll}0.000004 & 0.000043 & 1049.98\end{array}$

$\begin{array}{lll}0.000010 & 0.000050 & 1059.18\end{array}$

$4.5804-0.000041$

5.11

0.47

0.03

0.23

0.51

0.77

0.26

1.02

2.10

6.81

6.04

19.64

15.34

$\begin{array}{lll}0.000025 & 0.000066 & 1079.70 \\ 0.000064 & 0.000097 & 1120.96\end{array}$

$6.7402-0.000041$

33.68

$\begin{array}{lll}0.000095 & 0.000128 & 1162.43\end{array}$

8.7292

45.43

$\begin{array}{lll}0.000100 & 0.000128 & 1162.43\end{array}$

$\begin{array}{lll}7.5656 & -0.000028\end{array}$

76.20

$\begin{array}{lll}0.000108 & 0.000129 & 1165.13\end{array}$ $1 \mathrm{~cm}^{3} / \mathrm{mol}$

volume

971.7978

971.7978

$317.00 \quad 971.7978$

971.7978

971.7978
971.7978

$416.90 \quad 971.7978$

971.7978

971.7978

971.7978

$509.50 \quad 971.7978$

971.7978

$594.20 \quad 971.7978$

$671.90 \quad 971.7978$

968.6203

968.6203

968.6203

968.6203

968.6203

$-2.201$

$104.80 \quad 965.3201$

$212.10 \quad 965.3201$

$317.20 \quad 965.3201$

$416.70 \quad 965.3201$

$509.10 \quad 965.3201$

$594.50 \quad 965.3201$

$674.40 \quad 965.3201$

961.9004

$-3.15$

$-4.71$

$-3.63$

$-2.686$

961.9004

961.9004

961.9004

958.3637

958.3637

958.3637

958.3637

958.3637

958.3637

958.3637

$105.40 \quad 958.3637$

958.3637

958.3637

$212.80 \quad 958.3637$

958.3637

958.3637 
molality molarity

\begin{tabular}{|c|c|c|}
\hline $1{ }^{\circ} \mathrm{C}$ & ass frac & $\begin{array}{r}\text { Density } \\
\exp / \\
\mathrm{kg} / \mathrm{m}^{3}\end{array}$ \\
\hline 100 & 0.2400 & 1212.40 \\
\hline & 0.2800 & 254.60 \\
\hline 100 & 0.3000 & 1275.96 \\
\hline 100 & 0.3200 & 1296.00 \\
\hline 100 & 0.3298 & 1304.50 \\
\hline 100 & 0.3600 & 1336.00 \\
\hline 100 & 0.4000 & 1375.16 \\
\hline 100 & 0.4000 & 1375.00 \\
\hline 100 & 0.4400 & 1412.70 \\
\hline 100 & 0.4800 & 1450.30 \\
\hline 00 & 0.50 & 14 \\
\hline 100 & 0.50 & 9.00 \\
\hline 100 & 0.60 & 3.56 \\
\hline 00 & 0.70 & 5.46 \\
\hline 170 & 0.10 & 7.05 \\
\hline 170 & 0.4 & 1368 \\
\hline 110 & 0.5 & 1460 \\
\hline 110 & $0.6 c$ & 154 \\
\hline 110 & 0.7000 & 1630.95 \\
\hline 12 & 0.0099 & .80 \\
\hline 12 & & \\
\hline 120 & 0.0910 & 1039.00 \\
\hline 120 & 0.1000 & \\
\hline 120 & 0.2026 & 1156.10 \\
\hline 120 & & \\
\hline 12 & 0.6000 & \\
\hline & 0.7000 & 1626.28 \\
\hline
\end{tabular}

Ref

$\begin{array}{rr}\text { App vol } & \text { App vol } \\ \exp / & \text { Calc / } \\ \mathrm{m}^{3} / \mathrm{kg} & \mathrm{m}^{3} / \mathrm{kg}\end{array}$

Density

Density App Vol Square of Inconsistent Inconsistent $\begin{array}{rrrr}\mathrm{calc} / & \text { Residual / } & \text { Residual / } & \text { Density based on } \\ \mathrm{kg} / \mathrm{m}^{3} & \mathrm{~kg} / \mathrm{m}^{3} & \mathrm{~m}^{3} / \mathrm{kg} & \text { Res density }\end{array}$

(90)

0.0001320 .000156 $0.000164 \quad 0.000184$

1203.98

$8.4152-0.000024$

$9.1345-0.000021$

$9.8321-0.000020$

$9.2785-0.000017$

$\begin{array}{lll}7.7235 & -0.000014\end{array}$

$8.4101-0.000013$

$7.2624-0.000010$

$7.0988-0.000009$

$5.2193-0.000006$

$4.1495-0.000004$

$2.4753-0.000002$

$3.9116-0.000004$

$\begin{array}{ll}-1.3590 & 0.000001\end{array}$

$\begin{array}{ll}0.7321 & 0.000000\end{array}$

$6.5788-0.000059$

$10.6189-0.000014$

$4.8368-0.000005$

$\begin{array}{ll}-0.1809 & 0.000000\end{array}$

$0.7494 \quad 0.000000$

$1.1649-0.000130$

$4.0261-0.000086$

$\begin{array}{lll}6.6865 & -0.000069\end{array}$

$8.7870-0.000080$

$10.9644-0.000041$

$7.7504-0.000007$

$\begin{array}{lll}1.3891 & -0.000001\end{array}$

$0.8567 \quad 0.000000$

$0.1722-0.0000114606 .511$ Sum Chi sqr

$2.7159 \quad 0.000027$

Average Res

Std dev Res

Avg - 4std
Avg + 4std

$\begin{array}{ll}11.0359 & 0.000095\end{array}$
Apparent Delta density Density of based on molal volum apparent $/ \mathrm{cm} / \mathrm{mol}$

\begin{tabular}{|c|c|c|c|}
\hline 70.82 & & & 958.3637 \\
\hline 83.44 & & & 958.3637 \\
\hline 96.67 & & 317.60 & 958.3637 \\
\hline 86.09 & & & 958.3637 \\
\hline 59.65 & & & 958.3637 \\
\hline 70.73 & & & 958.3637 \\
\hline 52.74 & & 416.80 & 958.3637 \\
\hline 50.39 & & & 958.3637 \\
\hline 27.24 & & & 958.3637 \\
\hline 17.22 & & & 958.3637 \\
\hline 6.13 & & 509.20 & 958.3637 \\
\hline 15.30 & & & 958.3637 \\
\hline 1.85 & & 595.20 & 958.3637 \\
\hline 0.54 & & 677.10 & 958.3637 \\
\hline 43.28 & & 106.10 & 950.9471 \\
\hline 112.76 & & 417.30 & 950.9471 \\
\hline 23.39 & & 509.60 & 950.9471 \\
\hline 0.03 & & 596.30 & 950.9471 \\
\hline 0.56 & & 680.00 & 950.9471 \\
\hline 1.36 & $!$ & & 943.0825 \\
\hline 16.21 & & & 943.0825 \\
\hline 44.71 & & & 943.0825 \\
\hline 77.21 & & 107.00 & 943.0825 \\
\hline 120.22 & & & 943.0825 \\
\hline 60.07 & & 510.60 & 943.0825 \\
\hline 1.93 & & 597.90 & 943.0825 \\
\hline 0.73 & & 683.20 & 943.0825 \\
\hline
\end{tabular}

Curve fit for the model App vol $=\left(w+c 2+c 3^{\star} t{ }^{\circ} \mathrm{C}\right) /\left(c 0^{*} w+c 1\right) / e^{\wedge}\left(0.000001^{*}\left(t{ }^{\circ} \mathrm{C}+c 4\right)^{\wedge} 2\right)$

$\begin{array}{lr}\text { Curve fit for the model App vol= } \\ \text { c0 } \\ \text { c1 } & 385.5529 \\ \text { c2 } & 753.4695 \\ \text { c3 } & -0.10938 \\ \text { c4 } & 0.000695 \\ \text { Min T } & 542.8812 \\ \text { Max T } & 0 \\ \text { Min w } & 120 \\ \text { Max w } & 0.0005 \\ \text { Avg dens res } & 0.7000 \\ \text { Std dens res } & 0.172197 \\ & 2.715928\end{array}$


molality molarity

$t /{ }^{\circ} \mathrm{C}$ mass frac Density

$\exp /$

Ref

App vol

exp /

App vol Calc /

Dens

Density App Vol Square of Inconsistent Inconsistent calc / Residual / Residual / Density based on $\mathrm{kg} / \mathrm{m}^{3}$ $\mathrm{m}^{3} / \mathrm{kg}$

Res based on

apparen

Avg app vol res *10^3 $\quad-0.01094$

Std app vol res *10^3 0.026593

No of points in corr

623

No of inconsistent poir

12

Inconsistent data not used (Residual greater than average + /- $\mathbf{4}$ standard deviations)

$\begin{array}{llllll}20 & 0.2026 & 1210.90 & (66) & 0.000133 & 0.000094\end{array}$

$\begin{array}{lll}20 & 0.3298 & 1344.50\end{array}$

$0.0005 \quad 990.00$

(66) $\quad 0.000219 \quad 0.000194$

$\begin{array}{lllll}0.4896 & 1485.90 & (66) & 0.005519 & -0.000077\end{array}$

$1222.72 \quad-11.8237 \quad 0.000039$

$\begin{array}{lll}1359.93 & -15.4308 & 0.000026\end{array}$

$\begin{array}{llllll}0.005519 & -0.000077 & 992.75 & -2.7499 & 0.005596\end{array}$

139.80

998.2041 $1473.07 \quad 12.8291-0.000012$

238.11
7.56

998.2041

992.2158

971.7978

$0.4896 \quad 1472.50$

$0.000299 \quad 0.000316$

$1455.28 \quad 17.2240-0.000016$

296.66

$\begin{array}{llllll}0.000173 & 0.000201 & 1255.80 & 13.4488 & -0.000028\end{array}$

180.87

$\begin{array}{ll}0.3000 & 1269.25 \\ 0.2000 & 1164.75\end{array}$

(54)

$0.4896 \quad 1457.50$

$\begin{array}{ll}0.000086 & 0.000131\end{array}$

$\begin{array}{llll}1152.69 & 12.0608 & -0.000045\end{array}$

145.46

469.10

$0.3298 \quad 1289.70$

$\begin{array}{lll}(66) & 0.000296 & 0.000317 \\ \text { (66) } & 0.000196 & 0.000222\end{array}$

$1435.84 \quad 21.6587-0.000021$

200.36

$0.4000 \quad 1361.18$

$0.000246 \quad 0.000266$

1275.55

$14.1549-0.000026$

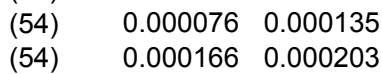

$\begin{array}{lll}14.2615 & -0.000019\end{array}$

$\begin{array}{ll}0.2000 & 1157.98 \\ 0.3000 & 1262.38\end{array}$

$1142.48 \quad 15.5036-0.000059$

203.39

240.36

301.70

318.30

$213.80 \quad 950.9471$ 943.0825 943.0825

$418.10 \quad 943.0825$ $214.90 \quad 943.0825$ $319.30 \quad 943.0825$ 
Density of aqueous solutions of $\mathrm{Na} 3 \mathrm{PO} 4$

References (122) Sohnel, P.; Novotny, P.; Solc, Z.; Densities of aqueous solutions of 18 inorganic substances, J Chem Eng Data, 1984, 29, 379-382

\begin{tabular}{|c|c|c|c|c|c|c|c|c|c|c|c|c|c|}
\hline molality molarity & $\mathbf{t} /{ }^{\circ} \mathrm{C}$ & ass frac & $\begin{array}{r}\text { Density } \\
\exp / \\
\mathrm{kg} / \mathrm{m}^{3}\end{array}$ & Ref & $\begin{array}{r}\text { App vol } \\
\exp / \\
\mathrm{m}^{3} / \mathrm{kg}\end{array}$ & $\begin{array}{r}\text { App vol } \\
\text { Calc / } \\
\mathrm{m}^{3} / \mathrm{kg}\end{array}$ & $\begin{array}{r}\text { Density } \\
\text { calc / } \\
\mathrm{kg} / \mathrm{m}^{3}\end{array}$ & $\begin{array}{r}\text { Density } \\
\text { Residual / } \\
\mathrm{kg} / \mathrm{m}^{3}\end{array}$ & $\begin{array}{r}\text { App Vol } \\
\text { Residual / } \\
\mathrm{m}^{3} / \mathrm{kg}\end{array}$ & $\begin{array}{r}\text { Square of } \\
\text { Density } \\
\text { Residual }\end{array}$ & $\begin{array}{l}\text { Inconsistent } \\
\text { based on } \\
\text { density }\end{array}$ & $\begin{array}{l}\text { Inconsistent } \\
\text { based on } \\
\text { apparent } \\
\text { volume }\end{array}$ & $\begin{array}{r}\text { Density of } \\
\text { water } / \mathrm{kg} / \mathrm{m}^{3}\end{array}$ \\
\hline & 40 & 0.0500 & 1047.3 & (122) & -0.000052 & -0.000058 & 1047.6 & -0.3156 & 0.000006 & 0.100 & & & 992.2158 \\
\hline & 40 & 0.1000 & 1106.1 & (122) & -0.000030 & -0.000027 & 1105.8 & 0.3377 & -0.000003 & 0.114 & & & 992.2158 \\
\hline & 40 & 0.1500 & 1166.5 & (122) & 0.000004 & 0.000002 & 1166.9 & -0.3890 & 0.000002 & 0.151 & & & 992.2158 \\
\hline & 60 & 0.0500 & 1037.7 & (122) & -0.000051 & -0.000056 & 1038.0 & -0.2507 & 0.000005 & 0.063 & & & 983.1989 \\
\hline & 60 & 0.1000 & 1095.5 & (122) & -0.000026 & -0.000025 & 1095.5 & 0.0351 & 0.000000 & 0.001 & & & 983.1989 \\
\hline & 60 & 0.1500 & 1155.4 & (122) & 0.000007 & 0.000004 & 1156.0 & -0.5846 & 0.000003 & 0.342 & & & 983.1989 \\
\hline & 60 & 0.2000 & 1221.5 & (122) & 0.000025 & 0.000031 & 1219.8 & 1.7187 & -0.000006 & 2.954 & & & 983.1989 \\
\hline & 60 & 0.2500 & 1286.5 & (122) & 0.000058 & 0.000056 & 1287.2 & -0.6596 & 0.000002 & 0.435 & & & 983.1989 \\
\hline & 80 & 0.0500 & 1025.5 & (122) & -0.000049 & -0.000054 & 1025.8 & -0.2665 & 0.000005 & 0.071 & & & 971.7978 \\
\hline & 80 & 0.1000 & 1083.1 & (122) & -0.000028 & -0.000023 & 1082.5 & 0.5850 & -0.000005 & 0.342 & & & 971.7978 \\
\hline & 80 & 0.1500 & 1141.6 & (122) & 0.000009 & 0.000005 & 1142.3 & -0.6975 & 0.000004 & 0.487 & & & 971.7978 \\
\hline & 80 & 0.2000 & 1206.5 & (122) & 0.000028 & 0.000032 & 1205.4 & 1.1004 & -0.000004 & 1.211 & & & 971.7978 \\
\hline & 80 & 0.2500 & 1270.6 & (122) & 0.000061 & 0.000057 & 1272.1 & -1.5427 & 0.000004 & 2.380 & & & 971.7978 \\
\hline & 80 & 0.3000 & 1343.5 & (122) & 0.000080 & 0.000081 & 1342.9 & 0.6100 & -0.000001 & 0.372 & & & 971.7978 \\
\hline & & & & & & $A v$ & rage Res & -0.0228 & 0.000001 & 9.022 & Sum chi sqr & & \\
\hline & & & & & & & dev Res & 0.8328 & 0.000004 & & & & \\
\hline & & & & & & & $\mathrm{vg}-4 \mathrm{std}$ & -3.3538 & -0.000015 & & & & \\
\hline & & & & & & & $\mathrm{vg}+4 \mathrm{std}$ & 3.3082 & 0.000016 & & & & \\
\hline
\end{tabular}

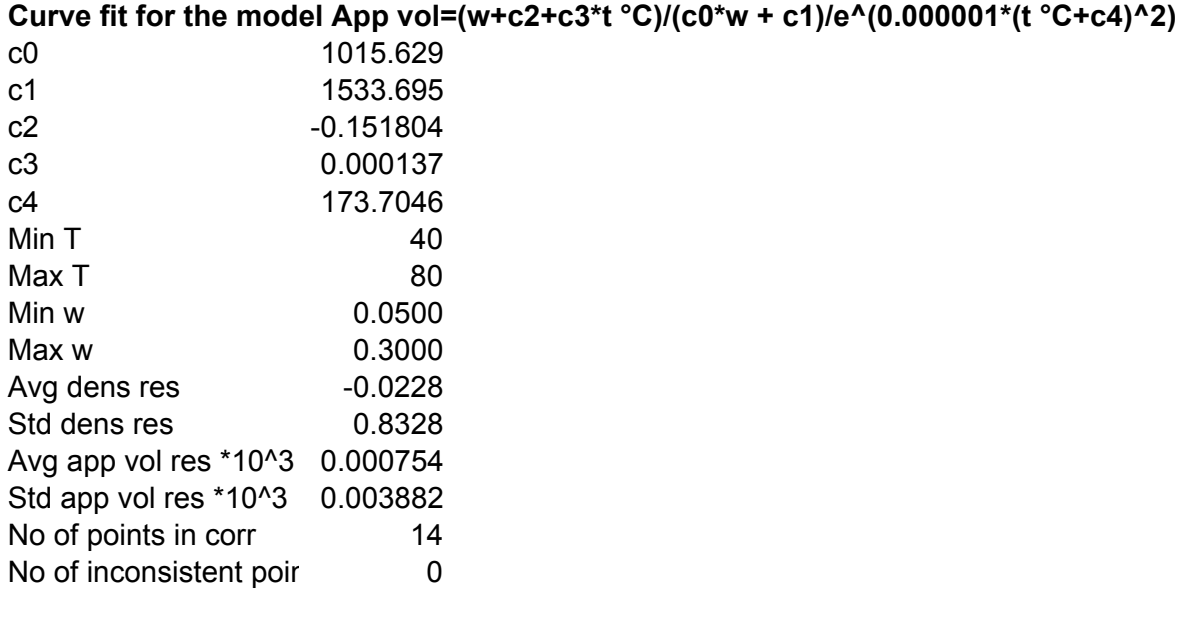

Inconsistent data not used (Residual greater than average $+/-4$ standard deviations) 
Density of aqueous solutions of $\mathrm{Na2SO}$

References (90) Perry, R. H.; Green, D. W. Perry's Chemical Engineers' Handbook; McGraw Hill: New York, 7th edition, 1997.

(122) Sohnel, P.; Novotny, P; Solc, Z.; Densities of aqueous solutions of 18 inorganic substances, J Chem Eng Data, 1984, 29, 379-382

(131) Vázquez, G.; Alvarez, E.; Cancela, A.; Navaza, J. M.; Density, Viscosity, and Surface Tension of Aqueous Solutions of Sodium Sulfite and Sodium Sulfite + Sucrose from 25 to $40^{\circ} \mathrm{C}$.; J. Chem. Eng. Data, 1995, 40, 1101-1105

\begin{tabular}{|c|c|c|c|c|c|c|c|c|c|c|c|c|c|c|}
\hline \multirow{8}{*}{\multicolumn{2}{|c|}{ molality }} & \multicolumn{2}{|c|}{$\mathrm{t} /{ }^{\circ} \mathrm{C}$ mass frac } & $\begin{array}{r}\text { Density } \\
\exp / \\
\mathrm{kg} / \mathrm{m}^{3}\end{array}$ & Ref & $\begin{array}{r}\text { App vol } \\
\exp / \\
\mathrm{m}^{3} / \mathrm{kg}\end{array}$ & $\begin{array}{r}\text { App vol } \\
\text { Calc / } \\
\mathrm{m}^{3} / \mathrm{kg}\end{array}$ & $\begin{array}{r}\text { Density } \\
\text { calc / } \\
\mathrm{kg} / \mathrm{m}^{3}\end{array}$ & $\begin{array}{r}\text { Density } \\
\text { Residual / } \\
\mathrm{kg} / \mathrm{m}^{3}\end{array}$ & $\begin{array}{r}\text { App Vol } \\
\text { Residual / } \\
\mathrm{m}^{3} / \mathrm{kg}\end{array}$ & $\begin{array}{r}\text { Square of } \\
\text { Density } \\
\text { Res }\end{array}$ & $\begin{array}{l}\text { Inconsistent } \\
\text { based on } \\
\text { density }\end{array}$ & $\begin{array}{c}\text { Inconsistent } \\
\text { based on } \\
\text { apparent } \\
\text { volume }\end{array}$ & $\begin{array}{r}\text { Density of } \\
\text { water } / \mathrm{kg} / \mathrm{m}^{3}\end{array}$ \\
\hline & & 19 & 0.0100 & 1007.8 & (90) & 0.000068 & 0.000269 & 1005.8 & 2.0384 & -0.000201 & 4.155 & & & 998.4052 \\
\hline & & 19 & 0.0200 & 1017.2 & (90) & 0.000076 & 0.000239 & 1013.8 & 3.3637 & -0.000163 & 11.315 & & & 998.4052 \\
\hline & & 19 & 0.0400 & 1036.3 & (90) & 0.000086 & 0.000206 & 1031.2 & 5.1280 & -0.000120 & 26.296 & & & 998.4052 \\
\hline & & 19 & 0.0800 & 1075.1 & (90) & 0.000108 & 0.000176 & 1068.9 & 6.2027 & -0.000067 & 38.473 & & & 998.4052 \\
\hline & & 19 & 0.1200 & 1114.6 & (90) & 0.000131 & 0.000162 & 1110.1 & 4.5403 & -0.000031 & 20.614 & & & 998.4052 \\
\hline & & 19 & 0.1600 & 1154.9 & (90) & 0.000153 & 0.000154 & 1154.7 & 0.1550 & -0.000001 & 0.024 & & & 998.4052 \\
\hline & & 19 & 0.1800 & 1175.5 & (90) & 0.000163 & 0.000151 & 1178.5 & -3.0104 & 0.000012 & 9.063 & & & 998.4052 \\
\hline & 0.1 & 25 & 0.0125 & 1007.2 & (131) & 0.000195 & 0.000245 & 1006.6 & 0.6418 & -0.000051 & 0.412 & & & 997.0449 \\
\hline & 0.2 & 25 & 0.0248 & 1015.8 & (131) & 0.000257 & 0.000219 & 1016.8 & -0.9612 & 0.000038 & 0.924 & & & 997.0449 \\
\hline & 0.3 & 25 & 0.0369 & 1025.3 & (131) & 0.000254 & 0.000203 & 1027.3 & -1.9588 & 0.000050 & 3.837 & & & 997.0449 \\
\hline & 0.4 & 25 & 0.0487 & 1035.0 & (131) & 0.000248 & 0.000192 & 1037.9 & -2.9198 & 0.000056 & 8.525 & & & 997.0449 \\
\hline & & 25 & 0.0500 & 1044.6 & (122) & 0.000090 & 0.000191 & 1039.1 & 5.4999 & -0.000101 & 30.249 & & & 997.0449 \\
\hline & 0.5 & 25 & 0.0603 & 1044.7 & (131) & 0.000245 & 0.000184 & 1048.7 & -3.9900 & 0.000060 & 15.920 & & & 997.0449 \\
\hline & & 25 & 0.1000 & 1092.8 & (122) & 0.000124 & 0.000168 & 1087.6 & 5.1965 & -0.000044 & 27.004 & & & 997.0449 \\
\hline & & 25 & 0.1500 & 1143.3 & (122) & 0.000148 & 0.000158 & 1141.3 & 1.9555 & -0.000010 & 3.824 & & & 997.0449 \\
\hline & & 25 & 0.2000 & 1198.2 & (122) & 0.000161 & 0.000152 & 1200.9 & -2.6585 & 0.000009 & 7.068 & & & 997.0449 \\
\hline & 0.1 & 30 & 0.0125 & 1006.0 & (131) & 0.000179 & 0.000231 & 1005.3 & 0.6577 & -0.000052 & 0.433 & & & 995.6473 \\
\hline & 0.2 & 30 & 0.0248 & 1014.6 & (131) & 0.000249 & 0.000210 & 1015.6 & -1.0122 & 0.000040 & 1.025 & & & 995.6473 \\
\hline & 0.3 & 30 & 0.0369 & 1023.9 & (131) & 0.000254 & 0.000196 & 1026.1 & -2.2330 & 0.000058 & 4.986 & & & 995.6473 \\
\hline & 0.4 & 30 & 0.0488 & 1033.7 & (131) & 0.000246 & 0.000187 & 1036.8 & -3.0823 & 0.000059 & 9.501 & & & 995.6473 \\
\hline & 0.5 & 30 & 0.0604 & 1043.4 & (131) & 0.000243 & 0.000181 & 1047.5 & -4.1277 & 0.000063 & 17.038 & & & 995.6473 \\
\hline & 0.1 & 35 & 0.0125 & 1004.5 & (131) & 0.000170 & 0.000215 & 1003.9 & 0.5676 & -0.000045 & 0.322 & & & 994.0319 \\
\hline & 0.2 & 35 & 0.0249 & 1012.8 & (131) & 0.000257 & 0.000199 & 1014.3 & -1.4879 & 0.000058 & 2.214 & & & 994.0319 \\
\hline & 0.3 & 35 & 0.0370 & 1022.3 & (131) & 0.000254 & 0.000189 & 1024.8 & -2.5300 & 0.000065 & 6.401 & & & 994.0319 \\
\hline & 0.4 & 35 & 0.0489 & 1032.0 & (131) & 0.000248 & 0.000182 & 1035.5 & -3.4798 & 0.000067 & 12.109 & & & 994.0319 \\
\hline & 0.5 & 35 & 0.0605 & 1041.7 & (131) & 0.000245 & 0.000177 & 1046.2 & -4.5050 & 0.000068 & 20.295 & & & 994.0319 \\
\hline & 0.1 & 40 & 0.0126 & 1002.7 & (131) & 0.000170 & 0.000197 & 1002.3 & 0.3529 & -0.000028 & 0.125 & & & 992.2158 \\
\hline & 0.2 & 40 & 0.0249 & 1011.0 & (131) & 0.000257 & 0.000187 & 1012.8 & -1.7951 & 0.000070 & 3.222 & & & 992.2158 \\
\hline & 0.3 & 40 & 0.0371 & 1020.5 & (131) & 0.000254 & 0.000180 & 1023.4 & -2.8694 & 0.000074 & 8.234 & & & 992.2158 \\
\hline & 0.4 & 40 & 0.0489 & 1030.1 & (131) & 0.000251 & 0.000175 & 1034.0 & -3.9241 & 0.000075 & 15.399 & & & 992.2158 \\
\hline & & 40 & 0.0500 & 1039.4 & (122) & 0.000093 & 0.000175 & 1035.0 & 4.4164 & -0.000082 & 19.504 & & & 992.2158 \\
\hline & 0.5 & 40 & 0.0606 & 1039.9 & (131) & 0.000245 & 0.000172 & 1044.7 & -4.8263 & 0.000073 & 23.293 & & & 992.2158 \\
\hline & & 40 & 0.1000 & 1086.8 & (122) & 0.000131 & 0.000165 & 1082.7 & 4.0637 & -0.000035 & 16.514 & & & 992.2158 \\
\hline & & 40 & 0.1500 & 1137.1 & (122) & 0.000152 & 0.000161 & 1135.3 & 1.7907 & -0.000009 & 3.207 & & & 992.2158 \\
\hline & & 40 & 0.2000 & 1191.2 & (122) & 0.000166 & 0.000159 & 1193.3 & -2.1246 & 0.000007 & 4.514 & & & 992.2158 \\
\hline
\end{tabular}




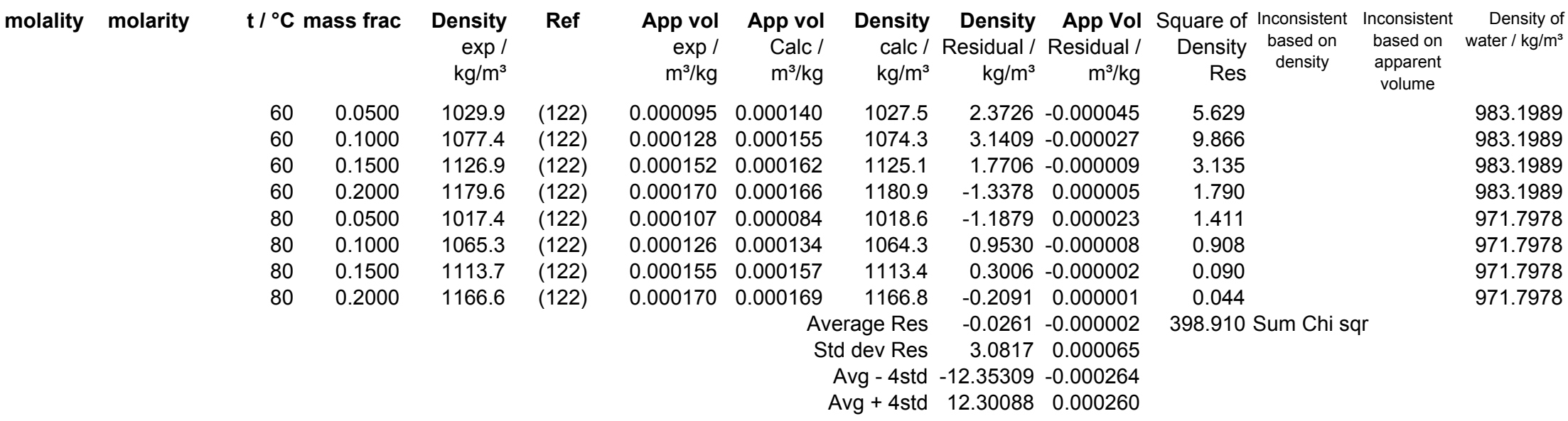

Curve fit for the model App vol $=\left(w+c 2+c 3^{*} t{ }^{\circ} \mathrm{C}\right) /\left(\mathrm{c0} 0^{*} \mathrm{w}+\mathrm{c} 1\right) / \mathrm{e}^{\wedge}\left(0.000001^{*}\left(\mathrm{t}{ }^{\circ} \mathrm{C}+\mathrm{c} 4\right)^{\wedge} 2\right)$

$\begin{array}{lr}\text { c0 } & 1.52 \mathrm{E}-05 \\ \text { c1 } & 4.38 \mathrm{E}-07 \\ \text { c2 } & 0.102961 \\ \text { c3 } & -0.001527 \\ \text { c4 } & -4500.85 \\ \text { Min T } & 19 \\ \text { Max T } & 80 \\ \text { Min w } & 0.0100 \\ \text { Max w } & 0.2000 \\ \text { Avg dens res } & -0.0261 \\ \text { Std dens res } & 3.0817 \\ \text { Avg app vol res *10^3 } & -0.002302 \\ \text { Std app vol res *10^3 } & 0.065464 \\ \text { No of points in corr } & 43 \\ \text { No of inconsistent poir } & 0\end{array}$

Inconsistent data not used (Residual greater than average +/- 4 standard deviations) 
Density of aqueous solutions of $\mathrm{Na2S2O3}$

References (78) Olofsson, I. V.; Spitzer, J. J.; Hepler, L. G. Apparent Molar Heat Capacities and Volumes of Aqueous Electrolytes at $25^{\circ} \mathrm{C}$ : $\mathrm{Na}_{2} \mathrm{SO}_{4}$, $\mathrm{K}_{2} \mathrm{SO}_{4}, \mathrm{Na}_{2} \mathrm{~S}_{2} \mathrm{O}_{3}, \mathrm{Na}_{2} \mathrm{~S}_{2} \mathrm{O}_{8}, \mathrm{~K}_{2} \mathrm{~S}_{2} \mathrm{O}_{8}, \mathrm{~K}_{2} \mathrm{CrO}_{4}, \mathrm{Na}_{2} \mathrm{MoO}_{4}$, and $\mathrm{Na}_{2} \mathrm{WO}_{4}$. Can. J. Chem. 1978, 56, 1871-1873

(90) Perry, R. H.; Green, D. W. Perry's Chemical Engineers' Handbook; McGraw Hill: New York, 7th edition, 1997.

(122) Sohnel, P.; Novotny, P; Solc, Z.; Densities of aqueous solutions of 18 inorganic substances, J Chem Eng Data, 1984, 29, 379-382

molality molarity $\quad t /{ }^{\circ} \mathrm{C}$ mass frac Density Ref App vol App vol Density Density App Vol Square of Inconsistent Inconsistent

\begin{tabular}{|c|c|c|c|c|c|c|c|c|c|c|c|c|c|c|c|}
\hline \multirow{13}{*}{\multicolumn{2}{|c|}{ molality }} & \multicolumn{2}{|c|}{$\mathrm{t} /{ }^{\circ} \mathrm{C}$ mass frac } & $\begin{array}{r}\text { Density } \\
\exp / \\
\mathrm{kg} / \mathrm{m}^{3}\end{array}$ & Ref & $\begin{array}{r}\text { App vol } \\
\text { exp / } \\
\mathrm{m}^{3} / \mathrm{kg}\end{array}$ & $\begin{array}{r}\text { App vol } \\
\text { Calc / } \\
\mathrm{m}^{3} / \mathrm{kg}\end{array}$ & $\begin{array}{r}\text { Density } \\
\text { calc / } \\
\mathrm{kg} / \mathrm{m}^{3}\end{array}$ & $\begin{array}{r}\text { Density } \\
\text { Residual / } \\
\mathrm{kg} / \mathrm{m}^{3}\end{array}$ & $\begin{array}{r}\text { App Vol } \\
\text { Residual / } \\
\mathrm{m}^{3} / \mathrm{kg}\end{array}$ & $\begin{array}{r}\text { Square of } \\
\text { Density } \\
\text { Res }\end{array}$ & $\begin{array}{l}\text { Inconsistent } \\
\text { based on } \\
\text { density }\end{array}$ & $\begin{array}{l}\text { Inconsistent } \\
\text { based on } \\
\text { apparent } \\
\text { volume }\end{array}$ & $\begin{array}{r}\text { Apparent } \\
\text { molal } \\
\text { volume / } \\
\mathrm{cm}^{3} / \mathrm{mol}\end{array}$ & $\begin{array}{r}\text { Density of } \\
\text { water } / \mathrm{kg} / \mathrm{m}^{3}\end{array}$ \\
\hline & & 20 & 0.0100 & 1006.5 & (90) & 0.000176 & 0.000238 & 1005.9 & 0.6247 & -0.000062 & 0.390 & & & & 998.2041 \\
\hline & & 20 & 0.0200 & 1014.8 & (90) & 0.000183 & 0.000239 & 1013.6 & 1.1610 & -0.000056 & 1.348 & & & & 998.2041 \\
\hline & & 20 & 0.0400 & 1031.5 & (90) & 0.000193 & 0.000242 & 1029.4 & 2.0517 & -0.000048 & 4.209 & & & & 998.2041 \\
\hline & & 20 & 0.0800 & 1065.4 & (90) & 0.000212 & 0.000247 & 1062.2 & 3.1752 & -0.000035 & 10.082 & & & & 998.2041 \\
\hline & & 20 & 0.1200 & 1100.3 & (90) & 0.000227 & 0.000253 & 1096.6 & 3.6990 & -0.000026 & 13.683 & & & & 998.2041 \\
\hline & & 20 & 0.1600 & 1136.5 & (90) & 0.000240 & 0.000259 & 1132.6 & 3.8603 & -0.000019 & 14.902 & & & & 998.2041 \\
\hline & & 20 & 0.2000 & 1174.0 & (90) & 0.000252 & 0.000265 & 1170.4 & 3.6036 & -0.000013 & 12.986 & & & & 998.2041 \\
\hline & & 20 & 0.2400 & 1212.8 & (90) & 0.000263 & 0.000271 & 1209.9 & 2.8848 & -0.000008 & 8.322 & & & & 998.2041 \\
\hline & & 20 & 0.2800 & 1253.2 & (90) & 0.000274 & 0.000278 & 1251.2 & 1.9759 & -0.000005 & 3.904 & & & & 998.2041 \\
\hline & & 20 & 0.3000 & 1273.9 & (90) & 0.000279 & 0.000282 & 1272.6 & 1.3474 & -0.000003 & 1.816 & & & & 998.2041 \\
\hline & & 20 & 0.3500 & 1327.3 & (90) & 0.000292 & 0.000291 & 1327.8 & -0.5215 & 0.000001 & 0.272 & & & & 998.2041 \\
\hline & & 20 & 0.4000 & 1382.7 & (90) & 0.000305 & 0.000301 & 1385.8 & -3.0837 & 0.000004 & 9.509 & & & & 998.2041 \\
\hline 0.02533 & & 25 & 0.0040 & 1000.34 & (78) & 0.000175 & 0.000239 & 1000.1 & 0.2554 & -0.000064 & 0.065 & & & 27.7 & 997.0449 \\
\hline 0.03377 & & 25 & 0.0053 & 1001.42 & (78) & 0.000178 & 0.000239 & 1001.1 & 0.3269 & -0.000061 & 0.107 & & & 28.14 & 997.0449 \\
\hline 0.04406 & & 25 & 0.0069 & 1002.73 & (78) & 0.000182 & 0.000240 & 1002.3 & 0.4032 & -0.000058 & 0.163 & & & 28.71 & 997.0449 \\
\hline 0.05071 & & 25 & 0.0080 & 1003.59 & (78) & 0.000181 & 0.000240 & 1003.1 & 0.4700 & -0.000059 & 0.221 & & & 28.62 & 997.0449 \\
\hline 0.05192 & & 25 & 0.0081 & 1003.75 & (78) & 0.000180 & 0.000240 & 1003.3 & 0.4856 & -0.000059 & 0.236 & & & 28.54 & 997.0449 \\
\hline 0.06768 & & 25 & 0.0106 & 1005.74 & (78) & 0.000184 & 0.000240 & 1005.1 & 0.6000 & -0.000056 & 0.360 & & & 29.09 & 997.0449 \\
\hline 0.06933 & & 25 & 0.0108 & 1005.95 & (78) & 0.000185 & 0.000240 & 1005.3 & 0.6088 & -0.000056 & 0.371 & & & 29.18 & 997.0449 \\
\hline 0.07364 & & 25 & 0.0115 & 1006.48 & (78) & 0.000186 & 0.000240 & 1005.9 & 0.6317 & -0.000054 & 0.399 & & & 29.4 & 997.0449 \\
\hline 0.08479 & & 25 & 0.0132 & 1007.91 & (78) & 0.000186 & 0.000240 & 1007.2 & 0.7301 & -0.000054 & 0.533 & & & 29.41 & 997.0449 \\
\hline 0.08802 & & 25 & 0.0137 & 1008.33 & (78) & 0.000185 & 0.000240 & 1007.6 & 0.7670 & -0.000055 & 0.588 & & & 29.32 & 997.0449 \\
\hline 0.10419 & & 25 & 0.0162 & 1010.35 & (78) & 0.000188 & 0.000241 & 1009.5 & 0.8664 & -0.000052 & 0.751 & & & 29.78 & 997.0449 \\
\hline 0.1227 & & 25 & 0.0190 & 1012.68 & (78) & 0.000190 & 0.000241 & 1011.7 & 1.0044 & -0.000052 & 1.009 & & & 29.98 & 997.0449 \\
\hline 0.13913 & & 25 & 0.0215 & 1014.69 & (78) & 0.000193 & 0.000241 & 1013.6 & 1.0790 & -0.000049 & 1.164 & & & 30.47 & 997.0449 \\
\hline 0.14701 & & 25 & 0.0227 & 1015.70 & (78) & 0.000192 & 0.000242 & 1014.5 & 1.1549 & -0.000049 & 1.334 & & & 30.4 & 997.0449 \\
\hline 0.14725 & & 25 & 0.0228 & 1015.74 & (78) & 0.000192 & 0.000242 & 1014.6 & 1.1703 & -0.000050 & 1.370 & & & 30.31 & 997.0449 \\
\hline 0.17441 & & 25 & 0.0268 & 1019.06 & (78) & 0.000196 & 0.000242 & 1017.8 & 1.2931 & -0.000046 & 1.672 & & & 30.94 & 997.0449 \\
\hline 0.24627 & & 25 & 0.0375 & 1027.91 & (78) & 0.000199 & 0.000244 & 1026.2 & 1.7445 & -0.000044 & 3.043 & & & 31.53 & 997.0449 \\
\hline & & 25 & 0.1000 & 1081.2 & (122) & 0.000222 & 0.000252 & 1077.7 & 3.4708 & -0.000030 & 12.047 & & & & 997.0449 \\
\hline & & 25 & 0.2000 & 1172.1 & (122) & 0.000254 & 0.000267 & 1168.5 & 3.6223 & -0.000013 & 13.121 & & & & 997.0449 \\
\hline & & 25 & 0.3000 & 1270.4 & (122) & 0.000284 & 0.000284 & 1270.0 & 0.3718 & -0.000001 & 0.138 & & & & 997.0449 \\
\hline & & 25 & 0.4000 & 1379.5 & (122) & 0.000308 & 0.000304 & 1382.4 & -2.9215 & 0.000004 & 8.535 & & & & 997.0449 \\
\hline & & 40 & 0.1000 & 1074.5 & (122) & 0.000236 & 0.000258 & 1072.0 & 2.5075 & -0.000022 & 6.288 & & & & 992.2158 \\
\hline & & 40 & 0.2000 & 1164.2 & (122) & 0.000263 & 0.000273 & 1161.5 & 2.6527 & -0.000010 & 7.037 & & & & 992.2158 \\
\hline
\end{tabular}


molality molarity

$\begin{array}{cccc}\mathbf{t} /{ }^{\circ} \mathrm{C} \text { mass frac } & \begin{array}{r}\text { Density } \\ \exp / \\ \mathrm{kg} / \mathrm{m}^{3}\end{array} & \text { Ref } \\ & & \\ 40 & 0.3000 & 1261.5 & (122) \\ 40 & 0.4000 & 1370.2 & (122) \\ 40 & 0.5000 & 1487.0 & (122) \\ 60 & 0.1000 & 1063.3 & (122) \\ 60 & 0.2000 & 1152.1 & (122) \\ 60 & 0.3000 & 1248.9 & (122) \\ 60 & 0.4000 & 1355.9 & (122) \\ 60 & 0.5000 & 1473.5 & (122) \\ 60 & 0.6000 & 1599.2 & (122) \\ 80 & 0.1000 & 1051.7 & (122) \\ 80 & 0.2000 & 1138.7 & (122) \\ 80 & 0.3000 & 1235.3 & (122) \\ 80 & 0.4000 & 1341.8 & (122) \\ 80 & 0.5000 & 1458.8 & (122) \\ 80 & 0.6000 & 1584.8 & (122)\end{array}$

\section{App vol App vol}

$\exp /$

$\exp /$
$\mathrm{m}^{3} / \mathrm{kg}$

Calc /
$\mathrm{m}^{3} / \mathrm{kg}$

$\begin{array}{ll}0.000291 & 0.000291\end{array}$

$0.000313 \quad 0.000311$

$0.000337 \quad 0.000334$

$0.000251 \quad 0.000263$

$0.000272 \quad 0.000278$

$0.000296 \quad 0.000296$

$0.000318 \quad 0.000316$

$0.000340 \quad 0.000339$

$0.000364 \quad 0.000367$

$0.000247 \quad 0.000263$

$0.000275 \quad 0.000279$

$0.000297 \quad 0.000296$

$0.000320 \quad 0.000316$

$0.000342 \quad 0.000340$

$0.000366 \quad 0.000367$
Density Density App Vol Square of Inconsistent Inconsistent calc / Residual / Residual /

$\mathrm{kg} / \mathrm{m}^{3}$ $\mathrm{kg} / \mathrm{m}^{3}$

$\mathrm{m}^{3} / \mathrm{kg}$

$\begin{array}{lll}1261.5 & -0.0125 & 0.000000\end{array}$

$\begin{array}{lll}1371.8 & -1.5887 & 0.000002\end{array}$

$\begin{array}{llll}1490.8 & -3.7745 & 0.000003\end{array}$

$\begin{array}{lll}1062.0 & 1.3217 & -0.000012\end{array}$

$\begin{array}{lll}1150.3 & 1.7597 & -0.000007\end{array}$

$\begin{array}{lll}1248.9 & 0.0279 & 0.000000\end{array}$

$\begin{array}{lll}1357.4 & -1.5248 & 0.000002\end{array}$

$\begin{array}{lll}1474.4 & -0.8523 & 0.000001\end{array}$

$1595.3 \quad 3.9414-0.000003$

$1049.9 \quad 1.7585-0.000016$

$1137.7 \quad 0.9754-0.000004$

$\begin{array}{llll}1235.8 & -0.5135 & 0.000001\end{array}$

$\begin{array}{lll}1344.2 & -2.3860 & 0.000003\end{array}$

$1461.4 \quad-2.5888 \quad 0.000002$

$1583.3 \quad 1.5029-0.000001$

$0.8424-0.000026$

$\begin{array}{rrr}\text { Average Res } & 0.8424 & -0.000026 \\ \text { Std dev Res } & 1.838677 & 0.000025\end{array}$

Avg - 4std -6.512313 -0.000126

Avg + 4std $8.197105 \quad 0.000075$
Apparent Density of molal water $/ \mathrm{kg} / \mathrm{m}^{3}$ volume /

$\mathrm{cm}^{3} / \mathrm{mo}$

992.2158

992.2158

992.2158

983.1989

983.1989

983.1989

983.1989

983.1989

983.1989

971.7978

971.7978

971.7978

971.7978

971.7978

971.7978

Curve fit for the model App vol $=\left(w+c 2+c 3^{*} t{ }^{\circ} \mathrm{C}\right) /\left(\mathrm{c} 0^{*} w+c 1\right) / e^{\wedge}\left(0.000001^{*}\left(t^{\circ} \mathrm{C}+\mathrm{c} 4\right)^{\wedge} 2\right)$

$\mathrm{CO}$

c1

c3

c4

Min T

$\operatorname{Max} T$

Min w

Max w

Avg dens res

Std dens res

Avg app vol res *10^3 -0.025726

Std app vol res *10^3 0.025159

No of points in corr

No of inconsistent poir

0.844616

$-1.514245$

$-42.94899$

0.19335

$-3425.907$

20

0.003989

0.6

0.842396

$-0.025726$

50
0

Inconsistent data not used (Residual greater than average $+/-4$ standard deviations) 
Density of aqueous solutions of $\mathrm{Na2SO}$

References (13) Chen, C.-T. A.; Chen, J. H.; Millero, F. J. Densities of $\mathrm{NaCl}, \mathrm{MgCl}_{2}, \mathrm{Na}_{2} \mathrm{SO}_{4}$, and $\mathrm{MgSO}_{4}$ Aqueous Solutions at 1 atm. from 0 to $50^{\circ} \mathrm{C}$ and from 0.001 to $1.5 \mathrm{~m}$. J. Chem. Eng. Data 1980, 25, 307-310.

(20) Dedick, Eugene A.; Hershey, J. Peter; Sotolongo, Sara; Stade, David J.; Millero, Frank J. The PVT Properties of Concentrated Aqueous Electrolytes IX. The Volume Properties of $\mathrm{KCl}$ and $\mathrm{K} 2 \mathrm{SO} 4$ and their Mixtures with $\mathrm{NaCl}$ and $\mathrm{Na} 2 \mathrm{SO} 4$ as a Function of Temperature, $\mathrm{J}$. Solution Chem. 1990, 19, 353-374

(27) Fabuss, B. M.; Korosi, A.; Huq, A. K. M. S.; Densities of Binary and Ternary Aqueous Solutions of NaCl, $\mathrm{Na2SO4,} \mathrm{and} \mathrm{MgSO4,} \mathrm{of} \mathrm{Sea}$ Waters, and Sea Water Concentrates, J. Chem. Eng. Data, 1966, 3, 325-331

(45) Isono, T. Densities, Viscosities, and Electrolytic Conductivity of Concentrated Aqueous Electrolyte Solutions at Several Temperatures. Alkaline-Earth Chlorides, $\mathrm{LaCl}_{3}, \mathrm{Na}_{2} \mathrm{SO}_{4}, \mathrm{NaNO}_{3}, \mathrm{NaBr}, \mathrm{KNO}_{3}, \mathrm{KBr}$, and $\mathrm{Cd}\left(\mathrm{NO}_{3}\right)_{2}$. J. Chem. Eng. Data 1984, 29 , 45-52.

(47) Kaminsky, M. Experimental Investigations of the Concentration and Temperature Dependence of the Viscosity of Aqueous Solutions of Strong Electrolytes. I. Potassium lodide, Ammonium Chloride, and Sodium Sulphate Solutions. Z. Physik. Chem. N. F. 1955, 5, 154191.

(52) Korosi, A.; Fabuss, B. M. Viscosities of Binary Aqueous Solutions of $\mathrm{NaCl}, \mathrm{KCl}, \mathrm{Na}_{2} \mathrm{SO}_{4}$, and $\mathrm{MgSO}_{4}$ at Concentrations and Temperatures of Interest in Desalination Processes. J. Chem. Eng. Data 1968, 13, 548-552.

(72) Millero, F. J.; Knox, J. H. Apparent Molal Volumes of Aqueous $\mathrm{NaF}, \mathrm{Na}_{2} \mathrm{SO}_{4}, \mathrm{KCl}, \mathrm{K}_{2} \mathrm{SO}_{4}, \mathrm{MgCl}_{2}$, and $\mathrm{MgSO}_{4}$ Solutions at $0^{\circ}$ and $50^{\circ} \mathrm{C}$. J. Chem. Eng. Data 1973, 18, 407-411.

(88) Perron, G., Desnoyers, J. E., Millero, F. J., "Apparent molal volumes and heat capacities of some sulfates and carbonates in water at 25 ${ }^{\circ} \mathrm{C} . ”$, Can. J. Chem. , 1975, 53, 1134-1138

(90) Perry, R. H.; Green, D. W. Perry's Chemical Engineers' Handbook; McGraw Hill: New York, 7th edition, 1997.

(114) Saluja, P. P. S.; Lemire, R. J.; LeBlanc, J. C.; High-Temperature Thermodynamics of Aqueous Alkali-Metal Salts; J. Chem. Thermodynamics, 1992, 24, 181-203

(134) Wirth, H. E.; Lo Surdo, A. Temperature Dependence of Volume Changes of Aqueous Solutions of Ammonium Chloride and Ammonium Nitrate at $25^{\circ} \mathrm{C}$. J. Chem. Eng. Data 1968, 13, 226-231.

\begin{tabular}{|c|c|c|c|c|c|c|c|c|c|c|c|}
\hline molality & molarity & \multicolumn{2}{|c|}{$\mathbf{t} /{ }^{\circ} \mathrm{C}$ mass frac } & $\begin{array}{r}\text { Density } \\
\exp / \\
\mathrm{kg} / \mathrm{m}^{3}\end{array}$ & Ref & $\begin{array}{r}\text { App vol } \\
\exp / \\
\mathrm{m}^{3} / \mathrm{kg}\end{array}$ & $\begin{array}{r}\text { App vol } \\
\text { Calc / } \\
\mathrm{m}^{3} / \mathrm{kg}\end{array}$ & $\begin{array}{r}\text { Density } \\
\text { calc / } \\
\mathrm{kg} / \mathrm{m}^{3}\end{array}$ & $\begin{array}{r}\text { Density } \\
\text { Residual / } \\
\mathrm{kg} / \mathrm{m}^{3}\end{array}$ & $\begin{array}{r}\text { App Vol } \\
\text { Residual / } \\
\mathrm{m}^{3} / \mathrm{kg}\end{array}$ & $\begin{array}{r}\text { Square of } \\
\text { Density } \\
\text { Residual }\end{array}$ \\
\hline 0.0034 & & 0 & 0.0005 & 1000.32 & (13) & 0.000004 & 0.000067 & 1000.29 & 0.0305 & -0.000063 & 0.001 \\
\hline 0.003567 & & 0 & 0.0005 & 1000.34 & (72) & 0.000019 & 0.000067 & 1000.31 & 0.0245 & -0.000048 & 0.001 \\
\hline 0.008254 & & 0 & 0.0012 & 1000.99 & (72) & 0.000022 & 0.000067 & 1000.93 & 0.0539 & -0.000046 & 0.003 \\
\hline 0.008351 & & 0 & 0.0012 & 1001.00 & (72) & 0.000021 & 0.000067 & 1000.95 & 0.0550 & -0.000046 & 0.003 \\
\hline 0.01055 & & 0 & 0.0015 & 1001.30 & (13) & 0.000023 & 0.000068 & 1001.24 & 0.0669 & -0.000045 & 0.004 \\
\hline 0.011673 & & 0 & 0.0017 & 1001.46 & (72) & 0.000022 & 0.000068 & 1001.39 & 0.0756 & -0.000046 & 0.006 \\
\hline 0.013831 & & 0 & 0.0020 & 1001.76 & (72) & 0.000023 & 0.000068 & 1001.67 & 0.0883 & -0.000045 & 0.008 \\
\hline 0.01399 & & 0 & 0.0020 & 1001.79 & (13) & 0.000017 & 0.000068 & 1001.69 & 0.1019 & -0.000051 & 0.010 \\
\hline 0.014836 & & 0 & 0.0021 & 1001.90 & (72) & 0.000022 & 0.000068 & 1001.80 & 0.0958 & -0.000045 & 0.009 \\
\hline 0.015569 & & 0 & 0.0022 & 1002.00 & (72) & 0.000023 & 0.000068 & 1001.90 & 0.0988 & -0.000045 & 0.010 \\
\hline 0.017031 & & 0 & 0.0024 & 1002.20 & (72) & 0.000023 & 0.000068 & 1002.09 & 0.1085 & -0.000045 & 0.012 \\
\hline 0.017661 & & 0 & 0.0025 & 1002.29 & (72) & 0.000023 & 0.000068 & 1002.18 & 0.1123 & -0.000045 & 0.013 \\
\hline 0.017808 & & 0 & 0.0025 & 1002.31 & (72) & 0.000024 & 0.000068 & 1002.20 & 0.1126 & -0.000044 & 0.013 \\
\hline 0.019987 & & 0 & 0.0028 & 1002.61 & (72) & 0.000024 & 0.000068 & 1002.48 & 0.1258 & -0.000044 & 0.016 \\
\hline 0.020799 & & 0 & 0.0029 & 1002.72 & (72) & 0.000025 & 0.000068 & 1002.59 & 0.1295 & -0.000044 & 0.017 \\
\hline 0.024185 & & 0 & 0.0034 & 1003.19 & (72) & 0.000025 & 0.000068 & 1003.04 & 0.1485 & -0.000043 & 0.022 \\
\hline 0.024998 & & 0 & 0.0035 & 1003.30 & (72) & 0.000026 & 0.000069 & 1003.15 & 0.1506 & -0.000042 & 0.023 \\
\hline 0.024998 & & 0 & 0.0035 & 1003.30 & (72) & 0.000026 & 0.000069 & 1003.15 & 0.1515 & -0.000043 & 0.023 \\
\hline 0.026919 & & 0 & 0.0038 & 1003.56 & (72) & 0.000026 & 0.000069 & 1003.40 & 0.1631 & -0.000043 & 90 \\
\hline
\end{tabular}

Apparent Delta density Density of $\quad / \mathrm{kg} / \mathrm{m}^{3}$ water at $\mathrm{t}^{\circ} \mathrm{C}$ $\mathrm{kg} / \mathrm{m}^{3}$

0.481999 .8395 0.4971999 .8395

1.1471999 .8395

1.1611999 .8395

1.464999 .8395

1.6213999 .8395

1.9194999 .8395

$1.954 \quad 999.8395$

2.0598999 .8395

$2.1597 \quad 999.8395$

$2.3627 \quad 999.8395$

$2.4497 \quad 999.8395$

2.4695999 .8395

$2.7706 \quad 999.8395$

$2.8816 \quad 999.8395$

3.3478999 .8395

$3.4572 \quad 999.8395$

3.4581999 .8395

3.7232999 .8395 
molality molarity

t $/{ }^{\circ} \mathrm{C}$ mass frac Density

$$
\exp /
$$$$
\mathrm{kg} / \mathrm{m}^{3}
$$

0.030351

0.03178

0.034785

0.040737

0.049147

0.0505

0.054676

0.05553

0.061606

0.070549

0.082529

0.09941

0.10019

0.105998

0.126706

0.147918

0.16373
0.1777

0.1777

0.19951

0.259374

0.34718

0.46965

0.50208

0.557229

0.5676

0.70189

0.705014

0.921454

1.3334

0.0034

0.01055

0.01399

0.03178

0.0505

0.05553

0.16373

0.19951

0.30182
Ref

App

App vol App vol Den $\exp / \quad$ Calc /

$$
\mathrm{m}^{3} / \mathrm{kg} \quad \mathrm{m}^{3} / \mathrm{kg}
$$

Density Density App Vol Square of Inconsistent Inconsistent $\mathrm{kg} / \mathrm{m}^{3}$ Residual/Residual Density density apparent molal volume $/ \mathrm{kg} / \mathrm{m}^{3}$ water at $\mathrm{t}^{\circ} \mathrm{C}$

$\begin{array}{llllll}0.000029 & 0.000069 & 1004.04 & 0.1796 & -0.000040 & 0.032\end{array}$

$\begin{array}{llllll}0.000028 & 0.000069 & 1004.44 & 0.2029 & -0.000041 & 0.041\end{array}$

$\begin{array}{llllll}0.000030 & 0.000070 & 1005.22 & 0.2273 & -0.000039 & 0.052\end{array}$

$\begin{array}{llllll}0.000032 & 0.000070 & 1006.33 & 0.2682 & -0.000038 & 0.072\end{array}$

$\begin{array}{lllllll}0.000035 & 0.000070 & 1006.51 & 0.2516 & -0.000035 & 0.063\end{array}$

$\begin{array}{llllll}0.000034 & 0.000070 & 1007.05 & 0.2832 & -0.000036 & 0.080\end{array}$

$\begin{array}{llllll}0.000035 & 0.000070 & 1007.17 & 0.2825 & -0.000036 & 0.080\end{array}$

$\begin{array}{llllll}0.000035 & 0.000071 & 1007.96 & 0.3137 & -0.000036 & 0.098\end{array}$

$\begin{array}{llllll}0.000038 & 0.000071 & 1009.14 & 0.3396 & -0.000034 & 0.115\end{array}$

$\begin{array}{lllllll}0.000053 & 0.000071 & 1009.21 & 0.1895 & -0.000019 & 0.036\end{array}$

$\begin{array}{llllll}0.000041 & 0.000072 & 1010.71 & 0.3683 & -0.000031 & 0.136\end{array}$

$\begin{array}{llllll}0.000045 & 0.000073 & 1012.91 & 0.4097 & -0.000029 & 0.168\end{array}$

$\begin{array}{llllll}0.000045 & 0.000073 & 1013.01 & 0.4101 & -0.000028 & 0.168\end{array}$

$\begin{array}{llllll}0.000047 & 0.000074 & 1013.77 & 0.4046 & -0.000027 & 0.164\end{array}$

$\begin{array}{llllll}0.000051 & 0.000075 & 1016.46 & 0.4384 & -0.000024 & 0.192\end{array}$

$\begin{array}{llllll}0.000065 & 0.000076 & 1018.66 & 0.2362 & -0.000011 & 0.056\end{array}$

$\begin{array}{llllll}0.000054 & 0.000076 & 1019.22 & 0.4735 & -0.000022 & 0.224\end{array}$

$\begin{array}{lllllll}0.000055 & 0.000077 & 1021.26 & 0.5241 & -0.000022 & 0.275\end{array}$

$\begin{array}{lllllll}0.000058 & 0.000078 & 1023.06 & 0.5138 & -0.000020 & 0.264\end{array}$

$\begin{array}{llllll}0.000060 & 0.000080 & 1025.87 & 0.5570 & -0.000019 & 0.310\end{array}$

$\begin{array}{llllll}0.000068 & 0.000083 & 1033.51 & 0.5588 & -0.000015 & 0.312\end{array}$

$\begin{array}{lllllll}0.000079 & 0.000085 & 1037.81 & 0.2876 & -0.000007 & 0.083\end{array}$

$\begin{array}{llllll}0.000079 & 0.000089 & 1044.59 & 0.5126 & -0.000010 & 0.263\end{array}$

$\begin{array}{llllll}0.000090 & 0.000096 & 1059.77 & 0.3922 & -0.000006 & 0.154\end{array}$

$\begin{array}{lllllll}0.000093 & 0.000098 & 1063.74 & 0.3597 & -0.000005 & 0.129\end{array}$

$\begin{array}{lllllll}0.000098 & 0.000101 & 1070.44 & 0.2360 & -0.000003 & 0.056\end{array}$

$\begin{array}{llllll}0.000099 & 0.000102 & 1071.69 & 0.2566 & -0.000003 & 0.066\end{array}$

$\begin{array}{llllll}0.000101 & 0.000104 & 1077.04 & 0.2629 & -0.000003 & 0.069\end{array}$

$\begin{array}{llllll}0.000109 & 0.000109 & 1087.70 & 0.0288 & 0.000000 & 0.001\end{array}$

$\begin{array}{llllll}0.000109 & 0.000109 & 1088.07 & 0.0014 & 0.000000 & 0.000\end{array}$

$\begin{array}{llllll}0.000124 & 0.000121 & 1113.06 & -0.3592 & 0.000003 & 0.129\end{array}$

$\begin{array}{llllll}0.000123 & 0.000123 & 1117.40 & 0.0016 & 0.000000 & 0.000\end{array}$

$\begin{array}{llllll}0.000148 & 0.000143 & 1157.96 & -1.1543 & 0.000005 & 1.332\end{array}$

$\begin{array}{llllll}0.000144 & 0.000143 & 1158.74 & -0.2379 & 0.000001 & 0.057\end{array}$

$\begin{array}{llllll}0.000163 & 0.000163 & 1200.85 & -0.0463 & 0.000000 & 0.002\end{array}$

$\begin{array}{llllll}0.000181 & 0.000184 & 1243.46 & 0.8436 & -0.000002 & 0.712\end{array}$

$\begin{array}{llllll}0.000031 & 0.000079 & 1000.41 & 0.0232 & -0.000048 & 0.001\end{array}$

$\begin{array}{lllllll}0.000040 & 0.000080 & 1001.34 & 0.0587 & -0.000039 & 0.003\end{array}$

$\begin{array}{lllllll}0.000041 & 0.000080 & 1001.79 & 0.0764 & -0.000038 & 0.006\end{array}$

$\begin{array}{llllll}0.000047 & 0.000081 & 1004.11 & 0.1540 & -0.000034 & 0.024\end{array}$

$\begin{array}{llllll}0.000055 & 0.000082 & 1006.55 & 0.1983 & -0.000027 & 0.039\end{array}$

$\begin{array}{llllll}0.000056 & 0.000082 & 1007.20 & 0.2116 & -0.000027 & 0.045\end{array}$

$\begin{array}{lllllll}0.000072 & 0.000089 & 1021.11 & 0.3993 & -0.000017 & 0.159\end{array}$

$\begin{array}{lllllll}0.000077 & 0.000091 & 1025.66 & 0.4016 & -0.000014 & 0.161\end{array}$

$\begin{array}{llllll}0.000089 & 0.000097 & 1038.52 & 0.3780 & -0.000009 & 0.143\end{array}$
4.1948999 .8395

4.381999 .8395

4.8005999 .8395

5.6091999 .8395

6.7567999 .8395

6.918999 .8395

7.4985999 .8395

7.61999 .8395

$8.439 \quad 999.8395$

9.6379999 .8395 999.8395

$11.2352 \quad 999.8395$

13.4818999 .8395

13.584999 .8395

$14.3357 \quad 999.8395$

$17.0637 \quad 999.8395$ 999.8395

$19.8493 \quad 999.8395$

21.944999 .8395

$23.7353 \quad 999.8395$

$26.583 \quad 999.8395$

$34.2313 \quad 999.8395$ 999.8395

$45.2643 \quad 999.8395$

60.3261999 .8395

$64.261 \quad 999.8395$

$70.8333 \quad 999.8395$

$72.106 \quad 999.8395$ 999.8395

$87.887 \quad 999.8395$

$88.2276 \quad 999.8395$

$112.8616 \quad 999.8395$

999.8395

999.8395

999.8395

999.8395

999.8395

$0.468 \quad 999.9638$

$1.438 \quad 999.9638$

1.905999 .9638

$4.302 \quad 999.9638$

$6.78 \quad 999.9638$

$7.446 \quad 999.9638$

21.547999 .9638

$26.097 \quad 999.9638$

38.931999 .9638 
molality molarity $\exp /$
$\mathrm{kg} / \mathrm{m}^{3}$

0.50208

0.5676

0.65

0.70189

1.3334

0.0034

0.01055

0.01399

0.01399

0.03178

0.05

0.0505

0.1

0.10019

0.16373

0.19951

0.30182

0.33307

0.40356

0.5
0.50208

0.5676

0.70189

1.3334

0.05

0.5

0.7

1
1.2

0.01948

0.05442

0.09547

0.20961

0.31478

0.39192

0.49767
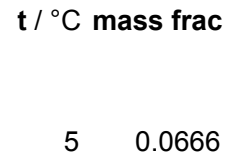

$\begin{array}{rrrc}5 & 0.0666 & 1063.22 & (13) \\ 5 & 0.0746 & 1070.98 & (13) \\ 5 & 0.0845 & 1082.39 & (20) \\ 5 & 0.0907 & 1086.60 & (13) \\ 5.12 & 0.1593 & 1155.02 & (134) \\ 15 & 0.0005 & 999.56 & (13) \\ 15 & 0.0015 & 1000.49 & (13) \\ 15 & 0.0020 & 1000.95 & (13) \\ 15 & 0.0020 & 1000.96 & (13) \\ 15 & 0.0045 & 1003.27 & (13) \\ 15 & 0.0071 & 1005.63 & (45) \\ 15 & 0.0071 & 1005.68 & (13) \\ 15 & 0.0078 & 1006.33 & (13) \\ 15 & 0.0140 & 1012.01 & (45) \\ 15 & 0.0140 & 1012.04 & (13) \\ 15 & 0.0227 & 1020.02 & (13) \\ 15 & 0.0276 & 1024.49 & (13) \\ 15 & 0.0411 & 1037.01 & (13) \\ 15 & 0.0452 & 1040.78 & (13) \\ 15 & 0.0542 & 1049.19 & (13) \\ 15 & 0.0663 & 1060.39 & (45) \\ 15 & 0.0666 & 1060.80 & (13) \\ 15 & 0.0746 & 1068.41 & (13) \\ 15 & 0.0907 & 1083.74 & (13) \\ 15.08 & 0.1593 & 1151.16 & (134) \\ 20 & 0.0071 & 1004.66 & (45) \\ 20 & 0.0100 & 1007.30 & (90) \\ 20 & 0.0140 & 1010.96 & (45) \\ 20 & 0.0200 & 1016.40 & (90) \\ 20 & 0.0400 & 1034.80 & (90) \\ 20 & 0.0663 & 1058.90 & (45) \\ 20 & 0.0800 & 1072.40 & (90) \\ 20 & 0.0904 & 1081.54 & (45) \\ 20 & 0.1200 & 1110.90 & (90) \\ 20 & 0.1244 & 1114.24 & (45) \\ 20 & 0.1456 & 1135.22 & (45) \\ 20 & 0.1600 & 1150.60 & (90) \\ 20 & 0.2000 & 1191.50 & (90) \\ 20 & 0.2400 & 1233.60 & (90) \\ 24.04 & 0.0028 & 999.80 & (114) \\ 24.04 & 0.0077 & 1004.23 & (114) \\ 24.04 & 0.0134 & 1009.37 & (114) \\ 24.04 & 0.0289 & 1023.41 & (114) \\ 24.04 & 0.0428 & 1036.04 & (114) \\ 24.04 & 0.0527 & 1045.17 & (114) \\ 24.04 & 0.0660 & 1057.51 & (114) \\ & & & \\ & & \end{array}$

$\begin{array}{cc}\exp / & \text { Calc } / \\ \mathrm{m}^{3} / \mathrm{kg} & \mathrm{m}^{3} / \mathrm{kg}\end{array}$

Density

Density App Vol Square of Inconsistent Inconsistent $\begin{gathered}\text { Apparent Delta density } \\ \text { bensity of } \\ \text { based on molal volume }\end{gathered} / \mathrm{kg} / \mathrm{m}^{3}$ water at $t^{\circ} \mathrm{C}$
based on

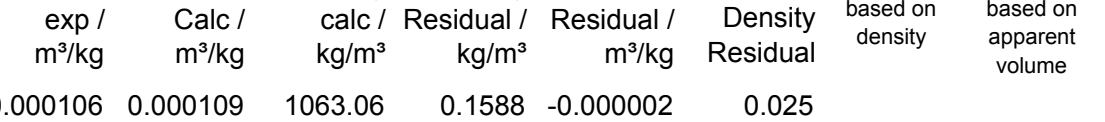
$\begin{array}{llllll}0.000111 & 0.000112 & 1070.91 & 0.0729 & -0.000001 & 0.005\end{array}$ $\begin{array}{llllll}0.000099 & 0.000117 & 1080.65 & 1.7366 & -0.000018 & 3.016\end{array}$ $\begin{array}{llllll}0.000121 & 0.000120 & 1086.72 & -0.1187 & 0.000001 & 0.014\end{array}$ $\begin{array}{llllll}0.000121 & 0.000120 & 1086.72 & -0.1187 & 0.000001 & 0.014\end{array}$ $\begin{array}{rrrrrr}0.000051 & 0.000099 & 999.53 & 0.0233 & -0.000048 & 0.001\end{array}$ $\begin{array}{llllll}0.000073 & 0.000100 & 1000.45 & 0.0400 & -0.000027 & 0.002\end{array}$ $\begin{array}{llllll}0.000068 & 0.000100 & 1000.89 & 0.0630 & -0.000032 & 0.004\end{array}$ $\begin{array}{llllll}0.000064 & 0.000100 & 1000.89 & 0.0720 & -0.000036 & 0.005\end{array}$ $\begin{array}{llllll}0.000075 & 0.000101 & 1003.15 & 0.1172 & -0.000026 & 0.014\end{array}$ $\begin{array}{llllll}0.000079 & 0.000102 & 1005.47 & 0.1616 & -0.000023 & 0.026\end{array}$ $\begin{array}{llllll}0.000081 & 0.000102 & 1005.53 & 0.1508 & -0.000021 & 0.023\end{array}$ $\begin{array}{lllllll}0.000082 & 0.000102 & 1006.17 & 0.1639 & -0.000021 & 0.027\end{array}$ $\begin{array}{llllll}0.000089 & 0.000105 & 1011.79 & 0.2227 & -0.000016 & 0.050\end{array}$ $\begin{array}{llllll}0.000089 & 0.000105 & 1011.81 & 0.2294 & -0.000016 & 0.053\end{array}$ $\begin{array}{llllll}0.000098 & 0.000108 & 1019.77 & 0.2526 & -0.000011 & 0.064\end{array}$ $\begin{array}{llllll}0.000101 & 0.000110 & 1024.21 & 0.2719 & -0.000009 & 0.074\end{array}$ $\begin{array}{lllllll}0.000111 & 0.000116 & 1036.79 & 0.2219 & -0.000005 & 0.049\end{array}$ $\begin{array}{lllllll}0.000114 & 0.000118 & 1040.58 & 0.1958 & -0.000004 & 0.038\end{array}$ $\begin{array}{llllll}0.000120 & 0.000121 & 1049.08 & 0.1149 & -0.000002 & 0.013\end{array}$ $\begin{array}{llllll}0.000129 & 0.000127 & 1060.54 & -0.1530 & 0.000002 & 0.023\end{array}$ $\begin{array}{llllll}0.000126 & 0.000127 & 1060.79 & 0.0165 & 0.000000 & 0.000\end{array}$ $\begin{array}{llllll}0.000131 & 0.000130 & 1068.47 & -0.0575 & 0.000001 & 0.003\end{array}$ $\begin{array}{llllll}0.000139 & 0.000137 & 1083.94 & -0.2039 & 0.000002 & 0.042\end{array}$ $\begin{array}{llllll}0.000171 & 0.000167 & 1151.98 & -0.8172 & 0.000004 & 0.668\end{array}$ $\begin{array}{llllll}0.000089 & 0.000110 & 1004.51 & 0.1516 & -0.000021 & 0.023\end{array}$ $\begin{array}{llllll}0.000097 & 0.000112 & 1007.15 & 0.1454 & -0.000014 & 0.021\end{array}$ $\begin{array}{llllll}0.000099 & 0.000113 & 1010.76 & 0.1963 & -0.000014 & 0.039\end{array}$ $\begin{array}{llllll}0.000105 & 0.000115 & 1016.19 & 0.2143 & -0.000010 & 0.046\end{array}$ $\begin{array}{llllll}0.000116 & 0.000123 & 1034.49 & 0.3128 & -0.000007 & 0.098\end{array}$ $\begin{array}{lllrrr}0.000136 & 0.000134 & 1059.05 & -0.1472 & 0.000002 & 0.022\end{array}$ $\begin{array}{llllll}0.000135 & 0.000140 & 1072.02 & 0.3789 & -0.000004 & 0.144\end{array}$ $\begin{array}{llllll}0.000148 & 0.000144 & 1082.02 & -0.4821 & 0.000005 & 0.232\end{array}$ $\begin{array}{llllll}0.000155 & 0.000156 & 1110.73 & 0.1695 & -0.000001 & 0.029\end{array}$ $\begin{array}{llllll}0.000163 & 0.000158 & 1115.05 & -0.8054 & 0.000005 & 0.649\end{array}$ $\begin{array}{llllll}0.000172 & 0.000167 & 1136.12 & -0.8966 & 0.000005 & 0.804\end{array}$ $\begin{array}{llllll}0.000173 & 0.000173 & 1150.51 & 0.0931 & 0.000000 & 0.009\end{array}$ $\begin{array}{llllll}0.000189 & 0.000190 & 1191.20 & 0.2970 & -0.000001 & 0.088\end{array}$ $\begin{array}{llllll}0.000205 & 0.000208 & 1232.63 & 0.9733 & -0.000003 & 0.947\end{array}$ $\begin{array}{llllll}0.000090 & 0.000115 & 999.73 & 0.0686 & -0.000025 & 0.005\end{array}$ $\begin{array}{llllll}0.000099 & 0.000117 & 1004.09 & 0.1392 & -0.000018 & 0.019\end{array}$ $\begin{array}{llllll}0.000106 & 0.000119 & 1009.19 & 0.1760 & -0.000013 & 0.031\end{array}$ $\begin{array}{llllll}0.000118 & 0.000125 & 1023.19 & 0.2173 & -0.000007 & 0.047\end{array}$ $\begin{array}{llllll}0.000126 & 0.000130 & 1035.87 & 0.1685 & -0.000004 & 0.028\end{array}$ $\begin{array}{llllll}0.000132 & 0.000134 & 1045.04 & 0.1295 & -0.000002 & 0.017\end{array}$ 0.009 $1 \mathrm{~cm}^{3} / \mathrm{mol} \quad \mathrm{kg} / \mathrm{m}^{3}$

63.252999 .9638 71.015999 .9638 82.424999 .9638 86.634999 .9638 999.9618 $0.458 \quad 999.0996$ 1.388999 .0996

1.85999 .0996

1.859999 .0996

4.171999 .0996 999.0996

$6.583 \quad 999.0996$

7.234999 .0996 999.0996

12.941999 .0996 $20.922 \quad 999.0996$ $25.387 \quad 999.0996$ 37.908999 .0996 $41.68 \quad 999.0996$ 50.095999 .0996 999.0996 61.705999 .0996 69.311999 .0996 84.64999 .0996 999.0875 998.2041 998.2041 998.2041 998.2041 998.2041 998.2041 998.2041 998.2041 998.2041 998.2041 998.2041 998.2041 998.2041 998.2041 997.2867 997.2867 997.2867 997.2867 997.2867 997.2867 997.2867 
molality molarity $\exp /$ $\mathrm{kg} / \mathrm{m}^{3}$

1.3334

0.00259

0.0034

0.00376

0.00504

0.01

0.01055

0.0127

0.01301

0.01682

0.02244

0.03022

0.03244

0.0333

0.03581

0.04293

0.05

0.05

0.0505

0.05553

0.05958

0.06485

0.07698

0.08861

0.0985

0.1

0.1
0019

0.11256

0.13073

0.13651

0.15

0.16801

0.19572

0.19951

0.2356

0.24072

0.2623

0.30182

0.32785

0.33307

0.35894

0.40356

0.4712

0.5

0.50061

$3 / 28 / 2004$
App vol App vol Density Density App Vol Square of Inconsistent Inconsistent Apparent Delta density Density of exp / Calc/ calc/ Residual / Residual / Density based on based on molal volume $1 / \mathrm{kg} / \mathrm{m}^{3}$ water at to $t^{\circ} \mathrm{C}$

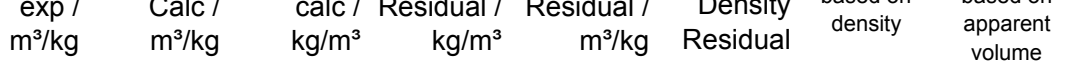
$\begin{array}{llllll}0.000180 & 0.000177 & 1147.60 & -0.6080 & 0.000003 & 0.370\end{array}$ $\begin{array}{llllll}0.000087 & 0.000115 & 997.37 & 0.0103 & -0.000028 & 0.000\end{array}$ $\begin{array}{llllll}0.000095 & 0.000115 & 997.47 & 0.0098 & -0.000020 & 0.000\end{array}$ $\begin{array}{llllll}0.000088 & 0.000115 & 997.52 & 0.0147 & -0.000028 & 0.000\end{array}$ $\begin{array}{llllll}0.000088 & 0.000115 & 997.68 & 0.0193 & -0.000027 & 0.000\end{array}$ $\begin{array}{llllll}0.000256 & 0.000116 & 998.30 & -0.1978 & 0.000140 & 0.039\end{array}$ $\begin{array}{llllll}0.000089 & 0.000116 & 998.37 & 0.0392 & -0.000026 & 0.002\end{array}$ $\begin{array}{llllll}0.000116 & 0.000116 & 998.64 & 0.0001 & 0.000000 & 0.000\end{array}$ $\begin{array}{llllll}0.000087 & 0.000116 & 998.67 & 0.0533 & -0.000029 & 0.003\end{array}$ $\begin{array}{lllllll}0.000093 & 0.000116 & 999.15 & 0.0556 & -0.000023 & 0.003\end{array}$ $\begin{array}{llllll}0.000092 & 0.000116 & 999.85 & 0.0780 & -0.000025 & 0.006\end{array}$ $\begin{array}{llllll}0.000096 & 0.000117 & 1000.83 & 0.0864 & -0.000020 & 0.007\end{array}$ $\begin{array}{lllllll}0.000095 & 0.000117 & 1001.10 & 0.1024 & -0.000022 & 0.010\end{array}$ $\begin{array}{lllllll}0.000077 & 0.000117 & 1001.21 & 0.1902 & -0.000040 & 0.036\end{array}$ $\begin{array}{llllll}0.000098 & 0.000117 & 1001.52 & 0.0970 & -0.000019 & 0.009\end{array}$ $\begin{array}{llllll}0.000099 & 0.000117 & 1002.41 & 0.1116 & -0.000018 & 0.012\end{array}$ $\begin{array}{llllll}0.000102 & 0.000118 & 1003.29 & 0.1095 & -0.000015 & 0.012\end{array}$ $\begin{array}{llllll}0.000098 & 0.000118 & 1003.29 & 0.1395 & -0.000020 & 0.019\end{array}$ $\begin{array}{llllll}0.000100 & 0.000118 & 1003.35 & 0.1292 & -0.000018 & 0.017\end{array}$ $\begin{array}{llllll}0.000100 & 0.000118 & 1003.98 & 0.1416 & -0.000018 & 0.020\end{array}$ $\begin{array}{llllll}0.000103 & 0.000118 & 1004.48 & 0.1322 & -0.000016 & 0.017\end{array}$ $\begin{array}{llllll}0.000101 & 0.000118 & 1005.14 & 0.1627 & -0.000018 & 0.026\end{array}$ $\begin{array}{llllll}0.000105 & 0.000119 & 1006.64 & 0.1572 & -0.000014 & 0.025\end{array}$ $\begin{array}{llllll}0.000106 & 0.000120 & 1008.08 & 0.1693 & -0.000013 & 0.029\end{array}$ $\begin{array}{llllll}0.000107 & 0.000120 & 1009.30 & 0.1849 & -0.000013 & 0.034\end{array}$ $\begin{array}{lllllll}0.000113 & 0.000120 & 1009.49 & 0.1118 & -0.000008 & 0.012\end{array}$ $\begin{array}{llllll}0.000108 & 0.000120 & 1009.49 & 0.1718 & -0.000012 & 0.030\end{array}$ $\begin{array}{llllll}0.000107 & 0.000120 & 1009.51 & 0.1852 & -0.000013 & 0.034\end{array}$ $\begin{array}{llllll}0.000109 & 0.000121 & 1011.04 & 0.1923 & -0.000012 & 0.037\end{array}$ $\begin{array}{llllll}0.000110 & 0.000122 & 1013.27 & 0.2203 & -0.000012 & 0.049\end{array}$ $\begin{array}{llllll}0.000112 & 0.000122 & 1013.98 & 0.1984 & -0.000010 & 0.039\end{array}$ $\begin{array}{llllll}0.000115 & 0.000123 & 1015.64 & 0.1620 & -0.000008 & 0.026\end{array}$ $\begin{array}{llllll}0.000116 & 0.000124 & 1017.84 & 0.1964 & -0.000008 & 0.039\end{array}$ $\begin{array}{llllll}0.000117 & 0.000125 & 1021.22 & 0.2324 & -0.000008 & 0.054\end{array}$ $\begin{array}{llllll}0.000118 & 0.000125 & 1021.68 & 0.2055 & -0.000007 & 0.042\end{array}$ $\begin{array}{lllllll}0.000123 & 0.000127 & 1026.06 & 0.1446 & -0.000004 & 0.021\end{array}$ $\begin{array}{llllll}0.000123 & 0.000128 & 1026.67 & 0.1698 & -0.000005 & 0.029\end{array}$ $\begin{array}{llllll}0.000123 & 0.000129 & 1029.28 & 0.2198 & -0.000006 & 0.048\end{array}$ $\begin{array}{lllllll}0.000127 & 0.000131 & 1034.02 & 0.1578 & -0.000004 & 0.025\end{array}$ $\begin{array}{lllllll}0.000128 & 0.000132 & 1037.13 & 0.1857 & -0.000004 & 0.034\end{array}$ $\begin{array}{llllll}0.000129 & 0.000132 & 1037.75 & 0.1402 & -0.000003 & 0.020\end{array}$ $\begin{array}{llllll}0.000131 & 0.000134 & 1040.82 & 0.1156 & -0.000002 & 0.013\end{array}$ $\begin{array}{llllll}0.000134 & 0.000136 & 1046.09 & 0.0780 & -0.000001 & 0.006\end{array}$ $\begin{array}{llllll}0.000139 & 0.000139 & 1054.00 & -0.0048 & 0.000000 & 0.000\end{array}$ $\begin{array}{llllll}0.000142 & 0.000140 & 1057.35 & -0.1189 & 0.000002 & 0.014\end{array}$ $\begin{array}{llllll}0.000141 & 0.000140 & 1057.42 & -0.0147 & 0.000000 & 0.000\end{array}$
997.1442

0.335997 .0449

$0.436 \quad 997.0449$

$0.486 \quad 997.0449$

0.651997 .0449 997.0449

1.361997 .0449

1.591997 .0449

1.683997 .0449

2.162997 .0449

$2.887 \quad 997.0449$

$3.867 \quad 997.0449$

$4.16 \quad 997.0449$ 997.0449

$4.575 \quad 997.0449$

$5.477 \quad 997.0449$

997.0449 997.0449

$6.437 \quad 997.0449$

$7.075 \quad 997.0449$

$7.569 \quad 997.0449$

$8.254 \quad 997.0449$

$9.753 \quad 997.0449$

$11.205 \quad 997.0449$

$12.443 \quad 997.0449$ 997.0449 997.0449

12.652997 .0449

$14.185 \quad 997.0449$

$16.449 \quad 997.0449$

$17.137 \quad 997.0449$ 997.0449

$20.993 \quad 997.0449$

$24.407 \quad 997.0449$

24.841997 .0449 997.0449

$29.799 \quad 997.0449$

$32.451 \quad 997.0449$

$37.131 \quad 997.0449$

$40.266 \quad 997.0449$

$40.842 \quad 997.0449$

43.89997 .0449

$49.122 \quad 997.0449$

997.0449

997.0449

$60.36 \quad 997.0449$ 


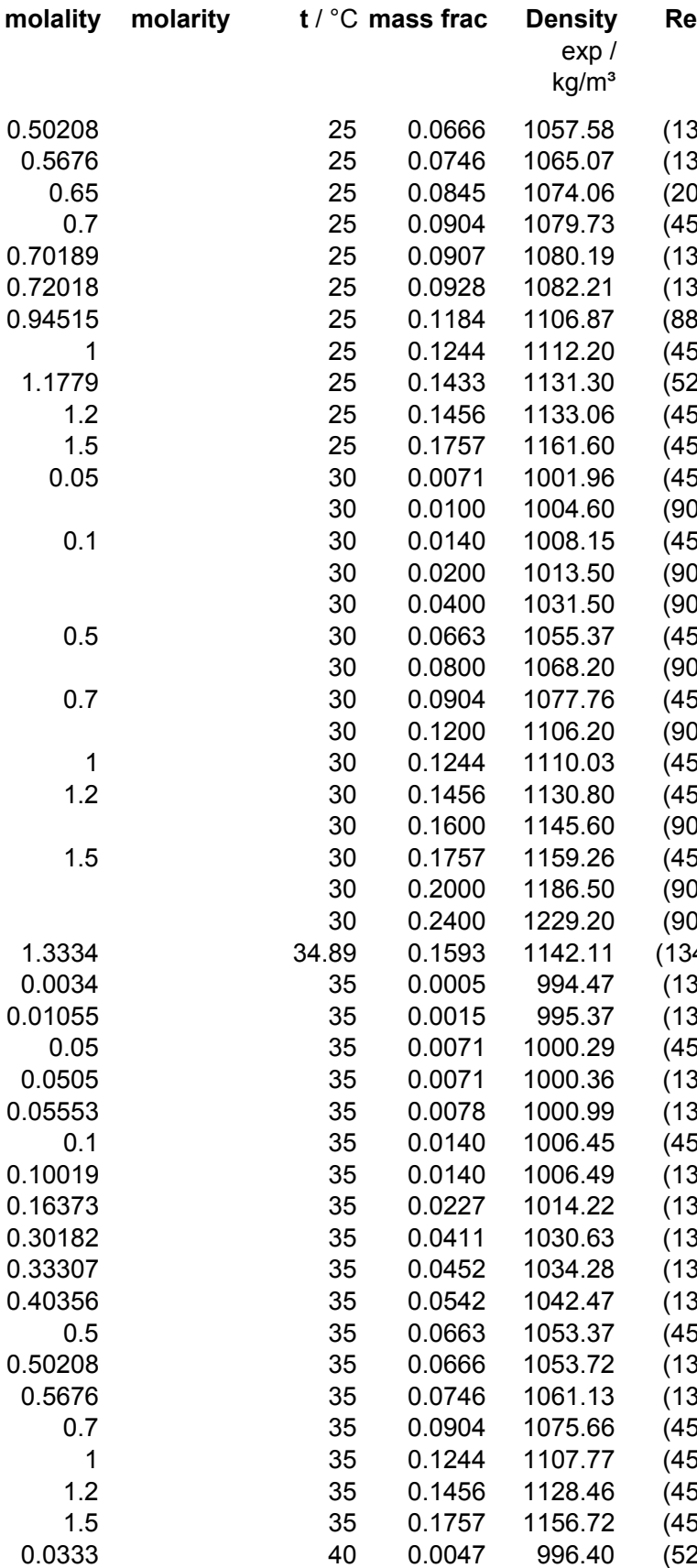

App vol App vol Density Density App Vol Square of Inconsistent Inconsistent Apparent Delta density Density of

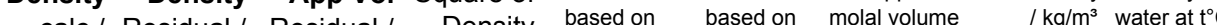

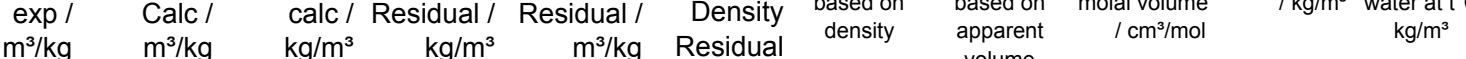
$\begin{array}{llllll}0.000141 & 0.000141 & 1057.59 & -0.0079 & 0.000000 & 0.000\end{array}$ $\begin{array}{llllll}0.000144 & 0.000144 & 1065.14 & -0.0646 & 0.000001 & 0.004\end{array}$ $\begin{array}{llllll}0.000152 & 0.000148 & 1074.51 & -0.4563 & 0.000005 & 0.208\end{array}$ $\begin{array}{llllll}0.000154 & 0.000150 & 1080.14 & -0.4090 & 0.000004 & 0.167\end{array}$ $\begin{array}{llllll}0.000152 & 0.000150 & 1080.35 & -0.1649 & 0.000002 & 0.027\end{array}$ $\begin{array}{llllll}0.000153 & 0.000151 & 1082.40 & -0.1858 & 0.000002 & 0.035\end{array}$ $\begin{array}{llllll}0.000162 & 0.000161 & 1107.05 & -0.1850 & 0.000001 & 0.034\end{array}$ $\begin{array}{llllll}0.000168 & 0.000163 & 1112.92 & -0.7196 & 0.000005 & 0.518\end{array}$ $\begin{array}{llllll}0.000173 & 0.000171 & 1131.57 & -0.2749 & 0.000001 & 0.076\end{array}$ $\begin{array}{llllll}0.000194 & 0.000184 & 1163.90 & -2.3011 & 0.000010 & 5.295\end{array}$ $\begin{array}{llllll}0.000107 & 0.000124 & 1001.84 & 0.1202 & -0.000017 & 0.014\end{array}$ $\begin{array}{llllll}0.000109 & 0.000125 & 1004.44 & 0.1606 & -0.000016 & 0.026\end{array}$ $\begin{array}{lllllll}0.000115 & 0.000127 & 1007.99 & 0.1646 & -0.000012 & 0.027\end{array}$ $\begin{array}{llllll}0.000120 & 0.000129 & 1013.31 & 0.1866 & -0.000009 & 0.035\end{array}$ $\begin{array}{llllll}0.000132 & 0.000136 & 1031.30 & 0.1950 & -0.000005 & 0.038\end{array}$ $\begin{array}{llllll}0.000147 & 0.000146 & 1055.47 & -0.0994 & 0.000001 & 0.010\end{array}$ $\begin{array}{llllll}0.000152 & 0.000151 & 1068.24 & -0.0449 & 0.000000 & 0.002\end{array}$ $\begin{array}{llllll}0.000158 & 0.000155 & 1078.10 & -0.3383 & 0.000003 & 0.114\end{array}$ $\begin{array}{llllll}0.000168 & 0.000166 & 1106.41 & -0.2125 & 0.000001 & 0.045\end{array}$ $\begin{array}{llllll}0.000172 & 0.000168 & 1110.67 & -0.6422 & 0.000004 & 0.412\end{array}$ $\begin{array}{llllll}0.000180 & 0.000176 & 1131.49 & -0.6904 & 0.000004 & 0.477\end{array}$ $\begin{array}{llllll}0.000183 & 0.000182 & 1145.72 & -0.1250 & 0.000001 & 0.016\end{array}$ $\begin{array}{llllll}0.000197 & 0.000188 & 1161.40 & -2.1424 & 0.000009 & 4.590\end{array}$ $\begin{array}{llllll}0.000197 & 0.000198 & 1186.07 & 0.4342 & -0.000002 & 0.189\end{array}$ $\begin{array}{llllll}0.000209 & 0.000215 & 1227.28 & 1.9211 & -0.000005 & 3.691\end{array}$ $\begin{array}{llllll}0.000187 & 0.000185 & 1142.54 & -0.4263 & 0.000002 & 0.182\end{array}$ $\begin{array}{llllll}0.000078 & 0.000127 & 994.45 & 0.0237 & -0.000050 & 0.001\end{array}$ $\begin{array}{llllll}0.000102 & 0.000128 & 995.33 & 0.0387 & -0.000026 & 0.001\end{array}$ $\begin{array}{llllll}0.000114 & 0.000130 & 1000.18 & 0.1136 & -0.000016 & 0.013\end{array}$ $\begin{array}{llllll}0.000112 & 0.000130 & 1000.24 & 0.1252 & -0.000018 & 0.016\end{array}$ $\begin{array}{llllll}0.000112 & 0.000130 & 1000.85 & 0.1396 & -0.000018 & 0.020\end{array}$ $\begin{array}{llllll}0.000120 & 0.000132 & 1006.28 & 0.1747 & -0.000012 & 0.031\end{array}$ $\begin{array}{llllll}0.000118 & 0.000132 & 1006.30 & 0.1945 & -0.000014 & 0.038\end{array}$ $\begin{array}{llllll}0.000125 & 0.000135 & 1013.98 & 0.2333 & -0.000010 & 0.054\end{array}$ $\begin{array}{llllll}0.000137 & 0.000142 & 1030.43 & 0.2064 & -0.000005 & 0.043\end{array}$ $\begin{array}{llllll}0.000139 & 0.000143 & 1034.10 & 0.1808 & -0.000004 & 0.033\end{array}$ $\begin{array}{llllll}0.000144 & 0.000146 & 1042.32 & 0.1466 & -0.000002 & 0.022\end{array}$ $\begin{array}{llllll}0.000152 & 0.000151 & 1053.43 & -0.0551 & 0.000001 & 0.003\end{array}$ $\begin{array}{llllll}0.000150 & 0.000151 & 1053.66 & 0.0561 & -0.000001 & 0.003\end{array}$ $\begin{array}{llllll}0.000153 & 0.000154 & 1061.11 & 0.0229 & 0.000000 & 0.001\end{array}$ $\begin{array}{llllll}0.000162 & 0.000160 & 1075.91 & -0.2547 & 0.000002 & 0.065\end{array}$ $\begin{array}{llllll}0.000176 & 0.000172 & 1108.31 & -0.5447 & 0.000004 & 0.297\end{array}$ $\begin{array}{llllll}0.000183 & 0.000180 & 1129.04 & -0.5803 & 0.000003 & 0.337\end{array}$ $\begin{array}{llrrrr}0.000201 & 0.000191 & 1158.85 & -2.1282 & 0.000009 & 4.529\end{array}$ $\begin{array}{llllll}0.000109 & 0.000134 & 996.28 & 0.1157 & -0.000025 & 0.013\end{array}$
60.537997 .0449 $68.027 \quad 997.0449$ 77.011997 .0449 997.0449 83.141997 .0449 $85.166 \quad 997.0449$ 997.0449 997.0449 997.0449 997.0449 995.6473 995.6473 995.6473 995.6473 995.6473 995.6473 995.6473 995.6473 995.6473 995.6473 995.6473 995.6473 995.6473 995.6473 995.6473 994.0696

$0.443 \quad 994.0319$

$1.339 \quad 994.0319$ 994.0319 6.331994 .0319 6.961994 .0319 994.0319

12.461994 .0319 $20.184 \quad 994.0319$ $36.603 \quad 994.0319$ $40.251 \quad 994.0319$

$48.438 \quad 994.0319$ 994.0319 $59.687 \quad 994.0319$ $67.098 \quad 994.0319$ 994.0319 994.0319 994.0319 994.0319 992.2158 109.821997 .0449 
molality molarity

$$
\text { exp / }
$$$$
\mathrm{kg} / \mathrm{m}^{3}
$$

0.2356

0.4712

1.1779

0.01

0.05
0.05

0.1

0.1

0.15

0.5
0.7

0.7

1.2

1.5

1.3334
0.09898

0.11341

0.20351

0.50228

0.99188

0.00969

0.02305

0.05339

0.10154

0.19785

0.49852

1.01884

1.53238

0.002226

0.00622

0.006274

0.012695

0.014306

0.026513

0.044254

0.085346

0.15125

0.2266

$t /{ }^{\circ} \mathrm{C}$ mass frac
$\begin{array}{r}\exp / \\ \mathrm{kg} / \mathrm{m}^{3}\end{array}$

$\begin{array}{lll}40 & 0.0200 & 1009.80 \\ 40 & 0.0324 & 1020.80 \\ 40 & 0.0400 & 1027.60 \\ 40 & 0.0627 & 1048.00 \\ 40 & 0.0800 & 1063.90 \\ 40 & 0.1200 & 1101.50\end{array}$

$\begin{array}{ll}0.1433 & 1124.20 \\ 0.1600 & 1140.60\end{array}$

$0.2000 \quad 1181.30$

$0.2400 \quad 1223.70$

$0.0014 \quad 991.30$

$0.0071 \quad 996.40$

$0.0140 \quad 1002.30$

$0.0140 \quad 1002.48$

$\begin{array}{lll}0.0209 & 1008.30\end{array}$

$0.0663 \quad 1048.96$

$0.0904 \quad 1071.06$

$0.1244 \quad 1102.94$

$0.1456 \quad 1123.44$

$\begin{array}{ll}0.1757 & 1151.68\end{array}$

$0.1593 \quad 1136.79$

$0.0139 \quad 1000.70$

$0.0159 \quad 1002.45$

$\begin{array}{ll}0.0281 & 1013.19\end{array}$

$0.0666 \quad 1047.54$

$0.1235 \quad 1100.37$

$0.0014 \quad 989.65$

0.0033

991.30

0.0075

995.10

$\begin{array}{ll}0.0142 & 1000.83 \\ 0.0273 & 1012.29\end{array}$

1012.29

$0.1264 \quad 1102.82$

$\begin{array}{rr}0.1788 & 1154.56 \\ 0.0003 & 988.32\end{array}$

$0.0009 \quad 988.82$

$0.0009 \quad 988.82$

$0.0018 \quad 989.63$

$0.0038 \quad 991.34$

$0.0062 \quad 993.53$

$0.0120 \quad 998.53$

$0.0210 \quad 1006.44$

$0.0312 \quad 1015.33$
Ref

App

exp / Calc /

$\begin{array}{cc}\exp / & \text { Calc / } \\ \mathrm{m}^{3} / \mathrm{kg} & \mathrm{m}^{3} / \mathrm{kg}\end{array}$

Density

sity Density App Vol Square of Inconsistent Inconsistent

Apparent Delta density Density of

(90)

$0.000130 \quad 0.000139$

$\begin{array}{lll}0.000136 & 0.000143\end{array}$

1000.88

1009.63

$0.000140 \quad 0.000146$

$0.000153 \quad 0.000153$

1020.58

1027.37

1047.95

$0.0071 \quad 996.39$

$0.0020 \quad 989.83$ $\begin{array}{ll}0.000175 & 0.000174\end{array}$

$0.000182 \quad 0.000182$

$0.000201 \quad 0.000203$

$0.000213 \quad 0.000218$

$0.000229 \quad 0.000137$

$\begin{array}{lll}0.000121 & 0.000139\end{array}$

$0.000122 \quad 0.000139$

$0.000140 \quad 0.000141$

$0.000128 \quad 0.000141$

$0.000142 \quad 0.000143$

$0.000167 \quad 0.000166$

$0.000180 \quad 0.000178$

$0.000188 \quad 0.000185$

$0.000204 \quad 0.000196$

$0.000192 \quad 0.000190$

$0.000128 \quad 0.000143$

1063.85
1101.63

1124.26

1140.65

1180.82

1222.01
991.43

996.28

996.28

1002.30
1002.30

1008.27

1048.89

1071.16

1103.30

1123.90
1153.58

1137.14

1000.49

$0.000129 \quad 0.000144$

$0.000137 \quad 0.000148$

1012.88

1047.29

$\begin{array}{lll}0.000179 & 0.000179 & 1100.45\end{array}$

$\begin{array}{lll}0.000087 & 0.000140 & 989.58\end{array}$

$\begin{array}{lll}0.000108 & 0.000142 & 994.85\end{array}$

$\begin{array}{lll}0.000129 & 0.000144 & 1000.62\end{array}$

$\begin{array}{lll}0.000139 & 0.000148 & 1012.04\end{array}$

$\begin{array}{lll}0.000158 & 0.000160 & 1046.67\end{array}$

$\begin{array}{lll}0.000182 & 0.000180 & 1103.06\end{array}$

$\begin{array}{llll}0.000197 & 0.000198 & 1154.44\end{array}$

988.31

$\begin{array}{lll}0.000104 & 0.000140 & 988.31 \\ 0.000105 & 0.000140 & 988.79\end{array}$

$0.000107 \quad 0.000140$

988.80

989.57

989.77

$0.000109 \quad 0.000140$

$0.000112 \quad 0.000141$

991.24

$\begin{array}{lll}0.000117 & 0.000142 & 993.37\end{array}$

$\begin{array}{lll}0.000124 & 0.000143 & 998.30\end{array}$

$0.000132 \quad 0.000146$

1006.14

$\begin{array}{lll}0.000140 & 0.000149 & 1015.02\end{array}$
$0.000188 \quad 0.000188$

$0.000157 \quad 0.000158$

$0.000157 \quad 0.000160$

$\begin{array}{lll}0.000108 & 0.000140 & 991.20\end{array}$

$$
\begin{array}{rrrr} 
& \mathrm{kg} / \mathrm{m}^{3} & \begin{array}{r}
\mathrm{m}^{3} / \mathrm{kg} \\
\mathrm{m}^{3}
\end{array} & \begin{array}{r}
\text { Residual density } \\
\text { Reparent } \\
\text { volume }
\end{array}
\end{array}
$$

$0.1209-0.000012 \quad 0.015$

$\begin{array}{lll}0.1738 & -0.000009 & 0.030\end{array}$

$\begin{array}{lll}0.2241 & -0.000007 & 0.050\end{array}$

$\begin{array}{lll}0.2287 & -0.000005 & 0.052\end{array}$

$\begin{array}{lll}0.0500 & -0.000001 & 0.002\end{array}$

$\begin{array}{lll}0.0452 & 0.000000 & 0.002\end{array}$

$\begin{array}{lll}-0.1331 & 0.000001 & 0.018\end{array}$

$\begin{array}{lll}-0.0590 & 0.000000 & 0.003\end{array}$

$\begin{array}{lll}-0.0486 & 0.000000 & 0.002\end{array}$

$0.4848-0.000002 \quad 0.235$

$\begin{array}{lll}1.6875 & -0.000005 & 2.848\end{array}$

$\begin{array}{lll}-0.1292 & 0.000093 & 0.017\end{array}$

$\begin{array}{lll}0.1241 & -0.000018 & 0.015\end{array}$

$\begin{array}{lll}0.1141 & -0.000016 & 0.013\end{array}$

$\begin{array}{lll}0.0048 & 0.000000 & 0.000\end{array}$

$\begin{array}{lll}0.1848 & -0.000013 & 0.034\end{array}$

$\begin{array}{lll}0.0290 & -0.000001 & 0.001\end{array}$

$\begin{array}{lll}0.0687 & -0.000001 & 0.005\end{array}$

$\begin{array}{lll}-0.1002 & 0.000001 & 0.010\end{array}$

$\begin{array}{lll}-0.3593 & 0.000002 & 0.129\end{array}$

$\begin{array}{lll}-0.4576 & 0.000002 & 0.209\end{array}$

$\begin{array}{lll}-1.9027 & 0.000008 & 3.620\end{array}$

$\begin{array}{lll}-0.3556 & 0.000002 & 0.126\end{array}$

$\begin{array}{lll}0.2136 & -0.000015 & 0.046\end{array}$

$\begin{array}{lll}0.2439 & -0.000015 & 0.059\end{array}$

$\begin{array}{lll}0.3075 & -0.000011 & 0.095\end{array}$

$\begin{array}{lll}0.2521 & -0.000003 & 0.064\end{array}$

$\begin{array}{lll}-0.0788 & 0.000001 & 0.006\end{array}$

$\begin{array}{lll}0.0703 & -0.000052 & 0.005\end{array}$

$\begin{array}{lll}0.1031 & -0.000032 & 0.011\end{array}$

$\begin{array}{lll}0.2464 & -0.000033 & 0.061\end{array}$

$\begin{array}{lll}0.2130 & -0.000015 & 0.045\end{array}$

$\begin{array}{lll}0.2528 & -0.000009 & 0.064\end{array}$

$\begin{array}{lll}0.1862 & -0.000003 & 0.035\end{array}$

$\begin{array}{rrr}-0.2459 & 0.000002 & 0.060\end{array}$

$\begin{array}{lll}0.1162 & 0.000000 & 0.014\end{array}$

$\begin{array}{lll}0.0111 & -0.000036 & 0.000\end{array}$

$\begin{array}{lll}0.0302 & -0.000035 & 0.001\end{array}$

$\begin{array}{lll}0.0291 & -0.000033 & 0.001\end{array}$

$\begin{array}{lll}0.0560 & -0.000032 & 0.003\end{array}$

$\begin{array}{lll}0.0627 & -0.000032 & 0.004\end{array}$

0.011

$\begin{array}{lll}0.2938 & -0.000014 & 0.086\end{array}$

$\begin{array}{lll}0.3167 & -0.000010 & 0.100\end{array}$ $\begin{array}{lll}0.2310 & -0.000019 & 0.053\end{array}$

$-2$

992.2158

992.2158

992.2158

992.2158

992.2158

992.2158

992.2158

992.2158

992.2158

992.2158

992.2158

990.2132

990.2132

990.2132

990.2132

990.2132

990.2132

990.2132

990.2132

990.2132

990.2132

990.2132

990.1084

18.17

988.5785

988.5785

988.5785

988.5785

988.5785

988.4093

988.4093

988.4093

988.4093

988.4093

988.4093

$\begin{array}{rr}22.39 & 988.4093 \\ 25.8 & 988.4093 \\ 28.03 & 988.4093\end{array}$

28.03

$0.2804 \quad 988.0363$

$0.7824 \quad 988.0363$

$0.7878 \quad 988.0363$

$1.5905 \quad 988.0363$

1.7918988 .0363

$3.3076 \quad 988.0363$

$5.4907 \quad 988.0363$

10.4949988 .0363

18.3991988 .0363

$27.2975 \quad 988.0363$ olal volume $\quad / \mathrm{kg} / \mathrm{m}^{3}$ water at $\mathrm{t}^{\circ} \mathrm{C}$ 
molality molarity

0.28814

0.41164

0.53857

0.69983

0.79824

1.00476

1.00476

0.05
0.1
0.5
0.65
0.7
1
1.2
1.5
0.0333

0.0333

0.2356

0.4712

1.1779

0.01

0.05

0.1
0.15

0.05156

0.06016

0.0715

0.20229

0.52286

0.70028

0.01948

0.05442

0.09547

0.20961

0.31478

0.39192

0.49767

0.10641

0.20393

0.4941

$0.1200 \quad 1091.50$ $\exp /$ $\mathrm{kg} / \mathrm{m}^{3}$

$\mathrm{t} /{ }^{\circ} \mathrm{C}$ mass frac Density Ref

$\begin{array}{lll}50 & 0.0393 & 1022.53 \\ 50 & 0.0552 & 1036.68 \\ 50 & 0.0711 & 1050.96\end{array}$

$0.0711 \quad 1050.96$

$0.0904 \quad 1068.69$

$0.1018 \quad 1079.30$

$0.1249 \quad 1101.08$

$0.1249 \quad 1101.09$

$0.0071 \quad 991.84$

$0.0140 \quad 997.86$

$0.0663 \quad 1044.04$

$0.0845 \quad 1060.56$

$0.0904 \quad 1066.01$

$0.1244 \quad 1097.67$

$0.1456 \quad 1118.13$

$0.1757 \quad 1146.17$

$0.0047 \quad 987.30$

$0.0100 \quad 991.90$

$0.0200 \quad 1000.70$

$0.0324 \quad 1011.30$

$0.0400 \quad 1018.40$

$0.0627 \quad 1038.30$

$0.0800 \quad 1054.40$

$0.1433 \quad 1113.40$

$0.1600 \quad 1129.90$

$0.2000 \quad 1169.60$

$0.0014 \quad 981.80$

$0.0071 \quad 986.70$

$0.0140 \quad 992.60$

$0.0209 \quad 998.50$

$0.0073 \quad 982.45$

$0.0085 \quad 983.39$

$0.0101 \quad 984.76$

0.0279

0.0691

0.0905

1000.11

1036.37

1055.69
977.37

0.0077

0.0077

0.0134
0.0289

981.62

986.53

$0.0428 \quad 1012.05$

$0.0527 \quad 1020.76$

0.0660

1020.76

0.0149

0.0282

$987.73 \quad(114)$

$999.19 \quad(114)$

$\begin{array}{ll}0.0656 & 1032.09\end{array}$ $\begin{array}{rr}\text { App vol } & \text { App vol } \\ \exp / & \text { Calc / } \\ \mathrm{m}^{3} / \mathrm{kg} & \mathrm{m}^{3} / \mathrm{kg}\end{array}$

Density Density App Vol Square of Inconsistent Inconsistent

Apparent Delta density Density of calc / Residual / Residual / Density based on based on molal volume $/ \mathrm{kg} / \mathrm{m}^{3}$ water at t ${ }^{\circ} \mathrm{C}$ $\mathrm{kg} / \mathrm{m}^{3} \quad \mathrm{~kg} / \mathrm{m}^{3} \quad \mathrm{~m}^{3} / \mathrm{kg}$ Residual density $\begin{gathered}\text { apparent } \\ \text { volume }\end{gathered}$

$/ \mathrm{cm}^{3} / \mathrm{mol}$

34.4945988 .0363 $48.6398 \quad 988.0363$ 62.9239988 .0363

80.651988 .0363

91.2671988 .0363

113.0439988 .0363

113.0488988 .0363

985.6952

985.6952

985.6952

74.869985 .6952

985.6952

985.6952

985.6952

985.6952

983.1989

983.1989

983.1989

983.1989

983.1989

983.1989

983.1989

983.1989

983.1989

983.1989

983.1989

980.5548

980.5548

980.5548

980.5548

976.0625

976.0625

17.56

17.67

20.29

23.05

24.21

15.74

16.91

18.13

19.78

20.98

21.76

22.43

18.33

19.76

22.55
976.0625

976.0625

976.0625

976.0625

974.9683

974.9683

974.9683

974.9683

974.9683

974.9683

974.9683

974.8729

974.8729

974.8729 
molality molarity

0.72002

1.001

1.5106

0.01
0.0333

0.05

0.1

0.15

0.236

0.4721
1.1802

1.1802

0.65

0.20186

0.20229

0.52286

0.70028

0.01

0.05

0.15
0.2367

0.4784

1.1836

0.01

0.0336

0.05
0.1

0.15

0.2381

0.4761

1.1904 $\mathrm{t} /{ }^{\circ} \mathrm{C}$ mass frac Density Ref

$$
\exp /
$$$$
\mathrm{kg} / \mathrm{m}^{3}
$$
$\mathrm{kg} / \mathrm{m}^{3}$

74.96
74.96
74.96

74.96

$\begin{array}{lll}0.0928 & 1056.59 & (114) \\ 0.1245 & 1086.03 & (114) \\ 0.1767 & 1136.77 & (114)\end{array}$

$0.1767 \quad 1136.77$

$0.0014 \quad 976.10$

$0.0047 \quad 979.20$

$0.0071 \quad 981.10$

$0.0140 \quad 987.00$

$0.0209 \quad 992.90$

$0.0324 \quad 1003.10$

$0.0629 \quad 1030.30$

$0.1436 \quad 1105.10$

$0.0100 \quad 980.50$

$0.0200 \quad 989.20$

$0.0400 \quad 1006.80$

$0.0800 \quad 1042.60$

$0.1200 \quad 1079.50$

$0.1600 \quad 1117.60$

$0.2000 \quad 1156.90$

$0.0845 \quad 1035.84$

$\begin{array}{lll}98.67 & 0.0279 \quad 983.30\end{array}$

$\begin{array}{lll}98.67 & 0.0279 & 983.35\end{array}$

$\begin{array}{llr}98.67 & 0.0279 & 983.35 \\ 98.67 & 0.0691 & 1019.45\end{array}$

0.0905
0.0014

0.0014

0.0071

0.0100

0.0140

0.0200

0.0209

0.0325

0.0400

0.0636

0.0800

0.1200

0.1439
0.1600

0.0014

0.0048

0.0071

0.0140

0.0209

0.0327

0.0634

0.1446
Density Density App Vol Square of Inconsistent Inconsistent

Apparent Delta density Density of

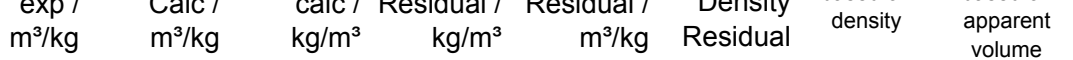

$\begin{array}{llllll}0.000171 & 0.000173 & 1056.39 & 0.1998 & -0.000002 & 0.040\end{array}$

$\begin{array}{llllll}0.000182 & 0.000181 & 1086.19 & -0.1630 & 0.000001 & 0.027\end{array}$

$\begin{array}{llllll}0.000199 & 0.000196 & 1137.48 & -0.7129 & 0.000003 & 0.508\end{array}$

$\begin{array}{llllll}0.000099 & 0.000149 & 976.03 & 0.0674 & -0.000050 & 0.005\end{array}$

$\begin{array}{llllll}0.000058 & 0.000150 & 978.78 & 0.4153 & -0.000092 & 0.173\end{array}$

$\begin{array}{llllll}0.000099 & 0.000150 & 980.75 & 0.3477 & -0.000051 & 0.121\end{array}$

$\begin{array}{llllll}0.000124 & 0.000152 & 986.62 & 0.3808 & -0.000028 & 0.145\end{array}$

$\begin{array}{llllll}0.000132 & 0.000154 & 992.45 & 0.4505 & -0.000022 & 0.203\end{array}$

$\begin{array}{llllll}0.000135 & 0.000157 & 1002.39 & 0.7074 & -0.000022 & 0.500\end{array}$

$\begin{array}{llllll}0.000147 & 0.000165 & 1029.14 & 1.1608 & -0.000017 & 1.347\end{array}$

$\begin{array}{llllll}0.000184 & 0.000187 & 1104.61 & 0.4937 & -0.000003 & 0.244\end{array}$

$\begin{array}{llllll}0.000116 & 0.000151 & 980.16 & 0.3426 & -0.000036 & 0.117\end{array}$

$\begin{array}{llllll}0.000124 & 0.000154 & 988.61 & 0.5861 & -0.000030 & 0.343\end{array}$

$\begin{array}{llllll}0.000135 & 0.000159 & 1005.82 & 0.9789 & -0.000024 & 0.958\end{array}$

$\begin{array}{llllll}0.000156 & 0.000169 & 1041.44 & 1.1648 & -0.000013 & 1.357\end{array}$

$\begin{array}{llllll}0.000173 & 0.000179 & 1078.69 & 0.8107 & -0.000006 & 0.657\end{array}$

$\begin{array}{llllll}0.000190 & 0.000190 & 1117.62 & -0.0248 & 0.000000 & 0.001\end{array}$

$\begin{array}{llllll}0.000206 & 0.000201 & 1158.27 & -1.3719 & 0.000005 & 1.882\end{array}$

$\begin{array}{llllll}0.000162 & 0.000167 & 1035.39 & 0.4530 & -0.000005 & 0.205\end{array}$

$\begin{array}{llllll}0.000131 & 0.000153 & 982.68 & 0.6129 & -0.000023 & 0.376\end{array}$

$\begin{array}{llllll}0.000130 & 0.000153 & 982.73 & 0.6222 & -0.000023 & 0.387\end{array}$

$\begin{array}{llllll}0.000153 & 0.000162 & 1018.80 & 0.6576 & -0.000009 & 0.432\end{array}$

$\begin{array}{lllllll}0.000161 & 0.000167 & 1038.21 & 0.5346 & -0.000005 & 0.286\end{array}$

$\begin{array}{llllll}-0.000134 & 0.000147 & 959.53 & 0.3674 & -0.000281 & 0.135\end{array}$

$\begin{array}{llllll}-0.000020 & 0.000148 & 962.26 & 0.7370 & -0.000168 & 0.543 \\ -0.000004 & 0.000149 & 964.20 & 1.0041 & -0.000153 & 1.008\end{array}$

$\begin{array}{rrrrrr}-0.000004 & 0.000149 & 964.20 & 1.0041 & -0.000153 & 1.008 \\ 0.000101 & 0.000149 & 966.65 & 0.4518 & -0.000048 & 0.204\end{array}$

$\begin{array}{llllll}0.000074 & 0.000150 & 970.00 & 1.0029 & -0.000076 & 1.006\end{array}$

$\begin{array}{llllll}0.000111 & 0.000151 & 975.04 & 0.7627 & -0.000040 & 0.582\end{array}$

$\begin{array}{llllll}0.000095 & 0.000151 & 975.77 & 1.1326 & -0.000057 & 1.283\end{array}$

$\begin{array}{llllll}0.000116 & 0.000154 & 985.70 & 1.2000 & -0.000038 & 1.440\end{array}$

$\begin{array}{llllll}0.000123 & 0.000156 & 992.13 & 1.2654 & -0.000032 & 1.601\end{array}$

$\begin{array}{llllll}0.000144 & 0.000161 & 1012.90 & 1.0980 & -0.000017 & 1.206\end{array}$

$\begin{array}{llllll}0.000146 & 0.000164 & 1027.64 & 1.5559 & -0.000018 & 2.421\end{array}$

$\begin{array}{llllll}0.000165 & 0.000173 & 1064.98 & 1.1198 & -0.000008 & 1.254\end{array}$

$\begin{array}{lllllll}0.000176 & 0.000178 & 1088.24 & 0.4640 & -0.000003 & 0.215\end{array}$

$\begin{array}{llllll}0.000182 & 0.000182 & 1104.23 & -0.0309 & 0.000000 & 0.001\end{array}$

$\begin{array}{llllll}-0.000384 & 0.000140 & 940.14 & 0.6569 & -0.000524 & 0.431\end{array}$

$\begin{array}{llllll}-0.000126 & 0.000140 & 942.87 & 1.1258 & -0.000266 & 1.267\end{array}$

$\begin{array}{llllll}-0.000055 & 0.000140 & 944.77 & 1.2313 & -0.000195 & 1.516\end{array}$

$\begin{array}{llllll}0.000025 & 0.000142 & 950.53 & 1.4727 & -0.000116 & 2.169\end{array}$

$\begin{array}{llllll}0.000067 & 0.000143 & 956.26 & 1.4399 & -0.000075 & 2.073\end{array}$

$\begin{array}{llllll}0.000079 & 0.000145 & 966.30 & 2.0015 & -0.000065 & 4.006\end{array}$

$\begin{array}{lllll}0.000109 & 0.000150 & 993.02 & 2.5820 & -0.000041\end{array}$

$0.000157 \quad 0.000165 \quad 1069.76$

Average Res $\begin{array}{ll}1.2366 & -0.000007\end{array}$

$0.1661-0.000019$
6.667
1.529

99.37 Sum chi sqr
$/ \mathrm{kg} / \mathrm{m}^{3}$
$\mathrm{~cm} / \mathrm{mol}$
$\mathrm{kg} / \mathrm{m}^{3}$

24.27

974.8729

974.849
974.849

974.849

974.849

974.849

974.849

974.849

974.849

971.7978

971.7978

971.7978

971.7978

971.7978

971.7978

971.7978

$73.938 \quad 961.9004$

959.3157

959.3157

959.3157

959.3157

958.3637

958.3637

958.3637

958.3637

958.3637

958.3637

958.3637

958.3637

958.3637

958.3637

958.3637

958.3637

958.3637

958.3637

938.9841

938.9841

938.9841

938.9841

938.9841

938.9841

938.9841

938.9841

Page 8 
molality molarity

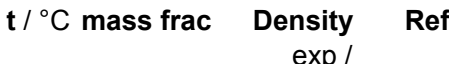

$$
\text { exp / }
$$

Ref

$\mathrm{kg} / \mathrm{m}^{3}$

$\exp /$
$\mathrm{m}^{3} / \mathrm{kg}$

\section{App vol Calc /} $\mathrm{m}^{3} / \mathrm{kg}$

Std dev Res

-4 std

Avg + 4std
Density Density App Vol Square of Inconsistent Inconsistent (t) App Vol Square of lnconsist calc/ Residual /

$\mathrm{kg} / \mathrm{m}^{3}$

$\mathrm{m}^{3} / \mathrm{k}$

$\begin{array}{ll}0.5152 & 0.000042\end{array}$

$-1.8948-0.000188$

$2.2270 \quad 0.000150$

Curve fit for the model App vol $=\left(w+c 2+c 3^{*} t{ }^{\circ} \mathrm{C}\right) /\left(\mathrm{c} 0^{*} w+c 1\right) / \mathrm{e}^{\wedge}\left(0.000001^{*}\left(\mathrm{t}^{\circ} \mathrm{C}+\mathrm{c} 4\right)^{\wedge} 2\right)$

$\begin{array}{lr}\text { c0 } 1 & -1.2 \mathrm{E}-07 \\ \text { c2 } & 4.35 \mathrm{E}-07 \\ \text { c3 } & 0.153639 \\ \text { c4 } & 0.007251 \\ \text { Min T } & 4731.543 \\ \text { Max T } & 0 \\ \text { Min w } & 125 \\ \text { Max w } & 0.000316 \\ \text { Avg dens res } & 0.24 \\ \text { Std dens res } & 0.1661 \\ \text { Avg app vol res *10^3 } & 0.515223 \\ \text { Std app vol res *10^3 } & -0.01924 \\ \text { No of points in corr } & 0.04224 \\ \text { No of inconsistent poir } & 340 \\ & 9\end{array}$

No of inconsistent poir $\quad 9$

Inconsistent data not used (Residual greater than average $+/ \mathbf{4}$ standard deviations) $\begin{array}{lllllll}0.001 & 15 & 0.0001 & 1000.4 & (47) & -0.008158 & 0.000099\end{array}$ $\begin{array}{lllllll}0.005 & 15 & 0.0007 & 1005.6 & (47) & -0.008158 & 0.000099\end{array}$

0.01001

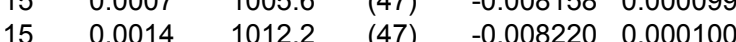

$999.23 \quad 1.1722-0.008258 \quad 1.374$ $\begin{array}{llll}999.74 & 5.8640 & -0.008258 & 34.386\end{array}$

0.02002

$\begin{array}{lllll}0.0014 & 1012.2 & (47) & -0.008220 & 0.000100 \\ 0.0028 & 1025.1 & (47) & -0.008149 & 0.000100\end{array}$

1000.37

$\begin{array}{lll}11.8346 & -0.008319 & 140.058\end{array}$

0.05006

$0.0067 \quad 1063.6$

$\begin{array}{lll}(47) & -0.008149 & 0.000100 \\ (47) & -0.008077 & 0.000102\end{array}$

1001.60

$\begin{array}{lll}11.8346 & -0.008319 & 140.058 \\ 23.4995 & -0.008250 & 552.227\end{array}$

0.06007

$0.0079 \quad 1077.1$

$\begin{array}{lll}(47) & -0.008148 & 0.000102 \\ \text { (47) } & -0.008007 & 0.000103\end{array}$

$1005.14 \quad 58.4633 \quad-0.008179 \quad 3417.954$

0.08009

$0.0103 \quad 1101.5$

$\begin{array}{llll}1006.26 & 70.8430 & -0.008250 & 5018.730\end{array}$

0.10012

0.0126

1129.1

(47) -0.00

$\begin{array}{lllll}1008.44 & 93.0591 & -0.008111 & 8659.988\end{array}$

0.14016

$15 \quad 0.0168$

1183.2

(47) $\quad-0.008254 \quad 0.000106$

$1010.50 \quad 118.5969-0.00825214065 .213$

$\begin{array}{llll}1014.36 & 168.8377 & -0.008360 & 28506.178\end{array}$

$1.3 \quad 999.0996$

6.5999 .0996

13.1999 .0996

26999.0996

64.5999 .0996

$78 \quad 999.0996$

102.4999 .0996

$130 \quad 999.0996$

184.1999 .0996 
Density of aqueous solutions of $\mathrm{NH} 3$

References (90) Perry, R. H.; Green, D. W. Perry's Chemical Engineers' Handbook; McGraw Hill: New York, 7th edition, 1997.

(106) Rashkovskaya, E. A.; Chernen'kaya, E. I. Densities of Solutions of NH4HCO3, NaHCO3, NH4Cl and Ammonia in the 20-100 Range.J. Appl. Chem. USSR 1967, 40, 301-308.

\begin{tabular}{|c|c|c|c|c|c|c|c|c|c|c|c|c|c|}
\hline \multirow[t]{37}{*}{ molality } & \multicolumn{2}{|c|}{$\mathrm{t} /{ }^{\circ} \mathrm{C}$ mass frac } & $\begin{array}{r}\text { Density } \\
\exp / \\
\mathrm{kg} / \mathrm{m}^{3}\end{array}$ & Ref & $\begin{array}{r}\text { App vol } \\
\exp / \\
\mathrm{m}^{3} / \mathrm{kg}\end{array}$ & $\begin{array}{r}\text { App vol } \\
\text { Calc / } \\
\mathrm{m}^{3} / \mathrm{kg}\end{array}$ & $\begin{array}{r}\text { Density } \\
\text { calc / } \\
\mathrm{kg} / \mathrm{m}^{3}\end{array}$ & $\begin{array}{r}\text { Density } \\
\text { Residual / } \\
\mathrm{kg} / \mathrm{m}^{3}\end{array}$ & $\begin{array}{r}\text { App Vol } \\
\text { Residual / } \\
\mathrm{m}^{3} / \mathrm{kg}\end{array}$ & $\begin{array}{l}\text { Square of } \\
\text { Density } \\
\text { Residual }\end{array}$ & $\begin{array}{l}\text { Inconsistent } \\
\text { based on } \\
\text { density }\end{array}$ & $\begin{array}{c}\text { Inconsistent } \\
\text { based on } \\
\text { apparent } \\
\text { volume }\end{array}$ & $\begin{array}{r}\text { Density of } \\
\text { water } / \mathrm{kg} / \mathrm{m}^{3}\end{array}$ \\
\hline & 0 & 0.0100 & 995.9 & (90) & 0.001396 & 0.001417 & 995.7 & 0.2141 & -0.000022 & 0.046 & & & 999.8395 \\
\hline & 0 & 0.0200 & 991.9 & (90) & 0.001400 & 0.001414 & 991.6 & 0.2682 & -0.000014 & 0.072 & & & 999.8395 \\
\hline & 0 & 0.0400 & 984.2 & (90) & 0.001397 & 0.001408 & 983.8 & 0.3949 & -0.000010 & 0.156 & & & 999.8395 \\
\hline & 0 & 0.0800 & 969.5 & (90) & 0.001391 & 0.001396 & 969.2 & 0.3270 & -0.000004 & 0.107 & & & 999.8395 \\
\hline & 0 & 0.1200 & 956.1 & (90) & 0.001381 & 0.001385 & 955.7 & 0.3694 & -0.000003 & 0.136 & & & 999.8395 \\
\hline & 0 & 0.1600 & 943.5 & (90) & 0.001373 & 0.001375 & 943.3 & 0.1931 & -0.000001 & 0.037 & & & 999.8395 \\
\hline & 0 & 0.2000 & 931.6 & (90) & 0.001366 & 0.001366 & 931.8 & -0.1629 & 0.000001 & 0.027 & & & 999.8395 \\
\hline & 0 & 0.2400 & 920.2 & (90) & 0.001361 & 0.001357 & 921.0 & -0.7842 & 0.000004 & 0.615 & & & 999.8395 \\
\hline & 0 & 0.2800 & 909.4 & (90) & 0.001355 & 0.001349 & 910.9 & -1.4760 & 0.000006 & 2.179 & & & 999.8395 \\
\hline & 0 & 0.3000 & 904.0 & (90) & 0.001354 & 0.001345 & 906.0 & -2.0481 & 0.000008 & 4.195 & & & 999.8395 \\
\hline & 5 & 0.0100 & 995.8 & (90) & 0.001418 & 0.001424 & 995.7 & 0.0623 & -0.000006 & 0.004 & & & 999.9638 \\
\hline & 5 & 0.0200 & 991.7 & (90) & 0.001417 & 0.001421 & 991.6 & 0.0942 & -0.000005 & 0.009 & & & 999.9638 \\
\hline & 5 & 0.0400 & 983.7 & (90) & 0.001413 & 0.001416 & 983.6 & 0.0903 & -0.000002 & 0.008 & & & 999.9638 \\
\hline & 5 & 0.0800 & 968.6 & (90) & 0.001405 & 0.001405 & 968.6 & 0.0101 & 0.000000 & 0.000 & & & 999.9638 \\
\hline & 5 & 0.1200 & 954.8 & (90) & 0.001394 & 0.001395 & 954.7 & 0.0928 & -0.000001 & 0.009 & & & 999.9638 \\
\hline & 5 & 0.1600 & 942.0 & (90) & 0.001385 & 0.001386 & 941.8 & 0.1973 & -0.000001 & 0.039 & & & 999.9638 \\
\hline & 5 & 0.2000 & 929.6 & (90) & 0.001379 & 0.001378 & 929.7 & -0.1465 & 0.000001 & 0.021 & & & 999.9638 \\
\hline & 5 & 0.2400 & 917.9 & (90) & 0.001373 & 0.001370 & 918.4 & -0.5315 & 0.000003 & 0.282 & & & 999.9638 \\
\hline & 5 & 0.2800 & 906.7 & (90) & 0.001367 & 0.001363 & 907.8 & -1.0687 & 0.000005 & 1.142 & & & 999.9638 \\
\hline & 5 & 0.3000 & 901.2 & (90) & 0.001365 & 0.001359 & 902.7 & -1.4581 & 0.000006 & 2.126 & & & 999.9638 \\
\hline & 10 & 0.0100 & 995.8 & (90) & 0.001392 & 0.001431 & 995.4 & 0.3827 & -0.000039 & 0.146 & & & 999.6996 \\
\hline & 10 & 0.0200 & 991.7 & (90) & 0.001404 & 0.001428 & 991.2 & 0.4765 & -0.000024 & 0.227 & & & 999.6996 \\
\hline & 10 & 0.0400 & 983.7 & (90) & 0.001407 & 0.001423 & 983.1 & 0.6129 & -0.000016 & 0.376 & & & 999.6996 \\
\hline & 10 & 0.0800 & 968.6 & (90) & 0.001402 & 0.001413 & 967.7 & 0.8686 & -0.000012 & 0.755 & & & 999.6996 \\
\hline & 10 & 0.1200 & 954.8 & (90) & 0.001392 & 0.001405 & 953.5 & 1.3473 & -0.000012 & 1.815 & & & 999.6996 \\
\hline & 10 & 0.1600 & 942.0 & (90) & 0.001383 & 0.001397 & 940.1 & 1.8944 & -0.000013 & 3.589 & & & 999.6996 \\
\hline & 10 & 0.2000 & 929.6 & (90) & 0.001377 & 0.001389 & 927.6 & 2.0290 & -0.000012 & 4.117 & & & 999.6996 \\
\hline & 10 & 0.2400 & 917.9 & (90) & 0.001372 & 0.001382 & 915.8 & 2.1499 & -0.000011 & 4.622 & & & 999.6996 \\
\hline & 10 & 0.2800 & 906.7 & (90) & 0.001367 & 0.001376 & 904.6 & 2.1394 & -0.000009 & 4.577 & & & 999.6996 \\
\hline & 10 & 0.3000 & 901.2 & (90) & 0.001365 & 0.001373 & 899.2 & 2.0195 & -0.000008 & 4.078 & & & 999.6996 \\
\hline & 20 & 0.0100 & 993.9 & (90) & 0.001436 & 0.001440 & 993.9 & 0.0422 & -0.000004 & 0.002 & & & 998.2041 \\
\hline & 20 & 0.0200 & 989.5 & $(90)$ & 0.001442 & 0.001438 & 989.6 & -0.0860 & 0.000004 & 0.007 & & & 998.2041 \\
\hline & 20 & 0.0200 & 989.4 & (106) & 0.001448 & 0.001438 & 989.6 & -0.1860 & 0.000009 & 0.035 & & & 998.2041 \\
\hline & 20 & 0.0400 & 981.1 & $(90)$ & 0.001438 & 0.001434 & 981.3 & -0.1553 & 0.000004 & 0.024 & & & 998.2041 \\
\hline & 20 & 0.0400 & 981.1 & (106) & 0.001438 & 0.001434 & 981.3 & -0.1553 & 0.000004 & 0.024 & & & 998.2041 \\
\hline & 20 & 0.0600 & 973.0 & (106) & 0.001434 & 0.001431 & 973.2 & -0.1921 & 0.000003 & 0.037 & & & 998.2041 \\
\hline
\end{tabular}


molality molarity

\begin{tabular}{|c|c|c|c|c|c|}
\hline $1{ }^{\circ} \mathrm{C}$ & ISs frac & $\begin{array}{r}\text { Density } \\
\exp / \\
\mathrm{kg} / \mathrm{m}^{3}\end{array}$ & Ref & $\begin{array}{r}\text { App vol } \\
\exp / \\
\mathrm{m}^{3} / \mathrm{kg}\end{array}$ & $\begin{array}{r}\text { App vol } \\
\text { Calc / } \\
\mathrm{m}^{3} / \mathrm{kg}\end{array}$ \\
\hline 20 & 0.0800 & 965.1 & (90) & 0.001431 & 0.001428 \\
\hline 20 & 0.0800 & 965.1 & (106) & 0.001431 & 0.001428 \\
\hline 20 & 0.1000 & 957.5 & (106) & 0.001428 & 0.001424 \\
\hline 20 & 0.1200 & 950.1 & (90) & 0.001424 & 0.001421 \\
\hline 20 & 0.1200 & 950.1 & (106) & 0.001424 & 0.001421 \\
\hline 20 & 0.1400 & 943.0 & (106) & 0.001421 & 0.001418 \\
\hline 20 & 0.1600 & 936.2 & (90) & 0.001416 & 0.001416 \\
\hline 20 & 0.1600 & 936.7 & (106) & 0.001413 & 0.001416 \\
\hline 20 & 0.2000 & 922.9 & (90) & 0.001411 & 0.001410 \\
\hline 20 & 0.2400 & 910.1 & (90) & 0.001406 & 0.001406 \\
\hline 20 & 0.2800 & 898.0 & (90) & 0.001401 & 0.001401 \\
\hline 20 & 0.3000 & 892.0 & (90) & 0.001399 & 0.001399 \\
\hline 25 & 0.0200 & 988.2 & (106) & 0.001452 & 0.001441 \\
\hline 25 & 0.0400 & 979.9 & (106) & 0.001442 & 0.001439 \\
\hline 25 & 0.0600 & 971.7 & (106) & 0.001439 & 0.001436 \\
\hline 25 & 0.0800 & 963.6 & (106) & 0.001438 & 0.001433 \\
\hline 25 & 0.1000 & 955.9 & (106) & 0.001435 & 0.001431 \\
\hline 25 & 0.1200 & 948.2 & (106) & 0.001434 & 0.001428 \\
\hline 25 & 0.1400 & 941.0 & (106) & 0.001430 & 0.001426 \\
\hline 25 & 0.1600 & 934.9 & (106) & 0.001420 & 0.001424 \\
\hline 30 & 0.0200 & 986.8 & (106) & 0.001455 & 0.001444 \\
\hline 30 & 0.0400 & 978.5 & (106) & 0.001444 & 0.001442 \\
\hline 30 & 0.0600 & 970.4 & (106) & 0.001440 & 0.001440 \\
\hline 30 & 0.0800 & 962.1 & (106) & 0.001442 & 0.001438 \\
\hline 30 & 0.1000 & 954.2 & (106) & 0.001441 & 0.001436 \\
\hline 30 & 0.1200 & 946.3 & (106) & 0.001441 & 0.001435 \\
\hline 30 & 0.1400 & 939.1 & (106) & 0.001436 & 0.001433 \\
\hline 30 & 0.1600 & 933.0 & (106) & 0.001426 & 0.001432 \\
\hline 35 & 0.0200 & 985.2 & (106) & 0.001457 & 0.001445 \\
\hline 35 & 0.0400 & 977.0 & (106) & 0.001444 & 0.001444 \\
\hline 35 & 0.0600 & 968.8 & (106) & 0.001443 & 0.001443 \\
\hline 35 & 0.0800 & 960.5 & (106) & 0.001445 & 0.001442 \\
\hline 35 & 0.1000 & 952.4 & (106) & 0.001446 & 0.001441 \\
\hline 35 & 0.1200 & 944.4 & (106) & 0.001447 & 0.001440 \\
\hline 35 & 0.1400 & 937.0 & (106) & 0.001443 & 0.001439 \\
\hline 35 & 0.1600 & 931.0 & (106) & 0.001432 & 0.001438 \\
\hline 40 & 0.0200 & 983.6 & (106) & 0.001449 & 0.001444 \\
\hline 40 & 0.0400 & 975.4 & (106) & 0.001442 & 0.001444 \\
\hline 40 & 0.0600 & 967.1 & (106) & 0.001444 & 0.001444 \\
\hline 40 & 0.0800 & 958.8 & (106) & 0.001447 & 0.001444 \\
\hline
\end{tabular}
calc / Residual /$$
\mathrm{kg} / \mathrm{m}^{3} \quad \mathrm{~kg} / \mathrm{m}^{3}
$$$$
\text { App Vol }
$$

Vol Square of Inco $\mathrm{m}^{3} / \mathrm{kg}$

$965.4-0.2783$

$965.4-0.2783$

$957.8-0.2974$

$950.4-0.3348$

$950.4 \quad-0.3348$

$943.3-0.2770$

$936.3-0.1118$

$936.3 \quad 0.3882$

$922.9-0.0152$

$910.2-0.0667$

$898.0 \quad 0.0001$

$892.1-0.1168$

$988.4-0.2028$

$980.0-0.1178$

$971.9-0.1734$

$964.0-0.3546$

$956.2-0.3480$

$948.7-0.5412$

$941.4-0.4230$

$934.3 \quad 0.6169$

$987.0 \quad-0.2128$

$978.6 \quad-0.1021$

$970.4-0.0026$

$962.4-0.3028$

$954.6 \quad-0.3921$

$947.0-0.6609$

$939.5-0.4004$

$932.2 \quad 0.7976$

$985.4-0.2370$

$977.0 \quad-0.0297$

$968.8-0.0015$

$960.7-0.2445$

$952.9-0.4515$

$945.1-0.7159$

$937.5-0.5312$

$930.1 \quad 0.9082$

$983.7 \quad-0.0930$

$975.3 \quad 0.0814$

$967.1 \quad 0.0116$

$959.0 \quad-0.1986$

0.000004

0.000004

0.000003

0.000003

0.000003

0.000002

0.000001

$-0.000003$

0.000000

0.000000

0.000000

0.000010

0.000003

0.000003

0.000005

0.000004

0.000005

0.000003

$-0.000004$

0.000011

0.000003

0.000000

0.000004

0.000004

0.000006

0.000003

$-0.000006$

0.000012

0.000001

0.000000

0.000003

0.000005

0.000007

0.000004

$-0.000007$

0.000005

$-0.000002$

0.000000

0.000003

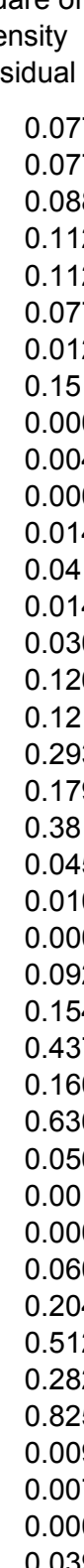

\begin{tabular}{l} 
sity \\
0.077 \\
0.077 \\
0.088 \\
0.112 \\
0.112 \\
0.077 \\
0.012 \\
0.151 \\
0.000 \\
0.004 \\
0.000 \\
0.014 \\
0.041 \\
0.014 \\
0.030 \\
0.126 \\
0.121 \\
0.293 \\
0.179 \\
0.381 \\
0.045 \\
0.010 \\
0.000 \\
0.092 \\
0.154 \\
0.437 \\
0.160 \\
0.636 \\
0.056 \\
0.001 \\
0.000 \\
0.060 \\
0.204 \\
0.512 \\
0.282 \\
0.825 \\
0.009 \\
0.007 \\
0.000 \\
0.039 \\
\hline
\end{tabular}

Density of ased on based on water $/ \mathrm{kg} / \mathrm{m}^{3}$ density apparent

998.2041 998.2041 998.2041 998.2041 998.2041 998.2041 998.2041 998.2041 998.2041 998.2041 998.2041

998.2041 997.0449 997.0449 997.0449 997.0449 997.0449 997.0449 997.0449 997.0449 995.6473 995.6473 995.6473 995.6473 995.6473 995.6473 995.6473 995.6473 994.0319 994.0319 994.0319 994.0319 994.0319 994.0319 994.0319 994.0319 992.2158 992.2158 992.2158 992.2158 
molality molarity

$\begin{array}{crrrrr}\mathbf{t} /{ }^{\circ} \mathrm{C} \text { mass frac } & \begin{array}{r}\text { Density } \\ \text { exp / }\end{array} & \text { Ref } & \begin{array}{r}\text { App vol } \\ \text { exp / }\end{array} & \begin{array}{r}\text { App vol } \\ \text { Calc } /\end{array} \\ & & & \mathrm{m}^{3} / \mathrm{kg} & \mathrm{m} / \mathrm{kg} \\ & & \mathrm{kg} / \mathrm{m}^{3} & & & \\ 40 & 0.1000 & 950.5 & (106) & 0.001450 & 0.001444 \\ 40 & 0.1400 & 935.0 & (106) & 0.001448 & 0.001444 \\ 40 & 0.1600 & 928.8 & (106) & 0.001438 & 0.001444 \\ 45 & 0.0200 & 981.8 & (106) & 0.001443 & 0.001443 \\ 45 & 0.0400 & 973.6 & (106) & 0.001441 & 0.001444 \\ 45 & 0.0600 & 965.3 & (106) & 0.001444 & 0.001445 \\ 45 & 0.0800 & 957.0 & (106) & 0.001448 & 0.001446 \\ 45 & 0.1000 & 948.6 & (106) & 0.001453 & 0.001446 \\ 45 & 0.1200 & 940.5 & (106) & 0.001455 & 0.001447 \\ 45 & 0.1400 & 932.9 & (106) & 0.001453 & 0.001448 \\ 45 & 0.1600 & 926.8 & (106) & 0.001442 & 0.001449 \\ 50 & 0.0200 & 979.9 & (106) & 0.001432 & 0.001440 \\ 50 & 0.0400 & 971.7 & (106) & 0.001437 & 0.001442 \\ 50 & 0.0600 & 963.4 & (106) & 0.001443 & 0.001444 \\ 50 & 0.0800 & 955.1 & (106) & 0.001448 & 0.001446 \\ 50 & 0.1000 & 946.7 & (106) & 0.001454 & 0.001447 \\ 50 & 0.1200 & 938.6 & (106) & 0.001456 & 0.001449 \\ 50 & 0.1400 & 930.9 & (106) & 0.001456 & 0.001451 \\ 50 & 0.1600 & 924.6 & (106) & 0.001446 & 0.001452 \\ 55 & 0.0200 & 977.9 & (106) & 0.001419 & 0.001435 \\ 55 & 0.0400 & 969.7 & (106) & 0.001433 & 0.001438 \\ 55 & 0.0600 & 961.5 & (106) & 0.001440 & 0.001441 \\ 55 & 0.0800 & 953.1 & (106) & 0.001448 & 0.001444 \\ 55 & 0.1000 & 944.8 & (106) & 0.001454 & 0.001447 \\ 55 & 0.1200 & 936.7 & (106) & 0.001457 & 0.001450 \\ 55 & 0.1400 & 928.9 & (106) & 0.001458 & 0.001452 \\ 55 & 0.1600 & 922.6 & (106) & 0.001448 & 0.001455 \\ 60 & 0.0200 & 975.8 & (106) & 0.001403 & 0.001429 \\ 60 & 0.0400 & 967.7 & (106) & 0.001424 & 0.001433 \\ 60 & 0.0600 & 959.5 & (106) & 0.001436 & 0.001437 \\ 60 & 0.0800 & 951.2 & (106) & 0.001445 & 0.001442 \\ 60 & 0.1000 & 943.0 & (106) & 0.001451 & 0.001445 \\ 60 & 0.1200 & 934.8 & (106) & 0.001456 & 0.001449 \\ 60 & 0.1400 & 926.8 & (106) & 0.001459 & 0.001453 \\ 60 & 0.1600 & 920.6 & (106) & 0.001449 & 0.001456 \\ 65 & 0.0200 & 973.5 & (106) & 0.001389 & 0.001421 \\ 65 & 0.0400 & 965.5 & (106) & 0.001417 & 0.001427 \\ 65 & 0.0600 & 957.4 & (106) & 0.001431 & 0.001432 \\ 65 & 0.0800 & 949.3 & (106) & 0.001440 & 0.001437 \\ 65 & 0.1000 & 941.2 & (106) & 0.001446 & 0.001442 \\ & & & & & \end{array}$

Den

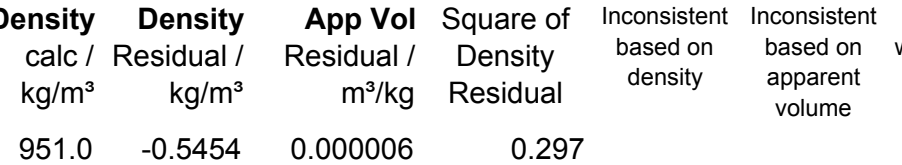
$\begin{array}{lll}951.0 & -0.5454 & 0.000006 \\ 935.5 & -0.5347 & 0.000004\end{array}$ $\begin{array}{lll}928.0 & 0.8295 & -0.000006\end{array}$ $\begin{array}{lll}981.8 & 0.0044 & 0.000000\end{array}$ $\begin{array}{lll}973.5 & 0.1159 & -0.000003\end{array}$ $\begin{array}{lll}965.3 & 0.0209 & 0.000000\end{array}$ $\begin{array}{lll}957.2 & -0.1811 & 0.000002\end{array}$ $\begin{array}{lll}949.2 & -0.5901 & 0.000007\end{array}$

$\begin{array}{lll}941.3 & -0.8058 & 0.000008\end{array}$ $\begin{array}{lll}933.5 & -0.6279 & 0.000005\end{array}$ $\begin{array}{lll}925.9 & 0.9444 & -0.000007\end{array}$ $\begin{array}{lll}979.8 & 0.1425 & -0.000007\end{array}$ $\begin{array}{lll}971.5 & 0.1606 & -0.000004\end{array}$ $\begin{array}{lll}963.4 & 0.0124 & 0.000000\end{array}$ $\begin{array}{lll}955.3 & -0.2066 & 0.000003\end{array}$ $\begin{array}{lll}947.3 & -0.6006 & 0.000007\end{array}$ $\begin{array}{lll}939.4 & -0.7728 & 0.000007\end{array}$ $\begin{array}{lll}931.5 & -0.6260 & 0.000005\end{array}$ $\begin{array}{lll}923.8 & 0.8374 & -0.000006\end{array}$ $\begin{array}{lll}977.6 & 0.3103 & -0.000016\end{array}$ $\begin{array}{lll}969.5 & 0.2037 & -0.000005\end{array}$ $\begin{array}{lll}961.4 & 0.0741 & -0.000001\end{array}$ $\begin{array}{lll}953.4 & -0.2877 & 0.000004\end{array}$ $\begin{array}{lll}945.4 & -0.5900 & 0.000007\end{array}$ $\begin{array}{lll}937.4 & -0.7397 & 0.000007\end{array}$ $\begin{array}{lll}929.5 & -0.6433 & 0.000005\end{array}$ $\begin{array}{lll}921.7 & 0.8940 & -0.000007\end{array}$ $\begin{array}{lll}975.3 & 0.4984 & -0.000026\end{array}$ $\begin{array}{llll}967.4 & 0.3350 & -0.000009\end{array}$ $\begin{array}{lll}959.4 & 0.0949 & -0.000002\end{array}$ $\begin{array}{lll}951.4 & -0.2360 & 0.000003\end{array}$ $\begin{array}{lll}943.5 & -0.4704 & 0.000005\end{array}$ $\begin{array}{lll}935.5 & -0.7193 & 0.000007\end{array}$ $\begin{array}{lll}927.6 & -0.7926 & 0.000007\end{array}$ $\begin{array}{lll}919.7 & 0.9012 & -0.000007\end{array}$ $\begin{array}{lll}972.9 & 0.5984 & -0.000032\end{array}$ $\begin{array}{lll}965.2 & 0.3455 & -0.000009\end{array}$ $\begin{array}{lll}957.3 & 0.0651 & -0.000001\end{array}$ $\begin{array}{lll}949.5 & -0.1621 & 0.000002\end{array}$ $\begin{array}{lll}941.6 & -0.3531 & 0.000004\end{array}$
0.286
0.688
0.000
0.013
0.000
0.033
0.348
0.649
0.394
0.892
0.020
0.026
0.000
0.043
0.361
0.597
0.392
0.701
0.096
0.041
0.005
0.083
0.348
0.547
0.414
0.799
0.248
0.112
0.009
0.056
0.221
0.517
0.628
0.812
0.358
0.119
0.004
0.026
0.125
Density of 992.2158 992.2158 992.2158 990.2132 990.2132 990.2132 990.2132 990.2132 990.2132 990.2132 990.2132 988.0363 988.0363 988.0363 988.0363 988.0363 988.0363 988.0363 988.0363 985.6952 985.6952 985.6952 985.6952 985.6952 985.6952 985.6952 985.6952 983.1989 983.1989 983.1989 983.1989 983.1989 983.1989 983.1989 983.1989 980.5548 980.5548 980.5548 980.5548 980.5548 
molality molarity

$\begin{array}{crrrrr}\text { t } /{ }^{\circ} \mathrm{C} \text { mass frac } & \begin{array}{r}\text { Density } \\ \text { exp / }\end{array} & \text { Ref } & \begin{array}{r}\text { App vol } \\ \text { exp / }\end{array} & \begin{array}{r}\text { App vol } \\ \text { Calc } /\end{array} \\ & & & \mathrm{m}^{3} / \mathrm{kg} & \mathrm{m} / \mathrm{kg} \\ & & \mathrm{kg} / \mathrm{m}^{3} & & & \\ 65 & 0.1200 & 933.0 & (106) & 0.001453 & 0.001447 \\ 65 & 0.1400 & 924.8 & (106) & 0.001459 & 0.001452 \\ 65 & 0.1600 & 918.8 & (106) & 0.001448 & 0.001456 \\ 70 & 0.0200 & 971.2 & (106) & 0.001369 & 0.001411 \\ 70 & 0.0400 & 963.3 & (106) & 0.001407 & 0.001418 \\ 70 & 0.0600 & 955.3 & (106) & 0.001424 & 0.001425 \\ 70 & 0.0800 & 947.4 & (106) & 0.001433 & 0.001431 \\ 70 & 0.1000 & 939.4 & (106) & 0.001440 & 0.001438 \\ 70 & 0.1200 & 931.2 & (106) & 0.001449 & 0.001444 \\ 70 & 0.1400 & 923.0 & (106) & 0.001456 & 0.001449 \\ 70 & 0.1600 & 917.1 & (106) & 0.001446 & 0.001455 \\ 75 & 0.0200 & 968.8 & (106) & 0.001346 & 0.001400 \\ 75 & 0.0400 & 961.0 & (106) & 0.001395 & 0.001408 \\ 75 & 0.0600 & 953.1 & (106) & 0.001416 & 0.001416 \\ 75 & 0.0800 & 945.5 & (106) & 0.001424 & 0.001424 \\ 75 & 0.1000 & 937.6 & (106) & 0.001433 & 0.001431 \\ 75 & 0.1200 & 929.4 & (106) & 0.001444 & 0.001439 \\ 75 & 0.1400 & 921.3 & (106) & 0.001452 & 0.001445 \\ 75 & 0.1600 & 915.4 & (106) & 0.001442 & 0.001452 \\ 80 & 0.0200 & 966.4 & (106) & 0.001316 & 0.001386 \\ 80 & 0.0400 & 958.8 & (106) & 0.001378 & 0.001396 \\ 80 & 0.0600 & 951.0 & (106) & 0.001404 & 0.001406 \\ 80 & 0.0800 & 943.7 & (106) & 0.001412 & 0.001415 \\ 80 & 0.1000 & 935.8 & (106) & 0.001425 & 0.001424 \\ 80 & 0.1200 & 927.6 & (106) & 0.001438 & 0.001432 \\ 80 & 0.1400 & 919.5 & (106) & 0.001447 & 0.001440 \\ 80 & 0.1600 & 913.8 & (106) & 0.001437 & 0.001448 \\ 85 & 0.0200 & 964.0 & (106) & 0.001280 & 0.001370 \\ 85 & 0.0400 & 956.5 & (106) & 0.001359 & 0.001382 \\ 85 & 0.0600 & 948.8 & (106) & 0.001392 & 0.001393 \\ 85 & 0.1000 & 934.1 & (106) & 0.001414 & 0.001414 \\ 85 & 0.1200 & 925.9 & (106) & 0.001429 & 0.001424 \\ 85 & 0.1400 & 917.8 & (106) & 0.001441 & 0.001433 \\ 85 & 0.1600 & 912.2 & (106) & 0.001431 & 0.001442 \\ 90 & 0.0200 & 961.5 & (106) & 0.001242 & 0.001353 \\ 90 & 0.0400 & 954.2 & (106) & 0.001338 & 0.001366 \\ 90 & 0.0600 & 946.5 & (106) & 0.001379 & 0.001379 \\ 90 & 0.0800 & 940.0 & (106) & 0.001385 & 0.001391 \\ 90 & 0.1000 & 932.4 & (106) & 0.001402 & 0.001402 \\ 90 & 0.1200 & 924.2 & (106) & 0.001420 & 0.001413\end{array}$

Den Density $\begin{array}{rr}\text { calc / } & \text { Residual / Rg/m } \\ \mathrm{kg} / \mathrm{m}^{3} & \text { R }\end{array}$

App Vol Square of

$933.6-0.6232$

$925.7-0.8860$

$\begin{array}{lll}917.8 & 1.0462 & -0.000008\end{array}$

$\begin{array}{lll}970.4 & 0.8028 & -0.000043\end{array}$

$\begin{array}{lll}962.9 & 0.4269 & -0.000012\end{array}$

$\begin{array}{lll}955.2 & 0.0756 & -0.000001\end{array}$

$\begin{array}{lll}947.5 & -0.0755 & 0.000001\end{array}$

$\begin{array}{lll}939.6 & -0.2483 & 0.000003\end{array}$

$\begin{array}{lll}931.8 & -0.5623 & 0.000005\end{array}$

$\begin{array}{lll}923.8 & -0.8352 & 0.000007\end{array}$

$\begin{array}{lll}915.9 & 1.2173 & -0.000009\end{array}$

$\begin{array}{lll}967.8 & 1.0053 & -0.000054\end{array}$

$\begin{array}{lll}960.5 & 0.4720 & -0.000013\end{array}$

$\begin{array}{lll}953.1 & 0.0183 & 0.000000\end{array}$

$\begin{array}{lll}945.5 & 0.0146 & 0.000000\end{array}$

$\begin{array}{lll}937.8 & -0.1658 & 0.000002\end{array}$

$\begin{array}{lll}929.9 & -0.5471 & 0.000005\end{array}$

$922.1-0.7510 \quad 0.000006$

$\begin{array}{lll}914.1 & 1.3029 & -0.000010\end{array}$

$\begin{array}{lll}965.1 & 1.3000 & -0.000070\end{array}$

$\begin{array}{lll}958.1 & 0.6740 & -0.000018\end{array}$

$\begin{array}{llll}950.9 & 0.0856 & -0.000002\end{array}$

$\begin{array}{llll}943.5 & 0.2000 & -0.000003\end{array}$

$\begin{array}{lll}935.9 & -0.1148 & 0.000001\end{array}$

$\begin{array}{lll}928.2 & -0.5874 & 0.000006\end{array}$

$\begin{array}{lll}920.3 & -0.8440 & 0.000007\end{array}$

$\begin{array}{lll}912.4 & 1.3919 & -0.000010\end{array}$

$\begin{array}{lll}962.3 & 1.6820 & -0.000091\end{array}$

$\begin{array}{lll}955.7 & 0.8270 & -0.000023\end{array}$

$\begin{array}{lll}948.7 & 0.0708 & -0.000001\end{array}$

$\begin{array}{lll}934.1 & -0.0039 & 0.000000\end{array}$

$\begin{array}{lll}926.5 & -0.5928 & 0.000006\end{array}$

$\begin{array}{lll}918.7 & -0.9244 & 0.000008\end{array}$

$\begin{array}{lll}910.8 & 1.3735 & -0.000010\end{array}$

$\begin{array}{lll}959.5 & 2.0466 & -0.000111\end{array}$

$\begin{array}{lll}953.2 & 1.0257 & -0.000028\end{array}$

$\begin{array}{lll}946.5 & -0.0328 & 0.000001\end{array}$

$\begin{array}{lll}939.6 & 0.4255 & -0.000006\end{array}$

$\begin{array}{lll}932.3 & 0.0584 & -0.000001\end{array}$

$\begin{array}{lll}924.9 & -0.6724 & 0.000007\end{array}$

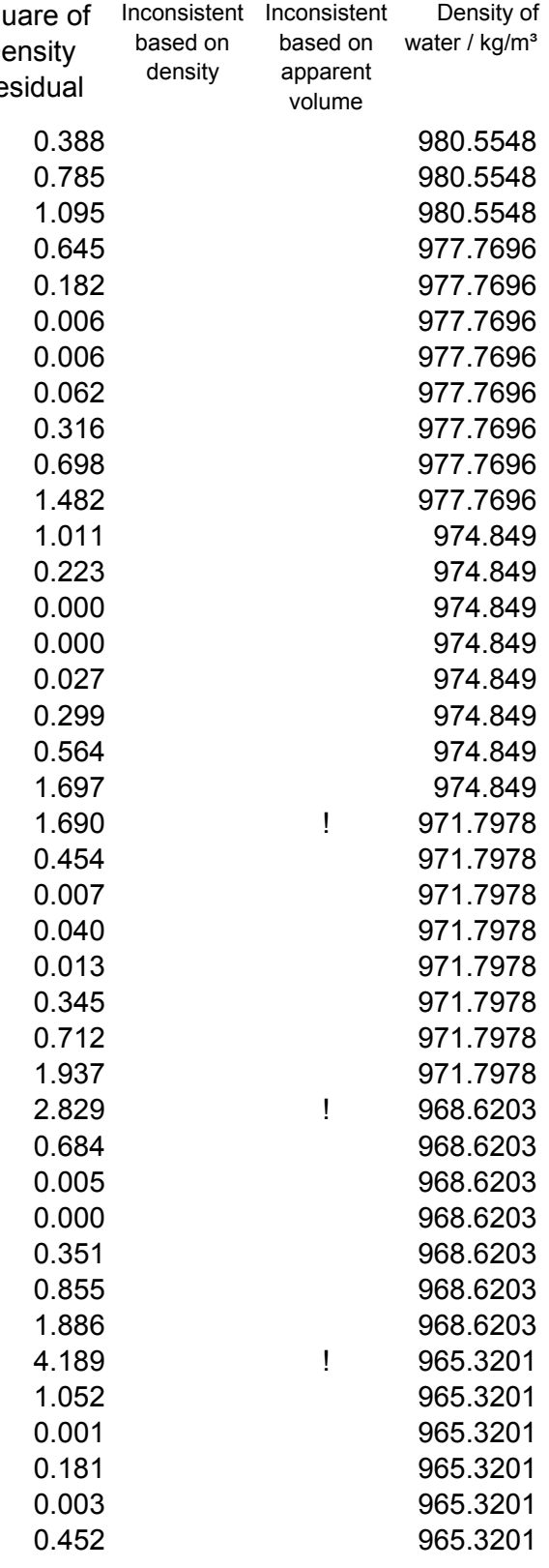


molality molarity

$\begin{array}{rrrr}\mathbf{t} /{ }^{\circ} \mathrm{C} \text { mass frac } & \begin{array}{r}\text { Density } \\ \text { exp } / \\ \mathrm{kg} / \mathrm{m}^{3}\end{array} & \text { Ref } \\ & & 916.5 & (106) \\ 90 & 0.1400 & 910.5 & (106) \\ 90 & 0.1600 & 91.8 & (106) \\ 95 & 0.0400 & 951.3 \\ 95 & 0.0600 & 944.3 & (106) \\ 95 & 0.0800 & 938.3 & (106) \\ 95 & 0.1000 & 930.8 & (106) \\ 95 & 0.1200 & 922.5 & (106) \\ 95 & 0.1400 & 914.5 & (106) \\ 95 & 0.1600 & 908.9 & (106) \\ 100 & 0.0400 & 949.5 & (106) \\ 100 & 0.0600 & 942.1 & (106) \\ 100 & 0.0800 & 936.6 & (106) \\ 100 & 0.1000 & 929.3 & (106) \\ 100 & 0.1200 & 920.9 & (106) \\ 100 & 0.1400 & 912.9 & (106) \\ 100 & 0.1600 & 907.3 & (106)\end{array}$

$\begin{array}{rr}\begin{array}{r}\text { App vol } \\ \text { exp / } \\ \mathrm{m}^{3} / \mathrm{kg}\end{array} & \begin{array}{r}\text { App vol } \\ \text { Calc } / \\ \mathrm{m}^{3} / \mathrm{kg}\end{array} \\ 0.001430 & 0.001424 \\ 0.001426 & 0.001434 \\ 0.001315 & 0.001348 \\ 0.001363 & 0.001362 \\ 0.001366 & 0.001376 \\ 0.001387 & 0.001389 \\ 0.001410 & 0.001401 \\ 0.001425 & 0.001414 \\ 0.001418 & 0.001425 \\ 0.001287 & 0.001327 \\ 0.001344 & 0.001343 \\ 0.001347 & 0.001359 \\ 0.001370 & 0.001373 \\ 0.001397 & 0.001387 \\ 0.001415 & 0.001401 \\ 0.001410 & 0.001414\end{array}$

Density Density

$\begin{array}{rr}\text { calc / } & \text { Residual / } \\ \mathrm{kg} / \mathrm{m}^{3} & \mathrm{~kg} / \mathrm{m}^{3}\end{array}$ $\mathrm{kg} / \mathrm{m}^{3}$

$\begin{array}{lr}917.2 & -0.7023 \\ 909.4 & 1.1369\end{array}$

$\begin{array}{ll}909.4 & 1.1369 \\ 950.6 & 1.1650\end{array}$

$944.3-0.0309$

$937.6 \quad 0.6513$

$930.6 \quad 0.1642$

$923.3-0.8354$

$915.8 \quad-1.2874$

$908.0 \quad 0.8716$

$948.1 \quad 1.4405$

$942.1-0.0292$

$935.8 \quad 0.8434$

$929.0 \quad 0.3058$

$921.9-0.9903$

$914.5 \quad-1.5896$

verage Res

Std dev Res

Avg - 4stc

Avg + 4std

$0.4670 \quad-0.000004$

$0.0543 \quad-0.000004$

$0.7350 \quad 0.000016$

$-2.8857-0.000067$

$2.9943 \quad 0.000059$

$\mathrm{m}^{3} / \mathrm{kg}$ Residual

$\mathrm{m}^{3} / \mathrm{kg}$

$-0.000009$

$-0.000032$

0.000009

$-0.000002$

0.000011

0.000007

0.000040

0.000001

$-0.000012$

000004

0.000059

\begin{tabular}{|c|c|c|}
\hline sidual & density & $\begin{array}{l}\text { apparent } \\
\text { volume }\end{array}$ \\
\hline 0.493 & & \\
\hline 1.293 & & \\
\hline 1.357 & & \\
\hline
\end{tabular}

965.3201

965.3201

961.9004

961.9004

961.9004

961.9004

961.9004

961.9004

961.9004

958.3637

958.3637

958.3637

958.3637

958.3637

958.3637

958.3637

\section{Curve fit for the model App vol $=\left(w+c 2+c 3^{*} t{ }^{\circ} \mathrm{C}\right) /\left(c 0^{*} w+c 1\right) / e^{\wedge}\left(0.000001^{*}\left(t{ }^{\circ} \mathrm{C}+c 4\right)^{\wedge} 2\right)$}

$\begin{array}{lr}\text { c0 } & 0.126926 \\ \text { c1 } 2 & 0.104704 \\ \text { c3 } & 1.030214 \\ \text { c4 } & -0.00508 \\ \text { Min T } & -2973.708 \\ \text { Max T } & 0 \\ \text { Min w } & 100 \\ \text { Max w } & 0.01 \\ \text { Avg dens res } & 0.3 \\ \text { Std dens res } & 0.054308 \\ \text { Avg app vol } & 0.734992\end{array}$

Avg app vol res *10^3 -0.003633

Std app vol res *10^3 0.015776

No of points in corr

No of inconsistent poir

172
4

Inconsistent data not used (Residual greater than average +/- 4 standard deviations)
40
0.1200
$947.4(106)$
$0.001405 \quad 0.001444$ 


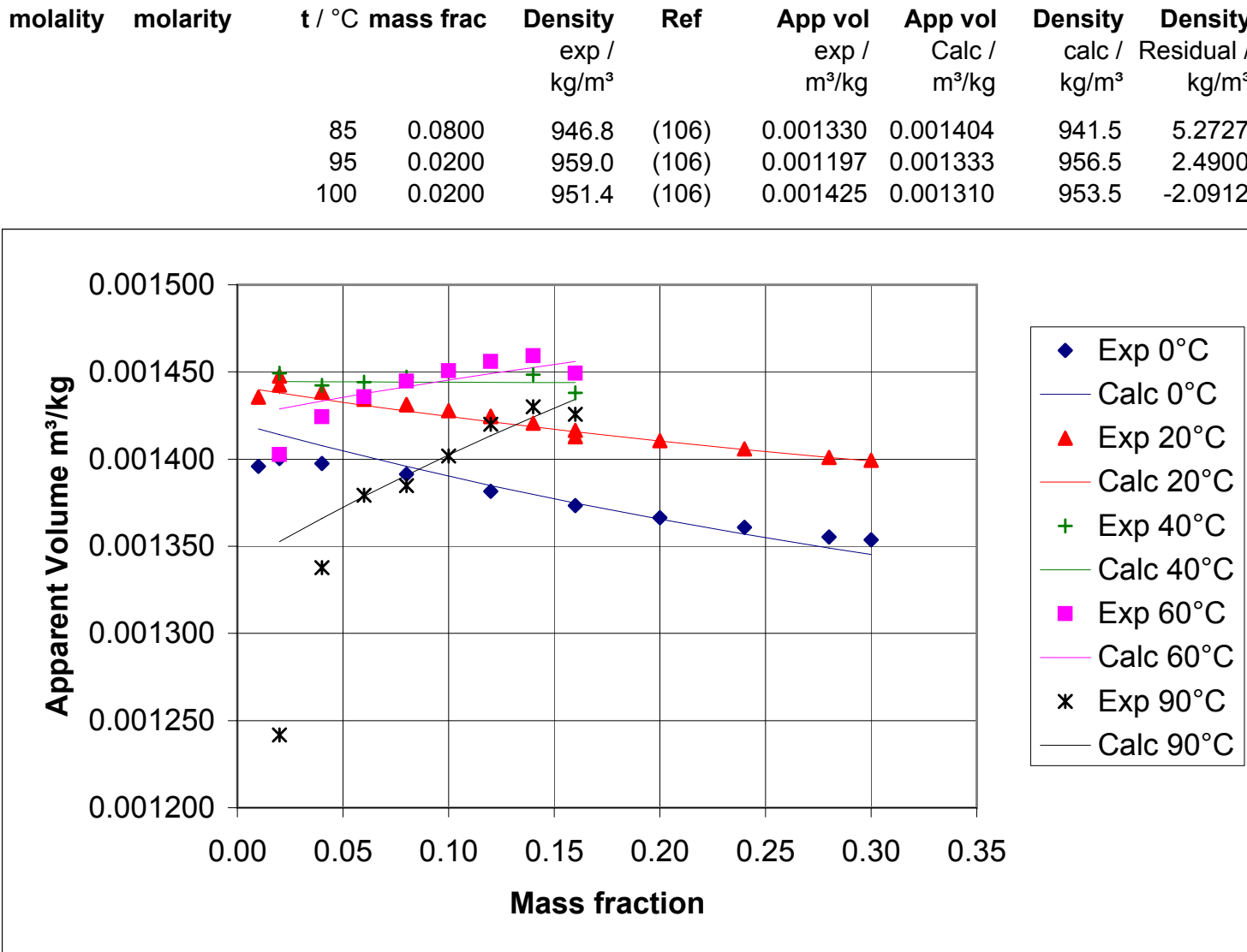


Density of aqueous solutions of $\mathrm{NH} 4 \mathrm{Cl}$

References $\quad$ (40) Herz, W. Internal Friction of Salt Solutions (C. A. Trans. from German). Z. Anorg. Chemie 1914, 89, $393-396$.

(45) Isono, T. Densities, Viscosities, and Electrolytic Conductivity of Concentrated Aqueous Electrolyte Solutions at Several Temperatures. Alkaline-Earth Chlorides, $\mathrm{LaCl}_{3}, \mathrm{Na}_{2} \mathrm{SO}_{4}, \mathrm{NaNO}_{3}, \mathrm{NaBr}, \mathrm{KNO}_{3}, \mathrm{KBr}$, and $\mathrm{Cd}\left(\mathrm{NO}_{3}\right)_{2}$. J. Chem. Eng. Data 1984, 29 , 45-52.

(47) Kaminsky, M. Experimental Investigations of the Concentration and Temperature Dependence of the Viscosity of Aqueous Solutions of Strong Electrolytes. I. Potassium lodide, Ammonium Chloride, and Sodium Sulphate Solutions. Z. Physik. Chem. N. F. 1955, 5, $154-191$.

(73) Millero, F. J.; Ward, G. K.; Chetirkin, P. V. Relative Sound Velocities of Sea Salts at $25^{\circ}$ C. J. Acoust. Soc. Am. 1977, $61,1492-1498$.

(74) Motin, M. A., Temperature and Concentration Dependence of Apparent Molar Volumes and Viscosities of $\mathrm{NaCl}, \mathrm{NH} 4 \mathrm{Cl}, \mathrm{CuCl}$, and MgSO4 in Pure Water and Water + Urea Mixtures, J. Chem. Eng. Data, 2004, 49, 94-98

(84) Pearce, J. N.; Pumplin, G. G. The Apparent and Partial Molal Volume of Ammonium Chloride and of Cupric Sulfate in Aqueous Solutions at $25^{\circ} \mathrm{C}$. J. Am. Chem. Soc. 1937, 59, 1221-1222.

(90) Perry, R. H.; Green, D. W. Perry's Chemical Engineers' Handbook; McGraw Hill: New York, 7th edition, 1997.

(106) Rashkovskaya, E. A.; Chernen'kaya, E. I. Densities of Solutions of $\mathrm{NH}_{4} \mathrm{HCO}_{3}, \mathrm{NaHCO}_{3}, \mathrm{NH}_{4} \mathrm{Cl}$ and Ammonia in the 20-100 Range. J. Appl. Chem. USSR 1967, 40, 301-308.

\begin{tabular}{|c|c|c|c|c|c|c|c|c|c|c|c|c|c|c|c|c|}
\hline \multirow[t]{17}{*}{ molality } & \multirow[t]{17}{*}{ molarity } & \multicolumn{2}{|c|}{$\mathrm{t} /{ }^{\circ} \mathrm{C}$ mass frac } & $\begin{array}{r}\text { Density } \\
\exp / \\
\mathrm{kg} / \mathrm{m}^{3}\end{array}$ & Ref & $\begin{array}{r}\text { App vol } \\
\exp / \\
\mathrm{m}^{3} / \mathrm{kg}\end{array}$ & $\begin{array}{c}\text { App vol } \\
\text { Calc / } \\
\mathrm{m}^{3} / \mathrm{kg}\end{array}$ & $\begin{array}{r}\text { Density } \\
\mathrm{calc} / \\
\mathrm{kg} / \mathrm{m}^{3}\end{array}$ & $\begin{array}{r}\text { Density } \\
\text { Residual / } \\
\mathrm{kg} / \mathrm{m}^{3}\end{array}$ & $\begin{array}{r}\text { App Vol } \\
\text { Residual / } \\
\mathrm{m}^{3} / \mathrm{kg}\end{array}$ & $\begin{array}{r}\text { Square of } \\
\text { Density } \\
\text { Res }\end{array}$ & $\begin{array}{l}\text { Inconsistent } \\
\text { based on } \\
\text { density }\end{array}$ & $\begin{array}{c}\text { Inconsistent } \\
\text { based on } \\
\text { apparent } \\
\text { volume }\end{array}$ & $\begin{array}{r}\text { (Dens rel - } \\
1)^{*} 10^{\wedge} 4\end{array}$ & $\begin{array}{r}\text { Apparent } \\
\text { molal volume } \\
/ \mathrm{cm}^{3} / \mathrm{mol}\end{array}$ & $\begin{array}{r}\text { Density of } \\
\text { water } / \mathrm{kg} / \mathrm{m}^{3}\end{array}$ \\
\hline & & 0 & 0.01 & 1003.3 & (90) & 0.000655 & 0.000695 & 1002.9 & 0.4013 & -0.000040 & 0.161 & & & & & 999.8395 \\
\hline & & 0 & 0.02 & 1006.7 & (90) & 0.000659 & 0.000696 & 1006.0 & 0.7418 & -0.000037 & 0.550 & & & & & 999.8395 \\
\hline & & 0 & 0.04 & 1013.5 & (90) & 0.000663 & 0.000698 & 1012.1 & 1.4218 & -0.000035 & 2.022 & & & & & 999.8395 \\
\hline & & 0 & 0.08 & 1026.6 & (90) & 0.000674 & 0.000701 & 1024.3 & 2.2824 & -0.000027 & 5.209 & & & & & 999.8395 \\
\hline & & 0 & 0.12 & 1039.1 & (90) & 0.000685 & 0.000705 & 1036.5 & 2.5503 & -0.000020 & 6.504 & $!$ & & & & 999.8395 \\
\hline & & 0 & 0.16 & 1051.0 & (90) & 0.000696 & 0.000709 & 1048.8 & 2.2342 & -0.000013 & 4.992 & & & & & 999.8395 \\
\hline & & 0 & 0.20 & 1062.5 & (90) & 0.000705 & 0.000712 & 1061.0 & 1.5429 & -0.000007 & 2.381 & & & & & 999.8395 \\
\hline & & 0 & 0.24 & 1073.9 & (90) & 0.000713 & 0.000716 & 1073.1 & 0.7855 & -0.000003 & 0.617 & & & & & 999.8395 \\
\hline & & 10 & 0.01 & 1002.9 & (90) & 0.000681 & 0.000700 & 1002.7 & 0.1923 & -0.000019 & 0.037 & & & & & 999.6996 \\
\hline & & 10 & 0.02 & 1006.2 & (90) & 0.000677 & 0.000701 & 1005.7 & 0.4856 & -0.000024 & 0.236 & & & & & 999.6996 \\
\hline & & 10 & 0.04 & 1012.6 & (90) & 0.000682 & 0.000703 & 1011.7 & 0.8768 & -0.000021 & 0.769 & & & & & 999.6996 \\
\hline & & 10 & 0.08 & 1025.1 & (90) & 0.000690 & 0.000707 & 1023.7 & 1.3815 & -0.000016 & 1.908 & & & & & 999.6996 \\
\hline & & 10 & 0.12 & 1037.0 & (90) & 0.000700 & 0.000711 & 1035.7 & 1.3239 & -0.000010 & 1.753 & & & & & 999.6996 \\
\hline & & 10 & 0.16 & 1048.5 & (90) & 0.000709 & 0.000715 & 1047.6 & 0.9140 & -0.000005 & 0.835 & & & & & 999.6996 \\
\hline & & 10 & 0.20 & 1059.6 & (90) & 0.000718 & 0.000718 & 1059.4 & 0.1619 & -0.000001 & 0.026 & & & & & 999.6996 \\
\hline & & 10 & 0.24 & 1070.5 & (90) & 0.000725 & 0.000722 & 1071.2 & -0.7221 & 0.000003 & 0.521 & & & & & 999.6996 \\
\hline 0.001 & & 12.5 & 0.0001 & 999.46 & (47) & 0.000645 & 0.000700 & 999.5 & 0.0030 & -0.000055 & 0.000 & & & 0.19 & & 999.4388 \\
\hline 0.002 & & 12.5 & 0.0001 & 999.48 & (47) & 0.000626 & 0.000700 & 999.5 & 0.0079 & -0.000074 & 0.000 & & & 0.4 & & 999.4388 \\
\hline 0.005 & & 12.5 & 0.0003 & 999.53 & (47) & 0.000664 & 0.000700 & 999.5 & 0.0098 & -0.000037 & 0.000 & & & 0.9 & & 999.4388 \\
\hline 0.01001 & & 12.5 & 0.0005 & 999.62 & (47) & 0.000664 & 0.000700 & 999.6 & 0.0194 & -0.000036 & 0.000 & & & 1.8 & & 999.4388 \\
\hline 0.02002 & & 12.5 & 0.0011 & 999.82 & (47) & 0.000645 & 0.000700 & 999.8 & 0.0590 & -0.000055 & 0.003 & & & 3.8 & & 999.4388 \\
\hline 0.05006 & & 12.5 & 0.0027 & 1000.38 & (47) & 0.000649 & 0.000701 & 1000.2 & 0.1387 & -0.000052 & 0.019 & & & 9.4 & & 999.4388 \\
\hline 0.10013 & & 12.5 & 0.0053 & 1001.28 & (47) & 0.000656 & 0.000701 & 1001.0 & 0.2417 & -0.000045 & 0.058 & & & 18.4 & & 999.4388 \\
\hline 0.20026 & & 12.5 & 0.0106 & 1002.93 & (47) & 0.000672 & 0.000701 & 1002.6 & 0.3110 & -0.000029 & 0.097 & & & 34.9 & & 999.4388 \\
\hline 0.50065 & & 12.5 & 0.0261 & 1007.78 & (47) & 0.000683 & 0.000703 & 1007.3 & 0.5305 & -0.000020 & 0.281 & & & 83.5 & & 999.4388 \\
\hline
\end{tabular}




\begin{tabular}{|c|c|c|c|}
\hline molality molarity & \multicolumn{2}{|c|}{$\mathrm{t} /{ }^{\circ} \mathrm{C}$ mass frac } & $\begin{array}{r}\text { Density } \\
\exp / \\
\mathrm{kg} / \mathrm{m}^{3}\end{array}$ \\
\hline \multirow[t]{6}{*}{0.60078} & 12.5 & 0.0311 & 1009.48 \\
\hline & 15 & 0.0001 & 999.11 \\
\hline & 15 & 0.0001 & 999.13 \\
\hline & 15 & 0.0003 & 999.18 \\
\hline & 15 & 0.0005 & 999.28 \\
\hline & 15 & 0.0011 & 999.47 \\
\hline \multirow[t]{2}{*}{0.05} & 15 & 0.0027 & 999.98 \\
\hline & 15 & 0.0027 & 1000.02 \\
\hline 0.07 & 15 & 0.0037 & 1000.34 \\
\hline \multirow[t]{2}{*}{0.1} & 15 & 0.0053 & 1000.85 \\
\hline & 15 & 0.0053 & 1000.92 \\
\hline \multirow[t]{2}{*}{0.2} & 15 & 0.0106 & 1002.41 \\
\hline & 15 & 0.0106 & 1002.58 \\
\hline 0.3 & 15 & 0.0158 & 1004.27 \\
\hline \multirow[t]{3}{*}{0.5} & 15 & 0.0260 & 1007.4 \\
\hline & 15 & 0.0261 & 1007.41 \\
\hline & 15 & 0.0311 & 1010.11 \\
\hline 1 & 15 & 0.0508 & 1015.12 \\
\hline 1.5 & 15 & 0.0743 & 1022.17 \\
\hline 2 & 15 & 0.0966 & 1028.6 \\
\hline 3 & 15 & 0.1383 & 1040.61 \\
\hline 4 & 15 & 0.1763 & 1051.83 \\
\hline 5 & 15 & 0.2110 & 1059.89 \\
\hline 0.05 & 20 & 0.0027 & 999.07 \\
\hline 0.07 & 20 & 0.0037 & 999.42 \\
\hline \multirow{2}{*}{0.1} & 20 & 0.0053 & 999.9 \\
\hline & 20 & 0.01 & 1001.3 \\
\hline 0.2 & 20 & 0.0106 & 1001.45 \\
\hline \multirow{2}{*}{0.3} & 20 & 0.0158 & 1003.29 \\
\hline & 20 & 0.02 & 1004.5 \\
\hline \multirow[t]{2}{*}{0.5} & 20 & 0.0260 & 1006.38 \\
\hline & 20 & 0.04 & 1010.7 \\
\hline 1 & 20 & 0.0508 & 1014 \\
\hline \multirow[t]{2}{*}{1.5} & 20 & 0.0743 & 1020.95 \\
\hline & 20 & 0.08 & 1022.7 \\
\hline \multirow[t]{2}{*}{2} & 20 & 0.0966 & 1027.31 \\
\hline & 20 & 0.12 & 1034.4 \\
\hline \multirow[t]{2}{*}{3} & 20 & 0.1383 & 1039.17 \\
\hline & 20 & 0.16 & 1045.7 \\
\hline \multirow[t]{2}{*}{4} & 20 & 0.1763 & 1050.28 \\
\hline & 20 & 0.20 & 1056.7 \\
\hline \multirow[t]{2}{*}{5} & 20 & 0.2110 & 1058.3 \\
\hline & 20 & 0.24 & 1067.4 \\
\hline
\end{tabular}
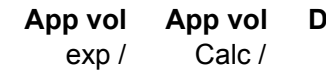

$$
\mathrm{m}^{3} / \mathrm{kg}
$$

$\mathrm{m}^{3} / \mathrm{kg}$

$\begin{array}{ll}0.000681 & 0.000703\end{array}$

$\begin{array}{ll}0.000814 & 0.000701\end{array}$

$0.000720 \quad 0.000701$

$\begin{array}{lll}0.000701 & 0.000701\end{array}$

$0.000664 \quad 0.000701$

$0.000655 \quad 0.000702$

$\begin{array}{lll}0.000671 & 0.000702\end{array}$

$\begin{array}{lll}0.000656 & 0.000702\end{array}$

$0.000668 \quad 0.000702$

$0.000672 \quad 0.000702$

$0.000660 \quad 0.000702$

$0.000689 \quad 0.000702$

$0.000673 \quad 0.000702$

$\begin{array}{lll}0.000675 & 0.000703\end{array}$

$0.000684 \quad 0.000704$

$0.000684 \quad 0.000704$

$0.000651 \quad 0.000705$

$\begin{array}{lll}0.000690 & 0.000706\end{array}$

$0.000697 \quad 0.000709$

$0.000704 \quad 0.000711$

$0.000712 \quad 0.000715$

$\begin{array}{ll}0.000716 & 0.000719\end{array}$

$0.000729 \quad 0.000722$

$0.000676 \quad 0.000704$

$0.000675 \quad 0.000704$

$0.000682 \quad 0.000704$

$\begin{array}{lll}0.000692 & 0.000704\end{array}$

$0.000695 \quad 0.000704$

$0.000680 \quad 0.000705$

$0.000688 \quad 0.000705$

$0.000689 \quad 0.000706$

$\begin{array}{lll}0.000692 & 0.000707\end{array}$

$0.000694 \quad 0.000709$

$\begin{array}{lll}0.000701 & 0.000711\end{array}$

$\begin{array}{ll}0.000702 & 0.000712\end{array}$

$0.000708 \quad 0.000713$

$0.000710 \quad 0.000716$

$\begin{array}{ll}0.000716 & 0.000717\end{array}$

$\begin{array}{ll}0.000717 & 0.000720\end{array}$

$\begin{array}{lll}0.000720 & 0.000721\end{array}$

$0.000725 \quad 0.000724$

$\begin{array}{ll}0.000732 & 0.000725\end{array}$

$\begin{array}{lll}0.000731 & 0.000728\end{array}$
App Vol Square of Inconsistent Inconsistent (Dens rel - Apparent Density of calc / Residual / Residual / Density based on based on 1$)^{\star} 10^{\wedge} 4$ molal volume $\mathrm{kg} / \mathrm{m}^{3} \quad \mathrm{~kg} / \mathrm{m}^{3} \quad \mathrm{~m}^{3} / \mathrm{kg} \quad$ Res density apparent

999.4388

$\begin{array}{llll}008.8 & 0.7167 & -0.000023 & 0.514\end{array}$

$\begin{array}{llll}999.1 & -0.0060 & 0.000112 & 0.000\end{array}$

$\begin{array}{llll}999.1 & -0.0020 & 0.000019 & 0.000\end{array}$

$\begin{array}{llll}999.2 & 0.0000 & 0.000000 & 0.000\end{array}$

$\begin{array}{llll}999.3 & 0.0199 & -0.000037 & 0.000\end{array}$

$\begin{array}{llll}999.4 & 0.0499 & -0.000047 & 0.002\end{array}$

$999.9 \quad 0.0831-0.000031 \quad 0.007$

$\begin{array}{llll}999.9 & 0.1209 & -0.000045 & 0.015\end{array}$

$\begin{array}{llll}1000.2 & 0.1254 & -0.000034 & 0.016\end{array}$

$\begin{array}{llll}1000.7 & 0.1602 & -0.000030 & 0.026\end{array}$

$\begin{array}{llll}1000.7 & 0.2261 & -0.000042 & 0.051\end{array}$

$\begin{array}{llll}1002.3 & 0.1474 & -0.000014 & 0.022\end{array}$

$\begin{array}{llll}10023 & 0.3098 & -0.000029 & 0.096\end{array}$

$\begin{array}{llll}1003.8 & 0.4517 & -0.000028 & 0.204\end{array}$

$\begin{array}{llll}1006.9 & 0.5208 & -0.000020 & 0.271\end{array}$

$\begin{array}{llll}1006.9 & 0.5231 & -0.000020 & 0.274\end{array}$

2.935

$\begin{array}{llll}1014.2 & 0.8711 & -0.000017 & 0.759\end{array}$

$\begin{array}{llll}1021.2 & 0.9320 & -0.000012 & 0.869\end{array}$

$\begin{array}{llll}1027.9 & 0.7266 & -0.000007 & 0.528\end{array}$

$\begin{array}{llll}040.2 & 0.4313 & -0.000003 & 0.186\end{array}$

$\begin{array}{llll}1051.3 & 0.4934 & -0.000003 & 0.243\end{array}$

$\begin{array}{rrrr}1061.5 & -1.6019 & 0.000007 & 2.566\end{array}$

$\begin{array}{rrrr}999.0 & 0.0728 & -0.000027 & 0.005\end{array}$

$\begin{array}{llll}999.3 & 0.1068 & -0.000029 & 0.011\end{array}$

$\begin{array}{llll}999.8 & 0.1141 & -0.000021 & 0.013\end{array}$

$\begin{array}{llll}1001.2 & 0.1237 & -0.000012 & 0.015\end{array}$

$\begin{array}{llll}1001.4 & 0.0999 & -0.000009 & 0.010\end{array}$

$\begin{array}{llll}1002.9 & 0.3929 & -0.000025 & 0.154\end{array}$

$\begin{array}{llll}004.1 & 0.3544 & -0.000018 & 0.126\end{array}$

$\begin{array}{llll}1005.9 & 0.4397 & -0.000017 & 0.193\end{array}$

$\begin{array}{llll}1010.1 & 0.6250 & -0.000015 & 0.391\end{array}$

$\begin{array}{llll}1013.3 & 0.7359 & -0.000014 & 0.541\end{array}$

$\begin{array}{llll}020.2 & 0.7448 & -0.000010 & 0.555\end{array}$

$\begin{array}{llll}1021.9 & 0.8079 & -0.000010 & 0.653\end{array}$

$\begin{array}{llll}1026.8 & 0.5192 & -0.000005 & 0.270\end{array}$

$\begin{array}{llll}1033.6 & 0.7555 & -0.000006 & 0.571\end{array}$

$\begin{array}{llll}039.0 & 0.1780 & -0.000001 & 0.032\end{array}$

$\begin{array}{llll}1045.3 & 0.3787 & -0.000002 & 0.143\end{array}$

$\begin{array}{llll}1050.0 & 0.2380 & -0.000001 & 0.057\end{array}$

$\begin{array}{llll}1056.9 & -0.2111 & 0.000001 & 0.045\end{array}$

$\begin{array}{llll}1060.1 & -1.7871 & 0.000008 & 3.194\end{array}$

$\begin{array}{llll}1068.4 & -1.0023 & 0.000004 & 1.005\end{array}$ 999.0996 999.0996 999.0996 999.0996 999.0996 999.0996 999.0996 999.0996 999.0996 999.0996 999.0996 999.0996 999.0996 999.0996 999.0996 999.0996 999.0996 999.0996 999.0996 999.0996 999.0996 999.0996 998.2041 998.2041

998.2041 998.2041 998.2041 998.2041 998.2041 998.2041 998.2041 998.2041 998.2041 998.2041 998.2041 998.2041 998.2041 998.2041 998.2041 998.2041 998.2041 998.2041 
molality molarity

\section{$\mathrm{t} /{ }^{\circ} \mathrm{C}$ mass frac Density}

\section{exp /}

$\mathrm{kg} / \mathrm{m}^{3}$

25

$\begin{array}{ll}25 & 0.01 \\ 25 & 0.01 \\ 25 & 0.01\end{array}$

0.05

0.07

0.1

0.2

0.2

0.20027

0.3

0.4

0.40722
0.49999

0.5

0.59872

0.6

0.69941

0.79957

0.8
0.89964

1
1

1.00084

1.268

3.83
0.0001

0.0001
0.0003

0.0005

$\quad 997.22$

$0.0011 \quad 997.40$

$0.0027 \quad 997.9$

$\begin{array}{ll}0.0027 & 997.92 \\ 0.0037 & 998.24\end{array}$

$0.0053 \quad 998.72$

$0.0053 \quad 998.79$

$0.0053 \quad 998.79$

$0.0106 \quad 1000.26$

$0.0106 \quad 1000.45$

$\begin{array}{ll}0.0106 & 1000.42\end{array}$

$0.0106 \quad 1000.44$

$\begin{array}{ll}0.0158 & 1002.07\end{array}$

$0.0209 \quad 1003.67$

$0.0213 \quad 1003.75$

$0.0260 \quad 1005.2$

$\begin{array}{ll}0.0260 & 1005.12\end{array}$

$0.0261 \quad 1005.30$

$0.0310 \quad 1006.71$

$0.0311 \quad 1006.76$

$\begin{array}{ll}0.0311 & 1006.87 \\ 0.0361 & 1007.23\end{array}$

$0.0310 \quad 1009.72$

$\begin{array}{ll}0.0410 & 1009.75\end{array}$

$0.0459 \quad 1011.19$

$0.0508 \quad 1012.65$

$0.0508 \quad 1012.64$

$0.0508 \quad 1012.65$

$0.0667 \quad 1016.40$

$\begin{array}{ll}0.0743 & 1019.54\end{array}$

$0.0966 \quad 1025.87$

$0.0966 \quad 1026.02$

$0.1311 \quad 1034.60$

$\begin{array}{ll}0.1383 & 1037.62 \\ 0.1383 & 1037.81\end{array}$

$0.1763 \quad 1048.63$

$0.1763 \quad 1048.32$

$\begin{array}{ll}0.1947 & 1052.00 \\ 0.2110 & 1056.59\end{array}$

$0.2110 \quad 1057.80$
$0.1383 \quad 1037.81$
Ref

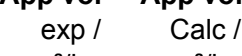

$\mathrm{m}^{3} / \mathrm{kg} \quad \mathrm{m}^{3} / \mathrm{kg}$

(47) $\quad 0.000815 \quad 0.000705$

$\begin{array}{lll}\text { (47) } & 0.000628 & 0.000705\end{array}$

$\begin{array}{ll}0.000703 & 0.000705\end{array}$

$0.000666 \quad 0.000705$

$\begin{array}{lll}0.000666 & 0.000705\end{array}$

$\begin{array}{lll}0.000681 & 0.000705\end{array}$

$0.000673 \quad 0.000705$

$0.000681 \quad 0.000705$

$0.000687 \quad 0.000706$

$0.000674 \quad 0.000706$

$0.000674 \quad 0.000706$

$\begin{array}{lll}0.000698 & 0.000706\end{array}$

$0.000680 \quad 0.000706$

$0.000683 \quad 0.000706$

$0.000682 \quad 0.000706$

$0.000685 \quad 0.000707$

$\begin{array}{lll}0.000687 & 0.000707\end{array}$

$\begin{array}{lll}0.000689 & 0.000707\end{array}$

$0.000691 \quad 0.000708$

$0.000694 \quad 0.000708$

$0.000687 \quad 0.000708$

$0.000693 \quad 0.000708$

$0.000692 \quad 0.000708$

0.0006890 .000708

$\begin{array}{lll}0.000722 & 0.000709\end{array}$

$\begin{array}{lll}0.000696 & 0.000709\end{array}$

$\begin{array}{lll}0.000695 & 0.000709\end{array}$

$0.000697 \quad 0.000710$

$0.000699 \quad 0.000710$

$0.000699 \quad 0.000710$

$0.000699 \quad 0.000710$

$\begin{array}{lll}0.000717 & 0.000712\end{array}$

$0.000705 \quad 0.000713$

$0.000711 \quad 0.000715$

$0.000710 \quad 0.000715$

$0.000725 \quad 0.000719$

$0.000719 \quad 0.000719$

$0.000718 \quad 0.000719$

$\begin{array}{lll}0.000723 & 0.000723\end{array}$

$0.000725 \quad 0.000723$

$0.000734 \quad 0.000725$

$0.000735 \quad 0.000727$

$0.000730 \quad 0.000727$
Density Density App Vol Square of Inconsistent Inconsistent (Dens rel - Apparent Density of calc / Residual / Residual / Density based on based on 1$)^{\star} 10^{\wedge} 4$ molal volume $\mathrm{kg} / \mathrm{m}^{3} \quad \mathrm{~kg} / \mathrm{m}^{3} \quad \mathrm{~m}^{3} / \mathrm{kg} \quad$ Res density 2 volum

$\begin{array}{llll}997.1 & -0.0059 & 0.000110 & 0.000\end{array}$

$\begin{array}{llll}997.1 & 0.0082 & -0.000077 & 0.000\end{array}$

$\begin{array}{llll}997.1 & 0.0006 & -0.000002 & 0.000\end{array}$

$997.2-0.0210 \quad-0.000039 \quad 0.000$

$\begin{array}{llll}997.4 & 0.0421 & -0.000040 & 0.002\end{array}$

$\begin{array}{llll}997.8 & 0.0652 & -0.000025 & 0.004\end{array}$

$\begin{array}{llll}997.8 & 0.0866 & -0.000033 & 0.007\end{array}$

$\begin{array}{llll}998.1 & 0.0905 & -0.000024 & 0.008\end{array}$

$\begin{array}{llll}998.6 & 0.0998 & -0.000019 & 0.010\end{array}$

$\begin{array}{llll}998.6 & 0.1688 & -0.000032 & 0.028\end{array}$

$\begin{array}{llll}998.6 & 0.1675 & -0.000032 & 0.028\end{array}$

$1000.2 \quad 0.0821 \quad-0.000008 \quad 0.007$

$\begin{array}{llll}000.2 & 0.2731 & -0.000026 & 0.075\end{array}$

$1000.2 \quad 0.2430 \quad-0.000023 \quad 0.059$

$\begin{array}{llll}1000.2 & 0.2579 & -0.000024 & 0.067\end{array}$

$\begin{array}{llll}1001.7 & 0.3518 & -0.000022 & 0.124\end{array}$

$0.4237-0.000020 \quad 0.179$

$\begin{array}{llll}1003.4 & 0.3993 & -0.000019 & 0.159\end{array}$

$\begin{array}{llll}1004.7 & 0.4525 & -0.000017 & 0.205\end{array}$

$\begin{array}{llll}1004.7 & 0.3723 & -0.000014 & 0.139\end{array}$

$\begin{array}{llll}1004.8 & 0.5430 & -0.000021 & 0.295\end{array}$

$\begin{array}{llll}1006.2 & 0.4916 & -0.000016 & 0.242\end{array}$

$\begin{array}{llll}1006.2 & 0.5216 & -0.000017 & 0.272\end{array}$

$\begin{array}{llll}1006.2 & 0.6168 & -0.000020 & 0.380\end{array}$

$\begin{array}{llll}1007.7 & -0.4721 & 0.000013 & 0.223\end{array}$

$\begin{array}{llll}009.2 & 0.5582 & -0.000013 & 0.312\end{array}$

$\begin{array}{llll}1009.2 & 0.5850 & -0.000014 & 0.342\end{array}$

$\begin{array}{llll}1010.6 & 0.5857 & -0.000012 & 0.343\end{array}$

$\begin{array}{llll}1012.0 & 0.6148 & -0.000012 & 0.378\end{array}$

$\begin{array}{llll}012.0 & 0.6088 & -0.000012 & 0.371\end{array}$

$\begin{array}{llll}1012.0 & 0.6028 & -0.000012 & 0.363\end{array}$

$\begin{array}{llll}1016.7 & -0.3246 & 0.000005 & 0.105\end{array}$

$\begin{array}{llll}1018.9 & 0.6023 & -0.000008 & 0.363\end{array}$

$\begin{array}{llll}1025.5 & 0.3873 & -0.000004 & 0.150\end{array}$

$\begin{array}{llll}1025.5 & 0.5323 & -0.000005 & 0.283\end{array}$

$\begin{array}{llll}1035.5 & -0.9199 & 0.000007 & 0.846\end{array}$

$\begin{array}{llll}1037.6 & 0.0221 & 0.000000 & 0.000\end{array}$

$\begin{array}{llll}1037.6 & 0.2121 & -0.000001 & 0.045\end{array}$

$\begin{array}{llll}048.6 & 0.0730 & 0.000000 & 0.005\end{array}$

$\begin{array}{llll}1048.6 & -0.2420 & 0.000001 & 0.059\end{array}$

$\begin{array}{llll}1053.9 & -1.8606 & 0.000009 & 3.462\end{array}$

$\begin{array}{llll}1058.5 & -1.9182 & 0.000008 & 3.679\end{array}$

$\begin{array}{llll}1058.5 & -0.7122 & 0.000003 & 0.507\end{array}$
997.0449

997.0449

997.0449

997.0449

997.0449

997.0449

997.0449

997.0449

997.0449

997.0449

997.0449

997.0449

997.0449

997.0449

997.0449

997.0449

997.0449

997.0449

997.0449

997.0449

997.0449

997.0449

997.0449

98.5

997.0449

997.0449

997.0449

997.0449

997.0449

997.0449

997.0449

997.0449

997.0449

997.0449

997.0449

997.0449

997.0449

997.0449

997.0449

997.0449

997.0449

997.0449

997.0449 
molality molarity

$\mathrm{t} /{ }^{\circ} \mathrm{C}$ mass frac Density

$$
\exp /
$$

$\mathrm{kg} / \mathrm{m}^{3}$

6
7.38
0.05
0.07

$$
0.1
$$

3

0.0625

0.125

$\begin{array}{rrr}25 & 0.2430 & 1066.46 \\ 25 & 0.2830 & 1077.30 \\ 30 & 0.0027 & 996.5 \\ 30 & 0.0037 & 996.84 \\ 30 & 0.0053 & 997.32 \\ 30 & 0.01 & 998.7 \\ 30 & 0.0106 & 998.84 \\ 30 & 0.0158 & 1000.64 \\ 30 & 0.02 & 1001.8 \\ 30 & 0.0260 & 1003.67 \\ 30 & 0.04 & 1007.7 \\ 30 & 0.0508 & 1011.16 \\ 30 & 0.0743 & 1017.98 \\ 30 & 0.08 & 1019.5 \\ 30 & 0.0966 & 1024.27 \\ 30 & 0.12 & 1031.0 \\ 30 & 0.1383 & 1035.95 \\ 30 & 0.16 & 1042.2 \\ 30 & 0.1763 & 1046.91 \\ 30 & 0.20 & 1053.2 \\ 30 & 0.2110 & 1054.84 \\ 30 & 0.24 & 1064.1 \\ 35 & 0.0001 & 994.04 \\ 35 & 0.0001 & 994.06 \\ 35 & 0.0003 & 994.11 \\ 35 & 0.0005 & 994.19 \\ 35 & 0.0011 & 994.37 \\ 35 & 0.0027 & 994.87 \\ 35 & 0.0027 & 994.86 \\ 35 & 0.0033 & 995.06 \\ 35 & 0.0037 & 995.22 \\ 35 & 0.0053 & 995.69 \\ 35 & 0.0053 & 995.66 \\ 35 & 0.0066 & 996.06 \\ 35 & 0.0106 & 997.2 \\ 35 & 0.0106 & 997.26 \\ 35 & 0.0132 & 998.05 \\ 35 & 0.0158 & 998.99 \\ 35 & 0.0260 & 1002.01 \\ 35 & 0.0260 & 1001.89 \\ 35 & 0.0261 & 1001.97 \\ 35 & 0.0311 & 1003.49 \\ 35 & 0.0508 & 1009.46\end{array}$

$\begin{array}{ll}0.0508 & 1009.46\end{array}$
Ref

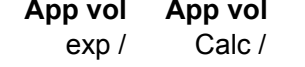

$\mathrm{m}^{3} / \mathrm{kg} \quad \mathrm{m}^{3} / \mathrm{kg}$

(84) $\quad 0.000734 \quad 0.000730$

$\begin{array}{lll}\text { (84) } & 0.000739 & 0.000734\end{array}$

$\begin{array}{lll}0.000682 & 0.000707\end{array}$

$\begin{array}{lll}0.000682 & 0.000707\end{array}$

$\begin{array}{lll}0.000688 & 0.000707\end{array}$

$0.000697 \quad 0.000708$

$0.000701 \quad 0.000708$

$\begin{array}{lll}0.000687 & 0.000708\end{array}$

$\begin{array}{ll}0.000696 & 0.000709\end{array}$

$\begin{array}{lll}0.000696 & 0.000709\end{array}$

$0.000704 \quad 0.000711$

$\begin{array}{ll}0.000701 & 0.000712\end{array}$

$0.000708 \quad 0.000714$

$\begin{array}{lll}0.000711 & 0.000715\end{array}$

$\begin{array}{ll}0.000714 & 0.000717\end{array}$

$\begin{array}{lll}0.000717 & 0.000719\end{array}$

$0.000722 \quad 0.000721$

$\begin{array}{lll}0.000724 & 0.000724\end{array}$

$0.000725 \quad 0.000725$

$0.000730 \quad 0.000728$

$0.000737 \quad 0.000729$

$0.000735 \quad 0.000732$

$0.000818 \quad 0.000708$

$0.000724 \quad 0.000708$

$0.000705 \quad 0.000708$

$0.000705 \quad 0.000708$

$\begin{array}{lll}0.000686 & 0.000708\end{array}$

$0.000688 \quad 0.000708$

$0.000694 \quad 0.000708$

$0.000695 \quad 0.000708$

$\begin{array}{lll}0.000684 & 0.000708\end{array}$

0.0006910 .000708

$0.000697 \quad 0.000708$

$0.000697 \quad 0.000708$

$0.000704 \quad 0.000709$

$0.000699 \quad 0.000709$

$0.000699 \quad 0.000709$

$\begin{array}{lll}0.000690 & 0.000709\end{array}$

$0.000699 \quad 0.000710$

$0.000703 \quad 0.000710$

$0.000700 \quad 0.000710$

$0.000702 \quad 0.000711$

$0.000703 \quad 0.000713$
Density Density App Vol Square of Inconsistent Inconsistent (Dens rel - Apparent Density of calc / Residual / Residual / Density based on based on 1$)^{\star} 10^{\wedge} 4$ molal volume $\mathrm{kg} / \mathrm{m}^{3} \quad \mathrm{~kg} / \mathrm{m}^{3} \quad \mathrm{~m}^{3} / \mathrm{kg} \quad$ Res density apparen

$\begin{array}{llll}1067.6 & -1.1214 & 0.000004 & 1.258\end{array}$

$\begin{array}{llll}1078.8 & -1.5351 & 0.000005 & 2.356\end{array}$

$996.4 \quad 0.0651 \quad-0.000025 \quad 0.004$

$996.7 \quad 0.0913 \quad-0.000025 \quad 0.008$

$\begin{array}{llll}997.2 & 0.1019 & -0.000019 & 0.010\end{array}$

$\begin{array}{llll}998.6 & 0.1014 & -0.000010 & 0.010\end{array}$

$\begin{array}{llll}998.8 & 0.0688 & -0.000007 & 0.005\end{array}$

$\begin{array}{llll}1000.3 & 0.3332 & -0.000021 & 0.111\end{array}$

$\begin{array}{llll}1001.5 & 0.2542 & -0.000013 & 0.065\end{array}$

$\begin{array}{llll}1003.3 & 0.3435 & -0.000013 & 0.118\end{array}$

$\begin{array}{llll}1007.4 & 0.2733 & -0.000007 & 0.075\end{array}$

$\begin{array}{llll}1010.6 & 0.5725 & -0.000011 & 0.328\end{array}$

$\begin{array}{llll}017.5 & 0.5192 & -0.000007 & 0.270\end{array}$

$1019.1 \quad 0.3701 \quad-0.000004 \quad 0.137$

$\begin{array}{llll}1024.0 & 0.2957 & -0.000003 & 0.087\end{array}$

$1030.7 \quad 0.2552 \quad-0.000002 \quad 0.065$

$\begin{array}{llll}0.0713 & 0.000000 & 0.005\end{array}$

$\begin{array}{llll}1042.3 & -0.0592 & 0.000000 & 0.004\end{array}$

$\begin{array}{llll}1046.9 & 0.0034 & 0.000000 & 0.000\end{array}$

$\begin{array}{llll}1053.7 & -0.4606 & 0.000002 & 0.212\end{array}$

$\begin{array}{llll}1056.8 & -1.9400 & 0.000008 & 3.764\end{array}$

$\begin{array}{llll}1064.9 & -0.8363 & 0.000003 & 0.699\end{array}$

$\begin{array}{llll}994.0 & -0.0058 & 0.000110 & 0.000\end{array}$

$\begin{array}{llll}994.1 & -0.0017 & 0.000016 & 0.000\end{array}$

$\begin{array}{llll}994.1 & 0.0007 & -0.000003 & 0.000\end{array}$

$\begin{array}{llll}994.2 & 0.0012 & -0.000002 & 0.000\end{array}$

$\begin{array}{llll}994.3 & 0.0225 & -0.000021 & 0.001\end{array}$

$\begin{array}{llll}994.8 & 0.0517 & -0.000020 & 0.003\end{array}$

$\begin{array}{llll}994.8 & 0.0377 & -0.000014 & 0.001\end{array}$

$\begin{array}{llll}995.0 & 0.0412 & -0.000012 & 0.002\end{array}$

$\begin{array}{llll}995.1 & 0.0884 & -0.000024 & 0.008\end{array}$

$\begin{array}{llll}995.6 & 0.0897 & -0.000017 & 0.008\end{array}$

$\begin{array}{llll}995.6 & 0.0598 & -0.000011 & 0.004\end{array}$

$\begin{array}{llll}996.0 & 0.0725 & -0.000011 & 0.005\end{array}$

$\begin{array}{llll}997.2 & 0.0493 & -0.000005 & 0.002\end{array}$

$\begin{array}{llll}997.2 & 0.1077 & -0.000010 & 0.012\end{array}$

$\begin{array}{llll}997.9 & 0.1296 & -0.000010 & 0.017\end{array}$

$\begin{array}{llll}998.7 & 0.3064 & -0.000019 & 0.094\end{array}$

$\begin{array}{llll}001.7 & 0.3126 & -0.000012 & 0.098\end{array}$

$\begin{array}{llll}1001.7 & 0.1975 & -0.000008 & 0.039\end{array}$

$\begin{array}{llll}1001.7 & 0.2671 & -0.000010 & 0.071\end{array}$

$\begin{array}{llll}1003.2 & 0.2947 & -0.000009 & 0.087\end{array}$

$\begin{array}{llll}1008.9 & 0.5185 & -0.000010 & 0.269\end{array}$
997.0449

997.0449

995.6473

995.6473

995.6473

995.6473

995.6473

995.6473

995.6473

995.6473

995.6473

995.6473

995.6473

995.6473

995.6473

995.6473

995.6473

995.6473

995.6473

995.6473

995.6473

995.6473

994.0319

994.0319

994.0319

994.0319

994.0319

994.0319

994.0319

$37.2 \quad 994.0319$

994.0319

994.0319

994.0319

$37.3 \quad 994.0319$

994.0319

994.0319

32.5

$37.4 \quad 994.0319$ 994.0319

994.0319

$37.6 \quad 994.0319$ 994.0319 994.0319 
molality molarity

$\mathrm{t} /{ }^{\circ} \mathrm{C}$ mass frac Density

$$
\exp /
$$

$\mathrm{kg} / \mathrm{m}^{3}$

1
1.5
2
3
4
5
0.05
0.07
0.1
0.2
0.3
0.5
1
1.5
2
3
4
5

0.05

0.0625

0.07
0.1
0.125

0.125

0.2

0.25
0.3

0.3
0.5
0.5

0.5

1
1
1.5
2

$\begin{array}{rrr}35 & 0.0508 & 1009.38 \\ 35 & 0.0743 & 1016.25 \\ 35 & 0.0966 & 1022.51 \\ 35 & 0.1383 & 1034.15 \\ 35 & 0.1763 & 1045.07 \\ 35 & 0.2110 & 1052.96 \\ 40 & 0.0027 & 993.05 \\ 40 & 0.0037 & 993.39 \\ 40 & 0.0053 & 993.86 \\ 40 & 0.0106 & 995.37 \\ 40 & 0.0158 & 997.17 \\ 40 & 0.0260 & 1000.17 \\ 40 & 0.0508 & 1007.61 \\ 40 & 0.0743 & 1014.39 \\ 40 & 0.0966 & 1020.63 \\ 40 & 0.1383 & 1032.25 \\ 40 & 0.1763 & 1043.13 \\ 40 & 0.2110 & 1051.01 \\ 42.5 & 0.0001 & 991.25 \\ 42.5 & 0.0001 & 991.27 \\ 42.5 & 0.0003 & 991.31 \\ 42.5 & 0.0005 & 991.40 \\ 42.5 & 0.0011 & 991.56 \\ 42.5 & 0.0027 & 992.02 \\ 42.5 & 0.0053 & 992.79 \\ 42.5 & 0.0106 & 994.37 \\ 42.5 & 0.0261 & 998.97 \\ 42.5 & 0.0311 & 1000.46 \\ 45 & 0.0027 & 991.04 \\ 45 & 0.0033 & 991.21 \\ 45 & 0.0037 & 991.38 \\ 45 & 0.0053 & 991.86 \\ 45 & 0.0066 & 992.19 \\ 45 & 0.0106 & 993.35 \\ 45 & 0.0132 & 994.13 \\ 45 & 0.0158 & 995.15 \\ 45 & 0.0260 & 998.16 \\ 45 & 0.0260 & 997.92 \\ 45 & 0.0508 & 1005.6 \\ 45 & 0.0508 & 1005.16 \\ 45 & 0.0743 & 1012.37 \\ 45 & 0.0966 & 1018.62 \\ 45 & 0.1383 & 1030.23\end{array}$

$0.1383 \quad 1030.23$
Ref

exp / Calc /

$$
\begin{array}{cc}
\exp / & \text { Calc } / \\
\mathrm{m}^{3} / \mathrm{kg} & \mathrm{m}^{3} / \mathrm{kg}
\end{array}
$$

(74)

$0.000705 \quad 0.000713$

$\begin{array}{ll}0.000710 & 0.000716\end{array}$

$0.000716 \quad 0.000718$

$0.000724 \quad 0.000723$

$\begin{array}{lll}0.000727 & 0.000727\end{array}$

$\begin{array}{lll}0.000739 & 0.000731\end{array}$

$0.000690 \quad 0.000709$

$\begin{array}{lll}0.000688 & 0.000709\end{array}$

$0.000694 \quad 0.000709$

$0.000706 \quad 0.000710$

$0.000691 \quad 0.000710$

$0.000700 \quad 0.000711$

$0.000705 \quad 0.000714$

$\begin{array}{lll}0.000711 & 0.000717\end{array}$

$0.000718 \quad 0.000719$

$0.000725 \quad 0.000724$

$0.000729 \quad 0.000728$

$\begin{array}{lll}0.000741 & 0.000732\end{array}$

$0.000820 \quad 0.000709$

$\begin{array}{lll}0.000726 & 0.000709\end{array}$

$\begin{array}{lll}0.000745 & 0.000709\end{array}$

$\begin{array}{lll}0.000707 & 0.000709\end{array}$

$0.000698 \quad 0.000709$

$\begin{array}{lll}0.000711 & 0.000709\end{array}$

$\begin{array}{lll}0.000712 & 0.000709\end{array}$

$0.000709 \quad 0.000710$

$\begin{array}{lll}0.000709 & 0.000712\end{array}$

$0.000710 \quad 0.000712$

$0.000694 \quad 0.000709$

$0.000705 \quad 0.000709$

$\begin{array}{lll}0.000691 & 0.000709\end{array}$

$0.000695 \quad 0.000710$

$0.000707 \quad 0.000710$

$0.000709 \quad 0.000710$

$0.000709 \quad 0.000710$

$0.000693 \quad 0.000711$

$\begin{array}{lll}0.000701 & 0.000712\end{array}$

$0.000710 \quad 0.000712$

$0.000706 \quad 0.000715$

$0.000714 \quad 0.000715$

$0.000712 \quad 0.000718$

$0.000718 \quad 0.000720$

$\begin{array}{lll}0.000726 & 0.000725\end{array}$
App Vol Square of Inconsistent Inconsistent (Dens rel- Apparent Density of ( Residual / Density based on based on 1$)^{\star} 10^{\wedge} 4$ molal volume water $/ \mathrm{kg} / \mathrm{m}^{3}$

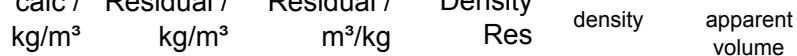

$\begin{array}{llll}1008.9 & 0.4364 & -0.000008 & 0.190\end{array}$

$\begin{array}{llll}1015.8 & 0.4549 & -0.000006 & 0.207\end{array}$

$\begin{array}{llll}1022.3 & 0.2235 & -0.000002 & 0.050\end{array}$

$\begin{array}{llll}034.3 & -0.1330 & 0.000001 & 0.018\end{array}$

$1045.1 \quad-0.0413 \quad 0.000000-0.002$

$\begin{array}{llll}1054.9 & -1.9631 & 0.000008 & 3.854\end{array}$

$\begin{array}{llll}993.0 & 0.0480 & -0.000018 & 0.002 \\ 993.3 & 0.0748 & -0.000020 & 0.006\end{array}$

$\begin{array}{llll}993.8 & 0.0763 & -0.000015 & 0.006\end{array}$

$\begin{array}{llll}995.3 & 0.0364 & -0.000003 & 0.001\end{array}$

$\begin{array}{llll}996.9 & 0.3044 & -0.000019 & 0.093\end{array}$

$999.9 \quad 0.2925 \quad-0.000011 \quad 0.086$

$\begin{array}{llll}1007.1 & 0.4956 & -0.000010 & 0.246\end{array}$

$\begin{array}{llll}1014.0 & 0.4322 & -0.000006 & 0.187\end{array}$

$\begin{array}{llll}1020.4 & 0.1937 & -0.000002 & 0.038\end{array}$

$1032.4 \quad-0.1501 \quad 0.000001 \quad 0.023$

$1043.2 \quad-0.0584 \quad 0.000000 \quad 0.003$

$\begin{array}{llll}1053.0 & -1.9445 & 0.000008 & 3.781\end{array}$

$\begin{array}{llll}991.3 & -0.0059 & 0.000112 & 0.000\end{array}$

$\begin{array}{llll}991.3 & -0.0018 & 0.000017 & 0.000\end{array}$

$\begin{array}{llll}991.3 & -0.0095 & 0.000036 & 0.000\end{array}$

$\begin{array}{llll}991.4 & 0.0008 & -0.000001 & 0.000\end{array}$

$\begin{array}{llll}991.6 & 0.0116 & -0.000011 & 0.000\end{array}$

$\begin{array}{llll}992.0 & -0.0044 & 0.000002 & 0.000\end{array}$

$\begin{array}{llll}992.8 & -0.0142 & 0.000003 & 0.000\end{array}$

$\begin{array}{llll}994.4 & 0.0095 & -0.000001 & 0.000\end{array}$

$\begin{array}{llll}998.9 & 0.0584 & -0.000002 & 0.003\end{array}$

$\begin{array}{cccc}1000.4 & 0.0630 & -0.000002 & 0.004\end{array}$

$\begin{array}{llll}991.0 & 0.0397 & -0.000015 & 0.002\end{array}$

$\begin{array}{llll}991.2 & 0.0148 & -0.000005 & 0.000\end{array}$

$\begin{array}{llll}991.3 & 0.0662 & -0.000018 & 0.004\end{array}$

$\begin{array}{llll}991.8 & 0.0773 & -0.000015 & 0.006\end{array}$

$\begin{array}{llll}992.2 & 0.0198 & -0.000003 & 0.000\end{array}$

$\begin{array}{llll}993.3 & 0.0161 & -0.000002 & 0.000\end{array}$

$\begin{array}{llll}994.1 & 0.0253 & -0.000002 & 0.001\end{array}$

$\begin{array}{llll}994.9 & 0.2828 & -0.000018 & 0.080\end{array}$

$\begin{array}{lllll}997.9 & 0.2789 & -0.000011 & 0.078\end{array}$

$\begin{array}{llll}997.9 & 0.0412 & -0.000002 & 0.002\end{array}$

$\begin{array}{llll}005.1 & 0.4794 & -0.000009 & 0.230\end{array}$

$\begin{array}{llll}1005.1 & 0.0395 & -0.000001 & 0.002\end{array}$

$\begin{array}{llll}1012.0 & 0.4068 & -0.000005 & 0.165\end{array}$

$\begin{array}{llll}1018.4 & 0.1820 & -0.000002 & 0.033\end{array}$

$\begin{array}{llll}1030.4 & -0.1570 & 0.000001 & 0.025\end{array}$
$37.7 \quad 994.0319$

994.0319

994.0319

994.0319

994.0319

994.0319

992.2158

992.2158

992.2158

992.2158

992.2158

992.2158

992.2158

992.2158

992.2158

992.2158

992.2158

992.2158

991.237

991.237

991.237

991.237

991.237

991.237

991.237

991.237

991.237

991.237

990.2132

$37.7 \quad 990.2132$

990.2132

990.2132

$\begin{array}{ll}37.8 & 990.2132\end{array}$

990.2132

$37.9 \quad 990.2132$

990.2132

990.2132

$38 \quad 990.2132$

990.2132

38.2990 .2132

990.2132

990.2132

990.2132 
molality molarity

$\mathrm{t} /{ }^{\circ} \mathrm{C}$ mass frac Density

$$
\exp /
$$

$\mathrm{kg} / \mathrm{m}^{3}$

0.05

0.05
0.07
0.1

0.1

0.2
0.3

0.5

2

3

4

0.05
0.0625
0.07
0.1
0.125
0.2
0.25
0.3
0.5
0.5
1

\begin{tabular}{|c|c|}
\hline 0.1763 & 1041.11 \\
\hline 0.2110 & 1048.99 \\
\hline 0.0027 & 988.88 \\
\hline 0.0037 & 989.23 \\
\hline 0.0053 & 989.7 \\
\hline 0.01 & 991.0 \\
\hline 0.0106 & 991.21 \\
\hline 0.0158 & 993.02 \\
\hline 0.02 & 994.0 \\
\hline 0.0260 & 996.03 \\
\hline 0.04 & 999.9 \\
\hline 0.0508 & 1003.48 \\
\hline 0.0743 & 1010.26 \\
\hline 0.08 & 1011.6 \\
\hline 0.0966 & 1016.52 \\
\hline 0.12 & 1023.1 \\
\hline 0.1383 & 1028.14 \\
\hline 0.1400 & 1028.7 \\
\hline 0.16 & 1034.3 \\
\hline 0.1600 & 1034.3 \\
\hline 0.1763 & 1039.05 \\
\hline 0.1800 & 1039.9 \\
\hline 0.20 & 1045.4 \\
\hline 0.2000 & 1045.4 \\
\hline 0.2110 & 1046.91 \\
\hline 0.2200 & 1050.9 \\
\hline 0.24 & 1056.4 \\
\hline 0.2400 & 1056.4 \\
\hline 0.2600 & 1062.0 \\
\hline 0.2800 & 1067.5 \\
\hline 0.3000 & 1073.0 \\
\hline 0.3200 & 1078.6 \\
\hline 0.0027 & 986.54 \\
\hline 0.0033 & 986.67 \\
\hline 0.0037 & 986.87 \\
\hline 0.0053 & 987.35 \\
\hline 0.0066 & 987.63 \\
\hline 0.0106 & 988.86 \\
\hline 0.0132 & 989.49 \\
\hline 0.0158 & 990.67 \\
\hline 0.0260 & 993.71 \\
\hline 0.0260 & 993.16 \\
\hline & 1001.17 \\
\hline
\end{tabular}

$0.0508 \quad 1001.17 \quad(45)$
Ref

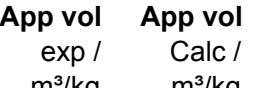

$$
\mathrm{m}^{3} / \mathrm{k}
$$

$\mathrm{m}^{3} / \mathrm{kg}$

$0.000730 \quad 0.000730$

$\begin{array}{lll}0.000742 & 0.000734\end{array}$

$\begin{array}{lll}0.000688 & 0.000709\end{array}$

$0.000685 \quad 0.000710$

$0.000692 \quad 0.000710$

$0.000709 \quad 0.000710$

$0.000706 \quad 0.000710$

$0.000690 \quad 0.000711$

$\begin{array}{lll}0.000708 & 0.000712\end{array}$

$0.000700 \quad 0.000712$

$\begin{array}{ll}0.000712 & 0.000714\end{array}$

$0.000705 \quad 0.000715$

$0.000712 \quad 0.000718$

$\begin{array}{ll}0.000717 & 0.000719\end{array}$

$0.000719 \quad 0.000721$

$0.000723 \quad 0.000724$

$0.000727 \quad 0.000726$

$0.000726 \quad 0.000726$

$0.000729 \quad 0.000728$

$0.000729 \quad 0.000728$

$0.000730 \quad 0.000730$

$0.000732 \quad 0.000731$

$\begin{array}{lll}0.000734 & 0.000733\end{array}$

$0.000734 \quad 0.000733$

$\begin{array}{lll}0.000742 & 0.000734\end{array}$

$0.000737 \quad 0.000736$

$\begin{array}{lll}0.000739 & 0.000738\end{array}$

$\begin{array}{lll}0.000739 & 0.000738\end{array}$

$\begin{array}{lll}0.000741 & 0.000740\end{array}$

$0.000743 \quad 0.000743$

$\begin{array}{lll}0.000745 & 0.000745\end{array}$

$0.000747 \quad 0.000747$

$0.000689 \quad 0.000709$

$\begin{array}{lll}0.000714 & 0.000709\end{array}$

$\begin{array}{lll}0.000691 & 0.000709\end{array}$

$0.000695 \quad 0.000710$

$\begin{array}{ll}0.000716 & 0.000710\end{array}$

$0.000708 \quad 0.000710$

$0.000720 \quad 0.000711$

$0.000692 \quad 0.000711$

$0.000700 \quad 0.000712$

$0.000722 \quad 0.000712$

$\begin{array}{lll}0.000706 & 0.000715\end{array}$
Density Density App Vol Square of Inconsistent Inconsistent (Dens rel - Apparent Density of calc / Residual / Residual / Density based on based on 1$)^{\star} 10^{\wedge} 4$ molal volume $\mathrm{kg} / \mathrm{m}^{3} \quad \mathrm{~kg} / \mathrm{m}^{3} \quad \mathrm{~m}^{3} / \mathrm{kg} \quad$ Res density apparent

$\begin{array}{llll}1041.2 & -0.0423 & 0.000000 & 0.002\end{array}$

$\begin{array}{llll}1050.9 & -1.8988 & 0.000008 & 3.605\end{array}$

$\begin{array}{llll}988.8 & 0.0549 & -0.000021 & 0.003\end{array}$

$\begin{array}{llll}989.1 & 0.0906 & -0.000025 & 0.008\end{array}$

$\begin{array}{llll}989.6 & 0.0907 & -0.000017 & 0.008\end{array}$

$\begin{array}{llll}991.0 & 0.0087 & -0.000001 & 0.000\end{array}$

$\begin{array}{llll}991.2 & 0.0460 & -0.000004 & 0.002\end{array}$

$992.7 \quad 0.3195 \quad-0.000021 \quad 0.102$

$993.9 \quad 0.0601 \quad-0.000003 \quad 0.004$

$\begin{array}{llll}995.7 & 0.3096 & -0.000012 & 0.096\end{array}$

$\begin{array}{llll}999.8 & 0.0829 & -0.000002 & 0.007\end{array}$

$\begin{array}{llll}1003.0 & 0.5079 & -0.000010 & 0.258\end{array}$

$0.809 .8 \quad 0.4364 \quad-0.000006 \quad 0.190$

$\begin{array}{llll}1011.5 & 0.1146 & -0.000001 & 0.013\end{array}$

$\begin{array}{llll}1016.3 & 0.2159 & -0.000002 & 0.047\end{array}$

$\begin{array}{llll}1023.0 & 0.0732 & -0.000001 & 0.005\end{array}$

$\begin{array}{lll}1023.0 & 0.0732 & -0.00001 \\ 1028.3 & -0.1162 & 0.000001\end{array}$

$\begin{array}{llll}1028.7 & -0.0454 & 0.000000 & 0.002\end{array}$

$\begin{array}{llll}1034.4 & -0.1268 & 0.000001 & 0.016\end{array}$

$\begin{array}{llll}1034.4 & -0.1268 & 0.000001 & 0.016\end{array}$

$\begin{array}{llll}1039.0 & 0.0346 & 0.000000 & 0.001\end{array}$

$\begin{array}{llll}1040.1 & -0.1691 & 0.000001 & 0.029\end{array}$

$\begin{array}{llll}1045.7 & -0.2704 & 0.000001 & 0.073\end{array}$

$\begin{array}{llll}1045.7 & -0.2704 & 0.000001 & 0.073\end{array}$

$\begin{array}{llll}1048.7 & -1.8285 & 0.000008 & 3.343\end{array}$

$\begin{array}{llll}1058.7 & -1.8285 & 0.000008 & 3.343\end{array}$

$\begin{array}{llll}1056.7 & -0.3429 & 0.000001 & 0.118\end{array}$

$\begin{array}{llll}1056.7 & -0.3429 & 0.000001 & 0.118\end{array}$

$\begin{array}{llll}1062.2 & -0.2101 & 0.000001 & 0.044\end{array}$

$\begin{array}{llll}1067.6 & -0.1289 & 0.000000 & 0.017\end{array}$

$\begin{array}{llll}1073.0 & 0.0027 & 0.000000 & 0.000\end{array}$

$\begin{array}{llll}1078.3 & 0.2866 & -0.000001 & 0.082\end{array}$

$\begin{array}{llll}986.5 & 0.0531 & -0.000020 & 0.003\end{array}$

$\begin{array}{llll}986.7 & -0.0154 & 0.000005 & 0.000\end{array}$

$\begin{array}{llll}986.8 & 0.0678 & -0.000019 & 0.005\end{array}$

$\begin{array}{llll}987.3 & 0.0761 & -0.000015 & 0.006\end{array}$

$\begin{array}{llll}987.7 & -0.0402 & 0.000006 & 0.002\end{array}$

$\begin{array}{llll}988.8 & 0.0261 & -0.000003 & 0.001\end{array}$

$\begin{array}{llll}989.6 & -0.1178 & 0.000009 & 0.014\end{array}$

$\begin{array}{llll}990.4 & 0.2943 & -0.000019 & 0.087\end{array}$

$\begin{array}{llll}993.4 & 0.3045 & -0.000012 & 0.093\end{array}$

$\begin{array}{llll}993.4 & -0.2409 & 0.000009 & 0.058\end{array}$

$\begin{array}{llll}1000.7 & 0.4906 & -0.000010 & 0.241\end{array}$
990.2132

990.2132

988.0363

988.0363

988.0363

988.0363

988.0363

988.0363

988.0363

988.0363

988.0363

988.0363

988.0363

988.0363

988.0363

988.0363

988.0363

988.0363

988.0363

988.0363

988.0363

988.0363

988.0363

988.0363

988.0363

988.0363

988.0363

988.0363

988.0363

988.0363

988.0363

988.0363

985.6952

38.2985 .6952

985.6952

985.6952

$38.3 \quad 985.6952$ 985.6952

$38.5 \quad 985.6952$

985.6952

985.6952

$38.6 \quad 985.6952$

985.6952 
molality molarity $\mathrm{t} /{ }^{\circ} \mathrm{C}$ mass frac

1
1.5
2
3

\section{$t /{ }^{\circ} \mathrm{C}$ mass frac Density
$\exp /$
$\mathrm{kg} / \mathrm{m}^{3}$ \\ $\mathbf{t} /{ }^{\circ} \mathrm{C}$ mass frac $\begin{array}{r}\text { Density } \\ \exp / \\ \mathrm{kg} / \mathrm{m}^{3}\end{array}$}

$55 \quad 0.0$

55
55
55

55

55
55

55

55

55

55
55

55

55

55

55

60

60
60
60

60

60

60

60

65

65
65

65

65

65

$\begin{array}{ll}65 & 0.2800\end{array}$

$65 \quad 0.3000$

0.3200

0.3400

0.3600

0.1400
0.1600

0.1800
Ref

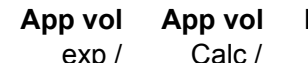

$\exp /$ Calc /

$\mathrm{m}^{3} / \mathrm{kg} \quad \mathrm{m}^{3} / \mathrm{kg}$

$\begin{array}{lll}0.000723 & 0.000715\end{array}$

$0.000713 \quad 0.000718$

$0.000719 \quad 0.000721$

$0.000727 \quad 0.000726$

$\begin{array}{lll}0.000726 & 0.000726\end{array}$

$\begin{array}{lll}0.000729 & 0.000729\end{array}$

$\begin{array}{lll}0.000731 & 0.000731\end{array}$

$\begin{array}{lll}0.000732 & 0.000731\end{array}$

$0.000735 \quad 0.000734$

$0.000743 \quad 0.000735$

$0.000737 \quad 0.000736$

$\begin{array}{lll}0.000739 & 0.000739\end{array}$

$0.000742 \quad 0.000741$

$0.000743 \quad 0.000743$

$0.000745 \quad 0.000746$

$0.000747 \quad 0.000748$

$0.000749 \quad 0.000751$

$\begin{array}{lll}0.000728 & 0.000726\end{array}$

$\begin{array}{ll}0.000731 & 0.000729\end{array}$

$0.000733 \quad 0.000731$

$\begin{array}{lll}0.000735 & 0.000734\end{array}$

$\begin{array}{lll}0.000738 & 0.000737\end{array}$

$\begin{array}{lll}0.000740 & 0.000739\end{array}$

$0.000742 \quad 0.000742$

$0.000744 \quad 0.000744$

$\begin{array}{lll}0.000746 & 0.000747\end{array}$

$\begin{array}{lll}0.000747 & 0.000749\end{array}$

$0.000749 \quad 0.000751$

$0.000727 \quad 0.000726$

$0.000730 \quad 0.000729$

$\begin{array}{lll}0.000733 & 0.000731\end{array}$

$\begin{array}{ll}0.000736 & 0.000734\end{array}$

$\begin{array}{lll}0.000738 & 0.000737\end{array}$

$0.000741 \quad 0.000739$

$\begin{array}{lll}0.000742 & 0.000742\end{array}$

$\begin{array}{lll}0.000744 & 0.000744\end{array}$

$\begin{array}{lll}0.000746 & 0.000747\end{array}$

$\begin{array}{lll}0.000748 & 0.000749\end{array}$

$0.000750 \quad 0.000752$

$\begin{array}{lll}0.000752 & 0.000754\end{array}$

$0.000724 \quad 0.000725$

$0.000729 \quad 0.000728$

$0.000732 \quad 0.000731$
App Vol Square of Inconsistent Inconsistent (Dens rel - Apparent Density of calc / Residual / Residual / Density based on based on

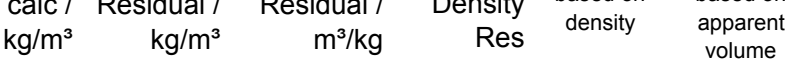

$1)^{\star} 10^{\wedge} 4$ molal volume water $/ \mathrm{kg} / \mathrm{m}^{3}$

$\begin{array}{llll}1000.7 & -0.4142 & 0.000008 & 0.172\end{array}$

$\begin{array}{llll}1007.5 & 0.4307 & -0.000006 & 0.185\end{array}$

$\begin{array}{llll}1014.0 & 0.2052 & -0.000002 & 0.042\end{array}$

$\begin{array}{llll}026.0 & -0.1081 & 0.000001 & 0.012\end{array}$

$\begin{array}{llll}1026.5 & -0.0080 & 0.000000 & 0.000\end{array}$

$\begin{array}{llll}1032.2 & -0.0961 & 0.000001 & 0.009\end{array}$

$\begin{array}{llll}1036.8 & 0.0516 & 0.000000 & 0.003\end{array}$

$\begin{array}{llll}1037.8 & -0.1428 & 0.000001 & 0.020\end{array}$

$\begin{array}{llll}1043.4 & -0.3462 & 0.000002 & 0.120\end{array}$

$\begin{array}{llll}1046.5 & -1.7843 & 0.000008 & 3.184\end{array}$

$\begin{array}{llll}1049.0 & -0.3044 & 0.000001 & 0.093\end{array}$

$\begin{array}{llll}1054.5 & -0.2154 & 0.000001 & 0.046\end{array}$

$\begin{array}{llll}060.0 & -0.1772 & 0.000001 & 0.031\end{array}$

$\begin{array}{llll}1065.4 & 0.0120 & 0.000000 & 0.000\end{array}$

$\begin{array}{llll}1070.7 & 0.1544 & 0.000000 & 0.024\end{array}$

$\begin{array}{llll}1076.0 & 0.4518 & -0.000001 & 0.204\end{array}$

$\begin{array}{llll}081.3 & 0.7062 & -0.000002 & 0.499\end{array}$

$1024.2 \quad-0.2734 \quad 0.000002 \quad 0.075$

$\begin{array}{llll}1029.9 & -0.2771 & 0.000002 & 0.077\end{array}$

$\begin{array}{llll}1035.5 & -0.2374 & 0.000001 & 0.056\end{array}$

$\begin{array}{llll}041.2 & -0.2522 & 0.000001 & 0.064\end{array}$

$\begin{array}{llll}1046.7 & -0.3196 & 0.000001 & 0.102\end{array}$

$\begin{array}{llll}1052.2 & -0.2375 & 0.000001 & 0.056\end{array}$

$\begin{array}{llll}1057.7 & -0.1038 & 0.000000 & 0.011\end{array}$

$\begin{array}{llll}1063.1 & -0.0166 & 0.000000 & 0.000\end{array}$

$\begin{array}{llll}1068.5 & 0.2263 & -0.000001 & 0.051\end{array}$

$\begin{array}{llll}1073.8 & 0.6269 & -0.000002 & 0.393\end{array}$

$\begin{array}{llll}1079.0 & 0.8872 & -0.000002 & 0.787\end{array}$

$\begin{array}{llll}1021.7 & -0.1495 & 0.000001 & 0.022\end{array}$

$\begin{array}{lll}1027.5 & -0.2779 & 0.000002\end{array}$

$\begin{array}{llll}1033.2 & -0.3611 & 0.000002 & 0.130\end{array}$

$\begin{array}{llll}1038.8 & -0.3968 & 0.000002 & 0.157\end{array}$

$\begin{array}{llll}1044.4 & -0.3830 & 0.000002 & 0.147\end{array}$

$\begin{array}{llll}1049.9 & -0.5175 & 0.000002 & 0.268\end{array}$

$\begin{array}{llll}1055.4 & -0.1983 & 0.000001 & 0.039\end{array}$

$\begin{array}{llll}1060.8 & -0.0232 & 0.000000 & 0.001\end{array}$

$\begin{array}{llll}1066.2 & 0.1100 & 0.000000 & 0.012\end{array}$

$\begin{array}{llll}1071.5 & 0.4032 & -0.000001 & 0.163\end{array}$

$\begin{array}{llll}076.7 & 0.7588 & -0.000002 & 0.576\end{array}$

$\begin{array}{llll}1081.9 & 1.0787 & -0.000003 & 1.164\end{array}$

$\begin{array}{llll}1019.2 & 0.1568 & -0.000001 & 0.025\end{array}$

$\begin{array}{llll}1025.0 & -0.2056 & 0.000001 & 0.042\end{array}$

$\begin{array}{llll}1030.7 & -0.2210 & 0.000001 & 0.049\end{array}$
$38.7 \quad 985.6952$

985.6952

985.6952

985.6952

985.6952

985.6952

985.6952

985.6952

985.6952

985.6952

985.6952

985.6952

985.6952

985.6952

985.6952

985.6952

985.6952

983.1989

983.1989

983.1989

983.1989

983.1989

983.1989

983.1989

983.1989

983.1989

983.1989

983.1989

980.5548

980.5548

980.5548

980.5548

980.5548

980.5548

980.5548

980.5548

980.5548

980.5548

980.5548

980.5548

977.7696

977.7696

977.7696 
molality molarity

$\begin{array}{crrc}\mathbf{t} /{ }^{\circ} \mathrm{C} \text { mass frac } & \begin{array}{r}\text { Density } \\ \text { exp } /\end{array} & \text { Ref } \\ & & \mathrm{kg} / \mathrm{m}^{3} & \\ & & & \\ 70 & 0.2000 & 1036.1 & (106) \\ 70 & 0.2200 & 1041.6 & (106) \\ 70 & 0.2400 & 1047.2 & (106) \\ 70 & 0.2600 & 1052.8 & (106) \\ 70 & 0.2800 & 1058.4 & (106) \\ 70 & 0.3000 & 1064.0 & (106) \\ 70 & 0.3200 & 1069.6 & (106) \\ 70 & 0.3400 & 1075.2 & (106) \\ 70 & 0.3600 & 1080.8 & (106) \\ 75 & 0.1400 & 1016.5 & (106) \\ 75 & 0.1600 & 1022.3 & (106) \\ 75 & 0.1800 & 1028.0 & (106) \\ 75 & 0.2000 & 1033.6 & (106) \\ 75 & 0.2200 & 1039.2 & (106) \\ 75 & 0.2400 & 1044.9 & (106) \\ 75 & 0.2600 & 1050.5 & (106) \\ 75 & 0.2800 & 1056.2 & (106) \\ 75 & 0.3000 & 1061.7 & (106) \\ 75 & 0.3200 & 1067.4 & (106) \\ 75 & 0.3400 & 1073.0 & (106) \\ 75 & 0.3600 & 1078.6 & (106) \\ 80 & 0.01 & 974.9 & (90) \\ 80 & 0.02 & 978.0 & (90) \\ 80 & 0.04 & 984.2 & (90) \\ 80 & 0.08 & 996.3 & (90) \\ 80 & 0.12 & 1008.1 & (90) \\ 80 & 0.1400 & 1014.0 & (106) \\ 80 & 0.16 & 1019.8 & (90) \\ 80 & 0.1600 & 1019.8 & (106) \\ 80 & 0.1800 & 1025.5 & (106) \\ 80 & 0.20 & 1031.2 & (90) \\ 80 & 0.2000 & 1031.2 & (106) \\ 80 & 0.2200 & 1036.9 & (106) \\ 80 & 0.24 & 1042.6 & (90) \\ 80 & 0.2400 & 1042.6 & (106) \\ 80 & 0.2600 & 1048.2 & (106) \\ 80 & 0.2800 & 1053.8 & (106) \\ 80 & 0.3000 & 1059.5 & (106) \\ 80 & 0.3200 & 1065.2 & (106) \\ 80 & 0.3400 & 1070.8 & (106) \\ 80 & 0.3600 & 1076.4 & (106) \\ 80 & 0.3800 & 1082.0 & (106) \\ 85 & 0.1400 & 1011.8 & (106)\end{array}$

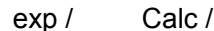

$$
\mathrm{m}^{3} / \mathrm{kg}
$$

Calc /
$\mathrm{m}^{3} / \mathrm{kg}$

$0.000735 \quad 0.000734$

$\begin{array}{lll}0.000738 & 0.000736\end{array}$

$\begin{array}{lll}0.000740 & 0.000739\end{array}$

$\begin{array}{lll}0.000742 & 0.000741\end{array}$

$\begin{array}{lll}0.000744 & 0.000744\end{array}$

$\begin{array}{ll}0.000746 & 0.000747\end{array}$

$0.000748 \quad 0.000749$

$0.000750 \quad 0.000752$

$0.000752 \quad 0.000755$

$\begin{array}{lll}0.000726 & 0.000724\end{array}$

$\begin{array}{ll}0.000728 & 0.000727\end{array}$

$\begin{array}{lll}0.000731 & 0.000730\end{array}$

$0.000734 \quad 0.000733$

$\begin{array}{lll}0.000737 & 0.000735\end{array}$

0.0007390 .000738

$0.000742 \quad 0.000741$

$0.000744 \quad 0.000744$

$\begin{array}{lll}0.000746 & 0.000746\end{array}$

$0.000748 \quad 0.000749$

$0.000750 \quad 0.000752$

$0.000752 \quad 0.000754$

$\begin{array}{lll}0.000702 & 0.000704\end{array}$

$0.000703 \quad 0.000706$

$0.000705 \quad 0.000709$

$0.000713 \quad 0.000714$

$0.000720 \quad 0.000720$

$0.000723 \quad 0.000723$

$0.000726 \quad 0.000726$

$0.000726 \quad 0.000726$

$0.000730 \quad 0.000729$

$\begin{array}{lll}0.000733 & 0.000732\end{array}$

$0.000733 \quad 0.000732$

$0.000735 \quad 0.000734$

$0.000738 \quad 0.000737$

$\begin{array}{lll}0.000738 & 0.000737\end{array}$

$\begin{array}{lll}0.000741 & 0.000740\end{array}$

$0.000743 \quad 0.000743$

$\begin{array}{lll}0.000745 & 0.000746\end{array}$

$0.000747 \quad 0.000748$

$0.000749 \quad 0.000751$

$0.000751 \quad 0.000754$

$\begin{array}{ll}0.000753 & 0.000757\end{array}$

$\begin{array}{lll}0.000718 & 0.000721\end{array}$
App Vol Square of Inconsistent Inconsistent (Dens rel - Apparent Density of calc / Residual / Residual / Density based on based on 1$)^{\star} 10^{\wedge} 4$ molal volume $\mathrm{kg} / \mathrm{m}^{3} \quad \mathrm{~kg} / \mathrm{m}^{3} \quad \mathrm{~m}^{3} / \mathrm{kg} \quad$ Res density $\begin{gathered}\text { apparent } \\ \text { volume }\end{gathered}$

$\begin{array}{llll}1036.4 & -0.2871 & 0.000001 & 0.082\end{array}$

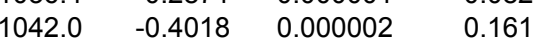

$\begin{array}{llll}1047.6 & -0.3629 & 0.000001 & 0.132\end{array}$

$\begin{array}{llll}053.1 & -0.2682 & 0.000001 & 0.072\end{array}$

$\begin{array}{llll}1058.5 & -0.1154 & 0.000000 & 0.013\end{array}$

$\begin{array}{llll}1063.9 & 0.0976 & 0.000000 & 0.010\end{array}$

$1069.2 \quad 0.3730 \quad-0.000001 \quad 0.139$

$\begin{array}{llll}1074.5 & 0.7130 & -0.000002 & 0.508\end{array}$

$\begin{array}{llll}1079.7 & 1.1199 & -0.000003 & 1.254\end{array}$

$\begin{array}{llll}1016.7 & -0.1607 & 0.000001 & 0.026\end{array}$

$\begin{array}{llll}1022.5 & -0.1663 & 0.000001 & 0.028\end{array}$

$\begin{array}{llll}1028.2 & -0.2234 & 0.000001 & 0.050\end{array}$

$\begin{array}{llll}1033.9 & -0.3296 & 0.000002 & 0.109\end{array}$

$\begin{array}{llll}1039.6 & -0.3826 & 0.000002 & 0.146\end{array}$

$\begin{array}{llll}1045.2 & -0.2803 & 0.000001 & 0.079\end{array}$

$\begin{array}{llll}1050.7 & -0.2202 & 0.000001 & 0.048\end{array}$

$\begin{array}{llll}056.2 & -0.0002 & 0.000000 & 0.000\end{array}$

$\begin{array}{llll}1061.6 & 0.0822 & 0.000000 & 0.007\end{array}$

$\begin{array}{llll}1067.0 & 0.4290 & -0.000001 & 0.184\end{array}$

$\begin{array}{llll}1072.3 & 0.7428 & -0.000002 & 0.552\end{array}$

$\begin{array}{llll}077.5 & 1.1256 & -0.000003 & 1.267\end{array}$

$\begin{array}{llll}974.9 & 0.0268 & -0.000003 & 0.001\end{array}$

$\begin{array}{llll}977.9 & 0.0595 & -0.000003 & 0.004\end{array}$

$\begin{array}{llll}984.0 & 0.1506 & -0.000004 & 0.023\end{array}$

$\begin{array}{llll}996.2 & 0.1433 & -0.000002 & 0.021\end{array}$

$\begin{array}{llll}1008.1 & -0.0016 & 0.000000 & 0.000\end{array}$

$\begin{array}{llll}1014.0 & -0.0073 & 0.000000 & 0.000\end{array}$

$\begin{array}{llll}1019.9 & -0.0655 & 0.000000 & 0.004\end{array}$

$\begin{array}{llll}1019.9 & -0.0655 & 0.000000 & 0.004\end{array}$

$\begin{array}{llll}025.7 & -0.1737 & 0.000001 & 0.030\end{array}$

$\begin{array}{llll}1031.4 & -0.2297 & 0.000001 & 0.053\end{array}$

$\begin{array}{llll}1031.4 & -0.2297 & 0.000001 & 0.053\end{array}$

$\begin{array}{llll}1037.1 & -0.2311 & 0.000001 & 0.053\end{array}$

$\begin{array}{llll}042.8 & -0.1754 & 0.000001 & 0.031\end{array}$

$\begin{array}{llll}1042.8 & -0.1754 & 0.000001 & 0.031\end{array}$

$\begin{array}{llll}1048.4 & -0.1603 & 0.000001 & 0.026\end{array}$

$\begin{array}{llll}1053.9 & -0.0835 & 0.000000 & 0.007\end{array}$

$\begin{array}{llll}059.3 & 0.1575 & 0.000000 & 0.025\end{array}$

$\begin{array}{llll}0.74 & 0.4650 & -0.000001 & 0.216\end{array}$

$\begin{array}{llll}1070.1 & 0.7414 & -0.000002 & 0.550\end{array}$

$\begin{array}{llll}1075.3 & 1.0891 & -0.000003 & 1.186\end{array}$

$\begin{array}{llll}1080.5 & 1.5104 & -0.000003 & 2.281\end{array}$

$\begin{array}{llll}1011.3 & 0.5123 & -0.000004 & 0.262\end{array}$
977.7696

977.7696

977.7696

977.7696

977.7696

977.7696

977.7696

977.7696

977.7696

974.849

974.849

974.849

974.849

974.849

974.849

974.849

974.849

974.849

974.849

974.849

974.849

971.7978

971.7978

971.7978

971.7978

971.7978

971.7978

971.7978

971.7978

971.7978

971.7978

971.7978

971.7978

971.7978

971.7978

971.7978

971.7978

971.7978

971.7978

971.7978

971.7978

971.7978

968.6203 
molality molarity

$\begin{array}{rrrr}\mathbf{t} /{ }^{\circ} \mathrm{C} \text { mass frac } & \begin{array}{r}\text { Density } \\ \text { exp } /\end{array} & \text { Ref } \\ & & \mathrm{kg} / \mathrm{m}^{3} & \\ & & & \\ 85 & 0.1600 & 1017.4 & (106) \\ 85 & 0.1800 & 1023.2 & (106) \\ 85 & 0.2000 & 1028.8 & (106) \\ 85 & 0.2200 & 1034.5 & (106) \\ 85 & 0.2400 & 1040.1 & (106) \\ 85 & 0.2600 & 1045.8 & (106) \\ 85 & 0.2800 & 1051.4 & (106) \\ 85 & 0.3000 & 1057.0 & (106) \\ 85 & 0.3200 & 1062.5 & (106) \\ 85 & 0.3400 & 1068.2 & (106) \\ 85 & 0.3600 & 1074.8 & (106) \\ 85 & 0.3800 & 1079.4 & (106) \\ 90 & 0.1400 & 1009.4 & (106) \\ 90 & 0.1600 & 1016.0 & (106) \\ 90 & 0.1800 & 1020.7 & (106) \\ 90 & 0.2000 & 1026.4 & (106) \\ 90 & 0.2200 & 1032.1 & (106) \\ 90 & 0.2400 & 1037.7 & (106) \\ 90 & 0.2600 & 1043.3 & (106) \\ 90 & 0.2800 & 1048.9 & (106) \\ 90 & 0.3000 & 1054.5 & (106) \\ 90 & 0.3200 & 1060.1 & (106) \\ 90 & 0.3400 & 1065.7 & (106) \\ 90 & 0.3600 & 1071.3 & (106) \\ 90 & 0.3800 & 1076.8 & (106) \\ 90 & 0.4000 & 1082.4 & (106) \\ 95 & 0.1400 & 1007.1 & (106) \\ 95 & 0.1600 & 1013.7 & (106) \\ 95 & 0.1800 & 1018.4 & (106) \\ 95 & 0.2000 & 1024.9 & (106) \\ 95 & 0.2200 & 1029.5 & (106) \\ 95 & 0.2400 & 1035.2 & (106) \\ 95 & 0.2600 & 1040.8 & (106) \\ 95 & 0.2800 & 1046.4 & (106) \\ 95 & 0.3000 & 1052.0 & (106) \\ 95 & 0.3200 & 1057.6 & (106) \\ 95 & 0.3400 & 1063.2 & (106) \\ 95 & 0.3800 & 1074.3 & (106) \\ 95 & 0.4000 & 1079.9 & (106) \\ 100 & 0.01 & 961.7 & (90) \\ 100 & 0.02 & 965.1 & (90) \\ 100 & 0.04 & 971.8 & (90) \\ 100 & 0.08 & 984.9 & (90)\end{array}$

\section{App vol App vol}

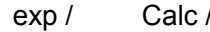

$$
\mathrm{m}^{3} / \mathrm{kg}
$$
$\mathrm{m}^{3} / \mathrm{kg}$

$0.000723 \quad 0.000724$

$\begin{array}{ll}0.000726 & 0.000727\end{array}$

$0.000730 \quad 0.000730$

$0.000734 \quad 0.000733$

$\begin{array}{lll}0.000737 & 0.000736\end{array}$

$\begin{array}{lll}0.000739 & 0.000739\end{array}$

$0.000742 \quad 0.000742$

$\begin{array}{lll}0.000745 & 0.000744\end{array}$

$\begin{array}{lll}0.000747 & 0.000747\end{array}$

$0.000749 \quad 0.000750$

$0.000749 \quad 0.000753$

$0.000754 \quad 0.000756$

$0.000713 \quad 0.000719$

$\begin{array}{lll}0.000713 & 0.000722\end{array}$

$0.000724 \quad 0.000725$

$0.000728 \quad 0.000728$

$0.000731 \quad 0.000731$

$\begin{array}{lll}0.000735 & 0.000734\end{array}$

$0.000738 \quad 0.000737$

$\begin{array}{lll}0.000741 & 0.000740\end{array}$

$0.000744 \quad 0.000743$

$0.000746 \quad 0.000746$

$\begin{array}{lll}0.000749 & 0.000749\end{array}$

$0.000751 \quad 0.000752$

$0.000754 \quad 0.000755$

$0.000756 \quad 0.000757$

$0.000706 \quad 0.000716$

$0.000708 \quad 0.000720$

$0.000719 \quad 0.000723$

$0.000720 \quad 0.000726$

$\begin{array}{lll}0.000729 & 0.000729\end{array}$

$0.000733 \quad 0.000732$

$0.000736 \quad 0.000735$

$0.000740 \quad 0.000738$

$\begin{array}{lll}0.000743 & 0.000741\end{array}$

$\begin{array}{lll}0.000746 & 0.000744\end{array}$

$\begin{array}{lll}0.000748 & 0.000747\end{array}$

$0.000753 \quad 0.000753$

$\begin{array}{ll}0.000756 & 0.000756\end{array}$

$0.000681 \quad 0.000693$

$\begin{array}{lll}0.000679 & 0.000694\end{array}$

$\begin{array}{lll}0.000683 & 0.000697\end{array}$

$\begin{array}{lll}0.000692 & 0.000704\end{array}$
App Vol Square of Inconsistent Inconsistent (Dens rel - Apparent Density of calc / Residual / Residual / Density based on based on 1$)^{\star} 10^{\wedge} 4$ molal volume

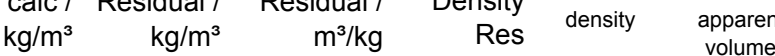

$\begin{array}{llll}1017.2 & 0.1922 & -0.000001 & 0.037\end{array}$

$\begin{array}{llll}1023.1 & 0.1231 & -0.000001 & 0.015\end{array}$

$\begin{array}{llll}1028.9 & -0.0926 & 0.000000 & 0.009\end{array}$

$\begin{array}{llll}1034.7 & -0.1522 & 0.000001 & 0.023\end{array}$

$\begin{array}{llll}1040.4 & -0.2535 & 0.000001 & 0.064\end{array}$

$\begin{array}{llll}1046.0 & -0.1939 & 0.000001 & 0.038\end{array}$

$\begin{array}{llll}1051.6 & -0.1709 & 0.000001 & 0.029\end{array}$

$\begin{array}{llll}1057.1 & -0.0820 & 0.000000 & 0.007\end{array}$

$\begin{array}{llll}1062.5 & -0.0249 & 0.000000 & 0.001\end{array}$

$\begin{array}{llll}1067.9 & 0.3030 & -0.000001 & 0.092\end{array}$

$\begin{array}{llll}1073.2 & 1.6042 & -0.000004 & 2.573\end{array}$

$\begin{array}{llll}1078.4 & 0.9811 & -0.000002 & 0.962\end{array}$

$\begin{array}{llll}008.5 & 0.8942 & -0.000006 & 0.800\end{array}$

$\begin{array}{llll}1014.5 & 1.5025 & -0.000009 & 2.258\end{array}$

$\begin{array}{llll}1020.4 & 0.2628 & -0.000001 & 0.069\end{array}$

$\begin{array}{llll}1026.3 & 0.0775 & 0.000000 & 0.006\end{array}$

$\begin{array}{llll}0.032 .2 & -0.0507 & 0.000000 & 0.003\end{array}$

$\begin{array}{llll}1037.9 & -0.2192 & 0.000001 & 0.048\end{array}$

$\begin{array}{llll}1043.6 & -0.3256 & 0.000001 & 0.106\end{array}$

$\begin{array}{llll}1049.3 & -0.3673 & 0.000001 & 0.135\end{array}$

$\begin{array}{llll}0.34 .8 & -0.3416 & 0.000001 & 0.117\end{array}$

$\begin{array}{llll}1060.3 & -0.2459 & 0.000001 & 0.060\end{array}$

$\begin{array}{llll}1065.8 & -0.0778 & 0.000000 & 0.006\end{array}$

$\begin{array}{llll}1071.1 & 0.1653 & 0.000000 & 0.027\end{array}$

$\begin{array}{llll}076.4 & 0.3860 & -0.000001 & 0.149\end{array}$

$\begin{array}{llll}1081.6 & 0.7868 & -0.000002 & 0.619\end{array}$

$\begin{array}{llll}1005.7 & 1.4349 & -0.000010 & 2.059\end{array}$

$\begin{array}{llll}1011.7 & 1.9621 & -0.000012 & 3.850\end{array}$

$\begin{array}{llll}1017.8 & 0.6418 & -0.000003 & 0.412\end{array}$

$\begin{array}{llll}1023.7 & 1.1768 & -0.000006 & 1.385\end{array}$

$\begin{array}{llll}1029.6 & -0.1302 & 0.000001 & 0.017\end{array}$

$\begin{array}{llll}1035.5 & -0.2767 & 0.000001 & 0.077\end{array}$

$\begin{array}{llll}1041.3 & -0.4599 & 0.000002 & 0.211\end{array}$

$\begin{array}{llll}047.0 & -0.5771 & 0.000002 & 0.333\end{array}$

$\begin{array}{llll}1052.6 & -0.6257 & 0.000002 & 0.391\end{array}$

$\begin{array}{llll}1058.2 & -0.6030 & 0.000002 & 0.364\end{array}$

$\begin{array}{llll}1063.7 & -0.5063 & 0.000001 & 0.256\end{array}$

$\begin{array}{llll}1074.5 & -0.1803 & 0.000000 & 0.032\end{array}$

$\begin{array}{llll}079.7 & 0.1543 & 0.000000 & 0.024\end{array}$

$\begin{array}{llll}961.6 & 0.1023 & -0.000011 & 0.010\end{array}$

$\begin{array}{llll}964.8 & 0.2766 & -0.000015 & 0.077\end{array}$

$\begin{array}{llll}971.2 & 0.5512 & -0.000015 & 0.304\end{array}$

$\begin{array}{llll}984.0 & 0.9140 & -0.000012 & 0.835\end{array}$
968.6203 968.6203 968.6203 968.6203 968.6203 968.6203 968.6203 968.6203

968.6203

968.6203

968.6203

968.6203

965.3201

965.3201

965.3201

965.3201

965.3201

965.3201

965.3201

965.3201

965.3201

965.3201

965.3201

965.3201

965.3201

965.3201

961.9004

961.9004

961.9004

961.9004

961.9004

961.9004

961.9004

961.9004

961.9004

961.9004

961.9004

961.9004

961.9004

958.3637

958.3637

958.3637

958.3637 
molality molarity

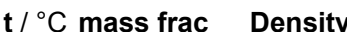

$t /{ }^{\circ} \mathrm{C}$ mass frac Density $\exp /$
$\mathrm{kg} / \mathrm{m}^{3}$

Ref

$\begin{array}{rrrc}100 & 0.12 & 997.5 & (90) \\ 100 & 0.1400 & 1004.6 & (106)\end{array}$

100
100
100
100

0.16

1009.6

1009.6

0.1600

0.20

1015.5

$0.2000 \quad 1021.3$

0.2200

0.24

0.2400

0.2600

0.3000

0.3200

0.3400

0.3600

0.3800

$100 \quad 0.4000$

1071.7

(106)
App vol App vol

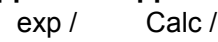

$\mathrm{m}^{3} / \mathrm{kg}$

$0.000700 \quad 0.000713$

$\begin{array}{ll}0.000712 & 0.000717\end{array}$

$\begin{array}{ll}0.000712 & 0.000717\end{array}$

$0.000717 \quad 0.000720$

$0.000722 \quad 0.000723$

$0.000722 \quad 0.000723$

$\begin{array}{lll}0.000726 & 0.000726\end{array}$

$\begin{array}{ll}0.000730 & 0.000729\end{array}$

$0.000730 \quad 0.000729$

$0.000734 \quad 0.000732$

$\begin{array}{ll}0.000738 & 0.000735\end{array}$

$\begin{array}{ll}0.000742 & 0.000739\end{array}$

$\begin{array}{lll}0.000745 & 0.000742\end{array}$

$0.000748 \quad 0.000745$

$0.000751 \quad 0.000748$

$0.000753 \quad 0.000751$

$0.000755 \quad 0.000754$
$0.000702 \quad 0.000710$
Density Density

calc/ Residual /

996.6

1002.8

1008.9

1008.9

1015.0

1021.1

1021.1
1021.1
1027.1

1027.1
1033.0

1033.0

1038.9

1044.7

1050.4

1056.1
1061.7

1067.2

1072.6 $\mathrm{kg} / \mathrm{m}^{3} \quad \mathrm{~kg} / \mathrm{m}^{3}$
Avg - 4std

Avg + 4std
0.9456

1.8315

0.6677

0.4569

0.2020

0.2020

0.0056

$-0.3294$

$-0.3294$

-0.6004
-0.8043

$-1.0386$

$-1.1004$

$-1.0869$

$-1.0953$

$-0.9228$

-0.6668
0.1037

0.5927

$-2.2669$

2.4744
App Vol Square of Inconsistent Inconsistent (Dens rel - Apparent Density of Ridual / Density based on based on 1)*10^4 molal volume water $/ \mathrm{kg} / \mathrm{m}^{3}$ $\mathrm{m}^{3} / \mathrm{kg}$ Res density volume

958.3637 958.3637 958.3637 958.3637 958.3637 958.3637 958.3637 958.3637 958.3637 958.3637 958.3637 958.3637 958.3637 958.3637 958.3637 958.3637 958.3637 958.3637

Curve fit for the model App vol $=\left(w+c 2+c 3^{*} t{ }^{\circ} \mathrm{C}\right) /\left(c 0^{*} w+c 1\right) / e^{\wedge}\left(0.000001^{*}\left(t^{\circ} \mathrm{C}+c 4\right)^{\wedge} 2\right.$

$\mathrm{cO}$

$\begin{array}{ll}\mathrm{c} 1 & 6.561521 \\ \mathrm{c} 2 & 89.77197 \\ \mathrm{c} 3 & 4.902429 \\ \mathrm{c} 4 & -0.01657 \\ \mathrm{M} & -2089.32\end{array}$

Min

$\operatorname{Min} T$

Min $w$

0

100

Max w

5.35E-05

Avg dens res $\quad 0.1037$

Std dens res $\quad 0.59267$

Avg app vol res *10^3 $\quad-0.00599$

Std app vol res *10^3 0.017782

No of points in corr

387

No of inconsistent poir

2

Inconsistent data not used (Residual greater than average $+\mathbf{+} \mathbf{4}$ standard deviations)

$\begin{array}{lllllll}5.071 & 25 & 0.2540 & 1068.00 & (40) & 0.000741 & 0.000731\end{array}$

$\begin{array}{llllll}95 & 0.3600 & 1087.0 & (106) & 0.000707 & 0.000750\end{array}$

1070.7

$-2.6848$

0.000009

7.208

997.0449

961.9004 
Density of aqueous solutions of NH4NO3

References (1) Adams, L. H. Equilibrium in Binary Systems Under Pressure. III. The Influence of Pressure on the Solubility of Ammonium Nitrate in Water at $25^{\circ} \mathrm{C}$. J. Am. Chem. Soc. 1932, 54, 4520-4537.

(10) Campbell, A. N.; Kartzmark, E. M. The Conductances of Strong Solutions of Strong Electrolytes at $95^{\circ} \mathrm{C}$. Can. J. Chem. 1952, $30,128-$ 134.

(90) Perry, R. H.; Green, D. W. Perry's Chemical Engineers' Handbook; McGraw Hill: New York, 7th edition, 1997.

(108) Roux, A.; Musbally, G. M.; Perron, G.; Singh, P. P.; Woolley, E. M.; Hepler, L. G. Apparent Molal Heat Capacities and Volumes of Aqueous Electrolytes at $25^{\circ} \mathrm{C}: \mathrm{NaClO} 3, \mathrm{NaClO} 4, \mathrm{NaNO} 3, \mathrm{NaBrO} 3, \mathrm{NaIO} 3, \mathrm{KClO} 3, \mathrm{KBrO} 3, \mathrm{KIO}, \mathrm{NH} 4 \mathrm{NO} 3, \mathrm{NH} 4 \mathrm{Cl}$, and $\mathrm{NH} 4 \mathrm{ClO} 4 . \mathrm{Can}$. J. Chem. 1978, 56, 24-28.

(110) Roy, Mahendra Nath; Jha, Anupam; Choudhury, Ankan, Densities, Viscosities and Adiabatic Compressibilities of Some Mineral Salts in Water at Different Temperatures, J. Chem. Eng. Data 2004, 49, 291-296.

(120) Sharma R. C.; Gaur, H. C. Densities and Molar Volumes of the Ammonium Nitrate-Water System. J. Chem. Eng. Data 1977, 22 , 41-44.

\begin{tabular}{|c|c|c|c|c|c|c|c|c|c|c|c|c|c|c|c|}
\hline \multirow{25}{*}{\multicolumn{2}{|c|}{ molality }} & \multicolumn{2}{|c|}{$\mathrm{t} /{ }^{\circ} \mathrm{C}$ mass frac } & $\begin{array}{r}\text { Density } \\
\exp / \\
\mathrm{kg} / \mathrm{m}^{3}\end{array}$ & Ref & $\begin{array}{r}\text { App vol } \\
\exp / \mathrm{m}^{3} / \mathrm{kg}\end{array}$ & $\begin{array}{c}\text { App vol } \\
\text { Calc / } \\
\mathrm{m}^{3} / \mathrm{kg}\end{array}$ & $\begin{array}{r}\text { Density } \\
\text { calc / } \\
\mathrm{kg} / \mathrm{m}^{3}\end{array}$ & $\begin{array}{r}\text { Density } \\
\text { Residual / } \\
\mathrm{kg} / \mathrm{m}^{3}\end{array}$ & $\begin{array}{r}\text { App Vol } \\
\text { Residual / } \\
\mathrm{m}^{3} / \mathrm{kg}\end{array}$ & $\begin{array}{l}\text { Square of } \\
\text { Density } \\
\text { Residual }\end{array}$ & $\begin{array}{l}\text { Inconsistent } \\
\text { based on } \\
\text { density }\end{array}$ & $\begin{array}{c}\text { Inconsistent } \\
\text { based on } \\
\text { apparent } \\
\text { volume }\end{array}$ & $\begin{array}{c}\text { Molar ratio } \\
\text { water / } \\
\text { nitrate }\end{array}$ & $\begin{array}{r}\text { Density of } \\
\text { water } / \mathrm{kg} / \mathrm{m}^{3}\end{array}$ \\
\hline & & 0 & 0.0100 & 1004.30 & (90) & 0.000556 & 0.000567 & 1004.2 & 0.1134 & -0.000011 & 0.013 & & & & 999.8395 \\
\hline & & 0 & 0.0200 & 1008.80 & (90) & 0.000556 & 0.000569 & 1008.5 & 0.2615 & -0.000013 & 0.068 & & & & 999.8395 \\
\hline & & 0 & 0.0400 & 1017.80 & (90) & 0.000559 & 0.000572 & 1017.3 & 0.5395 & -0.000013 & 0.291 & & & & 999.8395 \\
\hline & & 0 & 0.0800 & 1035.80 & (90) & 0.000566 & 0.000578 & 1034.8 & 1.0016 & -0.000012 & 1.003 & & & & 999.8395 \\
\hline & & 0 & 0.1200 & 1053.90 & (90) & 0.000573 & 0.000583 & 1052.5 & 1.4003 & -0.000011 & 1.961 & & & & 999.8395 \\
\hline & & 0 & 0.1600 & 1072.10 & (90) & 0.000579 & 0.000588 & 1070.4 & 1.6926 & -0.000009 & 2.865 & & & & 999.8395 \\
\hline & & 0 & 0.2000 & 1090.50 & (90) & 0.000584 & 0.000593 & 1088.6 & 1.9387 & -0.000008 & 3.759 & & & & 999.8395 \\
\hline & & 0 & 0.2400 & 1109.00 & (90) & 0.000590 & 0.000597 & 1107.0 & 2.0010 & -0.000007 & 4.004 & & & & 999.8395 \\
\hline & & 0 & 0.2800 & 1127.70 & (90) & 0.000595 & 0.000601 & 1125.8 & 1.9432 & -0.000005 & 3.776 & & & & 999.8395 \\
\hline & & 0 & 0.3000 & 1137.10 & (90) & 0.000598 & 0.000602 & 1135.3 & 1.8334 & -0.000005 & 3.361 & & & & 999.8395 \\
\hline & & 0 & 0.4000 & 1186.20 & (90) & 0.000607 & 0.000611 & 1184.3 & 1.9032 & -0.000003 & 3.622 & & & & 999.8395 \\
\hline & & 0 & 0.5000 & 1238.00 & (90) & 0.000615 & 0.000618 & 1236.2 & 1.8130 & -0.000002 & 3.287 & & & & 999.8395 \\
\hline & & 10 & 0.0100 & 1003.90 & (90) & 0.000582 & 0.000577 & 1003.9 & -0.0469 & 0.000005 & 0.002 & & & & 999.6996 \\
\hline & & 10 & 0.0200 & 1008.20 & (90) & 0.000579 & 0.000579 & 1008.2 & -0.0005 & 0.000000 & 0.000 & & & & 999.6996 \\
\hline & & 10 & 0.0400 & 1016.80 & (90) & 0.000580 & 0.000581 & 1016.7 & 0.0706 & -0.000002 & 0.005 & & & & 999.6996 \\
\hline & & 10 & 0.0800 & 1034.00 & (90) & 0.000586 & 0.000587 & 1033.9 & 0.1047 & -0.000001 & 0.011 & & & & 999.6996 \\
\hline & & 10 & 0.1200 & 1051.50 & (90) & 0.000590 & 0.000592 & 1051.2 & 0.2586 & -0.000002 & 0.067 & & & & 999.6996 \\
\hline & & 10 & 0.1600 & 1069.10 & (90) & 0.000594 & 0.000596 & 1068.8 & 0.2915 & -0.000002 & 0.085 & & & & 999.6996 \\
\hline & & 10 & 0.2000 & 1087.00 & (90) & 0.000599 & 0.000600 & 1086.6 & 0.3654 & -0.000002 & 0.134 & & & & 999.6996 \\
\hline & & 10 & 0.2400 & 1105.10 & (90) & 0.000603 & 0.000604 & 1104.8 & 0.3444 & -0.000001 & 0.119 & & & & 999.6996 \\
\hline & & 10 & 0.2800 & 1123.40 & (90) & 0.000607 & 0.000607 & 1123.2 & 0.1938 & -0.000001 & 0.038 & & & & 999.6996 \\
\hline & & 10 & 0.3000 & 1132.70 & (90) & 0.000609 & 0.000609 & 1132.6 & 0.1344 & 0.000000 & 0.018 & & & & 999.6996 \\
\hline & & 10 & 0.4000 & 1181.00 & (90) & 0.000616 & 0.000617 & 1180.9 & 0.1306 & 0.000000 & 0.017 & & & & 999.6996 \\
\hline & & 10 & 0.5000 & 1232.00 & (90) & 0.000623 & 0.000623 & 1232.1 & -0.0645 & 0.000000 & 0.004 & & & & 999.6996 \\
\hline 0.05619 & & 25 & 0.0045 & 998.85 & (108) & 0.000599 & 0.000591 & 998.9 & -0.0371 & 0.000008 & 0.001 & & & & 997.0449 \\
\hline 0.05726 & & 25 & 0.0046 & 998.88 & (108) & 0.000599 & 0.000591 & 998.9 & -0.0349 & 0.000008 & 0.001 & & & & 997.0449 \\
\hline
\end{tabular}


molality molarity

0.07591

0.10587

0.14494

0.16235

0.21516

\section{$\mathrm{t} /{ }^{\circ} \mathrm{C}$ mass frac Density

$$
\exp /
$$

$\mathrm{kg} / \mathrm{m}^{3}$ $\mathrm{m}^{3} / \mathrm{kg}$ $\mathrm{m}^{3} / \mathrm{kg}$ $0.000600 \quad 0.000591$ $0.000595 \quad 0.000592$ $\begin{array}{lll}0.000597 & 0.000592\end{array}$ $0.000600 \quad 0.000592$ $\begin{array}{lll}0.000601 & 0.000592\end{array}$ $\begin{array}{lll}0.000600 & 0.000592\end{array}$ $0.000601 \quad 0.000593$ $\begin{array}{lll}0.000603 & 0.000595\end{array}$ $\begin{array}{lll}0.000603 & 0.000596\end{array}$ $0.000605 \quad 0.000600$ $0.000608 \quad 0.000602$ $\begin{array}{lll}0.000609 & 0.000604\end{array}$ $0.000612 \quad 0.000607$ $0.000612 \quad 0.000608$ $\begin{array}{lll}0.000615 & 0.000611\end{array}$ $0.000615 \quad 0.000611$ $0.000618 \quad 0.000614$ $0.000618 \quad 0.000615$ $0.000618 \quad 0.000615$ $\begin{array}{lll}0.000621 & 0.000617\end{array}$ $\begin{array}{lll}0.000622 & 0.000619\end{array}$ $0.000624 \quad 0.000622$ $0.000627 \quad 0.000625$ $\begin{array}{lll}0.000629 & 0.000628\end{array}$ $0.000632 \quad 0.000631$ $0.000635 \quad 0.000633$ $0.000633 \quad 0.000635$ $\begin{array}{lll}0.000637 & 0.000635\end{array}$ $0.000635 \quad 0.000637$ $\begin{array}{lll}0.000640 & 0.000637\end{array}$ $\begin{array}{lll}0.000640 & 0.000637\end{array}$ $0.000641 \quad 0.000638$ $\begin{array}{lll}0.000636 & 0.000637\end{array}$ $\begin{array}{lll}0.000638 & 0.000639\end{array}$ $0.000642 \quad 0.000641$ $\begin{array}{lll}0.000643 & 0.000642\end{array}$ $0.000638 \quad 0.000639$ $0.000640 \quad 0.000641$ $0.000644 \quad 0.000643$
Density Density App Vol Square of Inconsistent Inconsistent Molar ratio Density of calc / Residual / Residual / Density based on based on water / water $/ \mathrm{kg} / \mathrm{m}^{3}$ $\mathrm{kg} / \mathrm{m}^{3} \quad \mathrm{~kg} / \mathrm{m}^{3} \quad \mathrm{~m}^{3} / \mathrm{kg}$ Residual density apparent nitrate

$\begin{array}{llll}999.5 & -0.0332 & 0.000005 & 0.001\end{array}$

$\begin{array}{llll}1000.5 & -0.0742 & 0.000009 & 0.006\end{array}$

$\begin{array}{llll}1001.1 & -0.0346 & 0.000003 & 0.001\end{array}$

$\begin{array}{llll}1001.2 & -0.0509 & 0.000005 & 0.003\end{array}$

$\begin{array}{llll}1001.8 & -0.0985 & 0.000009 & 0.010\end{array}$

$\begin{array}{llll}1002.3 & -0.1129 & 0.000009 & 0.013\end{array}$

$\begin{array}{llll}1004.0 & -0.1344 & 0.000008 & 0.018\end{array}$

$\begin{array}{llll}1005.3 & -0.1656 & 0.000008 & 0.027\end{array}$

$\begin{array}{llll}1013.5 & -0.3236 & 0.000008 & 0.105\end{array}$

$\begin{array}{llll}1017.7 & -0.3619 & 0.000007 & 0.131\end{array}$

$\begin{array}{llll}1030.2 & -0.4733 & 0.000006 & 0.224\end{array}$

$\begin{array}{llll}1038.2 & -0.6930 & 0.000006 & 0.480\end{array}$

$\begin{array}{llll}1047.0 & -0.6343 & 0.000005 & 0.402\end{array}$

$\begin{array}{llll}1059.9 & -0.8851 & 0.000005 & 0.783\end{array}$

$\begin{array}{llll}1064.1 & -0.8442 & 0.000005 & 0.713\end{array}$

$\begin{array}{llll}1081.5 & -0.9381 & 0.000004 & 0.880\end{array}$

$\begin{array}{llll}1081.6 & -0.9717 & 0.000004 & 0.944\end{array}$

$\begin{array}{llll}1099.2 & -1.0496 & 0.000004 & 1.102\end{array}$

$\begin{array}{llll}1103.7 & -0.9505 & 0.000003 & 0.903\end{array}$

$\begin{array}{llll}1103.8 & -0.9600 & 0.000003 & 0.922\end{array}$

$\begin{array}{llll}1117.3 & -1.2113 & 0.000003 & 1.467\end{array}$

$\begin{array}{llll}1126.5 & -1.2835 & 0.000003 & 1.647\end{array}$

$\begin{array}{llll}1149.9 & -0.8750 & 0.000002 & 0.766\end{array}$

$\begin{array}{llll}1173.9 & -1.2155 & 0.000002 & 1.478\end{array}$

$\begin{array}{llll}1197.3 & -0.9498 & 0.000001 & 0.902\end{array}$

$\begin{array}{llll}1224.3 & -1.4333 & 0.000002 & 2.054\end{array}$

$\begin{array}{llll}1250.7 & -1.4758 & 0.000002 & 2.178\end{array}$

$\begin{array}{llll}1276.6 & 1.6050 & -0.000002 & 2.576\end{array}$

$\begin{array}{llll}1278.2 & -1.9637 & 0.000002 & 3.856\end{array}$

$\begin{array}{llll}1300.9 & 1.6612 & -0.000002 & 2.760\end{array}$

$\begin{array}{llll}1306.3 & -2.5925 & 0.000002 & 6.721\end{array}$

$\begin{array}{llll}1307.8 & -2.6337 & 0.000002 & 6.936\end{array}$

$\begin{array}{llll}1322.9 & -3.0207 & 0.000003 & 9.125\end{array}$

$\begin{array}{llll}1273.6 & 1.4398 & -0.000001 & 2.073\end{array}$

$\begin{array}{llll}1297.8 & 1.3539 & -0.000001 & 1.833\end{array}$

$\begin{array}{llll}1326.9 & -0.9060 & 0.000001 & 0.821\end{array}$

$\begin{array}{llll}1354.6 & -1.0316 & 0.000001 & 1.064\end{array}$

$\begin{array}{llll}1270.4 & 1.3643 & -0.000001 & 1.861\end{array}$

$\begin{array}{llll}1294.7 & 1.1211 & -0.000001 & 1.257\end{array}$

$\begin{array}{llll}1323.7 & -0.9999 & 0.000001 & 1.000\end{array}$
997.0449

997.0449

997.0449

997.0449

997.0449

997.0449

997.0449

997.0449

997.0449

997.0449

997.0449

997.0449

997.0449

997.0449

997.0449

997.0449

997.0449

997.0449

997.0449

997.0449

997.0449

997.0449

997.0449

997.0449

997.0449

997.0449

997.0449

$3 \quad 997.0449$ 997.0449

$2.5 \quad 997.0449$

997.0449

997.0449

997.0449

$3 \quad 995.6473$

2.5995 .6473

2995.6473

$1.6 \quad 995.6473$
3

$\begin{array}{ll}3 & 994.0319\end{array}$

$2.5 \quad 994.0319$

$2 \quad 994.0319$ 
molality molarity

$\begin{array}{crrr}\mathbf{t} /{ }^{\circ} \mathrm{C} \text { mass frac } & \begin{array}{r}\text { Density } \\ \text { exp } /\end{array} & \text { Ref } \\ & & \mathrm{kg} / \mathrm{m}^{3} & \\ & & \\ 35 & 0.7117 & 1337.30 & (120) \\ 35 & 0.7353 & 1350.30 & (120) \\ 40 & 0.0100 & 996.10 & (90) \\ 40 & 0.0200 & 1000.00 & (90) \\ 40 & 0.0400 & 1007.90 & (90) \\ 40 & 0.0800 & 1023.80 & (90) \\ 40 & 0.1200 & 1040.00 & (90) \\ 40 & 0.1600 & 1056.50 & (90) \\ 40 & 0.2000 & 1073.40 & (90) \\ 40 & 0.2400 & 1090.70 & (90) \\ 40 & 0.2800 & 1108.20 & (90) \\ 40 & 0.3000 & 1117.10 & (90) \\ 40 & 0.4000 & 1164.00 & (90) \\ 40 & 0.5000 & 1213.60 & (90) \\ 40 & 0.5970 & 1268.70 & (120) \\ 40 & 0.6400 & 1292.60 & (120) \\ 40 & 0.6897 & 1319.00 & (120) \\ 40 & 0.7117 & 1334.00 & (120) \\ 40 & 0.7353 & 1346.90 & (120) \\ 40 & 0.7605 & 1365.10 & (120) \\ 45 & 0.5970 & 1265.30 & (120) \\ 45 & 0.6400 & 1289.40 & (120) \\ 45 & 0.6897 & 1315.70 & (120) \\ 45 & 0.7117 & 1331.10 & (120) \\ 45 & 0.7353 & 1343.60 & (120) \\ 45 & 0.7605 & 1361.80 & (120) \\ 50 & 0.5970 & 1261.90 & (120) \\ 50 & 0.6400 & 1286.00 & (120) \\ 50 & 0.6897 & 1312.30 & (120) \\ 50 & 0.7117 & 1327.80 & (120) \\ 50 & 0.7353 & 1340.10 & (120) \\ 50 & 0.7605 & 1358.50 & (120) \\ 50 & 0.7874 & 1377.00 & (120) \\ 55 & 0.5970 & 1258.80 & (120) \\ 55 & 0.6400 & 1282.60 & (120) \\ 55 & 0.6897 & 1309.10 & (120) \\ 55 & 0.7117 & 1324.50 & (120) \\ 55 & 0.7353 & 1336.50 & (120) \\ 55 & 0.7605 & 1354.50 & (120) \\ 55 & 0.7874 & 1373.20 & (120)\end{array}$

App vol App vol Density Density App Vol Square of Inconsistent Inconsistent Molar ratio Density of

$\exp / \mathrm{m}^{3} / \mathrm{kg} \quad$ Calc/ calc/ Residual/ Residual / Density based on based on water / water $/ \mathrm{kg} / \mathrm{m}^{3}$ $\mathrm{m}^{3} / \mathrm{kg}$ $\mathrm{m}^{3} / \mathrm{kg}$ $0.000645 \quad 0.000644$ 0.0006150 .000606 $\begin{array}{lll}0.000616 & 0.000607\end{array}$ $0.000616 \quad 0.000609$ $\begin{array}{lll}0.000619 & 0.000612\end{array}$ $0.000622 \quad 0.000616$ $0.000625 \quad 0.000619$ $0.000627 \quad 0.000622$ $0.000629 \quad 0.000624$ $\begin{array}{lll}0.000631 & 0.000627\end{array}$ $0.000632 \quad 0.000628$ $0.000636 \quad 0.000633$ $0.000640 \quad 0.000638$ $0.000640 \quad 0.000641$ $0.000642 \quad 0.000643$ $0.000646 \quad 0.000645$ $0.000645 \quad 0.000645$ $0.000647 \quad 0.000646$ $\begin{array}{lll}0.000646 & 0.000647\end{array}$ $0.000642 \quad 0.000644$ $0.000644 \quad 0.000645$ $0.000648 \quad 0.000646$ $0.000647 \quad 0.000647$ $0.000649 \quad 0.000648$ $0.000648 \quad 0.000648$ $0.000644 \quad 0.000646$ $0.000646 \quad 0.000647$ $0.000649 \quad 0.000648$ $0.000648 \quad 0.000649$ $\begin{array}{ll}0.000650 & 0.000649\end{array}$ $0.000649 \quad 0.000650$ $0.000649 \quad 0.000651$ $\begin{array}{ll}0.000646 & 0.000647\end{array}$ $0.000648 \quad 0.000649$ $0.000651 \quad 0.000650$ $0.000650 \quad 0.000650$ $0.000652 \quad 0.000651$ $0.000651 \quad 0.000652$ $0.000651 \quad 0.000652$ $\mathrm{kg} / \mathrm{m}^{3} \quad \mathrm{~kg} / \mathrm{m}^{3} \quad \mathrm{~m}^{3} / \mathrm{kg}$ Residual density apparent nitrate

$\begin{array}{llll}1337.0 & 0.3208 & 0.000000 & 0.103\end{array}$

$\begin{array}{llll}1351.4 & -1.0998 & 0.000001 & 1.210\end{array}$

$\begin{array}{llll}996.2 & -0.0911 & 0.000009 & 0.008\end{array}$

$\begin{array}{llll}1000.2 & -0.1777 & 0.000009 & 0.032\end{array}$

$\begin{array}{llll}1008.2 & -0.2875 & 0.000007 & 0.083\end{array}$

$\begin{array}{llll}1024.4 & -0.5699 & 0.000007 & 0.325\end{array}$

$\begin{array}{llll}1040.8 & -0.7995 & 0.000006 & 0.639\end{array}$

$\begin{array}{llll}1057.5 & -1.0105 & 0.000006 & 1.021\end{array}$

$\begin{array}{llll}1074.5 & -1.1356 & 0.000005 & 1.290\end{array}$

$\begin{array}{llll}1091.9 & -1.2060 & 0.000004 & 1.454\end{array}$

$\begin{array}{llll}1109.7 & -1.4526 & 0.000004 & 2.110\end{array}$

$\begin{array}{llll}1118.7 & -1.5764 & 0.000004 & 2.485\end{array}$

$\begin{array}{llll}1165.5 & -1.4533 & 0.000003 & 2.112\end{array}$

$\begin{array}{llll}1215.4 & -1.7514 & 0.000002 & 3.067\end{array}$

$\begin{array}{llll}1267.2 & 1.4668 & -0.000002 & 2.152\end{array}$

$\begin{array}{llll}1291.4 & 1.1521 & -0.000001 & 1.327\end{array}$

$\begin{array}{llll}1320.4 & -1.4478 & 0.000001 & 2.096\end{array}$

$\begin{array}{llll}1333.7 & 0.2781 & 0.000000 & 0.077\end{array}$

$\begin{array}{llll}1348.1 & -1.2399 & 0.000001 & 1.537\end{array}$

$\begin{array}{llll}1363.9 & 1.2424 & -0.000001 & 1.543\end{array}$

$\begin{array}{llll}1264.0 & 1.3381 & -0.000001 & 1.790\end{array}$

$1288.2 \quad 1.2382-0.000001 \quad 1.533$

$\begin{array}{llll}1317.2 & -1.4574 & 0.000001 & 2.124\end{array}$

$\begin{array}{llll}1330.4 & 0.6658 & -0.000001 & 0.443\end{array}$

$\begin{array}{llll}1344.9 & -1.2584 & 0.000001 & 1.583\end{array}$

$\begin{array}{llll}1360.6 & 1.2134 & -0.000001 & 1.472\end{array}$

$\begin{array}{llll}1260.6 & 1.2701 & -0.000001 & 1.613\end{array}$

$\begin{array}{llll}1284.8 & 1.1723 & -0.000001 & 1.374\end{array}$

$\begin{array}{llll}1313.8 & -1.5349 & 0.000001 & 2.356\end{array}$

$\begin{array}{llll}1327.1 & 0.6779 & -0.000001 & 0.459\end{array}$

$\begin{array}{llll}1341.6 & -1.4608 & 0.000001 & 2.134\end{array}$

$\begin{array}{llll}1357.3 & 1.1912 & -0.000001 & 1.419\end{array}$

$\begin{array}{llll}1374.6 & 2.4457 & -0.000002 & 5.982\end{array}$

$\begin{array}{llll}1257.2 & 1.5564 & -0.000002 & 2.422\end{array}$

$\begin{array}{llll}1281.5 & 1.1485 & -0.000001 & 1.319\end{array}$

$\begin{array}{llll}1310.5 & -1.3858 & 0.000001 & 1.920\end{array}$

$\begin{array}{llll}1323.8 & 0.7095 & -0.000001 & 0.503\end{array}$

$\begin{array}{llll}1338.3 & -1.7518 & 0.000001 & 3.069\end{array}$

$\begin{array}{llll}1354.0 & 0.4715 & 0.000000 & 0.222\end{array}$

$\begin{array}{llll}1371.3 & 1.8898 & -0.000001 & 3.572\end{array}$
$1.8 \quad 994.0319$

$1.6 \quad 994.0319$

992.2158 992.2158

992.2158

992.2158

992.2158

992.2158

992.2158

992.2158

992.2158

992.2158

992.2158

992.2158

$3 \quad 992.2158$

$2.5 \quad 992.2158$

2992.2158

$\begin{array}{ll}1.8 & 992.2158\end{array}$

$1.6 \quad 992.2158$

$1.4 \quad 992.2158$

$3 \quad 990.2132$

$\begin{array}{ll}2.5 & 990.2132\end{array}$

2990.2132

$\begin{array}{ll}1.8 & 990.2132\end{array}$

$\begin{array}{ll}1.6 & 990.2132\end{array}$

$1.4 \quad 990.2132$

$3 \quad 988.0363$

2.5988 .0363

2988.0363

$\begin{array}{ll}1.8 & 988.0363\end{array}$

$1.6 \quad 988.0363$

$1.4 \quad 988.0363$

1.2988 .0363

$3 \quad 985.6952$

$2.5 \quad 985.6952$

2985.6952

1.8985 .6952

1.6985 .6952

1.4985 .6952

1.2985 .6952 
molality molarity

$\begin{array}{crrr}\mathbf{t} /{ }^{\circ} \mathrm{C} \text { mass frac } & \begin{array}{r}\text { Density } \\ \text { exp } /\end{array} & \text { Ref } \\ & & \mathrm{kg} / \mathrm{m}^{3} & \\ & & \\ 60 & 0.0100 & 987.00 & (90) \\ 60 & 0.0200 & 990.80 & (90) \\ 60 & 0.0400 & 998.50 & (90) \\ 60 & 0.0800 & 1014.20 & (90) \\ 60 & 0.1200 & 1030.10 & (90) \\ 60 & 0.1600 & 1046.20 & (90) \\ 60 & 0.2000 & 1062.70 & (90) \\ 60 & 0.2400 & 1079.60 & (90) \\ 60 & 0.2800 & 1096.80 & (90) \\ 60 & 0.3000 & 1105.50 & (90) \\ 60 & 0.4000 & 1151.50 & (90) \\ 60 & 0.5000 & 1200.60 & (90) \\ 60 & 0.5970 & 1255.30 & (120) \\ 60 & 0.6400 & 1279.50 & (120) \\ 60 & 0.6897 & 1305.70 & (120) \\ 60 & 0.7117 & 1321.40 & (120) \\ 60 & 0.7353 & 1333.00 & (120) \\ 60 & 0.7605 & 1351.30 & (120) \\ 60 & 0.7874 & 1369.70 & (120) \\ 65 & 0.5970 & 1252.10 & (120) \\ 65 & 0.6400 & 1276.20 & (120) \\ 65 & 0.6897 & 1302.20 & (120) \\ 65 & 0.7117 & 1318.00 & (120) \\ 65 & 0.7353 & 1329.50 & (120) \\ 65 & 0.7605 & 1347.80 & (120) \\ 65 & 0.7874 & 1366.30 & (120) \\ 70 & 0.5970 & 1248.90 & (120) \\ 70 & 0.6400 & 1272.90 & (120) \\ 70 & 0.6897 & 1298.80 & (120) \\ 70 & 0.7117 & 1314.50 & (120) \\ 70 & 0.7353 & 1326.00 & (120) \\ 70 & 0.7874 & 1363.10 & (120) \\ 80 & 0.0100 & 975.50 & (90) \\ 80 & 0.0200 & 979.30 & (90) \\ 80 & 0.0400 & 986.90 & (90) \\ 80 & 0.0800 & 1002.40 & (90) \\ 80 & 0.1200 & 1018.10 & (90) \\ 80 & 0.1600 & 1034.20 & (90) \\ 80 & 0.2000 & 1050.60 & (90) \\ 80 & 0.2400 & 1067.30 & (90)\end{array}$

App vol App vol Density Density App Vol Square of Inconsistent Inconsistent Molar ratio Density of

$\exp / \mathrm{m}^{3} / \mathrm{kg} \quad$ Calc/ calc/ Residual/ Residual / Density based on based on water / water $/ \mathrm{kg} / \mathrm{m}^{3}$ $\mathrm{m}^{3} / \mathrm{kg}$ $\mathrm{m}^{3} / \mathrm{kg}$ $\begin{array}{ll}0.000625 & 0.000624\end{array}$ $\begin{array}{ll}0.000627 & 0.000624 \\ 0.000627 & 0.000626\end{array}$ $\begin{array}{ll}0.000627 & 0.000626 \\ 0.000628 & 0.000628\end{array}$ $\begin{array}{ll}0.000631 & 0.000631\end{array}$ $0.000634 \quad 0.000633$ $0.000637 \quad 0.000635$ $\begin{array}{ll}0.000639 & 0.000637\end{array}$ $\begin{array}{ll}0.000641 & 0.000639\end{array}$ $0.000642 \quad 0.000640$ $0.000645 \quad 0.000644$ $0.000649 \quad 0.000647$ $0.000648 \quad 0.000649$ $0.000649 \quad 0.000650$ $0.000653 \quad 0.000652$ $0.000651 \quad 0.000652$ $0.000654 \quad 0.000653$ $0.000653 \quad 0.000653$ $0.000653 \quad 0.000654$ $0.000649 \quad 0.000651$ $\begin{array}{ll}0.000651 & 0.000652\end{array}$ $0.000655 \quad 0.000653$ $0.000653 \quad 0.000654$ $0.000656 \quad 0.000654$ $0.000654 \quad 0.000655$ $0.000654 \quad 0.000655$ $0.000651 \quad 0.000653$ $0.000652 \quad 0.000654$ $0.000656 \quad 0.000655$ $0.000655 \quad 0.000655$ $0.000657 \quad 0.000656$ $\begin{array}{lll}0.000656 & 0.000657\end{array}$ $0.000638 \quad 0.000641$ $0.000635 \quad 0.000641$ $0.000635 \quad 0.000642$ $0.000636 \quad 0.000644$ $0.000639 \quad 0.000645$ $\begin{array}{ll}0.000641 & 0.000647\end{array}$ $0.000643 \quad 0.000648$ $0.000645 \quad 0.000649$ $\mathrm{kg} / \mathrm{m}^{3} \quad \mathrm{~kg} / \mathrm{m}^{3} \quad \mathrm{~m}^{3} / \mathrm{kg}$ Residual density apparent nitrate

$\begin{array}{llll}987.0 & -0.0170 & 0.000002 & 0.000\end{array}$

$\begin{array}{llll}990.9 & -0.0503 & 0.000003 & 0.003\end{array}$

$\begin{array}{llll}998.6 & -0.0647 & 0.000002 & 0.004\end{array}$

$\begin{array}{llll}1014.2 & 0.0015 & 0.000000 & 0.000\end{array}$

$\begin{array}{llll}1030.1 & -0.0320 & 0.000000 & 0.001\end{array}$

$\begin{array}{llll}1046.4 & -0.1952 & 0.000001 & 0.038\end{array}$

$\begin{array}{llll}1063.0 & -0.3173 & 0.000001 & 0.101\end{array}$

$\begin{array}{llll}1080.0 & -0.4271 & 0.000002 & 0.182\end{array}$

$\begin{array}{llll}1097.5 & -0.6532 & 0.000002 & 0.427\end{array}$

$\begin{array}{llll}1106.3 & -0.8314 & 0.000002 & 0.691\end{array}$

$\begin{array}{llll}1152.5 & -1.0198 & 0.000002 & 1.040\end{array}$

$\begin{array}{llll}1202.1 & -1.4568 & 0.000002 & 2.122\end{array}$

$\begin{array}{llll}1253.8 & 1.4915 & -0.000002 & 2.225\end{array}$

$\begin{array}{llll}1278.0 & 1.4618 & -0.000001 & 2.137\end{array}$

$\begin{array}{llll}1307.1 & -1.4141 & 0.000001 & 2.000\end{array}$

$\begin{array}{llll}1320.4 & 0.9567 & -0.000001 & 0.915\end{array}$

$\begin{array}{llll}1334.9 & -1.9349 & 0.000001 & 3.744\end{array}$

$\begin{array}{llll}1350.7 & 0.5511 & 0.000000 & 0.304\end{array}$

$\begin{array}{llll}1368.1 & 1.6236 & -0.000001 & 2.636\end{array}$

$\begin{array}{llll}1250.3 & 1.7710 & -0.000002 & 3.136\end{array}$

$\begin{array}{llll}1274.6 & 1.6081 & -0.000002 & 2.586\end{array}$

$\begin{array}{llll}1303.7 & -1.5236 & 0.000001 & 2.321\end{array}$

$\begin{array}{llll}1317.1 & 0.9161 & -0.000001 & 0.839\end{array}$

$\begin{array}{llll}1331.6 & -2.1131 & 0.000002 & 4.465\end{array}$

$\begin{array}{llll}1347.5 & 0.3273 & 0.000000 & 0.107\end{array}$

$\begin{array}{lllll}1364.9 & 1.4446 & -0.000001 & 2.087\end{array}$

$\begin{array}{llll}1246.8 & 2.0909 & -0.000002 & 4.372\end{array}$

$\begin{array}{llll}1271.1 & 1.7839 & -0.000002 & 3.182\end{array}$

$\begin{array}{llll}1300.3 & -1.5171 & 0.000001 & 2.302\end{array}$

$\begin{array}{llll}1313.7 & 0.7851 & -0.000001 & 0.616\end{array}$

$\begin{array}{llll}1328.3 & -2.2890 & 0.000002 & 5.239\end{array}$

$\begin{array}{llll}1361.6 & 1.4511 & -0.000001 & 2.106\end{array}$

$\begin{array}{llll}975.5 & 0.0214 & -0.000002 & 0.000\end{array}$

$\begin{array}{llll}979.2 & 0.1214 & -0.000006 & 0.015\end{array}$

$\begin{array}{lllll}986.6 & 0.2619 & -0.000007 & 0.069\end{array}$

$\begin{array}{llll}1001.8 & 0.5933 & -0.000007 & 0.352\end{array}$

$\begin{array}{llll}1017.3 & 0.7698 & -0.000006 & 0.593\end{array}$

$\begin{array}{llll}1033.2 & 0.9649 & -0.000006 & 0.931\end{array}$

$\begin{array}{llll}1049.5 & 1.0526 & -0.000005 & 1.108\end{array}$

$\begin{array}{llll}1066.3 & 1.0062 & -0.000004 & 1.012\end{array}$
983.1989

983.1989

983.1989

983.1989

983.1989

983.1989

983.1989

983.1989

983.1989

983.1989

983.1989

983.1989

$3 \quad 983.1989$

2.5983 .1989

2983.1989

$\begin{array}{ll}1.8 & 983.1989\end{array}$

1.6983 .1989

1.4983 .1989

1.2983 .1989

$\begin{array}{ll}3 & 980.5548\end{array}$

$2.5 \quad 980.5548$

2980.5548

$1.8 \quad 980.5548$

$1.6 \quad 980.5548$

$1.4 \quad 980.5548$

1.2980 .5548

$3 \quad 977.7696$

$2.5 \quad 977.7696$

2977.7696

1.8977 .7696

$1.6 \quad 977.7696$

1.2977 .7696

971.7978

971.7978

971.7978

971.7978

971.7978

971.7978

971.7978

971.7978 
molality molarity

$\begin{array}{rrr}\mathbf{t} /{ }^{\circ} \mathrm{C} \text { mass frac } & \begin{array}{r}\text { Density } \\ \text { exp } / \\ \mathrm{kg} / \mathrm{m}^{3}\end{array} \\ & & 1084.40 \\ 80 & 0.2800 & 1083.10 \\ 80 & 0.3000 & 1093.50 \\ 80 & 0.4000 & 1138.50 \\ 80 & 0.5000 & 1186.80 \\ 95 & 0.0161 & 967.70 \\ 95 & 0.0804 & 991.80 \\ 95 & 0.1212 & 1007.10 \\ 95 & 0.1990 & 1036.50 \\ 95 & 0.2696 & 1069.00 \\ 95 & 0.3112 & 1086.00 \\ 95 & 0.3813 & 1118.00 \\ 95 & 0.4589 & 1157.00 \\ 95 & 0.5312 & 1198.00 \\ 95 & 0.5750 & 1217.00 \\ 95 & 0.6465 & 1253.00 \\ 95 & 0.6954 & 1281.00\end{array}$

Ref App vol
$\exp / \mathrm{m}^{3} / \mathrm{kg}$

\section{(90)}

(90)

$0.000647 \quad 0.000650$ $0.000648 \quad 0.000651$ $0.000652 \quad 0.000653$

$\begin{array}{lll}\text { (90) } \quad 0.000656 & 0.000655\end{array}$

$\begin{array}{lll}(10) & 0.000651 & 0.000653\end{array}$

(10) $\quad 0.000650 \quad 0.000655$

(10) $\quad 0.000655 \quad 0.000655$

(10) $\quad 0.000664 \quad 0.000657$

(10) $\quad 0.000653 \quad 0.000658$

(10) $\quad 0.000658 \quad 0.000658$

(10) $\quad 0.000659 \quad 0.000659$

(10) $0.000658 \quad 0.000660$

$\begin{array}{lll}\text { (10) } & 0.000654 & 0.000661\end{array}$

(10) $\quad 0.000661 \quad 0.000661$

(10) $\quad 0.000666 \quad 0.000662$ $\begin{array}{ll}0.000666 & 0.000662 \\ 0.000667 & 0.000662\end{array}$

Average Res

Std dev Res

Avg - 4std

Avg + 4std
Density Density App Vol Square of Inconsistent Inconsistent Molar ratio kg/m $\mathrm{kg} / \mathrm{m}^{3}$ $\mathrm{m}^{3} / \mathrm{kg}$ Density

density

based o apparent water /

Density of

1083.5

1092.3

$0.8989-0.000003$

$0.8139-0.000002$

\subsection{8}

0.662

0.109

0.760

$\begin{array}{llll}967.7 & 0.0254 & -0.000002 & 0.001 \\ 991.4 & 0.3737 & -0.000005 & 0.140\end{array}$

$\begin{array}{llll}1007.0 & 0.0974 & -0.000001 & 0.009\end{array}$

$\begin{array}{llll}1037.9 & -1.4405 & 0.000007 & 2.075\end{array}$

$\begin{array}{llll}1067.6 & 1.4153 & -0.000005 & 2.003\end{array}$

$\begin{array}{llll}1085.8 & 0.2339 & -0.000001 & 0.055\end{array}$

$\begin{array}{llll}1117.8 & 0.1961 & 0.000000 & 0.038\end{array}$

$\begin{array}{llll}1155.4 & 1.5967 & -0.000003 & 2.550\end{array}$

$\begin{array}{llll}1192.7 & 5.3194 & -0.000007 & 28.296\end{array}$

$\begin{array}{llll}1216.4 & 0.5868 & -0.000001 & 0.344\end{array}$

$\begin{array}{lll}-4.1863 & 0.000004 & 17.525\end{array}$

$\begin{array}{lll}-5.6386 & 0.000005 & 31.794\end{array}$

$\begin{array}{ll}-0.0027 & 0.000000\end{array}$

$\begin{array}{ll}1.3507 & 0.000004\end{array}$

$\begin{array}{ll}-5.4054 & -0.000017\end{array}$

$\begin{array}{ll}5.4000 & 0.000017\end{array}$

volume

971.7978

971.7978

971.7978

971.7978

961.9004

961.9004

961.9004

961.9004

961.9004

961.9004

961.9004

961.9004

961.9004

961.9004

961.9004

961.9004

Curve fit for the model App vol $=\left(w+c 2+c 3^{*} t{ }^{\circ} \mathrm{C}\right) /\left(\mathrm{c} 0^{*} \mathrm{w}+\mathrm{c} 1\right) / \mathrm{e}^{\wedge}\left(0.000001^{*}\left(\mathrm{t}^{\circ} \mathrm{C}+\mathrm{c} 4\right)^{\wedge} 2\right)$

$\begin{array}{lr}\text { c0 } 1 & 1379.293 \\ \text { c2 } & 1124.381 \\ \text { c3 } & 0.655978 \\ \text { c4 } & 0.001411 \\ \text { Min T } & 176.4124 \\ \text { Max T } & 0 \\ \text { Min w } & 95 \\ \text { Max w } & 0.004478 \\ \text { Avg dens res } & 0.787404 \\ \text { Std dens res } & -0.002685 \\ \text { Avg app vol res *10^3 } & 1.350673 \\ \text { Std app vol res *10^3 } & 0.000372 \\ \text { No of points in corr } & 0.00426 \\ \text { No of inconsistent poir } & 162 \\ & 26\end{array}$

Inconsistent data not used (Residual greater than average $+/ \mathbf{- 4}$ standard deviations)

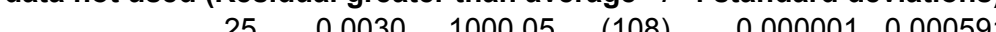


Density of aqueous solutions of (NH4)2SO4

References (30) Goldsack, D. E.; Franchetto, A. A. The Viscosity of Concentrated Electrolyte Solutions. III. A Mixture Law. Electrochimica Acta 1977, 22, 1287-1294.

(46) Isono, T. Densities, Viscosities, and Electrolytic Conductivities of Concentrated Aqueous Electrolyte Solutions of 31 Solutes in the Temperature Range $15-55^{\circ} \mathrm{C}$ and Empirical Equations for the Relative Viscosity. Rikagaku Kenkyusho Hokoku 1985, 61, 53-79

(90) Perry, R. H.; Green, D. W. Perry's Chemical Engineers' Handbook; McGraw Hill: New York, 7th edition, 1997.

\begin{tabular}{|c|c|c|c|c|c|c|c|c|c|c|c|c|c|c|}
\hline \multirow[t]{13}{*}{ molality } & \multirow[t]{13}{*}{ molarity } & $\mathrm{t} /{ }^{\circ} \mathrm{C}$ & ass frac & \multicolumn{2}{|l|}{$\begin{array}{r}\text { Density } \\
\exp / \mathrm{kg} / \mathrm{m}^{3}\end{array}$} & \multirow{2}{*}{$\begin{array}{r}\text { App vol } \\
\text { exp / } \\
\mathrm{m}^{3} / \mathrm{kg} \\
0.000378\end{array}$} & \multirow[t]{2}{*}{$\begin{array}{r}\text { App vol } \\
\text { Calc / } \\
\mathrm{m}^{3} / \mathrm{kg} \\
0.000417\end{array}$} & \multirow{2}{*}{$\begin{array}{r}\text { Density } \\
\text { calc / } \\
\mathrm{kg} / \mathrm{m}^{3} \\
1005.7\end{array}$} & \multirow{2}{*}{$\begin{array}{r}\text { Density } \\
\text { Residual / } \\
\mathrm{kg} / \mathrm{m}^{3} \\
0.3958\end{array}$} & \multirow{2}{*}{$\begin{array}{r}\text { App Vol } \\
\text { Residual / } \\
\mathrm{m}^{3} / \mathrm{kg} \\
-0.000039\end{array}$} & \multirow{2}{*}{$\begin{array}{r}\text { Square of } \\
\text { Density } \\
\text { Res } \\
0.157\end{array}$} & $\begin{array}{l}\text { Inconsistent } \\
\text { based on } \\
\text { density }\end{array}$ & $\begin{array}{c}\text { Inconsistent } \\
\text { based on } \\
\text { apparent } \\
\text { volume }\end{array}$ & $\begin{array}{r}\text { Density of } \\
\text { water } / \mathrm{kg} / \mathrm{m}^{3}\end{array}$ \\
\hline & & 0 & 0.0100 & 1006.10 & (90) & & & & & & & & & 999.8395 \\
\hline & & 0 & 0.0200 & 1012.40 & (90) & 0.000380 & 0.000419 & 1011.6 & 0.8113 & -0.000040 & 0.658 & & & 999.8395 \\
\hline & & 0 & 0.0400 & 1024.80 & (90) & 0.000391 & 0.000424 & 1023.4 & 1.3862 & -0.000033 & 1.922 & & & 999.8395 \\
\hline & & 0 & 0.0800 & 1049.50 & (90) & 0.000409 & 0.000434 & 1047.3 & 2.2405 & -0.000025 & 5.020 & & & 999.8395 \\
\hline & & 0 & 0.1200 & 1074.00 & (90) & 0.000425 & 0.000444 & 1071.3 & 2.6952 & -0.000020 & 7.264 & & & 999.8395 \\
\hline & & 0 & 0.1600 & 1098.00 & (90) & 0.000441 & 0.000454 & 1095.5 & 2.5342 & -0.000013 & 6.422 & & & 999.8395 \\
\hline & & 0 & 0.2000 & 1121.50 & (90) & 0.000458 & 0.000465 & 1119.6 & 1.8547 & -0.000007 & 3.440 & & & 999.8395 \\
\hline & & 0 & 0.2400 & 1144.80 & (90) & 0.000472 & 0.000476 & 1143.7 & 1.0678 & -0.000003 & 1.140 & & & 999.8395 \\
\hline & & 0 & 0.2800 & 1167.70 & (90) & 0.000487 & 0.000487 & 1167.6 & 0.0996 & 0.000000 & 0.010 & & & 999.8395 \\
\hline & & 0 & 0.3500 & 1207.20 & (90) & 0.000509 & 0.000507 & 1208.4 & -1.2097 & 0.000002 & 1.463 & & & 999.8395 \\
\hline & & 0 & 0.4000 & 1235.00 & (90) & 0.000524 & 0.000522 & 1236.4 & -1.4017 & 0.000002 & 1.965 & & & 999.8395 \\
\hline & & 0 & 0.5000 & 1289.90 & (90) & 0.000550 & 0.000553 & 1287.9 & 2.0248 & -0.000002 & 4.100 & & & 999.8395 \\
\hline 0.05 & & 15 & 0.0066 & 1003.17 & (46) & 0.000382 & 0.000425 & 1002.9 & 0.2811 & -0.000043 & 0.079 & & & 999.0996 \\
\hline 0.07 & & 15 & 0.0092 & 1004.72 & (46) & 0.000390 & 0.000425 & 1004.4 & 0.3270 & -0.000035 & 0.107 & & & 999.0996 \\
\hline 0.1 & & 15 & 0.0130 & 1007.09 & (46) & 0.000392 & 0.000426 & 1006.6 & 0.4532 & -0.000034 & 0.205 & & & 999.0996 \\
\hline 0.2 & & 15 & 0.0257 & 1014.72 & (46) & 0.000402 & 0.000429 & 1014.0 & 0.7098 & -0.000027 & 0.504 & & & 999.0996 \\
\hline 0.3 & & 15 & 0.0381 & 1022.06 & (46) & 0.000411 & 0.000432 & 1021.2 & 0.8368 & -0.000021 & 0.700 & & & 999.0996 \\
\hline 0.5 & & 15 & 0.0620 & 1036.16 & (46) & 0.000423 & 0.000438 & 1035.2 & 0.9795 & -0.000015 & 0.959 & & & 999.0996 \\
\hline 1 & & 15 & 0.1167 & 1068.17 & (46) & 0.000446 & 0.000452 & 1067.5 & 0.6812 & -0.000005 & 0.464 & & & 999.0996 \\
\hline 1.5 & & 15 & 0.1654 & 1096.74 & (46) & 0.000462 & 0.000464 & 1096.4 & 0.3297 & -0.000002 & 0.109 & & & 999.0996 \\
\hline 2 & & 15 & 0.2090 & 1122.12 & (46) & 0.000476 & 0.000475 & 1122.3 & -0.1839 & 0.000001 & 0.034 & & & 999.0996 \\
\hline 3 & & 15 & 0.2839 & 1165.50 & (46) & 0.000498 & 0.000495 & 1166.3 & -0.7912 & 0.000002 & 0.626 & & & 999.0996 \\
\hline 4 & & 15 & 0.3458 & 1201.06 & (46) & 0.000514 & 0.000513 & 1201.7 & -0.6510 & 0.000001 & 0.424 & & & 999.0996 \\
\hline 5 & & 15 & 0.3978 & 1230.45 & (46) & 0.000528 & 0.000528 & 1230.4 & 0.0846 & 0.000000 & 0.007 & & & 999.0996 \\
\hline 0.05 & & 20 & 0.0066 & 1002.23 & (46) & 0.000389 & 0.000427 & 1002.0 & 0.2533 & -0.000038 & 0.064 & & & 998.2041 \\
\hline \multirow{2}{*}{\multicolumn{2}{|c|}{0.07}} & 20 & 0.0092 & 1003.79 & (46) & 0.000394 & 0.000428 & 1003.5 & 0.3159 & -0.000034 & 0.100 & & & 998.2041 \\
\hline & & 20 & 0.0100 & 1004.10 & (90) & 0.000414 & 0.000428 & 1004.0 & 0.1450 & -0.000014 & 0.021 & & & 998.2041 \\
\hline \multirow{2}{*}{\multicolumn{2}{|c|}{0.1}} & 20 & 0.0130 & 1006.11 & (46) & 0.000398 & 0.000429 & 1005.7 & 0.4020 & -0.000030 & 0.162 & & & 998.2041 \\
\hline & & 20 & 0.0200 & 1010.10 & (90) & 0.000412 & 0.000430 & 1009.7 & 0.3754 & -0.000018 & 0.141 & & & 998.2041 \\
\hline 0.2 & & 20 & 0.0257 & 1013.66 & (46) & 0.000409 & 0.000432 & 1013.0 & 0.6113 & -0.000023 & 0.374 & & & 998.2041 \\
\hline \multirow{2}{*}{\multicolumn{2}{|c|}{0.3}} & 20 & 0.0381 & 1020.94 & (46) & 0.000417 & 0.000435 & 1020.2 & 0.7105 & -0.000018 & 0.505 & & & 998.2041 \\
\hline & & 20 & 0.0400 & 1022.00 & (90) & 0.000419 & 0.000435 & 1021.3 & 0.6842 & -0.000016 & 0.468 & & & 998.2041 \\
\hline \multirow{2}{*}{\multicolumn{2}{|c|}{0.5}} & 20 & 0.0620 & 1034.91 & (46) & 0.000428 & 0.000440 & 1034.1 & 0.7856 & -0.000012 & 0.617 & & & 998.2041 \\
\hline & & 20 & 0.0800 & 1045.60 & (90) & 0.000434 & 0.000445 & 1044.7 & 0.9206 & -0.000011 & 0.848 & & & 998.2041 \\
\hline 1 & & 20 & 0.1167 & 1066.81 & (46) & 0.000450 & 0.000454 & 1066.3 & 0.5227 & -0.000004 & 0.273 & & & 998.2041 \\
\hline
\end{tabular}


$\mathrm{t} /{ }^{\circ} \mathrm{C}$ mass frac Density

$\exp / \mathrm{kg} / \mathrm{m}^{3}$

$\begin{array}{rr}0.1200 & 1069.10 \\ 0.1600 & 1092.40 \\ 0.1654 & 1095.13 \\ 0.2000 & 1115.40 \\ 0.2090 & 1120.40 \\ 0.2400 & 1138.30 \\ 0.2800 & 1160.90 \\ 0.2839 & 1163.70 \\ 0.3458 & 1199.22 \\ 0.3978 & 1228.64 \\ 0.4000 & 1227.70 \\ 0.5000 & 1282.50 \\ 0.0066 & 1001.02 \\ 0.0092 & 1002.56 \\ 0.0130 & 1004.86 \\ 0.0257 & 1012.36 \\ 0.0381 & 1019.61 \\ 0.0620 & 1033.20 \\ 0.0620 & 1033.46 \\ 0.1167 & 1065.00 \\ 0.1167 & 1065.20 \\ 0.1654 & 1092.70 \\ 0.1654 & 1093.40 \\ 0.2090 & 1117.70 \\ 0.2090 & 1118.66 \\ 0.2483 & 1140.40 \\ 0.2839 & 1160.70 \\ 0.2839 & 1161.86 \\ 0.3458 & 1195.90 \\ 0.3458 & 1197.34 \\ 0.3978 & 1226.77 \\ 0.0066 & 999.59 \\ 0.0092 & 1001.13 \\ 0.0130 & 1003.42 \\ 0.0257 & 1010.87 \\ 0.0381 & 1018.07 \\ 0.0620 & 1031.86 \\ 0.1167 & 1063.46 \\ 0.1654 & 1091.58 \\ 0.2090 & 1116.77 \\ 0.2839 & 1159.95 \\ 0.3458 & 1195.45 \\ & \end{array}$

App

$$
\begin{array}{rr}
\text { exp / } & \text { App vol } \\
\text { Calc / }
\end{array}
$$$$
\mathrm{m}^{3} / \mathrm{kg} \quad \mathrm{m}^{3} / \mathrm{kg}
$$

$\begin{array}{lll}0.000448 & 0.000455\end{array}$

0.0004620 .000465

$0.000466 \quad 0.000466$

$\begin{array}{ll}0.000476 & 0.000475\end{array}$

$\begin{array}{ll}0.000479 & 0.000477\end{array}$

$0.000488 \quad 0.000486$

$0.000500 \quad 0.000496$

$0.000500 \quad 0.000497$

$0.000516 \quad 0.000515$

$0.000530 \quad 0.000530$

$0.000534 \quad 0.000530$

$0.000558 \quad 0.000561$

$0.000396 \quad 0.000429$

$0.000401 \quad 0.000430$

$0.000405 \quad 0.000431$

$0.000414 \quad 0.000434$

$\begin{array}{ll}0.000421 & 0.000437\end{array}$

$0.000437 \quad 0.000442$

$\begin{array}{ll}0.000433 & 0.000442\end{array}$

0.0004550 .000456

$0.000453 \quad 0.000456$

$0.000472 \quad 0.000468$

$0.000469 \quad 0.000468$

$0.000485 \quad 0.000479$

$\begin{array}{ll}0.000481 & 0.000479\end{array}$

$\begin{array}{lll}0.000495 & 0.000490\end{array}$

$\begin{array}{ll}0.000505 & 0.000499\end{array}$

$0.000502 \quad 0.000499$

$0.000521 \quad 0.000516$

$\begin{array}{ll}0.000518 & 0.000516\end{array}$

$\begin{array}{lll}0.000531 & 0.000531\end{array}$

$0.000401 \quad 0.000431$

$0.000404 \quad 0.000432$

$0.000408 \quad 0.000433$

$\begin{array}{lll}0.000417 & 0.000436\end{array}$

$0.000424 \quad 0.000439$

$0.000436 \quad 0.000444$

$0.000456 \quad 0.000458$

$0.000471 \quad 0.000470$

$0.000483 \quad 0.000481$

$0.000503 \quad 0.0005$

0.0005190 .000518
Density

Density App Vol Square of Inconsistent Inconsistent

cased on besity of $\mathrm{kg} / \mathrm{m}^{3} \quad \mathrm{~kg} / \mathrm{m}^{3} \quad \mathrm{~m}^{3} / \mathrm{kg} \quad$ Res density apparent

$1068.2 \quad 0.8752-0.000006-0.766$

$\begin{array}{llll}1091.9 & 0.5295 & -0.000003 & 0.280\end{array}$

$\begin{array}{llll}1095.1 & 0.0517 & 0.000000 & 0.003\end{array}$

$\begin{array}{llll}1115.5 & -0.1226 & 0.000000 & 0.015\end{array}$

$\begin{array}{llll}1120.9 & -0.4557 & 0.000002 & 0.208\end{array}$

$\begin{array}{llll}1139.1 & -0.7735 & 0.000002 & 0.598\end{array}$

$\begin{array}{llll}1162.4 & -1.5019 & 0.000004 & 2.256\end{array}$

$\begin{array}{llll}1164.6 & -0.9497 & 0.000002 & 0.902\end{array}$

$\begin{array}{llll}1199.9 & -0.7014 & 0.000001 & 0.492\end{array}$

$\begin{array}{llll}1228.5 & 0.1753 & 0.000000 & 0.031\end{array}$

$\begin{array}{llll}1229.6 & -1.9187 & 0.000003 & 3.681\end{array}$

$\begin{array}{llll}1279.9 & 2.5771 & -0.000003 & 6.641\end{array}$

$\begin{array}{llll}1000.8 & 0.2180 & -0.000033 & 0.048\end{array}$

$\begin{array}{llll}1002.3 & 0.2667 & -0.000029 & 0.071\end{array}$

$\begin{array}{llll}1004.5 & 0.3420 & -0.000026 & 0.117\end{array}$

$\begin{array}{llll}1011.8 & 0.5313 & -0.000020 & 0.282\end{array}$

$\begin{array}{llll}1019.0 & 0.6298 & -0.000016 & 0.397\end{array}$

$\begin{array}{llll}1032.8 & 0.3816 & -0.000006 & 0.146\end{array}$

$\begin{array}{llll}1032.8 & 0.6416 & -0.000010 & 0.412\end{array}$

$\begin{array}{llll}1064.9 & 0.1485 & -0.000001 & 0.022\end{array}$

$\begin{array}{llll}1064.9 & 0.3485 & -0.000003 & 0.121\end{array}$

$\begin{array}{llll}1093.5 & -0.8287 & 0.000004 & 0.687\end{array}$

$\begin{array}{llll}1093.5 & -0.1287 & 0.000001 & 0.017\end{array}$

$\begin{array}{llll}1119.2 & -1.5075 & 0.000006 & 2.273\end{array}$

$\begin{array}{llll}1119.2 & -0.5475 & 0.000002 & 0.300\end{array}$

$\begin{array}{llll}1142.2 & -1.8144 & 0.000006 & 3.292\end{array}$

$\begin{array}{llll}1162.8 & -2.1446 & 0.000006 & 4.599\end{array}$

$\begin{array}{llll}1162.8 & -0.9846 & 0.000003 & 0.969\end{array}$

$\begin{array}{llll}1198.0 & -2.1044 & 0.000004 & 4.428\end{array}$

$\begin{array}{llll}1198.0 & -0.6644 & 0.000001 & 0.441\end{array}$

$\begin{array}{llll}1226.5 & 0.2986 & 0.000000 & 0.089\end{array}$

$\begin{array}{llll}999.4 & 0.1998 & -0.000030 & 0.040\end{array}$

$\begin{array}{llll}1000.9 & 0.2541 & -0.000028 & 0.065\end{array}$

$\begin{array}{llll}1003.1 & 0.3277 & -0.000025 & 0.107\end{array}$

$\begin{array}{llll}1010.4 & 0.4945 & -0.000019 & 0.244\end{array}$

$\begin{array}{llll}1017.5 & 0.5696 & -0.000014 & 0.324\end{array}$

$\begin{array}{llll}1031.3 & 0.5724 & -0.000009 & 0.328\end{array}$

$\begin{array}{llll}1063.2 & 0.2539 & -0.000002 & 0.064\end{array}$

$\begin{array}{llll}1091.8 & -0.2059 & 0.000001 & 0.042\end{array}$

$\begin{array}{llll}1117.4 & -0.6130 & 0.000002 & 0.376\end{array}$

$\begin{array}{llll}1160.9 & -0.9478 & 0.000002 & 0.898\end{array}$

$\begin{array}{llll}1196.0 & -0.5301 & 0.000001 & 0.281\end{array}$
998.2041

998.2041

998.2041

998.2041

998.2041

998.2041

998.2041

998.2041

998.2041

998.2041

998.2041

998.2041

997.0449

997.0449

997.0449

997.0449

997.0449

997.0449

997.0449

997.0449

997.0449

997.0449

997.0449

997.0449

997.0449

997.0449

997.0449

997.0449

997.0449

997.0449

997.0449

995.6473

995.6473

995.6473

995.6473

995.6473

995.6473

995.6473

995.6473

995.6473

995.6473

995.6473 
molality molarity

$t /{ }^{\circ} \mathrm{C}$ mass frac Density

$\exp / \mathrm{kg} / \mathrm{m}^{3}$

5
0.05
0.07
0.1
0.2
0.3
0.5
1
1.5
2
3
4
5
0.05
0.07

0.1

0.2

0.3

0.5

1

1.5

2

3

5

0.05

0.07

0.1

0.2

0.3

$0.0130 \quad 997.88$

$0.0257 \quad 1005.25$

$0.0381 \quad 1012.40$
Ref

App vol App vol
$\exp / \quad$ Calc

$$
\mathrm{m}^{3} / \mathrm{kg} \quad \mathrm{m}^{3} / \mathrm{kg}
$$

0.0005320 .000533

$0.000404 \quad 0.000433$

$0.000408 \quad 0.000434$

$0.000412 \quad 0.000435$

$0.000427 \quad 0.000440$

$0.000438 \quad 0.000446$

$0.000457 \quad 0.000459$

$0.000472 \quad 0.000471$

$\begin{array}{lll}0.000485 & 0.000482\end{array}$

$0.000504 \quad 0.000502$

$0.000520 \quad 0.000519$

$0.000533 \quad 0.000534$

$0.000404 \quad 0.000435$

$0.000410 \quad 0.000435$

$0.000424 \quad 0.000435$

0.0004120 .000436

$\begin{array}{lll}0.000421 & 0.000438\end{array}$

$0.000422 \quad 0.000439$

0.0004290 .000442

$0.000430 \quad 0.000442$

$0.000439 \quad 0.000447$

0.0004440 .000452

$0.000459 \quad 0.000461$

$0.000457 \quad 0.000461$

$\begin{array}{lll}0.000470 & 0.000471\end{array}$

$\begin{array}{lll}0.000473 & 0.000473\end{array}$

$0.000482 \quad 0.000481$

$0.000486 \quad 0.000484$

$0.000495 \quad 0.000492$

$\begin{array}{lll}0.000506 & 0.000502\end{array}$

$0.000505 \quad 0.000503$

$0.000521 \quad 0.000520$

$0.000525 \quad 0.000521$

$0.000533 \quad 0.000535$

$0.000538 \quad 0.000535$

$0.000561 \quad 0.000565$

$0.000407 \quad 0.000436$

$0.000413 \quad 0.000436$

$0.000415 \quad 0.000437$

$0.000423 \quad 0.000440$

0.0004290 .000443
$0.000420 \quad 0.000437$

Density

App Vol Square of Inconsistent Inconsistent

( / Residual / Residual / Density based on based on waty of $\mathrm{kg} / \mathrm{m}^{3} \quad \mathrm{~kg} / \mathrm{m}^{3} \quad \mathrm{~m}^{3} / \mathrm{kg} \quad$ Res density apparent

$1224.4-0.5060-0.000001$

$0.2375-0.000026$

$0.2988-0.000023$

$0.4602-0.000018$

$0.5294-0.000013$

$0.5375-0.000008$

$0.2190-0.000002$

$\begin{array}{ll}-0.2191 & 0.000001\end{array}$

$\begin{array}{ll}-0.5908 & 0.000002\end{array}$

$-0.8866 \quad 0.000002$

$-0.3943 \quad 0.000001$

$0.7136-0.000001$

$0.1959-0.000030$

$0.2299-0.000025$

$0.1159-0.000012$

$0.3080-0.000024$

$0.3287-0.000016$

$0.4517-0.000017$

$0.5121-0.000013$

$0.5010-0.000012$

$0.5404-0.000008$

$0.6593-0.000008$

$0.2274-0.000002$

$0.6250-0.000005$

$0.2750-0.000001$

$\begin{array}{ll}-0.1741 & 0.000001\end{array}$

$\begin{array}{ll}-0.3017 & 0.000001\end{array}$

$\begin{array}{ll}-0.5060 & 0.000002\end{array}$

$\begin{array}{ll}-0.9036 & 0.000003\end{array}$

$\begin{array}{ll}-1.5156 & 0.000004\end{array}$

$\begin{array}{lll}1154.4 & -1.5156 & 0.000004 \\ 1156.6 & -0.7845 & 0.000002\end{array}$

$\begin{array}{lll}1191.7 & -0.2388 & 0.000000\end{array}$

$1194.0 \quad-2.1086 \quad 0.000004$

$1220.10 .9408-0.000002$

$\begin{array}{lll}1221.3 & -1.6507 & 0.000003\end{array}$

$1271.7 \quad 2.8405-0.000004$

$\begin{array}{llll}993.9 & 0.1889 & -0.000029\end{array}$

$\begin{array}{llll}995.4 & 0.2171 & -0.000024\end{array}$

997.6

$0.2912-0.000022$

1004.8

$\begin{array}{lll}0.4447 & -0.000017\end{array}$

$0.5340-0.000014$

0.256

0.035

0.056

0.089

0.212

0.280

0.289

0.048

0.048

0.349

0.786

0.155

0.509

0.038

0.053

0.013

0.095

0.108

0.204

0.262

0.251

0.292

0.435

0.052

0.391

0.076

0.030

0.091

0.256

0.817

2.297

0.615

0.057

4.446

0.885

2.725

8.069

0.036

0.047

0.085

0.198

0.285
995.6473

994.0319

994.0319

994.0319

994.0319

994.0319

994.0319

994.0319

994.0319

994.0319

994.0319

994.0319

994.0319

992.2158

992.2158

992.2158

992.2158

992.2158

992.2158

992.2158

992.2158

992.2158

992.2158

992.2158

992.2158

992.2158

992.2158

992.2158

992.2158

992.2158

992.2158

992.2158

992.2158

992.2158

992.2158

992.2158

992.2158

990.2132

990.2132

990.2132

990.2132

990.2132 
$\mathbf{t} /{ }^{\circ} \mathrm{C}$ mass frac Density

$\exp / \mathrm{kg} / \mathrm{m}^{3}$

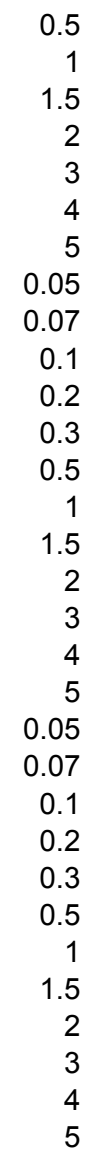

$\begin{array}{rrr}45 & 0.0620 & 1026.09 \\ 45 & 0.1167 & 1057.49 \\ 45 & 0.1654 & 1085.43 \\ 45 & 0.2090 & 1110.59 \\ 45 & 0.2839 & 1153.74 \\ 45 & 0.3458 & 1189.37 \\ 45 & 0.3978 & 1219.04 \\ 50 & 0.0066 & 991.95 \\ 50 & 0.0092 & 993.45 \\ 50 & 0.0130 & 995.72 \\ 50 & 0.0257 & 1003.08 \\ 50 & 0.0381 & 1010.22 \\ 50 & 0.0620 & 1023.90 \\ 50 & 0.1167 & 1055.27 \\ 50 & 0.1654 & 1083.23 \\ 50 & 0.2090 & 1108.37 \\ 50 & 0.2839 & 1151.61 \\ 50 & 0.3458 & 1187.29 \\ 50 & 0.3978 & 1217.02 \\ 55 & 0.0066 & 989.59 \\ 55 & 0.0092 & 991.09 \\ 55 & 0.0130 & 993.36 \\ 55 & 0.0257 & 1000.74 \\ 55 & 0.0381 & 1007.84 \\ 55 & 0.0620 & 1021.56 \\ 55 & 0.1167 & 1052.88 \\ 55 & 0.1654 & 1080.87 \\ 55 & 0.2090 & 1106.07 \\ 55 & 0.2839 & 1149.33 \\ 55 & 0.3458 & 1185.11 \\ 55 & 0.3978 & 1214.93 \\ 80 & 0.0100 & 977.70 \\ 80 & 0.0200 & 983.60 \\ 80 & 0.0400 & 995.30 \\ 80 & 0.0800 & 1018.70 \\ 80 & 0.1200 & 1042.10 \\ 80 & 0.1600 & 1065.30 \\ 80 & 0.2000 & 1088.30 \\ 80 & 0.2400 & 1111.10 \\ 80 & 0.2800 & 1133.80 \\ 80 & 0.3500 & 1173.10 \\ 80 & 0.4000 & 1201.10 \\ & & \end{array}$

Ref

App$$
\text { exp / Calc / }
$$$$
\mathrm{m}^{3} / \mathrm{kg} \quad \mathrm{m}^{3} / \mathrm{kg}
$$

$\begin{array}{ll}0.000440 & 0.000449\end{array}$

0.0004590 .000462

$\begin{array}{lll}0.000474 & 0.000474\end{array}$

$0.000486 \quad 0.000485$

$\begin{array}{ll}0.000506 & 0.000504\end{array}$

$0.000521 \quad 0.000521$

$0.000533 \quad 0.000535$

$0.000404 \quad 0.000437$

$0.000410 \quad 0.000438$

$\begin{array}{lll}0.000413 & 0.000439\end{array}$

$0.000423 \quad 0.000441$

$0.000429 \quad 0.000444$

$0.000440 \quad 0.000450$

$0.000460 \quad 0.000463$

$0.000474 \quad 0.000474$

$0.000486 \quad 0.000485$

$\begin{array}{ll}0.000506 & 0.000505\end{array}$

$0.000521 \quad 0.000521$

$\begin{array}{ll}0.000533 & 0.000536\end{array}$

$0.000406 \quad 0.000438$

0.0004120 .000439

$0.000414 \quad 0.000439$

$0.000422 \quad 0.000442$

$0.000430 \quad 0.000445$

$0.000440 \quad 0.000451$

$\begin{array}{lll}0.000460 & 0.000463\end{array}$

$0.000474 \quad 0.000475$

$0.000486 \quad 0.000486$

$0.000506 \quad 0.000505$

$\begin{array}{ll}0.000521 & 0.000522\end{array}$

$0.000533 \quad 0.000536$

$0.000408 \quad 0.000441$

0.0004120 .000443

$0.000422 \quad 0.000447$

$\begin{array}{lll}0.000437 & 0.000456\end{array}$

$0.000451 \quad 0.000465$

$\begin{array}{lll}0.000465 & 0.000475\end{array}$

$\begin{array}{lll}0.000478 & 0.000484\end{array}$

$0.000491 \quad 0.000494$

$0.000504 \quad 0.000504$

$0.000525 \quad 0.000522$

$0.000538 \quad 0.000535$
Density

App Vol Square of Inconsistent Inconsisten

bensity of $\mathrm{kg} / \mathrm{m}^{3} \quad \mathrm{~kg} / \mathrm{m}^{3} \quad \mathrm{~m}^{3} / \mathrm{kg} \quad$ Res density apparent

$\begin{array}{llll}1025.5 & 0.5570 & -0.000009 & 0.310\end{array}$

$1057.2 \quad 0.2959-0.000002$

$\begin{array}{lll}1085.6 & -0.1435 & 0.000001\end{array}$

$\begin{array}{lll}1111.0 & -0.4307 & 0.000002\end{array}$

$\begin{array}{lll}1154.4 & -0.6223 & 0.000002 \\ 1189.4 & -0.0331 & 0.000000\end{array}$

$\begin{array}{lll}1189.4 & -0.0331 & 0.000000\end{array}$

$\begin{array}{lll}1217.9 & 1.1597 & -0.000002\end{array}$

$\begin{array}{lll}991.7 & 0.2152 & -0.000033\end{array}$

$\begin{array}{llll}993.2 & 0.2470 & -0.000027\end{array}$

$995.4 \quad 0.3266-0.000025$

$1002.6 \quad 0.4874-0.000019$

$1009.6 \quad 0.5830-0.000015$

$1023.3-0.6259-0.000010$

$1054.9 \quad 0.3933-0.000003$

$\begin{array}{lll}1083.2 & 0.0120 & 0.000000\end{array}$

$\begin{array}{lll}1108.6 & -0.2748 & 0.000001\end{array}$

$\begin{array}{lll}1152.0 & -0.3784 & 0.000001\end{array}$

$\begin{array}{lll}1187.1 & 0.2158 & 0.000000\end{array}$

$\begin{array}{lll}1215.6 & 1.3947 & -0.000002\end{array}$

$\begin{array}{llll}989.4 & 0.2044 & -0.000032\end{array}$

$\begin{array}{lll}990.9 & 0.2394 & -0.000027\end{array}$

$\begin{array}{lll}993.0 & 0.3237 & -0.000025\end{array}$

$1000.2 \quad 0.5196-0.000020$

$1007.3 \quad 0.5892-0.000015$

$1020.9 \quad 0.6971-0.000011$

$1052.4 \quad 0.4604-0.000004$

$\begin{array}{lll}1080.7 & 0.1338 & -0.000001\end{array}$

$\begin{array}{lll}1106.2 & -0.0862 & 0.000000\end{array}$

$\begin{array}{lll}1149.5 & -0.1997 & 0.000001\end{array}$

$1184.7 \quad 0.4225-0.000001$

$1213.3 \quad 1.5914-0.000003$

$\begin{array}{llll}977.4 & 0.3153 & -0.000033\end{array}$

$\begin{array}{llll}983.0 & 0.6056 & -0.000031\end{array}$

$994.3 \quad 1.0209-0.000026$

$1017.1 \quad 1.6106-0.000019$

$1040.2 \quad 1.9248-0.000015$

$1063.5 \quad 1.8268-0.000010$

$1086.9 \quad 1.3908-0.000006$

$\begin{array}{lll}1110.4 & 0.7027 & -0.000002\end{array}$

$\begin{array}{lll}1133.8 & -0.0389 & 0.000000\end{array}$

$\begin{array}{lll}1174.4 & -1.3053 & 0.000003\end{array}$

$\begin{array}{lll}11202.7 & -1.5953 & 0.000003\end{array}$
0.310
0.088

0.021

0.186

0.387

0.001

1.345

0.046

0.061

0.107

0.238

0.340

0.392

0.155

0.000

0.076

0.143

0.047

1.945

0.042

0.057

0.105

0.270

0.347

0.486

0.212

0.018

0.007

0.040

0.179

2.533

0.099

0.367

1.042

2.594

3.705

3.337

1.934

0.494

0.002

1.704

2.545
990.2132

990.2132

990.2132

990.2132

990.2132

990.2132

990.2132

988.0363

988.0363

988.0363

988.0363

988.0363

988.0363

988.0363

988.0363

988.0363

988.0363

988.0363

988.0363

985.6952

985.6952

985.6952

985.6952

985.6952

985.6952

985.6952

985.6952

985.6952

985.6952

985.6952

985.6952

971.7978

971.7978

971.7978

971.7978

971.7978

971.7978

971.7978

971.7978

971.7978

971.7978

971.7978 
molality molarity

$t /{ }^{\circ} \mathrm{C}$ mass frac

Density
$\exp / \mathrm{kg} / \mathrm{m}^{3}$

App vol App vol

$\mathrm{m}^{3} / \mathrm{kg} \quad \mathrm{m}^{3} / \mathrm{kg}$

0.0005620 .000563

$0.000390 \quad 0.000440$

$0.000391 \quad 0.000442$

$0.000400 \quad 0.000446$

$0.000418 \quad 0.000454$

$0.000436 \quad 0.000463$

$0.000452 \quad 0.000472$

$0.000468 \quad 0.000481$

$0.000483 \quad 0.000491$

$0.000497 \quad 0.000501$

0.0005190 .000518

$0.000534 \quad 0.000531$

$\begin{array}{ll}0.000561 & 0.000558\end{array}$

Average Res

Std dev Res

Avg - 4std

Avg + 4std
Density Density

calc / Residual / Residual / Square of Inconsistent

$\mathrm{kg} / \mathrm{m}^{3}$

$\mathrm{kg} / \mathrm{m}^{3}$

$\mathrm{m}^{3} / \mathrm{kg}$

$0.5769-0.000001$

$0.4574-0.000049$

$0.9525-0.000051$

$1.7673-0.000046$

$2.9130-0.000036$

$\begin{array}{lll}3.4187 & -0.000027\end{array}$

$3.5392-0.000020$

$3.1393-0.000014$

$2.3954-0.000008$

$1.3959-0.000004$

$-0.54890 .000001$

$\begin{array}{ll}-1.7506 & 0.000003\end{array}$

$\begin{array}{ll}-2.3882 & 0.000003\end{array}$

$0.2771-0.000011$

$1.0300 \quad 0.000014$

$-3.8430-0.000065$

$\begin{array}{ll}4.3973 & 0.000044\end{array}$

Curve fit for the model App vol $=\left(w+c 2+c 3^{\star} t{ }^{\circ} \mathrm{C}\right) /\left(\mathrm{c} 0^{*} w+c 1\right) / e^{\wedge}\left(0.000001^{*}\left(t^{\circ} \mathrm{C}+\mathrm{c} 4\right)^{\wedge} 2\right)$

\section{$\mathrm{cO}$}

c1

452.5876

$\begin{array}{ll}\mathrm{c} 4 & 0.016292 \\ & 1692.395\end{array}$

$\operatorname{Max} T$

Min w

0

Max w

0.006564

Avg dens res $\quad 0.277127$

Std dens res $\quad 1.030043$

Avg app vol res *10^3 -0.01061

Std app vol res *10^3 0.013539

No of points in corr

174
0

No of inconsistent poir

0

Inconsistent data not used (Residual greater than average +/- 4 standard deviations) 
Density of aqueous solutions of $\mathbf{N i C l} 2$

References (23) Dolian, F. E. The Viscosities of Solutions of Chlorides in Certain Solvents. J. Phys. Chem. 1937, 41, 1129-1138.

(37) Herrington, Thelma M.; Roffey, Michael G.; Smith, David P.; Densities of Aqueous Electrolytes $\mathrm{MnCl}_{2}, \mathrm{CoCl}_{2}, \mathrm{NiCl}_{2}, \mathrm{ZnCl}_{2}$ and $\mathrm{CdCl}_{2}$ from 25 to $72^{\circ} \mathrm{C}$ at $1 \mathrm{~atm}, \mathrm{~J}$. Chem. Eng. Data, 1986, 31, 221-225

(83) Pearce, J. N.; Eckstrom, H. C. The Vapor Pressure and some Thermodynamic Properties of Aqueous Solutions of Nickel Chloride at $25^{\circ}$ C. J. Phys. Chem. 1937, 41, 563-565.

(90) Perry, R. H.; Green, D. W. Perry's Chemical Engineers' Handbook; McGraw Hill: New York, 7th edition, 1997.

(91) Phang, S. Viscosity and Transference Number Measurements of Concentrated Nickel Chloride Solutions at 298.15K. Aust. J. Chem. 1979, 32, 1149-1153.

(97) Pogue, R. F.; Atkinson, G. Solution Thermodynamics of First-Row Transition Elements. 1. Apparent Molal Volumes of Aqueous NiCl ${ }_{2}$, $\mathrm{Ni}\left(\mathrm{ClO}_{4}\right)_{2}, \mathrm{CuCl}_{2}$, and $\mathrm{Cu}\left(\mathrm{ClO}_{4}\right)_{2}$, from 15 to $55^{\circ} \mathrm{C}$. J. Chem. Eng. Data 1988, 33, 370-376.

(105) Rard, J. A. Densities and Apparent Molal Volumes of Aqueous Nickel Chloride at $25^{\circ} \mathrm{C}$. J. Chem. Eng. Data 1986, $31,183-185$.

(117) Schmelzer, N.; Einfeldt, J. Density Measurements in Some Aqueous and Non-Aqueous Electrolyte Solutions at $25^{\circ} \mathrm{C}$. Wiss. Z. Uni. Rostock 1989, 38, 81-82.

(123) Spitzer, J. J.; Singh, P. P.; McCurdy, K. G.; Hepler, L. G. Apparent Molar Heat Capacities and Volumes of Aqueous Electrolytes: CaCl ${ }_{2}$, $\mathrm{Cd}\left(\mathrm{NO}_{3}\right)_{2}, \mathrm{CoCl}_{2}, \mathrm{Cu}\left(\mathrm{ClO}_{4}\right)_{2}, \mathrm{Mg}\left(\mathrm{ClO}_{4}\right)_{2}$, and $\mathrm{NiCl}_{2}$. J. Sol. Chem. 1978, 7, 81-86.

(124) Stokes, R. H.; Phang, S.; Mills, R. Density, Conductance, Transference Numbers, and Diffusion Measurements in Concentrated Solutions of Nickel Chloride. J. Sol. Chem. 1979, 8, 489-500.

\begin{tabular}{|c|c|c|c|c|c|c|c|c|c|c|c|c|c|c|c|}
\hline molality & molarity & $\mathrm{t} /{ }^{\circ} \mathrm{C}$ & lass frac & $\begin{array}{r}\text { Density } \\
\exp / \mathrm{kg} / \mathrm{m}^{3}\end{array}$ & Ref & $\begin{array}{r}\text { App vol } \\
\exp / \\
\mathrm{m}^{3} / \mathrm{kg}\end{array}$ & $\begin{array}{r}\text { App vol } \\
\text { Calc / } \\
\mathrm{m}^{3} / \mathrm{kg}\end{array}$ & $\begin{array}{r}\text { Density } \\
\text { calc / } \\
\mathrm{kg} / \mathrm{m}^{3}\end{array}$ & $\begin{array}{r}\text { Density } \\
\text { Residual / } \\
\mathrm{kg} / \mathrm{m}^{3}\end{array}$ & $\begin{array}{r}\text { App Vol } \\
\text { Residual / } \\
\mathrm{m}^{3} / \mathrm{kg}\end{array}$ & $\begin{array}{r}\text { Square of } \\
\text { Density } \\
\text { Res }\end{array}$ & $\begin{array}{l}\text { Inconsistent } \\
\text { based on } \\
\text { density }\end{array}$ & $\begin{array}{c}\text { Inconsistent } \\
\text { based on } \\
\text { apparent } \\
\text { volume }\end{array}$ & $\begin{array}{l}\text { Apparent } \\
\text { molal volume } \\
/ \mathrm{cm}^{3} / \mathrm{mol}\end{array}$ & $\begin{array}{r}\text { Density of } \\
\text { water } / \mathrm{kg} / \mathrm{m}^{3}\end{array}$ \\
\hline 0.00906 & & 15 & 0.0012 & 1000.21 & (97) & 0.000051 & 0.000085 & 1000.17 & 0.0398 & -0.000034 & 0.002 & & & 6.5600 & 999.0996 \\
\hline 0.01948 & & 15 & 0.0025 & 1001.49 & (97) & 0.000052 & 0.000085 & 1001.41 & 0.0835 & -0.000033 & 0.007 & & & 6.7000 & 999.0996 \\
\hline 0.02981 & & 15 & 0.0038 & 1002.76 & (97) & 0.000052 & 0.000085 & 1002.63 & 0.1258 & -0.000033 & 0.016 & & & 6.8000 & 999.0996 \\
\hline 0.0491 & & 15 & 0.0063 & 1005.10 & (97) & 0.000055 & 0.000085 & 1004.91 & 0.1908 & -0.000030 & 0.036 & & & 7.1900 & 999.0996 \\
\hline 0.07449 & & 15 & 0.0096 & 1008.18 & (97) & 0.000058 & 0.000086 & 1007.91 & 0.2727 & -0.000028 & 0.074 & & & 7.4900 & 999.0996 \\
\hline 0.1023 & & 15 & 0.0131 & 1011.55 & (97) & 0.000060 & 0.000086 & 1011.19 & 0.3570 & -0.000027 & 0.127 & & & 7.7400 & 999.0996 \\
\hline 0.2025 & & 15 & 0.0256 & 1023.59 & (97) & 0.000064 & 0.000088 & 1022.95 & 0.6400 & -0.000024 & 0.410 & & & 8.3500 & 999.0996 \\
\hline 0.3925 & & 15 & 0.0484 & 1046.02 & (97) & 0.000073 & 0.000092 & 1045.05 & 0.9761 & -0.000018 & 0.953 & & & 9.5200 & 999.0996 \\
\hline 0.6 & & 15 & 0.0722 & 1070.10 & (97) & 0.000081 & 0.000096 & 1068.86 & 1.2417 & -0.000015 & 1.542 & & & 10.4500 & 999.0996 \\
\hline 0.8003 & & 15 & 0.0940 & 1092.92 & (97) & 0.000087 & 0.000099 & 1091.52 & 1.3985 & -0.000012 & 1.956 & & & 11.2400 & 999.0996 \\
\hline 0.9881 & & 15 & 0.1135 & 1113.91 & (97) & 0.000092 & 0.000102 & 1112.49 & 1.4238 & -0.000010 & 2.027 & & & 11.9600 & 999.0996 \\
\hline 1.497 & & 15 & 0.1625 & 1169.36 & (97) & 0.000104 & 0.000111 & 1167.92 & 1.4315 & -0.000006 & 2.049 & & & 13.5000 & 999.0996 \\
\hline 1.9982 & & 15 & 0.2057 & 1221.58 & (97) & 0.000115 & 0.000118 & 1220.58 & 1.0084 & -0.000003 & 1.017 & & & 14.8800 & 999.0996 \\
\hline 2.5079 & & 15 & 0.2453 & 1273.04 & (97) & 0.000123 & 0.000125 & 1272.17 & 0.8636 & -0.000002 & 0.746 & & & 15.9400 & 999.0996 \\
\hline 2.9976 & & 15 & 0.2798 & 1321.41 & (97) & 0.000128 & 0.000131 & 1319.93 & 1.4721 & -0.000003 & 2.167 & & & 16.6500 & 999.0996 \\
\hline \multirow{5}{*}{\multicolumn{2}{|c|}{3.3543}} & 15 & 0.3030 & 1354.21 & (97) & 0.000135 & 0.000136 & 1353.63 & 0.5733 & -0.000001 & 0.329 & & & 17.4700 & 999.0996 \\
\hline & & 18 & 0.0100 & 1008.20 & (90) & 0.000047 & 0.000086 & 1007.81 & 0.3892 & -0.000038 & 0.152 & & & & 998.5956 \\
\hline & & 18 & 0.0200 & 1017.90 & (90) & 0.000052 & 0.000087 & 1017.16 & 0.7350 & -0.000035 & 0.540 & & & & 998.5956 \\
\hline & & 18 & 0.0400 & 1037.50 & (90) & 0.000063 & 0.000091 & 1036.30 & 1.1987 & -0.000028 & 1.437 & & & & 998.5956 \\
\hline & & 18 & 0.0800 & 1078.50 & (90) & 0.000074 & 0.000097 & 1076.36 & 2.1367 & -0.000023 & 4.566 & & & & 998.5956 \\
\hline 0.003506 & & 25 & 0.0005 & 997.47 & (117) & 0.000062 & 0.000083 & 997.46 & 0.0097 & -0.000022 & 0.000 & & & & 997.0449 \\
\hline 0.00366 & & 25 & 0.0005 & 997.50 & (124) & 0.000038 & 0.000083 & 997.48 & 0.0215 & -0.000046 & 0.000 & & & & 997.0449 \\
\hline 0.008446 & & 25 & 0.0011 & 998.07 & (117) & 0.000061 & 0.000084 & 998.05 & 0.0246 & -0.000023 & 0.001 & & & & 997.0449 \\
\hline
\end{tabular}


$\mathbf{t} /{ }^{\circ} \mathrm{C}$ mass frac $\begin{array}{r}\text { Density } \\ \exp / \mathrm{kg} / \mathrm{m}^{3}\end{array}$

\subsection{4}

0.01031

0.0198

0.02149

0.02467

0.02912

0.039987

0.04212

0.04488

0.04962

0.07489

0.07527

0.07571

0.07941

0.0828

0.089987

0.09012

0.1

0.1023

0.104

0.1167

0.1264

0.13234

0.1498

0.1511

0.15996

0.1997

0.2

0.2015
0.24988

0.2985

0.3001

0.35101

0.35986

0.4
0.4045 molarity

0.0494

0.1266

782

0.3532 t/ ${ }^{\circ} \mathrm{C}$ mass frac $\begin{array}{r}\text { Density } \\ \exp / \mathrm{kg} / \mathrm{m}^{3}\end{array}$

Ref

998.27

$998.31 \quad(124)$

$999.45 \quad(97)$

$999.68 \quad(124)$

$\begin{array}{lrl}0.0028 & 999.68 & (124) \\ 0.0032 & 1000.05 & (117)\end{array}$

$0.0038 \quad 1000.58$

$0.0052 \quad 1001.89 \quad(105)$

$0.0054 \quad 1002.18 \quad(124)$

$0.0058 \quad 1002.46 \quad$ (123)

$0.0064 \quad 1003.05 \quad$ (91)

$0.0064 \quad 1003.05 \quad(97)$

$0.0097 \quad 1006.14$

$0.0097 \quad 1006.12$

$0.0102 \quad 1006.84 \quad(117)$

$0.0106 \quad 1007.10 \quad(124)$

$0.0114 \quad 1007.48$

$0.0115 \quad 1007.91$

$0.0115 \quad 1007.90 \quad(123)$

$0.0128 \quad 1009.11 \quad$ (83)

$0.0131 \quad 1009.37 \quad(97)$

$0.0133 \quad 1009.66 \quad(124)$

$0.0149 \quad 1010.96 \quad(124)$

$0.0161 \quad 1012.20 \quad(123)$

$0.0162 \quad 1012.27 \quad(91)$

$0.0169 \quad 1013.36 \quad(117)$

$\begin{array}{lll}0.0190 & 1014.97 & (123) \\ 0.0192 & 1015.19 & (123)\end{array}$

$0.0203 \quad 1016.25 \quad(105)$

$0.0225 \quad 1018.48 \quad$ (23)

$\begin{array}{lll}0.0252 & 1020.95 & (97)\end{array}$

$0.0253 \quad 1020.93 \quad$ (83)

$0.0255 \quad 1021.12 \quad(123)$

$\begin{array}{lll}0.0314 & 1026.81 \quad(105)\end{array}$

1030.24

$\begin{array}{lll}0.0373 & 1032.49 \quad(124)\end{array}$

$0.0374 \quad 1032.77 \quad(37)$

$0.0435 \quad 1040.34 \quad(117)$

$0.0441 \quad 1039.07 \quad(91)$

$0.0441 \quad 1039.74$

$0.0446 \quad 1039.66 \quad(105)$

$0.0493 \quad 1044.19 \quad(83)$

$0.0498 \quad 1044.89$
App vol App vol

$\exp / \quad$ Calc $/$

$$
\mathrm{m}^{3} / \mathrm{kg} \quad \mathrm{m}^{3} / \mathrm{kg}
$$

0.0000550 .000084

$\begin{array}{ll}0.000051 & 0.000084\end{array}$

$0.000059 \quad 0.000084$

$0.000051 \quad 0.000084$

$0.000057 \quad 0.000084$

$0.000060 \quad 0.000084$

$0.000062 \quad 0.000084$

$0.000057 \quad 0.000084$

$0.000066 \quad 0.000084$

0.0000620 .000084

$0.000063 \quad 0.000084$

$0.000069 \quad 0.000085$

$0.000065 \quad 0.000085$

$\begin{array}{ll}0.000072 & 0.000085\end{array}$

$0.000045 \quad 0.000085$

$0.000060 \quad 0.000085$

$0.000092 \quad 0.000085$

$0.000065 \quad 0.000085$

$0.000067 \quad 0.000085$

$0.000066 \quad 0.000086$

$0.000067 \quad 0.000086$

$\begin{array}{ll}0.000061 & 0.000086\end{array}$

$0.000077 \quad 0.000086$

$0.000071 \quad 0.000086$

$0.000072 \quad 0.000086$

0.0000450 .000086

$\begin{array}{ll}0.000073 & 0.000087\end{array}$

$0.000070 \quad 0.000087$

$\begin{array}{ll}0.000070 & 0.000087\end{array}$

$\begin{array}{lll}0.000065 & 0.000087\end{array}$

$0.000072 \quad 0.000088$

$0.000074 \quad 0.000088$

$\begin{array}{ll}0.000074 & 0.000088\end{array}$

$\begin{array}{lll}0.000076 & 0.000089\end{array}$

$0.000080 \quad 0.000089$

$0.000079 \quad 0.000090$

$0.000076 \quad 0.000090$

$\begin{array}{ll}0.000044 & 0.000091\end{array}$

$0.000082 \quad 0.000091$

$0.000069 \quad 0.000091$

$0.000080 \quad 0.000091$

$0.000084 \quad 0.000092$

$\begin{array}{ll}0.000081 & 0.000092\end{array}$
Density Density App Vol Square of Inconsistent Inconsistent Apparent Density of \begin{tabular}{rrrrrr} 
calc / & Residual / & Residual / & Density \\
$\mathrm{kg} / \mathrm{m}^{3}$ & $\mathrm{~kg} / \mathrm{m}^{3}$ & $\mathrm{~m}^{3} / \mathrm{kg}$ & Res density & $\begin{array}{r}\text { based on } \\
\text { apparent }\end{array}$ & molal volume \\
\hline $\mathrm{cm}^{3} / \mathrm{mol}$
\end{tabular}

$\begin{array}{llll}998.23 & 0.0365 & -0.000028 & 0.001\end{array}$

$\begin{array}{llll}998.27 & 0.0439 & -0.000033 & 0.002\end{array}$

$\begin{array}{llll}999.39 & 0.0628 & -0.000025 & 0.004\end{array}$

$\begin{array}{lllll}999.59 & 0.0906 & -0.000033 & 0.008\end{array}$

$\begin{array}{lllll}999.97 & 0.0844 & -0.000026 & 0.007\end{array}$

$\begin{array}{llll}1000.49 & 0.0894 & -0.000024 & 0.008\end{array}$

$\begin{array}{llll}1001.78 & 0.1164 & -0.000022 & 0.014\end{array}$

$\begin{array}{llll}1002.03 & 0.1514 & -0.000028 & 0.023\end{array}$

$\begin{array}{llll}1002.35 & 0.1064 & -0.000018 & 0.011\end{array}$

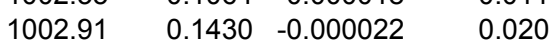

$\begin{array}{lll}0.1392 & -0.000022 & 0.019\end{array}$

$\begin{array}{llll}1005.90 & 0.1595 & -0.000016 & 0.025\end{array}$

$\begin{array}{llll}1005.94 & 0.1966 & -0.000020 & 0.039\end{array}$

$\begin{array}{llll}1005.99 & 0.1303 & -0.000013 & 0.017\end{array}$

$\begin{array}{lll}0.4111 & -0.000040 & 0.169\end{array}$

$\begin{array}{llll}1006.83 & 0.2716 & -0.000025 & 0.074\end{array}$

$\begin{array}{llll}1007.55 & -0.0744 & 0.000006 & 0.006\end{array}$

$\begin{array}{llll}1007.67 & 0.2370 & -0.000020 & 0.056\end{array}$

$\begin{array}{lll}0.2105 & -0.000018 & 0.044\end{array}$

$\begin{array}{llll}1008.85 & 0.2512 & -0.000019 & 0.063\end{array}$

$\begin{array}{llll}1009.12 & 0.2457 & -0.000018 & 0.060\end{array}$

$\begin{array}{llll}1009.32 & 0.3356 & -0.000025 & 0.113\end{array}$

$\begin{array}{lll}0.1420 & -0.000009 & 0.020\end{array}$

$\begin{array}{llll}1011.96 & 0.2452 & -0.000015 & 0.060\end{array}$

$\begin{array}{llll}1012.04 & 0.2268 & -0.000014 & 0.051\end{array}$

$\begin{array}{llll}1012.66 & 0.7045 & -0.000041 & 0.496\end{array}$

$\begin{array}{llll}1014.70 & 0.2660 & -0.000014 & 0.071\end{array}$

$0.3300-0.000017$

$\begin{array}{llll}1017.96 & 0.5205 & -0.000022 & 0.271\end{array}$

$\begin{array}{llll}1020.55 & 0.4060 & -0.000015 & 0.165\end{array}$

$\begin{array}{llll}1020.58 & 0.3481 & -0.000013 & 0.121\end{array}$

$\begin{array}{llll}1020.76 & 0.3677 & -0.000014 & 0.135\end{array}$

$\begin{array}{llll}1026.40 & 0.4033 & -0.000012 & 0.163\end{array}$

$\begin{array}{llll}1029.89 & 0.3537 & -0.000010 & 0.125\end{array}$

$\begin{array}{llll}1032.06 & 0.4349 & -0.000011 & 0.189\end{array}$

$\begin{array}{llll}1032.24 & 0.5292 & -0.000013 & 0.280\end{array}$

$\begin{array}{llll}1038.14 & 2.2007 & -0.000047 & 4.843\end{array}$

$\begin{array}{llll}1038.67 & 0.4015 & -0.000008 & 0.161\end{array}$

$\begin{array}{llll}1038.71 & 1.0328 & -0.000022 & 1.067\end{array}$

$\begin{array}{llll}1039.16 & 0.4975 & -0.000010 & 0.248\end{array}$

$\begin{array}{llll}1043.80 & 0.3976 & -0.000007 & 0.158\end{array}$

$\begin{array}{llll}1044.31 & 0.5741 & -0.000011 & 0.330\end{array}$
$7.1800 \quad 997.0449$ 997.0449

$7.6800 \quad 997.0449$ 997.0449 997.0449

$7.8100 \quad 997.0449$ 997.0449 997.0449

$8.5600 \quad 997.0449$ 997.0449

$8.1400 \quad 997.0449$

$8.8900 \quad 997.0449$

$8.4100 \quad 997.0449$

$9.3000 \quad 997.0449$ 997.0449 997.0449 997.0449 997.0449

$8.7300 \quad 997.0449$ 997.0449

$8.7000 \quad 997.0449$ 997.0449 997.0449

$9.2300 \quad 997.0449$

997.0449 997.0449

$9.4600 \quad 997.0449$

$9.0600 \quad 997.0449$ 997.0449 997.0449

$9.3500 \quad 997.0449$ 997.0449

$9.5600 \quad 997.0449$ 997.0449 997.0449 997.0449 997.0449 997.0449 997.0449 997.0449 997.0449 997.0449

$10.5200 \quad 997.0449$ 
$\mathbf{t} /{ }^{\circ} \mathrm{C}$ mass frac $\begin{array}{r}\text { Density } \\ \exp / \mathrm{kg} / \mathrm{m}^{3}\end{array}$

$$
\exp / \mathrm{kg} / \mathrm{m}^{3}
$$

\begin{tabular}{|c|c|c|c|c|c|}
\hline 0.4677 & & 25 & 0.0572 & 1052.28 & (37) \\
\hline 0.48997 & & 25 & 0.0597 & 1054.66 & (105) \\
\hline & 0.5406 & 25 & 0.0660 & 1060.95 & (91) \\
\hline 0.5957 & & 25 & 0.0717 & 1066.87 & (97) \\
\hline 0.6 & & 25 & 0.0722 & 1067.01 & (83) \\
\hline & 0.6375 & 25 & 0.0771 & 1072.15 & (91) \\
\hline 0.64587 & & 25 & 0.0773 & 1072.42 & (105) \\
\hline 0.6996 & & 25 & 0.0831 & 1078.78 & (37) \\
\hline 0.7891 & & 25 & 0.0928 & 1088.73 & (97) \\
\hline 0.8 & & 25 & 0.0940 & 1089.48 & (83) \\
\hline 0.81012 & & 25 & 0.0950 & 1090.87 & (105) \\
\hline & 0.8545 & 25 & 0.1010 & 1097.06 & (91) \\
\hline 0.9188 & & 25 & 0.1064 & 1103.33 & (37) \\
\hline 0.9881 & & 25 & 0.1135 & 1110.80 & (97) \\
\hline 0.99961 & & 25 & 0.1147 & 1111.84 & (105) \\
\hline 1 & & 25 & 0.1147 & 1111.58 & (83) \\
\hline 1.159 & & 25 & 0.1306 & 1129.69 & (37) \\
\hline 1.2097 & & 25 & 0.1355 & 1134.71 & (105) \\
\hline & 1.2360 & 25 & 0.1405 & 1140.19 & (91) \\
\hline 1.4107 & & 25 & 0.1546 & 1156.75 & (37) \\
\hline 1.4398 & & 25 & 0.1573 & 1159.30 & (105) \\
\hline 1.4973 & & 25 & 0.1625 & 1166.01 & (97) \\
\hline 1.5 & & 25 & 0.1628 & 1165.33 & (83) \\
\hline 1.6526 & & 25 & 0.1764 & 1182.23 & (37) \\
\hline 1.6661 & & 25 & 0.1776 & 1183.01 & (105) \\
\hline & 1.6270 & 25 & 0.1782 & 1183.67 & (91) \\
\hline 1.8985 & & 25 & 0.1975 & 1207.64 & (37) \\
\hline 1.9982 & & 25 & 0.2057 & 1218.01 & (97) \\
\hline 1.9994 & & 25 & 0.2058 & 1217.28 & (105) \\
\hline 2 & & 25 & 0.2059 & 1216.74 & (83) \\
\hline 2.0001 & & 25 & 0.2059 & 1217.34 & (105) \\
\hline 2.148 & & 25 & 0.2178 & 1232.95 & (37) \\
\hline 2.3331 & & 25 & 0.2322 & 1250.67 & (105) \\
\hline 2.3916 & & 25 & 0.2366 & 1257.23 & (37) \\
\hline 2.5 & & 25 & 0.2447 & 1266.39 & (83) \\
\hline 2.5075 & & 25 & 0.2453 & 1269.04 & (97) \\
\hline 2.6336 & & 25 & 0.2545 & 1280.97 & (37) \\
\hline 2.6652 & & 25 & 0.2568 & 1283.14 & (105) \\
\hline 2.8917 & & 25 & 0.2726 & 1305.88 & (37) \\
\hline 3 & & 25 & 0.2800 & 1314.34 & (83) \\
\hline 3.0008 & & 25 & 0.2800 & 1315.19 & (105) \\
\hline 3.0023 & & 25 & 0.2801 & 1316.71 & (97) \\
\hline 3.3082 & & 25 & 0.3001 & 1343.93 & (105) \\
\hline
\end{tabular}

\section{App vol App vol}

exp / Calc/

$$
\mathrm{m}^{3} / \mathrm{kg} \quad \mathrm{m}^{3} / \mathrm{kg}
$$

$\begin{array}{ll}0.000082 & 0.000093\end{array}$ $\begin{array}{ll}0.000085 & 0.000093\end{array}$ $0.000088 \quad 0.000094$ $0.000087 \quad 0.000095$ $0.000092 \quad 0.000096$ $0.000091 \quad 0.000096$ $0.000090 \quad 0.000096$ $\begin{array}{lll}0.000089 & 0.000097\end{array}$ $0.000093 \quad 0.000099$ 0.0000970 .000099 $0.000095 \quad 0.000100$ $0.000097 \quad 0.000101$ $0.000095 \quad 0.000102$ $0.000098 \quad 0.000103$ $0.000100 \quad 0.000103$ $0.000102 \quad 0.000103$ $\begin{array}{ll}0.000101 & 0.000106\end{array}$ $0.000105 \quad 0.000107$ $0.000107 \quad 0.000108$ $0.000107 \quad 0.000110$ $0.000110 \quad 0.00$ $0.000109 \quad 0.000112$ $0.000113 \quad 0.000112$ $0.000112 \quad 0.000114$ $0.000115 \quad 0.000114$ $0.000115 \quad 0.000115$ $0.000117 \quad 0.000118$ $0.000119 \quad 0.000120$ $\begin{array}{ll}0.000121 & 0.000120\end{array}$ $0.000123 \quad 0.000120$ $0.000121 \quad 0.000120$ $0.000122 \quad 0.000122$ $\begin{array}{lll}0.000127 & 0.000125\end{array}$ $0.000126 \quad 0.000126$ $\begin{array}{ll}0.000131 & 0.000127\end{array}$ $0.000127 \quad 0.000127$ $0.000129 \quad 0.000129$ $0.000132 \quad 0.000130$ $0.000133 \quad 0.000133$ $0.000138 \quad 0.000134$ $0.000137 \quad 0.000134$ $0.000134 \quad 0.000134$ $0.000140 \quad 0.000138$
Density Density App Vol Square of Inconsistent Inconsistent Apparent Density of calc / Residual / Residual / Density based on based on molal volume $\mathrm{kg} / \mathrm{m}^{3} \quad \mathrm{~kg} / \mathrm{m}^{3} \quad \mathrm{~m}^{3} / \mathrm{kg} \quad$ Res density apparent volume

$1 \mathrm{~cm}^{3} / \mathrm{mol}$

$1051.58 \quad 0.7004 \quad-0.000011 \quad 0.490$

$1054.13 \quad 0.5279-0.000008 \quad 0.279$

$\begin{array}{llll}1060.49 & 0.4620 & -0.000006 & 0.213\end{array}$

$\begin{array}{llll}1066.20 & 0.6755 & -0.000008 & 0.456\end{array}$

$\begin{array}{llll}1066.68 & 0.3248 & -0.000004 & 0.106\end{array}$

$\begin{array}{llll}1071.70 & 0.4452 & -0.000005 & 0.198\end{array}$

$\begin{array}{llll}1071.89 & 0.5321 & -0.000006 & 0.283\end{array}$

$\begin{array}{llll}1077.96 & 0.8185 & -0.000008 & 0.670\end{array}$

$\begin{array}{llll}1088.03 & 0.7033 & -0.000006 & 0.495\end{array}$

$\begin{array}{llll}1089.25 & 0.2353 & -0.000002 & 0.055\end{array}$

$\begin{array}{llll}1090.38 & 0.4892 & -0.000004 & 0.239\end{array}$

$\begin{array}{llll}1096.66 & 0.3965 & -0.000003 & 0.157\end{array}$

$\begin{array}{llll}1102.50 & 0.8335 & -0.000006 & 0.695\end{array}$

$\begin{array}{llll}1110.17 & 0.6301 & -0.000005 & 0.397\end{array}$

$\begin{array}{llll}1111.44 & 0.3970 & -0.000003 & 0.158\end{array}$

$\begin{array}{llll}1111.49 & 0.0929 & -0.000001 & 0.009\end{array}$

$\begin{array}{llll}1128.93 & 0.7561 & -0.000005 & 0.572\end{array}$

$\begin{array}{llll}1134.45 & 0.2557 & -0.000001 & 0.065\end{array}$

$\begin{array}{llll}1140.05 & 0.1439 & -0.000001 & 0.021\end{array}$

$\begin{array}{llll}1156.14 & 0.6142 & -0.000003 & 0.377\end{array}$

$\begin{array}{llll}1159.25 & 0.0522 & 0.000000 & 0.003\end{array}$

$\begin{array}{lllll}1165.38 & 0.6307 & -0.000003 & 0.398\end{array}$

$\begin{array}{llll}1165.66 & -0.3299 & 0.000001 & 0.109\end{array}$

$\begin{array}{llll}1181.80 & 0.4322 & -0.000002 & 0.187\end{array}$

$\begin{array}{llll}1183.22 & -0.2062 & 0.000001 & 0.043\end{array}$

$\begin{array}{llll}1183.89 & -0.2211 & 0.000001 & 0.049\end{array}$

$\begin{array}{llll}1207.40 & 0.2353 & -0.000001 & 0.055\end{array}$

$\begin{array}{llll}1217.65 & 0.3629 & -0.000001 & 0.132\end{array}$

$\begin{array}{llll}1217.77 & -0.4930 & 0.000002 & 0.243\end{array}$

$\begin{array}{llll}1217.83 & -1.0944 & 0.000004 & 1.198\end{array}$

$\begin{array}{llll}1217.84 & -0.5046 & 0.000002 & 0.255\end{array}$

$\begin{array}{llll}1232.90 & 0.0535 & 0.000000 & 0.003\end{array}$

$\begin{array}{llll}1251.49 & -0.8227 & 0.000002 & 0.677\end{array}$

$\begin{array}{llll}1257.31 & -0.0843 & 0.000000 & 0.007\end{array}$

$\begin{array}{llll}1268.03 & -1.6428 & 0.000004 & 2.699\end{array}$

$\begin{array}{llll}1268.77 & 0.2723 & -0.000001 & 0.074\end{array}$

$\begin{array}{llll}1281.12 & -0.1460 & 0.000000 & 0.021\end{array}$

$\begin{array}{llll}1284.19 & -1.0508 & 0.000002 & 1.104\end{array}$

$\begin{array}{llll}1306.01 & -0.1270 & 0.000000 & 0.016\end{array}$

$\begin{array}{llll}1316.30 & -1.9621 & 0.000004 & 3.850\end{array}$

$\begin{array}{llll}1316.38 & -1.1868 & 0.000002 & 1.409\end{array}$

$\begin{array}{llll}1316.52 & 0.1892 & 0.000000 & 0.036\end{array}$

$\begin{array}{llll}1345.12 & -1.1866 & 0.000002 & 1.408\end{array}$
997.0449

997.0449

997.0449

$11.3000 \quad 997.0449$

997.0449

997.0449

997.0449

997.0449

$12.0200 \quad 997.0449$

997.0449

997.0449

997.0449

997.0449

$12.7400 \quad 997.0449$

997.0449

997.0449

997.0449

997.0449

997.0449

997.0449

997.0449

$14.1000 \quad 997.0449$

997.0449

997.0449

997.0449

997.0449

997.0449

$15.3600 \quad 997.0449$

997.0449

997.0449

997.0449

997.0449

997.0449

997.0449

997.0449

$16.4100 \quad 997.0449$

997.0449

997.0449

997.0449

997.0449

997.0449

$17.3400 \quad 997.0449$

997.0449 
molality molarity

$\mathbf{t} /{ }^{\circ} \mathrm{C}$ mass frac $\begin{array}{r}\text { Density } \\ \exp / \mathrm{kg} / \mathrm{m}^{3}\end{array}$

1349.79

3.3543

3.6128

3.9221

4.2549

4.4271

4.9116

4.9832

0.00906

0.01947

0.02975

0.04911

0.07449

0.1023

0.2025

0.3001

0.3925

0.4677

0.6

0.6996

0.8003

0.9188

0.9881

1.159

1.4107

1.497

1.6526

1.8985

1.9982

2.148

2.3916

2.5079

2.6336

2.8917

2.9976

3.3543

0.00906

0.01991

0.03033

0.04911

0.0752

0.1026

0.2025

$\begin{array}{rrr}25 & 0.3030 & 1349.79 \\ 25 & 0.3189 & 1371.87 \\ 25 & 0.3370 & 1399.64 \\ 25 & 0.3414 & 1406.07 \\ 25 & 0.3555 & 1428.90 \\ 25 & 0.3646 & 1443.80 \\ 25 & 0.3890 & 1485.42 \\ 25 & 0.3924 & 1490.71 \\ 35 & 0.0012 & 995.13 \\ 35 & 0.0025 & 996.39 \\ 35 & 0.0038 & 997.62 \\ 35 & 0.0063 & 999.94 \\ 35 & 0.0096 & 1002.97 \\ 35 & 0.0131 & 1006.28 \\ 35 & 0.0256 & 1018.12 \\ 35 & 0.0374 & 1029.51 \\ 35 & 0.0484 & 1040.20 \\ 35 & 0.0572 & 1048.90 \\ 35 & 0.0722 & 1063.92 \\ 35 & 0.0831 & 1075.24 \\ 35 & 0.0940 & 1086.37 \\ 35 & 0.1064 & 1099.65 \\ 35 & 0.1135 & 1107.14 \\ 35 & 0.1306 & 1125.88 \\ 35 & 0.1546 & 1152.80 \\ 35 & 0.1625 & 1161.90 \\ 35 & 0.1764 & 1178.16 \\ 35 & 0.1975 & 1203.46 \\ 35 & 0.2057 & 1213.23 \\ 35 & 0.2178 & 1228.66 \\ 35 & 0.2366 & 1252.85 \\ 35 & 0.2453 & 1264.46 \\ 35 & 0.2545 & 1276.49 \\ 35 & 0.2726 & 1301.30 \\ 35 & 0.2798 & 1311.67 \\ 35 & 0.3030 & 1344.96 \\ 45 & 0.0012 & 991.31 \\ 45 & 0.0026 & 992.61 \\ 45 & 0.0039 & 993.85 \\ 45 & 0.0063 & 996.09 \\ 45 & 0.0097 & 999.19 \\ 45 & 0.0131 & 1002.42 \\ 45 & 0.0256 & 1014.17\end{array}$

App vol App vol $\begin{array}{cc}\exp / & \text { Calc / } \\ \mathrm{m}^{3} / \mathrm{kg} & \mathrm{m}^{3} / \mathrm{kg}\end{array}$

$\begin{array}{ll}0.000138 & 0.000139\end{array}$ $0.000144 \quad 0.000142$ $0.000147 \quad 0.000146$

$0.000148 \quad 0.000147$ $0.000150 \quad 0.000150$ 0.0001520 .000152 $0.000155 \quad 0.000157$ $0.000157 \quad 0.000158$ $0.000059 \quad 0.000082$ $0.000061 \quad 0.000082$ $0.000064 \quad 0.000082$ $0.000066 \quad 0.000083$ $0.000069 \quad 0.000083$ $0.000071 \quad 0.000084$ $0.000075 \quad 0.000086$ $0.000080 \quad 0.000088$ $0.000084 \quad 0.000090$ $\begin{array}{lll}0.000085 & 0.000092\end{array}$ $0.000090 \quad 0.000094$ $\begin{array}{ll}0.000092 & 0.000097\end{array}$ $0.000096 \quad 0.000099$ $\begin{array}{lll}0.000098 & 0.000101\end{array}$ $0.000101 \quad 0.000102$ $0.000104 \quad 0.000106$ $0.000110 \quad 0.000110$ $\begin{array}{ll}0.000112 & 0.000112\end{array}$ $0.000115 \quad 0.000115$ $0.000120 \quad 0.000119$ $0.000123 \quad 0.000120$ $0.000124 \quad 0.000123$ $0.000128 \quad 0.000127$ $0.000129 \quad 0.000129$ $0.000131 \quad 0.000131$ $\begin{array}{lll}0.000135 & 0.000134\end{array}$ $0.000135 \quad 0.000136$ $\begin{array}{ll}0.000140 & 0.000141\end{array}$ $0.000062 \quad 0.000078$ $\begin{array}{ll}0.000063 & 0.000079\end{array}$ $0.000065 \quad 0.000079$ $0.000068 \quad 0.000079$ $0.000070 \quad 0.000080$ $\begin{array}{lll}0.000073 & 0.000081\end{array}$ $\begin{array}{lll}0.000077 & 0.000083\end{array}$
Density Density App Vol Square of Inconsistent Inconsistent Apparent Density of calc / Residual / Residual / Density based on based on molal volume $\mathrm{kg} / \mathrm{m}^{3} \quad \mathrm{~kg} / \mathrm{m}^{3} \quad \mathrm{~m}^{3} / \mathrm{kg} \quad$ Res density apparent volume

$\begin{array}{llll}1372.91 & -1.0385 & 0.000002 & 1.079\end{array}$

$\begin{array}{llll}1400.44 & -0.8047 & 0.000001 & 0.647\end{array}$

$\begin{array}{llll}1407.27 & -1.2048 & 0.000002 & 1.452\end{array}$

$\begin{array}{llll}1429.32 & -0.4193 & 0.000001 & 0.176\end{array}$

$\begin{array}{llll}1443.96 & -0.1591 & 0.000000 & 0.025\end{array}$

$\begin{array}{llll}1484.08 & 1.3401 & -0.000002 & 1.796\end{array}$

$\begin{array}{llll}1489.87 & 0.8362 & -0.000001 & 0.699\end{array}$

$\begin{array}{llll}995.10 & 0.0259 & -0.000022 & 0.001\end{array}$

$\begin{array}{llll}996.34 & 0.0521 & -0.000021 & 0.003\end{array}$

$\begin{array}{llll}997.55 & 0.0708 & -0.000019 & 0.005\end{array}$

$\begin{array}{llll}999.84 & 0.1053 & -0.000017 & 0.011\end{array}$

$\begin{array}{llll}1002.83 & 0.1404 & -0.000015 & 0.020\end{array}$

$\begin{array}{llll}1006.10 & 0.1731 & -0.000013 & 0.030\end{array}$

$\begin{array}{llll}1017.84 & 0.2830 & -0.000011 & 0.080\end{array}$

$\begin{array}{llll}1029.19 & 0.3180 & -0.000008 & 0.101\end{array}$

$\begin{array}{llll}1039.87 & 0.3356 & -0.000006 & 0.113\end{array}$

$\begin{array}{llll}1048.50 & 0.4018 & -0.000006 & 0.161\end{array}$

$\begin{array}{llll}1063.57 & 0.3451 & -0.000004 & 0.119\end{array}$

$\begin{array}{llll}1074.82 & 0.4202 & -0.000004 & 0.177\end{array}$

$\begin{array}{llll}1086.11 & 0.2627 & -0.000002 & 0.069\end{array}$

$\begin{array}{llll}1099.28 & 0.3685 & -0.000003 & 0.136\end{array}$

$\begin{array}{llll}1106.93 & 0.2126 & -0.000002 & 0.045\end{array}$

$0.2598-0.000002$

$\begin{array}{llll}1161.86 & 0.0366 & 0.000000 & 0.001\end{array}$

$\begin{array}{llll}1178.22 & -0.0615 & 0.000000 & 0.004\end{array}$

$\begin{array}{llll}1203.67 & -0.2076 & 0.000001 & 0.043\end{array}$

$\begin{array}{llll}1213.84 & -0.6171 & 0.000002 & 0.381\end{array}$

$\begin{array}{llll}1228.98 & -0.3164 & 0.000001 & 0.100\end{array}$

$\begin{array}{llll}1253.20 & -0.3464 & 0.000001 & 0.120\end{array}$

$\begin{array}{llll}1264.59 & -0.1296 & 0.000000 & 0.017\end{array}$

$\begin{array}{llll}1276.78 & -0.2933 & 0.000001 & 0.086\end{array}$

$\begin{array}{llll}1301.43 & -0.1257 & 0.000000 & 0.016\end{array}$

$\begin{array}{lllll}1311.38 & 0.2846 & -0.000001 & 0.081\end{array}$

$\begin{array}{llll}1344.29 & 0.6720 & -0.000001 & 0.452\end{array}$

$\begin{array}{llll}991.29 & 0.0194 & -0.000017 & 0.000\end{array}$

$\begin{array}{lllll}992.57 & 0.0393 & -0.000015 & 0.002\end{array}$

$\begin{array}{lllll}993.80 & 0.0537 & -0.000014 & 0.003\end{array}$

$\begin{array}{lll}0.0750 & -0.000012 & 0.006\end{array}$

$\begin{array}{llll}999.09 & 0.0938 & -0.000010 & 0.009\end{array}$

$\begin{array}{llll}1002.32 & 0.1087 & -0.000008 & 0.012\end{array}$

$\begin{array}{lll}0.1561 & -0.000006 & 0.024\end{array}$
$17.8900 \quad 997.0449$

997.0449

997.0449

997.0449

997.0449

997.0449

997.0449

997.0449

$7.6900 \quad 994.0319$

$7.9100 \quad 994.0319$

$8.2400 \quad 994.0319$

$8.5400 \quad 994.0319$

$8.8800 \quad 994.0319$

$9.1600 \quad 994.0319$

$9.7600 \quad 994.0319$

994.0319

$10.8500 \quad 994.0319$ 994.0319

$11.7000 \quad 994.0319$ 994.0319

$12.4700 \quad 994.0319$ 994.0319

$13.0600 \quad 994.0319$ 994.0319

994.0319 994.0319 994.0319

$15.8800 \quad 994.0319$ 994.0319 994.0319

$16.7200 \quad 994.0319$ 994.0319 994.0319

$17.5500 \quad 994.0319$

$18.1200 \quad 994.0319$

$7.9900 \quad 990.2132$

$8.2000 \quad 990.2132$

$8.4400 \quad 990.2132$

$8.7500 \quad 990.2132$

$9.1200 \quad 990.2132$

$9.4000 \quad 990.2132$

$10.0100 \quad 990.2132$
$14.4700 \quad 994.0319$ 
molality molarity

$\mathbf{t} /{ }^{\circ} \mathrm{C}$ mass frac $\begin{array}{r}\text { Density } \\ \exp / \mathrm{kg} / \mathrm{m}^{3}\end{array}$

0.3925

0.4677

0.6

0.6996

0.8025

0.9188

0.9925

1.159
1.4107

1.497

1.6526

1.8985

1.9982

2.148

2.3916

2.5186

2.6336

2.8917

3.0275

3.3595

0.00977

0.02005

0.03209

0.04935

0.07582

0.103

0.2036

0.3001

0.3958

0.4677

0.6012

0.6996

0.8043

0.9188

1.0032

1.159

1.4107

1.497

1.8985

1.9982

2.148
1025.56

$\begin{array}{rrr}45 & 0.0374 & 1025.56 \\ 45 & 0.0484 & 1036.16 \\ 45 & 0.0572 & 1044.88 \\ 45 & 0.0722 & 1059.74 \\ 45 & 0.0831 & 1071.14 \\ 45 & 0.0942 & 1082.34 \\ 45 & 0.1064 & 1095.48 \\ 45 & 0.1140 & 1103.21 \\ 45 & 0.1306 & 1121.62 \\ 45 & 0.1546 & 1148.47 \\ 45 & 0.1625 & 1157.19 \\ 45 & 0.1764 & 1173.77 \\ 45 & 0.1975 & 1199.01 \\ 45 & 0.2057 & 1208.36 \\ 45 & 0.2178 & 1224.15 \\ 45 & 0.2366 & 1248.28 \\ 45 & 0.2461 & 1260.45 \\ 45 & 0.2545 & 1271.88 \\ 45 & 0.2726 & 1296.63 \\ 45 & 0.2818 & 1309.40 \\ 45 & 0.3034 & 1339.93 \\ 55 & 0.0013 & 986.87 \\ 55 & 0.0026 & 988.09 \\ 55 & 0.0041 & 989.52 \\ 55 & 0.0064 & 991.57 \\ 55 & 0.0097 & 994.69 \\ 55 & 0.0132 & 997.88 \\ 55 & 0.0257 & 1009.64 \\ 55 & 0.0374 & 1020.89 \\ 55 & 0.0488 & 1031.76 \\ 55 & 0.0572 & 1040.15 \\ 55 & 0.0723 & 1054.97 \\ 55 & 0.0831 & 1066.36 \\ 55 & 0.0944 & 1077.50 \\ 55 & 0.1064 & 1090.66 \\ 55 & 0.1151 & 1099.27 \\ 55 & 0.1306 & 1116.79 \\ 55 & 0.1546 & 1143.63 \\ 55 & 0.1625 & 1151.77 \\ 55 & 0.1764 & 1168.91 \\ 55 & 0.1975 & 1194.14 \\ 55 & 0.2057 & 1202.78 \\ 55 & 0.2178 & 1219.25\end{array}$

Ref

(37)

(97)

(37)

$(97)$
$(37)$

(97)

(37)

$(97)$

$(37)$
$(37)$

(97)

(37)

(37)

(97)

(37)

$(37)$
$(97)$
$(37)$

(37)

(37)

(97)

(97)

(97)

(97)

(97)

(97)

(97)

(97)

(97)

(37)

(97)

(37)

(97)

(37)

(97)

(37)

(97)

$(37)$
(37)

(97)

(37)

(37)

(97)

(37)
App vol App vol D exp / Calc $\mathrm{m}^{3} / \mathrm{kg} \quad \mathrm{m}^{3} / \mathrm{kg}$

$0.000080 \quad 0.000085$ 0.0000850 .000088 $\begin{array}{ll}0.000086 & 0.000089\end{array}$ $0.000092 \quad 0.000092$ $0.000092 \quad 0.000095$ $\begin{array}{ll}0.000098 & 0.000097\end{array}$ $0.000098 \quad 0.000099$ $0.000102 \quad 0.000101$ $0.000104 \quad 0.000104$ $\begin{array}{ll}0.000110 & 0.000109\end{array}$ $\begin{array}{lll}0.000113 & 0.000111\end{array}$ $0.000115 \quad 0.000114$ $0.000119 \quad 0.000118$ $0.000124 \quad 0.000120$ $0.000124 \quad 0.000123$ $0.000128 \quad 0.000127$ $0.000130 \quad 0.000129$ $\begin{array}{lll}0.000131 & 0.000131\end{array}$ $0.000135 \quad 0.000135$ $0.000136 \quad 0.000137$ $0.000141 \quad 0.000142$ $0.000064 \quad 0.000074$ $0.000065 \quad 0.000074$ $0.000067 \quad 0.000075$ $0.000069 \quad 0.000075$ 0.0000720 .000076 $0.000075 \quad 0.000076$ $0.000079 \quad 0.000079$ $0.000080 \quad 0.000081$ $0.000086 \quad 0.000084$ $0.000085 \quad 0.000086$ $0.000093 \quad 0.000089$ $\begin{array}{lll}0.000091 & 0.000091\end{array}$ $\begin{array}{lll}0.000099 & 0.000094\end{array}$ $0.000097 \quad 0.000096$ $0.000104 \quad 0.000098$ $0.000103 \quad 0.000102$ $0.000108 \quad 0.000107$ $0.000114 \quad 0.000109$

$0.000113 \quad 0.000112$ $0.000118 \quad 0.000117$ $0.000124 \quad 0.000119$ 0.0001220 .000122
Density Density App Vol Square of Inconsistent Inconsistent Apparent Density of calc / Residual / Residual / Density based on based on molal volume $\mathrm{kg} / \mathrm{m}^{3} \quad \mathrm{~kg} / \mathrm{m}^{3} \quad \mathrm{~m}^{3} / \mathrm{kg} \quad$ Res density apparent

$1 \mathrm{~cm}^{3} / \mathrm{mol}$

990.2132

$\begin{array}{llll}1025.36 & 0.2040 & -0.000005 & 0.042\end{array}$

$\begin{array}{llll}1036.02 & 0.1390 & -0.000003 & 0.019\end{array}$

$\begin{array}{llll}1044.64 & 0.2392 & -0.000004 & 0.057\end{array}$

$\begin{array}{llll}1059.69 & 0.0542 & -0.000001 & 0.003\end{array}$

$\begin{array}{llll}1070.92 & 0.2217 & -0.000002 & 0.049\end{array}$

$\begin{array}{llll}1082.43 & -0.0833 & 0.000001 & 0.007\end{array}$

$\begin{array}{llll}1095.32 & 0.1571 & -0.000001 & 0.025\end{array}$

$\begin{array}{llll}1103.43 & -0.2192 & 0.000002 & 0.048\end{array}$

$\begin{array}{llll}1121.58 & 0.0385 & 0.000000 & 0.001\end{array}$

$\begin{array}{llll}1148.56 & -0.0859 & 0.000000 & 0.007\end{array}$

$\begin{array}{llll}1157.68 & -0.4866 & 0.000002 & 0.237\end{array}$

$\begin{array}{llll}1173.96 & -0.1906 & 0.000001 & 0.036\end{array}$

$\begin{array}{llll}1199.27 & -0.2571 & 0.000001 & 0.066\end{array}$

$\begin{array}{llll}1209.38 & -1.0211 & 0.000003 & 1.043\end{array}$

$\begin{array}{llll}1224.41 & -0.2650 & 0.000001 & 0.070\end{array}$

$\begin{array}{llll}1248.46 & -0.1793 & 0.000000 & 0.032\end{array}$

$\begin{array}{llll}1260.80 & -0.3490 & 0.000001 & 0.122\end{array}$

$\begin{array}{llll}1271.85 & 0.0261 & 0.000000 & 0.001\end{array}$

$\begin{array}{llll}1308.90 & 0.4947 & -0.000001 & 0.245\end{array}$

$\begin{array}{lllll}1339.15 & 0.7739 & -0.000001 & 0.599\end{array}$

$\begin{array}{lllll}986.85 & 0.0126 & -0.000010 & 0.000\end{array}$

$\begin{array}{lll}0.0231 & -0.000009 & 0.001\end{array}$

$\begin{array}{llll}989.49 & 0.0321 & -0.000008 & 0.001\end{array}$

$\begin{array}{llll}991.53 & 0.0383 & -0.000006 & 0.001\end{array}$

$\begin{array}{lllll}994.65 & 0.0367 & -0.000004 & 0.001\end{array}$

$\begin{array}{llll}997.85 & 0.0249 & -0.000002 & 0.001\end{array}$

$\begin{array}{llll}1009.63 & 0.0025 & 0.000000 & 0.000\end{array}$

$\begin{array}{llll}1020.85 & 0.0411 & -0.000001 & 0.002\end{array}$

$\begin{array}{llll}1031.89 & -0.1242 & 0.000002 & 0.015\end{array}$

$\begin{array}{llll}1040.13 & 0.0212 & 0.000000 & 0.000\end{array}$

$\begin{array}{llll}1055.30 & -0.3297 & 0.000004 & 0.109\end{array}$

$\begin{array}{llll}1066.39 & -0.0265 & 0.000000 & 0.001\end{array}$

$\begin{array}{llll}1078.08 & -0.5842 & 0.000005 & 0.341\end{array}$

$\begin{array}{llll}1090.76 & -0.0977 & 0.000001 & 0.010\end{array}$

$\begin{array}{llll}1100.03 & -0.7540 & 0.000005 & 0.568\end{array}$

$\begin{array}{llll}1116.96 & -0.1733 & 0.000001 & 0.030\end{array}$

$\begin{array}{llll}1143.86 & -0.2334 & 0.000001 & 0.054\end{array}$

$\begin{array}{llll}1152.96 & -1.1816 & 0.000005 & 1.396\end{array}$

$\begin{array}{llll}1194.38 & -0.2357 & 0.000001 & 0.056\end{array}$

$\begin{array}{llll}1204.44 & -1.6543 & 0.000006 & 2.737\end{array}$

$\begin{array}{llll}1219.39 & -0.1439 & 0.000000 & 0.021\end{array}$
$11.0100 \quad 990.2132$ 990.2132

$11.8800 \quad 990.2132$ 990.2132

$12.6400 \quad 990.2132$ 990.2132

$13.2700 \quad 990.2132$ 990.2132 990.2132

$14.6700 \quad 990.2132$ 990.2132 990.2132

$16.0300 \quad 990.2132$ 990.2132 990.2132

$16.8700 \quad 990.2132$ 990.2132 990.2132

$17.6800 \quad 990.2132$

$18.2800 \quad 990.2132$

8.2600985 .6952

$8.4400 \quad 985.6952$

$8.6400 \quad 985.6952$

$8.9300 \quad 985.6952$

9.3200985 .6952

$9.6600 \quad 985.6952$

$10.2300 \quad 985.6952$ 985.6952

$11.1800 \quad 985.6952$ 985.6952

$12.0500 \quad 985.6952$ 985.6952

$12.8300 \quad 985.6952$ 985.6952

$13.4300 \quad 985.6952$ 985.6952 985.6952

$14.8200 \quad 985.6952$ 985.6952 985.6952

$16.1300 \quad 985.6952$ 985.6952 
molality molarity $\mathbf{t} /{ }^{\circ} \mathrm{C}$ mass frac $\begin{array}{r}\text { Density } \\ \exp / \mathrm{kg} / \mathrm{m}^{3}\end{array}$

$\begin{array}{r}2.3916 \\ 2.5079 \\ 2.6336 \\ 2.8917 \\ 2.9976 \\ 0.3001 \\ 0.4677 \\ 0.6996 \\ 0.9188 \\ 1.159 \\ 1.4107 \\ 1.6526 \\ 1.8985 \\ 2.148 \\ 2.3916 \\ 2.6336 \\ 2.8917 \\ 0.3001 \\ 0.4677 \\ 0.6996 \\ 0.9188 \\ 1.159 \\ 1.4107 \\ 1.6526 \\ 1.8985 \\ 2.148 \\ 2.3916 \\ 2.6336 \\ 2.8917 \\ \hline\end{array}$

Ref

1243.33 1253.53 $0.2545 \quad 1266.86$

$0.2726 \quad 1291.50$

$0.2798 \quad 1300.33$

$0.0374 \quad 1015.69$

$0.0572 \quad 1034.94$

$0.0831 \quad 1061.13$

$0.1064 \quad 1085.43$

$0.1306 \quad 1111.55$

$0.1546 \quad 1138.38$

$0.1764 \quad 1163.67$

$0.1975 \quad 1188.89$

$0.2178 \quad 1214.00$

$0.2366 \quad 1238.06$

$0.2545 \quad 1261.56$

$0.2726 \quad 1286.17$

$0.0374 \quad 1009.95$

$0.0572 \quad 1029.21$

$0.0831 \quad 1055.43$

$0.1064 \quad 1079.76$

$0.1306 \quad 1105.93$

$0.1546 \quad 1132.81$

$0.1764 \quad 1158.14$

$0.1975 \quad 1183.39$

$0.2178 \quad 1208.52$

$0.2366 \quad 1232.59$

$0.2545 \quad 1256.07$

$0.2726 \quad 1280.62$

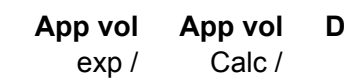

$\exp / \quad$ Calc

$\mathrm{m}^{3} / \mathrm{kg}$

$0.000126 \quad 0.000126$

0.0001310 .000128

$0.000130 \quad 0.000131$

$0.000133 \quad 0.000135$

$\begin{array}{ll}0.000137 & 0.000137\end{array}$

0.0000780 .000076

$0.000082 \quad 0.000080$

$0.000088 \quad 0.000086$

$0.000094 \quad 0.000092$

$0.000100 \quad 0.000098$

$0.000105 \quad 0.000103$

$0.000110 \quad 0.000109$

$\begin{array}{lll}0.000115 & 0.000114\end{array}$

$0.000119 \quad 0.000119$

$0.000123 \quad 0.000124$

$\begin{array}{ll}0.000127 & 0.000129\end{array}$

$0.000131 \quad 0.000133$

$\begin{array}{ll}0.000074 & 0.000069\end{array}$

$0.000078 \quad 0.000073$

$0.000084 \quad 0.000080$

0.0000890 .000086

0.0000950 .000092

$0.000101 \quad 0.000098$

$0.000106 \quad 0.000104$

$0.000110 \quad 0.000109$

0.0001150 .000115

0.0001190 .000120

$0.000123 \quad 0.000125$

$0.000127 \quad 0.000130$
Density Density App Vol Square of Inconsistent Inconsistent Apparent Density of calc / Residual / Residual / Density based on based on molal volume water $/ \mathrm{kg} / \mathrm{m}^{3}$ $\mathrm{kg} / \mathrm{m}^{3} \quad \mathrm{~kg} / \mathrm{m}^{3} \quad \mathrm{~m}^{3} / \mathrm{kg} \quad$ Res density apparent $1 \mathrm{~cm}^{3} / \mathrm{mol}$

985.6952

$16.9700 \quad 985.6952$ 985.6952 985.6952

$17.7900 \quad 985.6952$ 980.5548 980.5548 980.5548 980.5548 980.5548 980.5548 980.5548 980.5548 980.5548 980.5548 980.5548 980.5548 974.849 974.849 974.849 974.849 974.849 974.849 974.849

974.849

974.849 974.849 974.849 974.849

\section{Curve fit for the model App vol $=\left(w+c 2+c 3^{*} t{ }^{\circ} \mathrm{C}\right) /\left(\mathrm{c} 0^{*} w+c 1\right) / \mathrm{e}^{\wedge}\left(0.000001^{*}\left(t{ }^{\circ} \mathrm{C}+\mathrm{c} 4\right)^{\wedge} 2\right)$}

$\begin{array}{lr}\text { c0 } & -1.4 \mathrm{E}-06 \\ \text { c1 } & 4.19 \mathrm{E}-06 \\ \text { c2 } & 0.777343 \\ \text { c3 } & -0.00669 \\ \text { c4 } & -4638.13 \\ \text { Min T } & 15 \\ \text { Max T } & 75 \\ \text { Min w } & 0.000454\end{array}$


molality molarity $\mathbf{t} /{ }^{\circ} \mathrm{C}$ mass frac $\begin{array}{r}\text { Density } \\ \exp / \mathrm{kg} / \mathrm{m}^{3}\end{array}$
Ref

0.392438

\section{Max w}

Avg dens res

0.122376

Std dens res

Avg app vol res *10^3

Std app vol res *10^3 $\quad 0.011085$

No of points in corr

No of inconsistent poin

Inconsistent data not used (Residual greater than average +/- 4 standard deviations)

$\begin{array}{llllll}18 & 0.1200 & 1121.70 & (90) & 0.000086 & 0.000104\end{array}$

$\begin{array}{llllll}18 & 0.1600 & 1167.40 & (90) & 0.000096 & 0.000111\end{array}$

$\begin{array}{lllll}0.2000 & 1216.3 & (90) & 0.000105 & 0.000118\end{array}$

$\begin{array}{lllll}0.3000 & 1353.0 & (90) & 0.000127 & 0.000136\end{array}$

$\begin{array}{llllll}0.0029 & 999.91 & (23) & 0.000012 & 0.000084\end{array}$

$-0.000018$

$3.0793-0.000014$

1212.64

$3.6619-0.000012$

$4.9998-0.000009$

999.70

$0.2084-0.000072$

$0.000011 \quad 0.000084$

1002.28

$0.4225-0.000074$

7.459

9.482

13.409

24.998

0.043

0.178

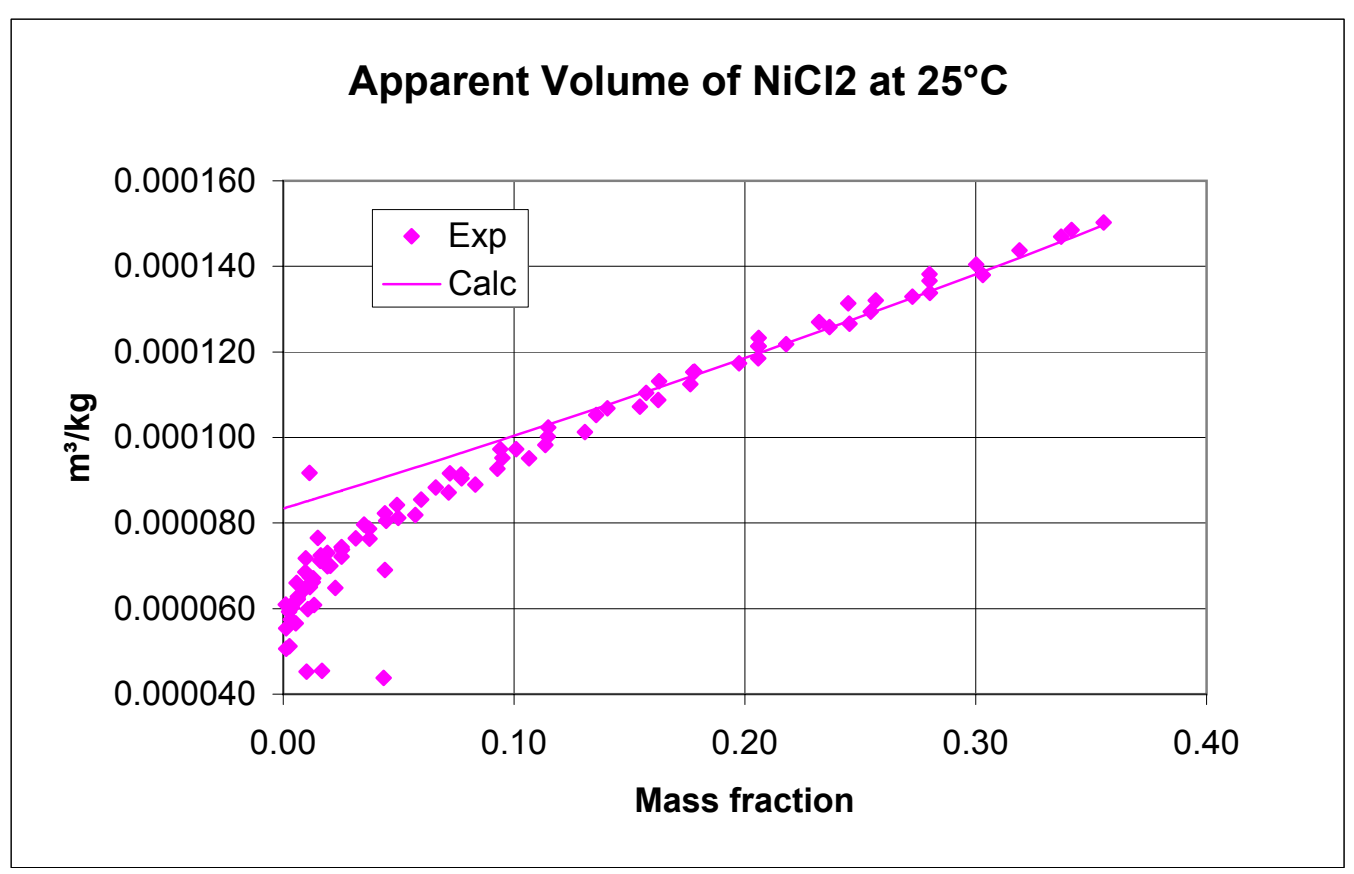


Density of aqueous solutions of $\mathrm{NiSO} 4$

References (44) Isono, T. Measurements of Density, Viscosity, and Electrolytic Conductivity of Concentrated Aqueous Electrolyte Solutions. Rikagaku Kenkyusho Hokoku 1980, 56, 103-114

(90) Perry, R. H.; Green, D. W. Perry's Chemical Engineers' Handbook; McGraw Hill: New York, 7th edition, 1997.

(94) Phillips, V. R. Specific Gravity, Viscosity, and Solubility for Aqueous Nickel Sulfate Solutions. J. Chem. Eng. Data 1972, 17, 357-360.

(117) Schmelzer, N.; Einfeldt, J. Density Measurements in Some Aqueous and Non-Aqueous Electrolyte Solutions at $25^{\circ} \mathrm{C}$. Wiss. Z. Uni. Rostock 1989, 38, 81-82.

\begin{tabular}{|c|c|c|c|c|c|c|c|c|c|c|c|c|c|c|c|}
\hline molality & molarity & \multicolumn{2}{|c|}{$\mathrm{t} /{ }^{\circ} \mathrm{C}$ mass frac } & $\begin{array}{r}\text { Density } \\
\exp / \\
\mathrm{kg} / \mathrm{m}^{3}\end{array}$ & Ref & $\begin{array}{r}\text { App vol } \\
\exp / \mathrm{m}^{3} / \mathrm{kg}\end{array}$ & $\begin{array}{r}\text { App vol } \\
\text { Calc / } \\
\mathrm{m}^{3} / \mathrm{kg}\end{array}$ & $\begin{array}{r}\text { Density } \\
\text { calc / } \\
\mathrm{kg} / \mathrm{m}^{3}\end{array}$ & $\begin{array}{r}\text { Density } \\
\text { Residual / } \\
\mathrm{kg} / \mathrm{m}^{3}\end{array}$ & $\begin{array}{r}\text { App Vol } \\
\text { Residual / } \\
\mathrm{m}^{3} / \mathrm{kg}\end{array}$ & $\begin{array}{r}\text { Square of } \\
\text { Density } \\
\text { Res }\end{array}$ & $\begin{array}{c}\text { Inconsistent } \\
\text { based on } \\
\text { density }\end{array}$ & $\begin{array}{l}\text { Inconsistent } \\
\text { based on } \\
\text { apparent } \\
\text { volume }\end{array}$ & $\begin{array}{c}\text { wt \% } \\
\text { NisO4.6H2O }\end{array}$ & $\begin{array}{r}\text { Density of } \\
\text { water } / \mathrm{kg} / \mathrm{m}^{3}\end{array}$ \\
\hline 0.05 & & 15 & 0.008 & 1007.4 & (44) & -0.000067 & -0.000040 & 1007.1 & 0.2096 & -0.000027 & 0.044 & & & & 999.0996 \\
\hline 0.1 & & 15 & 0.015 & 1015.4 & (44) & -0.000055 & -0.000037 & 1015.1 & 0.2817 & -0.000018 & 0.079 & & & & 999.0996 \\
\hline 0.5 & & 15 & 0.072 & 1078.0 & (44) & -0.000019 & -0.000019 & 1078.0 & -0.0423 & 0.000001 & 0.002 & & & & 999.0996 \\
\hline 1 & & 15 & 0.134 & 1153.8 & (44) & 0.000000 & 0.000001 & 1153.5 & 0.3095 & -0.000002 & 0.096 & & & & 999.0996 \\
\hline 1.5 & & 15 & 0.188 & 1225.7 & (44) & 0.000019 & 0.000020 & 1225.4 & 0.2418 & -0.000001 & 0.058 & & & & 999.0996 \\
\hline 2 & & 15 & 0.236 & 1295.1 & (44) & 0.000033 & 0.000036 & 1293.9 & 1.2484 & -0.000003 & 1.559 & & & & 999.0996 \\
\hline & & 18 & 0.010 & 1009.1 & (90) & -0.000041 & -0.000037 & 1009.1 & 0.0379 & -0.000004 & 0.001 & & & & 998.5956 \\
\hline & & 18 & 0.020 & 1019.8 & (90) & -0.000040 & -0.000034 & 1019.7 & 0.1149 & -0.000006 & 0.013 & & & & 998.5956 \\
\hline & & 18 & 0.040 & 1041.5 & (90) & -0.000030 & -0.000028 & 1041.4 & 0.0891 & -0.000002 & 0.008 & & & & 998.5956 \\
\hline 0.05 & & 20 & 0.008 & 1006.4 & (44) & -0.000061 & -0.000037 & 1006.2 & 0.1841 & -0.000024 & 0.034 & & & & 998.2041 \\
\hline 0.1 & & 20 & 0.015 & 1014.5 & (44) & -0.000053 & -0.000035 & 1014.2 & 0.2844 & -0.000018 & 0.081 & & & & 998.2041 \\
\hline & & 20 & 0.059 & 1062.2 & (94) & -0.000023 & -0.000021 & 1062.0 & 0.1512 & -0.000002 & 0.023 & & & 10 & 998.2041 \\
\hline 0.5 & & 20 & 0.072 & 1076.6 & (44) & -0.000014 & -0.000017 & 1076.9 & -0.2251 & 0.000003 & 0.051 & & & & 998.2041 \\
\hline & & 20 & 0.118 & 1131.7 & (94) & -0.000002 & -0.000002 & 1131.8 & -0.0853 & 0.000001 & 0.007 & & & 20 & 998.2041 \\
\hline 1 & & 20 & 0.134 & 1152.3 & (44) & 0.000002 & 0.000003 & 1152.2 & 0.0987 & -0.000001 & 0.010 & & & & 998.2041 \\
\hline & & 20 & 0.177 & 1208.0 & (94) & 0.000017 & 0.000017 & 1208.0 & 0.0085 & 0.000000 & 0.000 & & & 30 & 998.2041 \\
\hline 1.5 & & 20 & 0.188 & 1223.9 & (44) & 0.000021 & 0.000021 & 1224.1 & -0.2006 & 0.000001 & 0.040 & & & & 998.2041 \\
\hline & & 20 & 0.236 & 1291.9 & (94) & 0.000035 & 0.000036 & 1291.3 & 0.6310 & -0.000002 & 0.398 & & & 40 & 998.2041 \\
\hline 2 & & 20 & 0.236 & 1293.2 & (44) & 0.000035 & 0.000037 & 1292.5 & 0.6708 & -0.000002 & 0.450 & & & & 998.2041 \\
\hline 0.000329 & & 25 & 0.000 & 997.1 & (117) & -0.000086 & -0.000037 & 997.1 & 0.0025 & -0.000050 & 0.000 & & $!$ & & 997.0449 \\
\hline 0.002991 & & 25 & 0.000 & 997.5 & (117) & -0.000073 & -0.000036 & 997.5 & 0.0168 & -0.000036 & 0.000 & & & & 997.0449 \\
\hline 0.006661 & & 25 & 0.001 & 998.1 & (117) & -0.000046 & -0.000036 & 998.1 & 0.0099 & -0.000010 & 0.000 & & & & 997.0449 \\
\hline 0.02936 & & 25 & 0.005 & 1001.9 & (117) & -0.000065 & -0.000035 & 1001.7 & 0.1345 & -0.000030 & 0.018 & & & & 997.0449 \\
\hline 0.05 & & 25 & 0.008 & 1005.2 & $(44)$ & -0.000058 & -0.000034 & 1005.0 & 0.1834 & -0.000024 & 0.034 & & & & 997.0449 \\
\hline 0.1 & & 25 & 0.015 & 1013.3 & (44) & -0.000050 & -0.000032 & 1013.0 & 0.2830 & -0.000018 & 0.080 & & & & 997.0449 \\
\hline 0.20735 & & 25 & 0.031 & 1031.1 & (117) & -0.000061 & -0.000027 & 1029.9 & 1.1200 & -0.000034 & 1.254 & & & & 997.0449 \\
\hline & & 25 & 0.059 & 1060.9 & (94) & -0.000022 & -0.000019 & 1060.7 & 0.2295 & -0.000003 & 0.053 & & & 10 & 997.0449 \\
\hline 0.5 & & 25 & 0.072 & 1075.1 & (44) & -0.000011 & -0.000015 & 1075.4 & -0.3133 & 0.000004 & 0.098 & & & & 997.0449 \\
\hline & & 25 & 0.118 & 1129.9 & (94) & 0.000002 & -0.000001 & 1130.2 & -0.3287 & 0.000002 & 0.108 & & & 20 & 997.0449 \\
\hline 1 & & 25 & 0.134 & 1150.5 & (44) & 0.000005 & 0.000004 & 1150.6 & -0.0862 & 0.000000 & 0.007 & & & & 997.0449 \\
\hline & & 25 & 0.177 & 1206.0 & (94) & 0.000019 & 0.000018 & 1206.3 & -0.3261 & 0.000001 & 0.106 & & & 30 & 997.0449 \\
\hline
\end{tabular}


molality molarity

$\mathrm{t} /{ }^{\circ} \mathrm{C}$ mass frac Density

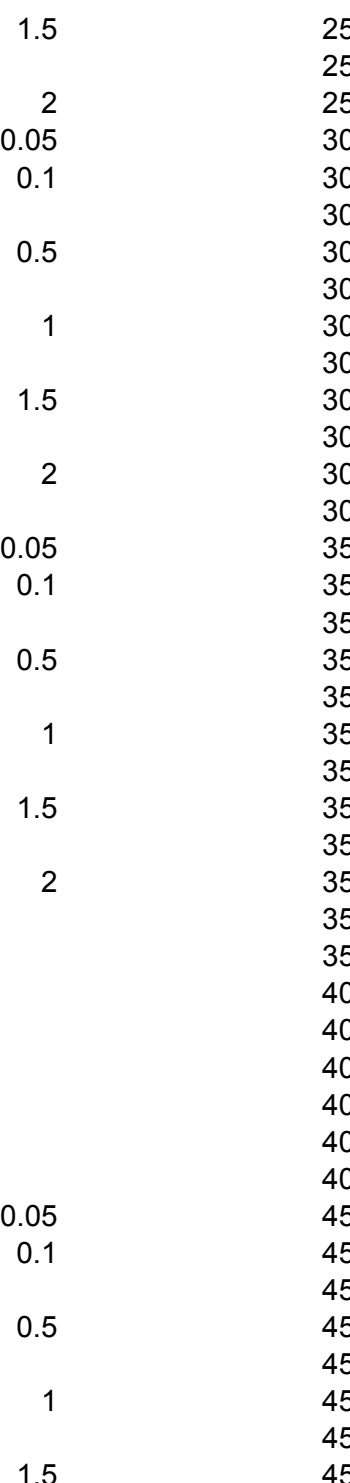

$\exp$

$\mathrm{kg} / \mathrm{m}^{3}$

$0.188 \quad 1221$.

0.236

$0.015 \quad 1011.8$

$0.059 \quad 1059.1$

$0.072 \quad 1073.4$

$0.118 \quad 1128.1$

$0.134 \quad 1148.6$

$0.177 \quad 1203.9$

$0.188 \quad 1219.7$

$0.236 \quad 1287.6$

$0.236 \quad 1288.8$

$0.294 \quad 1379.1$

$0.008 \quad 1002.1$

$0.015 \quad 1010.1$

$0.059 \quad 1057.2$

$0.072 \quad 1071.6$

$0.118 \quad 1125.9$

$0.134 \quad 1146.5$

$0.177 \quad 1201.6$

$0.188 \quad 1217.6$

$0.236 \quad 1285.2$

$0.236 \quad 1286.6$

$0.294 \quad 1376.7$

$0.309 \quad 1401.2$

$0.059 \quad 1055.3$

$0.118 \quad 1123.7$

$0.177 \quad 1199.4$

$0.236 \quad 1282.7$

$0.294 \quad 1374.2$

$0.309 \quad 1398.8$

$0.008 \quad 998.2$

$0.015 \quad 1006.2$

$0.059 \quad 1053.1$

$0.072 \quad 1067.3$

$0.118 \quad 1121.4$

$0.134 \quad 1142.0$

$0.177 \quad 1196.9$

$0.188 \quad 1212.9$
Ref $\exp / \mathrm{m}^{3} / \mathrm{kg}$

0.000024

$\begin{array}{ll}0.000024 & 0.000022\end{array}$

$\begin{array}{ll}0.000036 & 0.000037\end{array}$

$\begin{array}{ll}0.000037 & 0.000037\end{array}$

$-0.000053-0.000032$

$-0.000046-0.000030$

$-0.000018-0.000017$

$-0.000009-0.000013$

$0.000003 \quad 0.000001$

$0.000007 \quad 0.000006$

$0.000021 \quad 0.000019$

$0.000025 \quad 0.000023$

$\begin{array}{ll}0.000037 & 0.000037\end{array}$

$0.000038 \quad 0.000038$

$0.000056 \quad 0.000056$

$-0.000053-0.000029$

$-0.000045-0.000027$

$-0.000015-0.000015$

$-0.000007-0.000011$

$\begin{array}{ll}0.000005 & 0.000002\end{array}$

$\begin{array}{lll}0.000008 & 0.000007\end{array}$

$0.000022 \quad 0.000020$

$0.000026 \quad 0.000023$

$0.000038 \quad 0.000038$

$0.000038 \quad 0.000038$

$0.000056 \quad 0.000056$

$0.000060 \quad 0.000061$

$-0.000015-0.000013$

$0.000006 \quad 0.000004$

$0.000022 \quad 0.000021$

$0.000039 \quad 0.000038$

$0.000056 \quad 0.000056$

$0.000060 \quad 0.000060$

$-0.000048-0.000025$

$-0.000042-0.000023$

$-0.000014-0.000011$

$-0.000006-0.000008$

$0.000007 \quad 0.000005$

$0.000009 \quad 0.000009$

$0.000023 \quad 0.000021$

$0.000026 \quad 0.000025$
Density Density App Vol Square of Inconsistent Inconsistent wt \%

$\mathrm{kg} / \mathrm{m}^{3} \quad \mathrm{~kg} / \mathrm{m}^{3} \quad \mathrm{~m}^{3} / \mathrm{kg} \quad$ Res density apparent

volume

$1289.6-0.2949-0.000001-0.087$

$1290.9 \quad 0.2036-0.000001 \quad 0.041$

$\begin{array}{lllll}1003.6 & 0.1620 & -0.000021 & 0.026\end{array}$

$\begin{array}{llll}1011.5 & 0.2517 & -0.000016 & 0.063\end{array}$

$1059.0 \quad 0.0535-0.000001-0.003$

$\begin{array}{llll}1073.8 & -0.3639 & 0.000004 & 0.132\end{array}$

$\begin{array}{llll}1128.4 & -0.3174 & 0.000002 & 0.101\end{array}$

$\begin{array}{llll}1148.7 & -0.1437 & 0.000001 & 0.021\end{array}$

$\begin{array}{llll}1204.4 & -0.4953 & 0.000002 & 0.245\end{array}$

$\begin{array}{llll}1220.4 & -0.6917 & 0.000002 & 0.478\end{array}$

$\begin{array}{llll}1287.7 & -0.0622 & 0.000000 & 0.004\end{array}$

$\begin{array}{llll}1288.9 & -0.0844 & 0.000000 & 0.007\end{array}$

$\begin{array}{llll}1378.9 & 0.1634 & 0.000000 & 0.027\end{array}$

$1002.0 \quad 0.1791-0.000023 \quad 0.032$

$\begin{array}{lllll}1009.8 & 0.2698 & -0.000017 & 0.073\end{array}$

$\begin{array}{llll}1057.2 & 0.0016 & 0.000000 & 0.000\end{array}$

$\begin{array}{llll}1071.9 & -0.3190 & 0.000004 & 0.102\end{array}$

$\begin{array}{llll}1126.4 & -0.4746 & 0.000003 & 0.225\end{array}$

$\begin{array}{llll}1146.6 & -0.1072 & 0.000001 & 0.012\end{array}$

$\begin{array}{llll}1202.2 & -0.6232 & 0.000002 & 0.388\end{array}$

$\begin{array}{llll}1218.2 & -0.6848 & 0.000002 & 0.469\end{array}$

$\begin{array}{llll}1285.5 & -0.2654 & 0.000001 & 0.070\end{array}$

$\begin{array}{llll}1286.7 & -0.1382 & 0.000000 & 0.019\end{array}$

$\begin{array}{llll}1376.9 & -0.1741 & 0.000000 & 0.030\end{array}$

$\begin{array}{llll}1401.1 & 0.1033 & 0.000000 & 0.011\end{array}$

$1055.1-0.1556-0.000002-0.024$

$\begin{array}{llll}1124.1 & -0.4191 & 0.000003 & 0.176\end{array}$

$\begin{array}{llll}1199.8 & -0.4295 & 0.000002 & 0.185\end{array}$

$1283.0 \quad-0.3350 \quad 0.000001$

$\begin{array}{llll}1374.6 & -0.3620 & 0.000001 & 0.131\end{array}$

$\begin{array}{llll}1398.8 & -0.0466 & 0.000000 & 0.002\end{array}$

$\begin{array}{llll}998.1 & 0.1730 & -0.000023 & 0.030\end{array}$

$\begin{array}{llll}1005.9 & 0.2973 & -0.000019 & 0.088\end{array}$

$1052.9 \quad 0.2007 \quad-0.000003 \quad 0.040$

$\begin{array}{llll}1067.5 & -0.1422 & 0.000002 & 0.020\end{array}$

$\begin{array}{llll}1121.7 & -0.2665 & 0.000002 & 0.071\end{array}$

$\begin{array}{lllll}1141.8 & 0.1447 & -0.000001 & 0.021\end{array}$

$\begin{array}{llll}1197.2 & -0.3306 & 0.000001 & 0.109\end{array}$

$\begin{array}{llll}1213.2 & -0.3564 & 0.000001 & 0.127\end{array}$
997.0449

$40 \quad 997.0449$

997.0449

995.6473

995.6473

$10 \quad 995.6473$ 995.6473

$20 \quad 995.6473$

995.6473

$30 \quad 995.6473$ 995.6473

$40 \quad 995.6473$ 995.6473

$50 \quad 995.6473$

994.0319 994.0319

$10 \quad 994.0319$

994.0319

$20 \quad 994.0319$ 994.0319

$30 \quad 994.0319$ 994.0319

$40 \quad 994.0319$ 994.0319

$50 \quad 994.0319$

$52.5 \quad 994.0319$

$\begin{array}{ll}10 & 992.2158\end{array}$

$20 \quad 992.2158$

$30 \quad 992.2158$

$\begin{array}{ll}40 & 992.2158\end{array}$

$\begin{array}{ll}50 & 992.2158\end{array}$

52.5992 .2158 990.2132 990.2132

$10 \quad 990.2132$ 990.2132

$20 \quad 990.2132$ 990.2132

$30 \quad 990.2132$ 990.2132 
molality molarity

2

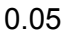

0.1

0.5

1.5 $t /{ }^{\circ} \mathrm{C}$ mass frac Density

$\exp /$

$\mathrm{kg} / \mathrm{m}^{3}$

0.236

0.236

0.294

$0.309 \quad 1396.2$

$0.324 \quad 1421.1$

$0.059 \quad 1050.9$

$0.118 \quad 1119.0$

$0.177 \quad 1194.4$

$0.236 \quad 1277.5$

$0.294 \quad 1368.9$

$0.309 \quad 1393.5$

$0.324 \quad 1418.4$

$0.339 \quad 1444.4$

$0.008 \quad 993.7$

$0.015 \quad 1001.6$

$0.059 \quad 1048.5$

$0.072 \quad 1062.5$

$0.118 \quad 1116.4$

$0.134 \quad 1136.9$

$0.177 \quad 1191.7$

$0.188 \quad 1207.7$

$0.236 \quad 1274.8$

$0.236 \quad 1276.3$

$0.294 \quad 1366.1$

$0.309 \quad 1390.7$

$0.324 \quad 1415.7$

$0.339 \quad 1441.7$

$0.059 \quad 1046.0$

$0.118 \quad 1113.8$

$0.177 \quad 1188.9$

$0.236 \quad 1272.1$

$0.294 \quad 1363.3$

$0.309 \quad 1387.9$

$0.324 \quad 1412.8$

$0.339 \quad 1438.9$

$0.353 \quad 1465.0$
Ref $\exp / \mathrm{m}^{3} / \mathrm{kg}$

0.000039

(94)

$(44)$

$(94)$
$(94)$

(94)

(94)

$(94)$
$(94)$

(94)

$(94)$
$(94)$

(94)

$(94)$

(94)
(44)

(44)

(94)

$(44)$
$(94)$

$(94)$

(44)
(94)

$(44)$
$(94)$

(44)

$(94)$

(94)

$(94)$
$(94)$

$(94)$

$(94)$
$(94)$

(94)

(94)

(94)

$(94)$
$(94)$

$(94)$
$(94)$

\begin{tabular}{rr}
0.000039 & 0.000038 \\
0.000039 & 0.000038 \\
0.000056 & 0.000055 \\
0.000060 & 0.000060 \\
0.000064 & 0.000064 \\
-0.000016 & -0.000010 \\
0.000006 & 0.000006 \\
0.000022 & 0.000022 \\
0.000038 & 0.000038 \\
0.000056 & 0.000055 \\
0.000059 & 0.000059 \\
0.000064 & 0.000063 \\
0.000068 & 0.000068 \\
-0.000047 & -0.000021 \\
-0.000044 & -0.000019 \\
-0.000018 & -0.000008 \\
-0.000006 & -0.000005 \\
0.000006 & 0.000007 \\
0.000008 & 0.000011 \\
0.000022 & 0.000023 \\
0.000025 & 0.000026 \\
0.000038 & 0.000038 \\
0.000037 & 0.000039 \\
0.000055 & 0.000055 \\
0.000059 & 0.000059 \\
0.000063 & 0.000063 \\
0.000067 & 0.000067 \\
-0.000020 & -0.000007 \\
0.000004 & 0.000008 \\
0.000021 & 0.000023 \\
0.000036 & 0.000039 \\
0.000054 & 0.000054 \\
0.000058 & 0.000058 \\
0.000062 & 0.000062 \\
0.000066 & 0.000066 \\
0.000070 & 0.000070 \\
& \\
\hline
\end{tabular}

$0.000070 \quad 0.000070$
Std dev Res

Avg - 4std

Avg $+4 s t d$
Density Density App Vol Square of Inconsistent Inconsistent wt \% $\mathrm{kg} / \mathrm{m}^{3} \quad \mathrm{~kg} / \mathrm{m}^{3} \quad \mathrm{~m}^{3} / \mathrm{kg} \quad$ Res density apparent

$\begin{array}{llll}1280.4 & -0.1881 & 0.000000 & 0.035\end{array}$

$\begin{array}{llll}1281.6 & -0.0516 & 0.000000 & 0.003\end{array}$

$\begin{array}{llll}1372.0 & -0.4179 & 0.000001 & 0.175\end{array}$

$\begin{array}{llll}1396.4 & -0.1597 & 0.000000 & 0.025\end{array}$

$\begin{array}{llll}1421.3 & -0.2061 & 0.000000 & 0.042\end{array}$

$\begin{array}{llll}1050.5 & 0.4242 & -0.000007 & 0.180\end{array}$

$\begin{array}{llll}1119.0 & -0.0299 & 0.000000 & 0.001\end{array}$

$\begin{array}{llll}1194.4 & -0.0399 & 0.000000 & 0.002\end{array}$

$\begin{array}{llll}1277.5 & -0.0387 & 0.000000 & 0.001\end{array}$

$\begin{array}{llll}1369.3 & -0.3561 & 0.000001 & 0.127\end{array}$

$\begin{array}{llll}1393.7 & -0.1505 & 0.000000 & 0.023\end{array}$

$\begin{array}{llll}1418.7 & -0.2644 & 0.000000 & 0.070\end{array}$

$\begin{array}{llll}1444.3 & 0.0848 & 0.000000 & 0.007\end{array}$

$\begin{array}{lllll}993.5 & 0.1982 & -0.000026 & 0.039\end{array}$

$\begin{array}{llll}1001.2 & 0.3880 & -0.000025 & 0.151\end{array}$

$1047.9 \quad 0.6154-0.000010 \quad 0.379$

$\begin{array}{llll}1062.4 & 0.1444 & -0.000002 & 0.021\end{array}$

$0.1795-0.000001 \quad 0.032$

$0.6620-0.000004 \quad 0.438$

$0.2309-0.000001 \quad 0.053$

$0.3084-0.000001 \quad 0.095$

$0.3014-0.000001 \quad 0.091$

$\begin{array}{lll}0.5579 & -0.000001 & 0.311\end{array}$

$\begin{array}{lll}-0.1887 & 0.000000 & 0.036\end{array}$

$\begin{array}{lll}-0.0312 & 0.000000 & 0.001\end{array}$

$\begin{array}{lll}-0.1076 & 0.000000 & 0.012\end{array}$

$\begin{array}{lll}0.1631 & 0.000000 & 0.027\end{array}$

$0.8653-0.000013 \quad 0.749$

$0.5523-0.000004 \quad 0.305$

$0.5721-0.000002-0.327$

$\begin{array}{lll}0.8222 & -0.000002 & 0.676\end{array}$

$\begin{array}{lll}0.1741 & 0.000000 & 0.030\end{array}$

$\begin{array}{lll}0.2881 & 0.000000 & 0.083\end{array}$

$\begin{array}{lll}0.0540 & 0.000000 & 0.003\end{array}$

$\begin{array}{lll}0.3516 & -0.000001 & 0.124\end{array}$

$\begin{array}{lll}-0.0400 & 0.000000 & 0.002\end{array}$

$0.0476-0.000005$

13.468 Jum Chi sqr
$40 \quad 990.2132$

990.2132

$\begin{array}{ll}50 & 990.2132\end{array}$

52.5990 .2132

$\begin{array}{ll}55 & 990.2132\end{array}$

$10 \quad 988.0363$

$20 \quad 988.0363$

$30 \quad 988.0363$

$40 \quad 988.0363$

$\begin{array}{ll}50 & 988.0363\end{array}$

$52.5 \quad 988.0363$

$55 \quad 988.0363$

57.5988 .0363 985.6952

985.6952

$10 \quad 985.6952$ 985.6952

$20 \quad 985.6952$ 985.6952

$30 \quad 985.6952$

985.6952

$40 \quad 985.6952$ 985.6952

$50 \quad 985.6952$

52.5985 .6952

$55 \quad 985.6952$

$57.5 \quad 985.6952$

$10 \quad 983.1989$

$20 \quad 983.1989$

$30 \quad 983.1989$

$40 \quad 983.1989$

$50 \quad 983.1989$

$52.5 \quad 983.1989$

55983.1989

$57.5 \quad 983.1989$

$60 \quad 983.1989$ 
molality molarity

$t /{ }^{\circ} \mathrm{C}$ mass frac $\begin{array}{rlr}\text { Density } & \text { Ref } & \begin{array}{r}\text { App vol } \\ \exp /\end{array} \\ \mathrm{kg} / \mathrm{m}^{3} & & \exp / \mathrm{m}^{3} / \mathrm{kg}\end{array}$

$A$

App vol

Calc /
$\mathrm{m}^{3} / \mathrm{kg}$

\section{Density}

calc /

Density

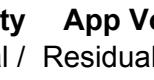

$\mathrm{m}^{3} / \mathrm{kg}$

Squa

Density

Res

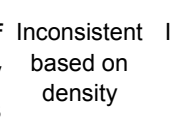

Inconsisten

based on

apparent

Curve fit for the model App vol $=\left(w+c 2+c 3^{*} t{ }^{\circ} \mathrm{C}\right) /\left(c 0^{*} w+c 1\right) / e^{\wedge}\left(0.000001^{*}\left(t^{\circ} \mathrm{C}+\mathrm{c} 4\right)^{\wedge} 2\right)$

$\begin{array}{lr}\text { c0 } & -0.038942 \\ \text { c2 } & 0.221094 \\ \text { c3 } & -0.144428 \\ \text { c4 } & 0.000987 \\ \text { Min T } & 3073.747 \\ \text { Max T } & 15 \\ \text { Min w } & 60 \\ \text { Max w } & 5.09 \mathrm{E}-05 \\ \text { Avg dens res } & 0.353294 \\ \text { Std dens res } & 0.047639 \\ \text { Avg app vol res *10^3 } & -0.00453227 \\ \text { Std app vol res *10^3 } & 0.010116 \\ \text { No of points in corr } & 107 \\ \text { No of inconsistent poir } & 8\end{array}$

\section{Inconsistent data not used (Residual greater than average +/- 4 standard deviations)}

$\begin{array}{rrrrrrr} & 18 & 0.080 & 1085.2 & (90) & 0.000002 & -0.000015 \\ & 18 & 0.120 & 1132.5 & (90) & 0.000015 & -0.000002 \\ & 18 & 0.160 & 1182.5 & (90) & 0.000028 & 0.000011 \\ 0.00075 & 18 & 0.180 & 1209.0 & (90) & 0.000033 & 0.000018 \\ 0.45858 & 25 & 0.000 & 997.2 & (117) & -0.000168 & -0.000037 \\ 0.83634 & 25 & 0.066 & 1072.8 & (117) & -0.000066 & -0.000017 \\ 0.87034 & 25 & 0.115 & 1141.8 & (117) & -0.000106 & -0.000002 \\ & 25 & 0.119 & 1150.2 & (117) & -0.000122 & 0.000000\end{array}$

$\begin{array}{rrrrr}1086.9 & -1.6559 & 0.000018 & 2.742 & ! \\ 1135.1 & -2.6038 & 0.000017 & 6.780 & ! \\ 1186.3 & -3.8339 & 0.000017 & 14.699 & ! \\ 1213.1 & -4.1245 & 0.000016 & 17.012 & ! \\ 997.2 & 0.0152 & -0.000132 & 0.000 & \\ 1069.1 & 3.7582 & -0.000049 & 14.124 & ! \\ 1126.3 & 15.4422 & -0.000105 & 238.462 & ! \\ 1131.4 & 18.8295 & -0.000122 & 354.552 & !\end{array}$


Density of aqueous solutions of $\mathrm{SrCl} 2$

References (40) Herz, W. Internal Friction of Salt Solutions (C. A. Trans. from German). Z. Anorg. Chemie 1914, 89, 393-396

(45) Isono, T. Densities, Viscosities, and Electrolytic Conductivity of Concentrated Aqueous Electrolyte Solutions at Several Temperatures.

Alkaline-Earth Chlorides, $\mathrm{LaCl}_{3}, \mathrm{Na}_{2} \mathrm{SO}_{4}, \mathrm{NaNO}_{3}, \mathrm{NaBr}, \mathrm{KNO}_{3}, \mathrm{KBr}$, and $\mathrm{Cd}\left(\mathrm{NO}_{3}\right)_{2}$. J. Chem. Eng. Data 1984, $29,45-52$.

(73) Millero, F. J.; Ward, G. K.; Chetirkin, P. V. Relative Sound Velocities of Sea Salts at 25C. J. Acoust. Soc. Am. 1977, 61, $1492-1498$.

(87) Perron, G.; Desnoyers, J. E. Apparent Molal Volumes and Heat Capacities of Alkaline Earth Chlorides in Water at $25^{\circ} \mathrm{C}$. Can. J. Chem. 1974, $52,3738-3741$

(96) Pilar Peña, M.; Vercher, E.; Martínez-Andreu, A. Apparent Molar Volumes of Strontium Chloride in Ethanol + Water at 298.15 K. J. Chem. Eng. Data 1997, 42, 187-189.

(113) Saluja, P. P. S.; LeBlanc, J. C. Apparent Molar Heat Capacities and Volumes of Aqueous Solutions of $\mathrm{MgCl}_{2}, \mathrm{CaCl}_{2}$, and $\mathrm{SrCl}_{2}$ at Elevated Temperatures. J. Chem. Eng. Data 1987, 32, 72-76.

molality

molarity

$t /{ }^{\circ} \mathrm{C}$ mass frac Density Ref

$$
\exp / \mathrm{kg} / \mathrm{m}^{3}
$$

0.0500

0.1000

0.5000

1.0000

1.5000

2.0000

2.5000

0.0500

0.1000

0.5000

1.0000

1.5000

2.0000

2.5000

0.02747

0.10044

0.20459

0.68309

1.00093

0.00374

0.0126

0.0219

0.0325

0.0500

0.0652

0.1000

0.1292

0.1975

0.2002

0.2513

0.2998

$\begin{array}{rrrr}15 & 0.0079 & 1006.1 & (45) \\ 15 & 0.0156 & 1013.2 & (45) \\ 15 & 0.0734 & 1067.5 & (45) \\ 15 & 0.1368 & 1131.9 & (45) \\ 15 & 0.1921 & 1192.3 & (45) \\ 15 & 0.2407 & 1239.5 & (45) \\ 15 & 0.2838 & 1288.4 & (45) \\ 20 & 0.0079 & 1005.2 & (45) \\ 20 & 0.0156 & 1012.2 & (45) \\ 20 & 0.0734 & 1066.2 & (45) \\ 20 & 0.1368 & 1130.2 & (45) \\ 20 & 0.1921 & 1190.2 & (45) \\ 20 & 0.2407 & 1237.3 & (45) \\ 20 & 0.2838 & 1286.0 & (45) \\ 24.04 & 0.0043 & 1001.10 & (113) \\ 24.04 & 0.0157 & 1011.10 & (113) \\ 24.04 & 0.0314 & 1024.89 & (113) \\ 24.04 & 0.0977 & 1087.28 & (113) \\ 24.04 & 0.1369 & 1125.89 & (113) \\ 25 & 0.0006 & 997.6 & (87) \\ 25 & 0.0020 & 998.8 & (87) \\ 25 & 0.0035 & 1000.1 & (87) \\ 25 & 0.0051 & 1001.5 & (87) \\ 25 & 0.0079 & 1004.0 & (45) \\ 25 & 0.0102 & 1006.0 & (87) \\ 25 & 0.0155 & 1010.8 & (96) \\ 25 & 0.0156 & 1011.0 & (45) \\ 25 & 0.0201 & 1014.8 & (87) \\ 25 & 0.0304 & 1024.0 & (87) \\ 25 & 0.0308 & 1024.3 & (73) \\ 25 & 0.0383 & 1031.2 & (87) \\ 25 & 0.0411 & 1033.8 & (96) \\ 25 & 0.0454 & 1037.6 & (73) \\ & & & \end{array}$

0.2678
App vol App vol

$$
\text { exp / Calc / }
$$$$
\mathrm{m}^{3} / \mathrm{kg} \quad \mathrm{m}^{3} / \mathrm{kg}
$$

$0.000110 \quad 0.000123$

$\begin{array}{lll}0.000109 & 0.000125\end{array}$

$\begin{array}{ll}0.000128 & 0.000135\end{array}$

$0.000143 \quad 0.000150$

$0.000157 \quad 0.000167$

$\begin{array}{ll}0.000195 & 0.000187 \\ 0.000209 & 0.000211\end{array}$

$0.000118 \quad 0.000126$

$0.000115 \quad 0.000127$

$\begin{array}{lll}0.000132 & 0.000137\end{array}$

$0.000147 \quad 0.000152$

$\begin{array}{lll}0.000160 & 0.000169\end{array}$

$0.000198 \quad 0.000189$

$0.000212 \quad 0.000213$

$0.000121 \quad 0.000127$

$\begin{array}{ll}0.000129 & 0.000129\end{array}$

$0.000143 \quad 0.000131$

$0.000153 \quad 0.000144$

$0.000166 \quad 0.000154$

$0.000122 \quad 0.000127$

$0.000122 \quad 0.000127$

$\begin{array}{ll}0.000123 & 0.000127 \\ 0.000126 & 0.000127\end{array}$

$\begin{array}{ll}0.000126 & 0.000127 \\ 0.000121 & 0.000128\end{array}$

$0.000127 \quad 0.000128$

$0.000125 \quad 0.000129$

$\begin{array}{ll}0.000118 & 0.000129\end{array}$

$0.000131 \quad 0.000130$

$0.000134 \quad 0.000131$

$0.000134 \quad 0.000131$

$0.000136 \quad 0.000133$

$0.000136 \quad 0.000133$

$0.000138 \quad 0.000134$

Density App Vol Square of Inconsistent Inconsistent

calc / Residual / Residual / Density based on based on

$\mathrm{kg} / \mathrm{m}^{3} \mathrm{~kg} / \mathrm{m}^{3} \quad \mathrm{~m}^{3} / \mathrm{kg}$. density apparen volume

$\begin{array}{lr}\text { Apparent } & \text { Delta density } \begin{array}{r}\text { Density of } \\ \text { al volume } \quad / \mathrm{kg} / \mathrm{m}^{3} \text { water } / \mathrm{kg} / \mathrm{m}^{3}\end{array}\end{array}$

$006.04 \quad 0.1042-0.000013$

1012.94

1066.88

1130.66

$0.2511-0.000016$

$0.6070-0.000007$

$1.2696-0.000007$

$2.7437-0.000010$

$\begin{array}{lll}-2.8343 & 0.000008\end{array}$

$0.7424-0.000002$

1005.12

1012.00

1065.75

$0.0627-0.000008$

$0.1929-0.000012$

$0.4327-0.000005$

$0.8861-0.000005$

$2.3986-0.000009$

$\begin{array}{ll}-3.0368 & 0.000008\end{array}$

$0.6511-0.000001$

$\begin{array}{llll}001.08 & 0.0241 & -0.000006\end{array}$

$\begin{array}{lll}011.10 & 0.0008 & 0.000000\end{array}$

$\begin{array}{ll}-0.3928 & 0.000012\end{array}$

$1088.33 \quad-1.0426 \quad 0.000009$

$\begin{array}{llll}1128.05 & -2.1679 & 0.000012\end{array}$

$\begin{array}{llll}997.56 & 0.0025 & -0.000004\end{array}$

$\begin{array}{llll}998.79 & 0.0094 & -0.000005\end{array}$

$\begin{array}{ll}0.0094 & -0.000005 \\ 0.0149 & -0.000004\end{array}$

Res
0.011

0.063

0.368

1.612
7.528

8.033

0.551

0.004

0.037

0.187
0.785

5.753

9.222

0.424

0.001

0.000
0.154
1.087

0.154
1.087

4.700

0.000

0.000

0.000

1001.53

$0.0091-0.000002$

0.000

$0.0557-0.000007$

0.003

$\begin{array}{llll}1006.03 & 0.0093 & -0.000001 & 0.000\end{array}$

$1010.71 \quad 0.0636-0.000004$

$\begin{array}{ll}0.0636 & -0.000004 \\ 0.1799 & -0.000011\end{array}$

0.004

0.032
0.001

$\begin{array}{lll}-0.0224 & 0.000001 & 0.001 \\ -0.0807 & 0.000003 & 0.007\end{array}$

$\begin{array}{lll}-0.0807 & 0.000003 & 0.007 \\ -0.0808 & 0.000003 & 0.007\end{array}$

$\begin{array}{llll}1024.42 & -0.0808 & 0.000003 & 0.007\end{array}$

$\begin{array}{llll}1031.32 & -0.1362 & 0.000003 & 0.019\end{array}$

$\begin{array}{llll}1033.86 & -0.0995 & 0.000002 & 0.010 \\ 1037.84 & -0.2033 & 0.000004 & 0.041\end{array}$
$/ \mathrm{cm}^{3} / \mathrm{mol}$

999.0996

999.0996

999.0996

999.0996

999.0996

999.0996

999.0996

998.2041

998.2041

998.2041

998.2041

998.2041

998.2041

998.2041

997.2867

997.2867

997.2867

997.2867

22.68

24.29

997.2867

$0.519 \quad 997.0449$

$\begin{array}{rr}1.75 & 997.0449\end{array}$

3.031997 .0449

4.491997 .0449

997.0449

8.992997 .0449

997.0449

997.0449

$17.715 \quad 997.0449$

$26.937 \quad 997.0449$

$27.293 \quad 997.0449$

$34.142 \quad 997.0449$

997.0449

$40.593 \quad 997.0449$ 


\begin{tabular}{|c|c|c|c|c|}
\hline molality & molarity & \multicolumn{2}{|c|}{$\mathrm{t} /{ }^{\circ} \mathrm{C}$ mass frac } & $\begin{array}{r}\text { Density } \\
\exp / \mathrm{kg} / \mathrm{m}^{3}\end{array}$ \\
\hline 0.3286 & & 25 & 0.0495 & 1041.5 \\
\hline 0.4002 & & 25 & 0.0597 & 1050.9 \\
\hline & 0.4536 & 25 & 0.0679 & 1058.8 \\
\hline 0.4996 & & 25 & 0.0734 & 1063.8 \\
\hline 0.5000 & & 25 & 0.0734 & 1064.7 \\
\hline & 0.5625 & 25 & 0.0832 & 1072.2 \\
\hline 0.6000 & & 25 & 0.0868 & 1076.7 \\
\hline 0.6990 & & 25 & 0.0998 & 1089.3 \\
\hline & 0.7633 & 25 & 0.1100 & 1099.6 \\
\hline 0.8000 & & 25 & 0.1125 & 1102.0 \\
\hline 0.8996 & & 25 & 0.1248 & 1114.4 \\
\hline 0.9962 & & 25 & 0.1364 & 1126.4 \\
\hline 1.0000 & & 25 & 0.1368 & 1128.4 \\
\hline & 1.0254 & 25 & 0.1434 & 1133.9 \\
\hline & 1.125 & 25 & 0.1557 & 1145.6 \\
\hline & 1.2109 & 25 & 0.1658 & 1157.8 \\
\hline 1.5000 & & 25 & 0.1921 & 1188.2 \\
\hline & 1.4484 & 25 & 0.1932 & 1188.4 \\
\hline & 1.6458 & 25 & 0.2150 & 1213.4 \\
\hline & 1.6875 & 25 & 0.2197 & 1217.6 \\
\hline 2.0000 & & 25 & 0.2407 & 1235.1 \\
\hline 2.5000 & & 25 & 0.2838 & 1283.5 \\
\hline 0.0500 & & 30 & 0.0079 & 1002.6 \\
\hline 0.1000 & & 30 & 0.0156 & 1009.5 \\
\hline 0.5000 & & 30 & 0.0734 & 1063.0 \\
\hline 1.0000 & & 30 & 0.1368 & 1126.4 \\
\hline 1.5000 & & 30 & 0.1921 & 1186.0 \\
\hline 2.0000 & & 30 & 0.2407 & 1232.7 \\
\hline 2.5000 & & 30 & 0.2838 & 1281.0 \\
\hline 0.0500 & & 35 & 0.0079 & 1000.9 \\
\hline 0.1000 & & 35 & 0.0156 & 1007.9 \\
\hline 0.5000 & & 35 & 0.0734 & 1061.1 \\
\hline 1.0000 & & 35 & 0.1368 & 1124.3 \\
\hline 1.5000 & & 35 & 0.1921 & 1183.8 \\
\hline 2.0000 & & 35 & 0.2407 & 1230.3 \\
\hline 2.5000 & & 35 & 0.2838 & 1278.4 \\
\hline 0.0500 & & 45 & 0.0079 & 997.1 \\
\hline 0.1000 & & 45 & 0.0156 & 1004.0 \\
\hline 0.5000 & & 45 & 0.0734 & 1056.9 \\
\hline 1.0000 & & 45 & 0.1368 & 1119.8 \\
\hline 1.5000 & & 45 & 0.1921 & 1178.9 \\
\hline 2.0000 & & 45 & 0.2407 & 1225.2 \\
\hline 2.5000 & & 45 & 0.2838 & 1273.0 \\
\hline 0.02747 & & 48.75 & 0.0043 & 992.34 \\
\hline 0.10044 & & 48.75 & 0.0157 & 1002.17 \\
\hline 0.20459 & & 48.75 & 0.0314 & 1015.67 \\
\hline 0.68309 & & 48.75 & 0.0977 & 1076.90 \\
\hline
\end{tabular}

App vol App vol De$$
\begin{array}{r}
\exp / \\
\mathrm{m}^{3} / \mathrm{kg}
\end{array}
$$

$$
\begin{aligned}
& \text { Calc / } \\
& \mathrm{m}^{3} / \mathrm{kg}
\end{aligned}
$$

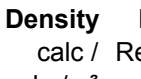

Apparent Delta density Density of

0.0001390 .000135 1041.68 $0.000142 \quad 0.000137$ 1041.68
1051.21 $\mathrm{kg} / \mathrm{m}^{3}$ $\mathrm{m}^{3} / \mathrm{kg}$ Density $\begin{array}{ccc}\text { of Inconsistent } & \begin{array}{c}\text { Inconsistent } \\ \text { based on } \\ \text { based on } \\ \text { apparent } \\ \text { volume }\end{array} & \begin{array}{c}\text { Apparent Dolal } \\ \text { molume } \\ \text { ( } \mathrm{cm}^{3} / \mathrm{mol}\end{array} \\ \text { Res } & \text { density } & \\ & & \end{array}$ $\begin{array}{lllll}0.000142 & 0.000138 & 1059.05 & -0.2586 & 0.000003\end{array}$ 0.049 0.119

$\begin{array}{llllll}0.000142 & 0.000138 & 1059.05 & -0.2586 & 0.000003 & 0.067 \\ 0.000146 & 0.000139 & 1064.29 & -0.5101 & 0.000006 & 0.260\end{array}$ $\begin{array}{llllll}0.000136 & 0.000139 & 1064.34 & 0.3264 & -0.000004 & 0.107\end{array}$ $\begin{array}{ll}-1.5483 & 0.000016\end{array}$ $0.000149 \quad 0.000142$ $0.000151 \quad 0.000145$ 1077.34 $\begin{array}{ll}-0.6426 & 0.000006\end{array}$ $\begin{array}{ll}-0.7480 & 0.000006\end{array}$ $\begin{array}{ll}-0.6753 & 0.000005\end{array}$ $\begin{array}{ll}-0.8199 & 0.000006\end{array}$ $\begin{array}{lll}0.000154 & 0.000148 & 1102.84\end{array}$ $\begin{array}{lll}0.000157 & 0.000151 & 1115.27\end{array}$ $-0.8478 \quad 0.000005$ $\begin{array}{ll}-0.7249 & 0.000004\end{array}$ $0.7563-0.000004$ $\begin{array}{cc}-0.4900 & 0.000003\end{array}$ $\begin{array}{ll}-1.6158 & 0.000008\end{array}$ $0.000150-0.000154$ $0.000159 \quad 0.000156 \quad 1134.36$ $0.000167 \quad 0.000160 \quad 1147.22$ $0.000163 \quad 0.000163 \quad 1157.87$ $-0.0312 \quad 0.000000$ 1185.92 $\begin{array}{lll}0.000167 & 0.000172 & 1187.10\end{array}$ $\begin{array}{lll}0.000171 & 0.000180 & 1210.57\end{array}$ $\begin{array}{lll}0.000176 & 0.000182 & 1215.60\end{array}$ $0.000200 \quad 0.000192$ 1215.60 $2.2323-0.000008$ $1.2679-0.000005$ $2.7849-0.000009$ $1.9977-0.000006$ $-3.0388 \quad 0.000008$ $0.5962-0.000001$ $0.000214 \quad 0.000216 \quad 1282.91$ $\begin{array}{llll}0.000124 & 0.000130 & 1002.51\end{array}$ $\begin{array}{lll}0.000121 & 0.000131 & 1009.34\end{array}$ $\begin{array}{lll}0.000138 & 0.000141 & 1062.70\end{array}$ $\begin{array}{lll}0.000152 & 0.000156 & 1125.71\end{array}$ $\begin{array}{lll}0.000165 & 0.000173 & 1183.78\end{array}$ $\begin{array}{lll}0.000202 & 0.000194 & 1235.79\end{array}$

$0.0476-0.000006$

$0.1661-0.000010$

$0.2800-0.000003$

$0.6786-0.000004$

$2.1991-0.000008$

$\begin{array}{ll}-3.0806 & 0.000008\end{array}$

$0.6323-0.000001$

$0.0575-0.000007$

$0.1906-0.000012$

$0.2899-0.000004$

$0.7163-0.000004$

$2.3084-0.000009$

$\begin{array}{ll}-2.9471 \quad 0.000008 \\ 0.291 & -0.000002\end{array}$

$0.7291-0.000002$

$0.0650-0.000008$

$0.1966-0.000013$

$0.3833-0.000005$

$0.8998-0.000005$

$2.4915-0.000009$

$\begin{array}{ll}-2.7559 & 0.000008\end{array}$

$0.7807-0.000002$

$0.0225-0.000005$

$\begin{array}{lll}-0.0037 & 0.000000\end{array}$

$\begin{array}{ll}-0.4302 & 0.000013\end{array}$

$\begin{array}{ll}-1.1409 & 0.000010\end{array}$
0.107
2.397

0.413

0.559
0.456

0.672

0.719

0.525

0.572

0.240

2.611

4.983
1.607

7.755

3.991

0.234

0.002

0.028

0.078

0.460

4.836

9.490

0.003

0.036

0.084

0.513

5.329

8.685

0.532
0.004

0.039

0.147

0.810

6.207

7.595
0.609

0.001

0.000

0.185

1.302
$44.417 \quad 997.0449$

$53.819 \quad 997.0449$ 997.0449

$66.731 \quad 997.0449$

997.0449

997.0449

$79.651 \quad 997.0449$

92.261997 .0449

997.0449

$104.972 \quad 997.0449$

$117.377 \quad 997.0449$

$129.373 \quad 997.0449$

997.0449

997.0449

997.0449

997.0449

997.0449

997.0449

997.0449

997.0449

997.0449

997.0449

995.6473

995.6473

995.6473

995.6473

995.6473

995.6473

994.0319

994.0319

994.0319

994.0319

994.0319

994.0319

994.0319

990.2132

990.2132
990.2132

990.2132

990.2132

990.2132

990.2132

20.66

21.81

24.28

988.5963

9885963

988.5963 


\begin{tabular}{rrrrr} 
molality & molarity & $\mathbf{t} /{ }^{\circ} \mathrm{C}$ mass frac & $\begin{array}{r}\text { Density } \\
\text { exp } / \mathrm{kg} / \mathrm{m}^{3}\end{array}$ & Ref \\
& \multicolumn{5}{c}{} & \\
1.00093 & 48.75 & 0.1369 & 1114.79 & $(113)$ \\
0.0500 & 55 & 0.0079 & 992.5 & $(45)$ \\
0.1000 & 55 & 0.0156 & 999.4 & $(45)$ \\
0.5000 & 55 & 0.0734 & 1052.2 & $(45)$ \\
1.0000 & 55 & 0.1368 & 1114.8 & $(45)$ \\
1.5000 & 55 & 0.1921 & 1173.7 & $(45)$ \\
2.0000 & 55 & 0.2407 & 1219.8 & $(45)$ \\
2.5000 & 55 & 0.2838 & 1267.4 & $(45)$ \\
0.02747 & 73.77 & 0.0043 & 979.31 & $(113)$ \\
0.10044 & 73.77 & 0.0157 & 989.07 & $(113)$ \\
0.20459 & 73.77 & 0.0314 & 1002.51 & $(113)$ \\
0.68309 & 73.77 & 0.0977 & 1063.31 & $(113)$ \\
1.00093 & 73.77 & 0.1369 & 1100.91 & $(113)$ \\
0.0998 & 98.81 & 0.0156 & 972.37 & $(113)$ \\
0.2058 & 98.81 & 0.0316 & 986.19 & $(113)$ \\
0.5013 & 98.81 & 0.0736 & 1024.24 & $(113)$ \\
1.0454 & 98.81 & 0.1422 & 1091.08 & $(113)$ \\
1.9989 & 98.81 & 0.2406 & 1200.20 & $(113)$
\end{tabular}

App vol App vol Densit

$$
\begin{array}{cc}
\exp / & \text { Calc } / \\
\mathrm{m}^{3} / \mathrm{kg} & \mathrm{m}^{3} / \mathrm{kg}
\end{array}
$$
( apparent

Apparent Delta density Density of $\begin{array}{llllll}0.000175 & 0.000162 & 1117.07 & -2.2850 & 0.000013\end{array}$ $\begin{array}{rrr}0.000125 & 0.000138 & 992.44\end{array}$ $\begin{array}{lll}0.000123 & 0.000139 & 999.15\end{array}$ $\begin{array}{lll}0.000142 & 0.000149 & 1051.59\end{array}$ $\begin{array}{lll}0.000156 & 0.000163 & 1113.58\end{array}$ $\begin{array}{lll}0.000169 & 0.000179 & 1170.85\end{array}$ $\begin{array}{lll}0.000206 & 0.000199 & 1222.35\end{array}$ $\begin{array}{lll}0.000220 & 0.000221 & 1266.80\end{array}$ $\begin{array}{lll}0.000126 & 0.000140 \quad 979.25\end{array}$ $\begin{array}{lll}0.000133 & 0.000141 & 988.94\end{array}$ $\begin{array}{lll}0.000148 & 0.000144 \quad 1002.66\end{array}$ $\begin{array}{lll}0.000159 & 0.000155 & 1063.84\end{array}$ $\begin{array}{lll}0.000173 & 0.000163 & 1102.57\end{array}$ $\begin{array}{lll}0.000137 & 0.000137 & 972.37\end{array}$ $\begin{array}{lll}0.000140 & 0.000139 & 986.23\end{array}$ $\begin{array}{lll}0.000144 & 0.000143 & 1024.25\end{array}$ $\begin{array}{lll}0.000156 & 0.000153 & 1091.63\end{array}$ $\begin{array}{lll}0.000173 & 0.000174 & 1199.63\end{array}$ Average Res Std dev Res Avg - 4std Avg + 4std $\begin{array}{rr}-2.2850 & 0.000013 \\ 0.1000 & -0.000013\end{array}$ $0.2481-0.000016$ $0.5805-0.000007$ Res 5.221 5.221
0.010 0.062
0.337 $1.2162-0.000007-1.479$ $\begin{array}{lll}2.8603 & -0.000011 \quad 8.181\end{array}$ $\begin{array}{lll}-2.5546 & 0.000007 & 6.526\end{array}$ $\begin{array}{lll}0.6346 & -0.000001 & 0.403\end{array}$ $\begin{array}{lll}0.0593 & -0.000014 & 0.004 \\ 0.1319 & -0.000009 & 0.017\end{array}$

$\begin{array}{lll}0.1319 & -0.000009 & 0.017\end{array}$

$\begin{array}{lll}-0.1537 & 0.000005 & 0.024\end{array}$

$\begin{array}{lll}-0.5233 & 0.000005 & 0.274 \\ -1.6609 & 0.000010 & 2.759\end{array}$

$\begin{array}{lll}-0.0029 & 0.000000 & 0.000\end{array}$

$\begin{array}{lll}-0.0426 & 0.000001 & 0.002\end{array}$

$\begin{array}{lll}-0.0149 & 0.000000 & 0.000\end{array}$

$\begin{array}{lll}-0.5463 & 0.000003 & 0.298\end{array}$

$0.5657-0.000002$

$\begin{array}{rr}-0.0056 & -0.000001 \\ 1.2503 & 0.000007\end{array}$

0.298
0.320

151.631 Sum Chi sq

$-5.0067-0.000031$

$4.9954 \quad 0.000028$ volume $/ \mathrm{kg}^{3} \mathrm{~m}^{3}$ ater $/ \mathrm{kg}^{3} \mathrm{~m}^{3}$ $1 \mathrm{~cm}^{3} / \mathrm{mol}$

27.8

988.5963

985.6952

985.6952

985.6952

985.6952

985.6952

985.6952

975.5797

975.5797

975.5797

975.5797

975.5797

959.2159

959.2159

959.2159

959.2159

959.2159
Curve fit for the model App vol $=\left(w+c 2+c 3^{*} t{ }^{\circ} \mathrm{C}\right) /\left(\mathrm{c} 0^{*} \mathrm{w}+\mathrm{c} 1\right) / \mathrm{e}^{\wedge}\left(0.000001^{*}\left(\mathrm{t}^{\circ} \mathrm{C}+\mathrm{c} 4\right)^{\wedge} 2\right)$

c0

c1 $-7.49 \mathrm{E}-07$

c2 $\quad-1.935633$

c3 0.010704

c4 -4882.145

Min T 15

$\begin{array}{lr}\text { Max } T & 98.81\end{array}$

$\begin{array}{ll}\text { Min } w & 0.000593 \\ \text { Max w } & 0.283822\end{array}$

Avg dens res $\quad-0.005642$

Std dens res $\quad 1.250269$

Avg app vol res *10^3 $\quad-0.001313$

Std app vol res *10^3 $\quad 0.007377$

No of points in corr

No of inconsistent points

Inconsistent data not used (Residual greater than average + /- $\mathbf{4}$ standard deviations)

$\begin{array}{rrrrrrrrrrr}2.25 & 25 & 0.2769 & 1287.9 & (40) & 0.000185 & 0.000211 & 1276.00 & 11.8974 & -0.000026 & 141.549 \\ 1.8667 & 25 & 0.2384 & 1241.2 & (96) & 0.000175 & 0.000190 & 1235.68 & 5.5386 & -0.000015 & 30.676 \\ 2.1147 & 25 & 0.2634 & 1272.5 & (96) & 0.000179 & 0.000203 & 1262.13 & 10.3573 & -0.000024 & 107.273 \\ 2.4265 & 25 & 0.2934 & 1311.1 & (96) & 0.000184 & 0.000222 & 1292.29 & 18.7783 & -0.000038 & 352.626 \\ 2.9214 & 25 & 0.3375 & 1372.3 & (96) & 0.000190 & 0.000259 & 1330.15 & 42.1108 & -0.000068 & 1773.321 \\ 3.2561 & 25 & 0.3652 & 1413.3 & (96) & 0.000194 & 0.000291 & 1346.21 & 67.1037 & -0.000097 & 4502.903\end{array}$


Density of aqueous solutions of $\mathrm{ZnCl} 2$

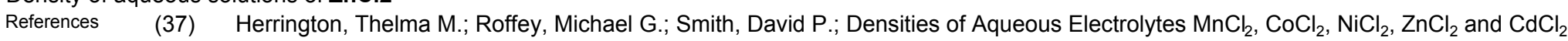
from 25 to $72^{\circ} \mathrm{C}$ at $1 \mathrm{~atm}, \mathrm{~J}$. Chem. Eng. Data, 1986, 31, 221-225

(90) Perry, R. H.; Green, D. W. Perry's Chemical Engineers' Handbook; McGraw Hill: New York, 7th edition, 1997.

(100) Pogue, R. F.; Atkinson, G. Solution Thermodynamics of First-Row Transition Elements. 3. Apparent Molal Volumes of Aqueous ZnCl2 and $\mathrm{Zn}(\mathrm{ClO} 4) 2$ from 15 to $55^{\circ} \mathrm{C}$ and an Examination of Solute-Solute and Solute-Solvent Interactions. J. Sol. Chem. 1989, 18, $249-264$.

(104) Rard, J. A.; Miller, D. G. Densities and Apparent Molal Volumes of Aqueous Manganese, Cadmium, and Zinc Chlorides at $25^{\circ} \mathrm{C} . \mathrm{J}$. Chem. Eng. Data 1984, 29, 151-156.

(132) Weingärtner, H.; Müller, K. J.; Hertz, H. G.; Edge, A. V. J.; Mills, R. Unusual Behavior of Transport Coefficients in Aqueous Solutions of Zinc Chloride. J. Phys. Chem. 1984, 88, 2173-2178.

\begin{tabular}{|c|c|c|c|c|c|c|c|c|c|c|c|c|c|c|c|}
\hline \multirow[t]{6}{*}{ molality } & \multirow[t]{6}{*}{ molarity } & \multicolumn{2}{|c|}{$\mathbf{t} /{ }^{\circ} \mathrm{C}$ mass frac } & \multicolumn{2}{|l|}{$\begin{array}{r}\text { Density } \\
\exp / \\
\mathrm{kg} / \mathrm{m}^{3}\end{array}$} & \multirow{2}{*}{$\begin{array}{r}\text { App vol } \\
\text { exp / } \\
\mathrm{m}^{3} / \mathrm{kg} \\
0.000050\end{array}$} & \multirow{2}{*}{$\begin{array}{r}\text { App vol } \\
\text { Calc / } \\
\mathrm{m}^{3} / \mathrm{kg} \\
0.000013\end{array}$} & \multirow{2}{*}{$\begin{array}{r}\text { Density } \\
\text { calc / } \\
\mathrm{kg} / \mathrm{m}^{3} \\
1020.0\end{array}$} & \multirow{2}{*}{$\begin{array}{r}\text { Density } \\
\text { Residual / } \\
\mathrm{kg} / \mathrm{m}^{3} \\
-0.7718\end{array}$} & \multirow{2}{*}{$\begin{array}{r}\text { App Vol } \\
\text { Residual / } \\
\mathrm{m}^{3} / \mathrm{kg} \\
0.000037\end{array}$} & \multirow{2}{*}{$\begin{array}{r}\text { Square of } \\
\text { Density } \\
\text { Res } \\
0.596\end{array}$} & \multirow[t]{2}{*}{$\begin{array}{l}\text { Inconsistent } \\
\text { based on } \\
\text { density }\end{array}$} & $\begin{array}{l}\text { Inconsistent } \\
\text { based on } \\
\text { apparent } \\
\text { volume }\end{array}$ & $\begin{array}{r}\text { Delta density } \\
\qquad / \mathrm{kg} / \mathrm{m}^{3}\end{array}$ & $\begin{array}{r}\text { Density of } \\
\text { water } / \mathrm{kg} / \mathrm{m}^{3}\end{array}$ \\
\hline & & 0 & 0.0200 & 1019.20 & (90) & & & & & & & & & & 999.8395 \\
\hline & & 0 & 0.0400 & 1038.40 & (90) & 0.000072 & 0.000049 & 1039.4 & -0.9594 & 0.000022 & 0.920 & & & & 999.8395 \\
\hline & & 0 & 0.0800 & 1076.90 & (90) & 0.000106 & 0.000104 & 1077.1 & -0.1754 & 0.000002 & 0.031 & & & & 999.8395 \\
\hline & & 0 & 0.1200 & 1115.90 & (90) & 0.000133 & 0.000142 & 1114.6 & 1.3279 & -0.000009 & 1.763 & & & & 999.8395 \\
\hline & & 0 & 0.1600 & 1155.80 & $(90)$ & 0.000157 & 0.000171 & 1152.7 & 3.0564 & -0.000014 & 9.342 & & & & 999.8395 \\
\hline 0.01012 & & 15 & 0.0014 & 1000.37 & (100) & 0.000078 & 0.000011 & 1000.5 & -0.0924 & 0.000067 & 0.009 & & $!$ & 1.27 & 999.0996 \\
\hline 0.02017 & & 15 & 0.0027 & 1001.64 & (100) & 0.000075 & 0.000014 & 1001.8 & -0.1671 & 0.000061 & 0.028 & & $!$ & 2.54 & 999.0996 \\
\hline 0.02986 & & 15 & 0.0041 & 1002.84 & $(100)$ & 0.000080 & 0.000017 & 1003.1 & -0.2559 & 0.000063 & 0.065 & & $!$ & 3.74 & 999.0996 \\
\hline 0.07469 & & 15 & 0.0101 & 1008.38 & (100) & 0.000087 & 0.000030 & 1009.0 & -0.5856 & 0.000057 & 0.343 & & & 9.28 & 999.0996 \\
\hline 0.09463 & & 15 & 0.0127 & 1010.83 & (100) & 0.000089 & 0.000035 & 1011.5 & -0.7001 & 0.000054 & 0.490 & & & 11.73 & 999.0996 \\
\hline 0.2009 & & 15 & 0.0267 & 1023.82 & (100) & 0.000094 & 0.000060 & 1024.8 & -0.9563 & 0.000034 & 0.915 & & & 24.72 & 999.0996 \\
\hline 0.2322 & & 15 & 0.0307 & 1027.56 & (100) & 0.000097 & 0.000067 & 1028.6 & -0.9971 & 0.000031 & 0.994 & & & 28.46 & 999.0996 \\
\hline 0.5014 & & 15 & 0.0640 & 1058.94 & (100) & 0.000117 & 0.000111 & 1059.3 & -0.3829 & 0.000005 & 0.147 & & & 59.84 & 999.0996 \\
\hline 0.7432 & & 15 & 0.0920 & 1085.46 & (100) & 0.000135 & 0.000140 & 1085.0 & 0.5068 & -0.000005 & 0.257 & & & 86.36 & 999.0996 \\
\hline 0.9842 & & 15 & 0.1183 & 1110.03 & (100) & 0.000155 & 0.000161 & 1109.2 & 0.8757 & -0.000006 & 0.767 & & & 110.93 & 999.0996 \\
\hline 1.9458 & & 15 & 0.2096 & 1198.44 & (100) & 0.000207 & 0.000212 & 1196.9 & 1.5287 & -0.000005 & 2.337 & & & 199.34 & 999.0996 \\
\hline 2.814 & & 15 & 0.2772 & 1270.09 & (100) & 0.000231 & 0.000235 & 1267.9 & 2.1872 & -0.000005 & 4.784 & & & 270.99 & 999.0996 \\
\hline & & 20 & 0.0200 & 1016.70 & $(90)$ & 0.000091 & 0.000060 & 1017.3 & -0.6307 & 0.000030 & 0.398 & & & & 998.2041 \\
\hline & & 20 & 0.0400 & 1035.00 & (90) & 0.000111 & 0.000091 & 1035.9 & -0.8860 & 0.000021 & 0.785 & & & & 998.2041 \\
\hline & & 20 & 0.0800 & 1071.50 & (90) & 0.000145 & 0.000137 & 1072.3 & -0.7949 & 0.000009 & 0.632 & & & & 998.2041 \\
\hline & & 20 & 0.1200 & 1108.50 & (90) & 0.000171 & 0.000169 & 1108.8 & -0.3004 & 0.000002 & 0.090 & & & & 998.2041 \\
\hline & & 20 & 0.1600 & 1146.80 & (90) & 0.000191 & 0.000193 & 1146.2 & 0.6154 & -0.000003 & 0.379 & & & & 998.2041 \\
\hline & & 20 & 0.2000 & 1186.60 & (90) & 0.000207 & 0.000212 & 1185.0 & 1.6237 & -0.000006 & 2.636 & & & & 998.2041 \\
\hline & & 20 & 0.3000 & 1292.80 & (90) & 0.000241 & 0.000245 & 1290.7 & 2.0788 & -0.000004 & 4.321 & & & & 998.2041 \\
\hline & & 20 & 0.4000 & 1417.30 & (90) & 0.000261 & 0.000266 & 1413.5 & 3.7983 & -0.000005 & 14.427 & $!$ & & & 998.2041 \\
\hline & & 20 & 0.7000 & 1962.00 & $(90)$ & 0.000299 & 0.000299 & 1960.1 & 1.8545 & -0.000001 & 3.439 & & & & 998.2041 \\
\hline 0.01012 & & 25 & 0.0014 & 998.30 & (100) & 0.000084 & 0.000038 & 998.4 & -0.0638 & 0.000046 & 0.004 & & & 1.26 & 997.0449 \\
\hline 0.02018 & & 25 & 0.0027 & 999.56 & (100) & 0.000081 & 0.000040 & 999.7 & -0.1120 & 0.000041 & 0.013 & & & 2.52 & 997.0449 \\
\hline 0.02986 & & 25 & 0.0041 & 1000.76 & (100) & 0.000083 & 0.000043 & 1000.9 & -0.1638 & 0.000040 & 0.027 & & & 3.72 & 997.0449 \\
\hline
\end{tabular}




$$
\begin{array}{r}
\text { molality } \\
\\
\hline 0.04001 \\
0.04595 \\
0.07469 \\
0.089958 \\
0.09463 \\
0.09964 \\
0.15994 \\
0.1968 \\
0.2009 \\
0.2322 \\
0.2348 \\
0.23987 \\
0.28986 \\
0.29985 \\
0.29985 \\
0.3279 \\
0.33191 \\
0.33194 \\
0.37981 \\
0.398 \\
0.41978 \\
0.4654 \\
0.47991 \\
0.5014 \\
0.51976 \\
0.592 \\
0.6969 \\
0.7439 \\
0.74978 \\
0.787 \\
0.92673 \\
0.9333 \\
0.94975 \\
0.97261 \\
0.9842 \\
0.99563 \\
1.1759 \\
1.2275 \\
1.2324 \\
1.2711 \\
1.4209
\end{array}
$$$$
\text { molarity }
$$$$
\mathrm{t} /{ }^{\circ} \mathrm{C} \text { mass frac Density }
$$$$
\exp
$$$$
\mathrm{kg} / \mathrm{m}^{3}
$$

Ref

$\begin{array}{rr}\text { App vol } & \text { App vol } \\ \exp / & \text { Calc / }\end{array}$

$\mathrm{m}^{3} / \mathrm{kg} \quad \mathrm{m}^{3} / \mathrm{kg}$

$\begin{array}{ll}0.000088 & 0.000046\end{array}$ $0.000087 \quad 0.000047$ $\begin{array}{ll}0.000090 & 0.000054\end{array}$ $0.000092 \quad 0.000058$ $0.000093 \quad 0.000059$ $0.000094 \quad 0.000060$ $0.000098 \quad 0.000074$ $0.000102 \quad 0.000081$ $0.000103 \quad 0.000082$ $0.000107 \quad 0.000088$ 0.0001090 .000088 $0.000107 \quad 0.000089$ 0.0001110 .000098 $\begin{array}{ll}0.000113 & 0.000100\end{array}$ 0.0001120 .000100 $0.000115 \quad 0.000104$ $0.000115 \quad 0.000105$ 0.0001150 .000105 $0.000122 \quad 0.000112$ $0.000122 \quad 0.000115$ $0.000124 \quad 0.000118$ $0.000131 \quad 0.000124$ $0.000130 \quad 0.000126$ $0.000128 \quad 0.000129$ $0.000134 \quad 0.000131$ $0.000142 \quad 0.000140$ 0.0001510 .000150 $\begin{array}{lll}0.000151 & 0.000155\end{array}$ $0.000156 \quad 0.000155$ 0.0001590 .000159 $\begin{array}{lll}0.000170 & 0.000170\end{array}$ $0.000169 \quad 0.000171$ 0.0001720 .000172 $\begin{array}{lll}0.000174 & 0.000174\end{array}$ $0.000171 \quad 0.000174$ $0.000175 \quad 0.000175$ $\begin{array}{lll}0.000185 & 0.000187\end{array}$ $0.000190 \quad 0.000190$ $0.000190 \quad 0.000190$ $0.000193 \quad 0.000192$ $0.000198 \quad 0.000200$
Density Density App Vol Square of Inconsistent Inconsistent Delta density Density of calc / Residual / Residual / Density based on based on $/ \mathrm{kg} / \mathrm{m}^{3}$ water $/ \mathrm{kg} / \mathrm{m}^{3}$ $\mathrm{kg} / \mathrm{m}^{3} \quad \mathrm{~kg} / \mathrm{m}^{3} \quad \mathrm{~m}^{3} / \mathrm{kg} \quad$ Res density $\begin{gathered}\text { apparent } \\ \text { volume }\end{gathered}$

$\begin{array}{llll}1002.2 & -0.2339 & 0.000043 & 0.055\end{array}$

$\begin{array}{llll}1003.0 & -0.2495 & 0.000040 & 0.062\end{array}$

$\begin{array}{llll}1006.6 & -0.3652 & 0.000036 & 0.133\end{array}$

$\begin{array}{llll}1008.6 & -0.4148 & 0.000034 & 0.172\end{array}$

$\begin{array}{llll}1009.1 & -0.4329 & 0.000033 & 0.187\end{array}$

$\begin{array}{llll}1009.8 & -0.4615 & 0.000034 & 0.213\end{array}$

$\begin{array}{llll}1017.2 & -0.5426 & 0.000025 & 0.294\end{array}$

$\begin{array}{llll}1021.6 & -0.5732 & 0.000021 & 0.329\end{array}$

$\begin{array}{llll}1022.1 & -0.5958 & 0.000021 & 0.355\end{array}$

$\begin{array}{llll}1025.8 & -0.6106 & 0.000019 & 0.373\end{array}$

$\begin{array}{llll}1026.1 & -0.6703 & 0.000021 & 0.449\end{array}$

$\begin{array}{llll}1026.7 & -0.5839 & 0.000018 & 0.341\end{array}$

$\begin{array}{llll}1032.4 & -0.5358 & 0.000013 & 0.287\end{array}$

$\begin{array}{llll}1033.6 & -0.5605 & 0.000013 & 0.314\end{array}$

$\begin{array}{llll}1033.6 & -0.5205 & 0.000012 & 0.271\end{array}$

$\begin{array}{llll}1036.8 & -0.4835 & 0.000011 & 0.234\end{array}$

$\begin{array}{llll}1037.2 & -0.4474 & 0.000010 & 0.200\end{array}$

$\begin{array}{llll}1037.2 & -0.4608 & 0.000010 & 0.212\end{array}$

$\begin{array}{llll}1042.6 & -0.5035 & 0.000009 & 0.254\end{array}$

$\begin{array}{llll}1044.6 & -0.3936 & 0.000007 & 0.155\end{array}$

$\begin{array}{llll}1047.0 & -0.3575 & 0.000006 & 0.128\end{array}$

$\begin{array}{llll}1052.0 & -0.4724 & 0.000007 & 0.223\end{array}$

$\begin{array}{llll}1053.6 & -0.2943 & 0.000004 & 0.087\end{array}$

$\begin{array}{llll}1055.9 & 0.0841 & -0.000001 & 0.007\end{array}$

$\begin{array}{llll}1057.9 & -0.2092 & 0.000003 & 0.044\end{array}$

$\begin{array}{llll}1065.5 & -0.1734 & 0.000002 & 0.030\end{array}$

$\begin{array}{llll}1076.4 & -0.0622 & 0.000001 & 0.004\end{array}$

$\begin{array}{llll}1081.2 & 0.3810 & -0.000004 & 0.145\end{array}$

$\begin{array}{llll}1081.8 & -0.0600 & 0.000001 & 0.004\end{array}$

$\begin{array}{lllll}1085.6 & -0.0465 & 0.000000 & 0.002\end{array}$

$\begin{array}{llll}1099.4 & 0.0046 & 0.000000 & 0.000\end{array}$

$\begin{array}{llll}1100.1 & 0.2718 & -0.000002 & 0.074\end{array}$

$\begin{array}{llll}1101.7 & -0.0542 & 0.000000 & 0.003\end{array}$

$\begin{array}{llll}1103.9 & 0.0018 & 0.000000 & 0.000\end{array}$

$\begin{array}{llll}1105.0 & 0.5425 & -0.000004 & 0.294\end{array}$

$\begin{array}{llll}1106.1 & -0.0088 & 0.000000 & 0.000\end{array}$

$\begin{array}{llll}1123.3 & 0.4154 & -0.000002 & 0.173\end{array}$

$\begin{array}{lll}1128.1 & -0.0618 & 0.000000\end{array}$

$\begin{array}{llll}1128.6 & -0.0582 & 0.000000 & 0.003\end{array}$

$\begin{array}{llll}1132.2 & -0.0517 & 0.000000 & 0.003\end{array}$

$\begin{array}{llll}1145.9 & 0.3745 & -0.000002 & 0.140\end{array}$
997.0449

$5.7 \quad 997.0449$

$9.23 \quad 997.0449$

997.0449

$11.66 \quad 997.0449$

997.0449

997.0449

997.0449

24.42997 .0449

28.1997 .0449

997.0449

997.0449

997.0449

997.0449

997.0449

997.0449

997.0449

997.0449

997.0449

997.0449

997.0449

997.0449

997.0449

$58.95 \quad 997.0449$

997.0449

997.0449

997.0449

997.0449

997.0449

997.0449

997.0449

997.0449

997.0449

108.51997 .0449

997.0449

997.0449

997.0449

997.0449

997.0449

997.0449
$84.54 \quad 997.0449$ 


$\begin{array}{r}\text { molality } \\ \\ \\ 1.4733 \\ 1.5253 \\ 1.6722 \\ 1.7505 \\ 1.9309 \\ 1.9317 \\ 1.9458 \\ 1.965 \\ 1.9693 \\ 1.9893 \\ 2.0298 \\ 2.2667 \\ 2.4457 \\ 2.4998 \\ 2.6199 \\ 2.814 \\ 2.956 \\ 2.9664 \\ 2.9867 \\ 3.0332 \\ 3.4979 \\ 3.93 \\ 3.9653 \\ 4.0332 \\ 4.4945 \\ 4.911 \\ 5.0018 \\ 5.4975 \\ 5.902 \\ 6.001 \\ 6.5043 \\ 6.9026 \\ 7.106 \\ 7.3213 \\ 7.933 \\ 0.01012 \\ 0.02018 \\ 0.02986 \\ 0.04595 \\ 0.07469 \\ 0.09462 \\ \hline\end{array}$

$t /{ }^{\circ} \mathrm{C}$ mass frac Density

$\exp /$

150.45

25
25

25
25
25

25

25
25

25

25
25

25

25

25
25

25
25

25

25

25

5

25

25

25

25
25

25

5

25

25

25

25

5

35

5

35

35
35

35
Ref

$\begin{array}{rr}\text { App vol } & \text { App vol } \\ \exp / & \text { Calc / }\end{array}$

$\mathrm{m}^{3} / \mathrm{kg} \quad \mathrm{m}^{3} / \mathrm{kg}$

$0.000203 \quad 0.000202$

$0.000205 \quad 0.000205$

$0.000210 \quad 0.000211$

$0.000215 \quad 0.000214$

$0.000221 \quad 0.000220$

$0.000220 \quad 0.000220$

$0.000219 \quad 0.000221$

$0.000222 \quad 0.000221$

$0.000222 \quad 0.000221$

$0.000223 \quad 0.000222$

$0.000224 \quad 0.000223$

$0.000230 \quad 0.000230$

$0.000234 \quad 0.000234$

$0.000237 \quad 0.000236$

$0.000237 \quad 0.000238$

$0.000240 \quad 0.000242$

$0.000246 \quad 0.000245$

$0.000246 \quad 0.000245$

$0.000240 \quad 0.000245$

$\begin{array}{ll}0.000247 & 0.000246\end{array}$

$0.000254 \quad 0.000253$

$0.000260 \quad 0.000259$

$0.000260 \quad 0.000259$

$0.000261 \quad 0.000260$

$0.000265 \quad 0.000265$

$0.000269 \quad 0.000268$

$0.000270 \quad 0.000269$

$0.000273 \quad 0.000273$

$0.000276 \quad 0.000275$

$0.000277 \quad 0.000276$

$0.000279 \quad 0.000278$

$0.000281 \quad 0.000280$

$0.000282 \quad 0.000281$

$0.000283 \quad 0.000282$

$0.000286 \quad 0.000284$

0.0000890 .000063

$0.000090 \quad 0.000066$

$0.000091 \quad 0.000068$

$0.000092 \quad 0.000072$

$0.000096 \quad 0.000078$

$\begin{array}{lll}0.000097 & 0.000083\end{array}$
Density Density App Vol Square of Inconsistent Inconsistent Delta density Density of calc / Residual / Residual / Density based on based on $/ \mathrm{kg} / \mathrm{m}^{3}$ water $/ \mathrm{kg} / \mathrm{m}^{3}$ $\mathrm{kg} / \mathrm{m}^{3} \quad \mathrm{~kg} / \mathrm{m}^{3} \quad \mathrm{~m}^{3} / \mathrm{kg} \quad$ Res density apparent

$\begin{array}{llll}1150.6 & -0.1798 & 0.000001 & 0.032\end{array}$

$\begin{array}{llll}1155.3 & -0.1656 & 0.000001 & 0.027\end{array}$

$1168.3 \quad 0.1995-0.000001 \quad 0.040$

$\begin{array}{llll}1175.2 & -0.1918 & 0.000001 & 0.037\end{array}$

$\begin{array}{llll}1190.7 & -0.2462 & 0.000001 & 0.061\end{array}$

$\begin{array}{llll}1190.7 & 0.0057 & 0.000000 & 0.000\end{array}$

$\begin{array}{llll}1191.9 & 0.5208 & -0.000002 & 0.271\end{array}$

$\begin{array}{llll}1193.6 & -0.3149 & 0.000001 & 0.099\end{array}$

$\begin{array}{llll}1193.9 & -0.2996 & 0.000001 & 0.090\end{array}$

$\begin{array}{llll}1195.6 & -0.2938 & 0.000001 & 0.086\end{array}$

$\begin{array}{llll}1199.1 & -0.2831 & 0.000001 & 0.080\end{array}$

$\begin{array}{llll}1218.8 & 0.0127 & 0.000000 & 0.000\end{array}$

$\begin{array}{llll}1233.3 & 0.2433 & -0.000001 & 0.059\end{array}$

$\begin{array}{llll}1237.7 & -0.3886 & 0.000001 & 0.151\end{array}$

$\begin{array}{llll}1247.3 & 0.7128 & -0.000002 & 0.508\end{array}$

$\begin{array}{llll}1262.5 & 0.7900 & -0.000002 & 0.624\end{array}$

$\begin{array}{llll}1273.5 & -0.4244 & 0.000001 & 0.180\end{array}$

$\begin{array}{llll}1274.3 & -0.4529 & 0.000001 & 0.205\end{array}$

$\begin{array}{llll}1275.8 & 2.7408 & -0.000006 & 7.512\end{array}$

$\begin{array}{llll}1279.4 & -0.4934 & 0.000001 & 0.243\end{array}$

$\begin{array}{llll}1314.1 & -0.4493 & 0.000001 & 0.202\end{array}$

$\begin{array}{llll}1345.2 & -0.4884 & 0.000001 & 0.239\end{array}$

$\begin{array}{llll}1347.6 & -0.5254 & 0.000001 & 0.276\end{array}$

$\begin{array}{llll}1352.4 & -0.4988 & 0.000001 & 0.249\end{array}$

$\begin{array}{llll}1384.1 & -0.5903 & 0.000001 & 0.348\end{array}$

$\begin{array}{llll}1411.7 & -0.6409 & 0.000001 & 0.411\end{array}$

$\begin{array}{llll}1417.6 & -0.6918 & 0.000001 & 0.479\end{array}$

$\begin{array}{llll}1449.1 & -0.8327 & 0.000001 & 0.693\end{array}$

$\begin{array}{llll}1473.9 & -1.0017 & 0.000001 & 1.003\end{array}$

$\begin{array}{llll}1479.9 & -1.0211 & 0.000001 & 1.043\end{array}$

$\begin{array}{llll}1509.6 & -1.2426 & 0.000001 & 1.544\end{array}$

$\begin{array}{llll}1532.4 & -1.4829 & 0.000001 & 2.199\end{array}$

$\begin{array}{llll}1543.7 & -1.5769 & 0.000001 & 2.487\end{array}$

$\begin{array}{llll}1555.6 & -1.7621 & 0.000001 & 3.105\end{array}$

$\begin{array}{llll}1588.4 & -2.7913 & 0.000002 & 7.791\end{array}$

$\begin{array}{llll}995.3 & -0.0351 & 0.000026 & 0.001\end{array}$

$\begin{array}{llll}996.6 & -0.0656 & 0.000024 & 0.004\end{array}$

$\begin{array}{llll}997.8 & -0.0916 & 0.000023 & 0.008\end{array}$

$\begin{array}{llll}999.8 & -0.1293 & 0.000021 & 0.017\end{array}$

$\begin{array}{llll}1003.4 & -0.1748 & 0.000017 & 0.031\end{array}$

$\begin{array}{llll}1005.8 & -0.1846 & 0.000014 & 0.034\end{array}$
997.0449

997.0449

997.0449

997.0449

997.0449

997.0449

195.42997 .0449

997.0449

997.0449

997.0449

997.0449

997.0449

997.0449

997.0449

997.0449

$266.25 \quad 997.0449$

997.0449

997.0449

997.0449

997.0449

997.0449

997.0449

997.0449

997.0449

997.0449

997.0449

997.0449

997.0449

997.0449

997.0449

997.0449

997.0449

997.0449

997.0449

997.0449

$1.25 \quad 994.0319$

$2.49 \quad 994.0319$

$3.68 \quad 994.0319$

$5.65 \quad 994.0319$

$9.15 \quad 994.0319$

$11.57 \quad 994.0319$ 


molality


0.2009
0.2322
0.2348
0.4654
0.5014
0.6969
0.7439
0.9333
0.9842
1.1759
1.4209
1.6722
1.9317
1.9458
2.2667
2.4457
2.6199
2.814
2.9867

0.01012

0.02018

0.02986

0.04595

0.07469

0.09463

0.2009

0.2322

0.2348

0.4654

0.5014

0.6969

0.7439 $\mathrm{t} /{ }^{\circ} \mathrm{C}$ mass frac Density

$\exp /$

$\mathrm{kg} / \mathrm{m}^{3}$

35

0.0267

0.0307

$\begin{array}{lll}0.0310 & 1021.87 \quad(37)\end{array}$

$0.0640 \quad 1051.92 \quad(100)$

$0.0867 \quad 1071.82 \quad(37)$

$0.0921 \quad 1076.84 \quad(100)$

$0.1129 \quad 1095.31 \quad$ (37)

$0.1183 \quad 1100.26 \quad(100)$

$0.1381 \quad 1118.24 \quad(37)$

$0.1622 \quad 1140.36$

$0.1856 \quad 1162.25$

$0.2084 \quad 1184.19 \quad(37)$

$0.2096 \quad 1185.86 \quad(100)$

$0.2360 \quad 1211.95 \quad$ (37)

$0.2500 \quad 1226.69 \quad(37)$

$0.2631 \quad 1241.07 \quad(37)$

$\begin{array}{lll}0.2772 & 1255.89 \quad(100)\end{array}$

$0.2893 \quad 1271.84 \quad(37)$

$\begin{array}{lll}0.0200 & 1009.99 & (90)\end{array}$

$0.0400 \quad 1027.40 \quad(90)$

$0.0800 \quad 1062.40 \quad(90)$

$0.1200 \quad 1098.00 \quad(90)$

$0.1600 \quad 1135.00 \quad(90)$

$\begin{array}{lll}0.2000 & 1173.60 \quad(90)\end{array}$

$0.3000 \quad 1277.80 \quad(90)$

$\begin{array}{lll}0.4000 & 1400.30 \quad(90)\end{array}$

$0.5000 \quad 1549.50 \quad(90)$

$0.0014 \quad 991.46 \quad(100)$

$0.0027 \quad 992.70 \quad(100)$

$0.0041 \quad 993.88 \quad(100)$

$0.0062 \quad 995.84 \quad(100)$

$0.0101 \quad 999.30 \quad(100)$

$0.0127 \quad 1001.67 \quad(100)$

$\begin{array}{lll}0.0267 & 1014.06 \quad(100)\end{array}$

$\begin{array}{llll}0.0307 & 1017.53 \quad(100)\end{array}$

$0.0310 \quad 1017.52$

$0.0597 \quad 1042.68$

$0.0640 \quad 1046.78 \quad(100)$

$0.0867 \quad 1066.52 \quad(37)$

$0.0921 \quad 1071.08 \quad(100)$
App vol App vol
$\exp / \quad$ Calc /

$\mathrm{m}^{3} / \mathrm{kg} \quad \mathrm{m}^{3} / \mathrm{kg}$

$0.000110 \quad 0.000103$

$0.000114 \quad 0.000109$

0.0001220 .000109

$0.000145 \quad 0.000142$

0.0001410 .000146

$0.000164 \quad 0.000166$

$0.000166 \quad 0.000170$

$0.000182 \quad 0.000184$

0.0001850 .000187

$0.000197 \quad 0.000199$

$0.000210 \quad 0.000210$

$0.000222 \quad 0.000220$

$0.000231 \quad 0.000229$

$0.000230 \quad 0.000229$

$0.000240 \quad 0.000238$

$0.000243 \quad 0.000242$

$0.000245 \quad 0.000245$

$0.000249 \quad 0.000249$

$0.000246 \quad 0.000252$

$0.000121 \quad 0.000105$

$0.000145 \quad 0.000130$

$0.000176 \quad 0.000168$

$0.000199 \quad 0.000195$

$0.000215 \quad 0.000215$

$0.000229 \quad 0.000230$

$0.000257 \quad 0.000257$

$0.000274 \quad 0.000274$

0.0002830 .000286

$0.000086 \quad 0.000088$

$0.000086 \quad 0.000090$

$0.000090 \quad 0.000092$

$0.000093 \quad 0.000096$

$0.000098 \quad 0.000102$

$0.000103 \quad 0.000106$

$0.000119 \quad 0.000124$

$0.000126 \quad 0.000129$

$0.000136 \quad 0.000129$

$0.000158 \quad 0.000159$

$0.000157 \quad 0.000163$

$\begin{array}{ll}0.000177 & 0.000180\end{array}$

$\begin{array}{lll}0.000182 & 0.000184\end{array}$
Density Density App Vol Square of Inconsistent Inconsistent Delta density Density of calc / Residual / Residual / Density based on based on $/ \mathrm{kg} / \mathrm{m}^{3}$ water $/ \mathrm{kg} / \mathrm{m}^{3}$ $\mathrm{kg} / \mathrm{m}^{3} \quad \mathrm{~kg} / \mathrm{m}^{3} \quad \mathrm{~m}^{3} / \mathrm{kg} \quad$ Res density apparent

$\begin{array}{llll}1018.4 & -0.1955 & 0.000007 & 0.038\end{array}$

$\begin{array}{llll}1022.0 & -0.1842 & 0.000006 & 0.034\end{array}$

$\begin{array}{llll}1022.3 & -0.4240 & 0.000013 & 0.180\end{array}$

$\begin{array}{llll}1047.7 & -0.2030 & 0.000003 & 0.041\end{array}$

$\begin{array}{llll}1051.5 & 0.3811 & -0.000005 & 0.145\end{array}$

$1071.7 \quad 0.1159-0.000001 \quad 0.013$

$\begin{array}{llll}1076.4 & 0.4177 & -0.000004 & 0.174\end{array}$

$\begin{array}{llll}1095.0 & 0.2905 & -0.000002 & 0.084\end{array}$

$\begin{array}{llll}1099.9 & 0.3506 & -0.000002 & 0.123\end{array}$

$\begin{array}{llll}1118.0 & 0.2597 & -0.000002 & 0.067\end{array}$

$\begin{array}{llll}1140.4 & 0.0098 & 0.000000 & 0.000\end{array}$

$\begin{array}{llll}1162.6 & -0.3154 & 0.000001 & 0.100\end{array}$

$\begin{array}{llll}1184.8 & -0.6335 & 0.000002 & 0.401\end{array}$

$\begin{array}{llll}1186.0 & -0.1527 & 0.000001 & 0.023\end{array}$

$\begin{array}{llll}1212.6 & -0.6951 & 0.000002 & 0.483\end{array}$

$\begin{array}{llll}1227.1 & -0.4354 & 0.000001 & 0.190\end{array}$

$\begin{array}{llll}1241.0 & 0.0933 & 0.000000 & 0.009\end{array}$

$\begin{array}{llll}1256.1 & -0.2518 & 0.000001 & 0.063\end{array}$

$\begin{array}{llll}1269.4 & 2.4277 & -0.000005 & 5.894\end{array}$

$\begin{array}{llll}1010.3 & -0.3313 & 0.000016 & 0.110\end{array}$

$\begin{array}{llll}1028.0 & -0.6312 & 0.000015 & 0.398\end{array}$

$\begin{array}{llll}1063.1 & -0.7096 & 0.000008 & 0.504\end{array}$

$\begin{array}{llll}1098.6 & -0.6021 & 0.000004 & 0.362\end{array}$

$\begin{array}{llll}1135.2 & -0.1784 & 0.000001 & 0.032\end{array}$

$\begin{array}{lllll}1173.3 & 0.2950 & -0.000001 & 0.087\end{array}$

$\begin{array}{llll}1277.8 & -0.0024 & 0.000000 & 0.000\end{array}$

$\begin{array}{llll}1399.8 & 0.5411 & -0.000001 & 0.293\end{array}$

$1545.5 \quad 4.0161-0.000003 \quad 16.129$

$\begin{array}{llll}991.5 & 0.0032 & -0.000002 & 0.000\end{array}$

$\begin{array}{llll}992.7 & 0.0101 & -0.000004 & 0.000\end{array}$

$\begin{array}{llll}993.9 & 0.0095 & -0.000002 & 0.000\end{array}$

$\begin{array}{llll}995.8 & 0.0194 & -0.000003 & 0.000\end{array}$

$\begin{array}{llll}999.3 & 0.0329 & -0.000003 & 0.001\end{array}$

$\begin{array}{llll}1001.6 & 0.0380 & -0.000003 & 0.001\end{array}$

$\begin{array}{llll}1013.9 & 0.1485 & -0.000005 & 0.022\end{array}$

$\begin{array}{lllll}1017.4 & 0.0949 & -0.000003 & 0.009\end{array}$

$\begin{array}{llll}1017.7 & -0.2093 & 0.000007 & 0.044\end{array}$

$\begin{array}{llll}1042.6 & 0.0499 & -0.000001 & 0.002\end{array}$

$1046.4 \quad 0.4051-0.000006 \quad 0.164$

$\begin{array}{llll}1066.2 & 0.3138 & -0.000003 & 0.098\end{array}$

$\begin{array}{llll}1070.9 & 0.2281 & -0.000002 & 0.052\end{array}$
$24.16 \quad 994.0319$

$27.78 \quad 994.0319$

994.0319

994.0319

$57.89 \quad 994.0319$ 994.0319

82.81994 .0319 994.0319

$106.23 \quad 994.0319$ 994.0319

994.0319 994.0319 994.0319

$191.83 \quad 994.0319$ 994.0319 994.0319 994.0319

$261.86 \quad 994.0319$ 994.0319 992.2158 992.2158 992.2158 992.2158 992.2158 992.2158 992.2158 992.2158 992.2158

$1.25 \quad 990.2132$

$2.49 \quad 990.2132$

$3.67 \quad 990.2132$

$5.63 \quad 990.2132$

$9.09 \quad 990.2132$

$11.46 \quad 990.2132$

23.85990 .2132

$27.32 \quad 990.2132$ 990.2132 990.2132

$56.57 \quad 990.2132$ 990.2132

$80.87 \quad 990.2132$ 


molality



0.9333
0.9842
1.1759
1.4209
1.6722
1.9317
1.9458
2.2667
2.4457
2.6199
2.814
2.9867
0.01012
0.02018
0.02986
0.04595
0.07469
0.09463
0.2009
0.2322
0.2348
0.4654
0.5014
0.6969
0.7439
0.9333
0.9842
1.1759
1.4209
1.6722
1.9317
1.9458
2.2667
2.4457
2.6199
2.814
2.9867

$\begin{array}{crrc}\mathbf{t} /{ }^{\circ} \mathrm{C} \text { mass frac } & \begin{array}{r}\text { Density } \\ \text { exp } /\end{array} & \text { Ref } \\ & & \mathrm{kg} / \mathrm{m}^{3} & \\ & & \\ 45 & 0.1129 & 1089.59 & (37) \\ 45 & 0.1183 & 1093.93 & (100) \\ 45 & 0.1381 & 1112.11 & (37) \\ 45 & 0.1622 & 1133.89 & (37) \\ 45 & 0.1856 & 1155.45 & (37) \\ 45 & 0.2084 & 1177.12 & (37) \\ 45 & 0.2096 & 1178.07 & (100) \\ 45 & 0.2360 & 1204.61 & (37) \\ 45 & 0.2500 & 1219.25 & (37) \\ 45 & 0.2631 & 1233.56 & (37) \\ 45 & 0.2772 & 1247.13 & (100) \\ 45 & 0.2893 & 1264.27 & (37) \\ 55 & 0.0014 & 986.94 & (100) \\ 55 & 0.0027 & 988.17 & (100) \\ 55 & 0.0041 & 989.34 & (100) \\ 55 & 0.0062 & 991.28 & (100) \\ 55 & 0.0101 & 994.69 & (100) \\ 55 & 0.0127 & 997.06 & (100) \\ 55 & 0.0267 & 1009.25 & (100) \\ 55 & 0.0307 & 1012.67 & (100) \\ 55 & 0.0310 & 1012.49 & (37) \\ 55 & 0.0597 & 1037.17 & (37) \\ 55 & 0.0640 & 1041.82 & (100) \\ 55 & 0.0867 & 1060.59 & (37) \\ 55 & 0.0921 & 1065.01 & (100) \\ 55 & 0.1129 & 1083.27 & (37) \\ 55 & 0.1183 & 1087.44 & (100) \\ 55 & 0.1381 & 1105.45 & (37) \\ 55 & 0.1622 & 1126.93 & (37) \\ 55 & 0.1856 & 1148.22 & (37) \\ 55 & 0.2084 & 1169.66 & (37) \\ 55 & 0.2096 & 1170.43 & (100) \\ 55 & 0.2360 & 1196.92 & (37) \\ 55 & 0.2500 & 1211.46 & (37) \\ 55 & 0.2631 & 1225.69 & (37) \\ 55 & 0.2772 & 1238.94 & (100) \\ 55 & 0.2893 & 1256.27 & (37) \\ 60 & 0.0200 & 1000.30 & (90) \\ 60 & 0.0400 & 1017.20 & (90) \\ 60 & 0.0800 & 1050.80 & (90) \\ 60 & 0.1200 & 1085.30 & (90) \\ & & & \end{array}$

$\begin{array}{rr}\text { App vol } & \text { App vol } \\ \exp / & \text { Calc / }\end{array}$

$\mathrm{m}^{3} / \mathrm{kg} \quad \mathrm{m}^{3} / \mathrm{kg}$

$0.000194 \quad 0.000197$

$0.000200 \quad 0.000200$

$0.000209 \quad 0.000210$

$0.000221 \quad 0.000221$

$\begin{array}{lll}0.000232 & 0.000229\end{array}$

$\begin{array}{lll}0.000240 & 0.000237\end{array}$

$\begin{array}{lll}0.000242 & 0.000237\end{array}$

$\begin{array}{lll}0.000248 & 0.000245\end{array}$

$\begin{array}{lll}0.000251 & 0.000249\end{array}$

$0.000253 \quad 0.000252$

$0.000259 \quad 0.000255$

$0.000253 \quad 0.000258$

$0.000089 \quad 0.000112$

$0.000090 \quad 0.000114$

$0.000094 \quad 0.000116$

$0.000097 \quad 0.000119$

$0.000105 \quad 0.000124$

$\begin{array}{lll}0.000107 & 0.000128\end{array}$

$\begin{array}{lll}0.000126 & 0.000144\end{array}$

$0.000134 \quad 0.000149$

$0.000149 \quad 0.000149$

$0.000170 \quad 0.000175$

$0.000160 \quad 0.000179$

0.0001890 .000194

$0.000194 \quad 0.000198$

$0.000205 \quad 0.000209$

$\begin{array}{lll}0.000212 & 0.000212\end{array}$

$\begin{array}{lll}0.000219 & 0.000221\end{array}$

$0.000231 \quad 0.000230$

$\begin{array}{lll}0.000241 & 0.000238\end{array}$

$0.000249 \quad 0.000245$

$0.000251 \quad 0.000245$

$0.000256 \quad 0.000252$

$0.000258 \quad 0.000255$

$0.000260 \quad 0.000258$

$\begin{array}{lll}0.000266 & 0.000261\end{array}$

$0.000259 \quad 0.000263$

$\begin{array}{lll}0.000148 & 0.000147\end{array}$

$\begin{array}{lll}0.000167 & 0.000167\end{array}$

$\begin{array}{lll}0.000199 & 0.000197\end{array}$

$0.000220 \quad 0.000218$
Density Density App Vol Square of Inconsistent Inconsistent Delta density Density of calc/ Residual / Residual / Density based on based on $/ \mathrm{kg} / \mathrm{m}^{3}$ water $/ \mathrm{kg} / \mathrm{m}^{3}$ $\mathrm{kg} / \mathrm{m}^{3} \quad \mathrm{~kg} / \mathrm{m}^{3} \quad \mathrm{~m}^{3} / \mathrm{kg} \quad$ Res density $\begin{gathered}\text { apparent } \\ \text { volume }\end{gathered}$

$\begin{array}{llll}1089.2 & 0.3964 & -0.000003 & 0.157\end{array}$

$\begin{array}{llll}1094.0 & -0.0900 & 0.000001 & 0.008\end{array}$

$\begin{array}{llll}1111.9 & 0.2295 & -0.000001 & 0.053\end{array}$

$\begin{array}{llll}1134.0 & -0.1310 & 0.000001 & 0.017\end{array}$

$\begin{array}{llll}1156.0 & -0.5876 & 0.000002 & 0.345\end{array}$

$\begin{array}{llll}1178.1 & -1.0010 & 0.000003 & 1.002\end{array}$

$\begin{array}{llll}1179.3 & -1.2300 & 0.000004 & 1.513\end{array}$

$\begin{array}{llll}1205.8 & -1.1424 & 0.000003 & 1.305\end{array}$

$\begin{array}{llll}1220.1 & -0.8943 & 0.000002 & 0.800\end{array}$

$\begin{array}{llll}1233.9 & -0.3571 & 0.000001 & 0.128\end{array}$

$\begin{array}{llll}1249.0 & -1.8711 & 0.000004 & 3.501\end{array}$

$\begin{array}{llll}1262.2 & 2.0620 & -0.000004 & 4.252\end{array}$

$\begin{array}{llll}986.9 & 0.0308 & -0.000023 & 0.001\end{array}$

$\begin{array}{llll}988.1 & 0.0645 & -0.000024 & 0.004\end{array}$

$\begin{array}{llll}989.2 & 0.0887 & -0.000022 & 0.008\end{array}$

$\begin{array}{llll}991.1 & 0.1353 & -0.000022 & 0.018\end{array}$

$\begin{array}{llll}994.5 & 0.1963 & -0.000020 & 0.039\end{array}$

$\begin{array}{llll}996.8 & 0.2664 & -0.000021 & 0.071\end{array}$

$\begin{array}{llll}1008.8 & 0.4917 & -0.000018 & 0.242\end{array}$

$\begin{array}{llll}1012.2 & 0.4719 & -0.000015 & 0.223\end{array}$

$\begin{array}{llll}1012.5 & 0.0125 & 0.000000 & 0.000\end{array}$

$\begin{array}{llll}1036.9 & 0.3112 & -0.000005 & 0.097\end{array}$

$1040.5 \quad 1.2773-0.000018 \quad 1.631$

$\begin{array}{llll}1060.0 & 0.5541 & -0.000006 & 0.307\end{array}$

$\begin{array}{llll}1064.6 & 0.3904 & -0.000004 & 0.152\end{array}$

$\begin{array}{llll}1082.7 & 0.5700 & -0.000004 & 0.325\end{array}$

$\begin{array}{llll}1087.5 & -0.0333 & 0.000000 & 0.001\end{array}$

$\begin{array}{llll}1105.1 & 0.3332 & -0.000002 & 0.111\end{array}$

$\begin{array}{llll}1127.0 & -0.1006 & 0.000000 & 0.010\end{array}$

$\begin{array}{llll}1148.9 & -0.6312 & 0.000003 & 0.398\end{array}$

$\begin{array}{llll}1170.8 & -1.1020 & 0.000004 & 1.214\end{array}$

$\begin{array}{llll}1171.9 & -1.5104 & 0.000005 & 2.281\end{array}$

$\begin{array}{llll}1198.2 & -1.2856 & 0.000004 & 1.653\end{array}$

$\begin{array}{llll}1212.5 & -1.0504 & 0.000003 & 1.103\end{array}$

$\begin{array}{llll}1226.2 & -0.5159 & 0.000001 & 0.266\end{array}$

$\begin{array}{llll}1241.2 & -2.2793 & 0.000005 & 5.195\end{array}$

$\begin{array}{lllll}1254.4 & 1.9155 & -0.000004 & 3.669\end{array}$

$\begin{array}{llll}1000.3 & -0.0093 & 0.000000 & 0.000\end{array}$

$\begin{array}{llll}1017.2 & 0.0048 & 0.000000 & 0.000\end{array}$

$\begin{array}{llll}1051.0 & -0.1751 & 0.000002 & 0.031\end{array}$

$\begin{array}{llll}1085.5 & -0.1760 & 0.000001 & 0.031\end{array}$
990.2132

103.72990 .2132

990.2132

990.2132

990.2132

990.2132

$187.86 \quad 990.2132$

990.2132

990.2132

990.2132

$256.92 \quad 990.2132$ 990.2132

$1.24 \quad 985.6952$

$2.47 \quad 985.6952$

3.64985 .6952

$5.58 \quad 985.6952$

8.99985 .6952

$11.36 \quad 985.6952$

$23.55 \quad 985.6952$

$26.97 \quad 985.6952$

985.6952

985.6952

$56.12 \quad 985.6952$ 985.6952

$79.31 \quad 985.6952$

985.6952

101.74985 .6952 985.6952 985.6952 985.6952 985.6952

184.73985 .6952 985.6952 985.6952 985.6952

$253.24 \quad 985.6952$ 985.6952 983.1989 983.1989 983.1989 983.1989 


$\begin{array}{rrrr}\mathbf{t} /{ }^{\circ} \mathrm{C} \text { mass frac } & \text { Density } & \text { Ref } \\ & & \exp / & \\ & & \mathrm{kg} / \mathrm{m}^{3} & \\ 60 & 0.1600 & 1121.20 & (90) \\ 60 & 0.2000 & 1159.00 & (90) \\ 60 & 0.3000 & 1261.40 & (90) \\ 60 & 0.4000 & 1382.40 & (90) \\ 60 & 0.5000 & 1530.00 & (90) \\ 65 & 0.0310 & 1006.79 & (37) \\ 65 & 0.0597 & 1031.03 & (37) \\ 65 & 0.0867 & 1054.06 & (37) \\ 65 & 0.1129 & 1076.41 & (37) \\ 65 & 0.1381 & 1098.31 & (37) \\ 65 & 0.1622 & 1119.56 & (37) \\ 65 & 0.1856 & 1140.65 & (37) \\ 65 & 0.2084 & 1161.91 & (37) \\ 65 & 0.2360 & 1188.96 & (37) \\ 65 & 0.2500 & 1203.39 & (37) \\ 65 & 0.2631 & 1217.51 & (37) \\ 65 & 0.2893 & 1247.83 & (37) \\ 75 & 0.0310 & 1000.34 & (37) \\ 75 & 0.0597 & 1024.08 & (37) \\ 75 & 0.0867 & 1046.77 & (37) \\ 75 & 0.1129 & 1068.89 & (37) \\ 75 & 0.1381 & 1090.64 & (37) \\ 75 & 0.1622 & 1111.79 & (37) \\ 75 & 0.1856 & 1132.79 & (37) \\ 75 & 0.2084 & 1153.92 & (37) \\ 75 & 0.2360 & 1180.67 & (37) \\ 75 & 0.2500 & 1194.84 & (37) \\ 75 & 0.2631 & 1208.64 & (37) \\ 75 & 0.2893 & 1237.93 & (37) \\ 80 & 0.0200 & 988.20 & (90) \\ 80 & 0.0400 & 1004.40 & (90) \\ 80 & 0.0800 & 1036.90 & (90) \\ 80 & 0.1200 & 1070.40 & (90) \\ 80 & 0.1600 & 1105.50 & (90) \\ 80 & 0.2000 & 1142.80 & (90) \\ 80 & 0.3000 & 1243.80 & (90) \\ 80 & 0.4000 & 1363.70 & (90) \\ 80 & 0.5000 & 1509.70 & (90) \\ 100 & 0.0200 & 973.90 & (90) \\ 100 & 0.0400 & 989.40 & (90) \\ 100 & 0.0800 & 1021.10 & (90) \\ & & & \end{array}$

$\begin{array}{rr}\text { App vol } & \text { App vol } \\ \exp / & \text { Calc / }\end{array}$ $\mathrm{m}^{3} / \mathrm{kg} \quad \mathrm{m}^{3} / \mathrm{kg}$

$0.000235 \quad 0.000234$ $\begin{array}{lll}0.000246 & 0.000247\end{array}$ $0.000269 \quad 0.000268$ $0.000283 \quad 0.000282$ $\begin{array}{lll}0.000290 & 0.000291\end{array}$ $0.000163 \quad 0.000168$ $0.000183 \quad 0.000191$ $0.000200 \quad 0.000208$ $\begin{array}{lll}0.000215 & 0.000221\end{array}$ $0.000228 \quad 0.000231$ 0.0002390 .000240 $\begin{array}{lll}0.000249 & 0.000247\end{array}$ $0.000256 \quad 0.000253$ $\begin{array}{lll}0.000262 & 0.000259\end{array}$ $0.000264 \quad 0.000262$ $0.000266 \quad 0.000264$ $\begin{array}{lll}0.000265 & 0.000269\end{array}$ $\begin{array}{lll}0.000183 & 0.000187\end{array}$ $0.000199 \quad 0.000207$ $\begin{array}{lll}0.000213 & 0.000221\end{array}$ $\begin{array}{lll}0.000226 & 0.000233\end{array}$ $0.000237 \quad 0.000242$ $\begin{array}{lll}0.000247 & 0.000249\end{array}$ $0.000255 \quad 0.000255$ $0.000262 \quad 0.000260$ $0.000268 \quad 0.000266$ $\begin{array}{lll}0.000270 & 0.000268\end{array}$ $0.000272 \quad 0.000270$ $\begin{array}{lll}0.000272 & 0.000274\end{array}$ $\begin{array}{lll}0.000175 & 0.000187\end{array}$ $0.000194 \quad 0.000202$ $0.000221 \quad 0.000225$ $\begin{array}{lll}0.000239 & 0.000241\end{array}$ $0.000251 \quad 0.000253$ $0.000259 \quad 0.000262$ $\begin{array}{lll}0.000279 & 0.000278\end{array}$ $\begin{array}{lll}0.000290 & 0.000289\end{array}$ $0.000296 \quad 0.000296$ $\begin{array}{lll}0.000211 & 0.000225\end{array}$ $\begin{array}{lll}0.000225 & 0.000235\end{array}$ $0.000242 \quad 0.000251$
Density Density App Vol Square of Inconsistent Inconsistent Delta density Density of calc/ Residual / Residual / Density based on based on $/ \mathrm{kg} / \mathrm{m}^{3}$ water $/ \mathrm{kg} / \mathrm{m}^{3}$ $\mathrm{kg} / \mathrm{m}^{3} \quad \mathrm{~kg} / \mathrm{m}^{3} \quad \mathrm{~m}^{3} / \mathrm{kg} \quad$ Res density $\begin{gathered}\text { apparent } \\ \text { volume }\end{gathered}$

$\begin{array}{llll}1121.3 & -0.0597 & 0.000000 & 0.004\end{array}$

$\begin{array}{llll}1158.7 & 0.2672 & -0.000001 & 0.071\end{array}$

$\begin{array}{llll}1262.0 & -0.6108 & 0.000001 & 0.373\end{array}$

$\begin{array}{llll}1383.2 & -0.7957 & 0.000001 & 0.633\end{array}$

$\begin{array}{llll}1528.6 & 1.4013 & -0.000001 & 1.964\end{array}$

$\begin{array}{llll}1006.6 & 0.1708 & -0.000005 & 0.029\end{array}$

$\begin{array}{llll}1030.5 & 0.5381 & -0.000008 & 0.290\end{array}$

$\begin{array}{llll}1053.3 & 0.7818 & -0.000008 & 0.611\end{array}$

$\begin{array}{llll}1075.6 & 0.7849 & -0.000006 & 0.616\end{array}$

$\begin{array}{llll}1097.8 & 0.5334 & -0.000003 & 0.284\end{array}$

$\begin{array}{llll}1119.5 & 0.0923 & 0.000000 & 0.009\end{array}$

$\begin{array}{llll}1141.1 & -0.4454 & 0.000002 & 0.198\end{array}$

$\begin{array}{llll}1162.8 & -0.9264 & 0.000003 & 0.858\end{array}$

$\begin{array}{llll}1190.1 & -1.1354 & 0.000003 & 1.289\end{array}$

$\begin{array}{llll}1204.3 & -0.9246 & 0.000003 & 0.855\end{array}$

$\begin{array}{llll}1217.9 & -0.4241 & 0.000001 & 0.180\end{array}$

$1245.9 \quad 1.8867-0.000004 \quad 3.560$

$1000.2 \quad 0.1260-0.000004 \quad 0.016$

$\begin{array}{llll}1023.6 & 0.4892 & -0.000008 & 0.239\end{array}$

$\begin{array}{llll}1046.0 & 0.7744 & -0.000008 & 0.600\end{array}$

$\begin{array}{llll}1068.0 & 0.8575 & -0.000007 & 0.735\end{array}$

$\begin{array}{llll}1089.9 & 0.7157 & -0.000004 & 0.512\end{array}$

$\begin{array}{llll}1111.4 & 0.3929 & -0.000002 & 0.154\end{array}$

$\begin{array}{llll}1132.8 & -0.0457 & 0.000000 & 0.002\end{array}$

$\begin{array}{llll}1154.4 & -0.4901 & 0.000002 & 0.240\end{array}$

$\begin{array}{llll}1181.5 & -0.8179 & 0.000002 & 0.669\end{array}$

$\begin{array}{llll}1195.6 & -0.7831 & 0.000002 & 0.613\end{array}$

$\begin{array}{llll}1209.2 & -0.5281 & 0.000001 & 0.279\end{array}$

$\begin{array}{llll}1237.0 & 0.8893 & -0.000002 & 0.791\end{array}$

$\begin{array}{lllll}988.0 & 0.2404 & -0.000012 & 0.058\end{array}$

$\begin{array}{llll}1004.1 & 0.3404 & -0.000008 & 0.116\end{array}$

$\begin{array}{llll}1036.6 & 0.3014 & -0.000004 & 0.091\end{array}$

$\begin{array}{llll}1070.1 & 0.2515 & -0.000002 & 0.063\end{array}$

$1105.2 \quad 0.3309-0.000002 \quad 0.110$

$\begin{array}{llll}1142.0 & 0.7888 & -0.000003 & 0.622\end{array}$

$\begin{array}{llll}1244.1 & -0.3150 & 0.000001 & 0.099\end{array}$

$\begin{array}{llll}1364.6 & -0.8829 & 0.000001 & 0.779\end{array}$

$\begin{array}{llll}1509.8 & -0.0613 & 0.000000 & 0.004\end{array}$

$\begin{array}{llll}973.6 & 0.2655 & -0.000014 & 0.070\end{array}$

$\begin{array}{llll}989.0 & 0.4047 & -0.000010 & 0.164\end{array}$

$\begin{array}{llll}1020.4 & 0.7371 & -0.000009 & 0.543\end{array}$
983.1989

983.1989 983.1989 983.1989 983.1989 980.5548 980.5548 980.5548 980.5548 980.5548 980.5548 980.5548 980.5548 980.5548 980.5548 980.5548 980.5548 974.849 974.849

974.849 974.849 974.849

974.849 974.849 974.849

974.849 974.849 974.849 974.849 971.7978 971.7978 971.7978 971.7978 971.7978 971.7978 971.7978 971.7978 971.7978 958.3637 958.3637 958.3637 
molality molarity

$\begin{array}{cccc}\mathbf{t} /{ }^{\circ} \mathrm{C} \text { mass frac } & \begin{array}{r}\text { Density } \\ \exp / \\ \mathrm{kg} / \mathrm{m}^{3}\end{array} & \text { Ref } \\ & & & \\ 100 & 0.1200 & 1054.10 & (90) \\ 100 & 0.1600 & 1088.80 & (90) \\ 100 & 0.2000 & 1125.50 & (90) \\ 100 & 0.3000 & 1225.20 & (90) \\ 100 & 0.4000 & 1344.10 & (90) \\ 100 & 0.5000 & 1489.20 & (90)\end{array}$

App vol App vol exp / Calc /$$
\mathrm{m}^{3} / \mathrm{k}
$$

$\begin{array}{ll}0.000254 & 0.000262\end{array}$

0.0002620 .000270

0.0002690 .000276

$\begin{array}{ll}0.000286 & 0.000287\end{array}$

0.0002950 .000295

$0.000300 \quad 0.000299$
Dens calc Density App Vol $\mathrm{kg} / \mathrm{m}^{3}$

Average Res

Std dev Res

Avg - 4std

Avg +4 std

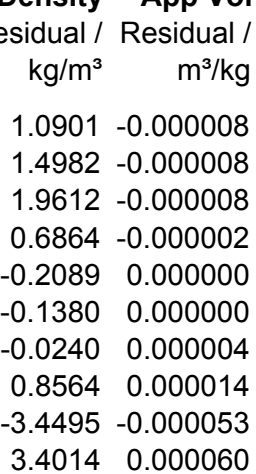

Square of Inconsistent Inconsistent Delta density quare of Inconsistent Inconsistent Delta density $\begin{gathered}\text { Density of } \\ \text { Density based on based on }\end{gathered} / \mathrm{kg} / \mathrm{m}^{3}$ water $/ \mathrm{kg} / \mathrm{m}^{3}$ $/ \mathrm{kg} / \mathrm{m}^{3}$ water $/ \mathrm{kg} / \mathrm{m}^{3}$ Res

1.188

2.245

3.846

0.471

0.044

0.019

175.410 Sum Chi sqr
958.3637

958.3637 958.3637 958.3637 958.3637 958.3637

Curve fit for the model App vol= $\left(w+c 2+c 3^{\star} t{ }^{\circ} \mathrm{C}\right) /\left(\mathrm{c} 0^{*} \mathrm{w}+\mathrm{c} 1\right) / \mathrm{e}^{\wedge}\left(0.000001^{*}\left(\mathrm{t}^{\circ} \mathrm{C}+\mathrm{c} 4\right)^{\wedge} 2\right)$

\begin{tabular}{lr} 
c0 & 1943.596 \\
c1 2 & 304.335 \\
c3 & -0.013753 \\
c4 & 0.001154 \\
Min T & 573.7902 \\
Max T & 0 \\
Min w & 100 \\
Max w & 0.0014 \\
Avg dens res & 0.7000 \\
Std dens res & -0.0240 \\
Avg app vol res *10^3 & 0.856359 \\
Std app vol res *10^3 & 0.003574 \\
No of points in corr & 0.014136 \\
No of inconsistent poir & 240 \\
\hline
\end{tabular}

Inconsistent data not used (Residual greater than average + /- 4 standard deviations) $\begin{array}{llllll}0 & 0.2000 & 1197.00 & (90) & 0.000176 & 0.000193\end{array}$

$\begin{array}{llllll}0 & 0.3000 & 1306.20 & (90) & 0.000218 & 0.000232\end{array}$

$\begin{array}{llllll}0 & 0.4000 & 1432.90 & (90) & 0.000244 & 0.000257\end{array}$

0.04595

$\begin{array}{lllll}0.4000 & 1432.90 & (90) & 0.000244 & 0.000257 \\ 0.5000 & 1586.00 & (90) & 0.000261 & 0.000274\end{array}$

$\begin{array}{lllll}0.0062 & 1004.81 & (100) & 0.000087 & 0.000022\end{array}$

$\begin{array}{lllll}0.5000 & 1568.10 & (90) & 0.000274 & 0.000281\end{array}$

$1192.2 \quad 4.8176-0.000017$

$1299.1 \quad 7.0519-0.000014$

$1422.8 \quad 10.1245-0.000012$

$1569.5 \quad 16.5145-0.000013$

$\begin{array}{llll}1005.2 & -0.4098 & 0.000065\end{array}$

$1559.7-8.4381-0.000007$

$11.2883-0.000006$

23.209

49.729

102.506

272.730

0.168

71.202

127.425

999.8395

999.8395

999.8395

999.8395

5.71999 .0996

998.2041

998.2041 
Density of aqueous solutions of ZnSO4

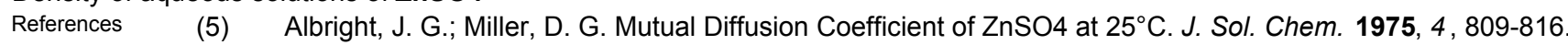

(7) Bakeev, M. I.; Zharmenov, A. A.; Andamasov, R. S.; Baikenova, N. A.; Abdygalimova, S. Sh. Electrical Conductivity and Viscosity of the Binary Systems MeSO4-H2O (Me=Mn2+, Fe2+, Co2+, Zn2+, Cd2+) at 25-75 Degree and the Structure of Electrolyte Solutions. IzV. Nats. Akad. Nauk Resp. Kaz., Ser. Khim. 1994, 6, 25-30.

(44) Isono, T. Measurements of Density, Viscosity, and Electrolytic Conductivity of Concentrated Aqueous Electrolyte Solutions. Rikagaku Kenkyusho Hokoku 1980, 56, 103-114.

(90) Perry, R. H.; Green, D. W. Perry's Chemical Engineers' Handbook; McGraw Hill: New York, 7th edition, 1997.

(101) Puchalska, D.; Atkinson, G.; Routh, S. Solution Thermodynamics of First-Row Transition Elements. 4. Apparent Molal Volumes of Aqueous $\mathrm{ZnSO} 4$ and CuSO4 Solutions from 15 to $55^{\circ} \mathrm{C}$. J. Sol. Chem. 1993, 22, 625-639.

(126) Suryanarayana, C. V.; Alamelu, S. Electrical Conductance of Concentrated Aqueous Solutions of Zinc Sulphate. Acta Chim. Hung. 1959, 20, 91-102.

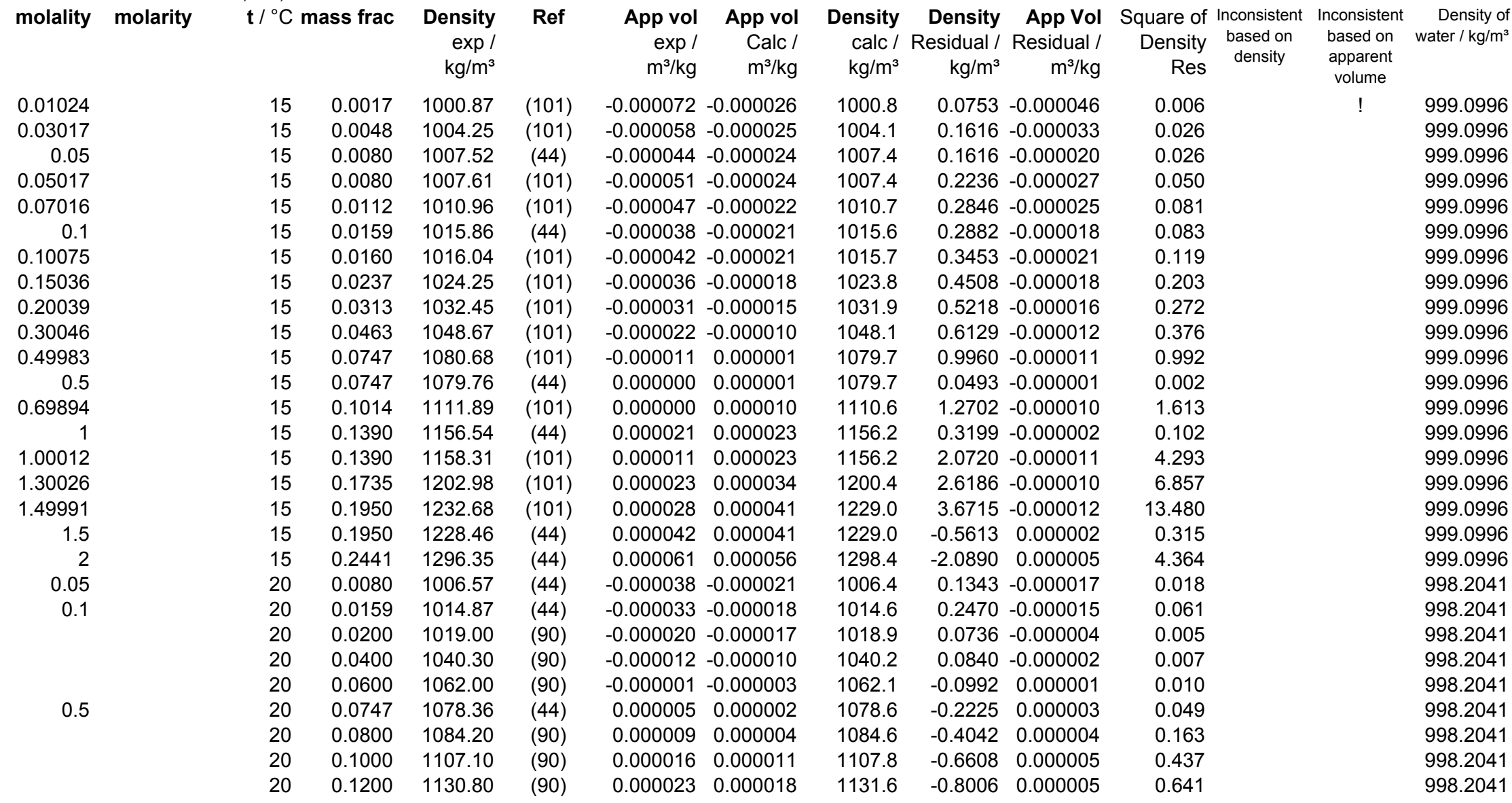


molality molarity

$\mathrm{t} /{ }^{\circ} \mathrm{C}$ mass frac Density

$\exp /$

$\mathrm{kg} / \mathrm{m}^{3}$

$0.1390 \quad 1154.83$

$0.1400 \quad 1155.30$

$0.1600 \quad 1180.60$

$0.1950 \quad 1226.47$

$0.2441 \quad 1294.37$

$0.0017 \quad 998.78$

$0.0048 \quad 1002.12$

$0.0080 \quad 1005.37$

$0.0080 \quad 1005.45$

$0.0112 \quad 1008.75$

$0.0150 \quad 1012.79$

$0.0159 \quad 1013.62$

$0.0160 \quad 1013.77$

$0.0237 \quad 1021.89$

$0.0313 \quad 1030.02$

$0.0463 \quad 1046.07$

$0.0702 \quad 1071.81$

$0.0747 \quad 1077.73$

$0.0747 \quad 1076.81$

$0.1014 \quad 1108.84$

$0.1300 \quad 1141.82$

$0.1390 \quad 1152.96$

$0.1390 \quad 1154.78$

$0.1735 \quad 1198.92$

$0.1824 \quad 1208.66$

$0.1950 \quad 1228.10$

$0.1950 \quad 1224.37$

$0.2283 \quad 1272.74$

$0.2441 \quad 1292.14$

$0.2505 \quad 1305.09$

$0.2693 \quad 1333.45$

$0.2894 \quad 1365.05$

$0.3054 \quad 1391.07$

$0.3219 \quad 1418.29$

$0.3379 \quad 1445.33$

$0.3546 \quad 1474.55$

$0.3639 \quad 1490.93$

$0.3677 \quad 1497.93$

$0.0080 \quad 1003.92$

$0.0159 \quad 1012.14$
Ref

App vol App vol exp / Calc /

$\mathrm{m}^{3} / \mathrm{kg} \quad \mathrm{m}^{3} / \mathrm{kg}$

(44) $\quad 0.000024 \quad 0.000024$

(90) $\quad 0.000029 \quad 0.000024$

$\begin{array}{lll}\text { (90) } \quad 0.000034 & 0.000031\end{array}$

$\begin{array}{lll}\text { (44) } & 0.000046 \quad 0.000041\end{array}$

(44) $0.000063 \quad 0.000056$

(101) $\quad-0.000053-0.000021$

(101) $\quad-0.000045-0.000020$

(44) $\quad-0.000034-0.000019$

(101) $\quad-0.000040-0.000019$

(101) $\quad-0.000036-0.000018$

(5) $\quad-0.000034-0.000016$

(44) $\quad-0.000029-0.000016$

(101) $\quad-0.000031-0.000016$

(101) $\quad-0.000026-0.000013$

(101) $\quad-0.000021-0.000011$

(101) $\quad-0.000013-0.000005$

(5) $\quad 0.000006 \quad 0.000003$

$\begin{array}{lll}(101) & -0.000002 & 0.000004\end{array}$

(44) $\quad 0.000008 \quad 0.000004$

$\begin{array}{lll}(101) & 0.000006 & 0.000013\end{array}$

(5) $\quad 0.000025 \quad 0.000022$

(44) $\quad 0.000027 \quad 0.000025$

$\begin{array}{lll}(101) & 0.000018 & 0.000025\end{array}$

$\begin{array}{lll}\text { (101) } \quad 0.000030 & 0.000036\end{array}$

(5) $\quad 0.000040 \quad 0.000039$

(101) $\quad 0.000035 \quad 0.000042$

(44) $\quad 0.000048 \quad 0.000042$

(5) $0.000051 \quad 0.000052$

(44) $0.000065 \quad 0.000057$

(5) $\quad 0.000058 \quad 0.000059$

(5) $0.000063 \quad 0.000064$

$\begin{array}{lll}\text { (5) } & 0.000069 & 0.000069\end{array}$

(5) $\quad 0.000073 \quad 0.000074$

$\begin{array}{lll}\text { (5) } & 0.000078 & 0.000078\end{array}$

(5) $\quad 0.0000820 .000082$

$\begin{array}{lll}\text { (5) } & 0.000087 & 0.000087\end{array}$

(5) $0.000090 \quad 0.000089$

(5) $\quad 0.000091 \quad 0.000090$

(44) $\quad-0.000029-0.000017$

(44) $\quad-0.000026-0.000014$
Density Density App Vol Square of Inconsistent Inconsistent Density of calc / Residual / Residual / Density based on based on water $/ \mathrm{kg} / \mathrm{m}^{3}$ $\mathrm{kg} / \mathrm{m}^{3} \quad \mathrm{~kg} / \mathrm{m}^{3} \quad \mathrm{~m}^{3} / \mathrm{kg} \quad$ Res density apparent

$1156.2-0.8574-0.000005$

0.012

0.735

0.752

1.417

7.248

0.003

0.014

0.015

0.030

0.043

0.077

0.045

0.058

0.095

0.129

0.141

0.089

0.336

0.134

0.808

0.160

0.167

1.943

2.343

0.184

4.478

2.646

0.111

10.435

0.078

0.033

0.135

0.339

0.170

0.009

0.033

0.412

0.330

0.010

0.036
998.2041

998.2041

998.2041

998.2041

998.2041

997.0449

997.0449

997.0449

997.0449

997.0449

997.0449

997.0449

997.0449

997.0449

997.0449

997.0449

997.0449

997.0449

997.0449

997.0449

997.0449

997.0449

997.0449

997.0449

997.0449

997.0449

997.0449

997.0449

997.0449

997.0449

997.0449

997.0449

997.0449

997.0449

997.0449

997.0449

997.0449

997.0449

995.6473

995.6473 
molality molarity

\section{$\mathrm{t} /{ }^{\circ} \mathrm{C}$ mass frac}

Density

$\exp /$
$\mathrm{kg} / \mathrm{m}^{3}$

0.2266

0.4652

0.5

0.6428

0.8347

1.016

1.19

1.48

1.5

1.77

2.06

2.35

2.64

2.93

3.22

3.51
0.01024

0.01024
0.03017

0.05

0.05017

0.07016

0.1

0.10075

0.15036

0.20039

0.2266

0.30046

0.4652

0.49983

0.5

0.6428

0.69894

0.8347

1.00012

1.016

1.19

1.30026

$\begin{array}{lrc}0.0353 & 1032.00 & (126) \\ 0.0699 & 1069.00 & (126) \\ 0.0747 & 1075.08 & (44) \\ 0.0940 & 1097.00 & (126) \\ 0.1188 & 1127.00 & (126) \\ 0.1390 & 1150.95 & (44) \\ 0.1409 & 1153.00 & (126) \\ 0.1612 & 1180.00 & (126) \\ 0.1929 & 1222.00 & (126) \\ 0.1950 & 1222.20 & (44) \\ 0.2223 & 1261.00 & (126) \\ 0.2441 & 1289.77 & (44) \\ 0.2496 & 1302.00 & (126) \\ 0.2751 & 1341.00 & (126) \\ 0.2989 & 1379.00 & (126) \\ 0.3212 & 1416.00 & (126) \\ 0.3421 & 1452.00 & (126) \\ 0.3617 & 1486.00 & (126) \\ 0.0017 & 995.76 & (101) \\ 0.0048 & 999.05 & (101) \\ 0.0080 & 1002.28 & (44) \\ 0.0080 & 1002.35 & (101) \\ 0.0112 & 1005.66 & (101) \\ 0.0159 & 1010.48 & (44) \\ 0.0160 & 1010.64 & (101) \\ 0.0237 & 1018.68 & (101) \\ 0.0313 & 1026.73 & (101) \\ 0.0353 & 1030.00 & (126) \\ 0.0463 & 1042.62 & (101) \\ 0.0699 & 1068.00 & (126) \\ 0.0747 & 1074.04 & (101) \\ 0.0747 & 1073.18 & (44) \\ 0.0940 & 1095.00 & (126) \\ 0.1014 & 1104.78 & (101) \\ 0.1188 & 1124.00 & (126) \\ 0.1390 & 1148.83 & (44) \\ 0.1390 & 1150.55 & (101) \\ 0.1409 & 1152.00 & (126) \\ 0.1612 & 1178.00 & (126) \\ 0.1735 & 1194.85 & (101) \\ & & \end{array}$

App vol App vol Density Density App Vol Square of Inconsistent Inconsistent Density of $\exp /$ Calc / calc/ Residual / Residual / Density based on based on water $/ \mathrm{kg} / \mathrm{m}^{3}$ $\mathrm{m}^{3} / \mathrm{kg} \quad \mathrm{m}^{3} / \mathrm{kg}$

$0.000002-0.000007$ $0.000018 \quad 0.000004$ 0.0000110 .000006 $0.000018 \quad 0.000012$ $0.000019 \quad 0.000020$ $0.000030 \quad 0.000026$ $\begin{array}{ll}0.000032 & 0.000027\end{array}$ $0.000031 \quad 0.000033$ $0.000040 \quad 0.000043$ $0.000050 \quad 0.000043$ $0.000054 \quad 0.000051$ $0.000066 \quad 0.000057$ $0.000058 \quad 0.000059$ $0.000064 \quad 0.000066$ $0.000070 \quad 0.000072$ $\begin{array}{ll}0.000076 & 0.000078\end{array}$ $0.000082 \quad 0.000083$

$0.000088 \quad 0.000088$ $-0.000052-0.000017$ $-0.000036-0.000016$ $-0.000028-0.000015$ $-0.000033-0.000015$ $-0.000032-0.000013$ $-0.000025-0.000012$ $-0.000027-0.000012$ $-0.000021-0.000009$ $-0.000016-0.000007$ $0.000011-0.000005$ $-0.000007-0.000002$ $0.000009 \quad 0.000006$ $0.000003 \quad 0.000008$ $0.000013 \quad 0.000008$ $0.000020 \quad 0.000014$ $0.000012 \quad 0.000016$ $0.000027 \quad 0.000021$ $\begin{array}{lll}0.000031 & 0.000027\end{array}$ $0.000022 \quad 0.000028$ $0.000027 \quad 0.000028$ $0.000031 \quad 0.000034$ $\begin{array}{lll}0.000032 & 0.000038\end{array}$ $\begin{array}{rrr}\text { calc / } & \text { Residual / } & \text { Residual / } \\ \mathrm{kg} / \mathrm{m}^{3} & \mathrm{~kg} / \mathrm{m}^{3} & \mathrm{~m}^{3} / \mathrm{kg}\end{array}$

$\begin{array}{lll}1032.4 & -0.3510 & 0.000009 \\ 1070.1 & -1.0911 & 0.000014\end{array}$

$\begin{array}{lll}1070.1 & -1.0911 & 0.000014\end{array}$

$\begin{array}{lll}1075.5 & -0.4407 & 0.000005\end{array}$

$\begin{array}{lll}1097.6 & -0.6065 & 0.000005\end{array}$

$1126.8 \quad 0.1923-0.000001$

$\begin{array}{lll}1151.5 & -0.5855 & 0.000003\end{array}$

$\begin{array}{lll}1153.9 & -0.9086 & 0.000005\end{array}$

$1179.5 \quad 0.5116-0.000002$

$1221.2 \quad 0.7780-0.000003$

$\begin{array}{lll}1224.1 & -1.8599 & 0.000006\end{array}$

$\begin{array}{lll}1261.9 & -0.8799 & 0.000002\end{array}$

$\begin{array}{ll}-3.6251 & 0.000009\end{array}$

$1301.5 \quad 0.4866-0.000001$

$1340.2 \quad 0.8310-0.000002$

$1377.9 \quad 1.1105-0.000002$

$\begin{array}{lll}1414.7 & 1.2858 & -0.000002\end{array}$

$1450.7 \quad 1.3203-0.000002$

$\begin{array}{lll}1485.8 & 0.1801 & 0.000000\end{array}$

$0.0571-0.000035$

$0.0999-0.000021$

$0.1056-0.000013$

$0.1480-0.000018$

$0.2144-0.000019$

$0.2048-0.000013$

$0.2436-0.000015$

$0.2874-0.000012$

$0.3140-0.000010$

$\begin{array}{lll}1030.6 & -0.6032 & 0.000016\end{array}$

$1042.3 \quad 0.2762-0.000005$

$\begin{array}{lll}1068.2 & -0.2231 & 0.000003\end{array}$

$1073.6 \quad 0.4297-0.000005$

$\begin{array}{lll}1073.6 & -0.4567 & 0.000005\end{array}$

$1095.7 \quad-0.6613 \quad 0.000006$

$1104.2 \quad 0.5423-0.000004$

$\begin{array}{lll}1124.8 & -0.7902 & 0.000005\end{array}$

$\begin{array}{lll}1149.5 & -0.6347 & 0.000003\end{array}$

$1149.5 \quad 1.0675-0.000006$

$1151.8 \quad 0.1669-0.000001$

$1177.4 \quad 0.6337-0.000003$

$1193.3 \quad 1.5113-0.000006$

\begin{tabular}{|c|c|c|}
\hline $\begin{array}{r}\text { Density } \\
\text { Res }\end{array}$ & $\begin{array}{cc}\text { based on } & \text { based on } \\
\text { density } & \text { apparent } \\
& \text { volume }\end{array}$ & water $/ \mathrm{kg} / \mathrm{m}^{3}$ \\
\hline 0.123 & & 995.6473 \\
\hline 1.191 & & 995.6473 \\
\hline 0.194 & & 995.6473 \\
\hline 0.368 & & 995.6473 \\
\hline 0.037 & & 995.6473 \\
\hline 0.343 & & 995.6473 \\
\hline 0.826 & & 995.6473 \\
\hline 0.262 & & 995.6473 \\
\hline 0.605 & & 995.6473 \\
\hline 3.459 & & 995.6473 \\
\hline 0.774 & & 995.6473 \\
\hline 13.141 & & 995.6473 \\
\hline 0.237 & & 995.6473 \\
\hline 0.691 & & 995.6473 \\
\hline 1.233 & & 995.6473 \\
\hline 1.653 & & 995.6473 \\
\hline 1.743 & & 995.6473 \\
\hline 0.032 & & 995.6473 \\
\hline 0.003 & & 994.0319 \\
\hline 0.010 & & 994.0319 \\
\hline 0.011 & & 994.0319 \\
\hline 0.022 & & 994.0319 \\
\hline 0.046 & & 994.0319 \\
\hline 0.042 & & 994.0319 \\
\hline 0.059 & & 994.0319 \\
\hline 0.083 & & 994.0319 \\
\hline 0.099 & & 994.0319 \\
\hline 0.364 & & 994.0319 \\
\hline 0.076 & & 994.0319 \\
\hline 0.050 & & 994.0319 \\
\hline 0.185 & & 994.0319 \\
\hline 0.209 & & 994.0319 \\
\hline 0.437 & & 994.0319 \\
\hline 0.294 & & 994.0319 \\
\hline 0.624 & & 994.0319 \\
\hline 0.403 & & 994.0319 \\
\hline 1.140 & & 994.0319 \\
\hline 0.028 & & 994.0319 \\
\hline 0.402 & & 994.0319 \\
\hline 2.284 & & 994.0319 \\
\hline
\end{tabular}


molality molarity

1.48
1.49991
1.5
1.77
2
2.06
2.35
2.64
2.93
3.22
3.51
0.2266
0.4652
0.6428
0.8347
1.016
1.19
1.48
1.77
2.06
2.35
2.64
2.93
3.22
3.51
0.01024
0.03017
0.05
0.05017
0.07016
0.1
0.10075
0.15036
0.20039
0.2266
0.30046
0.4652
0.49983

0.5

0.6428 $\mathbf{t} /{ }^{\circ} \mathrm{C}$ mass frac Density Ref

Density
$\exp /$

$\mathrm{kg} / \mathrm{m}^{3}$

0.1929

0.1950

0.1950

0.2223

0.2441

0.2496

0.2751

0.2989

0.3212

0.3421

0.3617

0.0353

0.0699

0.0940

0.1188

0.1409

0.1612

0.1929

0.2223

0.2496

0.2751

0.2989

0.3212

0.3421

0.3617

0.0017

0.0048

0.0080

0.0080

0.0112

0.0159

0.0160

0.0237

0.0313

0.0353

0.0463

0.0699

0.0747

0.0747

0.0940
$1219.00 \quad$ (126)

$1223.20 \quad(101)$

$1219.91 \quad(44)$

$1259.00 \quad(126)$

$1300.00 \quad(126)$

$1339.00 \quad(126)$

$1377.00 \quad(126)$

$1414.00 \quad(126)$

$1449.00 \quad(126)$

$1484.00 \quad(126)$

$1028.00 \quad(126)$

$1066.00 \quad(126)$

$093.00 \quad(126)$

$1149.00 \quad(126)$

(126)

$217.00 \quad(126)$

$1256.00 \quad(126)$

(126)

(126)

$1410.00 \quad(126)$

$1446.00 \quad(126)$

$1481.00 \quad(126)$

991.93

995.22

998.41

998.48

1001.70

1006.55

1006.63

014.60

1022.58

1026.00

(038.32

1069.34

1068.91

1091.00
$1122.00 \quad(126)$
App vol App vol Density Density App Vol Square of Inconsistent Inconsistent Density of $\exp /$ Calc / calc/ Residual / Residual / Density based on based on water $/ \mathrm{kg} / \mathrm{m}^{3}$ $\mathrm{m}^{3} / \mathrm{kg} \quad \mathrm{m}^{3} / \mathrm{kg}$

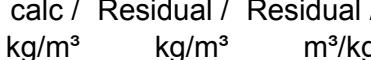
Res

$\begin{array}{cc}\text { Res density } & \begin{array}{c}\text { apparent } \\ \text { volume }\end{array} \\ 0.002 & \end{array}$

$0.000043 \quad 0.000043$ 0.0000390 .000044

$\begin{array}{lll}1219.0 & -0.0408 & 0.000000\end{array}$

1.788

3.863

0.438

$0.000053 \quad 0.000052$

$0.000067 \quad 0.000058$

$0.000057 \quad 0.000059$

$0.000064 \quad 0.000066$

$0.000070 \quad 0.000072$

$0.000076 \quad 0.000078$

$0.000083 \quad 0.000083$

$0.000088 \quad 0.000088$

$0.000014-0.000003$

$0.000009 \quad 0.000008$

$0.000020 \quad 0.000015$

$0.000026 \quad 0.000023$

$0.000032 \quad 0.000029$

$0.000035 \quad 0.000035$

$0.000043 \quad 0.000044$

$0.000056 \quad 0.000052$

0.0000590 .000059

$0.000065 \quad 0.000066$

$0.000071 \quad 0.000072$

$0.000078 \quad 0.000078$

$0.000083 \quad 0.000083$

$0.000088 \quad 0.000088$

$-0.000049-0.000013$

(101) $\quad-0.000038-0.000012$

(44) $\quad-0.000025-0.000011$

(101) $\quad-0.000031-0.000010$

(101) $\quad-0.000024-0.000009$

(44) $\quad-0.000022-0.000008$

(101) $\quad-0.000019-0.000008$

$-0.000014-0.000005$

$1221.9 \quad 1.3373-0.000005$

$\begin{array}{lll}1259.7 & -0.6617 & 0.000002\end{array}$

14.609

$\begin{array}{rrrr}1291.2 & -3.8221 & 0.000009 & 14.609 \\ 1299.3 & 0.7213 & -0.000002 & 0.520\end{array}$

$\begin{array}{llll}1337.9 & 1.0629 & -0.000002 & 1.130\end{array}$

$\begin{array}{llll}1375.7 & 1.3217 & -0.000002 & 1.747\end{array}$

$1412.5 \quad 1.4593-0.000002 \quad 2.130$

$\begin{array}{lllll}1448.6 & 0.4405 & -0.000001 & 0.194\end{array}$

$\begin{array}{llll}1483.8 & 0.2323 & 0.000000 & 0.054\end{array}$

$\begin{array}{llll}1028.7 & -0.6506 & 0.000017 & 0.423\end{array}$

$\begin{array}{llll}1066.1 & -0.1461 & 0.000002 & 0.021\end{array}$

$\begin{array}{llll}1093.5 & -0.5039 & 0.000004 & 0.254\end{array}$

$\begin{array}{llll}1122.6 & -0.5571 & 0.000004 & 0.310\end{array}$

$\begin{array}{llll}1149.5 & -0.5388 & 0.000003 & 0.290\end{array}$

$\begin{array}{llll}1175.0 & -0.0222 & 0.000000 & 0.000\end{array}$

$\begin{array}{llll}1216.6 & 0.3676 & -0.000001 & 0.135\end{array}$

$\begin{array}{llll}1257.2 & -1.2110 & 0.000003 & 1.466\end{array}$

$\begin{array}{llll}1296.8 & 0.1937 & 0.000000 & 0.038\end{array}$

$\begin{array}{llll}1335.5 & 0.5378 & -0.000001 & 0.289\end{array}$

$1373.2 \quad 0.7810 \quad-0.000001 \quad 0.610$

$\begin{array}{llll}1410.1 & -0.1138 & 0.000000 & 0.013\end{array}$

$\begin{array}{llll}1446.2 & -0.1808 & 0.000000 & 0.033\end{array}$

$\begin{array}{llll}1481.5 & -0.4518 & 0.000001 & 0.204\end{array}$

$\begin{array}{llll}991.9 & 0.0591 & -0.000036 & 0.003\end{array}$

$995.1-0.1275-0.000027$

$\begin{array}{llll}998.3 & 0.1184 & -0.000015 & 0.014\end{array}$

$\begin{array}{llll}998.3 & 0.1610 & -0.000020 & 0.026\end{array}$

$1001.5-0.1625-0.000014 \quad 0.026$

$\begin{array}{llll}1006.3 & 0.2200 & -0.000014 & 0.048\end{array}$

$\begin{array}{llll}1006.5 & 0.1797 & -0.000011 & 0.032\end{array}$

$1014.4 \quad 0.2135-0.000009 \quad 0.046$

$\begin{array}{llll}1022.4 & 0.2290 & -0.000007 & 0.052\end{array}$

$\begin{array}{llll}1026.5 & -0.5081 & 0.000014 & 0.258\end{array}$

$\begin{array}{llll}1038.2 & 0.1536 & -0.000003 & 0.024\end{array}$

$\begin{array}{llll}1063.9 & 0.1246 & -0.000002 & 0.016\end{array}$

$1069.2 \quad 0.1110-0.000001 \quad 0.012$

$\begin{array}{llll}1069.3 & -0.3453 & 0.000004 & 0.119\end{array}$

$\begin{array}{llll}1091.1 & -0.1499 & 0.000001 & 0.022\end{array}$
994.0319

994.0319

994.0319

994.0319

994.0319

994.0319

994.0319

994.0319

994.0319

994.0319

994.0319

992.2158

992.2158

992.2158

992.2158

992.2158

992.2158

992.2158

992.2158

992.2158

992.2158

992.2158

992.2158

992.2158

992.2158

990.2132

990.2132

990.2132

990.2132

990.2132

990.2132

990.2132

990.2132

990.2132

990.2132

990.2132

990.2132

990.2132

990.2132

990.2132 
molality molarity

0.8347

1

1.00012

1.016

1.19
1.30026

1.48

1.49991

1.5
1.77

2

2.06

2.35

2.64

2.93

3.22

0.2266

0.4652

0.6428

0.8347

1.016

1.19

1.48

1.77

2.06

2.35

2.64

2.93

3.22

3.51

0.01024

0.03017

0.05

0.05017

0.07016

0.1

0.10075

0.15036 $t /{ }^{\circ} \mathrm{C}$ mass frac

\section{Density}

$\exp /$

$\mathrm{kg} / \mathrm{m}^{3}$

0.1014

0.1188

0.1390

1144.15

$0.1390 \quad 1145.15$

$0.1409 \quad 1148.00$

0.1612

0.1735

0.1929

0.1950

0.1950

0.2223

0.2441

0.2496

0.2751

0.2989

0.3212

0.3421

0.3617

0.0353

0.0699

0.0940

0.1188

0.1409

0.1612

0.1929

0.2223

0.2496

0.2751

0.2989

0.3212

0.3421

0.3617

0.0017

0.0048

0.0080

0.0080

0.0112

0.0159

0.0160

0.0237
$73.00 \quad(126)$

(126)

$1218.02 \quad(101)$

$1214.87 \quad(44)$

(126)

$282.12 \quad$ (44)

$1333.00 \quad(126)$

$1371.00 \quad$ (126)

$1408.00 \quad(126)$

$1444.00 \quad(126)$

$1478.00 \quad(126)$

$1024.00 \quad(126)$

(126)

$1118.00 \quad(126)$

$1145.00 \quad$ (126)

$1170.00 \quad(126)$

$1212.00 \quad(126)$

(126)

$1330.00 \quad$ (126)

$1369.00 \quad$ (126)

$1405.00 \quad$ (126)

$1441.00 \quad(126)$

$1474.00 \quad$ (126)

987.40

990.66

993.85

997.12

1001.95

1002.04

1009.98
App vol App vol Density Density App Vol Square of Inconsistent Inconsistent Density of exp / Calc / calc/ Residual / Residual / Density based on based on water $/ \mathrm{kg} / \mathrm{m}^{3}$

$\mathrm{m}^{3} / \mathrm{kg} \quad \mathrm{m}^{3} / \mathrm{kg}$

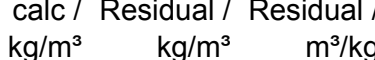

$$
\text { Density }
$$

Res

\subsection{6}

0.015

0.285

0.201

0.918

0.0000450 .000045

$0.000052 \quad 0.000045$

$0.000057 \quad 0.000053$

$0.000068 \quad 0.000058$

$0.000058 \quad 0.000060$

$0.000066 \quad 0.000066$

0.0000710 .000072

$0.000077 \quad 0.000078$

0.0000820 .000083

$0.000089 \quad 0.000087$

$0.000005 \quad 0.000000$

$0.000016 \quad 0.000011$

$0.000023 \quad 0.000018$

$0.000022 \quad 0.000025$

$0.000028 \quad 0.000031$

$\begin{array}{ll}0.000036 & 0.000037\end{array}$

$0.000042 \quad 0.000045$

$0.000055 \quad 0.000053$

$0.000058 \quad 0.000060$

$0.000066 \quad 0.000066$

$0.000070 \quad 0.000072$

$\begin{array}{lll}0.000077 & 0.000078\end{array}$

$0.000082 \quad 0.000082$

$\begin{array}{ll}0.000090 & 0.000087\end{array}$

$-0.000047-0.000009$

(101) $\quad-0.000034-0.000008$

(44) $\quad-0.000025-0.000007$

(101) $\quad-0.000029-0.000007$

(101) $-0.000023-0.000006$

(44) $\quad-0.000021-0.000004$

(101) $\quad-0.000019-0.000004$

(101) $\quad-0.000015-0.000002$
0.278

0.506

0.000

1.421

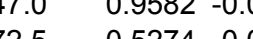

$\begin{array}{llll}1188.4 & 0.7110 & -0.000003\end{array}$

$\begin{array}{lll}1214.0 & -0.0137 & 0.000000\end{array}$

$1.1919-0.000004$

3.884

$\begin{array}{llll}1254.5 & -1.5451 & 0.000004 & 2.387\end{array}$

$\begin{array}{llll}1286.0 & -3.8840 & 0.000010 & 15.085\end{array}$

$\begin{array}{lllll}1294.1 & 0.8863 & -0.000002 & 0.785\end{array}$

$\begin{array}{llll}1332.8 & 0.2377 & 0.000000 & 0.057\end{array}$

$\begin{array}{llll}1370.5 & 0.4704 & -0.000001 & 0.221\end{array}$

$1407.5 \quad 0.5482-0.000001 \quad 0.301$

$\begin{array}{llll}1443.6 & 0.4381 & -0.000001 & 0.192\end{array}$

$\begin{array}{llll}1478.9 & -0.8908 & 0.000001 & 0.794\end{array}$

$\begin{array}{llll}1024.2 & -0.1880 & 0.000005 & 0.035\end{array}$

$\begin{array}{llll}1061.4 & -0.4237 & 0.000005 & 0.180\end{array}$

$\begin{array}{llll}1088.6 & -0.6124 & 0.000005 & 0.375\end{array}$

$\begin{array}{llll}1117.5 & 0.4947 & -0.000003 & 0.245\end{array}$

$1144.4 \quad 0.6441-0.000003 \quad 0.415$

$1169.7-0.2686-0.000001-0.072$

$\begin{array}{llll}1211.2 & 0.8011 & -0.000003 & 0.642\end{array}$

$\begin{array}{llll}1251.7 & -0.6785 & 0.000002 & 0.460\end{array}$

$1291.20 .7842-0.000002 \quad 0.615$

$\begin{array}{llll}1329.9 & 0.1479 & 0.000000 & 0.022\end{array}$

$\begin{array}{llll}1367.6 & 1.3747 & -0.000002 & 1.890\end{array}$

$\begin{array}{llll}1404.6 & 0.4300 & -0.000001 & 0.185\end{array}$

$\begin{array}{llll}1440.7 & 0.2816 & 0.000000 & 0.079\end{array}$

$\begin{array}{llll}1476.1 & -2.1004 & 0.000003 & 4.412\end{array}$

$987.3 \quad 0.0609-0.000038 \quad 0.004$

$\begin{array}{llll}990.5 & 0.1261 & -0.000027 & 0.016\end{array}$

$\begin{array}{llll}993.7 & 0.1433 & -0.000018 & 0.021\end{array}$

$\begin{array}{llll}993.7 & 0.1762 & -0.000022 & 0.031\end{array}$

$\begin{array}{llll}996.9 & 0.1939 & -0.000017 & 0.038\end{array}$

$\begin{array}{llll}1001.7 & 0.2700 & -0.000017 & 0.073\end{array}$

$\begin{array}{llll}1001.8 & 0.2407 & -0.000015 & 0.058\end{array}$

$\begin{array}{llll}1009.7 & 0.3074 & -0.000013 & 0.094\end{array}$
990.2132

990.2132

990.2132

990.2132

990.2132

990.2132

990.2132

990.2132

990.2132

990.2132

990.2132

990.2132

990.2132

990.2132

990.2132

990.2132

990.2132

990.2132

988.0363

988.0363

988.0363

988.0363

988.0363

988.0363

988.0363

988.0363

988.0363

988.0363

988.0363

988.0363

988.0363

988.0363

985.6952

985.6952

985.6952

985.6952

985.6952

985.6952

985.6952

985.6952 
molality molarity

0.20039

0.2266

0.30046

0.4652

0.49983

0.5
0.6428

0.69894

0.8347

1
0

1.00012

1.19

1.30026

1.48

1.49991

1.5
1.77

2

2.06

2.35

2.64

2.93

3.22

3.51

0.2266

0.6428

0.8347

1.016

1.19

1.48
1.77

2.06

2.35

2.64

2.93

3.22

3.51

\section{$t /{ }^{\circ} \mathrm{C}$ mass frac}

Density

$\exp /$
$\mathrm{kg} / \mathrm{m}^{3}$

0.0313

0.0353

0.0463

0.0699

0.0747

0.0747

0.0940

0.1014

0.1188

0.1390

0.1390

0.1409

0.1612

0.1735

0.1929

0.1950

0.1950

0.2223

0.2441

0.2496

0.2751

0.2989

0.3212

0.3421

0.3617

0.0353

0.0699

0.0940

0.1188

0.1409

0.1612

0.1929

0.2223

0.2496

0.2751

0.2989

0.3212

0.3421

0.3617
1017.72

1022.00

(101)

$1059.00 \quad(126)$

064.20

1064.03

1086.00

(1)

1114.00

1138.91
1139.77

1141.00

1168.00

1183.93

1209.00

1212.49

1209.53

1248.00

1276.39

(1)

(327.00

1403.00

1437.00

1472.00

1020.00

1056.00

1082.00

$1111.00 \quad$ (126)

$1139.00 \quad(126)$

$1163.00 \quad(126)$

$1205.00 \quad(126)$

$1245.00 \quad(126)$

$1286.00 \quad(126)$

$1324.00 \quad(126)$

$1362.00 \quad(126)$

$1399.00 \quad(126)$

$1434.00 \quad(126)$
(126)
App vol App vol Density Density App Vol Square of Inconsistent Inconsistent Density of $\exp /$ Calc / calc / Residual / Residual / Density based on based on water $/ \mathrm{kg} / \mathrm{m}^{3}$

$\mathrm{m}^{3} / \mathrm{kg} \quad \mathrm{m}^{3} / \mathrm{kg} \quad \mathrm{kg} / \mathrm{m}^{3} \quad \mathrm{~kg} / \mathrm{m}^{3} \quad \mathrm{~m}^{3} / \mathrm{kg} \quad$ Res density apparent

$-0.000004 \quad 0.0000$ $-0.000006 \quad 0.000002$

$0.000004 \quad 0.000005$

$0.000009 \quad 0.000012$

0.0000120 .000013

$0.000015 \quad 0.000013$

$0.000018 \quad 0.000019$

$0.000016 \quad 0.000021$

$0.000031 \quad 0.000026$

$0.000033 \quad 0.000031$

$0.000028 \quad 0.000031$

$0.000035 \quad 0.000032$

$0.000032 \quad 0.000037$

$0.000036 \quad 0.000041$

$0.000043 \quad 0.000046$

0.0000410 .000046

$0.000052 \quad 0.000046$

$0.000055 \quad 0.000053$

$0.000068 \quad 0.000059$

$0.000058 \quad 0.000060$

$0.000066 \quad 0.000066$

$0.000069 \quad 0.000072$

$\begin{array}{ll}0.000075 & 0.000077\end{array}$

$0.000083 \quad 0.000082$

$\begin{array}{ll}0.000088 & 0.000087\end{array}$

$\begin{array}{ll}-0.000023 & 0.000003\end{array}$

$0.000013 \quad 0.000013$

$0.000029 \quad 0.000020$

$0.000032 \quad 0.000027$

$0.000030 \quad 0.000033$

$0.000042 \quad 0.000038$

$0.000046 \quad 0.000046$

$0.000055 \quad 0.000054$

$0.000058 \quad 0.000060$

0.0000650 .000066

$0.000071 \quad 0.000072$

$0.000076 \quad 0.000077$

$0.000082 \quad 0.000082$

$0.000087 \quad 0.000086$

Average Res

$0.2990-0.000008$

0.021

$\begin{array}{lll}1033.7 & 0.2990-0.000008 & 0.089\end{array}$

$\begin{array}{lllll}1058.8 & 0.1979 & -0.000003 & 0.039\end{array}$

$1064.1 \quad 0.0798-0.000001 \quad 0.006$

$\begin{array}{llll}1064.1 & -0.1163 & 0.000001 & 0.014\end{array}$

$1085.9 \quad 0.0974-0.000001 \quad 0.009$

$\begin{array}{llll}1094.4 & 0.5593 & -0.000005 & 0.313\end{array}$

$\begin{array}{llll}1114.7 & -0.7113 & 0.000005 & 0.506\end{array}$

$\begin{array}{llll}1139.1 & -0.2356 & 0.000001 & 0.056\end{array}$

$\begin{array}{llll}1139.2 & 0.6067 & -0.000003 & 0.368\end{array}$

0.243

1.416

1.604

0.640

2.204

2.215

0.388

13.218

0.766

0.065

2.194

2.307

0.440

1.195

0.891

0.000

1.061

0.566

0.290

0.517

0.001

0.152

1.320

0.303

0.608

0.646

0.166

0.776

210.878 Jum Chi sqr
985.6952

985.6952

985.6952

985.6952

985.6952

985.6952

985.6952

985.6952

985.6952

985.6952

985.6952

985.6952

985.6952

985.6952

985.6952

985.6952

985.6952

985.6952

985.6952

985.6952

985.6952

985.6952

985.6952

985.6952

985.6952

983.1989

983.1989

983.1989

983.1989

983.1989

983.1989

983.1989

983.1989

983.1989

983.1989

983.1989

983.1989

983.1989

983.1989 
molality molarity

$\mathrm{t} /{ }^{\circ} \mathrm{C}$ mass frac Density Ref $\exp /$ $\exp /$
$\mathrm{kg} / \mathrm{m}^{3}$
App vol
$\exp /$
$\mathrm{m}^{3} / \mathrm{kg}$
App vol

Calc /
$\mathrm{m}^{3} / \mathrm{kg}$
Dens

Density App Vol Square of Inconsistent Inconsistent Density of calc / Residual / Residual / Density based on calc/ Residual / Residua / $\mathrm{kg} / \mathrm{m}^{3} \quad \mathrm{~m}^{3} / \mathrm{kg}$ Res

$\begin{array}{rrr}\text { Std dev Res } & 0.9653 & 0.000010 \\ \text { Avg - 4std } & -3.8263 & -0.000044 \\ \text { Avg + 4std } & 3.8963 & 0.000036\end{array}$

Curve fit for the model App vol $=\left(w+c 2+c 3^{\star} t{ }^{\circ} \mathrm{C}\right) /\left(\mathrm{c} 0^{*} w+c 1\right) / e^{\wedge}\left(0.000001^{*}\left(t^{\circ} \mathrm{C}+c 4\right)^{\wedge} 2\right)$

\begin{tabular}{lr} 
c0 & 18.37777 \\
c1 & 35.92677 \\
c2 & -0.089193 \\
c3 & 0.001077 \\
c4 & 2066.297 \\
\hline
\end{tabular}

Min

$\operatorname{Max} T$

Min w

Max w

Avg dens res

Std dens res

res $10^{\wedge} 3-0.004011$

Std app vol res *10^3 0.009897

No of points in corr

227

No of inconsistent poir

Inconsistent data not used (Residual greater than average $+/ \mathbf{4}$ standard deviations)

$\begin{array}{rrrrrr}25 & 0.0050 & 1001.10 & (7) & 0.000189 & -0.000020 \\ 25 & 0.0200 & 1014.80 & (7) & 0.000123 & -0.000015 \\ 25 & 0.0899 & 1075.30 & (7) & 0.000191 & 0.000009 \\ 25 & 0.1098 & 1093.10 & (7) & 0.000200 & 0.000016 \\ 25 & 0.1498 & 1134.50 & (7) & 0.000192 & 0.000029 \\ 25 & 0.1789 & 1173.60 & (7) & 0.000160 & 0.000038 \\ 25 & 0.2098 & 1191.20 & (7) & 0.000224 & 0.000047 \\ 25 & 0.2298 & 1216.20 & (7) & 0.000217 & 0.000053 \\ 25 & 0.2499 & 1237.70 & (7) & 0.000223 & 0.000058 \\ 25 & 0.2699 & 1251.20 & (7) & 0.000248 & 0.000064 \\ 25 & 0.2994 & 1293.90 & (7) & 0.000234 & 0.000072 \\ 50 & 0.0200 & 1004.90 & (7) & 0.000161 & -0.000005 \\ 50 & 0.0050 & 992.60 & (7) & 0.000080 & -0.000010 \\ 50 & 0.0899 & 1066.90 & (7) & 0.000179 & 0.000016 \\ 50 & 0.1098 & 1086.50 & (7) & 0.000177 & 0.000022 \\ 50 & 0.1498 & 1123.20 & (7) & 0.000199 & 0.000034 \\ 50 & 0.1789 & 1151.60 & (7) & 0.000209 & 0.000042 \\ 50 & 0.2098 & 1180.30 & (7) & 0.000226 & 0.000050\end{array}$

$1002.1 \quad-1.0455 \quad 0.000209$

$\begin{array}{llll}1017.6 & -2.8444 & 0.000138\end{array}$

$\begin{array}{lll}1094.5 & -19.1761 & 0.000181\end{array}$

$\begin{array}{lll}1117.9 & -24.7522 & 0.000184\end{array}$

$\begin{array}{lll}1166.9 & -32.3948 & 0.000163\end{array}$

$\begin{array}{llll}1204.5 & -30.8947 & 0.000122\end{array}$

$\begin{array}{lll}1246.4 & -55.1512 & 0.000177\end{array}$

$\begin{array}{lll}1274.6 & -58.4384 & 0.000164\end{array}$

$\begin{array}{llll}1303.9 & -66.2003 & 0.000164\end{array}$

$\begin{array}{lll}1334.2 & -82.9763 & 0.000184 \\ 1380.7 & -86.7951 & 0.000162\end{array}$

$\begin{array}{lll}1380.7 & -86.7951 & 0.000162\end{array}$

$\begin{array}{lll}1008.2 & -3.3464 & 0.000166\end{array}$

$\begin{array}{lll}993.0 & -0.4384 & 0.000089\end{array}$

$\begin{array}{lll}1083.8 & -16.9347 & 0.000163\end{array}$

$\begin{array}{llll}1106.9 & -20.3994 & 0.000154\end{array}$

$\begin{array}{llll}1155.4 & -32.1924 & 0.000166\end{array}$

$\begin{array}{llll}1192.7 & -41.0675 & 0.000167\end{array}$

$\begin{array}{lllll}1234.3 & -53.9624 & 0.000177 & 2911.9\end{array}$
997.0449

997.0449

997.0449

997.0449

997.0449

997.0449

997.0449

997.0449

997.0449

997.0449

997.0449

988.0363

988.0363

988.0363

988.0363

988.0363

988.0363

988.0363 
molality molarity

$\mathrm{t} /{ }^{\circ} \mathrm{C}$ mass frac Density

$\exp /$

$\mathrm{kg} / \mathrm{m}^{3}$

0.2298

0.2499

0.2699

0.2994

0.0200

0.0050

0.0899

0.1098

0.1498

0.1789

0.2098

0.2298

0.2499

0.2699
Ref

$\begin{array}{rr}\text { App vol } & \text { App vol } \\ \exp / & \text { Calc / }\end{array}$

$\mathrm{m}^{3} / \mathrm{kg} \quad \mathrm{m}^{3} / \mathrm{kg}$

0.0002190 .000055

$0.000223 \quad 0.000060$

$\begin{array}{ll}0.000231 & 0.000065\end{array}$

0.0002390 .000072

$0.000132 \quad 0.000003$

$-0.000430-0.000001$

$0.000168 \quad 0.000022$

$0.000129 \quad 0.000027$

$\begin{array}{lll}0.000194 & 0.000037\end{array}$

$0.000206 \quad 0.000044$

$0.000224 \quad 0.000052$

$0.000215 \quad 0.000056$

$0.000194 \quad 0.000061$

$0.000234 \quad 0.000065$

$0.000234 \quad 0.000072$
Density Density App Vol Square of Inconsistent Inconsistent Density of calc / Residual / Residual / Density based on based on water $/ \mathrm{kg} / \mathrm{m}^{3}$ $\mathrm{kg} / \mathrm{m}^{3} \quad \mathrm{~kg} / \mathrm{m}^{3} \quad \mathrm{~m}^{3} / \mathrm{kg} \quad$ Res density apparent

$\begin{array}{llll}1262.4 & -57.4332 & 0.000164 & 3298.567\end{array}$

$\begin{array}{llll}1291.6 & -64.5262 & 0.000163 & 4163.632\end{array}$

$\begin{array}{lllll}1321.9 & -73.7867 & 0.000166 & 5444.471\end{array}$

$\begin{array}{llll}1368.5 & -87.2926 & 0.000166 & 7619.999\end{array}$

$\begin{array}{llll}994.6 & -2.5290 & 0.000128 & 6.396\end{array}$

$\begin{array}{llll}979.7 & 2.0575 & -0.000429 & 4.233\end{array}$

$\begin{array}{llll}1068.8 & -14.8114 & 0.000146 & 219.378\end{array}$

$\begin{array}{llll}091.5 & -13.2115 & 0.000102 & 174.545\end{array}$

$\begin{array}{llll}139.3 & -29.6374 & 0.000156 & 878.378\end{array}$

$1176.2 \quad-38.7920 \quad 0.000162 \quad 1504.820$

$1217.4 \quad-51.4129 \quad 0.000173 \quad 2643.291$

$1245.4 \quad-54.0878 \quad 0.000159 \quad 2925.491$

$\begin{array}{llll}1274.4 & -51.9270 & 0.000133 & 2696.413\end{array}$

$\begin{array}{llll}1304.6 & -72.9809 & 0.000168 & 5326.213\end{array}$

$\begin{array}{llll}1351.1 & -83.0286 & 0.000162 & 6893.756\end{array}$
988.0363

988.0363

988.0363

988.0363

974.849

974.849

974.849

974.849

974.849

974.849

974.849

974.849

974.849

974.849

974.849 